KOMMUNIKATION - PARTIZIPATION INKLUSION

\title{
Handbuch \\ Barrierefreie Kommunikation
}

Christiane Maaß/Isabel Rink (Hg.)

TFrank \& Timme 
Christiane Maaß/Isabel Rink (Hg.) Handbuch Barrierefreie Kommunikation 
Bettina M. Bock/Ulla Fix/Daisy Lange (Hg.)

Kommunikation - Partizipation - Inklusion

Band 3 
Christiane Maaß/Isabel Rink (Hg.)

\section{Handbuch Barrierefreie Kommunikation}

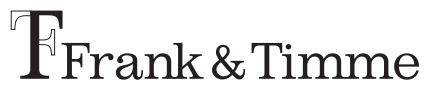

Verlag für wissenschaftliche Literatur 


\section{(c) (1) () $\Theta$}

BY NC ND CC-BY-NC-ND

ISBN 978-3-7329-9235-5

ISSN 2509-3215

DOI $10.26530 / 20.500 .12657 / 43216$

(C) Frank \& Timme GmbH Verlag für wissenschaftliche Literatur Berlin 2020. Alle Rechte vorbehalten.

Das Werk einschließlich aller Teile ist urheberrechtlich geschützt. Jede Verwertung außerhalb der engen Grenzen des Urheberrechtsgesetzes ist ohne Zustimmung des Verlags unzulässig und strafbar. Das gilt insbesondere für Vervielfältigungen, Übersetzungen, Mikroverfilmungen und die Einspeicherung und Verarbeitung in elektronischen Systemen.

Herstellung durch Frank \& Timme GmbH, Wittelsbacherstraße 27a, 10707 Berlin.

Printed in Germany.

Gedruckt auf säurefreiem, alterungsbeständigem Papier.

www.frank-timme.de 
Inhaltsverzeichnis

EINLEITUNG UND ÜBERBLICK

VOLKER SIEGER

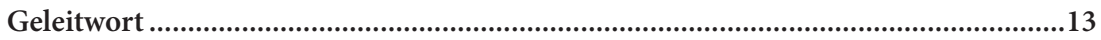

CHRISTIANE MAAß/ISABEL RINK

Über das Handbuch Barrierefreie Kommunikation. .17

\section{ANFORDERUNGEN AN WAHRNEHMBARKEIT UND VERSTÄNDLICHKEIT}

ISABEL RINK

Kommunikationsbarrieren .29

KATRIN LANG

Die rechtliche Lage zu Barrierefreier Kommunikation in Deutschland .67

KeRstin AleXANDER

Barrierefreies Grafikdesign . .95

Ursula Christmann/Norbert Groeben

Verständlichkeit: die psychologische Perspektive.

BENEDIKT LUTZ

Verständlichkeit aus fachkommunikativer Sicht 147

Silvia Hansen-Schirra/Silke Gutermuth

Empirische Überprüfung von Verständlichkeit. 163 
Julia DobroschKe/Thomas KaHLISCH

Barrierefreie Informations- und Kommunikationsangebote für blinde

und sehbehinderte Menschen

JOHANNES HENNIES

Prälinguale Hörbehinderung und Schriftsprachkompetenz 201

Saskia SChuppener/BetTina M. Bock

Geistige Behinderung und barrierefreie Kommunikation .221

\section{FORMEN DER BARRIEREFREIEN KOMMUNIKATION}

URSUla BRedel/Christiane MAaß

Leichte Sprache 251

CHRISTIANE MAAß

Übersetzen in Leichte Sprache 273

JUTTA WiTZEL

Ausprägungen und Dolmetschstrategien beim Schriftdolmetschen 303

NATHalie MäLZER/MARIA WÜNSCHE

Untertitelung für Hörgeschädigte (SDH)

KRISTIAN FOLTA-SCHOOFS

Apparategestützte Kommunikations- und Feedbacksysteme. 345

Oliver Musenberg

Unterstützte Kommunikation 361

UTA BENNER/ANNIKA HERRMANN

Gebärdensprachdolmetschen 381 
Conchita Otero Moreno

Community Interpreting zur Überwindung von

Kommunikationsbarrieren für Personen mit Deutsch als Zweitsprache:

Einsatzbereiche, Anforderungen, Professionalisierung 403

\section{MEDiAle Bereiche Und TEXTSORTEN}

AleXAnder Kurch

Produktionsprozesse der Hörgeschädigten-Untertitelungen und

Audiodeskription: Potenziale teilautomatisierter Prozessbeschleunigung

mittels (Sprach-)Technologien

BERND BENECKE

Audiodeskription - Methoden und Techniken der Filmbeschreibung 455

NATHALIE MÄlZER/MARIA WÜNSCHE

Audioeinführungen

NICOLA PRIDIK

Visualisierung rechtlicher Inhalte in Leichte-Sprache-Texten

Jan Hellbusch

Mit barrierefreiem Webdesign zu einer besseren User Experience .507

CHRISTA WOMSER-HACKER

Barrierefreie Mensch-Maschine-Interaktion

MARIE-LUISE SCHÜTT

Alternativtexte als wesentliches Gestaltungselement zugänglicher

(barrierefreier) Bildungsprozesse.

Dino CAPOVILla

Technologiegestützte Kommunikation bei Beeinträchtigungen des Sehens 565 
Inhaltsverzeichnis

CHRISTIANE ZEHRER

Schreib- und Übersetzungswerkzeuge

für die Barrierefreie Kommunikation 583

NATHAlie MÄlZER/MARIA WÜNSCHE

Barrierefreiheit und Inklusion am Theater:

Das Projekt Inklusives Theater an der Uni Hildesheim 599

EEva Rantamo/SwENJa SCHum

Museumstexte -

Zum Abbau sprachlicher Barrieren in Museen und Ausstellungen 615

JOHANNES SCHÄDLER

Barrierefreie Verwaltung - Grundlagen und Handlungskonzepte .637

THOMAS M. SCHINDLER

Ansätze für barrierefreie Kommunikation

im medizinisch-pharmazeutischen Bereich 657

ANTJE BAUMANN

Rechtstexte als Barrieren -

Einige Merkmale der Textsorte ,Gesetz` und die Verständlichkeit. 679

FRANZISKA HEIDRICH

Fachexterne technische Kommunikation als Barriere 703

\section{STIMMEN AUS DER PRAXIS}

USCHI HEERDEGEN-WESSEL

Barrierefreie Angebote des NDR und der ARD - Stand, Aufgaben, Ziele .725

LAURA M. SCHWENGBER

Barrierefreiheit bei Kulturevents und Live-Veranstaltungen .741 
DANA APEL/JÖRG APEL

„Die Gesellschaft soll uns so akzeptieren, wie wir sind“

THOMAS WidMAYER

„Da muss noch viel passieren, aber wir sind auf einem guten Weg!“

Cornelia Plagge

„Das barrierefreie Angebot ist immer noch zu gering“

JANNA HINRICHS

Auf dem Weg zur Selbstvertretung von Menschen mit

Beeinträchtigungen in der Gesellschaft

STEFANIE SCHRUHL

Audiodeskription in der Praxis -

Bericht einer blinden Rezipientin und Hörfilmautorin .771

RUDOLF SCHEPS

„Die Bürokratie im Behindertenrecht muss aufgelockert werden.“ 781

OXANA Kulikova/GÖKHAN ŞILfELER

Mündliche Einfache Sprache im DaZ-Unterricht. 785

Über die Beiträgerinnen und Beiträger . .791 

Einleitung und Überblick 



\section{Geleitwort}

Liebe Leserin, lieber Leser,

als ich im Herbst 2017 gefragt wurde, ob die Bundesfachstelle Barrierefreiheit ein Geleitwort zum Handbuch Barrierefreie Kommunikation schreiben würde, das zum Beginn des gleichnamigen Master-Studiengangs an der Universität Hildesheim erscheinen wird, habe ich schnell zugesagt. Ich gebe zu, da schwang auch ein wenig Egoismus mit, denn die Bundesfachstelle Barrierefreiheit ist zwar älter als der Master-Studiengang, aber seit ihrer Gründung im Juli 2016 mit 2 Jahren immer noch so jung, dass ihr ein höherer Bekanntheitsgrad nicht schaden kann.

Vor allem aber habe ich zugesagt, weil das vorliegende Handbuch in mehrfacher Hinsicht einen wichtigen Beitrag liefert - in Bezug auf den behinderungsübergreifenden Ansatz, in Bezug auf die Verbreitung des Themas Barrierefreiheit und schließlich in Bezug auf dessen Professionalisierung.

Barrierefreiheit ist ein anspruchsvolles Konzept, das unterschiedlichste und sich zum Teil sogar widersprechende Anforderungen überein bringen muss. Das Handbuch wird dieser Komplexität gerecht, indem es Barrierefreie Kommunikation aus der Perspektive vieler und ganz unterschiedlicher Beeinträchtigungsformen diskutiert.

Barrierefreiheit gelingt am Ende nur, wenn sie in allen Teilen der Gesellschaft selbstverständlich gelebt wird; eine Aufgabe, die kaum größer sein könnte. Die Gleichberechtigung von Menschen mit Behinderungen - und nicht ihre wohlmeinende, aber häufig bevormundende fürsorgliche Behandlung steht seit rund 20 Jahren auf der politischen Agenda. Ausgangspunkt war der Wille zu einem echten Paradigmenwechsel. Dieser muss sich auch im Diskurs über das Thema Barrierefreiheit sowie in der gelebten Praxis widerspiegeln. Denn ohne Barrierefreiheit ist eine gleichberechtigte Teilhabe undenkbar. 
Jeder Paradigmenwechsel aber bleibt ohne Verbreitung bloße Theorie. Für eine solche Verbreitung sind Ausbildungs- und Studiengänge ideale Orte. Der Master-Studiengang Barrierefreie Kommunikation ist der erste seiner Art und kann damit Vorbild sein - auch für andere Fachdisziplinen, die den Ruf, Barrierefreiheit zum verpflichtenden Ausbildungsinhalt zu machen, seit Jahren überhören und damit den anspruchsvollen Charakter der Barrierefreiheit unterschätzen.

Eine erfolgreiche Verbreitung verlangt aber auch eine hohe Qualität der tatsächlich umgesetzten Barrierefreiheit und damit auch eine Professionalisierung. Nur eine hohe Qualität wird auf Dauer die für die Umsetzung verantwortlichen Personen überzeugen. Auch in dieser Hinsicht sind Ausbildungs- und Studiengänge ideale Orte.

Um einen möglichen Einwand gegen eine Professionalisierung zu entkräften: sie ist aus unserer Sicht kein Gegensatz zur Einbindung von Menschen, die auf Barrierefreiheit angewiesen sind, in erster Linie der Menschen mit den unterschiedlichsten Beeinträchtigungen. Barrierefreiheit braucht am Ende beides: eine hohe Fachlichkeit und eine Einbindung der Zielgruppen. Wer nicht Gefahr laufen will, die Aufgabe zu verfehlen, wird sich bei den Menschen als Expertinnen und Experten in eigener Sache vergewissern wollen, ob wissenschaftlich begründete Ergebnisse praxistauglich sind. In anderen Zusammenhängen heißt das etwas nüchterner: die Perspektive der Nutzenden zu berücksichtigen. Das Handbuch zeigt mit Beiträgen zur empirischen Verständlichkeitsforschung eine - wenn auch nicht die einzige - Möglichkeit einer solchen Einbindung auf.

Dass es unter den Menschen mit Beeinträchtigungen eine Vielzahl hochqualifizierter und erfahrener Personen gibt, die schon seit Jahren an der Professionalisierung der Barrierefreiheit arbeiten, belegt gerade auch die Liste der Autorinnen und Autoren des Handbuchs. Aber auch wenn jemand beide Rollen wahrnehmen kann, gilt es auseinanderzuhalten, welche Rolle sie oder er gerade einnimmt.

Wenn ich Sie mit diesen wenigen Worten für die herausfordernde, aber auch anspornende Aufgabe Barrierefreier Kommunikation motiviert haben sollte, freut mich das. Auch die Bundesfachstelle Barrierefreiheit steht jeden Tag vor der Aufgabe, die Träger öffentlicher Gewalt sowie Wirtschaft und Gesellschaft 
durch beratende Unterstützung zu motivieren, das Thema Barrierefreiheit als großes gesamtgesellschaftliches Thema anzunehmen.

Ich wünsche dem Handbuch viele engagierte und kritische Leserinnen und Leser, dem Studiengang lern- und wissbegierige Studierende und uns allen den Willen, aber auch das Durchsetzungsvermögen und den Spaß, die Welt barrierefrei zu machen. Ich bin mir sicher, das vorliegende Handbuch wird seinen Beitrag dazu leisten.

Dr. Volker Sieger

Leiter der Bundesfachstelle Barrierefreiheit 



\section{Über das Handbuch Barrierefreie Kommunikation}

Kommunikative Barrierefreiheit hat Konjunktur: Das Angebot an Texten in Leichter und in Einfacher Sprache, Informationen in Gebärdensprache - die via QR-Code oder mittels Fernverdolmetschung abgerufen werden können - Schriftverdolmetschung, Community Interpreting oder die Auswahl an Filmen und Serien mit Untertiteln und/oder Hörfassung, kurz: das Angebot an Barrierefreier Kommunikation ist gegenwärtig umfangreicher als je zuvor. Entsprechend ist Barrierefreie Kommunikation in den vergangenen Jahren zunehmend in den Fokus gerückt. Es zeichnet sich ab, dass der Bedarf an professionellen Kommunikationsangeboten, die der Diversität und Pluralität unserer sich immer weiter ausdifferenzierenden Informationsgesellschaft Rechnung tragen, weiter steigen wird.

Aspekte der Wahrnehmbarkeit und Verständlichkeit von Kommunikation sind seit Jahrzehnten Gegenstand der Forschung. Gegenwärtig ist jedoch zu beobachten, dass sich die wissenschaftliche Auseinandersetzung mit Barrierefreier Kommunikation als eigener Forschungsbereich etabliert. Dies wird in einem erhöhten Publikationsvolumen in diesem Feld deutlich, zeigt sich aber auch in der beginnenden Vernetzung vormals getrennter disziplinärer Ansätze.

Ein wichtiger Impulsgeber hierfür war die Verabschiedung der UN-Behindertenrechtskonvention (UN-BRK) und ihre Ratifizierung in zahlreichen Ländern weltweit. Die UN-Behindertenrechtskonvention hat jedoch auch weitreichende Auswirkungen auf die deutsche Gesetzgebung gehabt. Das Behindertengleichstellungsgesetz wurde novelliert (BGG, Juni 2016), weitere Veränderungen sind geplant. Außerdem wurde mit dem Bundesteilhabegesetz (BTHG, Dezember 2016) eine Vorlage für den Eintrag des Themas in die unterschiedlichsten Rechtsbereiche geschaffen. Auf dieser Grundlage etabliert sich im Moment ein expandierender Markt für barrierefreie Kommunikationsangebote. Dieser Markt ist teilweise ungeregelt und intuitiv, zur Verfügung stehende 
Expertise wird nicht in allen Bereichen umfassend genutzt. Die Kommunikationsangebote sind folglich von heterogener Qualität. Bei den ausschreibenden Stellen, die teilweise bereits gesetzlich dazu verpflichtet sind, barrierefreie Kommunikationsangebote vorzuhalten, bestehen $u$. a. deshalb viele Unsicherheiten.

Hier situiert sich das vorliegende Handbuch. Es stellt sich einerseits das Ziel, die Forschung zur Barrierefreien Kommunikation aus den unterschiedlichen Disziplinen zusammenzutragen und ihren gegenwärtigen Stand sowie bestehende Desiderate sichtbar zu machen. Es leistet damit einen Beitrag zur interdisziplinären Vernetzung im eben skizzierten Sinne und entsprechend auch zu einer Konsolidierung der Barrierefreien Kommunikation als Forschungsdisziplin. Auf der anderen Seite ist dieses Handbuch auch Grundlage für professionelles Handeln am Markt, indem neben theoretischem auch anwendungsorientiertes Wissen zugänglich gemacht wird. Der Band enthält deshalb auch Beiträge von Praktiker(inne)n, die ihr professionelles Handeln reflektieren, sowie von Nutzer(inne)n Barrierefreier Kommunikation, die ihren Umgang mit den Kommunikaten erläutern und Bedarfe und Dysfunktionalitäten aufzeigen. Zwischen Theorie und Praxis liegt das Feld der akademischen Ausbildung zukünftiger Marktakteure. Hier etablieren sich momentan erste Studiengänge und Ausbildungsformate, für die das vorliegende Handbuch einen Überblick bietet.

\section{Barrierefreie Kommunikation: Eine Gegenstandsbestimmung}

Das Behindertengleichstellungsgesetz des Bundes definiert den Begriff „barrierefrei“ wie folgt:

Barrierefrei sind bauliche und sonstige Anlagen, Verkehrsmittel, technische Gebrauchsgegenstände, Systeme der Informationsverarbeitung, akustische und visuelle Informationsquellen und Kommunikationseinrichtungen sowie andere gestaltete Lebensbereiche, wenn sie für Menschen mit Behinderungen in der allgemein üblichen Weise, ohne besondere Erschwernis und grundsätzlich ohne fremde Hilfe auffindbar, zugänglich und nutzbar sind. Hierbei ist die Nutzung behinderungsbedingt notwendiger Hilfsmittel zulässig. (BGG $2016 \$ 4$ ) 
Als zentral sind hier die Aspekte der Teilhabe und der eigenständigen Nutzung gesetzt. Ausbuchstabiert wird der Begriff „barrierefrei“ über die Adjektive „auffindbar“, „zugänglich“ und „nutzbar“. Es wird die Möglichkeit eröffnet, dass „behinderungsbedingt notwendige [...] Hilfsmittel“ zum Einsatz kommen können, es erfolgt also eine Positionierung im Spannungsfeld zwischen Universal Design und assistiven Technologien. Gemäß der UN-BRK ist Universal Design grundsätzlich zu bevorzugen, d. h. die Welt soll insgesamt inklusiver und barriereärmer werden sowie Menschen mit unterschiedlichen Voraussetzungen $\mathrm{Zu}$ gang gewähren. Das ist aber nicht immer möglich; bisweilen müssen Behinderungen über assistive Technologien ausgeglichen werden, um Barrierefreiheit zu ermöglichen, weshalb sich mehrere Beiträge im Handbuch diesem Thema widmen.

Vergleichsweise vage bleibt im BGG der Aspekt des Zugangs „ohne besondere Erschwernis“, der sich potentiell auf sehr unterschiedliche Dimensionen beziehen kann: mit Blick auf körperliche oder geistige Anstrengung, aber auch bezogen auf finanziellen Aufwand bzw. eine ganz allgemeine Zugriffsfähigkeit. Diese Vagheit macht eine Einforderung von Ansprüchen nicht leicht. Entsprechend ist die tatsächliche Erlangung angemessener barrierefreier Kommunikationsangebote für Personen mit Behinderungen in der Praxis häufig alles andere als einfach.

Auf den ersten Blick konkret sind dagegen die Ausführungen in der UNBehindertenrechtskonvention:

Kommunikation [schließt] Sprachen, Textdarstellung, Brailleschrift, taktile Kommunikation, Großdruck, leicht zugängliches Multimedia sowie schriftliche, auditive, in einfache Sprache übersetzte, durch Vorleser zugänglich gemachte sowie ergänzende und alternative Formen, Mittel und Formate der Kommunikation, einschließlich leicht zugänglicher Informations- und Kommunikationstechnologie, ein. (UN-BRK 2008, Artikel 2: Begriffsbestimmungen)

Die UN-Behindertenrechtskonvention korreliert jedoch nicht mit einer tatsächlichen Rechtslage für in Deutschland lebende Personen mit Behinderungen. Sie ist vielmehr eine moralische Setzung im Sinne eines gesellschaftlichen Ziels und 
nicht direkt in konkrete Rechtsansprüche umzumünzen. Hinzu kommt, dass es sich bei der Aufzählung im Zitat um eine recht heterogene und repetitive Liste handelt, die nicht ebenengleiche Begrifflichkeit listet:

- Brailleschrift gehört zu den taktilen Formen der Kommunikation.

- Großdruck ist Teil der Textdarstellung.

- Leicht zugängliches Multimedia umfasst auditive Formen und basiert auf Informations- und Kommunikationstechnologie.

- „Durch Vorleser zugänglich gemacht“ ist ebenfalls identisch mit „auditiv“.

- Der Begriff „Sprache“ ist wiederum in seiner Generizität Oberbegriff für eine ganze Reihe der folgenden Aufzählungsglieder.

Die Ausführungen in der UN-BRK weisen folglich begriffliche Unschärfen auf und sind allenfalls ein Ausgangspunkt für Forschung und Praxis.

Eine weitere Präzisierung des Gegenstands tut deshalb not. Die Beiträge im vorliegenden Handbuch subsummierend kann Barrierefreie Kommunikation wie folgt bestimmt werden:

Barrierefreie Kommunikation umfasst alle Maßnahmen zur Eindämmung von Kommunikationsbarrieren in unterschiedlichen situationalen Handlungsfeldern. Kommunikationsbarrieren können mit Blick auf die Sinnesorgane und/oder die kognitiven Voraussetzungen der Kommunikationsteilnehmer(innen) bestehen sowie mit Blick auf die sprachlichen, fachsprachlichen, fachlichen, kulturellen und medialen Anforderungen, die Texte an die Rezipient(inn)en stellen. Kommunikationsbarrieren entstehen immer dann, wenn Kommunikationsangebote nicht in der erforderlichen Weise an die Zielsituation und die intendierte Adressatenschaft angepasst sind. 
Im weitesten Sinne kann jede Form von Interaktion durch Barrieren behindert werden, z. B. zwischen Expert(inn)en und fachlichen Laien ohne kommunikative Beeinträchtigungen. Andere Forschungsansätze fassen den Begriff der Barrierefreien Kommunikation enger und begrenzen ihn auf eine Rezipientenschaft, die durch Behinderung, divergierende Bildungschancen bzw. eingreifende Lebensereignisse für die eigenständige Partizipation an der Gesellschaft einer angepassten Textpraxis bedarf.

In diesem Sinne widmen sich einige Beiträge im vorliegenden Band konkreten Anforderungen, die unterschiedliche Nutzergruppen an Wahrnehmbarkeit und Verständlichkeit von Kommunikationsangeboten stellen. Andere Beiträge untersuchen insbesondere fachliche Kommunikationsbereiche daraufhin, inwiefern sie Barrieren für Nutzer(innen) darstellen, wie beispielsweise Medizinund Rechtskommunikation sowie technische Fachkommunikation. Insgesamt eröffnet das Handbuch den Blick auf diverse Formen, Bereiche und Textsorten Barrierefreier Kommunikation. Das Feld ist grundsätzlich offen und wird sich auch zukünftig weiter entwickeln.

Das vorliegende Handbuch hat sich das Ziel gesetzt, ein möglichst breites Spektrum repräsentativer Ausprägungen solcher Formen, Bereiche und Textsorten abzubilden, wie sie sich im Augenblick in der Textpraxis realisiert finden und wie sie teilweise auch bereits in der Wissenschaft reflektiert werden.

Dabei ist „Barrierefreiheit“ im wörtlichen Sinne eine Utopie. Tatsächliche Barrierefreiheit für alle Nutzergruppen wird sich häufig nicht realisieren lassen, schon weil sich die Anforderungen unterschiedlicher Nutzergruppen widersprechen. Außerdem sind die Ressourcen für die Herstellung der Kommunikate nicht selten begrenzt. Hinzu kommt, dass die Expertise der im Feld Handelnden an vielen Stellen noch nicht optimal ausgeprägt ist, auch wenn sich hier aktuell eine Professionalisierung abzeichnet. Im Allgemeinen wird mit „Barrierefreier Kommunikation“ auch die Schaffung „barrierearmer“ bzw. mit Bezug auf den Ausgangstext „barriereärmerer“ bzw. wahrnehmungs- und/oder verständlichkeitsoptimierter Kommunikate bezeichnet. Da „barrierefrei/Barrierefreiheit“ der auch im Rechtskontext gesetzte Terminus ist, wird dieser Begriff im vorliegenden Band synonym zu „barrierearm“ verwendet, auch wenn streng semantisch keine Synonymie besteht. 
Der vorliegende Band macht Barrierefreie Kommunikation in ihren Voraussetzungen und unterschiedlichen Facetten greifbar. Die Erkenntnisse der verschiedenen Bereiche sollen in ihren Überschneidungen und Ansatzpunkten herausgestellt werden, damit das Feld als Ganzes erschließbar wird. Auf dieser Basis lassen sich neue Erkenntnisse gewinnen, um zukünftig in noch ausgeprägterem Maße Barrierefreiheit in der Kommunikation herstellen zu können.

\section{Profil des Handbuchs}

Barrierefreie Kommunikation ist nicht nur ein Gegenstand wissenschaftlicher Auseinandersetzung, sondern auch ein breit ausdifferenziertes Praxisfeld, dessen Akteure die Produzent(inn)en und Nutzer(innen) barrierefreier Kommunikate sind. Dabei haben noch nicht alle Formen der praktischen Problemlösung und tatsächlichen Textangebote, aber auch der Bedarfe und Rezeptionsweisen dieser Textangebote bereits eine Reflexion durch die Wissenschaft erfahren.

Häufig sind die Artikulation von Bedarfen und die Erarbeitung von praktischen Lösungen erste Schritte, denen eine Theoretisierung um mehrere Jahre nachgeschaltet ist. Beispielsweise wurde das Konzept der Leichten Sprache aus den Reihen des Empowerment entwickelt, ebenso wie die ersten Regelwerke, die Basis für eine erste Textpraxis waren. Erst mit einem Abstand von ca. fünf Jahren begann die wissenschaftliche Auseinandersetzung mit diesem Gegenstand. Inzwischen ist Leichte Sprache ein viel beforschtes Thema in der Wissenschaft. Dabei findet neben rein theoretischer Auseinandersetzung auch anwendungsorientierte Forschung statt, die wiederum auf die Praxis zurückwirkt und zu einer Professionalisierung der am Markt Handelnden beiträgt. Aktuell tritt die Forschung in eine empirische Phase ein, in der mittels Probandenstudien Verstehensvoraussetzungen für und Verarbeitungsweisen von Kommunikaten ermittelt werden. Gleiches gilt für viele andere Formen und Aspekte der Barrierefreien Kommunikation (z. B. Audiodeskription, Untertitelung für Hörgeschädigte, Gebärdensprachdolmetschen etc.).

Für den Gegenstand der Barrierefreien Kommunikation ist in Theorie und Praxis die Einbeziehung der Nutzerperspektive essentiell, denn das Eingehen auf die Adressatenschaft ist in jeder Konstellation Voraussetzung für gelingende 
Kommunikation. Weist das Kommunikat eine Barriere für die Nutzer(innen) auf, so kann es nicht rezipiert werden. Damit ein Angebot wirklich barrierefrei ist, muss es folglich an die konkreten Bedarfe der Adressatenschaft angepasst werden. Diese Bedarfe können empirisch ermittelt werden. Durch die Zeitverzögerung und Selektivität der Forschung liegen jedoch aktuell nicht für alle Gegenstandsbereiche belastbare Studien vor.

Die Bedarfe können auch diskursiv ermittelt werden: Personen mit Kommunikationseinschränkungen können hinsichtlich ihrer Bedürfnisse, der Nutzungsformen und bestehenden Desiderate befragt werden. Eine solche Diskursivierung bleibt notwendig fragmentarisch und kann nicht den Anspruch auf Repräsentativität erheben. Solche Stimmen aus der Praxis hörbar zu machen, eröffnet jedoch häufig unerwartete Perspektiven und ermöglicht Einsichten, die so in anderer Form nicht zugänglich wären. Gleiches gilt für die Produzent(inn)en Barrierefreier Kommunikation: Aufgrund ihrer Expertise können sie nicht nur aus aktueller Praxis berichten, sondern häufig sogar auch über konkrete Bedarfe der Adressatenschaft und über ihr Vermitteln zwischen Auftraggeber und Bedarfsträgern Auskunft geben.

Entsprechend haben sich die Herausgeberinnen dazu entschlossen, den wissenschaftlichen Teil des Handbuchs mit einer Sektion "Stimmen aus der Praxis“ zu flankieren. Hier berichten Produzent(inn)en und Nutzer(innen) über ihre Erfahrungen und Wünsche bezüglich Barrierefreier Kommunikation. Manche von ihnen sind in beiden Rollen tätig, indem sie neben der Rezeption auch in die Produktion solcher Kommunikate mit einbezogen sind.

\section{Auffindbar > wahrnehmbar > verständlich > verknüpfungsfähig $>$ handlungsorientierend}

Die UN-Behindertenrechtskonvention und auch ihre Umsetzungen in die nationale Gesetzgebung (BGG und weitere) zielen darauf ab, Personen mit Behinderungen zur eigenständigen Teilhabe zu ermächtigen. Teilhabe ist ein aktives Konzept, das auf Interaktion im gesellschaftlichen Kontext ausgerichtet ist. Separation soll überwunden und ein Miteinander von Menschen mit und ohne 
Behinderung ermöglicht werden. Um dies zu ermöglichen, bedarf es barrierefreier Kommunikate, denn nur über sie haben Personen mit Behinderungen den vom Gesetz geforderten eigenständigen Zugang zu Informationen aller Art. In einer Informationsgesellschaft ist dies Voraussetzung für Teilhabe. Auch für Personen mit nicht behinderungsbedingten Kommunikationseinschränkungen ist Zugang zu Informationen Voraussetzung für Teilhabe.

Je nach Perspektive untergliedert sich der Prozess der Zugänglichkeit von Kommunikaten in die folgenden fünf Aspekte:

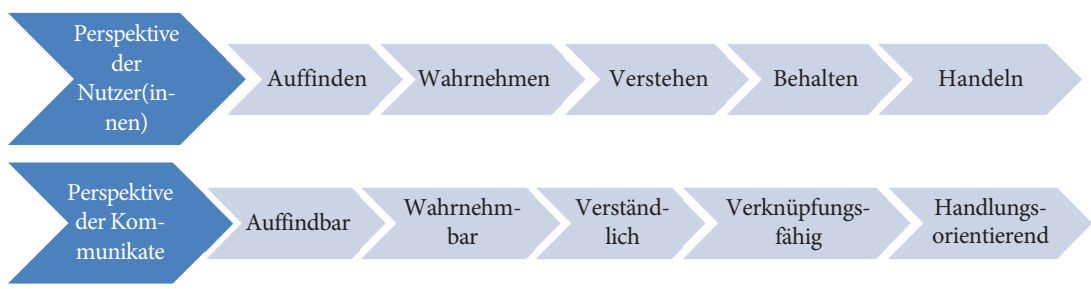

Abb. 1: Zugänglichkeit von Kommunikaten

Nutzer(innen) müssen Kommunikate auffinden können. Sie müssen sie wahrnehmen können, denn dies ist die Voraussetzung für die weitere Prozessierung der enthaltenen Informationen. Dabei darf der Wahrnehmungsprozess jedoch nur so viele Ressourcen binden, dass für die sich anschließenden Stufen noch ausreichend Verarbeitungskapazität vorhanden ist. Die wahrgenommenen Informationen müssen verstanden und semantisch sowie syntaktisch integriert werden. Auch dieser Schritt muss noch ausreichend Ressourcen verfügbar lassen, damit eine Verknüpfung mit bestehenden Wissensbeständen und eine Ablage der neu hinzugekommenen Informationen möglich sind. Nur wenn die Gesamtheit dieser Schritte die Verstehensressource der Nutzer(innen) nicht überbeansprucht, ist im nächsten Schritt ein wie auch immer geartetes Handeln möglich, das bereits Teilhabe ist.

Daraus ergeben sich Anforderungen an die Kommunikate: Sie müssen auffindbar sein, um die nächsten Stufen überhaupt zu ermöglichen. Sind sie für die Nutzer(innen) nicht wahrnehmbar, so können sie in der Folge nicht verstanden werden. Sind sie nicht verständlich, so ist ein Behalten nicht möglich. Werden 
Wissensressourcen nicht in einer Weise präsentiert, die ein Verknüpfen mit vorhandenen Wissensbeständen ermöglichen, so ist keine Grundlage für ein Handeln gegeben. Idealerweise wird die zielsituative Einbettung in den Kommunikaten selbst mit aufgenommen und so eine Handlungsorientierung angebahnt.

Die Beiträge des Handbuchs situieren sich entlang dieser Aspekte - entweder aus Perspektive der Nutzer(innen) oder aus Perspektive der Kommunikate.

\section{$4 \quad$ Ein Dank}

Ein Projekt wie das vorliegende kann nicht ohne tatkräftiges Mittun einer Vielzahl von Personen erfolgreich abgeschlossen werden. Das Handbuch wurde erst im Sommer 2017 erdacht, als uns das Fehlen eines entsprechenden Bands schmerzlich bewusst wurde: Für den neuen Masterstudiengang „Barrierefreie Kommunikation" sollte grundlegende Literatur zusammengestellt werden. Wir haben dann einschlägig forschende bzw. in der Praxis erfolgreiche Kolleginnen und Kollegen aus dem deutschsprachigen Raum angeschrieben und sie um Beiträge gebeten, ihnen aber auch den besonders engen Zeitrahmen erläutert - das Handbuch sollte noch vor Beginn des Studiengangs im Oktober 2018 erscheinen. Wir waren freudig überrascht, für ein derartig kurzfristiges Projekt so viele hochkarätige Zusagen zu erhalten. Noch überraschter waren wir jedoch, als diese Zusagen fast ausnahmslos eingehalten wurden, so dass das Projekt tatsächlich in der angesetzten ambitionierten Frist abgeschlossen werden konnte.

Wir bedanken uns an dieser Stelle bei allen Mitwirkenden für den disziplinierten Umgang mit Fristen und vor allem für die überaus inspirierenden, informativen und inhaltsreichen Beiträge, die das Feld der Barrierefreien Kommunikation als Ganzes zugänglich machen sollen.

Die Sektion „Stimmen aus der Praxis“ wurde überwiegend von Loraine Keller und Sergio Andres Hernández Garrido betreut. Loraine Keller hat überdies das Lektorat des gesamten Bandes übernommen und mit großer Sorgfalt ausgeführt. An beide geht ein herzlicher Dank. 

Anforderungen an Wahrnehmbarkeit und Verständlichkeit 

ISABEL RINK

\section{Kommunikationsbarrieren}

\section{$1 \quad$ Einleitung}

Zu den Maximen inklusiver Gesellschaften zählt die „barrierefreie[ ] Gestaltung ihrer Zugänge" (Bieling et al. 2012: 32). Zugänge sollen nicht nur in motorischer Hinsicht durchdringbar sein, sondern auch mit Bezug auf ihr perzeptives und kognitives Design. So wurden in den vergangenen Jahren verschiedene Formen von Zugängen bearbeitet, die auch die Kommunikation einschließen; denn an einer Gesellschaft teilhaben kann nur, wer Zugriff auf ihre Kommunikationsangebote hat. Nicht selten erschweren oder verwehren Kommunikationsbarrieren vielen Rezipient(inn)en den Zugang zu Information, weshalb der Fokus des vorliegenden Beitrags auf der Absenkung von Barrieren liegt, die die Kommunikation betreffen.

\section{Typen von Kommunikationsbarrieren}

Texte als Kommunikationsangebote können verschiedene Formen von Barrieren bergen, „die einer kommunikativen Teilhabe entgegenstehen“ (Schubert 2016: 15). In Abhängigkeit von ihrer Ausprägung können diese Barrieren Rezipient(inn)en mit und ohne Einschränkungen (s. Kap. 3) den Zugang zum Textgegenstand erschweren oder gar verwehren, da sie negativ mit der Perzeptibilität und der Verständlichkeit korrelieren. $\mathrm{Zu}$ diesen Kommunikationsbarrieren gehören nach Schubert (2016) und Rink (in Vorbereitung) die Sinnesbarriere, die Fachbarriere, die Fachsprachenbarriere, die Kulturbarriere, die Kognitionsbarriere, die Sprachbarriere und die Medienbarriere, die in der Folge in den Fokus der Auseinandersetzung rücken. 


\section{a. Die Sinnesbarriere}

Ist ein zur Aufnahme von Information notwendiger Sinneskanal in seiner Funktion erheblich beeinträchtigt, so ist eine erfolgreiche Rezeption über diesen Sinneskanal ausgeschlossen (Schubert 2016: 18). Angesprochen ist also die Modalität respektive die Art der Informationsaufnahme durch die verschiedenen Sinnesorgane als Wahrnehmungskanäle. Texte als Kommunikate können auditiv, visuell oder haptisch rezipiert werden, wobei auch eine Verschränkung der Modi denkbar ist (s. Abschnitt g). Stehen Auge, Ohr oder Tastsinn als Wahrnehmungskanäle zur Informationsaufnahme nicht zur Verfügung, während ein Text eine Wahrnehmung über eben diese Kanäle erforderlich macht, so liegt eine Sinnesbarriere vor.

\section{b. Die Fachbarriere}

Eine Fachbarriere wird durch Fachlichkeit, die sich auch in der Verwendung der Sprache niederschlägt (s. Abschnitt c und f), konstituiert. Liegt eine Fachbarriere vor, so wird ein Textgegenstand nicht verstanden, weil fachliches Wissen die Voraussetzung zur Erschließung des Aussageninhalts ist: „[...] die betroffenen Menschen [verstehen] den Inhalt einer Mitteilung nicht, weil ihnen fachliches, also inhaltliches Wissen fehlt" (Schubert 2016: 18). Laut Kalverkämper (1998a: 12) ist „[m]it ,Fach“ und seiner Qualität, nämlich der ,Fachlichkeit‘, [...] in höchstem Maße die ,Verständlichkeit' als eine in Texten verankerte kommunikative Qualität verbunden“, die vorrangig der Kommunikation unter Experten dient und eben aus diesem Grund zur Kommunikationsbarriere für ein Laienpublikum werden kann (vgl. Kalverkämper 1998b, Kap. 3.2.3) [Hervorhebung im Original].

\section{c. Die Fachsprachenbarriere}

Ähnlich der Fachbarriere korrelieren auch Fachsprachenbarriere und Verständlichkeit negativ. Ist eine Fachsprachenbarriere vorhanden, so wird potenziell die Sprache, nicht jedoch die besondere Fachsprache, die den Aussageninhalt trägt, verstanden. Schubert (2016: 19) weist darauf hin, dass diese beiden Kommunikationsbarrieren „zusammen auftreten [können], doch [...] nicht notwendigerweise gekoppelt [sind].“ 
Die Fach- und die Fachsprachenbarriere treten bei Fachkommunikation auf. Fachtexte stellen in der Barrierefreien Kommunikation aktuell eine der größten Herausforderungen dar, weshalb sie in diesem Beitrag systematisch mitberücksichtigt werden. Gehört ein Text nicht den Fachtexten zu, so weist er entsprechend keine Fach- und Fachsprachenbarriere auf; sein Barriereindex (dazu s.u.) ist folglich geringer. Solche Texte erfordern naturgemäß dann auch nicht die entsprechenden sprachlichen, medialen und konzeptuellen Maßnahmen.

\section{d. Die Kulturbarriere}

Kultur kann mit Vermeer (1990: 36) ganz allgemein als die Menge aller Konventionen einer Gesamtgesellschaft beschrieben werden. Liegt eine Kulturbarriere vor, so ist das für die Texterschließung notwendige kulturelle Wissen nicht in ausreichendem Maße vorhanden. Dieses Wissen schließt die Kenntnis um Diskurse und Textsorten sowie deren sprachliche, mediale und konzeptuelle Gestaltung ein (Schubert 2016: 18).

\section{e. Die Kognitionsbarriere}

Wenn „die sprachliche oder inhaltliche Komplexität der Mitteilung“ die Verarbeitungskapazität der Adressatenschaft übersteigt, so liegt nach Schubert (2016: 18f.) eine kognitive Barriere vor. In diesem Falle scheitern Adressat(inn)en auf der Ebene des Verstehens, weil „Aussagen indirekt wiedergegeben, verneint, bedingt, oder miteinander verknüpft sind“ (ebd.); die Argumentationsstruktur überfordert also die Verarbeitungskapazität der Rezipient(inn)en (s. hierzu auch cognitive load theory, Sweller et al. 1998).

\section{f. Die Sprachbarriere}

Die Sprachbarriere zielt auf die Ebene des sprachlichen Codes als Trägermedium von Information. Der Begriff des Codes kann mit Weidenmann (2002: 45f.) und Fröhlich (2015: 98) als System charakterisiert werden, das aus heterogenen (Schrift-)Zeichen, Bildern oder Symbolen besteht. Eine Sprachbarriere liegt insbesondere dann vor, wenn Deutsch nicht Erstsprache ist (s. Abschnitt d und g). 


\section{g. Die Medienbarriere}

Die Medienbarriere kann Ausprägungen in den Bereichen Codalität, Modalität und Medium aufweisen (vgl. Rink in Vorbereitung). Ist die Ebene des Codes betroffen, so können sprachliche Informationen, die in bestimmter Weise codiert, i.e. mittels verschiedener Zeichen- respektive Symbolsysteme realisiert sind, nicht aufgelöst werden (s. Abschnitt f). Auf der Ebene des Modes liegt eine Barriere dann vor, wenn das als Wahrnehmungskanal zur Informationsaufnahme erforderliche Sinnesorgan nicht funktionstüchtig ist (s. Abschnitt a). Texte können auditiv, visuell oder haptisch rezipiert werden, wobei auch eine Verschränkung der Modi, d. h. audiovisuell oder audiohaptisch, denkbar ist. Ist die Ebene des Modes betroffen, scheitert die Informationsaufnahme bereits hinsichtlich der Perzeption. Neben Code und Mode kann auch das Medium selbst zur Barriere werden, das mit Weidenmann (2002: 46) als (Über-)Träger im technischen Sinne gefasst wird. Es geht also um das Trägermedium als Übermittler von Information und seine Zugänglichkeit. Diese Ausprägungsform ist vor dem Hintergrund einer heterogenen Adressatenschaft besonders relevant, da ihre speziellen Bedarfe und Nutzungsformen den präferierten Zugriff auf Inhalte über bestimmte Medien determinieren (vgl. Rink in Vorbereitung).

In Abhängigkeit von ihrer sprachlichen, medialen und konzeptuellen Gestaltung können Texte die beschriebenen Barrieren in unterschiedlich starkem Ausmaß ausprägen. Ob die Ausprägungsformen negativ auf die Perzeptibilität und die Verständlichkeit wirken, entscheidet sich entsprechend dem spezifischen Anforderungsprofil der Adressatenschaft.

\section{Personen mit verschiedenen Arten von Kommunikationseinschränkungen}

In Anlehnung an Christmann (2006: 612f.) wird Textverstehen als Ergebnis der Interferenz textseitiger und rezipientenseitiger Parameter konzeptualisiert:

[D]er Text-Verstehensprozess [wird] als Wechselwirkung (Interaktion) zwischen zwei parallel ablaufenden Verarbeitungsrichtungen modelliert: 
die aufsteigende textgeleitete Verarbeitung (bottom-up), die durch Merkmale des Textes gesteuert wird; und die absteigende konzept- bzw. erwartungsgeleitete Verarbeitung (top-down), die durch die Merkmale der kognitiven Struktur der Rezipienten geprägt ist.

Während der Fokus des vorhergehenden Kapitels auf den primär von Texten ausgehenden, sich negativ auf die Perzeptibilität und die Verständlichkeit auswirkenden Kommunikationsbarrieren lag, richtet sich das Interesse nun auf die Rezipientenschaft, „deren Vorwissen, Ziele, Erwartungen und Interessen“ ebenfalls in prominenter Weise am Textverstehensprozess beteiligt sind (ebd.).

\subsection{Personen mit Perzeptionseinschränkungen}

Die Verständlichkeit von Texten ist ihrer Perzipierbarkeit nachgeordnet: Nur wenn Texte ohne Aufwendung einer zu großen Ressource wahrgenommen werden können, können ihre Inhalte in der Folge semantisch integriert und verarbeitet werden. Hier ist auf eine erste Gruppe von Kommunikationsbeeinträchtigungen einzugehen: die sogenannten Sinnesbehinderungen (Sehschädigung, Hörschädigung). Auf die Gruppe der Personen mit Sehschädigung gehen die Beiträge von Kahlisch/Dobroschke, Schütt, Benecke, Mälzer/Wünsche und Capovilla im vorliegenden Band ein, weshalb sie hier nur kurz vorgestellt werden soll:

\section{a. Personen mit Sehschädigung}

Nach deutschem Recht lässt sich das heterogene Feld der Sehschädigungen in „sehbehindert“, „hochgradig sehbehindert" und „blind“ differenzieren (vgl. Degenhardt 2007: 41 und DBSV 2018: Zahlen \& Fakten):

- Als sehbehindert gelten Personen, die trotz optischer Hilfen auf dem besseren Auge über einen Sehrest von nicht mehr als 30\% verfügen,

- als hochgradig sehbehindert gelten Personen, die trotz optischer Hilfen auf dem besseren Auge über einen Sehrest von nicht mehr als 5\% verfügen,

- als blind gelten Personen, die trotz optischer Hilfen auf dem besseren Auge über einen Sehrest von nicht mehr als 2\% verfügen oder bei denen trotz besserer Sehschärfe eine Einschränkung des Gesichtsfeldes vorliegt (ebd.). 
Inwieweit verschiedene Einschränkungsarten das Sehen erschweren, kann mit dem Sehbehinderungssimulator des ABSV geprüft werden (ABSV 2017: Sehbehinderungssimulator).

In Abhängigkeit von Ursache, Ausmaß und Alter sind betroffene Personen auf verschiedene Formen der Unterstützung angewiesen, die auch rechtlich untermauert sind (s. z. B. Sozialgesetzbuch, insb. SGB IX und XII, Behindertengleichstellungsgesetz, insb. $\$ 3,4,10$ und 12 oder Einkommenssteuergesetz, s. den Beitrag von Lang im vorliegenden Band). Da bei dieser Gruppe zugehörigen Personen ein Sinnesorgan als Wahrnehmungskanal zur Aufnahme von Information funktionseingeschränkt ist, ergeben sich besondere Anforderungsprofile bezüglich Kommunikation und Text.

Die zweite Form von Sinnesbehinderungen sind Hörschädigungen. Auf diese Gruppe gehen die Beiträge von Hennies, Witzel, Mälzer/Wünsche, Benner/ Herrmann, Kurch sowie Schwengber im vorliegenden Band ein. In den Beiträgen wird auch herausgearbeitet, dass es grundlegende Unterschiede zwischen der Gruppe der Personen mit prälingualer und postlingual erworbener Hörschädigung gibt. Im vorliegenden Beitrag wird insbesondere die Gruppe der Personen mit prälingualer Hörschädigung in den Blick genommen.

\section{b. Personen mit prälingualer Hörschädigung}

Gehörlosigkeit wird in der Medizin über den Grad des Hörverlusts klassifiziert; als gehörlos gilt, „wer im Bereich zwischen 125 und $250 \mathrm{~Hz}$ einen Hörverlust von mehr als $60 \mathrm{~dB}$ sowie im übrigen Frequenzbereich von mehr als $100 \mathrm{~dB}$ hat" (DGLB o.J.: Gehörlosigkeit). Demgegenüber definieren sich Personen mit prälingualer Hörschädigung meist selbst nicht über ihr fehlendes Hörvermögen. Vielmehr identifizieren sich Gehörlose als sprachlich und kulturell einer anderen Diakultur, nämlich der Gehörlosen-Community, zugehörig.

In Deutschland leben etwa 80000 prälingual gehörlose Personen, von denen der Großteil die Deutsche Gebärdensprache (DGS) zur Kommunikation nutzt. Zwar ist die DGS seit 2002 als Sprache offiziell anerkannt, nichtsdestotrotz leben gehörlose Personen als sprachliche Minderheit inmitten einer von Laut- und Schriftsprache dominierten Informationsgesellschaft (vgl. Rink in Vorbereitung). Das Beherrschen der deutschen Schriftsprache ist also die Voraussetzung zur gleichberechtigten Teilhabe dieses Personenkreises ohne die Hilfe Dritter. 
Jedoch müssen gehörlose Personen die deutsche Sprache, die in vielen Aspekten von der DGS abweicht, als Zweitsprache erst erlernen, was keine Trivialität darstellt, wenn der auditive Kanal eingeschränkt ist (vgl. Prillwitz/Wudtke 1988, Bouvet 1990, Wudtke 1993a und b, Krammer 2001, Brotzmann 2004, Hennies 2009 und Hennies in vorliegendem Band). Aus den Studien von Wudkte (1993a und b), Krammer (2001) und Hennies (2009 und im vorliegenden Band) geht hervor, dass die Lese- und Schreibkompetenz vieler gehörloser Personen aufgrund verschiedener Umstände nicht selten stark eingeschränkt ist. Aus der Natur der Beeinträchtigung resultiert also ein spezifischer Kommunikationsbedarf (s. Kap. 4).

\section{c. Personen mit Hör- und Sehbeeinträchtigung}

Hör- und Sehbeeinträchtigungen als Formen von Sinnesbehinderung können einzeln auftreten, sie können jedoch auch zusammen vorkommen. Dies ist gerade mit fortschreitendem Alter der Fall. Das gemeinsame Auftreten angeborener Formen hochgradiger Seh- und Hörschädigung betrifft eine relativ kleine Gruppe von Personen in Deutschland (nach Schätzungen der Gesellschaft für Taubblindheit zwischen 4000 und 9000 Personen, Gesellschaft für Taubblindheit 2014), die jedoch bezüglich ihrer Ansprüche an barrierefreie Texte sehr ausgeprägte Anforderungen hat (vgl. Nonn/Päßler-van Rey 2011: 53f.).

Erst seit Dezember 2016 ist Taubblindheit mit einem eigenen Zeichen (TBI) im Schwerbehindertenausweis versehen, was die Möglichkeit des Abrufs angemessener Leistungen zur Folge hat. Taubblinde Menschen kommunizieren über den taktil-kinästhetischen Kanal, wobei das Lormen respektive Daktylieren, also das Abtasten des Fingeralphabets auf der Hand bzw. bestimmten Handarealen als taktile Form der Kommunikation, dem Austausch von Informationen dient (vgl. BAT 2008: Taktiles Fingeralphabet, Deutsches Taubblindenwerk o.J.: Lormen). Insgesamt ist für diesen Bereich ein ausgeprägter Forschungsbedarf zu konstatieren, der v.a. die kommunikativen Anforderungen taubblinder Personen betrifft. Jüngere Bemühungen zielen auf die Entwicklung von LormHandschuhen (Bieling et al. 2012), die die kommunikative Teilhabe dieses Personenkreises erleichtern sollen. 


\subsection{Personen mit Verstehenseinschränkungen}

Obwohl sich Barrierefreie Kommunikation hauptsächlich an den Bedarfen einer Adressatenschaft mit besonderem Anforderungsprofil orientiert, profitieren darüber hinaus viele weitere Rezipient(inn)en von in zugänglicher Form aufbereiteten Texten.

Grotlüschen und Riekmann $(2011,2012)$ haben in ihrer Level-One Studie zur Literalität von Erwachsenen auf den unteren Kompetenzniveaus nachgewiesen, dass etwa $40 \%$ der erwachsenen und als erwerbsfähig geltenden Bevölkerung in Deutschland (Personen zwischen 18 und 64 Jahren mit hinreichender Sprachkompetenz) eine defizitäre Lese- und Schreibkompetenz aufweist (Grotlüschen/Riekmann 2011: 2ff.; 2012: 19ff.). Das entspricht einer Größenordnung von knapp 21000000 Personen. Als i.e.S. den funktionalen Analphabet(inn)en zugehörig klassifizieren Grotlüschen/Riekmann (2012: 19ff.) Personen, die Alpha-Level 4 bzw. die Textebene unterschreiten, wie die folgende Tabelle (1) zeigt:

\begin{tabular}{|l|c|c|c|}
\hline Literalität & Alpha-Level & $\begin{array}{c}\text { Anteil der erwachsenen } \\
\text { Bevölkerung }\end{array}$ & $\begin{array}{c}\text { Anzahl } \\
\text { (hochgerechnet) }\end{array}$ \\
\hline \multirow{2}{*}{$\begin{array}{l}\text { Funktionaler } \\
\text { Analphabetismus }\end{array}$} & $\alpha 1$ & $0,6 \%$ & 0,3 Mio. \\
\cline { 2 - 4 } & $\alpha 2$ & $3,9 \%$ & 2,0 Mio. \\
\hline Zwischensumme & $\alpha 3$ & $10,0 \%$ & 5,2 Mio. \\
\hline $\begin{array}{l}\text { Fehlerhaftes } \\
\text { Schreiben }\end{array}$ & $\alpha 4$ & $14,5 \%$ & 7,5 Mio. \\
\hline & $>a 4$ & $25,9 \%$ & 13,3 Mio. \\
\hline Summe & & $59,7 \%$ & 30,8 Mio. \\
\hline
\end{tabular}

Tab. 1: Funktionaler Analphabetismus und fehlerhaftes Schreiben

Quelle: leo. - Level-One Studie, n=8 436 Deutsch sprechende Personen im Alter von 18-64 Jahren. Abweichung der Summe von $100 \%$ aufgrund von Rundungseffekten

Während etwa 300000 Personen in Deutschland beim Lesen und Schreiben die Wortebene unterschreiten und damit dem Alpha-Level 1 (a1) zugehören, erreichen etwa 2000000 Personen die Wort- aber nicht die Satzebene: Personen auf 
Alpha-Level 2 sind imstande, einige Wörter zu lesen und zu schreiben, sie scheitern jedoch an der syntaktischen Integration (Grotlüschen/Riekmann 2012: 20). Auf Alpha-Level 3 können die betroffenen Personen (etwa 5,2 Mio.) „mit kurzen Sätzen umgehen“, scheitern jedoch an der Textebene (ebd.). Zu diesen 7500000 Personen kommen auf Alpha-Level 4 weitere 13,3 Mio. Menschen hinzu, die zwar die Wort-, Satz- und Textebene meistern, „deren Schriftsprache [jedoch] auch bei gebräuchlichem Wortschatz fehlerhaft ist" (ebd.). Grotlüschen/ Riekmann (2011: 2) ergänzen: „Die Rechtschreibung, wie sie bis zum Ende der Grundschule unterrichtet wird, wird nicht hinreichend beherrscht. Typische Betroffene vermeiden das Lesen und Schreiben häufig.“

Die sprachlichen Kompetenzen der teilnehmenden Proband(inn)en waren also insoweit ausgeprägt, dass sie an der Erhebung sowie damit einhergehenden Kompetenztests teilnehmen konnten (Grotlüschen/Riekmann 2011: 8). Dies legt, wie Bredel/Maaß (2016: 167) anmerken, den Schluss nahe, dass Personen mit gravierenden „Sprach- und/oder kognitiven Beeinträchtigungen“, wie sie im Fokus Barrierefreier Kommunikation und des vorliegenden Beitrags stehen, aus systematischen Erwägungen überwiegend nicht an der Studie teilgenommen haben. Die Ergebnisse berücksichtigen also solche Personengruppe nur partiell, deren Anforderungen an barrierefreie, i.e. leicht perzipierbare sowie leicht verständliche, Texte aus der Natur ihrer Beeinträchtigung resultieren und deren Schriftsprachkompetenz deshalb in besonderer Weise eingeschränkt ist.

Das Feld der Personen mit verschiedenen Arten von Kommunikationseinschränkungen ist divers. Um hier eine Eingrenzung vornehmen zu können, soll die Rezipientenschaft in den Fokus der Betrachtungen rücken, die Bredel/Maaß (2016) als primäre Adressat(inn)en von Texten in Leichter Sprache fassen:

Primäre Adressat(inn)en sind für alle oder die meisten Textsorten der Schriftlichkeit auf Leichte Sprache angewiesen und haben keinen oder nur sehr eingeschränkten Zugriff auf allgemein- oder fachsprachliche Texte. Die Bereitstellung von Informationen in Leichter Sprache ermöglicht ihnen eine selbstbestimmte und direkte Teilhabe an den Kommunikationsprozessen in allen Bereichen des öffentlichen Lebens (Bredel/Maßß 2016: 139) [Hervorhebung im Original]. 
Im Fokus stehen damit also Personen, deren Schriftsprachkompetenz dergestalt ist, dass sie auf Texte in Leichter Sprache als eine Form Barrierefreier Kommunikation angewiesen sind, um überhaupt partizipieren zu können. Hierzu zählen laut Bredel/Maaß (2016, Kap. 5) Personen mit geistiger Behinderung, Personen mit Lernschwierigkeiten, von demenziellen Erkrankungen Betroffene, prälingual Hörgeschädigte, Aphasiker und Personen mit Deutsch als Zweitsprache. Überdies profitieren die bereits erwähnten Analphabet(inn)en von Texten in Leichter Sprache sowie Barrierefreier Kommunikation insgesamt, wobei Bredel/Maaß (2016: 166) herausstellen, dass der Terminus Analphabetismus quer zu den vorherigen Zielgruppen Leichter Sprache liegt,

[d]enn je nach Schweregrad der Beeinträchtigung gehören auch Personen mit Lernschwierigkeiten, Demenzkranke, von Aphasie Betroffene oder prälingual Gehörlose zu den Analphabet(inn)en.

Diese Zielgruppen sollen in der Folge kurz in ihren (Anforderungs-)Profilen vorgestellt werden.

\section{d. Personen mit geistiger Behinderung}

Um die Benennung dieser Gruppe wird ein Diskurs geführt, da der Begriff "geistige Behinderung" von der Gruppe selbst als diskriminierend empfunden wird (s. ausführlich Schuppener/Bock im vorliegenden Band). Während sich Angehörige dieser Gruppe als „Menschen mit Lernschwierigkeiten“ bezeichnen, um einer Stigmatisierung entgegenzuwirken (Netzwerk Leichte Sprache o.J.: Das Prüfen), wird dieser Terminus in der Forschungsliteratur für eine andere Gruppe verwendet (s.u. Abschnitt e: Personen mit Lernschwierigkeiten).

Vor dem Hintergrund der rechtlichen Lage ist die im gesellschaftlichen Diskurs stattfindende Vermischung beider Konzepte (Menschen mit geistiger Behinderung vs. Menschen mit Lernschwierigkeiten) jedoch nicht unproblematisch, denn im juristischen Sinne Anspruch auf Barrierefreiheit und Verständlichkeit gemäß $\$ 4,10,11,12$ u.a. haben lediglich Personen, 
die langfristige körperliche, seelische, geistige oder Sinnesbeeinträchtigungen haben, welche sie in Wechselwirkung mit einstellungs- und umweltbedingten Barrieren an der gleichberechtigten Teilhabe an der Gesellschaft hindern können. Als langfristig gilt ein Zeitraum, der mit hoher Wahrscheinlichkeit länger als sechs Monate andauert (BMJV 2016: BGG).

Demgegenüber haben Personen mit Lernschwierigkeiten einen Rechtsanspruch auf zugängliche Kommunikationsangebote also nur dann, wenn die Dauer der Beeinträchtigung nachweislich vorliegt (s. BGG); i.e. mit der Verwendung der Termini sind juristische Ansprüche verbunden, die gesetzlich festgelegt sind.

Nach Fornefeld (2002: 44) sind Personen, die dieser Gruppe zugehören,

in Folge einer Hirnschädigung in ihren intellektuellen Fähigkeiten (in der Analyse und Synthese von Wahrnehmungen, Erfahrungen, Einsichten und Erkenntnissen) gravierend beeinträchtigt [...], was wiederum Auswirkungen auf ihr Lernen und ihre Lebensgestaltung hat.

Aus der Art der Einschränkung können sich spezifische Anforderungsprofile bezogen auf Kommunikationsangebote ergeben und eine stark verminderte Schriftsprachkompetenz kann ein Resultat der angesprochenen Schädigung sein. Ein Großteil dieser Gruppe gehört den Nicht-Leser(inne)n an bzw. benötigt verständlichkeitsoptimierte Kommunikationsangebote, um gleichberechtigt teilhaben zu können (vgl. Bredel/Maaß 2016, Kap. 5.2.4; Ratz 2013). Schuppener/Bock (im vorliegenden Band) betonen, dass dies wegen der Heterogenität dieser Gruppe nicht auf alle Menschen mit geistiger Behinderung zutrifft, nichtsdestotrotz sind es aus den primären Zielgruppen vorrangig „Menschen mit geistigen Behinderungen“, die qua Gesetz einen Anspruch auf Inhalte in Leichter und Einfacher Sprache haben (BMJV 2016: BGG \$11).

\section{e. Personen mit Lernschwierigkeiten}

Der Terminus Lernschwierigkeiten bündelt verschiedene Ausprägungsformen von Lernproblemen, die mit Heimlich (2016: 33) nach „Umfang, Schweregrad, Dauer, Inhalte, Beeinflussbarkeit und Ursachen“ kategorisiert und entsprechend 
in "allgemeine“ und "gravierende Lernschwierigkeiten“ differenziert werden können (s.a. Heimlich 2016: 31ff.):

Gravierende Lernschwierigkeiten unterscheiden sich von allgemeinen Lernschwierigkeiten dadurch, dass ein Bedarf an Unterstützung, Förderung und Begleitung zur Überwindung der Lernschwierigkeiten besteht" (Heimlich 2016: 13f.).

Die Abgrenzung richtet sich also nach dem ermittelten Bedarf an Unterstützung.

$\mathrm{Zu}$ den besonderen Charakteristika von Lernschwierigkeiten in der Grundschule gehören Heimlich (2016: 38) zufolge „Probleme beim Erlernen des Lesens, Schreibens und Rechnens" [Hervorhebung im Original], die sich konsequenterweise auswachsen, wird ihnen nicht rechtzeitig entgegengewirkt. Da jedoch gerade das Lesen Voraussetzung für die Informationsaufnahme bzw. den Aufbau von Wissen ist, sind Bredel/Maßß (2016: 151) der Annahme, dass

Texte in Leichter Sprache [...] Schülern und Erwachsenen mit Lernschwierigkeiten die Partizipation an der Informationsgesellschaft [erleichtern], da sie Informationen in zugänglicher und leicht rezipierbarer Form zur Verfügung stellen [...].

\section{f. Personen mit demenziellen Erkrankungen}

Laut Kurz (2013: 5) werden etwa „80\% aller Demenzzustände [...] durch Krankheiten des Gehirns hervorgerufen", die mit einem Zellsterben verbunden und durch den Alterungsprozess des Menschen bedingt sind:

Eine Demenz tritt mit wenigen Ausnahmen erst im Alter auf, weil sie meist mit degenerativen Erkrankungen verbunden ist, deren Auswirkungen erst mit steigendem Alter deutlich werden (Schindelmeiser 2016: 133). 
Es handelt sich bei Demenzen also um degenerative neurologische Erkrankungen, die etwa ab dem sechzigsten Lebensjahr einsetzen (Schindelmeiser 2016: 133; Kurz 2013: 7). Zu den Symptomen, die in Abhängigkeit vom jeweiligen Demenzstadium variieren, zählen sich auswachsende Gedächtnisstörungen, eine Beeinträchtigung der kognitiven Fähigkeiten sowie der Sprach- und Sprechfähigkeiten, Orientierungsschwierigkeiten und motorische Funktionsverluste (vgl. Kurz 2013: 10ff.; Schindelmeiser 2016: 136). Erworbene Fertigkeiten und Kompetenzen werden also sukzessive abgebaut und damit einher gehen spezifische Bedarfe, die auch die Kommunikation betreffen. So bereitet insbesondere die Konzeptbildung vielen Betroffenen Schwierigkeiten, was zu ausgeprägten Kommunikationseinschränkungen führt (vgl. Gröning 2012: 41ff.). Texte in Leichter Sprache machen die konzeptuelle Struktur der Inhalte transparent und sind voraussetzungsarm; es besteht die Annahme, dass sie auch bei demenziellen Abbauprozessen länger zugänglich sind als die nicht verständlichkeitsoptimierten Ausgangstexte (Bredel/Maaß 2016: 155).

\section{g. Personen mit Aphasie}

Bei einer Aphasie handelt es sich um eine erworbene Störung in der Sprache und Kommunikation infolge einer Erkrankung des zentralen Nervensystems:

Geschädigt wird das sog. Sprachzentrum mit den Sprachfunktionen des Gehirns. Es befindet sich bei mehr als 90\% der Menschen in der linken Hirnhälfte. Die aphasischen Störungen zeigen sich in allen sprachlichen Modalitäten, also beim Sprechen und Verstehen, Lesen und Schreiben. (Huber et al. 2006: 7, 2013: 24)

Häufigste Ursache einer Aphasie ist der Schlaganfall, jedoch können auch Bluthochdruck, Diabetes, durch Gefäßfehlbildungen ausgelöste Blutungen oder Unterversorgungen, aber auch Hirnverletzungen (z. B. nach Unfällen) zu Aphasien führen (ebd.). Während die Sprachstörungen in den ersten Wochen des Krankheitsverlaufs bei etwa einem Drittel der Patienten zurückgehen, verfestigen sich diese bei den übrigen zwei Dritteln und wachsen sich zu anhaltenden Kommunikationsbehinderungen aus, die die Wort-, Satz- und Textebene betreffen können. Das folgende Beispiel, das aus Huber et al. (2006: 40) entnommen ist, zeigt 
einen Ausschnitt eines Gesprächs mit einem Patienten, der unter globaler Aphasie leidet:

U: Wie ist das gekommen mit der Krankheit?

P: $\quad$ mh ... de ... de dodo ... und eh ... do dodo ... eh

$\mathrm{U}: \quad$ Ist das schon lange her?

P: $\quad$ nee ... mh ... dodo ... dodo ... (zeigt 4 Finger)

U: 4 Wochen?

P: $\quad$ ja ... dodo ... und dudu

$\mathrm{U}: \quad$ Und das ist plötzlich gekommen?

P: $\quad \mathrm{mh} \ldots \mathrm{mh}$

$\mathrm{U}: \quad$ Wie ist das denn gekommen?

P: $\quad$ ja ... dada (zeigt gelähmte rechte Seite)

U: Sind Sie da morgens aufgewacht und Sie konnten nicht mehr gehen?

P: $\quad$ nee dududu ... und eh ... dudodo und dodo

Aphasien können - in Abhängigkeit von der Ursache - in verschiedenen Ausprägungsformen vorkommen, wobei die „charakteristische[n] Einzelsymptome in Kombination mit anderen Symptomen immer wieder[kehren] “ (Huber et al 2006: 39). Zu den vier Standardtypen zählen die globale Aphasie, die Wernicke-Aphasie, die Broca-Aphasie und die amnestische Aphasie (ebd.: 39). Pro Jahr erkranken in Deutschland etwa 50000 Personen an einer Aphasie, die aus einem Schlaganfall rührt. Addiert man diese Ziffer um weitere Krankheitsursachen, so liegt die Zahl der an Aphasie Erkrankten bei mehr als 100000 Personen jährlich (ebd.: 7).

Bredel/Maaß (2016: 166) vermuten, dass insbesondere

motorischen Aphasiker(inne)n, bei denen das Broca-Areal geschädigt ist, die syntaktische Einfachheit [von Leichte-Sprache-Texten] entgegenkommt, während für sensorische Aphasiker(innen), bei denen das Wernicke-Areal beeinträchtigt ist, die Beschränkung auf den Basiswortschatz und die umfangreichen Erläuterungen von Wortbedeutungen hilfreich sein dürfte.

Eine entsprechende empirische Überprüfung steht noch aus. 


\section{h. Personen mit Deutsch als Zweitsprache}

Während das Fremdsprachenlernen i.d.R. gesteuert, „im institutionellen, zumeist schulischen Kontext" mit relativ homogenen Lerngruppen stattfindet (Lütke 2011: 26), sind unter dem Begriff des Zweitspracherwerbs solche Sprachlernprozesse gefasst, die ungesteuert, i.e. ohne institutionelle Einbindung erfolgen (Rösch 2011: 13). Laut Lütke (2011: 26) ist die

Erwerbssituation [...] dadurch gekennzeichnet, dass die Sprache zu einem großen Teil oder ausschließlich [...] ohne unterrichtliche Unterstützung erworben wird, wobei die Lernenden häufig über unterschiedliche zweitsprachliche Voraussetzungen verfügen.

Im Zentrum stehen also Personen, die in Deutschland leben und ihren Alltag bewältigen, ohne die Amtssprache Deutsch in einem didaktisch geprägten Lernumfeld in Wort und Schrift erworben zu haben (ebd.). Bei dieser sehr inhomogenen Personengruppe liegt also i.e.S. eine Sprachbarriere vor (s. Kap. 2, Abschnitt f und g), die die Ebene des sprachlichen Codes betrifft. Texte in verständlichkeitsoptimierter Form können gerade zu Beginn des Aufenthalts helfen, bestehende Defizite hinsichtlich des Sprach-, Diskurs- und Weltwissens zu kompensieren und dabei zugleich einen Anreiz schaffen, die eigenen schriftsprachlichen Kompetenzen auszubauen, die die Voraussetzung zur Rezeption standard- sowie fachsprachlicher Texte bilden (vgl. Bredel Maßß 2016, Kap. 5.2.9 und Rink in Vorbereitung). Diese Gruppe hat qua Gesetz keinen Anspruch auf barrierefreie Texte; gleichzeitig ist ein Großteil dieser Personen gerade am Anfang ihres Aufenthalts sehr häufig mit verschiedenen Anträgen und Formblättern konfrontiert, die als Texte mehrere Kommunikationsbarrieren bergen (s. Kap. 2) und die Adressierten schnell an ihre Verstehensgrenzen bringen, da sie bislang kaum Gelegenheit zum Spracherwerb hatten (vgl. Bredel/Maaß 2016: 171f., Rink in Vorbereitung).

Die Zahl der Zuwanderungen nach Deutschland ist seit dem Jahr 2014 merklich gestiegen, wobei in dieser Personengruppe sowohl die lautsprachlichen und schriftsprachlichen Fähigkeiten in der Muttersprache (nicht alle sind alphabetisiert) als auch die Kenntnisse mit Blick auf den Zweitspracherwerb großen Abweichungen unterliegen (BPB 2016: Datenreport 2016, S. 8). Daraus 
folgt, dass diese Adressatengruppe mit verschiedenen Kommunikationsbarrieren konfrontiert ist (Rink in Vorbereitung).

\section{i. Personen mit Mehrfachbehinderung}

In den vorhergehenden Abschnitten, $\mathrm{c}$ ausgenommen, wurden die verschiedenen Einschränkungsarten in heuristischer Weise, i.e. als Prototypen von Einfachbehinderung bzw. einer Art von Kommunikationseinschränkung konzeptualisiert. Zwar geht nicht jede Form von Kommunikationseinschränkung zwangsläufig mit einer Beeinträchtigung einher, jedoch kann mit Vorliegen einer der oben genannten Barrieretypen die Kommunikation und damit der $\mathrm{Zu}$ griff auf Textgegenstände in verschieden starkem Ausmaß beeinträchtigt sein. Darauf liegt der Fokus dieses Beitrags.

Die beschriebenen Einzelphänomene können separat vorkommen, sie können aber auch in Kombination auftreten bzw. kommen als Mischformen vor, z. B.:

- Taubblindheit (c)

- Demenz und Hörschädigung

- Deutsch als Zweitsprache und geistige Behinderung

- Deutsch als Zweitsprache und Lernschwierigkeiten.

Dies sind nur einige Beispiele möglicher Kombinationen von Kommunikationseinschränkungen, deren Ausprägung individuell verschieden sein kann. Laut Schnoor (2007: 247) treten

[d]ie Behinderungsbilder, die als schwere, schwerste oder Mehrfachbehinderung bezeichnet werden, [...] infolge unterschiedlicher Schädigungen auf und zeigen hinsichtlich ihrer Formen und Ausprägungen große Varianz.

Aufgrund dieser Heterogenität kann Mehrfachbehinderung als Beeinträchtigungsart nicht „als einheitliches Behinderungsbild“ klassifiziert werden (ebd.). Entsprechend wurde Taubblindheit im neuen Teilhabegesetz mit einem eigenen Maßnahmenpaket ausgestattet. 
So divers die Ursachen sind, so verschieden sind auch die Ausprägungsformen, die „in der Summe [...] zu gravierenden Einschränkungen des ganzen Menschen in allen seinen Lebensvollzügen [führen]" (ebd.). Betroffene sind nicht nur in ihrer Wahrnehmung und Kommunikation beeinträchtigt, sondern in ihren „Erlebens- und Ausdrucksmöglichkeiten“ insgesamt (ebd.). Dass solch multiple Beeinträchtigungsformen besondere Anforderungsprofile bezogen auf kommunikative Teilhabe ausprägen, konstatieren neben Schnoor (2007) auch Folta-Schoofs, Musenberg und Schuppener/Bock (im vorliegenden Band).

\section{Kommunikationsbarrieren und Beeinträchtigungsformen}

Die Barrieretypen nach Schubert (2016) und Rink (in Vorbereitung) wurden in Kapitel 2 vorgestellt. Barrieren haben auf die Kommunikation potentiell negative Auswirkungen, umso mehr, wenn ein Text gleichzeitig in mehrerlei Hinsicht Barrieren für die Adressat(inn)en darstellt. In Abhängigkeit von ihrer Ausprägung können die Barrieren den Rezipient(inn)en mit und ohne Beeinträchtigung den Zugang zu den Inhalten erschweren oder unmöglich machen; Missverstehen oder Nicht-Verstehen können die Folge sein (vgl. Schnotz 1994: 32ff.). Die verschiedenen Barrieretypen hemmen die Kommunikation jedoch nicht zwangsläufig im Sinne einer absoluten (Verstehens-)Grenze, vielmehr handelt es sich um ein dynamisches Konzept: die Barrieren weisen „graduelle Abstufungen“ auf, die je nach Ausprägung den Zugriff auf Kommunikationsinhalte mehr oder minder stark einschränken (Schubert 2016: 17). Zudem hängen sie von den rezipientenseitigen Determinanten ab, die in Kapitel 3 exemplarisch für verschiedene Adressatengruppen vorgestellt wurden.

In welcher Weise Kommunikationsbarrieren und verschiedene Arten von Einschränkungen korrelieren, soll die Tabelle (2) zeigen. Während der Tabellenkopf die verschiedenen Arten von Einschränkungen darstellt - darunter Sehschädigung, prälinguale Hörschädigung, geistige Behinderung, Lernschwierigkeiten, Demenz, Aphasie sowie Deutsch als Zweitsprache und funktionaler Analphabetismus - zeigt die linke Tabellenspalte die verschiedenen Barrieretypen, die die Kommunikation beeinträchtigen können. Hierzu zählen die Sinnesbarriere in ihren Ausprägungen auditiv, visuell und haptisch, die Fach- und 
die Fachsprachenbarriere, die Kultur-, die Kognitions- und die Sprachbarriere sowie die Medienbarriere in ihren Ausprägungsformen phonisch, grafisch, haptisch und Medium (vgl. Rink in Vorbereitung).

$\mathrm{Da}$ in vorliegendem Beitrag das Augenmerk auf Barrieren liegt, die von den Texten selbst ausgehen, steht primär die Modalität respektive die Realisierungsform der Inhalte im Fokus der Betrachtungen. Nach Burger (2005: 143) kann Text „phonisch, mit Lauten (das heißt zugleich akustisch), oder grafisch, mit Schriftzeichen (das heißt zugleich visuell), realisiert [sein]“. Außerdem sind audiohaptische und audiovisuelle Realisierungsformen möglich, nämlich wenn Texte zugleich phonisch und haptisch bzw. phonisch und grafisch zugänglich sind. Die Termini „phonisch“ und „grafisch“ sollen in Anlehnung an Burger (2005) und Koch/Oesterreicher (1994) übernommen werden: Während sich "phonisch“ auf die auditive Ebene bezieht, also das Hören meint, bezieht sich "grafisch" auf die visuelle Ebene und zielt auf das Sehen. Demgegenüber zielt die haptische Ebene auf das Tasten, das in der Regel mit den Händen erfolgt. Da es sich bei der Brailleschrift um ein besonderes System von Schriftzeichen handelt, das zwar nicht visuell wahrnehmbar ist, jedoch haptisch perzipiert werden kann, soll es hier unter der „haptischen“ Realisierungsform gefasst werden; auch das Lormen weist eine haptische Realisierungsform auf. Neben den Ausprägungsformen „phonisch“, „grafisch“ und „haptisch“ kann auch das Medium selbst, i.S. eines Gerätezugangs, eine Barriere darstellen (vgl. Rink in Vorbereitung). Informationen sind also nicht zugänglich, wenn für eine Adressatengruppe die für die Rezeption notwendigen Medien nicht verfügbar sind respektive von ihr üblicherweise nicht genutzt werden. Bezogen auf diese Ausprägungsform spielen Faktoren wie Alter, soziale Schicht, Infrastruktur oder Endgeräte eine entscheidende Rolle, da sie Einfluss auf den präferierten Zugang zu Inhalten über bestimmte Medien haben (vgl. Rink in Vorbereitung). Die Spalten folgen jeweils einer Beeinträchtigungsart, wobei davon ausgegangen wird, dass bei Mehrfachbehinderung bzw. bei multiplen Beeinträchtigungsformen Maßnahmen bezüglich aller dann vorliegenden Kategorien zu treffen sind.

In welcher Weise Barrieretypen und Einschränkungsarten kovariieren, zeigt die folgende Tabelle (2) im Überblick: 


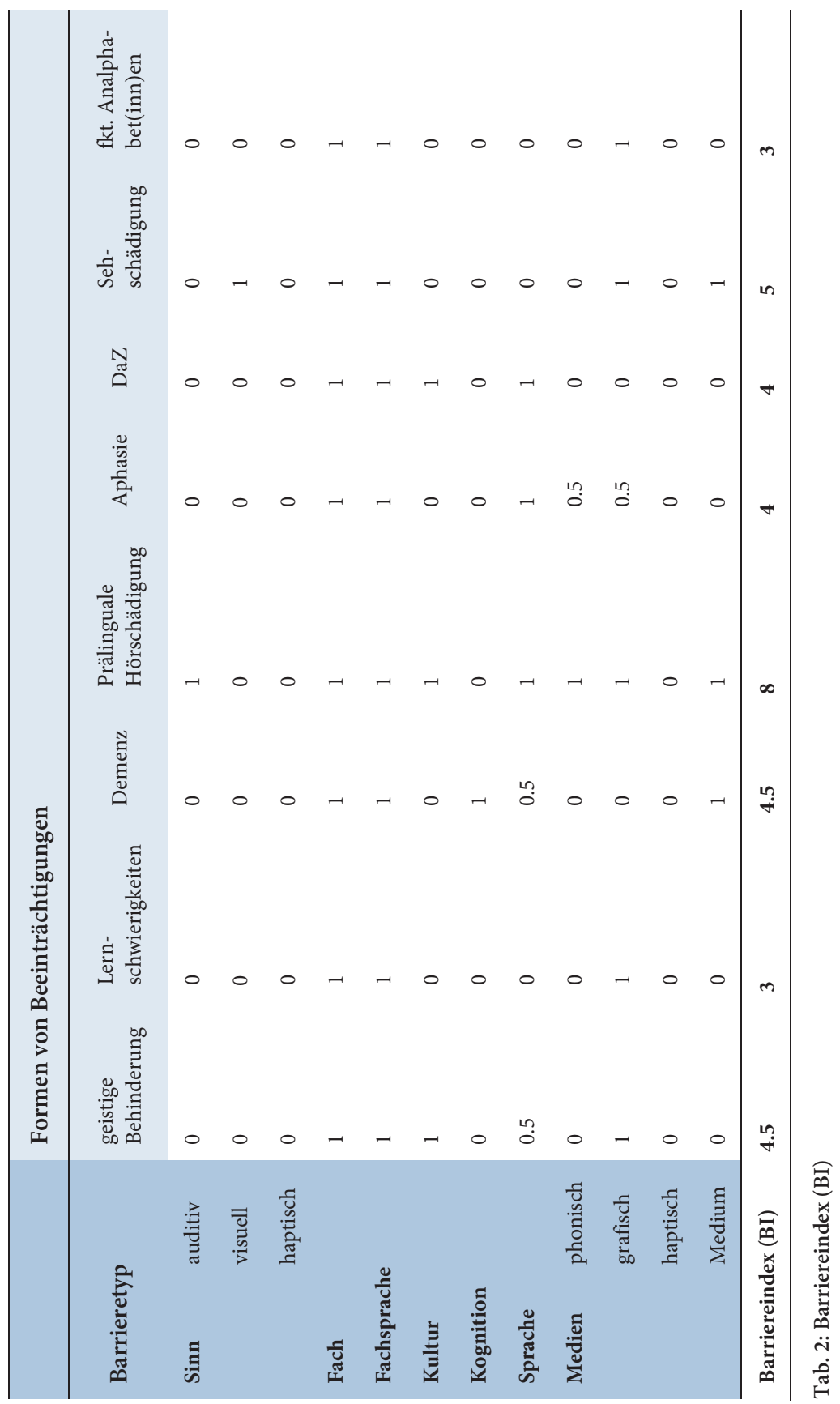


Die in der Tabelle dargestellten Ausprägungen werden aufgrund der vorliegenden Erkenntnisse zu den jeweiligen Adressatengruppen und Barrieretypen postuliert. Um hier weitere Präzisierungen und ggf. Korrekturen vornehmen zu können, bedarf es für jede einzelne Adressatenschaft weiterer empirischer Forschung.

\subsection{Adressat(inn)enprofil}

Das Adressat(inn)enprofil stellt die Ausprägung der jeweiligen Einschränkungsart bezogen auf die verschiedenen Barrieretypen dar. Im Zentrum stehen also die Spezifika je Einschränkungsart, wie sie sich in den Spalten der Tabelle abbilden.

\section{a. Sehgeschädigte Personen}

Bei sehgeschädigten Personen ist ein Sinnesorgan als Wahrnehmungskanal zur Aufnahme von Information eingeschränkt (1). Inhalte, die nicht in medial angemessener Weise aufbereitet sind, können also nicht rezipiert werden. Während sehgeschädigte Personen bezüglich der phonischen Realisierung keine Einschränkung haben, werden für sie Inhalte, die ausschließlich grafisch realisiert sind, schnell zur Hürde (1) bzw. müssen von ihnen weiter prozessiert werden, um sie in einer anderen Medialität aufzubereiten (z. B. per Scanner). Überdies resultiert aus der Natur der Behinderung eine präferierte Nutzungsart in Bezug auf das Medium, die die Informationsaufnahme restringiert (1). Eine weitere Hürde kann bezogen auf die Fach- (1) und die Fachsprachenbarriere (1) konstatiert werden, die im Übrigen sämtlich Adressat(inn)en mit und ohne Einschränkungen vor Herausforderungen stellen, wenn sich diese als fachliche Laien über komplexe und abstrakte Sachverhalte oder Gegenstände beispielsweise im Bereich der juristisch-administrativen oder medizinischen Kommunikation informieren wollen (vgl. Rink in Vorbereitung, Schaeffer et al. 2016, Bredel/Maaß 2016, Bromme/Jucks/Rambow 2004, Eckhardt 2000 und Kalverkämper a und b 1998). Hinsichtlich der Kultur- (0), der Kognitions- (0) und der Sprachbarriere (0) liegen keine Einschränkungen vor. 
Der Barriereindex für die Gruppe der sehgeschädigten Personen liegt damit bei einem Wert von 5 und ist unter den Gruppen mit Einfachbehinderung der zweithöchste nach der Gruppe der prälingual Hörgeschädigten.

\section{b. Prälingual Hörgeschädigte}

Bei prälingual hörgeschädigten Personen liegt eine auditive Sinnesbarriere vor (1), die dazu führt, dass phonisch realisierte Texte respektive Inhalte nicht wahrgenommen werden können (1). In Kapitel 3 wurde aufgezeigt, dass auch mit Blick auf grafisch realisierte Inhalte eine Barriere besteht (1), denn durch den unangemessenen Umgang der Gesellschaft mit dieser Form von Behinderung liegt bei gehörlosen Personen häufig auch eine gravierende Leseeinschränkung vor. Inhalte von fachlicher (1) sowie fachsprachlicher (1) Prägung stellen die Adressatenschaft vor große Herausforderungen, da sehr häufig die für die Texterschließung notwendigen Frames und Scripts zu fachlichen Themen nicht angelegt sind (s. Kap. 3, Bredel/Maaß 2016, Kap. 5.2.6). Die Art der Einschränkung determiniert den präferierten Zugang zu Informationen über bestimmte Medien, weshalb Texte, die nicht in entsprechend medial aufbereiteter Weise (z. B. DGS-Verdolmetschung, DGS-Video, verständlichkeitsoptimierte Sprache) vorliegen, schlicht nicht rezipiert werden können (1). Neben der Sinnes- (1), der Fach- (1), der Fachsprachen- (1) sowie der Medienbarriere in ihren verschiedenen Ausprägungsformen phonisch, grafisch, Medium $(1+1+1)$ sind auch die Sprach- (1) und die Kulturbarriere (1) betroffen, denn Gehörlose als Angehörige der Gehörlosen-Gemeinschaft unterscheiden sich in Sprache und Kultur von den Mitgliedern der Mehrheitsgesellschaft (s. Kap. 3). Einzig die Kognition ist bei gehörlosen Personen nicht eingeschränkt (0), sodass sie üblicherweise voll geschäftsfähig sind (vgl. Bredel/Maaß 2016: 159 und Maaß/Rink/Zehrer 2014: 59).

Der Blick in die Tabelle zeigt, dass der BI dieser Gruppe mit 8 von 10 möglichen Punkten der höchste unter den Gruppen mit einfacher Beeinträchtigung ist.

\section{c. Personen mit Hör- und Sehbehinderung}

Bei Personen mit einer Hör- und Sehbehinderung sind zwei Sinnesorgane als Wahrnehmungskanäle zur Aufnahme von Information eingeschränkt, weshalb 
die Spezifik dieser Gruppe in ihrer Mehrfachbehinderung liegt; es treffen die Ausprägungen zweier Spalten zu: Hörschädigung und Sehschädigung. Der Barriereindex dieser Adressatengruppe ist deshalb mit einem Wert von 9 besonders hoch. In Abhängigkeit von der Ausprägung der Einschränkung liegen eine visuelle und eine auditive Sinnesbarriere (2), eine Fach- (1), eine Fachsprachen- (1), eine Kultur- (1) sowie eine Sprachbarriere (1) vor. Mit Blick auf die Medienbarriere in ihren verschiedenen Ausprägungsformen ist einzig die haptische Realisierung (0) nicht betroffen, weshalb Kommunikation insbesondere über diesen Kanal erfolgen muss. Die Anforderungen an die sprachlichen, medialen und konzeptuellen Strategien sind aufgrund des besonderen Anforderungsprofils dieses Personenkreises besonders ausgeprägt.

\section{d. Personen mit geistiger Behinderung}

Bei dieser Adressatenschaft kann weder eine Sinnes- (0) noch eine Kulturbarriere (0) konstatiert werden; die Barriere liegt vielmehr im Bereich der Kognition (1). Personen mit geistiger Behinderung sind i.d.R. dazu in der Lage, sich mittels Gemeinsprache in den sie betreffenden Lebensbereichen kommunikativ zu orientieren. Probleme bezogen auf die Sprache entstehen v.a. dann, wenn Informationen in sprachlich oder in sprachlich und inhaltlich komplexer Weise realisiert sind (0.5). Diese Schwierigkeiten sind umso ausgeprägter, wenn es um die Aufnahme und Verarbeitung komplexer Gegenstände geht, denn oft ist die Natur der Beeinträchtigung dergestalt, dass Fach- (1) sowie Fachsprachenbarriere (1) nicht selbständig überwunden werden können (vgl. Kap. 3 und Rink in Vorbereitung). Mit Blick auf die Medienbarriere stellt nur eine von drei Ausprägungsformen Probleme dar, die die grafische Realisierung betrifft: Da ein Großteil der Personen mit geistiger Behinderung den Nicht-Leser(inne)n angehört, wird insbesondere Schrift als Träger von Information für diesen Teil der Adressatenschaft zur unüberwindbaren Hürde (1) (s. Kap. 3 und Rink in Vorbereitung). Inwiefern mit Blick auf die Kategorie „Medium“ eine Barriere vorliegt, wäre mittels Nutzungsstudien zu prüfen. Der Barriereindex der Personen mit geistiger Behinderung erreicht nach dieser Kalkulation einen Wert von 4.5. 


\section{e. Personen mit Lernschwierigkeiten}

Personen mit Lernschwierigkeiten haben sehr häufig Probleme bei der Aufnahme und Verarbeitung von grafisch realisierten Inhalten (1). Die Schwierigkeiten wachsen sich aus, wenn die Inhalte zudem eine fachliche (1) sowie eine fachsprachliche Prägung (1) aufweisen. Betroffene finden ohne die Hilfe Dritter häufig keinen Zugang zu solchen Inhalten (s. Kap. 3 und Rink in Vorbereitung). Von diesen drei Barrieretypen abgesehen, ist die Gruppe nicht von weiteren Kommunikationsbarrieren betroffen, weshalb der Barriereindex dieser Gruppe bei 3 liegt.

Im Vergleich fällt auf, dass sich Personen mit Lernschwierigkeiten und Personen mit geistiger Behinderung hinsichtlich der Barrieretypen Sprache und Kognition voneinander unterscheiden: Während die Sprach- (0) und die Kognitionsbarriere (0) auf Personen mit Lernschwierigkeiten nicht zutreffen, liegen aufgrund der kognitiven Einschränkung bei Personen mit geistiger Behinderung Restriktionen hinsichtlich beider Barrieretypen $(0,5+1)$ vor, die insbesondere den Zugang zu Text betreffen.

\section{f. Personen mit demenziellen Erkrankungen}

Bei Personen, die an demenziellen Erkrankungen leiden, ist die Art der Einschränkung in erheblicher Weise vom Stadium der Krankheit abhängig. Die Ausprägungen zu Beginn der Krankheit sind also weniger gravierend als mit fortschreitendem Krankheitsverlauf; die Einschränkungen verstärken sich und Angehörige dieser Gruppe scheiden sukzessive aus der Rezeption von Kommunikationsangeboten aus. Aus diesem Grund liegt der Fokus hier auf der Betrachtung der leichtgradigen und mittelschweren Formen von demenziellen Erkrankungen. Bei diesem Personenkreis kann weder eine Sinnes- (0) noch eine Kulturbarriere (0) festgestellt werden. Da jedoch infolge von Zellabbauprozessen verschiedene Fähigkeiten allmählich verloren gehen, die insbesondere die Kognition (1) und mit zunehmender Ausprägung auch die Sprache (0.5) betreffen, sind u.a. Gedächtnisstörungen sowie Veränderungen der Sprach- und Sprechweise die Folge (s. Kap. 3). Ebenso können fachliche (1) sowie fachsprachliche Informationen (1) aufgrund ihrer Komplexität nicht mehr hinreichend verarbeitet werden. Mit Blick auf die Medienbarriere sind die phonische 
(0) und die grafische (0) Realisierungsform zunächst nicht eingeschränkt, jedoch ist bezüglich des Mediums selbst eine Barriere zu konstatieren, die die zu Beginn dieses Kapitels erwähnten Faktoren Alter, Infrastruktur und präferierte Nutzung von Medien respektive Endgeräten betrifft: Inhalte sind nur dann rezipierbar, wenn sie in einer medial zugänglichen Weise vorliegen (vgl. Mollenkopf/Doh 2002 und Rink in Vorbereitung).

Insgesamt liegt der Barriereindex dieser Adressatenschaft bei 4.5, wobei ein Blick in die Tabelle zeigt, dass sich dieser Wert mit dem der Gruppe der Personen mit geistiger Behinderung deckt. Eine Abweichung lässt sich lediglich in Bezug auf die Ausprägungsformen der Medienbarriere konstatieren: Während Personen mit demenziellen Erkrankungen auf Inhalte, die bestimmte Endgeräte erfordern oder nicht in präferierter Weise vorliegen, keinen Zugriff haben, stellt für Personen mit geistiger Behinderung v.a. die grafische Realisierung eine Barriere dar.

\section{g. Personen mit Aphasie}

An Aphasie erkrankte Personen sind in Abhängigkeit von Ausprägung und Dauer der Erkrankung v.a. in den sprachlichen Modalitäten (Sprechen, Verstehen, Lesen, Schreiben, s. Kap. 3) eingeschränkt. Daraus lassen sich Barrieren bezogen auf die Sprache (1), das Fach (1), die Fachsprache (1) sowie die phonische $(0,5)$ und die grafische $(0,5)$ Realisierung von Inhalten ableiten. Für die übrigen Barrieretypen lassen sich zunächst keine Einschränkungen feststellen. Je nach Alter der betroffenen Personen kann eine Barriere in Bezug auf das Medium hinzukommen.

Diese Gruppe erreicht damit einen BI von 4 von 10 möglichen Punkten.

\section{h. Personen mit Deutsch als Zweitsprache}

Personen mit Deutsch als Zweitsprache gehören einer anderen Sprach- (1) und Kulturgemeinschaft (1) an, weshalb hier beide entsprechenden Barrieretypen zutreffen. Aus den Einschränkungen bezogen auf die Sprache resultieren auch Konsequenzen hinsichtlich der Inhalte, die eine fachliche (1) und/oder eine fachsprachliche (1) Prägung aufweisen. Zum Verstehen komplexerer Texte ist nämlich ein bestimmtes Maß an (schrift-)sprachlicher Kompetenz die Voraus- 
setzung (vgl. Bredel/Maaß 2016, Kap. 5.2.9). Für die übrigen Barrieretypen können keine Einschränkungen konstatiert werden, sodass der Barriereindex dieser Gruppe bei 4 liegt.

\section{i. Funktionale Analphabet(inn)en}

Zuletzt rückt die heterogene Gruppe der funktionalen Analphabet(inn)en in den Fokus der Betrachtungen, die - wie in Kapitel 3 beschrieben - eine Querstruktur (Bredel/Maaß 2016: 166) zu den übrigen Adressatengruppen bildet. Diese Gruppe erreicht einen Barriereindex von 3, denn Fachlichkeit (1) und Fachsprache (1) sowie Inhalte, die in grafischer Realisierung vorliegen (1), stellen diese Adressatenschaft vor Herausforderungen in Hinblick auf Perzeptibilität und Verständlichkeit von Texten. Die übrigen Barrieretypen treffen auf funktionale Analphabet(inn)en nicht zu, wenn man davon ausgeht, dass keine weitere Einschränkungsart vorliegt.

Im Vergleich zeigt sich, dass diese Gruppe den relativ geringen Barriereindex von 3 von 10 möglichen Punkten mit der Gruppe der Personen mit Lernschwierigkeiten teilt (s. Tabelle 2 ).

\subsection{Barriereprofil}

Den verschiedenen Zeilen der Tabelle ist zu entnehmen, inwieweit die unterschiedlichen Kommunikationsbarrieren eine Einschränkung bezogen auf die heterogenen Anforderungsprofile der ausgewählten Adressatengruppen darstellen. Das Barriereprofil ermöglicht einen vergleichenden Blick hinsichtlich der Ausprägung der einzelnen Barrieretypen quer durch die verschiedenen Adressatengruppen. Anhand des Barriereprofils lässt sich also ermitteln, welche Barrieren mit Blick auf eine barrierefreie Umsetzung von Text immer zu bearbeiten respektive zu kompensieren sind (s. Kap. 5).

\section{Barrieretyp: Sinnesbarriere}

Mit Blick auf die Tabelle stellen Texte, die nicht in multimodal aufbereiteter Weise vorliegen, eine Barriere für prälingual Hörgeschädigte und sehgeschädigte Personen sowie für Personen mit Hör- und Sehschädigung dar. Denkbar sind darüber hinaus Beeinträchtigungen bezüglich der Haptik, etwa wenn durch eine 
Lähmung Informationen nicht ertastet werden können. Bei allen diesen Arten von Beeinträchtigungen können Informationen bestimmter Modalität nicht aufgenommen werden, da die Ebene der Perzeption restringiert ist. In den vergangenen Jahren wurden verstärkt gesetzliche Maßnahmen getroffen, die sich auf die Absenkung von derartigen Sinnesbarrieren richten (s. hierzu Lang in vorliegendem Band und Rink in Vorbereitung). Die UN-Behindertenrechtskonvention (2009) sowie das novellierte Behindertengleichstellungsgesetz (2016) zielen konkret auf die gleichberechtigte Teilhabe von Menschen mit Behinderung ab und rücken ihre Bedarfe als gesellschaftspolitische Aufgabe in den Fokus. Liegt eine Mehrfachbehinderung vor, etwa im Sinne einer Taubblindheit oder einer mit Lähmung einhergehenden Hör- und/oder Sehbeeinträchtigung (zu schweren Formen der Mehrfachbehinderung und korrelierenden Formen der Barrierefreien Kommunikation s. den Beitrag von Folta-Schoofs im vorliegenden Band), dann müssen die Texte mit Bezug auf alle diese Beeinträchtigungen bearbeitet werden, da sie sonst nicht perzipiert werden können. Hier kommen insbesondere mediale Strategien in Betracht.

Für die übrigen in der Tabelle berücksichtigten Adressat(inn)en kann hinsichtlich der Sinnesbarriere keine Einschränkung festgestellt werden. Dies schließt jedoch nicht aus, dass zusätzliche Rezeptionsvarianten, i.e. auditiv, visuell und haptisch, einen Benefit für Vertreter(innen) dieser Gruppen darstellen würden.

\section{Barrieretyp: Fachbarriere}

Während die Sinnesbarriere insbesondere für hör- und sehschädigte Personen eine Barriere darstellt, betrifft die Fachbarriere alle in der Tabelle aufgeführten Adressatengruppen. Personen mit geistiger Behinderung, Personen mit Lernschwierigkeiten, von demenziellen Erkrankungen oder Aphasien Betroffene, prälingual Hörgeschädigte, Personen mit Deutsch als Zweitsprache und sehgeschädigte Personen können auf Fachinhalte häufig nicht zugreifen, weil fachliches bzw. inhaltliches Wissen für die Texterschließung Voraussetzung ist (s. Kap. 3 und Rink in Vorbereitung). Inhalte, die der fachexternen Kommunikation zugehören, liegen mehrheitlich über dem Anforderungsprofil der Rezipientenschaft. 


\section{Barrieretyp: Fachsprachenbarriere}

Ebenso wie die Kategorie Fach birgt auch die Dimension Fachsprache für sämtliche Adressat(inn)en in der Tabelle Verstehensschwierigkeiten (s. Tabelle), weil Aussageninhalte, die in einer speziellen Fachsprache formuliert sind und die der Kommunikation unter Expert(inn)en dient (vgl. Kalverkämper 1998b: 34f.), i.d.R. fachfremde Rezipient(inn)en vor Herausforderungen bei der Rezeption stellen (s. Kap. 3 und Rink in Vorbereitung).

\section{Barrieretyp: Kulturbarriere}

Im Kontrast zur Fach- und zur Fachsprachenbarriere stellt die Kulturbarriere v.a. für Personen mit prälingualer Hörschädigung sowie für Personen mit Deutsch als Zweitsprache eine Barriere dar, weil Angehörige beider Gruppen jeweils anderen (dia- oder parakulturellen) Gemeinschaften angehören (s. Kap. 3). Inhalte können deshalb nicht verstanden oder missverstanden werden, weil kulturgebundene Frames (vgl. Bredel/Maaß 2016, Fischer 2011, Rickheit et al. 2010 und Groeben 1982), die zur Auflösung der Textsemantik notwendig sind, nicht angelegt sind. Zwar kann kulturelles Wissen erworben werden, jedoch bedarf es dafür entsprechend aufbereiteter Texte (s. Kap. 5).

\section{Barrieretyp: Kognitionsbarriere}

Die Kognitionsbarriere stellt insbesondere Rezipient(inn)en mit kognitiven Beeinträchtigung vor Herausforderungen bei der Aufnahme und Verarbeitung von Informationen; gemäß Tabelle sind Personen mit geistiger Behinderung und Personen mit demenziellen Erkrankungen betroffen. Aufgrund der Natur der Beeinträchtigung, die angeboren oder erworben sein kann (vgl. Bredel/ Maaß 2016 Kap. 5.2), können sprachlich und/oder inhaltlich komplexe Sachverhalte nicht (mehr) oder nicht in Gänze verarbeitet werden. Das spezifische Anforderungsprofil erfordert also eine Textpraxis, die solchen Bedarfen mit Blick auf die Kognitionsbarriere Rechnung trägt. Eine Anpassung der Rechtslage (s. Lang in vorliegendem Band) ist ein Schritt in diese Richtung. 


\section{Barrieretyp: Sprachbarriere}

Sprache als komplexes Gebilde aus verschiedenen Zeichenressourcen stellt eine Hürde für viele Adressat(inn)en dar. Während Personen mit Deutsch als Zweitsprache und prälingualer Hörschädigung eine von der deutschen Laut- und Schriftsprache abweichende Erstsprache haben und deshalb auf Herausforderungen in Bezug auf die Kategorie Sprache stoßen, liegt das Problem bei Aphasie, Demenz und geistiger Behinderung vielmehr in der Erkrankung respektive Beeinträchtigung begründet, die den Umgang mit Sprache an sich in erheblicher Weise erschweren kann (s. Kap. 4.1 und Rink in Vorbereitung). Die Barriere ist in Abhängigkeit von der Komplexität des sprachlichen Codes mehr oder minder stark ausgeprägt.

\section{Barrieretyp: Medienbarriere}

Die auf die mediale Realisierung von Inhalten fokussierende Medienbarriere kann verschiedene Ausprägungen in den Bereichen von Codalität, Modalität und Medium aufweisen.

Die phonische Realisierungsform erweist sich für prälingual Hörgeschädigte als Barriere, weil die Ebene der Perzeption eingeschränkt ist. Für an Aphasie Erkrankte kann die phonische Realisierung Hürden in Bezug auf die Verständlichkeit von Informationen bergen.

Demgegenüber stellt die grafische Realisierung eine Barriere für fast alle Adressat(inn)en der Tabelle dar: Personen mit geistiger Behinderung, Personen mit Lernschwierigkeiten, prälingual Hörgeschädigte, an Aphasie Erkrankte, Personen mit Sehschädigung und funktionale Analphabet(inn)en weisen Einschränkungen hinsichtlich dieser Kategorie auf (s. Kap. 3). Weil die Rezipient(inn)en mit Bezug auf das Deutsche eine verminderte Schriftsprachkompetenz aufweisen, sind verbale Inhalte, die ausschließlich in grafisch realisierter Weise vorliegen, nicht oder nicht vollumfänglich zugänglich.

Informationen, die nicht in haptischer Realisierung vorliegen, stellen sehgeschädigte Personen vor Herausforderungen bezüglich der Perzeption; für Personen mit kombinierter Hör- und Sehschädigung ist die haptische Informationsaufnahme sogar vorrangig. Da das Auge als Wahrnehmungskanal für die Informationsaufnahme nicht zur Verfügung steht, müssen Inhalte in entsprechend haptischer und/oder phonischer Realisierung aufbereitet sein. 
Das Medium als Überträger von Information stellt eine Herausforderung hinsichtlich der Rezeption für von demenziellen Erkrankungen Betroffene, prälingual Hörgeschädigte und sehgeschädigte Personen dar. Hör- und sehgeschädigte Personen weisen bezogen auf diese Kategorie eine Ausprägung auf, weil die Natur ihrer Behinderung den präferierten Zugang zu Informationen über bestimmte Medien determiniert (z. B. Videos mit Untertiteln/Audiodeskription, Texte in Brailleschrift, Gebärdensprachverdolmetschung, etc.). Personen mit demenziellen Erkrankungen weisen bezüglich dieser Kategorie i.d.R. eine Einschränkung auf, die aus den Faktoren Alter, Infrastruktur respektive präferierte Nutzung von Medien/Endgeräten resultiert (vgl. Mollenkopf/Doh 2002). Stellt das Medium eine Hürde dar, so können die entsprechenden Inhalte nicht perzipiert werden.

Im Vergleich lässt sich feststellen, dass insbesondere hör- und sehgeschädigte Personen von der Medienbarriere betroffen sind, weil Inhalte, die nicht in multimodal aufbereiteter Weise vorliegen, aufgrund der Einschränkung (mindestens) eines Sinnesorgans nicht perzipiert werden können (jeweils 3 von 4 Ausprägungen). Bezogen auf die einzelnen Ausprägungsformen der Medienbarriere lässt sich konstatieren, dass die grafische Realisierung mit 5.5 Punkten die größte Hürde darstellt, weil Inhalte, die in Form von Text realisiert sind, von vielen Adressat(inn)en mit eingeschränkter Schriftsprachkompetenz nicht aufgenommen und verarbeitet werden können. Demgegenüber stellen Informationen, die nicht in haptischer Form realisiert sind, Personen mit Sehschädigung bzw. Personen mit kombinierter Hör- und Sehschädigung vor Herausforderungen bei der Perzeption.

\subsection{Barriereindex}

Im Anschluss an die qualitative Betrachtung der Korrelation von Kommunikationsbarrieren und Beeinträchtigungsformen soll der Blick nun auf das quantitative Ausmaß der Einschränkung gerichtet werden: Der Barriereindex als Ergebnis der Verschränkung von Adressat(inn)enebene (horizontal) mit Barrieretypenskala (vertikal) zeigt, dass der Barriereindex respektive das Anfor- 
derungsprofil einer Adressatengruppe umso höher ist, je mehr Kommunikationsbarrieren für eine Adressatenschaft bestehen. Mit anderen Worten: Je mehr Barrieretypen den Zugang zu Information restringieren, desto umfassender müssen die Strategien zur Kompensation sein, die bezogen auf eine barrierefreie und adressatengerechte Kommunikation Anwendung finden.

Von den in Tabelle 2 betrachteten Adressatengruppen weist die Gruppe der prälingual Hörgeschädigten mit einem Wert von 8 den höchsten Barriereindex auf: Sie sind - bezogen auf die Rezeption von Inhalten - von sämtlichen Barrieren betroffen; lediglich die Kognition stellt für diese Adressatenschaft keine Hürde dar. Gehörlose Personen haben jedoch aufgrund ihrer Beeinträchtigung besonders ausgeprägte Anforderungen an perzipierbare und verständliche Texte. Da bei dieser Rezipientenschaft keine Einschränkung der kognitiven Fähigkeiten konstatiert werden kann, sind die anzuwendenden Kompensationsstrategien zur Ermöglichung kommunikativer Teilhabe v. a. sprachlicher und medialer Art (s. Kap. 5); konzeptuelle Strategien kommen zum Einsatz, wenn über die Texte Wissensbestände neu angelegt werden müssen, die bei den Ausgangstexten vorausgesetzt werden.

Mit einem Barriereindex von 5 erreichen Personen mit Sehschädigung den zweithöchsten Wert gemäß Tabelle. Aus der Natur der Behinderung resultiert eine besondere Anforderung hinsichtlich der sprachlichen und medialen Gestaltung von Informationen, die v.a. auf die phonische und haptische Realisierung von Texten zielt (s. Kap. 5). Aber auch konzeptuelle Strategien greifen, beispielsweise, wenn der Kontext, in dem ein Text steht, anzumoderieren ist oder Text ausgelagert wird (z. B. Audioeinführung).

Liegt bei Personen eine Hör- und Sehbehinderung vor, so sind sprachliche, mediale und konzeptuelle Strategien anzuwenden, um Text perzipierbar und verständlich vorzuhalten.

Einen Barriereindex von 4.5 erreichen Personen mit geistiger Behinderung und Personen mit der Krankheit Demenz. Während Angehörige beider Gruppen von der Fach-, der Fachsprachen-, der Kognitions- sowie der Sprachbarriere betroffen sind und diesbezüglich auf Herausforderungen bei der Rezeption stoßen, divergieren ihre Anforderungen mit Blick auf die Medienbarriere: Personen mit geistiger Behinderung haben häufig Probleme mit der grafischen Realisierung von Text und ein Großteil dieser Gruppe beherrscht das verstehende 
Lesen nicht (vgl. Bredel/Maaß 2016, Ratz 2013 und Schuppener/Bock in vorliegendem Band, die jedoch auf das große Spektrum in der Ausprägung der Lesefähigkeit dieser Gruppe verweisen). Im Gegensatz dazu haben Personen mit demenziellen Erkrankungen i. d. R. einen regulären Sprach- und Schriftspracherwerb durchlaufen, scheitern jedoch bezüglich des Zugangs zu Information häufig am Medium selbst (vgl. Mollenkopf/Doh 2002). Faktoren wie Alter, Infrastruktur bzw. präferierte Nutzung determinieren den Umgang mit Information, weshalb für diese Rezipientenschaft Inhalte, die nur mittels bestimmter Medien konsumiert werden können (z. B. neuere Medien), mitunter nicht zugänglich sind. Um diesen Adressatengruppen Kommunikationsangebote vorhalten zu können, die perzipierbar, verständlich und handhabbar sind, bedarf es medialer, sprachlicher sowie konzeptueller Strategien (s. Kap. 5).

Für Personen mit Aphasie sowie für Personen mit Deutsch als Zweitsprache liegt der Barriereindex bei 4; er konvergiert für Angehörige beider Gruppen hinsichtlich der Barrieretypen Fach, Fachsprache sowie Sprache (s. Tabelle 2). Eine Divergenz liegt hinsichtlich der übrigen Barrieretypen vor: Wenn es um die Perzeption phonisch bzw. grafisch realisierter Inhalte geht, scheitern Personen mit Aphasie häufig aufgrund ihrer Erkrankung an diesen Ausprägungen der Medienbarriere. Personen mit Deutsch als Zweitsprache sind diesbezüglich weniger eingeschränkt; vielmehr liegt die Hürde für sie in der Absenz von bestimmten Beständen kulturellen Wissens, das für das Verständnis von Information notwendig ist; hier kommen konzeptuelle Strategien zum Einsatz. Um den kommunikativen Bedarfen dieser heterogenen Gruppen gerecht zu werden, bedarf es mit Blick auf eine Barrierefreie Kommunikation aber v.a. auch medialer und sprachlicher Strategien (s. Kap. 5).

Der geringste Barriereindex mit einem Wert von 3 wurde für Personen mit Lernschwierigkeiten sowie für funktionale Analphabet(inn)en festgestellt werden. Angehörige beider Gruppen sehen sich Schwierigkeiten gegenüber, wenn Inhalte eine fachliche und/oder fachsprachliche Prägung aufweisen und zudem in grafischer Realisierung vorliegen. Um hier kommunikative Teilhabe zu gewährleisten, müssen insbesondere mediale und sprachliche Strategien zum Tragen kommen (s. Kap. 5). Mit Bezug auf Wissensbestände, die in den Ausgangstexten vorausgesetzt werden und in den barrierefreien Themen neu angelegt werden, kommen auch konzeptuelle Strategien zum Tragen. 
Im Rahmen der quantitativen Auswertung wurde deutlich, dass den sieben Barrieretypen in ihren verschiedenen Ausprägungsformen drei grundlegende Strategien zur Kompensation gegenüberstehen. Diese werden im folgenden Kapitel betrachtet.

\section{Strategien zur Kompensation von Kommunikationsbarrieren}

Die in Kapitel 2 vorgestellten Barrieretypen beeinträchtigen die kommunikative Teilhabe bezogen auf die Perzeption von Inhalten und/oder mit Blick auf ihre Verständlichkeit. Ihnen stehen drei Strategien zur Kompensation entgegen; diese sind sprachlicher, medialer und konzeptueller Art.

$\mathrm{Zu}$ den sprachlichen Strategien zählen all jene Maßnahmen, die dazu beitragen, die Verständlichkeit von Inhalten auf Wort-, Satz- und Textebene zu erhöhen. Das betrifft z. B. die Verwendung zentraler Wörter und Satzstrukturen mit überschaubarer Buchstabenlänge respektive Anzahl von Lexemen je Satz, die Darstellung von Sachverhalten mittels anschaulicher Beispiele, die Adressierung, die Situierung sowie die Handlungsorientierung oder die Einführung und Erläuterung von komplexen, abstrakten bzw. insgesamt fachlichen Konzepten (vgl. Bredel/Maaß 2016: 520ff.). Im vorliegenden Band wird in den Beiträgen von Hennies, Bredel/Maaß, Maaß, Mälzer/Wünsche, Benner/Herrmann, Otero Moreno, Zehrer und Schwengber auf mögliche sprachliche Strategien zur barrierefreien Aufbereitung von Medientexten eingegangen.

Die medialen Strategien zielen insbesondere auf die Perzeption, d. h. die Wahrnehmbarkeit der Textoberfläche. Verschiedene Mittel der typografischen Gliederung (Einrückung, Mediopunkt, Hervorhebung) und Vernetzung (Bebilderung, Color Coding) können eingesetzt werden (Bredel/Maaß 2016: 520ff.), um die Perzeptibilität und damit die Informationsaufnahme zu befördern. Stehen Sinnesorgane als Wahrnehmungskanäle zur Aufnahme und Verarbeitung von Information nicht zur Verfügung, müssen alternative Kanäle bedient bzw. angewählt werden (z. B. Untertitel, Audiodeskription, Alternativtexte für Grafiken, Brailleschrift, Text-to-Speech-Systeme, Audiospur, QR-Code, Blicksteuerung, Talker, Lormen). 
Die medialen Strategien zielen damit auf die multimodale Aufbereitung von Text. Im vorliegenden Band widmen sich die Beiträge von Alexander, Dobroschke/Kahlisch, Witzel, Folta-Schoofs, Schütt, Kurch, Benecke, Mälzer/ Wünsche, Pridik, Hellbusch, Womser-Hacker, Capovilla und Rantamo/Schum solchen medialen Strategien.

$\mathrm{Zu}$ den konzeptuellen Strategien zählen solche Maßnahmen, die auf die Absenkung der kognitiven Komplexität von Inhalten zielen. Dies kann einerseits mittels sprachlicher und medialer Strategien erreicht werden, die zur Anwendung kommen, oder sie können sich andererseits auf strukturelle Eingriffe beziehen, die die Art und Weise der Informationsdarbietung betreffen (vgl. Christmann/Groeben in vorliegendem Band). Dazu zählen bspw. Metatexte, die i.S.e. Advance Organizers Informationen über ein Kommunikationsangebot vorhalten bzw. einen Digest über dieses liefern (z. B. Audioeinführungen, Leichte-Sprache-Zusammenfassungen etc.) oder die Inhalte eines Texts in komprimierter Form darstellen. Ebenso ist es möglich, dass Inhalte im Zuge der multimodalen Aufbereitung das Medium wechseln; so kann beispielsweise ein grafisch realisierter Text zu einem Video oder ein Audioangebot grafisch realisiert werden. Im vorliegenden Band gehen die Beiträge von Christmann/ Groeben, Lutz, Maaß, Mälzer/Wünsche, Musenberg, Schädler, Hellbusch und Heidrich auf konzeptuelle Strategien der Aufbereitung von Medientexten ein. 
Isabel Rink

\section{Literaturverzeichnis}

Allgemeiner Blinden- und Sehbehindertenverein Berlin (2017): Sehbehinderungs-Simulator. <http://www.absv.de/sehbehinderungs-simulator> (Verifizierungsdatum: 30.03.2018)

Bundesarbeitsgemeinschaft der Taubblinden e.V. (2008): Taktiles Fingeralphabet - Erläuterung. <http://bundesarbeitsgemeinschaft-taubblinden.de/?page_id=214> (Verifizierungsdatum: 30.03.2018)

Bieling, Tom/ Gollner, Ulrike/ Joost, Gesche (2012): „Schnittstelle Hand - Kommunikation mit Gefühl“. In: Journal of Interactive Media, 11/2, 32-36.

Bouvet, Danielle (1990): The Path to Language. Bilingual Education for Deaf Children. Clevedon: Multilingual Matters LDT.

Bredel, Ursula/MaAß, Christiane (2016): Leichte Sprache. Theoretische Grundlagen. Orientierung für die Praxis. Berlin: Dudenverlag (Sprache im Blick).

Bromme, Rainer/Jucks, REgina/RAmbow, RiKLEF (2004): „Experten-Laien-Kommunikation im Wissensmanagement“. In: Reinmann, Gabi/MandL, Heinz (Hrsg.): Der Mensch im Wissensmanagement: Psychologische Konzepte zum besseren Verständnis und Umgang mit Wissen. Göttingen: Hogrefe, 114-126.

BrotzMann, Hans (2004): „Kommunikationshilfen ermöglichen Teilhabe“. In: Schlenker-Schulte, Christa (Hrsg.): Barrierefreie Information und Kommunikation. Hören Se hen - Verstehen in Arbeit und Alltag. Villingen-Schwenningen: Neckar-Verlag $\mathrm{GmbH}, 63-78$.

Bundesministerium der Justiz und für Verbraucherschutz (2017a): Einkommensteuergesetz (EStG) <https://www.gesetze-im-internet.de/estg/BJNR010050934.html> (Verifizierungsdatum: 30.03.2018)

Bundesministerium der Justiz und für Verbraucherschutz (2017b): Sozialgesetzbuch Neuntes Buch - Rehabilitation und Teilhabe von Menschen mit Behinderungen (Neuntes Buch Sozialgesetzbuch - SGB IX) <https://www.gesetze-im-internet.de/ sgb_9_2018/BJNR323410016.html> (Verifizierungsdatum: 30.03.2018)

Bundesministerium der Justiz und für Verbraucherschutz (2017c): Sozialgesetzbuch (SGB) Zwölftes Buch (XII). <https://www.gesetze-im-internet.de/sgb_12/ BJNR302300003.html> (Verifizierungsdatum: 30.03.2018)

Bundesministerium der Justiz und für Verbraucherschutz (2016): Gesetz zur Gleichstellung von Menschen mit Behinderungen (Behindertengleichstellungsgesetz - BGG). <https://www.gesetze-im-internet.de/bgg/BJNR146800002.html> (Verifizierungsdatum: 30.03.2018)

Bundeszentrale für politische Bildung (2016): Datenreport 2016. Ein Sozialbericht für die Bundesrepublik Deutschland. <https://www.destatis.de/DE/Publikationen/ Datenreport/Downloads/Datenreport2016.pdf?_blob=publicationFile > (Verifizierungsdatum: 30.03 .2018 ) 
Burger, Harald (2005): Mediensprache. Eine Einführung in Sprache und Kommunikationsformen der Massenmedien. 3., völlig neu bearb.Aufl. Berlin u.a.: De Gruyter.

Christmann, Ursula (2006): „Textverstehen“. In: Funke, JoACHim/Frensch, Peter A. (Hrsg.): Handwörterbuch Allgemeine Psychologie: Kognition. Göttingen: Hogrefe, 612-620.

DEgENHARDT, SVEN (2007): „Blindheit und Sehbehinderung.“ In: BORCHERT, JOHANN (Hg.): Einführung in die Sonderpädagogik. München, Wien: Oldenbourg, 39-76.

Deutsche Gesellschaft für Taubblindheit (2014): Startseite. < https://www.gesellschafttaubblindheit.de> (Verifizierungsdatum: 30.03.2018)

Deutscher Blinden- und Sehbehindertenverband e.V. (2018): Zahlen \& Fakten. <https://www.dbsv.org/zahlen-fakten-669.html> (Verifizierungsdatum: 30.03.2018)

Deutscher Gehörlosen-Bund e.V. (o.J.): Gehörlosigkeit. <http://www.dglb.de/dgb/ index.php?option=com_content\&view=category\&id=38\&layout=blog\&Itemid $=$ $101 \&$ lang $=$ de $>$ (Verifizierungsdatum: 30.03.2018)

Deutsches Institut für Menschenrechte (2018): Behindertenrechtskonvention. <http://www.institut-fuer-menschenrechte.de/fileadmin/user_upload/PDF-Dateien/ Pakte_Konventionen/CRPD_behindertenrechtskonvention/crpd_b_de.pdf $>$ (Verifizierungsdatum: 30.03.2018)

Deutsches Taubblindenwerk (o.J.): Lormen. <http://www.taubblindenwerk.de/haeufiggestellte-fragen/lormen/> (Verifizierungsdatum: 30.03.2018)

ECKHARDT, BIRGIT (2000): Fachsprache als Kommunikationsbarriere? Verständigungsprobleme zwischen Juristen und Laien. Wiesbaden: DUV (Dissertation).

FISCHER, SYLVIA (2011): Verständlichkeit von Bedienungsanleitungen: dysfunktionale Rezeption von Instruktionen. Dissertationsschrift Universität Mainz. $<$ http://ubm.opus.hbz-nrw.de/volltexte/2011/2968/pdf/doc.pdf> (Verifizierungsdatum: 30.03.2018)

ForNEFELD, BARBARA (2002): Einführung in die Geistigbehindertenpädagogik. 2. Aufl. München u.a.: Reinhardt.

FRÖHLICH, UTA (2015): Facework in multicodaler spanischer Forenkommunikation. Bd. 66. Berlin: De Gruyter (Linguistik - Impulse \& Tendenzen).

Groeben, NORBERT (1982): Leserpsychologie: Textverständnis - Textverständlichkeit. Münster: Aschendorff.

Grotlüschen, Anke/Riekmann, Wibke (Hrsg.) (2012): Funktionaler Analphabetismus in Deutschland. Ergebnisse der ersten leo. - Level-One Studie. Münster: Waxmann.

Grotlüschen, ANKe/RieKmanN, WibKe (2011): leo. - Level-One Studie. Presseheft. Literalität von Erwachsenen auf den unteren Kompetenzniveaus. <http://blogs.epb.unihamburg.de/leo/files/2011/12/leo-Presseheft_15_12_2011.pdf> (Verifizierungsdatum: 30.03.2018)

GRÖNING, KATHARINA (2012): SPRECHEN SIE DEMENZISCH? Eine Einführung und Vorbereitung auf die Wissenschaftliche Weiterbildung Demenz im Krankenhaus. Bielefeld: Fakultät für Erziehungswissenschaft. <https://www.uni-bielefeld.de/ 
Isabel Rink

erziehungswissenschaft/ag7/familiale_pflege/materialien/studienbriefe/StB_Sprechen_ Sie_Demenzisch-2012.pdf> (Verifizierungsdatum: 30.03.2018)

Heimlich, Ulrich (2016): Pädagogik bei Lernschwierigkeiten. Sonderpädagogische Förderung im Förderschwerpunkt Lernen. 2. Aufl. Bad Heilbrunn: Julius Klinkhardt.

HENNIES, JOHANNES (2009): Lesekompetenz gehörloser und schwerhöriger SchülerInnen: Ein Beitrag zur empirischen Bildungsforschung in der Hörgeschädigtenpädagogik. Diss. Humboldt Universität Berlin, Manuskript der Dissertationsschrift.

Huber, Walter/Poeck, Klaus/Springer, Luise (2006/2013): Klinik und Rehabilitation der Aphasie. Stuttgart u.a.: Thieme (Forum Logopädie).

KALVERKÄMPER, HARTWIG (1998a): „Fach und Fachwissen“. In: HofFMANN, LOTHAR/ KAlverkäMPER, HARTWIg/Wiegand, Herbert ERnst (Hg.): Fachsprachen. Ein internationales Handbuch zur Fachsprachenforschung und Terminologiewissenschaft. 1. Halbband. Berlin: De Gruyter, 1-24.

KALVERKÄMPER, HARTWIG (1998b): „Rahmenbedingungen für die Fachkommunikation“. In: HofFmann, Lothar/Kalverkämper, Hartwig/Wiegand, HerberT ERNST (Hg.): Fachsprachen. Ein internationales Handbuch zur Fachsprachenforschung und Terminologiewissenschaft. 1. Halbband. Berlin: De Gruyter, 24-43.

Koch, Peter/Oesterreicher, Wulf (1985/1994): „Sprache der Nähe - Sprache der Distanz. Mündlichkeit und Schriftlichkeit im Spannungsfeld von Sprachtheorie und Sprachgeschichte“. In: Romanistisches Jahrbuch 36, 15-43.

KRAMMER, KLAUDIA (2001): Schriftsprachkompetenz gehörloser Erwachsener. <http://www.uni-klu.ac.at/zgh/downloads/krammer.pdf> (Verifizierungsdatum: 30.03.2018)

KurZ, AleXAnder (2013): Das Wichtigste über die Alzheimer-Krankheit und andere Demenzformen. Ein kompakter Ratgeber. 23. aktualisierte Auflage. Berlin: Deutsche Alzheimer Gesellschaft e. V.

LÜTKE, BEATE (2011): Deutsch als Zweitsprache in der Grundschule. Eine Untersuchung zum Erlernen lokaler Präpositionen. Bd. 2. DaZ-Forschung. Berlin/Boston: De Gruyter.

MaAß, Christiane/Rink, Isabel/Zehrer, Christiane (2014): „Leichte Sprache in der Sprach- und Übersetzungswissenschaft.“ In: JEKAT, Susanne J./JÜngST, HeIKE E./ Schubert, Klaus/Villiger, Claudia (Hrsg.): Sprache barrierefrei gestalten: Perspektiven aus der Angewandten Linguistik. Berlin: Frank \& Timme, 53-85 (TransÜD. Arbeiten zur Theorie und Praxis des Übersetzens und Dolmetschens Bd. 69).

Mollenkopf, Heidrun/DoH, Michael (2002): „Das Medienverhalten älterer Menschen". In: Sozialwissenschaften und Berufspraxis 25, 4, 387-408. <https://www.ssoar.info/ssoar/bitstream/handle/document/3790/ssoar-sub-2002-4mollenkopf_et_al-das_medienverhalten_alterer_menschen.pdf?sequence $=1>$

Netzwerk Leichte Sprache (o.J.): Das Prüfen. <https://www.leichte-sprache.org/daspruefen/> (Verifizierungsdatum: 30.03.2018)

Nonn, KeRstin/PÄßLER-VAN REY, DANIELA (2011): Unterstützte Kommunikation in der Logopädie. Stuttgart: Thieme. 
Prillwitz, Siegmund/Wudtke, Hubert (1988): Gebärden in der vorschulischen Erziehung gehörloser Kinder. 10 Fallstudien zur kommunikativ-sprachlichen Entwicklung gehörloser Kinder bis zum Einschulungsalter. Hamburg: Verl. Hörgeschädigte Kinder (Internationale Arbeiten zur Gebärdensprache und Kommunikation Gehörloser; Bd. 3).

RATZ, CHRistoph (2013): „Zur aktuellen Diskussion und Relevanz des erweiterten Lesebegriffs“. In: Empirische Sonderpädagogik. Nr. 4, 343-360.

RICKHEIT, GERT/WEISS, SABINE/EIKMEYER, HANS-JÜRGEN (2010): Kognitive Linguistik: Theorien, Modelle, Methoden. Stuttgart: A. Francke.

RINK, ISABEL (in Vorbereitung): Rechtskommunikation und Barrierefreiheit. Eine korpusgestützte Analyse der Übersetzung juristischer Informations- und Interaktionstexte in Leichte Sprache im Pilotprojekt „Leichte Sprache in der Niedersächsischen Justiz“ [Arbeitstitel]. Diss. Universität Hildesheim, Manuskript der Dissertationsschrift.

RÖSCH, HeIDI (2011): Deutsch als Zweit- und Fremdsprache. Berlin: Akademie.

Schaeffer, Doris/Vogt, Dominique/Berens, Eva-Maria/Hurrelmann, Klaus (2016): Gesundheitskompetenz der Bevölkerung in Deutschland. Ergebnisbericht. Bielefeld: Universität Bielefeld. <http://www.uni-bielefeld.de/gesundhw/ag6/ downloads/Ergebnisbericht_HLS-GER.pdf> (Verifizierungsdatum: 30.06.2018)

SCHINDELMEISER, JOCHEN (2016): Neurologie für Sprachtherapeuten. München: Elsevier.

SCHNOOR, HEIKE (2007): „Schwere Mehrfachbehinderung“. In: SCHNOOR, HEIKE (Hrsg.): Leben mit Behinderungen. Eine Einführung in die Rehabilitationspädagogik anhand von Fallbeispielen. Stuttgart: Kohlhammer, 247-249.

Schnotz, WolfGAng (1994): Aufbau von Wissensstrukturen: Untersuchungen zur Kohärenzbildung beim Wissenserwerb mit Texten. Vol. 20. Weinheim: Beltz. (Fortschritte der psychologischen Forschung).

SCHUBERT, KlaUs (2016): „Barriereabbau durch optimierte Kommunikationsmittel: Versuch einer Systematisierung“. In: Mälzer, NATHAliE (Hg.): Barrierefreie Kommunikation - Perspektiven aus Theorie und Praxis. Berlin: Frank \& Timme, 15-33 (Kommunikation - Partizipation - Inklusion Bd. 2).

Sweller, John /van Merriënboer, Jeroen J. G. / PaAs, Fred G. W. C. (1998): „Cognitive architecture and instructional design“. In: Educational Psychology Review 10, 251-296.

Vermeer, Hans J. (1990): Skopos und Translationsauftrag. 2. Aufl. Heidelberg: Selbstverlag.

WEIDENMANN, BERnd (2002): „Multicodierung und Multimodalität im Lernprozess“. In: Issing, LudWIG J./KLIMSA, PAUL (Hrsg): Information und Lernen mit Multimedia und Internet: Lehrbuch für Studium und Praxis. 3. vollst. überarb. Aufl. Weinheim: Beltz, 45-62.

WudTKe, Hubert (1993a): „Schriftspracherwerb: Schreibentwicklungen gehörloser Kinder“. In: Das Zeichen: Zeitschrift für Sprache und Kultur Gehörloser 24, 212-223.

WudTKe, HuberT (1993b): „Schriftspracherwerb: Schreibentwicklungen gehörloser Kinder“. In: Das Zeichen: Zeitschrift für Sprache und Kultur Gehörloser 25 332-341. 

KATRIN LANG

\section{Die rechtliche Lage zu Barrierefreier Kommunikation in Deutschland ${ }^{1}$}

\section{$1 \quad$ Einleitung}

Deutschland verfügt über ein hoch ausdifferenziertes Rechtssystem bestehend aus über 80.000 Einzelvorschriften (Bundesministerium der Justiz 2008: 16). Auch das Thema Barrierefreiheit der Kommunikation ist nicht in einem einzelnen Rechtstext erschöpfend dargelegt, sondern ist auf verschiedene Weise in unzähligen Normtexten bzw. Texten zu deren Implementierung enthalten. Neben der unserem Rechtssystem ohnehin inhärenten Komplexität und Intertextualität ist ein Grund hierfür darin zu vermuten, dass Kommunikation Bestandteil aller Lebensbereiche ist und sich in Konsequenz auch in vielen Rechtsbereichen niederschlägt. Zum anderen verändern sich mit neuen technischen Entwicklungen auch kontinuierlich die Möglichkeiten und Mittel, barrierefrei zu kommunizieren, was in den entsprechenden Rechtstexten berücksichtigt wird. Ein weiterer Grund kann darin gesehen werden, dass sich die gesellschaftlichen und politischen Bemühungen zur Gleichstellung und gleichberechtigten Teilhabe von Menschen mit Behinderung zunehmend verstärken und sich damit ihre Belange in immer mehr Normtexten Bahn brechen (Aichele 2015: 90).

Deutschland hat beim Thema Rechte von Menschen mit Behinderung und Barrierefreie Kommunikation in den letzten Jahren große Fortschritte gemacht (ebd.). Betrachtet man die Erweiterung des Grundgesetzes um das Benachteiligungsverbot von Menschen mit Behinderung im Jahr 1994 als Startpunkt, handelt es sich dabei jedoch um eine vergleichsweise junge Entwicklung. In diesem Beitrag soll die heutige Rechtslage zu Barrierefreier Kommunikation umrissen und demonstriert werden, wie stark das Thema den Rechtsbereich durchdringt.

1 Mit herzlichem Dank an die Bundesfachstelle Barrierefreiheit für die Sichtung dieses Beitrages und die juristischen Hinweise bei dessen Erstellung. 
Dafür wird im zweiten Teil zunächst ein grundlegender Einblick in das Gefüge der deutschen Rechtsordnung gegeben und beispielhaft dargelegt, in welchen Normtexten sich das Thema jeweils situiert. Für diejenigen, die im Feld Barrierefreie Kommunikation tätig sind, ist das Wissen um die rechtliche Lage wichtig, um Auswirkungen auf den eigenen Arbeitsbereich zu sehen. Daher werden im dritten Teil auch einige zentrale Normtexte auf verschiedenen Ebenen näher vorgestellt.

Die in diesem Beitrag fokussierten Rechtstexte zu Barrierefreier Kommunikation liegen im Wesentlichen in einem Überlappungsbereich aus drei verschiedenen Domänen (siehe Abbildung unten):

Zum einen sind sie Gegenstand und Ausdruck der Bemühungen um die allgemeine Gleichstellung von Menschen mit Behinderung. Dabei ist jedoch wenn man beispielsweise an das Kommunikationsmittel Leichte Sprache denkt - der Kreis potenzieller Nutznießer Barrierefreier Kommunikation sicher umfangreicher (z. B. Deutschlerner, Touristen, Senioren; siehe Adressatenkapitel in Bredel/Maaß (2016)). Wichtige rechtliche Grundlagen zur allgemeinen Gleichstellung und gleichberechtigten Teilhabe von Menschen mit Behinderung und damit auch von Barrierefreier Kommunikation sind zum Beispiel:

- das bereits erwähnte Grundgesetz (GG), das mit seinem Artikel 3 Absatz 3 Satz 2 („Niemand darf wegen seiner Behinderung benachteiligt werden") die verfassungsmäßige Grundlage für das Benachteiligungsverbot von Menschen mit Behinderung bildet,

- das Behindertengleichstellungsgesetz (BGG), das seit 2002 die Gleichstellung von Menschen mit Behinderung im öffentlichen Recht, also im Verhältnis zwischen den Bürgerinnen und Bürgern und der Verwaltung regelt,

- das Allgemeine Gleichbehandlungsgesetz (AGG), das die rechtliche Grundlage für die Gleichstellung von Menschen mit Behinderung im Privatrecht darstellt, 
- das Sozialgesetzbuch bzw. vor allem das Neunte Buch Sozialgesetzbuch (SGB IX), in dem Sozialversicherungsleistungen für Menschen mit Behinderung geregelt werden

- und die Behindertenrechtskonvention der Vereinten Nationen (UNBRK), die die uneingeschränkte Teilhabe und Selbstbestimmung von Menschen mit Behinderungen für sämtliche Lebensbereiche als Menschenrecht fordert und damit für einen Paradigmenwechsel in der internationalen Behindertenpolitik steht.

Zum anderen ragt das Thema Barrierefreie Kommunikation in den Bereich der Digitalisierung bzw. der zunehmenden Verlagerung von Serviceleistungen in das Internet und findet sich in Texten zur barrierefreien Gestaltung entsprechender Programmoberflächen und Internetseiten. Einschlägige Texte mit normativer Wirkung sind hier beispielsweise die Web Content Accessibility Guidelines (WCAG), die Barrierefreie-Informationstechnik-Verordnung (BITV), auf die jeweils an späterer Stelle noch eingegangen wird, und die ISONorm 9241 („Ergonomie der Mensch-System-Interaktion“). Dabei ist jedoch einzig die BITV als rechtlich verbindlich anzusehen, während die WCAG empfehlenden Charakter hat und eine Norm erst durch die Inbezugnahme durch ein Gesetz bzw. eine Verordnung rechtsverbindlich wird.

Der dritte Bereich, aus dem sich die Rechtstexte speisen, die für das Thema Barrierefreie Kommunikation besonders von Belang sind, ist die Verwaltungskommunikation. Dies betrifft sowohl Regelungen zur Übermittlung von Informationen zwischen der Behörde und den Bürgerinnen und Bürgern (z. B. zum Erlass von Bescheiden oder zur Kommunikation über das Internet) als auch teilweise zur verwaltungsinternen Kommunikation (z. B. zum Einreichen von Dokumenten bei Gericht oder zur Gestaltung von Intranetauftritten). Hier ergibt sich eine große Schnittmenge vor allem mit dem Thema Digitalisierung und Verbesserung behördlicher Online-Kommunikation. Wichtige Rechtstexte, die Verwaltungskommunikation und Digitalisierungsaspekte vereinen, sind beispielsweise das E-Government-Gesetz (EGovG) oder das Onlinezugangsgesetz (OZG). Besonders einschlägig für den Bereich der Barrierefreien Kommunikation sind darüber hinaus die Europäische Norm EN 301549 („Europäischer 
Standard zur Beschaffung von barrierefreien IKT-Produkten und Dienstleistungen") und die Richtlinie (EU) 2016/2102 des Europäischen Parlaments und des Rates vom 26. Oktober 2016 über den barrierefreien Zugang zu den Websites und mobilen Anwendungen öffentlicher Stellen (im Folgenden Richtlinie (EU) 2016/2102 genannt), die in Punkt 3 genauer beleuchtet wird.

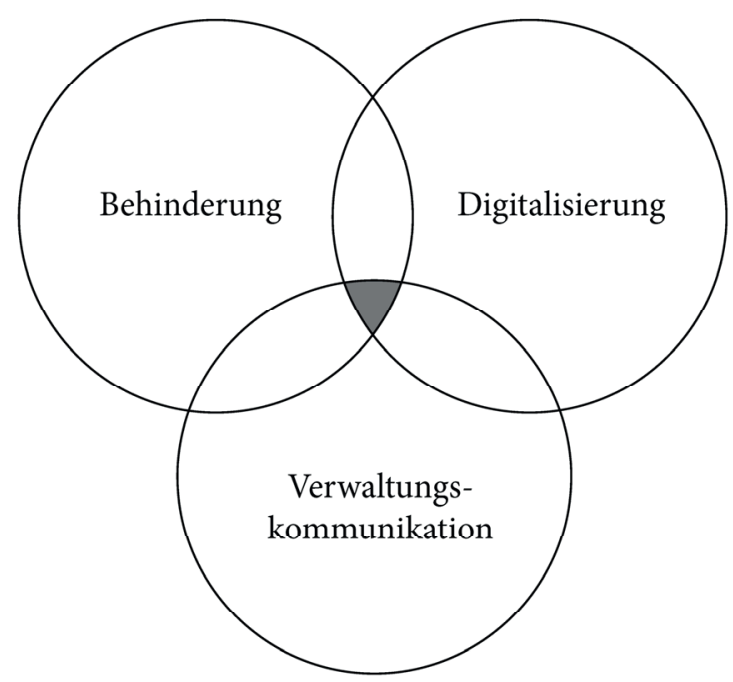

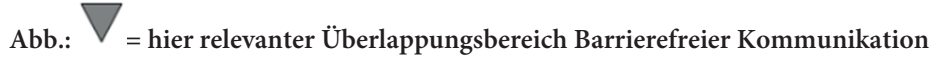

Die rechtliche Lage in dem in der Abbildung dargestellten Überlappungsbereich ist von besonderer Aktualität und Dynamik geprägt und wirkt sich auf den Arbeitsbereich vieler aus, die sich mit dem Thema Barrierefreie Kommunikation auseinandersetzen. Sie steht daher in diesem Beitrag im Mittelpunkt. Andere Rechtsgebiete wie zum Beispiel der Bereich Rundfunk (siehe dazu Heerdegen in diesem Band) oder weitere mögliche Bereiche des Privatrechts bleiben hier weitgehend ausgeklammert. 


\section{Zur Situierung des Gegenstands Barrierefreie Kommunikation in der deutschen Rechtsordnung}

Es sei hier zunächst grob skizziert, welche unterschiedlichen Arten von Normtexten in unserem Rechtssystem bestehen und wie sie miteinander interagieren:

Die Spitze der nationalen Normenpyramide bildet das Grundgesetz, zu dem alle anderen Rechtstexte nicht im Widerspruch stehen dürfen. Darunter stehen Bundesgesetze und Verordnungen. Der Unterschied zwischen beiden liegt darin, welches Organ die Rechtsregeln erlässt: Während Gesetze von den in der Verfassung vorgeschriebenen Gesetzgebungsorganen, also dem Bundestag und dem Bundesrat, erlassen werden, werden die in der Normenhierarchie unter dem Gesetz stehenden Rechtsverordnungen durch die Organe der Exekutive, also die Bundesregierung, Ministerien oder Landesregierungen, erlassen (Bundesministerium der Justiz 2008: 19). Gesetze und Verordnungen gibt es sowohl auf Bundes- als auch auf Länderebene, wobei Erstere aufgrund ihres übergeordneten Ranges - sofern konkurrierende Rechtsregeln auf beiden Ebenen bestehen, gilt „Bundesrecht bricht Landesrecht“ - den Fokus der nachfolgenden Ausführungen bilden. Unterhalb von Gesetz und Verordnung rangieren die Normentypen Satzung und Verwaltungsvorschrift, die hier jedoch ebenfalls vernachlässigbar sind.

\subsection{Einschlägige Rechtstexte auf Bundesebene}

Einschlägig für das Thema Barrierefreie Kommunikation ist auf Bundesebene vor allem das Behindertengleichstellungsgesetz (BGG bzw. Langtitel: „Gesetz zur Gleichstellung behinderter Menschen"). Obwohl es in Punkt 3 noch näher beleuchtet wird, sei das BGG an dieser Stelle bereits erwähnt, da es seit seinem erstmaligen Inkrafttreten im Jahr 2002 die rechtliche Grundlage für die Barrierefreiheit in der Kommunikation zwischen den Bürgerinnen und Bürgern und der Verwaltung und damit den hier fokussierten Kernbereich bildet. Mit $\$ 1$ BGG verpflichtet sich die Verwaltung außerdem, auf die Beseitigung der Benachteiligung von Menschen mit Behinderung hinzuwirken. Die aufseiten der Verwaltung Verpflichteten sind bislang die sogenannten „Träger öffentlicher Gewalt“ nach $\$ 1$ Absatz 2 Satz 1 BGG. Das sind Bundesbehörden wie zum Beispiel das 
Kraftfahrt-Bundesamt bzw. „bundesunmittelbare [...] Körperschaften, [...] Anstalten und [...] Stiftungen des öffentlichen Rechts" wie beispielsweise die Deutsche Rentenversicherung Bund. In $\$ 1$ Absatz 2 Satz 2 BGG sind außerdem entsprechende Institutionen auf Landesebene genannt, soweit diese Bundesrecht ausführen, wie zum Beispiel im Bereich des Sozialrechts (in etwa auf ein Bundesland beschränkte Sozialversicherungsträger) oder des Steuerrechts. Diese sind zwar zur Einhaltung des Benachteiligungsverbotes verpflichtet, jedoch momentan nicht zur Schaffung barrierefreier Kommunikationsangebote. Vermutlich ergibt sich eine Änderung dieser Lage mit Umsetzung der Richtlinie (EU) 2016/2102 im September 2018, auf die in Punkt 3 dieses Beitrages näher eingegangen wird und die Änderungen im BGG nach sich zieht. Zum Zeitpunkt der Erstellung dieses Beitrages ist das entsprechende Änderungsgesetz jedoch noch nicht verabschiedet, sodass hier nur der gegenwärtige Stand wiedergegeben werden kann.

Die Verpflichtung zur Beseitigung der Benachteiligung von Menschen mit Behinderung ergibt sich außerdem aus der UN-Behindertenrechtskonvention (UN-BRK), die in Deutschland 2009 ratifiziert wurde. Die gesetzlichen Grundlagen werden flankiert von entsprechenden Leitbildern und Regierungsprogrammen (z. B. Nationale Aktionspläne oder die Selbstverpflichtung im Koalitionsvertrag zur 19. Legislaturperiode). Dadurch findet Barrierefreie Kommunikation seit einigen Jahren nach und nach Eingang in sämtliche Rechtstexte, in denen es eine Rolle spielt, dass sich Bürgerinnen und Bürger im Bereich des öffentlichen Rechts eigenständig informieren bzw. Informationen von der Verwaltung erhalten.

Zwar ist in Anbetracht des von der Bundesregierung im Nationalen Aktionsplan zur UN-BRK formulierten Ziels der „inklusiven Gesellschaft“ Gleichstellung und damit auch Barrierefreiheit langfristig in allen Lebensbereichen zu gewährleisten. Besonders dringlich scheint ein uneingeschränkter Zugriff auf Informationen jedoch in den Bereichen, in denen die betreffenden Informationen (seien es z. B. Online-Wahlprogramme, Bescheide, Vordrucke, o. Ä.) zur Wahrnehmung der eigenen Rechte als Staatsbürgerin bzw. Staatsbürger dienen (dazu z. B. Bock 2015). Mit Blick auf die aktuelle rechtliche Lage soll hier jedoch nicht unerwähnt bleiben, dass es absehbar erscheint, dass auch die Privatwirt- 
schaft, also z. B. Dienstleister, Händler, Hersteller, bald zu Barrierefreiheit verpflichtet wird: Mit dem Allgemeinen Gleichbehandlungsgesetz (AGG) sind Weichenstellungen für die Gleichstellung von Menschen mit Behinderung im Privatrecht und insbesondere im Verhältnis zwischen Arbeitgeber und Arbeitnehmer erfolgt. Das BGG eröffnet mit seinem $₫ 5$ Behindertenverbänden bereits die Möglichkeit, private Unternehmen mittels Zielvereinbarungen dazu zu verpflichten, für Barrierefreiheit (z. B. in Form barrierefreier Webseiten) zu sorgen. Allerdings zeigte das Instrument der Zielvereinbarungen nach einer Evaluation der Bundesregierung zum BGG im Jahr 2014, zwölf Jahre nach dessen Inkrafttreten, noch nicht viel Wirkung (Evaluation des BGG; Abschlussbericht 2014: 288f). Auf europäischer Ebene ist seit 2015 ein European Accessibility Act zur Verpflichtung privater Wirtschaftsakteure zu Barrierefreiheit in Vorbereitung - zu Deutsch: „Vorschlag für eine Richtlinie des Europäischen Parlaments und des Rates zur Angleichung der Rechts- und Verwaltungsvorschriften der Mitgliedstaaten über die Barrierefreiheitsanforderungen für Produkte und Dienstleistungen“" (zum Stand der Vorbereitung im Jahr 2017 siehe z. B. Bundestagsdrucksache 18/13258). Bisher wurde jedoch noch keine endgültige Einigung und Beschlussfassung zu dem Entwurf unter den Mitgliedstaaten erzielt (Stand Februar 2018).

\subsection{Die Bandbreite der von Barrierefreier Kommunikation berührten Rechtstexte}

Da die Verwaltung - im Unterschied zur Privatwirtschaft - bereits dazu verpflichtet ist, in vielerei Hinsicht für Barrierefreiheit zu sorgen, gibt es Gesetze und Verordnungen, in denen Barrierefreie Kommunikation eine Rolle spielt, ohne dass Behinderung oder Gleichstellung den Hauptgegenstand der Regelung bildet. Es zeigen sich zudem verschiedene Ausdifferenzierungen des Gegenstands in den Rechtstexten, jeweils mit interner Verflechtung und unterschiedlicher Perspektivierung wie zum Beispiel: 
- Perspektivierung in Richtung der "Adressatenschaft“ (gemeint sind beispielsweise die jeweils in einer Regelung berücksichtigten Behinderungsarten oder Nutzergruppen; zur (Mehrfach-)Adressierung von Rechtstexten generell siehe Baumann in diesem Band)

- Perspektivierung auf die benötigten (Kommunikations-)Hilfen

- Perspektivierung auf die nötige Technik in den Behörden zur Herstellung von Barrierefreier Kommunikation (Content-ManagementSysteme etc.)

- Perspektivierung in Richtung unterschiedlicher Rechtsbereiche bzw. zur Umsetzung Verpflichteter (z. B. öffentliches Recht vs. Privatrecht)

Eine erschöpfende Darlegung der verschiedenen Regelungen mit Bezug zu Barrierefreier Kommunikation ist aufgrund der Komplexität und des Umfangs der Rechtsordnung nicht möglich. Um dennoch ein Schlaglicht auf die aktuelle Bandbreite der Regelungen und betroffenen Themengebiete zu werfen und die bestehenden Verflechtungen exemplarisch aufzuzeigen, seien die folgenden Rechtstexte genannt, die den Bereich gegenwärtig und in naher Zukunft besonders prägen:

- Das „Gesetz zur Verbesserung des Onlinezugangs zu Verwaltungsleistungen“ (Onlinezugangsgesetz - OZG), das darauf gerichtet ist, den digitalen Zugang zu Verwaltungsleistungen für Bürgerinnen und Bürger generell zu verbessern. Das OZG regelt, dass bis zum Jahr 2023 ein zentraler Portalverbund mit den gebündelten Serviceleistungen der Behörden von Bund, Ländern und Kommunen eingerichtet werden soll, sodass Bürgerinnen und Bürger künftig auf sämtliche Serviceleistungen von einer zentralen Internetseite aus zugreifen können. Dabei hat die Verwaltung gemäß $\$ 3$ Absatz 1 OZG auch Barrierefreiheit nach $₫ 12$ BGG, also eine grundsätzlich uneingeschränkte Nutzung dieses Portalverbundes durch Menschen mit Behinderung sicherzustellen und die 
Einhaltung der BITV (siehe Punkt 3) in ihrer jeweils geltenden Fassung zu gewährleisten (Bundestagsdrucksache 18/11135: 92).

- Das Gesetz gegen Wettbewerbsbeschränkungen (GWB), das Vorgaben zur Vergabe öffentlicher Aufträge enthält, wenn diese europaweit ausgeschrieben werden müssen. Es regelt u. a., dass bei der Beschreibung der zu erbringenden Auftragsleistungen in bestimmten Fällen die Zugänglichkeit für Menschen mit Behinderung oder eine „Konzeption für alle Nutzer" berücksichtigt werden muss ( $\$ 121$ Absatz 2 GWB). In Letzterem kann ein Schritt zur Förderung des sogenannten „Designs für alle“ oder „Universellen Designs“ nach Artikel 2 der UNBRK gesehen werden. Dieses Konzept meint die Nutzbarkeit eines Produktes für möglichst viele Nutzergruppen, ohne dass dabei Anpassungen an bestimmte Arten von Einschränkungen vonnöten wären. Zum GWB gehört außerdem die „Verordnung über die Vergabe öffentlicher Aufträge“ (Vergabeverordnung - VgV), in der geregelt wird, dass es ein Zuschlagskriterium darstellen kann, wenn die vom Wettbewerber angebotene Leistung für Menschen mit Behinderung zugänglich gestaltet ist ( $\$ 58$ Absatz 2 Nummer $1 \mathrm{VgV}$ ). Auch das Vergabeverfahren selbst bzw. die darin zum Einsatz kommenden elektronischen Mittel sind nach $₫ 11 \mathrm{VgV}$ barrierefrei im Sinne des BGG bzw. der BITV 2.0 zu gestalten, damit auch aufseiten der Wettbewerber eventuelle Einschränkungen keinen Hinderungsgrund für das Einreichen eines Angebots darstellen.

- Das „Gesetz zur Förderung der elektronischen Verwaltung“ (E-Government-Gesetz - EGovG), das dazu dient, eine einfachere elektronische Kommunikation zwischen der Verwaltung und den Bürgerinnen und Bürgern zu etablieren, bzw. das bundesrechtliche Hindernisse abbauen soll, um es den Behörden zu erleichtern, Online-Services anzubieten (Bundestagsdrucksache 17/11473: 2). Das größtenteils im Jahr 2013 in Kraft getretene Gesetz ermöglicht die Verwendung bestimmter sicherer elektronischer Verfahren zum Beispiel bei der Bereit- 
stellung von Formularen, der Datenübermittlung und der erforderlichen Nutzeridentifizierung und schafft damit die Voraussetzungen dafür, dass beispielsweise mobilitätseingeschränkte Personen langfristig nicht mehr den Gang zur Behörde auf sich nehmen müssen, sondern vom heimischen Computer aus ohne fremde Hilfe am Verwaltungsverfahren teilhaben können. Der Zugang über Internetseiten und entsprechende Programmoberflächen sollen nach dem EGovG gemäß dem BGG barrierefrei gestaltet werden (Bundestagsdrucksache 17/11473: 24).

- Das Gesetz zur Förderung des elektronischen Rechtsverkehrs mit den Gerichten (auch E-Justice-Gesetz genannt) ist ein Änderungsgesetz mit dem Ziel, den elektronischen Rechtsverkehr in gerichtlichen Verfahren zu vereinfachen und Zugangshürden abzubauen (Bundestagsdrucksache 17/12634: 1f.). Die verschiedenen Änderungen beziehen sich auf fast alle Gesetze und Verordnungen mit Bezug zu gerichtlichen Verfahren wie zum Beispiel die Zivilprozessordnung, das Arbeitsgerichtsgesetz, das Sozialgerichtsgesetz, die Verwaltungsgerichtsordnung oder die Bundesrechtsanwaltsordnung und treten bzw. traten im Zeitraum 2014 bis 2022 in Kraft.

Neben der sogenannten DE-Mail-Infrastruktur zum sicheren Versand elektronischer Dokumente sollen andere, technologieoffene Übermittlungswege etabliert und auch ein „besonderes elektronisches Anwaltspostfach“ für jeden Rechtsanwalt für gerichtliche Zustellungen eingerichtet werden. Barrierefreiheit findet hier insofern Erwähnung, als dass die Übermittlungswege für elektronische Dokumente oder zum Beispiel das besondere elektronische Anwaltspostfach barrierefrei zu gestalten sind. Damit ist Barrierefreiheit nicht nur in der Kommunikation zwischen den Bürgerinnen und Bürgern und der Verwaltung berücksichtigt, sondern bildet auch einen gerichtsinternen Anspruch, indem alle Verfahrensbeteiligten einheitliche Standards zur Einreichung elektronischer Dokumente bei Gericht erhalten. Das Gesetz enthält außerdem Verordnungsermächtigungen, d.h. die Länder 
und auch Ministerien sollen die hier festgelegten Bestimmungen mittels Rechtsverordnungen näher konkretisieren. Dies bedeutet, dass zahlreiche weitere Verordnungen aus dem Änderungsgesetz erwachsen sind, in denen u. a. die Barrierefreiheit von Registern wie im Falle der Zivilprozessordnung, des Anwaltspostfachs wie im Falle der Bundesrechtsanwaltsordnung oder der Übermittlungswege für elektronische Dokumente wie in allen genannten Gesetzen genauer reguliert wird. Zu nennen wäre hier beispielsweise die zum 1. Januar 2018 in Kraft getretene „Verordnung über die technischen Rahmenbedingungen des elektronischen Rechtsverkehrs und über das besondere elektronische Behördenpostfach“ (Elektronischer-RechtsverkehrVerordnung - ERVV), die wiederum in puncto Barrierefreiheit auf die BITV 2.0 verweist.

\subsection{Einschlägige Texte mit normativer Wirkung auf weiteren Ebenen}

Darüber hinaus bestehen auch Rechtsakte jenseits der nationalen Grenzen wie beispielsweise überstaatliche Völkerrechtsverträge oder EU-Verordnungen und -Richtlinien, die ebenfalls in Gesetze und Verordnungen auf Bundes- und Länderebene münden bzw. existierendes Recht modifizieren und die damit Einfluss auf die deutsche Rechtsordnung haben.

Völkerrechtsverträgen bzw. internationalen Übereinkommen kommt in der Normenhierarchie eine Sonderrolle zu. Nach den „Richtlinien für die Fassung von Vertragsgesetzen und vertragsbezogenen Verordnungen“ werden sie erst wirksam für die deutsche Rechtsordnung, wenn sie in nationales Recht umgesetzt und beschlossen werden. Sie stehen dann formal auf Ebene eines Bundesgesetzes. Zu nennen wäre hier insbesondere die bereits erwähnte UN-BRK, mit herausragender Bedeutung für die Gleichstellung von Menschen mit Behinderung und das Thema Barrierefreiheit, dazu mehr unter Punkt 3.

Während eine EU-Verordnung für alle Mitgliedstaaten verbindlich ist und unmittelbar gilt, wird in EU-Richtlinien ein für alle EU-Länder zu erreichendes Ziel vorgegeben, wobei es jedoch in der Verantwortung der Mitgliedstaaten 
selbst liegt, entsprechende nationale Rechtsvorschriften zu erlassen (Bundesministerium der Justiz 2008: 95). Das innerstaatliche Recht darf im Hinblick auf EU-Rechtsakte keine Widersprüche oder Lücken enthalten, sodass jeder deutsche Rechtsetzungsakt auf seine Vereinbarkeit mit dem Recht der Europäischen Union zu überprüfen ist (ebd.: 93f.). Für den Bereich der Barrierefreien Kommunikation besonders wichtig ist beispielsweise die bereits erwähnte Richtlinie (EU) 2016/2102, dazu ebenfalls weiter unten mehr.

Für die konkrete Umsetzung wird häufig in den jeweiligen Gesetzen und Verordnungen auf bestimmte Normen und Standards auf internationaler, europäischer oder nationaler Ebene verwiesen, die dadurch im jeweiligen Zusammenhang ebenfalls verbindlich werden. $\mathrm{Zu}$ nennen wären hier beispielsweise wiederum die WCAG oder die Europäische Norm EN 301 549. Die konkrete Umsetzung der verschiedenen Regelungen erfolgt zudem häufig über Aktionspläne oder Regierungsprogramme wiederum auf EU-, Bundes- und Länderebene (z. B. eEurope 2005, Nationaler Aktionsplan (NAP), gemeinsam einfach machen; Übersicht auch unter: https://www.gemeinsam-einfach-machen.de) oder in Form ganz konkreter Anleitungen wie beispielsweise des „Styleguides der Bundesregierung“ (https://styleguide.bundesregierung.de/) oder des „Leitfadens für eine barrierefreie Verwaltung“ des Landes Hessen (http://www.brk.hessen.de/).

\section{Zentrale Normtexte mit Bezug auf Barrierefreie Kommunikation}

Es sollen hier exemplarisch einige der vorerwähnten zentralen Vertreter der Normtexte vorgestellt werden, die die rechtliche Grundlage zu Barrierefreier Kommunikation in Deutschland bilden (weitere auch bei Kerkmann (2015)).

\section{UN-Behindertenrechtskonvention}

Einer der wichtigsten Regelungstexte zur Stärkung der Rechte von Menschen mit Behinderung weltweit ist die 2006 verabschiedete UN-BRK, die in Deutsch- 
land im Jahr 2009 in Form des Gesetzes „zu dem Übereinkommen der Vereinten Nationen vom 13. Dezember 2006 über die Rechte von Menschen mit Behinderungen sowie zu dem Fakultativprotokoll vom 13. Dezember 2006 zum Übereinkommen der Vereinten Nationen über die Rechte von Menschen mit Behinderungen“" in Kraft trat. Mit Unterzeichnung des Völkerrechtsvertrages verpflichten sich bislang 176 Staaten dazu (Stand Februar 2018 nach UN-Angaben), „den vollen und gleichberechtigten Genuss aller Menschenrechte und Grundfreiheiten durch alle Menschen mit Behinderungen zu fördern, zu schützen und zu gewährleisten" (Artikel 1 UN-BRK).

Eine der Haupterrungenschaften der UN-BRK ist der internationale Paradigmenwechsel im Verständnis von Behinderung durch die Umdeutung des Begriffs weg von einem defizitorientierten Ansatz, der Menschen eine Beeinträchtigung zuschreibt („behindert sein“), hin zu einer Auffassung von Behinderung als eine Beeinträchtigung, die nur in Wechselwirkung mit den in der Umwelt bestehenden Barrieren zum Tragen kommt („gehindert werden“) (Degener 2015: 58).

Die UN-BRK umfasst 50 Artikel, wobei die ersten 30 als inhaltlicher Kern gesehen werden können und neben einem allgemeinen Teil Rechte für verschiedene Lebensbereiche wie zum Beispiel Bildung, Gesundheit sowie Arbeit und Beschäftigung beinhalten. Die letzten 20 Artikel sind formaler Art und machen Vorgaben zur Umsetzung und Überwachung des Vertrages.

Wichtige Schlagworte stecken in den acht grundlegenden Prinzipien, die in Artikel 3 der UN-BRK genannt werden und die ihre übergeordneten Leitlinien darstellen wie die Achtung der Menschenwürde und der Autonomie des Einzelnen, die Nichtdiskriminierung, Teilhabe, Vielfalt, Chancengleichheit und Zugänglichkeit. Dies entspricht dem Wortlaut der amtlichen deutschen Fassung, die jedoch nach Artikel 50 der UN-BRK nicht zu den „verbindlichen“ Sprachfassungen zählt, die im Zweifel über die Auslegung herangezogen werden müssen (u. a. Englisch, Spanisch, Arabisch) (Kerkmann 2015: 18). Inzwischen zentrale Begriffe im Behinderungsdiskurs wie „Inklusion“ statt „Integration“ oder auch „Barrierefreiheit“ statt „Zugänglichkeit“ gehen zurück auf die sogenannte Schattenübersetzung der UN-BRK des Vereins Netzwerk Artikel 3 e. V. (2009), die in der Wortwahl zum Teil von der offiziellen Übersetzung abweicht und so 
zu mehr gesellschaftlicher Bewusstseinsbildung beitragen soll. Nähere Ausführungen zum Thema Schattenübersetzung finden sich u. a. bei Kerkmann (2015).

Die UN-BRK gilt für die Unterzeichnerstaaten zunächst für den öffentlichrechtlichen Bereich, wirkt sich aber auch auf das Privatrecht aus, da sich die Unterzeichner in Artikel 4 Absatz 1 e) dazu verpflichten, Maßnahmen zu ergreifen, um die Diskriminierung von Menschen mit Behinderung „durch Personen, Organisationen oder private Unternehmen“ zu beseitigen (Degener 2015: 58).

Zum Thema Barrierefreie Kommunikation sind folgende Punkte in der UNBRK zu nennen:

In der Präambel Buchstabe v) wird der uneingeschränkte Zugang zu Kommunikation und Information als Grundvoraussetzung zur Wahrnehmung der Menschenrechte und individuellen Freiheiten formuliert. Auch die Begriffsbestimmungen in Artikel 2 sind von Bedeutung, da hier ausdrücklich verschiedenste Mittel als „Kommunikation“ gefasst werden wie zum Beispiel Brailleschrift, taktile Kommunikation oder einfache Sprache. Auch die Gebärdensprache wird dort als eigenständige Sprache anerkannt. Wichtig ist außerdem Artikel 9 „Zugänglichkeit“ in der amtlichen Fassung bzw. „Barrierefreiheit“ in der Schattenübersetzung der UN-BRK. Dieser weitet ähnlich wie das deutsche BGG zunächst den Begriff der Barrierefreiheit bzw. Zugänglichkeit auf den Bereich der Kommunikation und Information aus. Er verpflichtet die Vertragsstaaten den gleichberechtigten Zugang auch zu neuen Informations- und Kommunikationsdiensten einschließlich des Internets für Menschen mit Behinderung zu fördern (Absatz 1 Buchstabe b), Absatz 2 Buchstabe g) und h)). Artikel 9 verpflichtet ähnlich wie auch Artikel 4 die Vertragsstaaten außerdem dazu, neue Entwicklungen und bezahlbare Technologien im Bereich Kommunikation und Information für Menschen mit Behinderung zu fördern. In Artikel 21 findet sich das Recht auf „für die Allgemeinheit bestimmte Informationen“ und die Anwendung von Gebärdensprache, Brailleschrift und alternativer Kommunikationsformen im Umgang mit Behörden, die zunehmend auch im deutschen Recht umgesetzt werden. In Artikel 24 „Bildung“ Absatz 3 werden die Vertragsstaaten dazu verpflichtet, sicherzustellen, dass Menschen mit Behinderung im Erlernen und Nutzen alternativer Kommunikationsformen, von Brailleschrift oder Gebärdensprache etc. gefördert werden. Die Umsetzung der Vorgaben in Deutschland ist dabei unterschiedlich weit, der größte Nachholbedarf besteht 
derzeit noch im Bereich der Information und Kommunikation durch private Anbieter (Bethke/Kruse/Rebstock/Welti 2015: 178ff.).

Behindertengleichstellungsgesetz (BGG) und zugehörige Verordnungen

Im Jahr 2002 trat das bereits mehrfach erwähnte BGG in Kraft, das für den Bereich des öffentlichen Rechts mit Zuständigkeit des Bundes gilt. Das Gesetz dient der Beseitigung der Benachteiligung von Menschen mit Behinderung und damit der Umsetzung des Benachteiligungsverbotes aus dem Grundgesetz in Bezug auf die Bundesverwaltung (Bundestagsdrucksache 14/7420: 1).

Hinsichtlich Barrierefreier Kommunikation ist $\$ 4$ BGG in der Fassung von 2002 hervorzuheben. Dort wird der Terminus „Barrierefreiheit“ neben der im allgemeinen Sprachgebrauch vorherrschenden Verwendung im Zusammenhang mit baulichen Anlagen ausdrücklich ausgeweitet auf „Systeme der Informationsverarbeitung, akustische und visuelle Informationsquellen und Kommunikationseinrichtungen“, wenn diese „für behinderte Menschen in der allgemein üblichen Weise ohne besondere Erschwernis und grundsätzlich ohne fremde Hilfe zugänglich und nutzbar sind“.

Mit $₫ 6$ Absatz 1 BGG wird darüber hinaus die Deutsche Gebärdensprache (DGS) als eigenständige Sprache anerkannt und mit $\$ 9$ Absatz 1 BGG wird Menschen mit Hör- und Sprachbehinderung das Recht eingeräumt, die DGS, lautsprachbegleitende Gebärden oder „,andere geeignete Kommunikationshilfen“ zur Verständigung in Verwaltungsverfahren zu verwenden, wobei die Verwaltung die entstehenden Kosten zu tragen hat. Nach einem jahrelangen Kampf um Anerkennung wurde dies in der Gebärdensprachgemeinschaft als großer Meilenstein empfunden, wobei auch hier die Ausweitung auf den privatrechtlichen Bereich gefordert wird (Gehörlosenbund e.V. 2012: 1). Näheres zu $\$ 9$ BGG regelt die zugehörige „Verordnung zur Verwendung von Gebärdensprache und anderen Kommunikationshilfen im Verwaltungsverfahren nach dem Behindertengleichstellungsgesetz" (Kommunikationshilfenverordnung KHV). So enthält die fünf Paragraphen umfassende KHV Regelungen zum Anwendungsbereich und zum Umfang des Anspruchs auf die Verwendung von 
Kommunikationshilfen. Darüber hinaus wird festgelegt, was als geeignete Kommunikationshilfe gilt und welches die Grundsätze für ihre Bereitstellung sowie die Vergütung bzw. Erstattung sind.

In $\$ 10$ Absatz 1 BGG wird festgelegt, dass für Menschen mit Sehbehinderung in Verwaltungsverfahren bestimmte Schriftstücke wie Bescheide, Vordrucke und Allgemeinverfügungen in einer "für sie wahrnehmbaren Form“ aufbereitet werden müssen. In der entsprechenden „Verordnung zur Zugänglichmachung von Dokumenten für blinde und sehbehinderte Menschen im Verwaltungsverfahren nach dem Behindertengleichstellungsgesetz" (Verordnung über barrierefreie Dokumente in der Bundesverwaltung - VBD) werden nähere Bestimmungen hierzu getroffen. Die VBD enthält sechs Paragraphen und regelt neben dem Anwendungsbereich, dem Umfang des Anspruchs und der Übernahme der Kosten, welche Dokumente zugänglich gestaltet werden müssen und welche Formen dafür zulässig sind.

Im Jahr 2016 erfolgte die Anpassung des BGG an die UN-BRK per Änderungsgesetz, wodurch $₫ 11$ „Verständlichkeit und Leichte Sprache“ neu hinzugekommen ist. $\$ 11$ BGG regelt das Recht von Menschen mit geistigen Behinderungen und Menschen mit seelischen Behinderungen auf einfache und verständliche Kommunikation im Kontakt mit der öffentlichen Verwaltung. Die Begrenzung auf diese Arten von Behinderung weicht dabei von den momentanen Erkenntnissen darüber ab, welche Zielgruppen tatsächlich von vereinfachter Kommunikation profitieren können (auch hierfür siehe Adressatenkapitel in Bredel/Maaß (2016)). Mit Wirkung zum 1. Januar 2018 müssen Bundesbehörden bzw. Körperschaften, Anstalten und Stiftungen des öffentlichen Rechts der genannten Zielgruppe auf Verlangen u. a. Bescheide, Vordrucke und Allgemeinverfügungen einfach und verständlich erläutern. Scheitert diese einfache „Erläuterung“, können die Betroffenen eine Erläuterung in Leichter Sprache fordern. Anders als im Falle der Verwendung von Kommunikationshilfen nach $₫ 9$ oder der Zugänglichmachung von Dokumenten nach $\$ 10$ regelt $\$ 11$ jedoch keinen - einklagbaren - gesetzlichen Anspruch der Betroffenen auf die Verwendung Leichter Sprache, sondern nur die Verpflichtung der Behörde, die Notwendigkeit sprachlicher Vereinfachung auf Verlangen zu prüfen und gegebenenfalls umzusetzen. Offen lässt der Gesetzgeber dabei, welche Anforderungen an die sprachliche Vereinfachung und an Leichte Sprache zu stellen sind und ob 
die jeweiligen Erläuterungen mündlich, schriftlich bzw. schriftgestützt erfolgen sollen. Hier eröffnet sich ein nicht unerheblicher Auslegungsspielraum für die betroffenen Behörden. Zum gegenwärtigen Zeitpunkt bleibt abzuwarten, wie genau Leichte Sprache in diesen Kontexten zum Einsatz kommen wird. $\$ 11 \mathrm{Ab}$ satz 4 BGG regelt zudem, dass von der Regelung umfasste Stellen vermehrt Informationen in Leichter Sprache bereitstellen sollen.

$\$ 12$ Absatz 1 BGG mit der Überschrift „Barrierefreie Informationstechnik“ verpflichtet die Behörden, ihre Internetauftritte so zu gestalten, dass sie von Menschen mit Behinderung "grundsätzlich uneingeschränkt genutzt werden können“. Klarstellend sei darauf verwiesen, dass „grundsätzlich“ im juristischen anders als im allgemeinsprachlichen Sinne nicht „,in jedem Fall“ meint, sondern Ausnahmen vom Grundsatz zulässt. Nach Absatz 2 sind darüber hinaus auch bestimmte verwaltungsinterne Informationen, Intranetseiten und elektronisch gestützte Verwaltungsabläufe für die Beschäftigten von Bundesbehörden und bundesunmittelbaren Körperschaften, Anstalten und Stiftungen des öffentlichen Rechts nach und nach barrierefrei aufzubereiten. Genauere Ausführungen zu $\$ 12$ BGG enthält die entsprechende „Verordnung zur Schaffung barrierefreier Informationstechnik nach dem Behindertengleichstellungsgesetz" (Barrierefreie-Informationstechnik-Verordnung - BITV), auf die im nächsten Punkt genauer eingegangen wird.

In vielen Bundesländern bestehen am BGG orientierte Landesgleichstellungsgesetze und entsprechende zugehörige Verordnungen. Einen Überblick über die Lage in den Ländern gibt zum Beispiel Aktion Mensch auf https://www.einfach-fuer-alle.de/artikel/bitv/bgg/ oder die Monitoring-Stelle UN-BRK auf http://www.institut-fuer-menschenrechte.de/monitoring-stelleun-brk/monitoring/koalitionsvertraege/.

\section{Barrierefreie-Informationstechnik-Verordnung - BITV 2.0}

Die erste Fassung der Barrierefreie-Informationstechnik-Verordnung trat im Jahr 2002 in Kraft. Sie konkretisiert die Anforderungen nach $\$ 12$ BGG an barrierefreie Internet- sowie öffentlich zugängliche Intranetseiten und andere grafische Programmoberflächen von Behörden der Bundesverwaltung sowie Institutionen auf Landesebene, die Bundesrecht ausführen. Die BITV orientiert sich 
im Wesentlichen an den weitverbreiteten und international anerkannten Empfehlungen der Web Content Accessibility Guidelines (WCAG), auf die weiter unten ebenfalls noch eingegangen wird. Nach einer Überarbeitung und Aktualisierung der WCAG wurde auch die BITV entsprechend geändert und trat im Jahr 2011 als BITV 2.0 in Kraft. Die nach der BITV 2.0 zu erfüllenden Standards sind nach zwei Prioritätsstufen getrennt. Anforderungen mit Priorität I müssen von allen Inhalten bzw. Internetseiten der Verpflichteten eingehalten werden. Die weitergehenden Anforderungen der Prioritätsstufe II hingegen sind nur von „Zentrale[n] Navigations- und Einstiegsangebote[n]“ zu erfüllen (\$ 3 Absatz 1 BITV 2.0). Dabei werden in Anlehnung an die WCAG vier grundlegende Prinzipien der Barrierefreiheit genannt und weiter ausdifferenziert: Die Webinhalte sind so zu gestalten, dass sie für den Benutzer 1. wahrnehmbar sind, 2. bedienbar sind, 3 . verständlich sind und 4 . so robust sind, dass sie mit verschiedenen assistierenden Techniken genutzt werden können. So werden mit Blick auf verschiedenartig eingeschränkte Nutzergruppen in Anlage 1 der BITV 2.0 beispielsweise das Bereitstellen von Alternativen für Nicht-Text-Inhalte, die durchgehende Bedienbarkeit per Tastatur, die Möglichkeit der variablen Einstellung von Textgröße oder Schrift sowie Richtwerte für Kontraste und Farben vorgegeben.

Anders als die WCAG regelt die BITV 2.0 außerdem in $\$ 3$ Absatz 2 sprachliche bzw. auf Verständlichkeit ausgerichtete Aspekte in Form der Vorgabe, in gewissem Umfang Leichte Sprache oder Gebärdensprache auf der Startseite eines Intranet- oder Internetangebots zu verwenden. In Anlage 2 der BITV 2.0 erfolgen konkrete Anweisungen zur Umsetzung in Gebärdensprache (Teil 1) bzw. Leichte Sprache (Teil 2). Bei Letzterem handelt es sich jedoch überwiegend um Vorgaben hinsichtlich des Layouts - nur fünf der 13 Vorgaben beziehen sich auf die tatsächliche sprachliche Umsetzung.

Entsprechende Landesgesetze und -verordnungen orientieren sich zum gegenwärtigen Zeitpunkt vorwiegend noch an der BITV-Fassung aus dem Jahr 2002, in der beispielsweise die Verwendung von Gebärdensprache und Leichter Sprache noch nicht als Bedingung enthalten war.

Kommentare zu aktuellen Entwicklungen in diesem Bereich sowie eine kritische Auseinandersetzung mit der BITV finden sich in den Veröffentlichungen 
von Hellbusch im Internet, so zum Beispiel auf https://www.barrierefreies-webdesign.de/, https://www.hellbusch.de/accessibility-checkliste/ oder http://webkrauts.de/artikel/2011/bitv-20-kraft (s. a. den Beitrag von Hellbusch im vorliegenden Band).

\section{Web Content Accessibility Guidelines - WCAG 2.0}

Die WCAG sind Empfehlungen für die Gestaltung barrierefreier Webinhalte der Arbeitsgruppe des World Wide Web Consortiums (W3C) und wurden erstmals im Jahr 1999 veröffentlicht. Sie richten sich an die Entwickler von Webinhalten und wurden nach einer Überarbeitung in ihrer aktuell gültigen Fassung im Jahr 2008 als WCAG 2.0 herausgegeben. Die WCAG haben keinen rechtlich bindenden Charakter, sind jedoch inzwischen - wie auch die vorangegangenen Ausführungen zeigen - in zahlreiche internationale und nationale Regelungstexte eingegangen und wurden im Jahr 2012 außerdem in einer internationalen Norm („ISO/IEC 40500: Informationstechnik - W3C Richtlinien für die Barrierefreiheit von Webinhalten (WCAG) 2.0“) umgesetzt (https://www.w3.org/2012/ 07/wcag2pas-pr.html).

Die WCAG sind pyramidenartig aufgebaut und basieren zunächst auf den vier bereits bei der BITV genannten Grundprinzipien der Wahrnehmbarkeit, Bedienbarkeit, Verständlichkeit und Robustheit (detailliert dazu Hellbusch/ Probiesch 2011). Diese Grundprinzipien sind wiederum in zwölf technikneutralen „Richtlinien“ konkretisiert, die zum Beispiel lauten:

„Richtlinie 1.1 Textalternativen: Stellen Sie Textalternativen für alle Nicht-Text-Inhalte zur Verfügung, so dass diese in andere vom Benutzer benötigte Formen geändert werden können, wie zum Beispiel Großschrift, Braille, Symbole oder einfachere Sprache." (WCAG 2.0 2009)

Oder: „Richtlinie 1.4 Unterscheidbar: Machen Sie es Benutzern leichter, Inhalt zu sehen und zu hören einschließlich der Trennung von Vorderund Hintergrund." (ebd.) 
Auf Grundlage der zwölf Richtlinien werden 61 sogenannte „Erfolgskriterien“ formuliert, die genaue Anweisungen zur Herstellung der Barrierefreiheit geben. Sie lauten beispielsweise:

\begin{abstract}
„3.1.5 Leseniveau: Wenn der Text nach der Entfernung von Eigennamen und Titeln Lesefähigkeiten voraussetzt, die über das Niveau der niedrigen, sekundären Schulbildung hinausgehen, dann gibt es ergänzenden Inhalt oder eine Version, die keine über die niedrige, sekundäre Schulbildung hinausgehenden Lesefähigkeiten verlangt (Stufe AAA)“ (ebd.).
\end{abstract}

Die Erfolgskriterien in den WCAG 2.0 sind einem dreistufigen Konformitätsmodell zugeordnet. Dabei ist Stufe A die mit dem geringsten Anforderungsniveau, ihr sind 25 Erfolgskriterien zugeordnet. Die mittlere Stufe AA umfasst 13 Erfolgskriterien und die höchste Konformität AAA zu den WCAG 2.0 erreicht man mit der Einhaltung weiterer 23 Erfolgskriterien.

In der deutschen BITV wurde das WCAG-Konformitätsmodell auf zwei Prioritätsstufen vereinfacht. Dabei entsprechen die WCAG-2.0-Stufen A und AA der Priorität I in der BITV 2.0 und die WCAG-2.0-Stufe AAA der Priorität II in der BITV 2.0.

Aktuell, 10 Jahre nach Erscheinen der letzten WCAG-Fassung, besteht auch ein Entwurf für eine Weiterentwicklung der Empfehlungen, die WCAG 2.1. Dieser enthält ca. 30 neue Erfolgskriterien und geht verstärkt auf die Anforderungen sehbehinderter und lernbehinderter Nutzer ein (https://www.w3.org/ TR/WCAG21/). Die Veröffentlichung ist laut W3C für Juni 2018 geplant (Stand Februar 2018: https://www.w3.org/blog/2018/01/wcag21-cr/).

Richtlinie (EU) 2016/2102

Auf europäischer Ebene wurde die „Richtlinie (EU) 2016/2102 des Europäischen Parlaments und des Rates vom 26. Oktober 2016 über den barrierefreien Zugang zu den Websites und mobilen Anwendungen öffentlicher Stellen“" erlassen, die bis zum 23. September 2018 in nationales Recht umzusetzen ist und damit zu Änderungen im BGG führt. Demnach müssen neue Webseiten von Gebietskörperschaften (also des Bundes und nun auch der Länder, Landkreise 
und Kommunen), von Einrichtungen des öffentlichen Rechts (z. B. Universitäten, Krankenkassen, Kirchen, Berufsgenossenschaften, Handwerkskammern) und von aus solchen bestehenden Verbänden ab September 2019 barrierefrei sein. Dabei werden auch einige Ausnahmen aufseiten der Verpflichteten genannt. So gilt die Richtlinie beispielsweise nicht für öffentlich-rechtliche Rundfunkanstalten und unter Umständen auch nicht nach Abwägung der „Verhältnismäßigkeit“ zwischen Aufwand für die öffentliche Stelle und Nutzen für die Menschen mit Behinderung. Ausgenommen sind auch bestimmte Inhalte wie zum Beispiel live übertragene zeitbasierte Medien oder Inhalte von Dritten, die von der betreffenden öffentlichen Stelle weder finanziert noch entwickelt werden noch deren Kontrolle unterliegen. Webseiten und mobile Anwendungen, die bereits vor September 2018 bestanden, müssen ab September 2020 bzw. Juni 2021 die notwendigen Anforderungen an Barrierefreiheit erfüllen. Auch „Büroanwendungen“ (z. B. PDF- oder Worddateien), die nach dem 23. September 2018 veröffentlicht werden, müssen dann barrierefrei sein.

Als technischer Standard liegt der Richtlinie (EU) 2016/2102 die europäische Norm EN 301549 („Accessibility requirements suitable for public procurement of ICT products and services in Europe") zugrunde, die sich jedoch wiederum an den WCAG 2.0 orientiert. Die entsprechenden Seiten müssen künftig eine „Erklärung zur Barrierefreiheit“ und einen „Feedback-Mechanismus“ für Nutzerinnen und Nutzer enthalten. Darüber hinaus muss jeder Mitgliedstaat die Umsetzung der Richtlinie mittels einer festzulegenden Überwachungsmethode wie zum Beispiel durch Sachverständigenanalyse oder Stichproben überprüfen. Jeder Mitgliedstaat ist außerdem dazu verpflichtet, zum Dezember 2021 einen ersten Bericht über die Ergebnisse der Überwachung an die EU-Kommission abzugeben und ab dann alle drei Jahre zu berichten. Ab September 2018 ist der Kommission außerdem eine Stelle zu nennen, die für die Durchsetzung der Richtlinie auf nationaler Ebene zuständig ist.

Sozialgesetzbuch und Bundesteilhabegesetz

Ein wichtiges und im Diskurs sehr präsentes Bundesgesetz, wenn es um das Thema Behinderung geht, ist das in zwölf Bücher unterteilte Sozialgesetzbuch, 
das Sozialversicherungsleistungen bzw. Sozialleistungen für Menschen mit und auch für Menschen ohne Behinderung regelt.

Den „Allgemeinen Teil“ des Sozialrechts bildet das Erste Buch des Sozialgesetzbuches (SGB I), das erstmals 1976 in Kraft trat. In SGB I, das nicht auf eine bestimmte Gruppe von Bedarfsträgern ausgerichtet ist, werden die Grundlagen des Sozialrechts sowie verschiedene Leistungsarten und Zuständigkeiten festgelegt. Auch der barrierefreie Zugang zu den jeweiligen Leistungen wird in SGB I berücksichtigt. So sind nach $\$ 17$ Absatz 1 SGB I Sozialleistungsträger dazu verpflichtet, den Zugang zu Sozialleistungen so weit wie möglich zu vereinfachen, zum Beispiel „durch Verwendung allgemein verständlicher Antragsvordrucke“ und den Abbau von Kommunikationsbarrieren und baulichen Barrieren. In Absatz 2 ist darüber hinaus festgelegt, dass Menschen mit Hör- bzw. Sprachbehinderung „bei der Ausführung von Sozialleistungen, insbesondere auch bei ärztlichen Untersuchungen und Behandlungen" das Recht haben, auf Kosten des zuständigen Leistungsträgers Gebärdensprache oder „andere geeignete Kommunikationshilfen" zu verwenden.

Nach $₫ 17$ Absatz 2a SGB I ist außerdem seit dem 1. Januar 2018 auch der bereits erwähnte $\$ 11$ BGG zur Leichten Sprache bei der Ausführung von Sozialleistungen anzuwenden. Die nach dem SGB I für die jeweiligen Leistungen zuständigen Stellen sind in den $\$ \$ 18$ bis 29 SGB I aufgeführt und reichen von den Ämtern für Ausbildungsförderung der Landkreise und kreisfreien Städte über die Unfallkassen bis zu den kommunalen Gesundheitsämtern oder die für die Kinder- und Jugendhilfe zuständigen Stellen der Landkreise, kreisfreien Städte und Gemeinden. Damit werden die im BGG genannten Stellen, die zur Erläuterung bestimmter Bescheide, Allgemeinverfügungen, Verträge und Vordrucke in Leichter Sprache verpflichtet sind, von Bundesbehörden bzw. bundesbehördliches Recht ausführenden Landesbehörden um alle Stellen erweitert, die nach dem SGB geregelte Sozialleistungen erbringen, und damit auch um den Bereich der Länder und Kommunen.

Diese Ausweitung ist auch in $\$ 19$ Absatz 1a des Zehnten Buches des Sozialgesetzbuches (SGB X) enthalten, das das sozialrechtliche Verwaltungsverfahren regelt, also die Tätigkeit einer Behörde, „die auf die Prüfung der Voraussetzungen, die Vorbereitung und den Erlass eines Verwaltungsaktes oder auf den Abschluss eines öffentlich-rechtlichen Vertrages gerichtet ist" ( $\$ 8$ SGB X). Eine 
Behörde im Sinne dieses Gesetzes ist dabei „jede Stelle, die Aufgaben der öffentlichen Verwaltung wahrnimmt“ ( $\$ 1$ Absatz 2 SGB X). Mit der in $\$ 19$ Absatz 1a SGB X enthaltenen Verweisung auf $₫ 11$ BGG geht damit eine potenziell weitreichende Erweiterung des Kreises der zur Verwendung Leichter Sprache Verpflichteten einher.

Das 2001 in erster Fassung in Kraft getretene Neunte Buch des Sozialgesetzbuches (SGB IX) regelt speziell die verschiedenen Arten von Sozialleistungen für Menschen mit Behinderung. Sie haben nach $\$ 164$ Absatz 4 Nummer 4 und 5 SGB IX beispielsweise Anspruch auf die individuell behinderungsgerechte Gestaltung ihres Arbeitsumfeldes, was auch Informations- und Kommunikationstechnik (z. B. Intranetseiten) einschließt. SGB IX enthält außerdem mit $\$ 209$ SGB IX die rechtliche Grundlage zur Erteilung von Nachteilsausgleichen für Menschen mit Behinderung, die zum Beispiel im Bereich Schule oder Ausbildung auch in Form von Kommunikationshilfen gewährt werden können (z. B. sprachlich vereinfachte Prüfungsaufgaben, siehe dazu Rink 2014).

Mit dem „Gesetz zur Stärkung der Teilhabe und Selbstbestimmung von Menschen mit Behinderungen“" (Bundesteilhabegesetz - BTHG) erfolgen im Zeitraum 2017 bis 2023 stufenweise tiefgreifende Änderungen zur Modernisierung des Sozialgesetzbuches und vor allem des SGB IX mit dem Ziel der Weiterentwicklung der nationalen Behindertenpolitik im Sinne der UN-BRK. Darüber hinaus soll die Position von Menschen mit Behinderung als Leistungsempfänger gestärkt werden. Wichtige Punkte im BTHG sind neben der Anpassung des Behinderungsbegriffs gemäß der UN-BRK die Neugestaltung der Eingliederungshilfe hin zu mehr Selbstbestimmungs- und Entscheidungsmöglichkeiten für die Betroffenen sowie die Verbesserung der Teilhabe im Arbeitsleben. 


\section{Literaturverzeichnis}

Aichele, VALEnTin (2015): „Unabhängig und kritisch: die Monitoring-Stelle zur UNBRK“. In: Degener, Theresia/DieHL, ElKe (Hrsg.): Handbuch Behindertenrechtskonvention. Teilhabe als Menschenrecht - Inklusion als gesellschaftliche Aufgabe. Bonn: Bundeszentrale für politische Bildung, S. 85-92. (Schriftenreihe Band 1506).

AKtion Mensch (о.J.): „Gleichstellungsgesetze im Bund und in den Ländern.“ In: https://www.einfach-fuer-alle.de/artikel/bitv/bgg/ (letzter Zugriff: 28.02.2018).

Bethre, Andreas/Kruse, Klemens/Rebstock, Markus/Welti, Felix (2015): „Barrierefreiheit“. In: Degener, Theresia/Diehl, ElKe (Hrsg.): Handbuch Behindertenrechtskonvention. Teilhabe als Menschenrecht - Inklusion als gesellschaftliche Aufgabe. Bonn: Bundeszentrale für politische Bildung, S. 170-188. (Schriftenreihe Band 1506).

BocK, BeTTINA M. (2015): „Barrierefreie Kommunikation als Voraussetzung und Mittel für die Partizipation benachteiligter Gruppen - Ein (polito-)linguistischer Blick auf Probleme und Potenziale von "Leichter" und "einfacher Sprache"“. In: Linguistik Online 73 (4) (https://bop.unibe.ch/linguistik-online/article/view/2196/3366; letzter Zugriff: 28.02.2018).

Bredel, Ursula/MaAß, Christiane (2016): Leichte Sprache. Theoretische Grundlagen. Orientierung für die Praxis. Berlin: Duden.

Bundesministerium der Justiz (2008): Handbuch der Rechtsförmlichkeit. Empfehlungen des Bundesministeriums der Justiz für die rechtsförmliche Gestaltung von Gesetzen und Rechtsverordnungen nach $\$ 42$ Absatz 4 und $\$ 62$ Absatz 2 der Gemeinsamen Geschäftsordnung der Bundesministerien. Köln: Bundesanzeiger Verlagsges. mbH. 3., neu bearbeitete Auflage.

Bundesministerium für Arbeit und Soziales (2014): „Evaluation des Behindertengleichstellungsgesetzes - Abschlussbericht“. In: http://www.bmas.de/SharedDocs/ Downloads/DE/PDF-Publikationen/Forschungsberichte/fb-445.pdf;jsessionid= A45261579D78572CC78BB7524C77ACDD?_blob=publicationFile\&v=2 (letzter Zugriff: 28.02.2018).

Bundesministerium für Arbeit und Soziales (2016): „Nationaler Aktionsplan 2.0 der Bundesregierung zur UN-Behindertenrechtskonvention“. In: https://www.gemeinsameinfach-machen.de/SharedDocs/Downloads/DE/AS/ NAP2/NAP2.pdf?_blob=publicationFile\&v=1 (letzter Zugriff: 28.02.2018).

Bundestagsdrucksache 14/7420: „Entwurf eines Gesetzes zur Gleichstellung behinderter Menschen und zur Änderung anderer Gesetze“. In: http://dipbt.bundestag.de/ doc/btd/14/074/1407420.pdf (letzter Zugriff: 28.02.2018).

Bundestagsdrucksache 17/11473: „Entwurf eines Gesetzes zur Förderung der elektronischen Verwaltung sowie zur Änderung weiterer Vorschriften“. In: http://dipbt.bundestag.de/ dip21/btd/17/114/1711473.pdf (letzter Zugriff: 28.02.2018). 
Bundestagsdrucksache 17/12634: „Entwurf eines Gesetzes zur Förderung des elektronischen Rechtsverkehrs mit den Gerichten“. In: http://dipbt.bundestag.de/dip21/ btd/17/126/1712634.pdf (letzter Zugriff: 28.02.2018).

Bundestagsdrucksache 18/13258: „Antwort der Bundesregierung auf Kleine Anfrage „Barrierefreiheit in der Privatwirtschaft ““. In: http://dip21.bundestag.de/dip21/btd/ 18/132/1813258.pdf (letzter Zugriff: 28.02.2018).

Bundestagsdrucksache 18/11135: „Entwurf eines Gesetzes zur Neuregelung des bundesstaatlichen Finanzausgleichssystems ab dem Jahr 2020 und zur Änderung haushaltsrechtlicher Vorschriften“. In: http://dip21.bundestag.de/dip21/btd/18/111/ 1811135.pdf (letzter Zugriff: 28.02.2018).

Degener, THERESIA (2015): „Die UN-Behindertenrechtskonvention - ein neues Verständnis von Behinderung“' In: DEGEner, Theresia/DieHL, ElKe (Hrsg.): Handbuch Behindertenrechtskonvention. Teilhabe als Menschenrecht - Inklusion als gesellschaftliche Aufgabe. Bonn: Bundeszentrale für politische Bildung, S. 55-74. (Schriftenreihe Band 1506).

EU-Richtlinie 2016/2102: „Amtsblatt der Europäischen Union 327/1. Richtlinie (EU) 2016/2102 des Europäischen Parlaments und des Rates vom 26. Oktober 2016 über den barrierefreien Zugang zu den Websites und mobilen Anwendungen öffentlicher Stellen“. In: http://eur-lex.europa.eu/legal-content/DE/TXT/PDF/?uri= CELEX:32016L2102\&from=DE (letzter Zugriff: 28.02.2018).

Gehörlosenbund e.V. (2012): „Pressemitteilung 07/2012: 10 Jahre Behindertengleichstellungsgesetz (BGG) - 10 Jahre Anerkennung der Deutschen Gebärdensprache!“. In: http://www.dglb.de/dgb/images/stories/pdfs_2012/03_pm_2012_07_

10jahrebgg_120502.pdf (letzter Zugriff: 28.02.2018).

HellbusCH, Jan ERIC (2001-2018): „Barrierefreies Webdesign. Ein zugängliches und nutzbares Internet gestalten.“ In: https://www.barrierefreies-webdesign.de/ (letzter Zugriff: 28.02.2018).

HellbUSCH, JAN ERIC (2014): „Accessibility-Blog und mehr. Accessibility Checklist gegen graue Haare. BITV 2.0 und ,BITV-Test' sind nicht kompatibel mit den internationalen WCAG 2.0.“ In: https://www.hellbusch.de/accessibility-checkliste/ (letzter Zugriff: 28.02.2018).

Hellbusch, Jan ERIC (2011): „BITV 2.0 in Kraft“ (Kommentar). In: http://webkrauts.de/artikel/2011/bitv-20-kraft (letzter Zugriff: 28.02.2018).

Hellbusch, Jan ERIC/Probiesch, Kerstin (2011): Barrierefreiheit verstehen und umsetzen. Webstandards für ein zugängliches und nutzbares Internet. Heidelberg: dpunkt Verlag.

Hessisches Ministerium für Soziales und Integration (2016): „Leitfaden für eine barrierefreie Verwaltung“. In: http://www.brk.hessen.de/global/show_document.asp?id= aaaaaaaaaacudc (letzter Zugriff: 28.02.2018).

Deutsches Institut für Menschenrechte (2018): Monitoring-Stelle UN-BRK. Auswertung der Koalitionsvereinbarungen in Bund und Ländern: Zentrale Anliegen der UN- 
BRK. In: http://www.institut-fuer-menschenrechte.de/monitoring-stelle-un-brk/ monitoring/koalitionsvertraege/ (letzter Zugriff: 28.02.2018).

Kerkmann, Friederike (2015): „Der rechtliche Rahmen - Ein Überblick über Gesetze, Verordnungen, Richtlinien und Normen zu barrierefreier Information und Kommunikation“. In: KERKMANN, FrIEDERIKe/LEWANDOWSKI, DiRK (Hrsg.): Barrierefreie Informationssysteme. Zugänglichkeit für Menschen mit Behinderung in Theorie und Praxis. Berlin u.a.: de Gruyter. S. 11-48. (Age of access? - Grundfragen der Informationsgesellschaft).

Koalitionsvertrag der Bundesregierung zur 19. Legislaturperiode: „Teilhabe von Menschen mit Behinderung“. In: https://www.bundesregierung.de/Content/DE/ Anlagen/2018/03/2018-03-14-koalitionsvertrag.pdf?_blob=publicationFile\&v=2 (letzter Zugriff: 15.04.2018).

Kommission der Europäischen Gemeinschaften (2002): „eEurope 2005: Eine Informationsgesellschaft für alle. Aktionsplan zur Vorlage im Hinblick auf den Europäischen Rat von Sevilla am 21./22. Juni 2002.“ In: http://ec.europa.eu/transparency/ regdoc/rep/1/2002/DE/1-2002-263-DE-F1-1.Pdf (letzter Zugriff: 28.02.2018).

Netzwerk Artikel 3 e.V. (2009): „Schattenübersetzung der UN-BRK“. In: http://www.nw3.de/ attachments/article/93/093_schattenuebersetzung-endgs.pdf (letzter Zugriff: 28.02.2018).

Presse- und Informationsamt der Bundesregierung (2018): Styleguide der Bundesregierung. In: https://styleguide.bundesregierung.de/Webs/SG/DE/Homepage/home_ node.html?__site=SG (letzter Zugriff: 28.02.2018).

RINK, IsABEL (2014): Nachteilsausgleich im Bereich Hörschädigung: Zur Übersetzung von Mathematikarbeiten in Leichte Sprache. Masterarbeit Universität Hildesheim. In: http://hildok.bsz-bw.de/frontdoor/index/index/docId/212 (letzter Zugriff: 28.02.2018).

UN-Behindertenrechtskonvention (2008): Übereinkommen über die Rechte von Menschen mit Behinderungen. Bundesgesetzblatt (BGBL) 2008 II, Nr. 35; 1419. In: https://www.bgbl.de/xaver/bgbl/start.xav?startbk=Bundesanzeiger_BGBl\&start=//* \%255B@attr_id=\%27bgbl208s1419.pdf\%27\%255D\#_bgbl_\%2F\%2F*\%5B\%40attr _id\%3D\%27bgbl208s1419.pdf\%27\%5D_1520501893038_(letzter Zugriff: 28.02.2018).

United Nations Treaty Collection (2006): „15. Convention on the Rights of Persons with Disabilities“. In: https://treaties.un.org/Pages/ViewDetails.aspx?src=TREATY\& mtdsg_no=IV-15\&chapter=4\&clang=_en (letzter Zugriff: 28.02 .2018 ).

WCAG 2.0 (2009): „Web Content Accessibility Guidelines (WCAG) 2.0. Autorisierte deutsche Übersetzung.“. In: http://www.w3.org/Translations/WCAG20-de (letzter Zugriff: 28.02.2018).

WCAG 2.1 (2018): „Web Content Accessibility Guidelines (WCAG) 2.1“. In: https://www.w3.org/TR/WCAG21/ (letzter Zugriff: 28.02.2018). 
W3C (2012): „W3C Web Content Accessibility Guidelines 2.0 Approved as ISO/IEC International Standard“. In: https://www.w3.org/2012/07/wcag2pas-pr.html (letzter Zugriff: 28.02.2018).

W3C (2018): „WCAG 2.1 is a Candidate Recommendation“. In: https://www.w3.org/ blog/2018/01/wcag21-cr/ (letzter Zugriff: 28.02.2018). 



\section{Barrierefreies Grafikdesign}

Grafikdesign hat die Aufgabe, mittels eines konsistenten Formen-, Farben- und Gliederungssystems eine leichte und zielgruppengerechte Informationsvermittlung zu ermöglichen. Barrierefreiheit ist somit per se sein Programm. In der Typografie und im Layout visualisiert Grafikdesign die Sprache, währenddessen es im Bild die Sprache auch um Informationen erweitern kann (vgl. van Leeuwen 2005; Stöckl 2011). Grafikdesign entwirft ein didaktisches Konzept, dessen Umsetzung dem Rezipienten eine möglichst fehlerfreie, erwartungskonforme Informationsaufnahme ermöglicht (vgl. EN ISO 9241-Teil 110, Grundsätze der Dialoggestaltung).

Ein konsistentes grafisches Konzept schont das Arbeitsgedächtnis des Lesers und ermöglicht ihm ein entspanntes Leseerlebnis. Er empfindet ein gut gestaltetes Layout als attraktiv und lesemotivierend. Schön ist, was funktioniert. Dieser Grundsatz gilt für die Produkte Leichter Sprache und einfacher Sprache genauso wie für die der Standardsprache.

Warum können wir dann die mikrostrukturellen Kriterien für gutes Grafikdesign (vgl. Alexander 2013: 51-62) nicht einfach auf die Leichte Sprache anwenden? Warum müssen wir Gestaltungsregeln überdenken und mit den Praxiserfahrungen, die das Netzwerk Leichte Sprache in seinen Regeln für Leichte Sprache verfasst hat, abgleichen? Die Empfehlungen des Netzwerks Leichte Sprache, Inclusion Europe und BITV 2.0 (vgl. Bredel/Maaß 2016: 110ff.) werden im Artikel als bekannt vorausgesetzt; deshalb werden die visuellen Regeln des Netzwerks Leichte Sprache in der folgenden Tabelle nur kurz zusammengefasst. 


\begin{tabular}{|c|c|c|c|}
\hline $\begin{array}{l}\text { Regeln zur } \\
\text { Typografie }\end{array}$ & Regeln zum Layout & $\begin{array}{l}\text { Regeln zur } \\
\text { Bildgestaltung }\end{array}$ & $\begin{array}{l}\text { Regeln zum } \\
\text { Umgang mit } \\
\text { Bildmedien }\end{array}$ \\
\hline $\begin{array}{l}\text { - einfache, gerade } \\
\text { Schrift } \\
\text { - nur eine Schriftart } \\
\text { - mind. Schriftgröße } \\
14 \text { pt } \\
\text { - Hervorhebung von } \\
\text { Text: } \\
\text { o durch dunkle } \\
\text { Schriftfarbe } \\
\text { o durch Hinter } \\
\text { legen des Textes } \\
\text { mit einer hellen } \\
\text { Farbe } \\
\text { o durch Fettdruck } \\
\text { - keine Hervor } \\
\text { hebung durch } \\
\text { Versalsatz oder } \\
\text { Kursivsatz, keine } \\
\text { Unterstreichung }\end{array}$ & $\begin{array}{l}\text { - deutlicher Kon- } \\
\text { trast zwischen } \\
\text { Schrift und Papier } \\
\text { - viele Absätze und } \\
\text { Überschriften } \\
\text { - Aufzählungs- } \\
\text { punkte } \\
\text { - Rahmung } \\
\text { - jeder Satz auf eine } \\
\text { neue Zeile } \\
\text { - } 1,5 \text {-facher Zeilen- } \\
\text { abstand } \\
\text { - linksbündig } \\
\text { - keine Silbentren- } \\
\text { nung am Zeilen- } \\
\text { ende } \\
\text { - kein Blocksatz }\end{array}$ & $\begin{array}{l}\text { - Bilder benutzen } \\
\text { - zum Text passende } \\
\text { Bilder benutzen } \\
\text { - scharfe und klare } \\
\text { Bilder benutzen }\end{array}$ & $\begin{array}{l}\text { - bei Bildeinsatz auf } \\
\text { Erkennbarkeit und } \\
\text { Kopierfähigkeit } \\
\text { achten: } \\
\text { o durch matte Pa- } \\
\text { pieroberfläche } \\
\text { o durch Verwen- } \\
\text { dung scharfer } \\
\text { und klarer Bilder } \\
\text { - Bilder nicht als } \\
\text { Hintergrund für } \\
\text { Text verwenden }\end{array}$ \\
\hline
\end{tabular}

Tab. 1: Regeln des Netzwerks Leichte Sprache für die visuelle Gestaltung nach BMAS (2014)

In der visuellen Kommunikation basieren alle grafischen Regeln auf den Gestaltgesetzen der Wahrnehmung (vgl. Arnheim 1954). Die Vertreter der Berliner Schule der Gestaltpsychologie entwickelten zu Beginn des 20. Jahrhunderts die "Gestalttheorie“ als eine neue psychologische Richtung. Die Wissenschaftler formulierten über 100 Regeln, nach denen das menschliche Gehirn visuelle Reize auswertet (vgl. Alexander 2013: 25-31). Ohne diese übereinstimmende Informationsauswertung wäre eine verlässliche visuelle Kommunikation unmöglich.

Ein Designer nutzt diese Regeln für den Gestaltungsprozess. Z. B. besagt das Gesetz der Nähe, dass Objekte, die sich in räumlicher Nähe zueinander befinden, vom Gehirn als Sinngemeinschaft gewertet werden. Dem durchschnittlichen Menschen ermöglicht dieses Gestaltgesetz, die Zusammengehörigkeit von Texten zu Bildern in horizontaler Anordnung oder in Spalten zu begreifen, Titel nachfolgenden Inhalten zuzuordnen, Bilder durch Beschriftungen zu ergänzen 
(vgl. Alexander 2013: 25ff.). Alle Abstandsregelungen im Layout fußen auf diesem Gestaltgesetz. Das Gesetz der Ähnlichkeit besagt, dass ähnlich aussehende Objekte als zusammengehörig erlebt werden. Deshalb zeichnet ein Designer Titel der gleichen Hierarchieebene mit der gleichen Form und Farbe aus, strukturiert Layouts durch Standardelemente wie Pagina, Rubriktitel, Piktogramme und setzt Farbe strukturbildend in Abbildungen ein.

Die eingeschränkten Wahrnehmungs- und Verarbeitungsmöglichkeiten der heterogenen Nutzergruppe „Menschen mit Lernschwierigkeiten“ stellen das gewohnte Wirken der Gestaltgesetze in Frage. Wir können uns nicht sicher sein, dass unser Gegenüber Bilddetails gemäß dem Gesetz der Prägnanz zu einem Ganzen ordnen kann, dass der sinnstiftende Zusammenhang farblicher Strukturierungen nach dem Gesetz der Ähnlichkeit erkannt wird, dass das Gesetz der guten Fortsetzung die Anordnung einer Bildfolge erschließbar macht usw. Dieser Umstand führt zu einem Desiderat, das ein erhebliches Forschungspotential aufweist.

Seminaristische Studien an der Hochschule Merseburg zu Typografie und Layout, Textgliederung, Darstellungsarten, Bild-Sprache-Beziehung und Bildfunktionen stellen Ansätze angehender Informationsdesignerinnen und Informationsdesigner dar, diese Forschungslücke zu schließen. Die Studien entspringen der praktischen Auseinandersetzung mit barrierefreiem Grafikdesign und nutzen Erkenntnisse der pädagogischen Psychologie und der Medienwissenschaft. Alle Studien wurden mit lernbehinderten Probanden getestet. Daraus können Hinweise zum barrierefreien Grafikdesign abgeleitet werden.

\section{Typografie und Layout im barrierefreien Grafikdesign}

Vereinfacht gesagt hat die Typografie (auch Mikrotypografie genannt) die Erkennbarkeit und Lesbarkeit einer Schrift zum Ziel. Mit Layout bezeichnet man das Beziehungsgeflecht von Schrift, Gliederungselementen, Abbildungen und den sie umgebenden Weißraum. Layout wird auch als auch Makrotypografie bezeichnet. Ausgangspunkt der Forschung zur Typografie an der Hochschule Merseburg sind die in der DIN 1450 (2013) formulierten typografischen Empfehlungen für eine gute Leserlichkeit von Schrift. Während die bisherige DIN 
1450 im Wesentlichen die Leserlichkeit von Schrift im öffentlichen Raum betraf, beinhaltet die Überarbeitung von 2013 nun auch Angaben zur Leserlichkeit in Büchern, Broschüren und Zeitungen. Insgesamt unterscheidet die DIN 1450 vier Textarten: Lesetext, Konsultationstext, Signalisationstext und Schautext. In der Studie von Rohde und Stechert (2016, vgl. Alexander 2017: 318ff.) liegt der Fokus auf den Lesetexten. Die DIN 1450 definiert für jede dieser Textarten Kriterien guter Leserlichkeit und trifft Schriftempfehlungen für die Praxis. Mit Blick darauf, wie diese Hinweise in der Praxis anzuwenden sind, ergeben sich jedoch gravierende Mängel. Zum einen sind die Kriterien relativ abstrakt formuliert und damit für „Nichttypografen“ eher untauglich. Zum anderen sind die empfohlenen Schriften veraltet und kostenpflichtig. Kurz nach der Veröffentlichung der neuen Norm kam die nach der DIN 1450 konzipierte und hinsichtlich Barrierefreiheit optimierte Schriftart Neue Frutiger 1450 auf den Markt, die eine gute Erkennbarkeit aufweist, aber kostenintensiv ist.

Die Studie von Rohde und Stechert (2016) vergleicht an Hand der Kriterien der Norm DIN 1450 vier kostenfreie Open-Source-Schriften mit der Neuen Frutiger 1450. Im Ergebnis sprechen Rohde und Stechert eine Empfehlung für den Testsieger, die Open-Source-Schrift Open Sans für Lesetexte aus. Rohde und Stechert weisen damit nach, dass auch kostenfreie Open-Source-Schriftarten die Anforderungen an barrierefreie Typografie erfüllen können. Auf Grund des geringen Abstands zum Testsieger erfüllen auch die zweit- und drittplatzierten Schriften Fira Sans und Roboto für das Lesen in Printform und am PC die Anforderungen an Barrierefreiheit. Neben der Frage nach der richtigen Schrift kann auch eine professionelle Satztechnik ihren Beitrag zu einer guten Erkennbarkeit und Differenzierbarkeit von Glyphen leisten. Eine gute Dokumentenschrift wie z. B. die Myriad Pro verfügt über Alternativglyphen wie z. B. eine Null mit Schrägstrich, welche die Zahl besser vom Versal-O differenziert.

Liquides Layout und Responsivität stellen neue Anforderungen an barrierefreies Grafikdesign. Auf die Frage, was für responsive Typografie wichtig ist, antwortet Kupferschmid: 
In Hinsicht auf Schrift: etwa gutes Rendering, gleichmäßige Strichstärken, nicht zu schmale, rhythmische Punzen, moderate bis große x-Höhe, deutliche Innenformen und Satzzeichen, Kerning, gute Akzentbuchstaben. (Kupferschmid 2014: 5)

Schrift- und Layoutempfehlungen für das Lesen auf Smartphones und anderen mobilen Endgeräten stellen derzeit ein Forschungsdesiderat dar.

In der Studie von Demagin, Donner, Kutschera und Thieme wird mit Hilfe eines Usability-Tests untersucht, wie typografische Gliederungselemente die Übersichtlichkeit sowie die Verständlichkeit eines Textes in Leichter Sprache beeinflussen und verbessern können (siehe Abb. 1). Die Makrotypografie bestimmt den Gesamteindruck des Textes, indem Informationen gegliedert, organisiert und visuell hervorgehoben werden. Dabei kehren bestimmte Anordnungsmuster immer wieder, sodass der Leser schon anhand der äußeren Form eine klare Vorinformation über die Textart bekommt (vgl. Hochuli 2011: 7). Mit diesem Ziel wurde das Layout eines nach den Regeln für Leichte Sprache gesetzten Textes verändert. 
Die Hilfe für Menschen

mit Behinderungen

in der Europäischen Union

\section{Das ist die Europäische Union}

Deutschland ist ein Land

Deutschland ist in der Mitte von Europa.

Das ist ein Teil von der Welt.

Europa hat viele Lănder.

Zum Beispiel: Deutschland.

Und Frankreich. Und Spanien.

Und noch viele andere $L$ ander.

28 Lānder in Europa arbeiten eng zusammen.

Sie sind eine Gruppe.

Die Gruppe heilt: Europäische Union.

Das kurze Wort dafür ist: EU.

So spricht man das: ee u.

\section{Was für Hilfe gibt es?}

Fur Menschen mit Behinderungen gibt es besondere Arbeits-Plätze. Wenn sie nicht in einer Firma

arbeiten können.

Menschen mit Behinderunge brauchen oft Hilfe mit Geld.

Die Länder in der EU helfen deswegen

zum Beispiel mit Geld:

Wenn ein Mensch nicht arbeiten kann.

oder wenn ein Mensch nicht.

für seine Medikamente bezahlen kann.

Leichte Sprache erklart alle Infos

auf einfache Art.

Zum Beispiel mit: Kurzen Sătzen.

Einfachen Wörtern.

Großer Schrift.

Und Bildern. 
Die Hilfe für Menschen

mit Behinderungen

in der Europäischen Union

\author{
Das ist die Europäische Union \\ Deutschland ist ein Land. \\ Deutschland ist in der Mitte von Europa. \\ Das ist ein Teil von der Welt. \\ Europa hat viele Länder. \\ Zum Beispiel: \\ Deutschland. \\ Und Frankreich. \\ Und Spanien. \\ Und noch viele andere Länder. \\ 28 Länder in Europa \\ arbeiten eng zusammen. \\ Sie sind eine Gruppe. \\ Die Gruppe heißt: Europäische Union. \\ Das kurze Wort dafür ist: EU. \\ So spricht man das: ee $u$.
}

Was für Hilfe gibt es?

- Für Menschen mit Behinderungen gibt es besondere Arbeits-Plätze. Wenn sie nicht in einer Firma arbeiten können.

- Menschen mit Behinderungen brauchen oft Hilfe mit Geld.

Die Länder in der EU helfen deswegen

zum Beispiel mit Geld:

Wenn ein Menserh nirht arheiten kann

Oder wenn ein Mensch nicht

für seine Medikamente bezahlen kann.

- Leichte Sprache erklärt alle Infos auf einfache Art.

Zum Beispiel mit: Kurzen Sătzen.

Einfachen Wörtern

Großer Schrift.

Und Bildern.

Abb. 1: Typografische Gliederungselemente im Vergleich (Quelle: https:// Rat-Geber für Menschen mit Behinderung in der Europäischen Union (BMAS 2015); Studie: () Donner/ Demagin/Kutschera/Thieme, HS Merseburg (2017))

Den Empfehlungen der Typografen Willberg und Forssman folgend wurde nach der Hauptüberschrift eine horizontale Linie eingefügt, um die hierarchische Überordnung zu verdeutlichen (vgl. Willberg/Forssman 2010). 
Es wurde ein Zeilenabstand von 135 \% gewählt, damit einzelne Absätze besser als Sinneinheit erkennbar sind. Des Weiteren wurden die Abstände vor und nach den Zwischenüberschriften geändert. So beträgt der Abstand vor diesen drei Leerzeilen und der Abstand danach eine Leerzeile. Damit wird die Zugehörigkeit zum jeweils folgenden Abschnitt verdeutlicht (vgl. Willberg/ Forssman 2010: 200). Der Schriftgrad wurde von 18 pt auf 20 pt erhöht.

Hauptüberschrift als auch Zwischenüberschriften wurden Grün ausgezeichnet. Als zusätzliche Gliederungsmaßnahme wurde der jeweils zugehörige Text unter beiden Zwischenüberschriften eingerückt und mit einem schwarzen Vertikalstrich links vom Zeilenanfang gekennzeichnet. Darüber hinaus wurden die Absätze nach der zweiten Zwischenüberschrift jeweils mit einem Punkt versehen. Suchbegriffe wurden in fetter Auszeichnung gesetzt.

Beide Texte wurden einem Lesetest unterzogen, dessen Lesezeit gemessen und durch Fragen zum Textüberblick und zum Textverständnis ergänzt wurde (vgl. Demagin/Donner/Kutschera/Thieme 2017: 6). Das Ergebnis zeigte eine signifikant schnellere Lesegeschwindigkeit für den optimierten Text. In der Beantwortung der Fragen zum Textüberblick und zum Textverständnis schnitt der optimierte Text dennoch in allen Fragen deutlich schlechter ab als der Ausgangstext. Auf Grund der Vielzahl an Veränderungen kann keine Aussage darüber getroffen werden, welches Gliederungselement als Barriere gewirkt hat. Ein möglicher Schluss ist, dass ungewohnte Gliederungselemente zwar beim Lesen helfen, aber die Probanden beim Aufsuchen von Informationen verunsichern.

In ihren Untersuchungen zur Typografie von Dokumenten berichten Lange, Römer und Schneider (2017), dass die Probanden stark auf das nach den Regeln der Leichten Sprache geformte Satzbild konditioniert sind. Vor die Wahl zweier Broschürencover gestellt, entschieden sich $57 \%$ der Probanden für das unoptimierte, deutlich weniger attraktive Titelmotiv und nur $43 \%$ für den optimierten Broschürentitel mit dem bunten Schirm (siehe Abb. 2). 

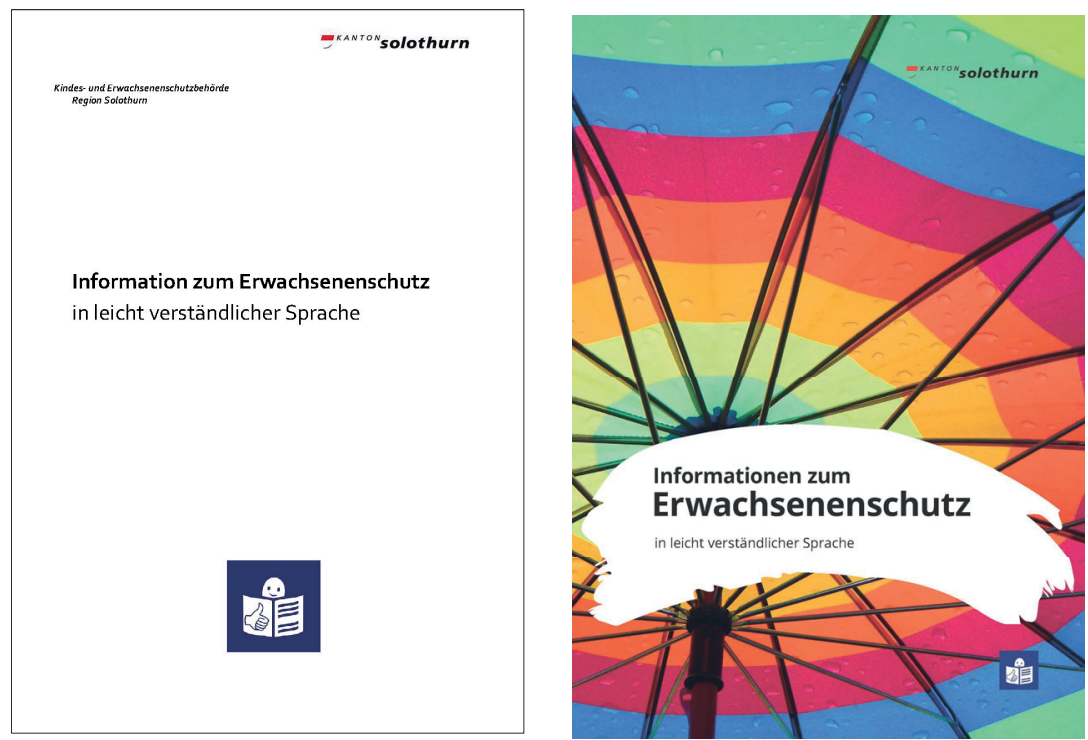

Abb. 2: Cover einer Broschüre, Design alt und neu im Vergleich (Quelle: Kindes- und Erwachsenenschutzbehörde Region Solothurn (Hrsg.) (2016): Information zum Erwachsenenschutz in leicht verständlicher Sprache. Solothurn; Studie: $\odot$ Lange/Schneider/Römer, HS Merseburg 2017)

Einige Probanden griffen nach dem schlichten weißen Cover der Ausgangsbroschüre und begründeten ihre Entscheidung mit den Worten: Das ist doch das für uns. Sie fühlten sich mit dem farbigen Motiv nicht gemeint. Es liegt zudem nahe, dass die Metapher „beschirmen“ nicht erkannt wurde. Die exemplifikationale Bildkompetenz kann bei Lernbehinderung nicht vorausgesetzt werden (vgl. Posner 2003: 20).

Die umfangreiche Broschüre zum Thema Erwachsenenschutz wurde durch ein Inhaltsverzeichnis mit farbigen Logogrammen zur Differenzierung der Kapitel strukturiert. Sowohl die Farben als auch die Logogramme erschienen in den jeweiligen Kapiteltiteln zur besseren Auffindbarkeit erneut (siehe Abb.3).

Auf die Frage zur Ausgangsbroschüre, „Was glauben Sie, welche Bedeutung die Farben im Inhaltsverzeichnis haben?", konnten 36 \% der Probanden die Bedeutung der Farben erkennen, die anderen $64 \%$ hingegen nicht. 
Auf die Frage zur optimierten Broschüre, „Was glauben Sie, warum im Inhaltsverzeichnis Symbole und Farben verwendet werden?", konnten in der optimierten Variante 57 \% der Probanden die Bedeutung der Farben und Piktogramme erkennen, $43 \%$ hingegen nicht.

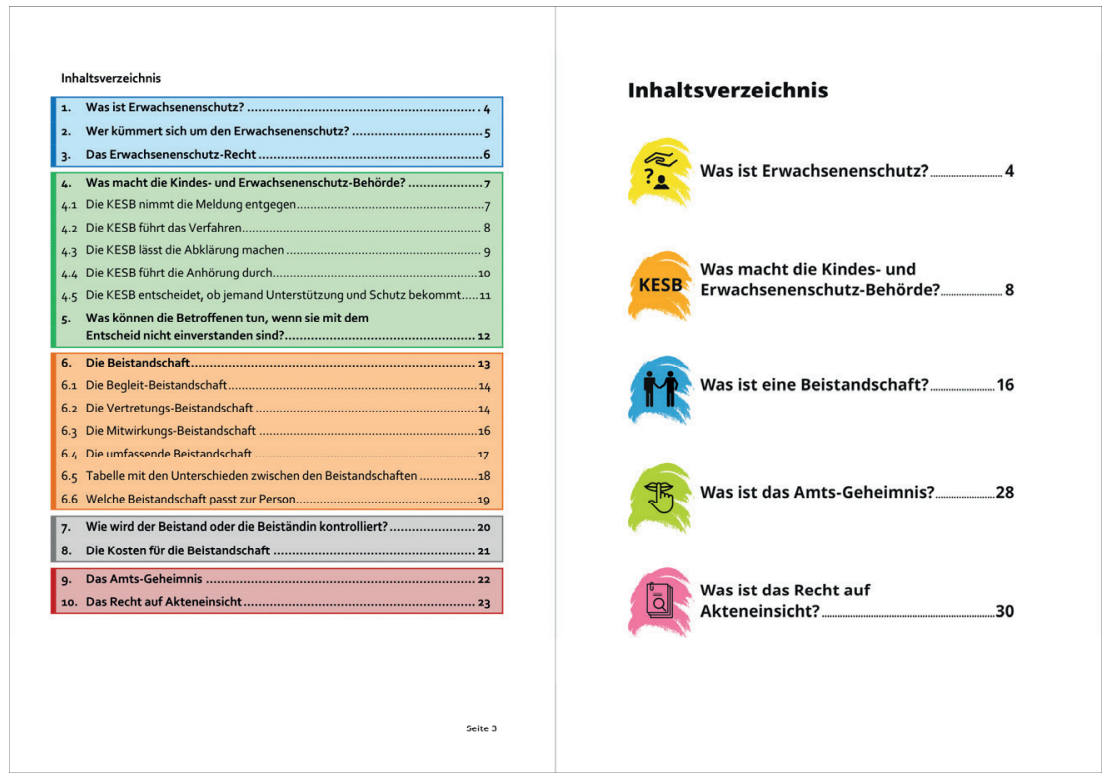

Abb. 3: Farbe und Symbole als strukturbildende Elemente einer Broschüre, Design alt und neu im Vergleich (Quelle: Kindes- und Erwachsenenschutzbehörde Region Solothurn (Hrsg.) (2016): Information zum Erwachsenenschutz in leicht verständlicher Sprache. Solothurn; Studie: (C) Lange/Schneider/Römer, HS Merseburg 2017)

Auf die Aufgabe „Finden Sie heraus, wer eine solche Beistandschaft bezahlt" konnten die Probanden nur unter Zuhilfenahme des Inhaltsverzeichnisses antworten.

Im Ausgangsdokument gelang es keinem der Probanden, die geforderte Information ohne Hilfe zu finden. 41 \% konnten die Information mit Hilfe auffinden, $42 \%$ haben es trotz Hilfe nur zum Teil geschafft und $17 \%$ haben es gar nicht geschafft. 
Im optimierten Dokument schafften es $7 \%$ der Probanden, die bestimmte Information ohne Hilfe zu finden. $14 \%$ haben sie zum Teil ohne Hilfe gefunden. Mit Hilfe konnten $22 \%$ die gesuchte Information finden. 50 \% hingegen konnten sie trotz Hilfestellung nicht finden.

Als ein Ergebnis der Studie kann festgestellt werden, dass der Einsatz von Piktogrammen (in Form und Farbe abstrahierte Zeichen mit Aufforderungscharakter) die Übersichtlichkeit der Broschüre verbessert hat. Farbe als strukturbildendes Element allein reicht nicht aus.

In ihren Aufzeichnungen zu den Usability-Tests notieren Lange, Römer und Schneider (2017), dass Probanden die Broschüre von vorn nach hinten durchlasen - inklusive des Impressums, wenn es dem Titel folgte. Andere Probanden lasen nur den Mengensatz und ignorierten die Titel. Wieder andere verwechselten die Seitenzahlen mit Kapitelnummerierungen.

Diese Ergebnisse lassen den Schluss zu, dass man die Wirkung typografischer Gliederungselemente der Standardsprache nur sehr sparsam auf Produkte der Leichten Sprache übertragen kann. Eine solche typografische Feinarbeit stellt die Abstandsregelung dar. Da das Regelwerk zur Leichten Sprache dazu auffordert, viele Absätze und Überschriften zu gestalten, ist es für die Wahrung des Textüberblicks von Vorteil, bei der Gestaltung auf die räumliche Nähe von Titeln zum nachfolgenden Text zu achten. So wird vom Leser fast unbemerkt eine inhaltliche Zusammengehörigkeit visualisiert, die auf dem Gestaltgesetz der Nähe beruht (vgl. Alexander 2013: 27). Auffallend oft begegnen einem in Texten Leichter Sprache lange, ja dreizeilige Titel. Dies wirkt sich beim Leser hinderlich auf das Erfassen der Titelinhalte aus. Kurze Titel, die sich optisch auch einem eventuellen Fettdruck überordnen, sind der attraktivere Leseeinstieg.

Das Interesse am Lesestoff ist für die Leseleistung von großer Bedeutung. Wichtige Kriterien der Lesbarkeit sind deshalb die Lesegewohnheit und die Motivation. „Wir lesen gut, was wir gewohnt sind - und noch besser, was wir lesen wollen" (Uebele 2013: 9).

Erwartungskonforme Layouts nach den Regeln der Leichten Sprache helfen schlechten Lesern beim Lesen, zugleich konditionieren sie aber auch auf empfohlene Elemente (vgl. Lange/Römer/Schneider 2017; Demagin/Donner/ Kutschera/Thieme 2017). 
Gerade in Hinblick auf die Einführung typografischer Elemente, die nicht in der Standardtypografie vorkommen, sollten die Gefahren der Exklusion als Folge von Konditionierung nicht unterschätzt werden.

\section{Bildkommunikation im barrierefreien Grafikdesign}

„Benutzen Sie Bilder. Bilder helfen Texte zu verstehen. Die Bilder müssen zum Text passen." (BMAS, 2014). Konkret wird vom Netzwerk Leichte Sprache sowie Inclusion Europe und der Barrierefreie-Informationstechnik-Verordnung gefordert, dass aussagekräftige Bilder und Symbole verwendet werden sollen (vgl. BITV 2.0) und die Bildmedien auch nach dem Kopieren noch gut erkennbar sein und nicht als Hintergrund verwendet werden sollen (vgl. BMAS, 2014).

Während die Anforderungen an scharfe, kopierbare Bilder einfach erfüllbar sind, steht für jeden Leser die Frage im Raum, was aussagekräftige Bilder und Symbole sind. Maaß schreibt dazu:

In Texten in Leichter Sprache sollen sie (Bilder) schwierige oder auch zentrale Konzepte stützen. Sie sollen die wichtigsten Informationen noch einmal in einem anderen Code darstellen. Die Bilder in Leichte-SpracheTexten dienen also nicht der Verschönerung, der Illustration des Texts, sondern sie sollen den Verstehensprozess stützen. (Maaß 2014: 153)

Die Darstellung der nicht-veränderlichen Bildmerkmale (Invarianten) (vgl. Böck, Wünsche 2013), die Bildfunktion, die Art der Text-Bild-Beziehung und die Bildqualität entscheiden darüber, ob ein Bild im entsprechenden Kontext verstehensfördernd wirkt. Gäbe es per se aussagekräftige Bilder, könnten sie in Bilddatenbanken vorgehalten werden und der Texter müsste sie nur noch richtig heraussuchen. Woran erkennt man, dass ein Bild zum Text passt? Reicht es, einen Häuserblock abzubilden, wenn von einem Wahllokal die Rede ist? Ist ein wenig hilfreiches Bild (wie das vom Häuserblock) noch besser als gar keine Abbildung? Ist es überhaupt möglich, an die individuellen Erfahrungen und Vorstellungen (z. B. vom Wahllokal) einer heterogenen Leserschaft bildlich anzuknüpfen? Bildkommunikation ist ein breites Fachgebiet, das in Anwendung auf 
die Zielgruppe Leichter Sprache noch an Komplexität gewinnt. Deshalb werden im Folgenden die Besonderheiten jeder Bildkommunikation vorgestellt.

\subsection{Vorzüge und Grenzen jeder Bilderkommunikation}

Bilderkommunikation weist gegenüber der sprachlichen Kommunikation Besonderheiten auf.

Denn als seinem Wesen nach immer zugleich äußeres und inneres Bild bleibt das Bild letztlich an das Subjekt gebunden und entzieht sich damit jeder Art von Wiederholbarkeit und Bestimmbarkeit. Im Stand der Unbestimmbarkeit wird das Bild immer ein Geheimnis bleiben, dem wir uns nähern können, wo es uns als äußeres Bild die Möglichkeit einräumt, mit ihm auf multipersonaler Ebene kommunizieren zu können. (Kregel 2011:33)

Das heißt, selbst wenn wir alle in einem Häuserblock zur Wahl gingen, würde jeder sein eigenes, konkretes Bild vom Häuserblock im Kopf haben.

Bilder sind nicht per se selbstbeschreibungsfähig, auch wenn dies manchmal im Volksmund behauptet wird. Auf Grund ihrer Polysemie benötigen sie einen Kontext und/oder eine Konvention zur Informationsentfaltung. Bilder verfügen über spezielle kommunikative Möglichkeiten, die einer Eigenschaft gleichgesetzt von Laien als „selbstbeschreibungsfähig“ bezeichnet werden. „Diagramme finden Bilder für Mengen. Logos finden Bilder für abstrakte Zusammenhänge“ (Alexander 2013: 131). Silhouetten fördern die Erkennbarkeit der Bildobjekte. „Die Stärke des Bildes liegt darin, für komplizierte Sachverhalte ein einprägsames Bild zu finden.“ (ebd.) Gestalter bemühen sich darum, diese kommunikativen Möglichkeiten herauszuarbeiten. Aber jenseits der Abbildungen mit hoher Ikonizität wie z. B. der Fotografie werden stark stilisierte Abbildungsarten wie Piktogramme (anweisende Zeichen) und grafische Symbole (konventionalisierte Zeichen) Bild für Bild gelernt (vgl. Alexander 2013: 209ff.). Äußere Bilder sind unter der Voraussetzung, dass Gestalter und Rezipient die gleichen gesellschaftlichen, sozialen, beruflichen oder andere 
Konventionen teilen, verständlich. Diese Möglichkeit der Verständlichkeit ist sprachübergreifend gegeben.

Nach Doelker (1997/2002: 52-60) lassen sich die Besonderheiten der Bildkommunikation in Abgrenzung zum Wort mit vier Eigenschaften beschreiben: 1) Vieldeutigkeit, 2) Konkretheit bis hin zu Ähnlichkeit, 3) Räumlichkeit und 4) Unmittelbarkeit der emotionalen Wirkung.

Hinzu kommen die Vorzüge der Bildverarbeitung im Gehirn. „Mühelosigkeit und Schnelligkeit der Bildverarbeitung sind ganz wesentlich auch dadurch bedingt, dass eine Umkodierung von wahrnehmungsfernen Zeichen (Phonemoder Graphemfolgen) in wahrnehmungsnahe hier im Unterschied zu Sprache entfällt" (Stöckl 2011: 49). Bilder können in kürzester Zeit aufgenommen und wiedererkannt werden. Es dauert oft nur Hundertstelsekunden, bis die wichtigsten Grundinformationen oder das Thema eines Bildes erfasst werden. Nach ungefähr zwei Sekunden schon kann man ein Bild sicher wiedererkennen (vgl. Behrens, Hinrichs 1986: 85). Bilder werden, verglichen mit Text, besonders schnell und effizient, aber auch in der Kapazität praktisch unbegrenzt gespeichert (vgl. Schweiger 1985: 229).

Empirischen Untersuchungen von Paivio (1971) zufolge verdanken Bilder diesen außerordentlich hohen Wiedererkennungswert der als „Picture Superiority-Effect“ bezeichneten Erscheinung. Diese kann, so Paivio (hier in den Worten von Schierl 2001) darauf zurückgeführt werden, „dass der sprachliche Kode in einen bildlichen übersetzt werden kann und umgekehrt. Konkrete Bilder werden nach diesem Ansatz besonders leicht doppelt kodiert und werden deshalb auch besonders leicht in das Gedächtnis übernommen" (Schierl 2001: 202).

Bildkommunikation hat Grenzen.

Ein Ergebnis der semantischen Dichte ist der Bedeutungsüberschuss in Bildern, vor allem im konnotativen Bereich. Die Botschaft eines Bildes ist daher schwer festzumachen, sie kann aus vielen aktivierbaren Einzelaussagen und beobachteten Wahrnehmungen bestehen. Der semantische Spielraum bildlicher Darstellungen ist in mehrfacher Hinsicht eingeschränkt. So sind Verneinungen nicht eindeutig möglich, Modalität (z. B. könnte, möchte, muss, will etc.) kann nicht adäquat zum Ausdruck gebracht werden, konkrete und eindeutige Sprechakte lassen sich nicht 
vollziehen und einzelne Bildaussagen können nicht klar logisch und rhetorisch (z. B. weil, nachdem, obwohl, daher etc.) miteinander verbunden werden. (Stöckl 2011: 50)

\subsection{Probleme der Bildkommunikation im barrierefreien Grafikdesign}

Auch Leser mit Lernbehinderungen profitieren von den Besonderheiten der Bildkommunikation. „In ihrem Überblick über die Forschung zu Illustrationswirkungen folgern Levie und Lentz (1982), dass ,Illustrationen für schlechte Leser etwas hilfreicher sein können als für gute Leser' (Levie/Lentz 1982: 226). Sie berichten, dass in den überprüften Studien die durchschnittliche Verbesserung des Textbehaltens durch Illustrationen bei schlechten Lesern $44 \%$ und bei guten Lesern 23\% betrug" (Peeck 1994: 76).

Im gleichen Artikel zum „Wissenserwerb mit darstellenden Bildern“ benennt Peeck Beispiele für die affektive und motivationale Funktion von Bildern beim Lernen. „Aber viele Illustrationen sind auch direkt für das Lernen relevant; sie sollen zum Lesen anregen, die Zeit verlängern, in der ein Leser sich mit dem Text beschäftigt usw." (Peeck 1994: 60).

Diese Anregung zur Auseinandersetzung mit Texten kommt schlechten Lesern zugute, genau wie die „kompensatorische“ Funktion, die Bildern beim Lesen zugesprochen wird (vgl. Levie/Lentz 1982).

Grafische Zeichen (wie das Symbol für Leichte Sprache, Piktogramme wie die Lupe zur Schriftgrößeneinstellung, der Lautsprecher für die Vorlesefunktion) spielen im barrierefreien Webdesign, im öffentlichen Raum (Piktogramm für barrierefreie Toiletten oder barrierefreie Wege), aber auch in Printmedien (Logogramme zur Kapitelstrukturierung) eine wichtige Rolle. Diese Zeichen sind durch ihre formale Reduktion auf das Wesentliche besonders gut memorierbar. Dem entspricht auch das Ergebnis der Studie zur Verständlichkeit bildlicher Darstellungsarten von Magaschütz, Schünzel und Teuber (2014). Die Studie vergleicht die Darstellungsarten unterschiedlicher Similarität: Fotografie, Strichzeichnung, Piktogramm. In der Begriff-Bild-Zuordnung konnten die lernbehinderten Probanden zwischen Zeichnung, Fotografie und schwarzweißem Piktogramm wählen. Alle Darstellungen visualisierten den gleichen 
Begriff. Am häufigsten wurden die Piktogramme für die Begriffszuordnung ausgewählt. Symbole und Piktogramme entfalten ihre Signalwirkung nur unter der Voraussetzung, dass viele Menschen sie kennen. Sie müssen konventionalisiert sein. Konventionen bürgern sich langsam über Verbreitungshäufigkeit ein. Im barrierefreien Grafikdesign geht es darum, nicht ständig neue Zeichen zu erfinden, sondern stark konventionalisierte Zeichen herauszufiltern und einzusetzen. Die Kommunikation über PC und Smartphone, Betriebssysteme, Programme und Apps geben in der Konventionalisierung den Ton an. Bien und Schmidt (2017) untersuchten mittels „Card Sorting“ den Grad der Konventionalisierung von Piktogrammen in der Durchschnittsbevölkerung. Die Probanden konnten ihren Favoriten unter fünf verschiedenen Piktogrammen zu einem Thema benennen. Beim Thema „barrierefrei“ zeigt sich, dass sich sowohl in der Altersgruppe unter 35 Jahren als auch über 35 Jahren noch kein Piktogramm eindeutig durchgesetzt hat.

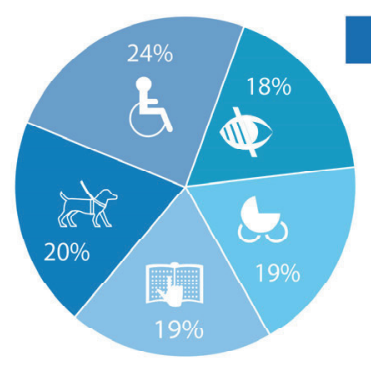

unter 35 Jahre

\section{Barrierefrei}

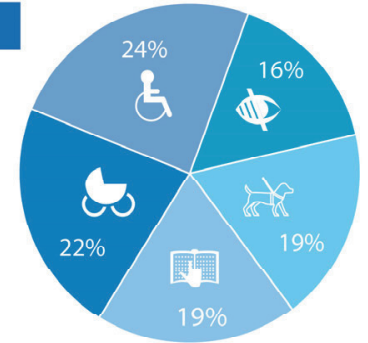

über 35 Jahre

Abb. 4: „Barrierefrei“, Fünf Piktogramme im Vergleich (Quelle: Studie: @Bien/Schmidt HS Merseburg 2017)

Trotz aller Vorzüge der Bildkommunikation gibt es in der Praxis Probleme, das aussagekräftige Bild für eine Text-Bild-Kombination zu finden, die schwierige Inhalte auflösen soll. Die Informationen in Text und Bild müssen sich im individuell sinnvollen Maße unterstützen. Alexander spricht von redundanter, komplementärer und diskrepanter Text-Bild Beziehung (vgl. Alexander 2013: 74ff.). 
Bredel und Maaß (2016: 273) sprechen von Passung und Bildern in unterschiedlichen Graden der Konvergenz.

Für die Informationsvermittlung im barrierefreien Design besteht das Problem im Besonderen darin, abstrakte Inhalte dieser Passung zu unterziehen.

Einen wichtigen Hinweis hierzu liefert Paivio $(1971,1977)$ mit dem „Picture Superiority-Effect“. Konkrete Begriffe können doppelt kodiert und somit relativ problemlos illustriert werden. Aber gerade wenn es um die Vermittlung abstrakter Inhalte geht, wird das Texten schwierig und es wird nach Bildern gesucht. Helfen, gewinnen, Betreuer, diskriminieren, Gleichberechtigung, Heimbeirat, Selbstbestimmung, Wahllokal, Schmerz, Gefahr etc. bezeichnen abstrakte $\mathrm{Zu}$ sammenhänge, von denen sich Texter Hilfe durch entsprechende Bilder aus Bilddatenbanken erhoffen - voraussehbar umsonst. So viele Erscheinungsformen es z. B. von Diskriminierung oder Liebe gibt - so viele individuell aussagekräftige Bilder existieren gleichberechtigt nebeneinander. Die Anzahl der nötigen Bilder, welche Datenbanken für eine sinnvolle Nutzung liefern müssten, ist extrem hoch. Für Abstrakta gibt es kein eineindeutiges Bild, das immer passt. Dies liegt, wie im obigen Abschnitt beschrieben, im Wesen des Bildes begründet.

\subsection{Bildfunktionen in der Bild-Sprache-Kombination - ein Lösungsansatz für abstrakte Inhalte}

Das Problem, ein aussagekräftiges Bild zu finden, besteht ausschließlich in der multicodalen Kommunikation, der Bild-Sprache-Kombination.

Bei einer Gestaltung steht die Frage im Vordergrund, was der Autor/die Autorin mit seinem/ihrem Design erreichen möchte. Bilder können unterschiedliche Funktionen erfüllen.

Obwohl die Forscher einzelne Funktionen von Bildern unterscheiden, sollte man beachten, dass ein Bild meistens mehrere verschiedene Funktionen gleichzeitig erfüllt, dass die Funktionen beträchtliche Überlappungen aufweisen und dass ein Bild - abhängig vom Lernenden, der Situation, der Aufgabe usw. - mehrere Funktionen erfüllen kann. Mit 
diesem Vorbehalt lassen sich die Funktionen von Bildern in zwei umfassende Kategorien gruppieren: in affektive/motivationale Funktionen und in kognitive Funktionen. (Peeck 1994: 60)

Die kognitiven Funktionen unterteilt Peeck weiterhin in die darstellende, die interpretierende und die organisierende Funktion.

Für barrierefreies Grafikdesign kann kein spezifisches Anwendungsgebiet ausgemacht werden. Vielmehr wird es für jegliche Informationen verwendet. Somit sind sowohl die affektive/motivationale Funktion als auch die kognitive Funktion in Bildern der Bild-Sprache-Beziehungen zu finden. Weidenmann formuliert für informierende und instruierende Multimediaangebote ein Modell aus drei verschiedenen Bildfunktionen, das sich zum großen Teil mit den Aussagen von Peeck deckt. Weidenmann differenziert drei Bildfunktionen (Weidenmann 2002: 85ff.), für die er auch Gestaltungshinweise entwickelt. Dies macht die Forschungen Weidenmanns für das barrierefreie Grafikdesign besonders relevant.

- Zeigefunktion - Veranschaulichen unbekannter oder bekannter Objekte, ein Schema wird angelegt

- Situierungsfunktion - Kontextwissen erweitert ein Schema oder Script, Vorwissen wird aktiviert, ein bestehendes Schema oder Script wird ergänzt

- Konstruktionsfunktion - Relationen und Zusammenhänge zwischen bekannten Elementen/Schemata werden dargestellt, aus bekannten Schemata bildet sich so ein neues Script

Epp und Speransky haben 2016 in einer Studie die Qualität von Bildern in der Leichten Sprache untersucht. Sie gingen folgender Frage nach: Sind die Bildfunktionen nach Weidenmann unterschiedlich gut geeignet, um abstrakte Begriffe in Bildern in der Leichten Sprache zu vermitteln? Ausgehend von dieser Fragestellung wurde die Hypothese aufgestellt: Nutzer der Leichten Sprache präferieren Bilder, die primär die Situierungsfunktion erfüllen. Die Studie baut auf eigens 
erstellten Forschungsobjekten auf, die sowohl Bild als auch Text enthalten; es werden die abstrakten Themen "Gefahr“, „Gewinnen“ und „Schmerz" untersucht.

Innerhalb eines Themas gibt es nur einen informativen (expositorischen) Text. Jedes Forschungsobjekt besteht aus drei Bild-Sprache-Kombinationen mit dem gleichen Text. Das Bild wurde jeweils in drei Varianten gezeichnet, so dass es die Bildfunktionen Zeigefunktion, Situierungsfunktion und Konstruktionsfunktion erfüllt (siehe Abb. 5,6,7).

Die Bilder wurden unter Einbeziehung der Empfehlungen von Weidenmann erarbeitet (vgl. Weidenmann 2002: 88ff.). Grundsätzlich empfiehlt er die Verwendung von Darstellungscodes (Lokalfarben, Schattierungen, Perspektive usw.) und Steuerungscodes (Pfeile, Umrandungen, Signalfarben usw.). Entscheidend für die Informationsvermittlung ist die Klarheit des zu vermittelnden Inhalts. Bei der Erstellung von Text und Bildern wurde deshalb großer Wert auf die Eindeutigkeit und die Reduzierung von Details gelegt. Inhaltlich wurden simple Informationen umgesetzt, die allerdings nicht konkret, sondern abstrakt sind.

Jedem Probanden werden alle drei Funktionen sowohl einzeln als auch am Ende gesammelt vorgelegt, so dass er seine Präferenz äußern kann. Es sind auch Mehrfachnennungen möglich.

Hier seien in Kürze die Ergebnisse für das Thema „Gewinnen“ dargestellt.

Als Konzept für die Umsetzung wurde ein Wettrennen gewählt, das in einer Turnier-Atmosphäre stattfindet. Das Konzept wird für alle drei Bildmedien verwendet, um die Vergleichbarkeit zu garantieren.

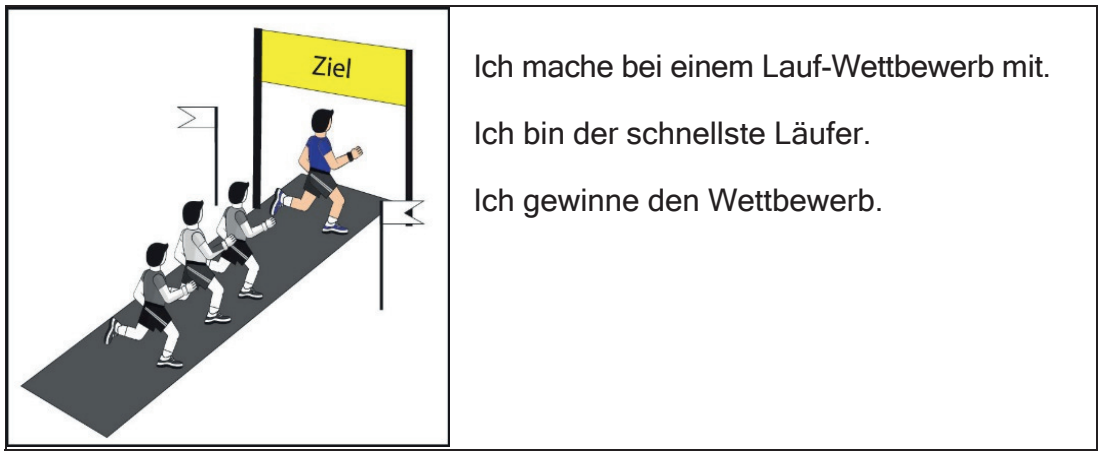

Abb. 5: Gewinnen - Zeigefunktion (Quelle: Studie @Epp/Speransky HS Merseburg 2016) 
Das erste Bildmedium erfüllt die Zeigefunktion. Es werden bekannte Schemata wie Personen in Laufkleidung, eine Laufbahn, die Reihenfolge der Personen, die Körperhaltung der Personen, die Zielfahne usw. aufgezeigt. Als Signalfarbe wurden das Zielbanner und die Kleidung des führenden Läufers eingefärbt. Im Bereich der Lokalfarben wurden die Haut und die Haare des führenden Läufers gefärbt.

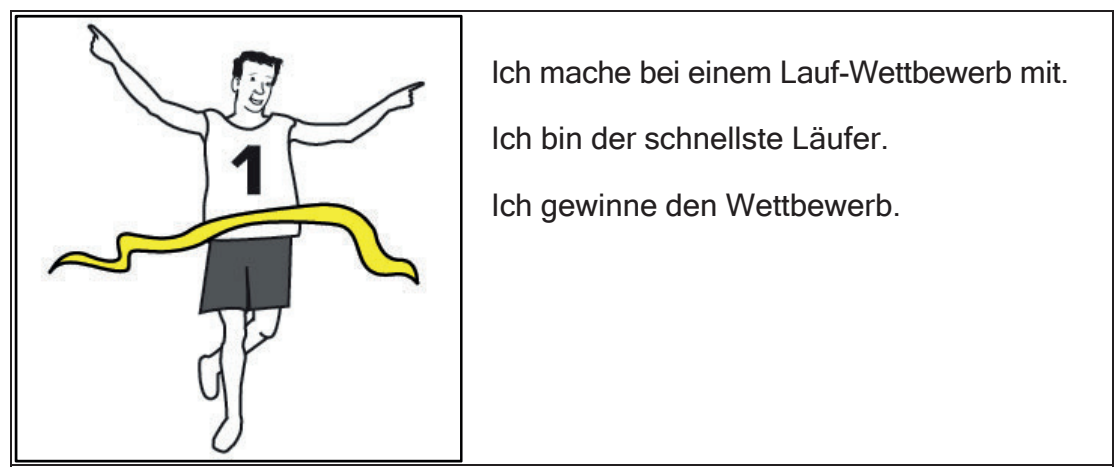

Abb. 6: Gewinnen - Situierungsfunktion (Quelle: Studie @Epp/Speransky, HS Merseburg 2016)

Das zweite Bildmedium wurde unter Anwendung der Situierungsfunktion umgesetzt. Die Situation ist stark emotionalisiert, da der Gewinner im Moment seines Triumphs zu sehen ist. Am Gesichtsausdruck und der Körperhaltung ist klar die Freude über das gewonnene Wettrennen zu erkennen. Im Bereich der Signalfarben wurde das Zielband eingefärbt. In diesem Bild wurde zusätzlich ein Steuerungscode eingesetzt. Dabei handelt es sich um die Ziffer „“" auf dem Trikot des Läufers. Sie soll verdeutlichen, dass es sich um die erste Person im Ziel, also den Gewinner, handelt. 


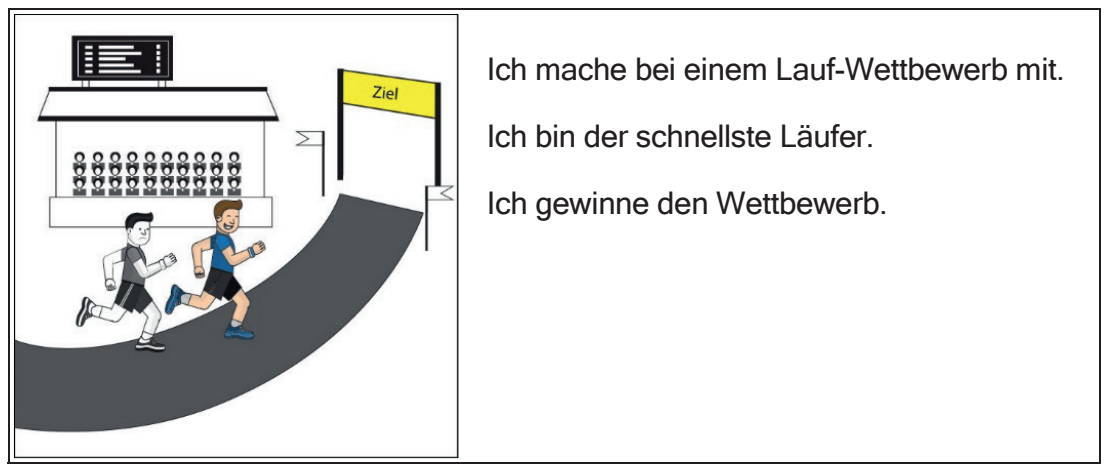

Abb. 7: Gewinnen - Konstruktionsfunktion (Quelle: Studie @Epp/Speransky, HS Merseburg 2016)

Das dritte Bildmedium erfüllt die Konstruktionsfunktion. Die Situation ist ähnlich gewählt wie bei der Zeigefunktion dieses Themas, allerdings wurden noch weitere Details hinzugefügt. Auf diese Weise kann ein bereits bekanntes Schema erweitert oder ein neues angelegt werden. $\mathrm{Zu}$ den hinzugefügten Details zählen die Tribüne mit den Zuschauern und die Anzeigetafel. Als Signalfarbe wurden wieder das Zielbanner und die Kleidung des führenden Läufers eingefärbt. Außerdem haben Haut und Haare des führenden Läufers die Lokalfarben erhalten.

Insgesamt haben 18 Probanden an der Studie teilgenommen. Sie konnten je drei Präferenzen vergeben (54 Präferenzen). Die Präferenzen konnten sowohl gedrittelt als auch halbiert auf mehrere Bilder verteilt werden. So entstehen die ungeraden Werte (Summe: 53,9).

- Gewinnen, 2a, Zeigefunktion: 1,8 Präferenzen

- Gewinnen, 2b, Situierungsfunktion: 13,3 Präferenzen

- Gewinnen, 2c, Konstruktionsfunktion: 2,8 Präferenzen

- Gefahr, Gewinnen, Schmerz, Zeigefunktion: 6,8 Präferenzen

- Gefahr, Gewinnen, Schmerz, Situierungsfunktion: 27,3 Präferenzen

- Gefahr, Gewinnen, Schmerz, Konstruktionsfunktion: 19,8 Präferenzen 
Epp und Speransky (2016) konnten ihre Hypothese, nach der Menschen mit Lernschwierigkeiten in Bild-Sprache-Beziehungen Bilder mit situativer Bildfunktion bevorzugen, anteilig verifizieren, da in den zwei Themen „Gewinnen“ und „Schmerz“ die Situierungsfunktion die meisten Präferenzen erhielt. Nur bei dem Thema "Gefahr“ wurde das Bildmedium, das die Konstruktionsfunktion erfüllt, als am besten bewertet.

Die durchgeführte Studie hat gezeigt, dass die Gestaltung von Bildmedien anhand der Bildfunktionen nach Weidenmann einen sinnvollen Ansatz für die Visualisierung von Abstrakta im barrierefreien Grafikdesign liefert. Weiterhin hat die Studie gezeigt, dass die Situierungsfunktion sich sehr gut dafür eignet, um die Zielgruppe der Leichten Sprache situativ in einem Kontext zu verorten. Für den Lehr-Lernkontext, für den diese Bildfunktionen im Speziellen gedacht sind (vgl. Weidenmann 2002: 84), eignet sich auch die Konstruktionsfunktion, die von den Probanden bei einem Thema präferiert wurde. Die Zeigefunktion hat sich als eher ungeeignet herausgestellt. Die Gestaltung von Bildmedien in Leichter Sprache kann und sollte sich nach Einschätzung der Forschungsgruppe zukünftig an den Regularien von Weidenmann orientieren.

Die Studie von Epp und Speransky (2016) legt nahe, dass zur Klärung von Abstrakta der Einsatz von Bildern mit situativer Bildfunktion sinnvoll ist. Es wirkt sich also vorteilhaft aus, wenn in der Textproduktion bewusst situative Bilder eingeplant werden. Die dabei anzuwendenden Strategien sind Gegenstand laufender Forschungen an der HS Merseburg.

Epp und Speransky (2016) konnten weiterhin belegen, dass die Probanden gut mit den Empfehlungen von Weidenmann hinsichtlich des Einsatzes von Darstellungscodes und Steuerungscodes zurechtkamen. Bedeutungszuschreibungen durch Farbeinsatz (rotes Feuer, gelbes Zielband, rotes Blut) konnten oft zugeordnet werden. Schwierigkeiten hatten sie dagegen mit der Bewertung der farblichen Hervorhebung des Siegerläufers gegenüber seinen in Schwarz-Weiß gestalteten Konkurrenten in der Zeigefunktion und der Konstruktionsfunktion.

Die Studie von Lange, Römer und Schneider (2017) zur Typografie von Dokumenten berichtete ebenfalls von Problemen, die Menschen mit Lernschwierigkeiten haben, Farbe als strukturbildendes Element zu identifizieren. Eine mögliche Lösung ist ein didaktisch reduzierter Farbeinsatz entsprechend der Ähnlichkeit. 
Sowohl in der Studie von Epp und Speransky (2016) als auch in der von Lange, Römer und Schneider (2017) wurden die Probanden ermutigt, ihren Empfindungen und Gedanken während der Tests laut Ausdruck zu verleihen. Diese als „lautes Denken“ bezeichnete Methode, Informationen zu gewinnen, floss in die Auswertung der Tests mit ein. Epp und Speransky (2016) berichteten von der starken Wirkung, die emotionale Bildinhalte wie Gesichter, welche Angst oder Schmerz zeigen, auf die Testpersonen haben. Diese emotionalen Bildinhalte haben vielfach Lösungsstrategien bei den Betrachtern wachgerufen und sie somit aktiviert. Dies bestätigt die positive Wirkung von Bildern.

Die Master-Thesis von Cordula Wünsche (2015) belegt, dass Instruktionstexte in komplementärer Bild-Sprache-Kombination schneller und fehlerfreier ausgeführt wurden als redundante Bild-Sprache-Kombination. Dies bestätigt Aussagen von Bredel und Maaß (2016: 273) zur Passung von Bildern. Wünsche (2015) berichtet zudem von einer aufmerksamen Durchmusterung der Bilder, die als Feedback der Handlung dienten.

\section{Zusammenfassung}

Barrierefreies Grafikdesign stellt eine komplexe Gestaltungsleistung aus typografischem Wissen, Layoutverständnis und Bildwissen dar, die zudem auf gestalterisches Talent angewiesen ist. Aber auch Wissen um die sprachliche Gestaltung ist vorteilhaft. Werden Text und Bild ganzheitlich als Informationseinheit betrachtet, können Bilder mit Bildfunktionen geplant werden, welche die Leser besonders ansprechen. Es hat sich herausgestellt, dass Motive mit situativer Bildfunktion am besten verstanden werden. Die komplementäre Bebilderung abstrakter Zusammenhänge hat Vorrang vor der redundanten Illustration von konkreten Objekten. Was sich leicht aus der Bild-Datenbank ziehen lässt, erfüllt eventuell eine affektive/motivationale Funktion, hilft aber nicht bei der Informationsvermittlung. Zudem begegnen dem Leser Datenbankbilder in unterschiedlichen Kontexten. Ob dies den Leser verwirrt, bleibt noch zu klären.

Alle Illustrationen für die Durchschnittsbevölkerung, ob Kinder oder Erwachsene, werden individuell angefertigt. Die individuelle Illustration ist auch für das barrierefreie Grafikdesign sinnvoll und Teil der Inklusion. 
Barrierefreies Grafikdesign orientiert sich an den Bedürfnissen der Zielgruppe „Menschen mit Lernschwierigkeiten“. Menschen mit Lernbehinderung, ältere Menschen und Migranten sind sehr unterschiedliche Zielgruppen. Aus Sicht des Grafikdesigns sind Informationsprodukte in Leichter Sprache weder für ältere Menschen noch für Migranten geeignet, denn beide Zielgruppen können z. B. Abstrakta mühelos verstehen und sind an effektive typografische Gliederungselemente zur Textorganisation gewöhnt. 


\section{Literaturverzeichnis}

AleXAnder, Kerstin (2013): Kompendium der visuellen Information und Kommunikation. 2. Aufl. Berlin, Heidelberg, New York: Springer.

AlEXANDER, KeRSTIN (2017): „Zur Lesbarkeit und Erkennbarkeit von Open-SourceSchriftarten im Umfeld ,Leichter Sprache“. In: Bock, BetTina/FiX, Ulla/LAnge, DAISY (Hrsg.): Leichte Sprache im Spiegel theoretischer und angewandter Forschung. Berlin: Frank und Timme, 317-328.

Arnheim, Rudolf (1954): Art and Visual Perception: A Psychology of the Creative Eye. Berkeley \& Los Angeles: University of California Press UCP.

Behrens, Gerold/Hinrichs, Alfred (1986): „Werben mit Bildern. Zum Stand der Bildwahrnehmungsforschung“. In: Werbeforschung \& Praxis, Nr. 3, 85-88.

BMAS (2014): Leichte Sprache. Ein Ratgeber. URL: http://www.bmas.de/SharedDocs/ Downloads/DE/PDF-Publikationen/a752-ratgeber-leichte-sprache.pdf?__blob= publicationFile (letzter Zugriff: 16.1.2018).

BITV 2.0 (2011): Verordnung zur Schaffung barrierefreier Informationstechnik nach dem Behindertengleichstellungsgesetz. (Barrierefreie-Informationstechnik-Verordnung BITV 2.0); (letzter Zugriff: 19.3.2018).

Bredel, URsula/MaAß, CHRISTIANE (2016): Leichte Sprache. Theoretische GrundlagenOrientierung für die Praxis. Berlin: Duden.

Doelker, Christian (1997/2002): Ein Bild ist mehr als ein Bild. Stuttgart: Klett-Cotta. DIN 1450 (2013): Schriften - Leserlichkeit. Berlin: Beuth.

EN ISO 9241-TeIL 110 (2008): Grundsätze der Dialoggestaltung. Berlin: Beuth Verlag.

Hochuli, Jost (2011): Das ABC eines Typografen. St. Gallen: VGS Verlagsgemeinschaft St. Gallen, 7.

Kindes- Und ERWACHSENEnSCHUTZBeHÖRdE REgion SOlothurn (Hrsg.) (2016): Information zum Erwachsenenschutz in leicht verständlicher Sprache. Solothurn.

Kregel, UlRIKe (2011): Bild und Begriff. Berlin: Kulturverlag Kadmos, 33.

KUPfERSCHMID, InDRA (2014): „Responsive Typography.“ In: Page eDOSSIER 09.2014, Typografie im Web. Ulm: Ebner Verlag GmbH \& Co. KG, 5.

LEEUWEN, THEO VAN (2005): Introducing social semiotics. London: Routledge.

LEVIE, W. HOWARD/LENTZ, RichARD (1982): „Effects of text illustrations: a review of research." In: Educational Communication and Technology Journal 30/4, 195-232.

MAAß, Christiane (2014): Leichte Sprache. Das Regelbuch - Universität Hildesheim. URL: https://www.uni-hildesheim.de/media/fb3/.../Leichte.../Regelbuch_komplett.pdf (letzter Zugriff: 19.3.2018).

Paivio, Allan (1971): Imagery and Verbal Process. New York: Holt, Reinhardt \& Winston.

Paivio, Allan (1977): „Images, Propositions, and Knowledge“. In: NiCHOlas, J. M. (Hrsg.) Images, Perception and Knowledge, Dordrecht, Boston, 47-71. 
Peeck, JoAn (1994): „Wissenserwerb mit darstellenden Bildern.“ In: WeIdenmann, BERND (Hrsg.): Wissenserwerb mit Bildern. Bern: Verlag Hans Huber, 59-94.

Posner, Roland (2003): „Ebenen der Bildkompetenz.“ In: SACHS-HombaCH, KlaUs (Hrsg.): Was ist Bildkompetenz? Studien zur Bildwissenschaft. Wiesbaden: Springer, 17-24.

SChierl, Thomas (2001): Text und Bild in der Werbung. Bedingungen, Wirkungen und Anwendungen bei Anzeigen und Plakaten. Köln: Halem.

SCHWEIGER, GÜNTER (1985): „Nonverbale Imagemessung.“ In: Werbeforschung \& Praxis, Nr. 4, 126-134.

StÖCKL, Hartmut (2011): „Sprache-Bild-Texte lesen.“ In: Diekmannshenke, H./ KLEMm, M./STÖCKL, H. (Hrsg.): Bildlinguistik: Theorien - Methoden - Fallbeispiele. Mainz: Erich Schmidt Verlag, 45-70.

UEBEle, ANDREAS (2013): „stützstrumpfschriften und der schiefe turm von pisa.“ In: FILEK, JAN: Read/ability - Typografie und Lesbarkeit. Sulgen: Niggli, 9.

WEIDENMANN, BERND (2002): „Abbilder in Multimediaanwendungen. “In: IssING, LUDWIG J./KLIMSA, PAUL (Hrsg.): Information und Lernen mit Multimedia und Internet. Lehrbuch für Studium und Praxis. Weinheim: Beltz PVU, 83-98.

Willberg, Hans P./Forssman, Friedrich (2010): Lesetypografie. 5. rev. Ausg. Mainz: Hermann Schmidt.

WÜnsche, Cordula (2015): Studie zum Einfluss der Text-Bild-Beziehung auf die Verständlichkeit von Instruktionstexten in Leichter Sprache. Masterthesis Hochschule Merseburg.

\section{Unveröffentlichte Studien}

Bien, Julia/Schmidt, Anita (2017): Nutzen Sie konventionalisierte Piktogramme? Studie zu Bild-Konventionen in Anwenderunterstützungen. Seminararbeit, Hochschule Merseburg.

BoecK, LAURA/WÜNSCHE, CORdUla (2013): Die Wahrnehmung von Invarianten - eine Studie zur Bildverständlichkeit in der Leichten Sprache. Seminararbeit, Hochschule Merseburg.

Demagin, Julia/Donner, Isabell/Kutschera, Thomas/Thieme, Hannes (2017): Leichte Sprache und typografische Gliederungselemente. Forschungsarbeit zur Übersichtlichkeit und Verständlichkeit von Texten in Leichter Sprache. Seminararbeit, Hochschule Merseburg.

EPP, ANNA/SPERANSKY, CHILJA (2016): Bildfunktionen in der Leichten Sprache - eine Studie. Seminararbeit, Hochschule Merseburg.

LANGE, RoXANA/RÖMER, JANICE/SCHNEIDER, REBEKKA (2017): Typografie \& Layout von Dokumenten. Usability-Testing zur Optimierung einer Informationsbroschüre zum Thema „Erwachsenenschutz“ in leicht verständlicher Sprache. Seminararbeit, Hochschule Merseburg. 
Magaschütz, Katja/Schünzel, Franz/Teuber, Laura (2014): Studie zur Verständlichkeit bildlicher Darstellungsarten. Seminararbeit, Hochschule Merseburg.

ROHDE, VERONIKA/STECHERT, LISA (2016): Leichte Sprache und Typografie. Ein Usability-Test zur Erkennbarkeit und Lesbarkeit ausgewählter Schriftarten. Seminararbeit, Hochschule Merseburg.

\section{Tabellenverzeichnis}

Tab. 1: Visuelle Regeln des Netzwerks Leichte Sprache, nach BMAS (2014) Leichte Sprache. Ein Ratgeber. URL: http://www.bmas.de/SharedDocs/Downloads/DE/PDFPublikationen/a752-ratgeber-leichte-sprache.pdf?_blob=publicationFile (letzter Zugriff: 16.1.2018).

\section{Abbildungsverzeichnis}

Abb. 1: Bundesministerium für Arbeit und Soziales (BMAS) (2014): Rat-Geber für Menschen mit Behinderung in der Europäischen Union. In: HS Merseburg (2017): Donner/ Demagin/Kutschera/Thieme. Leichte Sprache und typografische Gliederungselemente. Forschungsarbeit zur Übersichtlichkeit und Verständlichkeit von Texten in Leichter Sprache.

Abb. 2, 3: Kindes- und Erwachsenenschutzbehörde Region Solothurn (Hrsg.) (2016): Information zum Erwachsenenschutz in leicht verständlicher Sprache. Solothurn. In: HS Merseburg: Lange/Schneider/Römer (2017): Typografie \& Layout von Dokumenten. Usability-Testing zur Optimierung einer Informationsbroschüre zum Thema „Erwachsenenschutz" in leicht verständlicher Sprache.

Abb. 4: HS Merseburg: Bien/Schmidt (2017): Nutzen Sie konventionalisierte Piktogramme? Studie zu Bild-Konventionen in Anwenderunterstützungen.

Abb. 5, 6, 7: HS Merseburg: Epp/Speransky (2016): Bildfunktionen in der Leichten Sprache - eine Studie. 



\section{Verständlichkeit: die psychologische Perspektive}

\section{Psychologische Verständlichkeitsforschung: historische Entwicklungslinien}

Die psychologische Forschung zur Textverständlichkeit beginnt mit der klassischen Lesbarkeitsforschung, die sich bereits in den 1930er Jahren im angloamerikanischen Raum als eigene Forschungsrichtung etabliert hat (klassisches Werk: Gray/Leary 1935). Das primäre Ziel dieser Forschung bestand darin, die Lesbarkeit von Textmaterialien zu ermitteln und zu optimieren. Dazu wurde eine Fülle von objektiv auszählbaren sprachlich-stilistischen Textmerkmalen bestimmt (z. B. Wortlänge, Wortschwierigkeit, Satzlänge, Satzkomplexität) und deren Einfluss auf die Lesbarkeit eines Textes (Lesegeschwindigkeit, Expertenurteil, Verständnistests etc.) ermittelt (vgl. Groeben 1982). Die bedeutsamsten dieser Merkmale wurden dann regressionsanalytisch in Lesbarkeitsformeln transformiert. Dabei werden Textmerkmale mit Lesbarkeitsergebnissen korreliert und es wird mathematisch eine Funktion errechnet, die die Vorhersage der Lesbarkeit/Verständlichkeit der jeweiligen Texte erlaubt. Im Laufe der Jahre wurde eine ganze Reihe solcher Lesbarkeitsformeln entwickelt, die sich durch eine unterschiedliche Gewichtung von Wort-, Satz- und Silbenfaktoren auszeichnen. Die bekannteste Formel ist die Reading-Ease-Formel von Flesch (1948), die die Anzahl der Silben pro 100 Worte und die durchschnittliche Anzahl von Wörtern pro Satz (Satzlänge) berücksichtigt (Anpassung an deutschsprachige Texte: Amstad 1978). Eine aktuelle, komplexere Formel bietet der sog. Hohenheimer Verständlichkeitsindex (Klartext-Initiative der Universität Hohenheim), der die Lesbarkeit computergestützt (Software TextLab) mit vier für die deutsche Sprache validierten Lesbarkeitsformeln berechnet.

Die faktorenanalytische Aufbereitung der in dieser Tradition unterschiedenen Textmerkmale zeigt relativ übereinstimmend, dass die Faktoren Wort- und 
Satzschwierigkeit für die Lesbarkeit eines Textes eine besondere Bedeutung haben (Klare 1963; Groeben 1982). Entsprechend gelten z. B. die Verwendung kurzer, geläufiger Wörter sowie Sätze mit einfacher grammatikalischer Struktur als verständlichkeitsfördernd. Allerdings ist bereits früh deutlich geworden, dass die Lesbarkeitsforschung die Verständlichkeit eines Textes nur rudimentär erfassen kann, weil sie sich ausschließlich auf formale Merkmale der Textoberfläche bezieht und inhaltlich-strukturelle Aspekte von Texten ebenso ausblendet wie die Verarbeitungsprozesse von Leser(inne)n und deren individuelle Verstehensvoraussetzungen. Es überrascht daher kaum, dass die klassischen Lesbarkeitsformeln nur die Lesegeschwindigkeit, nicht aber das Verstehen und Behalten von Textinhalten vorhersagen können (Groeben 1982). Im Unterschied dazu berücksichtigt das derzeit aktuellste computergestützte Verfahren zur Beurteilung der Verständlichkeit - Coh-Metrix (z. B. McNamara et al. 2010) - nicht nur viele syntaktische, sondern auch aus der bisherigen Forschung abgeleitete semantische Kohäsionsmerkmale auf lokaler und globaler Ebene, die einen Einfluss auf die Leseleichtigkeit haben. Allerdings fehlen bislang empirische Validierungen.

\section{Text-Leser-Interaktion als theoretische Grundlage der Verständlichkeitsforschung}

Diese Beschränkungen der Lesbarkeitsforschung führten bereits zu Beginn der 1970er Jahre zur Entwicklung einer psychologischen Verständlichkeitsforschung. Kennzeichnend dafür war, dass versucht wurde, verstehensfördernde Textmerkmale nicht nur formal stilistisch, sondern auch in Bezug auf die Verarbeitungsprozesse der Rezipient(inn)en zu betrachten. Dabei lassen sich zwei Forschungszugänge unterscheiden, die sich weitgehend parallel entwickelt haben: ein theoretisch-deduktiver (Groeben, 1972; 1978) und ein empirisch-induktiver Zugang (Langer et al., 1974). Trotz der unterschiedlichen Vorgehensweisen gelangen die beiden Forschungsansätze relativ übereinstimmend zu vier übergeordneten Dimensionen der Textverständlichkeit: (1) sprachliche Einfachheit; (2) semantische Redundanz; (3) Gliederung-Ordnung; (4) motivationale Stimulanz. Diese vier Dimensionen werden auch heute noch als die bedeutsamsten 
Dimensionen der Textverständlichkeit angesehen. Sie gelten grundsätzlich als unabgeschlossen und können im Zuge der Forschungsentwicklung durch weitere Merkmale präzisiert und erweitert werden. Eine solche Ausdifferenzierung erfolgte seit Beginn der 1980er Jahre primär durch die neuere sprach- und kognitionspsychologische Textverarbeitungsforschung (Überblicke: Christmann 1989; Christmann/Groeben 1996; Christmann 2015). Im Mittelpunkt dieser Forschung steht die Frage, wie Rezipient(inn)en im Verarbeitungsprozess Wortund Satzfolgen integrieren und zu einer zusammenhängenden Repräsentation der Textbedeutung aufbauen. In diesem Kontext wurde über die bereits in der klassischen Verständlichkeitsforschung hinaus explizierten Textmerkmale eine Fülle weiterer Merkmale identifiziert, die den Integrationsprozess stützen, indem sie den Lesenden Hinweise geben, wie Sätze und Textteile aufeinander zu beziehen sind. Dabei werden auch Rezipientenmerkmale wie Vorwissen, Intelligenz, Interesse etc. berücksichtigt. Dieser Forschungsstrang repräsentiert die historisch vorläufig letzte Entwicklungsetappe der psychologischen Verständlichkeitsforschung, gilt aber ebenfalls als unabgeschlossen.

Die theoretisch übereinstimmende Grundlage für alle Entwicklungsstadien der psychologischen Verständlichkeitsforschung besteht in der Einsicht, dass das Verstehen eines Textes keinen passiven Prozess der Bedeutungsentnahme, sondern einen aktiven Prozess der Bedeutungskonstruktion darstellt, bei dem die Leser(innen) die Textinhalte gemäß ihren Erwartungen und Interessen mit dem Vorwissen verbinden. Aus diesem sog. kognitiven Konstruktivismus des sprachverarbeitenden Subjekts (nach Bartlett 1932) folgt, dass der Lesevorgang immer als Wechselwirkung oder Interaktion zwischen den Merkmalen des Textes und der Kognitionsstruktur des Rezipienten/der Rezipientin abläuft (TextLeser-Interaktion; z. B. Groeben 1982). Der Verarbeitungsprozess wird dabei als Zusammenspiel zwischen aufsteigender, textgeleiteter Verarbeitung und absteigender, erwartungsgeleiteter Verarbeitung modelliert. Die textgeleitete Verarbeitung wird durch die Merkmale des Textes (Syntax, Semantik; Gliederung etc.) beeinflusst, die erwartungsgeleitete Verarbeitung durch die Merkmale der Rezipient(inn)en, ihre Erwartungen, Zielsetzungen, Interessen und Vorkenntnissen. Die Qualität des Verstehens hängt demnach von den Merkmalen des Textes, aber auch von den kognitiven, emotionalen und motivationalen Voraussetzungen der Leser(innen) ab. Die psychologische Verständlichkeitsforschung 
ist dabei auf die Textseite dieses Wechselwirkungsprozesses konzentriert, indem sie fragt und empirisch prüft, welche Textmerkmale das Verstehen fördern oder erschweren.

\section{Der empirisch-induktive und der theoretisch-deduktive Ansatz der Verständlichkeitsforschung}

Zur Feststellung verständlichkeitsfördernder Textmerkmale sind zwei unterschiedliche Wege beschritten worden: ein empirisch-induktiver und ein theoretisch-deduktiver Weg.

Der empirisch-induktive Ansatz ging von 18 konkreten Merkmalsbeschreibungen von Texten aus (z. B. folgerichtig vs. zusammenhanglos; anregend vs. weitschweifig; flüssig vs. holprig), die sich in der bisherigen Forschung als relevant erwiesen hatten. Auf dieser Grundlage wurden Eindrucksdifferentiale gebildet, mit deren Hilfe Expert(inn)en Texte unterschiedlicher Schwierigkeit und Inhalte (Gebrauchstexte, Verwaltungstexte, wissenschaftliche Texte) bewerteten. Die faktorenanalytische Aufbereitung dieser Bewertungen führte zu einer Bündelung der ursprünglich 18 Merkmale zu vier Dimensionen der Verständlichkeit (Langer et al. 1974: 13ff.):

(1) Sprachliche Einfachheit (einfache Darstellung, kurze Sätze, geläufige Wörter, Erklärung von Fachwörtern, konkret, anschaulich);

(2) Gliederung-Ordnung (gegliedert, folgerichtig, übersichtlich, Unterscheidung von Wesentlichem und Unwesentlichem, roter Faden erkennbar, alles kommt der Reihe nach);

(3) Kürze-Prägnanz (zu kurz, aufs Wesentliche beschränkt, gedrängt, aufs Lernziel konzentriert, knapp, jedes Wort ist wichtig);

(4) Zusätzliche Stimulanz (anregend, interessant, abwechslungsreich, persönlich).

Der Lernerfolg konnte auf der Grundlage der Verständlichkeitswerte valide prognostiziert werden: Verständlichkeitsoptimierte Texte wurden signifikant besser behalten und verstanden als die Originalversionen der gleichen Texte 
(Langer et al. 1974; zusammenfassend: Groeben 1982; Groeben/Christmann 1989). Die Versuchsanlage erlaubte allerdings keine empirische Abschätzung, wie bedeutsam die einzelnen Dimensionen für den Lernerfolg sind. Die Autoren gewichten nach subjektivem Eindruck „sprachliche Einfachheit“ sowie „kognitive Gliederung und Ordnung“ als wichtiger im Vergleich zu „Kürze und Prägnanz“ sowie „zusätzliche Stimulanz“. Obgleich der als Hamburger Modell bekannte Ansatz häufig kritisiert wurde, weil er nur auf Expertenurteilen zur Feststellung von Verständlichkeit basiert und die einzelnen Textmerkmale unzureichend beschrieben sind (Groeben 1982), erlaubt er doch eine äußerst ökonomische Einschätzung von Verständlichkeit und wird bis heute in der Forschung und der Praxis eingesetzt. Eine Weiterführung dieses Ansatzes der subjektiven Verständlichkeitsbeurteilung bietet Friedrich (2017), der 10 Skalen zur Erfassung von Verständlichkeit unterscheidet und an Verstehensdaten (multiple choice-Aufgaben) validiert hat. Dabei haben sich in Überschneidung mit dem Hamburger Modell vier Skalen als besonders bedeutsam erwiesen:

- Wortschwierigkeit

- Satzschwierigkeit,

- Klarheit der Vorstellung und

- Aufwand für Reorganisationen.

Der theoretisch-deduktive Ansatz (Groeben 1972) geht von sprachpsychologischen (Theorien zur Satzgestaltung/Stilistik), lernpsychologischen (kognitive Lerntheorie) und motivationspsychologischen (Neugiertheorie) Modellen aus, formuliert auf dieser Grundlage Dimensionen der Verständlichkeit und leitet daraus Merkmale zur Textverständlichkeit ab. Groeben unterscheidet vier Dimensionen der Textverständlichkeit:

(1) stilistische Einfachheit (kurze Satzteile, aktive Verben, aktiv-positive Formulierungen, persönliche Wörter, keine Nominalisierungen, keine eingebetteten Nebensätze);

(2) semantische Redundanz (keine wörtliche Wiederholung, keine Weitschweifigkeit); 
(3) kognitive Gliederung und Ordnung (Gebrauch von Vorstrukturierungen, Hervorhebung wichtiger Konzepte, Verdeutlichung von Unterschieden und Ähnlichkeiten; Beispielgebung; Zusammenfassungen; sequentieller Textaufbau);

(4) konzeptueller Konflikt (Neuheit und Überraschung, Einfügen inkongruenter Konzepte, alternative Problemlösungen, Fragen).

Die empirische Überprüfung erfolgte mit 18 Versuchstexten, die inhaltlich identisch waren, sich jedoch hinsichtlich der Ausprägung der Dimensionen unterschieden (Groeben 1978: 87ff.). Als abhängige Variablen wurden Verständlichkeit, Behalten und Interesse erhoben. Dabei erwies sich der Faktor der kognitiven Gliederung und Ordnung sowohl für die Verständlichkeit als auch das Behalten am wichtigsten. Die besten Lernerfolge konnten mit einer Mischform der beiden Dimensionen „kognitive Gliederung“ und „konzeptueller Konflikt“ erreicht werden. Die Dimension der „stilistischen Einfachheit“ war zwar auch signifikant, aber nur für die Verständlichkeitsbewertung, nicht für das Behalten. Der Faktor der „semantischen Redundanz“ war nur in Kombination mit einer mittleren sprachlichen Einfachheit für die Verständlichkeit bedeutsam (Groeben 1978: 102ff.). Hervorzuheben ist ebenfalls, dass zwischen Verständlichkeit/Interesse und Behalten eine kurvilineare Beziehung besteht, dass also mittlere und nicht maximale Verständlichkeit für den Lernerfolg optimal ist - eine Konsequenz, die insbesondere für die Herstellung von Lernmaterial wichtig ist.

Ein Vergleich der beiden Ansätze zeigt Übereinstimmungen hinsichtlich der theoretischen Bedeutung der Dimension der kognitiven Gliederung/Ordnung, die von beiden am wichtigsten für die Realisierung verständlicher Texte angesehen wird. Unterschiede gibt es insbesondere bei der Dimension der sprachlichen Einfachheit, die allerdings vermutlich beim induktiven Ansatz wegen der Konzentration auf klassische Stilistikansätze überschätzt, beim deduktiven Ansatz hingegen möglicherweise nicht optimal realisiert und damit letztlich unterschätzt wurde (vgl. die ausführlichen Abwägungen bei Groeben 1982). Wenn auch die unter den Dimensionen subsumierten Textmerkmale in beiden Ansätzen noch nicht vollständig bzw. optimal präzise expliziert sind, dürfen die vier Dimensionen nach wie vor als Richtlinien zur Herstellung optimaler Verständlichkeit gelten. Präzisere und umfassendere Merkmalsexplikationen bieten die 
anschließenden Forschungsstadien der kognitionspsychologisch orientierten Textverarbeitungspsychologie, die im Folgenden mit einbezogen werden.

\section{$4 \quad$ Konkrete Merkmale der Verständlichkeitsdimensionen}

\subsection{Sprachliche Einfachheit}

Die Dimension der sprachlichen Einfachheit umfasst alle Merkmale der Textoberfläche. Empirische Untersuchungen liegen insbesondere zu Aspekten der Wortwahl sowie zur grammatikalisch-stilistischen Satzgestaltung vor.

Auf Wortebene ist vor allem die Wortschwierigkeit überprüft worden. Danach werden häufige und (subjektiv) geläufige Wörter schneller verarbeitet als seltene Wörter; Texte, in denen wenig geläufige durch geläufige Wörter ersetzt werden, führen zu besseren Verstehensleistungen (Überblick bei Groeben 1982). Ähnlich zeigen neuere Studien, dass die Fachwortdichte in populärwissenschaftlichen Texten (Korpus mit 465 Texten) bei subjektivem Eindruck von Schwerverständlichkeit zu einer Verlangsamung des Lesens führt (Wolfer/ Held/Hansen-Schirra 2015). Insgesamt ist die durchgängige Verwendung geläufiger Wörter aus motivationalen Gründen aber nur bedingt zu empfehlen, da dies zu langweiligen Texten führen kann. Unter didaktischer Perspektive besteht außerdem die Gefahr, dass keine neuen Wörter mehr gelernt werden (Christmann/Groeben 1999).

Besonders intensiv ist die Wirkung abstrakter im Vergleich zu konkreten Wörtern untersucht worden, da konkrete Wörter besser behalten werden als abstrakte (Marschark/Paivio 1977). Der Effekt wird im Rahmen der dualen Kodierungstheorie erklärt, wonach konkrete Wörter sowohl verbal als auch bildhaft kodiert werden (Sadoski/Paivio 2001). Ein Konkretheitseffekt im Sinne einer Verarbeitungserleichterung (z. B. besseres Behalten, präzisere Schlussfolgerungen) konnte dabei nicht nur für Wörter (z. B. Wippich 1987), sondern auch für Texte unterschiedlicher Länge nachgewiesen werden (Sadoski/Goetz/Rodriguez 2000). Als praktische Konsequenz lässt sich daraus ableiten, dass bei der Vermittlung abstrakter Informationen möglichst ein konkreter Interpretationskontext bereitgestellt werden sollte (empirisch: Beishuizen et al. 2002). Dabei sollte man auch berücksichtigen, dass sich Anschaulichkeit nicht nur durch konkrete 
Wörter, sondern auch mit Bildern und Graphiken erzeugen lässt. Der positive Einfluss von Bildern auf die illustrierte Textinformation konnte metaanalytisch gesichert werden und wird ebenfalls im Rahmen der dualen Kodierungstheorie erklärt (Überblick: Peeck 1993)

Neben der Wort- ist auch der Einfluss der Satzschwierigkeit (im Rahmen der Überprüfung der Generativen Grammatik von Chomsky) auf die Verständlichkeit empirisch nachgewiesen worden. Dabei zeigte sich, dass eingebettete Relativsätze, Nominalisierungen, überlange Sätze mit mehreren Teilsätzen, Sätze mit großer Informationsfülle (Überblick Groeben 1982) sowie syntaktisch mehrdeutige Sätze (zusammenfassend Christmann/Groeben 1999) einen eindeutig negativen Effekt auf die Verständlichkeit haben (zum spezifischen Einfluss von Nominalisierungen und deren Auflösung auf die Lesezeit bei juristischen Texten vgl. Wolfer 2016).

Bei der Zusammenstellung und Realisierung solcher Merkmale wird meist stillschweigend unterstellt, dass Leser(innen) den syntaktischen Input möglichst vollständig und korrekt analysieren. Das muss aber nicht so sein. Es ist durchaus möglich, dass in natürlichen Lesesituationen die syntaktische Analyse einem "Good-enough-Prinzip“ folgt (Ferreira/Bailey/Ferraro 2002), bei dem sich die Leser(innen) auf simple Heuristiken stützen und keine komplette Analyse der Syntax vornehmen. Für die Erfassung der Satzbedeutung ist nach diesem Ansatz die Semantik wichtiger. Die Syntax hat bei der Dekodierung der Satzbedeutung lediglich eine Art Hilfsfunktion, die nur im Falle von komplexen oder mehrdeutigen Sätzen zum Tragen kommt (Christmann 2006; Diskussion zum Einfluss der Syntax bei Poulson/Gravgaard 2016).

Zusammenfassend lässt sich festhalten, dass die Merkmale der sprachlichstilistischen Einfachheit und Konkretheit wichtig für eine unaufwändige und reibungslose Verarbeitung des Textes an der Oberfläche sind. Sie beeinflussen zwar eher das wörtliche als das sinnorientierte Verstehen, aber durch eine unaufwändige Rezeption werden kognitive Ressourcen für den Aufbau semantischer Sinnstrukturen frei, die im Zentrum des Verstehensprozesses stehen (Christmann/Groeben 1996:149ff.). 


\subsection{Semantische Redundanz}

Das Merkmal der semantischen Redundanz (Weitschweifigkeit) bezieht sich auf die Schwierigkeit bzw. Leichtigkeit der Verarbeitung in Abhängigkeit von dem umschreibenden Wiederholen von Textaussagen. Ebenso wie bei der sprachlichen Einfachheit geht es auch hier um die Gestaltung der Textoberfläche. Dabei stellt sich die Frage, ob eine Erhöhung oder Verringerung der semantischen Redundanz die Verarbeitung erleichtert. Insgesamt liegen zu dieser Dimension relativ wenig Untersuchungen vor, die überwiegend dafür sprechen, dass eine Verdichtung der Textinformation weder zu einer Verbesserung noch zu einer Verschlechterung der Verständlichkeit führt (vgl. bei Zeitungstexten: Bassin/ Martin 1976), dass aber eine Erhöhung der Redundanz (bis zu einem gewissen Grad) eher verarbeitungsfördernd ist (zusammenfassend Groeben/Christmann 1989). In einer Studie von Reder, Charney und Morgan (1986) führte ein elaboriertes gegenüber einem nicht elaborierten Manual zur Bedienung eines Computersystems zu einer Zeitersparnis bei der Aufgabendurchführung und zu weniger inkorrekten Befehlen.

Grundsätzlich kann davon ausgegangen werden, dass die Anreicherung eines Textes durch Wiederholung wichtiger Begriffe, Erläuterungen und Erklärungen die Verständlichkeit erhöhen dürfte. Die Wirksamkeit hängt dabei allerdings von der Textsorte (wissenschaftliche Texte, Gebrauchstexte, Handlungsanweisungen, Verwaltungstexte etc.) und dem Vorwissen der Leser(innen) ab. Prinzipiell darf der Auflösungsgrad nicht so hoch sein, dass die Rezipient(inn)en die übergeordneten Bezugspunkte verlieren.

\subsection{Gliederung/Ordnung}

Die Dimension der Gliederung/Ordnung bezieht sich auf die inhaltliche Strukturierung und Organisation von Texten und die Berücksichtigung der Wissensvoraussetzungen der Leser(innen). Dieses Zusammenspiel von Textinhaltsstruktur und kognitiver Struktur der Rezipient(inn)en ist bereits zu Beginn der 1960er Jahre im Rahmen der kognitiven Lerntheorie nach Ausubel (1963) modelliert worden und wird seit den 1970er Jahre bis in die Gegenwart hinein im Rahmen der kognitionspsychologischen Forschung zur Textverarbeitung kontinuierlich weiter ausdifferenziert. 


\subsubsection{Die kognitive Lerntheorie}

Die kognitive Lerntheorie hat den Rezeptionsprozess als Vorgang der Eingliederung von neuem Material in die kognitive Struktur des/der Lernenden aufgefasst und Maßnahmen zur Verbesserung des Textlernens expliziert und empirisch überprüft. Dazu gehören insbesondere Advance Organizers, das sequentielle Arrangieren von Textinhalten, die integrative Vereinigung und die Konsolidierung (zusammenfassend: Christmann/Groeben 1996).

Advance Organizers, oder auch Vorstrukturierungen, sind kurze, dem Text vorangestellte Einführungen, die die relevanten Textkonzepte auf einem umfassenderen Abstraktionsniveau einführen, als sie im Text selbst vorkommen. Es handelt sich dabei um Ankerideen, die die Integration der nachfolgenden Textinformation erleichtern sollen. Während die empirische Befundlage lange Zeit hinsichtlich der Frage des Verarbeitungsvorteils uneinheitlich war, ist durch neuere Studien mittlerweile geklärt, dass solche Vorstrukturierungen insbesondere dann wirksam sind, wenn sie gemäß der thematischen Struktur des Textes aufgebaut sind (Mannes/Kintsch 1987) und neben hochinklusiven, d.h. übergeordneten, auch konkrete Konzepte und Analogien enthalten (Corkill/ Bruning/Glover 1987). Sequentielles Arrangieren bezieht sich auf die Abfolge von Textinformationen. Bewährt hat sich z. B. ein hierarchisch-sequentieller Textaufbau, bei dem die Textinformation zunächst auf einem hohen Abstraktionsniveau beschrieben wird, bevor schrittweise auf detailliertere und konkretere Darstellungsebenen übergegangen wird (Christmann 1989). In der späteren Forschung hat sich auch eine Sequenzierung nach Superstrukturen (konventionalisierte Inhaltsorganisation für verschiedene Textsorten wie z. B. wissenschaftliche, juristische, narrative, etc. Texte) als effizient für das Behalten, das Beantworten von Fragen zum Text und die Verarbeitung der Textinformation erwiesen (Überblick: Christmann 2008). Integrative Vereinigung bezieht sich auf die Kenntlichmachung von Ähnlichkeiten und Unterschieden zwischen bereits etablierten und neuen Konzepten. Dies kann z. B. durch die Anreicherung von Texten durch Elaborationen, Beispiele und Analogien geschehen. Elaborationen und Beispiele gelten als verarbeitungsserleichternd, weil sie dem Aufbau einer reichhaltigen Wissensstruktur dienen, und Analogien fördern das Verstehen, weil sie inhaltliche oder strukturelle Entsprechungen zwischen Bekanntem und Neuem verdeutlichen (Übersicht über die empirische Befundlage: 
Christmann/Groeben 1999). Eine Konsolidierung des erworbenen Wissens kann schließlich durch Zusammenfassungen, Hervorhebungen und Unterstreichungen erreicht werden. Während ältere Studien hier nur vereinzelt Vorteile für die Verarbeitung nachweisen konnten, zeigen neuere kognitionspsychologische Studien im Rahmen des sog. Signaling (s.u.), dass durch solche Hinweise an der Textoberfläche der Aufbau einer kohärenten Textstruktur gefördert wird, was sich positiv auf Behalten, Inferenzbildung, Leseverstehen als zentrale Komponenten des Verarbeitungsprozesses auswirkt (z. B. Lorch 1989; Lorch/ Lorch 1996).

\subsubsection{Die kognitionspsychologische Textverarbeitungsforschung}

Der Aufbau einer solchen kohärenten mentalen Repräsentation der Textbedeutung stand für die kognitionspsychologische Forschung zum Textverstehen von Anfang an im Mittelpunkt (Überblick: Christmann 2008). Verstehen bedeutet dabei, Wort- und Satzfolgen aufeinander zu beziehen und in einen sinnvollen Zusammenhang zu stellen. Dieser Integrationsvorgang kann auf Textseite durch eine kohärente Inhaltsorganisation gestützt werden, die den Leser(inne)n Hinweise gibt, wie Sätze und Textteile aufeinander zu beziehen sind. Fehlen solche Strukturierungshinweise oder sind sie für die Rezipient(inn)en schwer erkennbar, entstehen Kohärenzlücken, die durch Schlussfolgerungen und Umstrukturierungen geschlossen werden müssen. Empirisch hat sich dabei immer wieder gezeigt, dass die Suche nach geeigneten Bezugspunkten zeitkonsumierend ist und die Verarbeitung erschwert (Überblick: Christmann/Groeben 1996). In der Forschung wird hier unterschieden zwischen Hinweisen zur Herstellung lokaler vs. globaler Kohärenz.

Lokale Kohärenz bezieht sich auf die Verknüpfungen innerhalb und zwischen einzelnen Sätzen. Im einfachsten Fall lassen sich die Bezüge zwischen Satzteilen und Sätzen durch Bezugnahme auf den gleichen Referenten (Koreferenz) herstellen. An der Sprachoberfläche kann dies realisiert werden durch Wortwiederholungen, pronominale Wiederaufnahmen, Wiederaufnahme von Satzsequenzen durch Proformen, Rückverweise und Vorverweise, aber auch durch Kontiguitätsrelationen, mit denen auf temporal, lokational oder strukturell verbundene Ereignisse, Situationen, Handlungen verwiesen wird (vgl. Dressler 
1972). Die Bedeutsamkeit solcher expliziten Hinweise für die reibungslose und schnelle Verknüpfung von Sätzen ist in vielen empirischen Untersuchungen belegt worden (Überblick: van Dijk/Kintsch 1983). Das Verstehen wird darüber hinaus grundsätzlich erleichtert, wenn sowohl die Beibehaltung eines Themas als auch dessen Wechsel sprachlich markiert werden (vgl. Schnotz 1993). Neben der Koreferenz werden Verknüpfungen zwischen Satzteilen und Sätzen auch durch sog. Kohärenzrelationen hergestellt. Das sind Relationen, die einen UrsacheWirkungszusammenhang, eine Addition, eine Verknüpfung von Bedingungen mit Folgen, Problemen mit Lösungen etc. beinhalten (Taxonomie bei Sanders/ Spooren/Noordman 1992) und die an der Sprachoberfläche z. B. durch die Verwendung von (kausalen, temporalen, adversativen, additiven etc.) Konnektiva markiert sein können. Innerhalb der diesbezüglich uneinheitlichen Befundlage (Überblick: Degand/Sanders 2002) lassen sich folgende Tendenzen erkennen (vgl. Christmann 2008): Das Einfügen koreferenzieller und kausaler Relationen führt gegenüber Texten ohne diese Relationen zu einer reibungsloseren und schnelleren Textrezeption und zu einer besseren Behaltensleistung (z. B. Loxterman/Beck/McKeown 1994), und zwar insbesondere dann, wenn die Leser(innen) keine expliziten Vorkenntnisse in einem Inhaltsbereich haben. Bei den Konnektiva haben sich vor allem explizite kausale Verknüpfungen (,weil', ,deshalb ',daher') zwischen Sätzen als verarbeitungswirksam erwiesen. Sie führen im Vergleich zu adversativen, additiven sowie fehlenden Verknüpfungen zu schnellerem Lesen und besserem Behalten der Textinformation (z. B. Millis/ Magliano 1999). Prozessual ist der Effekt darauf zurückzuführen, dass die jeweils antezedenten Sätze länger im Arbeitsgedächtnis bleiben und als Integrationsinstanz für nachfolgende Sätze zur Verfügung stehen (Millis/Just 1994). Darüber hinaus bewirken kausale Verknüpfungen eine schnellere und effizientere Inferenzbildung (Millis/Golding/Barker 1995), und zwar bei kürzeren und längeren, bei expositorischen und bei narrativen Texten (Singer/O' Connell 2003). Außerdem wirken sie sich (bei Informationstexten) positiv auf die Qualität des Verstehens aus (empirisch: Degand/Lefèvre/Bestgen 1999; Degand/ Sanders 2002). Allerdings haben diese Studien auch deutlich gemacht, dass Konnektiva sich nur dann positiv auf die Qualität des Leseprozesses auswirken, wenn die kausalen Bezüge zwischen den Sätzen nicht ohnehin transparent sind oder leicht erschlossen werden können (vgl. Linderholm et al. 2000). 
Die globale Kohärenz bezieht sich auf Verknüpfungen zwischen den Teilthemen und Abschnitten eines Textes. Entsprechend geht es darum, durch spezielle Hinweise im Text den Aufbau einer globalen Textbedeutungsstruktur und die Fokusnachführung bei den Leser(inne)n zu unterstützen. Zur Verfügung stehen insbesondere rhetorische Relationen und Signale. Rhetorische Relationen sind semantische Relationen, mit denen die Teilthemen eines Textes auf globaler Ebene verknüpft werden. Bekannt geworden sind insbesondere die von Meyer (2003) unterschiedenen Relationen: Problem/Problemlösung, Ursache/Folge bzw. Wirkung/Effekt, Vergleich (im Sinne einer Angabe von Ähnlichkeiten und Unterschieden zwischen Textgegenständen), Gruppierung und Beschreibung. Diese impliziten Strukturmerkmale können an der Sprachoberfläche durch bestimmte Signalwörter kenntlich gemacht werden (z. B. , weil', ,deshalb', führt zu', ,Konsequenz aus' etc. zur Verdeutlichung der Relation, Verursachung') und geben so dem Leser/der Leserin Hinweise auf die zugrundeliegende thematische Struktur. Positive Effekte konnten insbesondere in Bezug auf das Behalten und die Verarbeitungstiefe festgestellt werden (Überblick: Meyer 2003). Allerdings sind die von Meyer unterschiedenen Relationen nicht zur Beschreibung der inhaltlich-thematischen Struktur jedweder Textsorten/-strukturen geeignet. Vielmehr müssen für spezifische Textsorten die funktionalen Zusammenhänge und impliziten Strukturmerkmale herausgearbeitet und an der Textoberfläche expliziert werden.

Im Unterschied zu den eher impliziten rhetorischen Relationen sind Signale Hinweise an der Sprachoberfläche, die die zentralen Themen eines Textes und deren Interrelationen hervorheben, ohne dass weitere semantische Inhalte hinzugefügt werden. Als Signale gelten Titel, Kapitelüberschriften und -unterüberschriften, die Wiederholung von Inhaltsaspekten (zusammenfassende Aussagen, Wiederholung von Aussagen zur Emphase), Kapitelüberblicke und -einleitungen, Funktionsindikatoren (Hinweiswörter wie ,somit' und Hinweisphrasen wie ,zusammenfassend lässt sich festhalten ...'), Relevanzindikatoren (z. B. ,Hervorzuheben ist ... '), numerische Aufzählungen (z. B. von zentralen Gedanken und Argumenten), typographische Hinweise (z. B. Unterstreichungen, Kursivdruck etc.) (vgl. Lorch 1989), aber auch über den Text verteilte kurze Zusammenfassungen (van der Meij/van der Meij 2012). Nach der sehr umfang- 
reichen Forschung zur Wirkung von Signalen tragen sie dazu bei, dass die Leser(innen) einen Text als ein hierarchisch organisiertes Themengefüge repräsentieren, während ein Text ohne Signale als unverbundene Liste von Einzelthemen abgebildet wird. Und nur die hierarchische Struktur, nicht die unverbundene Liste stellt bei der Textwiedergabe eine effektive Abrufhilfe dar (Sanchez et al. 2001). Der verarbeitungserleichternde Effekt von Signalen konnte empirisch für verschiedene Komponenten des Verarbeitungsprozesses (Aufmerksamkeit, Lesegeschwindigkeit, Leseverstehen, Inferenzbildung, selektive Informationssuche und Wiedergabe) nachgewiesen werden (differenzierter Überblick über empirische Befunde zur Wirkung verschiedener Signaltypen bei Lorch 1989; Gaddy/van den Broek/Sung 2001; Lorch/ Lemarié/Grant 2011). Relativ gut gesichert ist dabei, dass Signale auf alle Fälle das Behalten der hervorgehobenen Information begünstigen und zu besser strukturierten Wiedergabeprotokollen führen (z. B. Loman/Mayer 1983; Lorch/ Lorch 1996; Sanchez et al. 2001). In Bezug auf die Gesamtbehaltensleistung sind positive Effekte von Signalen insbesondere dann nachzuweisen, wenn der Text ineinander verwobene Themen enthält und hinreichend komplex ist (Lorch/Lorch 1996; Sanchez et al. 2001). Generell profitieren insbesondere Leser(innen) mit geringem Vorwissen erheblich von einer durch Signale verbesserten Textstruktur (Xianyou 2004), und zwar sowohl hinsichtlich des wörtlichen als auch des inferenziellen Verstehens (McNamara/Kintsch 1996; Voss/Silfies 1996; Schmitz/Gräsel/Rothstein 2017). Das zeigt sich auch bei multimedialen Texten, bei denen die Text-BildIntegration durch Signale gestützt wird (Metaanalyse von Eitel et al. 2013). Ein gelegentlich beobachteter Umkehreffekt, wonach Leser(innen) mit hohen Vorkenntnissen bei inkohärenten Texten besser abschneiden als bei kohärenten, scheint nicht stabil zu sein (Kamalski et al. 2008; ausführliche Diskussion bei Schmitz 2016). Hingegen konnte in neueren Studien mit mehr als 700 Schüler(inne)n der verstehensfördernde Effekt von globalen Kohärenzhilfen in Texten („transparente Referenzstruktur, thematische Zwischenüberschriften, Betonung der übergeordneten Wissenseinheiten, Explikation funktionaler Relationen“; Schmitz 2016: 138) auch unabhängig von Vorwissen, der Fähigkeit zum sinnentnehmenden Lesen und dem thematischen Interesse festgestellt werden (Schmitz 2016: 189ff.). Eine weitere relevante Variable, die den Einfluss der 
globalen Textkohäsion auf das Verstehen moderiert, ist das Wissen von Leser(inne)n über Textstrukturen und die Bedeutung von rhetorischen Relationen, das eine relevante Moderatorvariable für den verstehensfördernden Effekt dieser Merkmale darstellt (Sánchez/García/Bustos 2017).

Insgesamt konnte im Rahmen der neueren Textverarbeitungsforschung immer wieder eindrucksvoll belegt werden, dass Texte umso besser verstanden werden, je klarer und deutlicher die Hinweise sind, wie die Textinformationen aufeinander zu beziehen sind. Aus diesem Grund darf heute gerade das Merkmal der globalen Textkohärenz als das bedeutsamste Merkmal verständlicher Sachtexte gelten (Schmitz 2016).

\subsection{Motivationale Stimulanz}

Die Frage nach dem Einfluss einer motivierenden Textgestaltung auf das Textverstehen ist im Rahmen von zwei unterschiedlichen Theorieansätzen behandelt worden (zusammenfassend: Christmann/Groeben 1999): der Neugiermotivationstheorie (Berlyne 1960) und der neueren Interessensforschung (Krapp/Prenzel 1992).

Nach der Neugiermotivationstheorie entsteht Wissensneugier durch kognitive Konflikte, die durch spezifische Merkmale eines Gegenstands (sog. kollative Variablen) ausgelöst werden, nämlich Neuheit, Überraschung, Unsicherheit und Widersprüchlichkeit. Die Theorie postuliert, dass solche Merkmale bei den Leser(inne)n zu Zweifel, Perplexität und Konfusion führen und dadurch die Suche nach neuen Informationen stimulieren. In der Terminologie der reformulierten Neugier-Theorie von Loewenstein (1994) entsteht eine Informationslücke in der kognitiven Struktur, die ein Bedürfnis nach Informationssuche auslöst (Überblick: Christmann 2004: 75 ff). Dabei können folgende Merkmale zur Schaffung von Konflikten eingesetzt werden:

(1) konfliktevozierende Fragen;

(2) Einführung neuer und überraschender Inhalte in Frageform;

(3) inkongruenter Rückzug auf Bekanntes (Einfügen von Informationen, die zum Wissens- und Überzeugungssystem der Rezipient(inn)en im Widerspruch stehen); 
(4) alternative Problemlösungen durch Aufzeigen möglichst gleichwahrscheinlicher Problemalternativen (ausführlicher: Groeben 1982: 267 ff; Groeben/Christmann 1989).

Allerdings darf durch das Einfügen solcher potenziell interesseauslösender Textmerkmale die übergeordnete Struktur der Textinhalte nicht gestört werden. Entsprechend wirkt sich eine konfliktevozierende Darstellung von Textinhalten nach der klassischen Verständlichkeitsforschung nur dann positiv auf die Verarbeitung aus, wenn sichergestellt ist, dass der Aufbau eines kognitiven Rahmens dadurch nicht behindert wird (Groeben 1982).

Die Richtung dieser Befunde wird auch von der Interessensforschung bestätigt (vgl. Krapp/Prenzel 1992; Sadoski 2001). Allerdings werden in diesem Ansatz keine interesseauslösenden Textmerkmale identifiziert, sondern die Interessantheit von Textelementen wird von Ratern eingeschätzt. Ein behaltensfördernder Effekt lässt sich mit dieser Methode z. B. dann nachweisen, wenn wichtige Textelemente zugleich in interessanter Form dargeboten werden (z. B. Wade/Adams 1990). Der im vorliegenden Zusammenhang wichtigste Befund ist, dass eine einfache Anreicherung von Texten mit interessanten, aber unwichtigen Details das Verstehen nicht verbessert und sich auf die Gesamtbehaltensleistung und das Problemlösen sogar negativ auswirkt (Harp/Mayer 1998). Es wird angenommen, dass solche Details das Verstehen und Behalten strukturell wichtiger Informationen behindern, weil sie Wissensschemata aktivieren, die mit dem Aufbau eines adäquaten kognitiven Rahmens konfligieren (,seductive detail effect'; empirisch: Garner et al. 1991).

Insgesamt lässt sich aus den vorliegenden Befunden der Schluss ableiten, dass eine motivierende Textgestaltung sparsam erfolgen sollte und auf keinen Fall die Verarbeitung strukturell wichtiger Informationen behindern darf. Unter dieser Prämisse hat sie zwar keinen direkten Effekt auf das Verstehen und Behalten eines Texts, kann aber dazu beitragen, dass die Aufmerksamkeit der Rezipient(inn)en konstant hoch bleibt und die Textrezeption nicht vorzeitig abgebrochen wird (vgl. auch Ainley/Hidi/Berndorff 2002). 
Obgleich die skizzierten verstehensfördernden Textmerkmale empirisch gut gesichert sind, stößt die auf Textoptimierung angelegte Verständlichkeitsforschung immer an eine strukturelle Grenze: Es gibt kein für alle Leser(innen) einheitliches Verständlichkeitsoptimum.

Das zeigt sich z. B. daran, dass in der Mehrzahl der Studien ein Effekt von verständlichkeitsfördernder Textgestaltung vor allem bei geringen Vorkenntnissen, niedrigem bis mittlerem Fähigkeitsniveau und unvertrauten Textinhalten nachweisbar ist. Bei vertrauten Inhalten und hohen Vorkenntnissen ist hingegen davon auszugehen, dass Rezipient(inn)en Mängel in der Gestaltung unter Rückgriff auf ihre Vorkenntnisse kompensieren können. Empirisch hat sich sogar gelegentlich gezeigt, dass ein inhaltlich völlig kohärenter und durchstrukturierter Text bei Rezipient(inn)en mit hohen Vorkenntnissen zu qualitativ schlechteren Verstehensleistungen führt, während er für Rezipient(inn)en mit geringen Vorkenntnissen verarbeitungserleichternd ist (Kintsch 1998: 307 ff). Vermutlich bieten hochkohärente Texte für Leser(innen) mit hohem Vorwissen keinen Anreiz mehr, sich aktiv mit der Textinformation auseinanderzusetzen. Für solche Leser(innen) dürfen Texte auch Kohärenzlücken enthalten, die durch leserseitige Inferenzen und Elaborationen zu schließen sind (Groeben 1972/1978; Christmann/Groeben 2002: 156). Das steht in Übereinstimmung mit der eingangs explizierten Rahmenkonzeption des Textverstehens als TextLeser-Interaktion. Wenn das Zusammenspiel zwischen der individuellen Kognitionsstruktur der Rezipient(inn)en und dem Text für die Konstruktion der Textbedeutung entscheidend ist, dann resultieren unterschiedliche Bedeutungsstrukturen in Abhängigkeit von je individuellen kognitiven Voraussetzungen, dem Vorwissen, den Erwartungen, den Interessen und den Zielsetzungen sowie den Rezeptionsstrategien(Christmann/Groeben1999).

Aus diesen Beschränkungen resultiert als praktische Konsequenz, dass man beim Problem der Verständlichkeitsoptimierung nicht nur am Text ansetzen sollte, sondern auch bei den Leser(inne)n. Die Leser(innen) müssen in die Lage versetzt werden, Informationstexte aus unterschiedlichen Bereichen effektiv zu verarbeiten. Und hier hat es sich im Bereich des schulischen Lernens in den letzten 20 Jahren als höchst effizient erwiesen, Schüler(inne)n Wissen über 
Textstrukturen und -relationen (z. B. Ursache-Wirkungszusammenhänge, Problem und Problemlösung etc.) zu vermitteln, so dass sie Textschemata aufbauen können, die die Rezeption leiten und erleichtern. Eine metaanalytische Aufbereitung von 45 Studien zur Effizienz der Instruktion zu den speziell von Meyer (z. B. 2003) unterschiedenen Textstrukturen hat in $85 \%$ der Fälle bei Schüler(inne)n unterschiedlicher Klassenstufen einen positiven Effekt von Textstrukturlernen auf verschiedene Maße des Textverstehens nachgewiesen (Herbert/Bohaty/Nelson/Brown 2016). 


\section{Literaturverzeichnis}

Ainley, Mary/Hidi, Suzanne/Berndorff, Dagmar (2002): „Interest, learning, and the psychological processes that mediate their relationship“. In: Journal of Educational Psychology 94, 545-561.

AMSTADT, TONI (1978): Wie verständlich sind unsere Zeitungen? [Dissertation] Zürich: Universität Zürich.

Ausubel, David P. (1963): The Psychology of Meaningful Verbal Learning. New York: Grune and Stratton.

Bassin, Carolyn B./Martin, Clessen J. (1976): „Effect of three types of redundancy reduction on comprehension, reading rate, and reading time of English prose“. In: Journal of Educational Psychology 68(5), 649-652.

Bartlett, Frederic C. (1932): Remembering: A study in Experimental and Social Psychology. Cambridge: Cambridge University Press.

Beishuizen, Jos/Stoutjesdijk, Evelien/Spuijbroek, Sandra/Bouwmeester, Samantha/ VAN DER GEest HANNEKE (2002): „Understanding abstract expository texts“. In: British Journal of Educational Psychology 72, 279-297.

Berlyne, DAniel E. (1960): Conflict, arousal, and curiosity. New York: McGraw-Hill.

Christmann, Ursula (1989): Modelle der Textverarbeitung: Textbeschreibung als Textverstehen. Münster: Aschendorff.

Christmann, Ursula (2004): „Information als Funktion des Lesens“. In: Norbert Groeben/Bettina Hurrelmann (Hg.): Lesesozialisation in der Mediengesellschaft. Weinheim et al.: Juventa, 61-94.

Christmann, Ursula (2006). Satz- und Textlernen. In Joachim Funke/Peter FRENSCH (Hg.): Handwörterbuch Allgemeine Psychologie: Kognition . Göttingen: Hogrefe, 254-259.

Christmann, Ursula (2008): „Rhetorisch-stilistische Aspekte moderner Verstehensund Verständlichkeitsforschung“. In: FIX, Ulla/GARDT, ANDREAS/KNAPE, JOACHIM (Hg.): Rhetorik und Stilistik. Ein internationales Handbuch historischer und systematischer Forschung: Berlin und New York: de Gruyter, 1092-1106.

Christmann, URsula (2015): „Kognitionspsychologische Ansätze des Lesens“. In: RAUTEnberg, Ursula/Schneider, Ute (Hg.): Lesen - Ein Handbuch. Berlin, München, New York: de Gruyter, 21-45.

Christmann, Ursula/Groeben, Norbert (1996): „Die Rezeption schriftlicher Texte“. In: GÜNTHer, Hartmut/Ludwig, OTTO (Hg.): Schrift und Schriftlichkeit. Ein interdisziplinäres Handbuch internationaler Forschung. Berlin: de Gruyter, 1536-1546.

Christmann, Ursula/Groeben, Norbert (1999): „Psychologie des Lesens“. In: Franzmann, Bodo/Hasemann, Klaus/Löffler, Dietrich/Schön, Erich (Hg.): Handbuch Lesen. München: Saur, 145-223. 
Christmann, Ursula/Groeben, Norbert (2002): „Anforderungen und Einflussfaktoren bei Sach- und Informationstexten“. In: Groeben, NorberT/HurrelmanN, BeTTINA (Hg.): Lesekompetenz: Bedingungen, Dimensionen, Funktionen. Weinheim et al.: Juventa, 150-173.

Corkill, Alice J./Bruning, Roger H./Glover, John A. (1988): „Advance organizers: Concrete versus abstract“. In: Journal of Educational Research 82 (2), 76-81.

Degand, Liesbeth/SANDERS, TeD (2002): „The Impact of Relational Markers on Expository Text Comprehension in L1 and L2“. In: Reading and Writing: An Interdisciplinary Journal 15, 739-757.

Degand, Liesbeth/Lefèvre, Nathalie/Bestgen, Yves (1999): „The Impact of Connectives and Anaphoric Expressions on Expository Discourse Comprehension“. In: Document Design 1, 39-51.

VAn DijK, Teun A./Kintsch, Walter (1983): Strategies of Discourse Comprehension. New York: Academic Press.

Dressler, Wolfgang U. (1972): Einführung in die Textlinguistik. Tübingen: Niemeyer.

Eitel, Alexander/Scheiter, Katharina/Schüler, Anne/Nyström, Markus/HolmQVIST, KENNETH (2013): „How a picture facilitates the process of learning from text: Evidence for scaffolding“. In: Learning and Instruction 28, 48-63.

Ferreira, Fernanda/Bailey, Karl G. D./Ferraro, Vittoria (2002): „Good enough representations in language comprehension“. In: Current Directions in Psychological Science 11, 11-15.

Flesch, Rudolf (1948): „A New Readability Yardstick“. In: Journal of Applied Psychology. 32 (3), 221-233.

FRIEDRICH, MARKUS (2017): Textverständlichkeit und ihre Messung. Münster: Waxmann.

Garner, Ruth/Alexander, Patricia A./Gillingham, Mark G./Kulikowich, Jonna M./BROWN, RACHEL (1991): „Interest and Learning from Text“. In: American Educational Research Journal 28, 643-659.

Gaddy, Michelle/van Den Broek, Paul/Sung, Yung-Chi (2001): „The Influence of text cues on the allocation of attention during reading“. In: SANDERS, TED/SCHILPEROORD, JoOst/SPOOREN, WiLPERT (Hg): Text Representation: Linguistic and Psycholinguistic Aspects. Amsterdam, Philadelphia: John Benjamins, 99-110.

Gray, William S./LEARY, BERniCE E. (1935): What makes a book readable. Chicago: University of Chicago Press.

Groeben, Norbert ([1972]/1978): Die Verständlichkeit von Unterrichtstexten. Münster: Aschendorff.

Groeben, Norbert (1982): Leserpsychologie: Textverständnis - Textverständlichkeit. Münster: Aschendorff.

Groeben, Norbert/Christmann, Ursula (1989): „Textoptimierung unter Verständlichkeitsperspektive“. In ANTOS, Gerd/KRINGs, HANS-PETER (Hg.): Textproduktion. Ein interdisziplinärer Forschungsüberblick. Tübingen: Niemeyer, 165-196. 
Harp, Shannon/Mayer, Richard (1998): „How Seductive Details do their Damage: A Theory of Cognitive Interest in Science Learning“. In: Journal of Educational Psychology $90,414-434$.

Herbert, Michael/Bohaty, Janet/Nelson, J. Ron/Brown, Jessica (2016): „The effects of text structure instruction on expository reading comprehension: A metaanalysis“. In: Journal of Educational Psychology 108(5), 609-629.

KAMAlski, Judith/LentZ, Leo/SAnders, Ted/ZwAan, Rolf A. (2008): „The forwarning effect of coherence markers in persuasive discourse: Evidence from persuasion and processing“. In: Discourse Processes 45(6), 547-579.

Kintsch, Walter (1998): Comprehension: A Paradigm for Cognition. Cambridge: University Press.

Klare, GeORGE R. (1963): The measurement of readability. Ames: Iowa State University Press.

[O.A.] Klartext-Initiative der Universität Hohenheim: https://www.uni-hohenheim.de/ politmonitor/methode.php (letzter Zugriff 19.2.2018)

Krapp, Andreas/Prenzel, Manfred (Hg.) (1992): Interesse, Lernen, Leistung. Neuere Ansätze einer pädagogisch-psychologischen Interessenforschung. Münster: Aschendorff.

LAnger, Inghard/Schulz von Thun, Friedemann/Tausch, Reinhard (1974): Verständlichkeit. München: Ernst Reinhardt.

Linderholm, Tracy/Everson, Michelle G./VAn den Broek, Paul/Mischinski, MAUREen/CritTEnden, AleX/SAmuels, Jay (2000): „Effects of Causal Text Revisions on More- and Less-Skilled Readers' Comprehension of Easy and Difficult Texts“. In: Cognition and Instruction 18, 525-556.

Loewenstein, George (1994): „The Psychology of Curiosity“. In: Psychological Bulletin 116, 75-98.

LOMAN, NANCY L./MAYER, RiCHARD E. (1983): „Signaling techniques that increase the understandability of expository prose“. In: Journal of Educational Psychology 75, 402412.

LORCH, Robert F. (1989): „Text-signaling devices and their effects on reading and memory processes“. In: Educational Psychology Review 1, 209-234.

LORCH, Robert/Elizabeth LORCH (1996): „Effects of Organizational Signals on Free Recall of Expository Text“. In: Journal of Educational Psychology 88, 38-48.

Lorch, Robert F., Lemarié, Julie/Grant, Russel (2011): „Signaling Hierarchical and Sequential Organization in Expository Text“. In: Scientific Studies of Reading 15(3), 267-284.

Loxterman, Jane A./Beck, Isabel L/McKeown, Margaret G. (1994): „The effects of thinking aloud during reading on students' comprehension of more or less coherent text“. In: Reading Research Quarterly 29, 353-367.

Mannes, Suzanne M./Kintsch, Walter (1987): „Knowledge organization and text organization“. In: Cognition and Instruction 4, 91-115. 
MARSCHARK, MARC/PAIVIO, Alan (1977): „Integrative processing of concrete and abstract sentences“. In: Journal of Verbal Learning and Verbal Behavior Vol 16 (2), 217 231.

McNamara, Danielle S./Kintsch, Walter (1996): „Learning from text: Effects of prior knowledge and text coherence“. In: Discourse Processes, 22, 247-287.

McNamara, Danielle S./Louwerse, Max M./McCarthy, Philip M./Graesser, ARThur C. (2010). „Coh-Metrix: Capturing linguistic features of cohesion“. In: Discourse Processes 47, 292-330.

VAN DER MEIJ, HANS/VAN DER MEIJ, JAN (2012): „Improving text recall with multiple summaries“. In: British Journal of Educational Psychology 82(2), 257-269.

Meyer, Bonnie J. F. (2003): „Text coherence and readability“. In: Topics in Language Disorders 23, 204-221.

Millis, Keith/Just, Marcel (1994): „The Influence of Connectives on Sentence Comprehension“. In: Journal of Memory and Language 33, 128-147.

Millis, Keith/Magliano, JosePh (1999): „The Co-Influence of Grammatical Markers and Comprehender Goals on the Memory for Short Discourse“. In: Journal of Memory and Language 41, 183-198.

Millis, Keith/Golding, Jonathan/Barker, Gregory (1995): „Causal Connectives Increase Inference Generation“. In: Discourse Processes 20, 29-49.

PEECK, JOAN (1993): „Increasing picture effects in learning from illustrated text“. In: Learning and Instruction 3(3), 227-238.

Poulson, Mads/Gravgaard, Amelie, K.D. (2016): „Who did what to whom? The relationsship between syntactic aspects of sentence comprehension and text comprehension“. In: Scientific Studies of Reading Vol 20(4), 325-338.

Reder, Lynne M./Charney, Davida, H./Morgan, Kim, I. (1986): „The role of elaborations in learning a skill from an instructional text". In: Memory and Cognition $14(1), 64-78$.

SADOSKI, MARK (2001): „Resolving the effects of concreteness on interest, comprehension, and learning important ideas from text“. In: Educational Psychology Review 13, 263-281.

SAdoski, Mark/Paivio, Allen (2001): Imagery and Text: A Dual Coding Theory of Reading and Writing. Mahwah: Lawrence Erlbaum.

SAdoski, MARK/GoetZ, ERnest/RodrigueZ, Maximo (2000): „Engaging Texts: Effects of Concreteness on Comprehensibility, Interest, and Recall in four Text Types“. In: Journal of Educational Psychology 92, 85-95.

SAnders, Ted/Spooren, Wilbert/Noordman, Leo (1992): „Toward a taxonomy of coherence relations“. In: Discourse Processes 15, 1-35.

SANChez, Rebecca Polley/Lorch, Elizabeth/Lorch, Robert (2001): „Effects of Headings on Text Processing Strategies“. In: Contempory Educational Psychology 26, $418-428$. 
SÁncheZ, Emilio/García, J. RicARdo/Bustos, Andrea (2017): „Does rhetorical competence moderate the effect of rhetorical devices on the comprehension of expository texts beyond general comprehension skills?"In: Reading and Writing 30 (3), 439-462.

SCHMITZ, ANKE (2016): Verständlichkeit von Sachtexten. Wirkung der globalen Textkohäsion auf das Textverständnis von Schülern. Wiesbaden: Springer VS.

SCHMitZ, ANKE/GRÄSEL, CORNELIA/RothSTEIN, BJÖRN B. (2017): „Students' genre expectations and the effects of text cohesion on reading comprehension". In: Reading and Writing. An Interdisciplinary Journal 30 (5), 1115-1135. Schnotz, Wolfgang (1993): Aufbau von Wissensstrukturen. München: Psychologie Verlagsunion.

Singer, Murray/O'CONNELl, Gordon (2003): „Robust inference processes in expository text comprehension“. In: European Journal of Cognitive Psychology 4, 607-631.

Voss, James/Silfies, LAURIE NeY (1996): „Learning from History Text: The Interaction of Knowledge and Comprehension Skill with Text Structure“. In: Cognition and Instruction 14, 45-68.

WADE, SUZANnE/ADAMS, ROBERT (1990): „Effects of Importance and Interest on Recall of Biographical Text“. In: Journal of Reading Behavior 22, 331-353.

WIPPICH, WERNER (1987): „Integrationsprozesse bei der Verarbeitung konkreter und abstrakter Texte“. In: Zeitschrift für Experimentelle und Angewandte Psychologie 34, 506-526.

WOLFER, SASCHA (2016). „The impact of nominalizations on the reading process: A case study using the Freiburg Legalese Reading Corpus“. In: HANSEN-SCHIRrA, Silvia/ GruCZA, SAmbor (Hg.): Eyetracking and Applied Linguistics. Berlin: Language Science Press, 163-186.

Wolfer, Sascha/Held, Uli/Hansen-Schirra, Silvia (2015): „Verstehen und Verständlichkeit von populärwissenschaftlichen Texten: Das Projekt PopSci - Understanding Science“. In: Information, Wissenschaft \& Praxis 66 (2-3), 111-119.

XIANYOU, He (2004): „The influence of text topic's familiarity and reading level on the effect of text signals“. In: Psychological Science (China) 27, 595-597. 



\section{Verständlichkeit aus fachkommunikativer Sicht}

\section{$0 \quad$ Einleitung}

Verständliche Fachkommunikation ist angesichts steigender Komplexität aller gesellschaftlichen Bereiche ein wichtiges Desiderat. Die Angewandte Linguistik kann im Zusammenspiel mit benachbarten Disziplinen einen Beitrag zur Verbesserung dieser Situation leisten. Zunächst wird hier der Problembereich dargestellt (Abschnitt 1), anschließend die interdisziplinäre Perspektive geöffnet (Abschnitt 2), sodann ein integratives Modell zur verständlichen Fachkommunikation vorgestellt (Abschnitt 3), und zum Schluss werden zwei Anwendungsfälle skizziert (Abschnitt 4).

\section{$1 \quad$ Verständlichkeit und Fachkommunikation}

In unserer modernen Wissensgesellschaft wird vieles immer komplexer, sei es im beruflichen Umfeld oder auch privat. Diese Komplexität äußert sich oft auch sprachlich; man denke an unterschiedlichste Textsorten wie Versicherungsverträge, Prozessbeschreibungen im Qualitätsmanagement, Gesetze, Allgemeine Geschäftsbedingungen, Artikel in Fachzeitschriften, elektronische Patientendokumentation im Krankenhaus, Lernunterlagen für das Studium, Antragsformulare, Powerpoint-Präsentationen, Handy-Apps für das persönliche Fitnessprogramm und viele andere mehr. Verständlichkeit scheint so etwas wie ein gesamtgesellschaftliches Anliegen zu sein, ähnlich wie der allgemeine Wunsch nach Reduktion von Komplexität.

Doch was ist eigentlich Verständlichkeit? - Dieser alltagssprachlich relativ klar wirkende Begriff entzieht sich einer eindeutigen Definition in einheitlicher 
Verwendung, und je nach Forschungstradition, Interessen und Zielen treten unterschiedliche Aspekte in den Vordergrund (Abschnitt 2; detailliert Lutz 2015: 49-233). Forschungsarbeiten der letzten Jahrzehnte haben jedenfalls deutlich gezeigt, dass Verstehen nicht auf einer tabula rasa des menschlichen Geistes funktioniert, sondern im Paradigma des kognitiven Konstruktivismus als $\mathrm{Zu}$ sammenspiel von Textmerkmalen und Lesermerkmalen modelliert werden sollte (Groeben 1982, aktuell Christmann/Groeben im vorliegenden Band): Wir verstehen immer auf der Basis dessen, was wir schon wissen. Dies betrifft sowohl kognitive Aspekte als auch emotionale Faktoren, formale Kenntnisse und Vertrautheit mit Textsorten ebenso wie persönliche Interessen und situationsbezogene Einflüsse. Insofern ist das Sprechen von der Verständlichkeit eines Textes immer eine generalisierende Abstraktion.

Methodisch kann man sich bei der Untersuchung des Verstehens eher auf Prozesse konzentrieren (Datenerfassung während des Kommunikationsakts, z. B. mittels Eye-tracking oder Thinking-aloud-Technik) oder auf Produkte des Verstehens (Überprüfung von Behaltensleistungen, Experteneinschätzungen, Folgehandlungen). Durch die Verwendung geeigneter empirischer Methoden lassen sich dann indirekt generalisierende Aussagen über die Verständlichkeit von Texten ableiten; zu einer Methodenübersicht siehe Christmann (2004). Solche Zusammenhänge sind am deutlichsten bei direkt handlungsanleitenden Texten nachweisbar (wie z. B. Gebrauchsanleitungen, Auspackanweisungen, Help-Texte), bei denen man den Verstehenserfolg unmittelbar an den Folgehandlungen erkennen kann; zur Diskussion der Validität solcher Methoden siehe Lutz (2015: 118-125).

Im Vergleich zu linguistisch und kognitionswissenschaftlich fundierteren Ansätzen zur Modellierung der Verständlichkeit (z. B. Göpferich 2002) setzen praxisorientierte Ansätze ganz stark auf Plausibilität, Didaktisierung und einfache Umsetzbarkeit. Im deutschen Sprachraum ist das sogenannte Hamburger Verständlichkeitsmodell (Langer et al. 1974) seit Jahrzehnten der am weitesten verbreitete Ansatz zur Optimierung von Fachtexten. Dabei wird folgendes Vorgehen vorgeschlagen: Zunächst sind vorliegende Texte nach vier Dimensionen der Verständlichkeit (Einfachheit - Gliederung/Ordnung - Kürze/Prägnanz anregende Zusätze) auf einer 5-stufigen Skala zu bewerten. Nach dem Erstellen eines Wunschprofils erfolgt dann die Umformulierung des Textes in Richtung 
der gewünschten Eigenschaften. Wenn z. B. ein Ausgangstext als besonders schlecht gegliedert bewertet wird (doch hinsichtlich der sprachlichen Einfachheit passend ist), so kann man sich beim Umformulieren besonders auf die Verbesserung der Gliederung konzentrieren.

Das Hamburger Verständlichkeitsmodell wurde wegen starker Vereinfachungen und fehlendem Eingehen auf konkrete sprachliche Gestaltungsoptionen stark kritisiert, hat aber vermutlich gerade aufgrund dieser Eigenschaften im populärwissenschaftlichen Umfeld großen Erfolg. Zu einer detaillierten Analyse der Vor- und Nachteile siehe Lutz (2015: 83-102). In Abschnitt 3 wird ein heuristisches Modell der Verständlichkeit vorgestellt, das die offensichtlichen Vorteile des Hamburger Verständlichkeitsmodells mit aktuellen Erkenntnissen aus verschiedenen Fachdisziplinen zusammenzuführen versucht, um so zu einer differenzierenden Sichtweise anzuleiten.

Wenn hier von Fachtexten und Fachkommunikation gesprochen wird, so erfolgt dies auf dem Hintergrund der Fachkommunikationswissenschaft (Schubert 2007); Heidrich (2017) stellt die historischen Entwicklungslinien aus Translationswissenschaft und Fachsprachenforschung dar, die sich in den letzten Jahren allmählich miteinander verflochten haben. Die Translationswissenschaft ist in diesem Kontext von Bedeutung, weil sprachliche Optimierungsprozesse als einsprachige Sonderfälle des Fachübersetzens modelliert werden können; und aus der Fachsprachenforschung sind aus Verständlichkeitsperspektive insbesondere die Textsortenlinguistik und die Terminologielehre von Bedeutung.

Die Verständlichkeitsforschung beschäftigt sich traditionell mit schriftlicher Kommunikation im fachlichen Kontext. Die Konzentration auf Schriftlichkeit hat keine ursächlichen Gründe; sie hängt vermutlich mit der Komplexität und dem Umfang der zu analysierenden und optimierenden Inhalte und Texte zusammen, bei denen man ohne Verschriftlichung kaum auskommt. In bestimmten Fällen, wie z. B. interkulturell bedingten Mehrdeutigkeiten oder Hesitationen und darauffolgenden Klärungsdialogen zur Auflösung von Missverständnissen, sind allerdings diskursanalytische Verfahren zur Analyse gesprochener Sprache sehr hilfreich, um ein besseres Verständnis der kommunikativen Dynamik zu erhalten.

Unter „fachlichem Kontext“ ist keinesfalls nur die Kommunikation zwischen Experten eines spezifischen Fachbereichs zu sehen. Das wäre die Experten- 
Experten-Kommunikation, die durch die spezifische Ausgestaltung von Fachsprachen optimiert werden kann. Die besondere Herausforderung der Kommunikation im fachlichen Kontext ist ja gerade, auch bei der Überschreitung enger Fachgrenzen von anderen Personen und Gruppen verstanden zu werden. Dies kann die Kommunikation zwischen Experten unterschiedlicher Fachbereiche betreffen, die Kommunikation zwischen Experten und Laien sowie alle möglichen Zwischenstufen und Kombinationen. Gerade diese Zwischenstufen sind besonders wichtig: Man denke etwa beim Hausbau an die unterschiedlichen sprachlichen und konzeptuellen Zugänge von Akteuren wie Architekt, Baupolier, Maurer, Installateur, Hilfsarbeiter, Heimwerker, Baubehörde oder Bauherr. Wir haben es dabei mit unterschiedlichsten Texten zu tun wie etwa Verträgen, Plänen, Anleitungen, Protokollen oder Bescheiden, die für alle betroffenen Personen möglichst gut verständlich und eindeutig interpretierbar sein sollten.

In diesem Zusammenhang ist auch die Forderung nach „Allgemeinverständlichkeit“" zu diskutieren, die in besonderem Maße gegenüber Gesetzen und der öffentlichen Verwaltung erhoben wird (Behörden-Bürger-Kommunikation), um die gesellschaftliche Teilhabe aller Bevölkerungsgruppen zu ermöglichen. Die Diskussion darüber ist teilweise sehr polemisch (zusammenfassend Eichhoff-Cyrus/Antos 2008) und nicht immer lösungsorientiert. Auf der einen Seite wäre es naiv anzunehmen, dass immer und überall Allgemeinverständlichkeit für alle erzielt werden könnte, auf der anderen Seite könnte man durch konkrete Maßnahmen deutliche Verbesserungen der Rechts- und Verwaltungssprache erreichen, indem man z. B. auf die langjährige Erfahrung Technischer Redakteure bei der Textoptimierung zurückgreift (Lutz 2017b).

Eine Forderung nach „Allgemeinverständlichkeit“ ist zudem theoretisch und praktisch problematisch. Im Rahmen eines funktional-pragmatischen und konstruktivistischen Zugangs zur Textverständlichkeit kann es theoretisch gar nicht den einen optimal verständlichen Text geben, sondern immer nur für unterschiedliche Zielgruppen oder sogar Einzelpersonen in spezifischen kommunikativen Situationen optimierte Texte, denn jeder und jede hat unterschiedliche Voraussetzungen für das Verstehen. Das gilt auch für die Ebene lokaler sprachlicher Strukturen. Es gibt zwar einige sprachliche Strukturen, die in jedem Fall und für jedes Zielpublikum die Textverarbeitung erschweren (z. B. 
überlange und komplexe Sätze oder ungegliederte längere Texte) und daher generell vermieden werden sollten. In diesen Fällen geht es im Wesentlichen um mangelnde fluency, also unnötige kognitive Anstrengung beim Lesen (im Detail Lutz 2015: 110ff.). In vielen anderen Fällen haben wir es eher mit GewinnVerlust-Relationen zu tun, die situationsbezogen unterschiedlich gestaltet („optimiert") werden können. Ein bekanntes Beispiel dafür ist die Verwendung des Passivs, die zwar in zahlreichen Stilratgebern aus Gründen der Verständlichkeit kritisiert wird, doch bei genauer Betrachtung eine ganze Reihe von Optionen zur flexiblen Positionierung von Informationen in einem Satz bietet, wie z. B. das Weglassen eines unwichtigen Agens oder das Betonen des sinntragenden Verbs in der Endposition. Auch sprachökonomische Überlegungen (z. B. Beibehalten eines logischen Objekts mit Aktiv- und Passivprädikaten) oder der Wunsch nach Verdeutlichung können Gründe für die Verwendung des Passivs sein (zahlreiche Beispiele bei Göpferich 2002: 104ff.). Ähnliches gilt für den Nominalstil, der textsortenspezifisch ein wichtiges Mittel der sprachlichen Ökonomie darstellt und komplexe syntaktische Strukturen vermeiden hilft.

Insofern sollten bei der praktischen Arbeit Überlegungen zum Zielpublikum mit seinen sprachlichen Kompetenzen, Kenntnissen, Erwartungen, Emotionen und der jeweiligen Kommunikationssituation am Anfang jeder Analyse stehen. Erst daran anschließend kann man sinnvollerweise ans inhaltliche und sprachliche Gestalten und Optimieren gehen, und das muss keinesfalls immer in einer sprachlich besonders einfachen Varietät des Deutschen enden. Bock (2014) unterscheidet in einem programmatischen Artikel zur Leichten Sprache zwischen leichter, einfacher und bürgernaher Sprache, wobei sie das Spannungsfeld zwischen sprachlicher Restringiertheit und Multifunktionalität im Zusammenhang mit dem Konzept der Angemessenheit adressiert. Ein stark normativ ausgerichtetes Konzept von Leichter Sprache - richtungweisend vorgelegt durch den Duden Leichte Sprache von Bredel/Maßß (2016) - stößt hier an gewisse Grenzen; vgl. dazu auch den aktuellen Sammelband von Bock et al. (2017) zu Leichter Sprache, in dem zu diesem Thema unterschiedliche Positionen vertreten werden.

Falls keine klare Identifikation eines Zielpublikums möglich ist (z. B. „die Öffentlichkeit“), so kann es sinnvoll sein, sich auf ein spezifisches imaginiertes 
Zielniveau und Zielpublikum zu einigen, dessen Bedürfnisse man bei der Textierung bestmöglich zu befriedigen sucht. So wurde z. B. bei einem größeren Projekt zur Neuherausgabe eines Gesetzes in Österreich folgende Vorstellung eines Zielpublikums entwickelt: „nicht juristisch gebildete Durchschnittsbürger auf Abitur-Niveau, die sich redlich bemühen“ (Lutz 2015: 311). Ob solche Ziele auch tatsächlich erreicht werden, könnte man dann ggf. durch empirische Tests mit geeigneten Stichproben überprüfen.

\section{Das weite und interdisziplinäre Feld des Optimierens}

Neben genuin linguistischen Themen soll hier auch auf einige weitere interdisziplinäre und praxisrelevante Zugänge zur verständlichen Fachkommunikation hingewiesen werden, die den Leser(inne)n vielleicht noch wenig bekannt sind. Aus Platzgründen kann vieles nur kurz angerissen werden.

Die Praktische Stilistik in Form von Stil- und Schreibratgebern erzielt hohe Auflagen im populärwissenschaftlichen Beratermarkt und ist schon aus diesem Grunde von Belang. Immer noch weit verbreitete Stilfibeln wie die von Reiners (1963) gehen auch für funktionale Textsorten von literarischen Stilidealen aus und wirken aus heutiger Sicht willkürlich und wenig hilfreich. Die aktuellen Bücher von Sick sind zwar amüsant zu lesen (z. B. Sick 2004), doch als Hilfestellung für das Verfassen verständlicher Fachtexte sind die an ein (journalistisches) Fachpublikum gerichteten Bücher von Autoren wie Wolf Schneider besser geeignet, wenn sie konkrete und begründete Lösungen anbieten (z. B. Schneider 1999).

Im weiteren Umfeld der Technischen Kommunikation gibt es zahlreiche praktische Ratgeber, die auch theoretisch gut fundiert sind. Beispielhaft seien hier das Buch von Baumert (2003) zum professionellen Texten und das Buch von Ballstaedt (2012) zum Visualisieren genannt. Die TEKOM, der Fachverband für Technische Kommunikation, muss in diesem Zusammenhang unbedingt erwähnt werden, da sie in den letzten zwei Jahrzehnten viel zur Professionalisierung des Berufsbilds „Technischer Redakteur“ beigetragen hat (im deutschen 
Sprachraum gibt es ungefähr 100.000 Technische Redakteure) und zwei Buchreihen herausgibt, die sich immer wieder mit Verständlichkeitsthemen befassen.

Im engeren Bereich der akademischen Linguistik sei besonders auf die Wichtigkeit der Textsortenlinguistik und Terminologielehre für die verständliche Fachkommunikation verwiesen. Textsortenspezifische Gestaltungskonventionen passen sich dynamisch geänderten Kommunikationsbedürfnissen an. Dies kann ungesteuert oder unterstützt erfolgen, z. B. durch Dokumententemplates mit vorgegebenen Gliederungen und Beispieldokumenten. Dadurch bilden sich kognitive Schemata heraus, die sowohl den Prozess der Textproduktion unterstützen als auch Erwartungshaltungen der Leser(innen) steuern und den Rezeptionsprozess erleichtern. Bhatia (2004) spricht auf diesem Hintergrund bei der Textsorte „Protokoll“ von der Kolonialisierung und Hybridisierung von Textsorten über Unternehmens- und Branchengrenzen hinweg.

Terminologiearbeit ist eine konzeptuelle und praktische Aufgabe, die im Alltag von Wirtschaftsunternehmen meist zu kurz kommt (Lutz 2015: 148ff.). Beispielhaft einige Fragen dazu: Was kennzeichnet einen guten Terminus, wie führt man Begriffsklärungen durch, wie baut man ein corporate wording oder ein projektspezifisches Glossar auf, wie geht man mit Abkürzungen um und wie erkennt man Termini in gedruckter und Online-Dokumentation?

Die Readability-Forschung (zusammenfassend DuBay 2004) ist in der akademischen Linguistik nicht hoch angesehen, da sie - theoretisch schwach fundiert - aufgrund relativ einfacher formaler Textmerkmale (wie durchschnittliche Wort- und Satzlänge, Worthäufigkeiten) Aussagen über die Verständlichkeit von Texten trifft. Dies geschieht meist in Form sogenannter Verständlichkeitsformeln, häufig auch normiert für die Bewertung der Eignung von Lehrtexten für bestimmte Schulstufen. Derartige Ansätze scheinen sich für eine grobe diagnostische Bewertung der Textkomplexität zu eignen, ähnlich wie SoftwareMetriken für die Voranalyse der Qualität des Quellcodes. Sie gewinnen in den letzten Jahren wieder an Bedeutung, da durchaus beeindruckende SoftwareLösungen entwickelt wurden, die auf der Basis großer Datenmengen nicht nur Verständlichkeitsindices errechnen, sondern auch für das Terminologiemanagement, die Entwicklung von Corporate Languages und SEO (search engine optimization) Unterstützung anbieten (für Einzelaspekte siehe z. B. Firmen und 
Tools wie SDL, TextQuest, LinguLab oder Searchmetrics). Für das Deutsche ist das Produkt TextLab (2018) besonders erwähnenswert, das mehrere dieser Aspekte attraktiv zusammenführt. Zu einem Überblick mit Einschätzung der Verwendungspotenziale siehe Lutz (2015: 71-83).

Im deutschen Sprachraum wurde die internationale Plain Language-Bewegung bisher wenig wahrgenommen. Die Vertreter dieser Bewegung sind in zwei Fachverbänden organisiert und verstehen sich als Lobbyisten für eine verständliche Sprache in Rechts- und Verwaltungstexten, in der Wirtschaft und in Kommunikationsberufen. Fachlinguisten sind relativ wenig vertreten; es handelt sich eher um eine engagierte Gruppe von Berufspraktikern und Consultants, die besonders in den englischsprachigen Ländern und in Skandinavien aktiv sind und praxisorientierte Lösungen vorantreiben. Umfangreiche Informationen über Aktivitäten, Kongresse und Publikationen finden sich auf den Websites der Fachverbände (Plain 2018, Clarity 2018).

Das Thema Multimodalität und Multimedialität gewinnt in der Fachkommunikation steigende Bedeutung, und hier ist ein interdisziplinärer Blick über den Tellerrand der Linguistik für die Praxis der Textoptimierung sehr nützlich: Die Fachdisziplin des Informationsdesigns beschäftigt sich schon seit Jahrzehnten intensiv mit der Gestaltung und Wirkung multimodaler und multimedialer Ausdrucksformen. Hier sei nur kurz auf den Fachverband IIID (2018) und das bei Benjamins erscheinende Information Design Journal verwiesen. Auch der aus der Tradition der Kommunikationswissenschaft stammende Ansatz der Knowledge Communication beschäftigt sich intensiv mit dem wirksamen $\mathrm{Zu}$ sammenspiel von Text und Bild, insbesondere in der Management-Kommunikation (Knowledge Communication 2018).

Zuletzt sei die praxisorientierte Disziplin des Usability Engineering erwähnt, die sich seit Mitte der 1990er Jahre etabliert hat. Hier geht es um die anwenderfreundliche Gestaltung von Benutzeroberflächen. Ein Beispiel: Wenn in einem Webshop auch Kaufwillige den Einkauf nicht abschließen, dann ist aus Sicht der Betreiber akuter Handlungsbedarf, und man muss mit geeigneten Mitteln herausfinden, warum das so ist und wie man das Interface besser gestalten könnte. Die Methoden des Usability Engineering sind auch für die Optimierung von Fachkommunikation sehr nützlich, da sie vielfach erprobt sind und mit relativ geringem Aufwand eingesetzt werden können. In den letzten Jahren hat sich der 
breitere Ausdruck User Experience (UX) durchgesetzt. Für einen ersten Überblick sei auf die umfangreiche Website der Nielsen and Norman Group verwiesen, des wohl bekanntesten Beratungsunternehmens in diesem Umfeld (NNg 2018).

Dieser kurze Überblick benachbarter relevanter Disziplinen und Methoden soll nicht den Eindruck von Beliebigkeit vermitteln. Ganz im Gegenteil: Eine genaue Analyse und strukturiertes methodisches Vorgehen im Sinne eines reflektierten Eklektizismus erscheinen bei interdisziplinärem Vorgehen besonders angemessen. Doch es wäre schade, wenn man relevante Erkenntnisse benachbarter Disziplinen nicht wahrnähme, nur weil sie einer anderen Forschungstradition entstammen. Wissenschaftstheoretisch könnte man die Verständlichkeitsforschung noch einen Schritt weiter in einem transdisziplinären Umfeld ansiedeln: Auch die handelnden Akteure (wie Fachexpert(inn)en, Autor(inn)en oder Lektor(inn)en) sollten in den Prozess einbezogen werden im Sinne einer Hilfe zur Selbsthilfe; Sprachexperten als Verständlichmacher ermöglichen dagegen keinen nachhaltigen Erfolg (siehe im Detail Lutz 2015: 33-47).

\section{Ein integratives Verständlichkeitsmodell}

Der hauptsächliche Beweggrund für die Erstellung dieses heuristischen Modells war, auf Basis aktueller Erkenntnisse ein Hilfsmittel zu entwickeln, das auch für Verfasser von Texten nützlich ist, die keine professionelle Schreibausbildung erhalten haben. Das Ziel dabei ist die Erhöhung der language awareness, also der Sensibilität für die Gestaltung verständlicher Texte. Eine wesentlich detailliertere Darstellung als hier möglich erfolgte in der Habilitationsschrift des Autors (Lutz 2015) und leicht überarbeitet online verfügbar (Lutz 2017a).

Die Randbedingungen in diesem Modell - in Abbildung 1 als umrahmende "Wolken" dargestellt - sollen den Gesamtkontext darstellen, in dessen Rahmen verständliche Texte produziert und rezipiert werden. Sie sind zum Teil durch situationelle und institutionelle Konstellationen vorgegeben, häufig aber auch durch die Kommunikationspartner mehr oder weniger flexibel gestaltbar. 
Die Dimensionen der Verständlichkeit - hier als Ellipsen innerhalb des Zyklus von Textproduktion, Textanalyse und Textoptimierung dargestellt - sind ähnlich zu verstehen wie im Hamburger Verständlichkeitsmodell und bei Göpferich (2002), als Mehr oder Weniger hinsichtlich der Ausprägung bestimmter Merkmale dieser Dimension. Die Dimensionen sind nicht komplett unabhängig voneinander, manche sollte man eher maximieren, andere in Abhängigkeit von den Randbedingungen austarieren. Der bei der Anwendung des Hamburger Verständlichkeitsmodells praktisch bewährte Ansatz des 5-stufigen Expertenratings für Ist-Analyse und Soll-Profil ist für die Einschätzung der Dimensionen durchaus möglich, aber nicht nötig.

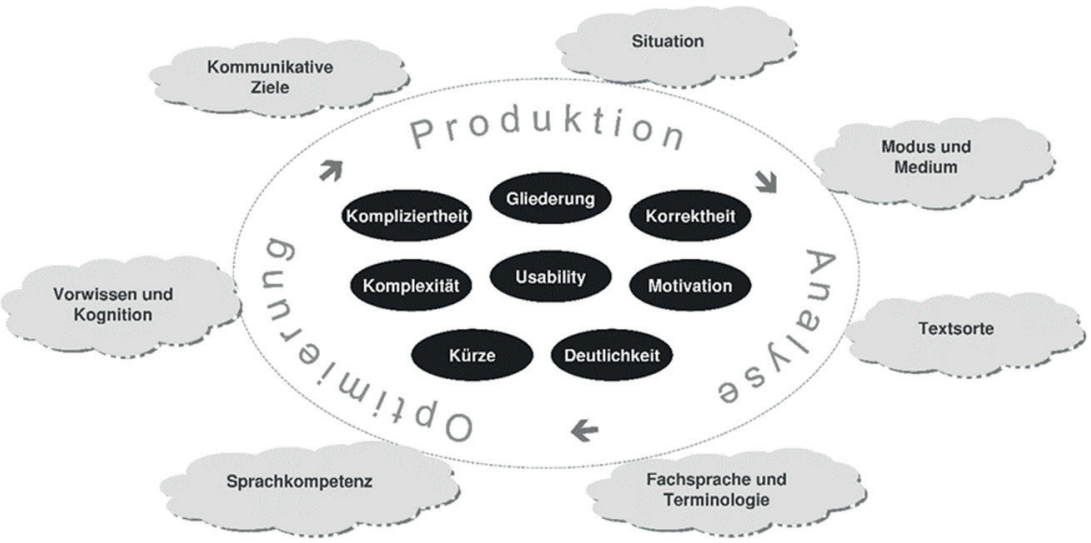

Abb. 1: Randbedingungen und Dimensionen der Textverständlichkeit

Die Analyse und ggf. der Ausgleich der kommunikativen Ziele von Autor(inn)en und Leser(inne)n ist eine wesentliche Voraussetzung für die zielgruppengerechte Gestaltung von Texten. Wenn die Leser(innen) andere Interessen verfolgen als der oder die Autor(in) von ihnen annimmt, dann sind Vorklärungen dringend vonnöten, z. B. zu folgenden Fragen: Geht es um Handlungskompetenz (etwa die Inbetriebnahme eines neuen Elektrogeräts), um Entscheidungskompetenz (etwa hinsichtlich der Wahl einer Alternative) oder lediglich um Wissenszuwachs (etwa durch das Lesen eines Lehrbuchs)? 
Die konkrete Situation im Prozess des Schreibens oder Lesens wird bei der Planung und Gestaltung von Texten häufig viel zu wenig berücksichtigt. Man denke beispielsweise an die unterschiedlichen Situationen beim Ausfüllen eines Formulars in einem Amt, beim Erlernen eines Gesellschaftsspiels in fröhlicher Runde oder beim Anlegen von Schneeketten in einem Schneesturm. Die Analyse dieser Faktoren kann zur Wahl unterschiedlicher Modi und Medien führen (verbal, visuell, Video; gedruckt, am Bildschirm, Smartphone etc.). Für bestimmte Zwecke eignen sich unterschiedliche Modus-Medium-Kombinationen unterschiedlich gut. So sind z. B. kurze Instruktionsvideos („how to ...") kommunikativ meist wesentlich effizienter als aufwändig erstellte textuelle oder Text-/Bild-Anleitungen. Derartige Videos sind z. B. in Plattformen wie Youtube sehr erfolgreich, und sie beginnen sich auch in „seriöseren“ Bereichen der Technischen Kommunikation durchzusetzen, wie man z. B. an Tagungsprogrammen der TEKOM oder an aktuellen Studienprogrammen zur Ausbildung Technischer Redakteure erkennen kann.

Die Beherrschung von Fachtextsorten sowohl auf Produzenten- als auch auf Rezipientenseite sowie deren Ausdifferenzierung im konkreten Arbeitsumfeld ist wesentlich für den kommunikativen Erfolg. Man baut dabei auf erlernte Muster auf, die das Schreiben und Verstehen unterstützen. Der Produktionsprozess wird typischerweise durch Dokumententemplates, Formulare, anzupassende Musterlösungen, Text-/Graphikbausteine oder automatisch generierte Textteile unterstützt. Entscheidend dabei ist auch der richtige Grad der Fachsprachlichkeit und Terminologie.

Die Sprachkompetenz der Adressat(inn)en sowie deren kognitive Fähigkeiten und Vorwissen sind eine weitere zu berücksichtigende Randbedingung. Themen wie funktionaler Analphabetismus (rund 20\% der Bevölkerung in Deutschland), Englisch als Esperanto der globalisierten Wirtschaft (English as a Lingua Franca), migrantische Kommunikation und Barrierefreiheit (Leichte Sprachen) spielen hier eine Rolle neben der immer zu berücksichtigenden fachlichen Vorbildung des Zielpublikums.

Bei den Dimensionen der Verständlichkeit ist die Differenzierung zwischen Komplexität und Kompliziertheit nach der Erfahrung des Autors besonders wichtig, da diese Ebenen in der Praxis oft vermischt werden. Komplexität be- 
trifft in diesem Modell die inhaltliche Ebene, Kompliziertheit die äußere Gestaltung. Komplexität sollte man optimieren, und Kompliziertheit minimieren. Ein Beispiel: Wenn ein Gesetz viele Ausnahmen zu allgemeinen Regelungen vorsieht (z. B. Übergangsbestimmungen, Berücksichtigung von spezifischen Gruppen und Härtefällen, etc.), so wird das Gesetz inhaltlich komplex. Dies kann als Ausdruck von politischen Kompromissen und Entscheidungsprozessen durchaus notwendig sein. Die überdurchschnittlich langen Sätze in Gesetzen ergeben sich allerdings nicht aus einer Notwendigkeit, sondern aus den historisch gewachsenen Traditionen der Textsorte Gesetz. Hier kann man ohne Verlust an Präzision durch die Vereinfachung syntaktischer Strukturen und Verkürzung der Satzlänge erhebliche Verbesserungen der Verständlichkeit erzielen. Ähnliches gilt für typische Verständlichmacher wie die Gliederung in überschaubare Einheiten, treffende Überschriften, einheitliche Terminologie, etc.

Die Dimensionen Gliederung, Kürze und Motivation sind ähnlich zu verstehen wie im Hamburger Verständlichkeitsmodell (Langer et al. 1974) die Dimensionen Gliederung/Ordnung, Kürze/Prägnanz sowie anregende Zusätze. Bei der Deutlichkeit geht es um die Hervorhebung wichtiger Inhalte durch geeignete Mittel, etwa typographisch (Fett- oder Kursivdruck, Schriftgröße), explizit verbalisiert („Wichtig! Beachten Sie bitte, dass ...") oder durch graphische Mittel. Die Korrektheit verdient in einem Verständlichkeitsmodell jedenfalls Beachtung als wichtiges Qualitätsmerkmal, das typischerweise im Reviewprozess überprüft wird.

Schließlich sei auf die besondere Bedeutung der Dimension Usability hingewiesen, die bei direkt handlungsorientierten Texten das entscheidende Außenkriterium für die Gebrauchstauglichkeit darstellt. Die in den letzten Jahren entwickelten Methoden des Usability Engineering sind auch für die Gestaltung verständlicher Texte nützlich; der/die Leser(in) wird dabei immer mehr als "User“ gesehen (man denke an Auskunftssysteme, Benutzerhandbücher oder Formulare). 
In diesem letzten Abschnitt soll kurz auf zwei Textsorten des beruflichen Alltags eingegangen werden, bei deren Analyse und Optimierung ein so breiter Zugang hilfreich ist.

Pflichtenhefte sind üblicherweise die Vertragsgrundlage zwischen Auftraggeber und Auftragnehmer und dadurch wichtige Brückendokumente. Sie sind sprachlich hochinteressant, da sie möglichst eindeutig formuliert und von allen Vertragspartnern verstanden werden sollten. Meist treffen hier mehrere Fachsprachen aufeinander: die der fachlichen Anwendungsdomäne, die der technischen Umsetzung, und für die rechtlich bindenden Bestandteile die juristische Fachsprache. Ein Pflichtenheft darf weder zu detailliert noch zu oberflächlich sein. Umfangreiche Pflichtenhefte benötigen sehr viel Aufwand für die Erstellung, doch sie verringern immerhin den Interpretationsspielraum der Vertragspartner. Sie werden allerdings aufgrund ihrer Komplexität selten im vollen Umfang gelesen und sind bei Beginn der Umsetzung inhaltlich oft schon überholt. Kurze und wenig detaillierte Pflichtenhefte hingegen erlauben große Interpretationsspielräume und eröffnen (gewollt oder ungewollt) Raum für Claim Management und Nachverhandlungen.

Für die Optimierung von Pflichtenheften empfiehlt sich das Entwickeln einer möglichst eindeutigen Terminologie (Glossare, Normen) sowie das Verwenden von Beschreibungssprachen, die beide Seiten verstehen. Das bedeutet je nach Anwendungsdomäne und Beschreibungstiefe z. B. Pläne, Diagramme, Visualisierungen, Szenarien, Use Cases, Personas oder formale Sprachen wie UML. Formale Beschreibungen sind exakter als natürliche Sprache, doch für Laien häufig schwer nachvollziehbar; visuelle Beschreibungen sind besonders gut geeignet für die Darstellung prozessualer Information, benötigen aber zusätzlich immer natürliche Sprache.

Einheitliche Gliederungen von Pflichtenheften mit Vorgaben hinsichtlich der Inhalte führen zur Standardisierung von Dokumentenstrukturen. Damit werden von den Autoren wichtige Themen weniger leicht vergessen, und für die Leser(innen) sind spezifische Inhalte leichter auffindbar. Solche Standardisierungen können noch weiter gehen: In der Rechtsinformatik sind smart contracts derzeit ein spannendes Thema, nämlich die (halb) automatisierte Erstellung von 
Verträgen, in denen sich IT-Systeme über „das Kleingedruckte“ einigen und die Menschen nur noch die komplexeren Themen ausformulieren müssen (Clack et al. 2017).

Prozessbeschreibungen spielen in vielen Branchen eine entscheidende Rolle für die Gestaltung von Arbeitsabläufen, von Produktionsbetrieben über Krankenhäuser bis zu reinen Dienstleistern wie etwa Call Centers (Wagner/Käfer 2017). Der enge funktionale Zusammenhang zwischen Beschreibung und Umsetzung ist dabei zentral, sonst droht das im Qualitätsmanagement gefürchtete Auseinanderdriften von „dokumentierten" und "gelebten "Prozessen. Alle oben erwähnten Randbedingungen und Dimensionen der Verständlichkeit sind von Belang, insbesondere Zielgruppenorientierung, Visualisierungen, Terminologie, situationelle Faktoren und die richtige Balance zwischen Detailliertheit und Abstraktion. Sprachliche Verständlichkeit im engeren Sinne bleibt dabei wichtig, allerdings im engen Zusammenspiel mit anderen Faktoren, um in Summe $\mathrm{zu}$ anwendergerechten Prozessen zu gelangen, die man am besten mittels Usability Tests auf ihre Tauglichkeit überprüfen sollte. 


\section{Literaturverzeichnis}

Ballstaedt, StefFen-Peter (2012): Visualisieren. Bilder in wissenschaftlichen Texten. Wien: Huter \& Roth.

Baumert, Andreas (2003): Professionell texten. Tipps und Techniken für den Berufsalltag. München: dtv.

Bhatia, Vijay K. (2004): Worlds of Written Discourse. A Genre-based View. New York: Continuum.

Bock, Bettina M. (2014): „,Leichte Sprache': Abgrenzung, Beschreibung und Problemstellungen aus Sicht der Linguistik“. In: JeKAT, SuSANNE J. ET AL. (Hg.): Sprache barrierefrei gestalten. Perspektiven aus der Angewandten Linguistik. Berlin: Frank \& Timme, 17-51.

Bock, Bettina M./Fix, Ulla/Lange, Daisy (Hg.) (2017): „Leichte Sprache“ im Spiegel theoretischer und angewandter Forschung. Berlin: Frank \& Timme.

Bredel, Ursula/MaAß, Christiane (2016): Leichte Sprache. Theoretische Grundlagen, Orientierung für die Praxis. Berlin: Dudenverlag.

Christmann, URsula (2004). „Verstehens- und Verständlichkeitsmessung: methodische Ansätze in der Anwendungsforschung“. In LERCH, KENT D. (Hg.): Recht verstehen. Verständlichkeit, Missverständlichkeit und Unverständlichkeit von Recht. Berlin: de Gruyter, 33-62.

Christmann, Ursula/Groeben, Norbert (2018, in diesem Band): „Verständlichkeit die psychologische Perspektive“.

Clack, Christopher. D./Bakshi, Vikram A./Braine, LeE (2017). „Smart contract templates: foundations, design landscape and research directions".

URL: https://arxiv.org/abs/1608.00771 (letzter Zugriff: 8.1.2018).

ClARITY (2018): URL: http://www.clarity-international.net/ (letzter Zugriff: 28.1.2018).

DuBAY, William (2004): The Principles of Readability. Costa Mesa: Impact Information. URL: https://eric.ed.gov/?id=ED490073 (letzter Zugriff: 30.3.2018).

EICHHOFF-Cyrus, Karin/Antos, Gerd (Hg.) (2008): Verständlichkeit als Bürgerrecht? Die Rechts- und Verwaltungssprache in der öffentlichen Diskussion. Mannheim: Dudenverlag.

GÖPFERICH, SUSANNE (2002): Textproduktion im Zeitalter der Globalisierung. Entwicklung einer Didaktik des Wissenstransfers. Tübingen: Stauffenburg.

Groeben, Norbert (1982): Leserpsychologie: Textverständnis - Textverständlichkeit. Münster: Aschendorff.

HEIDRICH, FrANZISKA (2017): „Die Fachkommunikationswissenschaft. Einführung in das Themenheft von trans-kom“. In: trans-kom 10 (3), 271-283.

IIID (2018): URL: http://www.iiid.net/ (letzter Zugriff: 28.1.2018).

KNOWLEDGE COMMUNICATION (2018): URL: http://www.knowledge-communication.org/ (letzter Zugriff: 28.1.2018). 
LANGer, Inghard et AL. (1974): Verständlichkeit in Schule, Verwaltung, Politik, Wissenschaft. 10. Aufl. 2015 unter dem Titel: Sich verständlich ausdrücken. München: Reinhardt.

LUTZ, BENEDIKT (2015): Verständlichkeitsforschung transdisziplinär. Plädoyer für eine anwenderfreundliche Wissensgesellschaft. Göttingen: V\&R unipress.

LUTZ, BENEDiKT (2017a): „Modelle für die verständliche Fachkommunikation. Das Spannungsfeld zwischen wissenschaftlichem Anspruch und praktischer Anwendbarkeit“. In: trans-kom 10 (3), 284-314.

LUTZ, BENEDIKT (2017b): „Verständliche Gesetze: Was Legisten von technischen Redakteuren lernen können“. In: SCHWEIGHOFER, ERICH ET AL. (Hg.): Trends und Communities der Rechtsinformatik. Tagungsband des 20. Internationalen RechtsinformatikSymposiums IRIS 2017. Wien: OCG, 209-216.

NNG (2018): URL: https://www.nngroup.com/ (letzter Zugriff: 28.1.2018).

PLAIN (2018): URL: http://plainlanguagenetwork.org/ (letzter Zugriff: 28.1.2018).

REINERS, Ludwig (1963): Stilfibel. Der sichere Weg zum guten Deutsch. München: dtv.

SCHneIder, Wolf (1999): Deutsch für Profis. Wege zu gutem Stil. München: Goldmann.

Schubert, Klaus (2007): Wissen, Sprache, Medium, Arbeit. Ein integratives Modell der ein- und mehrsprachigen Fachkommunikation. Tübingen: Narr.

SICK, BASTIAN (2004): Der Dativ ist dem Genitiv sein Tod. Ein Wegweiser durch den Irrgarten der deutschen Sprache. Köln: Kiepenheuer und Witsch.

TeXTLAB (2018): URL: https://www.text-lab.de/ (letzter Zugriff: 28.1.2018).

WAGNER, KARL/KäFER, ROMAN (2017). PQM-Prozessorientiertes Qualitätsmanagement: Leitfaden zur Umsetzung der ISO 9001. München: Hanser. 


\section{Empirische Überprüfung von Verständlichkeit}

\section{$1 \quad$ Methoden zur Verständlichkeitsmessung}

Bei der Produktion barrierefreier Texte geht es in erster Linie um die Optimierung der Verständlichkeit für den Rezipienten/die Rezipientin. Für die Leichte Sprache gibt es beispielsweise Regeln (vgl. Maßß 2015, Bredel/Maaß 2016), von denen man annimmt, dass sie sich verständlichkeitsfördernd auf die Textproduktion auswirken. Die Auswirkungen dieser Regeln auf die Komplexität der Texte lassen sich produktbasiert messen. Unklar bleibt jedoch, ob sich die reduzierte Komplexität tatsächlich auch positiv auf den Verarbeitungsaufwand bei der Textrezeption auswirkt. Auch dies lässt sich empirisch untersuchen. Hierfür werden allerdings prozessbasierte Methoden benötigt, die die Textrezeption näher beleuchten. Zusammenfassend lässt sich also sagen, dass man erst durch die empirische Erforschung der Textproduktion und -rezeption Aussagen über die Effizienz verständlichkeitsfördernder Regeln in der Barrierefreien Kommunikation ableiten kann.

Validität, Güte und Aussagekraft empirischer Forschung hängen in hohem Maße sowohl vom methodischen Zuschnitt als auch von der Auswahl des Instrumentariums ab.

So ist Text als Ergebnis eines Produktionsprozesses für empirische Forschung sehr gut unmittelbar zugänglich, wohingegen kognitive Rezeptionsprozesse sowie individuelle Lesermerkmale, wie z. B. Intention oder Motivation, in der Regel nur mittelbar beobachtbar bzw. messbar sind und daher eine aufwändigere Operationalisierung erfordern. Diese Überprüfung der Effektivität der Textproduktion und Effizienz der Rezeption kann sich je nach Forschungsfrage und -kontext unterschiedlich gestalten. Daher ist es von Vorteil, sich einen möglichst großen Überblick über das für diesen Zweck zur Verfügung stehende Instrumentarium zu verschaffen. 
Einen Kategorisierungsvorschlag dieser Methoden im Kontext empirischer Forschung und damit einen ersten Einstieg in das Thema bietet das von Krings (2005) ursprünglich im Rahmen der Translationsprozessforschung erstellte Übersichtsschema verschiedener Datenerhebungsverfahren (siehe Abbildung 1), das auch aus sprachwissenschaftlicher und psycholinguistischer Sicht für die Messbarkeit von Textkomplexität und Textverstehen in großen Teilen adaptiert werden kann (vgl. Hansen-Schirra/Gutermuth 2015):

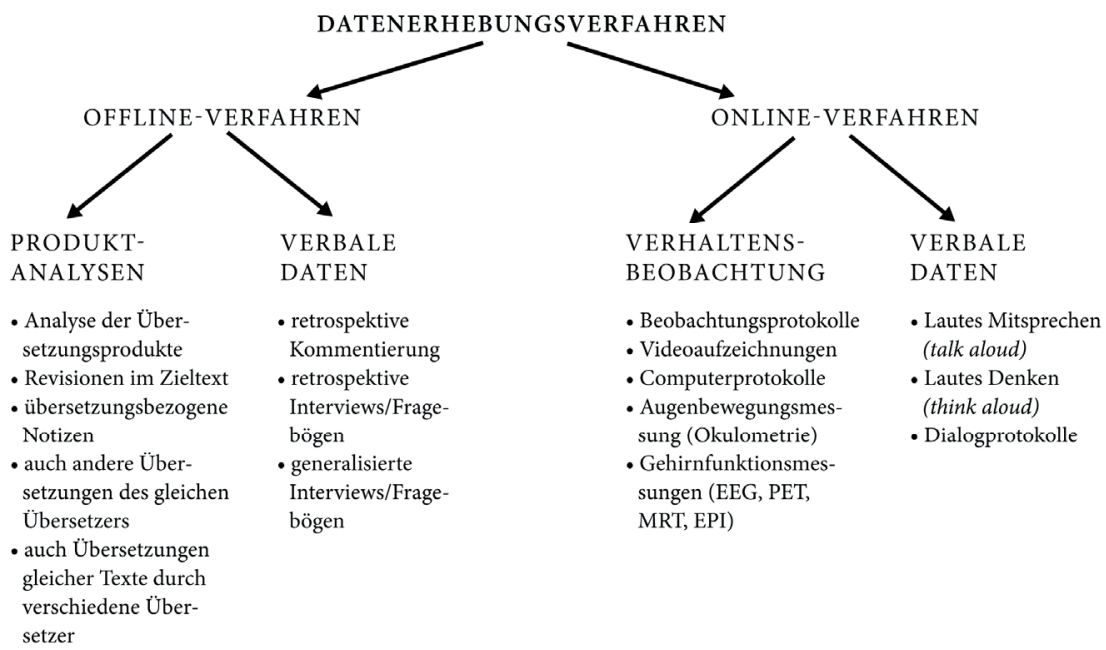

Abb. 1: Typologie von Datenerhebungsverfahren in der Translationsprozessforschung nach Krings (2005: 348)

Gemessen am Zeitpunkt der Datenerhebung unterscheidet Krings in erster Instanz zwischen Offline- und Online-Verfahren. Innerhalb der jeweiligen Verfahren differenziert er dann in zweiter Instanz zwischen produkt- und prozessorientierten Methoden einerseits sowie zwischen der Erhebung retrospektiver und introspektiver verbaler Daten andererseits. Für beide Verfahren lassen sich sowohl qualitative als auch quantitative Daten erheben.

Krings weist den Offline-Verfahren einen zeitlich nachgeordneten Charakter zu und definiert sie als Methoden, die im Anschluss an ein Experiment 
(postaktional) angewendet werden können. Hierunter fallen sowohl Produktanalysen als auch retrospektive verbale Daten (s.u.). Letztere ergänzen die reinen Produktdaten um zusätzliche qualitative Informationen und erhöhen somit deren Validität.

Die Datenerhebungsverfahren hingegen, die den (Rezeptions-)Prozess dokumentieren, müssen parallel zu diesem ablaufen (peraktional). Online-Verfahren finden somit in Echtzeit statt und haben laut Krings Beobachtungscharakter. Daher fasst er diese Verfahren unter dem Oberbegriff Verhaltensbeobachtung zusammen. Die hier verwendeten Verfahren sind meist maschinengestützt, erfordern größtenteils hochtechnisierte Geräte und sind daher oft mit einem größeren Untersuchungsaufwand verbunden. Die Gleichzeitigkeit bei der Erfassung der verbalen Daten wird hier durch Introspektion als Pendant zur Retrospektion ausgedrückt.

Da nicht alle von Krings aufgeführten Datenerhebungsverfahren gleichermaßen zielführend für die empirische Erforschung von Verständlichkeit sind, wird die folgende Vorstellung und Differenzierung der Methoden in ihrer Orientierung am State-of-the-Art der Sprach- und Kommunikationswissenschaft ohne Anspruch auf Vollständigkeit entsprechend angepasst.

\section{$2 \quad$ Messung der Produktionskomplexität}

Die Lesbarkeitsforschung kann als Beginn der empirischen Erforschung von Textverständlichkeit betrachtet werden (siehe auch Christmann/Groeben in diesem Band). Sie analysiert Texte auf Oberflächenebene, macht sich objektive Textmerkmale zunutze und entwickelt auf Basis deren Quantifizierbarkeit bestimmte sprachstatistische Formeln, um daraus Rückschlüsse auf die Lesbarkeit der Texte ziehen zu können. In der Annahme, dass die Lesbarkeit bei längeren Sätzen sowie bei langen und komplexen Wörtern leidet, bewertet ein Großteil der Formeln die Texte in Abhängigkeit der durchschnittlichen Satzlänge, Wortbzw. Silbenanzahl oder Satzkomplexität. 


\subsection{Lesbarkeitsformeln}

Die wohl bekannteste Lesbarkeitsformel ist der Flesch-Reading-Ease-Index (FRE), der in den 1930er Jahren für den anglo-amerikanischen Raum entwickelt und dessen Anpassung für das Deutsche 1978 unter dem Namen AmstadFormel bekannt wurde, wobei beide Formeln nach ihren Entwicklern benannt wurden. Die Amstad-Formel berücksichtigt die durchschnittliche Satzlänge in Wörtern (ASL, aus dem Englischen: Average Sentence Length) und die durchschnittliche Silbenanzahl pro Wort (ASW, aus dem Englischen: Average number of Syllables per Word) und lautet:

$F R E_{\text {Amstad }}=180-A S L-(58,5 \cdot A S W)$

Für die Auswertung wird eine Skala von 0-100 zugrunde gelegt, wobei gilt: Je höher der ermittelte Wert, desto leichter verständlich der Text.

Weitere bekannte Lesbarkeitsformeln für den deutschsprachigen Raum sind der SMOG-Index, der LIX Lesbarkeitsindex und die Wiener Sachtextformeln (siehe Wolfer 2015 für einen Überblick).

Lange Zeit galten die Lesbarkeitsformeln als einziger Zugang und Gradmesser für die Verständlichkeit von Texten. Auch heute noch werden sie vielfach eingesetzt und haben nach wie vor ihre Berechtigung; man muss sich aber ihrer doch sehr eingeschränkten Aussagekraft bewusst bleiben. Legt man die Kriterien des Flesch-Reading-Ease-Index zugrunde, so können auch durchaus inhaltlich anspruchsvolle Texte die Forderung nach kurzen Wörtern in kurzen Sätzen und den damit einhergehenden hohen Lesbarkeitsindex erfüllen. Ein hoher Lesbarkeitsindex bedeutet somit nicht automatisch verbesserte Verständlichkeit; ein niedriger Lesbarkeitsindex kann andererseits durchaus auf Probleme bei der Dekodierung hinweisen.

\subsection{Korpuslinguistische Analysen}

Technischer Fortschritt und Digitalisierung ermöglichen die Verfügbarkeit und Verarbeitung großer Mengen an elektronischen Daten, speziell großer natürlicher Textkorpora, welche mithilfe computerlinguistischer und korpusanalytischer Verfahren auf bestimmte grundlegende linguistische Fragestellungen 
und vermutete Gesetzmäßigkeiten und Frequenzmuster hin untersucht werden können.

Ein einfaches Verfahren, das auch in der Sprachtypologie verwendet wird, ist die Verdichtung des zu messenden Objekts mittels eines Computeralgorithmus wie z. B. bei einem ZIP-Programm (vgl. Juola 2008). Mit aufwändigeren Verfahren können die unterschiedlichen sprachlichen Ebenen abgebildet und deren Komplexität modelliert werden (vgl. Lüdeling/Kytö 2009 für einen Überblick). Auf morphologischer und lexikalischer Ebene sind Merkmalsanalysen und Part-of-Speech-Tagging etablierte Methoden. Dadurch können Komposita zerlegt und Wortbildungsphänomene wie Derivation, Flexion etc. untersucht werden. Auf phrasaler und syntaktischer Ebene wendet man Chunker und Parser zur Phrasen- und Satzgliederkennung und zur Analyse syntaktischer Dependenzstrukturen und Einbettungsebenen an. Auf semantischer Ebene sind die Disambiguierung semantischer Relationen sowie die automatische Erkennung anaphorischer Bezüge (z. B. Kohäsionsketten) möglich (vgl. Lemnitzer/ Zinsmeister 2006 für eine Einführung).

In parsing-orientierten Komplexitätsanalysen spielt die Entfernung von zusammenhängenden Teilen eines Satzes sowie die Einbettungstiefe eine Rolle. Durch solche Messungen kann vorhergesagt werden, dass der Verarbeitungsaufwand von weit voneinander entfernten Satzteilen höher sein sollte. Andere Ansätze verfolgen ein „nicht-lokales“ Gegenmodell (z. B. Federmeier 2007), bei dem die Verarbeitung von der Antizipation bzw. Projektion der noch ausstehenden Teile einer Äußerung abhängt. Das Parsen sollte demnach zum Satzende hin weniger komplex sein, weil der Inhalt meistens schon teilweise von dem Rezipienten/der Rezipientin vorhergesagt werden kann (Konieczny 2000: 627-629 für eine Literaturübersicht).

Alle diese Verfahren fokussieren auf sprachliche Ausdrucksmittel; sie blenden jedoch deren Wirkung auf die Rezeption und die Verarbeitungsprozesse aus. Methoden, die Letzteres näher beleuchten, werden im Folgenden vorgestellt. 
Im Zuge der Weiterentwicklung der Lesbarkeitsforschung entstanden in den 1970er Jahren die ersten kognitiv orientierten Verständlichkeitsmodelle unter expliziter Berücksichtigung der Rezipient(inn)en. Textexterne Faktoren wie Vor- und Weltwissen, Motivation, Intention, situativer Kontext oder physische und psychische Disposition werden als Einflussfaktoren für ein Gelingen der Text-Leser-Interaktion (vgl. Christmann/Groeben 1999) ausgemacht und untersucht. Verstehen wird als Wechselwirkungsprozess zwischen der individuellen Erwartungshaltung der Rezipient(inn)en und dem tatsächlichen Textangebot gesehen (vgl. Dermarmels 2010: 110).

\subsection{Verständlichkeitsratings}

Eine etablierte Methode stellt die Bewertung der Textverständlichkeit durch Ratings dar. Der Begriff „Rating“ kommt aus dem Englischen und bedeutet „einschätzen“ bzw. „beurteilen“. Die Ergebnisse aus Ratingverfahren bilden somit eine momentane subjektive Meinung ab (vgl. Lerch 2008: 66). In der Regel werden sie in Form von Skalierungsmodellen präsentiert wie z. B. Likert-Skalen oder Polaritätsprofile. Hier kann der Zeitpunkt der Beurteilung von Bedeutung sein. Dabei werden spezielle Aspekte der Verständlichkeit abgefragt, die von einer im Hinblick auf die zu untersuchende Zielgruppe repräsentativen Auswahl an Proband(inn)en bewertet werden. Als theoretischer Rahmen zur Definition dieser Aspekte werden oft Verständlichkeitsmodelle als Basis genommen. So werden beispielsweise gemäß dem Hamburger Verständlichkeitsmodell (vgl. Langer et al. 1993, s. auch Christmann/Groeben in diesem Band) die folgenden vier relevanten Textdimensionen beschrieben, die sich empirisch auf ihren Einfluss auf die Verständlichkeit untersuchen lassen:

- Einfachheit/Korrektheit

- Gliederung/Struktur

- Kürze/Prägnanz

- Motivationale Stimulanz 
Andere Modelle legen den Fokus auf andere Perspektiven, z. B. interaktional (Groeben 1982) oder kommunikationsorientiert (Göpferich 2002). Letzteres geht explizit sowohl auf textinterne (Textsorte) als auch textexterne Bezugsgrößen (Rezipient(inn)en) ein und integriert verschiedene Ansätze aus der Verständlichkeitsforschung und benachbarten Wissenschaften (z. B. durch den Einbezug mentaler Modelle).

Die aktuellste Weiterentwicklung auf diesem Gebiet ist der Hohenheimer Verständlichkeits-Index (HIX). Ihm liegen mehrere Lesbarkeitsformeln zugrunde und er integriert zusätzlich noch weitere Textmerkmale (vgl. Kercher 2013). Durch den Zugriff auf verschiedene Messgrößen und die Bereitstellung einer Auswahl an Einflussvariablen (Kommunikatorfaktoren, Textfaktoren, Kanalfaktoren etc.) ist eine sehr differenzierte und passgenaue Analyse möglich.

\subsection{Verbale Daten}

Unter dem Begriff Verbale Daten fasst Krings Datenerhebungsmethoden zusammen, die den Probanden/die Probandin explizit dazu auffordern, entweder während des Experiments oder danach alle Gedanken und Gefühle die Testsituation betreffend zu äußern. Diese Verbalisierungen werden dokumentiert und später analysiert und finden Eingang in die Gesamtanalyse der Daten. In diese Kategorie fallen auch verbalisierte Erinnerungsprotokolle, die sogenannte Reproduktion.

Introspektion (Lautes Denken (LD)), in der Fachliteratur oft unter der englischen Bezeichnung Think-Aloud Protocols (TAP) oder Concurrent Think-Aloud Protocol (CTAP) zu finden, ist ein weit verbreitetes psycholinguistisches Verfahren (vgl. Göpferich 2008: 10-11). Bei diesem Verfahren soll der Proband/die Probandin, parallel zur Ausführung der ihm/ihr gestellten Aufgabe, spontan und ungefiltert alle Gedanken und Gefühle verbalisieren, die ihm bzw. ihr gerade in den Kopf kommen. Sie erlauben direkte Einblicke in die bewusst ablaufenden Rezeptionsprozesse im Kurzzeitgedächtnis (vgl. Ericsson/Simon 1993) und liefern Informationen bezüglich Selbsteinschätzung und -wahrnehmung des jeweiligen Probanden/der jeweiligen Probandin. Die Aufzeichnungen dieser verbalen Daten werden in der Regel vor der Auswertung transkribiert. 
Häufig geäußerte Kritik an der Methode bezieht sich auf die Vollständigkeit der verbalisierten Daten: Einerseits sprechen einige Proband(inn)en nicht alle Gedanken auch wirklich aus, da die Erhebungssituation für sie unnatürlich ist, andererseits könnte es zu Interferenzen (vgl. Göpferich 2008: 23) zwischen der Ausführung der Aufgabe und der Verbalisierung kommen, so dass Verzerrungseffekte auftreten und im schlimmsten Fall weder die Aufgabe noch die Verbalisierung bewältigt werden kann, was wiederum deren Validität in Frage stellen würde.

Retrospektion, d.h. Retrospektives Lautes Denken (engl. Retrospective Think-Aloud Protocol (RTAP)) wird oft eingesetzt, wenn man den Probanden/ die Probandin bittet, anhand eines Mediums (Ton- oder Bildaufzeichnungen, Fragebogen) die bereits getroffenen und dokumentierten Entscheidungen im Nachhinein zu kommentieren, und zwar analog zu den TAP ebenfalls auf der Basis der spontanen, unstrukturierten Äußerung aller Gedanken und Gefühle dazu.

Im Gegensatz dazu stehen Retrospektive Interviews, die ebenfalls auf schon getroffenen und dokumentierten Entscheidungen basieren, die aber eher konkrete punktuelle Nachfragen (probing) des Interviewers zu bestimmten Entscheidungen einfordern.

Bei allen retrospektiven Verfahren ist der Zeitpunkt wichtig, da Speicherzeiten und -kapazitäten des Arbeitsgedächtnisses und des Langzeitgedächtnisses berücksichtigt werden müssen. Je länger der Abstand zwischen Experiment und Retrospektion, desto eher können Informationen verlorengehen (Auslassungen) oder verzerrt (Elaborationen) reproduziert werden (vgl. Norman/Rumelhart 1975).

Reproduktion lässt sich in freie (free recall) und gebundene Reproduktion (cued recall) unterscheiden. Bei der freien Reproduktion der Proband/die Probandin aufgefordert wird, das Gehörte oder Gelesene so gut und so vollständig wie möglich wiederzugeben (vgl. Rickheit/Strohner 1993: 103), wohingegen dem Probanden/der Probandin bei der gebundenen Reproduktion jeweils ein Wort vorgegeben wird, zu dem er/sie den Satz im Text reproduzieren muss, in dem das Wort vorkam. Beide Varianten sind einfach in der Anwendung, haben jedoch den Nachteil, dass nicht genau nachvollzogen werden kann, ob sich die Proband(inn)en erinnern, weil sie ein gutes Gedächtnis haben oder weil sie den 
Text verstanden haben und inwieweit Vor- und Weltwissen sowie Inferenzprozesse einen Einfluss auf das Ergebnis haben.

\subsection{Vor- und Nachwissenstests}

Vor- und Nachwissenstests sind weit verbreitete Methoden in der empirischen Forschung. Beide gehören zu den Offline-Verfahren, wobei an dieser Stelle Krings Definition um die Option der Vorzeitigkeit erweitert werden muss (präaktional), da Vorwissenstests zeitlich vor dem Experiment oder der zu bewältigenden Aufgabe liegen. Sie werden meistens in Form von Lückentests (engl. cloze-test), Multiple-Choice-Verfahren oder Fragebögen umgesetzt.

Vorwissenstests dienen in der Regel zur Aktivierung oder zur Dokumentation vorhandenen Wissens vor einem Experiment oder einer zu bewältigenden Aufgabe als Vergleichsbasis zu dem Wissensstand nach der Durchführung des Experiments, um die Frage nach einem messbaren Wissenszuwachs beantworten zu helfen. Diese Vorher-Nachher-Szenarien kommen oft in den Bereichen Sprachkompetenz oder Fachwissen zur Anwendung.

In Lückentests werden sowohl der Wortschatz als auch das Textverstehen der Proband(inn)en getestet, in dem sie Lücken in einem vorgegebenen Text mit der jeweils richtigen Ersetzung füllen müssen (z. B. Tuinman 1971).

Bei Multiple-Choice-Tests sind mehrere Aussagen zu einem Sachverhalt oder mögliche Antworten auf eine Frage bereits vorgegeben und der Proband/ die Probandin muss je nach Sachkenntnis bzw. Textverstehen die jeweils korrekte(n) Antwort(en) markieren. Hier gibt es die Möglichkeit, mit Glück und durch Raten die richtige(n) Antwort(en) zu treffen: Diese Methode ist daher für die Abfrage des Wissenstandes oder von Textverstehen nur eingeschränkt aussagekräftig. Sie findet Anwendung in der empirischen Kompetenzforschung (z. B. Zlatkin-Troitschanskaia et al. 2017).

Bei Fragebögen hängt die Auswahl und die Art der Fragen sowie das damit verbundene Antwortformat sehr eng mit der Forschungsfrage zusammen.

Handelt es sich beispielsweise um quantitative (hypothesentestende) oder qualitative (hypothesengenerierende) Forschung? Zielt die Forschungsfrage eher auf ein Merkmal oder eine Merkmalsausprägung ab? Oft werden Fragebögen ergänzend zu quantitativen Messmethoden eingesetzt, um dem Datenmaterial 
subjektive Aspekte hinzuzufügen und somit eine weitere Perspektive auf den Forschungsgegenstand zu ermöglichen. Die Antwortmöglichkeiten können in Form von Lückentext, Multiple-Choice, Ja/Nein-Optionen, offenen oder geschlossenen Antwortformaten etc. vorliegen. Fragebögen werden typischerweise in der Sprachkompetenzforschung eingesetzt (z. B. Hansen-Schirra et al. 2017). Im Kontext der Barrierefreien Kommunikation müssen sie allerdings der Probandengruppe entsprechend angepasst bzw. vereinfacht werden (z. B. durch den Einsatz von Bildmaterial; vgl. Bredel et al. 2016).

\subsection{Verhaltensbeobachtung}

Im Gegensatz zu den größtenteils subjektiv-qualitativen Methoden aus dem vorangegangenen Kapitel zählen die hier vorgestellten Verfahren der Verhaltensbeobachtung zu den objektiven, quantitativen Messmethoden, da die Daten in der Regel von Geräten oder außenstehenden Personen (Versuchsleiter(inne)n) im Zeitverlauf erfasst werden. Verzerrungen durch subjektive Einflussnahme des Probanden/der Probandin auf den Prozess der Datenerhebung werden dadurch extrem minimiert (vgl. Döring/Bortz 2016: 502). Gleichzeitig haben die Messdaten keine Aussagekraft bezüglich Motivation oder subjektiven Erlebens des Probanden/der Probandin.

Screen-Recording-Software zeichnet in Echtzeit im Hintergrund alle Bildschirmseiten auf, die der Proband/die Probandin besucht hat (vgl. Göpferich 2008: 53). Außerdem werden auch verbale Daten mit aufgezeichnet. So lassen sich z. B. Recherchewege im Kontext der Textrezeption nachvollziehen.

Beim Keylogging erfasst eine Software alle Tastaturanschläge sowie Mausoder Cursorbewegungen des Probanden/der Probandin. Sie liefert ein Protokoll des Schreibprozesses, das auf Pausen, Fehler, Löschungen und Korrekturen hin ausgewertet werden kann. So lassen sich beispielsweise das Anfertigen von Notizen bei der Textrezeption aufzeichnen oder Verarbeitungsstrategien bei der Textproduktion (siehe z. B. Carl et al. 2008).

Eyetracking ist eine gerätegestützte Methode, die es ermöglicht, die genauen Blickverläufe eines Probanden/einer Probandin auf einer Systemoberfläche (Bildschirm, Mobilgerät etc.) zu registrieren und aufzuzeichnen (vgl. Holmqvist et al. 2011; Duchowski 2003). Dabei werden Infrarotwellen von der Hornhaut 
im Auge reflektiert, über entsprechende Kameratechnik aufgenommen und darüber kann die Pupillenposition bestimmt und wiedergegeben werden. Just und Carpenter (1980) gehen bei der von ihnen aufgestellten Eye-Mind-Hypothese (vgl. ebd. 1980) davon aus, dass das, was mit dem Auge fixiert wird, sofort auch syntaktisch-semantisch eingeordnet und kognitiv verarbeitet wird. Basierend auf dieser Annahme können Parameter wie Länge, Anzahl oder Richtung der Fixation sowie Lesezeit Rückschlüsse auf die Lesbarkeit von Texten sowie auf kognitive Verarbeitungsprozesse zulassen (z. B. Carl/Dragsted 2012). Neuere Eyetracker sind nichtinvasiv und erlauben somit eine Datenerhebung im Versuchskontext, die vom Probanden/von der Probandin in der Regel als nicht störend empfunden wird, da eine möglichst natürliche Lesesituation hergestellt wird. Dies wiederum ist wichtig für die generelle Übertragbarkeit der Ergebnisse. Man spricht in diesem Zusammenhang oft von ökologischer Validität. Ökologische Validität gehört zu den Gütekriterien empirischer Forschung und bezieht sich auf die Ähnlichkeit zwischen Untersuchungsbedingungen und Lebenswelt der Proband(inn)en. Je ,natürlicher' (ähnlicher) die Bedingungen sind, desto ,gültiger` und damit übertragbarer werden die Ergebnisse angesehen. Oftmals werden die beiden letztgenannten Methoden auch kombiniert, um Produktions- und Rezeptionsprozesse integriert zu erforschen (z. B. Kruger 2016).

\subsection{Physiologische und neurophysiologische Methoden}

Physiologische Methoden untersuchen im experimentellen Kontext Reaktionen und Veränderungen des peripheren Nervensystems in Echtzeit wie z. B. die Herzschlagfrequenz, den Hautwiderstand oder die Pupillenveränderung (vgl. Rickheit und Strohner 1993: 120):

Das Elektrokardiogramm (EKG) zeichnet die elektrische Aktivität des Herzmuskels auf, indem es die elektrischen Spannungsänderungen direkt an der Körperoberfläche über Elektroden abnimmt und aufzeichnet. Für die Messung werden Elektroden und ein EKG-Aufzeichnungsgerät benötigt. Die Ergebnisse sind somit entweder mit Blick auf das emotionale Verhalten des Probanden/der Probandin oder dessen/deren Leistungsfähigkeit interpretierbar (siehe Katkin et al. 1993). 
Die Hautwiderstandsmessung (elektrodermale oder psychogalvanische Reaktion, EDR/PGR) macht sich den Umstand zunutze, dass sich der elektrische Hautwiderstand messbar verringert, wenn sich aufgrund von Anstrengung oder emotionaler Erregung die Schweißdrüsenaktivität verstärkt. Für die Messung werden Elektroden und ein Aufzeichnungsgerät, der Polygraph, benötigt. Auch hier sind die Ergebnisse somit entweder mit Blick auf das emotionale Verhalten des Probanden/der Probandin oder dessen/deren Leistungsfähigkeit interpretierbar (siehe Langner et al. 2015).

Die Pupillenreaktion, genauer die Pupillengröße wird als geeignetes Maß für kognitive Beanspruchung diskutiert. Je höher die mentale Beanspruchung, desto größer ist die Pupille (vgl. Beatty 1982). Der Vorteil der schnellen Reaktionszeit ist gleichzeitig ein Nachteil, da die Pupille extrem sensibel nicht nur auf psychosensorische, sondern auch auf andere sensorische Reize reagiert. Pupillenreaktionen können nichtinvasiv mithilfe von Eyetracking aufgezeichnet werden (siehe z. B. Seeber 2013).

Neurophysiologische Methoden basieren auf der Möglichkeit, aufgrund bestimmter neurophysiologischer Reaktionen Rückschlüsse auf Verarbeitungsprozesse im Gehirn ziehen zu können: Ein Elektroenzephalogramm (EEG) macht Ereigniskorrelierte Potentiale (EKP, auch engl. event-related potentials (ERP) im Gehirn messbar, die sich in der Änderung der positiven oder negativen Spannungsanteile als Reaktion auf einen Stimulus ausdrücken. Dadurch kann gezeigt werden, welche Gehirnareale im Experimentverlauf wie stark beansprucht werden (siehe z. B. Kutas/Hillyard 1980, Christoffels et al. 2007).

Neurophysiologische Verfahren, die es ermöglichen, bestimmte Regionen im Gehirn bei deren Aktivierung visuell zu lokalisieren, nennt man bildgebende Verfahren:

Die Funktionelle Magnetresonanztomographie (fMRT) ist ein Verfahren zur Darstellung der Stoffwechselaktivität im Gehirn durch Erzeugung eines starken Magnetfeldes. Dabei wird der Anstieg der Sauerstoffkonzentration im Blut, der bei einer Aktivierung von Gehirnarealen während der Sprachproduktion oder -rezeption stattfindet, sichtbar gemacht (vgl. beispielsweise Nagels et al. 2012). Die neuronale Aktivität kann durch die hohe räumliche Auflösung präziser als beim EEG dargestellt werden (vgl. Döring/Bortz 2016:499). Diese Methode ist ungefährlich, allerdings für die Proband(inn)en eher unnatürlich, 
da sie für die Dauer des Experiments möglichst regungslos liegend in einer engen Röhre verharren müssen. Zudem erzeugt das fMRT laute Klopfgeräusche, die als unangenehm empfunden werden können.

Die Positronen-Emissions-Tomographie (PET) zeigt mithilfe radioaktiver Marker im Blut Stellen erhöhter Gehirnaktivität während der Sprachverarbeitung an (siehe z. B. Young Kim et al. 2017). Bei dieser Methode werden dem Probanden/der Probandin schwach radioaktive Substanzen in ungefährlicher Dosierung in die Blutbahn gespritzt, deren Verteilung mit einem PET-Scanner aufgezeichnet und über einen Computertomographen ausgegeben wird (Acatech 2017: 23). Der Proband/die Probandin muss auch hier in unnatürlicher Haltung möglichst bewegungslos verharren.

Für alle bildgebenden Verfahren gilt, dass sie sehr untersuchungsaufwändig und kostenintensiv sind und sowohl für die Erhebung der Daten und Bedienung der Apparaturen als auch für die Auswertung der Ergebnisse eine hohe Expertise voraussetzen. Bildgebende Verfahren bedeuten einen massiven Eingriff in die Natürlichkeit der Umgebung der Proband(inn)en, daher ist ihre ökologische Validität (d.h. der Bezug zu authentischen Sprachverarbeitungssituationen) meist sehr gering. Hinzu kommt, dass sie für bestimmte Adressatengruppen der Barrierefreien Kommunikation nicht zumutbar sind.

Als Vorteil kann gesehen werden, dass man sehr detaillierte und vor allem zeitlich sehr genaue Messdaten bekommt. Da diese Daten nur quantitativ auswertbar sind, ist eine isolierte Betrachtung für die Frage nach Verständlichkeit nicht ausreichend aussagekräftig. In der Forschungspraxis findet man sie daher oft in Kombination mit anderen Methoden.

\section{$4 \quad$ Wechselwirkung zwischen Produktionskomplexität und Verarbeitungsaufwand}

Da jede Methode bestimmte Vor- und Nachteile hat, hat sich daher in der empirischen Forschung der Mixed-Methods-Ansatz bzw. die Datentriangulation (vgl. Döring/Bortz 2016, Alves 2003) etabliert. Daraus resultieren sogenannte Lesekorpora, die korpuslinguistische Annotationen mit Eyetracking-Daten zu den jeweiligen Texten integrieren. Für das Englische sind das UCL Corpus 
(Frank et al. 2013) und das Dundee Corpus (Kennedy 2003) zu nennen. Bedeutend für das Deutsche sind das Potsdam Sentence Corpus (Kliegl et al. 2006) sowie das Freiburg Legalese Reading Corpus (Wolfer 2017). Diese beiden deutschen Lesekorpora bestehen allerdings aus zusammenhangslosen Einzelsätzen. Dies ist zwar typisch für kognitionswissenschaftliche Experimente, durch den Mangel an Kontext können diese Daten aber nur wenig authentisch im Hinblick auf Verarbeitungskosten untersucht werden. Aus diesem Grund sollen im Folgenden exemplarisch zwei Projekte vorgestellt werden, in denen jeweils ein Lesekorpus mit authentischen Texten zur Erforschung des Zusammenspiels zwischen Texteigenschaften und deren Auswirkungen auf die Verständlichkeit erstellt wurde. Auf diese Weise kann die Wechselwirkung zwischen Produktionskomplexität und Verarbeitungsaufwand empirisch messbar gemacht werden. In dem einen Projekt geht es dezidiert um barrierefreie Sprache, in dem anderen wird mit populärwissenschaftlichen Texten ein breiterer Adressatenkreis angesprochen.

Im Projekt Understanding Science (Wolfer et al. 2015, Müller-Feldmeth et al. 2015) geht es um das komplexe Zusammenspiel von Texteigenschaften und dem Verstehensprozess im populärwissenschaftlichen Diskurs. Zur Untersuchung der Verständlichkeit werden Textanalysen mit Experimenten, die die Verarbeitung der Texte erfassen, kombiniert. Grundlage dieser Analysen ist ein Lesekorpus mit deutschen populärwissenschaftlichen Artikeln aus verschiedenen Wissenschaftsbereichen (ca. 500.000 Wörter), die über neue Entwicklungen berichten oder wissenschaftliche Themen erklären. Um textsortenspezifische Eigenschaften populärwissenschaftlicher Texte näher zu beleuchten, wurde das Korpus auf mehreren sprachlichen Ebenen annotiert (z. B. in Bezug auf die Fachwortdichte oder die Phrasen- und Satzkomplexität). Weiterhin wurde das Korpus um Informationen zu Leseverhalten und Textverstehen ergänzt, die über Experimente eruiert wurden: Eyetracking-Daten, Ergebnisse aus Vor- und Nachwissenstests von Laien und Verständlichkeitsratings von Experten und Laien. Dieser Methodenmix erlaubt die Quantifizierung typischer Textsortenmerkmale sowie Aussagen darüber, ob diese Merkmale das Leseverhalten und somit auch die Verständlichkeit der Texte positiv oder negativ für verschiedene Adressatengruppen beeinflussen. 
Die Ergebnisse zeigen (Wolfer et al. 2015, Müller-Feldmeth et al. 2015), dass Texte, die laut Fragebogen als verständlicher eingeschätzt werden, im Allgemeinen auch schneller gelesen werden als Texte, die als unverständlicher bewertet werden. Weiterhin führt eine höhere Fachwortdichte zu langsameren Lesezeiten. Dieser Effekt ist jedoch in Texten schwächer, die als verständlicher eingeschätzt wurden. Auch konnten die Proband(inn)en der Lesestudie dann besser von ihrem Vorwissen profitieren, wenn sie einen Text lasen, der laut Fragebogenstudie verständlicher war. Weiterhin lassen die Ergebnisse vermuten, dass Texte mit versteckter Komplexität, die ein hohes Maß an Sprachökonomie aufweisen und Inferenzleistung benötigen, für Fachexperten gut lesbar sind, sich jedoch für Laien als schlecht lesbar und unverständlich erwiesen. Diese Analysen zeigen einerseits, dass die subjektive Verständlichkeit eines populärwissenschaftlichen Textes Auswirkungen auf viele Ebenen des Verstehensprozesses zu haben scheint. Andererseits zeigen sie auch, wie komplex das Zusammenspiel von Texteigenschaften, dem individuellen Wissen und dem tatsächlichen Leseverhalten ist.

Diese Komplexität zeigt sich auch in empirischen Studien im Kontext des oben erwähnten Freiburg Legalese Reading Corpus zur Verständlichkeit in Rechtstexten. Die Triangulation von Eyetracking-Ergebnissen mit syntaktischer Annotation ergab, dass sich in manipulierten Sätzen mit drei syntaktischen Komplexitätsgraden die mittlere Komplexität als die am leichtesten lesbare erwiesen hat (Wolfer et al. 2015). Dies widerlegt, dass die angenommene Komplexitätsreduktion in der leichtesten Variante tatsächlich auch die geringsten Verarbeitungskosten erfordert.

Im Kontext der Barrierefreien Kommunikation wurde ein ähnlicher Methodenmix in Bezug auf die Rezeption von Einfacher und Leichter Sprache im Projekt LES is more (Hansen-Schirra/Gutermuth im Druck) mit der relevanten Rezipientengruppe getestet. Ein kleines Korpus, bestehend aus Webseiten des Ministeriums für Soziales, Arbeit, Gesundheit und Demografie in RheinlandPfalz, wurde in Einfache und Leichte Sprache umformuliert und korpuslinguistisch im Hinblick auf morphologische, terminologische, phrasale und syntaktische Komplexität analysiert. Zusätzlich wurden mittels Eyetracking Lesedaten von verschiedenen Adressatengruppen (Menschen mit kognitiven Einschränkungen, Menschen mit Migrationshintergrund, Senior(inn)en, Studierende als 
Kontrollgruppe) erhoben sowie Verständlichkeitstests durchgeführt und Erinnerungsprotokolle abgefragt.

Auf der Basis der Korpusannotation wurden typische Eigenschaften der verschiedenen Textvarianten quantifiziert und mit den gängigen Regelwerken zur Leichten Sprache (z. B. Maaß 2015) abgeglichen und empirisch validiert. Je leichter die Textvariante, desto weniger komplex waren die Textvarianten im Hinblick auf die verschiedenen sprachlichen Ebenen. Erste Pilotauswertungen in Bezug auf die Lesedaten der primären Zielgruppe (Menschen mit kognitiven Einschränkungen) zeigen, dass die Texte in Leichter Sprache die in Bezug auf die Verständlichkeit angenommenen Erwartungen mit der bei weitem kürzesten gemittelten Lesezeit im Vergleich zu der Einfachen Sprache und der OriginalWebseite erfüllen. Trianguliert man diese Lesezeiten mit den erhobenen Korpusergebnissen sowie den Ergebnissen der Verständlichkeitstests und der Erinnerungsprotokolle, so muss man davon ausgehen, dass dies an der Komplexitätsreduktion auf der phrasalen und syntaktischen Ebene liegt (vgl. HansenSchirra/Gutermuth im Druck). Wenn die Komplexitätsreduktion auf diesen Ebenen gewährleistet ist, scheint die Komplexität auf der morphologischen Ebene weniger problematisch zu sein und - in Abhängigkeit von der Adressatengruppe - keine bzw. nur kleine Effekte auf die Lesezeiten und die Verständlichkeit zu haben (vgl. Gutermuth im Druck).

Zusammenfassend lässt sich sagen, dass auf der einen Seite unter Zuhilfenahme computer- und korpuslinguistischer Verfahren die Eigenschaften Barrierefreier Kommunikation messbar gemacht werden können und sich auf dieser Basis Aussagen über die Textkomplexität ableiten lassen. Auf der anderen Seite können durch Methoden aus der Kognitionswissenschaft und Verständlichkeitsforschung die Wirkung dieser Eigenschaften und deren Komplexität auf die Verstehensprozesse und die Verständlichkeit der Texte nachvollzogen werden. Die beiden vorgestellten Projekte, in denen Lesekorpora aufgebaut und untersucht wurden, zeigen exemplarisch, wie diese Wechselwirkung empirisch aufgearbeitet werden kann, welche Vorhersagen auf dieser Basis gemacht werden können und wo Optimierungspotenzial im Hinblick auf die Verständlichkeit der Barrierefreien Kommunikation liegt. 


\section{Literaturverzeichnis}

ACATECH (Hg.) (2017): Individualisierte Medizin durch Medizintechnik. München: Herbert Utz Verlag GmbH.

Alves, FabIo (Hg.) (2003): Triangulating translation. Perspectives in process oriented research. Amsterdam/Philadelphia: John Benjamins (Benjamins Translation Library 45).

BEATTY, JACKSON (1982): „Task-evoked pupillary responses, processing load, and the structure of processing resources". In: Psychological Bulletin 91 (2), 276-292.

Bredel, Ursula/MaAß, Christiane (2016): Leichte Sprache. Theoretische Grundlagen. Orientierung für die Praxis. Berlin: Dudenverlag.

Bredel, Ursula/Lang, Katrin/MaAß, Christiane (2016): „Zur empirischen Überprüfbarkeit von Leichte-Sprache-Regeln am Beispiel der Negation“. In: MÄLZER, NAtHalie (Hg.): Barrierefreie Kommunikation - Perspektiven aus Theorie und Praxis. Berlin: Frank \& Timme, 95-116.

Carl, Michael/Jakobsen, Arnt Lykke/Hvelplund Jensen, Kristian Tangsgaard (2008): „Modelling human translator behaviour with user-activity data“. In: Proceedings of the 12th EAMT Conference. Hamburg: Hamburg University, 21-26.

Carl, Michael/Dragsted, Barbara (2012): „Inside the monitor model: Processes of default and challenged translation production". In: Translation: Corpora, Computation, Cognition 2 (1), 127-145.

Christmann, Ursula/Groeben, Norbert (1999): „Psychologie des Lesens“. In: Franzmann, Bodo/Hasemann, Klaus/Löffler, Dietrich/SchÖn, Erich (Hg.): Handbuch Lesen. München: Saur, 145-223.

Christoffels, Ingrid K./Firk, Christine/Schiller, Niels Olaf (2007): „Bilingual language control: An event-related brain potential study“. In: Brain and Research, $1147,192-208$.

DeRMARMELS, SASCHA (2010): „Was misst man eigentlich, wenn man Verständlichkeit misst? Der Zusammenhang von Verständlichkeit mit den Faktoren Hintergrundwissen und Motivation". In: Galliker, Esther/KleinerT, ANdrea (Hg.): Messen in der Linguistik: Beiträge zu den 5. Tagen der Schweizer Linguistik (Sprachenlernen Konkret! Angewandte Linguistik und Sprachvermittlung). Schneider Verlag Hohengehren, 105-121.

DÖRING, NICOLA/BORTZ, JÜRGEN (2016): Forschungsmethoden und Evaluation in den Sozial- und Humanwissenschaften (5. Auflage). Berlin, Heidelberg: Springer.

Duchowski, Andrew Ted (2003): Eye Tracking Methodology: Theory and Practice. Springer.

Ericsson, Karl Anders/Simon, Herbert A. (1993 [1984]): Protocol Analysis: Verbal Reports as Data. Rev. Ed. Cambridge (Mass.), London: MIT Press. 
FEDERMEIER, KARA D. (2007): „Thinking ahead: The role and roots of prediction in language comprehension“. In: Psychophysiology 44, 491-505.

Frank, Stefan L./Monsalve, Irene F./Thompson, Robin L./Vigliocco, Gabriella (2013): „Reading time data for evaluating broad-coverage models of English sentence processing“. In: Behavior Research Methods 45 (4), 1182-1190.

GÖPFERICH, SUSANNE (2002): Textproduktion im Zeitalter der Globalisierung. Entwicklung einer Didaktik des Wissenstransfers. Tübingen: Stauffenburg.

GöPfERICH, Susanne (2008): Translationsprozessforschung: Stand, Methoden, Perspektiven. Tübingen: Gunter Narr.

Groeben, Norbert (1982): Leserpsychologie: Textverständnis - Textverständlichkeit. Münster: Aschendorff Textverständlichkeit auf 148-277.

Gutermuth, Silke (im Druck): One for all? Eine zielgruppenorientierte Rezeptionsstudie zu Leichter und Einfacher Sprache. Dissertation. Germersheim: Johannes GutenbergUniversität Mainz.

HANSEN-Schirra, Silvia/Gutermuth, Silke (2015): „Approaching comprehensibility in translation studies“. In: MAKSYMSKI, KARIN/GUTERMUTH, SilKe/HANSENSCHIRra, Silvia (Hg.): Translation and Comprehensibility. Band 72. Berlin: Frank \& Timme, 227-262.

Hansen-Schirra, Silvia/Gutermuth, Silke (im Druck): „Modellierung und Messung Einfacher und Leichter Sprache“. In: Working Papers in Applied Linguistics 13.

Hansen-Schirra, Silvia/Hofmann, Sascha/Nitzke, Jean (2017): „Acquisition of Generic Competencies Through Project Simulation in Translation Studies“. In: ZLATKINTroitschanskaia, Olga/Wittum, Gabriel/Dengel, Andreas (Hg.): Positive Learning in the Age of Information: A Blessing or a Curse? Berlin: Springer, 267-280.

HolmQvist, KenNeth/Nyström, Marcus/Andersson, Richard/DEWHuRST, Richard/ JarotzKa, HalszKa/VAn de Weijer, Joost (2011): Eye Tracking: A Comprehensive Guide to Methods and Measures. Oxford University Press.

JuOla, PATRICK (2008): „Assessing linguistic complexity“. In: Miestamo, MatTi/SinneMÄKI, KaIUS/KARlsSON, Fred (Hg.): Language Complexity: Typology, Contact, Change, Amsterdam/Philadelphia: Benjamins, 89-108.

Just, Marcel Adam/CARPenter, Patricia A. (1980): „A Theory of Reading: From Eye Fixations to Comprehension“. In: Psychological Review 87, 329-354.

KATKIn, EdWARD S./DERmit, SusAn/Wine, SusAn K. F. (1993): „Psychophysiological assessment of stress“. In: Goldberger, Leo/Breznitz, SHlomo (Hg.): Handbook of stress: Theoretical and clinical aspects. New York, NY, US: Free Press, 142-157.

Kennedy, Alan (2003): The Dundee Corpus. [CD-ROM]. School of Psychology, The University of Dundee.

Kercher, Jan (2013): Verstehen und Verständlichkeit von Politikersprache. Wiesbaden: Springer VS. 
Kliegl, Reinhold/Nuthmann, Antje/Engbert, Ralf (2006): „Tracking the mind during reading: The influence of past, present, and future words on fixation durations“. In: Journal of Experimental Psychology, General 135 (1), 12-35.

KONIECZNY, LARS (2000): „Locality and parsing complexity“. In: Journal of Psycholinguistic Research 29, 627-645.

KRINGs, HANS Peter (2005): „Wege ins Labyrinth - Fragestellungen und Methoden der Übersetzungsprozessforschung im Überblick“. In: Meta - Journal des Traducteurs (Montréal) 50.2, 342-358.

KRUGER, HaideE (2016): „What's happening when nothing's happening? Combining eyetracking and keylogging to explore cognitive processing during pauses in translation production“. In: Across Languages and Cultures 17, 25-52.

Kutas, Marta/Hillyard, Steven A. (1980): „Reading senseless sentences: brain potentials reflect semantic incongruity“. In: Science 207, 203-205.

Langer, Inghard/Schulz von Thun, Friedemann/Tausch, Reinhard (1993): Sich verständlich ausdrücken. 5., verb. Aufl. München, Basel: Ernst Reinhardt.

LANGner, Tobias/Schmidt, Jennifer/Fischer, Alexander (2015): „Is It Really Love? A Comparative Investigation of the Emotional Nature of Brand and Interpersonal Love“. In: Psychology and Marketing 32, 624-634.

LEMnitzer, LOTHAR/Zinsmeister, HeIKe (2006): Korpuslinguistik. Eine Einführung. Tübingen: Narr.

LERCH, KENT D. (2008): „Ultra posse nemo obligatur. Von der Verständlichkeit und ihren Grenzen“. In: EICHHOFF-CYRUS, KARIN M./AnTOS, GERD (Hg.): Verständlichkeit als Bürgerrecht? Die Rechts- und Verwaltungssprache in der öffentlichen Diskussion. Mannheim: Dudenverlag, 54-80.

LÜdeling, Anke/Kytö, MerJa (Hg.) (2009): Corpus Linguistics. Berlin, New York: De Gruyter.

MaAß, Christiane (2015): Leichte Sprache. Das Regelbuch. Münster: Lit-Verlag.

Müller-Feldmeth, Daniel/Held, Uli/Auer, Peter/Hansen-Morath, Sandra/ Hansen-Schirra, Silvia/Maksymski, Karin/Wolfer, Sascha/Konieczny, Lars (2015): „Investigating comprehensibility of German popular science writing“. In: Maksymski, Karin/Gutermuth, Silke/Hansen-Schirra, Silvia (Hg.): Translation and Comprehensibility. Band 72. Berlin: Frank \& Timme, 227-262.

Nagels, Arne/Kircher, Tilo/Dietsche, Bruno/Backes, Heidelore/Marquetand, JUSTUS/KRUG, AXEL (2012): „Neural processing of overt word generation in healthy individuals: the effect of age and word knowledge". In: Neuroimage 61, 4, 832-840.

Norman, Donald A./Rumelhart, David E. (1975): Explorations in Cognition. San Francisco: Freeman.

Rickheit, Gert/Strohner, HAns (1993): Grundlagen der kognitiven Sprachverarbeitung: Modelle, Methoden, Ergebnisse. Tübingen: UTB für Wissenschaft.

SEeber, Kilian (2013): „Cognitive load in simultaneous interpreting: Measures and methods“. In: Target 25, 1, 18-32. 
TUINMAN, JAAP (1971): „The removal of information procedure“. In: Journal of Reading Behaviour 3, 2, 44-50.

Wolfer, Sascha/Hansen-Morath, SAndra/Konieczny, Lars (2015): „Are shorter sentences always simpler? Discourse level processing consequences of reformulating jurisdictional texts“. In: MAKSYMSKI, KARIN/Gutermuth, Silke/HANSEN-Schirra, Silvia (Hg.): Translation and Comprehensibility. Band 72. Berlin: Frank \& Timme, 263-287.

WOLFER, SASCHA (2015): „Comprehension and comprehensibility“. In: MAKSYMSKI, KARIN/ Gutermuth, Silke/HANSEN-SCHIRRA, Silvia (Hg.): Translation and Comprehensibility. Band 72. Berlin: Frank \& Timme, 33-52.

WOLFER, SASCHA (2017): Verstehen und Verständlichkeit juristisch-fachsprachlicher Texte (Korpuslinguistik und interdisziplinäre Perspektiven auf Sprache, CLIP 7). Tübingen: Narr.

Wolfer, Sascha/Held, Uli/Hansen-Schirra, Silvia (2015): „Verstehen und Verständlichkeit von populärwissenschaftlichen Texten“. In: Information. Wissenschaft \& Praxis 66 (De Gruyter), 1-9.

Young Kim, NA/An, Young-Sil/Shin, Ji-Cheol/Wook KIm, Yong (2017): „Recovery of language function and brain metabolism in a patient with crossed aphasia: 2-year follow-up of brain FDG PET images“. In: Aphasiology, 1-9.

Zlatkin-Troitschanskaia, Olga/Molerov, DimităR/ToepPer, Miriam/Brückner, Sebastian/Hansen-Schirra, Silvia/Hofmann, Sascha (2017): „Adapting and Validating the Collegiate Learning Assessment to Measure Generic Academic Skills of Students in Germany - Implications for International Assessment Studies in Higher Education“. In: Zlatkin-Troitschanskaia, Olga/Pant, Hans Anand/ LaUtenbach, Corinna/Molerov, Dimităr/Toepper, Miriam/BrÜCKNER, SEbastian (Hg.): Modeling and Measuring Competencies in Higher Education - Approaches to Challenges in Higher Education Policy and Practice. Wiesbaden: Springer, 245-266. 
JULIA DOBROSCHKE/THOMAS KAHLISCH

\section{Barrierefreie Informations- und Kommunikationsangebote für blinde und sehbehinderte Menschen}

\section{Gesetzliche Grundlagen}

Der Begriff Barrierefreiheit ist im Behindertengleichstellungsgesetz des Bundes seit 2002 sowie in den entsprechenden Gesetzen der Bundesländer definiert. Für blinde und sehbehinderte Menschen stehen, neben den Anforderungen an bauliche und räumliche Gestaltung der Umwelt, die Themenfelder des barrierefreien Zugangs zu Informations- und Kommunikationsangeboten im Vordergrund. Besonders technische Systeme der Informationsverarbeitung und deren Benutzungsoberflächen sind so zu entwickeln, dass sie „grundsätzlich ohne fremde Hilfe" von Menschen mit Seheinschränkungen genutzt werden können (Behindertengleichstellungsgesetz 2002).

Die Behindertengleichstellungsgesetze verpflichten Behörden und Einrichtungen des Bundes und der Länder dazu, ihre Informations- und Kommunikationsangebote barrierefrei zu gestalten. In der Barrierefreie-Informationstechnik-Verordnung (BITV) sind die Kriterien für eine barrierefreie Gestaltung von Informationssystemen definiert. Die BITV 2.0 basiert auf den international gültigen Web Content Accessibility Guidelines (WCAG), die vom World Wide Web Consortium (W3C) erarbeitet wurden (Bundesministerium der Justiz und für Verbraucherschutz 2011; Web Content Accessibility Guidelines 2009). Seit 2016 existiert außerdem die Richtlinie 2016/2102 der Europäischen Union über den barrierefreien Zugang zu Websites und mobilen Anwendungen öffentlicher Stellen (Richtlinie EU 2016), welche bis September 2018 in der Bundesrepublik umzusetzen ist (Barrierefreies Webdesign o.J.).

Neben den allgemeinen gesetzlichen Grundlagen sind auch urheberrechtliche Regelungen für den barrierefreien Zugang zu Literatur zu nennen. So gibt 
es im deutschen Urheberrecht seit 2004 eine Schrankenregelung, die es ermöglicht, Werke, die nicht barrierefrei nutzbar sind, für Menschen mit Behinderung zugänglich aufzubereiten (Gesetz über Urheberrecht und verwandte Schutzrechte 2003). Mit dem im Jahr 2013 verabschiedeten sog. Marrakesch-Vertrag im Rahmen der Weltorganisation für intellektuelles Eigentum (WIPO) kommt eine entscheidende internationale Urheberregelung hinzu. Sie erleichtert Menschen mit Lese- und Sehbehinderung den Zugang zu veröffentlichten Werken, indem sie entsprechende gesetzliche Regelungen vorgibt (Institut für Menschenrechte 2018). Entscheidende Neuerungen, die bis Oktober 2018 von der Bundesrepublik umgesetzt werden müssen, sind (Richtlinie EU 2017; Verordnung EU 2017):

- die Erweiterung der betreffenden Personengruppe auf Menschen mit Behinderung, die gedruckte Werke nicht eigenständig lesen können,

- das Recht, Werke für den betreffenden Personenkreis öffentlich zugänglich zu machen sowie

- das Recht auf grenzübergreifenden Austausch der barrierefrei gestalteten Werke (ebd.).

\section{Kommunikation blinder und sehbehinderter Menschen}

In den nun folgenden Abschnitten werden sowohl die Besonderheiten des Schriftsystems für blinde Menschen als auch spezielle Anforderungen an die Gestaltung sehbehindertengerechter Texte vorgestellt.

\subsection{Wie blinde Menschen Braille lesen und schreiben}

(Geburts-)blinde Menschen nehmen ihre Umwelt zu einem großen Teil akustisch wahr, können aber auch durch haptische Informationen den fehlenden Sehsinn kompensieren. Für das Lesen und Schreiben wird die von Louis Braille 
entwickelte Punktschrift genutzt, die aus einem System von sechs Punkten gebildet wird. Daraus ergeben sich zunächst 64 Kombinationsmöglichkeiten. Es werden drei Blindenschriftnotationen unterschieden (Abbildung 1). In der sog. Basisschrift wird jeder Buchstabe aus einer bestimmten Kombination erhabener Punkte dargestellt. Die Systematik sieht verschiedene Buchstabengruppen vor, die aufeinander aufbauen und so das Erlernen der Punktschrift erleichtern. Zur 1. Gruppe gehören die Buchstaben a bis j. Diese sind aus relativ einfachen Punktkombinationen aufgebaut. In der 2. Gruppe mit den Buchstaben k bis $\mathrm{t}$ wird den Zeichen der Gruppe 1 der Punkt 3 hinzugefügt, in der 3. Gruppe (Buchstaben u bis z) zusätzlich der Punkt 6. Die Vollschrift (Gruppen 3 und 4) enthält bereits Braillezeichen für Buchstabenkombinationen, wodurch sich der Umfang der Brailleschrift reduzieren lässt (z. B. ie, sch). Die ökonomischste Form der Punktschrift basiert jedoch auf der Kurzschriftnotation, bei welcher häufige Silben oder kurze Wörter oft mit nur einem Braillezeichen dargestellt werden (Mellor 2009: 98f.).

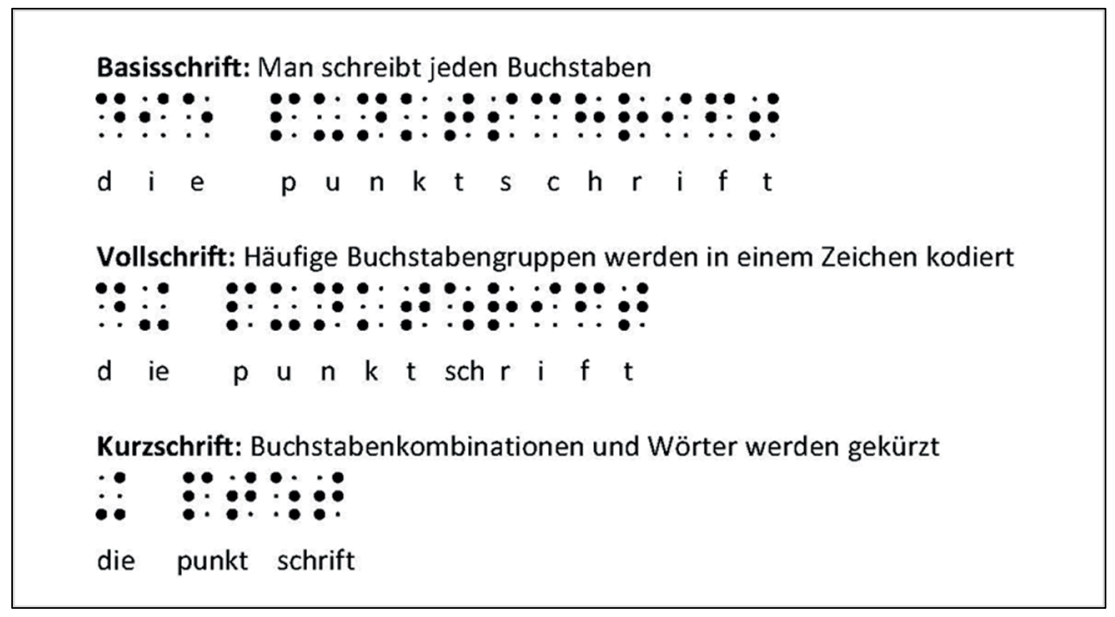

Abb. 1: Kürzungspotenzial der verschiedenen Braillenotationen, Quelle: Kahlisch et al. (2011:4)

Wenn Brailletexte gedruckt werden, ist auch bei der Darstellung von Inhalten z. B. in Kurzschrift zu berücksichtigen, dass der Umfang von Büchern wesentlich 
größer ist als bei Schwarzschriftbüchern. Die Braillezeichen besitzen fest definierte Größen für Punktdurchmesser, Punktabstände innerhalb der Braillezelle sowie eine taktile Mindesthöhe. Die Vollform der Braillezelle ist so gestaltet, dass sie unter eine Fingerkuppe passt. Alle Parameter zur Größe und zum Einsatz sind in der DIN 32976:2007-08 festgelegt und für Buchinhalte, aber auch Informationstexte auf Orientierungsplänen einzuhalten (Beuth Verlag $\mathrm{GmbH}$ 2014, Beuth Verlag GmbH 2015). Da der Zeichenvorrat von 64 Punktkombinationen begrenzt ist, werden Braillezeichen häufig kontextabhängig eingesetzt. Auch für die Darstellung von mathematischen Gleichungen oder Musiknoten sind deshalb Spezialnotationen definiert (Staupendahl 1998: 68ff.).

Blinde Menschen lesen, indem sie durch die Rezeptoren in den Fingerspitzen die Punktkombinationen der erhabenen Punkte wahrnehmen und erfassen. Häufig ist der Zeigefinger einer Hand der Hauptlesefinger, die anderen Finger dienen zur Orientierung auf der Seite, um beispielsweise das Zeilen- oder Absatzende zu erkunden. Einen Gesamteindruck einer Seite, wie es sehenden Lesern möglich ist, kann sich ein blinder Leser nicht verschaffen, da anders als bei der visuellen Informationsaufnahme die Wahrnehmung von Inhalten nur zeilenweise und nacheinander möglich ist.

Um sich selbst Notizen zu machen, kann z. B. eine Brailleschreibmaschine genutzt werden. Mit den sechs zur Verfügung stehenden Tasten kann die jeweilige Punktkombination durch gleichzeitiges Betätigen der entsprechenden Tasten für den gewünschten Buchstaben gedrückt werden. Durch die Leertaste, die sich in der Mitte der Tastatur befindet, wird ein Leerzeichen erzeugt. Braille lässt sich auch über die umgestellte PC-Tastatur schreiben. Digitale Texte werden aber üblicherweise im 10-Fingersystem geschrieben. Tafel und Stichel sind ebenfalls Schreibmedien, die aber zu den analogen Hilfsmitteln zählen und eine seitenverkehrte Schreibweise erfordern (Mönnig 2016: 60f.; Lang 2003: 154ff.).

Die Aufbereitung von Informationen für blinde Menschen sollte sich so nah wie möglich am Original orientieren. Durch die notwendige Linearisierung der Informationen für blinde Leser ist das jedoch bei komplexeren Gestaltungen kaum vollständig durchführbar. Orientierung, Wiederauffindbarkeit und Zitierfähigkeit spielen deshalb eine besondere Rolle beim Lesen. Dazu dienen vor allem Seitenzahlen des Originaltitels und der Blindenschriftseite, Fußnoten sowie etwaige Kolumnentitel, die in einer Fußzeile untergebracht werden. 


\subsection{Anforderungen an Publikationen für sehbehinderte Menschen}

Menschen mit einer starken Sehbeeinträchtigung verwenden im Allgemeinen die Schwarzschrift zum Lesen und Schreiben. Im Unterschied zu gut sehenden Menschen sind die Prozesse des Lesens und Schreibens mit enormem zeitlichem und physischem Aufwand verbunden und erfordern aufgrund der stark visuellen und damit verbundenen motorischen Einschränkung die volle Konzentration des Lesers/der Leserin. Häufig werden assistive Technologien wie optische Vergrößerungsgeräte (z. B. Kameras, Lupen) eingesetzt, um die Seheinschränkung zu kompensieren (Hofer 2008: 52).

Um sehbehindertengerechte gedruckte oder digitale Inhalte zu entwickeln, sollten die Grundprinzipien typografisch guter Gestaltungen eingehalten werden. Jene Anforderungen an Lesbarkeit und Leserlichkeit eines Textes finden sich etwa in der DIN-Norm 1450:2013-04 (Deutsches Institut für Normung e. V. 2013: 4; DBSV 2018). Dennoch besteht die Schwierigkeit, dass Sehbehinderungen unterschiedliche Wahrnehmungseinschränkungen mit sich bringen, sodass spezifische Anpassungen erforderlich werden können (Zeun 1998; Hilderley 2013: 23f.). Dazu gehört u. a., dass Originaltexte eine ergänzende inhaltliche Strukturierung erhalten, indem (zusätzliche) Titeltexte eingefügt werden, die in der Kopf- oder Fußzeile bzw. in Marginalspalten präsentiert werden können. Wichtige Begriffe können abweichend vom Original vergrößert oder in einem fetten Schriftschnitt gesetzt werden. Ebenso ist es möglich, Kapitelanfänge und -enden mit Sonderzeichen auszustatten, um so die bessere Orientierung auf der Seite zu unterstützen. Einen Überblick über ergänzende Strukturelemente sind in Büchern beispielsweise im Buchvorspann kenntlich zu machen (Zeun 1998).

Grundsätzlich sollte auf eine einheitliche und einfach erkennbare Struktur und Gestaltung geachtet werden. Abbildungen sollten ausreichende Größen und Kontraste aufweisen (ebd.; National Council for the Blind of Ireland 2009: 6). Verstärkung der Konturen sowie Reduktion des Bildinhaltes auf wesentliche Inhalte unterstützen zusätzlich. Ist dies nicht möglich oder führt die Reduktion nicht zum gewünschten Ergebnis, kann eine Aufbereitung als taktile Grafik Abhilfe schaffen, Bildinhalte zugänglich zu machen. Unterstreichungen, Umrahmungen oder eine gut erkennbare Kontrastfarbe für Schlüsselwörter sowie eine präsente Paginierung am äußeren Blattrand helfen bei der Orientierung. Werden Tabellen oder Verzeichnisse für die Darstellung von Texten genutzt, können 
beispielsweise Hilfslinien zu den durchaus größer und ggf. auch fetter ausgezeichneten Textelementen führen (ebd). Bei der Ausstattung gedruckter Medien sollte auf matte und blendungsfreie Papiere zurückgegriffen werden. Ein gutes Aufschlagverhalten von Büchern für die Verwendung z. B. auf einem sog. Bildschirmlesegerät gilt als zusätzlicher Komfort für das Lesen (Zeun 1998; National Council for the Blind of Ireland 2009: 7).

Kriterienlisten, die für seh- bzw. lesebehinderte Menschen erstellt wurden, enthalten grundsätzlich Regeln für gute Text- und Layoutgestaltung, um die Orientierung auf den Seiten zu unterstützen. Berücksichtigung finden hier vor allem die Richtlinien von Zeun (1998), Hilderley (2013), des Deutschen Instituts für Normung e. V. (2013) sowie des National Council for the Blind of Ireland (2009). So sollte ein übersichtliches Layout, das klar, einfach und konsistent aufgebaut ist, mit linksbündigem Flattersatz entwickelt werden. Durch die unregelmäßigen Zeilenlängen beim Flattersatz wird das Auffinden der nächsten Zeile erleichtert. Die Zeilen sollten nicht mehr als ca. 40 Zeichen aufweisen (Hilderley 2013: 23).

Neben dem Begriff Großdruck, der vor allem im kommerziellen Bereich Eingang gefunden hat und Schriftgrößen zwischen 12 und 14 pt definiert, hat sich beim Textsatz für stark sehbeeinträchtige Menschen der Begriff „Maxidruck“ etabliert. Schriftgrößen für eine unterstützende Textgestaltung werden dort ab 20 pt empfohlen (DZB 2017). Zeun (1998) empfiehlt für den Fließtext zusätzlich, serifenlose Schriften zu verwenden. Untersuchungen zeigten jedoch, dass dies für sehbehinderte Leser nicht unbedingt Einfluss auf die Lesbarkeit hat (Beck 2014: 269ff.). Bei der Auswahl der Schrift sollten Faktoren wie Erkennbarkeit, Unterscheidbarkeit, Offenheit sowie der Strichstärkenkontrast im Zusammenhang mit dem geplanten Medium berücksichtigt werden (DBSV 2018a). Es gibt speziell entwickelte Schriften wie „Tiresias“ (RNIB 2013) oder „Frutiger 1450“. Letztere ist nach der DIN-Norm 1450 zur Leserlichkeit von Schrift benannt (Beuth Verlag GmbH 2013) und wird für gut lesbare Texte empfohlen (DBSV 2018b; DZB 2017). Beide Schriften verfügen über mehrere Schriftschnitte, sind klar, gut ausgebaut und verzichten auf Ligaturen, um die Erkennbarkeit einzelner Buchstaben zu gewährleisten (Dyslexia Re- 
search Center AG 2012: 7; Hilderley 2013: 23). Weitere Informationen zur Behandlung von Sonderzeichen wie Fußnoten oder mathematischen Zeichen finden sich ebenfalls in den genannten Leitfäden (Zeun 1998; DBSV 2018a).

Sehbehindertengerechte Gestaltung heißt auch, ausreichend hohe Kontraste im Schriftbild zu verwenden, z. B. eine deutliche Abhebung der Überschriften vom Fließtext sowie ausreichend große Zeilen- und Absatzabstände einzuplanen, um eine Unterschneidung von Ober- und Unterlängen zu vermeiden (Zeun 1998; National Council for the Blind of Ireland 2009: 4). Auf Abbildungen hinter dem Text sollte wegen des Kontrasts zwischen Text- und Hintergrundfarbe generell verzichtet werden. Ein sparsamer Einsatz von Versalien oder gesperrt gesetzten Begriffen für die Wortgestaltung wird ebenfalls empfohlen (Hilderley 2013: 23). Je nach Sehbeeinträchtigung kann mit inverser Darstellung von Texten (z. B. weiße Schrift auf schwarzem Grund) eine weitere Hervorhebungsvariante von Überschriften oder kurzen Hinweisen erreicht werden (Zeun 1998; National Council for the Blind of Ireland 2009: 7).

\section{$3 \quad$ Medien für blinde und sehbehinderte Menschen}

Sehbeeinträchtigungen können unterschiedlich starke Auswirkungen auf das visuelle Wahrnehmungsvermögen eines Menschen haben. Deshalb wurden spezielle Medien entwickelt, die auf bestimmte Bedürfnisse und Fähigkeiten, z. B. das Lesen der Punktschrift, der Nutzer abgestimmt sind. Daneben haben sich aber auch Angebote etabliert, die nicht nur für eine bestimmte Zielgruppe zugänglich gestaltet, sondern für viele Menschen nutzbar sind. Die folgenden $\mathrm{Ab}$ schnitte geben dazu einen Überblick.

\subsection{Bücher lesen - in Punktschrift, als Hörbuch oder E-Book}

Literatur in Punktschrift wird u. a. von Einrichtungen wie der blista Marburg oder der DZB Leipzig übertragen und zum Verleih angeboten. Die Punktschriftbibliotheken und Hörbüchereien im deutschen Sprachraum sind unter dem Dach der Mediengemeinschaft für blinde und sehbehinderte Menschen (MEDIBUS) zusammengeschlossen. Eine Zusammenstellung aller Mitglieder 
findet man auf der MEDIBUS-Seite (MEDIBUS o.J.) Die neun größten MEDIBUS-Bibliotheken sind:

- Berlin: Berliner Blindenhörbücherei gGmbH

- Bonn: Deutsche Katholische Blindenbücherei gGmbH (DKBB)

- Hamburg: Norddeutsche Blindenhörbücherei e. V. (NBH)

- Leipzig: Deutsche Zentralbücherei für Blinde (DZB)

- Marburg: Deutsche Blinden-Bibliothek (DBB) der Deutschen Blindenstudienanstalt e.V.

- München: Bayerische Blindenhörbücherei e. V. (BBH)

- Münster: Westdeutsche Blindenhörbücherei e. V. (WBH)

- Wien: Hörbücherei des Blinden- und Sehbehindertenverbandes Österreich

- Zürich: Schweizerische Bibliothek für Blinde, Seh- und Lesebehinderte (SBS)

Seit den 1950er Jahren bieten Spezialbibliotheken für blinde und sehbehinderte Menschen Hörbücher und Hörzeitschriften an, welche in den Studios der MEDIBUS-Bibliotheken von Sprecherinnen und Sprechern vollständig aufgelesen werden. Die dann auf Tonträger gespeicherten Titel werden kostenfrei an Nutzer verschickt, die zur Ausleihe berechtigt sind. Anfangs auf Tonbandspulen, später auf Kompaktkassetten aufgenommen, werden die Titel heute im Format Digital Accessible Information System (DAISY) produziert. Das DAISYFormat ist ein internationaler Standard, der das Navigieren in Audioinhalten ermöglicht. Der Hörer ist damit in der Lage, in einem Hörbuch zu blättern, gezielt eine Stelle aufzufinden und die Wiedergabegeschwindigkeit anzupassen. Alle MEDIBUS-Bibliotheken bieten seit einigen Jahren Hörbücher und -zeitschriften im DAISY-Format zum Download an. Über einen WebKatalog, verschiedene Apps für mobile Endgeräte oder spezielle Abspielgeräte wie Plextalk Lineo Pocket oder Victor Reader Stream können Nutzer aus dem Angebot wählen.

Die Funktionen zur Auszeichnung navigierbarer und multimedialer DAISY-Inhalte sowie die barrierefreie Gestaltung digitaler Publikationen fand Eingang in die Entwicklung des E-Book-Formates electronic publication 
(EPUB) (DAISY-Consortium o.J.). So wird das EPUB-Format aufgrund seiner plattformunabhängigen und barrierefreien Einsatzmöglichkeit auf bekannten E-Readern, Lese-Apps, Tablets und dem PC unterstützt (Börsenverein des Deutschen Buchhandels e.V. 2017). EPUB-Bücher können aber auch durch Web-Reader gelesen werden. Ein Beispiel für ein solches Web-Plugin ist „readium“, welches vom International Digital Publishing Forum IDPF und W3C als open source software entwickelt wurde und von verschiedenen internationalen Gremien schrittweise ausgebaut wird (Readium Foundation 2018). Werden die Richtlinien und Standards des barrierefreien Publizierens von Verlagen konsequent eingehalten, können deren Inhalte unmittelbar blinden und sehbehinderten Nutzern angeboten werden.

\subsection{Nutzung digitaler Inhalte durch assistive Technologien und mobile Geräte}

Blinde Menschen können dank assistiver Technologien genauso gut und effizient wie sehende Menschen mit dem Computer arbeiten. Dazu nutzen sie z. B. eine sog. Braillezeile (siehe Abbildung 2), die ergänzend zur Tastatur angeschlossen wird und können digitale Texte in Braillenotation lesen. Genutzt wird dazu Computerbraille, welches, abweichend von der 6-Punkt-Notation, mit 8 Punkten gebildet wird. Die beweglichen Stifte auf der Zeile bilden in erhabener Position die Braillebuchstaben und ermöglichen das taktile Lesen digitaler Inhalte. 


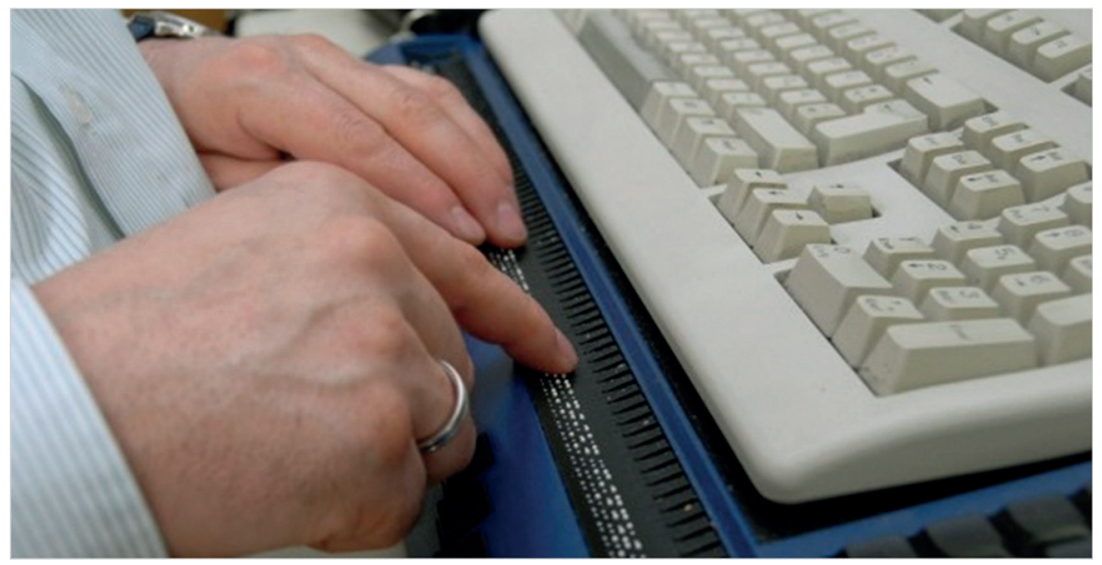

Abb. 2: Abbildung einer Braillezeile in Kombination mit der PC-Tastatur (DZB 2014)

Zur auditiven Unterstützung werden sog. Screenreader eingesetzt, um sich vor allem Texte im Internet, E-Mails oder Dokumente vorlesen zu lassen. Solche assistiven Technologien greifen auf die Struktur der Inhalte zurück, weshalb eine entsprechende Aufbereitung Voraussetzung für den gleichwertigen Zugang blinder und sehbehinderter Menschen ist. Medienneutrale Formate wie HTML oder XML stellen dafür eine technische Infrastruktur zur Verfügung und ermöglichen, dass Überschriften, Alternativtexte für Abbildungen oder interaktive Elemente wie Verweise oder Links als solche erkannt werden.

Stark sehbehinderte Menschen nutzen neben Lupen oder Vergrößerungskameras auch Vergrößerungssoftware auf dem Computer, um die für sie erforderliche Textgröße zu erreichen. In diesem Fall ist es von Vorteil, wenn Texte gut strukturiert und linearisiert erstellt werden. Ein Doppelseitenlayout mit vielzähligen Gestaltungselementen führt häufig zu Problemen bei der Orientierung in einem Dokument, wenn die Struktur stark visuell gestaltet ist.

Auch mobile Endgeräte bieten blinden und sehbehinderten Anwendern ein breites Spektrum an Möglichkeiten, sich eigenständig zu informieren und mit anderen zu kommunizieren. Neben Notebooks mit Braillezeile und Sprachausgabe sowie speziell auf die Bedürfnisse der Anwender ausgerichteten BrailleNotizgeräten nutzen viele blinde und sehbehinderte Menschen seit einigen 
Jahren auf dem Massenmarkt angebotene Tablets und Smartphones. Die verbreitetsten Systeme Android und iOS bieten viele Bedienhilfen, die es seheingeschränkten Anwendern ermöglichen, diese Geräte ohne fremde Hilfe zu verwenden, obwohl sie über einen Touchscreen bedient werden. Mit etwas Übung ist der Anwender in der Lage, diese Geräte mittels Gesten intuitiv zu nutzen. Der Anwender eines Tablets oder Smartphones kann selbst entscheiden, ob er die Unterstützungsmöglichkeiten einer vergrößernden Darstellung, einen Screenreader und/oder eine Braillezeile für die Interaktion gebraucht (DBSV 2018d).

Die von den Herstellern angebotenen Bedienhilfen stellen dabei sicher, dass die Geräte grundsätzlich zu bedienen sind und keine teure Zusatzsoftware gekauft werden muss. Damit auch die zahlreichen Apps von Fremdanbietern für alle nutzbar sind, müssen die Programme bestimmte Anforderungen an die barrierefreie Gestaltung erfüllen, die auf den Internetseiten der Hersteller veröffentlicht werden. In Arbeit befindet sich derzeit die WCAG 2.1, die die Richtlinien um Anforderungen an mobile Endgeräte erweitert (Web Content Accessibility Guidelines 2018).

\subsection{Nutzung taktiler Modelle und Audioguides}

Tastbare Reliefs und beschreibende Audioguides kommen zum Einsatz, um blinden Menschen eine Vorstellung von räumlichen Gegebenheiten zu vermitteln. Sowohl im schulischen als auch im Berufsbildungskontext sind Modelle, tastbare Abbildungen und dazugehörige Erläuterungen wichtig, um Zusammenhänge erkennbar zu machen. Dazu kommen in der Regel Schwellpapierkopien zum Einsatz. Für umfangreichere Pläne oder Atlanten wird das Folientiefziehverfahren eingesetzt, mit dem beispielsweise das Relief eines Gebirgszuges oder die Form eines Gebäudes haptisch erfahrbar wird. Für die Erstellung von Modellen, die z. B. im Unterricht verwendet werden, können auch Technologien wie das 3D-Druckverfahren eingesetzt werden (Isar-Projekt o.J.).

Zunehmend integrieren Museen und andere Kultureinrichtungen tastbare Modelle (ABSV o.J.). Damit sich Besucher einer Ausstellung oder einer Installation eigenständig in den Räumlichkeiten bewegen können, werden ergänzend Audioguides genutzt, welche die zu besichtigenden Exponate und die Wege zu den 
Ausstellungsstücken beschreiben. Bei Kombination eines Audioguides mit einem System zur Innenraumnavigation ist der Besucher in der Lage, sich frei im Ausstellungsbereich zu bewegen und bewusst zu entscheiden, mit welchen Exponaten er sich beschäftigen möchte. Audioguides funktionieren auf speziell dafür entwickelten Geräten oder als App auf dem privaten Smartphone. Mit einer App kann der Besucher im Vorfeld oder im Nachgang des Besuches den Audioguide weiter nutzen und sich zusätzliche Informationen erschließen. Ein Beispiel hierfür ist der barrierefrei gestaltete Audioguide des Bachmuseums in Leipzig (Bachmuseum o.J.).

\subsection{Rezeption von visuellen Inhalten durch Audiodeskription}

Als Audiodeskription (AD) bezeichnet man die ergänzende Beschreibung eines Filmes oder einer Veranstaltung. Seit den 1990er Jahren nutzt der öffentlichrechtliche Rundfunk diese Technik, um Filme mit einer Hörbeschreibung zu versehen. Diese Erläuterungen werden grundsätzlich nur in den Gesprächspausen eines Filmes eingebracht und sollen dem nichtsehenden Zuschauer Handlungen und Zusammenhänge verdeutlichen, die sich nicht aus der vorhandenen Audiospur erschließen. Mit der Verleihung des Deutschen Hörfilmpreises hat der Deutsche Blinden- und Sehbehindertenverband ein öffentlich wahrgenommenes Podium geschaffen, um Audiodeskription bekannter zu machen (Deutscher Hörfilmpreis o.J.; Hörfilminfo o.J.).

Seit einigen Jahren erweitern die Rundfunkanstalten regelmäßig ihr Angebot an Filmen und Dokumentationen (Müller 2013). Auch Kinobetreiber bieten immer mehr Filme mit zusätzlicher akustischer Bildbeschreibung an, die mittels eines Kopfhörers gehört werden kann. Ein gemeinsames Filmerlebnis vermittelt dabei die App „Greta“, die sich mit dem laufenden Kinofilm synchronisiert und die Filmbeschreibungen abspielt (Greta\&Starks o.J.).

Zunehmend findet AD auch in der Kunst- und Kulturszene oder bei Opernoder Theateraufführungen Anwendung. Dort werden dem blinden Besucher via Kopfhörer Erläuterungen zur Handlung und zum visuellen Erscheinungsbild der Schauspieler vermittelt. Das Schauspiel Leipzig ist eine der ersten Kultureinrichtungen, die regelmäßig Audiodeskriptionen für Theaterstücke erstellt 
(Schauspiel Leipzig o.J.). AD wird auch bei einigen Bundesligaspielen im Fußball oder im Handball (DHFK o.J.) angeboten, genau wie die kommenden Olympischen Spiele sowie die Paralympischen Spiele (einfach teilhaben o.J.).

Für blinde und sehbehinderte Menschen gibt es verschiedene Medien, die auf die jeweiligen Bedürfnisse und Fähigkeiten der Nutzer abgestimmt sind. Vom klassischen Punktschriftbuch über taktile Modelle bis hin zu mobilen Anwendungen und Audiodeskriptionen - die Angebote zur Nutzung barrierefreier Medien finden in kulturellen und gesellschaftlichen Bereichen mehr und mehr Anwendung. Um die Zugänglichkeit zu diesen Angeboten zu gewährleisten, gilt es, grundlegende Prinzipien zu berücksichtigen. Zunächst ist zu prüfen, inwieweit Kommunikationsangebote Punktschrift und Schwarzschrift enthalten sollen. In beiden Fällen ist in besonderem Maße darauf zu achten, dass eine Orientierung auf Buchseiten oder in digitalen Medien erfolgen kann. Dafür gibt es vor allem typografische Möglichkeiten, die den Anforderungen zur Herstellung solcher Inhalte entsprechen. Mit der Reduktion von Inhalten auf die wesentlichsten Informationen ist ein weiterer Schritt zu guter Orientierung getan. Um eine verbesserte Wahrnehmung zu unterstützen, ist der Einsatz kontrastreicher Gestaltungsmittel und Bilder nötig. Letztere sollen blinden Menschen auch zugänglich gemacht werden, weshalb taktile Grafiken oder bei digitalen Inhalten Alternativtexte zur Verfügung stehen müssen. Die Basisanforderungen sind in verschiedene Standards eingeflossen und im digitalen Bereich auch rechtlich verbindlich in Verordnungen eingegangen. Neben theoretischen Grundlagen ist es unerlässlich, die Zielgruppe in Produktentwicklungen einzubeziehen, da die Richtlinien und Standards nur eine Richtung weisen können, der Einzelfall aber stets zu prüfen ist.

Die Umsetzung der europäischen Richtlinie 2016/2102 in Bund, Ländern und Kommunen wird dazu beitragen, dass die Informations- und Kommunikationsangebote staatlicher Einrichtungen barrierefreier gestaltet werden. Wichtig dabei ist, die Möglichkeiten der Evaluation und Prüfung zu nutzen, um diesen Prozess zu befördern und die Qualität der Angebote zu sichern. 
Bislang existieren keine verbindlichen Regelungen des Gesetzgebers, die private Wirtschaft zu einer barrierefreien Gestaltung ihrer digitalen Dienstleistungen und Produkte anzuhalten. Ab Herbst 2018 wird sich zeigen, wie die genannten gesetzlichen Vorgaben dazu einen Beitrag leisten können. 
Barrierefreie Informations- und Kommunikationsangebote für Blinde und Sehbehinderte

\section{Literaturverzeichnis}

[ABSV] (o.J.): „Tastmodelle“. URL: http://www.absv.de/kultur-freizeit (letzter Zugriff: 07.02.2018).

[BACHMUSEUM LeIPZIG] (o.J.): „Audioguide“. URL: http://www.bachmuseumleipzig.de/ de/bach-museum/die-museums-app (letzter Zugriff: 02.01.2018).

[BARRIEREFREIES WEBDESIGN] (o.J.): URL: https://www.barrierefreies-webdesign.de/ richtlinien/eu-2016-2102/ (letzter Zugriff: 02.01.2018).

Beck, FranZ-Josef (2014): Lesen sehbehinderter Schülerinnen und Schüler. Diagnostik und Förderung. Dissertation. Marburg: Tectum.

[BeHINDERTENGLEICHSTELLUNGSGESETZ] (2002): \$4 Barrierefreiheit. URL: https://www.gesetze-im-internet.de/bgg/BGG.pdf (letzter Zugriff: 01.02.2018).

[Beuth VerlaG GmbH] (2013): „DIN 1450:2013-04. Lettering - Legibility“. URL: https://www.beuth.de/de/norm/din-1450/170093157 (letzter Zugriff: 07.02.2018).

[Beuth Verlag GmbH] (2014): „DIN 32976:2007-08. Blindenschrift - Anforderungen und Maße“. URL: http://www.beuth.de/de/norm/din-32976/98366852 (letzter Zugriff: 07.02.2018).

[BeUth VerLaG GmbH] (2015): „DIN 32986:2015-01: Taktile Schriften und Beschriftungen - Anforderungen an die Darstellung und Anbringung von Braille- und erhabener Profilschrift“. URL: https://www.beuth.de/de/norm/din-32986/224499905 (letzter Zugriff: 07.02.2018).

[BÖRSENVEREIN DES DEUTSCHEN BuChHANDELS E.V.] (2017): „Checkliste zur Barrierefreiheit von EPUB3-eBooks“. URL: https://www.igdigital.de/checkliste-zur-barrierefreiheit-von-ebooks/ (letzter Zugriff: 07.02.2018).

[BUNDESMINISTERIUM DER JUSTIZ UND FÜR VERBRAUCHERSCHUTZ] (2011): „Verordnung zur Schaffung barrierefreier Informationstechnik nach dem Behindertengleichstellungsgesetz (Barrierefreie-Informationstechnik-Verordnung - BITV 2.0)“. URL: https://www.gesetze-im-internet.de/bitv_2_0/BJNR184300011.html (letzter Zugriff: 07.02.2018).

[DAISY-CONSORTIUM] (o.J.): URL: http://www.daisy.org (letzter Zugriff: 07.02.2018).

[DBSV] (2018): „leserlich \& lesbar“. URL: http://www.dbsv.org/leserlich/kapitel/ leserlich-und-lesbar.php (letzter Zugriff: 07.02.2018).

[DBSV] (2018a): „Schriftart“. URL: http://www.dbsv.org/leserlich/kapitel/zeichen/ schriftart.php (letzter Zugriff: 07.02.2018).

[DBSV] (2018b): „Schriftgröße“. URL: http://www.dbsv.org/leserlich/kapitel/zeichen/ schriftgroesse.php (letzter Zugriff: 07.02.2018).

[DBSV] (2018d): „Smartphonenutzung“. URL: https://www.dbsv.org/computersmartphone-internet.html (letzter Zugriff: 07.02.2018).

[DEUTSCHER HÖRFILMPREIS] (o.J.): URL: https://deutscher-hoerfilmpreis.de/derhoerfilmpreis.html (letzter Zugriff: 01.02.2018). 
[DZB: Deutsche Zentralbücherei für Blinde ZU LeipZig] (2014): „Fotoarchiv“. URL: https://www.dzb.de/presseportal/fotoarchiv/pressefotos/bild96.html (letzter Zugriff: 07.02.2018).

DEUTSCHE ZENTRALBÜCHEREI FÜr BLINDE ZU LEIPZIG (2017): MAXI-Druck für hochgradig sehbehinderte Leserinnen und Leser. Leipzig: DZB.

[DeUtsches InstituT FÜR NORMUNG E.V.] (2013): „DIN 1450 Schriften - Leserlichkeit Lettering - Legibility Ecriture - Lisibilité“. URL: http://www.beuth.de/de/norm/ din-1450/170093157 (letzter Zugriff: 07.02.2018).

[DHFK] (o.J.): „Audiodeskription bei Handballspielen“. URL: www.scdhfk-handball.de/ aktuelles/details/audiodeskription-bei-allen-heimspielen (letzter Zugriff: 20.02.2018).

[Dyslexia Research CENTER AG] (2012): „Schriftarten für legasthene Menschen“. URL: http://www.legasthenie.at/broschure-schriftarten-fur-legasthene-menschen (letzter Zugriff: 07.02.2018).

[EINFACH TEILHABEN] (o.J.): „AD in Stadien“. URL: http://www.einfach-teilhaben.de/DE/ StdS/Mobilitaet/Sport/stadien_sportstaetten/stadien_und_arenen.html?nn=510720 (letzter Zugriff: 08.02.2018).

[GeSETZ Über URHEBERRECHT UND VERWANDTE SCHUtZRECHTE] (2003): „\$45a Behinderte Menschen“. URL: https://www.gesetze-im-internet.de/urhg/_45a.html, (letzter Zugriff: 01.02.2018).

[GRETA\&STARKS] (o.J.): „Audiodeskription für das Kino“. URL: http://www.gretaundstarks.de/ (letzter Zugriff: 01.02.2018).

[Hilderley, Sarah] (2013): „Accessible Publishing. Best Practice Guidelines for Publishers“. URL: http://www.accessiblebooksconsortium.org/inclusive_publishing/en/ accessible_best_practice_guidelines_for_publishers.html (letzter Zugriff: 07.02.2018).

Hofer, URsula (2008): „Sehen oder Nichtsehen: Bedeutung für Lernen und aktive Teilhabe in verschiedenen Bereichen des Lernens und Lebens“. In: LANG, MARKUs; Beyer, Friederike; Hofer, Ursula (Hrsg.): Didaktik des Unterrichts mit blinden und hochgradig sehbehinderten Schülern. Grundlagen. 1. Band. Stuttgart: Kohlhammer, 17-67.

[HÖRFILMINFO] (o.J.): „Audiodeskription“. URL: https:/hoerfilm.info/ hoerfilmprogramm. html (letzter Zugriff: 07.02.2018).

[INSTITUT FÜR MENSCHENRECHTE] (2018): „Vertrag von Marrakesch“. URL: http://www.institut-fuer-menschenrechte.de/monitoring-stelle-un-brk/staatenpruefung/ positionen-der-monitoring-stelle/vertrag-von-marrakesch/ (letzter Zugriff: 20.02.2018).

[ISAR-PROJEKT] (o.J.): „3D-Druck“. URL: (http://www.isar-projekt.de/literatur/ konstruktion-von-modellen-fuer-den-3d-druck-und-modelldruck-ammedienberatungszentrum-ilvesheim-6346.html (letzter Zugriff: 13.02.2018).

KAHLISCH, ThOMAS; SAMLAND, YvONnE; DobrosCHKe Julia (2011): Brailleschrift - Entwicklung, Notationen, Herstellung und Anwendung. Seminarunterlagen. Leipzig: DZB. 
LANG, Markus (2003): Haptische Wahrnehmungsförderung mit blinden Kindern. Möglichkeiten der Hinführung zur Brailleschrift. Dissertation. Pädagogische Hochschule Heidelberg, Regensburg: Roderer.

[MEDIBUS] (o.J.): „Mediengemeinschaft für blinde und sehbehinderte Menschen e.V.“. URL: http://www.medibus.info/index.php?article_id=26 (letzter Zugriff: 22.02.2018).

Mellor, C. Michael (2009): Louis Braille - Fühlbare Genialität. Paderborn: Blindenschrift-Verlag und -Druckerei „Pauline von Mallinckrodt“.

MÖnNIG, ANTJE (2016): Unmöglich. Aber machbar. Leipzig: DZB.

[MÜLlER, LUTZ] (2013): „Audiodeskription im MDR-Fernsehen“. URL: https://www.mdr.de/barrierefreiheit/audiodeskription106.html (letzter Zugriff: 07.02.2018).

[NATIONAL Council FOR THE Blind OF IRELAND] (2009): „Make it clear“. URL: https://www.sess.ie/sites/default/files/Categories/Sensory_Impairments/Accessibility_ NAD_NCBI.pdf (letzter Zugriff: 07.02.2018).

[REAdiUm Foundation] (2018): „Open Source Technology for EPUB 3 and the Open Web Platform“. URL: http://readium.org/ (letzter Zugriff: 07.02.2018).

[RICHTLINIE EU] (2016): „2016/2012 DES EUROPÄISCHE PARLAMENTS UND DES RATES über den barrierefreien Zugang zu den Websites und mobilen Anwendungen öffentlicher Stellen“. URL: http://eur-lex.europa.eu/legal-content/DE/TXT/ HTML/?uri=CELEX:32016L2102\&rid=1 (letzter Zugriff: 01.02.2018).

[RICHTLINIE EU] (2017): „2017/1564 DES EUROPÄISCHEN PARLAMENTS UND DES RATES“. URL: http://eur-lex.europa.eu/legal-content/DE/TXT/PDF/?uri= CELEX:32017L1564\&from=DE (letzter Zugriff: 01.02.2018).

[RNIB] (2013): „RNIB's free Tiresias font makes text easier to read for the partially sighted“. URL: https://www.digitalartsonline.co.uk/news/typography/rnibs-tiresiasfont-makes-text-easier-read-for-partially-sighted/ (letzter Zugriff: 07.02.2018).

[SCHAUSPIEL LEIPZIG] (o.J.): „Audiodeskription“. URL: https://www.schauspielleipzig.de/service/audiodeskription/ (letzter Zugriff: 06.02.2018).

Staupendahl, Frauke (1998): Musik mit Blinden. Eine Einführung in die Musik- und Instrumentalpädagogik. Münster: Waxmann.

[VERORDNUNG EU] (2017): „2017/1563 DES EUROPÄISCHEN PARLAMENTS UND DES RATES“. URL: http://eur-lex.europa.eu/legal-content/DE/TXT/PDF/ ?uri=CELEX:32017R1563\&from=DE (letzter Zugriff: 01.02.2018).

[Web Content Accessibility Guidelines] (2009): „WCAG 2.0“. URL: https://www.w3.org/Translations/WCAG20-de/ (letzter Zugriff: 01.02.2018).

[Web Content Accessibility Guidelines] (2018): „WCAG 2.1“. URL: https://www.w3.org/TR/WCAG21/ (letzter Zugriff: 07.02.2018).

[ZEUN, UlRICH] (1998): „Kriterien zur Umsetzung in einen sehbehindertengerechten Druck“. URL: http://www.sehgeschädigte.info/Gdk_main.htm (letzter Zugriff: 07.02.2018). 



\section{JOHANNES HENNIES}

\section{Prälinguale Hörbehinderung und Schriftsprachkompetenz}

Menschen, die von Geburt an gehörlos oder schwerhörig sind, haben heute deutlich bessere Bildungschancen als jemals zuvor. Einerseits erfahren die nationalen Gebärdensprachen, hierzulande also die Deutsche Gebärdensprache (DGS), eine vermehrte wissenschaftliche, gesellschaftliche und rechtliche Anerkennung, wodurch sie auch zunehmend in die pädagogische und therapeutische Arbeit einbezogen werden, andererseits ermöglichen die frühere Diagnostik und die modernen Hörgeräte und das Cochlea-Implantat (CI) vielen gehörlosen und schwerhörigen Menschen einen Zugang zum Hören und damit teilweise zu einem altersgemäßen Lautspracherwerb. Allerdings kann auch unter den verbesserten Bedingungen ihre Schriftsprachkompetenz eingeschränkt bleiben. Der Beitrag stellt die Zusammenhänge zwischen Hörbehinderung, Sprachennutzung und Schriftsprachkompetenz dar, skizziert Besonderheiten der Schriftsprachkompetenz von Menschen mit prälingualer Hörbehinderung und weist abschließend auf einige Konsequenzen für die pädagogische, therapeutische und soziale Arbeit hin.

\section{$1 \quad$ Menschen mit prälingualer Hörbehinderung}

Die Gruppe der Menschen mit einer prälingual oder im frühen Kindesalter erworbenen Hörbehinderung ist insgesamt sehr klein. Nach Daten des „Deutschen Zentralregisters für kindliche Hörstörungen" kommen in Deutschland von 1000 Geburten 1,2 Kinder mit einer beidseitigen Hörschädigung auf die Welt (Gross et al. 2000: 879). Zudem ist diese Gruppe in sich äußerst heterogen, mit verschiedenen Graden der Hörschädigung, unterschiedlichen Arten der hörtechnischen Versorgung (Hörgerät(e) und/oder CI) und verschiedenen Pri- 
märsprachen (Deutsch, Deutsche Gebärdensprache, andere Laut- und/oder Gebärdensprachen). Was sie jedoch verbindet, ist der eingeschränkte oder gar nicht existierende Zugang zum Hören, der sich u.a. negativ auf den Erwerb der Lautsprache (hierzulande also das gesprochene Deutsch) und nachfolgend der Schriftsprache auswirken kann. Um diese Zusammenhänge angemessen beschreiben zu können, sollen zuerst die allgemeinen sprachlichen Erwerbsbedingungen von Menschen mit einer Hörbehinderung dargestellt werden. Dabei gibt es eine „Vielzahl von Fachdisziplinen“ (Wisotzki 1994: 48), aus deren Perspektive die Gruppe beschrieben wird, wie etwa aus einem sprachwissenschaftlichen, soziologischen, medizinisch-therapeutischen oder pädagogischen Blickwinkel. Hinzu kommt die fachliche Beschreibung der „Deaf Studies“ (Fischer et al. 2009), die explizit die Sicht der selbst Betroffenen in die wissenschaftliche Konzeption aufnimmt. Entsprechend der jeweiligen fachlichen Orientierung können auch unterschiedliche Begriffe für den gleichen Personenkreis verwendet werden, mit denen sich auch unterschiedliche Wertehaltungen verbinden. Wenn z. B. von pädagogischer Seite ein Teil der gehörlosen Kinder als „resthörig“ beschrieben wird (Löwe 1996: 48 und 49), so soll damit auf die verbesserten hörtechnischen Möglichkeiten auch für audiologisch als „taub“ geltende Kinder aufmerksam gemacht werden. Wenn hingegen von Seiten erwachsener Gehörloser von „tauben Menschen“ als Oberbegriff aller Gehörloser und Schwerhöriger mit einem Zugang zur Gebärdensprache gesprochen wird, so ist der Begriff analog zu dem angelsächsischen „Deaf“ (Padden/Humphries 1991: 10) gewählt und soll verdeutlichen, dass es sich bei Gehörlosen um eine sprachliche und kulturelle Minderheit handelt. Um diese Perspektive zu betonen, wird dann gelegentlich nicht mehr von "gehörlosen“ und „hörenden“, sondern von „tauben“ und „taublosen“ Menschen gesprochen (Grote/Zäh 2016).

Da es also keine neutrale Bezeichnung der Zielgruppe geben kann, ist es notwendig, die eigenen sprachlichen Ausdrücke in einer Darstellung zu begründen. Der vorliegende Artikel orientiert sich an der Begrifflichkeit, die durch das Klassifikationsinstrument ICF (International Classification of Functioning, Disability and Health) der Weltgesundheitsorganisation (WHO) vorgegeben ist (DIMDI 2005), wonach erst durch die Einschränkung der gesellschaftlichen Teilhabe eine Behinderung entsteht und nicht notwendigerweise bereits durch eine Schädigung von Körperstrukturen und -funktionen, 
wie z. B. der Hörschädigung. Die Entscheidung, von „Menschen mit einer Hörbehinderung“ zu sprechen, orientiert sich zudem an dem einstimmigen Beschluss der Selbsthilfeverbände (u.a. Deutscher Gehörlosen-Bund und Deutscher Schwerhörigenbund) auf der Sitzung des Dachverbands „Deutsche Gesellschaft der Hörgeschädigten“ im November 2016 für diesen Sammelbegriff. Die Bezeichnungen "gehörlos“ und „schwerhörig“ werden verwendet, um von dem audiologisch feststellbaren Hörstatus auszugehen, wobei die Mitglieder der Gebärdensprachgemeinschaft überwiegend, wenn auch heute nicht mehr ausschließend, gehörlos und hochgradig schwerhörig sind.

\section{Erwerb einer Primärsprache von Menschen mit einer Hörbehinderung}

Es gibt drei mögliche Primärsprachen, die Menschen mit einer Hörbehinderung erwerben können, und zwar a) die Gebärdensprache der jeweiligen Gebärdensprachgemeinschaft, b) die Lautsprache der sie umgebenden hörenden Mehrheitsgesellschaft und c) die Schriftsprache, wobei es eher wenige historische Beispiele sind, bei denen gehörlose oder taubblinde Menschen den primären Sprachaufbau vollständig hierüber erworben haben.

a) Gebärdensprachen entwickeln sich vermutlich überall dort, wo eine ausreichende Anzahl Gehörloser in einer Gemeinschaft über mehrere Generationen zusammenlebt (Meir et al. 2010). In der Hörgeschädigtenpädagogik wurde sie bis Ende des 19. Jahrhunderts ebenfalls regulär eingesetzt, dann aber nach dem Beschluss des Mailänder Kongresses von 1880 über 100 Jahre weitgehend aus dem Unterricht verdrängt. Erst mit der Empowerment-Bewegung der Gehörlosen und der linguistischen und rechtlichen Anerkennung der nationalen Gebärdensprachen als vollwertigen Sprachen hat auch die DGS wieder einen Platz in den Hörgeschädigtenschulen gefunden, etwa in Form von bimodal-bilingualen Unterrichtskonzepten (erstmals ab 1992 in Hamburg) oder als reguläres Unterrichtsfach (erstmals ab 2001 in 
Bayern). Auch während der Zeit, als die Gebärdensprache in den Hörgeschädigtenschulen verpönt war, wurde sie in der Gebärdensprachgemeinschaft, etwa in den Gehörlosenvereinen, tradiert und weitergegeben. Die unmittelbare Weitergabe der Sprache und Kultur, die bei lautsprachlichen Kulturen als „mündliche Überlieferung“ bezeichnet wird, ist deswegen auch von besonderer Bedeutung, weil die Gebärdensprache keine Schriftform hat. Entsprechende Versuche hat es zuerst im 19. Jahrhundert gegeben (Bébian 1825). Mit der „Gebärdenschrift“ (Wöhrmann 2005), der deutschen Variante von „SignWriting“ (Sutton 1981), liegt auch ein zumindest weitgehend funktionaler Entwurf einer Alltagsschrift vor. Allerdings wird die Gebärdenschrift nur vereinzelt in pädagogischen Kontexten eingesetzt, etwa im sprachlichen Anfangsunterricht (Wöhrmann o.J.) und in der Deutschförderung erwachsener Gehörloser (Hänel-Faulhaber et al. 2012); es ist nicht dokumentiert, dass erwachsene Nutzer(innen) der DGS die Gebärdenschrift tatsächlich in ihrem Alltag verwenden.

Lange ist in der Hörgeschädigtenpädagogik davon ausgegangen worden, dass der Erwerb der Gebärdensprache den Erwerb der Lautsprache und nachfolgend der Schriftsprache stört sowie die Integration in die hörende Mehrheitsgesellschaft verhindert (z. B. SchmidGiovannini 1976: 25 und 26). Da es für diese Annahme keine empirischen Belege gibt, gilt sie heute als überholt (Marschark/Spencer 2009). In welchem Umfang gehörlose und schwerhörige Kinder DGS erwerben, hängt von zahlreichen individuellen Faktoren ab. Nur eine Minderheit der Kinder mit einer Hörbehinderung hat ein gehörloses oder schwerhöriges Elternteil und erwirbt somit die Gebärdensprache als natürliche Erstsprache: Dieser Anteil liegt in Untersuchungen zwischen 2,3\% und 8\% (Große 2003: 34; Mitchell/Karchmer 2004). Die überwiegende Mehrheit der gehörlosen Menschen, die DGS als Primärsprache verwenden, hat sie später erworben, z.T. auch erst im Erwachsenenalter. Trotz der immer größeren Offenheit gegenüber dem Einsatz der DGS in der Frühförderung (Günther et al. 2009) und der Hörgeschädigtenschule (Günther/Hennies 2015) ist es keine Selbst- 
verständlichkeit, dass hochgradig schwerhörige und gehörlose Menschen in unserem Bildungssystem DGS früh und umfassend angeboten bekommen.

b) Menschen mit einer Hörbehinderung sind von einer lautsprachlichen, hörenden Mehrheitsgesellschaft umgeben, weshalb lange Zeit der Erwerb einer möglichst guten Lautsprachkompetenz das vorrangige Ziel der Hörgeschädigtenpädagogik gewesen ist. Vor der Entwicklung von modernen Hörgeräten und dem CI wurde die Lautsprache im Rahmen des Artikulationsunterrichts über das Absehen sowie das Abfühlen der Lautbildung bei dem/der Lehrer(in) und der angeleiteten Eigenwahrnehmung der eigenen Sprechorgane vermittelt. Zugleich wurden alternative Wege, etwa über die Gebärdensprache, abgelehnt. Obwohl es immer einzelne Gehörlose gab, die auf diese Art erstaunlich gute lautsprachliche Kompetenzen erwerben konnten, stellte es für die meisten Schüler(innen) einen ineffektiven Weg zur Sprache dar, dessen zeitaufwendige Vermittlung zudem auf Kosten anderer Lernbereiche ging. Nach der Entwicklung von modernen Hörgeräten und der Innenohrprothese Cochlea-Implantat in Verbindung mit immer genauerer und früherer Diagnostik setzte sich in den 1980er Jahre die aurale oder hörgerichtete Methode durch, in der gehörlosen und schwerhörigen Kindern die Lautsprache über das nun zugänglich gewordene Gehör auf möglichst natürliche Weise vermittelt werden sollte, also über eine lautsprachliche Interaktion zwischen Eltern und Kind (Diller 2009). Insgesamt kann auf diese Art mehr Kindern der Zugang zu einer zumindest annähernd altersgemäßen lautsprachlichen Kompetenz ermöglicht werden, aber auch unter guten Bedingungen zeigt sich der Erwerb der Lautsprache als stark störanfällig. Es wird über 30\% bis 50\% der Kinder mit einer hochgradigen Schwerhörigkeit oder Gehörlosigkeit berichtet, deren lautsprachliche Entwicklung auch unter guten Bedingungen verzögert oder gestört bleibt (Geers 2006; Szagun 2010; Niparko et al. 2010). Selbst bei leicht- bis mittelgradig schwerhörigen Kindern sind einige Bereiche des Spracherwerbs, wie etwa die Grammatikentwicklung, zumindest gefährdeter als bei hörenden Kindern (Moeller et al. 2007; Delage/Tuller 2007; 
Wimmer et al. 2015). Vertreter(innen) der hörgerichteten Methode haben von ihren Vorgänger(inne)n der oralen Methode zumeist eine skeptische bis ablehnende Haltung gegenüber dem Einsatz von einzelnen Gebärden oder der DGS als Sprachsystem übernommen (Diller 2009: 175), es gibt aber keinen Grund anzunehmen, dass die parallele Vermittlung der Gebärdensprache einen Lautspracherwerb über das mit Hörhilfen ermöglichte Gehör verhindern sollte (Günther et al. 2009).

c) Es gibt wenige dokumentierte Fälle, in denen gehörlose oder taubblinde Menschen ihren initialen Sprachaufbau rein über die Schriftsprache erfahren haben (Günther 1985: 48); der bekannteste Fall ist sicherlich die taubblinde Schriftstellerin Helen Keller, die im Alter von 19 Monaten ihr Hör- und Sehvermögen verloren hatte und mit 7 Jahren einen vollständigen Sprachaufbau über die Schriftsprache erlangte, die ihr zuerst als abgefühltes Fingeralphabet und dann als Brailleschrift vermittelt wurde (Stern 1905). Jenseits von solchen Einzelbeispielen geht Günther (1993) davon aus, dass die Schriftsprache häufig die „wahre Basis der Verbalsprache Gehörloser" gewesen sei, weil diese im Rahmen des Artikulationsunterrichts üblicherweise auch die Schriftform eines Wortes erlernt und davon mehr profitiert hätten als von der Vermittlung lautsprachlicher Strukturen. Die Hörgeschädigtenpädagogik ist hingegen traditionell von einem umgekehrten Bedingungsgefüge ausgegangen. Walther (1895: 259) vertritt in dem ersten umfassenden "Handbuch der Taubstummenbildung" die Auffassung, dass auch bei gehörlosen Schüler(inne)n „der schriftliche Ausdruck i.a. stark vom mündlichen ab[hängt]“, und führt weiter aus: „[J]e flotter und gewandter die meisten Schüler sprechen können, desto besser geht auch das Schreiben vonstatten“. Deswegen wurde bis Ende der 1990er Jahre die „Sprechkorrekturhilfe: Schrift“ (Schulte/Schlenker-Schulte 1983, W1820) zwar zur Verbesserung der Lautsprache eingesetzt, aber zumeist nicht als ein eigenständiger Weg zur sprachlichen Kompetenz gehörloser Menschen angesehen (Schmid-Giovannini 1976: 63 und 64). 
Heute wird in der Hörgeschädigtenpädagogik zunehmend anerkannt, dass Menschen mit einer Hörbehinderung grundsätzlich davon profitieren, wenn sie mehrere Primärsprachen erwerben. Aufgrund der hörtechnischen Entwicklung und des seit 2009 flächendeckend eingeführten Neugeborenen-Hörscreenings ist für gehörlos oder schwerhörig geborene Kinder eine Diagnose, eine hörtechnische Versorgung und der Beginn der Frühförderung im ersten halben Lebensjahr möglich geworden (Hennies 2010). Zugleich kann die frühere Diagnose auch genutzt werden, um die DGS von Beginn an zu vermitteln. Beide Sprachen können sich gegenseitig positiv beeinflussen, wenn sie mit einem klaren Spracherwerbskonzept vermittelt werden, wie es sowohl für die Frühförderung (Günther et al. 2009; Hofmann/Hennies 2015) als auch die Schule (Günther/Schäfke 2004; Hennies/Günther 2015) vorliegt. Schriftsprache wird zwar teilweise immer noch als ergänzende Möglichkeit in der Früherziehung herangezogen (Stocker 2002), aber nicht mehr als Primärsprache vermittelt.

\section{Schriftsprachkompetenz von Menschen mit einer Hörbehinderung}

Die Schwierigkeiten, die Menschen mit einer prälingualen Hörbehinderung beim Erwerb der Schriftsprache haben, sind durch empirische Studien der letzten vierzig Jahre immer wieder dokumentiert worden (ein Überblick in Krammer 2001), wobei einige Kernaussagen bereit in Conrads (1979: 152) erster repräsentativer Studie der Lesekompetenz gehörloser Schulabgänger(innen) von Hörgeschädigtenschulen in England und Wales zu finden sind und zuletzt durch den USamerikanischen „Stanford Achievement Test“ erneut mit einer umfassenden Stichprobe bestätigt worden sind. Mit diesem Test wird für die Erhebung der Lesekompetenz gehörloser und schwerhöriger Schüler(innen) in regelmäßigen Abständen eine repräsentative Normierungsstichprobe mit mehreren tausend Kindern und Jugendlichen im Alter von 8 bis 18 Jahren erfasst (Holt 1993, Holt et al. 1997, Traxler 2000; Karchmer/Mitchell 2003): Gehörlose und schwerhörige Schulabgänger(innen) erreichen demzufolge im Durchschnitt Ergebnisse, wie sie bei hörenden Grundschüler(inne)n zu finden sind. Desweiteren hat der Hörstatus einen entscheidenden Einfluss auf das Abschneiden, wobei der Abstand zwischen 
stärken und schwächeren Schüler(inne)n über die Dauer des Schulbesuchs zunimmt. Es ist anzunehmen, dass es nicht der Hörstatus an sich, sondern die darauf aufbauende lautsprachliche Kompetenz ist, die zu den besseren Ergebnissen in der Schriftsprache führen. Deswegen wirken sich andere Faktoren, die bei Menschen mit einer Hörbehinderung den Lautspracherwerb erschweren, ebenfalls nachteilig auf den Schriftspracherwerb aus. Hierzu gehören etwa eine zusätzliche Beeinträchtigung, ein geringerer Bildungsstand der Eltern oder ein Migrationshintergrund mit entsprechender lautsprachlicher Mehrsprachigkeit in Kombination mit dem häufig geringeren sozio-ökonomischen Status (Hennies 2014). Dass sich in Untersuchungsgruppen von Kindern mit CI bessere Ergebnisse in der Schriftsprache finden als bei Vergleichsgruppen mit gehörlosen Kindern ohne CI (Archbold et al. 2008), ist ebenfalls darauf zurückzuführen, dass diese die Lautsprache im Durchschnitt besser erwerben. Allerdings bleiben auch sie deutlich hinter der Vergleichsgruppe gleichaltriger Kinder ohne Hörbehinderungen zurück und zeigen eine große Streuung in den Ergebnissen (Vermeulen et al. 2007: 289). Zahlreiche Studien belegen zudem, dass Gehörlose mit einer höheren Gebärdensprachkompetenz auch höhere schriftsprachliche Kompetenzen zeigen (Chamberlain/ Mayberry 2000) und dass ein früher Zugang zur Gebärdensprache ähnliche Kompetenzen in einem schriftsprachlichen grammatikalischen Bewertungstest ermöglicht, wie ihn hörende Erwachsene zeigen, die mit einer anderen Muttersprache aufgewachsen sind (Mayberry et al. 2002). Mayberry et al. (2011) weisen in einer Metastudie über die Lesekompetenz Gehörloser nach, dass der generelle Sprachstand (in Laut- oder Gebärdensprache) die Ergebnisse am stärksten beeinflusst, wodurch 35 \% der Varianz erklärt werden können; phonologisches Rekodieren und phonologische Bewusstheit der Lautsprache hingegen sagen nur $11 \%$ der Varianz in den Ergebnissen vorher. Deswegen lässt sich aus den zitierten Studien ableiten, dass eine möglichst gut entwickelte Primärsprache die beste Voraussetzung für einen gelingenden Erwerb der Schriftsprache bei Menschen mit einer Hörbehinderung darstellt. Bei entsprechenden Voraussetzungen und gelingender technischer Versorgung kann die Schriftsprache also auf der Lautsprache aufbauen, deren Erwerb bei Kindern mit einer Hörbehinderung aber potentiell störanfällig ist. Sie kann auch auf der Gebärdensprache aufbauen, die allen gehörlosen und schwerhörigen Kindern im Prinzip uneingeschränkt zu- 
gänglich ist, aber im Umfeld der meisten Kinder nicht von Geburt an zur Verfügung steht. Wenn Kinder zu beiden Sprachsystemen Zugang haben, können die beiden Primärsprachen im Schriftspracherwerb positiv miteinander interagieren (Günther/Hennies 2015: 98ff.).

\section{Schriftspracherwerb bei gehörlosen und schwerhörigen Kindern}

Neben den allgemeinen sprachlichen Voraussetzungen kann der Schriftspracherwerb gehörloser und schwerhöriger Kinder auch noch davon beeinflusst werden, in welchem Maße ihnen frühkindlich anregende Kontakte mit Schrift ermöglicht werden. Insgesamt zeigen hörende Eltern, die ein gehörloses oder hochgradig schwerhöriges Kind bekommen, die Tendenz, ein sprachlich weniger anregendes Umfeld zu schaffen, als sie dies bei einem hörenden Kind gemacht hätten. Welche Auswirkungen dies auf die Schriftsprachsozialisation haben kann, lässt sich anhand einer Studie von Swanwick und Watson (2005) illustrieren: Die untersuchten hörenden Mütter zeigen beim Vorlesen zwar Aufmerksamkeit und bauen Nähe zu ihren gehörlosen Kindern auf, animieren diese aber häufig, Bilder zu benennen und bestimmte Worte auszusprechen, anstatt über Inhalte zu sprechen. Gebärdensprachlich kommunizierende gehörlose Mütter konzentrieren sich dagegen eher auf die in den Büchern erzählten Geschichten. Es hat sich gezeigt, dass gehörlose Kinder in ihrer Wortschatzentwicklung signifikant profitieren, wenn ihre Eltern beim gemeinsamen Lesen (shared reading) spezifische sprachliche Verhaltensweisen verwenden, wie z. B. offene Fragen zu stellen und die Antworten der Kinder aufzugreifen und zu erweitern (Fung et al. 2005), wie dies zuvor auch schon bei Eltern hörender Kinder belegt worden ist (Whitehurst et al. 1988). Es ist also anzunehmen, dass ein kreativer und sprachlich anregender Umgang mit schriftlichem Material im frühen Kindesalter auch die spätere Schriftsprachkompetenz gehörloser und schwerhöriger Kinder fördert.

Es gibt verschiedene Modelle darüber, wie der Erwerb der Lese- und Schreibkompetenzen im engeren Sinne verläuft (Schründer-Lenzen 2013: 67, 73f.). Sie alle zeigen auf, dass hörende Kinder in der Regel eine Phase durchlaufen, in der 
sie Laut- und Schriftsprache eng aufeinander beziehen. Dabei erwerben sie Phonem-Graphem-Korrespondenz-Regeln und wenden diese aktiv beim Schreiben und Lesen an. Diese „alphabetische Phase“ hat eine hohe Bedeutung für Anfangsunterricht in der Deutschdidaktik und kann durch Übungen zur phonologischen Bewusstheit im Kindergarten vorbereitet sowie durch synthetische Anteile von Schriftspracherwerbslehrgängen didaktisch unterstützt werden (Hennies i.D.). Sie muss dann auch überwunden werden, um in der „orthographischen Phase“ morphembasierte Strategien beim Lesen und Schreiben zu weichen. Kinder, die einen eingeschränkten oder keinen Zugang zur (deutschen) Lautsprache haben, werden jedoch von einer Didaktik benachteiligt, die den lautbezogenen Erwerbsweg besonders stärkt. So können z. B. gehörlose Kinder durchaus zu kompetenten Schreiber(inne)n und Leser(inne)n werden, ohne einen Lautbezug dafür zu benötigen; sie zeigen dabei dann auch üblicherweise kaum spontan variierende Fehlschreibungen für das gleiche Wort, die für die alphabetische Phase bei hörenden Kindern typisch sind (Günther 2003). Da aber nicht nur gehörlose Kinder mit der alphabetischen Phase Schwierigkeiten haben, müsste eine inklusive Deutschdidaktik entsprechend neben dem synthetischen Wege auch gleichberechtigte analytische Zugänge eröffnen, durch die orthographische Regeln und Morphemstrukturen über visuelle Mustererkennung und Wortfelder, idealerweise auf einem großen Sichtwortschatz aufbauend, erworben werden können (Hennies i.D.). Insbesondere aus zwei bimodal-bilingualen Schulversuchen in Hamburg und Berlin, bei denen gehörlose Schüler(innen) erstmals unter gleichberechtigtem Einsatz von Gebärden-, Laut- und Schriftsprache gefördert worden sind, liegen umfangreiche Daten zur Lese- und Schreibentwicklung vor, die den Verlauf eines lautsprachunabhängigen Schriftspracherwerbs illustrieren (Günther 2011, Schäfke 2005). Zudem ist in diesem Projektzusammenhang eine große Sammlung von Vergleichstexten gehörloser und schwerhöriger Kinder entstanden, die nicht bimodal-bilingual gefördert worden sind (Schäfke 2005). Bei ihnen zeigt sich eine insgesamt niedrigere Textproduktionskompetenz, woraus sich die Notwendigkeit lautsprachunabhängiger, analytischer Erwerbswege als Ergänzung zu bestehenden Verfahren ableiten lässt. Allerdings liegt hierfür kaum didaktisches Material vor, wodurch der Schriftspracherwerb von gehörlosen und schwerhörigen Kindern zusätzlich erschwert wird (Brinkmann 2015). 

mit einer Hörbehinderung

Menschen mit einer Hörbehinderung haben heute durch bessere technische Möglichkeiten einen größeren Zugang zu lautsprachlicher Information. Zudem sind aufgrund der gesellschaftlichen und rechtlichen Anerkennung auch mehr Informationen in DGS zugänglich, etwa durch Gebärdensprachdolmetscher(innen) bei Veranstaltungen oder als Einblendungen in Fernsehsendungen (Prillwitz 2001, s.a. Mälzer/Wünsche, Heerdegen-Wessel und Witzel im vorliegenden Band) oder auf Internetseiten (BMGS \& Gebärdenwerk 2004). Dessen ungeachtet spielt Schriftsprache im Alltag von Menschen mit einer Hörbehinderung weiterhin eine wichtige Rolle; so finden Bosse und Hasebrink (2016: 77) in einer Befragung heraus, dass das Internet als (teil-)schriftliche Informationsquelle für diese Zielgruppe eine große Bedeutung hat und von gut 90\% der gehörlosen und knapp 60\% der schwerhörigen Teilnehmer(innen) mehrmals wöchentlich genutzt wird. Und auch die klassische Tageszeitung bleibt eine wichtige rein schriftliche Informationsquelle, auf die 71\% der Gehörlosen und 89\% der Schwerhörigen regelmäßig zurückgreifen. Da insbesondere bei gehörlosen Erwachsenen immer wieder eine durchschnittlich niedrige Lesekompetenz belegt worden ist (Krammer 2001) und ihnen offensichtlich viele relevante Informationen nur schriftlich zugänglich sind, können sie dadurch in ihrer gesellschaftlichen Teilhabe als deutlich beeinträchtigt gelten. Und auch für den beruflichen Erfolg von gehörlosen und schwerhörigen Menschen ist eine hohe Schriftsprachkompetenz eine der wichtigsten Ressourcen: Hintermair et al. (2017: 120ff.) stellen in der retrospektiven Befragung von 32 im Beruf erfolgreichen gehörlosen und schwerhörigen Menschen fest, dass diese fast ausnahmslos die Bedeutung der Schriftsprachkompetenz für ihren Werdegang betonen, ohne dass dies im Fokus der Befragung gestanden hat. Die Relevanz der Schriftsprache für das Berufsleben wird dabei als bedeutsamer eingeschätzt als für Ausbildung, Studium oder Schulzeit (Hintermair et al. 2017: 124).

Wenn insbesondere gebärdensprachkompetente gehörlose Erwachsene schriftliche Texte verfassen, kann es vorkommen, dass diese auf Außenstehende ungewöhnlich wirken. Die Schreiber(innen) können dabei durchaus in der Lage sein, Zusammenhänge in ihrer Primärsprache DGS gut auszudrücken, kennen 
aber in der Zweitsprache Deutsch nicht unbedingt immer die passenden Entsprechungen. Damit gleichen Gehörlose an vielen Stellen anderen Lerner(inne)n von Deutsch als Zweitsprache, indem sie Fehler wie Auslassung von Funktionswörtern und fehlerhafte Genuszuweisungen machen (Krausmann 1998: 585) oder die Kopula, also das Hilfsverb „sein“, auslassen (Plaza-Pust 2016: 410). Es gibt jedoch auch Unterschiede zu hörenden Lerner(inne)n des Deutschen als Zweitsprache, die zuerst eine andere Lautsprache erworben haben. Gebärdensprachen haben eine räumliche Grammatik, bei der zahlreiche sprachliche Einheiten simultan produziert werden. Wenn diese in die lineare Schriftsprache übertragen wird, können insbesondere morphosyntaktische Strukturmerkmale fehlen, die Leser(innen) im Deutschen benötigen, um alle Bezüge im Satz herzustellen. Zudem können andere Lexeme verwendet werden, als eigentlich notwendig, wenn es eine inhaltsgleiche Gebärde mit einem anderen Mundbild gibt. Krausmann (1998: 586ff.) führt eine Reihe von typischen Fehlern auf, die sich insbesondere in Texten gehörloser (und damit auch gebärdensprachkompetenter) Schreiber(innen) finden, und gibt hierfür Beispiele, wie etwa der Zusammenfall von Nomen und Verb („Danach tranken wir Kaffee und Kuchenessen“), die Auslassung von bereits bezeichneten Subjekten oder Objekten („Und danach auch über die Treueprämie gesprochen hatte“, gemeint ist die vorher genannte „Vorsitzende“), die Auslassung von Angaben zur Rollenübernahme, die in Gebärdensprachen durch Körperwendung und Kopfhaltung ausgedrückt wird („Außerdem braucht er für einen Spielfilm mit 1 1/2 Std. mindestens 4-5 Tage den UT zu bearbeiten. Das ist anstrengend. Gibt es eine Möglichkeit, mehr GL-Programme und UT zu bekommen?") und die Auslassung von lautsprachlich notwendigen Ergänzungen bei Kongruenzverben (wie BESCHEID-GEBEN), die in Gebärdensprachen durch die Bewegungsrichtung ausgedrückt werden („dann mir bescheid dieser Woche vielleicht am Freitag od. nächste“). Eine umfassende und systematische Beschreibung der schriftsprachlichen Fehler von gehörlosen Gebärdensprachnutzer(inne)n liegt jedoch nicht vor, was es hörenden Kommunikationspartner(inne)n ohne Gebärdensprachkompetenz nicht leichter macht, alle Aussagen korrekt zu interpretieren. Mit Kenntnis der DGS kann ein Text, der scheinbar wenig verständlich wirkt, z.T. jedoch leichter entschlüsselt werden, da die entsprechenden Leerstellen aufgrund der gebärdensprachlichen Kompetenz gefüllt werden können. 


\section{Konsequenzen für die pädagogische, therapeutische und soziale Arbeit}

Insgesamt können also gehörlose und schwerhörige Erwachsene als Menschen gelten, denen über eine schriftsprachliche Kommunikation nicht unbedingt alle Informationen zugänglich sind und die sich selbst nicht immer vollständig schriftsprachlich ausdrücken können. Wie andere Menschen, bei denen dies der Fall ist, geraten sie deshalb nicht selten in den Verdacht, dass sie insgesamt kognitiv und sprachlich nicht in der Lage sind, komplexere Sachverhalte zu verstehen. Deswegen ist es zunächst wichtig sich bewusst zu machen, dass Menschen mit einer Hörbehinderung aus einer Vielzahl von Gründen in Erwerb und Verwendung der Schriftsprache benachteiligt sein können, ohne dass dies in einem Zusammenhang mit ihren sonstigen (u.a. kognitiven, sprachlichen, sozialen oder berufsbezogenen) Kompetenzen stehen muss.

Um eine sichere Verständigung mit einem gehörlosen oder schwerhörigen Erwachsenen aufzubauen, ist eine unmittelbare Kommunikation häufig geeigneter als die mittelbare schriftliche Form. Dabei ist es wichtig, die Präferenzen des jeweiligen Menschen mit einer Hörbehinderung zu berücksichtigen, also ob sie gebärdensprachlich oder lautsprachlich kommunizieren und ob sie ggf. Zusatztechnik, wie z. B. eine elektronische Übertragungsanlage, verwenden. Bei einem gebärdensprachlich orientierten Menschen kann die Verständigung über eine(n) Gebärdensprachdolmetscher(in) abgesichert werden, der/die rechtzeitig über eine regionale Vermittlungszentrale für Gebärdensprachdolmetscher(innen) bestellt werden sollte und dessen Finanzierung ebenfalls in Absprache mit den Betroffenen geklärt werden muss. Menschen mit einer Hörbehinderung, die primär über Lautsprache kommunizieren, benötigen gute visuelle Bedingungen, um das Mundbild des/der Gesprächspartners/ Gesprächspartnerin zu sehen (gute Beleuchtung, kein Gegenlicht und Blickkontakt während der Kommunikation), einen Raum ohne Störschall, eine deutliche und nicht zu schnelle, aber nicht unbedingt besonders laute Sprache, eine deutliche Gestik und möglicherweise schriftliche Zusatzinformationen. Unabhängig von der Kommunikationsform sollten hörende Gesprächspartner(innen) absichern, dass alle relevanten Informationen verstanden worden sind. 
In einer rein schriftlichen Kommunikation sollte eine zu komplexe Schriftsprache vermieden werden, ohne dass dies auf Kosten der inhaltlichen Aussagen geht. Im Fach Hörgeschädigtenpädagogik wurde dafür das Konzept der Textoptimierung entwickelt, dessen Regeln helfen, schwierige in verständlichere Texte zu überführen (Wagner/Schlenker-Schulte 2009). 


\section{Literaturverzeichnis}

Archbold, Sue/Harris, Margaret/O'Donoghue, Gerard/Nikolopoulos, Thomas/ White, Alison/Lloyd Richmond, HAZEL (2008): „Reading abilities after cochlear implantation: The effect of age at implantation on outcomes at 5 and 7 years after implantation“. In: International Journal of Pediatric Otorhinolaryngology 10, 14711478.

Bébian, Roch-Ambroise-Auguste (1825): Mimographie, ou Essai d'écriture mimique propre à régulariser le langage des sourds-muets. Paris: Louis Colas.

BMGS - BUNDESMINISTERIUM FÜr GesUndHeIT UND SOZIALE Sicherung \& GebÄRDENWERK (2004): „Gebärdensprach-Filme im Internet: Eine Umfrage vom BMGS und Gebärdenwerk im Zeitraum vom 15. Januar bis 20. Februar 2004“. In: Das Zeichen 67, 224-229.

Bosse, Ingo/Hasebrink, Uwe (2016): Mediennutzung von Menschen mit Behinderungen. Bonn: Aktion Mensch. URL: http://kme.tu-dortmund.de/cms/de/Aktuelles/ aeltere-Meldungen/Studie-Mediennutzung-von-Menschen-mit-Behinderung-

_MMB16_/Studie-Mediennutzung_Langfassung_final.pdf (letzter Zugriff: 1.3.2018].

BRINKMANN, VERA (2015): Materialien für den Schriftspracherwerb - Anwendbarkeit für gehörlose und schwerhörige SchülerInnen. Pädagogische Hochschule Heidelberg [unveröff. Zulassungsarbeit].

Chamberlain, Charlene/Mayberry, Rachel (2000): „Theorizing About the Relationship Between American Sign Language and Reading“. In: Chamberlain, Charlene/Morford, Jill/MaYberRy, RACHel (Hg.): Language Acquisition by Eye. Mahwah, NJ: Lawrence Erlbaum Ass., 221-259.

CONRAD, RUdi (1979): The Deaf School Child: language and cognitive function. London: Harper and Row.

Delage, Helene/Tuller, LaURice (2007): „Language development and mild-to-moderate hearing loss: does language normalize with age?" In: Journal of Speech, Language, and Hearing Research 5, 1300-1313.

DilleR, GotTFRIED (2009): „Hörgerichtete Früherziehung und Förderung in Theorie und Praxis“. In: Frühförderung Interdisziplinär 28, 169-179.

DIMDI - DeUTSChes INSTITUT FÜr MEDIZINISChe DoKUMENTATION UND INFORMATION (Hg.) (2005): Internationale Klassifikation der Funktionsfähigkeit, Behinderung und Gesundheit. Köln: Deutsches Institut für Medizinische Dokumentation und Information. URL: http://www.dimdi.de/dynamic/de/klassi/downloadcenter/icf/ endfassung/ (letzter Zugriff: 11.6.2007).

Fischer, Katja/Vogel, Helmut/Fries, Sabine Geissler, Thomas/Rathmann, Christian/Peters, Christian/Goldschmidt, Stefan/Kollien, Simon/Von Meyenn, Alexander (2009): „Leitbild: Deaf Studies in Deutschland“. In: Das Zeichen 83, 438-439. 
Fung, Pan-Chung/Chow, Bonnie Wing-Yin/McBride-Chang, Catherine (2005): „The Impact of a Dialogic Reading Program on Deaf and Hard-of-Hearing Kindergarten and Early Primary School-Aged Students in Hong Kong“. In: Journal of Deaf Studies and Deaf Education 10, 82-95.

GEERS, ANN E. (2006): „Spoken language in children with cochlear implants“. In: SPENCer, Patricia Elizabeth/Marschark, Marc (Hg.): Advances in the Spoken Language Development of Deaf and Hard-Of-Hearing Children. New York: Oxford University Press, 244-270.

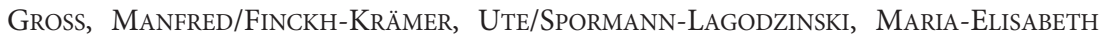
(2000): „Angeborene Erkrankungen des Hörvermögens bei Kindern Teil 1: Erworbene Hörstörungen“. In: HNO 12, 879-886.

Große, KlaUs-Dietrich (2003): Das Bildungswesen für Hörbehinderte in der Bundesrepublik Deutschland: Daten und Fakten zu Realitäten und Erfordernissen. Heidelberg: Universitätsverlag C. Winter (Edition S).

Grote, Klaudia/ZÄH, Kirsten (2016): „Schule \& Inklusion - Wie man Lehramtsstudenten erklärt, was taube Gehörlose und hörende Taublose sind“. In: Das Zeichen $104,2-10$.

GÜNTHER, KLAUS-B. (1985): Schriftsprache bei hör- und sprachgeschädigten Kindern: Bedeutung und Funktion für Sprachaufbau und Entwicklung, dargestellt am Beispiel gehörloser Kinder. 2., verbesserte Auflage, Heidelberg: Groos (Hörgeschädigtenpädagogik Beiheft; 9).

GÜNTHER, KLAUS-B. (1993): „Die Schrift als wahre Basis der Verbalsprache Gehörloser“. In: BAURMANN, JÜrgen/GÜNTHER, HARTMUt/KnOOP UlRICH (Hg): Homo scribens: Perspektiven der Schriftlichkeitsforschung. Tübingen: Niemeyer.

GÜNTHER, KLAUs-B. (2003): „Entwicklung des Wortschreibens bei gehörlosen und schwerhörigen Kindern“. In: dfgs forum 1, 35-70.

GÜNTHER, KlaUS-B. (2011): „Schriftsprachliche Kompetenzentwicklung (1): Schreiben“. In: GÜNTHER, Klaus-B./Hennies, Johannes (Hg.): Bilingualer Unterricht in Gebärden-, Schrift- und Lautsprache mit hörgeschädigten Schülerinnen in der Primarstufe: Zwischenbericht zum Berliner Bilingualen Schulversuch. Hamburg: Signum, 1557 (Sozialisation, Entwicklung und Bildung Gehörloser, 5).

Günther, Klaus-B./HÄnel-Faulhaber, Barbara/Hennies, Johannes (2009): „Bilinguale Frühförderung hochgradig hörgeschädigter Kinder - Entwicklungstheoretische Grundlagen und frühpädagogische Bildungspraxis“. In: Frühförderung Interdisziplinär 4, 179-186.

GÜNTHER, KLAUS-B./HENNIES, JOHANnes (2015): „Abschlussdokumentation zum Berliner Bilingualen Schulversuch: Zusammenfassung der Ergebnisse und Ausblick“. In: Hennies, Johannes/Günther, Klaus-B. (Hg.): Abschlussbericht zum Berliner Bilingualen Schulversuch (DFGS forum - Sonderheft), 86-107. 
GÜNTHER, KLAUS-B./SCHÄFKE, ILKA (2004): Bilinguale Erziehung als Förderkonzept für gehörlose SchülerInnen: Abschlussbericht zum Hamburger Bilingualen Schulversuch. Hamburg: Signum (Sozialisation, Entwicklung und Bildung Gehörloser; 1).

Hänel-Faulhaber, Barbara/Kleyboldt, Thimo/Hagemann, Katrin (2012): „delegs Deutsch lernen mit Gebärdenschrift“. In: Das Zeichen 91, 388-393.

HENNIES, JOHANNES (2010): „Frühförderung hörgeschädigter Kinder: ein aktueller Überblick“. In: dfgs forum 1, 52-57.

HENNIES, JOHANNES (2014): „Laut- und Schriftspracherwerb von gehörlosen und schwerhörigen Kindern“. In: CHILla, SOlveig/HABERZETTL, STEFANIE (Hg.): Mehrsprachigkeit. München: Elsevier, 225-237.

Hennies, Johannes (i.D./erscheint 2018): „Schriftspracherwerb“. In: HocHSTADT, Christiane/Olsen, Ralph (Hg.): Handbuch Inklusive Deutschdidaktik. Weinheim; Basel: Beltz.

Hennies, Johannes/Günther, Klaus-B. (Hg.) (2015): Abschlussbericht zum Berliner Bilingualen Schulversuch (dfgs forum - Sonderheft).

Hintermair, Manfred/Cremer, Inge/Gutjahr, Anja/Strauß, Hand Christoph/ LÖsCH, ANTONIA (2017): „Auf Augenhöhe“: Beruflich erfolgreiche gehörlose und schwerhörige Menschen. Median: Heidelberg.

Hofmann, KRistin/Hennies, JoHAnnes (2015): „Bimodal-bilinguale Frühförderung: Ein Modell zur Evaluation von Input, Sprachnutzung und Kompetenz (EvISK)“. In: Hörgeschädigtenpädagogik 5, 138-144.

Holt, Judith (1993): „Stanford Achievement Test - 8th Edition: reading comprehension subgroup results“. In: American Annals of the Deaf 2, 172-175.

Holt, Judith/TraXler, CAROL/Allen, ThOMAS (1997): Interpreting the Scores: a user's guide to the 9th edition stanford achievement test for educators of deaf and hard-ofhearing students. Washington, DC: Gallaudet University (Gallaudet Research Institute Technical Report: 97-1).

KARCHMER, Michael/MitChell, Ross (2003): „Demographic and Achievement: Characteristics of Deaf and Hard-of-Hearing Students“. In: MARSCHARK, MARC/SPENCER, Patricia Elizabeth (Hg.): Oxford Handbook of Deaf Studies, Language and Education. New York: Oxford University Press, 21-37.

KRAMMER, KLAUDIA (2001): Schriftsprachkompetenz gehörloser Erwachsener. Klagenfurt: Forschungszentrum für Gebärdensprache und Hörgeschädigtenkommunikation. (Veröffentlichungen des Forschungszentrums für Gebärdensprache und Hörgeschädigtenkommunikation der Universität Klagenfurt, 3). In: http://www.uniklu.ac.at/zgh/krammer.pdf (letzter Zugriff: 1.1.2006).

KRAUSMANN, BEATE (1998): „,anders, nicht selten sehr eigenwillig. Schriftsprachliche Kommunikation erwachsener Gehörloser zwischen Normverstößen und Selbstbewußtsein (Teil I)“. In: Das Zeichen 46, 581-591. 
LÖWE, ARMIN (1996): Hörerziehung für hörgeschädigte Kinder: Geschichte - Methoden Möglichkeiten - Eine Handreichung für Eltern, Pädagogen und Therapeuten. 2. überarb. Aufl., Heidelberg: Universitätsverlag C. Winter (Edition Schindele).

Marschark, Marc/Spencer, Patricia Elizabeth (2009): Evidence of best practice models and outcomes in the education of deaf and hard-of-hearing children: an international review. Dublin: NCSE (NCSE Research Reports).

Mayberry, Rachel I./Del Giudice, Alex A./Lieberman, Amy M. (2011): „Reading Achievement in Relation to Phonological Coding and Awareness in Deaf Readers: A Meta-analysis“. In: Journal of Deaf Studies and Deaf Education 16, 164-188.

MAYBERRY, RACHEL I./LOCK, ElizABETH/KAZMI, HenA (2002): „Development: Linguistic ability and early language exposure“. In: Nature 417, 38-38.

Meir, Irit/Sandler, Wendy/Padden, Carol/Aronoff, Mark (2010): „Emerging Sign Languages“. In: MARschark, Marc/Spencer, Patricia Elizabeth (Hg.): $O x-$ ford Handbook of Deaf Studies, Language, and Education: Volume 2. Oxford: Oxford University Press, 267-280.

Mitchell, Ross/Karchmer, Michael (2004): „Chasing the Mythical Ten Percent: parental hearing status of deaf and hard of hearing students in the united states“. In: Sign Language Studies 2, 138-63.

Moeller, Mary Pat/Tomblin, J. Bruce, Yoshinaga-Itano, Christine/McDonald CONNOR, CAROL/JERGER, SUSAN (2007): „Current State of Knowledge: Language and Literacy of Children with Hearing Impairment“. In: Ear and Hearing 6, 740-753.

Niparko, John K./Tobey, Emily A./Thal, Donna J./Eisenberg, Laurie S./Wang, Nae-Yuh/Quittner, Alexandra L./Fink, NAncy E. (2010): „Spoken Language Development in Children Following Cochlear Implantation“. In: JAMA 303, 14981506.

Padden, Carol/Humphries, Tom (1991): Gehörlose: Eine Kultur bringt sich zur Sprache. Hamburg: Signum (Internationale Arbeiten zur Gebärdensprache und Kommunikation Gehörloser; 16).

PlazA-Pust, CARolina (2016): Bilingualism and Deafness: On Language Contact in the Bilingual Acquisition of Sign Language and Written Language. Boston/Berlin: Walter de Gruyter (Sign Languages and Deaf Communities; 7).

Prillwitz, Siegmund (2001): Angebote für Gehörlose im Fernsehen und ihre Rezeption. Kiel: Unabhängige Landesanstalt für das Rundfunkwesen (ULR) (Themen-ThesenTheorien; 17).

SCHÄFKE, ILKA (2005): Untersuchungen zum Erwerb der Textproduktionskompetenz bei hörgeschädigten Schülern. Hamburg: Signum (Sozialisation, Entwicklung und Bildung Gehörloser, 4).

SCHMID-GIOVANNINI, SusAnNA (1976): Sprich mit mir: Eine ganzheitliche Lautsprachmethode für Kleinkinder von 0-7 Jahren: Für Eltern und Erzieher hörgeschädigter Kleinkinder. Berlin: Marhold. 
SCHRÜNDER-LENZEN, AgI (2013): Schriftspracherwerb und Unterricht. 4., überarb. Auflage. Wiesbaden: VS Verlag für Sozialwissenschaften.

Schulte, Klaus/Schlenker-Schule, Christa (1983): Sprech-Lehr-Programm Artikulation Hörgeschädigter (Begleitvideo). Göttingen: IWF.

STERN, L. William (1905): Helen Keller: Die Entwicklung und Erziehung einer Taubblinden als psychologisches, pädagogisches und sprachtheoretische Problem. Berlin: Reuther \& Reichard.

STOCKER, KURT (2002): Spracherwerb beim hörgeschädigten Kind: Cochlea-Implantat, Gebärden und Frühstschriftsprache. Luzern: Edition SZH/SCP

SutTon, VAlERIE (1981): Sign writing for everyday use. Boston \& Newport Beach: Sutton Movement Writing.

SWANWICK, RUTH/WATSON, LINDA (2005): „Literacy in the homes of young deaf children: common and distinct features of spoken language and sign bilingual environments“. In: Journal of Early Childhood Literacy 5, 53-78.

SZAGUN, GiSEla (2010): „Einflüsse auf den Spracherwerb bei Kindern mit Cochlea Implantat: Implantationsalter, soziale Faktoren und die Sprache der Eltern“. In: hörgeschädigte kinder - erwachsene hörgeschädigte 1, 8-36.

TRAXLER, CAROL (2000): „The Stanford Achievement Test, $9^{\text {th }}$ Edition: national norming and performance standards for deaf and hard-of-hearing students“. In: Journal of Deaf Studies and Deaf Education 4, 341-348.

Vermeulen, Anneke M./VAn Bon, Wim/Schreuder, Rob/Knoors, Harry/Snik, Ad (2007): „Reading comprehension of deaf children with cochlear implants“. In: Journal of Deaf Studies and Deaf Education 3, 283-302.

WAGner, SusAnne/SCHLENKER-SCHUlte, CHRISTA (2009): Textoptimierung von Prüfungsaufgaben: Handreichung zur Erstellung leicht verständlicher Aufgaben. 5., überarb. Auflage. Halle (Saale): Forschungsstelle zur Rehabilitation von Menschen mit kommunikativer Behinderung e. V. an der Martin-Luther-Universität Halle-Wittenberg. In: http://wcms.uzi.uni-halle.de/download.php?down=14524\&elem= 2279959 (letzter Zugriff: 25.3.2018).

Walther, Eduard (1895): Handbuch der Taubstummenbildung. Berlin: Staude.

Whitehurst, Grover J./FAlCo, Francie L./LOnigan, Christopher J./Fischel, JANet E./ DeBaryshe, Barbara D./Valdez-Menchaca, Marta C./Caulfield, Marie (1988): „Accelerating language development through picture book reading“. In: Developmental Psychology 24, 552-559.

Wimmer, Eva/Rothweiler, Monika/Hennies, Johannes/Hess, Markus/Penke, MARTINA (2015): „Comprehension of who questions in German children with hearing impairment“. In: HAMANN, CORNELIA/RuigendiJ, EsTHER (Hg.): Language Acquisition and Development: Proceedings of GALA. Cambridge [u.a.]: Cambridge Scholars Publishing, 584-603.

WisotZKI, KARL-HeINZ (1994): Grundriss der Hörgeschädigtenpädagogik. Berlin: Marhold (Grundrisse der Sonderpädagogik; 4). 
Johannes Hennies

WÖHRMANN, STEFAn (o.J.): „Einblicke in unseren Schulalltag“. In:

http://www.gebaerdenschrift.de/school/index_schule2.htm (letzter Zugriff: 20.3.2018).

WÖHRMANN, STEFAn (2005): Handbuch zur GebärdenSchrift. Hamburg: Birgit Jacobsen. 
SASKIA SCHUPPENER/BETTINA M. BOCK

\section{Geistige Behinderung und barrierefreie Kommunikation}

\section{Annäherung an den Personenkreis von Menschen mit sogenannter geistiger Behinderung}

Die Annäherung an Menschen in Form von Definitionen ist stets ein Blick von außen. Dies trifft im Besonderen auf eine definitorische Annäherung zu, die sich der Frage nach den Kriterien einer sogenannten "geistigen Behinderung“ stellt. Derartige Definitionsentwürfe stellen grundsätzlich Versuche externer Zuschreibungen dar: „Es gibt Menschen, die wir aufgrund unserer Wahrnehmung ihrer menschlichen Tätigkeit, im Spiegel der Normen, in dem wir sie sehen, einem Personenkreis zuordnen, den wir als ,geistigbehindert' bezeichnen " (Feuser 1996: 18; Hervorh. i. Orig.).

„Eine allgemein anerkannte Definition von Behinderung liegt bis zum heutigen Tag nicht vor, obwohl der Begriff seit einigen Jahrzehnten im allgemeinen Sprachgebrauch gängig und wissenschaftlich etabliert ist" (Dederich 2009: 15). Bei dem, was wir gesellschaftlich als „Behinderung“ markieren, handelt es sich

einerseits um einen Begriff[,] der deutungsbedürftig ist[,] und andererseits um einen Status[,] den ein Mensch erhalten bzw. zugewiesen bekommen kann. Es dreht sich also um die Differenz zwischen einer subjektiven und einer objektiven Sinnkonstruktion von Behinderung. Beides birgt Probleme, denn es gibt kein universelles Merkmal, anhand dessen festgestellt werden kann, ob ein Mensch ,behindert' ist oder nicht. Somit kann eine je individuell-subjektive Konstruktion von Behinderung zu objektiver Behinderung werden - muss sie aber nicht (Trescher 2014: o.S.). 
Vor dem Hintergrund eines „Sozialen Modells von Behinderung“ ist diese immer als negative Form der Abweichung von sozial gesetzten Normen zu verstehen (ebd.) und ist „in diesem Sinn und je nach Perspektive und Kontext das Ergebnis eines Wahrnehmungs- und Deutungsprozesses angesichts von erwartungswidrigen Merkmalen oder Eigenschaften eines Individuums" (Dederich 2009: 37).

Menschen, die im Speziellen als geistig behindert adressiert werden, sind oftmals in beträchtlichem Ausmaß auf Unterstützung, nicht selten auch auf Interpretationen ihrer individuellen Ausdrucksformen angewiesen, die wiederum auf einer Basis der Intersubjektivität und sozialen Interdependenz stattfinden sollte. Es sollte eine Sensibilität dafür bestehen, dass für Menschen, die im Laufe ihrer Biografie mit der Diagnose „geistig behindert“ konfrontiert wurden, auch ein hohes Risiko darin besteht, im „Bann der Defizite“(Haupt 1998: 100) wahrgenommen zu werden, da Definitionen einer "geistige[n] Behinderung“ z. T. eine Merkmalsbündelung von Defiziten und Beeinträchtigungen in nahezu allen Entwicklungsbereichen kennzeichnen. Feuser schlug schon 1976 eine Definition vor, die auch auf der Basis eines ableistischen Zugangs in aktuellen Diskursen Bedeutsamkeit hat:

Stellen wir fest, dass als geistigbehindert gilt, wer aufgrund organischer, genetischer oder anderweitiger Schädigungen, insbesondere durch Beeinträchtigungen infolge sozioökonomischer Benachteiligungen und sozialer Isolation, in seinen Aufnahme- und Verarbeitungskapazitäten, die sich besonders im Zusammenhang von Wahrnehmung, Denken und Handeln sowie in der Sensomotorik zeigen, derart beeinträchtigt ist, dass er angesichts der vorliegenden Lernfähigkeit zur Befriedigung seines besonderen Erziehungs- und Bildungsbedarfs voraussichtlich lebenslanger spezieller pädagogischer und sozialer Hilfen bedarf (Feuser 1976: 643f.).

Der Ableismus beschreibt Ideen, Überzeugungen, aber auch Praktiken, die ihren Ausgang von einem "nichtbehinderten Ideal“ nehmen und Behinderung auf der Basis fähigkeitsindividualistischer Praktiken (vgl. Buchner/Pfahl/Traue 2015) als „verminderter Zustand des Menschseins“ beschreiben (Campbell 2008 in Maskos 2015: o.S.). „Das aus den Disability Studies stammende Konzept 
des Ableism stellt die vermeintliche Normalität einer individualisierten Zurechnung von Fähigkeiten grundlegend in Frage und bietet dadurch wichtige analytische und kritische Möglichkeiten für Debatten um Inklusion“ (Meißner 2015: o.S.).

Im schulischen Kontext werden Kinder und Jugendliche mit dem Diagnosehintergrund einer geistigen Behinderung in der Regel mit dem Etikett des Förderschwerpunktes „Geistige Entwicklung“ (= FsgE) konfrontiert. Hierunter fallen dann auch Schüler(innen) mit einer sogenannten schweren und mehrfachen Behinderung (vgl. u.a. Dworschak/Kannewischer/Ratz/Wagner 2012). Insbesondere Einschränkungen in der Kommunikation kennzeichnen diesen Förderschwerpunkt, da „Beeinträchtigungen in der geistigen Entwicklung“ in der Regel auch Auswirkungen auf „die kommunikative Aufnahme-, Verarbeitungsund Darstellungsfähigkeit" haben (KMK 1998: o.S.).

Die Reduktion auf ein diagnostisches Etikett (= „geistige Behinderung“ oder „Förderschwerpunkt geistige Entwicklung“) wird seit Existenzbeginn an aufgrund stigmatisierender Wirkungen kontrovers diskutiert (vgl. Bock 2015). Das Selbstvertreter(innen)-Netzwerk „Mensch zuerst - Netzwerk People First Deutschland e.V." distanziert sich daher auch von dieser Begrifflichkeit und der damit verbundenen Zuschreibung und favorisiert den Terminus „Menschen mit Lernschwierigkeiten“in der Selbstbezeichnung: „Menschen mit Lern-Schwierigkeiten werden manchmal auch geistig behindert genannt. Das wollen wir nicht. Denken hat nichts mit dem Geist zu tun. Deshalb nennen wir uns Menschen mit Lern-Schwierigkeiten" (Mensch zuerst 2018).

\section{Teilhabe - Partizipation}

Vor dem Hintergrund eines gesellschaftlichen Ökonomisierungsdrucks sowie einer „rohen Bürgerlichkeit“ (Benkmann 2014) als gegenwärtiger Grundhaltung in unserer Gesellschaft erscheint die Frage nach Teilhabe oder Partizipation von Menschen in marginalisierten Lebenslagen als bedeutsam und herausfordernd gleichermaßen. „Partizipation wird häufig synonym mit den Begriffen Teilnahme, Teilhabe, Beteiligung oder Mitbestimmung verwendet" (Prosetzky 
2009: 88) und in ihrer Bedeutung oft unhinterfragt positiv besetzt und moralisch überhöht (ebd.; vgl. auch Niehr i.Dr.), was auch mit daran liegt, dass die Forderung nach Partizipation und Teilhabe doch eine der zentralen Forderungen innerhalb der Behindertenpädagogik darstellt. Mit Blick auf Menschen, die als behindert adressiert werden, wird Partizipation nicht nur als „aktive Beteiligung in einem sozialen System“, sondern gleichsam als ein „Recht auf Mitsprache, konkrete Mitgestaltungsmöglichkeiten sowie Mitbestimmung“ definiert (Schwalb/Theunissen 2018: 9) und sollte auch basale Formen der Mitwirkung von Menschen mit Komplexer Behinderung mitberücksichtigen (vgl. Schuppener 2016).

Bei der Frage nach der pragmatischen Realisierung von Partizipation muss zunächst berücksichtigt und reflektiert werden, dass der Diskurs um Teilhabe und Partizipation immer auch mit dem gesellschaftlichen Anspruch an Demokratie, Gerechtigkeit, Parteilichkeit und Institutionalisierung verbunden ist (vgl. Bärmig 2015). Der zuletzt genannte Aspekt ist im Speziellen für Menschen mit zugewiesener geistiger Behinderung von zentraler Bedeutung: Auch im Zeitalter der Post-Deinstitutionalisierung (vgl. Kremsner/Proyer 2016) findet ein hoher Anteil des Lebensalltags von Kindern, Jugendlichen und Erwachsenen mit dem Label "geistig behindert" in Institutionen statt; nicht selten lassen sich nach wie vor Strukturen „totaler Institutionen“ (Goffman 1993) finden. Institutionen der Behindertenhilfe sind Orte der Produktion und Reproduktion von Hierarchien und Machtstrukturen. Sie werden von Kremsner und Proyer (2016) in Anlehnung an Gramsci als „Hegemonialapparate“ bezeichnet, die zwangsläufig mit einem hohen $\mathrm{Ma}$ an Autonomieverlust verbunden sind. Es findet eine Regelunterwerfung statt, die für die dort lebenden Personen mit Unfreiheit und Unterdrückung einhergeht. Wenn nun beispielsweise von Dworschak/ Schmidt (2011) bei Teilhabe von einem Menschenrecht gesprochen wird, welches auf das „Vorhandensein der Zugangsmöglichkeiten zu Lebensbereichen, der Integration in Lebensbereiche und der Daseinsentfaltung in Lebensbereichen" rekurriert (Dworschak/Schmidt 2011: 276), dann stellt sich die Frage, ob dieser Anspruch für Menschen, die in institutionellen Strukturen leben, überhaupt einlösbar ist. Mit Bezugnahme auf Petersen (2007) spricht Prosetzky (2009) daher davon, dass von Partizipation nur die Rede sein kann, wenn 
Machtverhältnisse reflektiert und verändert werden. Die Veränderung der „Polarisierung zwischen einem Pol der Macht und einem Pol der Ohnmacht" (Jantzen 2005: 206) ist deshalb so herausfordernd, weil sich Einrichtungen der Behindertenhilfe gerne als Orte der „Autonomieförderung“ verstehen und den Anspruch der „Beziehungsarbeit“ betonen, aber fraglich bleibt, ob dies nicht auch eine gewisse Paradoxie darstellt: Jantzen spricht bezüglich eines autonomiefördernden institutionellen Selbstverständnisses auch von der „Vorderbühne der Einrichtung“, die auf der „Hinterbühne [...] Unterdrückung [...] gleichzeitig aufrecht erhält" (ebd.: 207; Hervorh. i. Orig.). Auf Partizipation ausgerichtete Veränderungen können nur stattfinden über Wege der Achtung, Anerkennung, des Vertrauens und der Akzeptanz, die sich in der kommunikativen Begegnung ausdrücken. Ausgangspunkt einer derartigen dialogischen Grundhaltung ist die Reflexion von Machtstrukturen im (kommunikativen) Miteinander: Dies beginnt damit, „so wie jede Freiheit beginnt, ,nein` zu Macht zu sagen: In diesem Fall zur eigenen Macht“ (ebd.: 221) und meinem Gegenüber emphatisch zu begegnen, seine Grenzen zu akzeptieren, zu versuchen, ihn vor dem Hintergrund seiner biografischen Erfahrungen zu verstehen. Nur darüber kann Partizipation erfolgen: durch Kommunikation mit „freundlichen Begleitern“ (vgl. Prosetzky 2009 mit Bezug auf Jantzen 2005). Daher kommt einer voraussetzungslosen Kommunikation elementare Bedeutung zu, wenn es um die Frage nach der Teilhabe, Teilnahme und der Partizipation von Menschen geht, die als geistig behindert wahrgenommen werden und in einem allerersten Schritt auf Parteilichkeit und Empathie angewiesen sind. Die Praxisstrukturen dürfen sich dann in einem weiteren Schritt nicht durch ein „Festhalten an Machtstrukturen“ kennzeichnen, da dies als „Handeln wider besseren Wissens entweder als unmoralisch, unvernünftig oder sinnlos“ zu bewerten ist (ebd.: 94) und eine zentrale Barriere im Rahmen der Realisierung von Partizipation verkörpert.

Den obigen Ausführungen folgend soll zusammenfassend von einem mehrdimensionalen Verständnis von Partizipation ausgegangen werden, welches von den Rechten von Menschen mit Behinderungserfahrungen ausgeht (vgl. Biewer 2017) und die häufig noch institutionalisierten Lebensbedingungen mitdenkt. Demzufolge ist Partizipation zum Einen „die Beteiligung an und die Identifikation mit bestimmten Institutionen, Werten und sozial relevanten Kräften einer Gesellschaft“ und zum Anderen ein ,engagiertes und sich in praktisch-politischer 
Arbeit manifestierendes Beteiligen an demokratischen Strukturen und Prozessen“ (Hillmann 2007: 667). Dieser Anspruch „verträgt sich nicht mit der traditionellen Behindertenarbeit, die von einem defekt- und defizitorientierten Denken und Handeln geprägt war und da und dort noch ist, welches Menschen mit Behinderungen als versorgungs-, behandlungs- und anweisungsbedürftige Mängelwesen betrachtet" (Schwalb/ Theunissen 2018: 9). Vor dem Hintergrund differenter (institutionell und strukturell geprägter) Grundhaltungen muss insbesondere eine „Scheinpartizipation“ vermieden werden, in welcher die Menschen mit sogenannter geistiger Behinderung lediglich innerhalb vorgegebener Konzepte und Institutionsstrukturen agieren können, außerhalb derer ein Agieren jedoch für sie nicht möglich ist (vgl. Schwab 2016).

\section{Sprach- und Schriftsprachkompetenzen}

Sprachliche und insbesondere literale Kompetenzen sind unbestritten eine wichtige Voraussetzung für gesellschaftliche Teilhabe (vgl. Dönges/Stegkemper/ Wagner i.Dr.; Tschernig/Vo Thi 2017; Bock i.Vorb.a). Kommt es zu einer Nichtpassung zwischen Handlungsdisposition des Einzelnen und der Alltagswirklichkeit, sind die Teilhabemöglichkeiten eingeschränkt (Dönges/Stegkemper/ Wagner i.Dr.).

Allgemeine Darstellungen zu den sprachlichen Kompetenzen des hier adressierten Personenkreises beinhalten oft nur unspezifische Hinweise: Es werden generelle Entwicklungsverzögerungen und besondere Entwicklungsverläufe im Spracherwerb benannt. Bei Kindern, Jugendlichen und Erwachsenen mit zugeschriebener geistiger Behinderung „muss man [...] - unabhängig vom Lebensalter - mit allen möglichen Sprachkompetenzniveaus rechnen und steht damit vor einer großen [...] Herausforderung" (Aktas 2012: 47). Grundsätzlich treten Sprachentwicklungsstörungen in allen Bereichen (Phonologie, Wortschatz, Morphologie, Syntax, Pragmatik) deutlich häufiger als bei Kindern ohne eine Diagnose aus dem Bereich geistiger Behinderung auf (vgl. Wagner/Kannewischer 2012: 103). Insgesamt liegen jedoch zu den Sprach- und Schriftsprachkompetenzen des gesamten Personenkreises, der als geistig behindert gilt, keine umfassenden empirischen Befunde vor. Die vorhandenen empirischen Studien 
beschränken sich meist auf Teilgruppen, die sich vermeintlich relativ gut abgrenzen lassen, vor allem der Bereich der sogenannten genetischen Syndrome wird in den Blick genommen (insbesondere Down-Syndrom, Williams-BeurenSyndrom etc. oder auch das Autismus-Spektrum).

Es werden im Folgenden einige Aspekte der Sprach- und Schriftsprachkompetenz näher betrachtet. Im Fokus stehen hier insbesondere empirische Studien, die nicht zuletzt mit Blick auf nach wie vor offene Fragen vorgestellt und diskutiert werden.

\subsection{Sprachliche und kommunikative Kompetenzen}

Die sprachlichen und kommunikativen Kompetenzen von Menschen mit zugewiesener geistiger Behinderung sind sehr unterschiedlich. Sie reichen von eingeschränkter kontextgebundener nicht-sprachlicher Kommunikationsfähigkeit bis zu „annähernd normalem Sprachgebrauch“ (Wilken 2009: 114). Diagnostizierte Beeinträchtigungen kognitiver Kompetenzen äußern sich häufig in Form von veränderten Wahrnehmungen und veränderter Verarbeitung von Reizen und Informationen. Dies wirkt sich mehr oder weniger stark auch auf die kommunikativen Kompetenzen und den Spracherwerb aus. Fast immer ist der Spracherwerb bei Kindern mit einer diagnostizierten geistigen Behinderung mehr oder weniger stark verzögert, wobei die sprachliche Retardierung meist nicht niveaugleich zur sonstigen Entwicklung verläuft (ebd.). Als potentiell betroffen von Einschränkungen werden alle Bereiche der Sprachkompetenz genannt: Einengung des produktiven und rezeptiven Wortschatzes, Schwierigkeiten im Bereich Grammatik sowie im Bereich Pragmatik. In der Kommunikation können allerdings Schwächen in einem Bereich durch Stärken in einem anderen ausgeglichen werden. So haben beispielsweise Menschen mit DownSyndrom oftmals „pragmatische Fähigkeiten, die in Verbindung mit der häufig guten sozialen Kompetenz trotz eingeschränkter Sprache eine relativ hohe Kommunikationsfähigkeit bewirken“ (Wilken 2000: 162). Das Niveau der Sozialkompetenz liegt bei diesem Personenkreis meist über dem Intelligenzniveau, was u.a. auch das aktive Einbringen und Lernen in inklusiven Strukturen erleichtern kann (Schuppener 2008: 99). 
Viele Menschen mit zugeschriebener geistiger Behinderung haben weitere Einschränkungen, wie Hör- und Sehbeeinträchtigungen, Wahrnehmungsschwächen im visuellen, auditiven, taktilen oder kinästhetischen Bereich. Dies macht entsprechend unterschiedliche Formen von Kommunikationsunterstützung und barrierefreier Kommunikation nötig (vgl. u.a. Musenberg im vorliegenden Band; Kahlisch/Dobroschke im vorliegenden Band). Zwischen dem Umfang der Sprachbeherrschung und den kognitiven Beeinträchtigungen besteht kein unmittelbarer Zusammenhang, auch wenn Diagnosen aus dem Spektrum sogenannter Komplexer Behinderung (vgl. Fornefeld 2008) oftmals mit entsprechend hohem Unterstützungsbedarf im Bereich der Kommunikation einhergehen. Es können jedoch große Unterschiede zwischen den rezeptiven und den produktiven sprachlichen Kompetenzen bestehen: Oft ist das Sprachverständnis „deutlich weiter entwickelt als die verbalen [d.h. produktivmündlichen, S.Sch./B.B.] Fähigkeiten vermuten lassen“ (Wilken 2009: 114; vgl. auch Schuppener 2008: 99). Das führt dazu, dass die kognitiven Fähigkeiten insgesamt häufig unterschätzt werden. Die Diskrepanz zwischen Sprachverständnis und Ausdrucksvermögen und die daraus resultierende Fehleinschätzung der tatsächlichen Kompetenzen führt auch dazu, dass die betreffenden Personen in Kommunikationssituationen entweder über- oder unterfordert werden (Wilken 2009: 120). Allgemein kann nicht von einer einfachen Entsprechung zwischen den Niveaus der sozialen, sprachlichen und kognitiven Fähigkeiten ausgegangen werden: Im Vergleich mit den sozialen und sprachlichen Kompetenzen werden speziell die Denkniveaus von Menschen mit sogenannter geistiger Behinderung „fast regelmäßig unterschätzt“" (Schuppener 2008: 98).

Es fehlen bislang empirische Studien, die die sprachlichen Kompetenzen und Schwierigkeiten, insbesondere bei Erwachsenen mit sogenannter geistiger Behinderung, detaillierter beschreiben (zu einer Grammatikstudie vgl. Kap. 4). Die einzige umfassende empirische Studie aus Deutschland hat die sprachlichen Kompetenzen der Schülerschaft im Förderschwerpunkt geistige Entwicklung in Bayern dokumentiert. Die Ergebnisse sind aus fragebogenbasierten Einschätzungen durch Lehrkräfte hervorgegangen; insgesamt wurden auf diesem Wege 1629 Kinder und Jugendliche erfasst, die an Förderschulen in Bayern beschult werden (Dworschak/Kannewischer/Ratz/Wagner 2012). Einer der abgefragten Bereiche waren die sprachlichen Kompetenzen (Wagner/Kannewischer 2012: 
105ff.). Dabei zeigte sich, dass im Bereich expressive mündliche Sprache fast 27\% der Schülerinnen und Schüler komplexe Sätze verwenden und mehr als ein Drittel Mehrwortsätze spricht. Weitere 19,5\% der Schüler(innen) verwenden lediglich Einwort- oder Zweiwortsätze und etwas mehr als 19\% verfügen über keine Lautsprache. Im Hinblick auf die rezeptiven Sprachkompetenzen haben die befragten Lehrkräfte bei fast $49 \%$ der Schüler(innen) angegeben, dass diese einzelne Wörter, einfache Sätze und Anweisungen verstehen können, etwas mehr als $41 \%$ verstehen auch komplexe Sätze und Anweisungen (bei den restlichen 9,9\% der Schülerinnen und Schüler konnten die Befragten das Sprachverständnis nicht einschätzen). Sprach- und Sprechstörungen liegen nach Einschätzung der Lehrkräfte bei $68,2 \%$ der Schülerinnen und Schüler vor (ebd.: 108), was deutlich über den Prävalenzangaben für die Gesamtbevölkerung liegt. Erfragt wurde in der Studie außerdem, welche Kommunikationsformen und -hilfen von Bedeutung sind: Nach Angabe der Lehrkräfte kommunizieren $16,3 \%$ der Schüler(innen) mit Hilfe von Gebärden, fast 32\% greifen zur Kommunikation auf Bilder und fast $26 \%$ auf Symbole zurück.

Darüber hinaus gibt es im englischsprachigen Raum eine Reihe empirischer Studien, die sich allerdings meist auf einzelne Teilgruppen beziehen. Es werden nach wie vor Forschungslücken beklagt (vgl. Abbeduto/Warren/Conners 2007: 257f.), insbesondere zu (allgemeinen und syndromspezifischen) Vorläuferfertigkeiten und nichtsprachlichen Faktoren, die den Spracherwerb beeinflussen (wie phonologisches Kurzzeitgedächtnis, Hörschärfe), zu Zusammenhängen zwischen sozialen Fähigkeiten (social cognition) und Wortschatzerwerb sowie zu den pragmatischen Fähigkeiten (vgl. auch Hatton 1998; Abbeduto/Hesketh 1997). Es fehlen Longitudinalstudien und insbesondere Studien zu den sprachlichen und kommunikativen Kompetenzen von Jugendlichen und Erwachsenen mit Diagnosen aus dem Spektrum sogenannter geistiger Behinderung (vgl. Abbeduto/Warren/Conners 2007: 257). Aufgrund dieser Forschungslage sind auch Erkenntnisse zu wirksamen Interventionen und Fördermaßnahmen weitgehend Desiderat (ebd.: 258). 


\subsection{Lesen und Schreiben}

Lange Zeit stand in Schulen zum Förderschwerpunkt geistige Entwicklung die sogenannte "praktische Bildbarkeit" der Schüler/-innen im Vordergrund, und der Erwerb schriftsprachlicher Kompetenzen spielte keine oder nur eine untergeordnete Rolle. Mittlerweile gilt das „Bildungsziel Schriftsprache als einer zentralen Kulturtechnik [...] heute auch im Förderschwerpunkt geistige Entwicklung (FsgE)“ als unbestritten (Ratz 2012: 111). Ähnliches gilt für den englischsprachigen Raum, allerdings wird beklagt, dass der Erwerb schriftsprachlicher Kompetenzen praktisch nur eine untergeordnete Rolle einnehme:

Despite the importance of literacy in today's society, surprisingly little attention has been given in schools to teaching reading to individuals with DS [Down-Syndrom; S.S./B.B.], perhaps because of the assumption that their limited language skills will make learning to read impossible. Nevertheless, many individuals with DS do acquire basic literacy skills, although they may do so by a different route than their typically developing peers. There is a need for more research on the strategies used by individuals with DS as they struggle with print (Abbeduto/Warren/ Conners 2007: 258).

Als einen Grund nehmen die Autor(inn)en also an, dass die erreichbaren Kompetenzen der Kinder unterschätzt werden. Darauf deuten auch empirische Studien aus diesem Bereich hin (s.u.). Ein anderer Grund, weshalb Kinder mit dem diagnostizierten Förderschwerpunkt geistige Entwicklung möglicherweise zu wenig mit Schriftlichkeit konfrontiert werden, könnte darin liegen, dass durch die lückenhafte Forschungslage wenig über zielgruppengemäße, effektive Methoden des Lesen- und Schreibenlernens bekannt ist. Die Forschung zu Wegen des Schriftspracherwerbs, zum Leseprozess, zu angemessener Förderung und zur Verteilung von Lese- und Schreibkompetenzen bei Menschen, die als geistig behindert gelten, ist noch deutlich ausbaufähig.

Wie schon bei den sprachlich-kommunikativen Kompetenzen sind also auch in diesem Bereich noch Forschungsdesiderate zu beklagen. Insgesamt werden eher die frühen Stufen des Schriftspracherwerbs und hierachieniedrige Prozess- 
ebenen sowie Vorläuferfertigkeiten des Lesens in den Blick genommen, während Forschungen zu Textkompetenzen bei Menschen mit zugewiesener geistiger Behinderung höchstens am Rande miterfasst werden. Vorliegende Studien zu Lesekompetenzen zielen häufig auf quantitativ messbare Teilfertigkeiten, d.h. es werden v.a. die hierarchieniedrigen Verarbeitungsprozesse erfasst. Aufwendigere qualitative Studien, die höhere Prozessebenen wie das Textverstehen und pragmatische Teilfertigkeiten erfassen würden, sind hier noch selten.

In der deutschsprachigen Forschung finden sich empirische Studien zur phonologischen Bewusstheit und zum Rekodieren: Koch (2008) hat untersucht, welche Teilfertigkeiten im Bereich phonologischer Bewusstheit für Kinder mit Down-Syndrom tatsächlich relevant sind für das Lesenlernen. Kuhl, Euker und Ennemoser (2015) haben die Wirkung eines silbenbasierten Rekodiertrainings auf das lautorientierte Lesen überprüft. Die einzige empirische Studie aus dem deutschsprachigen Raum, die die Lese- und Schreibkompetenzen des gesamten Personenkreises in seiner Heterogenität untersucht hat, ist dieselbe bereits oben zitierte Studie, die sich auf die Schülerschaft im Förderschwerpunkt geistige Entwicklung in Bayern bezieht (Ratz 2012). Auch die schriftsprachlichen Fähigkeiten wurden über fragebogenbasierte Einschätzungen der Lehrkräfte erfasst. Dokumentiert wurden auf diesem Wege die Lese- und Schreibkompetenzen von Schülerinnen und Schülern mit FsgE im Alter zwischen 6 und 21 Jahren in drei Schulstufen (Grundschul-, Hauptschul- und Berufsschulstufe). Die Kategorien im Lehrerfragebogen sind auf die Schriftsprach-Entwicklungsstufen nach Valtin bezogen. Die Studie ergab, dass 12\% der Schülerinnen und Schüler in der sog. Berufsschulstufe (10.-12. Schulbesuchsjahr) nach Einschätzung der Lehrkräfte sehr flüssig lesen, $21,1 \%$ „fortgeschritten“ lesen, aber immerhin 33\% (noch) überhaupt nicht lesen (vgl. Ratz 2012: 122). Der Anteil derjenigen, die überhaupt nicht lesen, ist in allen Schulstufen etwa gleichbleibend. Dieser Anteil entspricht grob dem Anteil von Schüler(inne)n mit Komplexer Behinderung (ebd.: 118). Hinsichtlich der Entwicklung der Fähigkeiten über die Schulzeit hinweg werde insgesamt „sehr gut sichtbar, dass die Schüler immer besser lesen können, je länger sie in der Schule sind“ (ebd.: 119). Im Bereich Schreiben sind die Ergebnisse vergleichbar: In der Berufsschulstufe zeigen 18,5 \% der Schülerinnen und Schüler eine phonetische Schreibweise, 17,3 \% verwenden ortho- 
grafische Muster und nur 4\% haben nach Einschätzung der Lehrkräfte gute orthografische Kenntnisse. Ein relativ großer Anteil von 31,1\% schreibt noch überhaupt nicht oder „kritzelt“.

Zusammenfassend ist festzuhalten, dass die schriftsprachlichen Fähigkeiten sehr streuen (ebd.: 127). Das Lesen ist dem Schreiben in der Fähigkeitsentwicklung zeitlich immer voraus. Zudem ist das Lesen

bei den Schülern des FsgE [...] deutlich weiter entwickelt als das Schreiben. Dieses Ergebnis stellt Fragen an das zugrundeliegende Entwicklungsmodell des Schriftspracherwerbs, das von einer parallelen Entwicklung ausgeht, in der sich Fortschritte im Lesen und Schreiben gegenseitig fördern (ebd.: 128).

Das Leseverständnis wurde in der Studie nicht erfasst, der Fragebogen enthielt aber zumindest eine Frage, die eine grobe Einschätzung des Leseprozesses fordert: Beurteilt werden sollte, ob mechanisches oder sinnerfassendes Lesen vorliegt. Insgesamt schätzen die Lehrkräfte ein, dass im Durchschnitt mehr als die Hälfte der Schülerinnen und Schüler sinnerfassend liest und dieser Anteil mit zunehmendem Alter steigt (ebd.: 123). Welche Konzepte von ,sinnerfassendem Lesen ' die Lehrkräfte hier heranziehen und worauf sie ihre Beurteilungen stützen, bleibt allerdings unbestimmt. Vergleicht man die Studienergebnisse mit empirischen Daten aus der regulären Grundschule zeigt sich, dass Schüler(innen) im FsgE insgesamt langsamer Lesen und Schreiben lernen als Grundschüler(innen) in Regelschulen (ebd.: 129).

Studien, die die Lesekompetenzen und insbesondere das Leseverstehen direkt erfassen und auf Textkompetenzen und Lesestrategien eingehen, gibt es in der deutschen Forschungslandschaft kaum. Derzeit liegen zwei qualitative Studien zu Lesekompetenzen von Erwachsenen mit zugeschriebener geistiger Behinderung vor: Im Rahmen des LeiSA-Projekts (http://research.unileipzig.de/leisa/de/) wurde eine qualitative Studie realisiert, die die Leseverstehens- und Textkompetenzen von Erwachsenen mit sogenannter geistiger Behinderung erfasst hat (vgl. Bock/Lange 2017, s. Kap. 4). Außerdem hat Wilke (2016) in einer Grounded-Theory-Studie empirisch untersucht, welche Bedeutung literalen Kompetenzen im Leben von Erwachsenen mit diagnostizierter 
geistiger Behinderung zukommt und in welche Alltagszusammenhänge ihre Lese- und Schreibhandlungen eingebettet sind. In dieser Studie kommt Wilke zu der Einschätzung, dass 32\% der Bewohner(innen) in stationären Wohneinrichtungen lesen können. Sie weist jedoch gleichzeitig auf die begrenzte Aussagekraft eines solchen Werts hin: „Dies ist ein nicht zu vernachlässigendes Drittel, jedoch verfügt der Großteil der Bewohner nicht über diese Kompetenz. Zugleich bedeutet die Fertigkeit selbst noch keine Praktizierung und aktives Lesen" (Wilke 2016: 261).

Im Rahmen der LeiSA-Studie standen die Leseverstehenskompetenzen im Mittelpunkt: Die Untersuchungsgruppe bestand aus 30 Erwachsenen mit sogenannter geistiger Behinderung, die in Werkstätten für Menschen mit Behinderung (WfbM) oder auf Außen- oder Integrationsarbeitsplätzen beschäftigt waren (eine zweite Untersuchungsgruppe bestand aus 20 funktionalen Analphabet(inn)en bzw. Menschen mit Leseschwierigkeiten) (vgl. Bock/Lange 2017). Eines der Samplingkriterien war ein möglichst breites Spektrum an Lesekompetenzen in der Untersuchungsgruppe. Getestet wurde das Leseverständnis mit der lea.-Diagnostik (Grotlüschen 2010). Mit dieser Diagnostik können insbesondere die niedrigen Kompetenzstufen bei jugendlichen und erwachsenen Leser(inne)n differenziert erfasst werden. Der Test erfasst fünf Leseniveaus: Leser(innen) auf Alphalevel 2 können konstruierend Wörter lesen; Leser(innen) auf Alphalevel 3 lesen Wörter und Sätze vorwiegend konstruierend, können aber Standardwörter auch bereits lexikalisch lesen. Auf Alphalevel 4 ist das konstruierende und lexikalische Lesen von kurzen Texten (aus ca. 2 Sätzen) möglich und auf Alphalevel 5 können auch längere Texte (5-8 Sätze) verstanden werden. Alphalevel 6 verweist auf unbeeinträchtigte Leseverstehenskompetenzen. Ab Alphalevel 4 verorten die Autorinnen der lea.-Studie funktionale Lesefähigkeiten, Lesefähigkeiten auf den Alphaleveln 3 oder weniger verweisen auf deutlich beeinträchtigte Leseverstehenskompetenzen bzw. funktionalen Analphabetismus.

Die Lesekompetenzen waren in der LeiSA-Untersuchungsgruppe folgendermaßen verteilt: 


\begin{tabular}{|c|c|c|c|}
\hline Alphalevel 2 & Alphalevel 3 & Alphalevel 4 & Alphalevel 5 und 6 \\
\hline $16,7 \%$ & $30 \%$ & $46,6 \%$ & $6,7 \%$ \\
\hline
\end{tabular}

Tab. 1: Verteilung der Lesekompetenzen in der Untersuchungsgruppe Erwachsene mit sog. geistiger Behinderung der LeiSA-Studie ( $N=30$; davon ein $\mathrm{TN}$, hier unter Alphalevel 2 gezählt, der sich nicht eindeutig Alphalevel 2 oder 3 zuordnen ließ)

Da es sich um ein qualitatives Sample handelt, können keine Schlüsse auf die Gesamtpopulation gezogen werden. Bemerkenswert ist jedoch, dass die Leseverstehenskompetenzen unter denjenigen, die lesen konnten, nicht selten ein hohes Niveau erreichten. So konnten in der Akquise ohne größere Suche 16 Studienteilnehmer(innen) mit diagnostizierter geistiger Behinderung gewonnen werden, die auf hohen bis nahezu unbeeinträchtigten Leseniveaus verstehend lesen konnten (Alpha-Level 4-5 nach lea-Diagnostik, vgl. Grotlüschen 2010). Die Schwierigkeiten beim Leseverstehen waren auf allen Niveaustufen sehr unterschiedlich; auch auf dem niedrigsten Leseniveau hatten die Studienteilnehmenden teilweise Lesestrategien zur Verfügung, die Rekodier- und Verstehensschwierigkeiten kompensieren konnten und die es ihnen ermöglichten, Texte zu erschließen (vgl. Bock/Lange 2017).

In der englischsprachigen Forschungslandschaft gibt es erste Studien, die Lesekompetenzen und Interventionsmaßnahmen mittels standardisierter und qualitativer Verfahren untersucht haben; Untersuchungsgruppen sind meist Kinder mit der Diagnose geistige Behinderung. Auch hier werden aber nach wie vor beträchtliche Forschungsdesiderate beklagt (zu Kindern mit DownSyndrom: Abbeduto/Warren/Conners 2007: 255f.). Eine US-amerikanische longitudinale Interventionsstudie hat den Leseprozess von Grundschulkindern mit einem IQ zwischen 40 und 69 in den Blick genommen (Allor et al. 2010). Die Intervention berücksichtigte eine große Breite an Leseparametern. Erhoben wurden sowohl quantifizierbare Leseparameter, u.a. in den Bereichen phonologische Bewusstheit, Lautieren und Worterkennen, aber auch Leseverstehensstrategien für verschiedene Textsorten. Die Intervention bewirkte deutliche Fortschritte in den einzelnen Parametern, allerdings war sie nicht in allen Bereichen gleichermaßen wirksam. Beim Leseverständnis zeigten sich beispielsweise keine signifikanten Effekte (ebd.: 460). Zudem fielen die interindividuellen Unterschiede sehr groß aus: Nicht bei allen Schüler(inne)n war 
die Intervention in gleichem Maße effektiv (ebd.: 464). $\mathrm{Zu}$ den wichtigsten Ergebnissen zählt aber, dass Schüler(innen) mit diagnostizierter geistiger Behinderung unabhängig vom IQ nach zwei bis drei Jahren überhaupt von Interventionen zur Leseförderung profitieren (ebd.: 456). Grundsätzlich gilt: „performance [in reading; S.Sch./B.B.] does not appear to be clearly predicted by IQ score" (ebd.: 463). Daraus lässt sich eine Notwendigkeit für die Entwicklung angemessener, langfristig angelegter Leseförderprogramme ableiten, was im ersten Schritt die genauere Erforschung von Leseprozess und Lesenlernen bei Schülerinnen und Schülern mit dem Förderschwerpunkt geistige Entwicklung voraussetzt. Die Studie zeigt allerdings nicht nur Forschungslücken auf, sondern verweist auch darauf, dass die potenziell möglichen Lesekompetenzen bei Kindern (und Erwachsenen) mit dem Label geistige Behinderung in der Breite mit adäquaten Instruktionstechniken und Förderinstrumenten eventuell höher liegen als es derzeit der Fall ist (vgl. ebd.: 463).

\section{Verständlichkeit und Angemessenheit „Leichter Sprache“ für Menschen mit sogenannter geistiger Behinderung}

Die Form barrierefreier Kommunikation, die derzeit wahrscheinlich die meiste öffentliche Aufmerksamkeit bekommt und teilweise kontrovers diskutiert wird, ist die sogenannte „Leichte Sprache“ (vgl. Bredel/Maaß i.d.Bd.). Da die empirische Fundierung "Leichter Sprache“ gerade in Bezug auf Menschen mit sogenannter geistiger Behinderung immer wieder gefordert wird (vgl. Bredel/Maaß 2016: 143; Christmann 2017: 46), soll im Folgenden ein Schlaglicht auf vorliegende empirische Studien geworfen werden, welche diesen Personenkreis fokussieren.

„Leichte Sprache“ entstand vorrangig intuitiv im Umfeld der sogenannten Behindertenbewegung und richtete sich primär an Menschen mit dem Etikett einer geistigen Behinderung. Sie ist in erster Linie eine schriftliche Kommunikationsform und setzt auf Textebene an. Insofern adressiert sie Personen, die zumindest basale Lesekompetenzen und Lesestrategien haben, um sich Texte selbständig anzueignen. Sehbeeinträchtigungen und motorische Beeinträchtigungen werden bis zu einem gewissen Maß mitberücksichtigt, indem Schriftgröße, Makrotypografie, Format, materieller Textträger an die Bedürfnisse des 
Adressatenkreises angepasst werden. Bei digitalen Texten gibt es weitere technische Möglichkeiten, Rezeptionsbarrieren abzubauen. Auf Webseiten in „Leichter Sprache“ wird oft zusätzlich eine Vorlesefunktion angeboten, die einen medialen Wechsel bewirkt: Die schriftlichen Texte werden zu mündlichen Kommunikationsangeboten.

Bisher liegen erst wenige empirische Studien zur Verständlichkeit von „Leichte Sprache“-Merkmalen mit der angesprochenen heterogenen Zielgruppe vor. Wir möchten an dieser Stelle zwei Merkmalsbereiche herausgreifen: die Ebene der Grammatik und die Textebene. Die folgenden Abschnitte sind angelehnt an die Darstellung in Bock (i. Vorb.b).

\subsection{Grammatik/Syntax}

Im Regelkatalog des Netzwerks Leichte Sprache nimmt die Ebene der Grammatik vergleichsweise viel Raum ein. Im Abschnitt „Sätze“ finden sich zwar nur wenige Hinweise, die - teilweise implizit - auf Satzkomplexität und Informationsstruktur zielen, weitere grammatische Phänomene sind allerdings im Kapitel „Wörter“ eingeordnet. Einige der Regeln:

- „Benutzen Sie aktive Wörter.“ (Netzwerk Leichte Sprache 2013: 9)

- „Vermeiden Sie den Genitiv.“ (ebd.: 10)

- „Benutzen Sie positive Sprache.“ (ebd.: 11)

- „Schreiben Sie kurze Sätze. Machen Sie in jedem Satz nur eine Aussage.“ Beispiel: „Wenn Sie mir sagen, was Sie wünschen, kann ich Ihnen helfen.“ („schlecht“) vs. „Ich kann Ihnen helfen. Bitte sagen Sie mir: Was wünschen Sie?“" (gut“) (ebd.: 18)

- „Benutzen Sie einen einfachen Satzbau.“ Beispiel: „Zusammen fahren wir in den Urlaub.“ („,schlecht“) vs. „Wir fahren zusammen in den Urlaub.“ („gut“) (ebd.) 
Diese Annahmen sind zunächst einmal grundsätzlich nachvollziehbar. Aus Spracherwerbsperspektive und beim Blick in die empirische Forschung zu Leseverstehen und Sprachverarbeitung wird aber bereits Differenzierung notwendig (vgl. Bredel/Maaß 2016). Lasch weist beispielsweise darauf hin, dass zwischen Zustands- und Vorgangspassiv bezüglich der Schwierigkeit unterschieden werden müsse: Das sein-Passiv wird von Kindern bereits sehr früh erworben, was nahelegt, dass es generell wenig Verstehensprobleme bereitet und demnach auch nicht vermieden werden müsste (Lasch 2017: 295f.). Zu "Leichter Sprache" und ihren Zielgruppen liegen erste exemplarische Studien zu grammatischen Phänomenen vor. Düver (2015) hat untersucht, wie Personen mit kognitiven Beeinträchtigungen Sätze unterschiedlicher Schwierigkeit beurteilen. In der Untersuchung ging es u.a. um Zustands- und Vorgangspassiv, die Satzschwierigkeit wurde außerdem durch Einbettung der PassivKonstruktionen in Konditionalsätze und durch Hinzufügung von Negation zusätzlich gesteigert (Lasch 2017: 294). Die Gewährspersonen waren in der Lage „nicht nur (negierte) Konditional- und Kausalsätze, sondern auch eingebettete Passivstrukturen zu verstehen und zu bewerten“ (ebd.: 296). Auch wenn die Satzverständlichkeit in dieser Studie als rezipientenseitige Bewertung erfasst wurde, decken sich die Befunde doch mit anderen Untersuchungen, in denen das Satzverständnis direkt erfasst wurde: Im Rahmen der LeiSAStudie wurde mit dem Grammatikverstehenstest TROG-D (Fox 2006) erhoben, wie gut Personen mit zugeschriebener geistiger Behinderung Passivsätze, Negation mit ,nicht' und, weder-noch', Typen von Nebensätzen, insbesondere Relativsätze, und weitere grammatische Phänomene verstehen. Die Untersuchungsgruppe bestand aus Erwachsenen mit diagnostizierter geistiger Behinderung und wurde bereits oben beschrieben (Abschnitt 3.2). Je Phänomen mussten vier Sätze selbständig gelesen und ein Bild zugeordnet werden. Ausgewertet wurden anschließend die Fehlerraten. Ein statistisches Clustering ergibt folgende Schwierigkeitsabstufungen nach Fehlerraten: 


\begin{tabular}{|l|l|}
\hline Durchschnittliche Fehlerraten & Phänomene in Sätzen des TROG-D \\
\hline \multirow{3}{*}{ nicht problematisch (5,9\%) } & $\begin{array}{l}\text { Negation mit nicht; 2-Element-Sätze; } \\
\text { 3-Element-Sätze, Präpositionen in, auf }\end{array}$ \\
\cline { 2 - 3 } wenig problematisch (12,5\%) & $\begin{array}{l}\text { Subjunktionen während, nachdem; Relativsätze; Plural; } \\
\text { Präpositionen unter, über; Personalpronomen (Nomina- } \\
\text { tiv, Akkusativ/Dativ) }\end{array}$ \\
\cline { 2 - 3 } mäßig problematisch (37,5\%): & $\begin{array}{l}\text { Passiv; Perfekt; Negation mit weder - noch; Doppel } \\
\text { objektkonstruktion; Koordination mit und (+Ellipse) } \\
\text { (z. B. Der Schuh ist auf dem Stift und ist blau.) }\end{array}$ \\
\cline { 2 - 3 } sehr problematisch (47,3\%): & $\begin{array}{l}\text { Topikalisierung; Subjunktion dass (z. B. Der Junge sieht, } \\
\text { dass die Frau sich sieht.) }\end{array}$ \\
\cline { 2 - 2 } äußerst problematisch (75,9\%) & Objektrelativsätze (Relativartikel im Dativ/Akkusativ) \\
\hline
\end{tabular}

Tab. 2: Überblick über Fehlerraten im Grammatikverstehenstest (TROG-D)

Als wenig bis gar nicht problematisch erwiesen sich also Negation mit ,nicht, kürzere Nebensätze mit den Konjunktionen, während' und ,nachdem' sowie Relativsätze mit Relativpronomen im Nominativ (vgl. Bock/Lange 2017, Bock 2017; zu Negation bei Hörgeschädigten vgl. Bredel/Lang/Maaß 2016). Bei Sätzen mit werden-Passiv machten die Studienteilnehmenden mäßig viele Fehler (vgl. Bock 2017), die meisten Schwierigkeiten verursachten Objektrelativsätze mit einem Relativpronomen im Dativ oder Akkusativ (z. B. „Das Buch, auf dem der Stift ist, ist rot.") (vgl. Bock/Lange 2017). Satzkomplexität ist also zweifellos eine potenzielle Verstehenshürde, das Gebot einer generellen Vermeidung von Nebensätzen lässt sich allerdings, wie es auch Lasch aus seinen Befunden schlussfolgert, nicht ableiten. Noch deutlicher fallen die Befunde für das Genitiv-Verbot aus: Lange hat das Verständnis von Sätzen mit Genitiv und von-Paraphrasen vergleichend mit Erwachsenen mit sogenannter geistiger Behinderung untersucht und konnte weder deutliche Verstehensschwierigkeiten beim Genitiv noch einen Vorteil der von-Alternativstrukturen gegenüber dem Genitiv nachweisen (Lange i.Vorb.).

Bezieht man verschiedene Erscheinungsweisen von Passiv, Negation oder Nebensätzen und den Textkontext mit ein, ergibt sich eine Notwendigkeit zur Differenzierung der Netzwerk-Regeln. Das zeigen auch Studien zur Textoptimierung aus einem anderen Bereich: Balling (2018) hat untersucht, inwieweit 
die Ersetzung von problematischen Konstruktionen wie Passiv, Nominalisierung etc. in Texten das Textverständnis von Studierenden verbessert. Dass sich hier kein positiver Effekt der Ersetzungen nachweisen ließ, erklärt sie u. a. damit, dass die Phänomene in der Untersuchung isoliert manipuliert wurden. Um das Textverständnis positiv zu beeinflussen sei es nötig, auch Aspekte der Kohärenz oder der Informationsstruktur, die z. B. beim Verständnis von Passivkonstruktionen von Bedeutung sind, einzubeziehen (Balling 2018: 117f.). Dies zeigt, wie komplex auch in der „Leichten Sprache“ die Umsetzung ist, und zwar bereits bei scheinbar gut abgrenzbaren Phänomenen der Grammatik.

\subsection{Leserseitiges Wissen: Textsorten}

Das Wissen der Adressat(inn)en ist praktisch auf allen sprachlichen Ebenen für das Textverstehen relevant. Exemplarisch sei hier nun die Textebene, und zwar das Textsortenwissen in den Blick genommen. Der Regelkatalog des Netzwerks Leichte Sprache gibt insgesamt wenige Hinweise auf der Ebene des Textes. Die Hinweise, die implizit oder explizit enthalten sind, bleiben meist allgemein oder betreffen Aspekte, die nicht zu den zentralen Problemen gehören, wenn es um Verständlichkeit auf der Textebene geht:

- „Sprechen Sie die Leser und Leserinnen persönlich an.“ (Netzwerk Leichte Sprache 2013: 20)

- „Schreiben Sie alles zusammen, was zusammen gehört.“ (bezogen auf das Vermeiden von Verweisen) (ebd.: 21)

- „Sie dürfen einen Text beim Schreiben in Leichter Sprache verändern.“ (ebd.: 22)

- „Machen Sie viele Absätze und Überschriften.“ (ebd.: 28)

- „Benutzen Sie immer die gleichen Wörter für die gleichen Dinge.“ (ebd.: 6) 
Dem Aspekt der Textsorte wird bisher noch zu wenig Aufmerksamkeit gewidmet. In der Wissenschaft wird aktuell diskutiert, welche Textsortenkompetenzen bei den Zielgruppen vorausgesetzt werden können (vgl. Bredel/Maaß 2016: 195f.). In empirischen Studien zeigt sich, dass Adressatinnen und Adressaten „Leichter Sprache“ über zum Teil ausgesprochen differenziertes und umfassendes Textsortenwissen verfügen, und zwar teilweise auch bei sehr geringen Lesekompetenzniveaus: Sie haben nicht nur ein Konzept von Textsorten und davon, dass diese unterschiedliche Funktionen erfüllen, sie erkennen Textsorten auch an typischen grafischen Merkmalen und verfügen über (teils ausgesprochen individuell geprägte) Textsortenbezeichnungen. Teilweise können sogar charakteristische Textsortenmerkmale selbständig benannt werden, was ein hohes Maß an Sprechreflexion und -bewusstheit belegt (Bock/ Lange 2017). Die Kenntnis von Textmustern ist wie bei allen Sprachbenutzer(inne)n maßgeblich von der individuellen kommunikativen Erfahrung abhängig.

Die bisherigen empirischen Erkenntnisse zur „Leichten Sprache“ deuten also etwas Ähnliches an wie schon vereinzelt die Forschung zu den Lesekompetenzen des Personenkreises mit sogenannter geistiger Behinderung: Zum einen gibt es ein Risiko, dass Kompetenzen unterschätzt bzw. falsch eingeschätzt werden. Zum anderen wird deutlich, wie komplex die Aufgabe der Textanpassung ist. Beides bietet Anlass zu weiterer kritischer Auseinandersetzung in Theorie, Forschung und Praxis mit der Entwicklung und Passfähigkeit „Leichter Sprache“.

\section{„Leichte Sprache“ und ihre partizipative Bedeutung}

Hinsichtlich der Bedeutung „Leichter Sprache“ mit Blick auf Barrierefreiheit lassen sich verschiedene Paradoxien kennzeichnen. Wenngleich das Konzept der „Leichten Sprache“ den Anspruch erhebt, „Texte in allen Kommunikationsbereichen adressatengerecht zugänglich machen zu können und damit Teilhabemöglichkeiten zu verbessern“ (Schuppener/Goldbach/Bock 2018: 361), wird gegenwärtig auch sensibel diskutiert, dass gleichsam Diskreditierungsrisiken bestehen: Die Nutzung von „Leichter Sprache“ geht immer auch „mit der Zuschreibung an das Gegenüber einher, auf ,Leichte Sprache` angewiesen zu sein 
und unterstellt damit ein Defizit" (Seitz 2014: o.S.; vgl. ähnlich Zurstrassen 2017: 61).

Aufgrund der Entwicklungskultur des Konzepts der "Leichten Sprache“ mit dem Fokus auf den Adressat(inn)enkreis von Menschen, die als geistig behindert bezeichnet werden - ist mit dieser Schriftsprachform auch eher eine Verstärkung ableistischer Konstruktionen als ein Beitrag zur Dekonstruktion von Behinderung zu konstatieren. So zeigt sich das Phänomen, dass ein Teil der potentiellen Nutzer(innen) nicht auf diese Sprachvarietät zurückgreift, weil dieser sich durch die zu starken Simplifizierungen stigmatisiert fühlt (vgl. Bergelt/ Goldbach/Seidel 2016).

Den Ergebnissen der LeiSA-Studie zufolge besteht ein Risiko darin, dass die Aufarbeitung und Bereitstellung von Informationen in „Leichter Sprache“ eine gewisse Alibifunktion erfüllt (vgl. Schuppener/Goldbach/Bock 2018), indem Einrichtungen damit die Berücksichtigung dieses Merkmals von Barrierefreiheit nachweisen, allerdings ohne dass ein tatsächlicher Mehrwert im Hinblick auf Selbstständigkeit und Selbstbestimmung für die Nutzer(innen) damit einhergeht (vgl. Bergelt/Goldbach/Leonhardt/Seidel i. Vorb.).

Auch stellt sich die Frage, inwieweit das bislang noch an einheitlichen Regelwerken ausgerichtete Konzept der „Leichten Sprache“ dem Anspruch an Individualisierung und Differenzierung in irgendeiner Form gerecht werden kann. Genau dieser Anspruch sollte jedoch zentrale Bedeutung haben mit Blick auf das Recht auf Partizipation von Menschen, die als geistig behindert gelten. Dies scheint mit einem dogmatischen Befolgen der Regeln im Rahmen der Erarbeitung und Prüfung von Texten in „Leichter Sprache“ allerdings in Anbetracht der Heterogenität des Personenkreises insgesamt - im Besonderen auch im Bereich der kommunikativen Kompetenzen und Unterstützungsbedarfe schwer einlösbar. Auch die Verständlichkeitsforschung stellt stark in Frage, ob "Leichte Sprache“-Texte in der bisherigen Gestaltungsform überhaupt zielgruppenangemessene Lösungen im Sinne eines Beitrages zur Barrierefreiheit darstellen. Vor dem Hintergrund einer sehr uneinheitlichen Praxis muss somit auch der universelle Geltungsanspruch von Regelwerken zur Disposition gestellt werden: 
Unterschiedliche Kommunikationssituationen fordern unterschiedliche sprachliche (und typografische) Mittel. Das wird bisher - anders als beispielsweise in den skandinavischen Ländern - kaum berücksichtigt oder sogar dogmatisch ausgeschlossen. Um für die Adressat*innen kommunikative Teilhabe zu ermöglichen, scheint eine Flexibilisierung bei der Umsetzung Leichter Sprache dringend nötig (Schuppener/Goldbach/ Bock 2018: 366).

Analog zur Erweiterung des Lese- und Schreibbegriffes kommt der „Leichten Sprache" jedoch auch eindeutig das Verdienst zu, ein Bewusstsein im Diskurs zu kommunikativer Barrierefreiheit (im Bereich der Schriftlichkeit) zu schaffen. Damit ist ein zusätzlicher Reflexionsanspruch im Hinblick auf die Diversität im Bereich von kommunikativen Grundvoraussetzungen - verbunden mit dem grundlegenden Anspruch an unterschiedliche schriftsprachliche Textgestaltungsniveaus - markiert. Dennoch bleibt die Frage, ob und in welcher (Gestaltungs-)Form die bisherige Praxis „Leichter Sprache“ einen tatsächlichen Beitrag zur Partizipation für Menschen mit Lernschwierigkeiten darstellt oder nicht vereinzelt z.T. sogar „exklusionsverschärfend“ wirkt, da durch die Zielgruppenorientierung eher ein Beitrag zur Produktion und Reproduktion von (geistiger) Behinderung geleistet wird und eine ,inklusions- und diversitätssensible Funktion“ des Konzeptes eher zu hinterfragen ist (ebd.; vgl. auch Zurstrassen 2017). 


\section{Literaturverzeichnis}

Abbeduto, LeONARd/Hesketh, Linda J. (1997): „Pragmatic development in individuals with mental retardation: Learning to use leanguage in social interactions". In: Mental retardation and developmental disabilities research reviews 3, 323-333.

Abbeduto, Leonard/Warren, Steven F./Conners, Frances A. (2007): „Language development in Down syndrome: From prelinguistic period to the acquisition of literacy“. In: Mental retardation and developmental disabilities research reviews 13, 247261.

ALLOR, JiLL H., U.A. (2010): „Comprehensive reading instruction for students with intellectual disabilities: Findings from the first three years of a longitudinal study". In: Psychology in the Schools 47 (5), 445-466.

AKTAS, MAREN (2012): Entwicklungsorientierte Sprachdiagnostik und -förderung bei Kindern mit geistiger Behinderung: Theorie und Praxis. München: Urban \& Fischer.

Balling, LaURA W. (2018): „No Effect of Writing Advice on Reading Comprehension“. In: Journal of Technical Writing and Communication 48 (1), 104-122.

BÄrmig, Sven (2015): „Teilhabe - Begriff(e) und Vermittlung“. In: SCHNell, IRMTRAUd (Hrsg.): Herausforderung Inklusion. Theoriebildung und Praxis. Bad Heilbrunn: Klinkhardt, 138-147.

BENKMANn, RAINER (2014): „Inklusive Bildung in Zeiten roher Bürgerlichkeit“. In: Gemeinsam leben 22 (2), 68-77.

Bergelt, Daniel/Goldbach, Anne/Seidel, Anja (2016): „Leichte Sprache im Arbeitsleben. Analyse der derzeitigen Nutzung von Texten in Leichter Sprache im beruflichen Kontext von Menschen mit Lernschwierigkeiten“. In: Teilhabe 55 (3), 106-113.

Bergelt, Daniel/Goldbach, Anne/Leonhardt, Nico/Seidel, Anja (i. Vorb.): Die berufliche Teilhabesituation von Menschen mit Lernschwierigkeiten in unterschiedlichen beruflichen Kontexten.

BIEWer, GotTfried (2017): Grundlagen der Heilpädagogik und Inklusiven Pädagogik. Bad Heilbrunn: Klinkhard, utb.

Bock, Bettina M. (i.Vorb. a): „Sprache und Partizipation“. In: LiedtKe, Frank (Hrsg.): Handbuch Pragmatik. Stuttgart: Metzler.

BocK, BetTina M. (i.Vorb. b): „Was ist für wen leicht verständlich? Befunde zu Wortschatz, Grammatik und leserseitigem Wissen“. In: Der Deutschunterricht.

Bock, BetTina M. (2017): „Das Passiv- und Negationsverbot ,Leichter Sprache' auf dem Prüfstand: Empirische Ergebnisse aus Verstehenstest und Korpusuntersuchung “. In: Sprachreport H. 1/2017. URL: http://pub.ids-mannheim.de/laufend/sprachreport/ sr17.html (letzter Zugriff: 27.3.2018).

Bock, Bettina M./LANGe, Daisy (2017): „Empirische Untersuchungen zu Satz- und Textverstehen bei Menschen mit geistiger Behinderung und funktionalen Analphabeten“. In: Bock, Bettina M./Fix, Ulla/LANGe, Daisy (Hrsg.): „Leichte Sprache“ 
im Spiegel theoretischer und angewandter Forschung. Berlin: Frank \& Timme, 253274.

Bock, BetTina M. (2015): „Leichte Texte schreiben. Zur Wirksamkeit von Regellisten Leichter Sprache in verschiedenen Kommunikationsbereichen und im WWW“. In: trans-kom 8 (1), 79-102. URL: http://www.trans-kom.eu/bd08nr01/trans-kom_ 08_01_04_Bock_Leichte_Texte.20150717.pdf (letzter Zugriff: 27.3.2018).

Bredel, Ursula/MaAß, Christiane (2016): Leichte Sprache. Theoretische Grundlagen. Orientierung für die Praxis. Berlin: Duden.

Bredel, Ursula/LANG, KATRIN/MAaß, Christiane (2016): „Zur empirischen Überprüfbarkeit von Leichte-Sprache-Regeln am Beispiel der Negation“. In: MÄLZER, Nathalie (Hrsg.): Barrierefreie Kommunikation. Perspektiven aus Theorie und Praxis. Berlin: Frank \& Timme, 95-115.

Buchner, Tobias/Pfahl, Lisa/Traue, Boris (2015): „Zur Kritik der Fähigkeiten: Ableism als neue Forschungsperspektive der Disability Studies und ihrer Partner_innen“. In: Zeitschrift für Inklusion-online 2/2015: URL: https://www.inklusiononline.net/index.php/inklusion-online/article/view/273/256 (letzter Zugriff: 27.03.2018).

Campbell, Fiona Kumari (2008): „Refusing Able(ness): A Premliminary Conversation about Ableism.“ In: M/C Journal 11 (3) - „able“: URL: http://journal.mediaculture.org.au/index.php/mcjournal/article/viewArticle/46 (letzter Zugriff: 27.03.2018).

Christmann, Ursula (2017): „Wie leicht darf leichte Sprache sein? Empirische Lücken in einem gut gemeinten Konzept“. In: Bock, BeTTINA M./FIX, Ulla/LANGE, DAISY (Hrsg.): „Leichte Sprache“ im Spiegel theoretischer und angewandter Forschung. Berlin: Frank \& Timme, 35-52.

DEDERICH, MARKUS (2009): „Behinderung als sozial- und kulturwissenschaftliche Kategorie“. In: Dederich, Markus/JAntzen, WolfGang (Hrsg.), Behinderung und Anerkennung. Stuttgart: Kohlhammer, 15-39.

Dönges, Christoph/Stegkemper, Jan/WAgner, Michael (i.Dr.): „Sprache als eine Barriere politischer Partizipation von Menschen mit geistiger Behinderung“. In: Bock, Bettina M./Dreesen, Philipp (Hrsg.): Sprache und Partizipation in Geschichte und Gegenwart. Bremen: Hempen.

DÜVER, JUlia (2015): Empirische Untersuchung zu Formulierungen in Leichter Sprache. Grammatikalität und Akzeptabilität von Adverbialbestimmungen und Komposita. Kiel, unveröffentlichte Bachelorarbeit.

DWORSCHAK, WOLFGANG/KANNEWISCHER, Sybille/RATZ, CHRISTOPH/WAGNER, MichaEL (Hrsg.) (2012): Schülerschaft mit dem Förderschwerpunkt geistige Entwicklung (SFGE). Eine empirische Studie. Oberhausen: Athena-Verlag.

DWORSCHAK, WolfGANG/SCHMIDT, MATthias (2011): „Inklusion und Teilhabe Gleichbedeutende oder unterschiedliche Leitbegriffe in der Sonder- und Heilpädagogik“. In: Zeitschrift für Heilpädagogik 62 (7), 269-280. 
FEUser, GeOrG (1976): „Notwendigkeit und Möglichkeit einer pädagogischen Förderung von Kindern mit frühkindlichem Autismus in Sonderkindergarten und Sonderschule“. In: Zeitschrift für Heilpädagogik 27 (11), 643-657.

Feuser, GEOrG (1996): „,Geistig Behinderte gibt es nicht!'. Projektionen und Artefakte in der Geistigbehindertenpädagogik." Geistige Behinderung, 35 (1), 18-25.

FORNEFELD, BARBARA (2008): „Menschen mit Komplexer Behinderung - Klärung des Begriffs“. In: FORNEFELD, BARBARA (Hrsg.), Menschen mit Komplexer Behinderung: Selbstverständnis und Aufgaben der Behindertenpädagogik. München: Reinhardt, 5081.

FOX, ANNETTE V. (2006) (Hrsg.): TROG-D. Test zur Überprüfung des Grammatikverständnisses. Idstein: Schulz-Kirchner.

Goffman, ERving (1973): Asyle. Über die soziale Situation psychiatrischer Patienten und anderer Insassen. Frankfurt am Main: Suhrkamp.

Grotlüschen, ANKe (Hg.) (2010): Lea.-Literarlitätsentwicklung von Arbeitskräften. Diagnose. Münster: Waxmann.

HATTON, CHRIS (1998): „Pragmatic language skills in people with intellectual disabilities: a review“. In: Journal of Intellectual \& Developmental Disability 23 (1), 79-101.

Hillmann, Karl-Heinz (2007): Wörterbuch der Soziologie. Stuttgart: Kröner.

JANTZEN, WolfGang (2005): „Es kommt darauf an, sich zu verändern ... “Zur Methodologie und Praxis rehistorisierender Diagnostik und Intervention. Gießen: PsychosozialVerlag.

KMK (1998): Empfehlungen zum Förderschwerpunkt geistige Entwicklung. URL: https://www.kmk.org/fileadmin/veroeffentlichungen_beschluesse/1998/1998_06_ 20_FS_Geistige_Entwickl.pdf (letzter Zugriff: 08.03.2018).

KOCH, ARnO (2008): Die Kulturtechnik Lesen im Unterricht für Schüler mit geistiger Behinderung. Lesen lernen ohne Phonologische Bewusstheit? Dissertation. Justus-LiebigUniversität Gießen. Gießen. URL: http://geb.uni-giessen.de/geb/volltexte/2008/ 6247/pdf/KochArno-2008-28-05.pdf (letzter Zugriff: 27.3.2018).

Kremsner, Gertraud/Proyer, Michelle (2016): „,Wohnst Du noch oder lebst Du schon?' - Wohnen und Unterbringen von Menschen mit Lernschwierigkeiten aus postkolonialer Sicht: globale und europäische Entwicklungen“. In: HEDDERICH, IngEBORG/ZAHnd, RAPHAEL (Hg.): Teilhabe und Vielfalt: Herausforderungen einer Weltgesellschaft. Beiträge zur Internationalen Heil- und Sonderpädagogik. Bad Heilbrunn: Klinhardt, 433-445.

Kuhl, Jan/Euker, Nils/Ennemoser, MARCo (2015): „Förderung des lautorientierten Lesens bei Schülerinnen und Schülern mit intellektueller Beeinträchtigung. Phonological reading instruction for students with intellectual disability“. In: Empirische Sonderpädagogik 7 (1), 41-55.

LANGE, DAISY (i.Vorb.): Die Verständlichkeit von Genitivkonstruktionen.

LASCH, AleXANDER (2017): „Zum Verständnis morphosyntaktischer Merkmale in der funktionalen Varietät ,Leichte Sprache““. In: Bock, BetTina M./FIX, UlLa/LANGE, 
DAISY (Hrsg.): „Leichte Sprache“ im Spiegel theoretischer und angewandter Forschung. Berlin: Frank \& Timme, 275-300.

Maskos, Rebecca (2015): „Ableism und das Ideal des autonomen Fähig-Seins in der kapitalistischen Gesellschaft“. In: Zeitschrift für Inklusion-online 2/2015. URL: https://www.inklusion-online.net/index.php/inklusion-online/article/view/277/260 (letzter Zugriff: 27.03.2018).

MEIßNer, Hanna (2015): „Studies in Ableism - Für ein Vorstellungsvermögen jenseits des individuellen autonomen Subjekts“. In: Zeitschrift für Inklusion-online 2/2015. URL: https://www.inklusion-online.net/index.php/inklusion-online/article/view/ 276/259 (letzter Zugriff 27.03.2018).

MENSCH ZUERST (2018): URL: http://www.menschzuerst.de/pages/startseite/wer-sindwir/grundsatz-programm.php (letzter Zugriff: 27.3.2018).

NetzWerk LeICHTE Sprache (2013): Die Regeln für Leichte Sprache. URL: http://www.leichte-sprache.de/dokumente/upload/21dba_regeln_fuer_leichte_ sprache.pdf (letzter Zugriff: 08.03.2018).

Niehr, Thomas (i. Dr.): „Partizipation - zur Geschichte eines politischen Hochwertwortes“. In: Bock, Bettina M./Dreesen, Philipp (Hg.): Sprache und Partizipation in Geschichte und Gegenwart. Bremen: Hempen.

Prosetzky, Ingolf (2009): „Isolation und Partizipation“. In: Dederich, Markus/ Jantzen, WolfGang (Hg.): Behinderung und Anerkennung. Stuttgart: Kohlhammer, 87-95.

RATZ, CHRISTOPH (2012): „Schriftsprachliche Fähigkeiten von Schülern mit dem Förderschwerpunkt geistige Entwicklung“. In: DWORSCHAK, WOLFGANG/KANNEWISCHER, Sybille/RATZ, Christoph/Wagner, Michael (Hg.): Schülerschaft mit dem Förderschwerpunkt geistige Entwicklung (FSGE). Eine empirische Studie. Oberhausen: Athena, 111-132.

SCHuPpener, SASKia (2008): „Psychologische Ansätze“. In: Nussbeck, Susanne/AdAM, Heidemarie/BIERMAnn, AdRIEnNe (Hg.), Sonderpädagogik der geistigen Entwicklung. Göttingen: Hogrefe. Im Rahmen der Reihe: GoETZE, HerberT/BorCHERT, Johann (Hg.): Handbuch der Pädagogik und Psychologie bei Behinderungen. Göttingen: Hogrefe, 89-114.

SCHUPPEnER, SASKia (2016): „Selbstbestimmung“. In: HEdDERICH, INGEBORG/Biewer, Gottfried/Hollenweger, Judith/Markowetz, Reinhard (Hg.): Handbuch Inklusion und Sonderpädagogik. Bad Heilbrunn: Klinkhardt/utb, 108-112.

SCHuppener, SASkia/GoldbaCH, ANNe/Bock, BetTina (2018): „LEICHTE SPRACHE - ein inklusionssensibles Konzept zur Förderung beruflicher Teilhabe?" In: FEYERER, Ewald/Prammer, Wilfried/Prammer-Semmler, Eva/Kladnik, Christina/ Leibetseder, Margit/Wimberger, Richard (Hg.): System. Wandel. Entwicklung. Akteurinnen und Akteure inklusiver Prozesse im Spannungsfeld von Institution, Profession und Person. Bad Heilbrunn: Klinkhardt, 361-367. 
Schwab, Susanne (2016): „Partizipation“. In: Hedderich, Hedderich/Biewer, GotTFried/Hollenweger, Judith/Markowetz, Reinhard (Hg.): Handbuch Inklusion und Sonderpädagogik. Bad Heilbrunn: Klinkhardt/utb, 127-131.

Schwalb, Helmut/Theunissen, GeOrg (2018): Inklusion, Partizipation und Empowerment in der Behindertenarbeit: Best Practice-Beispiele: Wohnen - Leben - Arbeit - Freizeit. Stuttgart: Kohlhammer (3. Auflage).

SEITZ, SimOnE (2014): Leichte Sprache? Keine einfache Sache. URL: http://www.bpb.de/ apuz/179339/leichte-sprache-keine-einfache-sache (letzter Zugriff: 07.03.2018).

TRESCHER, HENDRIK (2014): „Behinderung als demokratische Konstruktion. Zum objektiven Sinn und ,cultural impact' der UN-Konvention über die Rechte von Menschen mit Behinderungen“. In: Zeitschrift für Inklusion 4/2013. URL: https://www.inklusion-online.net/index.php/inklusion-online/article/view/ 195 (letzter Zugriff: 08.03.2018).

Tschernig, Kerstin/Vo Thi, My Hanh (2017): „Die Diversitätsdimension Sprache als Schlüsselqualifikation im Fachunterricht: diskusbestimmende Konzepte und inklusionsspezifische Paradigmen“. In: Zeitschrift für Inklusion 3/2017. URL: https://www.inklusion-online.net/index.php/inklusion-online/article/view/449 (letzter Zugriff: 06.03.2018).

Wagner, Michael/Kannewischer, Sybille (2012): „Einschätzung fer Kompetenzen im Bereich Sprache/Kommunikation“. In: DwORSCHAK, WOLFGANG/KANNEWISCHER, Sybille/Ratz, Christop/Wagner, Michael (Hg.): Schülerschaft mit dem Förderschwerpunkt geistige Entwicklung (SFGE). Eine empirische Studie. Oberhausen: Athena, 99-110.

Wilke, Julia (2016): Literacy und geistige Behinderung. Eine Grounded-Theory-Studie. Wiesbaden: Springer VS.

Wilken, ETTA (2000): Sprachförderung bei Kindern mit Down-Syndrom. Mit ausführlicher Darstellung des GuK-Systems. Berlin: Ed. Marhold.

WiLKEN, ETTA (2009): „Sprachentwicklungsstörungen und geistige Behinderung“. In: Grohnfeldt, MANFred (Hg.): Lehrbuch der Sprachheilpädagogik und Logopädie. Stuttgart: Kohlhammer, 112-122.

Zurstrassen, BetTina (2017): „Leichte Sprache - eine Sprache der Chancengleichheit?" In: Bock, Bettina M./Fix, Ulla/LAnge, Daisy (Hg.): „Leichte Sprache“ im Spiegel theoretischer und angewandter Forschung. Berlin: Frank \& Timme, 53-70. 

Formen der Barrierefreien Kommunikation 



\section{Leichte Sprache}

Standardsprachlich verfasste Allgemein- und Fachtexte enthalten für Personen mit Leseeinschränkungen Barrieren unterschiedlicher Art, die die Rezeption, das Verstehen und das Behalten erheblich erschweren oder sogar unmöglich machen können (s. die Beiträge von Rink und von Christmann/Groeben im vorliegenden Handbuch). In Christmann/Groeben sowie in Lutz (beide im vorliegenden Band) wird dargestellt, welche Eigenschaften von Texten der Verständlichkeit entgegenstehen bzw. welche Eigenschaften das Wahrnehmen und Verstehen erleichtern.

Leseeinschränkungen können verschieden verursacht sein und sowohl ihrer Art als auch ihrer Intensität nach verschiedene Ausprägungen annehmen (vgl. im Überblick Bredel/Maaß 2016a und 2016b). Die Frage, ob Leichte Sprache als maximal verständlichkeitsoptimierte Varietät des Deutschen für alle Personen mit Leseeinschränkung eine angemessene Lösung darstellt, muss empirisch beantwortet werden. Die Voraussetzungen für eine solche empirische Prüfung ist die Erfassung des Konstrukts der Leichten Sprache und ihrer gesellschaftlichen Verortung. Wir konzentrieren uns im folgenden Beitrag auf die Entstehungsgeschichte, die aktuelle Rechtslage, Ansätze zur wissenschaftlichen Bearbeitung sowie auf die Frage nach der gesellschaftlichen Relevanz und Akzeptanz der Leichten Sprache.

\section{$1 \quad$ Leichte Sprache: \\ Ursprung im Empowerment und erste Regelwerke}

Leichte Sprache ist eine Varietät des Deutschen, die gegenüber dem voll ausgebauten Standarddeutschen eine erhöhte Wahrnehmbarkeit und Verständlichkeit für Personen mit Leseeinschränkungen aufweist. Sie wurde von Akteur(inn)en 
der Behindertenrechtsbewegung und dem Empowerment entwickelt (TjarksSobhani 2012, Maaß 2015), und zwar insbesondere aus dem Kreis der Personen mit geistiger Behinderung (die Gruppe reklamiert für sich die allerdings anderweitig besetzte Bezeichnung „Lernschwierigkeiten“; zur Problematik der Benennung s. Bredel/Maßß 2016a und Schuppener/Bock im vorliegenden Band). Dabei steht die deutsche Leichte Sprache neben verständlichkeitsoptimierten Varietäten anderer Einzelsprachen wie leichtem Finnisch („Selkokeskus“, http://selkokeskus.fi, s. a. Bredel/Maaß 2016a: 66) oder leichtem Schwedisch („Lättläst“, http://papunet.net/ll-sidor), die teilweise auf eine jahrzehntelange Textpraxis zurückblicken. Auch hinsichtlich anderer Einzelsprachen lässt sich aktuell eine Entwicklung feststellen, z. B. mit Blick auf das Spanische in Europa und Amerika (Becker im Druck) oder das Französische in Kanada (Potthoff 2016).

Für die deutsche Leichte Sprache wurden ab dem Jahr 2009 Regelwerke vorgelegt, und zwar vom Netzwerk Leichte Sprache sowie von Inclusion Europe. Das 2009er Regelwerk des Netzwerks Leichte Sprache ist inhaltlich unverändert auf der Homepage des Bundesministeriums für Arbeit und Soziales zugänglich (BMAS 2014), das Regelwerk von Inclusion Europe findet sich auf der europäischen Easy-to-Read-Plattform (http://easy-to-read.eu), die neben der deutschen Fassung des Regelwerks Varianten in weiteren 15 europäischen Sprachen vorhält. Dabei konvergieren die Regeln einzelsprachübergreifend in weiten Teilen und es sind nur für einige wenige Sprachen einzelsprachliche Regeln vorgesehen; etwa die Regel zur Aufgliederung langer Wörter für das Deutsche. Grund für die hohe Konvergenz ist die sprachübergreifende Geltung verständlichkeitsverbessernder Strategien, wie sie auch die Verständlichkeitsforschung seit langem ermittelt hat. Hierzu gehören beispielsweise sprachliche Einfachheit und informationelle Gegliedertheit, die sich sowohl auf die Wort-, als auch auf die Satz- und Textebene beziehen (zu einer umfassenden Beschreibung der Strukturmerkmale der Leichten Sprache vgl. Bredel/Maßß 2016a).

Sowohl das Regelwerk von Inclusion Europe als auch dasjenige des Netzwerks Leichte Sprache sind selbst in Leichter Sprache verfasst. Sie adressieren damit die Zielleserschaft von Leichter Sprache und richten sich nicht vorrangig an Übersetzer(innen) oder Redakteure/Redakteurinnen. In standardsprachlichem Deutsch sind die Regeln zur Leichten Sprache im Anhang an die Barrierefreie-Informationstechnik-Verordnung (BITV 2.0, s. den Beitrag von 
Lang in diesem Band) formuliert. Die drei genannten Praxisregelwerke (Netzwerk Leichte Sprache, Inclusion Europe, BITV 2.0) formulieren insgesamt 120 Regeln zur Verständlichkeitsoptimierung und konvergieren bezüglich 17 dieser Regeln (zu den Regelwerken ausführlich Bredel/Maaß 2016a: 82ff.):

\begin{tabular}{|c|c|}
\hline $\begin{array}{l}\text { Visuelle und mediale } \\
\text { Gestaltung }\end{array}$ & $\begin{array}{l}\text { 1. Größere Schriftgröße } \\
\text { 2. Jeder Satz auf eine neue Zeile } \\
\text { 3. Keine Worttrennung am Zeilenende } \\
\text { 4. Linksbündig }\end{array}$ \\
\hline Morphologie & $\begin{array}{l}\text { 5. Kurze Wörter } \\
\text { 6. Trennung von komplexen Wörtern durch Bindestriche } \\
\text { 7. Verbot von Abkürzungen } \\
\text { 8. Passiv vermeiden }\end{array}$ \\
\hline Lexik & $\begin{array}{l}\text { 9. Leicht verständliche Wörter } \\
\text { 10. Möglichst keine Fremdwörter } \\
\text { 11. Fremdwörter erklären }\end{array}$ \\
\hline Syntax & 12. Kurze Sätze \\
\hline Semantik & 13. Negation vermeiden \\
\hline Text & $\begin{array}{l}\text { 14. Konsistenz in der Bezeichnung auf Ebene der Substantive } \\
\text { 15. Relevante Informationen an den Anfang } \\
\text { 16. Zwischenüberschriften erwünscht } \\
\text { 17. Direkte Ansprache }\end{array}$ \\
\hline
\end{tabular}

Tab. 1: Konvergierende Regeln (Netzwerk Leichte Sprache, Inclusion Europe, BITV 2.0) gemäß Bredel/Maaß (2016a: 89)

Die Regeln zeigen eine gute Intuition bezüglich ihres Gegenstands und sind dazu geeignet, Texte in Leichter Sprache zu identifizieren. Für eine professionelle Textpraxis sind sie jedoch nicht differenziert genug, da sie nur einen gewünschten Zustand beschreiben („Kurze Sätze“), aber keinen Ansatz dafür enthalten, wie dieser Zustand erreicht werden kann:

- Wie ist mit einem Konditionalsatz umzugehen?

- Wie ist mit einer komplexen, Abstrakta enthaltenden Nominalphrase umzugehen, um sie in den Zustand zu überführen, der in den Regeln als „leicht verständlich“ beschrieben wird? 
Problematisch sind die Praxisregeln dort, wo sie in die deutsche Orthographie eingreifen (Bredel/Maaß 2016a: 330ff., Bredel/Maaß: 2017). Das betrifft beispielsweise den Ratschlag, „lange Wörter“ auch entgegen den Regeln der Orthographie in Leichter Sprache mittels Bindestrichen zu trennen. In der Textpraxis führt das zu Falschschreibungen vom Typ „Kranken-Haus“, „Unter-Suchung“ oder „BeiStand“, alles Beispiele, die offiziellen Seiten deutscher Behörden entnommen sind. Verstöße gegen Orthographie und Grammatik reduzieren nicht nur die Wahrscheinlichkeit auf ein Verstehen (Bredel/Maaß 2017), sondern vergrößern das Stigma, das für die Nutzer(innen) mit Leichter Sprache verbunden ist, und provozieren Vorurteile gegenüber der Leichten Sprache und ihren Rezipient(inn)en bei einer Leserschaft ohne Kommunikationseinschränkungen (s.u. und den Beitrag von Maaß im vorliegenden Handbuch).

Die Regelwerke vermitteln überdies, dass eine Anwendung der formulierten Regeln zu einer maximal verständlichen Textpraxis führe; dies ist jedoch nicht der Fall: Eine mechanische Anwendung der Regeln führt häufig zu Problemen auf der Textebene, da grundlegende Eigenschaften von Texten wie z. B. Kohäsion, Intertextualität oder Signalisierung der Textsortenzugehörigkeit nicht ohne Weiteres mit den Regeln auf Wort- und Satzebene in Einklang zu bringen sind (s. den Beitrag von Maaß im vorliegenden Band und die in diesem Beitrag die formulierten Forschungsdesiderate (Kap. 6)).

Bezüglich der Frage, an wen sich Texte in Leichter Sprache richten, sind die Regelwerke ambivalent. Das Regelwerk des Netzwerks Leichte Sprache etwa fasst den Kreis potentieller Leser(innen) zunächst weit:

Leichte Sprache hilft vielen Menschen.

Zum Beispiel:

- Menschen mit Lern-Schwierigkeiten,

- Menschen, die nicht so gut lesen können,

- Menschen, die nicht so gut Deutsch sprechen. (BMAS 2014: 5)

Auf der anderen Seite wird für „Menschen mit Lern-Schwierigkeiten“ (bzw. geistiger Behinderung) eine Gatekeeper-Funktion postuliert, etwa mit Bezug 
auf die Textprüfung, die nach Auffassung des Netzwerks Leichte Sprache Teil der Texterstellung ist:

Prüfen ist für Leichte Sprache sehr wichtig.

Prüfen gehört zur Leichten Sprache.

Das Prüfen machen Menschen mit Lern-Schwierigkeiten.

Nur sie können sagen, ob ein Text leicht genug ist. (BMAS 2014: 21)

Diese Position impliziert, dass sich Texte in Leichter Sprache in erster Linie an Personen mit geistiger Behinderung richten und für diese optimiert werden sollen, auch wenn es darüber hinaus weitere und zahlenmäßig deutlich über diese Gruppe hinausgehende potentielle Adressatengruppen von Leichter Sprache gibt.

\section{$2 \quad$ Leichte Sprache im Kontext der Rechtsetzung}

In politischer Perspektive ist Leichte Sprache ein Instrument zur Implementierung von Inklusion. Sie soll Personen mit Kommunikationseinschränkungen zur selbständigen Teilhabe befähigen. Von zentraler Bedeutung ist hier die UNBehindertenrechtskonvention (UN-BRK), die einer neuen Konzeptualisierung von Behinderung den Weg gebahnt hat (Degener 2015): Nicht der oder die einzelne Person mit Behinderung muss „rehabilitiert" werden, vielmehr soll die Gesellschaft als Ganze Barrieren abbauen und somit zugänglicher werden. Die UN-BRK wurde 2006 verabschiedet und trat in Deutschland 2009 in Kraft; inzwischen ist dieser völkerrechtliche Vertrag von 160 Staaten unterzeichnet (Stand Februar 2018, United Nations Treaty Collection). In Deutschland hatte die UN-Behindertenrechtskonvention erhebliche Auswirkungen auf die Rechtsetzung (s. den Beitrag von Lang im vorliegenden Band), wobei insbesondere die Novelle des Behindertengleichstellungsgesetzes (BGG) von 2016 zu erwähnen ist. Das BGG verfügt nun im neu hinzugekommenen $₫ 11$, dass „Träger öffentlicher Gewalt [...] auf Verlangen Menschen mit geistigen Behinderungen und Menschen mit seelischen Behinderungen Bescheide, Allgemeinverfügun- 
gen, öffentlich-rechtliche Verträge und Vordrucke in Leichter Sprache erläutern" sollen, sofern dieser Personenkreis diese benötigt. In der Folgekostenabschätzung zum Entwurf dieses Gesetzes wird von 600000 Anfragen pro Jahr aus dem Kreis der Berechtigten ausgegangen (BGG Entwurf 2016: 4, 25; für den Wortlaut s. den Beitrag von Maßß im vorliegenden Band), weshalb „[e]in Grundstock von Erläuterungen zu besonders relevanten Dokumenten [...] in Leichter Sprache erstellt und den Behörden als Basis zur Verfügung gestellt werden“ und in Form eines „Pools von Mustererläuterungen“ schrittweise erweitert werden solle (ebd.). Für Deutschland zeichnet sich damit ein Schwerpunkt für den Einsatz Leichter Sprache im juristisch-administrativen Bereich ab. In anderen Ländern wie etwa Finnland oder Schweden liegt der Fokus eher auf Nachrichten oder im Bereich der Literatur/Belletristik (Leskelä 2017, Bohman 2017).

$\$ 11$ des BGG beschränkt die Ansprüche auf Texte in Leichter Sprache auf „Menschen mit geistigen Behinderungen und Menschen mit seelischen Behinderungen" ( $\$ 11$ BGG neu) und geht von ca. 200000 Berechtigten aus (BGG Entwurf 2016: 4; 25), die im Schnitt drei Erläuterungen in Leichter Sprache pro Jahr anfordern. Von den potentiellen Adressat(inn)en hat damit nur ein kleiner Teil ein verbürgtes Recht auf „Bescheide, Allgemeinverfügungen, öffentlich-rechtliche Verträge und Vordrucke“ in Leichter Sprache. Wenn diese Texte aber in Form eines Pools von Mustererläuterungen vorlägen, dann könnte auch ein größerer Personenkreis von den einmal erstellten Texten profitieren. Hinzu kommt, dass in $\$ 6$ „Gebärdensprache und Kommunikation von Menschen mit Hör- und Sprachbehinderungen “ explizit darauf verwiesen wird, dass Personen mit Sprachbehinderungen ein Recht auf „geeignete Kommunikationshilfen" haben. Auch in der Barrierefreie-InformationstechnikVerordnung (BITV 2.0) von 2011 erfolgt für die Leichte-Sprache-Angebote keine Festlegung auf den Kreis der Personen mit geistiger respektive seelischer Behinderung.

Die Frage nach der Adressatenschaft von Leichter Sprache bleibt jedoch sowohl im Empowerment als auch im politischen Raum unbestimmt. Ein Teil der Personen oder Personengruppen, für die die Ausgangstexte nicht wahrnehmbar und/oder verständlich genug sind, würde auch mit weniger stark verständlichkeitsoptimierten Texten (etwa aus dem Spektrum der Einfachen Sprache, dazu s. Bredel/Maaß 2016a und 2016b) zurechtkommen; diese Texte 
sind jedoch für die primäre Adressatenschaft von Leichte-Sprache-Texten möglicherweise nicht verständlich genug. In ökonomischer Hinsicht stellt sich dann die Frage, ob die Ressourcen in mehrfach abgestufte Textniveaus oder in ein insgesamt breiteres Textangebot fließen sollten. In rechtlicher Hinsicht ist zu entscheiden, welche Gruppen tatsächlich Anspruch auf Texte in Leichter Sprache haben; diese Frage ist nicht gleichzusetzen mit der Frage, wer von vorhandenen Texten letztendlich profitiert.

\section{Wissenschaftliche Konzeptualisierung der Leichten Sprache}

Leichte Sprache ist seit einigen Jahren zunehmend Gegenstand der Wissenschaft, wobei sich unterschiedliche Arten von Ansätzen manifestieren. Unterschiede finden sich u.a. bezüglich der Fragen,

- an wen sich Texte in Leichter Sprache richten,

- ob eine Leichte-Sprache-Praxis lediglich beschreibend beobachtet werden sollte (so z. B. Bock 2015 und öfter) oder ob aus vorangehender Forschung etwa zur Verständlichkeit oder zu den Anforderungen der unterschiedlichen Adressatengruppen Schlussfolgerungen für eine Gestaltung der Leichten Sprache abgeleitet werden können bzw. sollten (so bei Bredel/Maaß 2016a und b)

- ob Leichte Sprache als überwiegend homogen oder in sich gestuft konzeptualisiert werden sollte

- und ob Leichte Sprache eher regel- oder eher funktionsbasiert strukturiert sein sollte (Bock/Fix/Lange 2017b)

Weitgehender Konsens besteht mit Blick auf das fortbestehende Desiderat empirischer Forschung.

Von Seiten der Leipziger Schule der Leichte-Sprache-Forschung wird die Tauglichkeit Leichter Sprache, der Adressatenschaft in ihrer Heterogenität 
Rechnung zu tragen, hinterfragt (s. dazu die Studie von Bock 2015 und öfter, Bock/Lange 2017 und den Beitrag von Schuppener/Bock im vorliegenden Band, s. aber auch Lasch 2017). Dem steht die Annahme der Hildesheimer Schule (Bredel/Maaß 2016 und öfter, Maaß 2015 und öfter, Maaß/Rink/Zehrer 2014) gegenüber, wonach die konkreten Anforderungen an die Texte bei verschiedenen Personengruppen zwar unterschiedlich sind, dass jedoch Prinzipien der Textverständlichkeit überindividuelle Gültigkeit beanspruchen können und dass deshalb unterschiedliche Personengruppen von den nach diesen Prinzipien aufbereiteten verständlichkeitsoptimierten Texten in Leichter Sprache profitieren.

Auch die Frage, wo sich die Leichte Sprache kategorial ansiedelt, wird uneinheitlich beantwortet: Wagner/Schlenker-Schulte (2006 und öfter) und Wagner (2015 und öfter) gehen von „Leichten Texten“ aus, die sowohl sprachlich als auch inhaltlich angepasst seien; ihnen stellen sie die Einfache Sprache gegenüber, die lediglich sprachliche, nicht aber inhaltliche Vereinfachungen vornehme. Sie definieren folglich unterschiedliche Komplexitätsstufen von Texten, wobei Leichte Sprache dort angesiedelt ist, wo keine Informationskonstanz mehr gegeben ist. Die von den Autorinnen in ihrem Ansatz identifizierten Sprachbarrieren, die mit Reformulierungsstrategien bearbeitet werden (siehe Wagner/Schlenker-Schulte 2015: 6ff.), konvergieren allerdings in hohem Maße mit dem, was Praxisregelwerke (Inclusion Europe, Netzwerk Leichte Sprache) wie auch wissenschaftlich basierte Empfehlungen (Duden Leichte Sprache: Bredel/Maaß 2016) als dem Verstehen abträglich identifizieren und folglich abwählen.

Bredel/Maaß (2016a und öfter) und Bock (2015 und öfter) sowie Bock/ Fix/Lange (2017b: 12) beschreiben Leichte Sprache als Varietät des Deutschen und verorten den Begriff damit auf der Ebene des Sprachsystems. Die Art Beschreibung dieser Varietät divergiert jedoch ebenso wie die Fragestellungen und die Methodik innerhalb des Forschungsraums: Während die Leipziger Schule (s. exemplarisch Bock 2014) sich die Aufgabe stellt, die sich entwickelnde Varietät zu beobachten und zu beschreiben, geht die Hildesheimer Schule (s. exemplarisch Bredel/Maaß 2016a) daran, die Varietät zu evaluieren, die bestehenden Regelwerke auf der Basis existierender Forschung zu Verstehen/Verständlichkeit und zu den Adressatengruppen zu modifizieren und zu ergänzen und in 
übersetzungswissenschaftlicher Perspektive Vorschläge für das Umgehen mit bestimmten Textsorten und Diskursbereichen zu erarbeiten (s. exemplarisch Rink in Vorbereitung).

Die empirische Forschung zu Leichter Sprache steht noch am Anfang. Zu verweisen ist auf die LeiSa-Studie der Leipziger Arbeitsgruppe, die den Einsatz Leichter Sprache im Arbeitsleben mit einem empirisch-partizipativen Ansatz untersucht hat (s. Bergelt/Goldbach/Seidel 2016, Goldbach/Schuppener 2016, Lange/Bock 2016). Ein Überblick über kleinere Teilstudien zum Verstehen und zur Verständlichkeit von Leichte-Sprache-Texten findet sich in den Forschungsberichten in Bock/Fix/Lange (2017a: 253ff.). Erste Lesestudien zur Überprüfung der Leichte-Sprache-Regeln mittels apparativ-quantitativer Messmethoden liegen mit Gutermuth (im Druck) und Hansen-Schirra/Gutermuth (im Druck) vor. Potentielle Probleme bei der Implementierung von Forschungsdesigns bei Proband(inn)en mit Behinderungen werden in Bredel/Lang/Maaß (2016) aufgezeigt.

\section{Stereotypen in den Diskursen um Leichte Sprache}

Leichte Sprache ist im Empowerment, in der Wissenschaft und sogar bis in die Rechtsetzung hinein als Mittel für die kommunikative Inklusion von Personen mit Leseeinschränkungen anerkannt. In der öffentlichen Wahrnehmung wird Leichte Sprache dagegen jedoch durchaus ambivalent konzeptualisiert: Neben ihrer Rolle als Instrument der Partizipation wird sie immer wieder auch als Angriff auf die Bildungskultur und als Indiz des Sprachverfalls wahrgenommen. In einem Interview mit der Neuen Zürcher Zeitung vom 8. September 2014 äußert sich der Bildungsforscher Rainer Bremer vom Institut Technik und Bildung ablehnend gegenüber der Leichten Sprache, die er als Angriff auf die sprachliche Bildung ansieht:

Texte werden inhaltsleer, wenn man nicht versucht, das Verständnis einer Sprache durch Bildung und Aufklärung heranzubilden, sondern umgekehrt, die Sprache an den Erkenntnisstand der Leute anpasst. Das ist im Kern bildungsfeindlich. Das ist zwar nicht neu. Neu ist aber, dass sich 
alle Welt davon überzeugen lässt und kaum jemand widerspricht. [...] Dahinter verbirgt sich natürlich ein Klientelismus. Es gibt Leute, die schlagen sich auf die Seite der Benachteiligten und wollen damit eigentlich nur ein Geschäft betreiben. Indem sie gegen Geld eine sogenannte Dienstleistung erbringen, die im besten Fall zu einer Verfälschung führt. Die Leute, die die „Leichte Sprache“ propagieren, lassen sich komische Sätze einfallen, die sich wie Parodien auf behinderte Menschen lesen. [...] Das ist noch schlimmer als Realsatire. Und es hat auch einen moralischen Aspekt: Man nimmt diesen Menschen die Würde. Vor 20, 30 Jahren wäre das undenkbar gewesen, da wären die Behindertenverbände aufgestanden und hätten gesagt: «Ihr vermittelt ein fatales Bild von uns!» Und heute sind sie stolz darauf. (Neue Zürcher Zeitung, 8.9.2014)

Leichte Sprache bedrohe die Standardsprache und sei Zeichen für einen Verfall der Kultur. Die Akteure und Akteurinnen im Feld der Leichten Sprache maßten sich eine nicht existente Kompetenz an und bereicherten sich auf Kosten der Allgemeinheit; sie täuschten damit ihre Kund(inn)en und verfälschten die Aussage der Texte. Die Adressat(inn)en sind nach Bremer Opfer der Produzent(inn)en Leichter Sprache und der unangemessenen Textpraxis, aber auch der unzureichenden Vertretung ihrer Interessen durch ihre eigenen Verbände. Damit greift der Text von Bremer alle drei Parameter auf, die im Diskurs um Leichte Sprache Angriffspunkte für Provokation und Stigmatisierung liefern:

1. die Leichte Sprache selbst,

2. die Produzent(inn)en Leichter Sprache und

3. die Adressat(inn)en Leichter Sprache.

Der Text steht dabei exemplarisch für eine in der Sozialpsychologie weithin bekannte Stereotypenbildung, wie sie u.a. im Ansatz von Fiske/Cuddy/Glick/Xu (2002) beschrieben wird. Die Autor(inn)en klassifizieren unterschiedliche Arten von Vorurteilen, und arbeiten heraus, dass diese abhängig davon sind, welchen Status die bewertende Gruppe einer anderen Gruppe zuschreibt und ob sie 
sie als Konkurrenz zur eigenen Gruppe wahrnimmt. Auf dieser Basis, so die Autor(inn)en weiter, würden der bewerteten Gruppe eine hohe bzw. niedrige Kompetenz sowie Wärme bzw. Kälte zugeschrieben.

\begin{tabular}{cll} 
& \multicolumn{1}{c}{ nompetenz } \\
\cline { 2 - 3 } Wärme & \multicolumn{1}{c}{ niedrig } & \multicolumn{1}{c}{ hoch } \\
\hline hoch & $\begin{array}{l}\text { Paternalistisches Vorurteil } \\
\text { Status: niedrig }\end{array}$ & $\begin{array}{l}\text { Bewunderung } \\
\text { Status: hoch }\end{array}$ \\
& $\begin{array}{c}\text { Konkurrenz: niedrig } \\
\text { Mitleid, Sympathie } \\
\text { (z. B. Ältere, Behinderte) }\end{array}$ & $\begin{array}{l}\text { Konkurrenz: niedrig } \\
\text { Bewunderung, Stolz } \\
\text { (z. B. Gleichgesinnte, Verbündete) }\end{array}$ \\
\hline niedrig & $\begin{array}{l}\text { Verachtendes Vorurteil } \\
\text { Status: niedrig }\end{array}$ & $\begin{array}{l}\text { Neidgeprägtes Vorurteil } \\
\text { Status: hoch }\end{array}$ \\
& Konkurrenz: hoch & $\begin{array}{l}\text { Konkurrenz: hoch } \\
\text { Verachtung, Abscheu, Zorn, }\end{array}$ \\
& $\begin{array}{l}\text { Ressentiment } \text { Eifersucht } \\
\text { (z. B. Sozialhilfeempfänger, }\end{array}$ & \\
& Arme) &
\end{tabular}

Tab. 2: Vier Arten von Out-Groups, Kombination von Status und Konkurrenz, und entsprechende Formen von Vorurteilen als Funktion der Dimensionen Wärme und Kompetenz (Fiske/Cuddy/Glick/Xu 2002: 881)

Bei Bremer werden die primären Adressat(inn)en mit einem paternalistischen Vorurteil belegt. Entsprechend wird ihnen niedrige Kompetenz und hohe Wärme zugewiesen („Man nimmt diesen Menschen die Würde“). Deshalb begegnet Bremer ihnen mit Mitleid und Sympathie (s. Tabelle 2). Die Leichte Sprache selbst und die Textproduzent(inn)en sowie auch die Behindertenverbände werden mit einem verachtenden Vorurteil belegt. Entsprechend werden ihnen niedrige Kompetenz und Kälte zugewiesen: „bildungsfeindlich“, „Geschäft betreiben“, „Klientelismus“, „Verfälschung“, „Parodien auf behinderte Menschen“ etc. Bremer begegnet ihnen, dem Muster entsprechend, mit Verachtung, Abscheu und Zorn (s. Tabelle 2).

Ähnliche Einlassungen, die in derselben Weise im angezeigten Stereotypenraster verortet werden können, sind im deutschsprachigen Feuilleton und insgesamt im Diskurs um die Leichte Sprache nicht selten. Fiske/Cuddy/Glick/Xu (2002) stellen heraus, dass sich diese Stereotype in Bezug auf Gender, Ethnizität, in den USA auch ,race', Schicht, Alter und Behinderung finden lassen, was auf 
eine hohe Generalisierungsfähigkeit dieser Muster hinweist. Deshalb ist es kein Wunder, dass sich die Argumente in den Feuilletons mit Bezug auf die Leichte Sprache, die Akteure und Akteurinnen und die Adressat(inn)en gleichen und die Stereotypen in der beschriebenen Form ubiquitär sind.

Eine solche stereotypengeprägte Wahrnehmung der Leichten Sprache behindert potentiell eine Ausweitung des Textangebots, beispielsweise wenn potentielle Textanbieter durch eigene Vorurteile oder in Erwartung negativer Rückmeldung aus der Öffentlichkeit von barrierefreien Kommunikationsangeboten Abstand nehmen. Eine Verbesserung der Situation kann über eine veränderte Wahrnehmung der Leichten Sprache erzielt werden (Bredel/Maßß 2016a: 46ff.), insbesondere durch eine Arbeit an der Variable „Konkurrenz“: Leichte Sprache stellt keine Konkurrenz zur deutschen Standardsprache dar, denn das Textangebot in Leichter Sprache ist grundsätzlich ein Zusatzangebot, es ersetzt keine allgemein- oder fachsprachlichen Ausgangstexte. Zentral ist hier auch, dass sich Leichte-Sprache-Texte bezüglich Orthographie und Grammatik im Rahmen des deutschen Standards bewegen und folglich auch hier keinen Angriff auf die Standardsprache darstellen.

Ebenfalls zuträglich ist eine Arbeit an der Variable „Status“: Der gesellschaftliche Diskurs über Behinderung kann mit einer Aufwertung von Konzepten wie Diversität und Barrierefreiheit in der Gesellschaft insgesamt einhergehen; hier haben die UN-Behindertenrechtskonvention und die auf sie aufstufenden Maßnahmenpläne (z. B. der Nationale Aktionsplan NAP 2.0) in den vergangenen Jahren zu einer neuen Sicht auf Behinderung beigetragen. Jedoch stößt dieses Vorhaben an Grenzen, da es in der allgemeinen Wahrnehmung trotz allem ein Stigma darstellt, keinen Zugriff auf allgemeinsprachliche Texte zu haben und der Leichten Sprache zu bedürfen.

\section{$5 \quad$ Leichte Sprache als Stigma}

Leichte-Sprache-Texte zu benötigen triggert negative Zuschreibungen, denn Beeinträchtigungen der Kommunikationsfähigkeit werden als Stigma wahrgenommen. Jones (1984, deutsche Terminologie nach Knigge 2009) identifiziert 
sechs Dimensionen, die Auswirkungen darauf haben, als wie stark stigmatisierend ein Zustand wahrgenommen wird (ausführlicher hierzu s. Bredel/Maaß 2016a: 50ff.):

1. Kaschierbarkeit (Concealability): Ein Stigma ist umso größer, je stärker es wahrnehmbar ist. Mit Leichter Sprache liegt hier ein Paradoxon vor: Einerseits können die Betroffenen durch die Leichte-Sprache-Texte ihr Stigma leichter kaschieren, weil sie auf die Inhalte eigenständig zugreifen und ohne Offenlegung ihrer Einschränkung in der Gesellschaft agieren können. Andererseits ist durch die Präsenz von Leichte-Sprache-Texten im öffentlichen Raum wahrnehmbar, dass es eine große Zahl von Personen gibt, die auf diese Texte angewiesen sind.

2. Dauer bzw. Entwicklung (Course): Ein dauerhaftes Stigma wird als schwerwiegender wahrgenommen als ein temporäres. Für die Öffentlichkeit ist Leichte Sprache als Durchgangsstufe zum Standard im allgemeinen akzeptabler, als wenn die Leserschaft dauerhaft auf Leichte Sprache angewiesen bleibt. Hier bestehen große Abweichungen zwischen den Adressatengruppen: Einige von ihnen können potentiell über kurz oder lang auf die Standardtexte zugreifen, andere bleiben absehbar dauerhaft auf Leichte Sprache angewiesen. Auch die verschiedenen Textsorten und Gegenstandsbereiche bieten hier ein je unterschiedliches Bild: Während allgemeinsprachliche Texte alltagsnahen Inhalts für manche Teile der Leserschaft in der allgemeinsprachlichen Fassung zugänglich sein oder (mit steigender Leseerfahrung) werden können, trifft dies auf Fachtexte beispielsweise aus dem juristisch-administrativen Bereich nicht in gleichem Maße zu. Insgesamt lässt sich beobachten, dass das Konzept der Leichten Sprache als weniger stigmatisierend wahrgenommen wird, wenn eine Progression der Lesefähigkeit möglich erscheint.

3. Isolationspotential (Disruptiveness): Das Stigma ist umso stärker, je mehr die sozialen Interaktionen der Betroffenen gestört sind. Hier wiederholt sich das Paradoxon: Die reine Präsenz der Leichte-Sprache-Texte im öffentlichen Raum ist der Beleg dafür, dass die Betroffenen nicht ohne Hilfsmittel 
auf Informationen und Diskurse zugreifen können. Gleichzeitig liegt in der Präsenz der Texte auch die Überwindung der Isolation, denn die LeichteSprache-Texte ermöglichen Teilhabe.

4. Ästhetische Beeinflussung (Aesthetic Qualities): Wird eine Beeinträchtigung als abstoßend wahrgenommen, so vergrößert sich das Stigma. Leichte Sprache optimiert Verständlichkeit zulasten der Ästhetik der Sprache. Varianz und Vielfalt, Ausschöpfung der lexikalischen und syntaktischen Möglichkeiten, Indirektheit und Andeutung, Spiel mit der Form - solche und weitere Strategien, die sprachliche Schönheit erfahrbar machen, sind in Leichter Sprache nicht oder nur sehr eingeschränkt zugänglich. Leichte Sprache wird damit als ästhetisch inadäquat wahrgenommen, was das Stigma vergrößert. Dieses Stigma ist kaum überwindbar, da der Eingriff in die Ästhetik der Sprache eine Tatsache darstellt und hier nur sehr begrenzte Handlungsmöglichkeiten bestehen.

5. Verursachung (Origin): Ein als selbstverschuldet wahrgenommenes Stigma ist größer als eines, das als nicht von den Betroffenen verursacht gilt. Deshalb vermindert der Verweis darauf, dass Leichte Sprache Personen mit Behinderungen adressiert, regelmäßig das Stigma. Das betrifft jedoch nur einen Teil der Adressatenschaft; funktionaler Analphabetismus wird häufig als selbstverschuldet wahrgenommen, was zu einer Vergrößerung des Stigmas für diese Gruppe führt.

6. Gefährdung der anderen (Peril): Geht von einer stigmatisierten Gruppe oder Sache potentiell eine Gefahr für andere aus, so wird das Stigma als besonders stark wahrgenommen. Leichte Sprache wird dann als besonders stark stigmatisierend empfunden, wenn sie als Bedrohung der deutschen Bildungssprache wahrgenommen wird. Dies kann durch Eingriffe ins Sprachsystem (z. B. Zulassen inkorrekter Orthographie wie etwa Bindestrichschreibungen vom Typ „Unter-Suchung“) oder durch Versuche geschehen, eine Leserschaft ohne Kommunikationsbeeinträchtigung mit Texten in Leichter Sprache zu adressieren und Leichte Sprache damit quasi als neuen Standard zu postulieren. Beides führt regelmäßig zu einer starken 
Ablehnung. Exemplarisch ist dies greifbar in den heftigen Reaktionen auf Wahlbenachrichtigungen in Leichter Sprache, die entsprechend dem neuen Wahlgesetz zur Landtagswahl 2017 an alle Wähler in Schleswig-Holstein versendet wurden. Das Ziel, hierdurch eine größere Wahlbeteiligung zu erreichen, scheiterte und bescherte den Mitarbeiter(inne)n in den Gemeindewahlbehörden stattdessen heftige Proteste der Wähler(innen) und der Landesregierung bundesweit schlechte Presse (zur Presseberichterstattung über die Leichte Sprache s. Diekmannshenke 2017). Im Oktober 2017 wurde deshalb ein Vorstoß zur erneuten Änderung des Wahlgesetzes vorgenommen, um die in der Landeswahlgesetzgebung verankerte Pflicht zur allgemeinen Wahlbenachrichtigung in Leichter Sprache rückgängig zu machen (Landtag Schleswig-Holstein, 11.10.2017).

Bereits Goffman (1967) hat aber auch darauf verwiesen, dass potentieller Stigmatisierung durch „situation management“ begegnet werden kann. Dies deckt sich mit der oben vorgebrachten These, dass ein Arbeiten an den Variablen „Konkurrenz" und „Status“ zu einer Abmilderung des Stigmas und der paternalistischen und verachtenden Vorurteile führen wird.

6

Forschungsdesiderate

Zwar beschäftigt sich die Forschung seit einigen Jahren mit dem Phänomen der Leichten Sprache und es ist insgesamt ein Zuwachs an Studien im Bereich der Barrierefreien Kommunikation zu verzeichnen, es bleiben jedoch gerade angesichts einer sich bereits manifestierenden, nicht immer funktionalen Praxis noch erhebliche Forschungsdesiderate bestehen. Hier sind insbesondere die folgenden Felder zu nennen:

1. Empirische Untersuchungen: Die Regelwerke aus Praxis und Wissenschaft beruhen im Moment auf Erfahrungen und (wissenschaftlich begründbaren) Annahmen. Es gibt aber bislang noch kaum empirische Studien zur Leichten Sprache mit Proband(inn)en aus den verschiedenen Zielgruppen. Hier gibt es zwar bereits erste Ansätze und Vorstudien (s.o., 
Kap. 3). Empirisch muss aber geprüft werden, ob, wie und inwieweit Leichte Sprache in der bisher praktizierten Form ein funktionierendes Instrument ist, um die Partizipation der Adressat(inn)en tatsächlich zu verbessern. Dafür sind einerseits Studien zum Verstehen und zur Verständlichkeit von Texten in Leichter Sprache erforderlich, andererseits aber auch Untersuchungen zum praktischen Ertrag etwa mit Bezug auf Formen der aktiven Beteiligung der Adressat(inn)en an gesellschaftlichen Diskursen oder mit Bezug auf eine mögliche Entlastung von Behörden.

2. Konzeptionelle Weiterentwicklung: Wenngleich das Konstrukt der Leichten Sprache in Umrissen gut beschrieben ist, führt die Umsetzung der Regeln noch keinesfalls zu widerspruchsfreien Textergebnissen: So führt die Einhaltung aller Reduktionserfordernisse auf Wort- und Satzebene zu manifesten Problemen auf der Textebene: Was lokal (also auf Wort- oder Satzebene) die Verständlichkeit erhöht, kann auf der Textebene der Verständlichkeit gerade entgegenstehen. Dies ist z. B. der Fall, wenn ausführliche oder wiederholte Erläuterungen die thematische Entfaltung eines Texts behindern oder wenn durch die homogenen Layout-Eigenschaften (z. B.: ein Satz pro Zeile; Einrückungen zur Verdeutlichung von hierarchischen Relationen, Beschränkung der Schriftarten) aus der visuellen Makrostruktur des Texts keine Informationen zur Textsorte entnommen werden können. Auch können Regeln der Leichten Sprache mit den Anforderungen von Textsorten kollidieren - beispielsweise widerspricht die Regel, wonach Ereignisse in chronologischer Reihenfolge dargestellt werden sollten, dem Erfordernis von Nachrichtentexten, die üblicherweise mit der Nachricht und nicht mit ihrer Vorgeschichte beginnen. Da Lösungen hier an die jeweiligen Textsorten und Zielsituationen gebunden sind, stellt die Bearbeitung der Textebene in ihrer Vielfalt ein Desiderat der Leichte-Sprache-Forschung dar. Von besonderem Interesse sind hier u.a. Erkenntnisse über die Hierarchie der Regeln auf den unterschiedlichen sprachlichen Ebenen, die Relation der Regeln zueinander und texsortensensitive Strategien zur Bearbeitung der Dilemmata. 
3. Analyse des gesellschaftlichen Diskurses (Stigma und Provokation): Wenn der öffentliche Diskurs über Leichte Sprache in soziologischer, sozialpsychologischer, diskurstheoretischer und politischer Hinsicht mit Blick auf die Dynamik von Stereotypisierungen und negativen Zuschreibungen besser verstanden wird, können daraus ggf. Strategien für eine problemreduzierende Implementierung der Leichten Sprache in der gesellschaftlichen Praxis abgeleitet werden.

Um den Forschungsraum wirkungsvoll auszumessen, bedarf es der Interdisziplinarität: Leichte Sprache ist Teil des Forschungsfelds Barrierefreie Kommunikation, das aus der Perspektive sehr unterschiedlicher Disziplinen (Sonderpädagogik, Psychologie, Sozial- und Organisationspädagogik, Kommunikationstheorie, Medientheorie, Übersetzungswissenschaft, Sprachwissenschaft, Sprachdidaktik etc.) bearbeitet wird. Bislang gibt es unter den Forschenden noch kaum Berührungspunkte; diese wären aber notwendig, um übergreifende Strategien der Kommunikation zu entwickeln, zu überprüfen und zu implementieren. Darüber hinaus werden enge Kooperationen zwischen Forschung und Textpraxis benötigt, die über Pilotstudien mit wissenschaftlicher Begleitung und Monitoringstrukturen Implementierungsprozesse dokumentieren und aufzeigen, wie eine gute und funktionierende Praxis aussehen kann. 


\section{Literaturverzeichnis}

BECKER, LIDIA (im Druck): „Lenguaje claro / llano / ciudadano y lectura fácil: ¿nuevas variedades de comunicación digital de masas más allá del español general / común / total o internacional / neutro?“. In: GREUßLICH, SEBASTIAN / LEBSANFT, FRANZ (Hrsg.): El pluricentrismo de la cultura lingüística hispánica. Reflejos en los medios de comunicación masiva, Göttingen: Vandenhoeck \& Ruprecht.

Bergelt, DAniel/GoldBaCh, Anne/Seidel, ANJA (2016): „Leichte Sprache im Arbeitsleben. Analyse der derzeitigen Nutzung von Texten in Leichter Sprache im beruflichen Kontext von Menschen mit Lernschwierigkeiten“. In: Teilhabe 3, 106-113.

BGG Entwurf (2016): Entwurf des Gesetzes zur Weiterentwicklung des Behindertengleichstellungsrechts (BGG), BT-Drs. 18/7824 09. 03. 2016, http://dip21.bundestag.de/ dip21/btd/18/078/1807824.pdf (letzter Zugriff 10.7.2018)

BGG neu (2016): Gesetz zur Weiterentwicklung des Behindertengleichstellungsrechts vom 19. 07. 2016, https://www.gesetze-im-internet.de/bgg/BGG.pdf (letzter Zugriff 10.7.2018)

BMAS (2014): Leichte Sprache. Ein Ratgeber. Abdruck der „Regeln für Leichte Sprache“ des Netzwerks Leichte Sprache e. V. http://www.bmas.de/SharedDocs/Downloads/ DE/PDF-Publikationen/a752-ratgeber-leichte-sprache.pdf?__blob=publicationFile (letzter Zugriff 10.8.2018)

Bock, Bettina M. (2014): „,Leichte Sprache‘. Abgrenzung, Beschreibung und Problemstellungen aus linguistischer Sicht“. In: JEKAT, SusAnne J./JÜngST, HeIKE ELISABeth/Schubert, Klaus/Villiger, Claudia (Hrsg.): Sprache barrierefrei gestalten. Perspektiven aus der Angewandten Linguistik. Berlin: Frank \& Timme, 17-52.

Bock, BetTinA M. (2015): „Barrierefreie Kommunikation als Voraussetzung und Mittel für die Partizipation benachteiligter Zielgruppen. Ein (polito-)linguistischer Blick auf Probleme und Potenziale von ,Leichter und, einfacher' Sprache“, in: Linguistik Online 73, 115-137. https://bop.unibe.ch/linguistik-online/article/view/2196/3366 (letzter Zugriff 10.7.2018)

Bock, Bettina M./Fix, Ulla/Lange, DAisy (Hrsg.) (2017a): „Leichte Sprache“ im Spiegel theoretischer und angewandter Forschung. Berlin: Frank \& Timme.

Bock, Bettina M./Fix, Ulla/Lange, Daisy (2017b): „Das Phänomen ,Leichte Sprache im Spiegel aktueller Forschung - Tendenzen, Fragestellungen und Herangehensweisen“. In: Bock, BetTina M. /FIX, Ulla/LAnge, DAisy (Hrsg.): „Leichte Sprache“ im Spiegel theoretischer und angewandter Forschung. Berlin: Frank \& Timme, 11-31.

Bock, BetTina M./LANGE, Daisy (2017): „Empirische Untersuchungen zu Satz- und Textverstehen bei Menschen mit geistiger Behinderung und funktionalen Analphabeten“. In: Bock, BeTTINA M./ FIX, UlLA/LANGE, DAISY (Hrsg.): „Leichte Sprache“ im Spiegel theoretischer und angewandter Forschung. Berlin: Frank \& Timme, 253-274. 
Bohman, Ulla (2017): „Easy-to-Read in Sweden“, in: Bock, BetTina/Fix, Ulla/ LANGE, DAISY (Hrsg.): „Leichte Sprache“ im Spiegel theoretischer und angewandter Forschung. Berlin: Frank \& Timme, 447-455.

Bredel, URsula/LANG, Katrin/MaAß, Christiane (2016): Zur empirischen Überprüfbarkeit von Leichte-Sprache-Regeln am Beispiel der Negation, in: MäLZER, NATALIE (Hrsg.): Barrierefreie Kommunikation. Perspektiven aus Theorie und Praxis. Berlin Frank \& Timme, 95-115.

Bredel, Ursula/MaAß, Christiane (2016a): Leichte Sprache. Theoretische Grundlagen, Orientierung für die Praxis. Berlin: Dudenverlag.

Bredel, Ursula/MaAß, Christiane (2016b): Ratgeber Leichte Sprache. Alle wichtigen Regeln, Empfehlungen für die Praxis. Berlin: Dudenverlag.

Bredel, Ursula/MaAß, Christiane (2017): „Wortverstehen durch Wortgliederung Bindestrich und Mediopunkt in Leichter Sprache." In: BoCK, BetTINA M./FIX, Ulla/LANGe, DaIsy (Hrsg.): "Leichte Sprache“ im Spiegel theoretischer und angewandter Forschung. Berlin: Frank \& Timme, 211-228.

Degener, Theresia (2015): „Die UN-Behindertenrechtskonvention - ein neues Verständnis von Behinderung", in: Degener, Theresia/Diehl, Elke (Hg.): Handbuch Behindertenrechtskonvention. Teilhabe als Menschenrecht, Inklusion als gesellschaftliche Aufgabe. Bonn: Bundeszentrale für politische Bildung, 55-73.

DieKMANNSHENKe, Hajo (2017): „Zwischen ,Leicht kompliziert ${ }^{\star}$ und ,Deutsch light‘. Der mediale Diskurs um die ,Leichte Sprache“", in: Bock, BetTina/FiX, Ulla/ LANGE, DAISY (Hrsg.): „Leichte Sprache“ im Spiegel theoretischer und angewandter Forschung. Berlin: Frank \& Timme, 111-127.

Fiske, Susan T./Cuddy, Amy J.C./Glick, Peter/Xu, Jun (2002): „A Model of (Often Mixed) Stereotype Content“. In: Journal of Personality and Social Psychology 82, 878902.

Goffman, ERVING (1967): Stigma. Über Techniken der Bewältigung beschädigter Identität. Frankfurt/M.: Suhrkamp.

Goldbach, Anne/SchupPener, SASKia (2016): „Leichte Sprache im Kontext beruflicher Teilhabe von Menschen mit Lernschwierigkeiten. Partizipative Forschung an der Universität Leipzig“. In: Hinz, AndreAs/Kinne, TANJA/Kruschel, Robert/ Winter, StePhanie (Hrsg.): Von der Zukunft her denken. Inklusive Pädagogik im Diskurs. Bad Heilbrunn: Klinkhardt, 200-209.

Gutermuth, Silke (im Druck). One for all? Eine zielgruppenorientierte Rezeptionsstudie zu Leichter und Einfacher Sprache. Dissertation. Germersheim: Johannes-GutenbergUniversität Mainz.

Hansen-Schirra, Silvia/Gutermuth, Silke (im Druck): „Modellierung und Messung Einfacher und Leichter Sprache." In: Working Papers in Applied Linguistics 13. Winterthur: ZHAW Zürcher Hochschule für Angewandte Wissenschaften. 
Inclusion Europe: Informationen für alle Europäische Regeln, wie man Informationen leicht lesbar und leicht verständlich macht, http://easy-to-read.eu/wp-content/ uploads/2014/12/DE_Information_for_all.pdf (letzter Zugriff 10.7.2018)

JONES, EDWARD E. (1984): Social stigma. The psychology of marked relationships. New York: Freeman.

KNIGGE, Michel (2009): Hauptschüler als Bildungsverlierer? Eine Studie zu Stigma und selbstbezogenem Wissen bei einer gesellschaftlichen Problemgruppe. Münster: Waxmann.

Landtag Schleswig-Holstein, http://www.landtag.ltsh.de/plenumonline/archiv/wp19/ 05/debatten/08.html (letzter Zugriff 11.10.2017)

LANGE, DAISY/Bock, BETtina (2016): „Was heißt ,Leichte‘ und ,einfache‘ Sprache? Empirische Untersuchungen zu Begriffssemantik und tatsächlicher Gebrauchspraxis“. In: Mälzer, Nathalie (Hrsg.): Barrierefreie Kommunikation - Perspektiven aus Theorie und Praxis. Berlin: Frank \& Timme, 117-134.

LASCH, AleXANDER (2017): „Zum Verständnis morphosyntaktischer Merkmale in der funktionalen Varietät ,Leichte Sprache““. In: Bock, BeTtina M./FIX, UlLA/LANGE, DAISY (Hrsg.): „Leichte Sprache“ im Spiegel theoretischer und angewandter Forschung. Berlin: Frank \& Timme, 275-299.

Leskelä, Leealaura (2017): „Textgenre-orientierte Prinzipien für Leichtes Finnisch“, in: Bock, Bettina/Fix, Ulla/LAnge, Daisy (Hrsg.): „Leichte Sprache“ im Spiegel theoretischer und angewandter Forschung. Berlin: Frank \& Timme, 431-446.

MAAß, ChristiAne (2015): Leichte Sprache. Das Regelbuch. Münster: Lit.

MaAß, Christiane/Rink, Isabel/Zehrer, Christiane (2014): „Leichte Sprache in der Sprach- und Übersetzungswissenschaft“. In: JeKAT, SusAnNE J./JÜNGST, HeIKE ElISabeth/Schubert, Klaus/Villiger, Claudia (Hrsg.): Sprache barrierefrei gestalten. Perspektiven aus der Angewandten Linguistik. Berlin: Frank \& Timme, 53-85.

Neue Zürcher Zeitung: „,Schlimmer als Realsatire‘. Der Bildungsexperte Rainer Bremer kritisiert die ,Leichte Sprache` als bildungsfeindlich und befürchtet eine Abwertung der sprachlichen Bildung“. Interview: Tobias Ochsenbein 8.9.2014. https://www.nzz.ch/wissenschaft/bildung/schlimmer-als-realsatire-1.18378993 (letzter Zugriff 10.7.2018)

POTTHOFF, SARA (2016): ,Leichte Sprache“ im frankokanadischen Sprachraum. Das Stufenmodell Texte Simplifié im Lichte des deutschen Diskurses über Leichte Sprache. Masterarbeit, Universität Hildesheim.

RINK, ISABEL (in Vorbereitung): Rechtskommunikation und Barrierefreiheit. Eine korpusgestützte Analyse der Übersetzung juristischer Informations- und Interaktionstexte in Leichte Sprache im Pilotprojekt „Leichte Sprache in der Niedersächsischen Justiz“. Manuskript der Dissertationsschrift.

TJARKS-SOBHANI, MARITA 2012: „Leichte Sprache gegen schwer verständliche Texte. Technische Dokumentation für Analphabeten“, in: Fachzeitschrift, technische kommunikation' 6, 25-30. 
United Nations Treaty Collection: Convention on the Rights of Persons with Disabilities, https://treaties.un.org/Pages/ViewDetails.aspx?src=TREATY\&mtdsg_no=IV-15\& chapter $=4 \&$ clang $=$ =en (letzter Zugriff 10.7.2018)

WAGNER, SUSANNE (2015): Im Spannungsfeld von fachlichen Anforderungen und sprachlichen Barrieren. Einfache Sprache in der Beruflichen Bildung. Vortragsmanuskript auf der Tagung „Barrierefreie Kommunikation in interdisziplinärer Perspektive“, 23.-25. Oktober 2015, Hildesheim. Folien online abrufbar unter: https://www.researchgate.net/profile/Susanne_Wagner (letzter Zugriff 10.7.2018)

Wagner, Susanne/SChlenKer-Schulte, Christa (2006): Textoptimierung von Prüfungsaufgaben. Handreichung zur Erstellung leicht verständlicher Prüfungsaufgaben. 2. überarbeitete Auflage, Halle: FST.

Wagner, Susanne/SChlenker-SChulte, Christa $\left({ }^{10} 2015\right)$ : Textoptimierung von Prüfungsaufgaben, Halle: Institut für Textoptimierung. https://www.zfamedien.de/ downloads/ZFA/TOP_Broschuere-gesamt.pdf (letzter Zugriff 10.7.2018) 

CHRISTIANE MAAß

\section{Übersetzen in Leichte Sprache}

\section{Leichte Sprache als verständlichkeitsoptimierte Varietät des Deutschen}

Leichte Sprache beginnt zunächst als Praxiskonzept, das seit den 2010er Jahren verstärkt eine wissenschaftliche Ausarbeitung erfährt (s. dazu den Beitrag von Bredel/Maaß im vorliegenden Band). Aus dem Empowerment ergingen frühzeitig nachdrückliche Forderungen nach Texten in Leichter Sprache, die einer Adressatenschaft mit Kommunikationsbeeinträchtigungen Teilhabe ermöglichen sollen. Ausgehend von der UN-Behindertenrechtskonvention (UN-BRK) ist in Deutschland inzwischen eine Rechtslage erwachsen, die zumindest in begrenztem Umfang diese Forderung nach Textvarianten insbesondere zu Texten der juristisch-administrativen und sonstigen behördlichen Kommunikation in Leichter Sprache stützt (s. den Beitrag von Lang im vorliegenden Band). Hinzu kommen zahlreiche und aktuell weiter wachsende Textangebote in Leichter Sprache in anderen gesellschaftlichen Bereichen, u.a. Medien (s. den Beitrag von Heerdegen-Wessel im vorliegenden Band), Medizin (s. den Beitrag von Schindler im vorliegenden Band) oder Kultur (hier insbesondere Museum, s. hierzu Al Masri-Gutternig/Reitstätter 2017 und den Beitrag von Schum/Rantamo im vorliegenden Band). Gelegentlich liegen hier Neuschaffungen von Texten vor, üblicherweise schließen sich die Leichte-Sprache-Texte jedoch an ein bestehendes ausgangssprachliches Textangebot an und sind damit intralinguale Übersetzungen (Begriffsbestimmung s.u.).

Leichte Sprache ist eine Varietät des Deutschen mit reduziertem lexikalischen und grammatischen Inventar. Sie setzt auf einen zentralen, alltagsnahen Wortschatz, also auf Kernwörter, die stilistisch neutral, präzise, möglichst arm an Nebenbedeutungen und nicht metaphorisch verwendet sind (zur Bestimmung dieses Wortschatzes s. Bredel/Maßß 2016a: 345ff. und Bredel/Maaß 
2016b: 75f.). Kann aufgrund der Thematik und Funktion der Texte nicht auf periphere, insbesondere fachsprachliche Begriffe verzichtet werden, so werden diese im Text erläutert. Schriftbasierte Abkürzungen vom Typ bzw. oder $z$. B. werden vermieden, lexikalisierte Abkürzungen des zentralen Wortschatzes vom Typ $L K W$ oder $W C$ können dagegen verwendet werden. Lange Komposita (und in bestimmten Grenzen auch Derivationen) können aufgegliedert werden, um die Fugen sichtbar zu machen und damit einerseits die Perzeption zu unterstützen und andererseits dem Wortverstehen Vorschub zu leisten, indem vorher bekannte Begriffe im Wort freigestellt werden (vgl. Bredel/Maaß 2017). Hierfür stehen der Bindestrich (Narkose-Facharzt) und der Mediopunkt (Zusammen·arbeit) zur Verfügung: im zweiten Fall werden in einem fünfsilbigen Wort zwei den meisten Leser(inne)n bekannte zwei- bzw. dreisilbige Wörter (zusammen; Arbeit) separat wahrnehmbar gemacht, das Kompositum ergibt sich semantisch weitgehend aus der Verbindung seiner beiden Teile. Dies ist auch beim ersten Beispiel der Fall, das jedoch ein Fachwort (Narkose) enthält, das durch den Bindestrich besser perzipierbar ist und im nächsten Schritt einer Erklärung zugeführt werden kann bzw. bereits vorab im Text erläutert wurde. Von großer Bedeutung ist dabei die Wahrung einer korrekten Orthographie, denn falsche Bindestrichschreibungen mit wortinterner Großschreibung, wie sie sich in der Praxis häufig finden ( ${ }^{\star}$ Kranken-Haus; ${ }^{\star}$ Eigen-Name; Unter-Suchung) stigmatisieren die Leserschaft und wirken auf viele Leser(innen) als Provokation (zur Stigmatisierung durch Leichte Sprache s. Bredel/Maaß 2016a: 45ff. und den Beitrag von Bredel/Maaß im vorliegenden Band). Außerdem stehen sie dem Übergang in die reguläre Schriftlichkeit für solche Leser(innen) entgegen, die Leichte Sprache nur temporär oder nicht für alle Textsorten benötigen und dann mit dem Problem konfrontiert sind, dass ein Teil der im Leichte-Sprache-Kosmos erlernten Wörter Falschschreibungen sind. Und schließlich erschweren sie das Wiederfinden der entsprechenden Wörter außerhalb des Leichte-Sprache-Kontexts, etwa wenn am Strand der Tageskurbeitragsautomat gesucht wird, auf den im Leichte-Sprache-Text als ${ }^{\star}$ Tages-Kur-Beitrags-Automat verwiesen wird.

Auf Satzebene wird auf Nebensätze und nach Möglichkeit auch auf Hauptsatzreihungen verzichtet, stattdessen werden einfache Hauptsätze verwendet. Dennoch notwendige Reihungen werden in Form von Aufzählungen mit einer visualisierten Gliederungsstruktur über das Layout salient gemacht: 


\section{Fastnacht in Heidelberg}

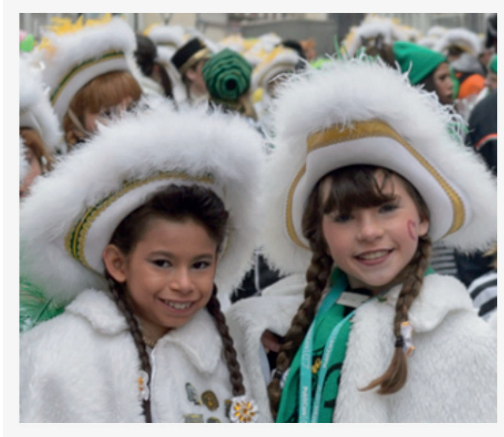

\section{Text vor-lesen $\theta$}

Gerade ist Fastnachts-zeit.

Wir erklären:

- Was ist Fastnacht?

- Wann ist der Fastnachts-umzug?

\section{Weiterlesen >}

Abb. 1: Teaser zu einem Nachrichtenartikel auf www.einfach-heidelberg.de, Text vom 11.2.2018

Die Informationsstruktur von Texten in Leichter Sprache ist wenig komplex, es wird der Verbal- gegenüber dem Nominalstil vorgezogen. Letzterer ist mit fachsprachlicher Schriftlichkeit assoziiert und bezeichnet eine syntaktische Struktur, bei der die meisten Informationen über komplexe nominale Strukturen in den Text eingespeist werden (s. Rink in Vorbereitung). Leichte Sprache verzichtet darüber hinaus auf weniger frequente oder gehäuft in der Schriftsprache vorkommende grammatische Kategorien wie den Genitiv, das Passiv, das Präteritum oder den Konjunktiv (zu Ersetzungsmöglichkeiten s. Bredel/Maaß 2016a: 299ff. und 439ff., Bredel/Maaß 2016b: 130ff.). Die Ereignisse werden in handlungsorientierter Form direkt an die Oberfläche geholt und vom Hier-und-Jetzt aus unter Bezugnahme auf die gegebene Welt (statt auf nicht Existentes) dargestellt.

Auf Textebene besteht die Herausforderung darin, dass je nach Textgegenstand teilweise höchst komplexe Gegenstände mit wenig komplexen Mitteln korrekt wiedergegeben werden müssen. Stimmen und Positionen werden im Text kenntlich gemacht, die Texte haben in der Regel eine klare und explizite Handlungsorientierung.

Texte in Leichter Sprache weisen eine gut erfassbare Makrostruktur auf; dabei helfen Zwischenüberschriften und/oder Randglossen, die Teilthemen wahrnehmbar machen. Häufig weisen Leichte-Sprache-Texte Bilder und visuelle 
Leitsysteme auf, um die Leser(innen) auf Textebene zu orientieren. Dabei wird in der Praxis vielfach eine kindlich anmutende Bebilderung eingesetzt (z. B. der Bildersatz der Bremer Lebenshilfe). Dies ist jedoch problematisch, weil eine offenkundig nicht altersangemessene bzw. markierte Bildlichkeit die Leser(innen) als anders herausstellt und damit stigmatisiert. Darüber hinaus werden die Textsorten gleichschaltet: Wenn alle Texte in ähnlicher Bebilderung gestaltet werden, so können Leser(innen) nicht auf einen Blick erste Informationen über die Textfunktion entnehmen. Die Ausgangstexte sind in der Regel je nach Textsorte visuell sehr unterschiedlich gestaltet und ermöglichen damit den Leser(inne)n Vorannahmen und Erwartungen, die für das Textverständnis hilfreich sind. Ist die Visualisierung für Leichte-Sprache-Texte immer gleich, so entfällt diese Verstehensressource.

Auch das Layout der Leichte-Sprache-Texte weist, folgt man den Regelvorgaben, eine große Homogenität auf (s. den Beitrag von Alexander im vorliegenden Band): Vergrößerte serifenlose Schrift, jeder Satz auf einer neuen Zeile, Einrückungen für Erläuterungen und direkte Rede. Ein solches Layout ist perzeptionserleichternd, es tilgt jedoch wiederum textsortenspezifische Unterschiede, die in der regulären Schriftlichkeit zum Textverständnis beitragen, weil die Texte durch ihr Layout bestimmte Erwartungen über Inhalt und Funktion beim Publikum wecken. Außerdem vergrößert dieses Layout das Textvolumen und beschränkt damit die Möglichkeiten für die Übersetzer(innen), Inhaltskonstanz zum Ausgangstext herzustellen. Leichte Texte in anderen Ländern (beispielsweise Finnland oder Norwegen) lehnen sich in Layout und Bebilderung generell enger an die Ausgangstexte an und verfolgen damit eine Strategie, die sich im Sinne der UN-BRK näher am Ideal des Universal Design ausrichtet bzw. an der Teilhabe an gleichen Angeboten wie alle anderen Menschen. Layout und Visualisierungsstrategien sind ein drängendes Desiderat der LeichteSprache-Forschung und -Praxis, es eröffnet sich jedoch aktuell ein Diskurs in der Wissenschaft (u.a. Hochschule Merseburg) und Praxis (s. exemplarisch https://www.inkl-design.de).

Durch die typische Erläuterungsstruktur (s. Strategien auf Wortebene) wird bei Leichte-Sprache-Texten der Textfluss mit vielen Einschüben durchbrochen. Hier setzen manche Texte auf Glossare; diese sind allerdings nur dann hilfreich, wenn die anvisierte Adressatenschaft nicht intensiv darauf zugreifen muss, da 
die Leser(innen) beim Konsultieren des Glossars ihre Lektüre unterbrechen und dann die richtige Stelle im Text wiederfinden müssen (Bredel/Maaß 2016b: 163).

Insgesamt werden durch die Leichte-Sprache-Regeln auf Wort-, Satz- und Textebene die Unterschiede zwischen den Textsorten eingeebnet, was zu eher gleichförmigen Texten führt. Durch die Erläuterungen, das perzeptionsorientierte Layout (jeder Satz auf einer neuen Zeile, Schriftgröße, Bebilderung) und den Verzicht auf indirekte Versprachlichung wird der Leichte-Sprache-Text bei informationskonstanter Übersetzung ein erheblich größeres Volumen aufweisen als der Ausgangstext. Dies ist einer Adressatenschaft mit Leseeinschränkungen jedoch im Allgemeinen nicht zuzumuten, weshalb andere Wege gefunden werden müssen, um Textinhalte korrekt, funktional sowie adressaten- und situationsangemessen in Leichte Sprache zu bringen. Das Übersetzen in Leichte Sprache stellt damit eine große Herausforderung für Übersetzer(innen) dar.

\section{2 Übersetzen in Leichte Sprache als intralinguale Übersetzung}

Das Übersetzen in Leichte Sprache hat in Deutschland inzwischen eine reiche Praxis ausgebildet: In den vergangenen Jahren haben sich bundesweit zahlreiche Büros für Leichte Sprache gegründet, die Übersetzungen allgemein- oder fachsprachlicher Texte in Leichte Sprache anbieten. Die Übersetzerverbände, insbesondere der Bundesverband der Dolmetscher und Übersetzer (BDÜ), der ca. 80\% der organisierten Übersetzer(innen) vertritt, sowie die Assoziierten Dolmetscher und Übersetzer in Norddeutschland (ADÜ), der größte Übersetzerund Dolmetscherberufsverband im Norden der Republik, haben ihren Tätigkeitsbereich um die Leichte Sprache erweitert. In seiner Pressemitteilung vom 8. Januar 2018 teilte der ADÜ Nord mit:

Ab sofort öffnet sich der ADÜ Nord für Leichte-Sprache-Übersetzer/ -innen. [...] Der Verband wird von nun an auch Sprachmittler aus dem Bereich der Leichte- Sprache-Übersetzung aufnehmen. Damit erweitert der ADÜ Nord sein verbandliches Tätigkeitsprofil um diesen jungen Arbeitsbereich. (ADÜ Nord: Pressemitteilung vom 9.1.2018) 
Der BDÜ hat ein Zertifizierungsprogramm für die eigenen Übersetzer(innen) implementiert, die ihr Tätigkeitsfeld um die Leichte Sprache ergänzen wollen.

Auch in den Bereich der universitären Übersetzerausbildung hat Leichte Sprache inzwischen Einzug gehalten. So ist das Übersetzen in Leichte Sprache seit 2011 Teil der Übersetzungsmasterstudiengänge „Medientext und Medienübersetzung“ sowie „Internationale Fachkommunikation: Sprache und Technik“ am Institut für Übersetzungswissenschaft und Fachkommunikation der Universität Hildesheim, wo am 1. Januar 2014 überdies die Forschungsstelle Leichte Sprache gegründet wurde, die einen sprach- und übersetzungswissenschaftlichen Ansatz verfolgt. Seit Oktober 2018 gibt es hier einen weiteren Masterstudiengang, „Barrierefreie Kommunikation“, der u.a. Übersetzen in Leichte Sprache zum Gegenstand hat.

Das Erstellen von Leichte-Sprache-Texten der Übersetzung zuzurechnen ist nicht mit jedem Übersetzungsbegriff kompatibel. Viele Übersetzungsdefinitionen fußen auf einem interlingualen Transferkonzept; exemplarisch sei hier Albrecht (2005) genannt, demzufolge Übersetzen „ein Akt sprachlichen Handelns ist und die Übersetzung ein Text, in dem sich ein Text in anderer Sprache spiegelt" (Albrecht 2005: XIII).

Auf der anderen Seite findet sich mit Jakobson bereits 1959 ein Autor, der den traditionellen Übersetzungsbegriff für weitere Formen öffnet. Jakobson (1959: 233) unterscheidet drei Übersetzungsarten:

- Interlinguale Übersetzung, die er als Überführung sprachlicher Zeichen einer Sprache in sprachliche Zeichen einer anderen Sprache definiert

- Intralinguale Übersetzung, verstanden als Substitution sprachlicher Zeichen einer Sprache durch andere Zeichen derselben Sprache und

- Intersemiotische Übersetzung, definiert als Übertragung sprachlicher Zeichen in ein nonverbales Zeichensystem. 
Im Anschluss an diese Unterteilung und die weitere Ausarbeitung des Modells in Siever (2010) unterscheiden Bredel/Maaß (2016a) die folgenden Übersetzungsdimensionen:

\begin{tabular}{|l|l|l|}
\hline Zeichensystem & Sprache & Kultur \\
\hline Intersemiotisch & Interlingual & Interkulturell \\
\hline Intrasemiotisch & Intralingual & Intrakulturell \\
\hline
\end{tabular}

Abb. 2: Übersetzungsdimensionen (Bredel/Maaß 2016a: 183 in Modifikation zu Siever 2010: 224)

In Anschluss an Jakobson und Siever kann das Übersetzen in Leichte Sprache also als intralinguales Übersetzen konzeptualisiert werden. Darüber hinaus ist es dann intersemiotisch, wenn bestimmte Inhalte, die im Ausgangstext nur verbal vorliegen, zusätzlich in Bildern umgesetzt werden, wie das in Leichter Sprache häufig geschieht (zur Visualisierung von verbal ausgedrückten Inhalten durch Bilder s. Pridik im vorliegenden Band sowie Bredel/Maaß 2016a: 271296). Interkulturell ist Übersetzen in Leichte Sprache dann, wenn die Adressatenschaft nicht derselben Kultur angehört wie die Leserschaft des Ausgangstexts. Das ist beispielsweise der Fall, wenn ein Text in Leichter Sprache für Personen mit Deutsch als Zweitsprache erstellt oder übersetzt wird. Richten sich die Leichte-Sprache-Texte dagegen an Personen, die mit der deutschen Kultur vertraut sind, so bleibt das Übersetzen in Leichte Sprache intrakulturell.

Leichte Sprache ist dabei nicht die einzige Form der intralingualen und (potentiell) intersemiotischen Übersetzung. In den vergangenen Jahren hat sich das Feld der Übersetzungs- und Verdolmetschungsarten diversifiziert und es ist im deutschsprachigen Raum und darüber hinaus eine rege Forschung zu Formen der Übersetzung und Verdolmetschung wie zum Beispiel Untertitelung für Hörgeschädigte (s. Mälzer/Wünsche im vorliegenden Band), Audiodeskription (s. Benecke im vorliegenden Band), Schriftdolmetschen (s. die Beiträge von Witzel und Kurch im vorliegenden Band) oder Theaterübertitelung (Griesel 2007) entstanden. Besondere Dynamik prägten dabei Theorieansätze zur Barrierefreien Kommunikation aus, die einen soliden translationstheoretischen Aspekt aufweisen (für einen Übertrag „klassischer" translatologischer Theorie auf die Gebärdensprachverdolmetschung s. exemplarisch den Beitrag von Benner/Herrmann im vorliegenden Band). 
Dieser Diversifizierung der übersetzungswissenschaftlichen Forschung entspricht eine Spezialisierung und Professionalisierung auf Seiten der beruflichen Tätigkeitsausübung von Übersetzer(inne)n, nicht zuletzt ermöglicht durch die neuen technologischen Entwicklungen im Bereich der Medientexte. Dies hat zu einer veränderten Marktlage geführt, die ihrerseits einer Anpassung an die Nachfrage durch die Bedarfsträger entspricht.

Im Bereich der Leichte-Sprache-Übersetzung steht die Professionalisierung jedoch noch am Anfang; viele Akteure im Feld sind keine ausgebildeten Übersetzer(innen), sondern sie sind im Rahmen ihrer Arbeit mit der Zielgruppe nun u.a. in Leichte-Sprache-Büros tätig. Insbesondere mit Blick auf die Rechtslage (s. die Artikel von Lang und Bredel/Maaß im vorliegenden Band) werden aber künftig verstärkt gerade Fachtexte in Leichte Sprache zu übersetzen sein; diese können von nichtprofessionellen, intuitiv handelnden Übersetzer(inne)n nicht adäquat bewältigt werden. Holz-Mänttäri (1984) stellt in ihrem Werk „Translatorisches Handeln“ die Notwendigkeit einer Professionalisierung der (interlingualen) Übersetzungspraxis heraus und sieht hier die übersetzungswissenschaftliche Forschung in der Pflicht. Unzureichende Forschung führe zu schlecht ausgebildeten Übersetzer(inne)n, die wiederum schlechte Texte produzierten; Holz-Mänttäri (1984: 165) spricht von einem „Teufelskreis“, der „nur im Wissenschaftsbereich wirklich durchbrochen werden" könne:

[...] weil nur dort ,Erkenntnis' der Bedingungen zu konstruktiven Maßnahmen führen kann. Bohrende Fragen, wie sie zum jetzigen historischen Zeitpunkt im Praxisbereich immer lauter gestellt werden, können den Erkenntnisprozess fördern. Insofern könnte ein gemeinsames Bemühen um die gemeinsame Sache seitens der vier Aspektvertreter

- translatorischer Bedarfsträger

- translatorischer Produzent

- translatologischer Ausbilder

- translatologischer Forscher 
zum gegenwärtigen historischen Zeitpunkt von großem Nutzen sein. Die Entwicklung hat wohl die Marge erreicht, wo naturwüchsiges und intuitives Handeln der vier Aspektvertreter den Anforderungen nicht mehr gerecht wird und für das Gesamtgefüge, Gemeinschaft' nicht mehr tragbar ist. (Holz-Mänttäri 1984: 165).

Die interlinguale Übersetzung hat sich inzwischen im skizzierten Sinne professionalisiert und es findet eine Verschränkung von Forschung und Praxis statt, greifbar u.a. in der Zusammenarbeit von Universitäten und Berufsverbänden der Übersetzer(innen) und Dolmetscher(innen) in Aus- und Weiterbildung. Ein ähnlicher Weg zeichnet sich nun auch, wie eben beschrieben, für das Übersetzen in Leichte Sprache ab, befindet sich jedoch noch an seinem Anfang.

Ein Ansatzpunkt für die Übersetzungstheorie zur Leichten Sprache besteht in der bisherigen Übersetzungswissenschaft, die in den Jahrzehnten ihres Bestehens ein umfangreiches Instrumentarium an Ansätzen und Methoden entwickelt hat. Für einen Überblick über die wichtigsten Strömungen insbesondere der deutschsprachigen Übersetzungswissenschaft sei auf Siever (2010) verwiesen. Nachfolgend werden vor allem äquivalenzorientierte und funktionale bzw. zielsituationsorientierte Übersetzungsansätze mit Blick auf ihre Übertragbarkeit auf die Leichte-Sprache-Übersetzung konsultiert.

\section{3 Äquivalenzorientierte Übersetzungsansätze}

Die traditionelle Übersetzungswissenschaft gründet auf der Annahme einer Äquivalenzrelation zwischen Ausgangs- und Zieltext, wobei der Zieltext den zielsprachlichen Leser(inne)n einen Zugriff auf den Ausgangstext vermittelt, dem er verpflichtet bleibt. Entsprechend waren äquivalenzzentrierte Übersetzungsansätze über Jahrzehnte in der Übersetzungswissenschaft dominant. Äquivalenzzentrierte Ansätze sind meist sprachenpaarbezogen und interlingual ausgerichtet; Siever (2010: 53) kritisiert, dass eine Reihe der Setzungen aus diesen Theorien nur für eng verwandte Sprachen funktioniere und konstatiert einen gewissen Eurozentrismus mancher dieser Ansätze. Für Leichte Sprache hält 
sich der Ertrag aus äquivalenzzentrierter Forschung im Rahmen, da beim Übersetzen in Leichte Sprache, anders als beim Transfer zwischen Sprachenpaaren, keine Entsprechungen für Fachwortschatz oder für bestimmte Muster von Textsorten o.ä. bestehen, weshalb die Möglichkeiten für Eins-zu-Eins-Entsprechungen zwischen Ausgangs- und Zielvarietät begrenzt sind. Entsprechend schließen Vertreter(innen) äquivalenzzentrierter Ansätze die intralinguale (aber auch die intersemiotische) Übersetzung im Allgemeinen aus ihren meist recht engen und spezifischen Übersetzungsbegriffen aus. Auf der anderen Seite werden äquivalenzzentrierte Ansätze für die Leichte-Sprache-Übersetzung gleichwohl benötigt, denn die grundlegende Annahme der Möglichkeit von Äquivalenz gleich welcher Ausprägung ist Voraussetzung für die Entwicklung und den Einsatz von übersetzerischen Hilfsmitteln (CAT-Tools, Glossare und Wörterbücher etc.), die stets auf einer Identifikation lokaler Entsprechungen zwischen Ausgangs- und Zieltext beruhen (s. dazu Bredel/Maaß 2016a: 196ff. und 2016b: 66ff. sowie Maaß/Rink/Zehrer 2014).

Prägend für die äquivalenzorientierten Ansätze waren die Studien von Nida (1964, s. auch Nida/Taber 1969 und weitere) zur dynamischen Äquivalenz bei der Bibelübersetzung in teilweise vorher nicht verschriftete Sprachen, die auch für die Leichte-Sprache-Übersetzung ein bislang nicht zur Geltung gebrachtes Potential haben. Zu nennen sind hier auch die Arbeiten von Albrecht (1990 und öfter) zu Äquivalenz und Invarianz, die texttypologischen Studien von Reiß (1976 und öfter) und insbesondere die Arbeiten der Leipziger Schule (u.a. Kade 1968 und öfter, Jäger 1968 und öfter, Neubert 1973 und öfter), die sich von einem linguistisch und sprachenpaarbezogenen zu einem textbezogenen Vorgehen entwickelte: in Neuberts späten Schriften (z. B. Neubert/Shreve 1992) liegt der Fokus auf der Textebene - der/die Übersetzer(in) erstellt einen funktionierenden Zieltext, dieser wird jedoch nach wie vor als (wenn auch in die Zielkultur eingebettetes) Abbild des Ausgangstexts entworfen. Die Betonung der Textebene ist ein wichtiger Aspekt der Leichte-Sprache-Übersetzung, bei der die besondere Herausforderung ebenfalls auf der Textebene liegt, während ein mechanisches Anwenden der Regeln auf Wort- und Satzebene im Allgemeinen zu überlangen, häufig wenig kohärenten sowie kohäsiven Zieltexten führt, die in der Zielsituation nicht funktional sind. 
Ein einflussreicher Ansatz der deutschsprachigen äquivalenzorientierten Übersetzungswissenschaft ist derjenige von Koller (2011: 63), der fünf „Bezugsoder Äquivalenzrahmen “ unterscheidet:

- Die denotative Äquivalenz bezieht sich auf den außersprachlichen Sachverhalt und stellt sicher, dass der Zieltext mit Bezug auf den Ausgangstext informationskonstant ist.

- Die konnotative Äquivalenz fokussiert auf die Auswahl der Ausdrucksmöglichkeiten und bezeichnet eine Gleichheit oder Vergleichbarkeit in Stil, Soziolekt etc. zwischen Ausgangs- und Zieltext.

- Textnormative Äquivalenz zwischen Ausgangs- und Zieltext besteht bei Vergleichbarkeit mit Bezug auf textgattungsspezifische Merkmale.

- Pragmatische Äquivalenz orientiert sich an den Verstehensvoraussetzungen der jeweiligen Empfängerschaft, auf die der Zieltext gleichermaßen wie der Ausgangstext einzugehen hat.

- Formal-ästhetische Äquivalenz schließlich ist vorhanden, wenn sich der Zieltext an die formalen und ästhetischen Vorgaben des Ausgangstexts anlehnt.

In der Leichte-Sprache-Übersetzung besteht denotative Äquivalenz zwischen Ausgangs- und Zieltext in einer grundsätzlich vergleichbaren Themensetzung. Ob Ausgangs- und Zieltext jedoch informationskonstant sind, hängt von unterschiedlichen Parametern ab (dazu s.u.); im Allgemeinen ist beim Übersetzen in Leichte Sprache die Funktionalität des Texts in der Zielsituation gegenüber dem Kriterium der Informationskonstanz prioritär, so dass denotative Äquivalenz im engeren Sinne auf Ebene des Gesamttexts kaum jemals gegeben sein wird. Lokal ist denotative Äquivalenz zwischen Ausgangs- und Zieltext jedoch auch in der Leichte-Sprache-Übersetzung durchaus gegeben, denn es werden natürlich in beiden Textvarianten gleiche Gegenstände abgebildet, wenn auch keine hundertprozentige Übereinstimmung besteht. Fachsprachliche Terminologie hat in 
Leichte-Sprache-Texten beispielsweise häufig eine Paraphrase oder Erläuterung als Entsprechung; diese referiert dann auf denselben außersprachlichen Gegenstand wie der Terminus, sodass an diesen Stellen im Text auch denotative Äquivalenz anzunehmen ist. Solche Terminus-Erläuterung-Entsprechungen können identifiziert und auch einer Terminologiearbeit zugeführt werden (s. den Beitrag von Zehrer im vorliegenden Band).

Konnotative Äquivalenz zwischen Ausgangs- und Zieltext ist im Regelfall nicht realisierbar, da nur zentrale sprachliche Mittel zur Verfügung stehen und Implizites an die Textoberfläche geholt wird. Gerade mit Bezug auf die Ausdrucksmöglichkeiten, Stil und Register etc. weist die Leichte Sprache eine extreme Reduktion auf, so dass konnotative Äquivalenz meist nicht oder nur lokal und rudimentär besteht. Infolgedessen kommt es insbesondere bei Ausgangstexten, die reich an Konnotationen sind, zu einer erheblichen und nicht kompensierbaren Einschränkung in den Zieltexten. Dies trägt auch zur negativen Haltung gerade bildungsbürgerlich orientierter Kreise gegenüber Leichter Sprache bei, die gerade im Feuilleton und in Kommentaren zu Berichten auf Online-Nachrichtenseiten häufig als Zeichen des Kulturverfalls interpretiert wird. Leichte Sprache ist allerdings nicht der neue Standard, sondern ein zusätzliches Textangebot, also kein Teil des Universal Design, sondern „behinderungsbedingt notwendiges Hilfsmittel“ im Sinne des BGG $\$ 4$, also ein Zusatzangebot zu bestehenden Informationsangeboten.

Auch textnormative Äquivalenz ist durch die Beschränkung auf wenige zentrale sprachliche Mittel nur begrenzt realisierbar, wobei die Gewinnung von Erkenntnissen über eine mögliche Gestaltung von Textsorten und Gattungen in Leichter Sprache aktuell eines der drängenden Desiderate der anwendungsbezogenen Leichte-Sprache-Forschung darstellt.

Im Gegensatz dazu ist pragmatische Äquivalenz zwischen allgemein- oder fachsprachlichem Ausgangstext und Zieltext in Leichter Sprache grundsätzlich möglich, denn so wie der Ausgangstext sich auf die eigene Adressatenschaft in bestimmten Zielsituationen ausrichtet, muss auch der Leichte-Sprache-Text den Verstehensvoraussetzungen seiner Leserschaft und der Rezeption in intendierten Zielsituationen gerecht werden. In der Praxis stellt sich jedoch häufig heraus, dass die Ausgangstexte keine explizite Strategie mit Blick auf die Verstehensvoraussetzungen ihrer Adressatenschaft verfolgen. Viele Texte insbesondere 
der fachexternen Kommunikation richten sich nicht nach den Bedürfnissen oder Verstehensvoraussetzungen ihrer Rezipient(inn)en und werden damit für eine große potentielle Leserschaft zur Barriere (s. den Beitrag von Rink im vorliegenden Band). Leichte-Sprache-Texte hingegen müssen sich in jedem Falle an den Verstehensvoraussetzungen ihrer Adressatenschaft orientieren, auch wenn dadurch gerade keine pragmatische Äquivalenz mit dem Ausgangstext besteht. Die pragmatische Äquivalenz besteht also eher zufällig, und zwar dann, wenn auch dem Ausgangstext eine adressatenspezifische Fokussierung inhärent ist.

Formal-ästhetische Äquivalenz kann, ebenso wie die textnormative Äquivalenz, nur begrenzt erreicht werden, ihre prinzipielle Realisierung im Rahmen der Regelvorgaben kann jedoch Teil der Übersetzungsstrategie sein, um die Brückenfunktion der Leichten Sprache zu wahren. Diese erfordert eine Strukturähnlichkeit zwischen Ausgangstext und Leichte-Sprache-Übersetzung auf der Ebene der textuellen Makrostruktur, um den Leser(inne)n ein Wechseln zwischen beiden Textvarianten zu ermöglichen (Bredel/Maaß 2016a: 57).

Zusammenfassend zeigt sich, dass Äquivalenz in der Leichte-Sprache-Übersetzung zwischen Ausgangs- und Zieltext nur bedingt und partiell erreichbar ist. Die Fokussierung auf die zumindest teilweise bestehende denotative Äquivalenz ist aber hilfreich, um die Möglichkeiten einer professionellen Terminologiearbeit auszuloten und damit Hilfsmittel für die Übersetzung in Leichte Sprache, etwa in Form von Wörterbüchern (Terminus > Paraphrase/Erläuterung), zur Verfügung stellen zu können.

\section{Zielsituationsorientierte Übersetzungsansätze}

In besonderem Maße dienlich für die Theoretisierung der Übersetzung in Leichte Sprache sind jedoch die funktionalistischen, zielsituationsorientierten Übersetzungsansätze, wie sie etwa in der Skopostheorie von Reiß/Vermeer (1984 und öfter) oder der funktionalistischen Übersetzungstheorie mit Fokussierung auf der Kreativität des Übersetzers/der Übersetzerin von Hönig/Kußmaul (1982) und handlungsorientierten Ansätzen wie dem von Holz-Mänttäri (1984) oder Risku (1998 und öfter) bestehen. Diese Ansätze zielen auf einen 
direkten Praxistransfer ab und nehmen insbesondere die Übersetzung als professionelle Tätigkeit, die Übersetzer(innen) als Experten(innen) in ihrem beruflichen Handlungskontext (inklusive Auftragsabwicklung, Kontakt zum Auftraggeber, Entwicklung einer angemessenen Übersetzungsstrategie) sowie die unterschiedlichen Verstehenshorizonte der Kommunikationspartner mit Bezug auf den Textgegenstand in den Blick:

In comparison to other translation theorists [...], the functionalist theorists have done the most to empower translators, elevating them to equal status with authors, editors, and clients, entrusting them to make appropriate, rational decisions that best realize the intended cross-cultural communication. (Gentzler 2001: 71)

Entsprechend sind diese Ansätze in besonderem Maße dazu geeignet, eine Professionalisierung der Übersetzer(innen) zu befördern, weil sie deren Berufsausübung theoretisch reflektieren und somit Einsichten über einen Best Practice ermöglichen, der auch für die akademische Ausbildung und/oder die professionelle Weiterbildung aufschlussreich ist.

Zentral ist für diese Ansätze nicht mehr der Begriff der Äquivalenz, also das Postulat einer möglichst großen Übereinstimmung mit dem Ausgangstext, sondern jener der Adäquatheit (so u.a. bei Reiß/Vermeer 1984) im Sinne einer Übereinstimmung des Zieltexts mit dem ihm zugewiesenen Zweck in der Zielsituation. Entsprechend unterscheidet sich in diesen Ansätzen der Status des Ausgangstexts grundlegend von den Postulaten äquivalenzzentrierter Theorie. Nach Reiß/Vermeer stellt der Ausgangstext im Übersetzungsprozess nur ein Informationsangebot für die Übersetzer(innen) dar, während der Zieltext als „Informationsangebot über ein Informationsangebot“ (Reiß/Vermeer 1984: 67) konzeptualisiert wird. Dies ist u.a. für die Leichte-Sprache-Übersetzung im juristisch-administrativen Kontext bedenkenswert: Rechtstexte verlieren bei der Übersetzung in Leichte Sprache im Allgemeinen ihre Justiziabilität, sind also selbst keine Rechtstexte mehr, sondern „nur“ noch Informationen über Rechtstexte. Sie können damit nicht benutzt werden, um vor einem Gericht - und damit in einem reinen Expertenkontext - Rechte einzuklagen; die eigentlichen 
Rechtstexte bleiben den Adressat(inn)en von Leichte-Sprache-Texten also weiterhin unzugänglich. Sie erhalten aber eine Vorstellung über den Inhalt der Texte sowie einen Überblick über ihre Handlungsoptionen (zur Rechtsübersetzung in Leichte Sprache s. Rink in Vorbereitung).

Hier zeichnen sich Funktionsänderungen der Zieltexte gegenüber den Ausgangstexten ab; zu diesem Aspekt hat insbesondere Nord (1993 und öfter) zahlreiche Publikationen vorgelegt. Nach ihrer Definition ist Übersetzen „eine Handlung [...], bei der Kommunikation zwischen Menschen verschiedener Sprach- und Kulturzugehörigkeit vermittelt wird“ (Nord 2011: 104). Der Übersetzer/die Übersetzerin ist damit „Teil einer kommunikativen Interaktion zur Überwindung von Sprach- und Kulturbarrieren“ (ebd.). Ziel des Übersetzens ist die „Produktion eines funktionsgerechten Zieltextes in Anbindung an einen vorhandenen Ausgangstext, wobei diese Anbindung je nach dem Translatskopos (der angestrebten oder geforderten Funktion des Zieltextes) unterschiedlich zu spezifizieren ist“ (Nord 2011: 17). Dabei ist die Funktion dem Text nicht inhärent, sondern sie wird ihm in der Zielsituation zugewiesen:

Der Text selbst „hat“ also nicht eine Funktion, sondern er „erhält“ eine Funktion in der Rezeptionssituation. (Nord 1993: 9)

Der Begriff des übersetzerischen Handelns ist in den Ansätzen von HolzMänttäri (1984) und Risku $(1998,2016)$ zentral. Diese Ansätze beziehen sich ursprünglich auf die interlinguale Fachübersetzung, sie sind jedoch auf die Leichte-Sprache-Übersetzung übertragbar und geben in besonders treffender Weise das translatorische Handeln des/der Leichte-Sprache-Übersetzers/ Übersetzerin wieder. Hauptkriterium für eine gelungene Übersetzung ist auch hier die Funktionalität des Zieltexts in einer konkreten Zielsituation. Der Zieltext wird als Teil der Zielsituation konzeptualisiert, innerhalb derer er konkrete Funktionen innehat; Risku (2016) etwa betont, dass der Zieltext in seiner sprachlichen Realisierung nur ein Teil der Zielsituation ist und sich den Erfordernissen der Rezeptionssituation mit Blick auf seine sprachliche und konzeptuelle Verfasstheit sowie seine mediale Gestaltung anpassen muss. 
Zwar zielt die deutsche Rechtsetzung im Anschluss an die UN-Behindertenrechtskonvention darauf ab, dass die Adressatenschaft mit Kommunikationsbehinderungen die Information aus Texten eigenständig und ohne Hilfe Dritter entnehmen kann (s. den Beitrag von Lang im vorliegenden Band), de facto sind insbesondere fachliche Texte in Leichter Sprache jedoch häufig Teil einer Vermittlungssituation bzw. Grundlage für einen mündlichen Austausch über den Textgegenstand, weil dieser für eine eigenständige Informationsentnahme zu komplex ist, ganz gleich, wie leicht die sprachliche Umsetzung ist. Oft sind die Texte auch in eine empraktische Interaktion eingebunden, bei der die Texte selbst nur Teil der Zieltextsituation sind, in der weitere kommunikative Ressourcen zum Einsatz kommen (zu solchen Formen der situierten Kommunikation s. Zehrer 2014). Die Anforderungen solcher Situationen haben unmittelbare Auswirkungen auf die Gestalt des Leichte-Sprache-Texts, wenn er für diese Situationen funktional sein soll.

Wilkes (2015) hat am Beispiel einer Leichte-Sprache-Übersetzung aufgezeigt, wie stark sich ein Zieltext entsprechend der Zielsituation und seiner konkreten Funktion darin verändern kann. Sie übersetzt einen Ausgangstext zur Geburtsvorbereitung für gehörlose Leser(innen) einerseits in eine illustrierte Broschüre in Leichter Sprache (Abb. 3), andererseits erstellt sie aus demselben Ausgangsmaterial ein Kartensystem, das in der Geburtssituation zum Einsatz kommen kann (Abb. 4). Während der Ratgeber für eine Lesesituation geschrieben ist, in der allein der Text den Gegenstand erschließt, ist das Kartensystem für eine angereicherte Situation mit mehreren ko-präsenten Interaktionspartnern konzipiert; dort ist er nur ein Teil eines mit weiteren situationalen Elementen angereicherten Handlungsgefüges. Dies hat durchgreifende Auswirkungen auf die Textgestalt: 
Übersetzen in Leichte Sprache

Teil 1 von der Geburt:

Für die Geburt muss sich der Muttermund öffnen.

Weil das Kind durch den Muttermund muss.

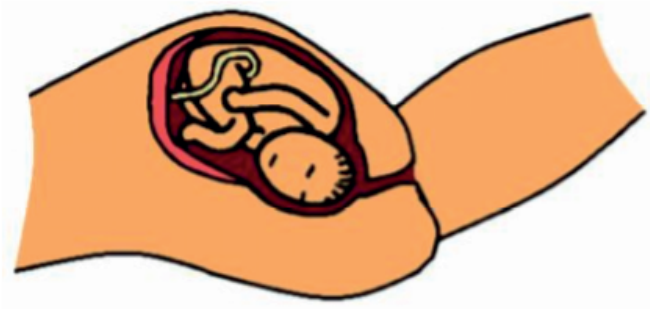

Sie bekommen Wehen

Wehen sind starke Schmerzen im Bauch.

Eine Wehe dauert jetzt 1 Minute.

Abb. 3: Wilkes (2015: 78) Ratgeber zur Orientierung für gehörlose Schwangere vor der Geburt

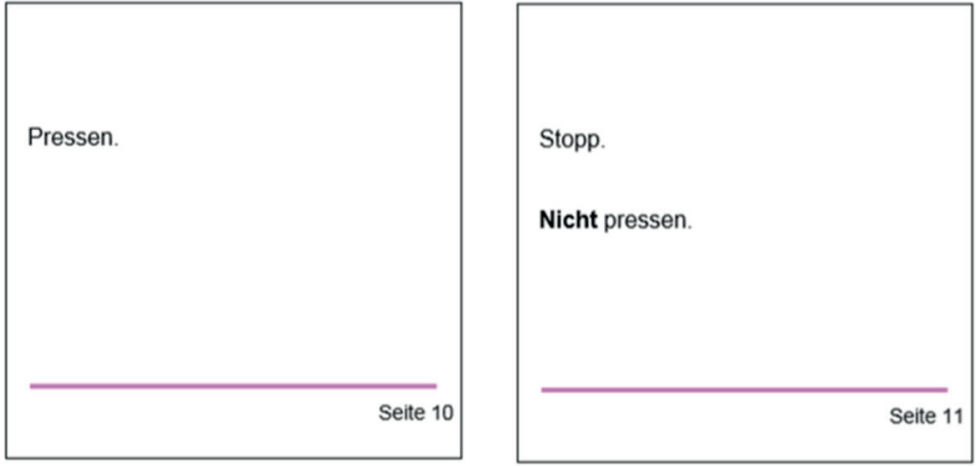

Abb. 4: Wilkes (2015: 100) Kartensystem zum Einsatz während der Geburt

() Frank \& Timme Verlag für wissenschaftliche Literatur 
Es zeigt sich hier, dass die zweite Übersetzungsvariante außerhalb der intendierten Zielsituation nicht mehr funktioniert, weil der Text zwingend um weitere situationale Elemente zu ergänzen ist, um Bedeutung entfalten zu können.

Dem eigentlichen Übersetzungsprozess vorgeschaltet ist nach Risku (2016) eine Zielanalyse, in der die Zielgruppe identifiziert und ihre konkreten Bedarfe ermittelt werden. Dabei orientieren sich die Übersetzer(innen) entlang den Wissensvoraussetzungen und den „Denkwelten“ der Adressat(inn)en und nicht notwendigerweise anhand der Kategorien des Ausgangstexts, denn die Kommunikate werden ,in den verschiedenen Umgebungen zu verschiedenen Zwecken eingesetzt“ (Risku 2016: 57). Dies hat Konsequenzen für ihre sprachliche, konzeptuelle und mediale Gestaltung (s. hierzu auch Rink im vorliegenden Band). Aus der Zielanalyse gewinnen die Übersetzer(innen) die Zielhypothese, die die Basis für eine übergreifende Makrostrategie bei der Texterstellung (Risku 2016: 59) ist mit dem Ziel, einen funktionalen Zieltext zu erstellen. Dabei ist stets der Verwendungszweck des Texts in der Zielsituation maßgeblich, der Text ist nicht allein „Sprachrohr zur reinen Weitergabe von Informationen“ (Risku 2016: 57).

Dies hat auch Auswirkungen auf den Umgang mit dem Ausgangstext - er ist nicht mehr notwendig die einzige Quelle für die Übersetzung:

Die Rolle des Ausgangstextes wird [...] neu gewichtet. Manchmal ist er nur unverbindliches Informationsmaterial als einer unter vielen anderen recherchierten Texten, manchmal bekommen Text, Autorin oder Entstehungssituation höchste Verbindlichkeit, möglicherweise bei einer literarischen Übersetzung oder bei einer Urkundenübersetzung. (Risku 2016: 47)

Risku beschreibt hier zugleich, wie neben weiteren schriftlichen Quellen auch Interaktionen mit dem Auftraggeber Orientierung über die Zielsituation, die in ihr zu vermittelnden Inhalte und die dafür nötige Gegebenheitsweise der Kommunikate bieten. Diese Situation ist Leichte-Sprache-Übersetzer(inne)n wohl bekannt: Häufig sind die Ausgangstexte nicht konkret und/oder handlungsleitend genug, um als einzige Quelle für einen funktionalen Zieltext zu dienen. 
Kontakte zum Auftraggeber sind hier von großer Bedeutung, wenn ein funktionaler Zieltext entstehen soll (s. dazu auch Bredel/Maaß 2016b: 69f.). Die Übersetzer(innen) antizipieren die Zielsituationen und beraten den Auftraggeber hinsichtlich der Möglichkeiten des Zieltexts. Es stellt sich dabei auch die für Leichte Sprache hoch relevante Frage, inwieweit es die anvisierte Textsorte mit ihrer ursprünglichen Funktionalität in Leichter Sprache überhaupt gibt bzw. geben kann. Auch diese Frage findet sich bei Risku theoretisiert, die die Rolle der Übersetzer(innen) so bestimmt:

Dabei kommt den Tranlatorinnen als Expertinnen für die Zielkultur eine beratende Funktion zu: Sie können erkennen, ob die Bestellung des Kunden so überhaupt sinnvoll ist. Wird die Form des Ausgangstextes auch der Funktion der Zielsituation in der Zielkultur gerecht oder muss dieser zunächst verändert werden? Gibt es die betreffende Textsorte in der Zielkultur überhaupt? Welche Wirkung kann sie haben, wenn sie dort neu eingeführt wird? Das sprachliche Material ist schließlich nur ein Teil des Ganzen. Eine Bearbeitung ist von geringerem Nutzen, wenn nicht berücksichtigt wird, ob und inwieweit die behandelten Sachverhalte, Illustrationen, fotografischen Abbildungen und die Verwendungsweise der Übersetzung in der Zielkultur zielführend sind. (Risku 2016: 45)

Übersetzungen sind damit nach Risku (2016: 51) „verantwortungsvolle Neuschöpfung“, Übersetzen selbst ein „Prozess des kreativen Loslassens“, der ein „hohes Vorstellungsvermögen“ erfordert, da die „Situation, für die ein Text erstellt werden soll, [...] immer grundlegend neu“ (ebd.) ist. Von den Übersetzer(inne)n ist also kein mechanisches Anwenden von Übersetzungsregeln gefragt, sondern ein Sich-Hineindenken in die Zielsituation mit dem Zweck der Erschaffung eines funktionalen, adressaten- und zielsituationsangemessenen Zieltexts. Die Texte, die aus einem solchen Prozess der Neuschöpfung entstehen, sind untereinander verschieden; zwei unterschiedliche Übersetzer(innen) werden mithin keine zwei gleichen Lösungen vorlegen. Angesichts der Restriktionen durch die Vorgaben der Leichten Sprache sowie der Anforderungen der Adressatenschaft besteht hierin die übersetzerische Kreativität und es wird deutlich, dass Übersetzen in Leichte Sprache eine große Herausforderung an die 
Übersetzer(innen) stellt, nicht zuletzt weil ihnen gegenüber der Adressatenschaft mit Kommunikationsbeeinträchtigungen eine besondere Verantwortung obliegt. Automatisierte Übersetzungsverfahren können folglich in der LeichteSprache-Übersetzung auf absehbare Zukunft nicht zum Einsatz kommen, da sie viel enger an den Ausgangstext angebundene und folglich für die Adressatenschaft nicht funktionale Zieltextangebote produzieren würden. Denkbar ist allerdings eine Anwendung von Instrumenten der Computer Aided Translation (s. den Beitrag von Zehrer im vorliegenden Band).

\section{$5 \quad$ Leichte-Sprache-Übersetzung als Gegenstand der Barrierefreien Kommunikation}

\section{1 Übersetzen als Überwinden kommunikativer Barrieren}

In Anlehnung an Rink (in Vorbereitung) lässt sich Übersetzung auch über die Barrieren definieren, die Texte für die Adressatenschaft darstellen: Man kann dann von einer Übersetzung sprechen, wenn der Ausgangstext für die intendierte Zielleserschaft eine Barriere (Sinnesbarriere, Fachbarriere, Fachsprachenbarriere, Kulturbarriere, Kognitionsbarriere, Sprachbarriere oder Medienbarriere) darstellt, die der Zieltext zu überwinden sucht. Dieser Ansatz ist auf die unterschiedlichen Übersetzungsarten (intra- und interlingual, intra- und intersemiotisch, intra- und interkulturell) anwendbar: Ist der Text beispielsweise auf Farsi verfasst und die intendierten Zieltextleser(innen) sind dieser Sprache nicht mächtig, so stellt der Text für sie eine Sprach- und wahrscheinlich auch Kulturbarriere dar, die durch eine Übersetzung überwunden werden kann. Handelt es sich um einen deutschsprachigen Text und eine Zielleserschaft, die des Deutschen grundsätzlich mächtig ist, so kann der Text dennoch Barrieren aufweisen, etwa wenn er einen Gegenstand behandelt, den die Zielleserschaft nicht ausreichend kennt, um die Informationen des Texts zu prozessieren, oder wenn er so fachsprachlich formuliert ist, dass er für das Zielpublikum nicht verständlich ist. Gleiches gilt, wenn er eine Medialität aufweist, die für das Zielpublikum nicht rezipierbar ist etc. Ein Zieltext ist dann angemessen, wenn er keine Barrieren für die intendierte Leserschaft aufweist, Übersetzen ist als Prozess der Überwindung kommunikativer Barrieren konzeptualisierbar. 
Je nach Art der Kommunikationseinschränkung kann ein und derselbe Text unterschiedliche Arten von Barrieren für die Zielleserschaft aufweisen. Deshalb ist es für den Übersetzer/die Übersetzerin wichtig zu wissen, welche Anforderungsprofile die jeweilige Zielleserschaft an die Texte stellt. Die Übersetzer(innen) sind „Expertinnen für die Zielkultur“ (Risku 2016: 45) und wählen ihre Übersetzungsstrategien in Abhängigkeit von Zielleserschaft und Zielsituation. Dafür stehen ihnen unterschiedliche Typen von Strategien zur Verfügen, nämlich sprachliche, konzeptuelle und mediale (Rink in Vorbereitung und im vorliegenden Band), aus denen sie entsprechend ihrer Übersetzungsstrategie auswählen. Beim Übersetzen in Leichte Sprache kommen in der Regel mehrere oder alle diese Strategietypen zum Einsatz, um adressatengerechte und zielsituationsadäquate Texte zu ermöglichen:

Um [...] Teilhabemöglichkeiten i.S. der selbständigen Texterschließung und damit Textverstehen zu schaffen, bedarf es perzeptibilitäts- und verständlichkeitsoptimierter Texte, deren konzeptuelle Planung von der Textebene ausgeht, deren sprachliche Mittel den semantisch-syntaktischen Bedarfen der Zieltextleserschaft Rechnung tragen und deren mediale Gestaltungsweisen der präferierten Informationsaufnahme der Adressat(inn)en entsprechen. Leichte Sprache, der alle diese Aspekte inhärent sind [...], kann den hohen Anforderungen einer beeinträchtigten, leseschwachen Adressatenschaft gerecht werden, jedoch bedarf es seitens der Übersetzer(innen) v.a. einer ausgeprägten Text- und Rezipientenexpertise, um ein funktionales Textangebot vorhalten zu können, das neben diesen die Verständlichkeit determinierenden Faktoren auch weitere Parameter (z. B. Situation, Medium, Kommunikator) der Texterstellung berücksichtigt. (Rink in Vorbereitung).

5.2 Angestrebte Eigenschaften von Leichte-Sprache-Texten:

Auffindbar, gut wahrnehmbar, leicht verständlich, korrekt und funktional

In den vorangehenden Abschnitten wurde hervorgehoben, dass die Übersetzer(innen) über Kompetenz verfügen und ihre Verantwortung wahrnehmen 
müssen und dass sich in der Übersetzung für sie ein kreativer Freiraum eröffnet. Dies ist die Grundlage für individuelle, einzeltextbezogene Lösungen entsprechend der Funktionalität der Texte in der Zielsituation. Die Strategien sind hierfür vielfältig, die Zieltexte weisen jedoch im Gelingensfall sämtlich die folgenden Eigenschaften auf: Sie sind auffindbar, gut wahrnehmbar, leicht verständlich, korrekt und funktional.

\section{Auffindbar}

Auffindbar zu sein scheint zunächst keine Eigenschaft des Texts selbst zu sein. Texte sind jedoch Teil von Rezeptionssituationen, die in idealtypischer Form bei ihrer Erstellung mitberücksichtigt werden. Wo und wie der Zieltext zugänglich gemacht wird, hat damit, wenn der Text in der Zielsituation funktional sein soll, in sprachlicher, konzeptueller und medialer Hinsicht Auswirkungen auf die Gestalt des Texts. Häufig tritt der Fall auf, dass Leichte-Sprache-Texte nur über den Umweg durch allgemein- oder fachsprachliche (Hyper-)Texte zugänglich sind. Das ist beispielsweise der Fall, wenn ein Angebot in Leichter Sprache nicht direkt oben auf der Startseite bei den Spracheinstellungen ausgewählt werden kann. Für die Adressatenschaft von Texten in Leichter Sprache stellt es eine Hürde dar, wenn sie sich auf der Suche nach Informationen in Leichter Sprache durch mehrere Seiten navigieren müssen.

\section{Gut wahrnehmbar}

Textangebote haben eine mediale Seite, die im Rahmen der Barrierefreien Kommunikation eine besondere Rolle spielt, denn die Adressatenschaft hat zumeist erhöhte Anforderungen an die mediale Gestaltung der Texte (s. den Beitrag von Rink im vorliegenden Band):

- ein wahrnehmungsfreundliches Layout (s. den Beitrag von Alexander im vorliegenden Band),

- die Visualisierung insbesondere schwieriger Textgegenstände (s. den Beitrag von Pridik im vorliegenden Band), 
- möglicherweise eine Audiofassung (s. die Beiträge von Mälzer/ Wünsche im vorliegenden Band) oder

- Alternativtexte für Bildressourcen (s. den Beitrag von Schütt im vorliegenden Band).

Übersetzer(innen) sind keine Layouter(innen) oder Grafiker(innen), sie sind auch keine professionellen Sprecher(innen); sie sind für die Erschaffung der verbalen Anteile der Zieltexte, nicht aber für deren Gestaltung oder Visualisierung zuständig. Als Expert(inn)en für die Zielkultur kommt ihnen aber wiederum beratende Funktion zu, denn sie sind durchaus imstande, dem Auftraggeber Empfehlungen zur Gestaltung des Texts zu geben. Die Ausgestaltung dieser Empfehlungen fällt dann jedoch in den Handlungsbereich weiterer Expert(inn)en für Textgestaltung, idealerweise in Zusammenarbeit bzw. in Abstimmung mit den Übersetzer(inne)n.

\section{Leicht verständlich}

Texte in Leichter Sprache sind verständlichkeitsoptimiert. Die Umsetzung eines Texts, insbesondere wenn er fachlich geprägt ist, in Leichter Sprache ist jedoch eine Herausforderung für die Übersetzer(innen): Die konzeptuelle Komplexität des Ausgangstexts bleibt bestehen - noch immer geht es etwa um Erbrecht -, allerdings stehen nur noch eingeschränkte sprachliche Mittel zur Verfügung. Außerdem führen Verfahren der Reduktion und Addition, die lokal die Verständlichkeit erhöhen, häufig auf Textebene zu Problemen, etwa wenn durch die Erklärung von Fachtermini der Fließtext wieder und wieder unterbrochen wird. Durch die Leichte-Sprache-Regeln verschwinden die Unterschiede zwischen Texten unterschiedlicher Textsorten; Leichte-Sprache-Texte sind nach diesem Konzept in Layout und Sprachregister weitgehend homogen. Das verbessert zwar die Wahrnehmbarkeit und die Verständlichkeit auf Wort- und Satzebene, nivelliert aber auch wichtige Merkmale auf der Makroebene, die Leser(innen) der Ausgangstexte zur Verfügung haben, um erste Annahmen über die Intention und Funktion von Texten zu entwickeln. Leichte-Sprache-Übersetzer(innen) als Expert(inn)en für die Adressatenschaft bewegen sich hier in einem Ermessensspielraum. 


\section{Korrekt}

Fachlich geprägte Kommunikation bedient sich im Allgemeinen auch der jeweiligen Fachsprache, um die Textgegenstände auszudrücken. In Leichter Sprache müssen solche fachlichen Gegenstände mit reduzierten sprachlichen Mitteln in den Text eingebracht und erläutert werden. Dies setzt voraus, dass die Übersetzer(innen) den fachlichen Gegenstand durchdrungen haben. Fachübersetzer(innen) im interlingualen Bereich weisen häufig eine Spezialisierung für einen bestimmten fachlichen Gegenstandsbereich auf. Das Übersetzen in Leichte Sprache hat diesen Spezialisierungsgrad noch nicht erreicht. Daher bedürfen Leichte-Sprache-Übersetzer(innen) bei Fachübersetzungen in der Regel der Abnahme durch Fachexpert(inn)en.

Das Gebot der Korrektheit gelangt dort an seine Grenzen, wo die sprachlichen Mittel der Leichten Sprache nicht ausreichen, um komplexe fachliche Gegenstände wiederzugeben. Dies ist bei juristischen Fachtexten regelmäßig der Fall und führt dann dazu, dass diese Texte nicht das volle Funktionsspektrum der Ausgangstexte erreichen können: Leichte-Sprache-Texte der juristischadministrativen Kommunikation sind überwiegend nicht justiziabel.

\section{Funktional}

Texte sind in Prozesse der Wissenskommunikation eingebunden: Der Textautor/die Textautorin hat eine Mitteilungsabsicht mit Bezug auf den Gegenstand des Texts und diese ist der Rezipientenschaft nicht vollständig bekannt, sonst wäre für den gegebenen Text das Kriterium der „Informativität“ (De Beaugrande/Dressler 1981) nicht gegeben und es wäre unwahrscheinlich, dass er gelesen wird. Dabei ist die Zielsituation als Ganze zu berücksichtigen. Der Zieltext wird also als Teil der Zielsituation konzeptualisiert und entsprechend an die Funktion angepasst, die er in der konkreten Zielsituation hat. Die Zielsituation, für die ein Text erstellt wird, eröffnet also einen Blick auf mögliche bzw. plausible Rezeptionsweisen des Texts. Ist ein Text für eine eigenständige Informationsentnahme konzipiert oder wird er vermutlich in Interaktion rezipiert? Soll sich beispielsweise ein Patient/eine Patientin eigenständig mit einem schriftlichen Text etwa in Form einer Patienteninformationsbroschüre über die eigene Krankheit und Behandlungsoptionen informieren oder dient der Text als Grundlage für ein Arzt-Patienten-Gespräch? Die Antworten auf diese Fragen 
haben Auswirkungen auf die Konzeptualisierung des Texts: Art und Umfang der Information, vorausgesetzte Wissensbestände, Erklärtiefe, Medialität (s. dazu die Ausführungen zu zielsituationsorientierten Übersetzungsansätzen in Abschnitt 4).

Dies betrifft im Grunde alle Texte; erfahrene Leser(innen) können es jedoch bis zu einem gewissen Grad kompensieren, wenn ein Text, wie das häufig vorkommt, dysfunktional ist. Ist das Verstehen und Behalten jedoch durch eine Kommunikationsbeeinträchtigung des Rezipienten/der Rezipientin bereits fragil, so können dysfunktionale Texte möglicherweise ihr Kommunikationsziel nicht erreichen.

Wer vor Gericht aussagen muss, erhält eine Zeugenladung, die auf mehreren eng beschriebenen Seiten viele wichtige Informationen enthält, um den Zeugen bzw. die Zeugin auf den Tag im Gericht und die Aussage vorzubereiten, aber auch über die (begrenzten) Möglichkeiten des Nichterscheinens und die Ansprüche auf Erstattung von Auslagen und Verdienstausfällen zu informieren. Zeugenladungen sind Texte der juristisch-administrativen Kommunikation, die einen erheblichen Grad an Fachlichkeit und Fachsprachlichkeit aufweisen. Sie stellen auch geübte Leser(innen) vor Herausforderungen. Wird eine Zeugenladung informationskonstant in Leichte Sprache übersetzt, so ergibt sich durch die hohe Dichte an Informationen und erläuterungsbedürftiger Fachterminologie für den Zieltext ein großer Umfang. Rink (in Vorbereitung) bringt das Beispiel einer informationskonstant übersetzten Zeugenladung in Leichter Sprache, die fast 40 Seiten Drucktext aufweist. Dieser Text ist für die Zielsituation nicht funktional, und das in doppelter Hinsicht:

- Zwar ist der Text auf Wort- und Satzebene verständlicher und damit leichter zugänglich, der große Gesamtumfang des Texts stellt für eine Adressatenschaft mit Leseeinschränkungen aber wiederum eine Barriere dar.

- Da Zeugenladungen postalisch versendet werden, müssen für die Zeugenladungen in Leichter Sprache ein anderes Umschlagformat, mehr Porto und insgesamt höhere Kosten vorgesehen werden, was in den behördlichen Abläufen so nicht vorgesehen ist. 
Entsprechend ist es unwahrscheinlich, dass Richter(innen) sich dazu entschließen, die Zeugenladung in Leichter Sprache auch tatsächlich einzusetzen. Der Leichte-Sprache-Text ist daher in der Zielsituation nicht funktional, und das, obwohl er sowohl leicht verständlich als auch im Rahmen des Möglichen korrekt ist.

\section{6 Ein Ausblick: Implementierung der Texte in der Zielsituation}

Die Rechtslage nimmt öffentliche Stellen und insbesondere Behörden in die Pflicht, Angebote in Leichter Sprache vorzuhalten (s. die Beiträge von Lang und Bredel/Maaß im vorliegenden Band). Hier handelt es sich um komplexe Organisationen, in denen viele professionelle Akteure aus unterschiedlichen Berufsfeldern zusammenwirken. Die Aufträge selbst ergehen häufig von der Hausspitze oder von der Kommunikationsabteilung bzw. Pressestelle. Die Personen, die dann in ihrem beruflichen Alltag mit den Texten arbeiten sollen, sind selten mit den Auftraggebern identisch. Um das Beispiel der Zeugenladung noch einmal aufzugreifen: Auftraggeber war hier das Niedersächsische Justizministerium (Pilotprojekt: Leichte Sprache in der Niedersächsischen Justiz, www.mj.niedersachsen.de; die wissenschaftliche Aufarbeitung des Projekts findet sich in Rink in Vorbereitung). Textnutzer(innen) auf Seiten der Institution sind jedoch die einzelnen Richter(innen), die Ladungen vornehmen und dabei in ihrem Content Management System auf die Leichte-Sprache-Fassung zugreifen oder eben nicht. Sind die eigentlichen Textnutzer(innen) auf Seiten der Behörde nicht mit den Leichte-Sprache-Texten bzw. mit dem Konzept Leichte Sprache insgesamt vertraut gemacht worden, so ist absehbar, dass die Texte nicht oder nur sporadisch zum Einsatz kommen werden. Maßnahmen zur Implementierung der Texte in der auftraggebenden Organisation bis hin zum Schreibtisch jedes einzelnen Sachbearbeiters bzw. jeder Textnutzerin sind bislang in der Leichte-Sprache-Übersetzung nicht die Regel. Dadurch werden bestehende Leichte-Sprache-Angebote in den intendierten Zielsituationen häufig nicht genutzt.

Aktuell besteht in den Organisationen zumeist keine Erfahrung mit LeichteSprache-Texten, so dass die Mitarbeiter(innen) in der Regel Schulungen zur 
Sensibilisierung und auch zur Verwendung der Texte benötigen, um mit diesen adäquat arbeiten zu können. Insbesondere bei größeren Aufträgen sind Maßnahmen wie Evaluation und Monitoring sinnvoll, dass also der tatsächliche Einsatz der Texte nach einer Weile überprüft und ggf. mit Schulungen oder Textüberarbeitungen nachgesteuert wird. Der Aufwand für solche Maßnahmen sollte bereits bei der Projektplanung mitberücksichtigt werden.

Insgesamt kann konstatiert werden, dass die Professionalisierung der Leichte-Sprache-Übersetzung insbesondere im Bereich der fachlichen Kommunikation, zu der auch die juristisch-administrative Kommunikation gehört, noch am Anfang steht; auch auf Seiten der Auftraggeber bestehen häufig noch viele Unsicherheiten. Die Zahl der zur Verfügung stehenden Texte ist begrenzt und die existierenden Texte werden oft nicht im wünschenswerten Umfang genutzt. Im Entwurf zur Novelle des Behindertengleichstellungsgesetzes vom Januar 2016 wird das Ziel formuliert, einen „Pool von Mustererläuterungen“ (BGG, Entwurf 2016: 4) in Leichter Sprache zu erstellen, der sukzessive erweitert wird und auf den die Mitarbeiter(innen) in den Verwaltungen in Beratungssituationen zugreifen können:

Ein Grundstock von Erläuterungen zu besonders relevanten Dokumenten soll in Leichter Sprache erstellt und den Behörden als Basis zur Verfügung gestellt werden. (BGG, Entwurf 2016: 4)

An gleicher Stelle wird die Zahl der jährlichen Anfragen nach Texten der in BGG $₫ 11$ erfassten Textsorten (Bescheide, Allgemeinverfügungen, öffentlichrechtliche Verträge und Vordrucke) in Leichter Sprache auf 600000 geschätzt:

Es wird davon ausgegangen, dass rund 200000 Bürgerinnen und Bürger davon [d.h. von der Möglichkeit, Erläuterungen in Leichter Sprache zu verlangen] dreimal jährlich Gebrauch machen. (BGG, Entwurf 2016: 25)

Das Ziel, eine derartige Textdatenbank für den behördlichen Bereich verfügbar zu machen und in eine funktionierende Praxis zu überführen, stellt eine große Herausforderung für die Barrierefreie Kommunikation insgesamt und für die 
Leichte-Sprache-Übersetzung im Besonderen dar, die nur durch eine Professionalisierung des gesamten Bereichs, entsprechende finanzielle Ressourcen sowie eine Flankierung durch die Forschung im Sinne Holz-Mänttäris (1984) erreicht werden kann. 


\section{Literaturverzeichnis}

Albrecht, JÖRN (1990): „Invarianz, Äquivalenz, Adäquatheit“. In: ReINER ARNTz/GISElA ThOMe (Hrsg.): Übersetzungswissenschaft. Ergebnisse und Perspektiven. Festschrift für Wolfram Wilss zum 65. Geburtstag, Tübingen: Narr, 71-81.

ALBRECHT, JÖRN (2005): Übersetzung und Linguistik: Grundlagen der Übersetzungsforschung II, Tübingen: Narr.

Al Masri-Gutternig, Nadja/Reitstätter, Luise (Hrsg.) (2017): Leichte Sprache. Sag es einfach. Sag es laut! Praxisbeispiel Salzburg Museum. Herausgegeben vom Salzburg Museum.

Assoziierte Dolmetscher und Übersetzer in Norddeutschland (ADÜ Nord): Pressemitteilung vom 9.1.2018. https:/www.adue-nord.de/wp-content/uploads/2016/11/ PM_ADUE_NORD_Januar-2018.pdf (letzter Zugriff 8.7.2018)

BGG, Entwurf (2016): Entwurf des Gesetzes zur Weiterentwicklung des Behindertengleichstellungsrechts (BGG), BT-Drs. 18/7824 vom 09. 03. 2016, http://dip21.bundestag.de/dip21/btd/18/078/1807824.pdf (letzter Zugriff 8.7.2018)

Bredel, Ursula/MaAß, Christiane (2016a): Leichte Sprache. Theoretische Grundlagen, Orientierung für die Praxis, Berlin: Duden.

Bredel, Ursula/MaAß, Christiane (2016b): Ratgeber Leichte Sprache, Berlin: Duden. BREDEL, URsula/MAAß, Christiane (2017): „Wortverstehen durch Wortgliederung Bindestrich und Mediopunkt in Leichter Sprache", in: BeTtina Bock, Ulla FiX, DAISY LANGE (Hg.): „Leichte Sprache“ im Spiegel theoretischer und angewandter Forschung, Berlin: Frank \& Timme, 211-228.

De Beaugrande, Robert-Alain/Dressler, Wolfgang-Ulrich (1981): Einführung in die Textlinguistik, Tübingen: Niemeyer.

Einfach Heidelberg (11.2.2018): „Fastnacht in Heidelberg“, www.einfach-heidelberg.de, Text vom 11.2.2018

GeNTZLER, Edwin (2001): Contemporary Translation Theories, Clevedon: Multilingual Matters.

Griesel, Yvonne (2007): Die Inszenierung als Translat: Möglichkeiten und Grenzen der Theaterübertitelung, Berlin: Frank \& Timme.

HolZ-MÄnTTÄRI, JusTa (1984): Translatorisches Handeln: Theorie und Methode, Helsinki: Suomalainen Tiedeakatemia.

HÖNIG, HANS G./KUßMAUL, PAUl (1982): Strategie der Übersetzung: Ein Lehr- und Arbeitsbuch, Tübingen: Narr.

JAKOBSON, ROMAN (1959): “On linguistic aspects of translation", in: BROWNER, REUBEN ARTHUR (Hrsg.): On Translation, Harvard University Press, 232-239.

JÄGER, GERD (1968): „Elemente einer Theorie der bilingualen Translation“. In: Grundfragen der Übersetzungswissenschaft, Beihefte zur Zeitschrift Fremdsprachen 3, 3552 . 
KADE, ОтTO (1968): Zufall und Gesetzmäßigkeit in der Übersetzung, Leipzig: Enzyklopädie.

KOLLER, WERNER ( ${ }^{1}$ 1979/2011): Einführung in die Übersetzungswissenschaft, Heidelberg: Quelle \& Meyer (Ausgabe 2011: UTB).

MaAß, Christiane/RinK, IsABel/Zehrer, Christiane (2014): „Leichte Sprache in der Sprach- und Übersetzungswissenschaft“. In: SusAnne J. JeKat, HeIKE EliSABETH JÜNGST, KlAus SCHUBERT, Claudia Villiger (Hrsg.): Sprache barrierefrei gestalten. Perspektiven aus der Angewandten Linguistik, Berlin: Frank \& Timme, 53-85.

NeUbert, Albrecht (1973): „Theorie und Praxis für die Übersetzungswissenschaft“. In: Linguistische Arbeitsberichte 7, 120-144.

Neubert, Albrecht/Shreve, Gregory M. (1992): Translation as Text, Kent, Ohio, London: Kent State University Press.

NidA, Eugene (1964): Toward a Science of Translating, Leiden: Brill.

Nida, Eugene/Taber, Charles R. (1969): The Theory and Practice of Translation, Leiden: Brill.

NoRD, CHRistiane (1993): Einführung in das funktionale Übersetzen: am Beispiel von Titeln und Überschriften, Tübingen: Francke.

Nord, Christiane (2011): Funktionsgerechtigkeit und Loyalität: Theorie, Methode und Didaktik des funktionalen Übersetzens, Berlin: Frank \& Timme.

REIß, KATHARINA (1976): Texttyp und Ubersetzungsmethode. Der operative Text, Kronberg: Scriptor.

ReIß, KATHARINA/VERMEer, HANS J. (1984): Grundlegung einer allgemeinen Translationstheorie, Berlin: De Gruyter.

RINK, ISABEL (in Vorbereitung): Rechtskommunikation und Barrierefreiheit. Eine korpusgestützte Analyse der Übersetzung juristischer Informations- und Interaktionstexte in Leichte Sprache im Pilotprojekt „Leichte Sprache in der Niedersächsischen Justiz“, Manuskript der Dissertationsschrift.

RISKU, HANNA (1998): Translatorische Kompetenz: kognitive Grundlagen des Übersetzens als Expertentätigkeit, Tübingen: Stauffenburg.

RISKU, HANNA ( $\left.{ }^{2} 2016\right)$ : Translationsmanagement. Interkulturelle Fachkommunikation im Informationszeitalter, Tübingen: Narr.

SIEVER, Holger (2010): Übersetzen und Interpretation: die Herausbildung der Übersetzungswissenschaft als eigenständige wissenschaftliche Disziplin im deutschen Sprachraum von 1960-2000, Frankfurt: Lang.

Wilkes, HannA (2015): Ratgeber in Leichter Sprache als Teil der fachexternen Kommunikation, Masterarbeit Universität Hildesheim.

Zehrer, Christiane (2014): Wissenskommunikation in der technischen Redaktion. Die situierte Gestaltung adäquater Kommunikation, Berlin: Frank \& Timme. 


\section{Ausprägungen und Dolmetschstrategien beim Schriftdolmetschen}

\section{Definition und Überblick über die Ausprägungen des Schriftdolmetschens}

Schriftdolmetschen ist eine Verschriftlichung eines mündlich dargebotenen Ausgangstextes nahezu in Echtzeit (simultan) wortwörtlich oder komprimiert. Die Dienstleitung richtet sich in erster Linie an schwerhörige oder gehörlose Menschen (Rezipient(inn)en). Daher werden Sprecher(innen) und Sprecherwechsel, Ironie, nonverbale Äußerungen und Geräusche ebenfalls wiedergegeben und in Form von Regieanweisungen in runden Klammern sichtbar gemacht, z. B.:

(Sprecherwechsel)

(Handy klingelt)

(Lachen)

D. h. für die Rezeption des Textes relevante paralinguale (bspw. Seufzen, Räuspern, Lachen), extralinguale (etwa wenn die Gesprächsteilnehmer(innen) aus dem Fenster schauen, weil eine Baumaschine laute Geräusche erzeugt) oder prosodische (bspw. Intonation) Informationen werden dem Rezipienten/ der Rezipientin mitgegeben. Darüber hinaus berücksichtigt der/die Schriftdolmetscher(in), sofern möglich, die Kommunikationsfähigkeiten der Rezipient(inn)en wie Schriftsprachkompetenz, kognitive Fähigkeiten, Sehfähigkeit, Lesegeschwindigkeit usw. Eine sehr differenzierte Definition unter situativen, theoretischen und berufspraktischen Gesichtspunkten von Schriftdolmetschen findet sich in Platter (2015: 4ff.).

Eine wichtige Rolle beim Schriftdolmetschen spielt die Unterscheidung zwischen mündlich dargebotenem Ausgangstext und der Verdolmetschung in 
schriftlichen Text. Platter spricht von einem „breit definierten Spannungsbereich in der Rezeption eines medial und konzeptionell mündlichen oder medial mündlichen (...) Ausgangstextes und der Produktion eines medial und konzeptionell schriftlichen oder medial schriftlichen Zieltextes (...)“ (Platter 2015: 293), dem die Schriftdolmetscher(innen) in der spezifischen Translationssituation gerecht werden müssen. Dabei müssen sie Widrigkeiten wie schlechte Audioqualität Braun (2004: 68) spricht von „Kommunikation unter widrigen Umständen“ oder ungünstige Positionierung im Raum und Widrigkeiten der Arbeitsmittel ausgleichen (Platter 2015: 293).

Schriftdolmetscher(innen) wenden unterschiedliche Methoden an: die konventionelle Methode (Eingabe des Textes über Tastatur), Spracherkennung (Respeaking des Textes mittels einer Software), Verwendung des Velotype oder computergestützter Maschinenstenographie (s.u.).

Im Englischen werden für das Schriftdolmetschen unterschiedliche Benennungen verwendet: „Speech-to-text-interpreting“, „Captioning“, „Speech-totext-reporting“. Die beiden letzteren Formen werden in den Vereinigten Staaten von Amerika verwendet, weil sie aus einer Zeit stammen, als die Tätigkeit eher der Protokollierung ähnelte (Eichmeyer im Druck). Die skandinavischen Länder Schweden, Finnland und Norwegen sowie die Vereinigten Staaten von Amerika gehören zu den Pionieren des Schriftdolmetschens. Als Beginn nennen Norberg et. al. die späten 1970er und frühen 1980er Jahre (Norberg et. al. 2015: 38).

\section{Für wen und wo arbeiten Schriftdolmetscher(innen)?}

Ein(e) Schriftdolmetscher(in) wird typischerweise von Schwerhörigen oder Gehörlosen angefragt, die schriftsprachkompetent sind (Witzel 2018: 16; zur Schriftsprachenkompetenz Gehörloser s. den Beitrag von Hennies im vorliegenden Band). Dies trifft vor allem für Menschen zu, die erst später im Leben aufgrund von Unfall oder Krankheit eine Hörbeeinträchtigung erlitten haben. Darüber hinaus werden die Dienste von schwerhörigen Menschen genutzt, die lautsprachorientiert sind. Von Geburt an Schwerhörige oder Gehörlose, die sich mit ihren Eltern oder in der Schule mit Gebärdensprache verständigen, fragen bei Bedarf eher eine(n) Gebärdensprachdolmetscher(in) an. Allerdings sind 
Hörschädigungen so individuell wie Fingerabdrücke, die Auswirkungen ebenso. Eine wichtige Gruppe potenzieller Rezipient(inn)en, die aus demografischen Gründen derzeit stark wächst, sind Altersschwerhörige. Darüber hinaus bietet das Schriftdolmetschen gegenüber dem Gebärdensprachdolmetschen in bestimmten Situationen Vorteile für alle Zielgruppen, beispielsweise wenn ein hoher Anteil von Fachsprache involviert ist und die Verschriftlichung des Textes (Mitschrift, s.u.) eine Nacharbeit ermöglicht. Dies ist etwa bei Vorlesungen an Hochschulen oder bei Fachkonferenzen der Fall.

Weitere Zielgruppen bei einer entsprechend angepassten (vereinfachten) Verschriftlichung sind Menschen mit kognitiven Einschränkungen oder Menschen, die der jeweiligen Sprache, die gesprochen wird, nur eingeschränkt mächtig sind. Das können beispielsweise Teilnehmer(innen) internationaler Konferenzen sein, welche die Konferenzsprache besser schriftlich verfolgen können, etwa wenn die Vortragenden schnell oder mit Akzent sprechen. Oder es können internationale Studierende sein, welche die Landessprache eingeschränkt beherrschen. Schriftdolmetscher(innen) werden bevorzugt für Gesprächssituationen in größeren Gruppen beauftragt: in der Schule, in der Ausund Weiterbildung, bei Betriebsversammlungen, Konferenzen und Teamsitzungen. Hier kommen verschiedene beeinträchtigende Faktoren zusammen: Der oder die Vortragende steht in diesen Situationen häufig weit entfernt vom bzw. von der Hörbehinderten, so dass ein Absehen vom Mund nicht oder nur eingeschränkt möglich ist; es gibt möglicherweise erhebliche Nebengeräusche; es sind viele Personen an der Gesprächssituation beteiligt.

Dank der Umsetzung von Inklusion bieten Schulen ein erweitertes Betätigungsfeld für Schriftdolmetscher(innen). Generell kommen Schriftdolmetscher(innen) in Situationen zum Einsatz, in denen es besonders wichtig ist, dass der oder die Hörbehinderte alles versteht, was gesprochen wird: beim Arzt, bei Gericht, bei Mitarbeitergesprächen, in Telefonkonferenzen. Die Gesprächsinhalte werden vertraulich behandelt. Dies ist in der Berufs- und Ehrenordnung von Schriftdolmetscher(inne)n festgelegt. Hier beispielhaft die entsprechende Passage aus der Berufsund Ehrenordnung des Deutschen Schwerhörigenverbunds e.V.: Schriftdolmetscher „verpflichten (sich), über alles, was ihnen bei der Ausübung ihrer Tätigkeit anvertraut worden oder bekannt geworden ist, Verschwiegenheit zu wahren ..." (ebd. 2009). Ein weiteres Merkmal ist die Neutralität des Schriftdolmetschers/der 
Schriftdolmetscherin. Er oder sie greift nicht in das Kommunikationsgeschehen ein, es sei denn, es betrifft unmittelbar seine bzw. ihre Dienstleistung (z. B. indem er/sie die Redner bittet, in das Mikrofon zu sprechen). Die Aufgabe des Schriftdolmetschers/der Schriftdolmetscherin ist, die mündlich dargebotenen Texte, inklusive anfallender Regieanweisungen, vollständig und unparteiisch zu verschriftlichen.

\section{$3 \quad$ Ist Schriftdolmetschen eine Dolmetschart?}

Schriftdolmetschen und Dolmetschen haben viele Gemeinsamkeiten (vgl. Gerzymisch 2013: 2):

- Es ist eine simultane Leistung,

- ein Text (mündlich) wird in einen anderen Text (schriftlich) transferiert,

- das Produkt kann nur eingeschränkt korrigiert werden,

- aufgrund der Einschränkung von Raum und Zeit muss ein Zusammenhang gebildet (Kohärenz) und Text verkürzt werden (Textkondensierung),

- thematische Sprünge in der Informationsgliederung müssen vermieden werden (Thema-Rhema-Gliederung).

In ihrem Gutachten kommt Gerzymisch zu dem Schluss, dass die Bezeichnung „Schriftdolmetschen“ verständlich und nachvollziehbar ist. Auch Tiittula (z. B. Tiittula 2006) und Platter (2015) weisen nach, dass Schriftdolmetschen eine Dolmetschart ist. Braun (2004) zählt das Schriftdolmetschen zur „multidimensionalen Translation“.

Das heißt, dass Schriftdolmetscher(innen) Kohärenz (Zusammenhang auf inhaltlicher Ebene) und Kohäsion (Zusammenhalt auf formaler Ebene) herstellen, wenn sie den mündlichen Ausgangstext in Schriftform umwandeln. 


\section{Intralinguales und interlinguales Schriftdolmetschen}

Derzeit ist Schriftdolmetschen in der Regel intralingual. Interlinguales Schriftdolmetschen ist aktuell noch kein eigenes Forschungsthema, wobei Konzepte aus dem Bereich Respeaking und Live-Untertitelung auf das Schriftdolmetschen angepasst werden könnten. In England wird an der Roehampton University an der didaktischen Entwicklung für interlinguales Respeaking geforscht (Romero-Fresco/Pöchhacker im Druck). Ferner gibt es internationale Zusammenarbeit in Bezug auf die Qualitätssicherung von interlingualer Live-Untertitelung (ebd.). Beispiele dafür, dass Schriftdolmetscher(innen) in der Praxis intralingual arbeiten, liefert Eichmeyer (im Druck).

\section{Dolmetschstrategien beim Schriftdolmetschen}

Qualitätsevaluation, Dolmetschstrategien und Kommunikationstechniken bilden den wissenschaftlichen Unterbau für die Weiterbildung im Bereich Schriftdolmetschen am SDI München. Dolmetschstrategien umfassen Verstehens-, Planungs-, Zieltextproduktions- und Monitoringstrategien (vgl. u.a. Kalina 1998).

Schriftdolmetscher(innen) wenden Verstehensstrategien an, indem sie

- Vorwissen aktiven (sie haben sich auf den Einsatz vorbereitet, sie aktivieren ihr Wissen während des Einsatzes),

- Schwierigkeiten im Verstehensprozess kompensieren (beispielweise indem sie Rückschlüsse aus dem Kontext ziehen),

- inferenzieren (implizit Gesagtes explizit machen) und

- antizipieren, was der Redner sagt.

Die Vorbereitung auf einen Einsatz, insbesondere wenn es sich um ein Fachthema handelt, trägt entscheidend zum besseren Verständnis des mündlichen Textes bei. Dazu gehört das Lesen und Hören von einschlägigen Texten 
zum Thema, das Einpflegen der (Fach-)Terminologie in die entsprechenden Systeme, das Anlegen von Kürzeln. Wenn das Thema, die Textsorte, die Zielgruppe (diese Merkmale kommen aus der Translationswissenschaft; vgl. Nord 1991) und die Sprecher(innen) bekannt sind, können Schriftdolmetscher(innen) antizipieren, welche Inhalte und welche Art von Terminologie, welcher Stil zu erwarten ist. Bei Parteitagen der SPD beispielsweise kann antizipiert werden, dass die Anrede „Liebe Genossinnen und Genossen“ gewählt wird, dass die Mitglieder sich duzen, dass mit hoher Wahrscheinlichkeit Wörter wie „soziale Gerechtigkeit", „Wahlen“, „sozialdemokratisch“, „Umverteilung“ etc. vorkommen werden. Auf der Mikroebene kann die Strategie Antizipation beispielweise eingesetzt werden, wenn der Redner/ die Rednerin Kollokationen wie „die Kuh vom Eis holen“ verwendet. Die Schriftdolmetscher(innen) können, wenn sie das Wort „Kuh“ hören, vorausahnen, wie der Satz weitergeht und damit ihre Aufmerksamkeit ggf. auf schwierigere Textpassagen lenken.

Entscheidend für ein gutes Dolmetschprodukt sind die Planungsstrategien. Diese Strategien werden rezeptiv (beim Verstehen des Textes) und produktiv (bei der Erstellung des Transkriptes) angewendet.

- Die Schriftdolmetscher(innen) segmentieren den Text und stellen Hierarchien und Bezüge her.

- Sie müssen die Textzusammenhänge erfassen und erkennen, wenn Sinneinheiten abgeschlossen sind.

- Damit das Schriftbild für die Rezipient(inn)en angenehm ist, sollte am Ende einer Sinneinheit - möglichst nach drei bis vier Sätzen mittlerer Länge - ein Absatz gesetzt werden.

- Die Sätze sollten nicht zu lang sein.

Insbesondere bei längeren Sätzen und Sätzen mit Einbettungsstrukturen können sich Schriftdolmetscher(innen) durch eine größere Décalage (zeitlicher Abstand zwischen Originalrede und Verdolmetschung) einen Überblick verschaffen, bevor sie den Satz möglicherweise mit einer anderen Segmentierung 
produzieren. Hier ein Beispiel; zunächst der Originaltext und dann eine Transkriptionsvariante:

Es ist jetzt erst mal eine ganz klare Rückendeckung für weitere Gespräche mit der CDU/CSU vom Parteitag gegeben worden. Sie werden aber sicher gemerkt haben: Es hat Stunden gedauert, diese Debatte zu führen, weil es natürlich auch eine Frage war, die jetzt plötzlich wieder auf dem Tisch stand, nachdem die Verhandlungen der Jamaika-Sondierer krachend gescheitert sind. (Deutschlandfunk 2017)

Es ist erst mal eine Rückendeckung für Gespräche mit der CDU/CSU vom Parteitag gegeben worden. Es hat Stunden gedauert, diese Debatte zu führen. Die Frage stand nämlich plötzlich wieder auf dem Tisch nach dem krachenden Scheitern der Jamaika-Verhandlungen. (Transkript in Schriftverdolmetschung)

Gerver (2002) untersuchte die Translationsleistung von Simultandolmetscher(inne)n bei mündlich dargebotenen Texten mit durchschnittlicher Sprechgeschwindigkeit und ermittelte einen zeitlichen Abstand von durchschnittlich 2 bis 3 Sekunden - das entspricht drei bis vier Wörtern - zwischen dem Vortrag und der Verdolmetschung. Andere Untersuchungen kamen zu vergleichbaren zeitlichen Abständen (Oléron/Nanpon 2002).

Ferner wenden Schriftdolmetscher(innen) Strategien der Zieltextproduktion an. Darunter fallen die folgenden Strategien:

- das Transkodieren (Umgang mit Namen und Zahlen),

- die Suche von Äquivalenten,

- die Herstellung von Kohärenz,

- die Kompression oder Expansion,

- Präsentationsstrategien und

- Notstrategien (vgl. Gile 1995, Kalina 1998 und 2002). 
Kompression eignet sich beispielsweise, wenn der Redner redundanten Text verwendet.

Notstrategien kommen zum Einsatz, wenn Schriftdolmetscher(innen) aus akustischen oder anderen Gründen einen Teil des mündlichen Ausgangstextes nicht wiedergeben können. Wie der Name besagt, sollte diese Strategie nur gewählt werden, wenn Schriftdolmetscher(innen) keine andere Möglichkeit sehen. Im äußersten Fall tilgen Schriftdolmetscher(innen) den Text(teil), d. h. sie lassen den Textteil aus und kennzeichnen die Auslassung beispielsweise mit „(...). Weitere Strategien sind die folgenden:

- Selektion: Die Schriftdolmetscher(innen) geben die Textteile wieder, die sie verstanden haben.

- Kompression: Die Schriftdolmetscher(innen) verdichten das Gesagte und erreichen dadurch, dass die wichtigsten Inhalte transportiert werden.

- Generalisierung: Die Schriftdolmetscher(innen) geben das Gesagte mit einer allgemeineren Formulierung wieder: Z. B. „mehrere Millionen“ statt „4 Millionen“, wenn sie die Zahl nicht genau verstanden haben.

Wenn die Schriftdolmetscher(innen) Notstrategien anwenden, sollten sie sich darauf konzentrieren, Subjekt, Prädikat und Objekt des Satzes - die Kernelemente des Satzes - zu transkribieren. Oberstes Ziel ist es, dass der rote Faden für die Rezipient(inn)en erkennbar bleibt.

Monitoringstrategien kommen sowohl bei der Rezeption als auch bei der Produktion des Textes zum Einsatz:

- Der/die Schriftdolmetscher(in) überprüft laufend den Output auf Kohärenz und Richtigkeit.

- Er/Sie korrigiert das Transkript, während er/sie dem mündlichen Text weiter folgt. 
Der Ausgangstext kann aus verschiedenen Gründen komprimiert wiedergegeben werden. Ein möglicher Fall wäre z. B. der folgende: Es gibt eine Vortragsfolie/Tafelaufzeichnung, auf die sich der oder die Vortragende bezieht und die dem Rezipienten/der Rezipientin vorliegt. In diesem Fall wird der Hinweis verschriftlicht, dass sich der oder die Vortragende auf die entsprechenden Referenzen bezieht. Weitere Gründe können in kognitiven Einschränkungen liegen, oder darin, dass Rezipient(inn)en einen komprimierten Text wünschen, um dem Text leichter folgen zu können, da für sie die inhaltliche Wiedergabe an erster Stelle steht.

Ferner findet beim Schriftdolmetschen eine Umwandlung eines mündlichen Textes in einen schriftlichen statt, für dessen Rezeption andere Regeln gelten. Würde man das, was Sprecher(innen) sagen, wortwörtlich schriftlich wiedergeben, wäre es in Schriftform oft unverständlich (vgl. Eichmeyer im Druck und Platter 2015). Sprecher(innen) brechen zeitweilig Sätze ab, korrigieren sich selbst (Reparationen) und sprechen redundant. Dresing beschreibt die Herausforderungen im Bereich Untertitelung, die beim Schriftdolmetschen ähnlich sind: „Einerseits möchte man das Gesprochene so detailgetreu wie möglich wiedergeben, um dem Leser einen möglichst guten Eindruck vom Gespräch zu geben (...), andererseits bewirken zu viele Details und Informationen, dass ein Transkript nur schwer lesbar wird. Die Aspekte Exaktheit und sinnvolle Umsetzbarkeit liegen mitunter an entgegengesetzten Polen" (Dresing 2018:16).

Bei der Verschriftlichung verbraucht der Schriftdolmetscher/ die Schriftdolmetscherin zusätzliche Zeit, um der Software die Interpunktion zu diktieren (Spracherkennungsmethode) und Zusatzinformationen wie Lachen, Beifall geben, Verweise auf Folien einzufügen, Tipp-/Erkennungsfehler zu korrigieren. Wenn das Sprechtempo des Redners oder der Rednerin schnell und die Informationsdichte hoch ist, lässt sich eine Kompression kaum vermeiden: Füllwörter und unabsichtliche Doppelungen können weggelassen werden. Kennen Schriftdolmetscher(innen) die Wortarten, können sie schneller reagieren und die Wortarten identifizieren, die am ehesten weggelassen werden können, wie Füllwörter und inhaltlich für das Verständnis der Botschaft weniger relevante 
Adjektive. Statt einen Namen zu wiederholen, können Schriftdolmetscher(innen) kürzere Personalpronomina verwenden. Haben Schriftdolmetscher(innen) einen flexiblen, umfassenden Wortschatz, können sie kürzere Synonyme wählen und somit zur Kompression beitragen. Sie können Dolmetschstrategien wie Paraphrasieren (mit anderen Worten umschreiben, Text sinngemäß übertragen) und grammatikalische Umformulierung (Passiv- in Aktivkonstruktion umwandeln, einen langen in zwei kurze Sätze umwandeln) anwenden.

Untersuchungen von Transkripten von Schriftdolmetscher(inne)n durch Elliot et al. (2001), Tiittula (2006), und Platter (2015) ergaben, dass die mündlichen Ausgangstexte und die Transkripte sich deutlich unterscheiden. In Tiittulas Material liegt die Wiedergabequote bei $60 \%$ des gesprochenen Textes. Ausgelassen wurden Wiederholungen, Satzabbrüche, Selbstkorrekturen, Füllwörter etc. Elliot et al. (2001: 285) kommen zu dem Ergebnis, dass die Schriftdolmetscher(innen) in dem von ihnen untersuchten Korpus 83\% der wichtigen Sinneinheiten erfassten. In ihrem Fazit nennen Norberg und Stachl-Peier (2017: 151) Kompression beim Schriftdolmetschen „nicht nur ein notwendiges Übel, sondern essentiell, wenn der Hauptinhalt des Ausgangstextes angemessen wiedergegeben werden soll“ (Übersetzung durch die Autorin dieses Beitrags).

Platter (2015) untersucht, inwieweit die Transkripte in der spezifischen Translationssituation ein Gelingen der Kommunikationssituation mit den jeweiligen Rezipient(inn)en erreichen. Schriftdolmetscher(innen) kommen häufig in Settings zum Einsatz, die aus einem/einer Sprecher(in) A, dem/der Dolmetscher(in) und einem/einer Sprecher(in) B (bzw. dem/der Rezipient(in)) bestehen. Entsprechend greift Platter in ihrer Dissertation auf das Diskursmodell nach Jiang, die sogenannte Dolmetschtriade (Jiang 2008), zurück und überträgt es auf das Schriftdolmetschen. Jiang geht davon aus, dass im Rahmen der Dolmetschtriade sämtliche Kommunikationspartner(innen) gleichberechtigt für den Erfolg der Kommunikation verantwortlich seien. Sprecher(innen) A und B sowie der/die Dolmetscher(in) verfügen über eine gemeinsame Schnittmenge an sprachlichem, kulturellem, fachspezifischem und allgemeinem Weltwissen im Sinne eines gemeinsamen für die Kommunikation notwendigen Wissensstandes (Jiang 2008: 96). Darüber hinaus verfügen sie über gleiches situationsspezifisches Wissen sowie über einen gemeinsamen Aufmerksamkeitsbereich der Kommunikationspartner(innen) unter Berücksichtigung des 
Kommunikationszweckes und der Kommunikationsabsichten (ebd. 96f; vgl. auch Platter 2015: 100). Die bilaterale Kommunikation findet über die Brücke der Dolmetscher(innen) statt, d.h. sie dolmetschen die Botschaften von A bzw. B. Beim Schriftdolmetschen benötigen die Rezipient(inn)en B die Dolmetschung zum Verständnis, äußern sich in der Regel selbst und sprechen direkt mit den Sprecher(inne)n A. Nach Jiang wenden die Dolmetscher(innen) Diskursfilter an: den Diskurzweck, die Kohärenz, das Weltwissen und die individuelle Ad-hocInteressenlage (Jiang 2008: 133-142). Unter Anwendung dieser Filter produzieren die Dolmetscher(innen) ihren Zieltext nahe am Ausgangstext, abgewandelt oder mit Auslassungen. D.h. diese Faktoren und die Dolmetschsituation haben einen Einfluss auf die Entscheidung, ob und wo sich das Kommunikat des Schriftdolmetschers/ der Schriftdolmetscherin auf dem Kontinuum zwischen einer 1:1Wiedergabe des Gesagten und einer starken Kompression ansiedelt.

Platter kommt beim Vergleich der Ausgangs- und Zieltexte aus ihrem Korpus zum Ergebnis, dass die Verständlichkeit des Zieltextes durch die bewusste Komprimierung der Schriftdolmetscher(innen) kaum beeinträchtigt ist. „Teilweise sind gegenüber dem medial und konzeptionell mündlichen Ausgangstext auch Textoptimierungen durch Berichtigungen grammatischer Strukturen feststellbar (...)“ (Platter 2015: 202). Sie stellt fest, „dass lexikalische Adaptierungen oft zu Gunsten des Zieltextes und dessen Verständlichkeit ausfallen und lexikalische Fehler kaum vorkommen" (ebd.).

\section{$7 \quad$ Ein komplexer Prozess}

Aufgrund der Kompression, also der Auswahl und Gliederung von Informationen innerhalb kürzester Zeit, wächst die Komplexität des Prozesses. Hinzu kommt, dass Schriftdolmetscher(innen) die Handlungen des Verstehens, Planens und der Zieltextproduktion weitgehend simultan umsetzen und den Output gleichzeitig kontrollieren und ggf. korrigieren. Ziegler und Eichmeyer (Ziegler 2017), die beide unter anderem als Konferenzdolmetscher(innen) und Dozent(inn)en für Schriftdolmetschen tätig sind, haben das Effort-Modell von Gile (1995) auf das Schriftdolmetschen übertragen. Gile entwickelt eine Formel für das Simultandolmetschen: 


$$
\mathrm{SI}=\mathrm{L}+\mathrm{P}+\mathrm{M}+\mathrm{C}
$$

$\mathrm{L}=$ Listening and Analysis Effort (mentale Energie für das Zuhören und die Analyse des Gehörten)

$\mathrm{P}=$ Production Effort (mentale Energie für die Produktion des Zieltextes)

$\mathrm{M}=$ Memory Effort (mentale Energie für die Memorisierung des Ausgangstextes/der Botschaft)

$\mathrm{C}=$ Coordination Effort (mentale Energie für die Koordination aller Handlungen)

Bei einer simultanen Dolmetschleistung muss die insgesamt verfügbare Kapazität des Schriftdolmetschers/der Schriftdolmetscherin größer sein aller erforderlichen Efforts, die für das Zuhören, Analysieren, Speichern im Kurzzeitgedächtnis, die Textproduktion inklusive der Zusatzinformationen und das Koordinieren aller Handlungen beansprucht werden. Andernfalls leidet die Qualität des Outputs. Die Formel lautet nach Gile (1995):

$$
\mathrm{LR}+\mathrm{MR}+\mathrm{PR}+\mathrm{CR} \geq \mathrm{TA}
$$

Die Abkürzungen sind wie folgt aufzulösen:

$$
\begin{aligned}
& \text { LR = Capacity Requirements for Listening } \\
& M R \text { = Capacity Requirements for Memory } \\
& \text { PR = Capacity Requirements for Production } \\
& \text { CR = Capacity Requirements for Coordination } \\
& \text { TA = Total Available Capacity }
\end{aligned}
$$

Beim Schriftdolmetschen kommen die Efforts Outputkontrolle (OR) und Korrektur (KR) noch hinzu, so dass Giles Formel wie folgt zu modifizieren wäre:

$$
\mathrm{LR}+\mathrm{MR}+\mathrm{PR}+\mathrm{CR}+\mathrm{OR}+\mathrm{KR} \geq \mathrm{TA} .
$$


Bei der konventionellen Methode (auch Schnellschreiben genannt) benutzen Schriftdolmetscher(innen) eine "normale“ Quertz-Tastatur und schreiben meist mit dem Zehnfingersystem und blind (d.h. ohne beim Tippen auf die Tastatur zu schauen). Um hohe Anschlagzahlen zu erreichen, die für eine zeitnahe Erfassung der mündlichen Texte erforderlich sind, ist ständige Übung erforderlich. Textverarbeitungsprogramme wie MS-Word ermöglichen die automatische Korrektur von häufigen Tippfehlern. Nach jedem Einsatz pflegen Schriftdolmetscher(innen) Tippfehler in das Computerprogramm ein, um das Schriftbild immer weiter zu verbessern.

Ferner können sich Schriftdolmetscher(innen) für Regieanweisungen und häufig vorkommende Wörter Kürzel oder Textmakros anlegen, womit die Anschlagzahl erhöht wird (vgl. Platter 2015: 29f.). Beispiele: „sw“ wird automatisch umgewandelt in „(Sprecherwechsel)“, „st“ in „(Student/in)“, „br“ in „Bundesregierung“. Das Kürzel- und Textmakrosystem muss so angelegt sein, dass es in der Dolmetschsituation rasch aus dem Gedächtnis abgerufen und umgesetzt werden kann. Es gibt Programme, die auf Betriebssystemebene ansetzen und die Kürzel in Textverarbeitungs-, Tabellenkalkulations- und Präsentationsprogrammen durch die entsprechende Langform ersetzen.

Die Anschlagzahl kann somit durch gut gepflegte Kürzel- und Textmakrosysteme erhöht werden sowie durch die Fähigkeit der Schriftdolmetscher(innen), durch Übung und mit zunehmender Praxiserfahrung mit hohen Geschwindigkeiten zu tippen. Erstrebenswert sind Geschwindigkeiten von 350 - 500 Anschlägen pro Minute (vgl. Platter 2015: 28f.). Einige Schriftdolmetscher(innen) erreichen weit höhere Anschlagzahlen und schreiben bei ihrer Arbeit mit einer Geschwindigkeit auf dem Niveau von Platz 10-34 der Weltmeisterschaften im Maschinenschnellschreiben (Intersteno 2017).

Dabei ist zu beachten, dass eine hohe Anschlagzahl alleine nicht hinreichend ist, um eine Aussage über die Qualität der Transkription zu treffen. Effizient komprimierte Transkripte können auch mit einer geringeren Anschlagzahl gute Ergebnisse liefern.

Der Kapitaleinsatz ist bei dieser Methode am geringsten, gewisse physische Grenzen sind allerdings vorhanden. 
Bei der computergestützten Maschinenstenografie wird die spezielle Tastatur einer Stenografiermaschine mit einer Softwarelösung kombiniert. Der/die Nutzer(in) betätigt mehrere Tasten gleichzeitig und schreibt damit ganze Silben bzw. Wörter. Allerdings benötigt man laut Aussage von Schriftdolmetscher(inne)n, die mit dieser Methode arbeiten, eine zweijährige Ausbildung, um diese Technik zu erlernen, sowie zusätzliche Berufspraxis, um die Technik optimal zu beherrschen (vgl. Platter 2015: 33f.). Diese Ausbildung wird derzeit in Deutschland nicht mehr angeboten. Der Zeit- und Kapitalaufwand ist bei dieser Methode folglich sehr hoch.

Eine andere Variante von Schnellschreibtastatur ist das Velotype, eine holländische Entwicklung, die von der Firma Velotype VOF angeboten wird. Die Tastaturen sind sprachspezifisch, weil sie mit Tasten für Vokale und Anfangs- und Endbuchstaben von Wörtern arbeiten. Es werden mehrere Tasten gleichzeitig gedrückt, um Wörter zu erzeugen. Die Anschaffungskosten sind niedriger als bei der computergestützten Maschinenstenografie und die Grundfertigkeit in dieser Technik kann laut Herstelleraussage innerhalb kurzer Zeit (Velotype 2018) erreicht werden. Schriftdolmetscher(innen), die mit dieser Methode arbeiten, verwenden ebenfalls Kürzel wie in der konventionellen Methode (Platter 2015: 32).

Eine relativ junge Softwareentwicklung ist die Spracherkennung, da die Computerprozessoren immer leistungsfähiger werden und zuletzt entscheidende technische Fortschritte auf diesem Gebiet erzielt wurden. Noch 2015 wird die Spracherkennung als „recent innovation“ beschrieben (Norberg et al. 2015: 38). Der/die Schriftdolmetscher(in) trainiert diese Software auf seine/ihre Stimme und die Wörter, die er/sie bei seinen/ihren Einsätzen verwendet. Der Dolmetscher/die Dolmetscherin hört den Sprecher/die Sprecherin über seinen/ihren Kopfhörer oder live und spricht den mündlichen Text in ein Mikrofon (Respeaking). Bei einem Präsenzeinsatz spricht der Dolmetscher/die Dolmetscherin in eine Stenomaske, damit die anderen Teilnehmer(innen) das Respeaking nicht hören. Das Computerprogramm setzt die Sprache in Text um und gibt den Text mit einer kleinen zeitlichen Verzögerung auf dem Bildschirm aus. Der/die Schriftdolmetscher(in) kann den ausgegebenen Text über die Eingabe per 
Tastatur oder durch Befehle im Programm korrigieren. Spracherkennungsprogramme weisen derzeit noch gewisse Schwächen auf, unter anderem bei der Erkennung von Deklinationen und Konjugationen sowie Komposita, doch werden mit den entsprechenden Korrekturen der Schriftdolmetscher(innen) gute Ergebnisse erzielt.

\section{Einsatzformen beim Schriftdolmetschen}

Schriftdolmetschen kann entweder vor Ort (Präsenzdolmetschen), als OnlineDolmetschen oder als Semi-Präsenzdolmetschen erfolgen. Beim Präsenzdolmetschen sind die Schriftdolmetscher(innen) direkt beim Geschehen dabei. Beim Online-Dolmetschen werden die Schriftdolmetscher(innen) über das Internet mittels einer Plattform zugeschaltet. Die Plattform ermöglicht den Schriftdolmetscher(inne)n, die Redner(innen) zu hören, den Text einzugeben und zu korrigieren und mittels Chat mit dem Rezipienten/der Rezipientin zu kommunizieren. Das Semi-Präsenz-Szenario sieht in der Regel folgendermaßen aus: Ein(e) Schriftdolmetscher(in) ist vor Ort und der/die zweite Schriftdolmetscher(in) ist elektronisch zugeschaltet.

Jede Form des Dolmetschens hat Vor- und Nachteile: Die Vorteile beim Präsenzdolmetschen sind, dass die Dolmetscher(innen) vor Ort direkten Kontakt zu den Rezipienten und Rezipientinnen und Rednern und Rednerinnen haben, dass sie die Atmosphäre direkt erleben und Einfluss auf die technischen Gegebenheiten nehmen können. Sie sind nicht unbedingt auf gute Internetverbindungen angewiesen. Die Herausforderungen für die Präsenzdolmetscher(innen) bestehen ggf. darin, dass Nebengeräusche die Audioqualität beeinträchtigen könnten und dass aufgrund der derzeit noch geringen Anzahl von Schriftdolmetscher(inne)n die Verfügbarkeit vor Ort eingeschränkt sein könnte.

Beim Online-Dolmetschen entfallen die Anreise des Schriftdolmetschers/der Schriftdolmetscherin sowie die Aufbauzeit für die Technik. Die Verfügbarkeit von Schriftdolmetscher(inne)n ist höher als beim Präsenzdolmetschen. Bei dieser Variante steht dem/der Dolmetscher(in) meist kein visueller Input, der für 
das Verständnis des Textes zusätzliche Informationen liefert, zur Verfügung und er/sie hat keinen direkten Einfluss auf das Geschehen. D.h. er/sie kann nicht um langsamere Sprechweise bitten oder die Redner(innen) erinnern, das Mikrofon korrekt zu verwenden. Außerdem muss eine stabile Internetverbindung mit hohen Übertragungsraten vorhanden sein.

Bei der Semi-Präsenz-Variante können die Vorteile aus beiden Szenarien vereint werden: Der Dolmetscher/die Dolmetscherin vor Ort kann für optimale Aufnahme- und Übertragungsbedingungen sorgen, der/die zugeschaltete Online-Dolmetscher(in) hat ggf. einen besseren Ton ohne Nebengeräusche. Die Verfügbarkeit der Dolmetscher(innen) ist besser und die Logistik für die Bereitstellung der Leistung ist einfacher als beim Präsenzdolmetschen.

Bei längeren Einsätzen von mehr als 60 Minuten arbeiten Schriftdolmetscher(innen) in der Regel in Doppelbesetzung. Eine entsprechende Empfehlung ist beispielsweise aus der Berufs- und Ehrenordnung von Konferenzdolmetschern abzuleiten (AIIC: Code of professional ethics, Art. 7.). Die Teamarbeit bietet den Vorteil, dass sich die Dolmetscher(innen) gegenseitig unterstützen können. Sie wechseln sich nach Vereinbarung, z. B. nach 15 Minuten, ab. Der/die nicht aktive Dolmetscher(in) unterstützt den Kollegen/die Kollegin, indem er/sie bei Bedarf editierend eingreift oder recherchiert (beispielsweise Eigennamen, Zahlen). Idealerweise haben beide Schriftdolmetscher(innen) Zugriff auf denselben Text, und zwar entweder über eine SchriftdolmetschPlattform oder über (kostenfreie oder kostenpflichte) Computerprogramme wie Gobby (https://gobby.github.io) und text on top (www.text-on-top.com). Dazu müssen die Computer über eine Kabelverbindung oder ein anderes System miteinander verbunden sein.

Die verschiedenen hier genannten Faktoren wie Einsatzformen, Schriftdolmetschmethode(n), Einzel- oder Teambesetzung, Einzel- oder Konferenzsetting, Grad der Hörbehinderung der Zielgruppe haben einen Einfluss auf die Dolmetschsituation (weitere Ausführungen siehe Platter 2015). 
Schriftdolmetschen wird derzeit in Deutschland als nebenberufliche Weiterbildung angeboten. Je nach Anbieter unterscheiden sich Art und Umfang der Weiterbildung sowie inhaltliche Gestaltung und die vermittelten Methoden. Am SDI München werden Themen wie psychologische Aspekte der Hörbehinderung, betriebswirtschaftliche Aspekte, Kommunikationstechniken und Dolmetschstrategien plus die konventionelle Methode und Spracherkennung gelehrt. Das SDI München kooperiert mit der Universität Hildesheim: Schriftdolmetschen wird als ein Kompaktwochenende durch Dozent(inn)en des SDI im Rahmen des Studiengangs Barrierefreie Kommunikation angeboten. Viele der vermittelten Inhalte sind darüber hinaus nützlich in anderen beruflichen Kontexten und im täglichen Umgang mit Menschen. Übersetzer(innen) und Dolmetscher(innen) sowie Gebärdensprachdolmetscher(innen) bringen eine gute Qualifikation für den Berufsstand mit und werden mit der Zusatzausbildung Schriftdolmetschen seitens aktiver Schriftdolmetschvermittler anerkannt (Platter 2015: 295). Dolmetscher(innen) etwa haben bereits konsekutives bzw. simultanes Dolmetschen erlernt. Die Weiterbildung eignet sich ebenfalls beispielsweise für andere Kommunikationsberufe, Inklusionsberufe, Logo- und Ergotherapeut(inn)en.

\section{Schriftdolmetscher(innen): Berufsaussichten}

Der Beruf des Schriftdolmetschers/der Schriftdolmetscherin ist stark nachgefragt: Nach Angaben des DSB gibt es ca. 55 DSB-zertifizierte Schriftdolmetscher(innen) in Deutschland (DSB 2018). Dem steht nach Angaben des DSB ein errechneter Bedarf von 20.000 Schriftdolmetscher(inne)n gegenüber (DSB. 2016). Andere Bedarfserhebungen liegen derzeit nicht vor. Dabei ist zu berücksichtigen, dass es unterschiedliche Zahlenangaben über den Anteil von Schwerhörigen an der Bevölkerung in Deutschland gibt (Verbraucherportal für Schwerhörige und Hörgeräte-Träger 2016). Wenn man von einer Nachfrage seitens der Schwerhörigen nur für bestimmte Situationen ausgeht, in denen ein gesetzlich geregelter Anspruch auf Übernahme der Kosten vorliegt, kommt man 
auf Bedarfszahlen von ca. 3.000 (vgl. Platter 2015: 39f.) Bei anspruchsvollen Aufträgen wie Schriftdolmetschen für den Landtag, auf internationalen Konferenzen oder bei Betriebsversammlungen werden nur Schriftdolmetscher(innen) mit einem DSB-Zertifikat oder einem Zertifikat eines anerkannten Bildungsanbieters gebucht.

Der Beruf kann in Festanstellung oder freiberuflich ausgeübt werden. Arbeitgeber sind beispielsweise Rundfunkanstalten, Vermittlungsagenturen oder Interessenverbände. Aufgrund der geforderten hohen Konzentrationsfähigkeit wird der Beruf meist in Teilzeit 20 bis 30 Stunden pro Woche ausgeübt.

\section{Honorierung der Schriftdolmetschleistung}

Im Zuge der Implementierung der EU-Richtlinie „zur Festlegung eines allgemeinen Rahmens für die Verwirklichung der Gleichbehandlung in Beschäftigung und Beruf" besteht in der Regel ein Rechtsanspruch auf Finanzierung der Dienstleistung Schriftdolmetschen (Richtlinie 2000/78/EG). Gesetzliche Grundlage in Deutschland ist $\$ 17$ Abs. 2 Sozialgesetzbuch I, Bundesbehindertengleichstellungsgesetz. Laut Kommunikationshilfen-Verordnung (KHV 2002) haben Personen mit Hör- oder Sprachbehinderung in Verwaltungsverfahren einen Anspruch auf Bereitstellung eines/einer Gebärdensprachdolmetschers/ -dolmetscherin, Schriftdolmetschers/-dolmetscherin oder Kommunikationsassistenten/-assistentin. Nach der KHV ist für die Schriftdolmetschleistung unter bestimmten Voraussetzungen eine Vergütung in Höhe des Honorars der Dolmetscher(innen) anwendbar, die laut $\$ 9$ Absatz 3 Satz 1 des Justizvergütungs- und -entschädigungsgesetzes (JVEG 2016) für simultanes Dolmetschen bezahlt werden.

Nach den vorgenannten Gesetzen und Verordnungen sind viele Bereiche des Bedarfs an Schriftdolmetschleistungen - außer im privaten Bereich - abgedeckt. Jedem hörbehinderten Menschen steht jährlich ein Budget für diese Unterstützung zu. Es gibt allerdings immer noch Graubereiche wie beispielsweise für Gespräche, die hörgeschädigte Eltern mit Lehrer(inne)n ihres Kindes in der Schule führen. Die Leistungen von Schriftdolmetscher(inne)n werden je nach Dolmetschsituation von 
unterschiedlichen Trägern bezahlt, z. B. von Krankenkassen, Rehabilitationsträgern, Agenturen für Arbeit, vom Integrations-/Inklusionsamt etc.

Die Honorarsätze bei Integrations-/Inklusionsämtern variieren je nach Region. Insgesamt richten sich die Honorare unter anderem nach den Anforderungen an die Dolmetschleistung und danach, ob die Leistung direkt vom Kunden oder beispielsweise von einer Vermittlungsagentur bezahlt wird. Laut Berufs- und Ehrenordnung sind Schriftdolmetscher(innen) gehalten, angemessene Honorare anzusetzen (Ehrenordnung/ Ehrenkodex 2018: $₫ 8$ ).

In diesem Honorar ist laut Berufsverbänden derzeit die Aushändigung einer Mitschrift der Dolmetschleistung nicht enthalten. Die Berufs- und Ehrenordnung des DSB vom 27.11.2009 schreibt vor, dass alle Datenaufzeichnungen nach Beendigung des Auftrags dauerhaft gelöscht werden, und verweist auf Ausnahmeregelungen, die bis dato (DSB 2009, Stand 26.2.2018) noch nicht veröffentlicht wurden. Derzeit gängige Praxis ist, dass Schriftdolmetscher(innen) auf Wunsch Mitschriften erstellen, die vergütet werden. Vor der Veröffentlichung der Mitschrift ist es erforderlich, das schriftliche Einverständnis bei den Betroffenen zur Veröffentlichung personenbezogener Daten sowie des Redners/der Rednerin zur Veröffentlichung einzuholen und Tipp- und Erkennungsfehler zu korrigieren. Ggf. entsteht zusätzlicher Recherche- oder Formatierungsaufwand. Die Mitschrift repräsentiert die geistige Leistung der Schriftdolmetscher(innen) und ist damit urheberrechtlich geschützt, d.h. die Verwertung ist zunächst für den persönlichen Bedarf ausgelegt. Darüberhinausgehende Verwertungen und damit ggf. anfallende Verwertungshonorare müssen gesondert vereinbart werden (vgl. Eichmeyer et al. 2017).

\section{Ausblick}

Der relativ junge Beruf des Schriftdolmetschers/der Schriftdolmetscherin befindet sich derzeit in der Professionalisierung. Tseng (1992) hat für Konferenzdolmetscher in Taiwan ein Professionalisierungsmodell entwickelt, das sich auf alle Translationsberufe anwenden lässt (Platter 2015: 207). Wendet man sein 4-Phasen-Modell auf das Schriftdolmetschen an, befindet sich der Beruf in Phase 1: Es herrscht ein undurchsichtiger Markt und starker Wettbewerb, kaum 
Sensibilität für die Qualität der Dienstleistung (Tseng 1992: 44). Die Ausbildungsprogramme sind uneinheitlich (ebd.).

Im Jahre 2018 wurde eine staatliche Prüfung für Schriftdolmetscher(innen)für Lautsprache und Schriftsprache genehmigt (veröffentlicht im Hessischen Amtsblatt). Als Zulassungsvoraussetzungen für die Prüfung werden „der Nachweis einer einschlägigen Ausbildung zur Schriftdolmetscherin oder zum Schriftdolmetscher für Lautsprache und Schriftsprache oder eine entsprechende mehrjährige Berufstätigkeit als Schriftdolmetscherin oder Schriftdolmetscher für Lautsprache und Schriftsprache" (Hessisches Amtsblatt 2018: 295) genannt. Weitere Informationen über die staatliche Prüfung finden sich auf der Website der Hessischen Lehrkräfteakademie (2018), welche die Prüfung durchführt. Legt man das Professionalisierungsmodell von Ju zugrunde (Ju 2009: 120), kann die Einrichtung dieser Prüfung einen Entwicklungsschub in der Professionalisierung des Berufes bedeuten. 


\section{Literaturverzeichnis}

AIIC: Code of professional ethics, https://aiic.net/page/6724, abgerufen am 27.2.2018.

Berufs- und Ehrenordnung des Deutschen Schwerhörigenverbunds e.V. (2009), https://www.schwerhoerigen-netz.de/fileadmin/user_upload/dsb/Dokumente/

Information/Service/Schriftdolmetscher/sd_berufs-ehrenordnung_170516.pdf (abgerufen am 6.2.2018, $\$ 6)$.

BRAUN, SABINE (2004): Kommunikation unter widrigen Umständen? Einsprachige und gedolmetschte Kommunikation der Videokonferenz. Tübingen: Gunter Narr.

Deutscher Schwerhörigenbund e.V. (2016): Eine arbeitsmarktpolitische Begrü̈dung zur Ausbildung von Schriftdolmetschern in Deutschland, 30.06.2016, http://www.schwerhoerigen-netz.de/DSB/SERVICE/SCHRIFTD/PDF/ arbeitsmarkt-analyse.pdf (abgerufen am: 02.02.2018).

Deutscher Schwerhörigenbund e.V. (2018): Zertifizierte Schriftdolmetscher. https://www.schwerhoerigen-netz.de/schriftdolmetscher/ (abgerufen am 18.05.2018).

Deutschlandfunk (2017): http://www.deutschlandfunk.de/spd-vor-gespraechen-mitder-union-nicht-mit-rucksack-voll.694.de.html?dram:article_id=402620 (abgefragt am 17.5.2018).

Dresing, Thorsten/Pehl, Thorsten (2018): Praxisbuch Interview, Transkription \& Analyse. Anleitungen und Regelsysteme für qualitativ Forschende. Marburg: Eigenverlag.

Ehrenordnung/Ehrenkodex des Bundesverbands der Schriftdolmetscher Deutschlands: Ehrenordnung / Ehrenkodex (2018): http://bsd-ev.org/index.php?id=92 (abgerufen am 18.5.2018.

EICHMEYER, DANIELA (im Druck): Schriftdolmetschen - eine neue Translationsform unter dem Einfluss der Entwicklung digitaler Technologien. In: Tagungsberichte der LICTRA 2017).

Eichmeyer, Daniela/Kaul, Mario/Nofftz, Birgit (2017): Schriftdolmetschen Handhabe von Mitschriften. Unveröffentlichtes Manuskript.

ElLIOT, LisA B. et al. (2001): College Students' Perceptions of the C-Print Speech-to-Text Transcription System. In: Journal of Deaf Studies and Deaf Education 6 (4), 285-298.

GERVER, DAVID (2002): The effects of Source Language presentation rate on the performance of simultaneous Conference interpreters. In: PÖCHHACKER, FRANZ/ Shlesinger, Miriam (Hrsg.): The Interpreting Studies Reader. London and New York: Routledge (Routledge Language Readers), 53-66.

GeRZYMisCH, HeIDRUn (2013): Gutachten zu der Bezeichnung Schriftdolmetschen, www.bsd-ev.org/fileadmin/gutachten_gerzymisch.pdf (abgerufen am 7.2.2018).

Gesetz zur Gleichstellung von Menschen mit Behinderungen (Behindertengleichstellungsgesetz - BGG): https://www.gesetze-im-internet.de/bgg/BGG.pdf (abgefragt am 6.2.2018). 
Hessisches Amtsblatt (2018): \$\$ 54-60 Prüfung für Schriftdolmetscherinnen und Schriftdolmetscher für Lautsprache und Schriftsprache. https://hessisches-amtsblatt.de/ wp-content/plugins/pdf-viewer/stable/web/viewer.html?file=/wp-content/uploads/ online_pdf/pdf_2018/03_2018.pdf (abgerufen am 31.3.2018).

Intersteno 2017: Ergebnisliste des Intersteno Kongresses 2017, http://intersteno2017.org/ berlin_resultlistTP.php (abgefragt am 23.2.2018).

JIANG, LIHUA (2008): From Community Interpreting to Discourse Interpreting - A Triadic Discourse Interpreting Model (TRIM). Dissertation: Universität des Saarlandes. https://d-nb.info/1009673432/34 (abgerufen am 12.12.2017).

JU, Elma Mingli (2009): The professionalization of interpreting in Taiwan: A critical review of Tseng's model. In: Compilation and Translation Review 2 (2): 105-125.

JVEG (2016) Justizvergütungs- und -entschädigungsgesetz von 2004, zuletzt geändert 2016, http://www.gesetze-im-internet.de/jveg/ (abgerufen am 28.5.2018).

Kalina, Sylvia (1998): Strategische Prozesse beim Dolmetschen. Tübingen: Gunter Narr.

Kalina, Sylvia (2002) Fragestellungen der Dolmetschwissenschaft. In: BeSt, JOANNA/ Kalina, Sylvia (Hrsg.): Übersetzen und Dolmetschen. Eine Orientierungshilfe. Uni Taschenbücher. Springer Verlag.

Kommunikationshilfen-Verordnung (KHV) (2002), http://www.gesetze-im-internet.de/ khv/KHV.pdf (abgerufen am 6.2.2018).

Lehrkräfteakademie Hessen (2018): Prüfungsteile Schriftdolmetscher. https://lehrkraefteakademie.hessen.de/sites/lehrkraefteakademie.hessen.de/files/

content-downloads/Prüfungsteile\%20Schriftdolmetscher_040418.pdf (abgefragt am 18.5.2018).

Norberg, Ulf/Stachl-Peier, Ursula/Titttula, LiIsa (2015): Speech-to-text interpreting in Finland, Sweden and Austria. In: Translation \& Interpreting, Vol. 7 No. 3, 36-49.

Norberg, Ulf/Stachl-PeIER, URsula (2017): Quality in speech-to-text-interpreting. A study of condensation strategies. Berlin: Frank \& Timme.

Nord, Christiane (1991): Textanalyse und Übersetzen. Theoretische Grundlagen, Methode und didaktische Anwendung einer übersetzungsrelevanten Textanalyse. Heidelberg: Groos.

Oléron, Pierre/NAnPon, Hubert (2002): Research into Simultaneous Translation. In: PÖCHHACKer, Franz/Shlesinger, Miriam (Hrsg.): Introducing interpreting studies. London: Routledge, 43-50.

PlatTER, JUdith (2015): Translation im Spannungsbereich von Mündlichkeit und Schriftlichkeit, Schriftdolmetschen in Österreich. Eine textbasierte Analyse. Unveröffentlichte Dissertation, Universität Wien.

Richtlinie 2000/78/EG des Rates vom 27. November 2000 zur Festlegung eines allgemeinen Rahmens für die Verwirklichung der Gleichbehandlung in Beschäftigung und Beruf: http://eur-lex.europa.eu/legal-content/DE/TXT/HTML/?uri=LEGISSUM: c10823\&from=DE (abgerufen am 31.3.2018). 
Romero-Fresco, Pablo/PöchHacker, Franz (im Druck): Quality assessment in interlingual live subtitling: The NTR model. Linguistica Antwerpiensia. New Series: Themes in Translation Studies, Vol. 14.

TirtTUla, LiISA (2006): Schriftdolmetschen - Mündlichkeit im schriftlichen Gewand. In: A Man of Measure. Festschrift in Honour of Fred Karlsson, 481-488.

TSENG, JOSEPH (1992): Interpreting as an emerging profession in Taiwan - A sociological model. Diplomarbeit: Fu Jen Catholic University Taipei, Taiwan.

Velotype (2018): Video, http://www.velotype.com/en/?Functioning_of_the_keyboard (abgerufen am 16.5.2018).

Verbraucherportal für Schwerhörige und Hörgeräte-Träger (2016): https://www.meinhoergeraet.de/de/news/schwerhoerige-in-deutschland-2016/631 (abgerufen am 16.5.2018).

Witzel, JutTa (2018): Schriftdolmetschen. Ein sinnvoller und vielseitiger Beruf. In: ADÜ Nord Infoblatt 1/2018, 14ff.

ZIEGLER, KLAUS (2017): Skript Schriftdolmetscherausbildung 2017/18. Grundlagen des Dolmetschens / Dolmetschstrategien. SDI München/BFW Würzburg. (unveröffentlicht).

\section{Internetquellen mit Informationen zu den Themen Schriftdolmetschen und Hörschädigung}

Berufsverband Deutscher Hörgeschädigtenpädagogen, http://www.b-d-h.de

Bundesverband der Schriftdolmetscher Deutschlands, http://www.bsd-ev.org/ index.php?id=75

Deutsche Gesellschaft der Hörgeschädigten, https://www.deutsche-gesellschaft.de

Deutscher Gehörlosenbund e.V., http://www.gehoerlosen-bund.de

Deutscher Schwerhörigenbund e.V. (DSB), www.schwerhoerigen-netz.de

Taubenschlag (Website mit Informationen und Einblicke in die Welt der Hörgeschädigten), www.taubenschlag.de

https://textgedanken.wordpress.com/schreibdolmetschen/ 



\section{Untertitelung für Hörgeschädigte (SDH)}

\section{$1 \quad$ Einleitung}

Untertitel für Hörgeschädigte werden in der Regel am unteren Bildrand eines audiovisuellen Medienprodukts eingeblendet und geben in geraffter Form schriftlich wieder, was in der Tonspur zu hören ist. Doch dienen sie nicht der Übersetzung von einer Sprache in eine andere wie die sogenannten interlingualen Untertitel, sondern hauptsächlich der Übertragung in einen anderen Modus, in den Schriftmodus. Untertitel für Hörgeschädigte richten sich, wie aus der Bezeichnung hervorgeht, in erster Linie an eine bestimmte Zielgruppe, an Menschen, die schwerhörig, ertaubt oder gehörlos sind und daher die Lautsprache und andere akustische Informationen eines Films nicht oder nur eingeschränkt wahrnehmen können. Über den Moduswechsel wird versucht, den dadurch entstehenden Informationsverlust auf verbaler, aber auch nonverbaler und paraverbaler Ebene zu kompensieren. Im Englischen werden Untertitel für Hörgeschädigte etwas differenzierter als subtitles for the D/deaf and hard of hearing, kurz SDH bezeichnet. Diese Bezeichnung soll hier übernommen werden, da sie sich international durchgesetzt hat und daran erinnert, dass die intendierte Zielgruppe heterogen ist, also sich aus Menschen mit unterschiedlichen Formen und Ausprägungen von Hörschädigungen und entsprechend unterschiedlichen Bedarfen zusammensetzt.

SDH werden in erster Linie im Fernsehen angeboten. Die Quote an untertitelten Sendungen bei den öffentlich-rechtlichen Rundfunkanstalten ist im Laufe des vergangenen Jahrzehnts rasant gestiegen. Während Jüngst 2010 noch darauf hinweist, dass nur $10 \%$ aller Sendungen im deutschen Fernsehen untertitelt werden (Jüngst 2010: 123), haben die öffentlich-rechtlichen Rundfunkanstalten ihre Untertitelquoten inzwischen deutlich gesteigert, das Erste erreicht seit 2013 regelmäßig Quoten von über 90\% (vgl. ARD 2016: 17 und Heerdegen- 
Wessel 2017: o. S. sowie Heerdegen-Wessel im vorliegenden Band). Der Hauptgrund für diese Entwicklung ist die seit 2013 geltende Regelung zum Rundfunkbeitrag, der seither auch von Menschen mit Sinnesbehinderungen gezahlt werden muss. Entsprechend sind die Rundfunkanstalten gehalten, dieser Zielgruppe einen barrierefreien Zugang zu audiovisuellen Medienprodukten und somit gesellschaftliche Teilhabe zu ermöglichen. SDH reihen sich damit in eine breite Palette von Maßnahmen ein, die der Herstellung von Accessibility im gesellschaftlich-kulturellen Bereich dient, wie im Übereinkommen der UN über die Rechte von Menschen mit Behinderungen (vgl. BRK 2008, s. auch Lang im vorliegenden Band) explizit gefordert wird.

Der Beitrag geht zunächst auf die Heterogenität der Zielgruppe (Punkt 2) sowie auf übliche Formen der SDH (Punkt 3) ein, bevor eine Einordnung von SDH als Translation vorgenommen wird (Punkt 4). Weiterhin werden einige zentrale Aspekte von SDH (Punkt 5) diskutiert und ungelöste Dilemmata bei der Gestaltung von SDH (Punkt 6) dargestellt, die mit der Lesegeschwindigkeit und der teils schwierigen Zuordnung der Untertitel zu den jeweils sprechenden Figuren zusammenhängen. Abschließend (Punkt 7) gibt der Beitrag einen kurzen Überblick über aktuelle Forschungstendenzen und -desiderate.

\section{Die Heterogenität der Zielgruppe}

Die primäre Zielgruppe für SDH sind Personen, die aufgrund einer Hörschädigung die Tonspur eines audiovisuellen Medienprodukts nicht oder nur eingeschränkt wahrnehmen können. Dieser durchaus logischen und pragmatischen Zielgruppenbestimmung muss jedoch die Perspektive der Zielgruppen selbst gegenübergestellt werden. Es zeigt sich, dass deren Bedarfe, Forderungen und Voraussetzungen äußerst heterogen sind und daher differenziert dargestellt werden müssen.

Die Zielgruppe lässt sich sowohl aus medizinischer als auch aus kultureller Perspektive beschreiben. Medizinisch betrachtet wird das Hörvermögen eines Menschen am Hörverlust, und zwar in Dezibel, gemessen. Üblicherweise erfolgt eine Einteilung in leicht-, mittel- und hochgradige Schwerhörigkeit oder Ertaubung. Ein weiteres Unterscheidungsmerkmal dieser Personengruppe ist der 
Zeitpunkt, zu dem die Hörschädigung eingetreten ist: prä- oder postlingual, also vor oder nach Abschluss des Lautspracherwerbs, der in etwa im vierten Lebensjahr angesetzt wird (vgl. Lim/Simser 2005: 308). Durch Hörscreenings, auf die seit 2009 alle Neugeborenen in Deutschland einen Anspruch haben, sowie durch eine stete technische Weiterentwicklung von Hörgeräten und CochleaImplantaten können Hörvermögen nachhaltig verbessert werden (vgl. u. a. Saß 2002).

Dieser medizinischen, hörend geprägten Perspektive steht der kulturelle Begriff von Gehörlosigkeit gegenüber. Menschen, die sich in diesem Sinne als gehörlos betrachten, verstehen sich als Teil einer kulturellen und sprachlichen Gemeinschaft, deren wichtigstes identitätsstiftendes Merkmal die Gebärdensprache ist. Gehörlosigkeit gilt in diesem Kontext allenfalls als relationale Behinderung, die nur im Aufeinandertreffen mit Hörenden auftritt und dann beide Seiten betrifft (vgl. Ugarte Chacón 2015: 33; 79).

Wir haben es innerhalb der Zielgruppe also sowohl mit unterschiedlichen Hörvermögen zu tun, die zu verschiedenen Zeitpunkten eingetreten sein können, als auch mit verschiedenen Kulturen. Das bedeutet, dass SDH nicht zwangsläufig von der gesamten Zielgruppe als bevorzugte Technik zur Schaffung von Barrierefreiheit angesehen werden. Vielmehr gibt es aus Zielgruppenverbänden regelmäßig Forderungen nach dem Ausbau weiterer AccessibilityTools, wie der Verdolmetschung in die Deutsche Gebärdensprache (vgl. Bosse/ Hasebrink 2016: 88f.).

Ein zentraler Aspekt bei der Betrachtung der Zielgruppe sind deren Leseund, daran gekoppelt, deren Schriftsprachkompetenzen, zu denen es seit den 1980er Jahren zahlreiche Forschungsarbeiten aus verschiedenen Disziplinen gibt (vgl. u. a. Jelinek/Jackson 2001, Krammer 2001, Rausch 2011). Wie Hennies (2009: 294 und im vorliegenden Band) darlegt, geht eine Hörbeeinträchtigung nicht grundsätzlich mit geringen Lese- oder Schriftsprachkompetenzen einher. Dennoch kann ein verzögerter Laut- oder Gebärdenspracherwerb zu Schwierigkeiten beim Erwerb von Lese- und Schriftsprachkompetenzen der Lautsprache führen (vgl. Marschark/Hauser 2011: 103), was wiederum die Rezeption von untertitelten Medien erschwert. Dies gilt erst recht für Kinder, die ab einem Alter von sieben oder acht Jahren zwar als Zielgruppe in Betracht kommen, sich aber noch im Lernprozess befinden. So ist es häufig der Fall, dass schwerhörige, 
seit Geburt gehörlose oder später ertaubte Kinder weniger gut ausgeprägte Leseund Schriftsprachkompetenzen haben als hörende Gleichaltrige (vgl. u. a. Hennies 2009 und im vorliegenden Band, Herman et al. 2017, Peltzer-Karpf 1994). Untertitel werden daher nur von wenigen Kindern (vgl. Jennewein 2016 und Mälzer/Wünsche in Vorbereitung) genutzt. Ein weiterer Grund liegt darin, dass Untertitel für Kinder erst seit wenigen Jahren von den Rundfunkanstalten produziert werden. Man muss also annehmen, dass das Angebot innerhalb der Zielgruppe noch weitgehend unbekannt ist. Um zielgruppengerechtere Untertitel anzubieten und zu einer vermehrten Nutzung der barrierefreien Angebote der öffentlich-rechtlichen Rundfunkanstalten beizutragen, wendet der KiKA seit 2016 spezifische Untertitelstandards an (vgl. Kap. 3), die anhand zweier kleinerer Studien, von Jennewein (2016) und Kramer (2016), erarbeitet wurden. Um statistisch belastbare Ergebnisse zu erhalten, muss der Abschluss eines größer angelegten empirischen Forschungsprojekts der Universität Hildesheim zur Untertitelung von TV-Sendungen für schwerhörige und gehörlose Kinder abgewartet werden. Die Studie wird voraussichtlich 2019 abgeschlossen sein und soll zur Erarbeitung wissenschaftlich fundierter Untertitelungsstandards für Kinder von 8 bis 12 Jahren beitragen (vgl. Mälzer/Wünsche in Vorbereitung).

\section{Formen und Einsatzbereiche von SDH}

Untertitel für schwerhörige und gehörlose Menschen werden zwar überwiegend im Fernsehen angeboten, aber längst nicht ausschließlich. Die öffentlich-rechtlichen Fernsehanstalten im deutschsprachigen Raum stellen sie über den Teletext zur Verfügung. Weil Teletext-Untertitel somit ganz nach Bedarf ein- oder ausgestellt werden können, spricht man im englischsprachigen Raum auch von closed captions (vgl. Jelinek/Jackson 2001: 43) im Unterschied zu open captions, die immer sichtbar sind. Auch in den Online-Mediatheken der Rundfunkanstalten können Untertitel optional hinzugeschaltet werden. Nicht zuletzt bieten viele DVD- oder Blu-Ray-Ausgaben audiovisueller Medienprodukte die Option, Untertitel abzuspielen.

Im deutschsprachigen Fernsehen, das in der Regel keine Originalfilme mit deutschen Untertiteln ausstrahlt, findet man Untertitel fast ausschließlich in 
Form von SDH, während DVDs oder Blu-Rays meist die Wahl zwischen unterschiedlichen Untertitelversionen lassen. SDH im Kino gibt es in Deutschland kaum, dort treten sie häufig in Form interlingualer Untertitel zu einem fremdsprachigen Film auf (vgl. Jüngst 2010: 124). Das Unternehmen Greta \& Starks bietet allerdings seit einigen Jahren die Möglichkeit, sich SDH dank einer speziellen App auf dem eigenen Smartphone anzeigen zu lassen (vgl. Starks o. J.). Außerdem wird seit jüngstem mit der Projektion von Untertiteln auf Datenbrillen experimentiert, sowohl im Kino (vgl. Starks Datenbrille o. J.), als auch im Theater (vgl. Panthea Smartglasses o. J.). Die Entwicklung einer markttauglichen Datenbrille steht bisher jedoch aus (vgl. Starks Datenbrille o. J.).

SDH können des Weiteren im Hinblick auf die Art ihrer Herstellung differenziert werden. Während SDH für Serien und Filme zum Zeitpunkt ihrer Ausstrahlung bereits vorproduziert sind, entstehen Untertitel für Nachrichtensendungen und live übertragene Shows erst während der Ausstrahlung mithilfe einer Speech-to-text-Software. Sie werden von Untertitler(inne)n live eingesprochen (vgl. Käber 2016: 300), eine Technik, die daher auch als Respeaking bezeichnet wird (Romero-Fresco 2011). Folglich erscheinen sie nicht synchron zum Sprecheinsatz der Figuren, sondern mit einigen Sekunden Verzögerung auf dem Bildschirm. Je nach Art der Sendung werden Live-Untertitel oder eine Mischform aus Live- und Semi-Live-Untertiteln ausgestrahlt (vgl. Lindner 2016: 312ff. und Kurch im vorliegenden Band). Live-Untertitel werden außer im Fernsehen auch in weiteren Kontexten eingesetzt: etwa bei Tagungen oder anderen öffentlichen Veranstaltungen. Untertitel, die für einen Vortrag begleitend an die (Lein-)Wand projiziert werden, sind das Produkt des sogenannten Schriftdolmetschens (s. Witzel im vorliegenden Band), einer mit der LiveUntertitelung im Fernsehen verwandten Form - auch wenn es sich bei den Kontexten um Live-Situationen handelt, in denen Redner(innen) und Rezipient(inn)en ko-präsent sind. Die Schriftdolmetscher(innen) selbst können hingegen auch online hinzugeschaltet sein. Eine andere Variante von Live- oder Semi-Live-Formen von Untertiteln kann man vereinzelt im Theater und in der Oper finden, wo sie üblicherweise als Übertitel bezeichnet werden. Intralinguale Formen gibt es häufiger in Großbritannien, in Deutschland bilden sie bisher die Ausnahme. Hier werden meist fremdsprachige Inszenierungen interlingual übertitelt. Übertitel am Theater, die sich sowohl an gehörlose als auch an 
schwerhörige und hörende Menschen richten, werden seit 2015 im Rahmen inklusiver Inszenierungen an der Universität Hildesheim in Kooperation mit verschiedenen Theaterhäusern entwickelt (vgl. Mälzer/Wünsche 2018, Universität Hildesheim o. J. und Mälzer/Wünsche in diesem Band).

Der mediale Kontext und die spezifische Gestaltung des Mediums prägen die hier erwähnten Erscheinungsformen von SDH wesentlich. So wurde bereits deutlich, dass die Gestalt der SDH variiert, je nachdem, ob sie live-, semi-live oder non-live produziert werden und ob die übersetzten Äußerungen in einen medial vermittelten oder einen Live-Kontext eingebettet sind. Doch damit ist die Spezifik von Untertiteln für Hörgeschädigte noch nicht hinreichend erfasst. Wie unterscheidet man also SDH von Untertiteln, die in fremdsprachigen Fassungen von Filmen im Kino oder auf DVD vorkommen?

\section{$4 \quad$ SDH als Translation}

SDH werden in der Regel als intralinguale Untertitel bezeichnet (Jüngst 2010: 123), um sie von interlingualen Formen abzugrenzen. Bei dieser Definition liegt der Fokus darauf, dass es sich um Untertitel handelt, die in derselben Sprache verfasst sind wie die Tonspur des Films oder der Sendung. Gottlieb nimmt darüber hinaus den mit jeder Untertitelung einhergehenden Kanalwechsel von auditiv wahrnehmbaren zu visuell wahrnehmbaren Äußerungen in den Blick, wenn er zwischen "diagonaler" (bei interlingualen Untertiteln) und „vertikaler“ Übersetzung (bei intralingualen Untertiteln) unterscheidet (Gottlieb 1994: 104). Unter Bezugnahme auf Jakobsons Unterscheidung zwischen drei Formen des Übersetzens (1959: 233) spricht er bei Untertiteln von intrasemiotischer, jedoch nicht von intersemiotischer Übersetzung, die Jakobson als „interpretation of verbal signs by means of signs of nonverbal sign systems“ (ebd.) bezeichnet. Damit ergänzt Gottlieb Jakobsons Formen der Übersetzung um eine intrasemiotische Variante. Er bezeichnet dabei das phonetische und das graphische System als zwei Subsysteme einer Sprache. Semiotisch betrachtet ist es allerdings nicht einleuchtend, den verbal-schriftlichen Code und den verbal-mündlichen Code als Subsysteme zu betrachten - zumindest stellt sich die Frage, auf welches übergeordnete System eigentlich rekurriert wird. Überdies werden dieser Logik 
folgend die Gebärdensprachen übersehen, die ebenfalls verbale Zeichensysteme bilden und, wie die Schriftsprache, visuell wahrgenommen werden. Aus streng semiotischer Sicht erscheint es daher sinnvoller, auf die Unterschiedlichkeit der Zeichensysteme hinzuweisen und demnach auch das Untertiteln als intersemiotische Übersetzung zu bezeichnen, ganz gleich ob es sich bei den Zeichensystemen um zwei verbale Systeme handelt oder nicht. Entscheidend ist, dass die Zeichenrepertoires wechseln. Bei SDH werden auditiv wahrnehmbare phonetische Zeichen in visuell wahrnehmbare graphische Schriftzeichen übersetzt: Der Code ändert sich - auch wenn es sich um verbale Codes handelt.

SDH übersetzen aber mehr Informationen als nur sprachliche Zeichen. Da gehörlose und schwerhörige Menschen die Tonspur eines Films nicht oder nur eingeschränkt bzw. modifiziert hören können, fehlen ihnen neben den auditiv vermittelten verbalen, auch die nonverbalen und paraverbalen bzw. prosodischen Informationen. Geräusche, Musik, Soundeffekte, nonverbale Laute aber auch prosodische Merkmale, die die Worte einer Person begleiten und bedeutungstragend sein können, werden ebenfalls über die Tonspur vermittelt. Sie alle prägen das Verständnis eines Films mit. Entsprechend gilt es bei der Erstellung von SDH nicht nur verbale mündliche Zeichen in verbale schriftliche Zeichen zu übertragen, sondern es werden eine Reihe von non-verbalen Zeichen in verbale Zeichen übersetzt, was nach Jakobson dem intersemiotischen Übersetzen in umgekehrter Richtung entspräche. SDH ließe sich also als eine Mischform aus intralingualem und intersemiotischem Übersetzen bezeichnen, auch wenn der Wechsel der Zeichensysteme auf den ersten Blick weniger deutlich hervorsticht als bei Hörfilmen (s. den Beitrag von Benecke im vorliegenden Band), in denen Bildzeichen durch verbale Zeichen ersetzt werden.

\section{5}

\section{Zentrale Aspekte von SDH}

Für die non-live Formen von SDH, die in diesem Artikel im Fokus stehen, haben die Fernsehanstalten im deutschsprachigen Raum gemeinsam mit Zielgruppenverbänden in den vergangenen Jahren Richtlinien entwickelt (vgl. ARD/ORF/SRF/ZDF 2015). Dabei handelt es sich um Grundsätze, die als „Basis 
der redaktionellen Arbeit" (ebd. 1) dienen sollen. Von einer einheitlichen Vorgehensweise aller deutschsprachigen Sender kann daher nicht gesprochen werden, da gestalterische und redaktionelle Eigenschaften von den jeweiligen Sendern individuell festgelegt werden. In anderen Ländern gelten überdies andere Konventionen (vgl. u. a. Muller 2012 für Frankreich, Zárate 2014 für Großbritannien).

Eine Einigung auf gemeinsame Standards würde dem Publikum die Rezeption sicherlich erleichtern, weil es sich beim Wechsel des Fernsehkanals nicht jedes Mal auf andere Konventionen einlassen müsste. Da die Umsetzung der Richtlinien jedoch auch von der jeweils verwendeten Technik abhängt, ist eine Vereinheitlichung der Standards zumindest auf europäischer Ebene unwahrscheinlich.

Relevante Vorgaben für die Untertitelredaktionen der Fernsehsender sind u. a. die Zeichenzahl pro Sekunde, die Platzierung der Untertitel im Bild, die Schriftart, -größe, -farbe sowie der Hintergrund oder die Umrandung der Untertitel. Diese formalen Parameter können in den Einstellungen verschiedener Untertitelungssoftwares (z. B. FAB, WinCAPS, EZTitles) weitestgehend festgelegt werden, sodass die Untertitler(innen) bei der Einhaltung der teilweise konfligierenden Richtlinien automatisierte Unterstützung erhalten. Ihnen obliegt es, innerhalb der manchmal sehr engen Spielräume regelgeleitete Entscheidungen zu treffen.

Die Geschwindigkeit, mit der Untertitel gezeigt werden, sollte den Richtlinien zufolge die Zahl von 15 Zeichen pro Sekunde nicht überschreiten (vgl. ARD/ORF/SRF/ZDF 2015: 2). Dieser Mittelwert wird bei Sendungen und Filmen für Erwachsene in der Regel eingehalten, obwohl Abweichungen von insgesamt bis zu 20\% durchaus zulässig sind. Das heißt, dass einzelne Untertitel auch mit 18 oder auch nur 12 Zeichen pro Sekunde angezeigt werden können. Auch die Zeichenzahl in einer Zeile und die Zeilenzahl sowie deren Form sind festgelegt. Derzeit wird meist von maximal 37 Zeichen pro Zeile auf zwei Zeilen ausgegangen, die häufig zentriert und vorzugsweise in Pyramidenform angeordnet oder unter den jeweils sprechenden Figuren platziert werden (vgl. ebd. 1f.). Auch für das Setzen sinnvoller Zeilenumbrüche gibt es Regeln, an denen sich Untertitler(innen) orientieren können (vgl. u. a. Ivarsson/Carroll 1998, Jüngst 2010, Perego 2008). Ob dabei die Syntax, die Wahrung von Sinneinheiten 
oder der Sprechrhythmus in den Vordergrund gestellt wird, ist szenenabhängig. Die Wahl der Schriftgröße, -art und -farbe basiert teilweise auf Untersuchungen zur Leserlichkeit von Schriftzeichen (De Linde/Kay 1998: 15 und Ivarsson/Carroll 1998: 39-48). So werden häufig serifenlose Schriftarten wie Tahoma oder Arial verwendet. Farben dienen in der derzeitigen Praxis der Kennzeichnung der Figuren und werden begrenzt eingesetzt. Die am häufigsten verwendeten Farben sind Weiß, Gelb, Grün und Cyan. Auf Magenta soll Baker et al. (1984) und auch den Standards zufolge weitgehend verzichtet werden (vgl. ARD/ORF/SRF/ZDF 2015: 2 und KiKA 2016: 30), dieser Empfehlung wird in der Praxis jedoch nicht immer gefolgt.

Zur Frage der Lesegeschwindigkeit und guten Wahrnehmbarkeit von Untertiteln gehören auch Fragen der Mindest- und Maximalstandzeit, sowie Regeln zum Mindestabstand zwischen zwei Untertiteln. Letztere wurden aus verschiedenen Beobachtungen abgeleitet: Neue Untertitel werden erst dann als neue Information wahrgenommen, wenn der vorhergehende Untertitel lange genug ausgeblendet war. Dennoch weichen die Angaben ab: Es werden mitunter ein bis vier Filmbilder als idealer Mindestabstand zwischen zwei Untertiteln empfohlen (vgl. ARD/ORF/SRF/ZDF 2015: 2, KiKA 2016: 6, Ivarsson/Carroll 1998: 76). Außerdem ist zu beachten, dass Untertitel vor und nach einem Filmschnitt lange genug (zwölf Bilder bis eine Sekunde, vgl. KiKA 2016: 6) eingeblendet sein müssen, da sie sonst als Flimmern wahrgenommen werden. Dies kann auch bei zu geringer Standzeit der Fall sein, weshalb 25 bis 37 Bilder als Mindeststandzeit empfohlen werden (vgl. KiKA 2016: 6 und Hezel 2009: 195). Die Maximalstandzeit sollte nicht länger als 6-8 Sekunden betragen (vgl. Ivarrson/Carroll 1998: 64 und KiKA 2016: 6), da der betreffende Untertitel sonst erneut gelesen wird. Diese Regel basiert auf einer von D'Ydewalle et al. (1987) unternommenen Studie.

Weitere Regeln beziehen sich auf jene Untertitel, die nonverbale oder paraverbale Informationen der Audiospur vermitteln. Für die Kennzeichnung von Geräuschen und Musik gibt es unterschiedliche Konventionen, Uneinigkeit besteht auch darin, wie und ob diese Informationen verbalisiert werden sollen. Es besteht die Wahl zwischen der Formulierung eines kurzen Satzes („sie hustet“), einer Ellipse („lacht“), einem Infinitiv oder einem substantivierten Verb („Klopfen"). Bei Kindersendungen und Animationsfilmen stößt man auch auf den 
Einsatz von Onomatopöien („Hihi“). Grundsätzlich gilt, dass nur unverzichtbare Informationen untertitelt werden sollen (vgl. KiKA 2016: 16 und ARD/ORF/SRF/ZDF 2015: 3) und den Rezipient(inn)en zugetraut wird, von einer im Bild sichtbaren Geräuschquelle auf ein Geräusch zu schließen. Musik wird häufig durch eine Note gekennzeichnet, wobei darauf entweder eine Beschreibung der Musik oder eine Wiedergabe des Textes folgen kann (mit oder ohne Übersetzung aus einer ggf. anderen Sprache). Musikuntertitel können Hinweise auf Interpret(inn)en oder Titel des Stücks enthalten, je nachdem, was im Kontext einer Szene relevant erscheint. Die Charakterisierung von atmosphärischer Filmmusik birgt die Gefahr, willkürlich und subjektiv zu wirken. Allerdings lässt sich ebenfalls diskutieren, wie informativ wenig differenzierte Beschreibungen wie etwa „spannende Musik“ oder gar nur „Musik“ sind. Untertitelredaktionen unterstützen ihre Mitarbeiter(innen) deswegen durch die Bereitstellung von Glossaren mit Standardbeschreibungen.

\section{Dilemmata}

\subsection{Vollständigkeit vs. Lesbarkeit}

Ein zentrales Dilemma beim Erstellen von Untertiteln und SDH stellt die Frage nach der Zeichenzahl pro Sekunde dar. Das Lesen einer Information dauert für gewöhnlich länger als dieselbe Information hörend zu perzipieren (wobei hiermit noch nichts zum Verstehen gesagt ist). Daraus ergibt sich, dass Untertitel, um in der vorgegebenen Zeit überhaupt gelesen werden zu können, die akustischen Informationen durch kürzere Paraphrasen oder durch Auslassungen komprimiert wiedergeben. Dem steht die Forderung der Zielgruppenverbände gegenüber, dass den Rezipient(inn)en keine Informationen vorenthalten werden und diese selbst entscheiden können sollen, welche Informationen ihnen relevant erscheinen (vgl. Romero-Fresco 2009: 111f.). Die praktische Umsetzung dieser Forderung erweist sich jedoch als ambivalent. Produziert man sogenannte verbatim subtitles, die jedes gesprochene Wort (sofern das überhaupt möglich ist) schriftlich wiedergeben, so werden die Untertitel deutlich umfangreicher und es ist unwahrscheinlich, dass sie in der Kürze der Zeit noch voll- 
ständig gelesen werden können. Fällt die Entscheidung hingegen auf sogenannte edited subtitles, bei denen bestimmte Informationen der Tonspur ausgelassen werden, geht dies unweigerlich mit einem Informationsverlust einher, der zwar kontrollierter ist, aber von Teilen der Zielgruppe als Bevormundung empfunden werden kann. Dieses ungelöste Dilemma hat Anlass zu mehreren Studien gegeben, die u. a. die Frage aufwerfen, ob sich die Lesegeschwindigkeit des durchschnittlichen Publikums in den vergangenen Jahren leicht erhöht hat (vgl. Iriarte 2017, Jensema 1998, Romero-Fresco 2009: 115). Das Dilemma verschärft sich noch bei der Zielgruppe Kinder (vgl. De Linde/Kay 1998, Mälzer/Wünsche in Vorbereitung, Zárate 2014). Die Frage nach einer verständnissichernden Lesegeschwindigkeit stellt sich bei dieser Gruppe noch deutlicher als bei Erwachsenen, da sich Kinder im Leselernprozess befinden.

In Abhängigkeit von der voreingestellten Lesegeschwindigkeit gilt es auch die anzuwendenden Kürzungsstrategien für Untertitel zu klären (vgl. Pedersen 2011: 75f.). Ideal könnte es erscheinen, knappe und dennoch treffende Paraphrasen für eine Äußerung zu formulieren. Der Informationsverlust wäre für die Rezipient(inn)en dann gering. Verfügen diese allerdings über ein Resthörvermögen, was bei der Zielgruppe überwiegend der Fall ist, wirkte die Abweichung zwischen dem Gehörten und dem Gesagten ablenkend und behinderte das Verstehen, selbst wenn die Lesezeit zunächst reduziert würde. In einer laufenden Studie von Mälzer/Wünsche (vgl. Universität Hildesheim - SDH4KIDS) deutet sich etwa an, dass einer sorgfältigen Wahl der auszulassenden Elemente gegenüber klugen Paraphrasen der Vorzug gegeben werden sollte.

\subsection{Kennzeichnung der Sprecher(innen)}

Der Aspekt der Kennzeichnung von Sprecher(inne)n ist für SDH ebenfalls zentral. Es gilt festzulegen, anhand welcher Zeichen die Rezipient(inn)en erkennen können, welcher Figur bzw. welchem Sprecher oder welcher Sprecherin der einzelne Untertitel oder die einzelne Untertitelzeile zugeordnet ist. Hierfür werden teilweise unterschiedliche Mittel eingesetzt. Untertitel können z. B. farbig gehalten werden. Problematisch daran ist, dass die Farbwahl Hierarchiekonventionen folgt, die für die Rezipient(inn)en arbiträr wirken, ja sogar störend sein können, wenn die Identität einzelner Figuren absichtlich im Ungewissen gelassen werden 
soll, wie etwa in Krimis (vgl. Mälzer 2015: 270ff.). Hinzu kommt, dass die Farbwahl falsche Assoziationen auslösen kann, wenn Magenta oder Cyan etwa als Gendermerkmal gedeutet werden. Sie kann außerdem mit der Ästhetik eines Films kollidieren, wenn ein Schwarzweißfilm mit bunten Untertiteln versehen wird. Nicht zuletzt nimmt die Leserlichkeit gegenüber schwarz auf weiß oder weiß auf schwarz bei farbigen Untertiteln zum Teil drastisch ab (vgl. z. B. für Magenta, Baker et al. 1984). Weiterhin werden Wechsel der Sprecher(innen) durch das Setzen von Dialogstrichen oder das Positionieren einzelner Untertitel unter den sprechenden Figuren gekennzeichnet. Letzteres wird etwa in den Richtlinien des Fernsehsenders KiKA (vgl. KiKA 2016: 7) beherzigt. Allerdings unterstützt die Positionierung des Untertitels weiter rechts oder links im Bild die Figurenzuordnung nur dann gut, wenn sich der Sprecher nicht allzu schnell durchs Bild bewegt und seine Position durch Filmschnitte nicht plötzlich verändert wird.

\section{$7 \quad$ Forschungsdesiderate}

Wenig Untersuchungen gibt es bisher zu der Frage, wie Hinweise auf Musik zu untertiteln sind (vgl. Neves 2010). Zwar gibt es in der Filmtheorie interessante Untersuchungen zum Zusammenwirken von Musik und Bild (vgl. Chion 1990), jedoch wenig Forschung dazu, wie und ob diese Wirkung verbalisiert werden könnte oder sollte. In der Praxis hängt diese Entscheidung von der filmwissenschaftlichen Vorbildung der Untertitler(innen) ab sowie davon, ob sie die $\mathrm{Zu}$ schauer(innen) mit einem absoluten Minimum an notwendigen Informationen versorgen wollen, um viel Raum für das Bild zu lassen, oder ob sie eine möglichst vollständige Vertextung des Mediums anstreben, was den oben erwähnten Grundsätzen der Rundfunkanstalten widerspräche.

Diskutiert wird derzeit auch der Einsatz von Emojis und Symbolen aller Art, um den geringen Raum, den Untertitel für Informationen bieten, effektiv und effizient zu nutzen. Die Verwendung mutmaßlich schnell perzipierbarer, informativer und Platz sparender Zeichen, wie Noten und Rauten, Avataren und Emojis (vgl. Civera/Orero 2010), ist vielversprechend, aber noch nicht genügend untersucht. Ihre potentiellen Funktionen sind vielfältig - als Figurenkennzeichnung, 
als Hinweis auf prosodische Merkmale, als Interpretationshilfe, als Hinweis auf Geräusche, Musik oder den Einsatz diverser technischer Dispositive für eine medial vermittelte Kommunikation. Es ist anzunehmen, dass die Akzeptanz von Untertiteln für Zielgruppen wie Kinder und Jugendliche durch den Einsatz verbessert werden kann. Ob die Verständlichkeit von Untertiteln durch sie unterstützt wird, lässt sich derzeit jedoch nicht sicher sagen.

Ein weiterer Aspekt, der bereits angesprochen wurde, ist die Unterstützung der Figurenzuordnung. So hat sich gezeigt, dass die Zielgruppe Kinder den Einsatz von Farben bei SDH zum Teil als störend empfinden (Mälzer/Wünsche in Vorbereitung). Es stellt sich daher die Frage nach alternativen Formen der Kennzeichnung. Untersuchungen, die mit unterschiedlichen Positionierungen, nicht nur rechts und links am unteren Bildrand, sondern auch oben und unten oder sogar unter Zuhilfenahme von Sprechblasen experimentieren, finden sich nur vereinzelt (vgl. Fox 2016). Ob alternativ zu diesen Möglichkeiten auch die erwähnte Verwendung von Emojis (vgl. Civera/Orero 2010) als Symbol für eine bestimmte Figur oder das Hinzufügen von Namen oder Informationen zu einer Figur in Klammern vor der eigentlichen Äußerung hilfreich sind oder aber das Lesen der Untertitel eher erschweren, müsste in empirischen Studien unter $\mathrm{Zu}$ hilfenahme von Eye-Tracking-Methoden erforscht werden.

Aktuelle Entwicklungen in der Branche hängen mit dem Auf- und Ausbau der Online-Mediatheken der Sender zusammen. Hier können, wie beim Digitalen Fernsehen, die Nutzer(innen) einige SDH-Parameter nach Bedarf selbst einstellen, wie die Schriftgröße, die Position und die Farbe. Nachrichtensendungen in der Mediathek des MDR können mit reduzierter Geschwindigkeit abgespielt werden. Dadurch eröffnet sich die Möglichkeit, ausführlichere Untertitel oder auch Untertitel mit Zusatzerklärungen zu produzieren.

Im Zusammenhang mit der Erforschung von Untertiteln im Hinblick auf den (Fremd-)Spracherwerb sind auch SDH zunehmend interessant. Studien wie Subtitles and Language Learning (Gambier et al. 2014) deuten an, dass nicht die Untertitelung eines fremdsprachigen Films in der Muttersprache der Rezipient(inn)en das Erlernen einer Fremdsprache am besten unterstützt, sondern die Nutzung von Untertiteln in derselben Sprache wie die der gewählten Tonspur, weil die Sprachenlerner(innen) von einen bimodalen Input der Fremdsprache profitieren (vgl. Gambier 2014: 161). 
Ein grundsätzliches Desiderat bleiben empirische Studien zur Rezeption, Verständlichkeit und Akzeptanz von Untertiteln. Diese werden zwar seit den 1980er Jahren u. a. in der Psychologie und Medienwissenschaft (vgl. Braverman/Hertzog 1980, Burnham et al. 2008, Jelinek/Jackson 2001) und vereinzelt auch in der Übersetzungswissenschaft (vgl. Romero-Fresco 2015) durchgeführt, fokussieren aber eher auf die Rezipient(inn)en und weniger auf die Gestaltung der Untertitelung selbst. Studien, v. a. für den deutschsprachigen Raum, mit genuin übersetzungswissenschaftlichem Ansatz, die sich empirischer Methoden bedienen und statistisch valide Ergebnisse liefern, stehen bisher weitestgehend aus. 


\section{Literaturverzeichnis}

ARD (2016): ARD Telemedien Bericht 2015/2016 und Leitlinien 2017/2018. URL: https://www.daserste.de/specials/ueber-uns/telemedien-bericht-2015-2016-ardleitlinien-2017-2018-100.pdf (letzter Zugriff: 27.02.2018).

ARD/ORF/SRF/ZDF (2015): „Untertitel-Standards von ARD, ORF, SRF, ZDF“. URL: http://www.daserste.de/service/kontakt-und-service/barrierefreiheit-im-ersten/ untertitel-standards/index.html (letzter Zugriff: 12.12.2017).

BaKer, R.G./Lambourne, A.D./Downton, A.C./King, A.W. (1984) (Hg.): Oracle Subtitling for the Deaf and Hard of Hearing. Department of Electronics and Information Engineering, University of Southampton.

Bogucki, Łukasz/Kredens, Krzysztof (2010) (Hg.): Perspectives on Audiovisual Translation. Frankfurt am Main u. a.: Peter Lang.

Bosse, INGO/HASEBRINK, UWE (2016): Mediennutzung von Menschen mit Behinderungen

- Forschungsbericht. URL: https://www.aktion-mensch.de/dam/jcr:8b186ca0-b0f146f8-acb1-a59f295b5bb4/aktion-mensch-studie-mediennutzung-langfassung-201703.pdf (letzter Zugriff: 23.02.2018).

Braverman, Barbara B./Hertzog, Melody (1980): „The Effects of Caption Rate and Language Level on Comprehension of a Captioned Video Presentation". In: American Annals of the Deaf. 125, 7, 943-948.

BRK (2008): „Die UN-Behindertenrechtskonvention. Übereinkommen über die Rechte von Menschen mit Behinderungen“. URL: https://www.behindertenbeauftragte.de/ SharedDocs/Publikationen/UN_Konvention_deutsch.pdf?_blob=publicationFile\&v=2 (letzter Zugriff: 26.02.2018).

Bruti, Silvia/DiGiovanni, Elena (2012) (Hg.): Audiovisual Translation across Europe. An Ever-changing Landscape. Bern u. a.: Peter Lang.

Burnham, Denis/Leigh, Greg/Noble, William/Jones, Caroline/Tyler, Michael/ Grebennikov, Leonid/VArLey, Alex (2008): „Parameters in Television Captioning for Deaf and Hard of Hearing People: Effects of Caption Rate versus Text Reduction on Comprehension". In: Journal of Deaf Studies and Deaf Education 13, 3, 391-404.

Chiaro, Delia/Heiss, Christine/Bucaria, Chiara (2008) (Hg.): Between Text and Image. Updating research in screen translation. Amsterdam/Philadelphia: John Benjamins

CHION, Michel (1990): L'audio-vision. Paris: Éditions Nathan.

Civera, Clara/Orero, Pilar (2010): „Introducing icons in subtitles for the deaf and hard of hearing: optimizing reception?"In: MATAMALA/ORERO (Hg.), 149-162.

De Linde, ZoÉ/KaY, NeIL (1998): The Semiotics of Subtitling. Manchester: St. Jerome.

D'Ydewalle, Géry/Rensbergen, Joris/Pollet, Johan VAn (1987): „Reading a message when the same message is available auditorily in another language: The case of subtitling“. In: O’REGAN/LÉVY-SCHOEN (Hg.), 313-321. 
FOX, WENDY (2016): „Integrated titles: An improved viewing experience?“ In: HANSENSCHIRRA/GRUCZA (Hg.), 6-30.

GAMBIER, Yves (2014): „Thirty years of research in subtitles and language learning. The knowns and unknowns“. In: GARZELLI/BALDO (Hg.), 145-168.

Gambier, Yves/Caimi, Annamaria/Mariotti, Cristina (2014): Subtitles and Language Learning Principles, strategies and practical experiences. Bern u. a.: Peter Lang.

Garzelli, Beatrice/BAldo, Michaela (2014) (Hg.): Subtitling and Intercultural Communication. Pisa: ETS.

GotTlieb, Henrik (1994): „Subtitling: Diagonal Translation“. In: Perspectives. Studies in Translatology 2, 1, 101-121.

HANSEN-Schirra, Silvia/Grucza, SAmbor (2016) (Hg.): Eyetracking and applied linguistics. Berlin: Language science press.

HeERdegen-Wessel, UsCHI (2017): „Barrierefreie Angebote des NDR. Erreichtes und zukünftige Aufgaben“. Vortrag auf der Tagung „Leichte Sprache in Politik und Medien: Die Situation in Deutschland und Finnland“, 09.11.17, Universität Hildesheim.

HENNIES, JOHANNES (2009): Lesekompetenz gehörloser und schwerhöriger SchülerInnen: Ein Beitrag zur empirischen Bildungsforschung in der Hörgeschädigtenpädagogik. Dissertation. Humboldt-Universität zu Berlin, Fachbereich Rehabilitationswissenschaften. URL: http://edoc.hu-berlin.de/dissertationen/hennies-johannes-2009-07-15/ PDF/hennies.pdf (letzter Zugriff: 12.12.2017).

Herman, Rosalind/Roy, Penny/Kyle, Fiona (2017): Reading and Dyslexia in Deaf Children. City, University of London. Nuffield Foundation.

HeZEL, Susanne (2009): „Untertitelung für Hörgeschädigte für das deutsche Fernsehen. Vorgehensweisen, Forderungen, Richtlinien“. In: NAGEL ET AL. (Hg.), 147-261.

IRIARTE, MARTA MIQUel (2017): The reception of subtitling for the deaf and hard of hearing: Viewers' hearing and communication profile \& subtitling speed of exposure. Dissertation. Universitat Autònoma de Barcelona, Departament de Traducció i d'Interpretació (letzter Zugriff: 26.02.2018).

Ivarsson, Jan/CARroll, Mary (1998): Subtitling. Simrishamn: TransEdit.

JAKOBSON, ROMAN (1959): „On Linguistic Aspects of Translation“. URL: http://complit.utoronto.ca/wp-content/uploads/COL1000H_Roman_Jakobson_ LinguisticAspects.pdf (letzter Zugriff: 22.02.2018).

Jankowska, Anna/Szarkowska, Agnieszka (2015) (Hg.): New Points of View on Audiovisual Translation and Accessibility. Oxford: Peter Lang.

Jelinek Lewis, Margaret S./Jackson, Dorothy W. (2001): „Television Literacy: Comprehension of Program Content using Closed Captions for the Deaf". In: Journal of Deaf Studies and Deaf Education, 6, 1, 43-53.

JENNEWEIn, NADJA (2016): Barrierefreiheit im Kontext audiovisueller Medien: Eine Untersuchung des Medienhandelns hörgeschädigter Kinder mit Schwerpunkt auf TV- und 
Telemedienangeboten. Masterarbeit. Universität Erfurt, Philosophische \& Erziehungswissenschaftliche Fakultät.

Jensema, Carl (1998): „Viewer Reaction to Different Television Captioning Speeds“. In: American Annals of the Deaf 143, 4, 318-324.

JÜNGST, HeIKE E. (2010): Audiovisuelles Übersetzen. Ein Lehr- und Arbeitsbuch. Tübingen: Narr.

KÄBER, MARCO (2016): „Die Geschichte der Liveuntertitelung - ein Praxisbericht.“ In: MÄLZER (Hg.), 295-305.

KIKA (2016): Styleguide für kindgerechte Untertitelung. Unveröffentlichtes Dokument des Senders.

KRAMER, EvElYN (2016): Barrierefreies Fernsehen für hörgeschädigte Kinder mit Fokus auf die Untertitelungspraxis. Masterarbeit. Universität Erfurt, Philosophische \& Erziehungswissenschaftliche Fakultät.

KRAMmer, KlaUdia (2001): Schriftsprachkompetenz gehörloser Erwachsener. Veröffentlichungen des Forschungszentrums für Gebärdensprache und Hörgeschädigtenkommunikation der Universität Klagenfurt. In: https://core.ac.uk/download/pdf/ 14515198.pdf (Letzter Zugriff: 20.02.18).

Lim, Syc/Simser, Judith (2005): „Auditory-Verbal Therapy for Children with Hearing Impairment“. In: Annals Academy of Medicine 34, 4, 307-312.

LINDNER, PETER (2016): „Vorproduktion - Semi Live - Live. Ein Arbeitstag in der Untertitelredaktion des NDR“. In: MÄLZER (Hg.), 307-316.

MÄlZER, NATHALIE/WÜNSCHE, MARIA (in Vorb.): „Comprehensibility, legibility and acceptance of TV subtitles for D/deaf and hard-of-hearing children: The pilot study of a two-year EFRD research project“. In: JÜNGST, HEIKE E./LINK, LISA/SCHUBERT, Klaus/Zehrer, Christiane (Hg.): Challenging Boundaries of Translation Studies. Berlin: Frank \& Timme.

MÄLZER, NATHALIE/WÜNSCHE, MARIA (2018): Inklusion am Theater. Übertitel zwischen Ästhetik und Translation. Bern u. a.: Peter Lang.

MÄlzer, NATHAlIE (2016) (Hg.): Barrierefreie Kommunikation - Perspektiven aus Theorie und Praxis. Berlin: Frank \& Timme.

MÄlZER, NATHALIE (2015): „It’s Crime Time. About the Use of Colour in SDH“. In: JANKOWSKa/SZARKOWSKa (Hg.), 267-278.

Marschark, Marc/Hauser, Peter C. (2011): How Deaf Children Learn. What parents and teachers need to know. New York: Oxford University Press.

Matamala, Anna/Orero, Pilar (2010) (Hg.): Listening to Subtitles. Bern u. a.: Peter Lang.

Muller, Tia (2012): „Subtitles for deaf and hard-of-hearing people on French television“. In: BRUTi/DiGiOVANNi (Hg), 257-273.

Nagel, Silke/Hezel, Susanne/Hinderer, Katharina/PiePer, Katrin (Hg.): Audiovisuelle Übersetzung. Filmuntertitelung in Deutschland, Portugal und Tschechien. Frankfurt am Main: Peter Lang. 
Neves, Joselia (2010): „Music to my eyes... Conveying Music for the Deaf and the Hard of Hearing“. In: Bogucki/Kredens (Hg.), 123-145.

O’Regan, John Kevin/Lévy-Schoen, Ariane (1987) (Hg.): Eye Movements: From Physiology to Cognition. Amsterdam/New York: Elsevier Science Publishers.

Panthea Smartglasses (o. J.): „Smartglasses. The Surtitles of Tomorrow“. URL: http://panthea.com/smartglasses.html (letzter Zugriff: 20.02.2018).

PEDERSEN, JAN (2011): Subtitling norms for television: an exploration focussing on extralinguistic cultural references. Amsterdam: John Benjamins.

PELTZER-KARPF, ANNEMARIE (1994): Spracherwerb bei hörenden, sehenden, hörgeschädigten, gehörlosen und blinden Kindern. Tübingen: Narr.

Perego, Elisa (2008): „Subtitles and line-breaks. Towards improved readability“. In: ChiARO ET AL. (Hg.), 211-223.

RAUSCH, CHRISTFried (2011): Hörbehinderung und Teilhabe. Hörschädigung: Einfluss auf Biografie und Potential für den Bildungsprozess. Dissertation. Martin-Luther-Universität Halle-Wittenberg, Philosophische Fakultät III - Erziehungswissenschaften. URL: http://bit.ly/2mF9tgF (letzter Zugriff: 26.02.2018).

Romero-Fresco, Pablo (2015): The reception of Subtitles for the Deaf and Hard of Hearing in Europe. Bern u. a.: Peter Lang.

Romero-Fresco, PABLO (2011): Subtitling through speech recognition: Respeaking. Manchester: St. Jerome Publishing.

Romero-Fresco, Pablo (2009): „More haste less speed: edited versus verbatim respoken subtitles“. In: Vial 6, 109-133.

SAß, ANKE CHRISTINE (2002): „Dekodierung emotionaler Sprechweisen durch Patienten mit Cochlear Implant“. In: Zeitschrift für Angewandte Linguistik 36, 81-91. URL: https://userpages.uni-koblenz.de/ diekmann/zfal/zfalarchiv/zfal36_4.pdf (letzter Zugriff: 22.02.2018).

STARKS (o. J.): „Barrierefreies Kino mit Untertiteln“. URL: http://www.gretaundstarks.de/starks/starks (letzter Zugriff: 20.02.2018).

StARKS DATENBRILle (o. J.): „Datenbrille Starks AR Headset ${ }^{\circledast * . ~ U R L: ~}$ https://www. gretaundstarks.de/starks/datenbrille (letzter Zugriff: 20.02.2018).

Ugarte Chacón, RAfael (2015): Theater und Taubheit. Ästhetiken des Zugangs in der Inszenierungskunst. Bielefeld: transcript.

UNIVERSITÄT HILDESHEIM (o. J.): „Inklusives Theater“. URL: https://www.uni-hildesheim.de/ fb3/institute/institut-fuer-uebersetzungswiss-fachkommunikation/forschung/ forschungsprojekte/inklusives-theater/ (letzter Zugriff: 22.02.2018).

UNIVERSITÄT HILDESHEIM - SDH4KIDS (o. J.): „EFRE-Projekt: TV-Gehörlosenuntertitel für Kindersendungen“. URL: https://www.uni-hildesheim.de/sdh4kids/ (letzter Zugriff: 26.02.2018).

ZÁrATE, SOLEDAD (2014): Subtitling for deaf children. Granting accessibility to audiovisual programmes in an educational way. Dissertation. University College London, Centre for Translation Studies. 
KRISTIAN FOLTA-SCHOOFS

\section{Apparategestützte Kommunikations- und Feedbacksysteme}

Der Mensch ist als soziales Lebewesen auf persönliche Beziehungen sowie die Kommunikation mit Mitmenschen und verlässlichen Bindungspartnern angewiesen, um die eigenen Fähigkeiten und Fertigkeiten entwicklungsgerecht entfalten zu können (Fröhlich/Haupt 1987; Mall 1995; Renner 2004). Neben Leistungen der Wahrnehmung, Aufmerksamkeit, des Lernens, Gedächtnisses, Denkens und Problemlösens entscheiden die Fähigkeiten zu sprachlicher und nichtsprachlicher Verständigung und Kommunikation in besonderer Weise über das Ausmaß einer aktiven und selbstbestimmten Lebensgestaltung sowie darüber, in welchem Ausmaß und in welcher Weise es Menschen gelingt, am gesellschaftlichen Leben (in der Öffentlichkeit, im Berufsleben, im Freundeskreis oder der Familie) teilzuhaben und soziale Anerkennung erfahren zu können.

Vor allem Menschen mit besonderem Assistenzbedarf, die eine angeborene oder erworbene und langfristige Mehrfachschädigung in sensorischen, kognitiven und/oder motorischen Funktionsbereichen aufweisen, sind aufgrund ihrer körperlichen und/oder geistigen Einschränkungen in der Regel nicht oder nicht ausreichend in der Lage, sich ohne fremde Hilfe und Hilfsmittel zu verständigen sowie einfache Kommunikationssituationen des Alltags eigenständig zu meistern (von Tetzchner/Martinsen 2000: 10). Neben Störungen der Aufmerksamkeit, der Wahrnehmung und der feinmotorischen Steuerung können bei den Betroffenen zusätzliche Antriebs-, Gedächtnis-, Denk- sowie Erlebens- und Verhaltensstörungen die Ausdrucksfähigkeit und Kommunikation in sozialen Interaktionen erschweren. Die Unfähigkeit oder verminderte Fähigkeit zu sozialer Verständigung und Kommunikation begünstigt Phänomene der sozialen Ausgrenzung, Vereinsamung und Verwahrlosung. Dies steht dem von der Generalversammlung der Vereinten Nationen (UN) im Dezember 2006 vereinbar- 
ten „Übereinkommen über die Rechte von Menschen mit Behinderungen (vereinfacht auch „UN-Behindertenrechtskonvention“, UN-BRK, genannt) und dem darin formulierten Anspruch auf volle und gleichberechtigte gesellschaftliche Teilhabe von Menschen mit Behinderung entgegen (vgl. Bundesgesetzblatt 2008, s. auch den Beitrag von Lang im vorliegenden Band). Tritt der Verlust von kommunikativen Ausdrucksmöglichkeiten zudem bereits in der frühen Kindheit der Betroffenen auf, so führen der Mangel an sozialen Interaktionsgelegenheiten sowie verminderte Handlungs-, Lern- und Entfaltungsmöglichkeiten zu einer erhöhten Vulnerabilität für die Entstehung von kindlichen Lern- und Entwicklungsstörungen sowie kognitiven und sozio-emotionalen Beeinträchtigungen, welche die Teilhabe am gesellschaftlichen Leben zusätzlich erschweren.

Um die Einbeziehung der Betroffenen in die Gemeinschaft gemäß den Zielen der UN-BRK und dem Rahmenkonzept der Inklusion (Ainscow et al. 2006; Hinz 2009; Amrhein 2011; Sturm 2013, Folta-Schoofs et al. 2017: 18ff.; FoltaSchoofs 2018) zu ermöglichen oder zu verbessern, sind insbesondere Menschen mit Mehrfachbeeinträchtigung auf Bezugs- bzw. Assistenzpersonen angewiesen, die ihnen bei der Erschließung ihrer personalen und gegenständlichen Umwelt behilflich sind. Darüber hinaus ist ein ökonomischer, praktikabler und an die jeweiligen Wünsche und konkreten Bedürfnisse der Betroffenen angepasster Einsatz von Methoden der unterstützten Kommunikation sinnvoll und notwendig. Hierunter fallen alle Maßnahmen, die geeignet sind, um die Ausdrucks-, Kommunikations-, Sprach- und Sprechfähigkeit von Menschen mit Behinderung sowie die Interaktion mit ihrer gegenständlichen und sozialen Umwelt zu ermöglichen oder zu verbessern (vgl. Kristen 1994: 15; von Tetzchner/Martinsen 2000: 48, s. auch den Beitrag von Musenberg im vorliegenden Band). Gemäß den Ausführungen in Artikel 2 („Begriffsbestimmungen“) der UN-BRK

schließt ,Kommunikation' Sprachen, Textdarstellung, Brailleschrift, taktile Kommunikation, Großdruck, leicht zugängliches Multimedia sowie schriftliche, auditive, in einfache Sprache übersetzte, durch Vorleser zugänglich gemachte sowie ergänzende und alternative Formen, Mittel und Formate der Kommunikation, einschließlich leicht zugänglicher Informations- und Kommunikationstechnologie, ein (Bundesgesetzblatt 2008). 
In diesem Sinne erkennt die UN-BRK die Notwendigkeit und Verwendung von unterschiedlichsten Formen der unterstützten Kommunikation und der Leichten Sprache (Bredel/Maaß 2016; s. auch die Beiträge von Bredel/Maaß und Maaß im vorliegenden Band) als Anspruch für Menschen mit Behinderung explizit an.

Der Gebrauch von ergänzenden und alternativen Ausdrucks- und Kommunikationsmitteln unterstützt ganz wesentlich die professionelle Beziehungsgestaltung und das Miteinander von Menschen mit Assistenzbedarf mit ihren Bezugs- bzw. Assistenzpersonen, und zwar auf zweifache Weise:

1. Diese Hilfsmittel erhöhen auf der Seite der Betroffenen den Kommunikationsanteil und tragen zu einer substanziellen Verbesserung der semantischen Klarheit und Verständlichkeit von kommunikativen Botschaften bei.

2. Auf der Seite der Bezugs- bzw. Assistenzpersonen führen sie zu eindeutigeren Rückmeldungen, ob kommunikative Botschaften vom Gegenüber richtig wahrgenommen oder verstanden wurden.

Durch den Einsatz von Methoden der unterstützten Kommunikation kann folglich das soziale Miteinander optimiert werden. Dies spielt insbesondere in Bereichen des betreuten Wohnens, beispielsweise im Kontext der gemeinsamen Gestaltung und Abstimmung von Tages- und Freizeitaktivitäten, aber auch für das Arbeitsleben von Menschen mit besonderem Assistenzbedarf eine Rolle. In diesem letzteren Bereich kommt dem korrekten Verständnis von Arbeitsanweisungen, der Einhaltung von Arbeitsabläufen sowie der sicheren Bedienung von Apparaten und Maschinen eine besonders große Bedeutung zu (Aßmann 2014: 58).

Zu Maßnahmen unterstützter Kommunikation zählen neben Dolmetscherleistungen (z. B. dem Einsatz von Schrift-, Simultan- und/oder Oraldolmetscher(inne)n) insbesondere auch 
1. die Verwendung von körpereigenen Ausdrucksformen, z. B. Mimik, Gestik, Veränderungen der Körperhaltung und von körperphysiologischen Parametern (Atmung, Pulsrate), Finger- und Handzeichen, Zeigebewegungen, Embleme, Lautäußerungen, Blinzelbewegungen der Augen, lautsprachbegleitende Gebärden, taktile Gebärden oder Lormen,

2. der Gebrauch von nicht-technischen Ausdrucks- und Kommunikationshilfen, z. B. Zeichnungen, Fotos, Buchstaben-, Bild- und Worttafeln, komplexe Symbolsysteme, Gebärdensprache oder Brailleschrift,

3. der Gebrauch von einfachen technischen Kommunikationsmitteln, z. B. akustische Signalgeber, Taster oder Talker und

4. der Einsatz von apparategestützten (d. h. elektronischen bzw. computergestützten) Ausdrucks- und Kommunikationshilfen, z. B. Talker mit Sprachausgabe, internetfähige PCs, Laptops, Tablets oder Smartphones mit Kommunikations- und Schreibsoftware, mobile Eye-Tracking- und Elektroencephalographie- bzw. NeurofeedbackSysteme (vgl. Aßmann 2014: 68ff.).

Die Verständigung über körpereigene Ausdrucksmittel stellt eine sehr kostengünstige Methode der unterstützten Kommunikation dar, die für die Betroffenen in der Regel aber nur mit sehr vertrauten Bezugs- bzw. Assistenzpersonen und erst nach einer längeren Zeit des gemeinsamen Miteinanders möglich ist (Aßmann 2014: 67). Zudem gehen Störungen in der Analyse der Lautstruktur und dem Verstehen der Wortbedeutung sowie der Planung, Kontrolle und Ausführung des Sprechaktes oder des stimmsprachlichen motorischen Ausdrucks häufig mit zusätzlichen Problemen der motorischen Steuerung und Kontrolle von nichtsprachlichen Kommunikationsformen einher. Betroffene können daher in vielen Fällen lautsprachliche Defizite nicht oder nicht ausreichend durch die Schriftsprache oder durch klassische nichtsprachliche Formen des Aus- 
drucks und der Kommunikation kompensieren, was den Nutzen von körpereigenen und auch von nicht-technischen Ausdrucks- und Kommunikationshilfen stark einschränkt.

Auch der Gebrauch von nicht-technischen Hilfsmitteln erfordert von allen beteiligten Interaktionspartnern ausreichend Übung und ein hohes Ausmaß an Konzentration und kognitiver Anstrengung, so dass die Verwendung von solchen Möglichkeiten der unterstützten Kommunikation in der Regel nur auf vergleichsweise wenige und kurze Interaktionssequenzen beschränkt bleibt. Außerdem setzt der erfolgreiche Gebrauch von nicht-technischen und auch von einfachen elektronischen Hilfsmitteln unterstützter Kommunikation voraus, dass die Betroffenen über intakte Fähigkeiten der Wahrnehmung, Motorik und Verhaltenskontrolle sowie ein ausreichend entwickeltes bzw. intaktes Symbolund Sprachverständnis verfügen.

Weitere wesentliche Nachteile von nicht-technischen und vielen elektronischen Hilfsmitteln bestehen darin, dass diese Methoden für Bezugs- bzw. Assistenzpersonen nicht oder nur unzureichend erkennen lassen, ob die Interaktionspartner fähig waren, den Inhalten einer Kommunikation aufmerksam zu folgen oder Anweisungen korrekt zu verstehen. Eine präzise Rückmeldung zum Ermüdungs- und Aufmerksamkeitsgrad sowie der Tiefe der Informationsverarbeitung ist bedeutsam, wenn man Menschen mit Behinderung bestmöglich in die Lage versetzen möchte, Anweisungen selbstständig in zielgerichtete Handlungen zu übersetzen. Bezugs- bzw. Assistenzpersonen müssen erkennen können, ob Anweisungen klar genug gestellt wurden. Zudem kann es nötig sein, das Verständnis von Handlungsanleitungen unmittelbar zu überprüfen. In solchen Situationen erweisen sich neuere apparategestützte Ausdrucks- und Kommunikationshilfen, deren Ansteuerung über Augen- und Blickbewegungen (das sogenannte „EyeTracking“) oder eine willkürlich induzierte Veränderung von elektrischen Erregungsmustern im Gehirn der Betroffenen (das sogenannte „Neurofeedback“) erfolgt, als geeignete Hilfsmittel. Solche Methoden ermöglichen:

- eine apparatebasierte Substitution von sprachersetzenden Gesten,

- eine zielgerichtete und bewusste Einwirkung auf die Umwelt (z. B. die Auswahl von Buchstaben, Wörtern, Zeichen oder Bildern), 
- die Rückmeldung (das sogenannte „Biofeedback“) über den physiologischen Erregungszustand (z. B. die Wachheit oder den Grad der kognitiven Anstrengung) der Interaktionspartner sowie über die Qualität ihrer kognitiven und affektiven Informationsverarbeitungsprozesse (z. B. hinsichtlich der Stärke der Aufmerksamkeit, der geistigen Verarbeitungstiefe von Informationen oder der emotionalen Involviertheit).

Zu den apparategestützten Ausdrucks- und Kommunikationshilfen, die gleichzeitig auch als Biofeedback-Systeme fungieren können, zählen mobile Augenund Blickbewegungssysteme (engl.: Eye-tracker) und Elektroencephalographie (EEG)-Systeme. Diese Methoden kamen lange Zeit vorwiegend in der biopsychologischen, neuropsychologischen, medizinischen und neurowissenschaftlichen Forschung und Diagnostik zum Einsatz. Inzwischen wurden sie erfolgreich zu mobilen apparategestützten Kommunikations- und Feedbackinstrumenten in Bereichen von Pflege bzw. Sorge weiterentwickelt. Aufgrund ihrer einfachen Handhabung werden sie zunehmend häufiger in Praxisfeldern der Eingliederungshilfe eingesetzt, um die soziale Teilhabe von Menschen mit besonderem Assistenzbedarf (insbesondere von Menschen mit Mehrfachbeeinträchtigungen) und die Interaktion mit ihren Bezugs- bzw. Assistenzpersonen in Wohn-, Förder- und Arbeitsbereichen zu verbessern.

Heute erlauben bereits handelsübliche Smartphones oder Mini-Tablets und die in diesen Geräten verbauten hochauflösenden Kameras eine einfache videobasierte Registrierung von Augen- und Blickbewegungen (Albrecht et al. 2013). Um zusätzliche Verbesserungen der zeitlichen und räumlichen Auflösung zu erzielen, werden in die handelsüblichen Kommunikationsgeräte oder in einfache Brillengestelle (sogenannte „Eye-Tracking-Brillen“) für Zwecke der unterstützten Kommunikation hochauflösende Frontkameras und Infrarot-LEDs verbaut. Diese Kameras belichten das Auge mit Infrarotlicht und ermöglichen dadurch eine sehr genaue Video-Detektion der dunklen Pupille, deren Position und Durchmesser mit einer Datenregistrierrate zwischen 80 und $300 \mathrm{~Hz}$ (bei mobilen Systemen) erfasst wird („Dark Pupil-Tracking“; Richardson/Spivey 2004). Dabei können Daten zur Position und Ausdehnung der Pupille als Maß 
für Aufmerksamkeits- und vegetative Erregungsprozesse registriert werden. $\mathrm{Zu}$ sätzlich wird häufig auch die Position der Cornealreflexion erfasst, die im Kamerabild des Eye-Trackers als kleiner heller Punkt im Bereich der Iris des Auges erscheint. Mit Hilfe der Daten zur Cornealreflexion können die Bewegungen des Kopfes, die als Bewegungsartefakte die Registrierung von Augen- und Blickbewegungen stören, ausgeglichen und kontrolliert werden (Duchowski 2003). Eine alternative und kostengünstige Methode des mobilen Eye-Trackings stellt die Elektrookulographie (EOG) dar. Bei dieser Methode werden zwischen zwei und vier Registrierelektroden oberhalb und unterhalb sowie seitlich des Auges positioniert. So können die Veränderungen von elektrischen Spannungen erfasst werden, die durch die Aktivität der Augenmuskeln, die das Auge in Bewegung versetzen, entstehen (Rohner 2002).

Grundsätzlich lassen sich während eines Eye-Trackings sprunghafte und schnelle ballistische Veränderungen von Blickzielen, genannt Sakkaden, erfassen. Den Sakkaden folgen relativ stabile Ruhephasen des Auges, sogenannte Fixationen, nach (Huey 1900). Mit Hilfe von Sakkaden können Menschen den Fokus ihrer visuellen Verarbeitung von einem eng umgrenzten Areal des Gesichtsfeldes auf ein alternatives Blickziel verlegen, das auf diese Weise einer tiefergehenden Verarbeitung zugänglich wird. Gesunde Erwachsene produzieren durchschnittlich etwa drei bis fünf Sakkaden pro Sekunde. In Abhängigkeit von organismischen Bedingungen, kognitiven Verarbeitungsanforderungen und Eigenschaften der wahrgenommenen Reize unterscheiden sich diese Sakkaden hinsichtlich ihrer Anzahl, Latenz, Dauer, Amplitude und ihres Geschwindigkeitsprofils (z. B. Durchschnittsgeschwindigkeit und Spitzengeschwindigkeit) (Folta/Mähler 2011; Folta-Schoofs et al. 2015: 109f.). Hierbei entspricht die Latenz einer Sakkade dem Zeitintervall zwischen der Darbietung eines Blickziels und dem Beginn der motorischen Ausführung einer Sakkade. Sie liegt üblicherweise zwischen ca. 250 und 1000 ms und gilt als sensitiver kognitiver Indikator, da sie neuronale Prozesse der Bewegungs-, Entscheidungs- und Aufmerksamkeitssteuerung widerspiegelt (Groner/Groner 1989). Latenzen von über $600 \mathrm{~ms}$ lassen auf eine Reduktion der allgemeinen Wachheit (Vigilanz) oder auf Ermüdung schließen. Sakkadenlatenzen zwischen ca. 250 und 600 ms können dagegen als Hinweis für die Augenfälligkeit (Salienz) oder Verhaltensrelevanz (z. B. 
im Sinne einer assoziierten Belohnung) von Reizen gewertet werden. Dabei gehen geringere Sakkadenlatenzen üblicherweise mit einer höheren Salienz und Verhaltensrelevanz von Reizen bzw. Reizereignissen einher. Längere Sakkadenlatenzen lassen sich als Zeichen für Entscheidungskonflikte und Unsicherheiten oder emotionale Blockaden werten. Sakkadenlatenzen von unter $80 \mathrm{~ms}$ werden üblicherweise als antizipatorische Sakkaden klassifiziert und spiegeln die folgenden Zustände wider:

- Unaufmerksamkeit,

- Ungeduld,

- Impulsivität des Verhaltens (als Zeichen einer unzureichenden zentralen Verhaltenskontrolle),

- Ermüdung.

Die Amplitude der Sakkade steht für die räumliche Distanz zwischen zwei Fixationen bzw. zwischen dem Ausgangs- und Zielpunkt einer Sakkade. Sie wird üblicherweise in Grad Sehwinkel $\left(^{\circ}\right)$ gemessen, wobei die Einheit den Winkel bezeichnet, der sich aus dem Betrachtungsabstand und der Größe von physikalischen Reizen ergibt. Die Dauer einer Sakkade bezieht sich auf die Zeit, die das Auge benötigt, um von einem Punkt einer Fixation zu einem nächsten Fixationspunkt zu gelangen. Mit der Dauer der Sakkade steigt typischerweise auch die sakkadische Spitzengeschwindigkeit an. Diese definiert sich als maximale Geschwindigkeit während der Ausführung einer Sakkade und wird in Grad Sehwinkel pro Sekunde ( $\%$ ) registriert. Im Allgemeinen zeichnen sich Sakkaden durch eine geringe zeitliche Dauer (ca. 15 bis $120 \mathrm{~ms}$ ) sowie eine Spitzengeschwindigkeit von ca. 100 bis $600 \%$ s aus (Kömpf/Heide 1998; Leigh/Zee 1999). Die Spitzengeschwindigkeit einer Sakkade wird durch die mentale Beanspruchung und den attentionalen Status eines Menschen beeinflusst (App/Debus 1998; DiStasi et al. 2010; Galley 1998). Sie steigt an, sobald die mentale Beanspruchung der Person zunimmt (bzw. sinkt bei Ermüdung oder Unaufmerksamkeit). Khan et al. (2000) berichteten zudem über einen Anstieg der sakkadischen Spitzengeschwindigkeit, der mit einer Zunahme an allgemeiner kognitiver Erregung (der sogenannten Alertness) korrelierte. Informationen zu Prozessen der Wahrnehmung und Informationsverarbeitung lassen sich über 
die Charakteristik von Sakkaden, aber auch durch die Rückmeldung der Position, Anzahl und Dauer von Fixationen gewinnen. Somit kann die Rückmeldung von verschiedensten Augen- und Blickbewegungsparametern ein „Echtzeit"-Profil der Informationsverarbeitung eines Menschen bereitstellen. Dieses Profil unterstützt Bezugs- bzw. Assistenzpersonen, die mit der Interpretation der zurückgemeldeten Parameter vertraut sind, in ihrem Bemühen um eine korrekte Einschätzung der geistigen Aufnahmebereitschaft, mentalen Beanspruchung sowie des kognitiven und affektiven Zustands ihrer Interaktionspartner.

Kognitive und affektive Verarbeitungsprozesse im Gehirn von Menschen gehen mit charakteristischen elektrischen Aktivitätsmustern (Spannungsschwankungen) von Nervenzellen einher, die sich im unmittelbar unterhalb der Schädeldecke liegenden Hirngewebe verortet befinden. Entsprechend lassen sich solche Prozesse - häufig begleitend zum Eye-Tracking mittels Eye-Tracking-Brillen oder EOG-Elektroden - auch mit Hilfe eines EEG-Neurofeedback-Systems zurückmelden. Ein solches Feedback-System besteht typischerweise aus zwei bis vier Registrierelektroden (identisch zu EOG-Elektroden), die an standardisierten Stellen der Kopfhaut, häufig links und rechts der Kopfmitte, aufgebracht werden. Zudem wird eine Referenzelektrode benötigt, die sich möglichst nicht durch aufgabenbezogene Hirnaktivität beeinflusst zeigt. Sie kann beispielsweise am Ohrläppchen oder über dem Mastoidknochen, der sich hinter der Ohrmuschel befindet, aufgebracht werden. In bestimmten Bereichen der Großhirnrinde (relativ zur Referenzelektrode) kann mit Hilfe der EEG-Registrierelektroden die hirnelektrische Aktivität, deren Amplituden sich im Mikrovolt-Bereich bewegen und die mit mentalen Prozessen assoziiert sind, mit hoher zeitlicher Auflösung (typischerweise zwischen 250 bis $1000 \mathrm{~Hz}$ ) registriert werden. Dies gelingt selbst durch den Schädelknochen und die Kopfhaut hindurch. Die auf diese Weise erfassten elektrischen Spannungsverläufe stellen sinusoidale Amplituden- und Frequenzveränderungen dar, die die „Grundaktivierung" von aktiven Nervenzellen widerspiegeln. Sie lassen sich „online“ (d. h. parallel zur Datenaufnahme und in der Regel parallel zur Rückmeldung von Eye-Tracking- oder EOG-Parametern) verfolgen. Anhand ihres charakteristischen Schwingungsbzw. Frequenzbereichs können sie als Delta-, Theta-, Alpha-, Beta- und Gammawellen kategorisiert und mittels Fourier-Analyse einzeln aus dem kon- 
tinuierlich registrierten EEG (sogenanntes „Spontan-EEG“) herausgefiltert werden (Berger 1929). Die so ermittelten Frequenzanteile können, vergleichbar zu den während des Eye-Trackings erfassten Augen- und Blickbewegungsparametern, mit bestimmten psychischen Zuständen assoziiert werden, z. B. Annäherungs- oder Vermeidungsmotivation, kognitive Anstrengung, Wachheit oder Ermüdung. In Bereichen von Pflege bzw. Sorge wird insbesondere der Anteil an Alpha- und Betawellen als Neurofeedbacksignal verwendet. Dabei stehen Alpha- und Beta-Wellen in einem inversen Verhältnis zueinander: Ein höherer Anteil an Alpha-Wellen (8 bis $13 \mathrm{~Hz}$ ) gilt als Zeichen für Ermüdung und geht typischerweise mit einer Reduktion des Anteils an Beta-Wellen (13 bis $30 \mathrm{~Hz}$ ) einher. Ein Anstieg des Anteils von Beta-Wellen (bei reduziertem Anteil von Alpha-Wellen) tritt hingegen unter der Einwirkung von Sinnesreizen sowie bei mentaler, emotionaler oder körperlicher Aktivität (bzw. geistiger Beanspruchung) auf. Gammawellen ( $>30 \mathrm{~Hz}$ ) lassen sich bei besonders anspruchsvollen kognitiven Tätigkeiten beobachten, die eine sehr fokussierte (bzw. konzentrierte) Aufmerksamkeit erfordern.

Neben der Verwendung des EEGs als Neurofeedback-System kann diese Methode auch ganz unmittelbar zur Kommunikation eingesetzt werden, insbesondere wenn Menschen unfähig oder unzureichend in der Lage sind, ihre Muskulatur bewusst und zielgerichtet zu kontrollieren. Dies ist beispielsweise bei Muskelerkrankungen oder dem sogenannten Locked-In-Syndrom der Fall. In solchen Fällen erlaubt das EEG das Auslesen der vormotorischen neuronalen Programme der Bewegungsplanung, deren Aktivierung einer konkreten Bewegungsausführung (durch die Muskeln) unmittelbar vorausgeht. Anstatt die motorischen Befehle über Motorneurone an die Muskulatur zu senden, geben EEG-Systeme die ausgelesenen neuronalen Signale an Steuerinstrumente weiter, mit denen Computer oder einfache technische Geräte zur unterstützen Kommunikation (z. B. Talker mit Sprachausgabe oder elektronische Buchstabentafeln) in einfacher Weise mit Hilfe der eigenen „Gedanken“ bedient werden können. Damit das Auslesen der „Gedanken“ gelingt, müssen die Betroffenen zuvor ein mehrwöchiges Neurofeedbacktraining absolvieren. Danach lassen sich einfache Ja/Nein-Unterscheidungen und Cursorsteuerungen (z. B. zur Auswahl von Buchstaben, Wörtern, Zeichen oder Bildern) vornehmen oder Apparate und Schalter (z. B. Fernsehgerät, Lichtschalter, etc.) mit Hilfe der eigenen 
"Gedanken" bedienen. Voraussetzung hierfür ist, dass die Betroffenen über intakte Fähigkeiten der Wahrnehmung, Handlungsplanung und Handlungskontrolle sowie ein ausreichend entwickeltes bzw. intaktes Symbol- und Sprachverständnis verfügen.

Bei den Methoden der Elektroencephalographie und des Eye-Trackings mittels Smartphone, Eye-Tracking-Brille oder EOG-Elektroden handelt es sich um nicht-invasive Registriermethoden, die selbst bei Neugeborenen sowie Kleinstund Kleinkindern gesundheitlich unbedenklich angewendet werden können. Zudem weisen diese Methoden als apparategestütztes Kommunikations- und Feedback-System eine hohe Praktikabilität auf. Mobile Eye-Tracking-Systeme müssen zu Beginn der Registrierung lediglich kalibriert werden, damit das System „erkennt“, welche Positionen von Pupille und Cornealreflexion auf welche Raumkoordinaten bezogen sind. Zur Kalibration ist es erforderlich, nacheinander wenigstens fünf Punkte im Raum zu fixieren (jeweils in den Ecken und in der Mitte). Dies erfordert bei gut erhaltenem Instruktionsverständnis üblicherweise einen zeitlichen Aufwand von wenigen Minuten, bevor die Datenregistrierung (und damit die Rückmeldung von Augen- und Blickbewegungsparametern) beginnen kann. Ein Eye-Tracking mittels EOG ist in der Vorbereitung geringfügig aufwändiger: Hier müssen die Registrierelektroden aufgebracht und die elektrische Leitfähigkeit der Elektroden hergestellt werden. Auch hier ist zu Beginn der Datenerfassung eine Kalibration des Systems erforderlich. Vergleichbar zum Eye-Tracking beschränkt sich der für kontinuierliche EEG-Messungen notwendige Vorbereitungsaufwand ebenfalls auf nur wenige Minuten, da nur eine geringe Anzahl von (bis zu vier) Registrierelektroden auf der Kopfhaut aufgebracht und die elektrische Leitfähigkeit der Elektroden hergestellt werden muss. Dieser Zeitaufwand kann durch den Einsatz von anwendungsfreundlichen (aber in der Anschaffung teureren) Aktiv- oder Trockenelektroden weiter verringert werden.

Geeignete mobile Eye-Tracking-Systeme sind auf dem Markt bereits für unter 10.000 EUR (Stand Frühjahr 2018) erhältlich. Ein 5-Kanal-EEG-Neurofeedbacksystem, mit dem parallel ein EOG und die Messung der Hirnaktivität über zwei EEG-Registrierelektroden erfolgen kann, bewegt sich auf einem ähnlichen Preisniveau. Obwohl die Systeme in den letzten Jahren deutlich günstiger geworden sind, ergibt sich damit für viele Anbieter der Eingliederungshilfe und 
die Kostenträger immer noch eine nicht unerhebliche monetäre Investition. $\mathrm{Zu}$ dem erfordert die Implementierung der Technik (z. B. in der Lernförderung oder im Werkstattbereich von Institutionen der Eingliederungshilfe) in der Anfangsphase eine mehrwöchige Einarbeitungs- und Betreuungszeit sowie eine regelmäßige Beratungs- und Schulungstätigkeit. Dies stellt sicher, dass die Methoden von Bezugs- bzw. Assistenzpersonen korrekt angewendet und die zurückgemeldeten Parameter in ihrer Gesamtheit richtig gedeutet werden können. Auf der anderen Seite lässt sich durch den Einsatz von Biofeedback-Systemen auf der relationalen Ebene eine deutliche Verbesserung der Interaktion und Intensivierung der Beziehung zwischen Anwender(in) und Bezugs- bzw. Assistenzperson erreichen. In Instruktions- und Lernkontexten lassen sich die zurückgemeldeten Parameter gut nutzen, um die Form der Vermittlung von Lerninhalten an die jeweilig vorherrschenden organismischen und geistigen Bedingungen des Lernenden anzupassen. Auf diese Weise wird das Biofeedback-System auch zu einem didaktischen Werkzeug, mit dem sich Lerninhalte „hirngerecht“ vermitteln lassen. Manche Menschen mit schweren motorischen Funktionseinschränkungen sind bei erhaltenem Sprachverständnis und erhaltenen zentralnervösen Voraussetzungen zu motorischer Steuerung und Kontrolle von sich heraus nicht mehr zu sprachlichen und nichtsprachlichen Kommunikationsformen fähig. Sie können körpereigene und nicht-technische Ausdrucks- und Kommunikationshilfen nicht nutzen. Für diesen Personenkreis eröffnen EEG-basierte Kommunikations- und Feedbacksysteme den gegenwärtig einzig verbleibenden Weg der Herstellung von (wenngleich auch beschränkten) Möglichkeiten der sozialen Mitteilung und Teilhabe. 


\section{Literaturverzeichnis}

Ainscow, Mel/Booth, Tony/Dyson, Alan (2006): Improving schools, developing inclusion. London: Routledge.

Albrecht, Urs-Vito/Khosravianarab, Khatereh/Folta-Schoofs, Kristian/ Teske, JÖrg/KanngIeßer, JÜrgen/Von Jan, Ute (2013): Mobile smarttracking Finding objective parameters for determining fitness to drive. In: Biomedical Engineering/Biomedizinische Technik (Berl.) 58 (Suppl. 1).

AmRHEIn, BetTINA (2011): Inklusion in der Sekundarstufe - Eine empirische Analyse. Bad Heilbrunn: Klinkhardt.

App, Eberhard/Debus, GunTer (1998): Saccadic velocity and activation: development of a diagnostic tool for assessing energy regulation. In: Ergonomics 41, S. 689-697.

AßMANN, KATI (2014): Die Anwendung alternativer und ergänzender Kommunikationsformen und -hilfen bei nicht und kaum sprechenden, mehrfach beeinträchtigten Erwachsenen in Wohnheimen für behinderte Menschen in den neuen Bundesländern und dem ehemaligen Ostteil Berlins. Unveröffentlichte Dissertationsschrift. Martin-Luther-Universität Halle-Wittenberg.

BERgER, HANS (1929): Über das Elektroencephalogramm des Menschen. In: Archiv für Psychiatrie und Nervenkrankheiten 87, S. 527-570.

Bredel, Ursula/MaAß, Christiane (2016): Leichte Sprache. Theoretische Grundlagen. Orientierung für die Praxis. Berlin: Dudenverlag.

BundeSGESETZBLATT (2008): Gesetz zu dem Übereinkommen der Vereinten Nationen vom 13. Dezember 2006 über die Rechte von Menschen mit Behinderungen sowie zu dem Fakultativprotokoll vom 13. Dezember 2006 zum Übereinkommen der Vereinten Nationen über die Rechte von Menschen mit Behinderungen. In: Bundesgesetzblatt Teil II Nr. 35, S. 1419-1457.

DiStasi, Leandro L./Renner, Rebekka/Staehr, Peggy/Helmert, Jens R./ Velichkovsky, Boris M./CAnas, José J./CATena, Andrés/Pannasch, Sebastian (2010): Saccadic peak velocity sensitivity to variations in mental workload. In: Aviation, Space, and Environmental Medicine 81, S. 413-417.

Duchowski, ANDrew T. (2003): Eye Tracking Methodology: Theory \& Practice. London: Springer.

FOLTA-SCHOOFS, KRISTIAN (2018): Leistung und Gerechtigkeit aus neurodidaktischer Perspektive. In: Sansour, Teresa/Musenberg, Oliver/Riegert, Judith (Hg.): Bildung und Leistung. Differenz zwischen Selektion und Anerkennung. Bad Heilbrunn: Klinkhardt. S. 130-139.

Folta-Schoofs, Kristian/Hesse-Zwillus, Marion/Kieslinger, Nina/Kruse, Julia/ SCHUlZ, REGINE (2017): Museen „inklusiv“ gestalten. Wissenschaftiche Evaluation von Maßnahmen für eine barrierefreie Museumsgestaltung. Hildesheim, Zürich, New York: Georg Olms. 
Folta-Schoofs, Kristian/Hilke, Carina/Bethge/Hans-Jörg/Felbel, Dieter (2015): Attention deficit hyperactivity disorder: precise time reproduction, but accelerated saccadic peak velocity of voluntary eye movements after MPH-medication. In: Heinen, Thomas (Ed.): Advances in Visual Perception Research. New York, NV: Nova Science Publishers. S. 107-140.

Folta, Kristian/Mähler, Claudia (2011): Schnelle Augenbewegungen und visuelle Fixation bei Kindern mit einer Aufmerksamkeitsdefizit-/Hyperaktivitätsstörung (ADHS). In: Kindheit und Entwicklung 20 (1), S. 21-30.

FRÖHLICH, ANDREAS/HAUPT, URSULA (1987): Förderdiagnostik mit schwerstbehinderten Kindern: eine praktische Anleitung zur pädagogisch-therapeutischen Einschätzung. Dortmund: Verlag Modernes Lernen.

GAlley, Nils (1998): An enquiry into the relationship between activation and performance using saccadic eye movement parameters. In: Ergonomics 41, S. 698-720.

Groner, Rudolf/Groner, Marina T. (1989): Attention and eye movement control: An overview. In: European Archives of Psychiatry and Clinical Neuroscience 239, S. 9-16.

HinZ, ANDREAS (2009): Inklusive Pädagogik in der Schule. In: Zeitschrift für Heilpädagogik 5, S. 171-179.

Huey, Edmund B. (1900): On the psychology and physiology of reading. I. In: American Journal of Psychology 11, S. 283-302.

Khan, Omar A./TAYlor, Simon R./JONES, J. GARETH (2000): Anaesthesia and saccadic eye movements. In: Anaesthesia 55, S. 877-882.

KÖMPF, Detlef/Heide, WolfGANG (1998): Zentralnervöse Strukturen - two goals, two modes, six systems. Eine Einführung. In: Huber, Alfred/KöMPF, DETLEF (Hg.): Klinische Neuroopthalmologie. Stuttgart: Thieme. S. 48-57.

Kristen, URsi (1994): Praxis Unterstützte Kommunikation. Eine Einführung. Düsseldorf: Verlag Selbstbestimmtes Leben.

LEIGH, JOHn. R./ZEE, DAVID S. (1999): The neurology of eye movements (3th ed.). Philadelphia: F.A. Davis Company.

MALL, WINFRIED (1995): Kommunikation mit schwer geistig behinderten Menschen. Ein Werkheft. Heidelberg: Edition Schindele.

RENNER, GREGOR (2004): Theorie der Unterstützten Kommunikation: Eine Grundlegung. Berlin: Edition Marhold.

Richardson, Daniel C./Spivey, Michael J. (2004): Eye Tracking: Characteristics and Methods. In: WNEK, GARY/BOWLIN, GARY (Eds.): Encyclopedia of Biomaterials and Biomedical Engineering. Abingdon: Taylor \& Francis. S. 568-572.

ROHNER, JENS-CHRIstophe (2002): The time-course of visual threat processing: High trait anxious individuals eventually avert their gaze from angry faces. In: Cognition and Emotion 16, S. 837-844.

Sturm, TANJa (2013): Lehrbuch Heterogenität in der Schule. München: Reinhardt. 
Apparategestützte Kommunikations- und Feedbacksysteme

von Tetzchner, Stephen/Martinsen, Harald (2000): Einführung in Unterstützte Kommunikation. Übersetzt aus dem Norwegischen von Sebastian Vogel. Heidelberg: Edition S. 



\section{Oliver MusenberG}

\section{Unterstützte Kommunikation}

\section{$1 \quad$ Begriff und Geschichte}

Mit der englischsprachigen Bezeichnung Augmentative and Alternative Communication (Abk.: AAC) und der deutschsprachigen Adaption Unterstützte Kommunikation (Abk.: UK) werden zusammenfassend kommunikationsbezogene Hilfen und Hilfsmittel für Kinder, Jugendliche und Erwachsene bezeichnet, die sich vorübergehend oder dauerhaft nicht oder nur eingeschränkt verbalsprachlich mitteilen können. Wenngleich die internationale Bezeichnung ergänzende (engl. augmentative) und alternative (engl. alternative) Kommunikation präziser ist, hat sich im deutschsprachigen Raum die Bezeichnung Unterstützte Kommunikation als Oberbegriff durchgesetzt. Mit „Alternative Communication “ sind alternative Formen der Kommunikation gemeint, wie z. B. die Braille-Schrift oder die Gebärdensprache, die den üblichen Sprachgebrauch ersetzen. Mit „Augmentative Communication“ sind hingegen ergänzende und zusätzliche Kommunikationsformen wie lautsprachbegleitende Gebärden oder strukturierende Visualisierungen durch Bilder gemeint (vgl. Wilken 2018).

Unterstützte Kommunikation hat sich in den vergangenen 30 Jahren zu einem eigenständigen, vor allem anwendungsorientierten Fachgebiet im Kontext der Sonderpädagogik entwickelt. Hinzu sind im Laufe der Zeit sowohl Bemühungen getreten, die Theoriebildung im Kontext Unterstützter Kommunikation weiterzuentwickeln (vgl. Renner 2004; Lage 2006), als auch die empirische Forschung zu stärken (vgl. z. B. Boenisch 2016a). Darüber hinaus lässt sich im Zusammenhang der bildungspolitischen und erziehungswissenschaftlichen Fokussierung eines inklusiven Bildungssystems die beginnende Entgrenzung des Fachdiskurses beobachten, da Unterstützte Kommunikation zunehmend auch außerhalb sonderpädagogischer Disziplingrenzen thematisiert wird, und ebenso die langsame Verbreitung Unterstützter Kommunikation außerhalb von 
Einrichtungen der Behindertenhilfe und Sonderschulen. Neben dem Begriff Sonderpädagogik als Bezeichnung für die Disziplin und Profession finden sich auch der ältere Begriff Heilpädagogik sowie die jüngeren Begriffe Behindertenpädagogik, Rehabilitationspädagogik und Förderpädagogik, die trotz ihrer sehr unterschiedlichen historischen Ursprünge oft synonym verwendet werden. Sonderpädagogik als Disziplin gliedert sich in Deutschland in verschiedene Fachrichtungen, die sich mit der historischen Genese des professionellen Feldes und personenkreisspezifischer Institutionen ausdifferenziert haben und sich auch in der aktuellen Diktion der Kultusministerkonferenz als unterschiedliche Förderschwerpunkte wiederfinden (vgl. Sekretariat der Ständigen Konferenz der Kultusminister 1994). Impulse für die Entwicklung Unterstützter Kommunikation gingen in den Anfängen in Deutschland primär von den Förderschwerpunkten körperlich-motorische Entwicklung (vgl. Boenisch 2016b) und geistige Entwicklung (vgl. Musenberg 2016) aus (zur kritischen Diskussion behinderungsspezifischer Kategorisierungen vgl. Walgenbach 2018).

Ein wesentlicher Motor der Verbreitung Unterstützter Kommunikation in pädagogischen Handlungsfeldern ist die International Society for Augmentative and Alternative Communication (ISAAC), eine 1983 gegründete Mitgliederorganisation mit Sitz in Toronto/Kanada, deren eigener Anspruch es ist, weltweite Aufmerksamkeit für die Belange von Menschen mit Kommunikationsbeeinträchtigungen zu schaffen, Informationen bereitzustellen und innovative Konzepte und Forschungsansätze zu unterstützen (vgl. ISAAC 2011-2018). Seit 1990 existiert mit der Gesellschaft für Unterstützte Kommunikation e.V. ein deutschsprachiger Ableger von ISAAC, der u.a. regionale und überregionale Tagungen ausrichtet und das Handbuch Unterstützte Kommunikation sowie die Zeitschrift Unterstützte Kommunikation herausgibt (vgl. Gesellschaft für Unterstützte Kommunikation e.V. 2015).

Vom Begriff der Unterstützten Kommunikation zu unterscheiden ist der von der Australierin Rosemary Crossley entwickelte Ansatz der Gestützten Kommunikation (Facilitated Communication, Abk. FC), bei der es um das gestützte Schreiben geht: „Bei der gestützten Kommunikation gibt es immer ein StützerSchreiber-Paar, das zusammen eine Kommunikationshilfe bedient. Meist handelt es sich dabei um eine Buchstabentafel, eine Schreibmaschine oder einen 
Computer, also um Mittel, die schriftsprachliche Äußerungen zulassen“ (Nußbeck 2018: 239). Die Methode wird kritisch dahingehend diskutiert, inwieweit der Stützer/die Stützerin inhaltlichen Einfluss auf die Textproduktion nimmt (Janz/Klauß 2012).

\section{$2 \quad$ Personenkreis und Systematik}

Die drei folgenden Beispiele vermitteln einen Eindruck von der Vielfalt sowohl des Adressatenkreises als auch der konkreten Maßnahmen Unterstützter Kommunikation:

Frau S. lebt in einer Einrichtung der Behindertenhilfe und besucht tagsüber den Förder- und Betreuungsbereich einer Werkstatt für Menschen mit Behinderung (WfbM). Sie ist motorisch und kognitiv beeinträchtigt und kommuniziert mit ihren Bezugspersonen, indem sie lautiert und nach Dingen und Personen greift. Die pädagogischen Mitarbeiter(innen) können die körpereigenen, als prä-intentional (ungezielt) interpretierten Signale von Frau S. häufig nicht verstehen und versuchen intentionales (gezieltes) Verhalten zu unterstützen. Für den Aufbau intentionalen Verhaltens werden u.a. einfache Schalter eingesetzt, mit denen Frau S. für sie interessante elektronische Geräte (z. B. Musik, Ventilator) an- und ausschalten kann, um auf diesem Wege Selbstwirksamkeit und Ursache-Wirkungs-Verhältnisse zu erfahren.

Der berühmte Physiker Stephan Hawking (1942-2018) hat aufgrund einer fortschreitenden Erkrankung des motorischen Nervensystems (Amyotrophe Lateralsklerose/ALS) u.a. die Fähigkeit verloren, lautsprachlich zu kommunizieren. Alternativ hat sich der Wissenschaftler über einen Computer mit Sprachausgabe mitgeteilt.

Jakob besucht die 4. Klasse einer Grundschule. Bei ihm ist frühkindlicher Autismus diagnostiziert worden und er ist bislang nicht in der Lage, verbalsprachlich zu kommunizieren. Er kann Zustimmung und Ablehnung gestisch klar mitteilen und verfügt über ein altersangemessenes Sprachverständnis. Damit auch komplexere 
Mitteilungen möglich werden und sich seine Mitschüler(innen) mit ihm austauschen können, nutzt Jakob eine analoge Kommunikationstafel mit vielen Symbolen und Satzbausteinen, auf die er zeigt, um Fragen zu stellen oder zu beantworten und auch um Situationen zu kommentieren. Die Komplexität der Kommunikationstafel wird sukzessive erweitert, wobei auch Anregungen der Mitschüler(innen) aufgegriffen werden.

Maßnahmen Unterstützter Kommunikation richten sich also an einen heterogenen Personenkreis, dessen Gemeinsamkeit darin besteht, dass verbalsprachliche Fähigkeiten dauerhaft fehlen oder vorübergehend eingeschränkt sind. Die Funktionen alternativer Kommunikationsformen variieren. Sie werden eingesetzt

- als Ersatz für die Lautsprache bei normalem Sprachverständnis,

- zum Erwerb symbolischer Fähigkeiten,

- zum verbesserten Sprachverständnis,

- als Ergänzung zu teilweise vorhandener Lautsprache,

- zum Schriftspracherwerb (vgl. Lage 2016: 375f.).

Einschränkungen der Kommunikationsfähigkeit können durch angeborene oder erworbene Beeinträchtigungen bedingt sein und unterschiedliche Ursachen haben, wie beispielsweise

- Cerebralparesen

- Autismus-Spektrum-Störungen

- Verzögerungen der Sprachentwicklung

- kognitive Beeinträchtigungen

- Schädel-Hirn-Trauma und Schlaganfall (in Folge z. B. Aphasie)

- Neurologische Erkrankungen

- Schlaganfall

- Ataxie, Dyspraxie,

- Temporäre medizinische Bedingungen, wie die Notwendigkeit einer Trachealkanüle

- ... (vgl. z. B. ISAAC 2011-2018) 
Während in den Anfangsjahren der Entwicklung Unterstützter Kommunikation stets das Individuum hinsichtlich seiner kommunikativen Kompetenzen in den Blick genommen und Kommunikationsförderung als Dyade, d.h. als Einszu-Eins-Beziehung und Einzelförderung verstanden wurde, sind in den vergangenen zehn Jahren verstärkt das Umfeld, die Kommunikationspartner(innen) und -situationen fokussiert (vgl. Light/McNaughton 2014; Hömberg 2018) sowie Aspekte wie Zwei- und Mehrsprachigkeit stärker in den Blick genommen worden.

Auch die Gesellschaft für Unterstützte Kommunikation setzt die Beschreibung des Personenkreises direkt mit dem sozialen Kontext und mit Fragen der Teilhabe in Beziehung:

Es gibt Menschen, die aufgrund von angeborenen oder erworbenen Schädigungen Beeinträchtigungen in der Kommunikation und sprachlichen Verständigung mit ihrer Umwelt erfahren. Einige von ihnen haben umfassende Behinderungen in vielen Entwicklungsbereichen, während andere nur in ihrer Kommunikation mit fremden Personen beeinträchtigt sind und von vertrauten Personen durchaus verstanden werden. Für alle aber gilt, dass die Teilhabe an der Gesellschaft und ein selbstbestimmtes Leben in erheblichem Maß eingeschränkt sein können. Durch den Einsatz von Unterstützter Kommunikation können diese Menschen eine erhebliche Verbesserung ihrer Verständigung erreichen (Gesellschaft für Unterstützte Kommunikation e.V. 2015 o. S.).

Zur Systematisierung der Unterstützten Kommunikation hat sich die Unterscheidung körpereigener Kommunikationsformen und externer Kommunikationshilfen sowie die Differenzierung der externen Hilfen in elektronische und nicht elektronische Hilfsmittel etabliert (vgl. Kristen 2005; Tetzchner/ Martinsen 2000). 


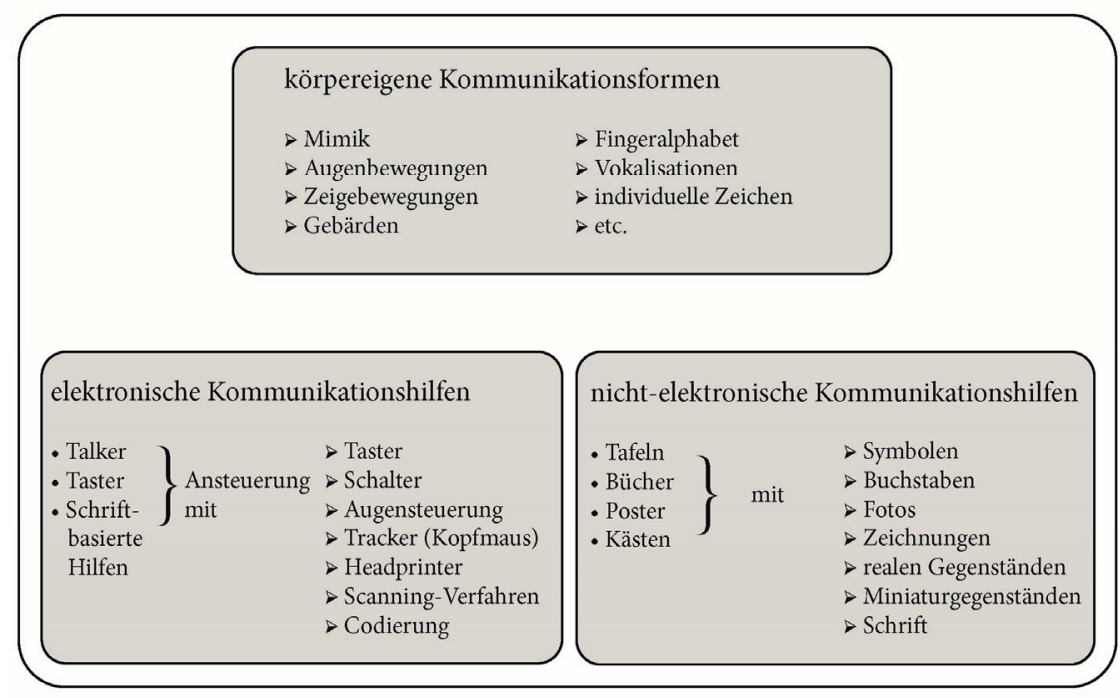

Abb. 1: Übersicht über Kommunikationshilfen (Heidtmann 2010: 347)

\section{Körpereigene Kommunikationsformen:}

$\mathrm{Zu}$ den körpereigenen Kommunikationsformen zählen Blicke, Körperbewegungen und -haltungen, Mimik, Gestik, Zeigebewegungen, Gebärden und Gebärdensprache, Laute, Lautsprachreste sowie individuelle Signale und Signalsysteme. Die körpereigene Kommunikation bildet somit ein breites Spektrum von sehr basalen (z. B. Körperbewegung) bis zu sehr komplexen Formen (Gebärdensprache) ab. Neben der Deutschen Gebärdensprache sind im Kontext Unterstützter Kommunikation einfachere Gebärdensysteme entwickelt worden, wie z. B. die aus der Deutschen Gebärdensprache (DGS) abgeleiteten Lautsprachbegleitenden Gebärden (LBG). Davon zu unterscheiden ist wiederum die von Etta Wilken entwickelte Gebärden-unterstützte Kommunikation (GuK): GuK-Gebärden sind zwar auch vornehmlich der DGS entnommen, „aber so ausgewählt, dass sie motorisch einfach und möglichst transparent sind. Dadurch können sie von kleinen Kindern und schwerer beeinträchtigten Personen leichter gelernt und behalten werden (...). Aufgrund der zwischen beiden Symbolsystemen - Sprache und Gebärde - gegebenen ähnlichen kognitiven 
Voraussetzungen haben Gebärden sprachanbahnende Funktion und fördern die wechselseitige Aufmerksamkeit von Kind und Bezugsperson“"(Wilken 2018: 77).

Ein zentraler Vorteil körpereigener Kommunikationsformen liegt in der Unabhängigkeit von zusätzlichen externen Hilfen und der spontanen Einsatzmöglichkeit. Von Nachteil ist, dass sie bestimmte Anforderungen an die Kommunikationspartner(innen) stellen, z. B. motorische und visuelle Fähigkeiten, Kenntnis der Gebärdensprache oder des individuellen Symbolsystems. Außerdem können mit den basalen körpereigenen Kommunikationsformen (z. B. Blicke, Mimik) keine komplexen Themen vermittelt werden (vgl. Heidtmann 2010: 347).

\section{Externe, nicht-elektronische und elektronische Kommunikationshilfen:}

$\mathrm{Zu}$ den nicht elektronischen, externen Kommunikationshilfen zählen verschiedene Symbolsammlungen, welche Schrift, reale Gegenstände (Bezugsobjekte, vgl. Wagner 2007), Fotos und Bilder beinhalten können. Symbolsammlungen werden z. B. als mobile Kommunikationstafel oder als Kommunikationsmappe (z. B. als erweiterbares Ringbuch) gestaltet und dazu standardisierte Symbolsammlungen (z. B. Picture Communication System) oder individuell gestaltete Fotos und Bilder genutzt. Mitteilungen können hier durch direkte oder indirekte Selektion realisiert werden. Bei der direkten Selektion wird z. B. ein Bild durch direktes Zeigen oder Anblicken ausgewählt. Fehlen die motorischen Fähigkeiten, um ein Symbol direkt auszuwählen oder ist die Fixierung des Blickes nicht eindeutig genug, können Symbole auch über indirekte Selektion ausgewählt werden, indem die Kommunikationspartnerin/der Kommunikationspartner z. B. mit dem Finger die Symbole der Kommunikationstafel entlangfährt (sog. Partner-Scanning), bis die UK-Nutzerin/der UK-Nutzer „Stop“ signalisiert, z. B. durch einen Laut oder einen motorischen Impuls.

Diese Unterscheidung der direkten und indirekten Selektion kann ebenso auf elektronische Kommunikationshilfen angewandt werden. So können die Bilder und Symbole auf einem einfach strukturierten Talker mit Sprachausgabe direkt z. B. mit dem Finger ausgewählt werden und das hinterlegte Wort wird per natürlicher oder synthetischer Sprachausgabe vom Talker erzeugt (z. B. „Go Talk“). Ebenso können auf einem Bildschirm gezeigte Symbole via Eye-Tracking direkt angesteuert werden: Eine Kamera erfasst die Blickbewegungen und die 
Nutzerin/der Nutzer kann Bilder, Symbole oder auch Buchstaben und Schrift direkt durch Fixierung mit den Augen auswählen. Verfügt die UK-Nutzerin/der UK-Nutzer nicht über die motorischen Fähigkeiten zur direkten Auswahl, kann bei Talkern auch die indirekte Auswahl ermöglicht werden, z. B. indem ein Curser nacheinander sämtliche auf einem Bildschirm gezeigten Symbole abwandert (Scanning) und die UK-Nutzerin/der UK-Nutzer den Curser an der passenden Stelle anhält (z. B. durch einen Kopf- oder Knieschalter oder durch Saugen oder Pusten eines Röhrchens). Herkömmliche PCs mit Touch-Screens und vor allem Tablet-PC's stellen teilweise Alternativen zu den speziellen Talkern dar. Sie werden mittlerweile verstärkt in der Unterstützten Kommunikation eingesetzt und haben zudem den Vorteil, dass sie keine ,besonderen' Geräte sind, sondern generell von allen, beispielsweise in der Schule, genutzt werden (vgl. Lamers/Terfloth 2013) (zum Spannungsfeld zwischen Universal Design und individuellen Lösungen s. den Beitrag von Womser-Hacker im vorliegenden Band).

$\mathrm{Zu}$ den elektronischen Hilfen gehören aber nicht nur komplexe Talker, sondern auch einfache, individuell einsetzbare Schalter, mit deren Hilfe Stromkreise unterbrochen und dadurch elektrische Geräte an- und ausgeschaltet werden können (z. B. „Power Link“). Ebenfalls häufig eingesetzt werden einfache „Talker“, mit denen ein zuvor eingesprochener Text abgespielt werden kann (z. B. „Big Mack“) oder auch mehrere kurze Texte nacheinander durch mehrfaches Drücken des Schalters abgespielt werden können (z. B. „Step-by-Step“).

Externe Kommunikationshilfen haben gegenüber den körpereigenen Kommunikationsformen den Nachteil, dass man solche externen Hilfen nicht automatisch mit sich führt und dass sie im Falle von elektronischen Hilfen Energie benötigen. Außerdem können sie kostspielig sein; sie haben allerdings auch viele Vorzüge. So kann mit Hilfe von Talkern mit Sprachausgabe räumliche Distanz überwunden werden und es können mit Talkern und PC's sehr komplexe Mitteilungen auf Basis verschiedener Codiersysteme realisiert werden. 
Für den sinnvollen Einsatz von Maßnahmen Unterstützter Kommunikation ist es wichtig, die kommunikativen Kompetenzen des Kindes, Jugendlichen oder Erwachsenen einschätzen zu können (vgl. zur Diagnostik Scholz/Wagner/ Haag/Herale 2014; Kane 2018). So ist z. B. der Einsatz verschiedener Symbole (z. B. ein Foto eines Balls als Zeichen für „Spielen“) bei Menschen mit sehr ausgeprägten kognitiven Einschränkungen dann möglicherweise nicht sinnvoll, wenn (noch) kein Symbolverständnis erkennbar ist.

Nach einer kurzen Beschreibung der Kommunikationsentwicklung im Anschluss an Wilken (2018) soll in einem zweiten Schritt mit Hilfe des „Partizipationsmodells“ (Beukelman/Mirenda 1998) der Blick auf konkrete Situationen und deren Kommunikationsbarrieren gerichtet und damit Entwicklung und Partizipation in ein enges Verhältnis gebracht werden.

Zunächst zu entwicklungsbezogenen Aspekten der Kommunikation: Wilken (2018) nimmt die Kommunikationsentwicklung bis zum Meilenstein der Sprachlichen Kommunikation im zweiten Lebensjahr in den Blick und unterscheidet dabei 1) Prä-intentionales Verhalten, 2) Intentionales Verhalten, 3) Gezielte Kommunikation, 4) Konventionelle Kommunikation, 5) Symbolische Kommunikation und schließlich 6) Sprachliche Kommunikation. Die folgende Tabelle gibt einen Überblick entlang dieser sechs Etappen der Kommunikationsentwicklung, ordnet diese jeweils einem Entwicklungsalter zu und beschreibt sie kurz hinsichtlich des Verhaltens des Kindes und der Bezugsperson.

\section{Prä-intentionales Verhalten}

Kind: zeigt Aktivität (Bewegung, Schreien, Lautieren), Laute drücken sich aus

Behinderung verändert Aktivität
Bezugsperson: versteht Verhalten und Lautäußerungen als "Mitteilungen«, gleiche Interpretation ermöglicht dem Kind zunehmend Intentionalität

Behinderung erschwert Interpretation 
2. Intentionales Verhalten (Entwicklungsalter ca. 5 Mon.)

Kind: Erfahrungen in Interaktionen führen zu Erwartungen, aktives Mitgestalten von Ritualen, Aufmerksamkeit ist noch eindimensional, differenzierte Lautäußerungen

Behinderung beeinträchtigt kooperatives Verhalten
Bezugsperson: gestaltet Rituale, ermöglicht aufeinander bezogenes Handeln in Alltagssituationen, unterstützt Aufmerksamkeit, fördert referentiellen Blickkontakt

Behinderung schränkt gemeinsame Handlungen ein

3. Gezielte Kommunikation (Entwicklungsalter ca. 9 Mon.)

Kind: beachtet Dinge und Partner, lenkt die Aufmerksamkeit (ref. Blickkontakt), Zeigen und zielbezogenes Verhalten, handlungsbezogenes Lautieren

Behinderung wirkt sich auf Aufmerksamkeit und Handlungsmöglichkeiten aus

4. konventionelle Kommunikation (Entwicklungsalter ca. 10 Mon.)

Kind: konventionelle Signale, Rituale und Gesten, deklaratives Zeigen, Imitation von Einzellauten und Lautverdoppellungen, Protowörter

Behinderung führt zu Diskrepanzen in der Entwicklung, eingeschränkte aktive Einflußnahme und reduziert Kompetenzerfahrung bezogene Sprache lungsangemessener Angebote
Bezugsperson: antwortet verbal und mit Handeln, kommentiert eigene und kindliche Aktivität, zeigt und macht vor

Behinderung erschwert gemeinsame Aktivität und sichere Interpretation

Bezugsperson: Zeigen und benennen, situationsgebundene und handlungs-

Behinderung erschwert Einschätzung kindlicher Kompetenzen und entwick- 
5. Symbolische Kommunikation (Entwicklungsalter ca. 13 Mon.)

Kind: konventionelle Gestik und Mimik, einzelne Wörter

Behinderung verändert Mimik und Gestik, beeinträchtigt den Spracherwerb in spezifischer Weise
Bezugsperson: verstärken, erweitern, unterstützen Verstehen und Nachsprechen

Angemessene Verstärkung bei alternativen Kommunikationsformen oft schwierig

6. Sprachliche Kommunikation (Entwicklungsalter ca. 24 Mon.)

Kind: mehrere Wörter werden verbunden, Aufbau von Syntax und Grammatik, zunehmend normentsprechende Artikulation
Bezugsperson: erweitert kindliche Äußerungen den intendierten Bedeutungen entsprechend, verstärkt richtige und korrigiert falsche Regelbildung

Behinderung bewirkt Probleme der angemessenen Abstimmung auf kindliche Interessen, Fragen und Bedürfnisse

Tab. 1: Kommunikationsentwicklung nach Wilken (2018).

Standen anfangs vor allem behinderte Menschen und ihre kommunikationsbezogenen, individuellen Defizite im Vordergrund, hat sich der Blick systemisch geweitet. Zunehmend stehen nicht mehr nur einzelne Personen und ihre individuellen Ausdrucksmöglichkeiten im Vordergrund, sondern kommunikative Situationen werden zum Ausgangspunkt von Maßnahmen Unterstützter Kommunikation genommen (vgl. Lage 2016: 375). Zwar ist eine entwicklungsorientierte Diagnostik und Förderplanung weiterhin notwendig und sinnvoll, sie wird aber ergänzt durch eine stärkere Fokussierung der Teilhabemöglichkeiten an konkreten Alltagssituationen. Einen entscheidenden Beitrag zur Etablierung 
dieser die konkreten Situationen und Barrieren fokussierenden Perspektive haben Beukelman/Mirenda (1998) mit ihrem Partizipationsmodell geleistet.

Grundlage des Partizipationsmodells ist die Fokussierung der Teilhabe einer Person an konkreten Situationen in verschiedenen Lebensbereichen: „Es geht darum, wie eine Person mit Kommunikationsbeeinträchtigung an den Alltagsaktivitäten und Tätigkeiten der verschiedenen Lebensbereiche teilnehmen kann und zwar im Vergleich zu nichtbehinderten Peers" (Antener 2012: 01-024-001).

Anhand des Beispiels der nicht sprechenden Schülerin Inge, die eine inklusive Grundschule besucht, werden im Folgenden in Anlehnung an Braun (2018: 156ff.) die im Partizipationsmodell beschriebenen Schritte dargestellt.

\section{Erstellen einer Aktivitätenliste:}

Als erster Schritt wird eine Aktivitätenliste des Schultages erstellt und darauf eine konkrete Beispielaktivität genauer beschrieben: 1) Morgenkreis, 2) Sachunterricht, 3) Pause, 4) Mathematik, 5) Mittagessen, 6) Sport, 7) Hort.

Aus der Aktivitätenliste wird eine Beispielaktivität herausgegriffen: Im Morgenkreis 1) begrüßen sich die Schüler(innen), 2) sie wählen ein Lied aus und singen, 3) sie erzählen von ihrem Wochenende und 4) sie beenden den Morgenkreis.

Einschätzung der Partizipationsmöglichkeiten von Inges Mitschüler(inne)n:

Die Mitschüler(innen) begrüßen jeweils ihren Sitznachbarn und wünschen sich einen guten Morgen. Sie bringen Liedvorschläge ein und erzählen vom Wochenende. Sie beenden den Morgenkreis mit einem lautsprachlichen Ritual.

\section{Einschätzung der Partizipationsmöglichkeiten von Inge:}

Inge wird mit Handführung am Begrüßungsritual beteiligt, begrüßt aber nicht verbal. Sie kann an der Liedauswahl mit Hilfe von Ja/Nein-Fragen beteiligt werden. Die Auswahl bleibt damit auf das beschränkt, was verbal angeboten wird. Inge erzählt nicht selbst vom Wochenende, sondern hört zu, wenn aus dem Mitteilungsheft vorgelesen wird, in das die Eltern einige Informationen zum Wochenende eingetragen haben. Am Abschluss des Morgenkreises wird Inge wiederum mittels Handführung beteiligt. 
Auf der Grundlage dieser Einschätzung der Partizipationsmöglichkeiten werden in einem weiteren Schritt Zugangsbarrieren und Gelegenheitsbarrieren identifiziert.

Zugangsbarrieren beziehen sich primär auf die gegenwärtigen Kompetenzen der unterstützt kommunizierenden Person. Gelegenheitsbarrieren bezeichnen die (einschränkenden) Rahmenbedingungen (z. B. politische, einstellungs- und wissensbezogene Bedingungen), mit denen gerechnet werden muss. Zugangsbarrieren zeigen sich bei Inge z. B. darin, dass sie aufgrund ihrer motorischen Einschränkungen keine Gebärden nutzen kann und dass direkte Selektion nur mit großformatigen Hilfsmitteln möglich ist. Gelegenheitsbarrieren zeigen sich in der nur sehr begrenzten Verfügbarkeit elektronischer Hilfsmittel in der Schule sowie in der Unerfahrenheit der Lehrerin mit UK. Weiterhin in Anlehnung an Braun (2018) lassen sich nun erste „Interventionsideen“ formulieren, z. B. dass Inge die Möglichkeit bekommen soll, selbst aktiv zu begrüßen (BigMack), selbständig Lieder auszuwählen (Liedertafel mit Bildern/Symbolen für verschiedene Lieder), selbstständig vom Wochenende zu erzählen (Etablierung von z. B. Personen- und Aktivitätentafeln) sowie aktiv den Morgenkreis zu beenden.

Durch die Teilhabe an den Aktivitäten des Schultages kann Kommunikation als sinnvoll erlebt werden und es können Motive für kommunikatives Handeln generiert werden. Es geht also gerade nicht um ein isoliertes Funktionstraining, sondern um Bildung. Dies soll auch im nun folgenden Kapitel zur Unterstützten Kommunikation mit Menschen mit sog. schweren und mehrfachen Behinderungen besondere Betonung erfahren.

\section{$4 \quad$ Unterstützte Kommunikation und schwere Behinderung}

Menschen mit schweren und mehrfachen Behinderungen standen in den Anfangsjahren Unterstützter Kommunikation noch nicht im Fokus der Entwicklung verschiedener technischer und nicht technischer Hilfen. Und auch aktuell beginnt erst langsam die systematische Berücksichtigung derjenigen Menschen im Diskurs Unterstützter Kommunikation, die (noch) kein intentionales Verhalten zeigen und auch nicht mit Hilfe von einfachen Symbolen kommunizieren 
können (vgl. Hennig 2011: 274). Generell ist die Thematisierung von Menschen mit schwerer Behinderung in der (Sonder-)Pädagogik erst jüngeren Datums, so wurde z. B. die Schulpflicht für Kinder und Jugendliche mit schweren Behinderungen erst im Laufe der 1980er Jahre in den „alten“ Bundesländern etabliert und umgesetzt. Als erstes Förderkonzept für Menschen mit motorischen und kognitiven Beeinträchtigungen kann im deutschsprachigen Raum die ab Ende der 1970er Jahre von Andreas Fröhlich entwickelte Basale Stimulation (1996) gelten. Fröhlich bezieht sich mit seinem Ansatz auf entwicklungspsychologische, physiotherapeutische sowie hirnphysiologische Erkenntnisse. Er bezeichnet den Adressatenkreis seines Konzepts als „schwerstbehindert“ und meint damit „immer eine komplexe Beeinträchtigung des ganzen Menschen in allen seinen Erlebnis- und Ausdrucksmöglichkeiten. Emotionale, kognitive und körperliche, aber auch soziale und kommunikative Fähigkeiten sind erheblich eingeschränkt oder verändert" (Fröhlich 1996: 11).

Die Kommunikation stellt Fröhlich schließlich ins Zentrum seines gemeinsam mit Ursula Haupt gestalteten Entwicklungsmodells (vgl. ebd. 50). Basale Stimulation zielt auf das In-Gang-Setzen von Wahrnehmung durch unterschiedliche Reizangebote und differenziert visuelle, taktile, vibratorische, geruchliche, geschmackliche, thermische und somatische Kommunikation (vgl. ebd. 51f.). Ungefähr zur gleichen Zeit entwickelt Winfried Mall das Konzept der Basalen Kommunikation auf der Basis gestaltpsychologischer Bezüge und adressiert vornehmlich Menschen mit sog. schwerer geistiger Behinderung (vgl. Mall 1998). Beide Konzepte, die viele Schnittmengen mit dem aktuellen Konzept Intensive Interaction aufweisen (vgl. Hewett 2018), können als wichtige Impulsgeber für die erst später erfolgende Einbeziehung von Menschen mit schweren Behinderungen bei der Entwicklung von Methoden und Maßnahmen Unterstützter Kommunikation gelten - die Basale Stimulation durch Betonung eines „somatischen Dialogs" und die Basale Kommunikation durch die Figur des „Kreislaufs der primären Kommunikation“ (Mall 1998: 3; vgl. auch „präreflexiver Dialog“ bei Fornefeld 1989). Mall visualisiert diesen Kreislauf wie folgt: 


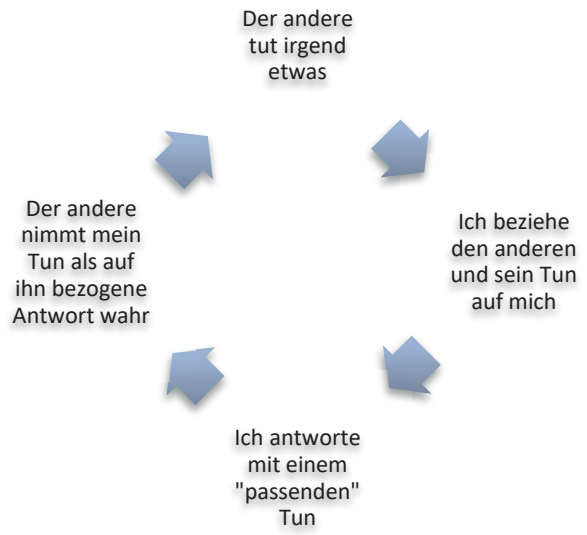

Abb. 2: Der Kreislauf der primären Kommunikation (nach Mall 1996: 34).

Diese primäre Kommunikation beschreibt den Versuch, prä-intentionales Verhalten in intentionales Verhalten und gezielte Kommunikation zu überführen.

Frau S. zeigt im oben genannten Fallbeispiel (siehe Seite 363) noch kein gezieltes Verhalten bzw. die Blicke und Bewegungen von Frau S. können für die Außenstehenden nicht als gezielte Kommunikation (z. B. gezieltes Zeigen auf einen Gegenstand im Sinne einer Aufforderung) identifiziert werden. Gezielte Kommunikation geht mit dem sukzessiven Ausbau der Triangulierung, des referentiellen Blicks und geteilter Aufmerksamkeit einher (vgl. Hennig 2011: 276). Das bedeutet z. B., sich in Anwesenheit einer Bezugsperson auf etwas Drittes (z. B. die auf dem Tisch stehende Tasse Kaffee) beziehen zu können und den Wunsch zu äußern, einen Schluck Kaffee zu trinken: „Der Meilenstein der Triangulierung wird bei Menschen mit schwerster Behinderung nicht selten zum Grenzstein der kommunikativen Entwicklung" (Hennig 2011: 276).

Das Verhalten von Frau S. bewegt sich vor allem im Bereich des prä-intentionalen Verhaltens und das entwicklungsbezogene Ziel ist es zunächst, die Voraussetzung für die Entwicklung intentionalen Verhaltens zu schaffen, d. h. Erfahrungen zu ermöglichen, selbst etwas bewirken zu können, mit dem eigenen Tun die Umwelt beeinflussen zu können. Zu diesem Zweck können z. B. die bereits 
erwähnten einfachen elektronischen Kommunikationshilfen (Power Link, BigMack, ...) eingesetzt werden. Ein Schalter (Power Link) kann beispielsweise an der Kopfstütze des Rollstuhls angebracht werden. Immer, wenn Frau S. mit dem Kopf den Schalter betätigt, wird z. B. der CD-Player eingeschaltet und ihre Lieblingsmusik ertönt. Betätigt Frau S. erneut den Schalter, geht die Musik wieder aus. Auch wenn Frau S. vielleicht zunächst nur zufällig, nicht intentional den Schalter betätigt, kann in dieser Situation bei mehrfacher Wiederholung die Erfahrung gemacht werden, dass das eigene Tun eine Wirkung hat und auf diesem Wege überhaupt erst die Erkenntnis möglich ist, dass es Sinn macht, zu kommunizieren. Hennig (2011) macht allerdings darauf aufmerksam, dass ähnliche Ursache-Wirkungs-Zusammenhänge im Sinne von Kontingenzerfahrung auch in spielerischen Formen sozialer Interaktionen erfahren werden können und deshalb der Mehrwert des Einsatzes technischer Geräte auf dieser basalen Ebene der Kommunikation nicht überbewertet werden sollte (vgl. ebd. 289). Zudem würden Menschen mit schwerer Behinderung nachweislich soziale Effekte gegenüber anderen Effekten vorziehen, so dass es sinnvoll ist, einfache Tasten mit Sprachausgabe vor allem zu nutzen, um soziale Prozesse auszulösen (jemanden rufen, auf sich aufmerksam machen, ...), um auf diese Weise soziale Resonanz und Selbstwirksamkeit zu erfahren (vgl. ebd.).

In der Sonderpädagogik, in der Unterstützte Kommunikation als Konzept entwickelt und in den vergangenen ca. 25 Jahren weiter ausdifferenziert wurde, ist im Anschluss an Paul Watzlawick ein sehr weites Kommunikationsverständnis favorisiert worden. In Anlehnung an das erste Axiom „Man kann nicht nicht kommunizieren" (Watzlawick/Beavin/Jackson 1996: 53, Hervorhebung im Original) wurde in der Gleichsetzung von Verhalten und Kommunikation die Möglichkeit eines nicht ausgrenzenden Kommunikationsverständnisses gesehen, das letzten Endes allen Menschen Teilhabe an Kommunikation ermöglicht. Auf der Rückseite dieser optimistischen Lesart wurde zeitweilig vergessen, dass Kommunikation auch Gründe, Anlässe und Inhalte braucht. Klauß (2002) hat bereits um die Jahrtausendwende darauf aufmerksam gemacht, dass das erste Axiom aus Watzlawicks Kommunikationstheorie wie ein „sonderpädagogischer Glaubenssatz" gewirkt habe. Wenn Kommunikation als voraussetzungslos gilt und mit jeglichem Verhalten gleichgesetzt werde, gehe dies aber mit der Gefahr einher, den kommunikationsbezogenen Bildungsbedarf $\mathrm{zu}$ vernachlässigen: 
Wenn jeder Mensch ständig und voraussetzungslos kommuniziert, so Klauß, muss Kommunikation eigentlich gar nicht gefördert werden. Es handelt sich dann nur noch um ein funktionales Problem: Die pädagogische Bezugsperson müsse dann nur noch lernen, die Mitteilungen zu verstehen, die der andere permanent von sich gibt und ihm die fehlenden Mittel (Techniken) zur Verfügung stellen. Damit würden aber wesentliche Bedingungen für Kommunikation ignoriert, nämlich dass ein Mensch:

1. etwas mitzuteilen hat (Kommunikation braucht einen Inhalt),

2. etwas mitteilen möchte (Kommunikation braucht einen Grund),

3. etwas mitteilen kann (Kommunikation braucht einen Kanal und ein verstehbares Zeichensystem) und

4. ein Gegenüber braucht, das sich für seine Mitteilung interessiert und diese verstehen kann (vgl. Klauß 2002).

Somit ist zwar ein dialogisches In-Beziehung-Treten (z. B. im Sinne „primärer Kommunikation") Voraussetzung für Kommunikation, allerdings müssen auch Möglichkeiten eröffnet werden, Bedürfnisse, Vorlieben und Geschmack auszubilden. Kommunikation und Bildung sind im Kontext schwerer Behinderung sehr eng miteinander verbunden, denn hier geht es darum, gerade „die einfachsten Formen von Symbolbildungen als solche zu identifizieren und als Bildung zu erkennen" (Ackermann 2010: 234), sowohl in ihrer kognitiven als auch in ihrer affektiv-emotionalen Dimension (vgl. ebd. 235). 


\section{Literaturverzeichnis}

ACKERMANN, KARL-ERNST (2010): „Zum Verhältnis von geistiger Entwicklung und Bildung". In: MusenberG, Oliver/RiegerT, Judith (Hrsg.): Bildung und geistige Behinderung. Bildungstheoretische Reflexionen und aktuelle Fragestellungen. Oberhausen: Athena, 225-244.

Antener, Gabriela (2012): „Interventionsmodelle der Unterstützten Kommunikation“. In: Handbuch der Unterstützten Kommunikation. Karlsruhe: Von Loeper Literaturverlag, 01.018.001-01.026.001.

Beukelman, David/Mirenda, Pat (1998): Augmentative and Alternative Communication. Baltimore: Paul Brooks Publishing.

BraUn, URsula (2018): „Unterstützte Kommunikation in der Sonderschule“. In: WILKEN, ETTA (Hrsg.): Unterstützte Kommunikation. Eine Einführung in Theorie und Praxis. 5. Aufl. Stuttgart: Kohlhammer, 151-165.

BoenisCH, JeNS (2016a): „Verständigung ermöglichen. Neue Ansätze zur Sprachförderung von Menschen mit schwerer und mehrfacher Behinderung". In: BERNASCONI, Tobias/BÖING, URsula (Hrsg.): Schwere Behinderung \& Inklusion. Facetten einer nicht ausgrenzenden Pädagogik. Oberhausen: Athena, 91-109.

BOENISCH, JeNs (2016b): „Körperlich-motorische Entwicklung“. In: HEDdERICH, INGEBORG/Biewer, GotTfried/Hollenweger, Judith/Markowetz, Reinhard (Hrsg.): Handbuch Inklusion und Sonderpädagogik. Bad Heilbrunn: Klinkhardt, 224-228.

FORNEFELD, BARBARA (1989): Elementare Beziehung und Selbstverwirklichung geistig Schwerstbehinderter in sozialer Integration. Reflexionen im Vorfeld einer leiborientierten Pädagogik. Aachen: Mainz-Verlag.

FrÖHLICH, ANDREAS (1996): Basale Stimulation. Düsseldorf: Verlag Selbstbestimmtes Leben. 8. Aufl.

Gesellschaft für Unterstützte Kommunikation e.V. (2015). Online: URL: http://www.gesellschaft-uk.de/index.php/unterstuetzte-kommunikation (24.04.2018).

Heidtmann, Hildegard (2010): „Unterstützte Kommunikation und Sprachheilpädagogik: Von der Sprache zu Kommunikation“. In: Sonderpädagogische Förderung heute $55,4,343-364$.

HeNnig, Birgit (2011): „Interaktion und Kommunikation zwischen Menschen mit schwerster Behinderung und ihren Bezugspersonen: Aspekte des Gelingens". In: Fröhlich, Andreas/Heinen, Norbert/Klauß, Theo/Lamers, WolfGang (Hrsg.): Schwere und mehrfache Behinderung - interdisziplinär. Oberhausen: Athena, 273-297.

HewetT, DAve (Hrsg.) (2018): The Intensive Interaction Handbook. London: Sage Publications. 
HÖMBERG, NinA (2018): „With a little help from my friends. Unterstützte Kommunikation im integrativen Unterricht“. In: WILKEN, ETTA (Hrsg.): Unterstützte Kommunikation. Eine Einführung in Theorie und Praxis. 5. Aufl. Stuttgart: Kohlhammer, 166187.

ISAAC - International Association for Alternative and Augmentative Communication (2011-2018). Online: URL: https://www.isaac-online.org (24.04.2018).

JanZ, Frauke/KlaUß, TheO (2012): Facilitated Communication. Interaktionsanalysen bei einer kontrovers diskutierten Methode. Heidelberg: Universitätsverlag Winter.

KANE, GUDRUN (2018): „Diagnose der Verständigungsfähigkeit bei nicht sprechenden Kindern“. In: WiLKen, ETTA (Hrsg.): Unterstützte Kommunikation. Eine Einführung in Theorie und Praxis. Stuttgart: Kohlhammer, 18-37.

Klauß, Theo (2002): „Können Menschen wirklich nicht nicht kommunizieren? - Anfragen zu einem an Watzlawick angelehnten sonderpädagogischen Glaubenssatz“. In: Vierteljahresschrift für Heilpädagogik und Ihre Nachbargebiete (VHN) 71, 3, 262-276.

KRISTEN, URSI (2005): Praxis Unterstützte Kommunikation. Eine Einführung. Düsseldorf: Verlag selbstbestimmtes Leben.

Lage, Dorothea (2006): Unterstützte Kommunikation und Lebenswelt: Eine kommunikationstheoretische Grundlegung für eine behindertenpädagogische Konzeption. Bad Heilbrunn: Klinkhardt.

LAGE, DorotheA (2016): „Unterstützte Kommunikation“. In: HedDERICH, INGEBORG/ Biewer, Gottfried/Hollenweger, Judith/Markowetz, Reinhard (Hrsg.): Handbuch Inklusion und Sonderpädagogik. Bad Heilbrunn: Klinkhardt, 375-379.

LAMERS, WolfGAng/Terfloth, KARIN (2013): „Tablet-PC - ein UK-Medium der Zukunft?" In: Teilhabe 52, 1, 33-42.

Light, Janice/McNaughton, David (2014): „Communication Competence for Individuals who require Augmentative and Alternative Communication. A New Definition for a New Era of Communication?" In: Augmentative and Alternative Communication 30, 1, 1-18.

MALL, WINFRIED (1998): Kommunikation mit schwer geistig behinderten Menschen. Heidelberg: Universitätsverlag Winter. 4. Aufl.

Musenberg, Oliver (2016): „Geistige Entwicklung“. In: Hedderich, Ingeborg/ Biewer, Gottfried/Hollenweger, Judith/Markowetz, Reinhard (Hrsg.): Handbuch Inklusion und Sonderpädagogik. Bad Heilbrunn: Klinkhardt, 213-217.

NußBecK, Susanne (2018): „Das Konzept der Gestützten Kommunikation. Beschreibung und kritische Bewertung“. In: Wilken, ETTA (Hrsg.): Unterstützte Kommunikation. Eine Einführung in Theorie und Praxis. Stuttgart: Kohlhammer, 238-261.

RENner, Gregor (2004): Theorie der Unterstützen Kommunikation. Eine Grundlegung. Edition Marhold.

Scholz, Markus/Wagner, Michael/HaAG, Kathrin/Herale, Philipp (2014): „Beobachtungsbogen zu kommunikativen Fähigkeiten - Revision (BKF-R): Ein Verfahren zur 
Diagnostik und Förderung in der Unterstützten Kommunikation“. In: Zeitschrift für Heilpädagogik, 5, 186-191.

Sekretariat der Ständigen Konferenz der Kultusminister der Länder in der Bundesrepublik Deutschland (1994). Online: URL: https://www.kmk.org/fileadmin/Dateien/ veroeffentlichungen_beschluesse/1994/1994_05_06-Empfehl-SonderpaedagogischeFoerderung.pdf (24.04.2018).

TetzChner, Stephen von/Martinsen, Harald (2000): Einführung in die Unterstützte Kommunikation. Heidelberg.

Wagner, Michael (2007): Wir sehen mit den Augen des Kollektivs!? Der Mensch mit schwerer Behinderung zwischen Individualität und Sozialität. Bad Heilbrunn: Klinkhardt.

WalgenbaCH, Katharina (2018): „Dekategorisierung - Verzicht auf Kategorien?“ In: Musenberg, Oliver/Riegert, Judith/SAnsour, Teresa (Hrsg.): Dekategorisierung in der Pädagogik? Notwendig und riskant? Bad Heilbrunn: Klinkhardt, 11-39.

Watzlawick, Paul/Beavin, Janet H./Jackson, Don D. (1996): Menschliche Kommunikation. Formen, Störungen, Paradoxien. Bern: Verlag Hans Huber.

Wilken, EtTA (2018): „Präverbale sprachliche Förderung und Gebärden-unterstützte Kommunikation in der Frühförderung“. In: Wilken, ETTA (Hrsg.): Unterstützte Kommunikation. Eine Einführung in Theorie und Praxis. Stuttgart: Kohlhammer, 6586. 


\section{UTA BENNER/ANNIKA HERRMANN}

\section{Gebärdensprachdolmetschen}

\section{$1 \quad$ Einleitung}

Gebärdensprachdolmetschen erfährt aktuell eine vorher nicht da gewesene Aufmerksamkeit, obwohl die Berufsausbildung bereits auf eine über 30 Jahre alte Tradition zurückblicken kann. Dies liegt an verschiedenen gesetzlichen, wissenschaftlichen sowie kulturellen Aspekten. Der Bedarf an Gebärdensprachdolmetscher(inne)n steigt nach wie vor, nicht zuletzt aufgrund des Behindertengleichstellungsgesetzes und der UN-Behindertenrechtskonvention. Zudem wird die wissenschaftliche Auseinandersetzung mit dem Bereich Gebärdensprachdolmetschen aus translationswissenschaftlicher Perspektive zunehmend wichtiger, vor allem auch im internationalen Kontext. Nicht zuletzt sind kulturelle Einflüsse und die damit verbundene Entwicklung von situativen Rollenverständnissen eines Dolmetschers / einer Dolmetscherin zentral. Wir stellen in diesem Artikel das Thema Gebärdensprachdolmetschen im Überblick vor.

\section{Gebärdensprachen und Gebärdensprachgemeinschaften}

Gebärdensprachen sind natürliche und komplexe Sprachen mit jeweils eigenständiger Grammatik. Sie sind sowohl länderspezifisch als auch innerhalb eines Landes z. T. dialektal geprägt, so dass man nicht von der Gebärdensprache spricht, sondern von verschiedenen Gebärdensprachen weltweit. Sie werden in der visuell-manuellen Modalität produziert und wahrgenommen, d. h. mit den Händen, dem Oberkörper, dem Kopf und der Mimik artikuliert und mit den Augen wahrgenommen (vgl. u.a. Bellugi/Fischer 1972). Laut- und Gebärdensprachen unterscheiden sich in sprachspezifischen Bereichen, jedoch nicht kognitiv, da sie auf gleiche neurologische Grundlagen zurückgreifen (vgl. u. a. 
Klima/Bellugi 1979, Poizner et al. 1987). Betrachtet man verschiedene Gebärdensprachen, so unterscheiden sie sich auf einigen Ebenen stark voneinander, z. B. in der Lexik, also den einzelnen Gebärden, und der Wortordnung, d. h. in Bezug auf den Satzaufbau (vgl. u. a. Woll 1983, Meier 2002, Perniss et al. 2007). In anderen Bereichen, wie der Morphologie, also der internen Gebärdenstruktur, gibt es auf der anderen Seite auch einige Gemeinsamkeiten über alle Gebärdensprachen hinweg (vgl. z. B. Wilbur 2000, Aronoff et al. 2005).

\subsection{Deutsche Gebärdensprache}

Die Deutsche Gebärdensprache (DGS) ist die Muttersprache tauber Menschen in Deutschland und wird innerhalb der Gemeinschaft tauber Menschen weitergegeben. Auch wenn sich das Alter von verschiedenen Gebärdensprachen aufgrund der fehlenden Schriftform und selten vorhandenen geschichtlichen Dokumenten nicht vollständig rekonstruieren lässt und die These besteht, dass Gebärdensprachen sogar vor Lautsprachen existierten (vgl. Armstrong et al. 1995, Armstrong/Wilcox 2003), wird im Allgemeinen angenommen, dass Gebärdensprachen im dokumentierten und später dann bildungspolitischen Sinn z. T. ca. 300-400 Jahre alt seien. Wissenschaftliche Untersuchungen zur DGS begannen in Deutschland in den 1970er und 1980er Jahren (vgl. Prillwitz 1986, 1988). Die DGS ist eine linguistisch eigenständige Sprache mit einer komplexen Grammatik und dabei unabhängig von der deutschen Lautsprache (vgl. Happ/Vorköper 2006, Papaspyrou et al. 2008, Eichmann et al. 2012). Seit 2002 ist die DGS im Behindertengleichstellungsgesetz (BGG $\$ 6$ ) offiziell als eigenständige Sprache anerkannt (vgl. Kapitel 5.2). In Österreich und dem deutschsprachigen Teil der Schweiz werden andere den Ländern eigene Gebärdensprachen verwendet: die Österreichische Gebärdensprache (ÖGS) und die Deutschschweizer Gebärdensprache (DSGS) (vgl. Skant et al. 2002, Boyes Braem 1995).

\subsection{Gebärdensprachgemeinschaften und Gebärdensprachkultur}

Gebärdensprachgemeinschaften definieren sich primär über eine gemeinsame Kultur, welche essentiell von den jeweils verwendeten Gebärdensprachen der 
visuell-gestischen Modalität geprägt ist. Die Gemeinschaft als Ganzes schließt taube, schwerhörige und hörende Gebärdensprachnutzer(innen) ein, wobei sich unterschiedliche Gruppen durchaus voneinander abgrenzen lassen und Unterkategorien bilden: z. B. taube Muttersprachler(innen), taube Spätlerner(innen), schwerhörige Personen, Children of Deaf Adults (CODAs), Sozialarbeiter(innen), Dolmetscher(innen), andere Nutzer(innen) der DGS, etc. Das konstituierende Merkmal der deutschen Gebärdensprachgemeinschaft und der Gebärdensprachkultur bleibt jedoch dabei immer die DGS und die visuelle Kommunikation. Ohne in diesem Rahmen im Detail auf die Gebärdensprachkultur eingehen zu können, soll hier erwähnt werden, dass es in Deutschland kulturelle Errungenschaften im Bereich Gebärdensprachgeschichte, -kunst, -theater, -poesie, -humor etc. gibt. Diese Kultur ermöglicht über spezifische Konventionen, die von Generation zu Generation weitergegeben werden, eine starke Identifikation (vgl. u. a. KUGG Bundesvereinigung zur Kultur und Geschichte Gehörloser e.V., Vogel 2010).

\section{Translationswissenschaft und Gebärdensprachdolmetschen}

Auch wenn die Translationswissenschaft eine eher junge Disziplin darstellt, so sind doch Übersetzen und Dolmetschen an sich ein uraltes Thema. Translation wird immer da notwendig, wo Menschen unterschiedlicher Sprachen und Kulturen aufeinandertreffen. Wenn diese Kommunikationsparteien nun nicht selbst die Möglichkeit haben, auf die Sprache des anderen einzugehen, werden Sprachmittler(innen) ins Boot geholt. Da dieses Phänomen ein sehr altes ist, wird davon ausgegangen, dass Translation mit zu den ältesten Themen und Tätigkeiten der Menschheitsgeschichte gehört (vgl. Černý 2002: 3, Woodsworth 2006: 39). Dies gilt jedoch nicht in gleichem Maße für die Erforschung derselben. Translationswissenschaft als Disziplin hat sich erst in den letzten Jahrzehnten etabliert (Snell-Hornby 2006: 37f.), auch wenn es in der Geschichte immer wieder Abhandlungen zu translatorischen Themen gab (wie z. B. Luthers Sendschreiben zum Dolmetschen oder Goethes theoretische Abhandlungen zu verschiedenen Formen der Übersetzung) (vgl. Woodsworth 2006: 41f.). 
Auch das Gebärdensprachdolmetschen gilt als eher junge Disziplin. In Deutschland setzte die Professionalisierung und Ausformung eines Berufsbildes erst in den 1970er Jahren ein (vgl. Hillert/Leven 2012: 425). Doch auch hier sind die Themen Taubheit und Gebärdensprache geschichtlich gesehen ebenfalls schon sehr lange bekannt. Nach einer ersten Blütezeit im 18. Jahrhundert mit ersten Gebärdensprachforschungen fristeten die Gebärdensprachen ab Ende des 19. Jahrhunderts ein mehr schlecht als recht geduldetes Dasein. Gebärdensprachen wurden als minderwertig betrachtet und soweit wie möglich aus der öffentlichen Wahrnehmung verbannt. Erst in der Mitte des 20. Jahrhunderts erwachte das wissenschaftliche Interesse an Gebärdensprachen erneut (vgl. Benner 2012: 151-156). Auch in Deutschland entstanden linguistische Forschungsarbeiten zur DGS (vgl. Prillwitz 1988). Damit einher ging eine Emanzipationsbewegung tauber Menschen, die mehr und mehr Mitspracherecht einforderten. Um für ihre Belange einzutreten, wurden Gebärdensprachdolmetscher(innen) benötigt. In diesem Zuge entstanden erste Prüfungen und Ausbildungen, die schließlich in erste Studiengänge mündeten (vgl. Hillert/Leven 2012: 425).

Aus translationstheoretischer Perspektive unterscheidet sich das Gebärdensprachdolmetschen nur unwesentlich vom Dolmetschen in Lautsprachen. Der Aspekt einer zusätzlichen Sprachmodalität sowie die vielfältigen Einsatzbereiche und -situationen liefern jedoch einen interessanten Beitrag zur Translationswissenschaft.

\subsection{Translationswissenschaft}

Der Begriff Translation geht auf das Lateinische translatio zurück, was für Übertragung, Übersetzung oder Umstellung steht, und gilt seit Otto Kade als Oberbegriff für Übersetzen und Dolmetschen (vgl. Snell-Hornby 2006: 37). Wurden die Begriffe Übersetzen und Dolmetschen in den letzten Jahrhunderten eher synonym verwendet, so gibt es seit Kade (1968) eine klare Aufteilung der Begriffe, denn auch wenn Übersetzen und Dolmetschen verwandte Tätigkeiten sind, so gibt es doch sehr markante Unterschiede. Kades Definition ist heutzutage in der Fachwelt unumstritten und so gilt das Übersetzen als Übertragen 
(Translation) eines fixierten Textes. Diese Eigenschaft zieht weitere Eigenschaften nach sich, so wird ein fixierter Text permanent dargeboten und ist demzufolge auch beliebig oft wiederholbar. Damit wird auch die Übertragung aus einer Ausgangssprache in eine Zielsprache, die eigentliche Übersetzung, kontrollierbar und jederzeit korrigierbar. Beim Dolmetschen hingegen wird das Ausgangsprodukt einmalig angeboten, damit ist das Translat auch nur bedingt kontrollierbar und die Korrigierbarkeit ist auch aufgrund des Zeitmangels stark eingeschränkt (vgl. Snell-Hornby 2006: 37). Dennoch hielt sich zunächst noch der Begriff Übersetzungswissenschaft als „Oberbegriff für die Wissenschaft vom Übersetzen und Dolmetschen“ (ebd.: 38). In der Zwischenzeit hat sich aber der Begriff Translationswissenschaft in der Fachwelt etabliert, der in sich eine interdisziplinäre Herangehensweise an das Dolmetschen und Übersetzen vereint (ebd.).

Gerade in dieser begrifflichen Unterscheidung liegt die Herausforderung im Bereich der Gebärdensprachen. Da diese visuellen Sprachen lange Zeit unterdrückte Minderheitensprachen waren und bis heute sind, hat sich bislang kein allgemein verwendetes Schriftsystem herauskristallisiert. Zwar gibt es seit den ersten Forschungen an Gebärdensprachen Vorschläge für Notationssysteme, doch wird bislang keines dieser Systeme im Alltag von einer Mehrzahl der jeweiligen Gebärdensprachnutzer(innen) verwendet. Translation im Bereich Gebärdensprachen spielte und spielt sich von daher nach wie vor primär im Dolmetschbereich ab. Technische Neuerungen ermöglichen zwar auch permanente Fixierungen sprachlicher Äußerungen, doch sind diese nur sehr schwer korrigierbar. Eine direkte Vergleichbarkeit zu Übersetzungen in Schriftsprachen ist hier von daher nicht gegeben. In vielen Fällen handelt es sich am ehesten um eine Mischform zwischen Dolmetschen und Übersetzen. Translationstheoretisch ist dieses Phänomen bisher jedoch kaum erforscht.

Im Bereich der Dolmetschwissenschaften fällt in den Veröffentlichungen der letzten Jahre auf, dass das Thema Gebärdensprachdolmetschen Einzug in die Disziplin gehalten hat (z. B. Gile 2009, Nicodemus/Swabey 2011). Forschung zu Gebärdensprachdolmetschen wird zunehmend ernst genommen und leistet einen wichtigen Beitrag zu Erkenntnisgewinnen innerhalb der Disziplin. Ein Grund hierfür ist, dass Gebärdensprachdolmetschen in so gut wie allen Lebensbereichen vorkommt. Das liegt nicht zuletzt daran, dass ein echter Abbau der 
Kommunikationsbarrieren von tauben Menschen fast ausschließlich über das Gebärdensprachdolmetschen erfolgen kann. So überrascht es auch nicht, dass die Internetseite Taubwissen http://www.taubwissen.de - eine Sammlung von Informationen über die Lebenswelt, Geschichte und Kultur tauber Menschen dieses Thema in den Bereich Alltag verortet (vgl. Taubwissen 2008). Die Unterschiedlichkeit der Einsatzbereiche wie auch die Unterschiedlichkeit der Kommunikationssituationen und -beteiligten bieten aus translationstheoretischer Perspektive interessante Forschungsbereiche. So hat gerade die Auseinandersetzung mit diesen Themen zu neuen Rollen- und Dolmetschmodellen geführt (u.a. Cokely 1995, Dean/Pollard 2011, Llewellyn-Jones/Lee 2013).

Das Verhältnis zum Kunden/zur Kundin findet in der Translationswissenschaft ebenfalls Berücksichtigung (vgl. Gile 2009: 26ff.), dennoch ist dieses Thema innerhalb des Gebärdensprachdolmetschens ungleich brisanter. Als Minderheitensprachen von recht kleinen Sprachgemeinschaften stellt sich immer wieder die Frage, wie Gebärdensprachen kultiviert und gepflegt werden können. Nach wie vor werden Gebärdensprachen von den meisten Nutzer(inne)n zwar als Erstsprachen, nicht zwingend jedoch als Muttersprachen erlernt. Hinzu kommt, dass es bislang nur vereinzelt Schulunterricht im Fach Gebärdensprache gibt. Auch wenn die meisten Gebärdensprachdolmetscher(innen) die jeweilige Gebärdensprache als Zweitsprache erlernen, so haben sie im Gegensatz zu den meisten tauben Menschen doch einen fundierten Sprachunterricht. Dennoch fehlt ihnen häufig die Möglichkeit, vollständig in die Sprache einzutauchen - ein Gehörlosenland gibt es eben nicht (die Ausgangssituation ist in vielen Ländern ähnlich, s. z. B. die Ausführungen von Napier et al. 2010: 5f.). An manchen Stellen wird deswegen innerhalb der Gebärdensprachgemeinschaft von einer „Dolmetscher-DGS“ gesprochen (vgl. Hillert/Leven 2012: 451). Hinzu kommt die Frage, inwieweit sich Dolmetscher(innen) innerhalb der Gemeinschaft beteiligen und engagieren sollen. Involviertheit scheint dabei generell erwünscht, die Frage bleibt jedoch, ab wann es angesagt ist, eine Grenze zwischen Beruflichem und Privatem zu ziehen. Innerhalb einer kleinen Sprachgemeinschaft wird es zwangsläufig dazu kommen, seinen Kunden/Kundinnen über den Weg zu laufen, möchte man sich innerhalb der Gemeinschaft bewegen. Napier et al. (2010) beschreiben das Verhältnis zwischen Dolmetscher(inne)n und der Gebärden- 
sprachgemeinschaft von daher auch als symbiotisch. Beide Parteien sind aufeinander angewiesen: die Dolmetscher(innen) zur Erlangung der sprachlichen und kulturellen Fertigkeiten und die Mitglieder der Gebärdensprachgemeinschaft für eine barrierearme Kommunikation (ebd. 9).

Im Kontext von Minderheitensprachen und einer langen Unterdrückungsgeschichte spielen auch Machtfragen eine nicht zu vernachlässigende Rolle. Häufig sind Gebärdensprachdolmetscher(innen) besser ausgebildet als viele ihrer tauben Kunden/Kundinnen (in den letzten Jahren hat glücklicherweise die Anzahl der sehr gut ausgebildeten tauben Menschen zugenommen). Hinzu kommen Dynamiken innerhalb der Kommunikationssituation und zwischen den Kommunikationspartner(innen)n, die ebenfalls häufig Machtaspekte beinhalten (vgl. Hillert/Leven 2012). Diese Fragen finden zunehmend in Untersuchungen zum Community Interpreting Eingang in die Translationswissenschaft.

\subsection{Formen des Gebärdensprachdolmetschens}

Dolmetschen an sich ist eine hochkomplexe Angelegenheit, die vielfältige Kompetenzen erfordert. Innerhalb der Translationswissenschaft beschäftigt sich deswegen der Bereich Dolmetschwissenschaft dezidiert mit den Anforderungen an das Dolmetschen. Dabei werden verschiedene Aspekte des Dolmetschens beleuchtet. Die Anforderungen können dabei auch je nach Dolmetschart sehr unterschiedlich sein. Im Bereich Gebärdensprachdolmetschen sind drei Arten des Dolmetschens vorrangig im Einsatz: Konsekutivdolmetschen, Simultandolmetschen und das sog. Vom-Blatt-Übersetzen, das schon im Begriff andeutet, dass es sich hier um eine Mischform zwischen Übersetzen und Dolmetschen handelt.

Simultandolmetschen ist sicherlich die innerhalb des Gebärdensprachdolmetschens verbreitetste Dolmetschart. Erfordert Simultandolmetschen in Lautsprachen immer zusätzliche Technik, ermöglichen die unterschiedlichen Modalitäten der beteiligten Sprachen im Gebärdensprachdolmetschen einen komplikationsloseren Einsatz dieser Art. Simultandolmetschen bezeichnet in der Fachwelt das fast gleichzeitige Übertragen der Botschaft. Es wird also sowohl in der Ausgangs- als auch in der Zielsprache kontinuierlich eine Ausgabe produziert. Diese Dolmetschart erfordert also das gleichzeitige Aufnehmen und 
Analysieren sowie Wiedergeben und Überwachen einer Botschaft (Napier et al. 2010: 28, BDÜ o.J.). Diverse Dolmetschmodelle beschäftigen sich von daher auch mit den Anforderungen an das Simultandolmetschen (u.a. Effort Model (vgl. Gile 2009), model for simultaneous conference interpretation nach Setton (vgl. Setton 1999)). Aufgrund der hohen Anforderungen arbeiten in der Regel zwei Dolmetscher(innen) im Team.

Auch wenn das Simultandolmetschen im Gebärdensprachdolmetschen den größten Raum einnimmt, so hat das Konsekutivdolmetschen ebenso seinen Platz. Beim Konsekutivdolmetschen findet die Übertragung in Abschnitten statt. Während der Darlegung der Botschaft durch den Redner/die Rednerin, fertigt der Dolmetscher/die Dolmetscherin Notizen an, die dann die Grundlage seiner Verdolmetschung darstellen (vgl. Andres 2002). Konsekutivdolmetschen kann für manche Dolmetschsituationen geeigneter sein und zur Akkuratesse der Übertragung beitragen. So berichtet Russel (2005: 151), dass im Gerichtskontext konsekutives Dolmetschen zu genaueren Translaten führt. Während des Dolmetschens werden Informationen strukturiert und gewichtet, hierfür muss die dargebotene Botschaft verstanden werden (vgl. Andres 2002: 214). Dazu gehört auch die Intention des Redners/der Rednerin, die leichter zu erfassen ist, wenn ganze Abschnitte dargeboten sind. Auch wenn Konsekutivdolmetschen im Gebärdensprachdolmetschbereich verwendet wird, so kommen Notationen hier deutlich seltener vor (Napier et al. 2010: 27). In Gebärdensprachen spielen der Blickkontakt zwischen Sender/Senderin und Empfänger/Empfängerin eine große Rolle, was das Erstellen von Notizen erschwert (ebd.).

Das Vom-Blatt-Übersetzen (andere Bezeichnungen sind Stegreifübersetzungen, Spontanübersetzung oder im Englischen sight translation) stellt auch im Gebärdensprachdolmetschen eine häufige Variante dar. Dabei „wird ein schriftlicher Text vom Dolmetscher direkt aus dem Stegreif - oder nach einer minimalen Vorbereitungszeit - mündlich übersetzt" (Viljanmaa 2012: 346). Diese Art kommt gelegentlich auch in der Variante Vom-Blatt-Dolmetschen vor, wenn der Text laut verlesen wird. Die Terminologie ist hier jedoch recht uneinheitlich. Dies liegt sicherlich auch daran, dass es sich beim Vom-Blatt-Übersetzen nach Otto Kades Definition weder um reines Dolmetschen noch reines Übersetzen handelt, sondern um die flüchtige Übertragung eines fixierten Textes. In der Translationswissenschaft wird diese Art jedoch zumeist dem Dolmetschen zugerechnet (ebd.). 
Im Berufsalltag eines Dolmetschers/einer Dolmetscherin kommt das Vom-BlattÜbersetzen in vielfältigen Situationen vor: beim Verlesen eines Manuskripts oder Protokolls sowie sehr häufig im medizinischen Kontext beim Ausfüllen von Formularen oder Aufklärungsbögen.

Die Wahl der Dolmetschart hängt maßgeblich von der Kommunikationssituation und den Bedürfnissen der Kommunikationsbeteiligten ab.

\subsection{Gebärdensprachdolmetschen studieren}

Schon bald nach der Etablierung nebenberuflicher Ausbildungen für Gebärdensprachdolmetscher(innen) zeigte sich, dass diese lediglich als Nachqualifizierung bereits erfahrener Dolmetscher(innen) geeignet sind. So entstanden in Deutschland ab den 1990er Jahren verschiedene Dolmetschstudiengänge. 1993/1994 wurde der bundesweit erste Studiengang Gebärdensprachdolmetschen an der Universität Hamburg etabliert. Es folgte 1997 der Studiengang an der Fachhochschule Magdeburg-Stendal sowie 2000 an der Westsächsischen Hochschule Zwickau und 2003/2004 an der Humboldt-Universität zu Berlin (Hillert/Leven 2012: 425). 2015 schließlich richtete die Hochschule Landshut den bislang einzigen Studiengang in Süddeutschland ein und 2017 nahm die Universität zu Köln Gebärdensprachdolmetschen ebenfalls in ihr Programm mit auf. Nebenberuflich kann Gebärdensprachdolmetschen an der Hochschule Fresenius in Idstein studiert werden. Die meisten Studiengänge bieten Gebärdensprachdolmetschen als grundständigen Bachelorstudiengang an (Hamburg, Köln, Landshut und Magdeburg). Vereinzelt wird Gebärdensprachdolmetschen auch als konsekutiver Master angeboten (Berlin, Idstein). An der Hochschule in Zwickau kann Gebärdensprachdolmetschen noch auf Diplom studiert werden. Die Universität Hamburg und die Hochschule in Magdeburg bieten darüber hinaus einen auf den Bachelor aufbauenden Master zur Spezialisierung an.

Problematisch scheint auch an vielen Hochschulen die Zuordnung des Studiengangs. So wird nach wie vor keiner der Studiengänge an Entitäten angeboten, die auch Lautsprachendolmetscher(innen) ausbilden. Insgesamt stehen alle Studiengänge vor der Herausforderung, die Kompetenzen des Dolmetschens innerhalb einer kurzen Zeitspanne aufbauen zu müssen. Nachteilig wirkt sich hier aus, dass Gebärdensprache zumeist nicht als Fremdsprache bereits in der Schule 
erworben werden kann. Die meisten Studienanfänger(innen) bringen daher entweder keine oder nur sehr geringe Sprachkenntnisse mit. Da das Dolmetschen eine hochkomplexe Angelegenheit darstellt, die vielfältiger und differenzierter Kompetenzen bedarf, benötigen Studienanfänger(innen) eine rasche Auffassungsgabe, ein hohes Maß an Reflexionsfähigkeit und -willigkeit, Flexibilität, Belastbarkeit, psychische und physische Stabilität sowie Offenheit. Darüber hinaus sind ein ausgeprägtes Interesse an der Kultur und Gemeinschaft der tauben Menschen sowie Freude am Erlernen einer Fremdsprache unabdingbar.

Da innerhalb kurzer Zeit sowohl Sprach- und Kultur- als auch Dolmetschkompetenzen erworben werden müssen, ist das Studium zumeist sehr zeitintensiv. Neben dem Besuch der Lehrveranstaltungen wird von den Studierenden sehr viel Eigenleistung und individuelles Training erwartet.

\section{Der Beruf Gebärdensprachdolmetscher(in)}

Beruflich ist für die meisten Gebärdensprachdolmetscher(innen) die Arbeit in Selbstständigkeit das Ziel. Es gibt jedoch auch einige Möglichkeiten der Festanstellung an universitären oder institutionellen Einrichtungen wie Beratungsstellen, etc. Beide Tätigkeitsfelder haben eigene Anforderungen und Spezifika in Bezug auf unterschiedliche Bedarfe und Kompetenzen sowohl des Dolmetschers/der Dolmetscherin als auch der Kunden/Kundinnen.

Für selbstständig Arbeitende gibt es verschiedene Landesdolmetscherzentralen für Gebärdensprache, welche die Verteilung von Anfragen organisieren und weiterleiten. Auch landesweite Gehörlosenvereine und einige Berufsfachverbände bieten die Vermittlung von Gebärdensprachdolmetscher(inne)n an. Meist orientieren sich diese Listen an Bundeslandgrenzen, sind bisher nicht deutschlandweit organisiert und nur selten an spezifische Bedarfe der Kunden/Kundinnen angepasst. Der Berufseinstieg im Übergang von Ausbildung und Praktika hin zum Berufsalltag ist häufig von Herausforderungen geprägt. Hier ist eine enge Verzahnung von Ausbildungsstätten und Berufsverbänden wünschenswert, um Bedarfe und Angebote anzupassen und zu verknüpfen. Im Alltag ist der Bedarf an Gebärdensprachdolmetscher(inne)n nach wie vor sehr hoch und die Auftragslage meist gut. Essentiell ist eine gute Teamarbeit mit 
der/dem sogenannten Co-Dolmetscher(in) und eine nachhaltige, sachliche und faire Feedback-Kultur (vgl. Werther 2015), die nach wie vor noch nicht regelmäßig produktiv genutzt wird. Eine stetige Weiterbildung und ggf. Spezialisierung sind für das Berufsbild im Bereich Gebärdensprachdolmetschen unumgänglich, werden meist initiativ durchgeführt und teils auch als Pflichtveranstaltung einer Qualitätskontrolle unterzogen (vgl. Kapitel 4.4).

\subsection{Hörende und taube Gebärdensprachdolmetscher(innen)}

Bisher sind alle BA- und MA-Studiengänge in Deutschland, die Gebärdensprachdolmetscher(innen) ausbilden, für hörende Gebärdensprachdolmetscher(innen) konzipiert. Die Arbeitssprachen dabei sind DGS und Deutsch und je nach Weiterbildung auch DGS, Deutsch und Englisch. Eine Ausbildung zum/zur tauben Gebärdensprachdolmetscher(in) (tGSD) wurde bisher in Hamburg als berufsbegleitende Weiterbildung angeboten, welche mit universitären Modulstrukturen auf die staatliche Prüfung beim Amt für Lehrerbildung (AfL) in Darmstadt vorbereitet (vgl. Mitterhuber 2016). Es gibt tGSD, welche zwischen zwei Gebärdensprachen dolmetschen (z. B. DGS und Türkische Gebärdensprache (TID)), solche, die Verdolmetschungen zwischen DGS und deutscher Schriftsprache anfertigen und auch solche, die z. B. DGS und International Sign jeweils in beide Richtungen als Schwerpunkt haben (vgl. generell zum Thema deaf interpreters auch Boudreault 2005). Mit der staatlichen Prüfung erhalten tGSD das Zertifikat, um als Dolmetscher(in) zu arbeiten. Zentral organisiert sind tGSD im Berufsverband der tauben Gebärdensprachdolmetscher e.V. (TGSD e.V.). Besonders interessant im aktuellen Kontext von Barrierefreier Kommunikation ist das Teamdolmetschen in einem hörenden und tauben GSD-Team, z. B. bei internationalen Zusammenkünften (vgl. Bentley-Sassaman/Dawson 2012). Auch für diese verschiedenen Konstellationen und Settings, in denen hörende und taube Dolmetscher(innen) gleichzeitig und in Abhängigkeit voneinander dolmetschen, ist eine besondere Feedback-Kultur und Reflexion der Teamarbeit essentiell, um langfristig die Zusammenarbeit zu fördern und für die Kunden/Kundinnen sowohl inhaltlich als auch soziokulturell die bestmöglichen Resultate gemeinsam zu erarbeiten (s. auch Kapitel 4.4 zu Supervision). 


\subsection{Berufs- und Ehrenordnung (BEO)}

Die Berufs- und Ehrenordnung (BEO) für Gebärdensprachdolmetscher(innen) wurde 1995 erstmals ins Leben gerufen (vgl. B.A.G. der GebärdensprachdolmetscherInnen 1997). Das aktuell vom BGSD überarbeitete Dokument enthält Leitlinien, Werte und Standards, denen sich ein Dolmetscher/eine Dolmetscherin, welche/-r z. B. in einem Verband angemeldet ist, zur Wahrung des Ansehens des Berufsstandes verpflichtet. Es geht um kollegiales und berufswürdiges Verhalten, die Auftragsabwicklung, das Verhalten gegenüber Kunden/Kundinnen und die Förderung des Vertrauens zwischen allen Beteiligten. Es gibt bisher keine institutionalisierte Einrichtung als Anlaufstelle zur Überprüfung der Einhaltung dieser Richtlinien, so dass die Qualitätskontrolle noch immer alleinig der freiwilligen Selbstverpflichtung unterliegt. Die BEO soll taube und hörende Gebärdensprachdolmetscher(innen) sowie freiberuflich arbeitende und abhängig beschäftigte Personen und Auszubildende einschließen.

\subsection{Dolmetschverbände}

Seit der Professionalisierung des Gebärdensprachdolmetschens haben sich verschiedene berufsständische Vereinigungen herausgebildet. Die meisten sind regional auf Landesebene tätig und gehen auf die Anfänge der Professionalisierung des Berufsstandes zurück. In diesen zu Beginn zumeist als Arbeitsgemeinschaft tätigen Vereinigungen wurden auch die ersten Berufs- und Ehrenordnungen entwickelt (vgl. Kapitel 4.2). Viele der regional tätigen Landesorganisationen haben sich dem Bundesverband der GebärdensprachdolmetscherInnen Deutschlands (BGSD) e.V. angeschlossen, der 1997 aus der Bundesarbeitsgemeinschaft der Gebärdensprachdolmetscher(innen) entstanden war (Hillert/Leven 2012: 426). In Deutschland gibt es die Besonderheit, dass es neben dem BGSD e.V. einen weiteren Nationalverband gibt, den Berufsverband der tauben GebärdensprachdolmetscherInnen (TGSD) e.V., der berufsständischen Interessensvertretung der tauben Gebärdensprachdolmetscher(innen) (vgl. Kapitel 4.1). Beide Nationalverbände sind Mitglied in der europäischen Vertretung EFSLI (European Forum of Sign Language Interpreters), die sich bereits 1987 gegründet hat. Auf internationaler Ebene kümmert sich der Weltverband WASLI (World Association of Sign Language Interpreters) um die 
Belange von Gebärdensprachdolmetschern_innen (eine Übersicht der Verbände findet sich auf http://www.dgsd.de/info/berufsstand/verbaende.html, siehe Braun et al. 2017).

Die Berufsverbände sehen es als ihre primäre Aufgabe, über das Berufsbild aufzuklären und die gesellschaftliche Relevanz des Berufsstandes zu erläutern. Sie setzen sich für einheitliche Regelungen im Umfeld des Dolmetschens ein und engagieren sich für einen reglementierten Berufszugang, eine qualitative Ausund Weiterbildung, angemessene Honorare und Arbeitsbedingungen.

Diese Berufsverbände, aber auch der Bundesverband der Dolmetscher und Übersetzer e.V. (BDÜ) und die Assoziierten Dolmetscher und Übersetzer (ADÜ), sind von daher wichtige Ansprechpartner(innen) von Kostenträgern und Gehörlosenverbänden und treten für ein flächendeckendes Angebot von Gebärdensprachdolmetscher(inne)n ein. Um diese Ziele erreichen zu können, wirken die berufsständischen Vereinigungen auch auf Entscheidungsträger ein.

In Deutschland herrscht in den meisten Regionen ein großer Mangel an Gebärdensprachdolmetscher(inne)n. Viele Einsätze können nicht auf Anhieb besetzt werden. Da die meisten Gebärdensprachdolmetscher(innen) freiberuflich tätig und zumeist sehr ausgebucht sind, kann es sich als schwierig erweisen, auch in Notsituationen Dolmetscher(innen) zur Verfügung zu haben. Einzelne Berufsverbände haben dafür aus Eigeninitiative einen Notfallbereitschaftsdienst etabliert (so z. B. der BGSD Bayern e.V. (BGSD Bayern o.J.)). Dieses Angebot stand jedoch regional nur sehr begrenzt und in zeitlich eingeschränktem Umfang zur Verfügung und wurde Ende Mai 2018 wieder eingestellt.

\subsection{Weiterbildung und Supervision}

Dolmetschen wird von Dean und Pollard (2013: XIII-XIV) auch als practice profession beschrieben. Damit geht einher, dass Erfahrung und Training einen wichtigen Aspekt der Berufsausübung darstellen. Der Erwerb des (akademischen) Titels stellt somit nicht das Ende der Ausbildung eines Gebärdensprachdolmetschers/einer Gebärdensprachdolmetscherin dar, sondern vielmehr den Startschuss. Ständige Weiterbildung gehört von daher zum Selbstverständnis eines Gebärdensprachdolmetschers/einer Gebärdensprachdolmetscherin. Viele berufsständische Vertretungen bieten deswegen auch Weiterbildungen für ihre 
Mitglieder an. In manchen Berufsverbänden besteht darüber hinaus eine Weiterbildungspflicht. Der Umgang mit der Pflicht kann je nach Verband unterschiedlich aussehen. In einigen Verbänden folgt bei Nichterfüllung die Streichung von einschlägigen Listen, über welche Dolmetscher(innen) bestellt werden, in anderen Verbänden wird bei Erfüllung ein Qualitätssiegel verliehen.

Da Gebärdensprachdolmetscher(innen) in ihrer Berufsausübung hohen Belastungen ausgesetzt sind und häufig in schwierigen Kommunikationssituationen eingesetzt werden, hat sich in den letzten Jahren das Bewusstsein entwickelt, dass tätige Dolmetscher(innen) für eine Berufsausübung auf ihre physische und psychische Gesundheit zu achten haben. Dabei hat sich Supervision als ein geeignetes Instrumentarium herausgestellt, um die eigene Belastung zu minimieren und in geschütztem Rahmen die eigene Arbeit reflektieren und damit auch verbessern zu können (vgl. Chase/Omoyele 2017, De Beer/Bruns-Heij 2017). Supervisionsangebote gibt es bislang jedoch nur vereinzelt und auch das Wissen um die Relevanz von Supervision ist bisher sowohl bei den Gebärdensprachdolmetscher(innen)n als auch in der Gebärdensprachgemeinschaft nur am Rande gegeben. Auch hier bedarf es weiterer verstärkter Aufklärungsarbeit (vgl. Benner/Böhm 2017).

\section{$5 \quad$ Barrierefreie Kommunikation für taube und schwerhörige Menschen}

\subsection{Anerkennung der DGS}

Wie bereits in Kapitel 2.1 erwähnt, wurde DGS in Deutschland im Jahre 2002 im Rahmen des Behindertengleichstellungsgesetzes als offizielle Sprache anerkannt. Verschiedene sprach- und neurowissenschaftliche Studien haben seit den 1960er Jahren sukzessive nachgewiesen, dass es sich bei Gebärdensprachen um komplexe und eigenständige Sprachen handelt (u.a. Stokoe 1960, Poizner et al. 1987, Huber/Klann 2005, Campbell et al. 2008). Die linguistische und auch neurologische Beweislage ist dabei eindeutig, die gesetzliche Grundlage für DGS nun ebenfalls. Eine offizielle Anerkennung von DGS als Minderheitensprache in Deutschland ist jedoch bisher politisch nicht erfolgt. 
5.2 Behindertengleichstellungsgesetz (BGG)

Das Gesetz zur Gleichstellung von Menschen mit Behinderungen (Behindertengleichstellungsgesetz - BGG) trat in Deutschland 2002 in Kraft (zur rechtlichen Lage im Bereich der Barrierefreien Kommunikation s. den Beitrag von Lang im vorliegenden Band). Das BGG soll Menschen mit Behinderungen die gleichberechtigte und selbstbestimmte Partizipation am gesellschaftlichen Leben gewährleisten. Im Rahmen dieses Gesetzes wird in $\$ 6$ die Deutsche Gebärdensprache offiziell als eigenständige Sprache anerkannt. Gleichzeitig wird in $\$ 9$ das Recht auf geeignete Kommunikationshilfen, d.h. Gebärdensprachdolmetscher(innen), verankert. Somit haben taube Menschen in Alltag, Ausbildung und Ausübung ihres Berufs das Recht auf Barrierefreie Kommunikation und können dies auch bei entsprechend zuständigen Trägern einfordern. Diese Beantragung ist nach wie vor mit enormen Mühen verbunden, hat jedoch in den letzten Jahren für große Fortschritte im Bereich der Gleichstellung von tauben Menschen gesorgt. Dennoch ist vor allem in verschiedenen beruflichen Bereichen die Abdeckung der Bedarfe über speziell ausgebildete Dolmetscher(innen) sowohl finanziell als auch fachlich nicht immer gegeben. Für eine gleichberechtigte Teilhabe aller Menschen am Leben in unserer Gesellschaft bedarf es weiteren aktiven Einsatzes für gesetzliche und gesellschaftliche Veränderungen.

\subsection{UN-Behindertenrechtskonvention}

Dieses Übereinkommen über die Rechte von Menschen mit Behinderungen (Convention on the Rights of Persons with Disabilities) wurde Ende 2006 von der Generalversammlung der Vereinten Nationen (UN) verabschiedet und trat im Mai 2008 in Kraft (s. dazu auch den Beitrag von Lang im vorliegenden Band). Es beinhaltet sowohl die Bekräftigung der allgemeinen Menschenrechte als auch spezifische Regelungen zu Menschenrechten für Menschen mit Behinderungen. In Artikel 21 geht es um das Recht auf Meinungsfreiheit und Meinungsäußerung, unabhängig von der Kommunikationsform. Das Recht auf Verwendung von Gebärdensprache (u.a. werden dort auch die Braille-Schrift und ergänzende Kommunikationsformen genannt) ist in Artikel 21 explizit verankert. Die Kosten tragen dabei die Behörden. Somit wird auf Ebene der UN im 
Hinblick auf den Anspruch auf Gebärdensprachdolmetscher(innen) der Rahmen gesetzt.

\subsection{Barrierefreie Kommunikation und Gebärdensprachdolmetschen in Alltag, Ausbildung, Beruf, akademischen und internationalen Kontexten}

Taube Menschen stoßen in ihrem Alltag immer wieder auf Kommunikationsbarrieren. Die Verfügbarkeit von Gebärdensprachdolmetscher(inne)n hat in vielen Bereichen dazu beigetragen, diese Barrieren abzubauen. Dabei ist der Einsatz von Gebärdensprachdolmetscher(inne)n sehr vielfältig. Die Einsätze decken die ganze Vielfalt menschlichen Lebens ab: ob Telefondolmetschen für alltägliche Besorgungen, ob Arzttermine, Elterngespräche oder Teamsitzungen, in allen diesen Bereichen tragen Gebärdensprachdolmetscher(innen) zu einer Barrierefreien Kommunikation bei. Gerade im Berufskontext konnten in den letzten Jahren Kommunikationsbarrieren abgebaut und die beruflichen Optionen für taube Menschen erhöht werden. Für taube Menschen gehören Dolmetscher(innen) von daher zu ihrem alltäglichen Leben. Das sieht bei den hörenden Kommunikationspartner(innen)n häufig anders aus. Viele sind mit dem Thema Dolmetschen nicht vertraut (vgl. Napier et al. 2010: 9). Bedenken und Ängste müssen daher häufig erst abgebaut werden.

Auch das Dolmetschen in akademischen Kontexten hat in den letzten Jahren zugenommen. Immer mehr taube Menschen sind akademisch tätig und verwenden Dolmetscher(innen) für ihre akademische Arbeit. Das umfasst auch die Teilnahme an internationalen Konferenzen und das Präsentieren von Vorträgen. Diese Kontexte stellen eine besondere Herausforderung dar, zumal Kenntnisse in der Fachsprache und oft auch in einer weiteren Fremdsprache (zumeist Englisch) notwendig sind. Von daher wird in diesen Kontexten immer eine $\mathrm{Zu}$ satzqualifikation benötigt. Da solche Angebote kaum existieren, nehmen taube Fachleute häufig die Weiterqualifikation in die eigene Hand (vgl. Vorträge von tauben Fachleuten in Deutschland, z. B. im MINT-Bereich). Der Ausbau der Programme für die Abstimmung von professionellen tauben Kunden/Kundinnen und spezialisierten Dolmetscher(inne)n stellt ein Desideratum dar (vgl. Hauser et al. 2015 zum Thema Designated Interpreters). 
5.5 Zuständigkeiten für Barrierefreie Kommunikation mit Gebärdensprachdolmetscher(innen) (Sozialamt, Integrationsamt, Firmen, Schulen, Universitäten, etc.)

Eine weitere Hürde auf dem Weg zur Barrierefreien Kommunikation ist, dass taube Menschen in den meisten Fällen selbst für eine gesicherte Kommunikation sorgen müssen. Zumeist ist es ihre Aufgabe für anstehende Termine nach Gebärdensprachdolmetscher(inne)n zu suchen. Je nach Kontext wird diese Aufgabe auch von Ämtern oder Firmen übernommen. Für taube Studierende oder Eltern inklusiv beschulter tauber Kinder ist jedoch die Organisation von Gebärdensprachdolmetscher(inne)n eine sehr belastende Herausforderung. Häufig gehört neben der Organisation der Termine auch die Kostenklärung. Dazu müssen Anträge gestellt und Bedarfe dargelegt werden. Für eine Barrierefreie Kommunikation in der Gesellschaft bedarf es neuer Konzepte, die aufzeigen, wie Gebärdensprachdolmetscher(innen) möglichst ohne großen Aufwand zur Verfügung gestellt werden können.

5.6 Herausforderungen beim Dolmetschen mit besonderen Gruppen (z. B. Kinder, Senioren/Seniorinnen, Geflüchtete)

Zusätzliche besondere Anforderungen beim Dolmetschen entstehen vor allem im Umgang mit mehrfachen Minderheitengruppen (multiple minorities, vgl. Leigh 2012), wie z. B. taube Kinder, Senioren/Seniorinnen und Geflüchtete. Hierbei sind besondere sprachliche sowie soziale Kompetenzen, jeweilige Erfahrungen und ein spezielles situatives Einfühlungsvermögen gefragt. Aktuelle Arbeiten von Studierenden und internationale Projekte beschäftigen sich u. a. mit den Herausforderungen für Dolmetscher(innen) im Umgang mit inklusiv beschulten Schulkindern oder dem Sprachgebrauch von tauben Senioren/ Seniorinnen, die durch die orale Erziehung bis heute häufig eine spezielle Mischform aus Sprechen, lautsprachbegleitenden Gebärden (LBG) und DGS verwenden. Auf der BEO-Tagung im Januar 2018 in Berlin wurde darüber diskutiert, zusätzlich zur allgemeinen BEO ergänzende Verhaltensrichtlinien für den Umgang mit den jeweiligen Gruppen zu etablieren und zu verschriftlichen. 


\section{Zusammenfassung: Diversität als Chance}

Gebärdensprachdolmetschen ist ein offiziell anerkanntes Berufsfeld, das sich aufgrund der derzeitigen hohen Bedarfe enormer Nachfrage erfreut. Es gibt deutschlandweit systematische Gebärdensprachdolmetscherausbildungen und -studiengänge, entsprechende Verbände und verschiedene Spezialisierungsmöglichkeiten. Gebärdensprachdolmetschen ist eine sowohl kognitive als auch sozial-kommunikative Herausforderung in Abhängigkeit von der Art der Verdolmetschung (simultan, konsekutiv, vom Blatt), den jeweiligen Bedarfsanforderungen in verschiedenen Situationen und den jeweiligen Kunden/Kundinnen. Gesetze und rahmengebende Regelungen bzgl. des Rechts auf Verwendung von Gebärdensprache als Kommunikationsform (BGG, UN-Konvention) ermöglichen mittels tauber und hörender Gebärdensprachdolmetscher(innen) die gesellschaftliche Teilhabe tauber Menschen in Deutschland. Somit werden zudem diverse Kompetenzen und gesellschaftlich relevante Qualifikationen tauber Menschen sichtbar. Eine starke deutschlandweite, aber auch internationale Vernetzung, standardisierte Richtlinien und eine konstruktive Feedback- und Supervisions-Kultur tragen zu einer Barrierefreien Kommunikation innerhalb der Gebärdensprachgemeinschaft bei. 


\section{Literaturverzeichnis}

ANDRES, DÖRTE (2002): „Die Funktion der Notation im Konsekutivdolmetschen“. In BeSt J./KALINA, S. (Hg.): Übersetzen und Dolmetschen: eine Orientierungshilfe. UTB. Tübingen: A. Francke. S. 209-215.

Armstrong, David F./Stokoe, William C./Wilcox, Sherman E. (1995): Gesture and the nature of language. Cambridge: Cambridge University Press.

Armstrong, David F./WilcoX, Sherman E. (2003): „Origins of sign languages“. In: Marschark, Marc/Spencer, Patricia E. (Hg.): Oxford handbook of deaf studies, language, and education. Oxford: Oxford University Press. S. 305-317.

Aronoff, MARK/Meir, IRIT/SANDler, Wendy (2005): „The paradox of sign language morphology“. In: Language 81:2, S. 301-344.

B.A.G. der GebärdensprachdolmetscherInnen (1997): „Berufs- und Ehrenordnung für GebärdensprachdolmetscherInnen“. In: Das Zeichen 11:39, S. 106-107.

BDÜ (o.J.), „Berufsbild Dolmetscher“. In: http://bdue.de/fuer-auftraggeber/derberuf/dolmetscher/ (letzter Zugriff: 26.02.2018).

BGG (2002): Gesetz zur Gleichstellung von Menschen mit Behinderungen. Behindertengleichstellungsgesetz vom 27. April 2002 (BGBl. I S. 1467, 1468).

BeLlugi, URSUla/FISCHER, SUSAN (1972): „A comparison of sign language and spoken language“. Cognition 1/2-3, S. 173-200.

BenNer, UtA E. (2012): Phonological processing of German sign language. Dissertation.

Benner, UtA E./BÖHM, CARMEN (2017): „Supervision von Gebärdensprachdolmetscher*innen - Anspruch und Berufsalltag einer jungen Profession“. In: Das Zeichen 31:106, S. 318-326.

Bentley-Sassaman, Jessica/DaWson, Christina (2012): „Deaf-Hearing Interpreter Teams: A Teamwork Approach“. In: Journal of Interpretation 22/1, S. 1-33.

BGSD Bayern e.V. (o.J.): „Notfallprojekt“. In: http://www.bgsd-bayern.de/Notfallprojekt/ (letzter Zugriff: 26.02.2018).

Boudreault, Patrick (2005): „Deaf interpreters“. In: Terry JANZEN (Hg.): Topics in signed language interpreting. Theory and practice. Amsterdam: Benjamins, S. 323355.

Boyes BRaem, Penny (1995): Einführung in die Gebärdensprache und ihre Erforschung. Seedorf: Signum.

Braun, Nicole/Kießing, Jette/Kirketerp, Christina/Paul, Ann-Katrin/WestPHAL, JENS (2017): „Berufsständische Vertretungen von GebärdensprachdolmetscherInnen in Deutschland“. In: http://www.dgsd.de/info/berufsstand/verbaende.html (letzter Zugriff: 26.02.2018).

CAMPBell, RUth et al. (2008): „Sign Language and the Brain: A Review“. In: Journal of Deaf Studies and Education 13, S. 3-20. 
ČERNÝ, LOTHAR (2002): „Zwischen den Zeichen: Zur Geschichte der Übersetzungstheorie“. In: Best, JoAnna/ Kalina, Sylvia (Hg.): Übersetzen und Dolmetschen: eine Orientierungshilfe. UTB Tübingen: A. Francke, S. 3-16.

Chase, Lyn/Omoyele, Thomas (2017): „Warum Gebärdensprachdolmetscher_innen professionelle Supervision brauchen“. In: Das Zeichen 31:107, S. 486-495.

COKELY, DenNis (1995): Gebärdensprach-Dolmetschen: ein soziolinguistisches Modell. Dt. Erstausg. Hamburg: Signum.

DeAn, Robyn K./Pollard, Robert Q. (2011): „Context-based Ethical Reasoning in Interpreting: A Demand Control Schema Perspective“. In: The Interpreter and Translator Trainer 5/1, S. 155-182.

DeAn, Robyn K./POllard, ROBERT Q. (2013): The demand control schema: interpreting as a practice profession. North Charleston, SC: CreateSpace.

De Beer, Winnie/Bruns-Heij, Anke (2017): „Professionalität stärken durch Gruppensupervision - An unterschiedlichen Perspektiven wachsen“. In: Das Zeichen 31: 107, S. 496-505.

Eichmann, Hanna/Hansen, Martje/Heßmann, Jens (Hg.) (2012): Handbuch Deutsche Gebärdensprache. Sprachwissenschaftliche und anwendungsbezogene Perspektiven. Seedorf: Signum.

Gile, DANiEL (2009): Basic concepts and models for interpreter and translator training. Rev. ed. Amsterdam; Philadelphia: John Benjamins Pub. Co.

Happ, Daniela/VorköPer, Marc-Oliver (2006): Deutsche Gebärdensprache. Ein Lehr- und Arbeitsbuch. Frankfurt: Fachhochschulverlag.

Hauser, Peter C./Finch, Karen L./Hauser Angela B. (Hg.) (2015): Deaf Professionals and Designated Interpreters: A New Paradigm. Gallaudet University Press; 1 Edition.

Hillert, Gudrun/Leven, Regina (2012): „Gebärdensprachdolmetschen“. In: EicHmann, Hanna/Hansen, Martje/Heßmann, Jens (Hg.): Handbuch Deutsche Gebärdensprache. Sprachwissenschaftliche und anwendungsbezogene Perspektiven. Internationale Arbeiten zur Gebärdensprache und Kommunikation Gehörloser. Seedorf: Signum, S. 425-453.

Huber, WALter/KlAnN, Juliane (2005): „Zerebrale Repräsentation der Gebärdensprache“. In: Leuninger, Helen/Happ, DAniela (Hg.): Gebärdensprachen: Struktur, Erwerb, Verwendung. Linguistische Berichte Sonderheft 13. Hamburg: Buske, 359-380.

Kade, Отто (1968): „Zufall oder Gesetzmäßigkeit in der Übersetzung“. Beihefte zur Zeitschrift Fremdsprachen Bd.1. Leipzig: VEB Verlag Enzyklopädie.

Klima, Edward S./Bellugi, Ursula (1979): The Signs of Language. Cambridge, MA: Harvard University Press.

KUGG (o.J.): Bundesvereinigung zur Kultur und Geschichte Gehörloser e.V., https://www.kugg.de/ 
Leigh, Irene W. (2012): „Not Just Deaf: Multiple Intersections“. In: NetTles, Reginald/Balter, Rochelle (Hg.): Multiple Minority Identities: Applications for Practice, Research, and Training. New York, NY: Springer, S. 59-80.

Llewellyn-Jones, Peter/Lee, Robert G. (2013): „Getting to the Core of Role: Defining Interpreters’ Role Space“. In: International Journal of Interpreter Education 5/2, S. 54-72.

MEIER, Richard P. (2002): „Why different, why the same? Explaining effects and noneffects of modality upon linguistic structure of sign and speech“. In: MEIER, RICHARD P./CORMIER, KeARsY/Quinto-Pozos, DAVID (Hg.): Modality and structure in signed and spoken languages. Cambrigde: Cambridge University Press, S. 1-25.

Mitterhuber, Thomas (2016): „Die Teamplayer von morgen“. In: Deutsche Gehörlosen Zeitung 144/10, S. 6-10.

Napier, Jemina/McKee, RaChel Locker/Goswell, Della (2010): Sign language interpreting: theory and practice in Australia and New Zealand. 2nd ed. Annandale, N.S.W: Federation Press.

NICODEMUS, BRENDA/SWABEY, LAURIE (2011): Advances in interpreting research: inquiry in action. Amsterdam; Philadelphia: John Benjamins Pub. Co.

Papaspyrou, Chrissostomos/von Meyenn, Alexander/Matthaei, Michaela/ Herrmann, Bettina (Hg.) (2008): Grammatik der Deutschen Gebärdensprache aus der Sicht gehörloser Fachleute. Seedorf: Signum.

Perniss, Pamela/Pfau, Roland/Steinbach, Markus (Hg.) (2007): Visible variation. Comparative studies on sign language structure. Trends in Linguistics. Berlin: Mouton de Gruyter.

Poizner, Howard/Klima, Edward/Bellugi, Ursula (1987): What the hands reveal about the brain. Cambridge, MA: MIT Press.

PRILLWITZ, SiEGMUND (1986): „Informationsbrief zum Thema: Die Deutsche Gebärdensprache und die Verwendung von Gebärden in der Erziehung Gehörloser“. In: PRILLWITZ, Siegmund (Hg.): Die Gebärde in Erziehung und Bildung Gehörloser. Internationaler Kongreß am 9. und 10. November 1985 im Congress Centrum Hamburg. Tagungsbericht. Hamburg: Verlag hörgeschädigte Kinder, S. 19.

PRILLWITZ, SiEGMUND (1988): Skizzen zu einer Grammatik der Deutschen Gebärdensprache. 2. Aufl. Hamburg: Forschungsstelle Deutsche Gebärdensprache.

RUSSEL, DEBRA (2005): „Consecutive and simultaneous interpreting“. In: JANZEN, TERRY (Hg.): Topics in signed language interpreting: theory and practice. Amsterdam; Philadelphia, Pa.: Benjamins, S. 135-164.

SETTON, RoBIN (1999): Simultaneous interpretation: a cognitive-pragmatic analysis. Amsterdam: Benjamins.

Skant, Andrea/Dotter, Franz/Bergmeister, Elisabeth/Hilzensauer, Marlene/ Hobel, Manuela/Krammer, Klaudia/OKorn, Ingeborg/Orasche, Christian/ ORTER, REINHOLD/UNTERBERGER, NATALIE (2002): Grammatik der Österreichischen 
Gebärdensprache. Vol. 4. Klagenfurt: Veröffentlichungen des Forschungszentrums für Gebärdensprache und Hörgeschädigtenkommunikation.

SNelL-Hornby, MARY (2006): „Translation (Übersetzen / Dolmetschen) / Translationswissenschaft / Translatologie“. In: Snell-Hornby, Mary/HÖnIG, Hans G./Kußmaul, Paul/Schmitt, Peter A. (Hg.): Handbuch Translation. Stauffenburg Handbücher. Tübingen: Stauffenburg Verl., S. 37-38.

Stokoe, William C. (1960): „Sign Language Structure. An Outline of the Visual Communication System of the American Deaf. Studies“. In: Linguistics Occasional Papers 8. Buffalo: University of Buffalo Press.

Taubwissen (2008): „Gebärdensprachdolmetscher“. In: http://www.taubwissen.de (letzter Zugriff: 25.02.2018).

VilJANMAA, ANU (2012): „Vom-Blatt-Übersetzen aus studentischer Perspektive - Erfahrungen eines VBÜ/VBD-Kurses“. In: NISSILÄ, N./ SIPONKOSKI N. (Hg.): VAKKI Symposium, Kielet liikkeessä, Språk i rörelse, Languages in Motion, Sprachen in Bewegung. VAKKI Publications, 1. Vaasa, S. 346-357.

Vogel, Helmut (2010): „Taubsein: Vom Aufbruch einer Bewegung“. In: Magazin MENSCHEN 2, S. 52-55.

WERTHER, SIMON (2015): Einführung in Feedbackinstrumente in Organisationen. Vom $360^{\circ}$-Feedback bis hin zur Mitarbeiterbefragung. Wiesbaden: Springer.

WILBUR, RONNIE B. (2000): „Phonological and prosodic layering of nonmanuals in American Sign Language“. In: Emmorey, Karen/LAne, Harlan (Hg.): The Signs of Language revisited: Festschrift für Ursula Bellugi and Edward Klima. Hillsdale, NJ: Lawrence Erlbaum Associates, S. 213-244.

Woll, Bencie (1983): Language in Sign: An International Perspective on Sign Language. London: Croom Helm.

Woodsworth, Judith (2006): „Geschichte des Übersetzens“. In: SNELL-Hornby, Mary/Hönig, Hans G./KußMaul, Paul/Schmitt, Peter A. (Hg.): Handbuch Translation. Stauffenburg Handbücher. Tübingen: Stauffenburg Verl., S. 39-43. 


\section{Community Interpreting zur Überwindung von Kommunikationsbarrieren für Personen mit Deutsch als Zweitsprache: Einsatzbereiche, Anforderungen, Professionalisierung}

\section{$1 \quad$ Einführung}

Wir können heutzutage in Deutschland nicht mehr abstreiten, dass wir in einer Migrationsgesellschaft leben, in der Tausende von Migrant(inn)en aufgrund der vorhandenen Sprachbarrieren große Schwierigkeiten bei ihrer Integration haben. Das Zuwanderungsgesetz von 2005 sieht zwar unterschiedliche Mechanismen zur Verbesserung einer aktiven Integration der neu Zugewanderten vor, die allesamt sinnvoll und notwendig sind, die jedoch erst längerfristig greifen können (Integrations- und Sprachkurse). Nicht vorgesehen in dem Gesetz sind aber Angebote, die von Anfang an unterstützend wirken und dem Abbau von Sprachbarrieren für diese Bevölkerungsgruppe mit Deutsch als Fremd- oder Zweitsprache in konkreten Alltagssituationen dienen. Texte aus dem Verwaltungsbereich wie Anträge oder Formulare bergen besonders am Anfang des Aufenthalts in Deutschland oft schwer zu überwindende Kommunikationsbarrieren (s. den Beitrag von Rink im vorliegenden Band), die zusammen mit dem Mangel an kulturellem Wissen für die meisten Migrant(inn)en erst nach vielen Jahren Aufenthalt und intensiver Auseinandersetzung mit der deutschen Sprache und Kultur abgebaut werden können. Überdies hat die Migrationslinguistik in zahlreichen Studien gezeigt, dass ein gewisser, häufig nicht kleiner Teil der Migrant(inn)en der Akkulturationsstrategie der Separation folgt und sich sprachlich wie kulturell nicht in das Gastland integriert. Dies ist für alle Migrationsgesellschaften $\mathrm{zu}$ konstatieren, wenn auch in unterschiedlich starkem Maße (Estévez Grossi 2018). 
Vor diesem Hintergrund und angesichts der Tatsache, dass in den letzten Jahren mehr Flüchtlinge und Migrant(inn)en aus den unterschiedlichsten Ländern nach Deutschland gekommen sind als in den Jahren davor, steht der Staat hinsichtlich der sprachlichen Hürden, vor denen die Migrant(inn)en stehen, vor einer immensen organisatorischen Herausforderung. Zwar werden Sprach- und Orientierungskurse angeboten, jedoch sind die Absolvent(inn)en dieser Kurse nicht direkt in der Lage, sprachlich alle Situationen zu meistern, die auf sie zukommen: In seinem „Bericht zur Integrationskursgeschäftsstatistik für das Jahr 2017“ legt das Bundesamt für Migration und Flüchtlinge (BAMF) dar, dass nur ca. die Hälfte der Teilnehmenden an den Integrationskursen die Prüfung für das Niveau B1 bestehen (BAMF 2017); ob das Sprachniveau B1 ausreicht, um die anfallende Kommunikation im behördlichen Kontext erfolgreich bestreiten zu können, sei einmal dahin gestellt. In konkreten Interaktionssituationen beispielsweise mit deutschen Behörden werden folglich Sprachmittler(innen) benötigt, die für eine gelingende Interaktion schriftliche oder mündliche Kommunikate übersetzen oder verdolmetschen. Auf das Fehlen geeigneter Sprachmittler(innen) oder Dolmetscher(innen) (in diesem Beitrag der Einfachheit halber als Synonyme gebraucht) für Sprachkombinationen, die bislang nicht oft benötigt wurden und die jetzt überproportional oft nachgefragt werden (Bsp. Arabisch, Paschtunisch etc.), muss in der Regel schnell und mit kurzfristig praktikablen Lösungen reagiert werden. Bereits in Deutschland lebende Flüchtlinge und Migrant(inn)en, die der deutschen Sprache durch ihren langjährigen Aufenthalt und den Besuch von Sprach- und Integrationskursen mächtig sind, stellen ein Potential dar, das man für diese Aufgabe nutzen könnte, indem man sie bspw. gezielt für diese sprachmittlerischen Aufgaben schult. Aufgrund fehlender Dolmetscher(innen) in bestimmten Sprachkombinationen kommt es überdies immer häufiger vor, dass nicht professionelle ehrenamtliche Sprachmittler(innen) als Dolmetscher(innen) zwischen deutschen Behörden und ausländischen Mitbürger(inne)n eingesetzt werden.

Beide Gruppen - sowohl professionelle als auch Laiendolmetscher(innen) benötigen für einen erfolgreichen Einsatz Wissen und Fertigkeiten, die über eine professionelle Ausbildung erworben werden. Neben den bereits etablierten universitären übersetzungs- und dolmetschwissenschaftlichen Ausbildungen, wie es sie seit Jahrzehnten an mehreren deutschen Universitäten gibt, gilt es 
daher, gezielte, punktuelle und praktikable Lösungen, die bedarfsorientiert konzipiert werden müssen, zu entwickeln und umzusetzen. Da curriculare Entwicklungsprozesse an den Hochschulen und anderen öffentlichen Bildungseinrichtungen langwierig und komplex sind, ist es angesichts der gegenwärtigen politischen Entwicklung an den betroffenen städtischen und anderweitigen Einrichtungen, Abhilfe zu schaffen und kurzfristig alternative Schulungsvorschläge durchzuführen, um dieser besonderen Lage Herr zu werden.

Im vorliegenden Beitrag soll zunächst der Begriff „Community Interpreting“ im Kontext alternativer, angrenzender und konkurrierender Begrifflichkeiten erläutert und Tätigkeiten sowie das Arbeitsfeld des Community Interpreters in Abgrenzung von anderen Dolmetscharten näher bestimmt werden. Im nächsten Schritt werden die Bereiche und Einsatzfelder des Community Interpreting mit einem Schwerpunkt auf der Behördenkommunikation vorgestellt. Schließlich wird der Blick auf die Notwendigkeit der Professionalisierung der Dolmetscher(innen) gelegt und es werden Wege zum Erreichen dieses Ziels aufgezeigt.

\section{Community Interpreting: eine Begriffsbestimmung}

Die Bezeichnungen Übersetzen und Dolmetschen sind Begriffe, die bereits seit dem Altertum bekannt sind. In den letzten Jahren haben jedoch sowohl die Tätigkeitsbereiche von Übersetzer(inne)n und Dolmetscher(inne)n große Veränderungen erfahren, als auch die damit einhergehenden vielfältigen Bezeichnungen, die hier zum Einsatz kommen. Für den hier beschriebenen Gegenstandsbereich sind das u.a. die folgenden:

Konferenzdolmetschen, Community Interpreting, Gerichtsdolmetschen, Konsekutiv-dolmetschen, Behördendolmetschen, Bilaterales Dolmetschen, Soziales Dolmetschen, Sprachmittlung, Kommunales Dolmetschen, Liaison Interpreting, Interkulturelles Übersetzen, Verhandlungsdolmetschen, Geschäftsdolmetschen, Dialogdolmetschen, Cultural Interpreting, Contact Interpreting, Public Service Interpreting, Ad hoc Dolmetschen, Sprach- und Integrationsmittlung, Kulturvermittlung, 
Krankenhaus-Dolmetschen, Medizinisches Dolmetschen, Escort Interpreting, Retour Dolmetschen, Delegations-Dolmetschen, Begleit- oder Betreuungsdolmetschen, Business Interpreting, Media Interpreting, Telephone Interpreting, Schriftdolmetschen, Flüsterdolmetschen, Sign Language Interpreting ...

Diese Aufzählung ließe sich noch um weitere Begriffe ergänzen; die in diesen Bezeichnungen vereinzelt genannten Arbeitsbereiche (z. B. Krankenhaus, Gericht, Business, Konferenz, Behörde) lassen Rückschlüsse auf den Kontext zu, in dem diese Dolmetschtätigkeit jeweils ausgeführt wird. So wird ein(e) Konferenzdolmetscher(in) vorrangig in internationalen Konferenzen oder Kongressen dolmetschen, während der Gerichtsdolmetscher in einer Gerichtsverhandlung mit ausländischen Zeugen oder Angeklagten dolmetschen wird usw. Andere Bezeichnungen wiederum nehmen Bezug auf die Art und Weise, d.h. die Modalität, in der gedolmetscht wird (z. B. ad hoc, retour, bilateral, konsekutiv).

Einigkeit herrscht dagegen bezüglich der Zielgruppe dieser Dolmetschart:

Community Interpreting enables people who are not fluent speakers of the official language(s) of the country to communicate with the providers of public services so as to facilitate full and equal access to legal, health, education, government, and social services. (Carr et al. 1995, zit. nach Mikkelson 1996)

Community Interpreting ermöglicht es demzufolge all jenen Personen, die die offizielle(n) Sprache(n) des Landes nicht beherrschen, mit den Anbietern öffentlicher Dienstleistungen zu kommunizieren und auf diese Weise idealerweise gleichberechtigten Zugang zu Leistungen im Gesundheits-, Rechts-, Verwaltungs- und Sozialwesen sowie im Bildungsbereich zu erlangen.

Bei den Studien zum Community Interpreting handelt es sich um eine relativ neue Disziplin innerhalb der Translationswissenschaften, der sich die Forschungsgemeinschaft erst seit den 1990er Jahren verstärkt widmet (für einen umfassenden Überblick s. Estévez Grossi 2018). Der Auftakt einer wissenschaftlichen Beschäftigung mit dem Community Interpreting kann auf das Jahr 1995 
datiert werden, als die erste Konferenz von The Critical Link in Kanada abgehalten wurde (vgl. Pöchhacker 2004: 41-42).

Bowen (1998: 319) definiert Community Interpreting als „das Dolmetschen für Einzelpersonen oder Kleingruppen (Familien) [...], meist Einwanderer, Flüchtlinge oder Wanderarbeiter, für Gespräche bei Behörden und Sozialämtern, auch in Schulen, im Gesundheitswesen usw. des Aufnahmelandes“. Der Begriff wurde durch eine Arbeitsgruppe des Instituts of Linguists in London geprägt und entstand in Anlehnung an den Ausdruck community work, der in den USA für unterschiedliche Dienstleistungen durch Laien verwendet wird (vgl. Bowen 1998: 319). Allerdings werden in verschiedenen Ländern unterschiedliche Bezeichnungen für dieses Phänomen verwendet, die sich in ihrem Begriffsumfang erheblich voneinander unterscheiden. So finden wir im englischsprachigen Raum vorwiegend Begriffe wie community interpreting, cultural interpeting, ad hoc interpreting, contact interpreting, public service interpreting, dialogue interpreting, liaison interpreting, escort interpreting, legal interpreting, medical interpreting usw. (vgl. Gentile et al. 1996: 110; Roberts 1997: 8). In Skandinavien finden wir die Begriffe contact interpreting oder dialogue interpreting (Wadensjö 1998), die interessanterweise auch das Gerichtsdolmetschen einschließen. In Spanien finden sich unter anderem die Begriffe interpretación social, interpretación comunitaria und interpretación en los servicios públicos, wobei sich letzterer Terminus im akademischen Bereich durchzusetzen scheint (vgl. Abril 2006: 24). Etwas komplexer ist diesbezüglich die Situation im deutschsprachigen Raum. Neben Community Interpreting wird in Deutschland auch der Begriff „Behördendolmetschen“verwendet, der nicht nur das Dolmetschen in sozialen Einrichtungen umfasst, sondern auch das Dolmetschen in Gesundheits- und Bildungseinrichtungen. In Österreich dagegen wird in diesem Kontext hauptsächlich von „Kommunaldolmetschen“ gesprochen, ein von Pöchhacker vorgeschlagener und inzwischen standarisierter Begriff (Pöchhacker 2000).

Daneben findet sich der Begriff der Sprach- und Kulturmittlung, der je nach Autor(in) oder Anwendungsbereich unterschiedlich definiert und verwendet wird. Oft wird die Sprachmittlung als ein übergeordneter Begriff des interlingualen Übersetzens und Dolmetschens betrachtet (Estévez Grossi 2018: 61). Andererseits wird durch die Kulturmittlung den soziokulturellen Unterschieden 
zwischen den betreffenden Kulturen und der interkulturellen Kommunikation Rechnung getragen und dadurch die Klärung kultureller Missverständnisse ermöglicht. Durch den Begriff „Mittlung“ wird gerade im Bereich des Community Interpreting der Fokus auf die Beihilfe zur Beseitigung von Konflikten seitens der Person gelegt, die die sprach- und kulturmittlerische Tätigkeit ausübt (vgl. Pöchhacker 2008: 18). In anderen Ländern wie z. B. Italien sind die Berufsbezeichnungen und Aufgaben der Mediator(inn)en und Dolmetscher(innen) streng voneinander getrennt und genau definiert. Die Schweiz verwendet ihrerseits besonders die Bezeichnung „Interkulturelles Übersetzen“. Auch hier wird der Fokus zum einen auf die interkulturelle Kommunikation und damit auf die sprachliche Vermittlung zwischen unterschiedlichen Kulturen gelegt; zum anderen soll durch das „Übersetzen“ auch die Schriftlichkeit in den Vordergrund gerückt werden, da die in den verschiedenen Einrichtungen tätigen Dolmetscher(innen) sehr häufig auch schriftliche Dokumente spontan (aus dem Stegreif) und „vom Blatt übersetzen“ müssen.

Aus dieser Begriffsvielfalt resultiert eine uneinheitliche Verwendung der Termini für diese Form der Sprachmittlung. Wie Ozolins (2010: 200ff.) anmerkt, handelt es sich hierbei keineswegs um ein triviales Problem. Die terminologische Vielfalt ist vielmehr als ein Ausdruck der Bemühungen zu sehen, diese Disziplin und die damit einhergehenden Aufgaben genauer zu definieren und von anderen benachbarten abzugrenzen. Bislang ist in dieser Hinsicht noch kein Konsens erreicht. Die Suche nach „Einheit innerhalb der Vielfalt" (Pöchhacker 1999: 127) sollte sich allerdings nicht auf die Bezeichnungsproblematik allein konzentrieren. Vielmehr stellt es ein Desiderat dar, Einsichten bezüglich des beruflichen Profils und der Aufgabenbeschreibung sowie der genauen Bestimmung der Voraussetzungen für eine Professionalisierung zu gewinnen.

Um jedoch diese Professionalisierung voranzutreiben, muss es einen zumindest grundsätzlichen Konsens darüber geben, was die Aktivität des Community Interpreting ausmacht und wie sie zumindest annäherungsweise definiert werden kann. Zu diesem Zweck müssen die dem Community Interpreting inhärenten Eigenschaften charakterisiert und systematisiert werden, damit Bildungsund Professionalisierungsziele klar formuliert und Qualitätsmerkmale festgelegt werden können. Eines der Hauptmerkmale von Community Interpreting ist ihr interdisziplinärer Charakter. Entsprechend ist jede sprachmittlerische 
Handlung auch als komplexe soziokommunikative Interaktion zu verstehen, die keineswegs innerhalb einer einzigen und gar stagnierenden Disziplin untersucht werden kann. Estévez Grossi (2018: 63ff.) verweist auf verschiedene Disziplinen, innerhalb derer Studien über Community Interpreting betrieben werden können und zitiert Wadensjös Begriff der dialogic discourse-based interaction. Wadensjö (1998) sucht die Rolle des Sprachmittlers durch Rückgriffe auf Ansätze aus der Soziologie und der Angewandten Sprachwissenschaft (bspw. Diskursanalyse) zu ergründen (Estévez Grossi 2018: 64f.). Auch Vargas Yupi (2011) zählt in seinem Artikel „The Interdisciplinary Approach in Community Interpreting Research“ fünf Disziplinen auf, deren Instrumente und Konzepte auf Forschungsarbeiten des Community Interpreting angewandt werden können: die Anthropologie, die Soziologie, die Angewandte Sprachwissenschaft, die Kommunikationswissenschaften und die Psychologie.

Der österreichische Berufsverband für Dolmetscher und Übersetzer definiert in Anlehnung an die AMS-Broschüre „Jobchancen - Studium - Sprachen“ (www.ams.at/biz) das Berufsbild des Community Interpreters folgendermaßen:

Community Interpreters ermöglichen Menschen, deren Muttersprache nicht die des Gastlandes ist, den Zugang zu öffentlichen Einrichtungen [...]. Sie übersetzen und dolmetschen im Dienst der Gemeinschaft für Einzelpersonen oder Kleingruppen (Familien), meist für MigrantInnen oder Flüchtlinge, bei Gesprächen mit Behörden und Sozialämtern, in Schulen oder in Institutionen des Gesundheitswesens. Besonders gefragt sind dabei DolmetscherInnen mit seltenen Sprachkombinationen. (AMS-Broschüre 2015: 62)

Pöchhacker geht noch näher auf den Tätigkeitsbereich des Community Interpreters ein, den er wie folgt ausweitet:

Community Interpreting bezieht sich [...] auf vermittelte Kommunikation in jeder Art von 'Institution' - im weitesten Sinn: nicht nur auf Ämter und Behörden, Gerichte und Krankenhäuser, sondern etwa auch auf Beratungsstellen 'privater' Vereine, Arztpraxen und Anwaltskanzleien, ja 
sogar auf kommerzielle Einrichtungen. Es geht also prinzipiell um Dolmetschen in kommunalen Institutionen, in jeglichen Situationen des Alltagslebens in der Gemeinde (Kommune). (Pöchhacker 1997: 52)

Gentile et al. (1996: 117) hatten bereits Ende der 1990er Jahre betont, dass der Terminus im Hinblick auf die vielen Tätigkeitsbereiche, die er umfasse, zu allgemein sei. Einen Beleg für diese Annahme sehen wir auch in den zahlreichen Bezeichnungen im englischsprachigen Raum (public service interpreting, community interpreting, dialogue interpreting usw., s.o.), wobei die Gemeinsamkeit der Begriffe darin bestehe, dass die Benennungen eine Dolmetschtätigkeit beschreiben, die als Schauplatz nicht die Konferenz habe (vgl. Roberts 1997: 8).

Ein weiteres wichtiges unterscheidendes Merkmal des Community Interpreters, insbesondere im Hinblick auf die interkulturellen Anforderungen, ist ferner die beträchtliche Bedeutung des Faktors Kultur, die Andres wie folgt darstellt:

Die Erkenntnis der besonderen Relevanz des Faktors Kultur in der Vermittlung unterscheidet das Community Interpreting vom Gerichtsdolmetschen, bei dem nach wie vor der Akzent auf der Übertragung von Worten liegt, die Vermittlung kultureller Unterschiede jedoch nur ein, häufig sogar umstrittener, Faktor unter anderen ist. (Andres 2008: 404f.)

Auch andere Autor(inn)en sehen die Vermittlung zwischen den Kulturen als besondere Aufgabe der Community Interpreter an, insbesondere im Vergleich zum Konferenzdolmetscher (Gentile 1996: 111). Diese besondere Aufgabe der Kulturmittlung beim Community Interpreting wird dadurch verstärkt, dass die Kommunikation in der Regel persönlicher abläuft als beim Konferenz- oder Gerichtsdolmetschen (Roberts 1997: 11), da wir es hier mit einer face-to-faceSituation zu tun haben und dadurch kulturelle Unterschiede deutlicher hervortreten können. Die Transkom-Studie fasst diesen Aspekt noch einmal zusammen und weist auf einen weiteren wichtigen Aspekt hin - das Bestehen unterschiedlicher Machtpositionen zwischen den Parteien: 
Die Sprach- und KulturmittlerInnen sind das Bindeglied oder die Brücke zwischen beiden Gesprächsparteien und haben die Aufgabe, das asymmetrische Machtgefälle zwischen beiden Parteien auszugleichen und zum Abbau sprachlicher und kultureller Barrieren beizutragen." (Transkom-Studie (2007): 58)

Hinzu kommen ein oft ungleicher Sprachstand und häufig ein ungleicher Wissens-, Bildungs- und Erfahrungshorizont, der zu Kommunikationsstörungen oder Missverständnissen führen kann.

Community Interpreting stellt somit eine besondere Art des Dolmetschens dar. In der Dolmetschsituation treffen zwei Seiten aufeinander, die im Gegensatz zu anderen Dolmetscharten eine einzigartige Relation aufweisen. In der Regel weisen kulturelle Aspekte, Erwartungen und Einstellungen keine gänzliche Übereinstimmung auf. Bahadir geht auf diesen besonderen Umstand ein:

Dolmetschen im medizinischen, sozialen und juristischen Bereich ist eine Dolmetschtätigkeit, in der Perspektivität und Positionalität eine große Rolle spielen. Dolmetscher nehmen während des Dolmetschens in einer spezifischen Situation vor dem Hintergrund ihrer ,dazwischenkulturellen Identität' eine bestimmte Position ein. Diese Stellungnahme ist verbunden mit ethnischen und politischen Entscheidungen, die sie bedingt durch ihre soziale Rolle in diesem Kommunikationskontext treffen. (Bahadir 2001: 40)

Durch die folgende Tabelle, die die Besonderheiten von Konferenzdolmetschen und Community Interpreting zusammenfasst und gegenüberstellt, werden die wesentlichsten Unterschiede deutlich veranschaulicht: 


\begin{tabular}{|l|l|}
\hline Konferenzdolmetscher & Community Interpreter \\
\hline Professionalisierung des Berufsstandes & Oft ohne Qualifikationsnachweis \\
\hline Angesehene Tätigkeit & Wenig Ansehen \\
\hline Gleichberechtigte Gesprächspartner & Unterschiedliche Machtpositionen \\
\hline Standesvertretungen & (noch) ohne Lobby \\
\hline Sprachen: Englisch, Französisch, Spanisch & Minderheitensprachen, exotische Sprachen \\
\hline Hoher Bildungsstand & Flüchtlings- und Migrantenbereich \\
\hline Gute Bezahlung & Bezahlung gering \\
\hline $\begin{array}{l}\text { Monologisches Dolmetschen in die Mutter- } \\
\text { sprache }\end{array}$ & Dolmetschen in beide Richtungen \\
\hline Arbeit im Team & Arbeit alleine \\
\hline Große Zuhörerschaft & Meist für zwei Personen \\
\hline Üblichste Technik: Simultandolmetschen & $\begin{array}{l}\text { Gesprächsdolmetschen (konsekutiv), nur } \\
\text { gelegentlich Flüsterdolmetschen }\end{array}$ \\
\hline Anwendung von Notizentechnik & $\begin{array}{l}\text { Notizentechnik als Gedächtnisstütze, aber } \\
\text { nicht ständig }\end{array}$ \\
\hline Sachthemen bekannt, Vorbereitung möglich & $\begin{array}{l}\text { Inhalte nicht vorhersehbar, kaum Vorberei- } \\
\text { tung möglich: Flexibilität }\end{array}$ \\
\hline Face-to-face weniger bedeutend & Face-to-face sehr wichtig \\
\hline
\end{tabular}

Im Folgenden werden wir Behördendolmetschen als das Dolmetschen auffassen, welches weder bei Gericht noch in einer Konferenz stattfindet und bei welchem der Schwerpunkt auf einer interpersonellen und interkulturellen Kommunikation liegt.

\section{Rollen des Community Interpreters in der Interaktion}

Community Interpreter können im Rahmen ihrer Tätigkeit - selbst innerhalb eines selben Gesprächs - mehrere unterschiedliche Rollen übernehmen, die über die reine Sprachmittlung hinausgehen. Im Gegensatz zu den Konferenzdolmetscher(inne)n, die in der schallisolierten Kabine in der Regel keinen direkten Kontakt zu ihren Gesprächspartnern haben, wirken Community Interpreter durch ihre Präsenz und ihre Sichtbarkeit bewusst oder unbewusst auf den Gesprächsverlauf ein: sie erklären beispielsweise bei Bedarf bestimmte Begriffe 
und legen dadurch schon eine eigene Interpretation zugrunde, und sie übernehmen häufig die Moderation von Gesprächen. Daran zeigt sich die durchaus aktive Rolle des Dolmetschers/der Dolmetscherin, die sich meist nicht auf eine passive neutrale Sprachvermittlung begrenzen lässt. Diese aktive Rolle nimmt je nach Situation unterschiedliche Formen an, die jedoch nicht alle mit der Berufsethik des Dolmetschens konvergieren (s.u.). In Anlehnung an Abril (2006: 96ff.) können wir folgende Rollen feststellen:

- Conduit: Dolmetscher(in) als „Sprachrohr“. Der Fokus liegt hier auf der wörtlichen Wiedergabe, und folglich neutralen Sprachmittlerrolle. Dies ist die Rolle per se des Dolmetschers/ der Dolmetscherin, die häufigste und auch die grundlegendste.

- Clarifier: Ist die Rolle als Conduit nicht ausreichend, muss der/die Dolmetscher(in) in diese Rolle schlüpfen, um die Verständigung zu garantieren. Sie besteht darin, bestimmte nicht verstandene Konzepte näher zu erklären, bspw., wenn sie in der Zielsprache nicht vorhanden sind (Bescheid, Jugendamt, Ehefähigkeitszeugnis sind bekannte Beispiele aus dem Deutschen) oder vom Klienten/ von der Klientin nicht verstanden werden. Der/die Dolmetscher(in) greift ein und kann eigenmächtig zusätzliche Erklärungen verlangen, die dann vermittelt werden.

- Cultural broker: In diese Rolle schlüpft der/die Dolmetscher(in), wenn er/sie einen kulturellen Rahmen schaffen muss, in dem eine bestimmte Botschaft zu verstehen ist. Diese Rolle wird nur ausgeübt, wenn der kulturelle Unterschied die Verständigung erschwert. Der/die Dolmetscher(in) erklärt, was sich ggf. hinter einzelnen Wörtern verbirgt, erklärt Unterschiede in der Körpersprache, etc.

- Advocate: Diese aktivste Rolle des Dolmetschers/der Dolmetscherin (d.h. das Einsetzen für die Interessen des Klienten/der Klientin oder Parteiergreifung) zielt darauf, die Qualität des Einsatzes zu garantieren und ist eher am Rande des Dolmetscheinsatzes angesiedelt. Sie darf im 
Prinzip nur dann eingenommen werden, wenn eine systemische Barriere festgestellt wird, die ein Hindernis für die Erfüllung des Anliegens der Klienten darstellt. Sie beschränkt sich in der Regel darauf, festgestellte fehlende und für die Partei wichtige Informationen zu liefern, damit die Situation zufriedenstellend geklärt werden kann.

Die Übergänge zwischen diesen Rollen können in der Praxis fließend sein und sind daher nicht eindeutig definiert:

The role of the community interpreter today is ill-defined or, more commonly, too vast. He is often expected to be not only a mediator between two languages, but also a help-mate and guide, cultural broker and even advocate or conciliator. In other words, he wears many hats. (Roberts 1997: 12)

So müssen die Sprachmittler(innen) in der jeweiligen Gesprächssituation oftmals intuitiv entscheiden, welche Rolle(n) sie einnehmen (Abril 2006: 96ff.). Die unterschiedlichen Rollen des Dolmetschers/der Dolmetscherin, wie sie tatsächlich in der Praxis auftreten, sind im nachfolgenden Schema in Anlehnung an Leanza (2005: 186f.) dargestellt: 


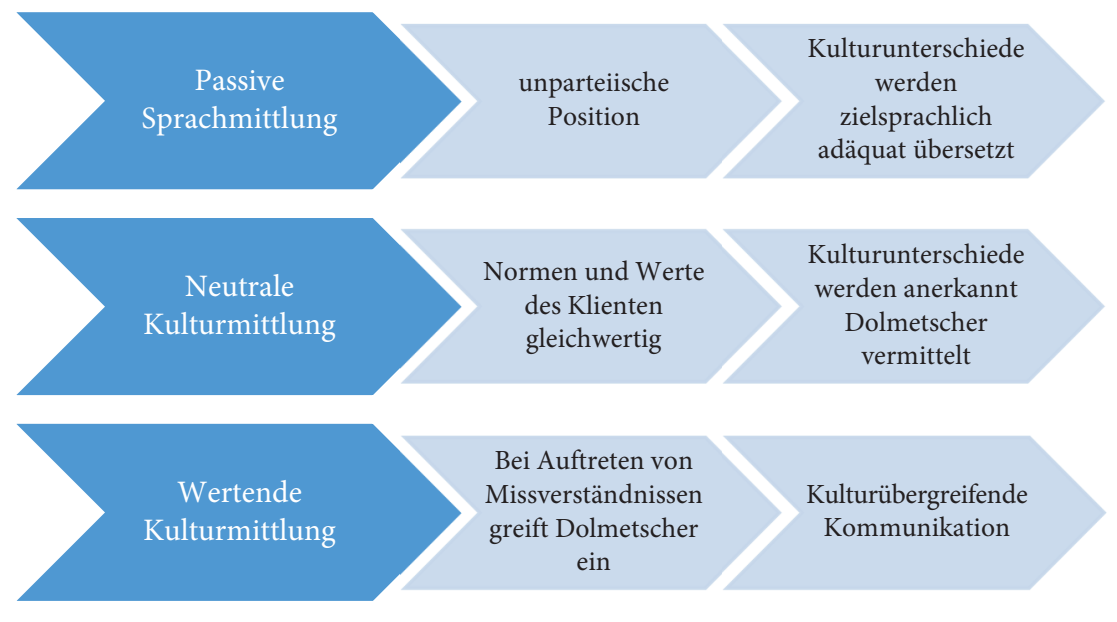

\section{Aktive Integrationshilfe - Advocate-Funktion}

Dolmetscher greift (z. B. bei Diskriminierung und Ungerechtigkeiten) aktiv zum Wohle der Klienten ein

Abb. 1: Dolmetscherrollen nach Leanza (2005)

Es sei noch einmal betont, dass die wertenden Formen der Interaktion mit Bezug auf berufsethische Vorgaben problematisch sind (dazu s.u.).

Würden wir dieses Modell als Pyramide abbilden, so befände auf dem untersten Teil der Pyramide die Rolle als Conduit und ganz oben auf der Spitze die des Advocate. Je weiter wir uns also nach oben innerhalb der Pyramide bewegen, umso mehr erfordert es das aktive Eingreifen des Dolmetschers/der Dolmetscherin. Seine/ihre Protagonistenrolle tritt deutlicher hervor und die Aufmerksamkeit des Einsatzes wird immer mehr auf ihn/sie gelenkt, wodurch er/sie in den Mittelpunkt rückt und sogar eigenmächtig FÜR seine/ihre Klienten spricht. Der Community Interpreter ist demnach immer Conduit und häufig nur Conduit, wenn er zusätzlich die anderen erwähnten Rollen einnimmt, um bspw. 
den kulturellen Rahmen für die Gesprächsparteien als Cultural Broker herzustellen oder um für die Parteien als Advocate eingreifen zu müssen. Dieses Modell basiert auf Roat et al. (1999: 15-23), die ein Handbuch für Dolmetscher(innen) im medizinischen Bereich erarbeitet haben, das von der California Healthcare Interpreter Association (CHIA) als Standard übernommen wurde und sich auch auf andere Bereiche des Community Interpreting übertragen lässt (vgl. CHIA 2002).

\section{Die Einsatzbereiche für Community Interpreter}

Im Zusammenhang mit Behördendolmetschen werden unter settings die verschiedenen Umgebungen verstanden, in denen der Community Interpreter seine Arbeit ausführt. Mit Blick auf den großen Unterschied zwischen den Begriffsumfängen innerhalb der Terminologie zum Gegenstand, aber auch mit Blick auf die zwischen den verschiedenen Ländern abweichende Praxis beschränken wir uns hier auf die Praxis in Deutschland und da auf den Bereich der Behördenkommunikation im Verwaltungsbereich, z. B. im Einwohnermeldeamt, in der Ausländerbehörde, im Standesamt, im Migrationsdienst oder in der Agentur für Arbeit.

Mit „Verwaltungsbereich“ sind diejenigen staatlichen Einrichtungen oder Ämter bezeichnet, die im weitesten Sinne für die Erfüllung von gesetzlich vorgeschriebenen Aufgaben zuständig sind. Da diese Einrichtungen öffentliche Aufgaben wahrnehmen und Gesetze oder Vorschriften ausführen, ist deren Handeln an genaue Regeln gebunden, die sich zunächst auf die unterschiedlichen Diskursformen des Dolmetschens auswirken und nicht zuletzt auch auf die jeweilige schriftliche und mündliche Informationsvermittlung, die bestimmte Merkmale aufweist. Es muss auch darauf hingewiesen werden, dass die Grenzen zwischen „Behörde“ und sonstigen Institutionen (Dienstleister, Unternehmen) zunehmend fließend sind und sich die im Folgenden genannten Eigenschaften mithin durchaus auch auf andere Bereiche anwenden lassen.

Im Behördenbereich wird der Community Interpreter mit einem besonderen Sprachgebrauch, sowohl in schriftlichen Texten wie Anträgen, Formularen, 
Gesetzestexten usw. als auch in mündlicher Form, konfrontiert, der sich erheblich vom alltäglichen Sprachgebrauch unterscheidet. Wissen um und Vertrautheit mit den Besonderheiten und Merkmalen der häufigsten Diskursformen der Verwaltung sind deshalb eine Voraussetzung dafür, Dolmetscheinsätze angemessen meistern zu können. Da dieser Bereich naturgemäß durch einen hohen Verschriftlichungsgrad geprägt ist, man denke nur an die zahlreichen Formulare und Merkblätter, kommt der Community Interpreter nicht umhin, sich Kenntnisse zu diesen schriftlichen Textsorten zu verschaffen (zu den Eigenschaften von Rechtstexten s. den Beitrag von Baumann im vorliegenden Band). Dies betrifft sowohl eine allgemeine Vertrautheit mit dem Bereich und seinen Textsorten als auch eine konkrete Vorbereitung auf die einzelnen Dolmetscheinsätze. Dabei können Dolmetscher(innen) vor einem Einsatz beispielsweise erwartbare Terminologie aus dem Bereich juristischer Begrifflichkeiten oder der Textsorten „Formulartext, Bescheid“ recherchieren. Ist der Einsatzort ein Einwohnermeldeamt, so können dies Begriffe wie z. B. Pflichtfeld, Widerspruchsrechte, Anmeldeformular sein. Häufig setzen solche Begriffe Kenntnisse des deutschen Verwaltungsapparats voraus (z. B. Rechtsstellung der Mutter, Datenübermittlungsgesetz). Manche Einzeltexte treten in diesem Kontext besonders häufig auf. So muss das Formular „Wohnungsgeberbestätigung“ von jedem/jeder Mieter(in) bei einer Anmeldung vorgelegt werden. Solche Texte können im Rahmen von Schulungen mit den Schulungsteilnehmer(inne)n weiter untersucht und Umgangsformen mit diesen Texten erarbeitet werden. Hier wären dann auch die juristischen Konsequenzen für die unterzeichnende Person ausdrücklich zu erörtern und insgesamt auf die Merkmale der Verwaltungssprache (eindeutige Informationsvermittlung, Genauigkeit, komprimiert-abstrakter und unpersönlicher Stil) einzugehen, um den Schulungsteilnehmer(inne)n eine Perspektive auf weitere, ähnlich gelagerte Texte zu eröffnen, mit denen sie im Rahmen ihrer Tätigkeit zu tun haben werden.

Häufig können die Dolmetscher(innen) die erforderlichen Dokumente im Vorfeld einsehen und vorbereiten. Dies gilt z. B. auch bei einem Einsatz im Standesamt und betrifft hier u.a. das Formular „Vollmacht Einverständniserklärung zur Anmeldung einer Eheschließung“, das ein ausgeprägtes Beispiel für die juristisch-administrative Kommunikation darstellt, etwa wenn ein „Antrag auf Befreiung von der Beibringung eines Ehefähigkeitszeugnisses“ oder ein „Antrag 
auf Anerkennung einer ausländischen Entscheidung in Ehesachen" gestellt werden muss. Bei Formularen muss in der Regel per Unterschrift die Korrektheit und Vollständigkeit der Daten bestätigt werden; eine Zuwiderhandlung stellt regelmäßig eine Ordnungswidrigkeit dar. Dolmetscher(innen) sind hier als Mittler gefragt, die Auskunft über die abgeforderte Handlung und ihre möglichen Konsequenzen geben.

Eine der ersten Anlaufstellen ausländischer Mitbürger(innen) sind die Migrationsberatungsstellen unterschiedlicher Träger (z. B. der Caritas) sowie die Ausländerbehörden. Dort erfahren die neu Zugewanderten Unterstützung beim Integrationsprozess in Deutschland und erhalten die notwendigen Informationen bezüglich ihrer sprachlichen, beruflichen und sozialen Integration. Dazu gehört auch die Vermittlung von Integrationskursen, die häufig Gegenstand individueller Beratungsangebote ist. Da viele ausländische Bürger(innen), insbesondere in der Anfangsphase ihres Aufenthalts, die deutsche Sprache nicht beherrschen, werden sie oft von Dolmetscher(inne)n begleitet.

Im Zusammenhang mit dem Antrag auf einen Integrationskurs können sich Dolmetscher(innen)auf mögliche Fragen ihrer Klienten durch die Webseite des Bundesamts für Migration und Flüchtlinge vorbereiten. Dort finden sich beispielsweise häufige Fragen zu den Integrationskursen und man kann sich die erforderlichen Formulare herunterladen.

Erfahrungsgemäß stellen die Anträge auf bestimmte Leistungen wie Arbeitslosen-, Kranken- oder Kindergeld häufig einen wichtigen Bestandteil dolmetschender Beratungseinsätze dar, sei es in den Migrationsberatungsstellen mit Sozialarbeiter(inne)n, die den ausländischen Klienten beratend zur Seite stehen, oder in den Jobcentern selbst. Das Formular für den Antrag auf Arbeitslosengeld ist ein in diesem Zusammenhang immer wieder zu bewältigender Text, über den Community Interpreter Kenntnisse erwerben sollten, um ihre Aufgabe angemessen erfüllen zu können. Dabei müssen die Dolmetscher(innen) u. U. erklärend einwirken und in ihrer Verdolmetschung weiter ausholen (zu den Dolmetschtechniken s.u.). 
Einige Stadtverwaltungen in Deutschland haben die gesellschaftliche Relevanz des hier erörterten Themas und den Handlungsbedarf erkannt und Qualifizierungsmaßnahmen konzipiert, die sich am konkreten Bedarf und an der Dringlichkeit der gegenwärtigen Situation orientieren. So werden bspw. in Braunschweig und Wolfsburg in regelmäßigen Abständen Schulungen für sprachmittlerische Tätigkeiten im Verwaltungsbereich durchgeführt (vgl. hierzu die Webseiten der Stadt Braunschweig und Wolfsburg). Unterrichtsinhalte und -ziele dieser Schulungsmaßnahmen entwickeln sich somit in diesem Falle aus einer besonderen Situation heraus, die am ehesten mit einer berufsbegleitenden Aus- oder Weiterbildung außerhalb der Universität vergleichbar ist, wo zweisprachige Personen von einer solchen Qualifizierung profitieren, die zwar nicht über eine Übersetzer- oder Dolmetscherausbildung, wohl aber über eine andere Ausbildung verfügen und unter Umständen bereits als Sprachmittler(in) tätig sind. Nach erfolgreicher Teilnahme an diesen Professionalisierungsmaßnahmen werden die Daten der Teilnehmer(innen) mit ihrer Sprachenkombination und weiteren für den Einsatz als Dolmetscher(innen) wichtigen Angaben (Geschlecht, Alter, berufliche Qualifizierung usw.) in eine Dolmetscherdatenbank eingegeben, auf die Mitarbeiter(innen) der unterschiedlichen städtischen Einrichtungen über das Büro für Migrationsfragen in Braunschweig und das Integrationsreferat der Stadt Wolfsburg Zugriff haben (s. auch Interkulturelle Stadtverwaltung der Stadt Wolfsburg).

\section{Inhalte von Professionalisierungsschulungen}

Da es dieser erwähnten Zielgruppe vor der Aus- oder Weiterbildung an verschiedenen Kompetenzen und Kenntnissen mangelt, gilt es also zunächst Inhalte für die Schulung festzulegen, mit denen die Teilnehmer(innen) in die entsprechenden Bereiche eingeführt werden und die erforderlichen Kompetenzen und Kenntnisse erwerben können. Insbesondere sind dies: 
- Dolmetschkompetenz

- Sprach- und Kulturkompetenz in der Muttersprache und im Deutschen

- Fachwissen und berufsethisches Wissen

Von Bedeutung sind aber auch:

- institutionelles Hintergrundwissen in den Bereichen Soziales, Bildung und Gesundheit

- Wissen zu Funktionsweisen und Zielen von Institutionen des Aufnahmelandes

- Kenntnisse und Funktionsweise der Gesetze des Landes

- Kenntnis der Rolle und der Anforderungen an Community Interpreter

- theoretische Grundkenntnisse in Bereichen wie Translationstheorie, Sprachwissenschaft, Soziolinguistik, Pragmatik und Diskursanalyse

- ein gutes Allgemeinwissen

Da der Sprachunterricht aufgrund des damit verbundenen Zeit- und Kostenaufwands innerhalb der Schulung entfällt, werden ausreichende Sprachkenntnisse der Teilnehmer(innen) vorausgesetzt. Entsprechende Sprachtests werden im Auswahlverfahren der Teilnehmer(innen) vor der Schulung durchgeführt.

Eine Schwierigkeit liegt sicherlich auch in den unterschiedlichen Vorkenntnissen der Teilnehmer(innen), deren Überprüfung vor Beginn des Ausbildungslehrganges äußerst müßig wäre. Es ist daher schwer abzuschätzen, welche Inhalte man in welcher Tiefe vermitteln sollte, um eine Unter- oder Überforderung einiger Teilnehmer(innen) auszuschließen (Hale 2005: 175f.).

Nachfolgend werden einige typische Typen von Inhalten dargestellt, die Gegenstand solcher Professionalisierungsschulungen sind: Neben einem kurzen Einblick in die Verständigungsstrategien sollen Dolmetschtechniken und die berufsethischen Grundlagen dargestellt werden. 


\subsection{Verständigungsstrategien}

Insgesamt müssen Dolmetscher(innen) in die Lage versetzt werden, Verständigungsstrategien anzuwenden, damit die Kommunikation funktionieren kann. Hier sind u.a. die folgenden zu benennen:

- Klientenorientiertes sprachliches Handeln

- Szenisches Erklären (Vorformulieren von sprachlichen Handlungen und Atomisierung von Handlungsschritten)

- Paraphrasen, Wiederholungen

- Perspektivenübernahmen ohne Übernahme von Entscheidungen durch den/die Dolmetscher(in)

- Der/die Klient(in) darf nicht auf eine passive und abhängige Rolle reduziert werden. Entsprechend muss jede Art von Paternalismus vermieden werden. Der/ die Dolmetscher(in) kann keine Entscheidungen treffen, das muss der/die Klient(in) bzw. der/die Behördenmitarbeiter(in) tun. Die Dolmetscher(innen) stellen die Kommunikation her und sind nur dafür verantwortlich.

Gerade letzterer Aspekt ist für Dolmetscher(innen), die selbst Teil der Gemeinschaft sind, für die sie Dolmetschaufgaben übernehmen, häufig nicht intuitiv erfassbar und muss entsprechend Teil der Professionalisierung sein. Darüber hinaus sind auch Dolmetschtechniken zu erlernen, die darauf angelegt sind, sprachliche, fachliche und fachsprachliche Barrieren (s. den Beitrag von Rink im vorliegenden Band) zu überwinden. 


\subsection{Dolmetschtechniken}

Ein wichtiger Gegenstandsbereich von Professionalisierungsschulungen für Community Interpreter sind naheliegenderweise die Dolmetschtechniken. Dabei kann zwischen Techniken auf der Makro-, Meso- und Mikroebene unterschieden werden.

\section{Makroebene}

Die Dolmetscher(innen) müssen es lernen:

- ... die Situationskohärenz zu erfassen. Dies ist eine wichtige Voraussetzung dafür, dass eine Kohärenz des Zieltextes in Konvergenz mit dem Ausgangstext und mit Blick auf die Rezeptionssituation erzielt werden kann. Besonders bei der Verdolmetschung von Formulartexten stellt dies eine Herausforderung dar, weil sie durch den Prozess des gemeinsamen Ausfüllens eine besonders ausgeprägte Zieltexteinbettung haben (Rink im Druck und den Beitrag von Maaß im vorliegenden Handbuch).

- ... mit den Gesprächspartner(inne)n steuernd und klärend zu interagieren. Gelegentliche Rückfragen werden meist von beiden Seiten notwendig sein.

- ... sprachliche Realisierung sukzessive an Gesprächspartner(innen) anzupassen: das fachsprachlich geprägte "Behördendeutsch" muss in vielen Situationen in eine sehr konkrete, einfache Ausdrucksweise in der Zielsprache überführt bzw. in der Gegenrichtung wieder an die entsprechende sprachliche Form der (deutschen) Formulare angepasst werden (vgl. Mikrotechnik).

\section{Mesoebene}

$\mathrm{Zu}$ den Besonderheiten der Behördenkommunikation gehört es, dass die Behördenvertreter(innen) auf ihre Funktionsrollen festgelegt sind und aus diesen 
Rollen heraus agieren. Die Kommunikation besteht dann häufig aus unterschiedlichen Bausteinen; beim Beispiel des gemeinsamen Ausfüllens eines Formulars sind das folgende:

$$
\text { Gespräch + Formular + Merkblatt }
$$

Das Behördendeutsch weist als ein Merkmal seiner Fachsprachlichkeit eine unpersönliche, meist sehr distanzierte Ansprache auf, die durch die Dolmetscher(innen) in der konkreten Gesprächssituation ausgeglichen werden muss. Eine weitere Herausforderung für Dolmetscher(innen) ist die Terminologisierung von Alltagssprache (Rink im Druck); Alltagsbezeichnungen bekommen in diesen Kontexten spezifisch fachliche Bedeutung (fachgeprägter Wortschatz), während alltagsnahe Begriffe mit Fachterminologie belegt werden:

\section{Terminologisierung von Alltagssprache:}

Unterbringung $\rightarrow$ Zwangseinweisung in die geschlossene Abteilung einer psychiatrischen Klinik

Betreuung $\rightarrow$ juristische Vertretung einer hilfsbedürftigen volljährigen

Person unter Beachtung ihres eigenen Willens

Fachterminologie für alltagsnahe Begriffe:

Eltern $\rightarrow$ Sorgeberechtigte

Kinder und Jugendliche $\rightarrow$ Minderjährige

Ältere $\rightarrow$ Senioren/Pflegebedürftige

\section{Mikroebene}

Es treten hier potentiell Herausforderungen auf allen sprachlichen Ebenen auf. Auf der Ebene der Phonetik bzw. Lautlichkeit ist das korrekte Verstehen und Wiedergeben von Namen und Fachtermini zu benennen. Dolmetscher(innen) müssen hier Notationstechniken sowie für ihre Arbeitssprachen übliche Transliterationen erlernen, aber auch Techniken des Umgangs mit derartigen Problemen (z. B. Bitten um Buchstabieren oder Aufschreiben). 
Auf der Ebene der Lexik/Wortwahl ist wiederum das Umgehen mit Fachterminologie zu benennen. Es wurde schon angeführt, dass dem Einsatz vorausgehende Auseinandersetzung mit der Fachterminologie sowie eine Einarbeitung mit den einzelnen Sach- und Fachgebieten zu den professionellen Standards gehört; gerade für fachfremde Community Interpreter ist aber das Umgehen mit Fachterminologie eine besondere Herausforderung, die in Schulungen einer Bearbeitung bedarf, um entsprechende Techniken und Strategien zu erlernen (z. B. Erklärung, Paraphrase, Generalisierung) sowie Techniken zum Ausgleich eines etwaigen Informationsverlusts später im Gesprächsverlauf.

Auf der Ebene der Syntax gehören Techniken des Bildens verständlicher Sätze (Vermeidung komplexer Nebensätze) sowie des Aufbaus einer Informationsstruktur zu den Schulungsgegenständen.

Auf der Ebene der Pragmatik gilt es, einen Blick auf Stil und Perspektive zu werfen: Adressierung, Umgang mit Fragen und Rückfragen, Verwendung unpersönlicher Ausdrücke, Kennzeichnung eigener Fragen.

Auf der Textebene geht es um die Entfaltung des gesamten Gesprächs, das Umgehen mit Nebenthemen und Nebensächlichkeiten, das Einbauen von $\mathrm{Zu}$ sammenfassungen und die Entfaltung eines auf beiden Seiten Verständnis hervorbringenden, erfolgreichen Gesprächs.

Es liegt nahe, den Umgang mit häufig auftretenden Dokumenten (dazu s.o.) im Rahmen von Schulungen zu vermitteln. Durch Rollenspiele lassen sich gezielt wesentliche Aspekte eines erfolgreichen Gesprächs mit Sprachmittler(innen) üben und bearbeiten. Die Gruppe kann das Gespräch zunächst im Hinblick auf das Verhalten der teilnehmenden Personen beobachten und dabei folgende Leitfragen beantworten:

1. Das Verhalten des Behördenmitarbeiters/ der Behördenmitarbeiterin oder Fachperson: Welches Verhalten wirkt sich positiv auf die Zusammenarbeit mit dem/ der Dolmetscher(in) aus? Welches Verhalten wird zum Störfaktor für die Kommunikation?

2. Analog zur Fachperson wird das Verhalten des Sprachmittlers/der Sprachmittlerin beobachtet: Welches Verhalten wirkt sich positiv auf die 
Zusammenarbeit zwischen Gesprächspartnern und Dolmetscher(in) aus? Welches Verhalten stört den Prozess der Kommunikation?

3. Das Verhalten des Klienten/der Klientin: Welche Auswirkungen hat das Verhalten des Behördenmitarbeiters/der Behördenmitarbeiterin und der dolmetschenden Person auf den/die Nutzer(in) bzw. den Klienten/ die Klientin?

4. Rollen der Gesprächspartner: Besteht eindeutige Klarheit über die jeweiligen Rollen der Gesprächsteilnehmer(innen) (Klient(in) und/oder - falls vorhanden - Begleitpersonen, Sprachmittler(innen), Behördenmitarbeiter(innen))? Werden diese Rollen im Vorfeld geklärt? Falls ja, wie wird das realisiert?

5. Mögliche Rollenwechsel: Gibt es im Gespräch einen Wechsel der Rollen? Nehmen die Personen im Gesprächsverlauf tatsächlich andere Rollen ein? Wie kommt es dazu? Ist dies förderlich für den Gesprächsverlauf oder eher hinderlich?

6. Falls es zu einem Rollenwechsel kommt: Werden die Gesprächsteilnehmer(innen) über den Rollenwechsel informiert und werden sämtliche Informationen an alle Teilnehmer(innen) weitergegeben?

Bei der Analyse des eigentlichen Gesprächs in der Dolmetschkonstellation müssen folgende Aspekte berücksichtigt werden:

- Gesprächsverlauf als Ganzes: einzelne Phasen, Gesprächsfluss, Gesprächssteuerung (Wer managt das Gespräch?), turn-taking (Wie wird der Wechsel der einzelnen Beiträge angezeigt?), Ausgewogenheit der Beiträge (Gibt es eine Dominanz zwischen den Gesprächspartnern? Wie wird diese Dominanz demonstriert und sprachlich artikuliert?); Involviertheit der Beteiligten (Fühlen sich Beteiligte fehl am Platz? Wodurch wird dies angezeigt?); Protagonismus der Gesprächsteilnehmer(innen). 
- Realisierung der verbalen Kommunikation: Wortwahl, Fachvokabular, Redefluss, einzelne Sequenzen, Pausen, Schweigen, Prosodie.

- Nonverbale Kommunikation: Welche Auffälligkeiten sind vorhanden? Wie ist die Körperhaltung, die Mimik und Gestik? Welche Schlussfolgerungen lassen sich aus der Körperhaltung ziehen?

\subsection{Berufsethische Grundlagen}

Neben den Verständigungsstrategien und den eigentlichen Dolmetschtechniken sind besonders die berufsethischen Grundlagen ein wichtiger Baustein von Professionalisierungsschulungen. Gerade für Dolmetscher(innen), die Teil der Gemeinschaft sind, aus der der Klient/die Klientin stammt, sehen sich intuitiv häufig als Interessenvertreter(innen) oder werden als solche vereinnahmt. Es ist von großer Bedeutung für eine professionelle Dolmetschhandlung, dass die zukünftigen Expert(inn)en verstehen, dass sie zwar die Funktion des Kulturmittlers/der Kulturmittlerin einnehmen können, jedoch keine Interessenvertreter für die Klient(inn)en sind. Daher ist die Vermittlung berufsethischer Grundlagen eine unverzichtbare Voraussetzung für eine professionelle Dolmetschpraxis.

Für Community Interpreter besteht derzeit (noch) kein berufsethischer Kodex, an dem sich die Dolmetscher(innen) orientieren können, obwohl unterschiedliche Berufsverbände ihre eigenen Richtlinien erarbeitet haben. Das Verhalten des Dolmetschers/der Dolmetscherin muss sich daher in Anlehnung an den anerkannten und bewährten berufsethischen Grundsätzen orientieren, wie sie bspw. von der International Association of Conference Interpreters (AIIC 2018 für Konferenzdolmetscher gefordert werden. Deren Code of Professional Ethics legt Standards für Professionalität, Vertraulichkeit und Integrität fest. In Deutschland vertritt der Bundesverband der Dolmetscher und Übersetzer e. V. (BDÜ) die in ihm zusammengeschlossenen Verbände und zielt mit seinen Richtlinien u.a. darauf, das Vertrauen zwischen den Parteien, die Qualität, das Ansehen des Berufsstandes und ethisch einwandfreies Verhalten sicherzustellen. Auch der ADÜ Nord, der Mitglieder vor allem in Hamburg, SchleswigHolstein und Niedersachsen hat, verfasste seine Berufs- und Ehrenordnung in 
der Absicht, das Ansehen des Verbandes und der in ihm assoziierten Dolmetscher(innen) und Übersetzer(innen) angesichts des ungeschützten Berufsbildes zu wahren. Die nachfolgend genannten Kategorien sind laut dieser Verbandsordnungen für professionelles dolmetscherisches Handeln zentral:

- Vertraulichkeit: Dolmetscher(innen) sind an das Berufsgeheimnis gebunden und müssen die Geheimhaltungspflicht einhalten. Sie dürfen erlangte Information nicht für eigene Zwecke verwenden.

- Berufsloyalität: Es werden nur Aufträge angenommen, denen man tatsächlich gewachsen ist. Um qualitativ hochwertige Arbeit gewährleisten zu können, benötigt der/die Dolmetscher(in) vom Auftraggeber die entsprechenden Informationsmaterialien.

- Neutralität: Es werden keine eigenen Meinungsäußerungen zum Ausdruck gebracht, weder über die eigene Arbeit noch über die Gesprächspartner.

- Respektvoller Umgang mit den Gesprächsteilnehmer(inne)n: Alle Gesprächsteilnehmer(innen) werden ungeachtet ihrer Nationalität, Alter, Hautfarbe, Sozialstatus, Religion und politischer oder ideologischer Einstellung gleichermaßen mit Respekt und Würde behandelt.

- Einsatz für würdige Arbeitsbedingungen: Verantwortungsvolles Verhalten gegenüber dem Berufsstand (für sich und die Kollegen(innen)) und Vermeidung illoyalen Wettbewerbsverhaltens.

Außerdem beachten Dolmetscher(innen) die folgenden Aspekte der Arbeitssituation:

- Das Arbeitsumfeld: Sie achten auf Pünktlichkeit und angemessene Kleidung; mögliche Pannen werden einkalkuliert. 
- Der Umgang mit den Gesprächspartnern: Dolmetscher(innen) machen klare Ausführungen zu ihrer Rolle, stellen mögliche Probleme dar und handeln vorausschauend; sie betreiben aktives Raummanagement (unterschiedliche Szenarien werden dabei stets berücksichtigt).

Die Aufgaben für den Dolmetscher/die Dolmetscherin leiten sich somit aus den erwähnten berufsethischen Richtlinien ab. Konkret hat er/sie dabei folgende Regeln zu beachten:

Der Dolmetscher/die Dolmetscherin

1. ... überträgt alles, was in dem Gespräch zum Ausdruck gebracht wird, ohne Hinzufügungen, Modifizierungen oder Auslassungen.

2. ... ist unparteiisch in seiner/ihrer Arbeit. Seine/ihre persönliche Einstellung darf die Dolmetscharbeit nicht beeinflussen.

3. ... hat sich nur um die Verdolmetschung zu kümmern und trifft in der Situation keine eigenen Entscheidungen.

4. ... unterliegt dem Verschwiegenheitsgebot.

5. ... trägt keine Verantwortung hinsichtlich der Wahrhaftigkeit von Informationen, die zwischen beiden Gesprächspartnern ausgetauscht werden.

6. ... repräsentiert keine der beiden im Gespräch involvierten Parteien.

7. ... ist verpflichtet zu sagen:

- ob er/sie mit einer der Parteien verwandt oder verheiratet ist

- ob er/sie im betreffenden Fall involviert ist

- ob er/sie in diesem Fall bereits für eine Partei tätig war

8. ... ist in der Pflicht Bescheid zu sagen, falls er/sie den Dolmetschauftrag nicht zur Zufriedenheit ausführen kann. 
Das Berufsgeheimnis impliziert:

1. Alle Informationen, zu denen der Dolmetscher/die Dolmetscherin durch seine/ihre Arbeit Zugang hat, sind absolut vertraulich.

2. Das Berufsgeheimnis schließt alle ein: Arbeitgeber, Kollegen und Familienangehörige.

Auch nach Beendigung der Arbeit bleibt das Berufsgeheimnis erhalten (vgl. ADÜ Nord 2009).

$\mathrm{Da}$ - wie bereits erwähnt - verbindliche Handlungsanweisungen für die im Verwaltungsbereich tätigen Dolmetscher(innen) fehlen, stellen die vorangegangenen Codes of Ethics der aufgeführten Verbände Orientierungsrichtlinien dar, die den Dolmetscher(inne)n in erster Linie dabei helfen sollen, individuelle Entscheidungen aus dem jeweiligen Kontext und der entsprechenden und immer einzigartigen Situation heraus zu treffen. In diesem Sinne äußert sich auch Hale (2005):

Interpreters will allways need to use their discretion and better judgement to make the appropriate decisions for each situation, led by the general guidelines of the code of ethics.

So kann es in der täglichen Arbeit trotz der empfohlenen Richtlinien für korrektes Verhalten zu Gewissenskonflikten und Grenzfällen kommen, die durch die Berufsordnungen oder Vorgaben nicht zu erfassen sind und, etwa bei Neutralitätskonflikten, einer besonderen ethischen Auseinandersetzung bedürfen. Dolmetscher(innen), die u. a. in der Verwaltung tätig sind, handeln in der Regel aus eigener Verantwortung heraus und meist situationsbedingt. Das macht es schwierig, Einzelfälle herauszugreifen und auf die Berufsordnungen zu verweisen, da in der Regel Kontrollinstanzen außerhalb der beruflichen $\mathrm{Zu}$ sammenschlüsse fehlen. So widersprechen einige Expert(inn)en der Möglichkeit völliger Neutralität in der Praxis und vertreten die Ansicht, ein/e Dolmetscher(in) könne nicht völlig zurücktreten und unsichtbar werden (Bahadir 
2001), denn allein durch seine bzw. ihre physische Präsenz entstünde eine „nonpossibility for interpreters to be nonpersons" (Bahadir 2001: 4). Letztlich seien sie im Konflikt zwischen neutralem Standort und Mitwissen eines Eingeweihten gefangen. Andere wiederum sehen die Neutralität des Dolmetschers/der Dolmetscherin dadurch gefährdet, dass Auftraggeber wiederholt die Dienste desselben Dolmetschers/derselben Dolmetscherin in Anspruch nehmen und mit der Zeit ein Vertrauensverhältnis zwischen Klient(in) und Dolmetscher(in) entsteht, welches die Unparteilichkeit in Frage stellen könnte (Stanek 2011: 150).

Mit Blick auf die hier dargestellten Dolmetschsituationen zeigt sich, dass der/die Dolmetscher(in) nicht nur nicht unsichtbar sein kann, sondern ein wichtiger aktiver Teil der Kommunikation ist, denn er/sie ist häufig für die Steuerung des Gesprächs zuständig, erklärt darüber hinaus missverständliche Konzepte und beseitigt kulturelle Verständnisprobleme. Dabei ist das Einnehmen einer moderaten Haltung erforderlich sowie ein auf ein Minimum beschränktes selbständiges Eingreifen in der kommunikativen Situation. Doch auch wenn Dolmetscher(innen) den oben aufgeführten berufsethischen Richtlinien Folge leisten, ist es häufig - auch aufgrund der Unmöglichkeit, alle denkbaren Einsatzmöglichkeiten zu antizipieren - dem persönlichen Urteilsvermögen des Dolmetschers/der Dolmetscherin überlassen, Entscheidungen selbständig zu treffen, um die vielfältigen Kommunikationssituationen meistern zu können.

\section{$7 \quad$ Fazit}

In diesem Beitrag wurde der Versuch unternommen, den Bereich des Community Interpreting zu definieren und gegenüber anderen Dolmetscharten abzugrenzen. Darüber hinaus wurden einige konkrete Beispiele zum strategischen Vorgehen im Verwaltungsbereich aufgezeigt und Schulungsinhalte für mögliche Professionalisierungsma $\beta$ nahmen für Community Interpreter, wie sie derzeit schon von einigen Stadtverwaltungen Niedersachsens erfolgreich durchgeführt werden, dargestellt. In den öffentlichen Einrichtungen einer Einwanderungsgesellschaft, der die Integration und Teilhabe ihrer Bürger wichtig sind, gewinnt die Förderung von Projekten dieser Art, die der Verbesserung der Verständigung zwischen den neu Zugewanderten und der einheimischen Bevölkerung 
dienen und letztlich den Integrationsprozess fördern, immer mehr an Bedeutung. Damit steht unsere Gesellschaft vor einer großen Verantwortung im Hinblick auf die Umsetzung und Etablierung solcher konstruktiven und notwendigen Angebote, von denen zu hoffen bleibt, dass sie nicht nur punktueller Natur sind, sondern von den politisch Verantwortlichen in nicht allzu ferner Zukunft institutionalisiert werden. 


\section{Literaturverzeichnis}

Abril, Martí I. (2006): La interpretación en los Servicios Públicos: Caracterización como Género, Contextualización y Modelos de Formación. Hacia unas bases para el Diseño Curricular. Dissertation, Universidad de Granada, online verfügbar unter: https://hera.ugr.es/tesisugr/16235320.pdf.

ADÜ Nord (2009): Berufs- und Ehrenordnung: http://www.adue-nord.de/wp-content/ uploads/2017/09/Berufs-undEhrenordnung.pdf (zuletzt aufgerufen am 08.06.2018).

AIIC: Code of professional ethics (2018): https://aiic.net/page/6724/code-of-professional-ethics-2018-version/lang/1 (zuletzt aufgerufen am 11.06.2018).

AMS-Broschüre (2015): „Jobchance - Studium Sprachen“; http://www.forschungsnetzwerk.at/downloadpub/JCS_2015_Sprachen.pdf (zuletzt aufgerufen am 11.06.2018).

ANDRES, DÖRTE (2008). Dolmetscher als literarische Figuren - Von Identitätsverlust, Dilettantismus und Verrat. München: Martin Meidenbauer.

BAHAdir, SEBnem (2001): The Empowerment of the (Community) Interpreter: The Right to Speak with a Voice of One's Own: https:/www.criticallink.org/s/ CL3_Bahadir.pdf (zuletzt aufgerufen am 11.06.2018).

BAMF 2017: Bundesamt für Migration und Flüchtlinge - Bericht zur Integrationskursgeschäftsstatistik für das Jahr 2017; Abfragestand: 4.4.2018: http://www.bamf.de/ SharedDocs/Anlagen/DE/Downloads/Infothek/Statistik/Integration/2017/2017-integrationskursgeschaeftsstatistik-gesamt_bund.pdf?_blob=publicationFile（zuletzt aufgerufen am 11.06.2018)

BDÜ: Berufs- und Ehrenordnung (2014): http://bdue.de/der-bdue/statuten/berufs-undehrenordnung (zuletzt aufgerufen am 24.07.2016).

Bowen, Margareta (1998): „Community Interpreting." In: SNell-Hornby, Mary/Hönig, Hans G./Kubmaul, Paul/Schmitt, Peter A. (Hg.): Handbuch Translation. Tübingen: Stauffenburg, 319-321.

Büro für Migrationsfragen der Stadt Braunschweig: https://www.braunschweig.de/ leben/soziales/migration/Servicestelle.html (zuletzt aufgerufen am 02.07.2018).

Carr, Sylvana, Roda RoberTs, Aideen Dufour, Dini Steyn. (1995): The critical link: Interpreters in the community. Papers from the 1st International Conference on Interpreting in Legal, Health, and Social Service Settings, Geneva Park, Canada, June 1-4, 1995. Amsterdam, Philadelphia: John Benjamins Publishing.

CHIA - California Healthcare Interpreters Association (2002): California Standards for Healthcare Interpreting: Ethical Principles, Protocols and Guidance on Roles \& Intervention, http://chiaonline.org/images/Publications/CA_standards_healthcare_ interpreters.pdf (zuletzt aufgerufen am 25.07.2009).

Estévez Grossi, M. (2018): Lingüística Migratoria e Inerpretación en los Servicios Públicos. La comunidad gallega en Alemania. Berlin: Frank \& Timme. 
Gentile, Adolfo/Ozolins, Uldis/Vasilakakos, Mary (1996) [with Leong Ko and Ton-That Quynh-Du]: Liaison Interpreting. A Handbook. Melbourne: Melbourne University Press.

HALE, SANDRA (2005): „Controversies over the role of the court interpreter“. Plenarkonferenz des Kongresses „Traducción como Mediación entre Lenguas y Culturas. II. Congreso Internacional sobre Traducción e Interpretación en los Servicios Públicos.” (Universidad de Alcalá de Henares, Madrid, 28 u. 29. April 2005).

Interkulturelle Stadtverwaltung der Stadt Wolfsburg (o.J.): https://www.wolfsburg.de/ / media/wolfsburg/.../interkulturelle-stadtverwaltung (zuletzt aufgerufen am 18.08.2018).

LEANZA, YVAN (2005): „Roles of community interpreters in pediatrics as seen by interpreters, physicians and researchers“. In: Interpreting, 7/2, 167-192.

Mikkelson, Holly (1996): “The professionalization of Community Interpreting”. In: Jérôme-O’Keeffe, Muriel M.: Global vision: Proceedings of the $37^{\text {th }}$ annual Conference of the American Translator Association. John Benjamins, 77-89. http://aiic.net/ page/1546/the-professionalization-of-community-interpreting/lang/1

OzOLINS, ULDIS (2010): "Factors that determine the provision of Public Service Interpreting: comparative perspectives on government motivation and language service implementation". In: The Journal of Specialized Translation 14: 194-215. http://jostrans.org/issue14/art_ozolins.php

PÖCHHACKER, FrANZ (1997): Kommunikation mit Nichtdeutschsprachigen in Wiener Gesundheits - und Sozialeinrichtungen. Teil 2. MA 15 / Dezernat für Gesundheitsplanung. Wien: Lischkar.

PÖCHHACKer, Franz (1998): „Simultandolmetschen.“ In: Snell-Hornby, Mary/ Hönig, Hans G./Kußmaul, Paul/Schmitt, Peter A. (Hg.): Handbuch Translation. Tübingen: Stauffenburg, 301-304.

PöchHaCKer, Franz (1999) "'Getting Organized': The Evolution of Community Interpreting". In: Interpreting 4:1, 125-140.

PÖCHHACKer, FranZ (2000): Dolmetschen. Konzeptuelle Grundlagen und deskriptive Untersuchungen. Tübingen: Stauffenburg. (= Reihe Translation; Bd. 7).

PÖCHHACKER, Franz (2004): Introducing Interpreting Studies. London \& New York: Routledge.

PÖChHACKer, Franz (2008): “Interpreting as Mediation.” In: VAlero GarCÉs, CARMEN/ Martin, Anne (Hg.): Crossing Borders in Community Interpreting: Definitions and Dilemmas. Amsterdam / Philadelphia: John Benjamins, 9-26.

Roat, Cynthia, Gheisar, Bookda, Putsch, Robert U. SenGupta, Ira (1999): Bridging the Gap: A Basic Training for Medical Interpreters. Interpreters Handbook. 3. Aufl. Seattle, WA: Cross Cultural Health Care Program (CCHCP).

Roberts, Roda P. (1997): “Community Interpreting Today and Tomorrow.” In: CARR, Silvana E./Roberts, Roda/Dufour, Aideen/Steyn, Dini (Hg.): The Critical Link: Interpreters in the Community: Papers from the First International Conference on 
Interpreting in Legal, Health, and Social Service Settings. Geneva Park, Canada, June 1-4, 1995. Amsterdam /Philadelphia: John Benjamins, 7-25.

StaneK, MaŁgorzata (2011): Dolmetschen bei der Polizei: Zur Problematik des Einsatzes unqualifizierter Dolmetscher. Berlin: Frank \& Timme.

Transkom-Studie. Vergleichende Studien zu Sprach- und Kulturmittlung in verschiedenen europäischen Ländern (2007): https://www.yumpu.com/de/document/view/ 4932327/vergleichende-studie-zu-sprach-und-kulturmittlung-in-transkom (zuletzt aufgerufen am 25.06.2018).

Valero Garcés, Carmen/Martin, Anne (Hg.): Crossing Borders in Community Interpreting: Definitions and Dilemmas. Amsterdam / Philadelphia: John Benjamins, 9-26.

WADENSJÖ, CECILIA (1998): Interpreting as Interaction. London: Edison Wesley Longman. 
Mediale Bereiche und Textsorten 

ALEXANDER KURCH

\section{Produktionsprozesse der Hörgeschädigten-Untertitelungen und Audiodeskription: Potenziale teilautomatisierter Prozessbeschleunigung mittels (Sprach-)Technologien}

\section{$1 \quad$ Einleitung}

Im Rahmen ihres Fernsehprogramms setzten die öffentlich-rechtlichen Rundfunkanstalten innerhalb der letzten Jahre ein beachtliches Mehrangebot an barrierefreien Inhalten um und stellten dies in ihren Mediatheken zur Verfügung (Landtag Rheinland-Pfalz 2017 und Heerdegen-Wessel im vorliegenden Band).Die barrierefreie Gestaltung audiovisueller Medienangebote für Menschen mit Sinneseinschränkungen in öffentlichen Einrichtungen und privaten Unternehmen sowie die Bewusstseinsbildung zur Notwendigkeit dieser Angebote befindet sich hingegen noch im Anfangsstadium. Dies betrifft Inhalte mit Audiodeskription, mit Untertiteln, Verdolmetschungen in Gebärdensprache und Übersetzungen in Leichte Sprache.

Gerade im Zeitalter der Digitalisierung nimmt das Medienangebot vor allem durch diverse Anbieter im privaten Bereich stetig zu. Diese Anbieter stehen jedoch hinsichtlich der Vision einer flächendeckenden Umsetzung und Produktion eines Mehrangebots barrierefreier Inhalte vor finanziellen und personellen Herausforderungen.

Zukünftig wird dies schnellere und kostengünstigere Produktionsprozesse erfordern, um hier den Bedarf nach Zugänglichkeit in allen Bereichen zu decken. Durch die verstärkte Forschung und Entwicklung technologischer Hilfsmittel und die zunehmende Anwendung maschineller Lernverfahren eröffnen sich in diesem Kontext neue Möglichkeiten, die besonders in den Bereichen der Hörgeschädigten-Untertitelung (engl. subtitles for the deaf and hard-of-hearing, Abk. SDH) und der Audiodeskription (engl. audio description, Abk. AD) künftig Abhilfe schaffen können. 
Dieser Beitrag soll einen Überblick über die derzeitigen Produktionsprozesse im deutschsprachigen Raum geben. Des Weiteren werden mit Blick auf die Zukunft mögliche und zum Teil bereits existierende Teilautomatisierungsprozesse (Abk. TAP) in Hinblick darauf beleuchtet, inwiefern die Potenziale verschiedener Technologien in Kombination mit maschinellen Lernverfahren für den Bereich der barrierefreien Übersetzung audiovisueller Medien ausgeschöpft werden können.

\section{Hörgeschädigten-Untertitelung (SDH) und Audiodeskription (AD)}

Die Hörgeschädigten-Untertitelung bietet hörgeschädigten und gehörlosen Menschen durch die Verschriftung und Ausstrahlung auditiver Inhalte in Form von Untertiteln einen Zugang zu Informationen, die aufgrund einer Hörbehinderung nicht oder nur unzureichend rezipiert werden können (Hezel 2009 und Mälzer/ Wünsche im vorliegenden Band). Bei der Audiodeskription erfolgt der Transfer von visuellen Informationen in gesprochener Sprache für blinde und sehbehinderte Menschen (vgl. Jekat 2016: 70 und Benecke im vorliegenden Band). Diese Formen des audiovisuellen und intralingualen Übersetzungstransfers lassen sich jenseits von Film und Fernsehen in den verschiedensten Bereichen anwenden, in denen barrierefreie Inhalte erstellt werden sollen.

Die Produktionsabläufe zur Schaffung dieser beiden barrierefreien Angebote erfordern eine Vielzahl von Schritten, die zum Teil äußerst zeit- und kostenintensiv sind. Daher stellt sich die Frage, wie (sprach-)technologische Werkzeuge eingesetzt werden können, um diese Prozesse zu beschleunigen, damit in kürzerer Zeit mehr Angebote mit SDH und $\mathrm{AD}$ umgesetzt werden können. Hierfür existieren mit der Spracherkennung und Sprachsynthese bereits Technologien, die eine automatisierte Umwandlung von Sprache in Text bzw. Text in Sprache erlauben und mittlerweile als bereits kommerziell erschwingliche Software-as-a-Service (Abk. SaaS) kosteneffizientere Produktionsvarianten erlauben. Zunächst sollen nun aber die aktuell in Deutschland angewandten Produktionsprozesse zur Erstellung von SDH und AD genauer betrachtet werden, um ein Verständnis für die qualitativen Ansprüche der derzeitigen SDH- 
und AD-Praxis und einen Überblick auf die Anwendbarkeit von Spracherkennung und Sprachsynthese in diesem Kontext zu schaffen. Anschließend werden Ansätze zur technisch gestützten Produktion von SDH und AD vorgestellt.

\subsection{Derzeitige Produktionsprozesse der SDH in Deutschland}

\subsubsection{SDH-Postproduktion}

Für die Postproduktion von Untertiteln gibt es inzwischen eine Bandbreite verschiedener computergestützter Untertitelungsprogramme, die eine schnelle Erstellung von SDH erlauben. Diese bieten den Nutzer(inne)n zahlreiche Funktionen, die eine relativ zügige Erstellung intralingualer Untertitelung ermöglichen. Dabei lassen sich auch erforderliche technische Vorgaben gemäß den Standards und Style Guides der Auftraggeber berücksichtigen.

Die technischen Komponenten einer Untertitelungssoftware ermöglichen die Eingabe von Basiswerten für die Standzeiten von Untertiteln, die eine optimale Lesegeschwindigkeit für die Rezipient(inn)en begünstigen (i. d. R. gemessen in Zeichen pro Sekunde). So kann diese Software automatisch die Standzeiten von Untertiteln nach Zeichen und Zeilenzahl berechnen und auch den zeitlichen Mindestabstand zwischen den einzelnen Untertiteln generieren. Außerdem verteilt das Programm den Text des Untertitels automatisch auf zwei Zeilen, die sogenannte Segmentierung, wobei dies ausschließlich nach Zeichenanzahl pro Zeile erfolgt und sich nicht an syntaktischen und semantischen Gesichtspunkten orientiert. Im nächsten Schritt wird der Text durch einen Redakteur/eine Redakteurin linguistisch bearbeitet, um hochwertige Untertitel für eine möglichst anstrengungsarme Rezeption zu produzieren, was von der Software nicht automatisiert übernommen werden kann.

Die linguistische Bearbeitung von Untertiteln umfasst verschiedenste Aufgaben: Sofern dem Untertitelredakteur kein Dialogbuch vorliegt, müssen zu Beginn alle gesprochenen und weiteren hörbaren Anteile des Sendematerials transkribiert werden, also alle gesprochenen Äußerungen und Geräusche. Bei der Übertragung des Originaltons in Untertiteln findet ein Transfer von Mündlichkeit zur Schriftlichkeit statt. Daher entspricht der Text des Untertitels nicht zwingend dem Text des Originaltons. Entsprechende Änderungen werden durch den 
Untertitelredakteur/die Untertitelredakteurin so vorgenommen, dass möglichst kein Informationsverlust entsteht.

Diese bringen gegebenenfalls die Tilgung von Merkmalen der Spontansprachlichkeit, wie Füllwörtern, inhaltsleeren Floskeln, Hesitationen sowie Reparaturen von Satzabbrüchen, ungünstiger, schwerverständlicher Syntax und weiteren linguistischen Überarbeitungen mit sich, um nur einige Beispiele zu nennen. Die wortwörtliche Übertragung des Originaltons in den Untertiteln ist aufgrund zeitlicher und räumlicher Restriktionen nicht möglich. Denn bei der Produktion von Untertiteln ist die Einhaltung von auftraggeberspezifischen Vorgaben bezüglich Anzeigedauer und Zeichenzahl der Untertitel erforderlich. Daher braucht es eine Selektion des auditiven Inputs nach primär inhaltstragenden Anteilen und häufig eine Neustrukturierung des Textes, um diesem die Form eines optimal lesbaren Untertitels zu verleihen.

Emotionen, Subtexte, Konnotation des Gesagten (z. B. (wütend), (ironisch), (laut)), sowie Geräusche und Musik gehören in Klammersetzungen als nonverbale, para- und extrasprachliche Elemente ebenfalls untertitelt. Des Weiteren müssen die Sprecherwechsel durch die Untertitelredakteure/-redakteurinnen ebenfalls in Klammern gesetzt werden und zum Teil farblich gekennzeichnet werden, um die einzelnen Untertitel den jeweiligen sprechenden Akteur(inn)en des Bildgeschehens schnellstmöglich zuordnen zu können.

Zudem müssen die textlichen Inhalte der Untertitel auf zwei Zeilen eines Untertitels verteilt werden (Segmentierung). Hier liefern Empfehlungen der „good subtitling practice“ (Ivarsson/Carroll 1998, Díaz-Cintas/Remael 2007) eine Handreiche hinsichtlich der linguistischen Bearbeitung im Sinne einer möglichst optimalen Segmentierung. Dadurch werden die Untertitel für Rezipient(inn)en leichter lesbar.

Zu den technischen Bearbeitungen der Untertitel durch die Redakteure/Redakteurinnen gehört vor allem die Zeitkodierung. Untertitel benötigen für ihre punktgenaue Ein- und Ausblendung einen Zeitstempel für den Start und das Ende des Untertitels, orientiert an den jeweiligen Vorgaben des Auftraggebers zu den Standzeiten eines Untertitels. Die Untertitel sollen i. d. R. so synchron wie möglich zum Bild und Ton des Ausgangsmaterials bzw. möglichst lippensychron zum gesprochenen Originalton erscheinen. 
Es wird deutlich, dass die Postproduktion von Hörgeschädigten-Untertiteln für die verantwortlichen Redakteure/Redakteurinnen sehr aufwendig ist, selbst wenn eine dafür ausgerichtete Untertitelungssoftware zum Einsatz kommt. Angesichts der schriftlichen Umsetzung aller gesprochenen und weiteren auditiven Inhalte in Form von Untertiteln, der Zeitkodierung, der Farbvergabe bei Sprecherwechseln, sowie der Segmentierung jedes einzelnen Untertitels nimmt die handwerkliche Arbeit entsprechend viel Zeit in Anspruch. Abhilfe kann hier die automatische Spracherkennung (engl. automatic speech recognition, Abk. ASR) bieten, die sich im Bereich der Live-Untertitelung bereits durchgesetzt hat, aber in der Postproduktion bisher nur vereinzelt zum Einsatz kommt.

\subsubsection{SDH-Produktion und Spracherkennung}

Spracherkennung (ASR) wird derzeit vor allem in Live-Untertitelungen für Sendungen bzw. Veranstaltungen eingesetzt, für die aufgrund des „Aktualitätsdrucks" (vgl. Lindner 2016: 313) keine Inhalte in Form von vorproduzierten Untertitelungen vorbereitet werden können, wie dies bei der Postproduktion von Untertiteln der Fall ist. Hier müssen Untertitelredakteure/-redakteurinnen „die Inhalte mittels einer Spracherkennung in dem Moment erzeugen, in dem sie [...] stattfinden“ (ebd. 312). Daher hielt die ASR-Software in den letzten Jahren Einzug in öffentlich-rechtliche Sendeanstalten für die Produktion von LiveUntertitelungen im Fernsehen (Käber 2016) und jenseits der Rundfunkanstalten in Einsatzbereichen des Schriftdolmetschens (VerbaVoice GmbH o. A., Witzel im vorliegenden Band). Die Aufgabe der ASR in diesem Kontext besteht in der Umsetzung „eines Sprachsignals in eine textliche Form“ (Pfister/Kaufmann 2017: 28), d. h. „ein gesprochener Text soll vom System erkannt und z. B. in Form eines Schrifttextes ausgegeben werden“ (Fellbaum 2012: 306). Doch es stellt sich die Frage, inwiefern die Spracherkennung als computergestütztes Hilfsmittel zur Produktivitätssteigerung in der Postproduktion von SDH integriert werden kann.

Zuvor muss zwischen sprecherunabhängiger, sprecheradaptiver und sprecherabhängiger ASR unterschieden werden. ASR-Software erzielt die besten Ergebnisse, wenn ein persönliches Benutzerprofil für den jeweiligen Nutzer erstellt wird, das für bestimmte Sach- und Fachgebiete (Domänen) angelegt wird (vgl. Pfister/Kaufmann 2017: 334, Fellbaum 2012: 307). 
Sprecherabhängige und -adaptive ASR-Systeme unterscheiden sich von sprecherunabhängigen Systemen in ihrer Vielzahl an Funktionen, die innerhalb weniger Schritte die Erweiterung und Pflege der benutzerspezifischen Sprachprofile erlauben. Dies begünstigt eine erhöhte Erkennungsquote bzw. Reduktion von Erkennungsfehlern, besonders bei Wörtern und Termini, die dem angelegten Sprachprofil und somit dem System unbekannt sind. Denn durch die sprecheradaptive Komponente der ASR passen sich die Systeme beim Gebrauch zunehmend besser an den jeweiligen Benutzer an (Fellbaum 2012:309).

Im Fall der Live-Untertitelung handelt es sich bei der angewandten ASR um eine hybride Form der sprecherabhängigen und sprecheradaptiven Spracherkennung. Die Übertragung auditiver Inhalte kann über den gleichzeitigen Einsatz einer Untertitelungs- und sprecherabhängigen und -adaptiven ASR-Software erfolgen. So kann mittels des sogenannten „Respeakings“ die Wiedergabe auditiver Inhalte durch das gesprochene Wort eines Untertitelredakteurs/einer Untertitelredakteurin mithilfe eines Mikrofons und den genannten Computeranwendungen in Untertitel umgesetzt werden. Laut Angaben eines Anbieters für ASR soll durch das Diktieren statt des Eintippens von Transkriptionen eine Zeitersparnis von bis zu $66 \%$ erreichbar sein (Nuance 2015).

Alternativ zu sprecheradaptiver ASR existieren heute einige Anbieter von sprecherunabhängigen, automatischen Transkriptionssystemen und Editoren, die das gesprochene Wort mit Zeitstempeln versehen und bei guter Audioqualität eine Erkennungsgenauigkeit von $80 \%$ bis $90 \%$ erreichen. Dank eines integrierten Editors und weiterer Funktionen sowie einer automatischen Generierung von Untertiteln versprechen diese Lösungen eine beträchtliche Produktivitätssteigerung (u.a. Nuance o. A., TRINT 2016, Myers 2018, 3Play Media 2018).

Doch wie bereits dargelegt sind, jenseits der reinen Wiedergabe der gesprochenen Inhalte und zusätzlicher Informationen, für die nächsten Arbeitsschritte weitere Mensch-Maschine-Interaktionen erforderlich. Dazu gehören:

- textliche Bearbeitung und Umstrukturierungen des Originaltons im Sinne vom Moduswechsel der Mündlichkeit zur Schriftlichkeit

- Transfer nonverbaler, para- und extralinguistischer Informationen 
- (Neu-)Strukturierung und Verdeutlichung von überlappenden Gesprächsanteilen

- das Filtern von Stör- und Hintergrundgeräuschen

- Einfügung oder Korrektur von Satzzeichen

- farbliche Kennzeichnung von Sprecherwechseln

- ggf. Segmentierungsoptimierungen der Untertitel für eine optimale Rezeption der Untertitel

- Korrekturen des Spracherkennungsoutputs bei fehlerhaften Erkennungen

Eine vollautomatische Spracherkennung würde in Bezug auf die SDH hier also in vielerlei Hinsicht an ihre Grenzen stoßen, was wiederum die menschliche Komponente somit unverzichtbar macht.

\subsection{Derzeitige Produktionsprozesse der AD in Deutschland}

\subsubsection{AD-Postproduktion}

Die Produktion einer Audiodeskription beginnt zunächst mit der Sichtung des Filmmaterials und der Zeitspannen, in denen Filmtonpausen für die Audiodeskription vorhanden sind oder in denen Geräusche, Musik u. Ä. vorkommen, die Bildbeschreibungen erfordern (Jekat 2016: 82). Dies geschieht im Idealfall in einem Team von ein oder zwei sehenden Beschreiber(inne)n und einem/ einer nicht-sehenden Teamkollegen/Teamkollegin, wobei die Zusammenstellung und Anzahl der Teammitglieder von AD-Dienstleister zu -Dienstleister variiert. Wird der $\mathrm{AD}$-Text durch nur einen Beschreiber/eine Beschreiberin erstellt, ist mindestens für die Abnahme und Kontrolle ein(e) blinde/r oder sehgeschädigte/r AD-Redakteur(in) hinzuzuziehen, der oder die den Text auf problemlose Funktionalität prüft. Nach Anpassung des AD-Skripts werden die einzelnen AD-Passagen von einem/einer professionellen Sprecher(in) in einem 
Tonstudio eingesprochen. Im Tonstudio sind gewöhnlich ein(e) Tontechniker(in), Sounddesigner(in) und Tonregisseur(in) in den Prozess involviert. Abschließend erfolgt eine ausbalancierte Zusammenlegung und Mischung des Originalfilmtons mit der AD für die finale Produktion der Hörfilmfassung des audiovisuellen Ausgangsmaterials (vgl. Benecke 2014: 14ff.).

Auch für diese Form der barrierefreien Postproduktion existieren einige wenige Softwaresysteme (Jekat 2016: 82). Diese erlauben wie bei der Untertitelungssoftware die Zeitkodierung. In diesem Fall liegt der Fokus jedoch auf den Zeitspannen, in denen kein gesprochener Inhalt vorhanden ist bzw. in dem kein Filmton zu hören ist und dies ich für das Einsprechen von AD-Passagen eignen. Nicolai beschreibt diesen Prozess treffend als die „akustische Untertitelung visueller Medien“ (ebd. o. A.). Der AD-Text kann in diesen zeitkodierten Zeitspannen schriftlich eingegeben und durch ein Mikrofon zu Testzwecken aufgesprochen werden, um zu überprüfen, ob die Beschreibung in die dafür vorgesehene Zeitspanne passt. So können ggf. Anpassungen vorgenommen werden, d.h. der Audiodeskriptionstext wird gekürzt oder überarbeitet. Bei einigen dieser AD-Programme kann die gesamte Hörfilmfassung produziert und als sendefähiges Format exportiert werden (Jekat 2016: 83).

\subsubsection{AD-Postproduktion und Sprachsynthese}

Als Alternative zur „traditionellen“ AD wurden in jüngster Zeit SoftwareLösungen entwickelt, die neben den genannten Funktionen den AD-Text durch eine künstliche Stimme in verschiedenen Sprachen und von Sprechern unterschiedlichen Geschlechts wiedergeben können. Dies ist einerseits durch eine lokal installierte Sprachsynthese-Software möglich. Anderseits besteht die Möglichkeit, Sprachsynthese-Stimmen zu nutzen (engl. text-to-speech synthesis, Abk. TTS), die per cloudbasierter Platform-as-a-Service-Anwendung (Abk. PaaS) verfügbar sind (u.a. Video To Voice GmbH o. A., Mieskes/Martínez Pérez 2011). Diese TTS-Software wandelt, im Gegensatz zur ASR, orthographische Texteingaben in ein gesprochenes Sprachsignal um (Pfister/Kaufmann2017: 27). Dieser Produktionsprozess wird auch englisch als TTS-AD bezeichnet (Szarkowska 2011). Im Rahmen dieses Beitrags wird für die Audiodeskription mit Text-toSpeech-Synthese die Abkürzung AD-TTS verwendet. 
Neben dem „reinen“ AD-Text können weitere Informationen zur Optimierung der prosodischen Ausdrucksweise des Sprachsignals hinzugefügt werden, z. B. zu Geschwindigkeit, Tonhöhe und Tonfall, Betonung für die Prosodie und Intonation einzelner Buchstaben, Wörter und Satzsegmente. Die Einbettung von TTS erlaubt also, alternativ zur Aufnahme der Stimme einer weiteren Person, die Wiedergabe von AD-Passagen mittels künstlicher Stimme. Die Nutzung dieser Sprachtechnologie kann ebenfalls für das Testen der optimalen Länge des $\mathrm{AD}$-Textes in den dafür verfügbaren Zeitspannen genutzt werden. Dies geschieht einerseits durch das Probehören des jeweiligen AD-Textfragments mittels künstlicher Stimme, andererseits durch eine automatisierte Kalkulation der ungefähren zeitlichen Länge des AD-Segments anhand der darin verwendeten Zeichen. So erhält der $\mathrm{AD}$-Redakteur Auskunft darüber, ob die wiederzugebende Audiodeskription via Sprachsynthese für die jeweilige ADPassage in die dafür vorhandene Zeitspanne passt.

Im Falle finanzieller Restriktionen können im Zuge dieses Produktionsprozesses die per Sprachsynthese wiedergegebenen AD-Segmente automatisiert mit dem audiovisuellen Originalmaterial zusammengelegt werden. Teilweise verfügen diese multifunktionalen Anwendungen über eine automatisierte Ausbalancierung von Originalton und AD-TTS. Diese Variante unterscheidet sich jedoch in ihrer Qualität bisher noch deutlich von der „traditionellen“ AD. Entscheidend ist daher, für welche Formate - oder in diesem Sinne für welche „ADTextsorten“ - diese alternative Produktionsmöglichkeit Anwendung finden kann, was weiterer Forschung bedarf. Festhalten lässt sich dennoch, dass diese Technologie als kosteneffizientere Variante zum Zugang zu audiovisuellen Medien für Menschen mit Seheinschränkung genutzt werden kann, bei denen aufgrund finanzieller Hürden eine AD sonst nicht umsetzbar wäre. In diesem Kontext betont auch Szarkowska (2011: 146):

TTS AD is by no means intended to replace the audio description practice currently in use. Rather, it aims to supplement it and to increase the number of audio described films and audiovisual programmes made available to people with visual impairments. 
Die Akzeptanz der AD-TTS als Alternative bestätigt sich im Zuge diverser Studien (Matamala 2016: 279ff.), u.a. auch einer Studie im deutschsprachigen Raum, die jedoch ebenfalls ergaben, dass eine menschliche Stimme allgemein präferiert wird (Kurch 2016: 59ff.).

Die Verwendung von Sprachtechnologien als Hilfsmittel für die intralinguale Übersetzung eines Übersetzers/einer Übersetzerin zeigt insgesamt jedoch, dass es sich lohnt, einen Blick auf jüngste technologische Innovationen zu werfen. Besonders die Symbiose von maschinellen Lernverfahren und Sprachtechnologie zur Erfassung, zum Verstehen und zur Generierung natürlicher Sprache hielt in den letzten Jahren verstärkt Einzug in Forschung, Industrie und Gesellschaft (Härtel 2016: 68). Die Kombination dieser beiden Bereiche und die intensive Forschung verzeichneten beachtliche Ergebnisse. Das folgende Kapitel befasst sich daher mit der Frage, inwieweit maschinelle Lernverfahren (Abk. ML) in Kombination mit Sprachtechnologien Übersetzer(innen) in der intralingualen Übersetzung unterstützen können.

\section{Teilautomatisierungsprozesse für die Postproduktion von $\mathrm{SDH}$ und $\mathrm{AD}$}

Im spanischsprachigen Raum wurde im Zuge diverser Forschungsprojekte mit äußerst innovativen Ansätzen in der Teilautomatisierung (Abk. TA) verschiedener intralingualer Übersetzungsaufgaben für die Produktion barrierefreier audiovisueller Medien experimentiert (Álvarez Muniain 2016: 205ff., Matamala 2016). Im Laufe der letzten Jahre wurden ML zunehmend für Teilautomatisierungsprozesse (TAP) verschiedener Arbeitsabläufe besonders in der Untertitelung erprobt. Hier wurden bereits einige kommerzielle Lösungen entwickelt, die eine teilautomatisierte Generierung von Untertitelungen von Audio- und Videodateien ermöglichen. Diese umfassen diverse Technologien, die automatisch das gesprochene Wort in Text und Text in Worte überführen, sowie $\mathrm{Zu}$ ordnungen zu jedem geschriebenen und gesprochenen Wort per Zeitinformationen erfassen und mit Zeitstempeln versehen. Die Eingangs- und Ausgangsstandzeiten bedürfen vereinzelt noch geringfügiger Nachbearbeitung durch die jeweiligen Redakteure/Redakteurinnen, zu denen die oben genannten humanen 
Transferleistungen gehören. Die folgenden Ausführungen sind jedoch als theoretischer Ausblick im Sinne eines Best Practice zu betrachten, in dem die heute existierenden Potenziale sichtbar gemacht werden.

Die Synergie aus diversen Sprachtechnologien und ML erzielten zum Teil äußerst vielversprechende Ergebnisse. Diese erlauben eine zeit- und somit kosteneffizientere Produktion barrierefreier Medieninhalte im Bereich der SDH und der AD (Álvarez Muniain2016, Matamala 2016). Nachfolgend soll eine zusammenfassende Erläuterung der Potenziale dieser Technologien im Sinne eines Best Practice-Workflows skizziert werden. In der folgenden Übersicht werden verschiedene erprobte TAP ausgeführt, die in der Lage sind, die obengenannten Anforderungen durch ML in Kombination mit diversen (Sprach-)Technologien zu einem gewissen Teil automatisiert zu erfüllen.

Audio- und Sprachverarbeitungstechnologien kommen heute bereits erfolgreich zum Einsatz, wie z. B. bei der Interaktion mit Smartphones und Navigationsgeräten mittels Sprachein- und -ausgabe. So ermöglichen Audioinhaltsanalysen heutzutage die automatische Extraktion semantischer Informationen von audiovisuellen Inhalten, die Analysen von Sprach-, Musik- und/oder Geräuschsegmenten beinhalten. Die damit verbundenen Technologien sind vielzählig. Im aktuellen Anwendungsbeispiel dieser Technologien werden umfassende Datensätze vorhandener, barrierefreier audiovisueller Produktionen benötigt. Das sind in diesem Fall Daten bereits produzierter SDH und AD. Die Daten werden in das jeweilige System eingespeist, um von ihnen zu lernen (ML: SDH- und AD-Datensatz). Anhand dieser Informationen kann dieses System das Erlernte auf neues audiovisuelles Material anwenden (AV-Text: $\mathrm{SDH} \pm \mathrm{AD}$ ). Dies bedeutet, es können automatisiert gesprochene Inhalte von Untertiteln und Audiodeskriptionen transkribiert und zeitlich zugeordnet werden (Zeitkodierungen), die nicht als Lernmaterial für das ML verwendet wurden und somit der Maschine unbekannt sind.

Die Sinnhaftigkeit der Nutzung eines AD-Transkripts lässt sich damit begründen, dass dies im Bedarfsfall einer interlingualen Übersetzung (Abk. hier: TL, für engl. translation) des jeweiligen AV-Textes verwendbar wäre. So müsste die AD nicht von Grund neu getextet werden (Jankowska 2015) und könnte ebenfalls als AD-TTS produziert werden. Bei dieser Produktionsmethode ist jedoch zu prüfen, ob diese mit den erwünschten zeit- und kosteneinsparenden 
Vorteilen einhergeht und gegebenen Qualitätsansprüchen an die AD-Produktion gerecht wird.

Mittels informationsangereichertem Material durch das maschinelle Lernverfahren können also die Transkription und die Zeitkodierung einer Audiodatei inklusive Metadaten automatisch generiert werden, die Informationen zu Interpunktion, Großschreibung, Sprechertrennung und Vergabe von SprecherIDs, Satzsegmenten, etc. beinhalten (Álvarez Muniain2016: 45). Dafür durchläuft die Bearbeitung des neuen audiovisuellen Materials mehrere Schritte, wodurch Untertitler(innen) oder Übersetzer(innen) von Audiodeskriptionen ein Großteil der Arbeit abgenommen werden kann.

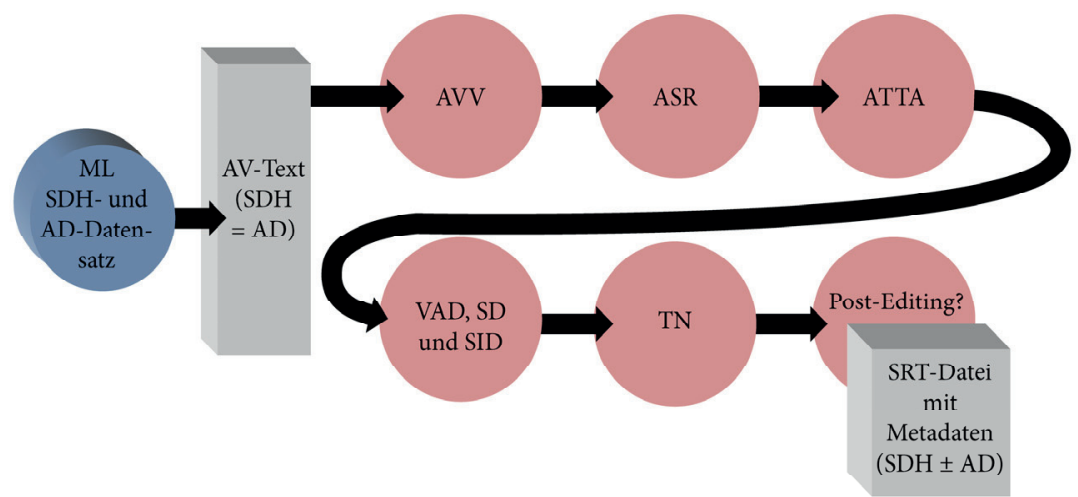

Abb. 1: Pipeline zur Vorverarbeitung des AV-Textes mittels ML und (Sprach-)Technologie

Per Audiovorverarbeitung (Abk. AVV) werden Inhalte des AV-Materials als sprachliche bzw. nicht-sprachliche Anteile erkannt. Daraufhin werden die sprachlichen Anteile per ASR transkribiert. Im Anschluss daran erfolgt die Zuordnung der transkribierten Sprachanteile zu dem gesprochenen Signal der Audiospur, wodurch Wort für Wort die akustischen und textlichen Anteile miteinander verknüpft und zeitkodiert werden (das sogenannte audio-to-text alignment, Abk. ATTA). Des Weiteren werden vom Output der ASR akustische, prosodische und linguistische Informationen extrahiert. Diese Informationen kommen ggf. einer Erkennung der verschiedenen Sprecher innerhalb des AV- 
Materials entgegen, um diese zu ermitteln (Sprechpausenerkennung, Sprechertrennung und Sprecheridentifikation, engl. voice activation detection, speaker diarization und speakeridentification, Abk. VAD, SD und SID, Matamala 2016: 273f.).

Im nächstem Schritt werden Textnormalisierungen (Abk. TN) wie Interpunktionen, Großschreibungen sowie die Konvertierung von Zahlen, Daten und Mengen (z. B. Geldeinheiten und Prozent), etc. umgesetzt. Da das System nicht fehlerfrei arbeitet, bedarf es hier jedoch weiterhin der menschlichen Kontrolle und Überarbeitung in Form von Post-Editing, um eine korrekte Transkription, Zeitkodierung und Sprecherzuordnung aller Inhalte sicher zu stellen. Dies lässt sich dann beispielsweise im SRT-Dateiformat inkl. Metadaten zur Weiterverarbeitung speichern.

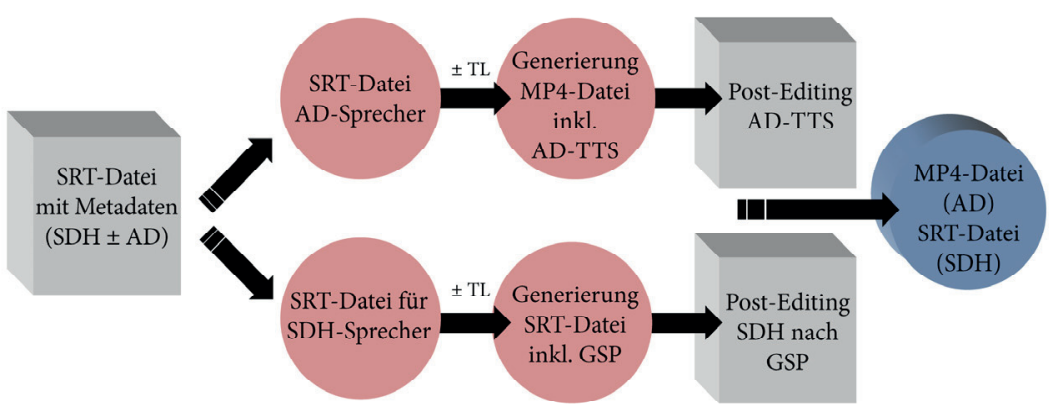

Abb. 2: Verarbeitung der SRT-Datei inklusive Post-Editing zu AD- und SDH-Dateien

Aus dieser SRT-Datei lassen sich durch die Metadaten die jeweiligen sprachlichen Einheiten extrahieren, die entweder der SDH oder der AD zuzuordnen sind. Hierbei ist ggf. eine manuelle Selektion der Sprecheranteile oder eine Kontrolle der korrekten Zuordnung der sprachlichen Einheiten zu den jeweiligen Sprecher-IDs (beispielsweise des/der AD-Sprechers/Sprecherin als SprecherID) durch einen/eine Redakteur(in) erforderlich (ebd. 274). Durch die „gelernte Erfahrung" eingespeister SDH-Dateien lassen sich per ML ebenfalls Merkmale gemäß einer "good subtitling practice“ (Abk., GSP, vgl. u.a. Ivarsson/Carroll 1998, Díaz-Cintas/Remael 2007) oder Vorgaben von vereinbarten Standards ei- 
ner intralingualen SDH (vgl. u.a. ARD o. A., Stand 2015) auf das zu verarbeitende Material anwenden. So kann eine automatische Generierung von SDH inkl. verschiedener Aspekte erstellt werden, wie:

- Synchronität der SDH zum Bild und Ton, die möglichst lippensynchron und am Originaltext bleibt

- adäquate Standzeitberechnung und Zeitkodierung einzelner SDH nach Zeichen und Zeilen gemäß erforderlicher Basiswerte für eine angemessene Lesegeschwindigkeit der SDH mit der Maßgabe lesbarer Zeichen in der Sekunde

- Vorgaben hinsichtlich Segmentierung

- farbliche Zuordnung von Sprecher(inne)n und Stimmen

- Einhaltung orthografischer Vorgaben, Standards, Richtlinien und Style Guides

Da auch hier die Vorarbeit des Systems keinesfalls fehlerfrei ist, sind die SDH im Sinne eines Post-Editing auf Fehler zu überprüfen und zu korrigieren. $\mathrm{Zu}$ dem sind nicht erfasste sprachliche Zusatzinformationen wie Geräusche, Musik, non- und paraverbale Inhalte als SDH in Klammersetzung hinzuzufügen. Auf diese Weise ließe sich in einem beschleunigten Verfahren eine entsprechende SRT-Datei für das zu bearbeitende AV-Material erstellen.

Im Falle der $\mathrm{AD}$ sind die jeweiligen ggf. intralingual übersetzten $\mathrm{AD}$ Segmente für die auditive Ausgabe mittels TTS zusätzlich als MP3-Dateien zu generieren und zu kontrollieren, ob die Ausgabe den qualitativen Vorstellungen entspricht. Ist dies nicht der Fall, können in einem Post-Editing-Prozess ebenfalls zuvor erwähnte Optimierungsmaßnahmen vorgenommen werden (siehe 2.2.2). Nach finaler Überprüfung der passenden Länge der AD-Anteile innerhalb der dafür vorhandenen Dialogpausen lässt sich dies mit dem AV-Material automatisiert zusammenlegen und als eine alternative AD-TTS-Hörfilmfassung zur „traditionellen“ AD als MP4-Datei exportieren. 


\section{$4 \quad$ Fazit und Ausblick}

Die zuvor theoretisch ausgeführten alternativen Produktionsweisen beleuchteten, inwiefern es anhand heutiger technischer Möglichkeiten möglich ist, kosten- und zeiteffizientere SDH und AD für audiovisuelles Material produzieren und welche weiteren Ansätze denkbar wären. Was jedoch vor allem bisher außerhalb des deutschsprachigen Raums erprobt wurde, ist hinsichtlich der Effizienz und Verbesserung der Produktivität auf deutsche SDH- und AD-Produktionen $\mathrm{zu}$ testen und anzuwenden, um hier die tatsächliche Kosten- und Zeitersparnis wissenschaftlich zu ergründen. Erst so ließe sich eine valide Aussage über eine etwaige Beschleunigung und Reduzierung des Arbeitsaufwands für Redakteure/Redakteurinnen in den Bereichen SDH und AD treffen. Hier bestehen folglich noch Forschungsdesiderate, die es zu schließen gilt. Zudem könnten aufgezeigte Umsetzungen positive Impulse für die Barrierefreiheit mit sich bringen und somit weitere Fortschritte im Hinblick auf eine barriereärmere Medienlandschaft erreichen. 
Alexander Kurch

\section{Literaturverzeichnis}

3Play Media (2018): „Solutions“. URL: https://www.3playmedia.com/solutions/ (letzter Zugriff: 21.2.2018).

Álvarez Muniain, Aitor (2016): Speech Technologies for the Audiovisual and Multimedia Interaction Environments. Donostia; San Sebastián: University of the Basque Country - Department of Computer Architecture and Technology; Vicomtech-IK4 Human Speech and Language Technologies Department. URL: https:/addi.ehu.es/ handle/10810/19491 (letzter Zugriff: 21.2.2018).

ARD (o. A.): „Untertitel-Standards“. URL: http://www.daserste.de/service/kontakt-undservice/barrierefreiheit-im-ersten/untertitel-standards/index.html (letzter Zugriff: 21.2.2018).

BENECKE, BERnd (2014): Audiodeskription als partielle Translation. Modell und Methode. Berlin: LIT.

Díaz-Cintas, Jorge/Remael, Aline (2007): Audiovisual Translation : Subtitling. Manchester: St. Jerome Publishing.

Fellbaum, Klaus (2012): Sprachverarbeitung und Sprachübertragung. Berlin, Heidelberg: Springer.

HÄRTEL, JOHANNES (2016): „Vollautomatisches Dolmetschen - Möglichkeiten und Grenzen“. In: Schmitt, Peter A./LeE-Jahnke, Hannelore (Hg.): Lebende Sprachen - Zeitschrift für interlinguale und interkulturelle Kommunikation 61 (1). Berlin: De Gruyter, 67-116.

HeZEL, SusANne (2009): „Untertitelung für Hörgeschädigte für das deutsche Fernsehen: Vorgehensweisen, Forderungen, Richtlinien“. In: Nagel, Silke/Hezel, Susanne/ Hinderer, Katharina/PiePer, Katrin (Hg.): Audiovisuelle Unterstützung: Filmuntertitelung in Deutschland, Portugal und Tschechien. Frankfurt am Main: Peter Lang, $147-264$.

IVARSSON, JAN/CARroll, MARY (1998): Subtitling. Simrishamn: TransEdit.

JankowsKa, Anna (2015): Translating Audio Description Script: Translation as a New Strategy of Creating Audio Description. Frankfurt am Main: Peter Lang.

Jekat, Susanne J. (2016): „Theorie und Methode der Audiodeskription: Ein Pilotprojekt“. In: Mälzer, NATHALIE (Hg.) (2016): Barrierefreie Kommunikation - Perspektiven aus Theorie und Praxis. Berlin: Frank \& Timme, 69-94.

KÄBER, MARCO (2016): „Die Geschichte der Liveuntertitelung - ein Praxisbericht“. In: Mälzer, Nathalie (Hg.): Barrierefreie Kommunikation - Perspektiven aus Theorie und Praxis. Berlin: Frank \& Timme, 295-306.

Kurch, Alexander (2016): Audiodeskription mit Sprachsynthese - Eine Perzeptionsund Rezeptionsstudie am Beispiel des Dokumentarfilms „buy buy st. pauli“. Universität Hildesheim: unpublizierte Masterarbeit. 
Landtag Rheinland-Pfalz (2017): „12. Sitzung des Ausschusses für Medien, Digitale Infrastruktur und Netzpolitik am 19.10.2017“. URL: http://www.landtag.rlp.de/ landtag/ausschuesse/mediena-12-17.pdf (letzter Zugriff: 21.2.2018).

LindNER, PETER (2016): „Vorproduktion - Semi-Live - Live. Ein Arbeitstag in der Untertitelredaktion des NDR“. In: MäLZER, NATHALIE (Hg.): Barrierefreie Kommunikation - Perspektiven aus Theorie und Praxis. Berlin: Frank \& Timme, 307-316.

Matamala, AnNa (2016): „The ALST Project: Technologies for Audio Description“. In: Matamala, Anna/Orero, Pilar: Researching Audio Description: New approaches. London: Palgrave Studies in Translating and Interpreting. Palgrave Macmillan, 269284.

Mieskes, Margot/Martínez Pérez, Juan (2011): „A Web-based Editor for Audio Titling using Synthetic Speech". 3rd International Symposium on Live Subtitling with Speech Recognition. Antwerp: European Media Laboratory, SWISS TXT. URL: http://www.respeaking.net/Antwerp\%202011/Webbased_editor.pdf (letzter Zugriff: 21.2.2018).

Myers, ERIN (2018): „Temi vs. Trint Comparison of Automatic Transcription Services“. URL: https:/www.temi.com/blog/2018/01/26/temi-vs-trint-comparison-automatictranscription/ (letzter Zugriff: 21.2.2018).

Nicolai, ANKe (o. J): Erschließung visueller Informationen für blinde und sehbehinderte Menschen am Beispiel Museum. Berlin: Hörfilm e. V.

NuANCE (o. A.): „Dragon Legal Individual : Rechtsdokumente effizienter erstellen durch Sprechen“. URL http://www.nuance.de/for-business/by-product/dragon/ dragon-for-the-pc/dragon-legal-individual/index.htm\#docturnaround (letzter Zugriff: 21.2.2018).

NuANCE (2015): „Nuance-Bericht für KMUs“. - http://www.nuance.de/business/ effizienter-arbeiten-im-buero-kleinunternehmen.pdf (letzter Zugriff: 21.2.2018).

Pfister, BeAT/KaUfmann, ToBias (2017): Sprachverarbeitung Grundlagen und Methoden der Sprachsynthese und Spracherkennung. Berlin: Springer Vieweg.

SZARKOWSKA, AGNIESKA (2011): „Text-to-speech audio description: towards wider availability of AD“. In: The Journal of Specialised Translation15, 142-162. URL: http://www.jostrans.org/issue15/art_szarkowska.pdf (letzter Zugriff: 21.2.2018).

TRINT (2016): „Trint launches tool that makes it easy to transcribe audio files to text instantly with funding from Knight Foundation and Google“. URL: https://blog.trint.com/ trint_launches_instant_transcription_tool (letzter Zugriff: 21.2.2018).

VerbaVoice GmbH (о. A.): „Dolmetscher online zuschalten“. URL: https://www.verba voice.de/dolmetscher (letzter Zugriff: 21.2.2018).

Video To Voice GmbH (o.A.): „Frazier - Audiodeskription mit Text-to-Speech-Technologies (TTS-AD)“. URL: https://videotovoice.com (letzter Zugriff: 21.2.2018). 



\section{Audiodeskription - Methoden und Techniken der Filmbeschreibung}

\section{$1 \quad$ Die Probleme bei der Erstellung eines Audiodeskriptionstextes}

Bei der Audiodeskription handelt es sich um eine vorwiegend für blinde und sehbehinderte Menschen entwickelte Technik, bei der optische Informationen in einem zumeist audiovisuellen Inhalt (Film, Theaterstück, Oper etc.) in einen geschriebenen Text transferiert werden, der dann akustisch präsentiert wird (siehe auch Benecke 2014a: 1f.). Das neu entstehende Produkt wird dann als Hörfilm, Hörtheater oder Höroper bezeichnet. Der aus den optischen Informationen entstandene Text soll die unverändert vorhandenen akustischen Informationen des Originalprodukts nicht behindern. Er muss so formuliert werden, dass er Dialoge sowie wichtige Musik- und Toneffekte (in der Oper z. B. auch die Arien) unberührt lässt, also in die verbleibenden Lücken eingepasst werden kann.

Der Autor bzw. die Autorin, im Folgenden als Beschreiber(in) bezeichnet, sieht sich also mit einigen eklatanten Problemen konfrontiert:

- Wann kann der Audiodeskriptionstext sinnvoll in die vorhandenen Pausen zwischen Dialogen sowie Musik- und Toneffekten eingefügt werden? Das Zusammenspiel mit den akustischen Informationen ist von herausragender Bedeutung, wird aber oft unterschätzt. Dabei kann der Ton dem/der Beschreiber(in) einiges an Arbeit abnehmen, weil viele Informationen im Bild auch durch den Ton erfasst werden (Benecke 2014b): So wird in vielen Filmen ein emotionales Wörterbuch wichtiger Figuren oder Schauplätze angelegt, d.h. wichtige Personen 
bekommen z. B. ein Musikthema, das immer erklingt, wenn sie auftauchen. Oder Schauplätze haben ein ganz bestimmtes Sounddesign aus Geräuschen und Tönen, die den Ort sofort akustisch wiedererkennbar machen. In diesem Fall kann sich die Audiodeskription zurückziehen. Musik ist auch entscheidend bei der Etablierung einer bestimmten Stimmung oder Emotion, sofern es sich um empathische Musik handelt - Musik, die die Bildinhalte unterstützt (im Gegensatz zu anempathischer Musik, die sich den visuellen Inhalten widersetzt, s. hierzu auch Chion 1990). Der Ton kann bei der Erstellung eines Audiodeskriptionstextes aber auch zusätzliche Probleme schaffen. So findet sich in vielen Filmen eine audio-visuelle Dissonanz (ebd.), d. h. im Ton ist etwas grundlegend anderes zu hören als im Bild gezeigt wird (z. B. vor dramatischen Bildern die sanfte Stimme eines Erzählers).

- Wie muss der Audiodeskriptionstext klingen, damit er dem Tonfall des Originalprodukts entspricht und sich homogen in die akustischen Informationen einfügt? Hier geht es um Fragen der Wortwahl, Syntax und Grammatik und natürlich des gewählten Sprachregisters. Dieses wird in einer anspruchsvollen Literaturverfilmung anders sein als bei einem Animations- oder Actionfilm, wobei sich durchaus die Frage stellt, ob ein eher vulgäres Sprachregister in den Dialogen eines Films sich auch in der Audiodeskription widerspiegeln muss.

- Was aus den umfangreichen Bildinhalten muss in der Audiodeskription auftauchen, damit gesprochener Text und Ton am Ende wieder ein sinnvolles Ganzes ergeben? Dieser Auswahlprozess ist für den/die Beschreiber(in) zumeist die umfangreichste und anspruchsvollste Arbeit. Zunächst wird er bzw. sie sich auf die Klärung folgender Punkte konzentrieren: Wo lassen sich die Bildinhalte verorten, sowohl zeitlich als auch räumlich? Es geht also um die Frage: Wo und zu welcher (Tages-)Zeit findet etwas statt? Dann ist zu klären, wer oder was präsent ist, welche Personen, welche wichtigen Gegenstände oder Gebäude etc. Und dann schließlich die Frage nach einer eventuellen 
Handlung: Was passiert im optischen Teil des Originalprodukts? Was davon muss im Text auftauchen?

In Kapitel 4 dieses Artikels wird dann noch die Frage nach dem Wozu einer Beschreibung auftauchen, sie soll hier erst einmal keine Rolle spielen.

\section{Lösungsansätze durch nationale Guidelines und Regelwerke}

Zur Klärung der in Kapitel 1 aufgestellten Fragen wurden im Laufe der Jahre eine Reihe von nationalen Guidelines und Regelwerken zusammengestellt. $\mathrm{Zu}$ ihnen gehören z. B. der ITC Guidance on Standards for Audio Description in Großbritannien oder in Spanien die Requisitos para la audiodescripción (AENOR). In Deutschland wurden Regeln zur Audiodeskription erstmals 1997 formuliert und 2004 überarbeitet (Dosch/Benecke 2004). Die letzte Formulierung von Regeln zur Audiodeskription erfolgte 2015 durch die öffentlich-rechtlichen Sender in Deutschland, Österreich und der Schweiz, sie gelten als verbindlich für alle Hörfilme in den Programmen dieser Sender (abrufbar auf der „Barrierefreiheit"-Seite von www.ndr.de) und sollen auch bei der Produktion von Audiodeskription für Kinofilme berücksichtigt werden.

Eine Reihe von Publikationen (Vercauteren 2007, Puigdomènech/Matamala/Orero 2010, Rai/Greening/Petré 2010) hat sich mit dem Vergleich verschiedener Guidelines beschäftigt und kommt im Wesentlichen zu einem durchaus überraschenden Ergebnis: Obwohl sich Audiodeskription in Stil, Wortwahl und Präsentation in vielen Ländern (aber z. B. auch innerhalb der deutschsprachigen Länder) durchaus unterscheidet und sich somit eine kulturelle Komponente in der Audiodeskription eines Landes nicht verleugnen lässt (was der Frage nach der Übersetzbarkeit von Audiodeskription eine interessante Färbung gibt), sind sich die existierenden Guidelines in ihren Vorgaben doch weitgehend einig. Das betrifft nicht nur die Grundregel, Audiodeskription nur in den Lücken zwischen Dialogen sowie Musik- und Toneffekten zuzulassen, sondern auch die Forderung nach der Beantwortung der oben erwähnten Wo/Wer/Was-Fragen. Auch in Detailfragen besteht meist Einigkeit: 
- So sollen Hauptpersonen und wichtige Schauplätze an geeigneter Stelle eine ausführlichere Beschreibung bekommen.

- Auch die Erwähnung von Farben sowie das Erklären unklarer Töne und Geräusche sind Teil der Audiodeskription.

- Schrift auf Gebäuden oder Schildern und auch Einblendungen müssen in der Beschreibung auftauchen, dazu gehören auch die Credits am Anfang oder Ende.

- Untertitelte Sequenzen werden mit einem Voice-Over versehen, gesprochen entweder von dem/der Sprecher(in) der Audiodeskription oder (je nach Film) von einer oder mehreren zusätzlichen Stimmen. Gleiches gilt für die Übertitelung im Theater oder in der Oper.

- Audiodeskription soll nicht erklären, nicht bewerten und nicht interpretieren.

Auch die eher formalen Vorgaben, wichtig bei der Frage nach dem Wie, ähneln sich in den meisten Fällen, z. B.:

- Der Text der Audiodeskription ist im Präsens geschrieben.

- Neue Begriffe werden mit unbestimmtem Artikel eingeführt.

- Fachbegriffe können verwendet werden, müssen aber eingeführt und erläutert werden.

- Auf filmtechnische Begriffe wird verzichtet, ihre Wirkung ist zu beschreiben.

Dass sich trotz dieser weitgehend gleichen Vorgaben insbesondere in Europa recht unterschiedliche Stile und Praktiken zur Audiodeskription entfalten konnten, zeigt, dass Guidelines zwar geschrieben und diskutiert werden, für den 
konkreten Alltag aber doch zweitrangig sind, weil die Vorgaben dem/der Beschreiber(in) noch einen großen Spielraum lassen. Insofern erschien es notwendig, einen europäischen Blick auf die Audiodeskription zu erhalten. Dieses geschah im Jahr 2011 mit dem von der EU geförderten Forschungsprojekt ADLAB (Audio Description Lifelong Access for the Blind), um das es im nächsten Kapitel gehen wird.

\section{Strategie statt Regeln: Die ADLAB-Guidelines}

Beantragt und geleitet von der Universität Triest fand von Oktober 2011 bis September 2014 das von der EU geförderte Forschungsprojekt ADLAB statt (www.adlabproject.eu). Neben Universitäten aus Portugal, Spanien, Belgien und Polen nahmen auch das öffentlich-rechtliche flämische Fernsehen VRT aus Belgien und der Bayerische Rundfunk aus Deutschland als TV-Sender an dem Projekt teil. Zunächst ging es wie in Kapitel 2 angedeutet darum, einen europäischen Überblick über die verschiedenen Aktivitäten und Schwerpunkte im Bereich Audiodeskription zu bekommen. Der Abschlussbericht (ADLAB 2013a) ist auf www.adlabproject.eu unter "deliverables “ abrufbar und bietet eine seitdem nicht wieder erreichte Informationsdichte zur Lage der Audiodeskription in Europa. In einem weiteren „work package“ ging es um die vielfältige Analyse eines Audiodeskriptionstextes (gewählt wurde der amerikanische Spielfilm „Inglourious Basterds“von Quentin Tarantino), deren Ergebnisse als Buch veröffentlicht werden konnten (Maszerowska/Matamala/Orero 2014).

Es folgte der erste europaweite Test zu Präferenzen der Zielgruppe. So wurden in allen Mitgliedsländer des Projekts einem Publikum aus blinden und sehbehinderten Menschen unterschiedliche Audiodeskriptionsfassungen (in der jeweiligen Landessprache) zu zwei Szenen aus „Inglourious Basterds“ gezeigt und anschließend anhand eines Fragebogens Fragen zum Verständnis und zu Vorlieben gestellt. Auch die Ergebnisse dieser Untersuchung sind sehr informativ: So gab es Einigkeit darüber, dass die Namen der Schauspieler bereits im Vorspann mit den Namen ihrer Figuren verbunden werden sollten, auch die Namen von Beschreiber(in) und Sprecher(in) werden schon im Vorspann gewünscht. Uneinigkeit herrschte dagegen bei den Fragen, ob geschriebener Text 
im Bild als solcher explizit angekündigt werden soll und wann und wie eine Figur im Film ihren Namen bekommt.

The respondents' preferences were as follows: actors' names should be given along with the characters they play in the opening credits (presumably to strengthen character identification), names of the AD author and the $\mathrm{AD}$ voice talent should be read out in the opening credits. There were no clear preferences as regards information sequencing and fragmentation as well as description/narration. The results were inconclusive in the case of announcement of text-on-screen and character identification, as neither the explicit announcing of text-on-screen or the lack of it nor naming characters immediately or only if introduced in the film had a significant effect on the replies to comprehension/information recall questions. (ADLAB 2013b)

Zum Abschluss des Projekts wurden dann europäische Guidelines zur Audiodeskription erstellt. Aus der Diskussion der Ergebnisse der Textanalyse und der Zielgruppentests ergab sich im Projekt die Folgerung, dass strenge Richtlinien oder Vorgaben wenig zielführend und nutzerfreundlich erscheinen. So ist in der deutschen Übersetzung zwar noch von „Richtlinien“ die Rede, diese verstehen sich aber im Sinne des Projekts eher als Strategien und Vorschläge bei wiederkehrenden Problemen. Es geht zunächst auch hier um eine detaillierte Analyse:

Diese Analyse besteht aus dem genauen Studium des Ausgangsmaterials, inklusive Hintergrundrecherche über das Material und seiner Produktion. [...] Alles, was Sie für die AD entscheiden, wird immer auch vom jeweiligen Kontext bestimmt, in dem ein bestimmtes narratives Ereignis (z. B. die Einführung einer Figur) stattfindet, und oft wird es mehr als eine Möglichkeit geben, es zu beschreiben. Abschnitt 2, das Erarbeiten eines AD-Skripts, soll Ihnen dabei helfen, Ihre eigenen Entscheidungen zu treffen und angemessene Strategien zu finden. Jedes Kapitel dieses Abschnitts geht auf eine besondere Schwierigkeit beim Erstellen eines ADSkripts ein (Figuren und Handlung, räumliches und zeitliches Setting, Genre, Filmsprache, Geräusche und Musik, Schrift im Bild, intertextuelle 
Bezüge, Wortwahl und Stil, Kohäsion) und hat die gleiche Grundstruktur. (Remael/Reviers/Vercauteren 2014: 19f.)

Die ADLAB Richtlinien entstammen einem universitären Umfeld und haben einen anderen sprachlichen Duktus als die aus der Praxis entstandenen und in Kapitel 2 erwähnten Guidelines. Durch diesen völlig anderen Ansatz können die ADLAB Richtlinien selbst einem/einer erfahrenen Beschreiber(in) neue Blickwinkel sowie Strategien aufzeigen, die Praktiker(inne)n bei der täglichen Audiodeskriptionsarbeit normalerweise entgehen. Das trifft auch auf das im folgenden Kapitel beschriebene Audiodeskriptions-Entwicklungsmodell ADEM zu, das auf wissenschaftlichen Theorien zur Übersetzung fußt und diese mit der langjährigen Praxis der Audiodeskription verwebt.

Von den in den vorgehenden Kapiteln beschriebenen Regelwerken und Strategien bei der Erstellung eines Audiodeskriptionstextes löst sich das Audiodeskriptions-Entwicklungsmodell ADEM (Benecke 2014a: 43f.) gänzlich. Hier werden stattdessen Erfahrungen aus der Praxis (u.a. auch die in Kapitel 1 erwähnte Wichtigkeit der Analyse des Tons) mit den von Gerzymisch/Mudersbach (1998) entwickelten „Methoden des wissenschaftlichen Übersetzens“ kombiniert.

Dafür werden zunächst die in Kapitel 1 erwähnten Fragen nach dem Wann, Wie und Was um die Frage nach dem Wozu ergänzt: Zu welchem Zweck wird etwas beschrieben? Dafür greift Benecke (2014a) auf den Begriff des erlebten Eindrucks (Willi Bühler 1937: 153f.) zurück. ADEM liegt somit die Annahme zugrunde, dass der/die Beschreiber(in) beim Betrachten des Originalprodukts ein ganz bestimmtes Erlebnis oder einen erlebten Eindruck hatte, das oder den er oder sie mithilfe der Audiodeskription an das blinde und sehbehinderte Publikum weitergeben möchte.

Danach wird die Audiodeskription in den Bereich der Übersetzungswissenschaften eingebettet und hierbei als partielle Translation (Benecke 2014a: 44) 
verortet. Zugrunde liegt ja ein aus Bild und Ton bestehendes kohärentes Ausgangsprodukt - der Film ohne Audiodeskription. Im Verlauf des Audiodeskriptionsprozesses wird die Bildinformation in (gesprochene) Textinformation übersetzt, wobei die Toninformation weitgehend unberührt bleibt. Beides, gesprochener Text und unveränderter Ton, muss im Zielprodukt wieder kohärent zusammenpassen, d.h. es wird hier nur partiell übersetzt und die nicht übersetzten Anteile müssen mit dem Translat wieder eine logische Einheit bilden. In Anlehnung an das Sprachzeichenmodell (Karl Bühler 1934: 28) entwickelt Benecke dann drei Kommunikationsmodelle, die die Stufen des Audiodeskriptionsprozesses beschreiben und visualisieren (Benecke 2014a: 49f.).

Diesen theoretischen Vorüberlegungen folgt eine methodische Umsetzung, die aus den folgenden Schritten besteht:

Zugrunde liegt das Handlungsziel eines/einer Beschreibers/Beschreiberin, einem blinden oder sehbehinderten Publikum seinen erlebten Eindruck beim Betrachten des Originalprodukts mittels der Audiodeskription zu vermitteln. Dafür sind zunächst drei Fragen zu klären:

1. Was ist der antizipierte Empfängerkreis der Audiodeskription? Welche Voraussetzungen bringt das blinde oder sehbehinderte Publikum für das Verständnis der Audiodeskription mit? Es könnte sich beispielsweise um einen Film für Kinder handeln, die entweder (weil geburtsblind) keinen oder nur einen kleinen Bildspeicher mitbringen im Gegensatz zu einem Erwachsenen, der viele Jahre gesehen hat und somit noch vieles aus seiner Erinnerung memorieren kann. Es könnte sich um ein Theaterstück für ein eher anspruchsvolles und informiertes erwachsenes Publikum handeln etc. Der intendierte Empfängerkreis hat großen Einfluss auf das notwendige Maß an Informationen in der Audiodeskription.

2. Was sind die Vorgaben und Restriktionen im Ton? Diese Frage beschäftigt sich mit den in Kapitel 1 erwähnten Fragen nach Erleichterungen durch emotionales Wörterbuch und empathische Musik oder zusätzliche Behinderungen durch audiovisuelle Dissonanz oder anempathische Musik. 
3. Was ist aus Sicht des Beschreibers der übergeordnete "Sinn“ des Originalprodukts? Diese Frage steht im Zusammenhang mit dem Erlebnis oder erlebten Eindruck des Beschreibers/der Beschreiberin, worum geht es nach seinem oder ihrem Empfinden im Originalprodukt? So kann z. B. der Film „Das Leben der Anderen“ als Film über die DDR in den 1980er-Jahren verstanden werden, aber auch über das Scheitern einer Schauspielerin am politischen System oder über den Wandel eines Stasi-Agenten zum „guten Menschen“. Alles ist richtig, die Entscheidung für eine der Möglichkeiten ändert aber den Inhalt der Audiodeskription. Diese Sinngebung kann im Sinne von Gerzymisch/Mudersbach (1998) als Wissenssystem oder Holon formuliert werden. Ein solches Holon sorgt für mehr Übersicht und wird auch für Schritt 6 benötigt. Ein Beispiel für ein Holon zum Film „Das Leben der Anderen“ findet sich bei Benecke (2014a: 89).

4. Nach dem Blick auf das Originalprodukt als Ganzes und der Klärung der Frage, worum es aus Sicht des Beschreibers geht, folgt der Blick auf die einzelnen Sequenzen der Beschreibung. Bei einem Film wäre das z. B. die Beschreibung der einzelnen Szenen des Films. Diese sollten genau analysiert und die Ergebnisse übersichtlich gesammelt werden. Hierfür bietet sich nach Gerzymisch/Mudersbach (1998) das Ausfüllen einer sogenannten Aspektmatrix an. Es handelt sich dabei um eine Tabelle, bei der vertikal die Szenen in chronologischer Folge angeordnet werden, während horizontal wichtige Aspekte im Film erscheinen, die Einfluss auf die Audiodeskription haben. Solche Aspekte sind u.a. die Länge der Dialogpausen und die Intensität der Handlung, die in den Dialogpausen in Worte gefasst werden muss. Es kann darüber hinaus festgehalten werden, ob es wichtige Ton- und Musikeffekte in der Szene gibt, ob Namen von Personen oder Schauplätzen fallen, ob es unklare Geräusche gibt o.ä. Bei Benecke (2014a: 91f.) findet sich die gesamte Aspektmatix für „Das Leben der Anderen“. 
5. Während Sinngebung und Aspektmatrix in die praktische Arbeit der Audiodeskription leicht integriert werden können (siehe dazu auch Kapitel 5), scheint das von Gerzymisch/Mudersbach (1998) vorgeschlagene Erstellen von sogenannten synchron-optischen Netzen für die Praxis wenig praktikabel. Zwar liefert ein solches Netz wichtige Informationen zu inhaltlichen Bezügen und Beziehungen innerhalb des Originalprodukts - die praktische Erstellung nimmt aber viel Zeit in Anspruch, sodass sich der effektive Nutzen meist in Grenzen halten wird. Bei Benecke (2014a: 96) findet sich zum Film „Das Leben der Anderen" ein synchron-optisches Netz, das einen wichtigen inhaltlichen Bezug (rotes Farbband sorgt für roten Fingerabdruck auf Stasibericht und entlarvt so den Stasi-Mann als Retter) übersichtlich visualisiert. Man erkennt aber auch, dass solch ein Netz nur mit erheblichem Aufwand erstellt werden kann, erst recht für einen ganzen Film.

6. Nun wird das in Schritt 3 erstellte Holon mit den Szenen der Aspektmatrix aus Schritt 4 verknüpft und es entsteht eine Grafik, die visualisiert, welche Unterpunkte des Holons entweder im Ton oder im Bild oder auch in beiden zu finden sind. Das erlaubt nun schnelle Rückschlüsse, welche Informationen für die Audiodeskription unter genau dieser Sinngebung und genau dieser Aspektanalyse wichtig sind - es sind nämlich jene, die nur im Bild auftauchen. Was im Ton auftaucht, kann für die Audiodeskription vernachlässigt werden - was nur im Bild auftaucht, muss aber mit hoher Wichtigkeit Bestandteil der Audiodeskription sein.

7. Wie die in Schritt 6 identifizierten wichtigen Inhalte nun in die Audiodeskription aufgenommen werden können, zeigt das Handlungsschema des Inhalts (Benecke 2014a: 68f.). Es handelt sich um ein Flussdiagramm, das prüft, wie und wo ein wichtiges Detail in die Audiodeskription eingefügt werden kann. Dabei durchläuft dieses Detail eine Reihe von Handlungsanweisungen, die mit Ja oder Nein/Teilweise beantwortet werden können. Handlungsanweisung A 
fragt z. B. noch einmal nach dem Auftauchen des zu beschreibenden Details in der Tonspur. Es folgen u.a. Fragen zum Auftauchen des Details in einer Kohärenzdeskription (eine erste spontane Audiodeskription, die einem blinden oder sehbehinderten Publikum ein kohärentes Verstehen ermöglicht) oder zur physischen Möglichkeit, die Beschreibung des Details an einer konkreten Stelle zwischen den Dialogpausen einzufügen.

Das Flussdiagramm erlaubt auch die Möglichkeit, ein wichtiges Detail mittels einer Intended Hyper Description (Benecke 2007; 2009) einzufügen. Diese kommt zum Zuge, wenn ein wichtiges Detail aus Platzgründen nicht direkt an der Stelle seines visuellen Auftauchens in die Audiodeskription aufgenommen werden kann und sucht nach einer Stelle im Originalprodukt, wo diese Information kohärent in der Audiodeskription auftauchen kann, obwohl es an dieser Stelle kein visuelles Pendant gibt. Ein Beispiel ist der oben erwähnte wichtige Hinweis auf das rot abfärbende Farbband in „Das Leben der Anderen“. Es wird im Film nur an einer Stelle visualisiert, an der aber aus Platzgründen keine Audiodeskription möglich ist. Mithilfe einer Intended Hyper Description und unter Verwendung der Aspektmatrix lassen sich aber Stellen im Film identifizieren, an denen zumindest die Schreibmaschine zu sehen und genug Dialogpause vorhanden ist, um das rot abfärbende Farbband in der Audiodeskription zu erwähnen, auch wenn dieser wichtige Aspekt an dieser Stelle im Bild nicht zu sehen ist.

Ähnliche Flussdiagramme helfen bei der Beschreibung formaler Auffälligkeiten (Schwarzweißfilm, Rückblende, Zeitlupe etc.), bei der Namensvergabe von Figuren und Schauplätzen oder beim Finden von längeren Dialogpausen, um dort wichtige Figuren und Schauplätze ausführlicher zu beschreiben (siehe Kapitel 1).

ADEM erkennt die individuelle Komponente bei der Erstellung einer Audiodeskription an; zudem macht es ADEM bei einer späteren Analyse der Audiodeskription möglich, die einzelnen Schritte und die dabei erfolgten Entscheidungen nachzuvollziehen. 
Schließlich eröffnet ADEM auch eine didaktische Perspektive. Die Ausbildung von Beschreiber(inne)n kann auf ein konkretes Handlungsgerüst gestützt werden, das den/die Lernende(n) Schritt für Schritt in die Notwendigkeiten bei der Erstellung einer Audiodeskription einführt. Wie sich ADEM auf das Training neuer Beschreiber(innen) in der Realität ausgewirkt hat, darum soll es im abschließenden Kapitel gehen.

\section{$5 \quad$ Der Einfluss von Guidelines und ADEM auf das Training neuer Beschreiber}

Das Vorstellen und Diskutieren von Guidelines ist zumeist fester Bestandteil in den Ausbildungsseminaren und Trainingseinheiten zur Audiodeskription. Inwieweit dies auch für die in Kapitel 3 beschriebenen ADLAB-Strategien gilt, bleibt zumindest fraglich. Das in Kapitel 4 vorgestellte Audiodeskriptions-Entwicklungsmodell ADEM ist jedoch inzwischen Gegenstand der Ausbildung neuer Filmbeschreiber(innen) und hat sich dort nach den bisherigen Erfahrungen auch gut bewährt. ADEM ist ein fester Programmpunkt in den Seminaren zur Audiodeskription der Hochschule für Angewandte Sprachen am SDI München (www.sdi-muenchen.de/seminare/film-uebersetzung/audiodeskription/):

Am ersten Tag des Seminars arbeiten die Teilnehmer(innen) zunächst auf der Basis einer allgemeinen Einführung ins Thema und der Diskussion von Regelwerken. Dies geschieht meist an einem eher wenig komplexen Filmbeispiel, zum Beispiel einem kurzen Clip aus einer Fernsehserie. Die Ergebnisse werden diskutiert und - falls vorhanden - mit der professionell erstellten Audiodeskription des Clips verglichen. Aufgrund dieser ersten praktischen Erfahrung mit Audiodeskription erfolgt am zweiten Tag eine ausführliche Einführung in Modell und Methode von ADEM.

Die Seminarteilnehmer(innen) erhalten dann einen zweiten, anspruchsvolleren Filmausschnitt, zumeist aus einem Kinofilm, und werden aufgefordert, nach dem ersten Sichten des Clips Holon und Aspektmatrix zu erstellen. Inwieweit das Holon dann wirklich ausformuliert wird, entscheidet sich je nach dem Fortgang des Seminars und dem individuellen Arbeitstempo der Teams. In jedem Fall sind die Beschreiber(innen) aufgefordert, das gewählte Holon bei der 
anschließenden Texterstellung präsent zu haben und zu berücksichtigen, damit der Einfluss des Holons auf den Text der Audiodeskription sichtbar wird - immer dann, wenn aufgrund einer knappen Dialogpause bei gleichzeitig hoher Bildinformation Entscheidungen dahingehend notwendig sind, auf welche Inhalte der Fokus in der Audiodeskription gelegt werden soll. Ein Beispiel aus der Praxis:

Die künftigen Filmbeschreiber(innen) bekamen die ersten 20 Minuten des Spielfilms „Inglorious Basterds“ als Arbeitsmaterial und waren aufgefordert, zu diesen 20 Minuten ein Holon zu erstellen und für die Beschreibung zu nutzen. Eine kurze Inhaltsangabe der 20 Minuten: Im besetzten Frankreich taucht SSOffizier Landa bei Monsieur LaPadite und seinen Töchtern auf, um sich nach dem Verbleib einer jüdischen Familie aus der Umgebung zu erkundigen. In einem langen Gespräch setzt Landa LaPadite so unter Druck, dass dieser schließlich das Versteck der Familie unter dem Fußboden seines Hauses verrät. Die in mehreren Seminaren gesammelten Holons zum Clip lassen sich mehrheitlich zwei Kategorien zuordnen: „Die Angst der Familie LaPadite“ und „Die Bedrohung durch die Nazis“.

Ein Beschreiberteam, das sich für das erste Holon entschieden hatte, schlug für eine Sequenz des Clips relativ zu Anfang die folgende Audiodeskription vor:

Der Vater sieht den Fahrzeugen entgegen. Er wischt sich mit einem dreckigen Taschentuch über Stirn und Bart. Seine Kleidung ist abgewetzt. Der Vater blickt sorgenvoll aus tiefblauen Augen und geht zum Haus.

Ein Team, das sich für das zweite Holon entschied, wählte folgende Beschreibung:

Der Vater senkt den Blick. Die eskortierte Limousine hat das Haus beinahe erreicht. Während die Fahrzeuge vorfahren, kühlt er sich mit dem Wasser Gesicht und Nacken. Die Militärfahrzeuge, zwei Motorräder und ein Auto, erreichen die Anhöhe. [...] Drei Soldaten stehen einem Oberst zugewandt, der auf der Rückbank des offenen Autos sitzt.

Ähnliche Unterschiede finden sich an einer anderen Stelle des Clips: 
Die Töchter, eine blond, zwei brünett stehen ernst und still mit verschränkten Händen im Raum. (vom Team mit dem Holon Die Angst der Familie LaPadite)

Oberst Landa trägt einen langen Ledermantel und eine Aktentasche. Er hat ein kantiges Gesicht und einen intensiven Blick (vom Team mit dem Holon Die Bedrohung durch die Nazis)

In beiden Fällen mussten sich die Beschreiber(innen) entscheiden, welche Information sie in der knappen Dialoglücke vermitteln wollten, damit das blinde oder sehbehinderte Publikum den gleichen erlebten Eindruck erfährt wie sie selber. Diese Entscheidung geschah durch die Wahl des Holons. Dieses Beispiel illustriert, wie sich mit der (auch unbewussten) Wahl eines Holons eine Audiodeskription in eine bestimmte Richtung bewegt und eben hier eher den Schwerpunkt auf die Angst der bedrohten Familie legt oder (was für den Film als Ganzes möglicherweise die bessere Wahl ist) auf die Bedrohung durch die Nazis und speziell diesen einen Offizier.

Aber auch das Ausfüllen der Aspektmatrix erleichtert die Arbeit der Beschreiber(innen), was bereits bei der vergleichsweise kurzen Sequenz von 20 Minuten Film deutlich wird. Die Aspektmatrix erlaubt ein schnelles Identifizieren der Stellen im Film, die mit knappen Dialoglücken bei intensiver Handlung eine größere Herausforderung darstellen (und dann eventuell die Hilfe des Holons benötigen), Namen und Beschreibungen wichtiger Personen (hier vor allem von SS-Offizier Landa) können notiert und für den späteren Film genutzt werden.

ADEM kann somit bei der Ausbildung neuer Autoren/Autorinnen ergänzend eingesetzt werden. Für die Analyse einer Audiodeskription liefern Holon und Aspektmatrix dem/der Trainer(in) Hinweise, warum eine Beschreibung auf genau diese Weise erfolgt ist.

Das Zusammenspiel von Regelwerken und Guidelines (Kapitel 2), von ADLAB-Strategien (Kapitel 3) und ADEM-Methode (Kapitel 4) bietet dem/der Beschreiber(in) nun ein breites Spektrum von Lösungsansätzen für die in Kapitel 1 dargelegten Probleme beim Erstellen des Textes einer Audiodeskription. 


\section{Literaturverzeichnis}

AENOR (2005): Audiodescripción para personas con discapacidad visual. Requisitos para la audiodescripción y elaboración de audioguías. Madrid: AENOR.

BENECKE, BERND (2007): „Audio Description: Phenomena of Information sequencing“. URL: www.euroconferences.info/proceedings/2007_Proceedings/2007_Benecke_ Bernd.pdf (letzter Zugriff: 2.3.2018).

BENECKE, BERND (2009): The use of Intended Hyper Descriptions in the Audio Description process. Vortrag bei ARSAD-Konferenz. Barcelona: UAB.

BeneCKe, Bernd (2014a): Audiodeskription als partielle Translation. Modell und Methode. Berlin/Münster: LIT.

Benecke, Bernd (2014b): Der Ton macht die Audiodeskription. In: Jekat, Susanne/ JÜngSt, Heike/SChubert, Klaus/Villiger, Claudia (Hg.): Sprache barrierefrei gestalten: Perspektiven aus der Angewandten Linguistik. Berlin: Frank \& Timme, 109126.

Maszerowska, Anna/Matamala, Anna/Orero, Pilar (Hg.) (2014): Audio Description: New Perspectives Illustrated. Amsterdam: Benjamins.

BÜHLER, KARL (1934): Sprachtheorie. Die Darstellungsfunktion der Sprache. Frankfurt am Main/Berlin/Wien: Ullstein.

BÜHLER, Willi (1937): Die „erlebte Rede“im englischen Roman. Zürich und Leipzig: Niehans.

Chion, Michel (1990): Audio-Vision: Sound on Screen. New York: Columbia University Press.

Dosch, Elmar/Benecke, Bernd (2004): Wenn aus Bildern Worte werden. Durch AudioDescription zum Hörfilm. München: Bayerischer Rundfunk.

Gerzymisch-Arbogast, Heidrun/Mudersbach, Klaus (1998): Methoden des wissenschaftlichen Übersetzens. Tübingen/Basel: Francke.

Puigdomènech, Laura/Matamala, Anna/Orero, Pilar (2010): „Audio Description of films: State of the art and protocol proposal“. In: BOGUCKI, LUKASZ/ Kredens, KrZYszTof (Hg.): Perspectives on Audiovisual translation. Series: Lodz Studies in Language. Band 20. Frankfurt am Main: Peter Lang, 27-44.

Remael, Aline/Reviers, Nina/Vercauteren, Gert (2014): Mit Wörtern Bilder malen: ADLAB Richtlinien für die Audiodeskription. Übersetzt von Birgit Roberts. Triest: EUT.

Rai, Sonali/Greening, Joan/Petré, Leen (2010): A Comparative Study of Audio Description Guidelines Prevalent in Different Countries. London: RNIB.

VERCAUTEREN, GERT (2007): „Towards a European guideline for audio description“. In: Díaz Cintas, Jorge/Orero, Pilar/Remael, Aline (Hg.): Media for All. Amsterdam: Rodopi. 175-188. 
Bernd Benecke

\section{Internetquellen}

ADLAB (2013a). Report on user needs assessment. Report no. 1, ADLAB (Audio Description: Lifelong Access to the Blind) project. URL: www.adlabproject.eu (letzter Zugriff: 2.3.2018).

ADLAB (2013b). Report on testing. Report no. 3, ADLAB (Audio Description: Lifelong Access to the Blind) project. URL: www.adlabproject.eu (letzter Zugriff: 2.3.2018).

ITC GUIDANCE ON STANDARds FOR AUdio DEscription (2000). URL: http://audiodescription.co.uk/uploads/general/itcguide_sds_audio_desc_word3.pdf (letzter Zugriff: 2.3.2018).

VORGABEN FÜR AUdIODESKRIPTION VON ARD, ZDF, ORF UND SRF (2015). URL: https:/www.ndr.de/fernsehen/service/audiodeskription/Vorgaben-fuer-Audiodeskriptionen,audiodeskription140.html (letzter Zugriff: 2.3.2018).

\section{Filmquellen}

Das Leben der Anderen, Florian Henckel von Donnersmarck (D 2006).

Inglorious Basterds, Quentin Tarantino (USA 2009). 


\section{Audioeinführungen}

\section{$1 \quad$ Einleitung}

Audioeinführungen stellen in der Regel ein auditiv vermitteltes zusätzliches Informationsangebot über audiovisuelle Medientexte dar, die zeitnah, jedoch nicht parallel zu dem jeweiligen Artefakt rezipiert werden. Audioeinführungen gibt es seit den 1980er Jahren. Zunächst wurde diese Textsorte in den USA erprobt, seit Beginn der 1990er ist sie auch in Europa zu finden (vgl. Di Giovanni 2014: 1). Während sie anfangs v. a. im Rahmen der Darstellenden Künste, also bei Opern-, Ballett- und Theateraufführungen (vgl. Orero/Matamala 2007: 7f., York 2007, Weaver 2010, Fryer/Romero-Fresco 2014: 11) genutzt wurden, gibt es seit einer Initiative von Fryer und Romero-Fresco im Jahr 2013 auch Audioeinführungen für Spiel- und Dokumentarfilme (vgl. Romero-Fresco/Fryer 2013, Di Giovianni 2014, Hammer et al. 2015).

Werkeinführungen, wie sie schon seit längerem aus dem Musik- und Theaterkontext bekannt sind, gehen einer Aufführung voraus. Sie werden entweder live vorgetragen oder als downloadbarer Podcast auf den Webseiten von Theaterhäusern angeboten (s. etwa das Schauspiel Frankfurt oder das Deutsche Theater Berlin). Solche Werkeinführungen, die sich auch als Audioeinführungen bezeichnen, wenden sich an ein heterogeneres Publikum. Die Art Audioeinführung, die in diesem Beitrag im Fokus steht, wird hingegen spezifisch für ein blindes und sehbeeinträchtigtes Publikum erstellt - was eine breitere Zielgruppe jedoch nicht ausschließen muss. Fryer und Romero-Fresco (2014: 26) weisen explizit darauf hin, dass sie auch von einem Publikum ohne Sinnesbehinderungen als Zusatzangebot genutzt werden kann. Inhaltlich ähneln sich Audioeinführungen und Werkeinführungen tatsächlich: Beide bieten eine Zusammenfassung wichtiger Informationen etwa zur Handlung, zum Hintergrund 
sowie zu formalen Aspekten eines Films, einer Opern- oder Theaterinszenierung (vgl. Benecke 2014: 21 und Orero/Matamala 2007: 7f.). Entsprechend gelten Audioeinführungen als komplementär zur Audiodeskription eines Films (Di Giovanni 2014: 1) oder eines Bühnenstücks (vgl. Szarkowska/Jankowska 2015: 261). Sie können darüber hinaus durch weitere Informationsangebote ergänzt werden. Dazu gehören sogenannte Touch-Touren für sehbehinderte Menschen im Theater (Orero/Matamala 2007: 11; Fryer 2016: 160), die von einigen Häusern vor der Vorstellung durchgeführt werden und die Möglichkeit bieten, die Bühne zu betreten, Bühnenbilder, Kostüme, Requisiten zu berühren. Bei Kinovorstellungen wird auch mit der Möglichkeit experimentiert, vorab Handstücke an blinde und sehbeeinträchtigte Rezipient(inn)en auszuteilen, die über den Tastsinn, ggf. aber auch über den Geruchs- und Geschmackssinn weitere Informationen zu Figuren eines Films wahrnehmen können (vgl. Jüngst 2016). Audioeinführungen werden vorzugsweise vor der Rezeption von Filmen oder Theateraufführungen mit Audiodeskription angehört bzw. gelesen. Inhaltlich liegt ihr Fokus auf dem Wie - also auf dem visuellen Stil - und weniger auf dem Was - der Handlung - eines Films oder einer Aufführung (vgl. Di Giovanni 2014: 2).

Der vorliegende Beitrag liefert eine Beschreibung der Textsortenmerkmale von Audioeinführungen und geht auf ihre wichtigsten Funktionen ein (Punkt 2). Neben Fragen des inhaltlichen Aufbaus von Audioeinführungen (Punkt 3) werden die verschiedenen Einsatzbereiche sowie die technische Umsetzung vorgestellt (Punkt 4). Punkt 5 befasst sich mit der sprachlichen Gestaltung von Audioeinführungen, bevor unter Punkt 6 ein Überblick über aktuelle Forschungsdesiderate gegeben wird.

\section{Textsortenmerkmale und Funktionen der Audioeinführung}

Bei einer Audioeinführung handelt es sich um einen meist auditiv vermittelten Text, der von einer Person oder mehreren Personen gesprochen sein und eine Länge von ca. fünf bis 15 Minuten haben kann (Fryer/Romero-Fresco 2014: 11). Neben einem Livevortrag ist auch die Bereitstellung als Audiodatei üblich. In letzterem Fall kann der Text auch aufwändig gestaltet sein: mit dem Einsatz von 
mehreren Sprecher(inne)n, Geräuschen, Musik oder Zitaten aus dem Medientext, auf den sich die Audioeinführung bezieht (Fryer/Romero-Fresco 2014: 11). Audioeinführungen sind in der Regel weder zeitlich noch medial mit dem Basistext verbunden, sondern bilden einen selbstständigen Metatext, der in sich geschlossen ist (vgl. Fryer/Romero-Fresco 2014: 12).

Im Unterschied zu dieser Textsorte sind Audiodeskriptionen (vgl. Benecke im vorliegenden Band) intersemiotische Teilübersetzungen eines Artefakts, oder, nach Benecke (2014), partielle Translationen, die sowohl zeitlich und (bei Filmen auch) medial mit dem zu übersetzenden Artefakt verbunden sind: Sie bestehen aus nicht-kohäsiven verbalen Textelementen, sind in den audiovisuellen Medientext eingebettet und werden entsprechend parallel zum Medientext rezipiert. Die visuellen Elemente eines audiovisuellen Produkts, etwa eines Filmes oder eines Bühnenstücks, die von einem blinden oder sehbeeinträchtigten $\mathrm{Pu}$ blikum nicht oder nur eingeschränkt wahrgenommen werden können, werden in verbale akustische Zeichen übersetzt. Für die Vermittlung dieser auditiven Zusatzinformationen werden die Pausen zwischen den Dialogen eines Films oder Theaterstücks genutzt. Musik und handlungstragende Geräusche sollen nicht übersprochen werden, damit das Erlebnis des Originals für die Rezipient(inn)en weitgehend gewahrt werden kann und die Audiodeskription andere wichtige Informationen nicht verdrängt. Aus diesen Notwendigkeiten ergibt sich, dass für eine Audiodeskription weitaus weniger Platz zur Verfügung steht, als dies für eine ideale Beschreibung aller wichtigen visuellen Details nötig erscheinen mag.

Audiodeskripteure(innen) müssen sich bei der Auswahl der zu beschreibenden Elemente daher auf zum Verständnis der Handlung unverzichtbare narrative Aspekte der visuellen Ebene eines Films oder einer Theateraufführung beschränken (vgl. Remael/Vercauteren 2007). Dazu gehören die Beschreibungen der Orte des Geschehens, der Figuren und ihrer äußeren Merkmale sowie die Beschreibungen ihres Handelns und ihrer Reaktionen (vgl. Benecke 2014: 16f.). Ästhetische Aspekte oder Hintergrundinformationen kommen dabei häufig zu kurz (Benecke 2014: 21). Benecke baut die Audioeinführung daher in sein Audiodeskriptions-Entwicklungsmodell (ADEM) ein - gewissermaßen als letzte Option, falls sich bei der Erstellung eines Hörfilms keine Möglichkeit ergibt, wichtige Informationen zu einer Szene in der Audiodeskription unterzubringen 
(Benecke 2014: 78 und 84). Das Zusammenspiel der Audioeinführung mit der vorhandenen Audiodeskription zu einem Medientext spielt bei ihrer Erstellung eine wichtige Rolle, weshalb Benecke dafür plädiert, dass die Audioeinführung am besten auch von den Autor(inn)en der entsprechenden Audiodeskription verfasst werden sollte (vgl. ebd. 21).

Es wäre allerdings ein Missverständnis, Audioeinführungen folglich als reine Notlösung zu betrachten. Auch vermögen sie sicherlich nicht, punktuell auftretende Dilemmata etwa bei einer Filmbeschreibung zu lösen und wichtige Hinweise auf die Handlung quasi vorab zu ergänzen. Ihre Aufgabe liegt vielmehr darin, einem blinden oder sehbeeinträchtigtem Publikum Informationen über das Artefakt verfügbar zu machen, die mit der Textsorte Audiodeskription tendenziell unvereinbar sind oder regelmäßig zu kurz kommen. Verschiedene empirische Studien deuten darauf hin, dass das Verständnis eines Hörfilms durch die zusätzliche Rezeption einer Audioeinführung deutlich verbessert werden kann (vgl. Fryer/Romero-Fresco 2014: 13f.). Darüber hinaus wird vermutet, dass selbst Audioeinführungen zu (noch) nicht audiodeskribierten Artefakten dem Abbau von Barrieren für ein blindes und sehbeeinträchtigtes Publikum dienen können.

Nach Remael/Reviers (2013) und Fryer/Romero-Fresco (2014) erfüllen Audioeinführungen regelmäßig mehrere Textfunktionen. Ihre übergeordnete Aufgabe besteht darin, zu einem besseren Verständnis des audiovisuellen Artefakts beizutragen. Audioeinführungen sind folglich in erster Linie informativ, da sie allgemeine Fakten zur Produktion des Films oder der Theater- bzw. Operninszenierung, zu seiner/ihrer Dauer, zu den Namen der Schauspieler(innen) bzw. Figuren oder die Drehorte bzw. Schauplätze oder zum Bühnenbild und den Kostümen liefern. Hinzu kommen Beschreibungen ästhetischer Merkmale eines Films oder einer Inszenierung.

Audioeinführungen können aber auch selbstreferentielle Instruktionen an die Zuhörer(innen) enthalten, wenn bspw. Hinweise auf das Abspielen der Audiodatei gegeben werden (vgl. Remael/Reviers 2013 und Fryer/Romero-Fresco 2014: 11). Nicht zuletzt sollen sie dem Publikum Lust machen, den betreffenden Film zu rezipieren oder die besprochene Aufführung zu besuchen (Fryer/ Romero-Fesco 2014: 12). 
Wie sind Audioeinführungen in der Regel aufgebaut? Da in einer Audioeinführung zeitlich die Möglichkeit gegeben ist, Informationen zu einem audiovisuellen Artefakt zu vermitteln, die über seine narrative Ebene hinausgehen, liegt der Fokus, wie bereits oben erwähnt, auf den stilistischen Besonderheiten und den Hintergrundinformationen desselbigen. Konkrete Richtlinien oder Empfehlungen, welche Informationen in eine Audioeinführung aufgenommen werden sollen, gibt es für den deutschsprachigen Raum nicht - aus den existierenden Audioeinführungen lassen sich jedoch Schlüsse für die Praxis ziehen. Grundsätzlich richtet sich deren inhaltliche Gestaltung immer nach dem jeweiligen Film oder der Inszenierung, die ihnen zugrunde liegen.

Beispiele für deutschsprachige Audioeinführungen für Filme sind u. a. über die Homepage der Universität Hildesheim (vgl. Universität Hildesheim o. J.). verfügbar. Zur Audioeinführung zum Film „Die Wand“ (Pölsler 2013) muss noch korrigierend angemerkt werden, dass sie nicht, wie behauptet (Fryer 2016: 155), auch auf DVD gefunden werden kann. Ebenso wenig handelt es sich um eine Übersetzung aus dem Englischen ins Deutsche (ebd.), sondern um einen von Studierenden des Studiengangs Medientext und Medienübersetzung originär erstellten Text, der anschließend ins Englische übersetzt und mit dem Original zusammen online gestellt wurde.

Grundsätzlich muss bei der Entscheidung, welche Inhalte vermittelt werden sollen, zwischen Audioeinführungen für Bühnenstücke und solchen für Filme unterschieden werden.

Audioeinführungen für das Theater oder die Oper enthalten meist relevante Informationen aus dem gedruckt vorliegenden Programm, d. h. sie geben Hinweise zur Dauer der Aufführung und führen die Namen der Schauspieler(innen) und des Produktionsteams auf. Außerdem werden regelmäßig Bühnenbilder, Kostüme und Figuren beschrieben. Darüber hinaus können auch der Stil der Inszenierung und besondere Merkmale zur Spielweise oder zur Regie beschrieben werden (vgl. Romero-Fresco/Fryer 2013: 289). Fryer empfiehlt, das Publikum ggf. auf Momente der Aufführung aufmerksam zu machen, die bei einer vorliegenden Sehbehinderung als beängstigend empfunden werden können. Dazu zählt sie u. a. plötzliche, laute Geräusche wie Schüsse oder haptisch schwer 
zu kontextualisierende Effekte wie der Einsatz von Nebelmaschinen, aber auch von starken Lichteffekten wie Stroboskoplicht (vgl. Fryer 2016: 158), die auch von Menschen mit einer Sehbeeinträchtigung wahrgenommen werden und fälschlich als Gefahr gedeutet werden können.

Die Inhalte von Audioeinführungen zu Filmen sind vergleichbar: Es lassen sich Beschreibungen von Figuren, Drehorten und - sofern vorhanden - Angaben zur (literarischen) Vorlage finden. Hintergrundinformationen zur Regie, zu Drehbuchautor(inn)en oder zu Auszeichnungen können ebenfalls vorkommen. Eine Beschreibung filmtechnischer Besonderheiten, etwa zur Kameraführung, zum Schnitt, Licht und Farbe sind darüber hinaus wünschenswert, da diese aus Audiodeskriptionen meist verbannt sind (Fryer/Romero-Fresco 2014: 12). Auch der Wunsch nach Informationen über bis dahin erschienene Filmkritiken wird von der Zielgruppe wiederholt geäußert, bislang aber nicht umgesetzt (vgl. Hammer et al. 2015, Fryer/Romero-Fresco 2014).

Romero-Fresco und Fryer (2011: Folie 13) empfehlen, sich bei der Erstellung einer Audioeinführung an folgende Struktur zu halten: Auf eine kurze Einleitung folgen eine Synopsis, Erläuterungen zum visuellen Stil, Beschreibungen der Figuren und Schauplätze und abschließend Hintergrundinformationen zur Besetzung und zum Produktionsteam.

\subsection{Die Einleitung}

Beim Anhören in Großbritannien oder Deutschland produzierter Audioeinführungen zu Filmen lässt sich feststellen, dass neben einer Begrüßung und dem selbstreferentiellen Hinweis darauf, um was für einen Text es sich handelt, zunächst einmal der Titel des Films genannt wird, auf den sich die Audioeinführung bezieht, und erwähnt wird, ob für diesen auch eine Audiodeskription zur Verfügung steht (vgl. Fryer/Romero-Fresco 2014: 23). Ein Hinweis zur Dauer der Audiodatei kann ebenfalls eine wichtige Information sein, die sinnvollerweise zu Beginn gegeben werden sollte. Regelmäßig werden die Regie und das Erscheinungsjahr des Films genannt. 


\subsection{Die Synopsis}

Von einer ausführlichen Darstellung des Handlungsverlaufs wird abgeraten (Fryer/Romero-Fresco 2014: 22). Eine zu lange Synopsis kann von den Rezipient(inn)en als unnötige Vorwegnahme angesehen werden, die den Aufbau von Spannungsmomenten im Film oder im Bühnenstück untergräbt. Ganz auf Hinweise zur Handlung zu verzichten, dürfte allerdings schwierig sein. Ohne das narrative Grundgerüst, den Ausgangskonflikt oder einzelne Figurenkonstellationen zu erwähnen, können Informationen zu Schauplätzen oder Figuren kaum sinnvoll verknüpft werden. Ergebnisse aus einer empirischen Studie legen nahe (Fryer/Romero-Fresco 2014: 13), dass sich Informationen zu den Figuren besser aufnehmen und memorieren lassen, wenn sie in einen logischen bzw. narrativen Zusammenhang gebracht und nicht bloß als Aufzählung präsentiert werden. Die Synopsis ist also kein Selbstzweck, sondern dient der Strukturierung der weiteren Informationen über Figuren und Schauplätze.

Bei der Formulierung der Synopsis wird auch diskutiert, ob inhaltliche Redundanz zwischen Audioeinführung und Audiodeskription eher als Vor- oder Nachteil betrachtet werden muss. Redundante Informationen in der Audioeinführung können als langweilige Vorwegnahme empfunden werden. Jedoch erscheint es geradezu sinnvoll, den Rezipient(inn)en durch bewusste Wiederaufnahme von Formulierungen einige "Ankerpunkte“ für die folgende Audiodeskription des Films oder Bühnenstücks zu liefern (vgl. Hammer et al. 2015: 166).

\subsection{Figurenbeschreibungen}

Die Beschreibung von Figuren kann der Chronologie ihres Erscheinens folgen oder nach ihrer Relevanz für die Handlung geordnet werden. $\mathrm{Zu}$ den äußeren Merkmalen, die veränderlich sein können, gehört auch die Beschreibung kennzeichnender Mimik, Gestik oder Proxemik, also des Raumverhaltens der Figuren (vgl. Fryer/Romero-Fresco 2014: 18). Solche Aspekte lassen sich allgemein oder beispielhaft anhand einer Szene oder Einstellung beschreiben. Auch die Besetzung der Rollen kann bedeutsam und daher erwähnenswert sein, etwa wenn mehrere Figuren von derselben Person verkörpert werden, die Besetzung ungewöhnlich erscheint oder berühmte Schauspieler(innen) zu sehen sind, die 
aufgrund einer neu gewählten Synchronstimme für blinde Zuschauer(innen) nicht erkennbar sind. Eine Herausforderung kann mitunter die Auswahl der zu beschreibenden Figuren darstellen. Wenn etwa, wie in Historienfilmen üblich, sehr viele Darsteller(innen) und Kompars(inn)en auftreten, können sich die Autor(inn)en der Audioeinführung von der Frage leiten lassen, wer eine handlungstragende Rolle spielt. Es erweist sich ebenfalls als sinnvoll, einheitlich agierende Personen zu Gruppen zusammenzufassen. Aber auch die Auswahl der Informationen, die zu einer Figur gegeben werden soll, wirft mitunter Probleme auf, z. B. wenn in einem Rätselkrimi oder Thriller die Identität einer Figur zunächst verschleiert wird oder den Zuschauer(inne)n relevante Informationen bewusst vorenthalten werden.

\subsection{Schauplätze}

Die Beschreibung von Schauplätzen kann, je nach Genre, mehr oder weniger aufwändig sein. Di Giovanni bemerkt jedoch, dass die Beschreibung der Schauplätze in einer Audioeinführung weniger ausgeführt wird, weil diese Informationen meist in der Audiodeskription geliefert werden (vgl. Di Giovanni 2014: 5). Wie bei der Beschreibung der Figuren gilt die Übernahme von Formulierungen aus der Audiodeskription als empfehlenswert, um das spätere Komplettieren der audiodeskribierten Szenen mit den memorierten Ausführungen der Audioeinführung $\mathrm{zu}$ befördern. Schließlich können die Beschreibungen der Schauplätze durch Hintergrundinformationen zu den tatsächlichen Drehorten ergänzt werden.

\subsection{Visueller Stil}

Manche Audioeinführungen erwähnen nicht erst die Figuren und Schauplätze, sondern gehen gleich auf den visuellen Stil des Films ein. Dabei kommt es auf den konkreten Fall an, welche Aspekte jeweils herausgearbeitet werden. Der Schnittrhythmus, die Farbgebung und die Lichtregie können ebenso relevante Informationen darstellen wie die Erwähnung der vorherrschenden Kameraeinstellungen, -perspektiven oder -bewegungen. Die Beschreibung von Zeitlupen, 
Zeitraffern, Split Screens oder Special Effects kann in Audioeinführungen ebenfalls ihren Platz finden. Bei Zeichentrick- oder Animationsfilmen rücken in der Regel noch weitere Aspekte in den Vordergrund.

Optional können zu jedem Film auch interessante Hintergrundinformationen oder Hinweise zu intertextuellen bzw. interpikturellen Anspielungen gegeben werden, die typischerweise schwer in Audiodeskriptionen unterzubringen sind. Um derartige Aspekte herauszuarbeiten, kann es sich als hilfreich erweisen, Filmkritiken zurate zu ziehen und diese, nach Prüfung der Fakten, in der Audioeinführung zu erwähnen (vgl. Fryer/Romero-Fresco 2014: 22).

Welche konkreten Informationen in die jeweilige Audioeinführung aufgenommen werden, in welcher Reihenfolge, mit welcher Gewichtung sie präsentiert werden und wie lang der gesamte Text ist, variiert (vgl. Fryer/RomeroFresco 2014: 17) und hängt letztlich vom einzelnen Artefakt und von den Entscheidungen der Autor(inn)en ab.

Bisher werden Audioeinführungen als zusammenhängende Datei angeboten. Ein Vorschlag der Zielgruppe im Rahmen einer empirischen Studie lautete, verschiedene Versionen anzubieten (vgl. Hammer et al. 2015): Neben einer knapp gehaltenen Version könnte es eine ausführlichere geben, die weitere Informationen wie etwa eine Presseschau enthielte. Eine in einzelne Kapitel mit Zwischenüberschriften unterteilte Audiodatei ließe sich kapitelweise ansteuern, sodass es den Nutzer(inne)n überlassen bliebe, zu welchem Aspekt sie mehr Informationen haben möchten und was sie ggf. lieber erst nach der Rezeption des Films oder der Theateraufführung zusätzlich erfahren möchten. Die Möglichkeit, einzelne Kapitel anzusteuern, müsste jeweils zu Beginn mit einer kurzen Auflistung der Zwischenüberschriften erwähnt werden, um die Navigation im Text zu erleichtern. Ist die Navigierbarkeit gewährleistet, spielt die Gesamtdauer der Audioeinführung auch eine geringere Rolle, da die Rezipient(inn)en die Möglichkeit hätten, für sie weniger relevante Textteile zu überspringen. 


\section{$4 \quad$ Einsatzbereiche und technische Umsetzung}

Audioeinführungen können in Live-Kontexten dem anwesenden Publikum unmittelbar vorgetragen oder direkt vor der Aufführung medial zur Verfügung gestellt werden (vgl. York 2007: 216f., Di Giovanni 2014: 2), sie können aber auch vorab als Audio- oder Textdatei an das Publikum verschickt oder auf einer Webseite, etwa als Download, angeboten werden. Audioeinführungen für Filme lassen sich ebenfalls sowohl als Audiodatei als auch als Textdatei abrufen, allerdings stellt sich hier verstärkt die Frage nach der Auffindbarkeit der Dateien durch die potenziellen Nutzer(innen). Wird eine Audioeinführung zu einem im Fernsehen ausgestrahlten Film angeboten, ist es nicht möglich, diese einfach vor der Sendung auszustrahlen (vgl. Fryer/Romero-Fresco 2014: 12). Eine sinnvolle Plattform wäre hingegen die Webseite der Rundfunkanstalten, die den Film ausstrahlen. So hat etwa der NDR die von der Universität Hildesheim erstellte Audioeinführung zu dem Film „A girl walks home alone at night“ (Amirpour 2014) produziert und einige Tage vor der Ausstrahlung auf seiner Webseite zur Verfügung gestellt (vgl. NDR o. J.). Dort ist sie weiterhin verfügbar. Es muss jedoch angezweifelt werden, dass potentielle Nutzer(innen), die den Film bspw. auf DVD oder im Kino ansehen möchten, beim NDR nach dieser Audioeinführung suchen. Eine begrüßenswerte Initiative wäre eine Webseite (wie etwa die mittlerweile nicht mehr betriebene Seite audiointros.org), auf der verschiedene Audioeinführungen gesammelt und dem Publikum in Form von Text- und Audiodateien zur Verfügung gestellt würden.

Für die potentiellen Nutzer(innen) von Audioeinführungen wäre daher eine neue Initiative insbesondere für den deutschen Sprachraum wünschenswert, um die leichte Auffindbarkeit und Verfügbarkeit von Audioeinführungen zu gewährleisten. So könnten Audioeinführungen auch unabhängig von konkreten Sendeterminen eines Films im Fernsehen rezipiert werden. Im Rahmen der bereits erwähnten empirischen Studie von Hammer et al. kam vom Zielpublikum auch der Vorschlag, Audioeinführungen über Apps wie GRETA zugänglich zu machen (vgl. Hammer et al. 2015: 174). Bisher werden über GRETA (vgl. Greta o. J.) Audiodeskriptionen für Spielfilme bereitgestellt, die sich über das Smartphone zum Film hinzuschalten lassen und sich automatisch mit diesem syn- 
chronisieren. Darüber hinaus ist aber auch die Bereitstellung von Audioeinführungen in den Mediatheken der Fernsehsender sinnvoll, insbesondere wenn der betreffende Film zeitnah ausgestrahlt wird und die Audioeinführung von den Fernsehsendern produziert wurde. Im Falle von Audioeinführungen zu Theaterinszenierungen ist der Webauftritt des jeweiligen Theaterhauses ein geeigneter Platz. Fryer und Romero-Fresco (2014: 26) empfehlen, sie auf der DVD zum Film als Bonusmaterial zur Verfügung zu stellen.

Abgesehen von der Problematik der Auffindbarkeit von Audioeinführungen stellt sich im Moment aber vor allem die Frage nach der Produktion weiterer Audioeinführungen für Film und Fernsehen. Zwar wird an der Textsorte regelmäßig hervorgehoben, dass ihre Produktion im Vergleich zu Audiodeskriptionen kostengünstiger und weniger aufwändig sei (vgl. Fryer/Romero-Fresco 2014: 27). Doch gilt dies vor allem für die Studioaufnahme und im Hinblick auf urheberrechtliche Fragen. Das Erstellen der Textfassung erfordert hingegen einen hohen Aufwand und setzt eine profunde Film- bzw. Theateranalyse voraus. Bei der sprachlichen Gestaltung steht die Audioeinführung den Herausforderungen an eine Audiodeskription in nichts nach, auch wenn die zeitlichen Restriktionen hier anderer Natur sind.

\section{$5 \quad$ Sprachliche Gestaltung}

Für die sprachlich-stilistische Gestaltung dieser Textsorte liegen für den deutschsprachigen Raum bisher keine Richtlinien vor, allerdings lässt sich sowohl aus der britischen Forschungsliteratur als auch aus den bereits in Deutschland entwickelten Audioeinführungen ableiten, welche Aspekte als wichtig angesehen werden. So gilt es zu beachten, dass es sich hierbei in erster Linie um auditiv vermittelte Texte handelt. Deshalb betonen Fryer/Romero-Fresco (2014: 23): „AIs [audiointroductions] must be written for the ear“. Akustisch rezipierte Texte sind flüchtig. Bei der sprachlichen Gestaltung werden daher aktivisch und im Präsens formulierten Hauptsätzen der Vorrang gegeben. Fryer/RomeroFresco (ebd.) empfehlen außerdem, den Text in der ersten Person Plural zu verfassen, um zwischen dem Sprecher oder der Sprecherin und den Rezipient(inn)en 
Nähe herzustellen - eine Vorgabe, der jedoch bei Weitem nicht alle Audioeinführungen folgen. Regelmäßig vermieden werden außerdem komplexe Satzkonstruktionen, darunter auch eingeschobene Relativsätze. Im Stil und bei der Wahl der Lexik lässt sich ein Streben nach konzisen und aussagekräftigen Formulierungen feststellen, während Redundanzen innerhalb der Audioeinführung vermieden werden. Zwar unterliegt eine Audioeinführung nicht den gleichen zeitlichen und räumlichen Restriktionen wie eine Audiodeskription. Dennoch gilt es auch bei Audioeinführungen, die Aufmerksamkeitsspanne der Rezipient(inn)en nicht übermäßig zu beanspruchen.

Im Hinblick auf die Verwendung von technischem Vokabular finden sich in der wissenschaftlichen Literatur widersprüchliche Angaben. Während nach Ansicht von Weaver technische Begriffe in Theater und Oper möglichst vermieden werden sollten, um den performativen und narrativen Charakter der Audioeinführung zu erhalten (vgl. Weaver 2010: 5), spricht sich Fryer (2016: 159) durchaus dafür aus, technisches Vokabular zu verwenden: zum Beispiel bei Ballettaufführungen, für die vorab bestimmte Tanzschritte erläutert werden können. Im Hinblick auf Filme haben empirische Studien ergeben, dass die Verwendung von spezifischem Vokabular zur Beschreibung von Kameraführung, Montage oder Beleuchtung in Audioeinführungen von einem überwiegenden Teil des Publikums sogar gewünscht wird (vgl. Fryer/Romero-Fresco 2014: 25 und Fryer 2016: 159), zumal ihre Verwendung in Audiodeskriptionen umstritten und von den meisten Richtlinien ausgeschlossen ist (vgl. Perego 2014: 88). Di Giovanni (2014: 5) schätzt die Verwendung von filmtechnischen Termini jedoch auch bei Audioeinführungen als problematisch ein und regt an, weitere empirische Studien durchzuführen. Insbesondere sollten die Ergebnisse der Umfragen zu den Vorlieben der Nutzer(innen) nach Eintritt und Ausmaß der Sehbehinderung differenziert werden.

\section{Forschungsdesiderate}

Audioeinführungen sind eine bisher wenig erforschte Textsorte innerhalb der Barrierefreien Kommunikation. Entsprechend lassen sich zum aktuellen Zeitpunkt zahlreiche Forschungsdesiderate formulieren. 
So wird in der Literatur mehrfach darauf hingewiesen, dass das Zusammenspiel zwischen Audiodeskription und Audioeinführung durch empirische Studien noch unerforscht ist. Dazu gehört auch die Frage, ob die Konzentration von Audiodeskriptionen und Audioeinführungen auf visuelle Elemente ausreicht, um die suggestiven Verknüpfungen zwischen Ton und Bild für ein blindes und sehbeeinträchtigtes Publikum verständlich zu machen, oder ob Audioeinführungen ggf. nicht auch auf diese Verknüpfungen eingehen sollten. Hinsichtlich der Akzeptanz dieser Textsorte und ihrer Gestaltung gilt, dass die bisherigen Studien mit wenigen Proband(inn)en durchgeführt wurden. Statistisch verlässliche Ergebnisse, die auch eine Ausdifferenzierung der Zielgruppe erlaubten, stehen also noch aus.

Zur Ausdifferenzierung des Zielpublikums gehört die Frage, auf welche speziellen Bedarfe eine Audioeinführung für sehbeeinträchtigte oder blinde Kinder abgestimmt werden müsste. Auch die Erforschung, wie Audiodeskriptionen für Kinder gestaltet werden müssen, liegt noch in den Anfängen. Ob Audioeinführungen von Kindern gern genutzt würden und verständnisfördernd wirkten, gilt es ebenso zu untersuchen wie die Frage nach der spezifischen sprachlichen Gestaltung für diese Zielgruppe.

Eine regelmäßig wiederkehrende Frage ist, ob diese Textsorte sich gut übersetzen lässt und welche Übersetzungsstrategien bei einer sprachlich-kulturellen Übertragung von Audioeinführungen beherzigt werden müssten (vgl. Di Giovanni 2014: 5). Fryer plädiert bspw. für das Übersetzen von Audioeinführungen und führt das wirtschaftliche Argument ins Feld, dass diese kostengünstig und schnell produziert werden könnten (vgl. Fryer 2016: 155), was dem Ausbau des Angebots dienen würde. Tatsächlich wurden mehrere empirische Studien zu Audioeinführungen auf Grundlage von übersetzten Audioeinführungen aus dem Englischen ins Italienische oder ins Polnische durchgeführt und erhielten ein sehr positives Feedback (vgl. Fryer/Romero-Fresco 2014: 12). Während grundlegende Hintergrundinformationen zum jeweiligen Artefakt sicher problemlos in eine andere Sprache übertragen werden können, müssen Elemente wie Filmkritiken, ggf. Angaben zu Synchronsprecher(inne)n und die Auskunft darüber, ob eine Audiodeskription vorliegt, zielsprachen- und -kulturspezifisch angepasst werden. Problematisch ist allerdings, dass der For- 
derung, zwischen Audioeinführung und Audiodeskription bewusst Ankerpunkte in Form redundanter Formulierungen und Bezeichnungen herzustellen, bei einer Übersetzung nicht mehr nachgekommen werden könnte. Das Zusammenspiel von Audiodeskription und Audioeinführung würde durch diese Vorgehensweise also unterlaufen.

Ein weiterer Aspekt, der im Zusammenhang mit der Textsorte Audioeinführung untersucht werden könnte, wäre die Ausweitung dieser Textsorte auf weitere audiovisuelle Formate. So ließen sich auch Audioeinführungen für Fernsehserien erstellen. Dies birgt eine Reihe spezifischer Herausforderungen, die mit ihrem seriellen und unabgeschlossenen Charakter zusammenhängen. Entsprechend müssten ggf. regelmäßige Anpassungen vorgenommen werden. Mit welchen Strategien Audioeinführungen für Serien erstellt werden können, ist bislang unerforscht.

Abschließend stellt sich noch die Frage, ob komplementäre Textsorten wie die Audioeinführung in abgewandelter Form nicht auch für die Zielgruppe gehörloser Menschen interessant wäre und ein komplementäres Angebot für SDH böte (vgl. Mälzer/Wünsche im vorliegenden Band). Entsprechend könnten derartige Einführungen als Videoeinführungen in Gebärdensprache verfasst sein und die auditiven Elemente eines Films oder eines Live-Events kommentieren: Musik, Geräusche, Stimmen, Prosodie - Aspekte, die in SDH regelmäßig zu kurz kommen. 


\section{Literaturverzeichnis}

BENECKE, BERnd (2014): Audiodeskription als partielle Translation. Modell und Methode. Berlin u. a.: LIT.

Di Giovianni, Elena (2014): „Audiodescription meets audio introduction: an Italian experiment“. In: TRAlinea Special Issue: Across Screens Across Boundaries. URL: http://www.intralinea.org/specials/article/audio_introduction_meets_audio_ description (letzter Zugriff: 21.02.2018).

Díaz-Cintas, Jorge/Orero, Pilar/Remael, Aline (2007) (Hg.): Media for All: Subtitling for the Deaf, Audio Description and Sign Language. Amsterdam/New York: Rodopi.

Fryer, Louise (2016): An Introduction to Audio Description. A practical guide. London/ New York: Routledge.

Fryer, Louise/Romero-Fresco, PABlo (2014): „Audiointroductions“. In: MASZERowSKA ET AL. (Hg.), 11-28.

GRETA (o. J.): „Kino einfach erleben mit GRETA“. URL: http://www.gretaundstarks.de/ greta (Letzter Zugriff: 21.02.2018).

Hammer, Philipp/Mälzer, NATHalie/WÜnsche, Maria (2015): „Audioeinführungen als Zusatzangebot zu Audiodeskriptionen?" In: trans-kom 8, 1, 164-178. URL: http://www.trans-kom.eu/bd08nr01/trans-kom_08_01_08_Hammer_Maelzer_ Wuensche_Audioeinfuehrung.20150717.pdf (letzter Zugriff: 21.02.2018).

JÜNGST, HEIKE E. (2016): „Taststücke als Ergänzung zur Audiodeskription - Zwischenbericht aus einer Studie“. In: MÄLZER (Hg.), 245-255.

Mälzer, Nathalie (2016) (Hg.): Barrierefreie Kommunikation - Perspektiven aus Theorie und Praxis. Berlin: Frank \& Timme.

Maszerowska, Anna/Matamala, Anna/Orero, Pilar (2014) (Hg.): Audio description: new perspectives illustrated. Amsterdam u. a.: John Benjamins.

NDR (o. J.): „Audioeinführung für ,A Girl Walks Home At Night“. URL: https:/www. ndr.de/info/Audioeinfuehrung-fuer-A-Girl-Walks-Home-At-Night, audio276972.html (letzter Zugriff: 23.02.2018).

Orero, Pilar/Matamala, Anna (2007): „Accessible Opera: Overcoming Linguistic and Sensorial Barriers“. In: Perspectives. Studies in Translatology 15, 4, 1-14. URL: http://ddd.uab.cat/pub/artpub/2007/117149/accessibleopera_FINAL.pdf(letzter Zugriff: 21.02 .2018$)$.

Perego, Elisa (2014): „Film language and Tools“. In: MASZEROWSKa ET AL. (Hg.), 81101.

Remael, Aline/Reviers, Nina (2013): „Audio description for live performances: The role of the introduction“. Vortrag im Rahmen des 4. Advanced Research Seminar on Audio Description (ARSAD), 13.-14.03.2013, Barcelona. 
Remael, Aline/Vercauteren, Gert (2007): „Audio Describing the Exposition Phase of a Film: Teaching Students What to Choose“. In: Trans 11, 73-93.

Romero-Fresco, Pablo/Fryer, Louise (2011): „Audio Introductions: Conveying the ,How' of Cinematic Storytelling“. Vortrag im Rahmen der 4. Media For All Konferenz, 28.06.-01.07.2011, London: Imperial College.

Romero-Fresco, Pablo/Fryer, Louise (2013): „Could Audio-Described Films Benefit from Audio Introductions? An Audience Response Study.“ In: Journal of Visual Impairment and Blindness 107, 4, 287-295.

SZARKOWSKA, AgNiESZKA/JANKOWSKA, ANNA (2015): „Audio describing foreign films“. In: The Journal of Specialised Translation 23, 243-269. URL: http://www.jostrans.org/issue23/art_szarkowska.pdf (letzter Zugriff: 26.02.2018).

Universität Hildesheim (o. J.): „Audioeinführungen als komplementäres Angebot zu Audiodeskriptionen“. URL: https://www.uni-hildesheim.de/fb3/institute/institut-fueruebersetzungswiss-fachkommunikation/mitglieder-des-instituts/maelzer/audio einfuehrungen-als-komplementaeres-angebot-zu-audiodeskriptionen/ (letzter Zugriff: 23.02.2018)

WeAVER, SARAH (2010): „Opening Doors to Opera: The Strategies, Challenges and General Role of the Translator". In: TRAlinea 12, 12. URL: http://www.intralinea.org/ archive/article/Opening_doors_to_opera_(letzter Zugriff: 21.02.2018).

YORK, GREgORY (2007): „Verdi Made Visible. Audio-introduction for Opera and Ballet“. In: DíAZ-CinTAS eT AL. (Hg.), 215-229.

\section{Filme}

Die Wand. Regie: Julian Pölsler. DE/AT 2012.

A Girl Walks Home Alone at Night. Regie: Ana Lily Amirpour. USA 2014. 
NICOLA PRIDIK

\section{Visualisierung rechtlicher Inhalte in Leichte-Sprache-Texten}

Juristisches Wissen wird seit jeher über das gesprochene oder geschriebene Wort vermittelt (Röhl/Ulbrich 2007: 12). Zu tun hat das vor allem damit, dass die verbale Sprache mit ihrer Fähigkeit zur Präzision, Klarheit, Eindeutigkeit und Argumentation der abstrakten und zugleich komplexen Materie am besten gerecht wird (vgl. Bergmans 2009: 4f.). Geht es allerdings darum, rechtliche Strukturen zu kommunizieren, über die sich die Inhalte häufig erst erschließen, stoßen selbst die besten Texte und Vorträge an ihre Grenze. Sie können die Strukturen nämlich nicht zeigen, sondern nur linear beschreiben (ebd.: 7). In der Rechtsdidaktik wird deshalb der ergänzende Einsatz juristischer Schaubzw. Strukturbilder empfohlen (ebd.: 23, Eickelberg 2017: Rn. 262ff., Kostorz 2016: 70f.).

Auch Leichte-Sprache-Texte mit rechtlichen Inhalten kommen bei der Darstellung von Strukturen an ihre Grenze. Es liegt deshalb nahe, hier auf vergleichbare Visualisierungen zu setzen. Zu bedenken ist dabei allerdings, dass juristische Schaubilder nicht nur das Verstehen erleichtern, sondern auch besondere Anforderungen an ihre Adressat(inn)en stellen. So müssen diese z. B. in der Lage sein, räumliche Anordnungen und Verbindungen von Textkästen in konzeptuelle Zusammenhänge zu übersetzen (vgl. Ballstaedt 2012: 51), eine nicht lineare Leserichtung zu erkennen und die Bedeutung von Piktogrammen/Icons im jeweiligen Kontext zu erfassen. Für Menschen mit kognitiven Beeinträchtigungen bzw. Lernschwierigkeiten können dies Barrieren sein, welche die Aufnahme der Informationen erschweren. Der vorliegende Beitrag geht deshalb der Frage nach, welche Anforderungen juristische Schaubilder erfüllen müssen, um für einen möglichst großen Personenkreis, der die Zielgruppen der Leichten Sprache einschließt, verständlich zu sein. Ergänzt werden die Ausführungen durch drei Schaubilder, die beispielhaft zeigen, wie eine Umsetzung in der Praxis aussehen könnte. 
Nicola Pridik

\section{Die Ausgangslage}

1.1 Die Bedeutung rechtlicher Strukturen

Wer Rechtsinformationen in Textform an andere weitergeben möchte, hat drei Aufgaben zu meistern:

- Er/Sie muss aus der Fülle an Informationen zu einem Thema diejenigen auswählen, die in der konkreten Situation für die jeweilige Zielgruppe relevant sind. Die in diesem Zusammenhang anzustellenden Überlegungen entsprechen denen zur didaktischen Reduktion, die z. B. Kostorz (2016: 35ff.) und Eickelberg (2017: Rn. 133ff.) für die juristische Lehre formulieren.

- Er/Sie muss die Inhalte verständlich in Worte fassen. Im Hamburger Verständlichkeitsmodell entspricht dies dem Merkmal der Einfachheit (Langer/Schulz von Thun/Tausch 2015: 22f.).

- Er/Sie muss die Inhalte strukturiert darbieten. Der Grund liegt darin, dass insbesondere die innere Ordnung und äußere Gliederung Einfluss auf die Verständlichkeit haben (ebd.: 24f., s. a. den Beitrag von Christmann/Groeben im vorliegenden Band). Im Recht gilt das ganz besonders, weil es sich zu einem großen Teil über seine Strukturen erschließt. So sind die meisten Vorschriften mitsamt den darin enthaltenen Regeln, Vorgaben, Informationen und Begriffen als Teile eines Gefüges miteinander verbunden, beziehen sich aufeinander oder setzen einander voraus. Besonders gut sieht man dies bei Verfahrensabläufen und Systematiken oder auch bei Rechtsmodellen, also Darstellungen, die zeigen, wie bestimmte Rechtsregeln oder -institute grundsätzlich funktionieren, z. B. eine Forderungsabtretung oder Bürgschaft (vgl. Bergmans 2009: 69). Wer diese Strukturen kennt, hat es leichter, sich im Recht zu orientieren, Inhalte zu verstehen und sie zu behalten. Dagegen lässt sich mit einzelnen Regelungen für sich genommen häufig nur scheinbar etwas anfangen. 


\subsection{Grenzen der verbalen Vermittlung rechtlicher Strukturen}

Vermitteln lassen sich rechtliche Strukturen in Texten insbesondere durch eine übersichtliche Gliederung, treffende Überschriften, Zwischenüberschriften und eine sinnvolle Absatzbildung (ausführlich zum strukturierten Schreiben für Juristen s. Haft 2009). Hinzu kommen ein logischer Gesamtaufbau des Textes, eine folgerichtige Vermittlung der Informationen und ihre logische Verknüpfung (Langer/Schulz von Thun/Tausch 2015: 24 und 69, Nussbaumer 2017, Christmann/Groeben im vorliegenden Band). Dennoch hat Text als Medium stets einen entscheidenden Nachteil: Es ist ihm nicht möglich, die Strukturen zu zeigen. Selbst die komplexesten Zusammenhänge kann er immer nur linear mit Worten beschreiben (Bergmans 2009: 7).

Bei juristischen Texten in Leichter Sprache kommt erschwerend folgendes Dilemma hinzu, das Bredel/Maaß (2016: 481 und 489f.) allgemein für Texte in Leichter Sprache beschreiben: Um Verständlichkeit auf Satz- und Wortebene zu erreichen, ist die Leichte Sprache auf ein maximal reduziertes Sprachsystem beschränkt. Zugleich werden Erläuterungen in den Text eingefügt, um fehlendes Sprach- und Weltwissen auszugleichen. Beides zusammen erschwert aber das Verstehen auf Textebene, denn der innere Zusammenhalt des Textes geht dadurch verloren. Beinhaltet der Text Rechtsinformationen, bedeutet das zwangsläufig, dass auch die rechtlichen Strukturen aus dem Blickfeld der Leser(innen) geraten. Umso wahrscheinlicher ist es, dass diese den Inhalt trotz Leichter Sprache nicht verstehen.

\subsection{Problem der Bebilderung von Leichte-Sprache-Texten mit rechtlichen Inhalten}

Viele Leichte-Sprache-Texte werden derzeit mit dem Bildersatz der Lebenshilfe Bremen bebildert (ebd.: 285). Es handelt sich eigens für Leichte-Sprache-Texte erstellte Illustrationen, die den Charakter von Abbildern haben. Sie stellen also Gegenstände, Personen, Handlungen oder Szenen als Ausschnitt aus der Wirklichkeit dar (Ballstaedt 2012: 20). Bei Texten mit rechtlichen Inhalten stößt diese Form der Bebilderung an Grenzen, denn Recht ist abstrakt und kann in diesem Sinne nicht abgebildet werden. Selbst wenn sich für einzelne Aspekte des Inhalts Bilder 
finden lassen, so wird dies in der Regel nicht ausreichen, um ein Konzept der Bebilderung für den gesamten Text umzusetzen. Ausgerechnet für Menschen, die sich mit dem Lesen schwertun, bleiben damit zur Vermittlung von Rechtsinformationen häufig nur bilderlose Texte mit schwer verständlichen Inhalten (zu den Grenzen der Visualisierbarkeit abstrakter Gegenstände s. Bredel/Maaß 2016: 278ff. und Alexander im vorliegenden Band).

\section{Was sind juristische Schaubilder?}

Juristische Schaubilder setzen bei der Informationsvermittlung genau da an, wo dem Medium Text Grenzen gesetzt sind, nämlich bei der Visualisierung rechtlicher Strukturen. Bergmans (2009: 21) und Eickelberg (2017: Rn. 262) bezeichnen sie deshalb treffend als Strukturbilder. Realisiert wird die Visualisierung, indem Text-, Form- und Bildelemente miteinander kombiniert, in einer speziellen Weise räumlich angeordnet und logisch miteinander verknüpft werden. Während sich der/die Adressat(in) beim Lesen eines Textes die Strukturen und Zusammenhänge erst durch eine syntaktische Analyse erarbeiten muss, springen sie ihm/ihr beim Anschauen eines Schaubildes förmlich ins Auge (vgl. Ballstaedt 2012: 51, allerdings spricht Ballstaedt nicht von Schaubildern, sondern von Charts). Schaubilder sind deshalb häufig leichter zu verstehen als informationsadäquater Text (ebd.). Zudem eignen sie sich, um im wahrsten Sinne des Wortes einen Überblick über Inhalte zu geben. Dabei stehen sie in aller Regel nicht für sich, sondern ergänzen Texte oder werden mündlich erläutert.

Die Beispiele auf den folgenden Seiten sollen einen Eindruck vermitteln, wie juristische Schaubilder im Leichte-Sprache-Kontext aussehen könnten. Eine farbige Fassung der Schaubilder mit Hintergrundinformationen zu ihrer Entstehung ist im Internet unter www.npridik.de/visualisierung-recht-leichte-sprache zu finden. 
Juristische Schaubilder in diesem Beitrag:

Abb. 1: Wenn Sie nicht mehr alles selbst entscheiden können

Das Schaubild erklärt die Begriffe Vorsorgevollmacht, rechtliche Betreuung und Betreuungsverfügung und setzt sie zueinander in Beziehung. Außerdem enthält das Schaubild den Appell, sich möglichst frühzeitig um eine Vorsorgevollmacht oder Betreuungsverfügung zu kümmern.

Abb. 2: Wer bekommt nach Ihrem Tod Ihr Geld und Ihre Sachen?

Das Schaubild vermittelt dem/der Betrachter(in), dass er/sie zu Lebzeiten Einfluss auf die Erbfolge nehmen kann, indem er/sie ein Testament macht oder einen Erbvertrag schließt. Außerdem erfährt er/sie, wie die Erbfolge geregelt ist, wenn er/sie ohne entsprechende Verfügungen von Todes wegen verstirbt.

Abb. 3: Strafverfahren: Was passiert in der Hauptverhandlung?

Das Schaubild vermittelt, wie die Hauptverhandlung vor dem Strafgericht abläuft. Dabei werden nicht nur die einzelnen Stationen des Ablaufs, sondern auch die Akteure benannt. 
Nicola Pridik

Wenn Sie nicht mehr alles selbst entscheiden können

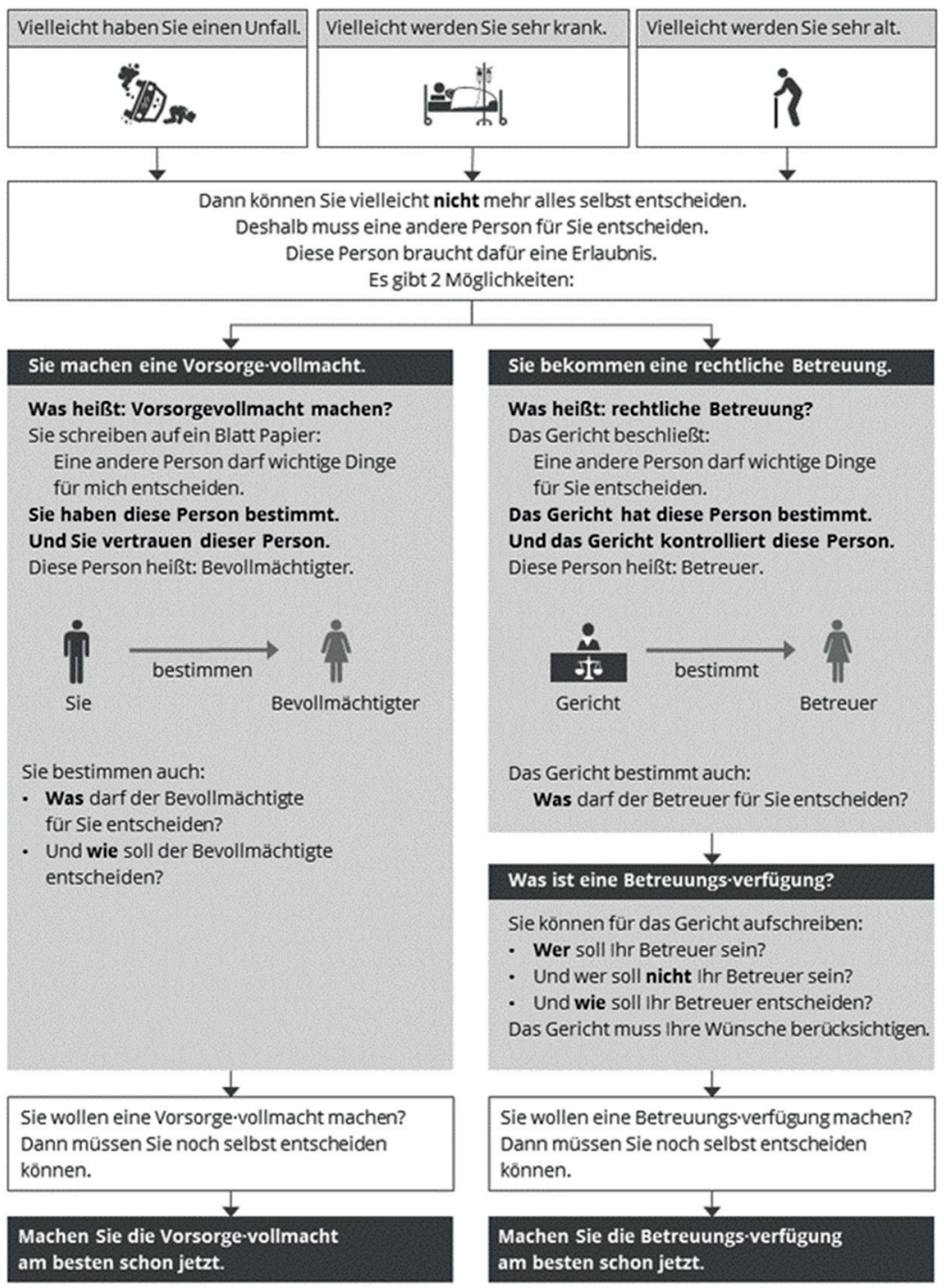

Onpridik.de

Abb. 1: Schaubild zur Vorsorgevollmacht und rechtlichen Betreuung (farbige Fassung: www.npridik.de/visualisierung-recht-leichte-sprache) 


\section{Wer bekommt nach Ihrem Tod Ihr Geld und Ihre Sachen?}

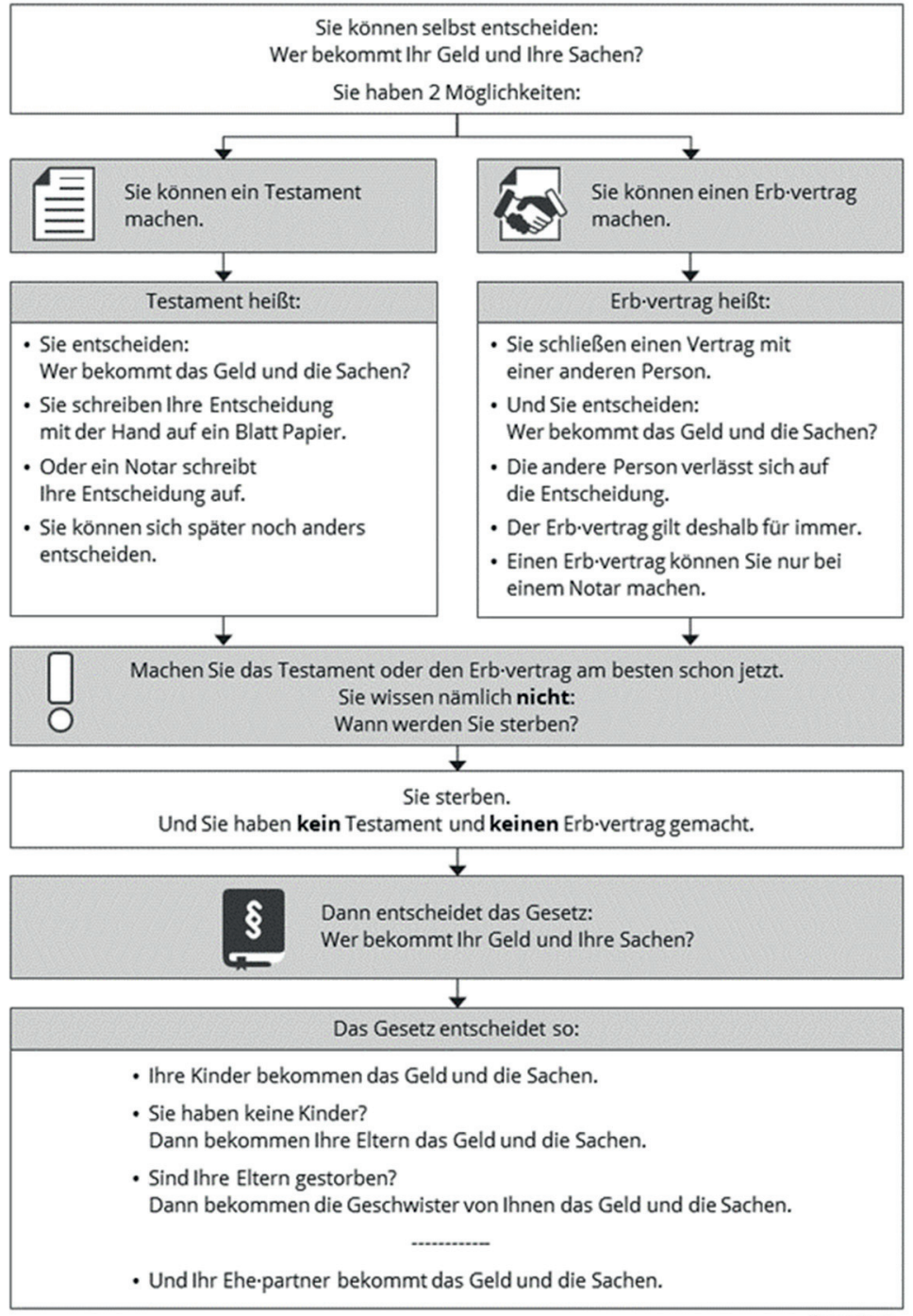

Snpridik.de

Abb. 2: Schaubild zur Erbfolge (farbige Fassung: www.npridik.de/

visualisierung-recht-leichte-sprache)

() Frank \& Timme Verlag für wissenschaftliche Literatur 
Nicola Pridik

\section{Straf-verfahren: Was passiert in der Haupt·verhandlung?}

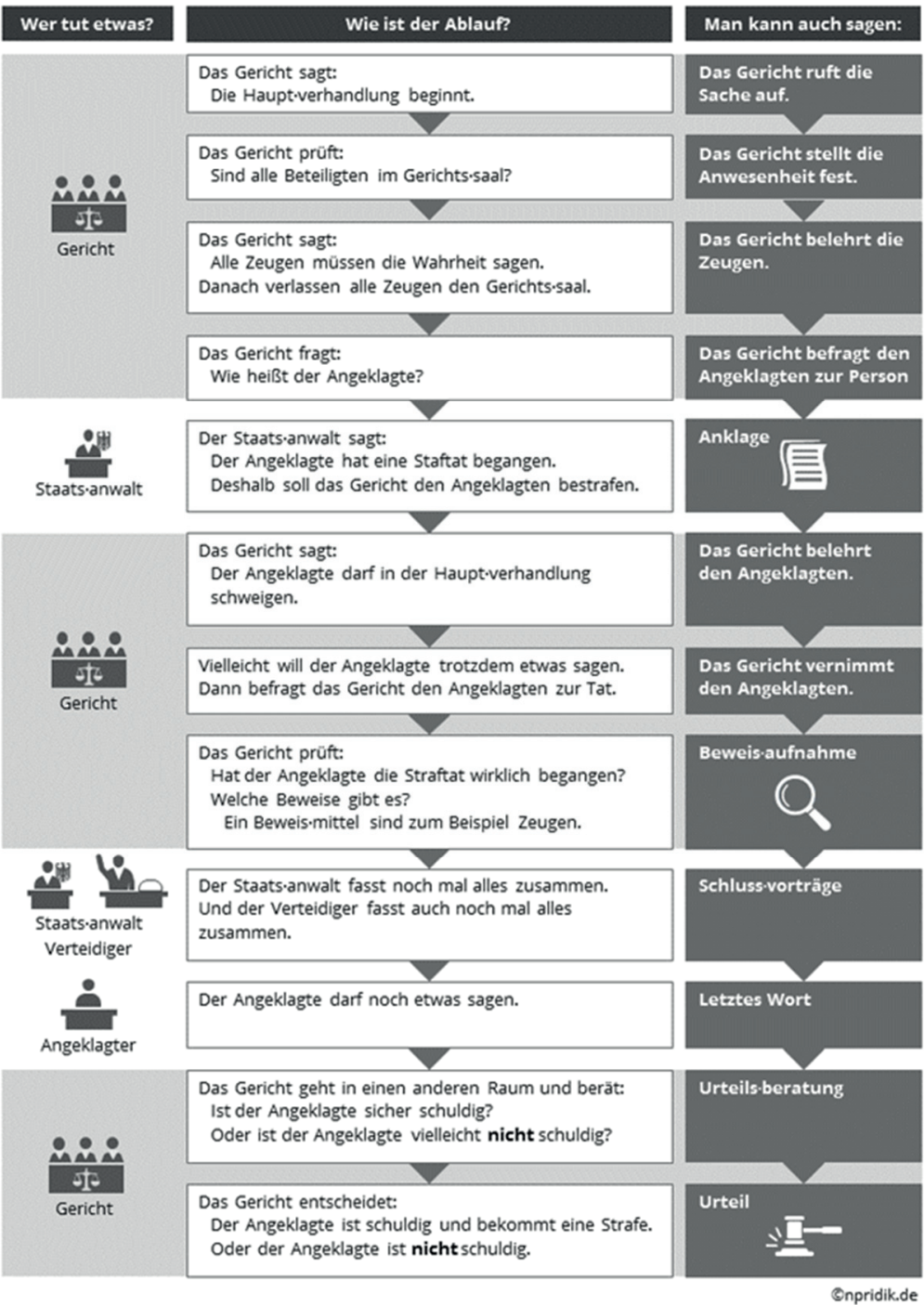

Abb. 3: Schaubild zur Hauptverhandlung (farbige Fassung: www.npridik.de/ visualisierung-recht-leichte-sprache) 
Noch nicht untersucht wurde allerdings bislang, welche Anforderungen juristische Schaubilder erfüllen müssen, die für möglichst viele Menschen - auch solche mit kognitiven Einschränkungen oder eingeschränkter Lesefähigkeit - verständlich sein sollen. Die nachfolgenden Ausführungen versuchen, eine erste Antwort zu geben. Sie bedürfen jedoch einer weiteren Theoretisierung und empirischen Überprüfung.

\subsection{Aussagekräftige Überschrift}

Eine Überschrift sollte mit möglichst wenigen Worten präzise auf den Punkt bringen, um was es im Schaubild geht. So kann der/die Betrachter(in) das Visualisierte besser einordnen und verstehen, denn die „Überschrift bietet [...] eine Vorstrukturierung und inhaltliche Einstimmung " (Lutz 2015: 296 zu Überschriften bei Gesetzestexten; zur Bedeutung von Titeln bei juristischen Abhandlungen Haft 2009: 142 und 143f.).

\subsection{Verständlicher Text}

Wie eingangs erwähnt, ist die verbale Sprache das wichtigste Werkzeug zur Vermittlung von Recht. Juristische Schaubilder enthalten deshalb immer Text. Ebenso klar ist, dass der Text in einem Schaubild stets so kurz und prägnant wie eben möglich gehalten und in sich verständlich sein muss, denn es handelt sich ja primär um eine Visualisierung. Im Leichte-Sprache-Kontext heißt verständlich, dass die Regeln der Leichten Sprache eingehalten werden. (Den Regeln widmen sich die Beiträge von Bredel/Maaß und Maaß im vorliegenden Band.) Trotzdem mit wenig Text auszukommen, ist folglich eine Herausforderung, denn die Erklärung von Begriffen gehört ebenso direkt ins Schaubild wie sie in einen Text in Leichter Sprache gehört. Darüber hinaus führt z. B. der Verzicht auf Pronomen der 3. Person zu einer Zunahme des Textvolumens. Gelöst werden können die daraus resultierenden Platzprobleme nur dadurch, dass Abstriche bei den Layout-Regeln (Schriftgröße 14 pt, jeder Satz auf eine neue Zeile, größerer Zeilenabstand) gemacht werden. Würde man sich an alle Vorgaben 
halten, wären die Schaubilder im Leichte-Sprache-Kontext nur als Poster denkbar. Die Beispiele in diesem Beitrag passen bei Verwendung der Schrift Open Sans, die von Alexander (2017 und im vorliegenden Band) als kostenfreie Alternative zu der für die Leichte-Sprache-Praxis optimierten kostenpflichtigen Schrift Neue Frutiger 1450 empfohlen wird, in einer Größe von 10-11 pt gerade auf ein DIN-A4-Blatt.

\subsection{Konzentration auf Wesentliches}

Angesichts der Platzprobleme ist es bei Schaubildern in Leichter Sprache noch wichtiger als bei anderen juristischen Schaubildern, dass sie sich auf die wesentlichen Inhalte beschränken. Dabei bedeutet die Reduktion der Komplexität in der Regel auch, dass weniger wichtige Inhalte weggelassen werden und eine inhaltliche Vereinfachung stattfindet (Lehner 2012: 125). Das hat der Prozess des Visualisierens mit dem des Übersetzens von Texten in Leichte Sprache gemeinsam (vgl. Bredel/Maaß 2016a: 173).

\subsection{Klare Struktur}

Die Visualisierung soll z. B. zeigen, wie ein Verfahren abläuft, in welchem hierarchischen Verhältnis bestimmte Begriffe zueinander stehen, was aus dem Eintritt bestimmter Bedingungen folgt oder was der Unterschied zwischen zwei Konzepten ist. Dagegen soll das Schaubild nicht vermitteln, dass alles mit allem irgendwie zusammenhängt und sich gegenseitig beeinflusst. Auch dies ist eine Erkenntnis, zu ihrer Vermittlung bedarf es jedoch keines Schaubildes. Juristische Schaubilder helfen also nur dann, rechtliche Inhalte besser zu verstehen, wenn sie sich auf eine ausgewählte Struktur konzentrieren und diese klar zutage treten lassen.

Im Kontext Leichter Sprache wird die Struktur immer eine sehr einfache sein. Das hat im Wesentlichen zwei Gründe:

1. Rechtsinformationen sind wegen ihres hohen Abstraktionsgrades und der schwierigen begrifflichen Konzepte für juristische Laien mit kog- 
nitiven Beeinträchtigungen oder eingeschränkter Lesefähigkeit immer schwer zu verstehen. Umso wichtiger ist es, die Komplexität so weit es eben geht zu reduzieren, um die Adressat(inn)en nicht zu überfordern.

2. Schaubilder sollten stets auf eine Seite passen. Dabei beanspruchen solche in Leichter Sprache, wie gesehen, schon allein wegen der ausgeprägten Erklärstruktur des Textes viel Raum. Für mehr als eine einfache Struktur ist deshalb kein Platz.

\subsection{Lesbarkeit konzeptueller Zusammenhänge}

Die Struktur muss nicht nur inhaltlich klar sein, der/die Betrachter(in) muss die räumliche Anordnung der einzelnen Schaubildelemente und ihre Verknüpfungen auch in konzeptuelle Zusammenhänge übersetzen können (vgl. Ballstaedt 2012: 51). Welche Bedeutung haben insbesondere Pfeile? Geben sie nur die Leserichtung vor und zeigen an, dass ein neuer Gedankenschritt folgt? Veranschaulichen sie ein kausales Verhältnis (Ursache $\rightarrow$ Wirkung)? Geht es um einen konditionalen Zusammenhang (Bedingung $\rightarrow$ Folge) oder um eine zeitliche Abfolge? Bei der Klärung dieser Fragen sollte das Schaubild dem Betrachter helfen.

Ballstaedt (ebd.: 115) empfiehlt, Pfeile mit unterschiedlicher Funktion in Form und/oder Farbe zu unterscheiden und ihre Bedeutung in einer Legende zu erklären (ähnlich Bergmans 2009: 38f.). Stary/Unger (o. J.: 13f.) gehen noch einen Schritt weiter und definieren in der Legende nicht nur verschiedene Pfeile, sondern auch Formen und Linien mit ihrer jeweiligen logischen Bedeutung und verbalen Aussage. All das schafft zweifellos Klarheit. Allerdings erschwert es dem/der Betrachter(in) auch das Lesen des Schaubildes, denn sein/ihr Blick muss mehrfach zwischen Schaubild und Legende wandern. Bei Texten in Leichter Sprache sind aus genau diesem Grund keine Fußnoten erlaubt und Begriffe werden an Ort und Stelle im Text erklärt (Bredel/Maaß 2016: 483). Die Verwendung einer Legende kommt deshalb auch bei Schaubildern im Leichte-Sprache-Kontext eher nicht in Betracht. 
Vorzuziehen sind Lesehilfen im Schaubild selbst, die entweder aus sich heraus verständlich sind oder deren Bedeutung sich ohne Weiteres aus dem Kontext ergibt. Soweit dies inhaltlich sinnvoll ist und der Platz ausreicht, können Pfeile z. B. in aller Kürze leserlich beschriftet werden. (Zur leserlichen Beschriftung von Charts Ballstaedt 2012: 59.) Ein typisches Beispiel ist die Beschriftung mit ja oder nein in Entscheidungsdiagrammen. Alternativ besteht die Möglichkeit, die Art der Verknüpfung zusätzlich im Text aufzugreifen. So verbalisiert z. B. in dem folgenden Beispiel das an sich überflüssige Dann im zweiten Kasten das konditionale Verhältnis, für das der Pfeil steht, und klärt damit dessen Bedeutung:

Sie sterben.

Und Sie haben kein Testament und keinen Erbvertrag gemacht.

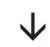

Dann entscheidet das Gesetz:

Wer bekommt Ihr Geld und Ihre Sachen.

Eine inhaltliche Interpretation ist allerdings trotzdem nötig, denn das Dann kann auch auf eine zeitliche Abfolge hindeuten. Dieselbe Interpretation ist aber erforderlich, wenn die Sätze ohne Pfeil als Fließtext miteinander verbunden werden. Die durch den Pfeil verbundenen Textkästen rücken nur stärker in den Blick, dass die Sätze überhaupt unmittelbar aufeinander bezogen sind. Mit anderen Worten: Die Strukturierung des Inhalts durch Kästen und Pfeile ersetzt die Verknüpfer auf verbaler Ebene nicht, sondern macht sie zusätzlich fürs Auge sichtbar und lenkt damit den Blick des Betrachters / der Betrachterin auf die Struktur.

\subsection{Erkennbare Leserichtung}

Eine Stärke von Schaubildern ist, dass sie bei der Vermittlung von Informationen nicht an eine lineare Abfolge gebunden sind. Allerdings führt gerade 
diese Nicht-Linearität auch leicht zur Verwirrung und Desorientierung des Betrachters / der Betrachterin. Das kann schon damit beginnen, dass das Schaubild keinen klaren Startpunkt aufweist (vgl. ebd.: 136 für Infografiken).

Schaubilder in Leichter Sprache wird der/die lesekundige Betrachter(in) (zumindest wenn er/sie aus einem Kulturkreis stammt, in dem von links nach rechts gelesen und geschrieben wird) schon aufgrund der umfangreichen Textanteile und einfachen Struktur intuitiv von oben nach unten und von links nach rechts lesen. Zusätzlich sollte im Schaubild ein „Blickpfad mit visuellen Leitplanken“ (vgl. ebd.) angelegt sein, der die Abfolge der Auswertung steuert. So können insbesondere Pfeile oder eine Nummerierung von Teilen des Schaubildes die Leserichtung vorgeben. Die Orientierung wird ansonsten vor allem durch die Anordnung der Elemente und ihre Farbgebung verbessert.

Wie oben dargelegt, sind die Strukturen von Schaubildern im LeichteSprache-Kontext sehr einfach gehalten. Die Probleme beim Erkennen der Leserichtung treten damit aller Voraussicht nach nur in zwei Konstellationen auf:

- Die erste betrifft den Fall, dass das Erkennen der Leserichtung mit dem Erkennen eines konzeptuellen Zusammenhangs zusammenfällt. So muss der/die Betrachter(in) im Schaubild zur Erbfolge (S. 493) den Hinweis mit den zwei Möglichkeiten im ersten Kasten verstanden haben, um die Lesart der beiden Pfeile darunter einordnen zu können. Ähnlich ist es bei Entscheidungsdiagrammen, in denen die weitere Leserichtung davon abhängt, ob der/die Betrachter(in) die im Schaubild gestellten Fragen jeweils mit ja oder nein beantwortet.

- Im zweiten Fall ergeben sich die Probleme daraus, dass zwei verschiedene Leserichtungen möglich (und gewollt) sind. Ein Beispiel dafür bietet das Schaubild zur Hauptverhandlung (vgl. S. 494). Hier gibt der Ablauf des Verfahrens die Haupt-Leserichtung von oben nach unten vor. Zugleich werden die einzelnen Stationen des Verfahrens aber in der rechten Spalte durch die fachliche Bezeichnung oder eine Formulierung, die dieser angenähert ist, ergänzt. Die Funktion dieser Spalte besteht darin, die relativ textlastigen Kästen in der mittleren Spalte, 
soweit dies möglich ist, mit kürzer gehaltenen Begriffen zusammenzufassen und dabei zugleich fachlichen Wissenserwerb zu ermöglichen (zum Aufbau von juristischem Wissen durch Concept Maps s. Stary/Unger o. J.). (Man könnte die rechte und mittlere Spalte auch umgekehrt anordnen. An dem Problem mit der Leserichtung ändert sich dadurch allerdings nichts.) Der/Die Betrachter(in) kann diese Spalte unbeachtet lassen, wenn sie ihm/ihr zu schwierig ist, er/sie kann sie aber auch mitlesen. Voraussetzung ist, dass sich für ihn/sie aus dem Kontext, insbesondere der Spaltenüberschrift „Man kann auch sagen: “ ergibt, wie die Informationen in das Lesen des Schaubildes einzubeziehen sind.

\section{7 Übersichtliche Gestaltung}

Angesichts zahlreicher Kästen und Pfeile wirken Schaubilder leicht unruhig und überladen. Das kann dem/der Betrachter(in) die Motivation nehmen, sich näher mit ihnen zu beschäftigen und behindert die Vermittlung der Inhalte. Auch bei der visuellen Organisation der Schaubildelemente und der Gestaltung als solcher ist deshalb mit Bedacht vorzugehen. Das Ziel ist, die Inhalte so aufzubereiten, dass schon auf den ersten Blick der Eindruck von Ordnung und Übersichtlichkeit entsteht und die Darstellung im Ganzen optisch ansprechend wirkt. Dafür müssen die Schaubildelemente unter Beachtung von Inhalt und Struktur ausgewogen auf der Fläche verteilt und so angeordnet werden, dass der Gestaltungswille klar zutage tritt (zur Ausgewogenheit und Eindeutigkeit als Mikrostruktur einer bildhaften Gestaltung Alexander 2013: 54 und 55). Zugleich ist auf genügend Weißraum zu achten (zur Bedeutung von Weißraum Koschembar 2009: 176ff.). Schließlich sind wenige miteinander harmonierende Farben einzusetzen und die Gestaltgesetze (Alexander 2013: 25ff., Turtschi 2010: 20ff.) zu beachten.

Die Gestaltgesetze (ebd.) beschreiben, nach welchen Gesetzmäßigkeiten bestimmte Gestaltungselemente vom Menschen wahrgenommen werden (Alexander 2013: 25 und im vorliegenden Band). Für juristische Schaubilder sind vor allem die folgenden vier Gesetze von Bedeutung: 
- Nach dem Gesetz der Ähnlichkeit werden gleich aussehende Elemente als zusammengehörig wahrgenommen. Aus diesem Grund sollten Textkästen und Formen mit gleicher Funktion in einem Schaubild auch gleich aussehen.

- Nach dem Gesetz der Nähe werden nahe beieinander liegende Elemente als Einheit wahrgenommen. Text-Bild-Kombinationen innerhalb des Schaubildes gehören deshalb räumlich eng zusammen.

- Nach dem Gesetz der Einfachheit tendiert unsere Wahrnehmung dazu, einfache Formen bevorzugt wahrzunehmen. Schaubilder sollten deshalb z. B. mit einfach gehaltenen Bildern bestückt und insgesamt nicht mit Informationen und Gestaltungselementen überladen werden.

- Das Gesetz des gemeinsamen Bereichs bedeutet schließlich, dass Elemente in abgegrenzten Gebieten als zusammengehörig wahrgenommen werden. Mithilfe des Gesetzes können und sollten zusammengehörende Informationen in einem Schaubild z. B. durch unterlegte Flächen gruppiert werden.

Die Gestaltgesetze zu beachten bedeutet nicht nur, dass sie gezielt bei der Erstellung des Schaubildes eingesetzt werden sollten, sondern auch, dass die „visuelle Organisation [...] nicht durch ungewollte Wirkungen von Gestaltgesetzen gestört werden [darf]“ (Ballstaedt 2012: 58). Was inhaltlich nicht zusammengehört, ist deshalb mit Abstand zueinander anzuordnen und Textkästen mit unterschiedlicher Funktion sollten nicht gleich aussehen.

Bei den Gestaltgesetzen handelt es sich um „angeborene ,Programme‘ zur spontanen Verarbeitung visueller Daten“ (ebd.: 25). Sie funktionieren beim Menschen also automatisch und müssen nicht gelernt werden. Ob das allerdings in vollem Umfang auch für die Zielgruppen der Leichten Sprache gilt, die eventuell eingeschränkte Wahrnehmungsmöglichkeiten haben, ist bislang nicht bekannt. 


\subsection{Bilder als Teil von Text-Bild-Kombinationen}

Ergänzend zum Text werden in juristischen Schaubildern häufig Piktogramme/Icons zur Vermittlung des Inhalts eingesetzt. Meist sollen diese die verbal formulierten Inhalte veranschaulichen. Sie können aber auch eine strukturierende Funktion haben, so wie z. B. in der ersten Spalte des Schaubildes zur Hauptverhandlung (S. 494). Ein weiterer Vorteil solcher Bildelemente ist, dass sie textlastige Schaubilder optisch auflockern, ansprechender gestalten und den/die Betrachter(in) in der Folge dazu motivieren, sich mit ihnen zu beschäftigen. Im Gegensatz zu Leichte-Sprache-Texten kommen Schaubilder in Leichter Sprache auch mit sehr wenigen Bildern aus, ohne den Eindruck zu vermitteln, dass es an Bildern gefehlt hat.

Unabhängig davon, in welcher Funktion Bilder im Schaubild eingesetzt werden, gilt, dass sie in aller Regel nicht für sich allein stehen, sondern innerhalb des Schaubildes Teil einer Text-Bild-Kombination sind (insofern besteht eine Parallele zur Erklärgrafik und Infografik, vgl. Ballstaedt 2012: 132 und 134ff.). Wichtig ist das deshalb, weil Piktogramme/Icons als Bilder mehrdeutig sein können, was es zunächst schwer macht, sie im Kontext des Schaubildes zu verstehen. Text kann das ändern, indem er dem Bild eine eindeutige Bedeutung zuschreibt. So weist Ballstaedt (ebd.: 142) darauf hin, dass Text dazu beitragen kann, das Bild in einer bestimmten Weise auszuwerten und zu interpretieren. Da das Bild zugleich den Text veranschaulicht, brauchen Text und Bild also einander (siehe auch Ballstaedt 2016: 7). Gemeinsam unterstützen sie das Verstehen und Erinnern der Inhalte.

Probleme entstehen allerdings dann, wenn die Inhalte so abstrakt und fachlich sind, dass nur Bilder mit einer metaphorischen oder symbolischen Bedeutung zur Veranschaulichung eingesetzt werden können. So muss der/die Betrachter(in) im Schaubild zur Hauptverhandlung (S. 494) beispielsweise im Piktogramm „Staatsanwalt“ den Bundesadler als Symbol für den Staat erkennen, um das Bild im Ganzen mit dem darunter stehenden Begriff „Staatsanwalt“ in Verbindung zu bringen. Noch schwieriger ist es, die Visualisierung von Fachbegriffen bzw. komplexerer Konzepte zu verstehen, wie z. B. die Piktogramme im Schaubild zur Erbfolge (S. 493). Leider gibt es bislang keine Erkenntnisse dazu, ob und inwiefern derartige Text-Bild-Kombinationen den Zielgruppen 
der Leichten Sprache zugänglich sind bzw. ob die Bilder in diesen Kombinationen tatsächlich dazu beitragen können, den Inhalt verständlich zu vermitteln.

Text und Bild sollten räumlich eng zusammenstehen, damit eine integrative Verarbeitung (Ballstaedt 2012: 137) möglich ist. Bleibt die Frage, wie Text und Bild in dieser Einheit zueinander anzuordnen sind. Zunächst ist es besser, sie nebeneinander anzuordnen als untereinander, da die horizontale Bewegung für die Augen weniger anstrengend ist (Alexander 2013: 88). In Schaubildern lässt sich das allerdings aus Platz- und Gestaltungsgründen nicht immer realisieren. So wäre es z. B. im Schaubild zur Vorsorgevollmacht und rechtlichen Betreuung nicht möglich, die oberen drei Bilder neben dem Text anzuordnen (vgl. S. 492). Da es stets nur einzelne Text-Bild-Kombinationen betrifft, ist es aber trotzdem unwahrscheinlich, dass die Anordnung zu einer Ermüdung der Augen des Betrachters / der Betrachterin führt.

Im Übrigen gilt: Bei horizontaler Anordnung von Text und Bild sollte das Leitmedium links platziert werden und bei vertikaler Anordnung oben (Alexander 2013: 87f.). Der Grund ist, dass das Leitmedium mehr Informationen enthält und deshalb zuerst ins Auge fallen sollte (ebd.: 87). Bei juristischen Schaubildern ist stets der Text das Leitmedium. Er sollte also über dem Bild bzw. links davon stehen. Auch hier kann es aber sein, dass Platz- oder Gestaltungsgründe im konkreten Fall eine andere Anordnung erfordern oder nahelegen, wie z. B. im Schaubild zur Erbfolge (S. 493).

\section{$4 \quad$ Resümee}

Auch wenn diverse Fragen noch unbeantwortet sind, so spricht doch viel dafür, dass juristische Schaubilder auch im Leichte-Sprache-Kontext ein geeignetes Medium sein können, um Rechtsinformationen zu vermitteln. Ihr Vorzug gegenüber dem Medium Text besteht darin, dass sie Strukturen sichtbar machen, die für das Verstehen des Inhalts wesentlich sind. Damit bieten sie zugleich eine Alternative zur üblichen Bebilderung von Leichte-Sprache-Texten, die bei rechtlichen Inhalten schnell an ihre Grenze stößt. Einen empirischen Beleg für den Schaubildnutzen gibt es freilich noch nicht. 
Die Visualisierungen bleiben aufgrund der stets einfachen Struktur und umfangreichen Textanteile nah an einer textlichen Darstellung, die Struktur tritt aber durch den Einsatz von Kästen, Pfeilen und Farben zusätzlich hervor. Die Schaubilder knüpfen damit unmittelbar an die Strategien an, die von Bredel/Maaß (2016: 502ff.) zur Herstellung von Textkohärenz in Leichte-SpracheTexten durch typografische Gestaltung empfohlen werden. Diese sehen den Einsatz von Zwischenüberschriften und Randglossen, Einrückungen, Listen und ergänzenden Bildern vor (ebd.). Bei Schaubildern geschieht im Ergebnis nichts anderes, nur die strukturierenden Mittel sind zum Teil andere und gelegentlich wird die Linearität des Textes durch eine abweichende Textanordnung durchbrochen.

Positiv kommt hinzu, dass juristische Schaubilder stets auf eine Seite beschränkt sind. Der/Die Betrachter(in) muss also nicht über mehrere Seiten der Entfaltung des Themas folgen, sondern hat eine überschaubare Sinneinheit vor sich. Diese Sinneinheit ist in sich abgeschlossen und nach Möglichkeit selbsterklärend, auch wenn sie weit davon entfernt ist, den Inhalt umfassend darzulegen. Da sie außerdem nur einen kleinen Ausschnitt aus einem größeren komplexen Zusammenhang darstellt, muss sie auf jeden Fall in einen textlichen Zusammenhang eingebettet sein oder mündlich erläutert werden. Am Ende wird es genau diese Wort-Bild-Kombination sein, die den Adressat(inn)en beim Verstehen der Inhalte hilft.

Abschließend bleibt festzuhalten, dass rechtliche Inhalte selbst bei noch so verständlicher Aufbereitung immer abstrakt und komplex bleiben. Das gilt für juristische Texte in Leichter Sprache ebenso wie für juristische Schaubilder. Beide werden also sicher nicht jeden Menschen erreichen. Sind Personen allerdings in der Lage, einen juristischen Text in Leichter Sprache zu verstehen, werden sie wahrscheinlich auch von ergänzenden Schaubildern profitieren. Zudem bieten Schaubilder unter Umständen für Menschen einen Zugang zum Inhalt, die eine überschaubare visuelle Aufbereitung eher anspricht als ein längerer Fließtext. Interessant ist sie auf jeden Fall für diejenigen, die den primären Zielgruppen der Leichten Sprache die Inhalte erklären, denn das Erklären fällt leichter, wenn man dabei auf eine Visualisierung Bezug nehmen kann. 


\section{Literaturverzeichnis}

AleXAnder, Kerstin (2013): Kompendium der visuellen Information und Kommunikation, 2. Auflage. Berlin, Heidelberg: Springer.

AlEXANDER, KERSTIN (2017): „Zur Lesbarkeit und Erkennbarkeit von Open-SourceSchriftarten im Umfeld ,Leichter Sprache“. In: Bock, BetTinA M./FIX, Ulla/ LANGE, DAISY (Hrsg.): „Leichte Sprache“ im Spiegel theoretischer und angewandter Forschung. Berlin: Frank \& Timme, 317-328.

Ballstaedt, STEFFEN-Peter (2012): Visualisieren. Konstanz: UVK.

Ballstaedt, StefFen-Peter (2016): Mit Bildern instruieren. Skript für Seminare und Workshops für technische Redakteure. URL: http://www.ballstaedt-kommunikation.de/ wp-content/uploads/Skript_Mit-Bildern-Instruieren.pdf (letzter Zugriff: 18.12.2017).

BERgmans, BERNHARD (2009): Visualisierungen in Rechtslehre und Rechtswissenschaft. Ein Beitrag zur Rechtsvisualisierung. Berlin: Logos.

Bredel, Ursula/MaAß, Christiane (2016): Leichte Sprache. Theoretische Grundlagen, Orientierung für die Praxis. Berlin: Duden.

Bredel, Ursula/MaAß, Christiane (2016a): Ratgeber Leichte Sprache. Die wichtigsten Regeln und Empfehlungen für die Praxis. Berlin: Duden.

EICKelBerg, JAN M. (2017): Didaktik für Juristen. Wissensvermittlung - Präsentationstechnik - Rhetorik. München: Vahlen.

HAFT, FRITJOF (2009): Juristische Schreibschule. Anleitung zum strukturierten Schreiben. München: Normalfall.

Koschembar, Frank (2009): Grafik für Nicht-Grafiker. Ein Rezeptbuch für den sicheren Umgang mit Gestaltung. Ein Plädoyer für besseres Design. Frankfurt/Main: Westend.

Kostorz, Peter (2016): Grundfragen der Rechtsdidaktik. Berlin: Lit.

LANGER, IngHaRdT/Schulz von Thun, FriedEMAnN/TAusch, Reinhard (2015): Sich verständlich ausdrücken. 10. Auflage. München: Reinhardt.

LEHNER, MARTIN (2012): Didaktische Reduktion. Berne: Haupt.

LUTZ, BENEDIKT (2015): Verständlichkeitsforschung transdisziplinär. Plädoyer für eine anwenderfreundliche Wissensgesellschaft. Göttingen: V\&R unipress.

Nussbaumer, Markus (2017): „Gesetze verständlich machen - Gesetze verständlich ,verkaufen“. In: Bock, BeTTINA/FIX, UlLa/LANGE, DAISY (Hrsg.): „Leichte Sprache“ im Spiegel theoretischer und angewandter Forschung. Berlin: Frank \& Timme, 351-372.

RÖHL, KLAUS F./UlbRich, STEFAN (2007): Recht anschaulich. Visualisierung in der Juristenausbildung. Köln: Halem.

StARY, JOACHIM/Unger, Werner (o. J.): „Concept Maps: Die Visualisierung juristischer Inhalte“. In: Neues Handbuch Hochschullehre. Berlin: DUZ, Kap. C 2.15. URL: https://www.nhhl-bibliothek.de/de/handbuch/gliederung/\#/Beitragsdetailansicht/ 251/750/Concept-Maps---Die-Visualisierung-juristischer-Inhalte (letzter Zugriff: 05.02.2018). 
Nicola Pridik

TurTschi, Ralf (2010): Typotuning 4, Bildgestaltung. Digitale Bilder kreativ gestalten und typografisch inszenieren. Winterthur: Publisher. 


\author{
Jan HeLlbusch
}

\title{
Mit barrierefreiem Webdesign zu einer besseren User Experience
}

\section{$1 \quad$ Einführung}

Damit digitale Inhalte barrierefrei nutzbar werden, müssen zuvor zahlreiche Aspekte bei deren Entwicklung berücksichtigt werden. Die Anforderungen der Barrierefreiheit sind sehr unterschiedlich und abhängig von den konkreten Nutzergruppen mit Behinderungen, den einzelnen Inhalten und dem Format. Für blinde Nutzer(innen) bedeutet Barrierefreiheit beispielsweise etwas ganz anderes als für Nutzer(innen) mit einer Sehbehinderung oder einer Lernbehinderung. Die Herangehensweise für die Anbieter der Inhalte ist bei einem wissenschaftlichen Artikel anders als bei interaktiven oder multimedialen Inhalten; genauso kommen verschiedene Werkzeuge zum Einsatz, je nachdem, ob HTML, ein Dokumentenformat oder eine andere Benutzungsschnittstelle genutzt wird, um die Inhalte zugänglich zu machen.

In diesem Beitrag werden einige zentrale Anforderungen der Barrierefreiheit an webbasierte Inhalte vorgestellt. Die Anforderungen für barrierefreie Webinhalte werden in den Web Content Accessibility Guidelines (WCAG) 2.0 gebündelt. Mit „Barrierefreiheit“ wird insbesondere die Konformität zu diesen Richtlinien verstanden. Die Erfüllung der Anforderungen wird dabei als Mindestanforderung für eine positive User Experience (UX) für Menschen mit Behinderungen bewertet.

Barrierefreie Webinhalte entstehen nicht von selbst. Nicht nur Entwickler(innen), Grafikdesigner(innen) oder Redakteure/Redakteurinnen müssen die Anforderungen im Blick haben. Es ist Aufgabe aller an einem Webprojekt Beteiligten, ihren Teil der Barrierefreiheit während der Entwicklungsprozesse zu verantworten. Insbesondere sollten folgende Punkte berücksichtigt werden: 
1. Digitale Inhalte können erst dann von allen konsumiert oder genutzt werden, wenn die Mindestanforderungen der Barrierefreiheit erfüllt sind.

2. Erst mit barrierefreien Inhalten können Aspekte wie Effizienz oder Zufriedenheit der Nutzer(innen) und letztlich die UX überprüft werden. Auch wenn die digitalen Inhalte selbst konform zu den Richtlinien sind, können Nutzer(innen) vor „Barrieren“ stehen, die sehr unterschiedliche Ursachen haben können; diese reichen von Inkompatibilitäten zwischen Hilfsmitteln und Browser bis hin zu UsabilityProblemen im Kontext einer Behinderung.

3. Um möglichst wenige Nutzer(innen) (mit Behinderung) auszugrenzen, müssen digitale Inhalte kontinuierlich und in einem inklusiven Entwicklungsprozess geplant und regelmäßig evaluiert werden.

\section{Richtlinien}

Die Barrierefreiheit von Webinhalten ist gleichbedeutend mit der Konformität zu den WCAG 2.0 (W3C 2008). Die WCAG 2.0 wurden bereits 2008 vom World Wide Web Consortium (W3C) veröffentlicht, stellen aber nach wie vor den Kriterienkatalog für barrierefreies Webdesign in zahlreichen Ländern der Welt dar.

Die WCAG 2.0 sind ein komplexes System an Dokumenten mit normativen und nicht-normativen (informativen) Inhalten. Die WCAG 2.0 selbst enthalten vier Prinzipien - untergliedert in zwölf einzelne Richtlinien - und formulieren 61 Erfolgskriterien. Entscheidend für die Bewertung der Barrierefreiheit ist die Erfüllung der Erfolgskriterien. Sie sind auf drei Konformitätsstufen verteilt (Konformitätsstufe A, AA und AAA), wobei Erfolgskriterien auf der Stufe A die wichtigsten Kriterien darstellen. Alle Erfolgskriterien sind als testbare Anweisungen formuliert und, um die Barrierefreiheit einer Webseite zu attestieren, müssen entweder alle Kriterien auf Stufe A oder alle Kriterien auf Stufe A und AA (Konformitätsstufe AA) oder alle Kriterien aus den WCAG 2.0 (Konformitätsstufe AAA) erfüllt sein (W3C 2016a). Im Übrigen ist die Erreichung von 
Konformitätsstufe AA ein realistisches Ziel in jedem Webprojekt. Die Erfüllung aller Erfolgskriterien ist in der Praxis hingegen nicht immer möglich.

In Deutschland gilt die Barrierefreie-Informationstechnik-Verordnung BITV 2.0 seit 2011. Verpflichtend ist sie nur für die öffentlichen Stellen des Bundes. In Anlage 1 der BITV 2.0 befindet sich ein Auszug der WCAG 2.0. Obwohl die Anlage 1 der BITV 2.0 nicht identisch mit den WCAG 2.0 ist, so kann sie weitgehend wie die WCAG 2.0 gelesen werden; ein wichtiger Aspekt wird in $₫ 3$ Abs. 1 BITV 2.0 festgelegt, wonach alle digitalen Inhalte Konformitätsstufe AA („Bedingungen der Priorität I“) erfüllen müssen. Ähnliche Regelungen finden sich in Deutschland auf Länderebene sowie in der Schweiz, in Österreich und vielen anderen Ländern Europas.

Die ausführlichste Dokumentation zur Barrierefreiheit vor allem in Webtechniken bietet das W3C selbst. Die WCAG 2.0 werden durch zahlreiche informative Dokumente ergänzt. Allen voran sind die erläuternden Dokumente einschließlich Best-Practice-Techniken zu den einzelnen Erfolgskriterien zu nennen (W3C 2016b), aber es gibt ebenfalls sogenannte „Notizen“, die einzelne Themen oder weitergehende Anforderungen beschreiben, die aus unterschiedlichsten Gründen nicht Teil der Webstandards geworden sind. Hierzu zählen beispielsweise über die WCAG 2.0 hinausgehende Anforderungen etwa von sehbehinderten Nutzer(inne)n oder formalisierte Rahmenbedingungen für die seitenübergreifende Evaluierung eines Webangebots.

Im Juni 2018 wurden die WCAG 2.1 veröffentlicht (W3C 2018). Die WCAG 2.1 schließen einige Lücken in den WCAG 2.0, die die Zugänglichkeit insbesondere für Sehbehinderte, Lernbehinderte und Nutzer(innen) mobiler Geräte mit Behinderungen betreffen. Die WCAG 2.1 sind als erster Schritt auf dem Weg zu WCAG 3.0 zu bewerten, die die Anforderungen der Barrierefreiheit auf Dokumente und andere Umgebungen (z. B. Zahlungssysteme oder Navigationsgeräte) ausdehnen (Hellbusch 2018). Dieses große Update der Webstandards für Barrierefreiheit ist für Ende 2020 geplant. Bis die WCAG 3.0 erscheinen, werden WCAG 2.0 und WCAG 2.1 parallel angewandt werden können.

Zunächst werden die WCAG 2.1 noch nicht verbindlich sein - das gilt in Deutschland und in Europa. Die Europäische Kommission hat im Oktober 2016 mit der Richtlinie 2102 Mindestanforderungen für die Barrierefreiheit von 
Webseiten festgelegt (s. den Beitrag von Lang im vorliegenden Band). Als technischer Standard ist die EN 301549 benannt, die sich auf die WCAG 2.0 stützt. Die Europäische Richtlinie legt derzeit über die EN 301549 fest, dass die WCAG 2.0, Konformitätsstufe AA, für die öffentlichen Stellen in den Mitgliedsstaaten und somit auch in Deutschland als Mindeststandard für die Barrierefreiheit von digitalen Inhalten anzuwenden ist.

Die Privatwirtschaft ist bisher nur in den wenigsten Fällen zu barrierefreiem Webdesign verpflichtet. Allerdings steht eine weitere europäische Richtlinie in einer Entwurfsfassung zur Diskussion, der sogenannte European Accessibility Act (EAA), der die Barrierefreiheit rund um Produkte und Dienstleistungen regeln wird. Auch im EAA werden die WCAG 2.0 als technischer Standard festgelegt, aber der Geltungsbereich umfasst aktuell „nur“ bestimmte Unternehmen des öffentlichen Interesses.

Obwohl die WCAG 2.0 speziell für webbasierte Inhalte entwickelt wurden, lassen sie sich auch auf andere digitale Inhalte, z. B. in Software oder Dokumenten, anwenden. Dabei gibt es einige Einschränkungen. Beispielsweise sind bei (nativen) Apps für Mobilgeräte zwar die Erfolgskriterien aus den WCAG 2.0 anwendbar, aber bestimmte Besonderheiten wie z. B. die Touch-Bedienung werden von den Anforderungen nicht abgedeckt. Auch die Displays auf HardwareGeräten im „Internet of Things“ oder die Besonderheiten einzelner Dokumente und Dokumentformate werden nicht ausreichend von den WCAG 2.0 abgedeckt.

Die WCAG 2.0 - Konformitätsstufe AA - stellen auf formaler Ebene zwar die Messlatte für Barrierefreiheit in Webinhalten dar, aber die Nutzeranforderungen werden in vielen konkreten Fällen darüber hinausgehen. Das liegt nicht nur an einzelnen Anforderungen in den WCAG 2.0, die teilweise minimalistischen Charakter haben, sondern selbstverständlich auch an Software oder Anforderungen der Nutzer(innen) selbst. Deswegen darf die Konformität zu den WCAG 2.0 lediglich als Ausgangspunkt für eine gut nutzbare Webseite für möglichst alle Nutzer(innen) angesehen werden. 
Ein Beispiel für minimale Anforderungen in den WCAG 2.0 ist die Tastaturbedienung. Es gibt einige wenige Kriterien, die nach den WCAG 2.0 für den barrierefreien Tastaturzugang auf einer Webseite erfüllt sein müssen, aber für die eigentliche Bedienung der Seite reicht es aus, wenn jede Funktion und Verlinkung auf der Seite grundsätzlich per Tastatur erreicht und aktiviert werden kann. So kann eine dynamische Navigation am Anfang der Seite per Tastatur nicht zugänglich sein, aber wenn am Ende der Seite eine weitere per Tastatur zugängliche Navigation mit den gleichen Links vorhanden ist, dann kann trotz der oberen Navigation Konformität erreicht werden. Für nicht umfangreiche Webseiten ist diese Herangehensweise an die Konformität nicht zwingend einschränkend, aber vor allem auf umfangreichen Webseiten könnte eine nur teilweise nutzbare Navigation für Tastaturnutzer(innen) zeitraubend sein. Dabei können dynamische Inhalte wie Menüs, Reiternavigationen oder sortierbare Tabellen mit einem passenden Fokus-Management auch für Tastaturnutzer(innen) gut nutzbar gemacht werden. Gerade das Thema der Tastaturbedienung auf Webseiten kann den Unterschied zwischen Barrierefreiheit (Konformität zu den WCAG 2.0) und einer positiven UX besonders verdeutlichen.

Dass die reine Konformität zu den WCAG 2.0 manchmal nicht ausreichend ist, wird durch den Einsatz von konkreten Hilfsmitteln durch Menschen mit Behinderungen ebenfalls verdeutlicht. Es gibt diverse Eingabegeräte als Mausund Tastaturersatz sowie Software z. B. für Bildschirmtastaturen oder Spracherkennung, die alle auf vorhandenen Schnittstellen des Betriebssystems aufsetzen. Für die Ausgabe gibt es Screenreader, die Inhalte in Sprache oder Braille umwandeln, und Vergrößerungssysteme (Hellbusch/Probiesch 2011: 11ff.). Nicht alle der Hilfsmittel unterstützen Webstandards auf ausreichende Weise und nicht alle Nutzer(innen) setzen die aktuellste Software ein. So kann es durchaus passieren, dass moderne, standardkonforme Webseiten mit einem fünf Jahre alten Screenreader genutzt werden und Kompatibilitätsprobleme dann dazu führen, dass eine Webseite oder einzelne Komponente der Webseite nicht oder nur eingeschränkt nutzbar sind. Es ist daher erforderlich, die Bedienbarkeit von Webseiten in verschiedenen Browsern und mit Hilfsmitteln zu überprüfen und gegebenenfalls alternative Umsetzungstechniken für bestimmte Inhalte einzusetzen - auch wenn die Konformität bereits attestiert wurde. 
Andere Themen sind in den WCAG 2.0 unterrepräsentiert, wozu die Verständlichkeit und die Berücksichtigung bestimmter Formatierungstechniken für eine bessere Leserlichkeit zählen; sie tauchen bestenfalls auf Konformitätsstufe AAA auf. Neben einigen wenigen Anforderungen zu Abkürzungen, Aussprache und ungewöhnlichen Wörtern enthalten die Anforderungen zur Verständlichkeit lediglich ein Erfolgskriterium zum Leseniveau:

\subsubsection{Leseniveau: Wenn der Text nach der Entfernung von Eigennamen und Titeln Lesefähigkeiten voraussetzt, die über das Niveau der niedri- gen, sekundären Schulbildung hinausgehen, dann gibt es ergänzenden Inhalt oder eine Version, die keine über die niedrige, sekundäre Schul- bildung hinausgehenden Lesefähigkeiten verlangt. (WCAG 2.0, Stufe AAA)}

Auch zur Leserlichkeit finden sich Anforderungen zur visuellen Präsentation lediglich auf Konformitätsstufe AAA und betreffen Farbe, Zeilenlänge, Zeilenfall, Zeilenzwischenraum und Textvergrößerung. Aspekte wie Schriftart und Auszeichnungsart sowie weitere Gestaltungsaspekte z. B. zum Hintergrund gehören nicht zu den Anforderungen - auch weil sie nicht universell festgelegt werden können. Bestimmte Nutzer(innen) werden beispielsweise bei einer serifenlosen Schrift wie Helvetica besser lesen können, während andere eine Serifenschrift bevorzugen. Dennoch: Die Anforderungen an die visuelle Präsentation legen nicht die Gestaltung einer Webseite fest, sondern sie bestimmen lediglich, dass Nutzer(innen) eine Anpassungsmöglichkeit haben sollten.

Die WCAG 2.0 umfassen nicht alle Anforderungen von Menschen mit Behinderungen an die Barrierefreiheit. Dafür gibt es verschiedene Gründe. Ein wichtiger Grund ist die Testbarkeit: Anforderungen, die objektiv getestet werden können, sind eher auf Konformitätsstufe A oder AA zu finden (je nach Auswirkung bei Nicht-Erfüllung), während Erfolgskriterien, die beispielsweise nur bedingt messbar sind oder die Gestaltung der Inhalte möglicherweise zu sehr beeinflussen, eher auf Stufe AAA oder gar nicht in den Richtlinien vorkommen (W3C 2016a). Diese Kompromisse sind insofern wichtig, als sie das Ziel repräsentieren, die Barrierefreiheit zu objektivieren, und die Akzeptanz der Anforderungen fördern sollen. 
Dass die Messlatte für Barrierefreiheit vor allem in einem individuellen Kontext viel zu kurz greifen kann, liegt auf der Hand. Um ein positives Nutzungserlebnis bei möglichst vielen Menschen mit Behinderungen zu erreichen, müssen die Anforderungen einzelner Nutzergruppen und Nutzer(innen) in die Gestaltungsüberlegungen für digitale Inhalte einfließen. Die Arbeitsweisen und spezifischen Anforderungen der verschiedenen Nutzergruppen können dabei auf der angestrebten Konformitätsstufe der WCAG 2.0 nicht vorkommen. Außerdem gibt es oft keine Schnittmengen zwischen den einzelnen Anforderungen, wodurch das Thema vor allem beim Einstieg viele Ressourcen in Anspruch nimmt. Deshalb sollten vor der Umsetzung eines barrierefreien Webdesigns die möglichen Anforderungen und die Schnittmengen zu den Richtlinien einzeln vergegenwärtigt werden:

- Sehbehinderte stellen eine heterogene Nutzergruppe dar. Wichtige Aspekte für die Webentwicklung sind insbesondere die Anpassung der Bildschirmeinstellungen und die Berücksichtigung ausreichender Kontrastverhältnisse für Text. Sehbehinderte Nutzer(innen) werden stärker von einer Konformität zu den WCAG 2.1 als von der Konformität zu den WCAG 2.0 profitieren.

- Blinde Nutzer(innen) verwenden Screenreader, um Bildschirminhalte mithilfe nicht-visueller Ausgabegeräte (akustisch über eine Sprachausgabe oder haptisch über eine so genannte Braillezeile) zu vermitteln. Screenreader wiederum stützen sich auf den Accessibility-Tree eines Betriebssystems. Screenreadernutzer profitieren bereits stark von einer Konformität zu den WCAG 2.0.

- Manche Nutzer(innen) mit körperlichen Einschränkungen können die Tastatur (oder den Mauszeiger) nicht benutzen. Wichtig für das Webdesign ist aber, dass die Nutzbarkeit sämtlicher Funktionen nicht nur per Mauszeiger, sondern ebenso per Tastatur gewährleistet wird. Vor allem für Mobilgeräte bieten die WCAG 2.1 einige weitergehende Anforderungen gegenüber den WCAG 2.0 für die barrierefreie Nutzung durch Menschen mit körperlichen Einschränkungen. 
- Menschen mit Lernbehinderungen benötigen u.a. Leichte Sprache. Anforderungen hierzu finden sich in den Webstandards zur Barrierefreiheit leider kaum. Die WCAG 2.1 umfassen einige Anforderungen zur ablenkungsfreien Interaktion z. B. bei einer Authentifizierung. Die BITV 2.0 legt darüber hinaus fest, dass die Startseite eines Webangebots einführende Informationen in Leichter Sprache enthalten muss.

- Für gehörlose Nutzer(innen) sind insbesondere akustische Informationen auch in Textform anzubieten. Die Übersetzung von Inhalten in Gebärdensprache wird nach den WCAG 2.0 nur für Audio-Inhalte in Videos empfohlen. Die BITV 2.0 legt hingegen fest, dass die Startseite eines Webangebots ein Gebärdensprachvideo mit Vorstellung der Inhalte und der Navigation enthalten muss.

Diese Übersicht ist nicht vollständig. Die Nutzergruppen lassen sich erweitern (z. B. kommen einige Behinderungsformen bzw. chronische Erkrankungen wie Epilepsie in der Liste gar nicht vor) und ebenso weiter differenzieren. Die Übersicht stellt lediglich den Ausgangspunkt für die Entwicklung barrierefrei nutzbarer Webangebote aus Nutzersicht dar. Die Konformität zu den WCAG 2.0 ermöglicht den Zugang zu Inhalten für viele Nutzer(innen) mit Behinderungen, aber um ein positives Nutzungserlebnis für möglichst viele Nutzer(innen) zu bewirken, müssen behinderungsbedingte und manchmal auch individuell geprägte Anforderungen stärker gewichtet werden.

\section{Ausgewählte Themen}

Barrierefreiheit betrifft jeden, der an der Entwicklung und Veröffentlichung digitaler Inhalte beteiligt ist. Es gibt jedoch verschiedene Schwerpunkte, die in konkreten Situationen insbesondere durch Designer(innen), Entwickler(innen) oder Redakteure/Redakteurinnen zu verantworten sind. Gleichzeitig lassen sich die Aufgaben oft nicht scharf abgrenzen und Aufgaben überlagern sich mit anderen Arbeitsabläufen in einer Organisation. 
Ein kleiner Aspekt der Barrierefreiheit ist beispielsweise der Seitentitel einer Webseite. Selbstverständlich muss am Ende eines Veröffentlichungsprozesses ein(e) Redakteur(in) den Text in einem Redaktionssystem eingeben und kontrollieren, aber zuvor muss ein(e) Entwickler(in) die Eingabe eines individuellen Seitentitels zu diesem Zweck auch ermöglichen. Gleichzeitig könnte das Marketing oder ein(e) Experte/Expertin für Suchmaschinenoptimierung bestimmte Vorgaben an Formulierungen in Seitentiteln haben, denn der Seitentitel beeinflusst die Auffindbarkeit von Webseiten in Suchmaschinen. Solche Aspekte ebenso wie die immer vorhandenen zeitlichen und kostenmäßigen Anforderungen müssen stets mit den Anforderungen der Barrierefreiheit abgeglichen werden.

Es gibt einige Anforderungen, die für bestimmte Nutzergruppen wichtig und für die Glaubwürdigkeit eines Informationsanbieters, der sich die Barrierefreiheit auf die Fahnen schreibt, zumindest empfehlenswert sind. Es handelt sich nicht zwingend um die wichtigsten Themen für jede(n) Nutzer(in) - was wichtig ist, hängt meist von einzelnen Nutzer(inne)n und Nutzergruppen ab aber auf der Basis der WCAG 2.0 handelt es sich um entscheidende Aspekte der Barrierefreiheit.

Wie die einzelnen Themen idealerweise umgesetzt werden, muss den ausführlichen Dokumentationen beim W3C (W3C 2016b) oder anderswo im Netz entnommen werden, denn es gibt einfach zu viele Szenarien, um „beste“ oder allgemein gültige Lösungen anzupreisen. Dabei wird die Konformität in verschiedenen Situationen durchaus unterschiedlich zu erreichen sein.

\subsection{Textalternativen}

Textalternativen spielen eine wichtige Rolle insbesondere für Grafiken und deren Wahrnehmung durch Screenreadernutzer(innen). Die Wahl der richtigen Textalternative ist dabei alles andere als trivial (Kitza 2015). Zum einen stellt sich die Frage, wie genau eine Grafik beschrieben werden muss, und zum anderen gibt es diverse Techniken für die Umsetzung einer Textalternative.

Zunächst geben die WCAG 2.0 vor, dass Grafiken eine Textalternative benötigen, die den äquivalenten Zweck wie die Grafik erfüllt. Der Zweck der Grafik 
ist also das, was in die Textalternative gehört. Dabei gibt es grundsätzlich drei Zwecke:

- Die Grafik ist Teil eines aktiven Elements (z. B. Link oder Schaltfläche). In diesem Fall ist die Grafik zweitrangig. Die Grafik (samt Textalternative) und der sonst mit dem aktiven Element verknüpfte Text müssen die dahinterliegende Funktion angeben.

- Die Grafik stellt einen Inhalt dar, der auf der Seite sonst nicht in Textform vorhanden ist. Die WCAG 2.0 unterscheiden dabei zwischen identifizierenden und beschreibenden Textalternativen. Identifizierende Textalternativen können für Grafiken eingesetzt werden, die im Wesentlichen im Kontext beschrieben werden, aber auch beispielsweise für Kunstwerke. Beschreibende Textalternativen sind hingegen vor allem dann notwendig, wenn wesentliche Inhalte in der Grafik dargestellt werden, die bei Fehlen der Grafik zu unvollständigen Informationen auf der Seite führen (TU Chemnitz 2016).

- Die Grafik wird zu dekorativen Zwecken eingesetzt. Wenn das Fehlen der Grafik zu keinerlei Informationsverlust führt, dann muss die Grafik vor allem von Screenreadern ignoriert werden können.

Wir sprechen hier von „Textalternativen“ und nicht etwa von „Alternativtexten“. Tatsächlich werden in den WCAG 2.0 Textalternativen verlangt und der Alternativtext (alt-Attribut einer Grafik) ist „nur“ eine mögliche Technik. Neben einer Bildunterschrift (mit den HTML-Elementen $<$ figure $>$ und $<$ figcaption>) gibt es weitere Techniken mit Accessible Rich Internet Applications (ARIA), um eine Textalternative zu bestimmen. In der Praxis ist der Alternativtext fast immer als Textalternative einzusetzen (s. dazu auch den Beitrag von Schütt im vorliegenden Handbuch). 
4.2 Anweisungen und Beschriftungen für Formulare

Bei der Barrierefreiheit muss oft unterschieden werden zwischen Barrierefreiheit im eigentlichen Sinn und Usability im Allgemeinen. Wenn beispielsweise in einem Formular keine Validierung stattfindet und jeder ein fehlerhaft ausgefülltes Formular abschicken kann, dann stehen alle vor dem gleichen Problem. Wenn jedoch Fehleingaben farblich, aber nicht textlich gekennzeichnet werden, dann stehen bestimmte Nutzergruppen mit Behinderung vor einer Barriere.

Die WCAG 2.0 legen bei Formularen einige Kriterien für die Interaktion mit Formularen und anderen Komponenten fest. Im Vordergrund steht die Fehlervermeidung. Obwohl jede(r) Nutzer(in) Fehler bei der Eingabe macht, fällt es manchen Menschen mit Behinderungen schwerer, fehlerfreie Eingaben vorzunehmen oder fehlerhafte Eingaben zu erkennen. Deswegen müssen Nutzer(innen) bei der Formulareingabe unterstützt werden, fehlerhafte Eingaben zu vermeiden. Sollten Fehler gemacht worden sein, dann sollten Nutzer(innen) dabei unterstützt werden, die Fehleingaben zu erkennen und Korrekturen vorzunehmen. Konkret geht es um die folgenden Aspekte und Strategien:

- Fehlermeldungen müssen textlich vorhanden sein (eine farbliche Markierung reicht nicht aus).

- Wenn eine Formularvalidierung beim Abschicken eines Formulars stattfindet, dann werden Algorithmen angewandt und meist werden Nutzer(innen) dann aufgefordert, Fehleingaben zu korrigieren. Der Grund für die Nicht-Validierung ist dann möglichst genau anzugeben; dies sollte sich von den Algorithmen ableiten. Beispielsweise sollte auf nicht ausgefüllte Pflichtfelder hingewiesen werden oder bei einer falschen Telefonnummer statt „Ungültige Telefonnummer“ besser „Buchstaben sind für Telefonnummern nicht gültig“ genutzt werden.

- Wenn das Abschicken eines Formulars rechtliche oder finanzielle Folgen hat, muss der/die Nutzer(in) das Abschicken des Formulars mindestens einmal bestätigen (oder nach dem Abschicken rückgängig machen) können. Dies garantiert, dass eine Verpflichtung nicht 
versehentlich eingegangen wird. Das gilt analog, wenn Nutzer(innen) z. B. ein Konto oder andere wichtige Daten zu löschen versuchen.

Darüber hinaus gibt es einige eher technische Anforderungen an Formulare, von denen insbesondere Screenreadernutzer(innen) profitieren. Generell gilt, dass native HTML-Elemente wie <input> oder <select> für Formularelemente zu bevorzugen sind, denn Browser ermöglichen standardmäßig die Tastaturbedienung von Formularelementen, und die technische Identifizierung anhand von Rolle und Wert wird ebenfalls vom Browser zuverlässig an den Accessibility-Tree des Betriebssystems übertragen. Wenn bedeutungslose <span>Elemente für Formularelemente eingesetzt und gestaltet werden, ist die Konformität zu den WCAG 2.0 nicht mehr so leicht zu erreichen.

Außerdem muss die sichtbare Beschriftung eines Formularfelds - falls vorhanden - explizit über ein <label> Element mit dem Steuerelement verknüpft sein. Ist keine textliche Beschriftung vorhanden (z. B. in einer Werkzeugleiste), dann müssen die Steuerelemente anderweitig z. B. mit ARIA-Attributen bezeichnet werden, denn ohne eine Bezeichnung wird der Zweck des aktiven Elements von Screenreadernutzer(inne)n nicht ermittelt werden können.

\subsection{Struktur und Semantik}

Strukturen in einer Webseite, einem Dokument oder einer Software sind der technische Unterbau für die Barrierefreiheit. Strukturen im Inhalt, die visuell am Bildschirm erkennbar sind, müssen ohne Präsentationsebene (im Web: ohne CSS) nachvollzogen werden können.

Die Strukturierung von Inhalten in HTML erfolgt zumeist mit:

- Absätzen

- Überschriften (mit insgesamt sechs Ebenen)

- Listen $(<\mathrm{ul}>,<\mathrm{ol}>$ und $<\mathrm{dl}>)$

- Datentabellen (mit der Unterscheidung zwischen Kopf- und Datenzellen) 
Dem Grunde nach sollten alle Inhalte in einem der Elemente stehen. Technisch ist der richtige Einsatz dieser HTML-Elemente das Handwerk, das jedes Redaktionssystem und jede(r) Redakteur(in) beherrschen muss. Allerdings können nicht alle Inhaltsformen mit den vier Arten von Elementen abgebildet werden. Mit HTML5 wurden weitere barrierefreiheitsrelevante Elemente eingeführt, etwa:

- $<$ figure $>$ (mit < figcaption>) für referenzierbare Inhalte (z. B. Bilder mit Bildunterschrift, aber auch andere Inhalte wie Code-Beispiele oder Multimedia)

- <main> für den Hauptinhalt einer Webseite

- Gliederungselemente, um die Regionen einer Webseite semantisch zu identifizieren, etwa mit $<$ nav $>$, $<$ header $>$, $<$ footer $>$ und $<$ aside $>$.

- Weniger wichtig, aber ebenfalls mit Semantik für Screenreader versehen, sind die Gliederungselemente $<$ section $>$ und $<$ article $>$.

Die Semantik ist nicht nur für die Erfassung der Struktur eines Inhalts wichtig. Weil es in einem Screenreader nicht ohne weiteres möglich ist, einen umfangreichen Inhalt zu „scannen“, bieten Screenreader eine sogenannte strukturelle Navigation an. So ist es beispielsweise möglich, von Überschrift zu Überschrift mit einer Taste zu springen (meist ist das die Taste „ $\mathrm{H}^{“}$ ). Mit anderen Tasten können Regionen, Tabellen, Formularelemente oder Grafiken angesteuert werden. Je besser die semantischen Elemente in einem Inhalt aufeinander abgestimmt sind, desto einfacher wird es, in einem Screenreader darin zu navigieren.

\subsection{Tastaturbedienung}

Die Tastaturbedienung wurde weiter oben bereits als Minimalanforderung eingeführt. Auf die Tastaturbedienbarkeit angewiesen sind zum Beispiel manche motorisch eingeschränkte sowie blinde Menschen. Für die Konformität geht es bei der Tastaturbedienung vor allem um die Bedienung mit der Tab-Taste, was 
im Web eine Browserfunktionalität darstellt (und keine Screenreaderfunktionalität). Die Tastaturbedienung ist für manche Nutzer(innen) essentiell und die konkreten Anforderungen sind durchaus überschaubar:

1. Allen voran müssen sämtliche Funktionen und Links der Webseite per Tastatur aktiviert werden können. Für Links und Formularelemente liefert der Browser die Funktionalität dann, wenn standardkonformes HTML eingesetzt wird. Für fortgeschrittene (dynamische) Komponenten wie Reiternavigationen, sortierbare Tabellen oder Filterfunktionen für Auswahllisten bietet HTML keine passenden Elemente. Worauf es hinsichtlich der Tastaturbedienung ankommt, ist ein autorenseitiges Fokus-Management mit Event-Handlern. Die Anforderungen für das Fokus-Management werden in einem informativen und die ARIA-Spezifikation ergänzenden Dokument (W3C 2017) beschrieben und mit funktionierenden Beispielen ergänzt.

2. Des Weiteren darf der Fokus nicht manipuliert werden. Wenn ein(e) Nutzer(in) durch eine Webseite per Tab-Taste navigiert, dürfen zwar kontextuelle Hilfen und ähnliche Ergänzungstexte zur Seite hinzugefügt werden, aber neue Inhalte dürfen erst durch Aktivierung (i.d.R. die Eingabe- oder Leertaste) angezeigt werden. Noch wichtiger ist, dass der Fokus keinesfalls durch JavaScript verändert werden darf, d.h. wenn ein(e) Nutzer(in) die Seite per Tab-Taste durchwandert, muss es möglich sein, vom Anfang bis zum Ende der Seite und wieder zurück zu navigieren.

3. Die Fokus-Manipulation ist auch bei der Bedienung von Steuerelementen (Eingabe oder Auswahl) zu vermeiden. Ein häufiger Fall ist eine Auswahlliste, die per Maus problemlos ausgeklappt werden kann und - nachdem per Click ein Eintrag ausgewählt wird - automatisch zu einer anderen Seite weitergeleitet wird. Bei einer solchen Auswahl haben Tastaturnutzer(innen) in längeren Auswahllisten kaum eine Chance, eine Auswahl zu treffen, denn sobald die Markierung nach unten oder oben wechselt, greift die automatische Weiterleitung. Mit 
passenden Event-Handlern für Tastaturdrücke können solche Barrieren abgebaut werden.

4. Darüber hinaus muss die Fokus-Reihenfolge stimmig sein, d.h. aktive Elemente in einzelnen Abschnitten einer Webseite werden nacheinander und generell von links nach rechts und von oben nach unten in die Fokus-Reihenfolge des Browsers aufgenommen. Das ist auch dann relevant, wenn Inhalte dynamisch zur Seite hinzugefügt werden. Dass der Fokus stets sichtbar sein sollte, ist dabei selbstverständlich.

\subsection{Farbe und Kontrast}

Wer keine Farbe sehen kann (wegen veränderter Bildschirmeinstellungen oder einer Fehlsichtigkeit oder weil ein Screenreader im Einsatz ist) kann Informationen, die durch Vordergrund- oder Hintergrundfarben vermittelt werden, nicht erkennen. Farbe darf und soll zur Hervorhebung eingesetzt werden, aber es müssen zusätzliche Unterscheidungsmerkmale für die Barrierefreiheit berücksichtigt werden. Es gilt, folgende zwei Szenarien zu berücksichtigen:

- Für sehbehinderte Nutzer(innen) ist mindestens eine weitere visuelle Hervorhebung (z. B. Fettung, vorangestelltes Symbol, Rahmen o.ä.) notwendig, um farblich hervorgehobene Inhalte zuverlässig wahrzunehmen.

- Für Screenreadernutzer(innen) sind visuelle Formatierungen ohne Belang. Screenreader benötigen strukturelle oder textliche Hervorhebungen, z. B. sollte ein hervorgehobener Eintrag in der Navigation (womit die aktuelle Seite angezeigt wird) ein passendes ARIA-Attribut (z. B. aria-current=,true“) erhalten, das die aktuelle Seite kennzeichnet.

In den WCAG 2.0 werden einige weitere Anforderungen an die visuelle Wahrnehmung gestellt. Insbesondere sollten die Kontrastanforderungen beachtet werden. Das W3C stellt Anforderungen an die Helligkeitskontraste und liefert 
dafür eine Formel für das Kontrastverhältnis zweier Farbwerte (Paciello Group o.J.). Um dieses Kontrastverhältnis zu messen, gibt es zahlreiche Werkzeuge im Netz zum kostenlosen Download, etwa der Color Contrast Analyzer.

In dem Algorithmus des W3C werden Farbunterschiede nicht berücksichtigt, die bei Farbblindheit relevant sein können. Wenn beispielsweise ein(e) Nutzer(in) eine Rotblindheit hat, so kann die Wahrnehmung von rot wie die Wahrnehmung von schwarz oder dunkelgrau sein, so dass ein mittlerer Rotton auf schwarz vielleicht ein ausreichendes Kontrastverhältnis nach dem Algorithmus des W3C aufweist, aber der Text dennoch von einzelnen Nutzer(inne)n nicht gelesen werden kann. Bei der Farbgestaltung ist es daher ratsam, Simulatoren für Farbwahrnehmung zusätzlich heranzuziehen.

Kontrastverhältnisse müssen an vielen Stellen gemessen werden. Neben Fließtexten, Überschriften, Tabellen oder Beschriftungen müssen Kontrastverhältnisse beispielsweise auch bei den folgenden Texten geprüft werden:

- Häufig weisen Fehlermeldungen in Formularen ein zu geringes Kontrastverhältnis auf.

- Bei Mouse-Over und Fokus werden Kontraste oft verringert statt verstärkt.

- Wenn Text auf einem mehrfarbigen Hintergrund steht, dann ist das geringste Kontrastverhältnis ausschlaggebend.

- Vor allem bei Text in Grafiken, die einen transparenten Hintergrund haben, müssen oft verschiedene Stellen mit verschiedenen Hintergründen überprüft werden.

\subsection{Multimedia}

Multimedia bietet sehr viele Möglichkeiten der Informationsvermittlung. Vor allem bei der Kombination von Audio und Video sind folgende Maßnahmen erforderlich, um akustische und visuelle Informationen barrierefrei anzubieten: 
- Zur Vermittlung von akustischen Informationen in Multimedia müssen nach den WCAG 2.0 Untertitel eingesetzt werden. Gesprochene Inhalte können automatisch in Text umgewandelt werden, aber eine redaktionelle Korrektur ist anschließend fast immer notwendig. Neben gesprochenen Inhalten muss darüber hinaus geprüft werden, ob weitere akustische Inhalte wesentliche Informationen vermitteln, die ebenfalls in den Untertiteln zu berücksichtigen sind (zur Untertitelung für Hörgeschädigte s. auch den Beitrag von Mälzer/Wünsche im vorliegenden Band).

- Zur Vermittlung von visuellen Informationen in Multimedia müssen ebenfalls Audiodeskriptionen eingesetzt werden. Audiodeskriptionen sind Audio-Dateien mit Beschreibungen der rein visuellen Anteile eines Videostreams (z. B. eingeblendeter Text, Gestik, Mimik, Handlungen oder Landschaft), die mit Multimedia synchronisiert werden und zu denen Multimedia optional zugeschaltet werden können. Zuverlässige automatisierte Verfahren zur Beschreibung von Informationen, die akustisch nicht verfügbar sind, wie informationsgebender Gestik oder Handlungen, gibt es bislang nicht (zum aktuellen Stand der Dinge s. den Beitrag von Kurch im vorliegenden Band); vielmehr ist die Audiodeskription eine Aufgabe für Spezialist(inn)en (s. dazu den Beitrag von Benecke im vorliegenden Band).

Diese Anforderungen gelten nicht für reine Audiodateien oder reine Videodateien. Obwohl Inhalte dieser Art genauso für gehörlose respektive blinde Nutzer(innen) unzugänglich sind, so genügt es nach den Richtlinien, ein Tontranskript (für Audio) bzw. eine vollständige Textalternative (für Video) bereitzustellen.

\section{$5 \quad$ Accessibility First}

Barrierefreies Webdesign stellt oft eine Herausforderung aus dem Grund dar, dass es sich um sehr breit gefächerte Anforderungen handelt. Designer(innen) oder 
Entwickler(innen) müssen aber selbst keine Expert(inn)en für Barrierefreiheit sein. Wichtig ist, dass sie um die Bedeutung der WCAG 2.0 als Ausgangspunkt wissen und dass die Notwendigkeit, die Erfüllung der Kriterien rechtzeitig zu kontrollieren bzw. kontrollieren zu lassen, auf Entscheiderebene erkannt wird.

Es ist in der Praxis oft so, dass die Konformität zu den WCAG 2.0 am Ende eines Prozesses - auch nach dem Launch - angesetzt wird und nur dann, wenn das Budget die Überprüfung und die Nachbesserungen zulässt. Diese Herangehensweise ist nicht zielführend, denn wenn festgestellt wird, dass die eingesetzten JavaScript-Frameworks unzugänglichen Code produzieren oder dass ein Redaktionssystem wichtige Voraussetzungen zur Erreichung der Barrierefreiheit nicht erfüllt, dann wird die Software normalerweise nicht nachgerüstet werden können. Einige eher redaktionelle Anpassungen können vorgenommen werden und eventuell wird der eine oder andere gestalterische Aspekt überarbeitet. Die technischen Anforderungen für Barrierefreiheit bleiben in dieser Situation unnötigerweise auf der Strecke. Und auch wenn die grundsätzliche Zugänglichkeit durch Menschen mit Behinderungen ermöglicht werden kann, so ist es immer noch ein weiterer Schritt, bis diese Nutzer(innen) ein positives Nutzungserlebnis erhalten.

Damit Konformitätsstufe AA der WCAG 2.0 überhaupt erreicht werden kann, müssen die Überprüfungen früh im Entwicklungsprozess angesetzt werden. Wenn Barrierefreiheit in frühen Phasen qualitätsgesichert wird - ein „Accessibility First“-Ansatz -, dann werden Webseiten auch Prinzipien des universellen Designs bzw. Design for All gerecht. Webseiten werden durch die Barrierefreiheit nicht schlechter nutzbar, im Gegenteil: Was die Nutzbarkeit durch Menschen mit Behinderungen verbessert, wird die Nutzbarkeit insgesamt steigern (s. dazu auch den Beitrag von Womser-Hacker im vorliegenden Band). Mit „Accessibility First“ wird Konformität zu den WCAG 2.0 zwar nicht garantiert, aber das Ergebnis wird voraussichtlich eine sehr gut nutzbare Seite sein. Wenn im Gestaltungs- und Entwicklungsprozess:

- Software von Drittanbietern (z. B. Frameworks) auf barrierefreien Output überprüft wird, 
- die Bedeutung der Leserlichkeit für Menschen mit Behinderungen bei der Gestaltung berücksichtigt wird,

- Entwickler(innen) eine einführende Schulung zu technischen Anforderungen erhalten oder

- Prototypen durch Nutzer(innen) mit verschiedenen Behinderungen getestet werden,

dann werden viele Hindernisse für ein barrierefreies Webdesign frühzeitig abgebaut. 
Jan Hellbusch

\section{Literaturverzeichnis}

Hellbusch, Jan (2018): „Neues in den WCAG 2.1“. In: Screenguide, 74-77.

Hellbusch, Jan/Probiesch, Kerstin (2011): Barrierefreiheit verstehen und umsetzen Webstandards für ein zugängliches und nutzbares Internet. Heidelberg: dpunkt.verlag. KITZA, SARAH (2015): Gestaltung von Alternativtexten - eine empirische Studie aus Nutzerinnen- und Nutzersicht. Dortmund: Masterarbeit. URL:

https://www.barrierefreies-webdesign.de/knowhow/textalternative/pdf/Gestaltungvon-Alternativtexten-Kitza/ (letzter Zugriff: 07.05.2018).

Paciello Group (o.J.): Accessibility testing tools. URL:

https://developer.paciellogroup.com/resources/ (letzter Zugriff: 07.05.2018).

TU Chemnitz (2016): Leitfaden: Alternativtexte für Grafiken. URL:

https://www.tu-chemnitz.de/urz/www/bf/alt.html (letzter Zugriff: 07.05.2018).

W3C (2008): Web Content Accessibility Guidelines (WCAG) 2.0. URL: http://www.w3.org/TR/WCAG20/ (letzter Zugriff: 07.05.2018).

W3C (2016a): Understanding Conformance Requirements. URL: http://www.w3.org/ TR/UNDERSTANDING-WCAG20/conformance.html (letzter Zugriff: 07.05.2018).

W3C (2016b): Understanding WCAG 2.0. URL: http://www.w3.org/TR/UNDER STANDING-WCAG20/ (letzter Zugriff: 07.05.2018).

W3C (2017): WAI-ARIA Authoring Practices 1.1. URL: http://www.w3.org/TR/ wai-aria-practices-1.1/ (letzter Zugriff: 07.05.2018).

W3C (2018): Web Content Accessibility Guidelines (WCAG) 2.1 - Proposed recommendation. URL: http://www.w3.org/TR/WCAG21/ (letzter Zugriff: 07.05.2018). 


\section{Barrierefreie Mensch-Maschine-Interaktion}

\section{$0 \quad$ Einleitung}

Barrierefreiheit (engl. Accessibility) etabliert sich immer mehr als wichtiges Thema in der Mensch-Maschine-Interaktion (MMI). MMI wird als interdisziplinärer Gegenstandsbereich angesehen, an dem verschiedene Disziplinen (Arbeitswissenschaft, Ergonomie, Informatik, Informationswissenschaft, Medizin, Psychologie, etc.) beteiligt sind. In der Informationswissenschaft, deren wissenschaftliche Perspektive hier eingenommen werden soll, ist das allgemeine Ziel, den Zugang zu Information und Wissen sowie die dafür eingesetzten Technologien und Werkzeuge für alle gleichermaßen bereitzustellen. Bei dieser Teilhabe geht es nicht nur um die Rezeption von Wissen, sondern auch um die Erzeugung eigener Inhalte z. B. in sozialen Netzwerken als User-generated Content oder im World Wide Web.

Die Reihung der Elemente im Kompositum Mensch-Maschine-Interaktion ist programmatisch: Es geht in der Informationswissenschaft primär um die Perspektive des Menschen, dem „die Maschine“ für die Erledigung seiner Aufgaben hilfreich sein soll. Im angloamerikanischen Sprachraum hat sich dafür die Bezeichnung Human-Computer Interaction (HCI) etabliert. Die ComputerHuman Interaction (CHI) hingegen sieht sich mehr systemgetrieben und stellt die Entwicklung neuer Technologien in den Vordergrund.

In diesem Beitrag wird zunächst erklärt, was unter MMI zu verstehen ist, um anschließend auf die besonderen Herausforderungen durch die Barrierefreiheit einzugehen. Ein besonderer Fokus liegt auf dem Entwicklungsprozess von interaktiven Benutzungsschnittstellen sowie auf Methoden und Werkzeugen für eine benutzergerechte Gestaltung und Evaluierung. Beispiele barrierefreier Ansätze sowie ein Fazit schließen den Beitrag ab. 


\section{Mensch-Maschine-Interaktion: Definition und Kontext}

Unter MMI wird das interaktive Zusammenwirken innerhalb von Bedien- und Kommunikationsprozessen zwischen Mensch und Maschine über eine Schnittstelle verstanden, wobei die Aufgaben zwischen beiden Interaktionspartner(inne)n verteilt sind. Intensive Diskussionen finden derzeit bzgl. der Erweiterung der Autonomieübertragung an Maschinen statt. Bei der Maschine handelt es sich um automatisierte Artefakte, meist um einen Computer. Da die genaue Differenzierung zwischen Maschine, Computer, System etc. heutzutage immer schwieriger wird, soll der Begriff der Mensch-Maschine-Interaktion beibehalten werden und stellvertretend für alle automatisierten, digitalen Systeme wie auch Smartphones, Tablets, Automaten, Datenbrillen, Smart Watches, Apps, Webapplikationen, Navigationssysteme etc. gelten. Auch sog. ubiquitäre Systeme (ubiquitous, ambient systems), wie sie sich z. B. in Alltags- bzw. Haushaltsgeräten verbergen oder in verschiedensten Lebenslagen z.T. realitätserweiternd Aufgaben übernehmen, stellen für die MMI eine neue Herausforderung dar.

Dabei ist die entscheidende Komponente die sichtbare oder unsichtbare Benutzungsschnittstelle bzw. das Interface, worüber die Interaktion abläuft. In der Regel müssen Nutzer(innen) nicht genau verstehen, was hinter diesem Interface passiert, um erfolgreich zu interagieren. Das Interface kann z. B. eine traditionelle Benutzungsoberfläche (engl. Graphical User Interface) mit graphischtextuellen Elementen (Fenster, Menüs, durch Ikonen repräsentierte Objekte, Dialogboxen, Buttons etc.) sein, die mit Steuerungsinstrumenten wie der Maus oder über Touchscreen direkt selektiert und manipuliert werden können. Hinter der direkten Manipulation steht das sog. WYSIWYG-Prinzip („What You See Is What You Get"), das getätigte Veränderungen sofort offensichtlich werden lässt (z. B. die Löschfunktion $\rightarrow$ ein Objekt wird in den Papierkorb befördert und verschwindet vom Desktop).

Ein anderes Interaktionsparadigma nutzt die natürliche Sprache in geschriebener oder gesprochener Form. Gerade durch aktuelle Sprachassistenten wie Amazon Alexa, Apple Siri, Google Assistent haben Sprachsteuerungssysteme derzeit hohe Popularität z. B. im Smart Home Bereich erreicht. 
Neuerdings ist auch die Steuerung der Interaktion über Gesten (per Hand oder Kopf) oder Augenbewegungen, die über eine Kamera aufgenommen werden, schon im Einsatz (z. B. bei Smartphone-Kameras, Spielekonsolen, Car Multimedia Systemen, Tastaturbedienung). Die sog. Stereoskopie (Gestenerkennung ohne Infrarotlicht) funktioniert auch im Freien. Bei berührungssensitiven Bildschirmen von Smartphones oder Tablets ist die Interaktion durch verschiedene Gesten z. B. zum Navigieren oder zum Vergrößern oder Verkleinern schon seit langem in der Praxis angekommen.

Die früheren kommandoorientierten, formalsprachlichen Schnittstellen wurden in allgemeinen Bereichen weitgehend abgelöst und finden sich $u$. U. noch in Datenbankabfragesprachen wie z. B. SQL oder MESSENGER, werden aber auch dort vorwiegend als interne Schnittstellen verstanden, die von Fachexpert(inn)en bedient werden.

\section{Barrierefreie Mensch-Maschine-Interaktion}

Die MMI unterliegt zum einen spezifischen Richtlinien und Gesetzesvorgaben zur Barrierefreiheit, zum anderen haben die Normen zur Gebrauchstauglichkeit bei der Gestaltung der MMI auch hier Gültigkeit und sollen durch eine positive Erfahrung bei der Benutzung ergänzt werden. Carstens (2015) fasst den rechtlichen Rahmen für den spezifischen Bereich der barrierefreien IT in der Justiz zusammen, dieser lässt sich aber sehr gut bzgl. der gesamten Informations- und Kommunikationstechnologie generalisieren. Das Grundgesetz (GG), die UNBehindertenrechtskonvention (UN-BRK) sowie Vorgaben aus dem EU-Recht verpflichten dazu, Menschen mit Behinderungen

eine selbstbestimmte Teilhabe an allen modernen Informations- und Kommunikationstechnologien, die elektronisch bereitgestellt werden oder zur Nutzung offen stehen, zu ermöglichen sowie vorhandene $\mathrm{Zu}$ gangshindernisse und -barrieren zu beseitigen (Art. 9, 13 und $27 \mathrm{UN}$ BRK) (Carstens 2015: 178). 
Das Behindertengleichstellungsgesetz des Bundes (BGG) (vgl. auch Durchführungsverordnungen und die Behindertengleichstellungsgesetze der Länder) verlangt in $₫ 12$ Abs. 1, dass der Zugang über Benutzungsoberflächen von behinderten Menschen uneingeschränkt und ohne fremde Hilfe genutzt werden kann. Für die hier relevanten Bereiche der web- und nicht-webbasierten MMI liegen Standards für die Barrierefreiheit vor. Für Web-Applikationen und Website-Gestaltung sind insbesondere die Web Content Accessibility Guidelines (WCAG 2.0) als offizielle Empfehlung der Web Accessibility Initiative (WAI) des World Wide Web Consortiums (W3C) zu beachten. Die seit Oktober 2009 vorliegende autorisierte deutsche Übersetzung umfasst einen normativen und einen nicht-normativen Teil. Auf die Rechtslage inklusive die Verordungstexte und den Best Practice vom Typ WCAG 2.0 geht der Beitrag von Lang im vorliegenden Band ein. $\mathrm{Zu}$ den WCAG gibt es inzwischen die Version WCAG 2.1 (auch WCAG 2018), auf die u.a. der Beitrag von Schütt im vorliegenden Band berichtet. Die konkreten Guidelines bzw. Erfolgskriterien werden im Abschnitt 3.2 zur Evaluierung aufgegriffen.

Hier zeigt sich, dass zwischen Accessibility und Usability im rechtlichen Rahmen ein großer Überschneidungsbereich besteht.

Wie oben bereits erläutert steht der/die Nutzer(in) bei der informationswissenschaftlichen Sicht auf die MMI immer im Vordergrund. So gibt die DIN EN ISO-Norm 9241-110 (2008) folgende Definition für Gebrauchstauglichkeit bzw. Usability:

Gebrauchstauglichkeit ist das Ausmaß, in dem ein Produkt durch bestimmte Benutzer in einem bestimmten Nutzungskontext genutzt werden kann, um bestimmte Ziele effektiv, effizient und zufriedenstellend zu erreichen.

D.h., ein Produkt/System wird nicht nur von seinen funktionalen Eigenschaften her betrachtet, sondern immer in Bezug auf die Interaktion mit realen Benutzer(inne)n. Diese sind ausschlaggebend, denn sie geben Nutzungskontext und Ziele vor. Während die Effektivität (Wie genau und vollständig lässt sich ein Ziel erreichen?) und die Effizienz (Welcher Aufwand muss für eine bestimmte Ef- 
fektivität geleistet werden?) quantifiziert werden können, ist der Zufriedenheitsaspekt ein eher subjektiv-qualitativer Wert, der über die Erfülltheit bzw. NichtErfülltheit von Benutzererwartungen erfasst werden kann. Die Usability wird über sieben Grundsätze der Dialoggestaltung (vgl. DIN EN ISO 9241-110 2008) (Aufgabenangemessenheit, Selbstbeschreibungsfähigkeit, Erwartungskonformität, Lernförderlichkeit, Steuerbarkeit, Fehlertoleranz, Individualisierbarkeit) eingelöst.

Immer mehr wird die Usability durch die sog. User Experience (UX) ersetzt. Dieser Begriff ist weiter gefasst und bezieht sich nicht nur auf die unmittelbare Nutzung, sondern auch auf das Davor und das Danach. Es geht um das Erlebnis, das der/die Nutzer(in) insgesamt mit dem Produkt/System verbindet. Dazu zählen alle Erfahrungen, die mit einem Produkt oder einer Webanwendung gemacht werden - auch Emotionen, subjektives ästhetisches Empfinden, Spaß bzw. Joy of Use.

Im Bereich der barrierefreien MMI ist das Ziel, dass Nutzer(innen) mit Behinderungen die Systeme genauso nutzen können wie Menschen ohne Behinderungen (vgl. BGG 2016). Deshalb müssen Entwickler(innen) von MMISchnittstellen alle Arten von Einschränkungen, die Zugang und Nutzen von Wissen und Information erschweren, gleichermaßen berücksichtigen. Nicht immer werden Barrieren durch Behinderungen ausgelöst, sondern können auch fehlende Kompetenzen wie das Nichtverstehen einer Sprache oder veraltete Hardware-Infrastrukturen bedeuten. Exemplarisch sollen Smartphones mit kleinen Displays angeführt werden, die verhältnismäßig wenig Raum für Inhalt und Interaktionselemente bieten. Der Nutzungskontext kann sich hier rasch verändern und z. B. verminderte Lichtverhältnisse, akustische Störquellen oder schlechte Netzabdeckung umfassen, was für Menschen mit und ohne Handicaps gleichermaßen problematisch ist.

Interessant ist die Diskussion, die hier zwischen Universal Design (vgl. Fuchs/Obrist 2010) und individuellen Lösungen ausgetragen wird. Die Quintessenz ist, dass die Gestaltung der Systeme auf die Nutzung durch möglichst viele Menschen ausgerichtet sein soll, d.h., es soll nicht ein bestimmtes Handicap kompensiert, sondern eine möglichst generelle barrierefreie Nutzbarkeit angestrebt werden. Bzgl. der nutzergerechten Gestaltung/Programmierung von 
MMI-Systemen werden sog. User Interfaces for all und benutzerspezifische Lösungen gegeneinander abgewogen. Der erste Ansatz vertritt die Position, dass eine „Benutzerschnittstelle von der Stange“ so einfach, intuitiv und natürlich gestaltet sein soll, dass sie dem/der "durchschnittlichen“ Benutzer(in) keine Probleme bereitet. Aus ökonomischen Gründen werden individuelle Notwendigkeiten dabei meist ausgeklammert. Vor dem Hintergrund einer immer älter werdenden Gesellschaft rücken aber bestimmte Behinderungsarten wie z. B. Hör- und Sehbehinderungen immer mehr in das Zentrum des Interesses und erscheinen in Anbetracht der hohen Zahlen auch für Entwickler(innen) und Hersteller(innen) wieder rentabel. Das heißt aber ebenso, dass in der MMI die Erreichung einer 100\%igen Barrierefreiheit kaum möglich ist.

Eine systematische Kategorisierung von alternativen MMI-Konzepten gibt es derzeit noch nicht, was nicht zuletzt der großen Komplexität geschuldet ist. In der MMI-Forschung liegt eine große Anzahl exemplarischer Lösungsansätze vor, die meist eine bestimmte Form von Beeinträchtigung ins Visier nehmen.

Im Folgenden werden die Barrieren angeführt, die in der MMI bedacht werden müssen (zu Kommunikationsbarrieren s. den Beitrag von Rink im vorliegenden Band):

- Sensorische Barrieren liegen vor, wenn die Nutzung eines oder mehrerer Sinne eingeschränkt oder vollständig abhandengekommen ist. Dies kann infolge einer Erkrankung/Verletzung oder von Geburt an der Fall sein und betrifft den Gesichtssinn und das Gehör. Durch altersbedingte Veränderungen der menschlichen Fähigkeiten ist eine große Anzahl von Menschen davon betroffen (vgl. Weber 2015: 50f.). Das Spektrum verläuft hier von leichten Einschränkungen der Sehund Hörfähigkeit bis zur vollständigen Erblindung bzw. zum kompletten Hörverlust. Auch Farbfehlsichtigkeit kann in der MMI eine Rolle spielen, da Farben mit Funktionen konnotiert sind (z. B. Ampelmetapher).

- Motorische Barrieren betreffen ebenso ein weites Spektrum an Beeinträchtigungen. Sie können völlige Unbeweglichkeit von Gliedmaßen 
sowie Spastiken umfassen, sodass z. B. die Navigation und Treffsicherheit auf Tastaturen oder Navigationselementen leiden (vgl. Sünkler 2015: 222f.).

- Geistig-kognitive Behinderungen sollen hier nicht im Vordergrund stehen. Die MMI kann aber ebenfalls beeinträchtigt sein, wenn z. B. das Textverständnis angebotener Inhalte nicht gegeben ist oder Informationsabläufe, -architekturen oder Workflows zu Orientierungsoder Verständnisschwierigkeiten führen.

Bei der herkömmlichen MMI fällt eine Priorisierung des Visuellen auf, d.h. der Input wird gemeinhin über die Tastatur, der Output über eine visuelle Ausgabe realisiert, was diesbezüglich gehandicapten Menschen die Teilhabe an Information und Wissen verwehrt. Die MMI-Forschung konzentriert sich derzeit auf diese visuellen Einschränkungen, was auch Weber (2015: 57) feststellt.

Vor dem Hintergrund des gesellschaftlichen Ziels, eine Teilhabe an Information und Wissen zu ermöglichen, erhalten Benutzungsschnittstellen die Funktion, entsprechende Beeinträchtigungen zu kompensieren. Da bei der herkömmlichen MMI der Input über die Tastatur, der Output über die visuelle Ausgabe realisiert wird, führt dies zum Ausschluss einer großen Anzahl von Menschen.

Um ein für den/die Nutzer(in) zufriedenstellendes Ergebnis zu erzielen, folgt die benutzerorientierte Gestaltung von interaktiven MMI-Systemen zunächst zwei grundlegenden Paradigmen:

1. Durch das sog. User-centered Design (vgl. Cato 2001, Preece et al. 2015), das verschiedene Phasen durchläuft, soll dem/der zukünftigen Nutzer(in) der zentrale Fokus eingeräumt und eine Beteiligung an der Gestaltung von Anfang an realisiert werden. Der erste Schritt beinhal- 
tet deshalb eine Analyse der Eigenschaften der zukünftigen Nutzer(innen) und der jeweiligen Nutzungskontexte. Die Ziele, Bedürfnisse, Vorlieben, Wünsche, Intentionen, aber auch mögliche Beeinträchtigungen werden vorweggenommen, um die MMI-Entwicklung daran zu orientieren. Ebenso werden die Charakteristika des Kontexts erhoben, sodass auch diese in den Gestaltungsprozess einfließen können. Im zweiten Schritt münden die Ergebnisse der Bedarfsanalyse in einen Anforderungskatalog, der die wesentlichen Funktionen und Gestaltungselemente vorgibt. Daraus wiederum lassen sich multimodale Bedienkonzepte in einem ersten Prototyp unter Berücksichtigung von Usability Guidelines bzw. Styleguides entwerfen, der einer iterativen Evaluierung unterzogen wird. Man spricht hier auch von rapid prototyping, was bedeutet, dass zu einem sehr frühen Zeitpunkt ein z.T. lauffähiger Prototyp entwickelt wird, mit dem sich die spätere reale Situation durch Benutzer(innen) gut nachvollziehen lässt.

2. Durch das Prinzip der Partizipation werden die Nutzer(innen) an dieser Evaluation beteiligt. Man spricht hier von formativer Evaluation, weil die Ergebnisse in die iterative Optimierung des Prototyps zurückgespielt werden und so in mehreren Zyklen eine verbesserte Version erarbeitet wird. Am Schluss jeder Systementwicklung steht eine summative Evaluation, die das Gesamtsystem einer Bewertung unterzieht. 


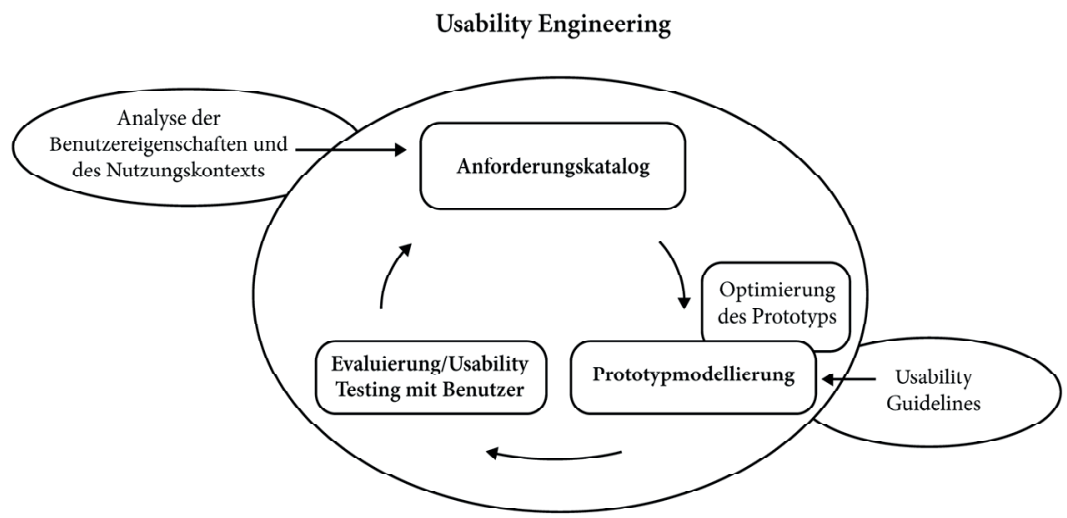

Abb. 1: Usability Engineering

Die Einbeziehung von $U X$-Aspekten erfordert eine wesentlich differenziertere Vorgehensweise, da hier neben der funktionalen Effektivität und Effizienz subjektive, hedonische, ästhetische und emotionale Faktoren ins Spiel kommen, die zusätzlich einer Dynamik unterliegen können (vgl. Hassenzahl/Tractinsky 2006: 91-97). Hier geht es darum, dem/der Nutzer(in) ein möglichst positives Erlebnis zu verschaffen.

\subsection{Methoden und Werkzeuge}

Für die verschiedenen Gestaltungsphasen stehen elaborierte Methoden und Werkzeuge zur Verfügung. In der Informationswissenschaft hat sich das Forschungsgebiet des sog. Information Behaviour etabliert, das gerade in den letzten Jahren eine intensive Verknüpfung mit der MMI - insbesondere im Bereich des interaktiven Information Retrieval - erfahren hat. Dabei geht es darum, das Informationsverhalten von Nutzer(inne)n sowie die zugehörigen Nutzungsszenarien zu erheben - nicht zuletzt mit dem Ziel, die Entwicklung der Systeme daran auszurichten. Wichtig ist, dass es nicht darum geht, den Nutzer(inne)n beizubringen, wie sie die Systeme richtig bedienen, sondern ihnen eine möglichst intuitive und natürliche Anwendung zu erlauben. 
Die Erhebung von Nutzereigenschaften und -kontexten kann durch Befragungen erfolgen, allerdings stellt es ein bekanntes Phänomen dar, dass sich Nutzer(innen) nicht immer ihres genauen Verhaltens bewusst sind und dieses auch nicht immer einer gezielten Strategie folgt. Vielfach wurde die Befragung deshalb durch Beobachtung oder durch sog. Shadowing (vgl. Görtz 2011) ersetzt oder ergänzt, wobei der/die Testende im Hintergrund bleibt. Oftmals sind die Möglichkeiten neuer Technologien noch nicht im Vorstellungsspektrum der Nutzer(innen) vorhanden. Deshalb ist es besonders wichtig, Entwickler(innen) und Nutzer(innen) in allen Phasen an dem Gestaltungsprozess zu beteiligen, z. B. in sog. Co-Creation-Workshops oder in Fokusgruppen. Sobald ein erster Prototyp (möglich auch als Mock-Up ohne vollständig ausgearbeitete Funktionalität) vorhanden ist, können Testszenarien erstellt werden, die den Kontext des/der Nutzer(in)s möglichst realistisch abbilden. Nutzer(innen) können vorgegebene Aufgaben (simulated tasks) (vgl. Borlund 2016) mit dem angebotenen Lösungsvorschlag ausführen und ihr Vorgehen durch Thinking aloud kommentieren. Diese Tests können unter Laborbedingungen in einem Usability-Labor oder in einer entsprechenden Remote-Umgebung stattfinden, wofür entsprechende Gerätschaften wie Eyetracker, Audio- und/oder Video-Aufzeichnungssoftware etc. notwendig sind. Manchmal bietet sich eine individuellere Atmosphäre an, z. B. bei Benutzertests im mobilen Bereich (Smartphones, Tablets, Datenbrillen etc.) oder im speziellen Bereich von Car-Multimedia-Tests mittels Fahrsimulatoren. Sollten Testpersonen und Testleiter(innen) nicht an einem Ort zusammentreffen können, liegen verschiedene Remote-Optionen vor, die z. B. zum Einsatz kommen, wenn Testpersonen von der Mobilität her eingeschränkt sind oder sich in einem anderen Land oder Kontinent befinden (z. B. bei der Entwicklung von Webapplikationen für neue Märkte). Um den Entwickler(inne)n stets einen Eindruck ihrer zukünftigen Anwender(innen) vor Augen zu führen, werden sog. Personas eingesetzt, die visualisierte Darstellungen von Nutzerstereotypen repräsentieren. Körperliche Einschränkungen kommen hier selten vor und Buß (2009) nennt noch weitere Tücken.

Wie oben dargestellt erfordern UX-Fragestellungen z.T. komplexere Techniken, um die Systeme daran auszurichten. Auch hier gibt es bereits Werkzeuge in Form von Fragebögen o.ä. Um z. B. die Emotionen oder das ästhetische Emp- 
finden von Nutzer(inne)n bei der Interaktion mit einem System zu erheben, haben Hassenzahl et al. (2003) z. B. den AttrakDiff2-Fragebogen vorgeschlagen, der mit semantischen Differentialen arbeitet und die pragmatische und hedonische Qualität bewertet. Moshagen und Thielsch (2013) entwickelten das sog. Visual Aesthetics of Websites Inventory (VisAWI), das vielfach genutzt wird. Ebenso wurde die Erfassung der Mimik (vgl. Fasel/Luettin 2003) zur Einschätzung der emotionalen Lage der Nutzer(innen) herangezogen. Auch die Self Assessment Manikin (SAM) Methode versucht eine Erhebung der Emotionen, indem die Nutzer(innen) eine auf sie zutreffende Darstellung selektieren. Weiterführende Experimente haben erprobt, welche Rolle z. B. die Ästhetik innerhalb von Benutzungsoberflächen spielt. Lindgaard et al. (2006) konnten zeigen, dass Nutzer(innen) bereits nach einer Betrachtungszeit von 50 Millisekunden aufgrund eines spontanen Ersteindrucks ein recht konsistentes Urteil über die Ästhetik fällten.

Gute Erfahrungen zeigten sich auch bei der Mischung von Methoden z. B. durch Aufzeichnung der Benutzertests sowie gleichzeitiges Messen von physiologischen Reaktionen (Hautleitfähigkeit, Puls, Pupillengröße etc.). Insbesondere die mit den Proband(inn)en durchgeführte gemeinsame Betrachtung der zusammengefügten Aufzeichnungen (stimulated recall Methode) erbrachte hier großes Potential (vgl. Irle 2017) für eine erfolgreiche Interpretation.

Die ständige Weiterentwicklung von neuartigen Schnittstellen z. B. im Bereich der Virtuellen Realität bzw. der immersiven Systeme führt zu einer Erweiterung der Informations- und Handlungsoptionen und zu einer abstrakteren und indirekteren MMI, was wiederum neuartige Methoden der Entwicklung und Evaluierung erforderlich macht. Allerdings muss in der MMI das Ziel bestehen bleiben, die Stärken der unterschiedlichen Interaktionspartner(innen) zu analysieren und zu unterstützen.

MMI-Systeme, die auf eine barrierefreie Interaktion und spezielle Handicaps von Nutzer(inne)n abzielen, erfordern meist eine Anpassung der Methoden. 


\subsection{Evaluierung bzgl. Barrierefreiheit}

Für die Evaluierung der MMI im Hinblick auf die Usability bzw. User Experience liegen verschiedene Methoden, Vorgehensweisen und Werkzeuge vor, die jedoch im Kontext barrierefreier MMI-Angebote angepasst werden müssen. Durch die Vielfältigkeit der Barrieren ist es oft nicht möglich, großangelegte Benutzertests mit einer adäquaten Anzahl an Proband(inn)en zu fahren. Hilfe bieten Befragungen in sog. User Panels oder neuerdings auch über Crowdsourcing. User Panels stellen einen Pool aus potentiellen Nutzer(inne)n zusammen, die an regelmäßigen Meetings teilnehmen und im Entwicklungsprozess zur Qualität eines Produkts beitragen. Sie identifizieren Problembereiche und geben Ideen für Verbesserungspotential. Möglich sind auch Online Panels, die entsprechend virtuell agieren. Mit Crowdsourcing (Kombination aus crowd und outsourcing) werden meist kleine Arbeitsprozesse an eine Menge von Freiwilligen ausgelagert, die gegen einen kleinen Obolus bestimmte Aufgaben erfüllen. Mehrfach werden dabei Simulationstests eingesetzt, die bestimmte Handicaps simulieren, z. B. mittels verzerrender Brillen oder mobilitätseinschränkenden Ganzkörperanzügen. Sog. Konformitätsprüfungen werden durchgeführt, um die Benutzerschnittstelle mit den Richtlinien abzugleichen.

Die WAI des W3C bietet einen Leitfaden an, der eine dreigliedrige Evaluierung vorschlägt, die einen heuristischen Experten Walk Through, eine manuelle oder automatisierte Konformitätsprüfung und einen realitätsnahen Benutzertest umfasst. Bei der ersten Komponente versetzen sich Expert(inn)en in die Situation der Nutzer(innen) und gehen allen Vorkommensvarianten der Interaktion nach. Die Konformitätsprüfung testet, ob alle Informationen und Komponenten der Benutzerschnittstelle aus den verschiedenen Perspektiven wahrnehmbar (d.h. evtl. auch mit alternativen Medien besetzt sind), bedienbar, verständlich und robust sind. Bei den Nutzertests muss dafür Sorge getragen werden, dass alle möglichen Nutzergruppen vertreten sind. Den vier genannten Prinzipien werden 12 allgemeine Guidelines zugeordnet, die sich wiederum in 61 Erfolgskriterien verzweigen (vgl. Carstens 2015: 194f.). Den Erfolgskriterien werden drei objektive Konformitätsstufen (A, AA oder AAA) zugeordnet, die eine graduelle Einstufung der erreichten Lösung ermöglichen. Weitere Richtlinien des $W 3 C$ formulieren Anforderungen an die Barrierefreiheit von Browsern und Autoren- und Entwicklungswerkzeugen (vgl. Carstens 2015: 196). 
Für die nicht-webbasierte MMI stehen die o.g. Standards für die Zugänglichkeit von Software der DIN EN ISO 9241-171 im Vordergrund, die in Deutschland 2008 als Leitlinie „Ergonomie der Mensch-System-Interaktion“ bekannt gemacht wurden. Die DIN EN ISO 9241-171 enthält insgesamt 142 Richtlinien mit Prüf-Checkliste (cf. Carstens 2015: 197f.). Wie oben gezeigt, treffen hier im Zuge des Universal Designs die Normen von Accessibility und Usability aufeinander.

Sünkler (2015) legt eine systematische Zusammenstellung von Evaluierungstools für automatisierte Accessibility-Tests bezogen auf das Web vor, die Entwickler(inne)n und Designer(inne)n Hinweise geben, wo evtl. Barrieren vorliegen. Sie umfassen Methoden aus den Bereichen von Software- und Usability-Tests. Ein Beispiel für derartige Tools, das auch für die deutsche Sprache anwendbar ist, nennt sich AChecker. Für eine Gesamtdarstellung sei auf Sünkler (2015: 236ff.) verwiesen.

\section{Prinzipien barrierefreier Benutzungsschnittstellen}

In der Forschungsliteratur finden sich viele verschiedene Ansätze, die zum einen auf bestimmte Barrieren abzielen, zum anderen Barrieren thematisieren, die individuelle Lösungsansätze erfordern. Obwohl neuere Entwicklungen wie Wearables, Smart Home, Internet der Dinge, Virtual Reality nicht an erster Stelle die Barrierefreiheit zum Ziel haben, ergeben sich durch sie vielfach Optionen, um menschliche Sinne zu ergänzen bzw. zu ersetzen, Handicaps auszugleichen oder andere Abläufe aufzuzeigen. Z.T. erweitern diese alternativen Schnittstellen auch für Menschen ohne Handicaps das Modalitätenspektrum (vgl. Weber 2015).

- Eine erste und recht vereinfachende Vorgehensweise besteht darin, die Sinne gegeneinander auszutauschen und andere Kanäle anzusprechen. D. h. im Fall von sehgeschädigten oder blinden Nutzer(inne)n wird insbesondere auf den Gehörsinn zurückgegriffen und umgekehrt. Kann ein/e Nutzer(in) z. B. die Tastatur nicht bedienen und visuellen 
Output wahrnehmen, so werden Screen Reader oder gesprochene Anweisungen eingesetzt. Bei Hörschädigungen übernehmen visuelle die Funktion akustischer Signale. Ist sowohl Sehen als auch Hören beeinträchtigt, werden haptische Sensoren auf ihre Einsatzmöglichkeit überprüft.

- Einen deutlichen Anstieg haben auch Personalisierungskonzepte zu verzeichnen, die individuelle Einstellungen z. B. bzgl. Buchstabengröße, Lautstärke, Farb- und Kontrastverwendung etc. vorsehen.

- Im Kontext der Web-Accessibility, die durch die rasche Entwicklung neuer Web-Technologien und -anwendungen gekennzeichnet ist, setzt man vor allem auf die Trennung von Inhalt und Layout und auf systematische Strukturierung und Beschreibung von inhaltlichen Medien (Text, Grafik, Audio- und Videofiles etc.), sodass verschiedene der Barrierefreiheit zuträgliche Optionen ermöglicht werden. Z. B. werden Abbildungen mit zusätzlicher textueller Information ergänzt, auf die Screen Reader zugreifen können (s. die Beiträge von Schütt und Hellbusch im vorliegenden Band). Dies geht $z$. T. einher mit weiteren Vorteilen z. B. für die Suchmaschinenoptimierung.

- Hinzu kommt der Einsatz sog. assistiver Technologien, die Menschen mit Behinderungen unterstützen können, z. B. Screen Reader, Braillezeilen, Text-to-Speech-Anwendungen, Bildschirmtastaturen mit Eyetrackern, Gaze-Technologie, Kopf- oder Fußmaus, haptisches Feedback (s. die Beiträge von Folta-Schoofs, Capovilla und Musenberg im vorliegenden Band).

\section{$5 \quad$ Beispiele}

Die Auswahl der hier vorgestellten Schnittstellen und Lösungsvorschläge kann keine Vollständigkeit anstreben, sondern versucht, einige Trends aufzuzeigen. 
Die rege Aktivität, die sich im Kontext der Barrierefreiheit beobachten lässt, zeigt, dass mit dem Thema aktiv „experimentiert“ wird.

Billah et al. (2017) hinterfragen in ihrem Beitrag die ubiquitäre Barrierefreiheit bei sehbehinderten Menschen. Sie stellen fest, dass Screen Reader noch große Beschränkungen haben, z.T. keine Vorteile bringen und hier insgesamt noch große Forschungsdefizite vorliegen.

Rozado et al. (2017) entwickelten ein Softwaresystem FaceSwitch, das bewegungseingeschränkten Personen hilft, ohne Hände effizient mit einem Computer zu interagieren. Sie kombinieren dafür Gaze-Tracking mit Mimik, wobei Gaze zum Zeigen auf interessante Objekte und unterschiedliche mimische Gesten (Lächeln, Öffnen des Mundes, Augenbrauen anheben, mit der Nase zucken) zur Funktionsauslösung eingesetzt werden. Im Vergleich mit anderen Systemen konnte eine höhere Geschwindigkeit bei der Aufgabenlösung erzielt werden.

Rodrigues et al. (2017) schlagen einen Service Hint Me! vor, der blinde Personen unterstützt, den Umgang mit einem Smartphone zu erlernen. Dabei liegt der Fokus nicht, wie bei früheren Studien, auf der Texteingabe, sondern es soll die generelle Akzeptanz eines Services in Form eines kollektiven Wissensaustauschs untersucht werden. Das zentrale Element ist ein ständig präsenter Hilfeknopf. Der/die Benutzer(in) kann nach dessen Betätigung eine Frage stellen, die zusammen mit dem Screenshot der Seite, auf der sich der/die Benutzer(in) befindet, an verschiedene Soziale Medien sowie an eine Wissensbasis weitergeleitet wird. Längeres Drücken des Knopfes gibt die Antwort wieder und kann mehrfach abgehört werden.

Ezaki et al. (2004) stellen ein System vor, das in natürlicher Umgebung (getestet mit Fotos) Text ausfindig macht, um sehbehinderte oder blinde Personen zu unterstützen. Die Autoren führen in erster Linie Beispiele von Verkehrsschildern oder Warnhinweisen (z. B. ein Stop-Schild an einer Kreuzung) an. Personen mit einem visuellen Handicap sollen sich unbegleitet im Freien besser bewegen können. Mittels einer Kamera, die auf der Schulter der sehbehinderten Person platziert wird, werden zunächst Texte in der Umgebung identifiziert und den Personen vorgelesen. Auch beim Einkaufen können Personen dadurch unterstützt werden, dass entsprechende Produktnamen oder Preise sprachlich mitgeteilt werden. Die notwendigen technologischen Grundlagen finden sich im Bereich der Texterkennung und -extraktion sowie in der Text-to-Speech Umsetzung. 


\section{$6 \quad$ Fazit}

Da immer mehr Informationsangebote und -dienste auf das Web verlagert werden und auch ein ständiges Wachstum an nicht-webbasierten Applikationen zu beobachten ist, kommt der barrierefreien MMI eine wichtige Rolle zu. Um allen Menschen die Teilhabe an Information und Wissen zu ermöglichen, ist ein barrierefreier Zugang zu gewährleisten. Aus der Usability- und UX-Forschung liegen elaborierte Methoden vor, die angewandt auf Barrierefreiheit meist angepasst werden müssen, aber die Anforderungen zwischen Accessibility und Usability weisen deutliche Überschneidungen auf und können gegenseitig von dem jeweiligen Methoden- und Erfahrungs-Know-How profitieren. Im Sinne der User Experience sollen barrierefreie Systeme jedoch über reine Effektivitätsgesichtspunkte hinausgehen. Wichtig ist, dass das Ziel der Barrierefreiheit von Beginn der Entwicklung an verfolgt wird, um Unzulänglichkeiten durch eine entsprechende Gestaltung proaktiv entgegenzuwirken. Dies könnte einer allgemeinen und selbstverständlichen Barrierefreiheit in der MMI zum Durchbruch verhelfen. 


\section{Literaturverzeichnis}

BGG (BEHINDERTENGLEICHSTELLUNGSGESETZ) (2002): Gesetz zur Gleichstellung von Menschen mit Behinderungen. Zuletzt geändert 07/2016.

Billah, Syed Masum/Ashok, Vikas/Porter, Donald E./Ramakrishnan, I.V. (2017): „Ubiquitous Accessibility for People with Visual Impairments: Are We There Yet?“. In: CHI 2017, May 6-11, 2017, Denver, CO, USA, 5862-5868.

BORLUND, PIA (2016): „A study of the use of simulated work task situations in interactive information retrieval evaluations: A meta-evaluation“. In: Journal of Documentation 72, 3, 394-413.

Buß, Anna (2009): Personas als Standardwerkzeuge des User Centered Design: Methode mit Tücken. I-com 2/2009, 58-60.

CARSTENS, ANDREAS (2015): „Grundlagen für eine barrierefreie IT in der Justiz“. In: Kerkmann, Friederike/LeWANDOWski, Dirk (Hg.): Barrierefreie Informationssysteme. Zugänglichkeit für Menschen mit Behinderung in Theorie und Praxis. Berlin/Boston: De Gruyter Saur, 177-216.

Cato, John (2001): User-centered Web Design. London et al.: Addison-Wesley.

DIN EN ISO 9241-110 (2008): Ergonomie der Mensch-System-Interaktion - Teil 110: Grundsätze der Dialoggestaltung (ISO 9241-110:2006); Deutsche Fassung EN ISO 9241-110:2006. Ausgabe 2008-09. Berlin.

Ezaki, Nobuo/Bulacu, Marius/Schomaker, Lambert (2004): Text Detection from Natural Scene Images: Towards a System for Visually Ipaired Persons. Proc. Of the 17th Int. Conf. On Pattern Recognition (ICPR 2004), IEEE Computer Society. Cambridge, UK, Bd. 2: 23. August-26. August 2004, 683-686.

FASEL, BeAT/Luettin, Juergen (2003): „Automatic facial expression analysis: a survey“. In: Pattern Recognition 36, 1, 259-275.

Fuchs, Christian/Obrist, MARIANna (2010): „HCI and Society: Towards a Typology of Universal Design Principles“. In: International Journal of Human-Computer Interaction 26, 6, 638-656.

GÖRTZ, MATTHIAS (2011): „Social Software as a Source of Information in the Workplace. Modeling Information Seeking Behaviour of Young Professionals in Management Consulting“. In: Schriften zur Informationswissenschaft 59. Glücksstadt: Hülsbusch.

Hassenzahl, Marc/Burmester, Michael/Koller, Franz (2003): „AttrakDiff. Ein Fragebogen zur Messung wahrgenommener hedonischer und pragmatischer Qualität“. In: ZIEGLER, JÜRGEN/SzWILliUs GERD (Hg.): Mensch und Computer 2003. Interaktion in Bewegung. Stuttgart/Leipzig: B.G. Teubner, 187-196.

Hassenzahl, Marc/Tractinsky, NoAm (2006): „User Experience - a Research Agenda“. In. Behaviour \& Information Technology 25, 2, 91-97.

HeINECKe, ANDREAS M. (2012): Mensch-Computer-Interaktion. Basiswissen für Entwickler und Gestalter. 2. Auflage. Berlin/Heidelberg et al.: Springer. 
IRLE, GABRIELE (2017): „Gefühlserleben bei der Informationssuche im Internet. Eine qualitative Studie zur Individualität und Alltäglichkeit der Sucherfahrung“. In: Schriften zur Informationswissenschaft 72. Glückstadt: Hülsbusch.

LindgaArd, Gitte/Fernandes, Gary/DudeK, Cathy/Brown, J. (2006): „Attention web designers: You have 50 milliseconds to make a good first impression!“. In: Journal of Behaviour \& Information Technology 25, 2, 115-126.

Moshagen, Morten/Thielsch, Meinald (2013): „A short version of the visual aesthetics of websites inventory“. In: Journal of Behaviour \& Information Technology 32, 12.

Preece, Jenny/Sharp, Helen/Rogers, Yvonne (2015): Interaction Design: Beyond Human-Computer Interaction. US: Wiley.

Rodrigues, André/Montague, Kyle/Nicolau, Hugo/Guerreiro, Joao/ Guerreiro, Tiago (2017): In-context Q\&A to Support Blind People Using Smartphones. ASSETS’17, Baltimore, MD, USA, 29. Oktober-1. November 2017.

Rozado, David/NiU, Jason/Lochner, MARTin (2017): „Fast Human-Computer Interaction by Combining Gaze Pointing and Face Gestures“. In: ACM Transactions on Accessible Computing 10, 3 Artikel 10.

SAROdNick, Florian/Brau, Henning (2010): Methoden der Usability Evaluation: Wissenschaftliche Grundlagen und praktische Anwendung. Bern: Hans Huber.

SÜNKLER, SEBASTIAN (2015): „Evaluierungstools für automatisierte Accessibility-Tests“. In: KERKMANN, FrIEDERIKE/LEWANDOWSKI, DiRK (Hg.): Barrierefreie Informationssysteme. Zugänglichkeit für Menschen mit Behinderung in Theorie und Praxis. Berlin/Boston: De Gruyter Saur, 221-261.

Web Content Accessibility Guidelines (WCAG) 2.0 (2008). W3C Recommendation 11 December 2008. URL: https://www.w3.org/TR/2008/REC-WCAG20-20081211/ (letzter Zugriff: 26.04.2018).

WEBER, KARSTEN (2015): „Alternative Benutzerschnittstellen als Möglichkeit der Kompensation sensorischer Handicaps“. In: KERKMANn, FrIEDERIKE/LEWANDOWSKI, DIRK (Hg.): Barrierefreie Informationssysteme. Zugänglichkeit für Menschen mit Behinderung in Theorie und Praxis. Berlin/Boston: De Gruyter Saur, 49-70. 


\section{Alternativtexte als wesentliches Gestaltungselement zugänglicher (barrierefreier) Bildungsprozesse}

\section{Ausgangslage: Zur allgemeinen Bedeutung von Bildmaterial beim Lernen und Lehren in Schule und Hochschule (sowie resultierende Konsequenzen für einzelne Lernerinnen und Lerner)}

Bildergeschichten im Fremdsprachenunterricht, Karikaturen im Geschichtsunterricht oder Balkendiagramme in der Vorlesung - beim Lernen und Lehren im Schul- oder Hochschulbereich kommt täglich eine Vielzahl von Bildern zum Einsatz, welche die Lehr- und Lernprozesse gezielt unterstützen sollen.

Die besondere Funktion von Bildern für den Lehr- und Lernprozess ist bereits länger bekannt. Historisch betrachtet werden bereits seit dem 17. Jahrhundert bewusst Bilder eingesetzt, um bspw. komplexe Sachverhalte zu illustrieren oder mnemonische Denkprozesse zu fördern (Reinmann 2015: 46). Auch die rasche Auf- bzw. Entnahme essentieller Informationen kann durch den Einsatz von übersichtlichen Darstellungen unterstützt werden. Um kognitive Denkprozesse anzuregen, dominiert die Kombination von Bild- und Textmaterial im Bildungsbereich (z. B. Lehrervortrag mit Präsentation, Text- und Bildmaterial in klassischen Lehrwerken oder Sachbüchern ...). „Neben diesen kognitiven Funktionen können Bilder auch Interesse wecken und zum Lernen motivieren oder dekorative Funktion haben und damit die emotionale Seite des Lernens berühren“ (Reinmann 2015: 46). So zielen spezifische Unterrichts- und Lehrmethoden wie der Bildimpuls auf die unmittelbare Wirkung von Bildern auf die Lernerinnen und Lerner ab. Zweifellos ist die Vermittlung von Bildungsinhalten ohne den Einsatz von Bildern - gerade auch im Zeitalter der Digitalisierung kaum noch denkbar. 
Allerdings profitieren längst nicht alle Lernerinnen und Lerner vom besonderen Potential bildhafter Darstellungen. Wenn Bildergeschichten oder Karikaturen ohne verbale oder schriftsprachliche Beschreibung zum Einsatz kommen, wird Lernenden mit Beeinträchtigung des Sehens der Zugang zum Bildungsinhalt verwehrt. Doch nicht nur bei sehbeeinträchtigten Lernerinnen und Lernern kann die Bildungsteilhabe eingeschränkt sein. Auch Lernende mit Lernbeeinträchtigung und Lernende mit Autismus-Spektrum-Störungen sind ggf. auf weiterführende Anmerkungen angewiesen, um die Karikatur im Unterrichtsgeschehen verorten zu können. Der ausschließliche Einsatz von bildhaften Darstellungen kann im Bildungsbereich zu maßgeblichen Ausschlussmechanismen führen, so dass die gleichberechtigte Bildungsteilhabe nicht gegeben ist.

\section{$2 \quad$ Kurzdefinition „Alternativtexte“ sowie Begründungslinien für die Umsetzung von Alternativtexten im Bildungsbereich}

Um die Bildungsteilhabe aller Lernerinnen und Lerner sicherzustellen, muss die bestmögliche Zugänglichkeit zum Bildmaterial gegeben sein. Diesbezüglich sind Alternativtexte geeignet, um die Bildinformationen in einem veränderten bzw. optionalen Format bereit zu stellen.

Alternativtexte bieten eine "textuelle Alternative zu Nicht-Text-Inhalten“ (Barrierefreies Webdesign 2018). Alternativtexte oder Textalternativen sind „Texte, die anstelle der Bilder benutzt werden und entsprechende Informationen vermitteln sollen“ (BITV-Lotse 2018). Unter Verwendung assistiver Technologien (Screenreader, Braillezeilen) wird die Zugänglichkeit zum Bildmaterial hergestellt, in dem der Alternativtext mittels Screenreader vorgelesen oder via Braillezeile ausgegeben wird.

Die Verwendung von Alternativtexten begründet sich einerseits in der menschenrechtlichen Vereinbarung der UN-Konvention über die Rechte von Menschen mit Behinderungen und andererseits erfüllt der Einsatz von Alternativtexten die vielfältige Herangehensweise an den Lerngegenstand im Sinne des universellen Designs. 
2.1 Begründungslinie I: UN-Konvention über die Rechte von Menschen mit Behinderungen

Mit der Unterzeichnung der UN-Konvention über die Rechte von Menschen mit Behinderungen (UN-BRK) hat sich Deutschland dazu verpflichtet, dass für Menschen mit Behinderung eine chancengleiche, diskriminierungsfreie und gleichberechtigte Teilhabe an Bildung ermöglicht wird (UN 2006/2008: Art. 24). Damit wird an das grundlegende Recht aller Menschen auf Bildung angeknüpft, welches in der Allgemeinen Erklärung der Menschenrechte verankert ist („Jeder hat das Recht auf Bildung", UN 1948: Art. 26). In der UN-BRK, wie auch in der Allgemeinen Erklärung der Menschenrechte (AEMR), haben sich die Unterzeichnerstaaten verpflichtet, dass der diskriminierungsfreie Zugang zu Bildungsangeboten für alle gegeben ist. In der UN-BRK werden nun die allgemeinen Menschenrechte auf die Personengruppe der Menschen mit Behinderung hin fokussiert und konkretisiert.

Um die gleichberechtigte Bildungsteilhabe zu erreichen, sind gemäß UNBRK geeignete Maßnahmen („angemessene Vorkehrungen“) zu ergreifen. Im Artikel 2 der UN-BRK sind ,angemessene Vorkehrungen“ definiert als „notwendige und geeignete Änderungen und Anpassungen, (...) die, wenn sie in einem bestimmten Fall erforderlich sind, vorgenommen werden, um zu gewährleisten, dass Menschen mit Behinderungen gleichberechtigt mit anderen alle Menschenrechte und Grundfreiheiten genießen oder ausüben können“ (UN 2006/2008: Art. 2). Wenn Bildmaterial ohne adäquate Alternative im Schul- und Hochschulbereich eingesetzt wird, kommt es zum Ausschluss einzelner Personengruppen. Die Bilder stellen eine Barriere beim Zugang zu Informationen dar. In diesem Zusammenhang sind Alternativtexte als „angemessene Vorkehrungen“"zu definieren (Degenhardt 2016a). Mit Hilfe der Alternativtexte wird sichergestellt, dass die visuellen Informationen verfügbar werden. Werden keine „angemessenen Vorkehrungen“ (hier: Alternativtexte) vorgehalten, ist die gleichberechtigte Bildungsteilhabe von einzelnen Personengruppen, z. B. von Menschen mit Sehbeeinträchtigung, gefährdet. In Anknüpfung an das normative Verständnis der UN-BRK führen die nicht vorhandenen Bildinformationen zur Benachteiligung einzelner Personengruppen im Bildungsprozess („Diskriminierung aufgrund von Behinderung"), was für das gemeinsame (inklusive) Lernen nicht förderlich und damit zu vermeiden ist (UN 2006/2008: Art. 2, Art. 24). 
Ergänzend ist auf den Marrakesch-Vertrag zu verweisen (WIPO 2013), welcher am 30.09.2016 in Kraft trat (DBSV 2018a). Das völkerrechtliche Übereinkommen legt Regelungen vor, welche die Herstellung sowie den (inter-) nationalen Austausch von barrierefreier Literatur erleichtern sollen. Wesentliche Zielstellung ist es hierbei, dass der barrierefreie Zugang zu Literatur für Menschen mit Seh- und Lesebeeinträchtigung verbessert wird. Deutschland setzt sich aktuell mit der Ratifizierung des Marrakesch-Vertrags auseinander. Das Bundesministerium für Justiz und Verbraucherschutz hat einen ersten Gesetzesentwurf vorgelegt (DBSV 2018b). Die erfolgreiche Umsetzung des Marrakesch-Vertrags kann einen wesentlichen Beitrag zur Realisierung grundlegender Forderungen der UN-BRK leisten (z. B. Art. 9: Zugang zu Informationen, Art. 24 Teilhabe an Bildung ...).

\subsection{Begründungslinie II: Universal Design for Learning}

Aber nicht nur um die Teilhabe für Menschen mit Sehbeeinträchtigung zu bewirken, sondern auch um die heterogenen Bedürfnisse von Gruppen von Lernerinnen und Lernern zu berücksichtigen, erfüllt der Alternativtext eine wichtige Funktion. Im Artikel 2 der UN-BRK wird gleichzeitig auf die Bedeutung des Universal Designs für die Gestaltung zukünftiger (Bildungs-)Prozesse näher eingegangen. Im Sinne der UN-BRK „bedeutet , universelles Design“ ein Design von Produkten, Umfeldern, Programmen und Dienstleistungen in der Weise, dass sie von allen Menschen möglichst weitgehend ohne eine Anpassung oder ein spezielles Design genutzt werden können. ,Universelles Design' schließt Hilfsmittel für bestimmte Gruppen von Menschen mit Behinderungen, soweit sie benötigt werden, nicht aus" (UN 2006/2008: Art. 2). Wie oben ausgeführt stellt der ausschließliche Einsatz von Bildern keine universelle Lösung dar. Vielmehr sind Adaptionen für einzelne Nutzerinnen und Nutzer notwendig, wobei sich die Kombination aus Bild und Alternativtext der Idee des Universal Designs annähert.

Gemäß dem Konzept des Universal Design for Learning, welches den Transfer der allgemeinen Prinzipien des universellen Designs auf die Gestaltung von Lernarrangements vornimmt, sind Lerninhalte stets so zu konzipieren, dass die Bedürfnisse aller Lernenden bestmöglich berücksichtigt werden (Schlüter/ 
Melle/Wember 2016: 274ff.). Das Universal Design for Learning beruft sich sowohl auf lerntheoretische und entwicklungspsychologische Grundlagen als auch neurowissenschaftliche Erkenntnisse (Hall/Meyer/Rose 2012: 5; Rapp 2014: 15; UDL 2018a). Insbesondere Anregungen aus den Arbeiten von Vygotsky zur Zone der nächsten Entwicklung („Scaffolding“) und entwicklungspsychologische Überzeugungen von Piaget haben die Konzeptentwicklung beeinflusst (Piaget 1988; Rapp 2014: 267, 313, 318; Vygotsky 1978). Neurowissenschaftlich gründet sich das Konzept u. a. auf wesentliche Erkenntnisse von Gazzangia, Ivry und Mangun (Gazzangia/Ivry/Mangun 1998). In enger Anknüpfung an diese und weitere wissenschaftstheoretische Befunde stehen die Betrachtungen zu den „interindividuellen Unterschieden“ beim Lernen im Mittelpunkt des UDLKonzepts (Schlüter/Melle/Wember 2016: 274). Das UDL-Konzept knüpft an die grundlegende Annahme an, dass die Lernaktivitäten jedes Menschen in drei Netzwerken organisiert sind (Recognition Network: „Was“ des Lernens, Strategic Network: „Wie“ des Lernens und Affective Network: „Warum“ des Lernens) (Hall/Meyer/Rose 2015: 2f.; Rapp 2014: 15ff.; UDL 2018b). Jedoch differieren die Netzwerke in ihrer spezifischen Ausprägung von Individuum zu Individuum. Um den individuellen Bedürfnissen der Lernenden zu entsprechen, sind Lernangebote möglichst vielfältig zu gestalten. Insgesamt stehen drei Prinzipien mit weiterführenden Anhaltspunkten (s. g. Checkpoints) für die gezielte Aufbereitung der Lernangebote zur Verfügung (Prinzip 1: Biete multiple Mittel der Repräsentation von Information, Prinzip 2: Biete multiple Mittel der Verarbeitung von Informationen und der Darstellung von Lernergebnissen, Prinzip 3: Biete multiple Möglichkeiten der Förderung von Lernengagement und Lernmotivation) (Schlüter/Melle/Wember 2016: 275). Anknüpfend an Prinzip 1 kann der grundlegende Bedarf an Alternativtexten einzelner Schülerinnen und Schüler als Hilfsmittel für alle Lernerinnen und Lerner aufgefasst werden. Nach Meier präferiert jeder Mensch beim Lernen einen anderen Sinn. Während einige Menschen am besten über das Hören neue Informationen erfassen, lernen andere Menschen wiederum besonders gut über das Beobachten oder aktive Anfassen und Berühren. Demnach existieren drei verschiedene Lerntypen: visueller, auditiver und haptischer Lerntyp (Meier 2006: 97f.). Da vorwiegend visuelle Lerntypen vom Bildeinsatz profitieren, sind für die anderen Lerntypen 
entsprechende Alternativen wünschenswert. In diesem Fall ermöglicht der Einsatz von Alternativtexten, dass Lernende neben der bildhaften Umsetzung auch Zugang zu einer verbalen oder schriftsprachlichen Beschreibung haben, welche mittels Sprachausgabe auditiv genutzt werden könnte. Nicht nur Schülerinnen und Schüler mit Sehbeeinträchtigung, sondern auch Lernende, die vornehmlich auditiv lernen, können so in ihrem individuellen Lernprozess unterstützt werden.

\section{Basisinformationen zur Gestaltung von Alternativtexten}

Alternativtexte (bzw. verbale Beschreibungen) zählen zu den wesentlichen Gestaltungsmitteln, um die Zugänglichkeit von Bildungsangeboten zu erhöhen. Im Rahmen des bildungspolitischen Bemühens, eine inklusive Schule in Deutschland zu etablieren, hat der Bedarf an vielfältig nutzbaren Lernmaterialien, welche der heterogenen Lernerschaft besser gerecht werden, zugenommen. Daran anknüpfend sind Alternativtexte ein angemessenes Hilfsmittel, um visuelle Inhalte in anderer Form darzulegen. Gegenwärtig findet die Verwendung von Alternativtexten jedoch nur exemplarisch, z. B. für Schülerinnen und Schüler mit Sehbeeinträchtigung, statt. Dies ist u. a. auf den Umstand zurückzuführen, dass das Bereitstellen von geeigneten Alternativtexten keineswegs einfach ist. Wesentliche Zielstellung beim Erstellen von Alternativtexten ist es, dass mit Hilfe des Alternativtextes die grundlegenden Bildinformationen erfasst werden können.

Im Folgenden werden wesentliche Kriterien vorgestellt, die bei der Anfertigung von Alternativtexten zu beachten sind. Dabei orientiert sich die Zusammenstellung an gesetzlichen Vorgaben (BITV 2.0 2011) sowie nationalen und internationalen Empfehlungen aus dem Bildungsbereich (Blista 2012; DIAGRAM Center 2018). 
3.1 Grundsätzliche Dilemmata bei der Gestaltung von Alternativtexten

Die Gestaltung von Alternativtexten ist keinesfalls unproblematisch, was sich auf zwei grundsätzliche Dilemmata zurückführen lässt.

\section{Kein Alternativtext ohne Interpretation!}

Die Verschriftlichung bzw. Verbalisierung der Bildinhalte sollte möglichst objektiv stattfinden. Jedoch hängt der Alternativtext von der Wahrnehmung des Ausgangsproduktes (Bildmaterials) ab (Kitza 2015: 16). Somit gehen mit der Wortwahl und dem Sprachgebrauch bestimmte Assoziationen beim Rezipienten/bei der Rezipientin des Alternativtexts einher, so dass der Alternativtext ohne jegliche Form der Interpretation durch Produzent(in) bzw. Rezipient(in) nicht umsetzbar ist.

Der Alternativtext ist die verbale bzw. schriftsprachliche Umsetzung der Bildwahrnehmung. Die Bildwahrnehmung wird durch Faktoren wie z. B. Vorwissen, Fachvokabular und Alter geprägt. Ähnlich verhält es sich mit der Aufnahme der wesentlichen Bildinformationen aus dem Alternativtext.

\section{Es gibt nicht "den" Alternativtext!}

Die genaue Ausführung des Alternativtexts ist von verschiedenen Faktoren (Kontext, Zielgruppe, Einsatzbereich...) abhängig, so dass eine individuelle Abstimmung auf die Bedürfnisse der Zielgruppe notwendig sein kann. In Abhängigkeit vom eigentlichen Nutzerkreis kann es bspw. zweckmäßig sein, dass differierende Alternativtexte zu einem Bild zur Verfügung gestellt werden.

\subsection{Empfehlungen zur Gestaltung von Alternativtexten}

In Anknüpfung an die bestehende Forschungslage sowie die Empfehlungen aus dem nationalen und internationalen Raum zur Gestaltung von Alternativtexten werden in der Literatur die folgenden Kriterien als handlungsleitend charakterisiert (Blista 2012; Degenhardt 2016a; Degenhardt 2016b; NCAM/DIAGRAM Center 2015). 
„Um den Alternativtext zu erstellen, müssen:

- $\quad$ der Typ (Foto, Gemälde, Diagramm, Cartoon, Organigramm, mind map, concept map...),

- die Struktur \& Leserichtung,

- die Funktion,

- die Details und

- die Daten der Abbildung

ermittelt werden.

Diese Informationen werden

- (möglichst) objektiv/wertfrei,

- verständlich,

- effektiv,

- $\quad$ kontextgebunden vollständig

sprachlich in einer für den Leserinnen- und Leserkreis passigen Version (Fließtext, Liste...) dargestellt“ (Degenhardt 2016a; 2016b).

Nachfolgend werden die Gestaltungsempfehlungen ausführlich erläutert (Blista 2012; Degenhardt 2016a; Degenhardt 2016b; NCAM/DIAGRAM Center 2015).

Typ: Welcher Bildtyp liegt vor? Ist es ein Foto, Gemälde, Cartoon, Diagramm oder Organigramm? Da mit dem konkreten Bildtyp fachspezifische Informationen verknüpft sind, ist die Einordnung des Bildes (Typisierung) empfehlenswert (bspw. im ersten Satz des Alternativtextes...). Dieses Vorgehen klärt den Nutzerinnenkreis frühzeitig über die zu erwartenden Informationen in der Beschreibung auf. So suggeriert der Bildtyp Balkendiagramm der Nutzerin bzw. dem Nutzer des Alternativtextes, dass empirische Daten zu erwarten sind.

Struktur \& Leserichtung von Bildern: An welche Struktur und Leserichtung des Bildes sollte der Alternativtext anknüpfen? Im Unterschied zum Textmaterial, welches von links nach rechts gelesen wird, ist die Leserichtung von Bildern nicht automatisch vorgegeben. Um die Bildinformationen möglichst logisch und strukturiert an die Nutzerin bzw. den Nutzer mit dem Alternativtext zu 
übermitteln, müssen Struktur und Leserichtung vom Ersteller/von der Erstellerin des Alternativtexts definiert werden.

Im Namen des amerikanischen Bildungsministeriums hat das DIAGRAM Center Empfehlungen für die Gestaltung von zugänglichen (barrierefreien) Lernmaterialien veröffentlicht (NCAM/DIAGRAM Center 2015; DIAGRAM Center 2018). Dazu zählen u.a. Empfehlungen zur Gestaltung von Alternativtexten. Gemäß dem DIAGRAM Center ist es ratsam, dass die Beschreibung vom Allgemeinen zum Spezifischen erfolgt. Im Einzelfall kann es jedoch auch vorteilhaft sein, wenn ein spezifisches Detail als wesentlicher Ausgangspunkt der Beschreibung verwendet wird, beispielsweise wenn es sich hierbei um das zentrale Motiv des Bildes handelt.

Funktion: Welche Funktion soll das Bild erfüllen? Zweifellos muss sich die Gestaltung des Alternativtexts an der Bildfunktion orientieren. Um die Funktion des zu beschreibenden Bildes zu definieren, sollte das zugeordnete Textmaterial analysiert werden. Wenn eine bestimmte Atmosphäre bzw. Stimmung mit dem Bild vermittelt werden soll, kann die Nennung von sorgfältig ausgewählten Adjektiven zweckmäßig sein. Bei einem Bild mit illustrativer Funktion kann ein Alternativtext mit wenigen Worten ausreichend sein, da im dazugehörigen Textmaterial ausführliche Erläuterungen abgegeben werden.

Details der Abbildung: Es müssen die wesentlichen Details des Bildes erschlossen werden, um entsprechende Bezüge in der Bildbeschreibung vornehmen zu können.

Daten der Abbildung: Die Daten, die mit dem Bild vermittelt werden sollen, sind zu erfassen und entsprechend in den Alternativtext zu integrieren.

(Möglichst) objektiv/wertfrei: Zu jeder Abbildung sind unterschiedlichste Ausführungen des Alternativtextes möglich. In Abhängigkeit von der Wortwahl verbindet der Nutzer bzw. die Nutzerin unterschiedliche Assoziationen mit der Abbildung (Kitza 2015: 45). Daran anknüpfend entwickelt sich die Bildvorstellung bei der Nutzerin bzw. dem Nutzer, „die von der Wahrnehmung und der 
Auswahl einer sprachlichen Formulierung der Erstellerin oder des Erstellers abhängig ist“ (Kitza 2015: 45). „[...] [D]a Wahrnehmung subjektiv ist und dabei nicht den Kriterien richtig oder falsch zugeordnet werden kann, sollte die Beschreibung einer Grafik oder eines Bildes nach Gombrich den Empfängerinnen und Empfängern keine Interpretation aufoktroyieren, sondern das Spektrum der Interpretationsmöglichkeiten erhalten“ (Gombrich 1984: 200; Kitza 2015: 45). Vorrangig sollte sich die Beschreibung darauf konzentrieren, was tatsächlich dargestellt ist. Interpretationen sind bestmöglich $\mathrm{zu}$ vermeiden (NCAM/DIAGRAM Center 2015: 11). Vielmehr sollte der Leserin bzw. dem Leser mit dem Alternativtext die Chance zur eigenen Bildinterpretation eröffnet werden.

Verständlich: Der Alternativtext sollte möglichst so formuliert sein, dass die eigenständige Auseinandersetzung mit dem Textmaterial gegeben ist. Beispielsweise sollten keine neuen Begrifflichkeiten oder Konzepte in den Alternativtext eingehen.

Effektiv: Beim Erstellen des Alternativtextes ist darauf zu achten, dass man sich auf die Wiedergabe der wesentlichen Informationen beschränkt. Unbedingt ist ein kognitiver Overload, d. h. eine Blockade des Arbeitsgedächtnisses aufgrund irrelevanter Informationen und damit unnötiger Verarbeitungsvorgänge, zu verhindern (Sweller 2005; Schütt 2015: 94). Gerade in Lehrbüchern sind häufig ergänzende Beschreibungen zum Bild in den weiterführenden Ausführungen vorhanden. Diese Ausführungen sollten bestmöglich einbezogen werden, um unzweckmäßige Wiederholungen zu vermeiden (NCAM/DIAGRAM Center 2015: 10).

Kontextgebunden vollständig: Die endgültige Ausführung des Alternativtexts ist vom Kontext, in welchem das Bild zum Einsatz kommt, abhängig.

Für den Leser_innenkreis passige Version (z. B. Fließtext, Liste): Die anschaulichen Beispiele des DIAGRAM Centers zeigen, dass die konkrete Ausgestaltung des Alternativtexts von der Zielgruppe abhängig ist (Alter, kultureller Hintergrund, Fachwissen, Fähigkeiten im Umgang mit assistiven Technologien, z. B. 
Brailledisplay...) (NCAM/DIAGRAM Center 2015: 9). Demzufolge muss sich der Alternativtext an den vorhandenen Fähigkeiten der Lernerinnen und Lerner orientieren (vgl. hierzu Abb. 1: Beispiel Baumdiagramm für Lernerinnen und Lerner im Primar- bzw. Sekundarbereich). Dies bedeutet u.a., dass der Alternativtext sprachlich angemessen zu formulieren ist. (Fach-)Vokabular, Satzbau und Wortwahl sollte an den Fähigkeiten der Nutzerinnen und Nutzer orientiert sein. Auch ist zu beachten, ob die potentiellen Leserinnen und Leser die strukturierende „Funktion“ von Aufzählungszeichen an einem Brailledisplay erkennen und nutzen können. Wie das Beispiel zeigt, werden Aufzählungszeichen erst in der Variante für die fortgeschrittenen Lernerinnen und Lerner im Sekundarbereich eingesetzt. Die Aufzählungszeichen bieten den Vorteil, dass die Bildinformationen in knappen Stichworten zur Verfügung gestellt werden können. Dahingegen erhalten die Lernerinnen und Lerner im Primarbereich die Bildbeschreibung als kurzen Text, um eine mögliche Überforderung auszuschließen.

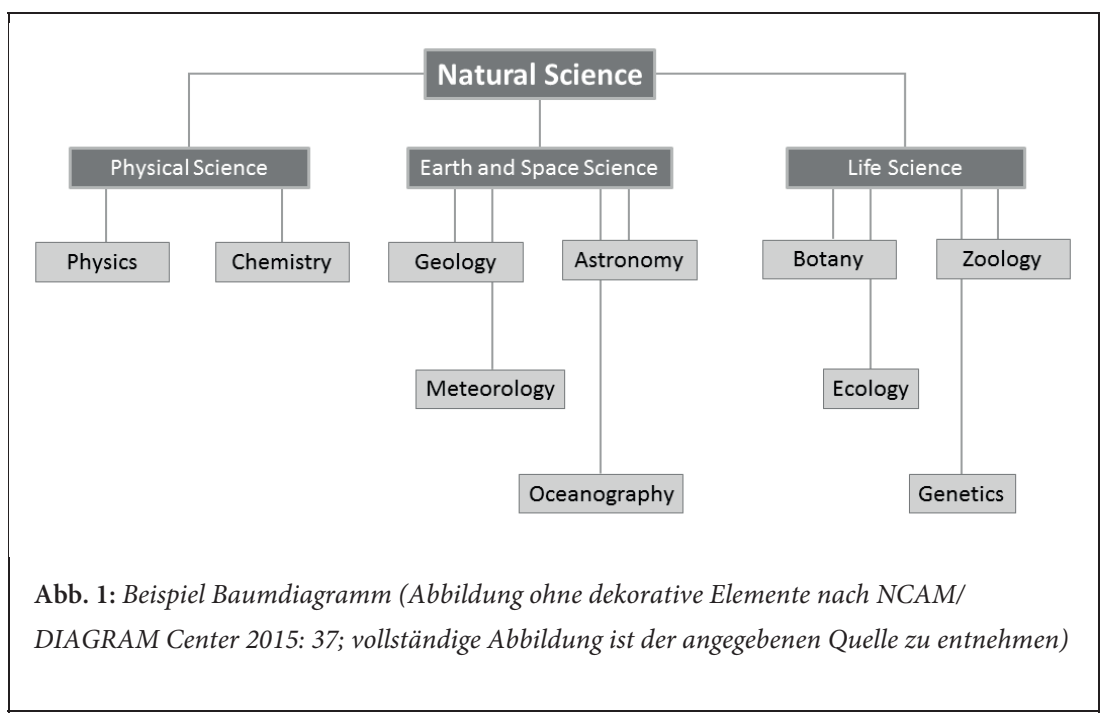

(C) Frank \& Timme Verlag für wissenschaftliche Literatur 


\begin{tabular}{|c|c|}
\hline $\begin{array}{l}\text { Alternativtext für Lernerinnen und } \\
\text { Lerner im Primarbereich: } \\
\text { "A tree diagram shows different bran- } \\
\text { ches of science. There are three levels, } \\
\text { from top to bottom. The highest level is } \\
\text { Natural Science. The next level has Phy- } \\
\text { sical Science, Earth and Space Science, } \\
\text { and Life Science. Physical Science in- } \\
\text { cludes Physics and Chemistry. Earth } \\
\text { and Space Science includes Geology, } \\
\text { Meteorology, Astronomy, and Ocea- } \\
\text { nography. Life Science includes Botany, } \\
\text { Ecology, Zoology, and Genetics" } \\
\text { (NCAM/DIAGRAM Center 2015: 37f.). }\end{array}$ & $\begin{array}{l}\text { Alternativtext für Lernerinnen und Ler- } \\
\text { ner höherer Klassenstufen (Sekundarbe- } \\
\text { reich): } \\
\text { "A tree diagram shows different branches } \\
\text { of science. There are three levels, from top } \\
\text { to bottom. The highest level is Natural Sci- } \\
\text { ence. The next level has Physical Science, } \\
\text { Earth and Space Science, and Life Science. } \\
\text { Physical Science } \\
\text { - Physics } \\
\text { - Chemistry } \\
\text { Earth and Space Science } \\
\text { - Geology } \\
\text { - Meteorology } \\
\text { - Astronomy } \\
\text { Life Science } \\
\text { - Botany } \\
\text { - Ecology } \\
\text { Genegraphy } \\
\text { Genetics" (NCAM/DIAGRAM Center }\end{array}$ \\
\hline
\end{tabular}

In der Handreichung des DIAGRAM Centers sind Empfehlungen zur sprachlichen Umsetzung von Alternativtexten ergänzt (NCAM/DIAGRAM Center 2015: 13). Demzufolge sollen vorrangig aktive Verben in der Präsensform für den Alternativtext verwendet werden (inkl. geläufiger Regeln der Grammatik und Interpunktion). Das DIAGRAM Center räumt ein, dass im Rahmen der Textreduktion Einschübe vorgenommen werden können. Um der Nutzerin bzw. dem Nutzer das Erfassen des Alternativtexts zu erleichtern, sollte die Orientierung am umgebenden Text stattfinden. Dies vereinfacht das Erfassen der Informationen im Alternativtext. Zahlreiche Anwendungsbeispiele sind den nationalen und internationalen Handreichungen $\mathrm{zu}$ entnehmen (Blista 2012; NCAM/DIAGRAM Center 2015). 
Der Anspruch, Bildungsangebote für eine heterogene Lernerschaft und damit u. a. Alternativtexte zur Verfügung zu stellen, ist vergleichsweise jung. Erste Erkenntnisse zur lernerspezifischen Ausgestaltung von Alternativtexten liegen aus nationalen und internationalen Forschungsprojekten vor (Blista 2012; DIAGRAM Center 2018). Ebenso verdeutlichen die Kriterien zur Erstellung von Alternativtexten, dass erstes Expertenwissen vorhanden ist. Jedoch sind Forschungsdefizite auszumachen (Degenhardt 2016a). Um in den kommenden Jahren weiterführende Erkenntnisse zur Gestaltung von Alternativtexten zu erlangen, sind gezielte Forschungsaktivitäten vorzunehmen.

Forschungsdesiderat I: Detaillierte Informationen zur Gestaltung von Alternativtexten im Bildungsbereich

Diesbezüglich sind auch Erkenntnisse zur Gestaltung von Alternativtexten im Bildungsbereich noch ausstehend.

Um den grundlegenden Zugang der Schülerinnen und Schüler - insbesondere der Schülerschaft mit Beeinträchtigung des Sehens - zu den Bildinformationen sicherzustellen, sind Alternativtexte ein mögliches Gestaltungsmittel. Doch sind Alternativbeschreibungen immer eine adäquate Alternative? Inwieweit lässt sich tatsächlich sagen, dass Alternativtexte eine „angemessene Vorkehrung" sind? Ohne Zweifel sind einige Umsetzungen nicht bzw. kaum möglich (z. B. das Auslösen von Emotionen bei der Bildbetrachtung).

Die Erhöhung des schriftsprachlichen (verbalen) Inputs verändert die Rezeption der Bildungsinhalte. Bislang liegen nur vereinzelt Erkenntnisse vor, welche maximale Länge für Alternativbeschreibungen für eine bestimmte Altersgruppe gegeben sein sollte. Außerdem muss geklärt werden, inwieweit dekorative Elemente in die Betrachtung mit eingehen. Sowohl den WCAG und der BITV 2.0 ist zu entnehmen, dass dekorative Elemente (bspw. ein wiederkehrendes Logo auf der PowerPoint-Präsentation) nicht zu beschreiben sind (BITV 2.0 2011; WCAG 2018). Das Auslassen der Beschreibung von dekorativen Elementen wird damit begründet, dass keine wesentlichen Informationen weitergegeben werden. Diesbezüglich stellt sich die Frage, wer auf welcher Grundlage 
über Sinnhaftigkeit bzw. Nicht-Sinnhaftigkeit entscheiden soll. Qualitative Befragungen von Nutzerinnen und Nutzern von Alternativtexten haben gezeigt, dass der Informationswunsch differiert, d. h. einige Nutzerinnen und Nutzer sind an der Beschreibung von dekorativen Elementen interessiert (Kitza 2015: 158).

Forschungsdesiderat II: Erkenntnisse zum Nutzen für alle Lernerinnen und Lerner Im Rahmen des gesetzlichen Nachteilsausgleichs für Schülerinnen und Schüler mit Sehbeeinträchtigung und für Schülerinnen und Schüler mit körperlicher Behinderung ist die Möglichkeit gegeben, dass digitale Schulbücher im Unterricht genutzt werden (siehe z. B. BSB Hamburg 2013). Um die bestmögliche Zugänglichkeit der Schulbücher zu gewährleisten, sind die digitalen Schulbuchversionen für blinde und sehbehinderte Schülerinnen und Schüler mit Alternativtexten ausgestattet. Um den tatsächlichen Mehrwert von Alternativtexten gemäß dem Ansatz des Universal Design for Learning in Erfahrung zu bringen, sind entsprechende Forschungsprojekte in der inklusiven Schule zu realisieren und alle potentiellen Nutzerinnen und Nutzer (Schülerinnen und Schüler mit Lernbehinderung, mit Sprachbehinderung oder DaZ ...) in den Erkenntnisprozess einzubeziehen. Diesbezüglich gilt es auch, dass die formalen Regelungen, wie z. B. die Urheberrechte der Schulbuchverlage, näher betrachtet und ggf. Lösungen erarbeitet werden.

\section{Forschungsdesiderat III: Alternativen zu schriftlichen Umsetzungen der Bildinformationen}

Im Unterschied zum „schnellen“ Blick auf das Bild ist das Erfassen des Alternativtexts oftmals mit einem hohen kognitiven und zeitlichen Aufwand verbunden. Im Schulalltag erhalten Lernende mit Sehbeeinträchtigung zahlreiche verbale Beschreibungen, damit der Zugang zum Lernangebot gesichert ist. Gerade für Lernerinnen und Lerner mit Beeinträchtigung des Sehens besteht daher das Risiko, dass ein „verbaler“ Overload stattfindet (Blista 2012: 5). Es sollte stets geprüft werden, inwieweit verbale Beschreibungen auch durch den Einsatz von taktilen oder nicht ausschließlich sprachlich dominierten akustischen Lernmaterialien ersetzt werden können, um weitere Zugangsmöglichkeiten zu schaffen. Die Option, das Bild resp. den Alternativtext als Audiodatei (Sound, 
Geräusch, Hörspiel, ...) abzuspielen, kann den Aneignungsprozess gezielt unterstützen. Der Einsatz von taktilen Abbildungen kann für vollsichtige Lernerinnen und Lerner durchaus hilfreich sein. Im Sinne des Universal Design for Learning muss geprüft werden, inwiefern diese Alternativen - neben Textmaterial - für Lernerinnen und Lerner nützlich sind und wie diese didaktisch eingebunden werden können. Im Vorhaben VISCH (Visualisierte Informationen in Schulbüchern) wird auf ein Beispiel aus dem Bereich Mathematik verwiesen (Blista 2012: 11). Die Schülerinnen und Schüler erhalten zur mathematischen Textaufgabe ein 3D-Modell, das zum Lösen der Aufgabe zur Verfügung gestellt wird. Dieses 3D-Modell kann auch für Schülerinnen und Schüler mit eingeschränktem räumlichen Vorstellungsvermögen eine besondere Chance darstellen, um die Aufgabe zu begreifen. Mit Hilfe von zugeordneten 3D-DruckDateien könnte der Ausdruck über den schuleigenen 3D-Drucker organisiert werden (Blista 2012: 25; DIAGRAM Center 2018). Weitere Projekte, wie z. B. das Projekt Tangram der TU Dresden, setzen an diesem Bedarf an (Tangram 2018).

\section{$5 \quad$ Fazit und Ausblick}

Angesichts der bildungspolitischen Zielstellung, eine inklusive Schul- bzw. Hochschullandschaft in Deutschland zu etablieren, kommt der Gestaltung von zugänglichen (barrierefreien) Bildungsangeboten eine besondere Bedeutung zu. In diesem Zusammenhang ist das Vorhandensein von Alternativtexten ein wesentlicher Baustein, um Bildungsangebote für eine heterogene Lernerschaft zugänglicher (barrierefreier) zu machen. In den nächsten Jahren sind geeignete Maßnahmen, welche die Zugänglichkeit der Bildungsangebote erhöhen, konsequenter im Handlungsfeld Schule bzw. Hochschule umzusetzen. Nicht nur der Bedarf für Schülerinnen und Schüler mit Sehbeeinträchtigung, sondern auch der allgemeine Nutzen für Schülerinnen und Schüler ohne sonderpädagogischen Förderbedarf im Sinne des universellen Designs muss dabei fokussiert werden. Die Notwendigkeit zum Handeln wird durch den Umstand verstärkt, dass die Wirkkraft von Bildern im Zeitalter der Digitalisierung auch im Bildungsbereich 
weiter zugenommen hat. In Forschungszusammenhängen wird vermehrt überlegt, inwiefern die Aussagekraft von Ergebnissen durch den Einsatz von Bildern unterstützt werden kann.

Täglich kommt es zur Weiterentwicklung von digitalen Werkzeugen, welche die gesellschaftliche Teilhabe von Menschen mit und ohne Behinderung optimieren. Auch im Bereich der Erstellung von Alternativtexten sind derartige Entwicklungen zu verzeichnen. Neue Medien, welche die automatische Erzeugung von Alternativtexten versprechen, befinden sich im Entwicklungs- bzw. Erprobungsprozess. Damit können Lehrende aus dem Bildungsbereich beim Erstellen von Alternativtexten maßgeblich unterstützt werden, was sich positiv auf die gezielte Vorbereitung von Bildungsangeboten auswirken kann. Jedoch gilt es zu beachten, dass Universaltexte nur begrenzt einsetzbar sind. Um die zielgerichtete Nutzung des Alternativtexts durch die Lernerin bzw. den Lerner sicher zu stellen, sind individuelle Anpassungen auch zukünftig nötig.

Zum jetzigen Zeitpunkt können Lehrende nur vereinzelt auf Empfehlungen zur Gestaltung von Alternativtexten - vorrangig aus dem amerikanischen Raum - zurückgreifen (Degenhardt 2016b; DIAGRAM Center 2018). Entsprechend den Empfehlungen lässt sich zusammenfassend formulieren, dass die Erstellung passgenauer Alternativtexte für die einzelne Nutzerin bzw. den einzelnen Nutzer zeitintensiv und fachlich anspruchsvoll ist. Im Idealfall sollten die Alternativtexte von qualifiziertem Fachpersonal erstellt werden. Jedoch stehen geschulte Ansprechpartner für Lehrende aus Schulen und Hochschulen im bundesweiten Vergleich kaum zur Verfügung (z. B. KIT: Karlsruher Institut für Technologie - Studienzentrum für Sehgeschädigte, Servicestelle InkluSoB an der Universität Hamburg, DoBuS an der TU Dortmund, Arbeitsgruppe Studium für Blinde und Sehbehinderte der TU Dresden oder Blinden- und Sehbehindertenpädagoginnen und -pädagogen im mobilen Dienst auf Landesebene). Die Einrichtung eines bundesweiten Expertennetzwerks, ähnlich dem DIAGRAM Center im amerikanischen Raum, welches Werkzeuge und Best Practice-Beispiele aufzeigt und auf regionale Unterstützungsinstrumente verweist, wäre zu begrüßen (DIAGRAM Center 2018). Gerade mit Blick auf die aktuellen Entwicklungen - der bevorstehenden Ratifizierung des MarrakeschVertrags - nimmt die Bedeutung von adäquaten Serviceangeboten, welche z. B. 
bei der Gestaltung von zugänglichen (barrierefreien) Dokumenten helfen, weiter zu (WIPO 2013).

Im Weiteren ist davon auszugehen, dass die Aufarbeitung der bestehenden Forschungsdefizite neue Erkenntnisse, bspw. zum allgemeinen Nutzen von und Bedarf an Alternativtexten, zur Verfügung stellt. Einerseits sind diese Forschungsbefunde hilfreich, um die allgemeinen Empfehlungen zur Gestaltung von Alternativtexten weiter zu konkretisieren. Und andererseits können die Hinweise genutzt werden, um im Sinne des universellen Designs die Chancen von Alternativtexten für Lernerinnen und Lerner ohne sonderpädagogischen Förderbedarf expliziter zu erforschen.

Unabhängig vom Nutzen der Alternativtexte für Lernerinnen und Lerner ohne sonderpädagogischen Förderbedarf bleibt die hohe Relevanz von Bildalternativen z. B. für Lernerinnen und Lerner mit Sehbeeinträchtigung bestehen, so dass der Alternativtext als angemessenes Mittel zur Gestaltung von zugänglichen (barrierefreien) Bildungsressourcen seine Legitimation hat. Ohne zugängliche (barrierefreie) Bildungsressourcen findet die Isolation von einzelnen Personengruppen statt. Da die Isolation bzw. der Ausschluss vom Bildungsangebot unbedingt zu vermeiden ist, müssen stets alle Möglichkeiten der Bildungsbeteiligung geprüft werden. 


\section{Literaturverzeichnis}

BARRIEREFREIES WEBDESIGN (2018): „Alternativtexte“. In: http://rubikon-seminare.com/ webaccessibility/alternative-text/ (letzter Zugriff: 23.02.2018).

BITV-LOTSE (2018): „Textalternativen für Bilder. Begründung“. In: http://www.bitvlotse.de/BL/DE/2_Bestandteile/2_1_Textalternativen/2_1_a_Bilder/

2_1_a_bilder_node.html (letzter Zugriff: 10.06.2018).

BITV 2.0 - BARRIEREFREIE INFORMATIONSTECHNIK-VERORDNUNG (2011): „Verordnung zur Schaffung barrierefreier Informationstechnik nach dem Behindertengleichstellungsgesetz." In: https://www.gesetze-im-internet.de/bitv_2_0/BJNR184300011.html (letzter Zugriff: 31.01.2018).

Blista - Deutsche Blindenstudienanstalt e.V. (Hg.) (2012): „VISCH - Visualisierte Informationen in Schulbüchern zugänglich machen. Leitfaden mit Beispielen“. In: https://www.blista.de/sites/default/files/visch-leitfaden.pdf (letzter Zugriff: 31.01.2018).

BSB Hamburg - BehÖRde FÜr SCHule und Berufsbildung der Freien und HanseSTADT HAMBURG (2013): „Handreichung Nachteilsausgleich“. In: http://li.hamburg.de/contentblob/3897592/fb84b9706cfa759bc2fb89ba6008d738/ data/download-pdf-handreichung-nachteilsausgleich.pdf (letzter Zugriff: 20.04.2018). DBSV - Deutscher BLINDEN- Und Sehbehindertenverband E. V. (2018a): „Der Vertrag von Marrakesch“. In: https://www.dbsv.org/vertrag-von-marrakesch.html (letzter Zugriff: 22.05.2018).

DBSV - Deutscher Blinden- Und Sehbehindertenverband E. V. (2018b): „Marrakesch-Vertrag - DBSV kritisiert Gesetzentwurf". In: https://www.dbsv.org/aktuell/ marrakesch-vertrag-dbsv-kritisiert-gesetzentwurf.html (letzter Zugriff: 22.05.2018).

DEGENHARDT, SVEN (2016a): „Alternativtexte als Baustein barrierefreier elektronischer Dokumente: Notwendigkeit, Erfahrungen und Forschungsbedarf. Vortrag am 17.11.2016: Campus Innovation 2016/Digitalisierung von Lehren und Lernen, Hamburg“. In: https://lecture2go.uni-hamburg.de/l2go/-/get/v/20550 (letzter Zugriff: 23.02.2018).

DEgENHARDT, SVEn (2016b): „Kurzinformationen zum Thema Alternativtexte“(Unveröffentlichtes Dokument).

DIAGRAM CENTER (2018): „Image Description“. In: http://diagramcenter.org/makingimages-accessible.html (letzter Zugriff: 31.01.2018).

GazZaniga, Michael S./Lvry, Richard B./Mangun, George R. (1998): Cognitive neuroscience. The biology of the mind. New York: W. W. Norton.

Gombrich, ERnst H. (1984): Bild und Auge. Neue Studien zur Psychologie der bildlichen Darstellung. Stuttgart: Klett-Cotta.

Hall, Tracey E./Meyer, Anne/Rose, David H. (2012): Universal Design for Learning in the Classroom. Practical Applications. New York: Guilford Press. 
KITZA, SARAH S. (2015): Gestaltung von Alternativtexten - eine empirische Studie aus Nutzerinnen- und Nutzersicht. Masterarbeit. Technische Universität Dortmund.

MeIER, Rolf (2006): Praxis E-Learning: Grundlagen, Didaktik, Rahmenanalyse, Medienauswahl, Qualifizierungskonzept, Betreuungskonzept, Einführungsstrategie, Erfolgssicherung. Offenbach: Gabal-Verlag.

NCAM/DiAgram Center - National Center for Accessible Media at WGBH/ Digital Image And Graphic Resources for AcCessible Materials (2015): „Image Description Guidelines“. In: http://diagramcenter.org/table-of-contents-2.html (letzter Zugriff: 31.01.2018).

Piaget, Jean (1988): Meine Theorie der geistigen Entwicklung. Frankfurt/M.: Fischer Verlag.

Rapp, Whitney (2014): Universal Design for Learning in Action: 100 Ways to Teach All Learners. Baltimore, Maryland: Brookes Publishing.

ReInMAnN, Gabi (2015): „Studientext Didaktisches Design“. In: http:/gabi-reinmann.de/ wp-content/uploads/2013/05/Studientext_DD_Sept2015.pdf (letzter Zugriff: 19.02.2018).

SCHLÜter, ANn-Kathrin/Melle, Insa/Wember, Franz B. (2016): „Unterrichtsgestaltung in Klassen des Gemeinsamen Lernens: Universal Design for Learning“. In: Sonderpädagogische Förderung 3, S. 270-285.

SCHÜTT, MARIE-LUISE (2015): E-Learning als Baustein im inklusiven Unterstützungs- und Beratungssystem in Deutschland. Konzeption, Implementierung und Evaluation des Onlineangebots ,MIT BISS' für Regelschullehrerinnen und Regelschullehrer. Münster: Waxmann.

SWELLER, JOHN (2005): „Implications of Cognitive Load Theory for Multimedia Learning“. In: MAYER, RICHARD E. (Hg.): The Cambridge Handbook of Multimedia Learning. Cambridge [u. a.]: Cambridge University Press, S. 19 - 30.

TANGRAM (2018): „Unterstützung der Fachbucherstellung durch multimediale Aufbereitung von technischen Grafiken“. In: https:/tu-dresden.de/ing/informatik/ai/ mci/forschung/forschungsgebiete/tangram (letzter Zugriff: 23.02.2018).

UDL - Universal DeSIGN FOR LEARNING (2018a): „Types of Evidence Supporting UDL“. In: http://www.udlcenter.org/aboutudl/udlevidence (letzter Zugriff: 03.05.2018).

UDL - Universal Design FOR LEARning (2018b): „The UDL Guidelines“. In: http://udlguidelines.cast.org/?utm_medium=web\&utm_campaign=launch\&utm_ source=cast-news\&utm_content=image-caption (letzter Zugriff: 23.02.2018).

UN - United Nations (2006/2008): „Gesetz zu dem Übereinkommen der Vereinten Nationen vom 13. Dezember 2006 über die Rechte von Menschen mit Behinderungen sowie zu dem Fakultativprotokoll vom 13. Dezember 2006 zum Übereinkommen der Vereinten Nationen über die Rechte von Menschen mit Behinderungen. “ In: http://www.un.org/depts/german/uebereinkommen/ar61106-dbgbl.pdf (letzter Zugriff: 23.02 .2018$)$. 
UN - United Nations (1948): „Resolution der Generalversammlung. Allgemeine Erklärung der Menschenrechte“. In: http://www.un.org/depts/german/ menschenrechte/aemr.pdf (letzter Zugriff: 23.02.2018).

Vygotsky, LeV SEMONOVICH (1978): Mind in society: The development of higher psychological processes. Cambridge, MA: Harvard University Press.

WCAG - Web CONTENT ACCESSibility Guidelines (2018): „Web Content Accessibility Guidelines (WCAG) Overview“. In: https://www.w3.org/TR/2018/CR-WCAG2120180130/ (letzter Zugriff: 23.02.2018).

WIPO - WelTORGANISATION FÜR GEISTIGeS EIGENTUM (2013): „Marrakesh Treaty to Facilitate Access to Published Works for Persons Who Are Blind, Visually Impaired or Otherwise Print Disabled“. In: http://www.wipo.int/wipolex/en/details.jsp?id=13169 (letzter Zugriff: 20.05.2018). 


\section{Technologiegestützte Kommunikation bei Beeinträchtigungen des Sehens}

Aus der Perspektive der Barrierefreien Kommunikation bedeutet eine Sehschädigung, dass die über den visuellen Sinneskanal gewonnenen und über andere Sinneskanäle ergänzten Reize nicht ausreichen, um eine Rekonstruktion der Wirklichkeit mit allen handlungsrelevanten visuellen Informationen herzustellen. Die hieraus folgenden Beeinträchtigungen sind vielfältig und weitreichend und verlangen nach Kompensation.

Assistive Technologien sind bei dieser Kompensation von zentraler und zunehmender Bedeutung. Mit assistiven Technologien kann die Qualität der wahrzunehmenden Reize verbessert oder durch andere, informationsgleiche Reizmuster ersetzt werden. Durch assistive Technologien kann der Interpretationsprozess der wahrgenommenen Reizmuster unterstützt und der Fokus auf relevante Informationen gelenkt werden. Schließlich ermöglichen assistive Technologien Menschen mit einer Beeinträchtigung des Sehens den Zugang zu zahlreichen Informationen und damit auch zum selbstbestimmten Erkenntnisgewinn.

Konkret bedeutet dies, dass assistive Technologien für Menschen mit einer Beeinträchtigung des Sehens einen selbstbestimmten Zugang zu Beipackzetteln, Straßenschildern, Kurznachrichten, Tafelbildern, Büchern, Anzeigetafeln, Kochrezepten, Zeitungen, Bankkonten, Produktbeschreibungen, Lexika, Speisekarten, sozialen Netzwerken usw. bieten. Neben dem Zugang zu Informationen erleichtern assistive Technologien die aktive Kommunikation. E-MailProgramme, Textverarbeitung, Webshops, Tabellenkalkulation, digitale Formulare, Online-Buchungssysteme usw. sind weitgehend auch von Menschen mit einer Beeinträchtigung des Sehens bedienbar. Der hier beschriebene Gewinn an Selbstbestimmung reicht weit über den praktischen Nutzen hinaus, da es bei- 
spielsweise Kommunikationsinhalte gibt, die vorzugsweise nicht mit einer Assistenzkraft geteilt werden (kompromittierende Recherchen, Kauf spezieller Medikamente, Liebesbriefe, Konsum bestimmter Medien, Finden bestimmter Etablissements etc.).

Grundsätzlich gibt es bei der Gestaltung technologiegestützter Kompensation zwei Herangehensweisen: Auf der einen Seite können die subjektiven Voraussetzungen der einzelnen Person an die Anforderungen des Informationsangebots angepasst werden. Auf der anderen Seite kann das Informationsangebot selbst so verändert werden, dass es für möglichst viele Personen zugänglich ist.

Bei der technologiegestützten Kompensation im Bereich der Anpassung der subjektiven Voraussetzungen wird durch Technologien versucht, die physischen, sensorischen oder kognitiven Fähigkeiten von Menschen mit Behinderung dahingehend zu stärken, dass sie in unterschiedlichen Umgebungen mehr Unabhängigkeit gewinnen und ihre behinderungsspezifischen Eigenarten in den Hintergrund rücken (Story/Mueller/Mace 1998). Demgegenüber stehen Ansätze, bei denen Technologien zum Abbau von Barrieren und im Sinne eines universellen Designs zur Gestaltung von Produkten und Lebensräumen eingesetzt werden, die von möglichst vielen Menschen jeden Alters und unterschiedlicher Fähigkeiten benutzbar sind (ebd.). Da das Ideal der universell designten Welt praktisch nicht vollständig erreicht werden kann, wird es auch weiterhin assistive Technologien geben müssen und genau um diese Technologien geht es im vorliegenden Artikel.

Mittlerweile gibt es ein breites Angebot an assistiven Technologien, mit welchen versucht wird, dem subjektiven Charakter der unterschiedlichen Beeinträchtigungen des Sehens zu begegnen. Nachfolgend sollen die wesentlichen Konzepte vorgestellt werden, auf welche diese konkreten assistiven Technologien aufbauen. 


\section{Screen Reader Technologie}

Während anfänglich assistive Technologien isolierte und speziell für Menschen mit Behinderung entwickelte Hilfsmittel waren (z. B. Prothesen, Blindenschriftschreibmaschinen, Farberkennungsgeräte), wird in den letzten Jahrzehnten versucht, handelsübliche Produkte durch assistive Technologien dahingehend zu erweitern, dass diese für Menschen mit Behinderung nutzbar werden. Ursächlich für diese Entwicklung ist vor allem der Wunsch der Menschen mit Behinderung selbst, die naturgemäß die gleiche Hard- und Software wie Menschen ohne Behinderung verwenden möchten (Shinohara/Wobbrock 2011). Dieser Wunsch erscheint durchaus nachvollziehbar: Die Verwendung von handelsüblicher Hard- und Software kann Stigmatisierung reduzieren (Böing 2013; Capovilla/Hubwieser 2013; Hofer 2008) und unterstützt damit ganz konkret die gesellschaftliche Teilhabe.

In diesem Entwicklungsprozess hat sich mittlerweile die Screen Reader Technologie als stabiler und ausgereifter Standard herausgebildet (Miesenberger 2015). Dies war vor allem deshalb möglich, da sich die grundlegenden Interaktionskonzepte in den letzten Jahrzehnten unwesentlich verändert haben (ebd.). Diese grundlegenden Interaktionskonzepte sind Fenster, Icons, Menüs und Zeiger (Müller-Prove 2002). Sie werden auf einem virtuellen Arbeitsbereich angeordnet und mit einem Zeigewerkzeug durch unterschiedliche Operationen (Point \& Click, Drag \& Drop etc.) angesteuert (Miesenberger 2015). Für dieses Steuerungskonzept hat sich der Begriff "grafische Benutzeroberfläche" etabliert. Deren Bedeutung zeigt sich darin, dass es mittlerweile so gut wie keine Endgeräte mehr gibt, die nicht darauf aufbauen.

Der Erfolg der grafischen Benutzeroberfläche begründet sich zum einen durch die intuitive Benutzerführung und die damit verbundene Maussteuerung (Capovilla/Hubwieser 2013) und zum anderen durch die klare Standardisierung der Bedienungselemente (Miesenberger 2015). Für Menschen mit einer Beeinträchtigung des Sehens entfallen die Vorteile der intuitiven Benutzerführung und der Maussteuerung, während die weitgehende Standardisierung der Interaktionskonzepte die Entwicklung wiederverwertbarer alternativer Steuerungsstrategien ermöglicht. 
Die entscheidende Schwierigkeit für Menschen mit einer Beeinträchtigung des Sehens besteht darin, dass ein maßgeblich visuell orientiertes System soweit abstrahiert werden muss, dass die enthaltenen Konzepte unabhängig von der konkreten Ausgestaltung am Bildschirm verstanden werden müssen. Ausgehend von diesem Verständnis müssen alternative, in der Regel nicht intuitive, Steuerungsstrategien erlernt werden. Nicht visuell-orientiertes Arbeiten mit Endgeräten besteht also im Wesentlichen darin: Identifizieren und Verstehen des Aufbaus und der abgebildeten Elemente der grafischen Benutzeroberfläche, Abrufen der bereits gelernten und damit bekannten Bedienungskonzepte für genau diese Elemente und Anwendung eines dieser bekannten Bedienungskonzepte.

Ein Screen Reader wird auf einem handelsüblichen Computer, einem Standard-Smartphone oder auch anderen Endgeräten installiert. Die wesentliche Aufgabe des Screen Readers besteht darin, den mehrdimensionalen Bildschirminhalt dahingehend zu vereinfachen, dass er mithilfe einer Sprachausgabe oder einer Braillezeile ausgegeben werden kann. Dies ist nur dann möglich, wenn die Informationen in linearer Form als einfacher Text angeordnet sind. Die entscheidende Aufgabe des Screen Readers besteht also entgegen der suggestiven Bezeichnung nicht darin, den Bildschirminhalt vorzulesen, sondern den Bildschirminhalt auf die notwendigen und relevanten Informationen zu reduzieren und für die Weiterverarbeitung zu linearisieren.

Inzwischen gibt es eine ganze Reihe kommerzieller und freier Screen Reader, die für unterschiedliche Plattformen auf stationären oder mobilen Geräten angeboten werden. In den letzten Jahren haben zudem die meisten großen Hersteller von mobilen Endgeräten begonnen, eigene Screen Reader als feste Bestandteile ihres Betriebssystems zu integrieren (Brady et al. 2013).

\section{Dateneingabe}

Bei der Dateneingabe kann die Eingabe von inhaltlichen Daten und die Eingabe von Steuerungsanweisungen unterschieden werden. Während es sich bei inhaltlichen Daten in der Regel um Text, aber mittlerweile beispielsweise auch um 
Sprachnachrichten oder Bilder handelt, wird mit Steuerungsanweisungen bestimmt, was mit diesen inhaltlichen Daten geschehen soll.

Bei der Eingabe von nicht primär textbasierten Daten wie Sprachnachrichten, Bildern, Videos usw. stoßen Menschen mit einer Beeinträchtigung des Sehens an die gleichen Grenzen wie alle anderen Menschen und profitieren gleichermaßen von Technologie zur Verbesserung der Qualität der Eingaben. Bezüglich der Eingabe von Daten in Textform ist die Tastatur nach wie vor die effizienteste Möglichkeit. Wesentlich ist hierbei, dass Menschen mit einer Beeinträchtigung des Sehens das 10-Finger-System zuverlässig beherrschen müssen, da eine visuelle Unterstützung des Eingabeprozesses gar nicht oder nur unter erheblichem Zeitverlust möglich ist. Alternativen wie die Spracheingabe mit anschließender Spracherkennung stoßen bei Menschen mit einer Beeinträchtigung des Sehens an die gleichen Grenzen wie bei allen anderen Menschen. Auf mobilen Endgeräten ist die Texteingabe für Menschen mit einer Beeinträchtigung des Sehens ohne Tastatur deutlich erschwert. Bei der Texteingabe über den Touchscreen wird ein Finger auf die Abbildung der Tastatur gelegt. Während der Touchscreen berührt wird, wiederholt die Sprachausgabe den unter dem Finger abgebildeten Buchstaben. Der Finger wird nun so lange verschoben, bis der gewünschte Buchstabe durch das akustische Feedback gefunden wird. Durch das Abheben des Fingers wird der Buchstabe eingegeben. Dieses Verfahren ist offenkundig zeitaufwändig und mühsam. Effiziente Texteingabe ohne den Touchscreen zu sehen, bleibt bis auf weiteres problematisch (Azenkot/Lee 2013).

Steuerungseingaben werden bei verfügbarer Tastatur in der Regel mit Tastenkombinationen durchgeführt. Vor diesem Hintergrund profitieren Menschen mit einer Beeinträchtigung des Sehens entscheidend von ergänzenden tastenbasierten Steuerungskonzepten, über die mittlerweile die meisten Betriebssysteme und Anwendungsprogramme verfügen. Erfahrungsgemäß sind diese Steuerungskonzepte aber nicht vollständig, weshalb mit ergänzenden Tastenkombinationen und einer tastaturbasierten Maussteuerung des Screen Readers versucht werden muss, die Funktionalität möglichst vollständig herzustellen.

Spezielle Tastenkombinationen des Screen Readers werden beispielsweise verwendet, um Texte auf bestimmte Art vorzulesen oder Worte zu buchstabieren, um die Inhalte bestimmter Fensterbereiche vorlesen zu lassen oder z B. 
auch Details zur Formatierung einzelner Zeichen zu erfahren. Bei der tastenbasierten Maussteuerung des Screen Readers wird nach der Aktivierung der Mauszeiger mit den Pfeiltasten über den Bildschirm bewegt, während die Bezeichnungen der darunterliegenden Objekte vorgelesen werden. Die linke und rechte Maustaste werden auf der Tastatur beispielsweise durch die Plus- und Minus-Tasten repräsentiert. Dadurch ist prinzipiell auch Drag \& Drop möglich. Einige Screen Reader bieten die Möglichkeit, eigene Steuerungsabläufe (Skripte) zu speichern und z. B. über eine Tastenkombination verfügbar zu machen. Der Vorteil solcher Skripte besteht darin, dass auch eine Software zugänglich gemacht werden kann, die aufgrund der geringen Verbreitung nicht aktiv vom Screen Reader unterstützt wird. Dies kann beispielweise in der Schule oder am Arbeitsplatz von Bedeutung sein, wenn eine spezielle Lernsoftware oder ein bestimmtes Datenverwaltungsprogramm eingesetzt werden soll, die nicht standardmäßig zugänglich sind.

Mobile Endgeräte verfügen in der Regel über keine Tastatur und seit der Einführung von Smartphones und von Tablet-PCs wurden auch Nummernblöcke auf mobilen Endgeräten verdrängt. Dies erscheint besonders für Menschen mit einer Beeinträchtigung des Sehens problematisch, da ohne festen Tastenblock wesentliche Referenzpunkte entfallen. Eine Alternative zur Tastatur ist die in den meisten mobilen Endgeräten enthaltene Sprachsteuerung, die allerdings aktuell nur ergänzend wirkt und keine vollständige Steuerung zulässt. Als ergänzendes Steuerungskonzept wird die Sprachsteuerung jedoch von Menschen mit einer Beeinträchtigung des Sehens im Vergleich zum Durchschnitt häufiger verwendet (Azenkot/Lee 2013).

Mittlerweise scheint sich die Multi-Touch-Technologie als zentrales Steuerungskonzept auch bei Menschen mit einer Beeinträchtigung des Sehens durchgesetzt zu haben. Bei dieser Technologie werden bestimmte Wischbewegungen und Berührungsfolgen festgelegt und mit Funktionen verbunden. Von entscheidendem Vorteil ist dabei, dass sich, abgesehen von Texteingaben, die Bedienung von mobilen Endgeräten im Wesentlichen auf die hierarchische Menüsteuerung reduzieren lässt (Kane et al. 2008). Eine solche hierarchische Menüsteuerung könnte folgendermaßen aufgebaut sein: In Menüs wird durch Wischen nach oben und unten navigiert, während die Menüeinträge vorgelesen werden. Durch ein Wischen nach rechts wird das entsprechende Untermenü geöffnet, 
während durch ein Wischen nach links das Untermenü geschlossen und eine Hierarchieebene höher gesprungen wird. Ein Menüpunkt könnte durch die Berührung mit einem zweiten Finger ausgewählt werden und das objektbezogene Kontextmenü durch eine kleine kreisende Wischbewegung geöffnet werden.

\section{Datenausgabe}

Bei der Datenausgabe für Menschen mit einer Beeinträchtigung des Sehens muss in der Regel ein durch den Screen Reader auf einfachen Text reduzierter Bildschirminhalt akustisch oder taktil ausgegeben werden.

Akustisch wird dies mit einer Sprachausgabe umgesetzt. Sprachausgaben verwenden Sprachen mit unterschiedlichen Qualitäten und Erzeugungsverfahren (Hess 1996). Die Wahl der Sprache hängt von subjektiven Bedürfnissen und Wünschen ab. Während bei einem gut geschriebenen Roman vermutlich auf den natürlichen Klang der Stimme wert gelegt wird, kann es bei einer ausschweifenden Einführung in die Betriebswirtschaftslehre wichtiger sein, dass die Stimme trotz sehr hoher Sprechgeschwindigkeit einfach nur verständlich bleibt.

Neben der Sprachausgabe kann auch die Brailleschrift für die Datenausgabe verwendet werden. Braille ist eine taktile Schrift, bei der Buchstaben durch Kombinationen von tastbaren Punkten repräsentiert werden. Eine Braillezeile dient nun dazu, die aufbereiteten Daten des Screen Readers in Braille auszugeben. Braillezeilen bestehen in der Regel aus 20 bis 80 Modulen (Einheiten, mit denen genau ein Zeichen repräsentiert werden kann), in denen jeweils 8 Stifte angeordnet sind (8-Punkt -Braille). Die Stifte werden abhängig vom Zeichen durch eine elektrische Spannung angehoben, wodurch die tastbaren Repräsentationen entstehen.

Sprachausgabe und Braillezeile haben unterschiedliche Vor- und Nachteile. Ein maßgeblicher Vorteil der Sprachausgabe ist das Erreichen einer hohen Lesegeschwindigkeit. Die durchschnittliche Lesegeschwindigkeit geübter Jugendlicher und junger Erwachsener beträgt etwa 200 bis 350 Wörter pro Minute (Denninghaus 1996). Geübte früherblindete Leserinnen und Leser von Braille 
erreichten 100 bis 150 Wörter in der gleichen Zeit, während hochgradig sehbehinderte Lesende in Schwarzschrift eine Geschwindigkeit von 40 bis 80 Wörtern pro Minute erreichen (ebd.). Mit einer Sprachausgabe lässt sich problemlos die Geschwindigkeit von geübten Schwarzschriftlesenden ohne Sehbeeinträchtigung erreichen. Ein maßgeblicher Vorteil der Braillezeile besteht in der Stärkung der orthographischen und grammatikalischen Kompetenz, die bei ausschließlicher Verwendung einer Sprachausgabe möglicherweise nicht ausreichend ausgebildet und gefördert wird. Auf der anderen Seite eignet sich die Sprachausgabe besonders gut, um Buchstabendreher oder Satzstellungsfehler zu finden (Mulloy et al. 2014), da sie alle Buchstaben sequentiell vorliest und im Unterschied zum Menschen nicht interpoliert. Vor allem in Bildungsprozessen oder an interaktionsorientierten Arbeitsplätzen bietet die Braillezeile den Vorteil, dass der auditive Sinneskanal nicht belegt wird. Mit laufender Sprachausgabe fällt es schwer, parallel den mündlichen Ausführungen einer Lehrkraft zu folgen oder die Wünsche von Callcenter-Anrufenden entgegenzunehmen. Außerdem kann eine stimmliche Dauerbeschallung durchaus anstrengend sein und die Braillezeile bietet hier eine Auszeit.

Schließlich stellt die Verwendung einer Vergrößerungssoftware eine dritte Möglichkeit zur Datenausgabe dar. Diese Möglichkeit bleibt jedoch auf den Personenkreis der Menschen mit entsprechend verwertbaren Sehvermögen beschränkt.

Vergrößerungssoftware vergrößert auf unterschiedliche Arten bestimmte Ausschnitte des Bildschirms (Kalina 2011) und damit auch alles, was auf dem Bildschirm abgebildet werden kann (Texte, gescannte Texte, Fotos, Computerspiele, Webcam-Bilder etc.). Dementsprechend ist der Komplexitätsgrad von Vergrößerungssoftware im Vergleich zur Screen Reader Technologie wesentlich niedriger. Diverse Betriebssysteme enthalten bereits standardmäßig Vergrößerungssoftware, beispielsweise in Form einer Bildschirmlupe. Im Unterschied zu diesen kostenlosen Ausgabehilfen bietet kommerzielle Vergrößerungssoftware deutlich höhere Vergrößerungsfaktoren, Kantenglättung zur Reduktion der sichtbaren Pixelstruktur, die Möglichkeit zur farblichen Invertierung oder Wahlmöglichkeiten von bestimmten Farbschemata, manchmal eine Vorlesefunktion und ein besseres Fokustracking (Verschiebung des Vergrößerungsausschnitts zum Mauszeiger oder zur Eingabeaufforderung). 
Der entscheidende Nachteil von Vergrößerungssoftware besteht im Vergleich zur Screen Reader Technologie in einem deutlich höheren Zeitaufwand im Arbeitsprozess, was z. B. der beruflichen Entwicklung einer konkurrenzfähigen Arbeitsleistung im Wege stehen kann. Der höhere Zeitbedarf ergibt sich durch die zusätzliche Sehleistung beim Fokustracking und vor allem durch die notwendigen zusätzlichen Interventionen, wenn das Fokustracking nicht gelingt. Außerdem kann sich der Zeitbedarf durch die notwendige gedankliche Rekonstruktion des nur ausschnitthaft wahrgenommenen Bildschirminhalts und die damit verbundenen zusätzlichen Erkundungshandlungen erhöhen. Schließlich entfallen durch die Aufbereitung des Screen Readers zahlreiche situativ nicht relevante Details, die beim Einsatz einer Vergrößerungssoftware erst durch zusätzliche Sehleistung erkannt und dann ignoriert werden müssen.

Auf der anderen Seite lassen sich mit Vergrößerungssoftware die Vorteile der grafischen Benutzeroberfläche (intuitive Führung, Maussteuerung etc.) nutzen, was auch bei Menschen mit einer Beeinträchtigung des Sehens die Anlernzeit erheblich verkürzt und die Zahl möglicher Nutzerinnen und Nutzer erweitert. Vergrößerungssoftware bietet zudem für Menschen, die mit ihrer moderaten Beeinträchtigung des Sehens keine Aufmerksamkeit auf sich ziehen wollen den Vorteil, dass sie in öffentlichen Situationen (z. B. während einer Bahnfahrt) weniger auffallen. Ohne Screen Reader und Vergrößerungssoftware ist eine Objektannäherung notwendig, was durchaus auffällt, stigmatisierend wirken kann und letztlich aufgrund der resultierenden unnatürlichen Körperhaltung (Rücken, Schultern, Knie ...) bedenklich ist.

Während beim Einsatz von Endgeräten die Screen Reader Technologie eine mächtige Alternative zum Vergrößerungsbedarf darstellt, fehlt diese Alternative häufig in der Lebenswirklichkeit. Während Menschen ohne verwertbares Sehvermögen auf nicht visuelle Wahrnehmungssurrogate, die über andere Sinneskanäle konstruiert werden, zurückgreifen müssen, können Menschen mit verwertbarem Sehvermögen das wahrnehmbare visuelle Reizmuster durch Vergrößerungsstrategien verbessern oder erweitern. Dies ist insofern relevant, 
da sich hieraus sehr unterschiedliche Konsequenzen für die zielgruppenspezifische Barrierefreie Kommunikation ergeben. Für die Zielgruppe der Menschen mit einem entsprechend verwertbaren Sehvermögen lässt sich eine Vergrößerung grundsätzlich durch Objektannäherung, durch Objektvergrößerung oder durch den Einsatz von optischen oder elektronischen assistiven Technologien erreichen.

\subsection{Objektannäherung und -vergrößerung}

Durch die Annäherung an ein Objekt lässt sich erfahrungsgemäß eine Vergrößerung desselben erzielen. Da diese Vergrößerung rein physiologisch erfolgt, beziehen sich assistive Technologien in diesem Kontext auf mechanische Vorrichtungen, mit denen diese Annäherung unterstützt wird. Solche Vorrichtungen sind z. B. Schwenkarme für Computerbildschirme, Buchständer oder schwenkbare Klemmbretter. In den Kontext der Objektannäherung fallen beispielsweise auch Anzeigetafeln auf Augenhöhe in Bahnhöfen oder der Einsatz von nicht vertieften Vitrinen und Schaukästen, die eine Annäherung an den Informationsträger erlauben.

Ein klassisches Beispiel für die Objektvergrößerung ist der Großdruck. Dabei wird der Schriftgrad eines Dokuments in der Regel auf 18 Punkte (AFB 2013) angehoben. Eine weitere Möglichkeit ist die Vergrößerung des Informationsträgers mithilfe eines Kopierers (z. B. von DIN-A4 auf DIN-A3). Außerdem kann versucht werden, die Schriftgröße an der Tafel oder am Projektor zu erhöhen.

Obwohl Großdruck tatsächlich zu einer durchschnittlichen Erhöhung der Lesegeschwindigkeit von Menschen mit Sehbeeinträchtigung führt (Lueck et al. 2003; Lovie-Kitchin/Bevanm/Hein 2001), stoßen diese Maßnahmen schnell an ihre Grenzen. Dies liegt vor allem an den begrenzten Möglichkeiten hinsichtlich des Vergrößerungsfaktors und der dadurch entstehenden Ausmaße der Informationsträger. Eine DIN-A4 Seite vergrößert mit dem Faktor 4 entspricht bereits dem Format DIN-A0 und liegt fernab jeder Handhabbarkeit. Mit zunehmendem Vergrößerungsfaktor werden zudem zusätzliche Kopf- und Augenbewegungen notwendig, durch welche auch die Lesegeschwindigkeit wieder deutlich abnimmt (Lueck et al. 2003). 
Alternativ zur Objektvergrößerung können assistive Technologien verwendet werden, die das Ausgangsobjekt unverändert lassen und die Vergrößerung optisch oder elektronisch erreichen. Da der Informationsträger in Größe, Kontrast, Farbschema etc. an die subjektiven Bedürfnisse angepasst werden kann, schneidet die Vergrößerung durch assistive Technologien im Vergleich zur Objektvergrößerung, insbesondere im Textverständnis beim Lesen, besser ab (Farmer/Morse 2007).

\subsection{Optische und elektronische Vergrößerung}

Im einfachsten Fall kann die Vergrößerung optisch erreicht werden. Für die Ferne können handelsübliche Monokulare oder Binokulare verwendet werden. Außerdem gibt es Aufsätze für die Ferne, die bereits in ein Brillengestell integriert sind oder aufgesteckt werden. Für Entfernungen unter einer Armlänge können z. B. Standlupen, die über eine Haltevorrichtung in stabiler Lage gehalten werden, Handlupen oder Lupenbrillen verwendet werden (Mulloy et al. 2014). Häufig werden bei Nahlupen Vergrößerungsfaktoren zwischen 6 und 10 verwendet. Vor allem Handlupen und Lupenbrillen haben den Vorteil, dass Sie überall und verhältnismäßig unauffällig eingesetzt werden können. Durch Lupenbrillen wird es z. B. möglich, ein ganz normales Buch am Strand zu lesen. Ein Nachteil ist, dass optische Vergrößerungssysteme Fehlhaltungen provozieren können. Ebenso erfordern diese Geräte Übung, da z. B. die Zeile gehalten oder die Informationen des zweiten Auges beim monokularen Lesen ignoriert werden müssen. Zudem ist die optische Vergrößerung technisch limitiert, da sich mit zunehmendem Vergrößerungsfaktor der Abstand zwischen Auge und Informationsträger verkleinert.

Bei elektronischen Sehhilfen kann zwischen Standgeräten, Handkameras und batteriebetriebenen mobilen Geräten unterschieden werden. Bei Standgeräten ist die Kamera modellabhängig ca. $20 \mathrm{~cm}$ über einem Kreuztisch zur Bewegung des Informationsträgers und unter einem Bildschirm fixiert. Neben dem Lesen von Texten eignen sich Standgeräte beispielsweise auch für Tätigkeiten wie das Ausmalen von Bilderbüchern, das Lösen von Kreuzworträtseln oder das Schneiden der Fingernägel. Handkameras sind in der Regel über ein Kabel 
mit einem Notebook verbunden, begrenzt mobil und eignen sich auch zum Vergrößern von Dingen, die nicht unter ein Standgerät passen wie Kochrezepte auf Verpackungen, Beschriftungen auf Staubsaugern, Globen usw. Außerdem können Handkameras beim Schminken, zur Betrachtung von Pickeln oder anderen Hautveränderungen oder zur Wundversorgung eingesetzt werden. Bei mobilen Geräten ist die Kamera in der Regel direkt in das Displaygehäuse integriert, über welches das Abbild des vergrößerten Objekts ausgegeben wird. Eigenständige mobile elektronische Vergrößerungssysteme haben inzwischen an Bedeutung stark verloren, da sie durch Smartphones und Tablet-PCs verdrängt werden. Für die Ferne gibt es Tafellesesysteme, die mit einem Endgerät verbunden und per Hand oder z. B. über einen Joystick gesteuert werden können. Solche Systeme kommen vor allem in Klassenzimmern, Seminarräumen und Konferenzsälen zum Einsatz.

Ein spannender neuerer Ansatz in diesem Kontext sind interaktive Whiteboards (Illinois State University 2012). Dabei wird das Bild des interaktiven Whiteboards in Echtzeit über eine kabellose Verbindung an einen Tablet-PC übertragen, wo es durch Standardfunktionen und zusätzliche Apps entsprechend der subjektiven Bedürfnisse angepasst werden kann.

Die Grenzen der Vergrößerung werden bei elektronischen Sehhilfen in erster Linie durch die Grenzen der Handhabung bestimmt. Mit zunehmendem Vergrößerungsgrad wird die Orientierung auf dem Informationsträger und die Handhabung allgemein (Zittern, Wackeln des Tisches, Fixierung etc.) schwieriger und die notwendigen Hand-, Kopf- und Augenbewegungen nehmen zu.

\section{$5 \quad$ Spezifische assistive Technologien im Alltag}

Im dritten Abschnitt sollen schließlich Technologien vorgestellt werden, mit welchen sich spezifischen Herausforderungen insbesondere in den Bereichen Orientierung, Mobilität, lebenspraktische Fähigkeiten und Freizeitgestaltung begegnen lässt.

Einige dieser Technologien sind nach wie vor als speziell gefertigte Geräte erhältlich. Hierzu gehören z. B. sprechende Uhren, sprechende Küchen- und 
Personenwaagen, sprechende Farbeerkennungsgeräte, geschlossene Vorlesesysteme (ein Sprachausgabesystem, das an einen Scanner gekoppelt ist und per Knopfdruck das eingelegte Blatt vorliest) und Notizgeräte. All diesen Geräten sind eine verhältnismäßig einfache Bedienbarkeit und aufgrund der geringen Absatzzahlen sehr hohe Produktkosten gemeinsam.

Viele dieser Geräte werden mittlerweile jedoch durch kostenlose oder erschwingliche Applikationen für Smartphones oder Tablets verdrängt. Dies ermöglicht den Rückgriff auf die bereits beschriebenen Bedienkonzepte (Screen Reader Technologie) und die Nutzung der auf den Endgeräten bereits verfügbaren Ein- und Ausgabesysteme. Dies unterstreicht die zentrale Bedeutung der Screen Reader Technologie für alle Menschen mit einer Beeinträchtigung des Sehens, da speziell gefertigte Geräte offenbar weiter an Bedeutung verlieren werden.

Während die im Internet verfügbaren und jederzeit testbaren Apps Weiterentwicklungen und Verdrängungsprozessen unterworfen sind, verändern sich die damit adressierten Herausforderungen kaum. Daher bleibt dieser Abschnitt auf eine Problemkonkretisierung mit entsprechenden konzeptuellen Lösungsansätzen beschränkt.

Mittlerweile sind zahlreiche Apps zur Unterstützung der Mobilität und Orientierung verfügbar. Aufbauend auf die herkömmlichen Navigationssysteme stellen diese Apps Benutzerschnittstellen für Menschen mit einer Beeinträchtigung des Sehens bereit. Vorzugsweise wird hier haptisches Feedback verwendet, um den auditiven Sinneskanal für die situative Regulation (Erkennen von Hindernissen, Verkehrsflussorientierung, Interaktion mit anderen etc.) nicht zu überdecken.

Ein zweiter zentraler Bereich sind Apps zur Texterkennung, bei denen der Informationsträger, idealerweise gestützt durch Funktionen zur Fokussierung, fotografiert, der enthaltene Text softwaregestützt erkannt und durch die Sprachausgabe vorgelesen wird. Vor allem für blinde Menschen bieten solche Apps Vorteile, wenn eingrenzbar ist, wo genau der zu erkennende Text abgebildet ist. Dies wäre beispielsweise bei einer Speisekarte oder einem Brief gegeben, während Straßenschilder aufgrund der fehlenden Möglichkeit zur konkreten Lokalisation in der Regel nicht effektiv erfasst werden können. Menschen, die mit 
ihrem Sehvermögen die Kontur des Straßenschildes lokalisieren können, werden hingegen ein optisches oder elektronisches Vergrößerungssystem (z. B. die Zoomfunktion des Smartphones) verwenden, um den Text auf dem Schild zu lesen.

Eine weitere Kategorie bilden Frage- \& Antwort-Applikationen (Q\&A Apps). Hier wird beispielsweise ein visuell nicht bestimmbares Objekt mit dem Smartphone fotografiert und für eine Gruppe Freiwilliger z. B. in einem sozialen Netzwerk gepostet. Eine gerade verfügbare Person transkribiert dann die visuelle Information in eine Textnachricht oder beantwortet Fragen (Welches Medikament ist das? Was enthält diese Konservendose? Sieht mein neuer Freund gut aus?). Der Erkennungsprozess in Q\&A-Systemen wurde mittlerweile teilweise automatisiert. In diesem Kontext, insbesondere beim Einkaufen, können auch Apps zum Lesen von QR Codes oder Barcodes oder Apps zur Farberkennung (Ist die Tomate reif?) hilfreich sein. Außerdem stehen inzwischen Apps zur Verfügung, bei denen Freiwillige bei einer laufenden Videokonferenz Unterstützung leisten, was z. B. beim Wiederauffinden eines davongerollten Apfels hilfreich sein kann.

Nicht nur für Menschen mit einer Beeinträchtigung des Sehens gibt es mittlerweile Apps, welche sich mit der Tonspur eines laufenden Kinofilms synchronisieren können und eine mit Audiodeskription ergänzte Audiospur über einen Kopfhörer anbieten. Außerdem gibt es inzwischen eine ganze Reihe von zugänglichen textbasierten Rollenspielen, Geschicklichkeitsspielen wie auditives Bogenschießen (je zentraler die Pfeilspitze, desto höher der wahrnehmbare Ton), auditiven Ego-Shootern oder Ratespielen.

Neben diesen speziell entwickelten Apps erfreuen sich zahlreiche zugängliche Standard-Apps, insbesondere im Bereich der Kommunikation und der sozialen Netzwerke, auch bei Menschen mit einer Beeinträchtigung des Sehens großer und weiter zunehmender Beliebtheit.

\section{Schluss}

In den vorangegangenen Abschnitten wurde eine Auswahl verfügbarer assistiver Technologien für Menschen mit einer Beeinträchtigung des Sehens vorgestellt. 
Mit der Beschreibung der konkreten Anwendung dieser Technologien wurden zahlreiche Herausforderungen deutlich, denen sich Menschen mit einer Beeinträchtigung des Sehens nach wie vor stellen müssen. Zu diesen Herausforderungen gehören beispielsweise das allgemein verständliche Kommunizieren auf Augenhöhe, der selbstbestimmte Zugang zu Informationen und die Verwendung von Standard-Hard- und Software und die damit verbundene Screen Reader Technologie. Die Bewältigung dieser Herausforderungen ist wesentlich für das Gelingen der gesellschaftlichen Partizipation. Die beschriebenen Lösungsansätze machen aber auch deutlich, dass sich zahlreiche dieser Herausforderungen durch den adäquaten Einsatz von assistiven Technologien bewältigen lassen. Damit gehört der Einsatz assistiver Technologien zu den wirksamsten Maßnahmen im Inklusionsprozess von Menschen mit Behinderung (Michaels/McDermott 2003).

Trotz dieser Erkenntnis lassen zahlreiche Studien darauf schließen, dass die Potentiale von assistiven Technologien nicht annähernd genutzt werden (Wong/Cohen 2012). Während durch den Trend hin zum Einsatz von StandardEndgeräten die hemmenden Faktoren der hohen Anschaffungskosten von assistiven Technologien und die damit verbundene fehlende Verfügbarkeit entspannt werden, gibt es nach wie vor kein flächendeckendes und systematisches Konzept, wie die Kompetenzen im Umgang mit diesen Technologien erworben werden können.

Bei diesem Verdrängungsprozess gilt es drei abschließende Aspekte zu berücksichtigen. Durch den Trend hin zum Einsatz von Standard-Endgeräten und der damit verbundenen einheitlichen Benutzerschnittstelle konvergieren die Bedienungskonzepte in Richtung Screen Reader Technologie, was als durchaus positiv zu bewerten ist. Der Komplexitätsgrad dieser Endgeräte und der Screen Reader Technologie liegt deutlich höher als jener von vielen speziell gefertigten Geräten, die insbesondere für Menschen mit erschwerenden Voraussetzungen und zusätzlichen Beeinträchtigungen sinnvolle Hilfsmittel darstellen können. Ohne sinnvolle Alternativen wird sich dann genau für diese Zielgruppe die Situation verschlechtern, da diese speziell gefertigten Geräte samt ihrer Herstellerfirmen aufgrund der fallenden Absatzzahlen verschwinden werden. Schließlich sollte kritisch bedacht werden, dass durch den aktuellen Trend Menschen mit Behinderung vom gesellschaftlichen Selbstverständnis abhängig werden, 
Dino Capovilla

dass Kommunikation barrierefrei sein soll. Die Förderung von Teilhabe durch die Gestaltung von Barrierefreier Kommunikation ist damit nicht nur eine spannende technische Herausforderung, sondern auch eine nachhaltige verantwortungsvolle gesellschaftliche Aufgabe. 
Technologiegestützte Kommunikation bei Beeinträchtigungen des Sehens

\section{Literaturverzeichnis}

AFB - AMERICAN FOUNDATION FOR THE BLIND (2013): „Tips for making print more readable“. URL: http://www.afb.org/section.aspx?TopicID=200\&DocumentID=210 (letzter Zugriff: 24.11.2015).

AZENKot, Shiri/LeE, NiCole B. (2013): „Exploring the use of speech input by blind people on mobile devices". In: Proceedings of the ASSETS'13. New York: ACM, 1-8.

BöING, URSUlA (2013). Schritte inklusiver Schulentwicklung. Würzburg: Edition Bentheim.

Brady, Erin/Ringel, Meredith/Yu Zhong, Morris/White, Samuel/Bigham, JeFFrey (2013): „Visual challenges in the everyday lives of blind people“. In: Proceedings of the CHI '13. New York: ACM, 2117-2126.

Capovilla, Dino/Hubwieser, Peter (2013): „Soziale Inklusion als fachdidaktisches Problem der Informatik“. In: blind-sehbehindert 4/2013, 226-235.

DenNinghaUS, ERWIN (1996): „Die Förderung der Lesegeschwindigkeit bei blinden und sehbehinderten Jugendlichen und jungen Erwachsenen“. blind-sehbehindert 2/1996, 95-100.

FARMER, JEANIE/MORSE, STEPHEN E. (2007): „Project magnify: Increasing reading skills in students with low vision“. In: Journal of Visual Impairment and Blindness 101 (12), $763-768$.

HeSs, WolfGang (1996): „Neuere Entwicklungen in der Sprachsynthese“. In Spektrum der Wissenschaft 12/1996, 100-103.

Hofer, URSUla (2008). „Sehen oder Nichtsehen: Bedeutung für Lernen und aktive Teilhabe in verschiedenen Bereichen des Lernens und Lehrens". In: LANG, MARKus/ Hofer, Ursula/Beyer, Friederike (Hg.): Didaktik des Unterrichts mit blinden und hochgradig sehbehinderten Schülerinnen und Schülern - Band 1: Grundlagen. Stuttgart: Kohlhammer, 17-67.

ILLINOIS STATE UNIVERISTY (2012): „SMART Boards for visually impaired“. URL: http://mediarelations.illinoisstate.edu/report/1213/july3/smartboards.asp (letzter Zugriff: 24.11.2015)

KALINA, UlRICH (2011): „Informationstechnologie“. In: LANG, MARKUS/Hofer, URSUla/Beyer, Friederike (Hg.): Didaktik des Unterrichts mit blinden und hochgradig sehbehinderten Schülerinnen und Schülern - Band 2: Fachdidaktiken. Stuttgart: Kohlhammer, 189-199.

Kane, Shaun K./Bigham, JefFrey P./Wobbrock, Jacob O. (2008): „Slide rule: making mobile touch screens accessible to blind people using multi-touch interaction techniques“. In: Proceedings of ASSETS'08. New York: ACM, 73-80.

Lovie-Kitchin, Jan E./Bevanm, Jennifer D./Hein, Bronwyn (2001): „Reading performance in children with low vision". In: Clinical and Experimental Optometry 84 (3), 148-154. 
Dino Capovilla

Lueck, Amanda Hall/Bailey, Ian L./Greer, Robert B./Tuan, Kuang Mon/ Bailey, VAlerie M./Dornbusch, Helen G. (2003): „Exploring print-size requirements and reading for students with low vision". In: Journal of Visual Impairment and Blindness 97 (6), 335-354.

Michaels, Craig A./McDermott, Jennifer (2003): „Assistive technology integration in special education teacher preparation: Program coordinators' perceptions of current attainment and importance“. In: Journal of Special Education Technology 18 (3), 29-44.

Miesenberger, Klaus (2015): „Advanced and Emerging Solutions: ICT and AT in Education of Low Vision and Blind Students“. In: Proceedings of ICEAPVI'15, 17-26.

Müller-Prove, Matthias (2002): „Vision and Reality of Hypertext and Graphical User Interfaces“. Masterarbeit an der Universität Hamburg.

Mulloy, Austin/Gevarter, Cindy/Hopkins, Megan/Sutherland, Kevin/Ramdoss, SATHIYAPRAKASH (2014): „Assistive Technology for Students with Visual Impairments and Blindness“. In: Lancioni, Giulio/Singh, Nirbhay, N. (Hg.): Assistive Technologies for People with Diverse Abilities. New York: Springer, 113-156.

Shinohara, Kristen/WOBвrock, JACOB (2011): „In the shadow of misperception: assistive technology use and social interactions“. In: Proceedings of the SIGCHI'11. New York: ACM, 705-714.

Story, Molly Follette/Mueller, James L./Mace, Ronald L. (1998): The Universal Design File: Designing for People of All Ages and Abilities. Revised Edition. URL: http://files.eric.ed.gov/fulltext/ED460554.pdf (letzter Zugriff: 4.2.2018).

Wong, Meng E./Cohen, Libby (2012): „Assistive technology use amongst students with visual impairments and their teachers: Barriers and challenges in special education“. In: Research Brief 12 (5), 1-4. 


\section{Schreib- und Übersetzungswerkzeuge für die Barrierefreie Kommunikation}

\section{$1 \quad$ Einleitung}

Barrierefreiheit ist mit der Verabschiedung der UN-Behindertenrechtskonvention im Jahr 2008 und deren bis heute andauernder Umsetzung in nationales Recht immer wichtiger geworden. Damit wuchs das Volumen an Dokumenten, die in Leichter Sprache verfasst werden müssen, sprunghaft an. Gleichzeitig erwachte auch das wissenschaftliche Interesse an kommunikativen Barrieren und deren Vermeidung, was zu steigenden Anforderungen an die Qualität barrierefreier Kommunikationsangebote geführt hat. An dieser Stelle kommen Schreibund Übersetzungswerkzeuge ins Spiel.

Schreib- und Übersetzungswerkzeuge werden in der technischen Kommunikation verwendet, um Gebrauchstexte wie Betriebsanleitungen durch eindeutige Wortwahl und konsistente Formulierungen zu optimieren. Im Umfeld Barrierefreier Kommunikation kommt diesem Funktionsprinzip, nämlich sprachliche Vielfalt gezielt zu begrenzen, eine zentrale Rolle zu, denn sprachliche Varianz kann ungeübte Rezipient(inn)en verwirren. Abhilfe schaffen hier Terminologiedatenbanken, in denen verständliche Definitionen gespeichert und aus ihnen wieder abgerufen werden können; Übersetzungs- und Textspeicher, die gelungene vereinfachte Formulierungen zur Wiederverwendung bereitstellen; und Autorentools, die Regeln auf Wort-, Phrasen- und Satzebene überprüfen und im Idealfall gezielte Vorschläge für die barrierefreie Umgestaltung geben. Der vorliegende Beitrag geht auf die Funktionsweise sowie den möglichen Einsatz dieser Werkzeuge für Leichte Sprache (und vereinfachte Varietäten anderer Sprachen) ein. 
Der Beitrag orientiert sich dabei am Stand der Technik im Februar 2018. Dazu gehört auch ein Ausblick auf die Entwicklung von Werkzeugen auf Basis der so genannten Künstlichen Intelligenz (KI), präziser bezeichnet als neuronale Netze.

\section{Leichte Sprache als Spielart Barrierefreier Kommunikation}

„Leichte Sprache ist eine Varietät [...] des Deutschen, die [...] im Bereich Satzbau und Wortschatz systematisch reduziert ist. Ebenso systematisch ist die Reduktion mit Bezug auf das Weltwissen, das für die Lektüre vorausgesetzt wird. [...] Außerdem zeichnen sich Leichte-Sprache-Texte durch eine besondere Form der visuellen Aufbereitung aus“ (Maßß 2015: 11-12).

Diese Definition von Maaß gibt alle für die Nutzung von Schreibwerkzeugen wesentlichen Merkmale wieder. Sowohl hinsichtlich des Wortschatzes als auch der Auswahl syntaktischer Konstruktionen gelten bestimmte Regeln, die eine Teilmenge der Regeln des Standarddeutschen bilden, ergänzt durch einige spezifische Standards, wie z. B. den Mediopunkt („“) (ebd. 12) oder die Zahlendarstellung durch arabische Ziffern (ebd. 93-94). Während diese Reduktion sich grundsätzlich mit Hilfe der unterschiedlichen computergestützten Werkzeuge umsetzen lässt, liegt ein angemessener Umgang mit reduziertem Weltund Vorwissen (der eine Addition der fehlenden Aspekte notwendig macht) aktuell außerhalb von deren Möglichkeiten.

Bevor sich dieser Artikel den Herausforderungen widmet, die eine „leichte“ Varietät an die Textproduktion stellt und auf die Hilfestellung, die computerbasierte Werkzeuge bieten, folgt hier ein kurzer Exkurs zum Zusammenhang zwischen Barrierefreiheit und verringerter Varianz des Zeicheninventars. 


\section{Besondere Herausforderungen bei der Produktion Barrierefreier Kommunikation}

Kommunikation ist nicht nur auf Rezipientenseite (wo Leichte Sprache ja eine Erleichterung schaffen soll) eine Herausforderung, sondern auch für die Produzenten. Grund sind die oben aufgeführten Restriktionen auf allen Ebenen des Sprachsystems und die Besonderheit der Kommunikationssituation. Im Umgang mit Texten sind der/die Übersetzer(in) von LS-Texten und deren Adressat(inn)en maximal weit voneinander entfernt: hier von Berufs wegen ausgesprochene Vertrautheit mit Schriftlichkeit, Textsorten und Diskursen; dort Angewiesensein auf Leichte Sprache zum Verständnis jeglicher formellen Kommunikation (vgl. Bredel/Maaß 2016: 139).

Aus der ausgeprägten Distanz zwischen den Kommunikationsvermögen auf Rezipienten- und Produzentenseite erwächst für letztere eine große Schwierigkeit. Leichte Sprache ist nämlich mitnichten leicht zu produzieren. Die konsequente Reduktion von Schreib- und Ausdrucksweisen erfordert einen hohen Konzentrationsaufwand und teilweise auch eine große Kreativität.

Software kann hier auf der Wort- und Satzebene erheblich unterstützen, wie die folgenden Abschnitte darlegen. Die derzeitigen Werkzeuge besitzen jedoch jenseits der Satz- oder spätestens der Absatzgrenze eine systematische Grenze. Was genau Schreib- und Übersetzungswerkzeuge leisten können und wie man sie für Leichte Sprache einsetzen kann, erklären die folgenden Abschnitte.

\section{$4 \quad$ Funktionsprinzipien der Schreib- und Übersetzungswerkzeuge}

Schreib- und Übersetzungswerkzeuge sind auf dem aktuellen Stand der Technik eben dies: Werkzeuge. Sie sind demnach Hilfsmittel, die einen qualifizierten Verfasser von Texten bei dessen Tätigkeit unterstützen. Nach der Taxonomie von Hutchins und Somers (1992: 148) handelt es sich demnach um so genannte maschinengestützte menschliche Übersetzung („machine-aided human translation") bzw. einen entsprechend geringen Automatisierungsgrad des technischen Schreibens. 
Die einzelnen Werkzeuge operieren dabei auf Ebene unterschiedlicher sprachlicher Einheiten: Terminologiedatenbanken verwalten inhaltliche Gegenstände sowie unterschiedliche - erlaubte und unerwünschte - Bezeichnungen für diese Gegenstände. Übersetzungs- und Textspeicher operieren auf Basis von Sätzen oder Satzsegmenten. Autorenwerkzeuge analysieren unterschiedliche Parameter der Sprachoberfläche und geben dann Hinweise zur Vereinfachung (s. hierzu Keller 2011, Arntz/Picht/Schmitz 2014: 232-233).

Die letztendliche Entscheidung über die sprachliche Gestaltung des Textes trifft jedoch stets ein Mensch. Die Werkzeuge vermindern dabei den cognitive load - die notwendigen Aufmerksamkeitsressourcen. Im Idealfall stehen diese dann für das Entwickeln, Behalten und die Reflexion der wirklich kreativen Anteile zur Verfügung (vgl. hierzu Hacker 1994: 85-86; Wickens/Carswell 2012: 134-135; Vidulich/Tsang 2012: 245, 263).

\subsection{Terminologiedatenbanken}

Terminologiedatenbanken (auch: „Terminologieverwaltungssysteme“ - „TVS“) sind die älteste und technisch einfachste Form von Schreibwerkzeugen (Arntz/Picht/Schmitz 2014: 221). Ihr Funktionsprinzip besteht darin, dass sie terminusbasiert, d.h. von der Bedeutung ausgehend, synonyme Ausdrücke verwalten (Arntz/Picht/Schmitz 2014: 235-236). Diese Ausdrücke können dabei aus einem oder mehreren Wörtern bestehen, oder es kann sich sogar um Mehrwortbildungen handeln, wie sie z. B. in romanischen Sprachen typisch sind.

In der Praxis besteht eine häufige Übersetzungsstrategie in Leichter Sprache darin, schwierige Ausdrücke zu paraphrasieren bzw. zu definieren. Das trifft auch für viele geläufige Wendungen $\mathrm{zu}$, die für typische Rezipienten von Leichte-Sprache-Texten genau dies längst nicht sind. Die Definition wiederum muss den Regeln der Leichten Sprache und den Anforderungen der Zielgruppengerechtigkeit ganz allgemein genügen.

Nehmen wir zum Beispiel einen Text über den Ausbildungsberuf „Näher/in im Damen- und Herrenschneiderhandwerk“. Dort heißt es z. B. 
„Wer Näher/in werden möchte, sollte manuelles Geschick und gutes Vorstellungsvermögen mitbringen."

(Landesbildungszentrum für Hörgeschädigte Hildesheim o.J. c)

Unser Übersetzungsteam fand damals die Lösung:

„Als Näher müssen Sie das können:

Sie müssen gut mit Ihren Händen arbeiten können.

Sie müssen sich Dinge gut vorstellen können.“

(Landesbildungszentrum für Hörgeschädigte Hildesheim 2013b)

Die Entscheidung fiel damals nach längerer Gruppendiskussion - erhebliche kreative Anstrengung also. Ein Terminologiemanagement-Tool hält nun diese einmal gefundene Übersetzung dauerhaft vor.

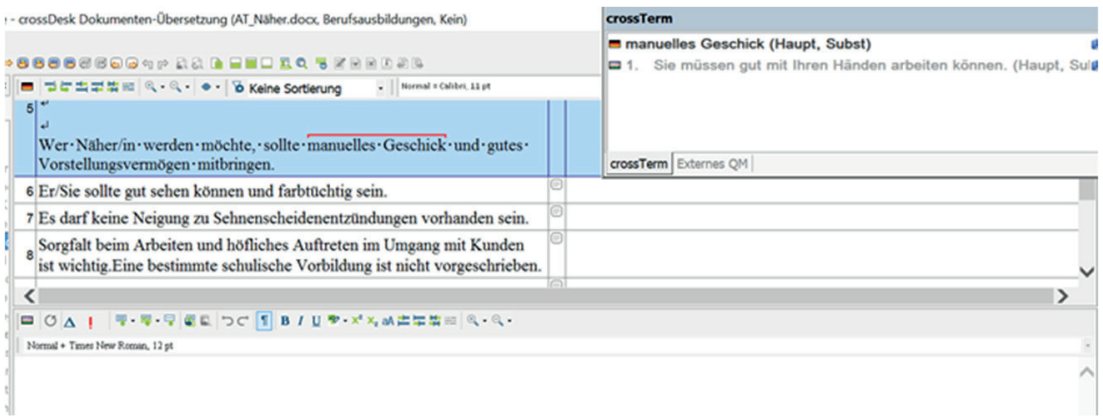

Abb. 1: Unterstützung durch Terminologiemanagement: wiedergefundener Ausdruck (li.) und Paraphrase als Übersetzung aus der Datenbank (Fenster oben re.) (Across 2014-2016)

Der Nutzen ist dabei doppelter Art: Erstens kann ein Übersetzer mit Fortschreiten eines Projekts auf einen Fundus vorhandener Lösungen zurückgreifen. Zweitens bleibt für die Adressaten die Konsistenz gewahrt: ein Ausdruck, eine identische Erklärung an allen entsprechenden Stellen im Text. Und ggf. kann der Übersetzer dann auch gezielt darauf verzichten, dieselbe Erklärung mehrfach zu wiederholen. Vor allem bei verschiedenen, aber ähnlich strukturierten 
Texten erfüllt das TVS seine Hauptfunktionen: Effizienzsteigerung und Vereinheitlichung.

\section{2 Übersetzungsspeicher}

Zentrales Funktionsprinzip von Übersetzungsspeichern (auch „Translation Memory Systeme“, kurz „TMS“ genannt) ist die Wiedererkennung ganzer Sätze (und ggf. auch Absätze) im Ausgangstext. Passend hierzu schlägt das Werkzeug dann diejenige Übersetzung vor, die dem wiedergefundenen Textabschnitt zugeordnet ist.

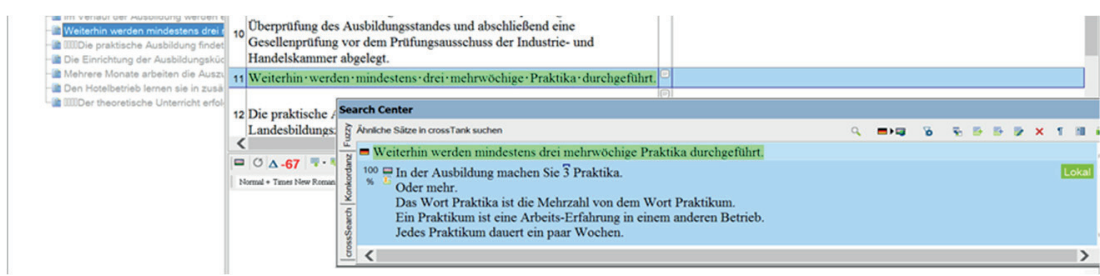

Abb. 2: In der Übersetzungsumgebung wird ein bereits übersetztes Textsegment grün markiert und die vorhandene Übersetzungslösung vorgeschlagen. (Across 2014-2016)

Eine Besonderheit von Übersetzungsspeichern besteht darin, dass sie auch so genannte „Fuzzy Matches“ erkennen können, d.h. Übersetzungseinheiten, die einem bereits übersetzten Textfragment nicht vollständig, sondern nur zu 90, 80 oder sogar 50 Prozent entsprechen. So ähneln sich beispielsweise folgende Sätze aus der Online-Darstellung von Ausbildungsberufen:

\begin{tabular}{|l|l|}
\hline Näher/in & Maler und Lackierer/in \\
\hline $\begin{array}{l}\text { Wer Näher/in werden möchte, sollte ma- } \\
\text { nuelles Geschick und gutes Vorstellungs- } \\
\text { vermögen mitbringen. }\end{array}$ & $\begin{array}{l}\text { Wer Maler und Lackierer/in werden } \\
\text { möchte, sollte manuelles Geschick } \\
\text { mitbringen und körperlich belastbar } \\
\text { sein. }\end{array}$ \\
\hline
\end{tabular}

Abb. 3: Zwei Sätze mit derselben Formulierung im Ausgangstext. (Landesbildungszentrum für Hörgeschädigte Hildesheim o.J. c, b) 
Stellt man die Schwelle für Matches im Übersetzungsspeicher niedrig genug ein, wird die Gleichförmigkeit der Sätze im Ausgangstext richtig erkannt. Somit wird dem Übersetzer die Formulierung „Als ... müssen Sie ... gut können:“vorgeschlagen (s. Abb.).

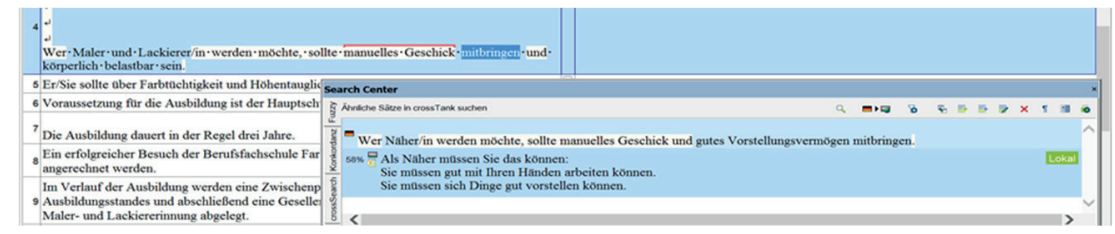

Abb. 4: Die Übersetzungsumgebung zeigt ein so genanntes „Fuzzy Match“ mit 58\% Übereinstimmung an. (Across 2014-2016)

Entsprechend wurde übersetzt:

„Als Maler und Lackierer müssen Sie das können:

Sie müssen gut mit Ihren Händen arbeiten können.

Sie müssen lange stehen können.

Sie müssen sich viel bewegen."

(Landesbildungszentrum für Hörgeschädigte Hildesheim 2013a)

Für den Übersetzer besteht der Vorteil darin, auf eine einmal gefundene - und womöglich bereits qualitätsgesicherte - Version zurückgreifen zu können. Gleichzeitig wird die Varianz der verwendeten sprachlichen Mittel reduziert. Dies wiederum vermeidet eine Überforderung der Rezipientinnen und Rezipienten durch zu viele Vokabeln und Strukturen.

Voraussetzung für eine sinnvolle Verwendung eines Übersetzungsspeichers für Leichte Sprache ist die Zuordnung der Paarungen aus Ausgangs- und Zielsprache nach Sinneinheiten. Dies ist nur schwer automatisch realisierbar, da in Leichter Sprache die Information eines standardsprachlichen Satzes quasi per definitionem auf mehrere Hauptsätze aufgeteilt werden muss

Entsprechend muss die Zuordnung für die Kombination Standardsprache Leichte Sprache (das „Alignment“) fast immer händisch durchgeführt werden. In 
einigen Systemen ist dies auch während des Übersetzens möglich. Die folgende Abbildung eines nachträglichen Alignments illustriert die wesentlichen Hürden.

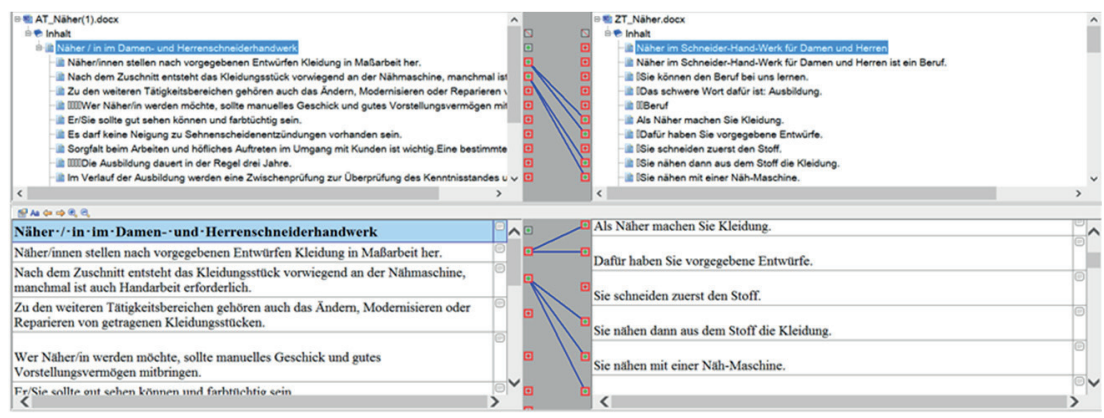

Abb. 5: Wesentliche Hürden bei der Nutzung von Übersetzungsspeichern für Leichte Sprache: Mehrere Sätze in Leichter Sprache bilden eine Übersetzungseinheit (unten); Einfügen erklärender Absätze ohne Gegenpart an der standardsprachlichen Textoberfläche (oben). (Across 20142016)

Die Arbeit mit Übersetzungsspeichern in Leichter Sprache hat jedoch Grenzen, wenn Texte sich zu unähnlich werden. Eine Lösung, die aber eine etwas andere Herangehensweise fordert, bieten Textspeicher bzw. Autorensysteme.

\subsection{Textspeicher}

Textspeicher funktionieren nach einem ähnlichen Prinzip wie Übersetzungsspeicher, durchsuchen aber Referenztexte in der Zielsprache nach Ähnlichkeiten. So findet ein Übersetzungsspeicher z. B. für die folgende Paarung auch kein Fuzzy Match mehr.

\begin{tabular}{|l|l|}
\hline Näher/in & Koch/Köchin \\
\hline Wer Näher/in werden möchte, sollte & Wer Koch/Köchin werden möchte, \\
manuelles Geschick und gutes Vorstel- & $\begin{array}{l}\text { sollte manuelles Geschick und Interesse } \\
\text { lungsvermögen mitbringen. }\end{array}$ \\
& \begin{tabular}{l} 
gen. \\
\hline
\end{tabular}
\end{tabular}

Abb. 6: Kein Fuzzy Match im Übersetzungsspeicher, Lösung durch Textspeicher. (Landesbildungszentrum für Hörgeschädigte Hildesheim o.J. c, a) 
Textspeicher weisen weitergehende Konfigurationsmöglichkeiten aus. So können die gesuchten Wörter in der eingegebenen Reihenfolge gesucht werden, oder es können alle Flexionsformen eines Satzes gesucht werden (s. Abb.).

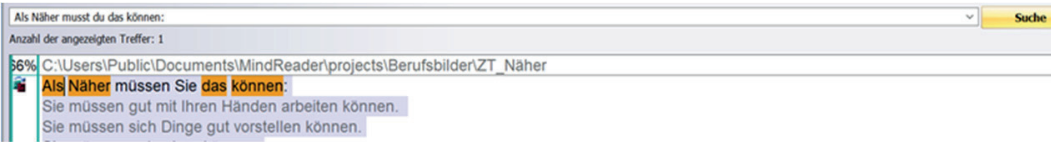

Abb. 7: Der Textspeicher findet weitere Flexionsformen einer Formulierung (MindReader Autorenunterstützung 2010)

Vor allem weist die Suche in Textspeichern in der Regel eine höhere Abweichungstoleranz auf. Dies liegt an der jeweiligen Implementierung, ist also nicht systematisch bedingt. Zusammen mit der Tatsache, dass die Zieltexte weniger Varianz aufweisen (sollten), ergibt sich eine höhere Trefferquote.

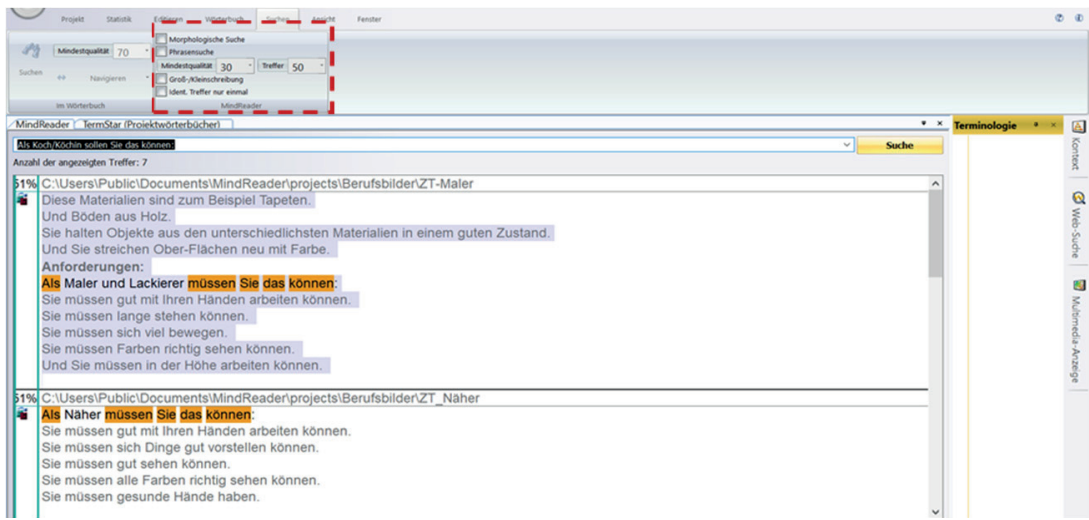

Abb. 8: Suche nach ähnlichen Segmenten im Textspeicher. Die gefundenen Entsprechungen weisen eine Übereinstimmung von $51 \%$ auf (s. Zeile ganz links). Theoretisch könnten sie so wenig wie 30\% aufweisen (s. Menüleiste). (MindReader Autorenunterstützung 2010)

Die Schwierigkeit eines Einsatzes von Textspeichern für die Leichte Sprache besteht darin, dass der Verfasser zumindest beginnen muss, einen Ziel-Satz zu formulieren, um dem System Input für dessen Suche zu liefern. Sowohl Übersetzungs- als auch Textspeicher basieren auf Referenzmaterial, das der Verfasser 
selbst zu besorgen und ins System einzustellen hat. Die systemseitige Hilfestellung beruht weitestgehend auf dem Abgleich mit bereits vorhandenem Material (Villiger 2014: 234-235; vgl. a. Bock 2014: 27-28). Den Weg zur Vertextungslösung weisen sie nur indirekt. Dazu kann ein Verfasser von Texten in Leichter Sprache auf regelbasierte Autorensysteme zurückgreifen.

\subsection{Autorensysteme}

Autorensysteme untersuchen den gerade in der Produktion begriffenen Text auf die Einhaltung bestimmter Regeln. Im Idealfall zeigt das System dann nicht nur an, welche Sätze, Absätze oder Fragmente nicht den Regeln z. B. Leichter Sprache entsprechen, sondern auch, wie diese optimiert werden können.

Im System sind Kriterien hinterlegt, auf die der eingegebene Text überprüft wird. Man unterscheidet im Allgemeinen eine statistische oder „flache" Analyse des sprachlichen Materials von einer „tiefen“. Letztere verfügt über mehr „Wissen “ über z. B. Wortarten, Phrasenkomplexität und Syntax (Carstensen 2017: 13 und 41-43). Ungeachtet des Namens kann eine flache Analyse viele Probleme des Schreibens in Leichter Sprache (und des Natural Language Processing im Allgemeinen) lösen helfen (Johnson 2009).

Die nachfolgende Abbildung illustriert den praktischen Einsatz eines Autorensystems. Dieses zeigt in einer Randleiste auf, in welchen Einzelkategorien der jeweilige Text die Kriterien sprachlicher Einfachheit überschreitet. Indem das Tool auf Klick alle entsprechenden Textstellen markiert, kann der Übersetzer gezielt an diesen arbeiten. Dabei werden z. B. lange Sätze durch Punkt in zwei Sätze aufgeteilt. Lange Wörter, Abstrakta und Metaphern werden durch kürzere und leichter verständliche Ausdrücke ersetzt. Das Autorentool selbst gibt hierzu vor allem allgemeine und prozedural formulierte Hinweise. Zusätzlich gibt es einen Lesbarkeitsindex aus. 


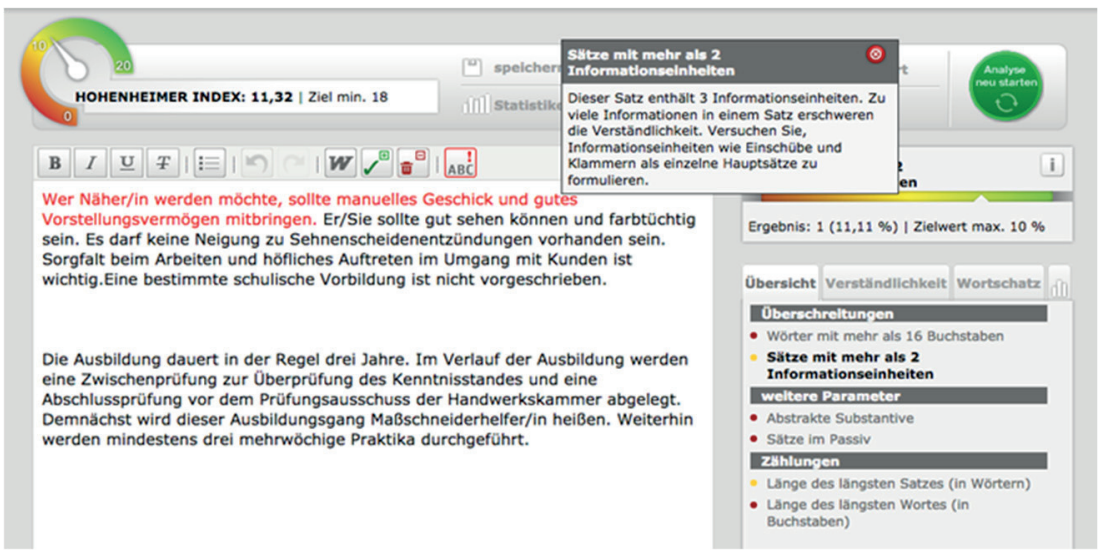

Abb. 9: Das Autorensystem zeigt Regelverstöße an und gibt Hinweise zur Verbesserung. (Textlab 2017)

Die meisten Tools bringen zusätzlich eine eigene Terminologieverwaltung mit. Auf einer „Whitelist“ können Anwender(innen) Ausdrücke eintragen, die in Texten verwendet werden sollen, also Vorzugsbenennungen. Auf eine schwarze Liste kommen unerwünschte Ausdrücke. Der Vorteil gegenüber alleinstehenden Terminologielösungen besteht in der Integration in die durchgeführten statistischen Analysen und in der leichten Anwendbarkeit für ungeübte Nutzer(innen). Der Nachteil besteht darin, dass das System von dem/der Nutzer(in) längst nicht so weitgehend konfiguriert werden kann wie ein dediziertes TVS.

Um die mit Hilfe des Autorensystems gefundenen Formulierungslösungen auf weitere Texte zu übertragen, müssten diese in einen Text- oder Übersetzungsspeicher übernommen werden. Die Werkzeug-Kombination muss also für das jeweilige Projektumfeld gezielt konfiguriert werden, um optimale Unterstützung zu erreichen. Doch selbst in Kombination stehen alle am Markt befindlichen Lösungen (und wohl das Übersetzen in Leichte Sprache allgemein) immer vor derselben Schwierigkeit: aus allen optimierten Einzelsätzen einen Text entstehen zu lassen (Bredel/Maaß 2016: 488-489). Eine neue Generation von Schreibwerkzeugen aus Basis der so genannten „Künstlichen Intelligenz“ (KI) könnte hier zumindest mittelfristig Abhilfe schaffen. 


\section{$5 \quad$ Schreibwerkzeuge auf Basis maschinellen Lernens}

Am 27.09.2016 gab Google die Umstellung seines Übersetzungsdienstes "Google translator" auf die so genannte „Neuronal Machine Translation“ bekannt. Damit kehrte das Thema (voll-)automatisches Übersetzen mit einem Schlag zurück ins Bewusstsein der Öffentlichkeit (Google 2016). Das Unternehmen DeepL veröffentliche 2018 seinen DeepL-Übersetzer (s. DeepL GmbH o.J. a). Das Programm übersetzt zwischen gängigen europäischen Sprachen sehr viel besser als bisher eingesetzte Algorithmen (DeepL GmbH o.J. b; Schwan 2017). Aktuell gibt es allerdings kein neuronales Netz, das sich explizit der Leichten Sprache widmet. Die am meisten fordernde Aufgabe beim Übersetzen in Leichte Sprache ist das Finden von passenden Leichte-Sprache-Realisierungen für komplexe Sachverhalte. Um diese Fähigkeit zu „erlernen“ benötigt auch das neuronale Netz große Mengen an so genannten Trainingsdaten. Dies sind aktuell noch Satzpaare, wie wir sie aus herkömmlichen Übersetzungsspeichern kennen (DeepL GmbH o.J. b). Für eine Erläuterung der Funktionsweise neuronaler Netze als Grundlage derartiger Werkzeuge verweise ich auf den Artikel von Krüger (2017). Der Gründer der Firma DeepL attestiert seinem Programm jedoch ein „hohes Textverständnis“, die Berichterstattung spekuliert in Richtung tatsächlichen inhaltlichen Verständnisses (Schwan 2017). Ein solches wäre Voraussetzung für die Überwindung der Herausforderung, die Leichte Sprache jenseits der Absatzgrenze darstellt.

\section{$6 \quad$ Fazit und Ausblick}

Dieser Beitrag stellt aktuelle und künftige Werkzeuge für die Erstellung von Texten in Leichter Sprache vor. Dabei geht er von den Besonderheiten dieser Varietät aus, die an anderer Stelle als Reduktion sprachlicher Mittel und notwendige Addition von Weltwissen vorgestellt wurden.

Das Ergebnis ist insofern ernüchternd, als bestehende Softwarewerkzeuge zwar einen Teil der monotonen Arbeit übernehmen (Terminologieverwaltungen, Übersetzungs- und Textspeicher) und auch Hilfestellungen bei der Umformulierung recht präzise platzieren können (Autorensysteme). Dazu bedarf es 
allerdings aufwändiger Vorarbeiten; die kreative Leistung beim Einsatz der verfügbaren Werkzeuge und beim Formulieren ist hoch anspruchsvoll - und verbleibt bei dem/der Nutzer(in). Die Systeme schlagen einfach in großen Datenmengen sprachliche Oberflächenphänomene nach. Ein wirkliches Eindringen in die Inhalte lässt aktuell die KI-Forschung erhoffen. Dies wäre nicht nur eine äußerst nützliche Anwendung, sondern auch eine Nagelprobe für die Algorithmen: Erkennen sie tatsächlich Informationen? Und können sie diese dann in Leichter Sprache erklären? 


\section{Literaturverzeichnis}

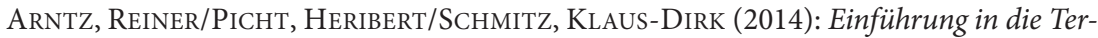
minologiearbeit. 7., vollständig überarbeitete und aktualisierte Auflage. Hildesheim, Zürich, New York: Olms.

Bock, BetTina M. (2014): „,Leichte Sprache`: Abgrenzung, Beschreibung und Problemstellungen aus Sicht der Linguistik“. In: JeKat, SusAnne/JÜngST, HeIKE EliSABETH/ SCHubert, Klaus/Villiger, Claudia (Hg.): Sprache barrierefrei gestalten. Perspektiven aus der Angewandten Linguistik. Berlin: Frank \& Timme (TRAnsÜD. Arbeiten zur Theorie und Praxis des Übersetzens und Dolmetschens, 69), 17-51.

Bredel, Ursula/MaAß, Christiane (2016): Leichte Sprache. Theoretische Grundlagen, Orientierung für die Praxis. Berlin: Dudenverlag (Sprache im Blick).

Carstensen, KaI-Uwe (2017): Sprachtechnologie. Ein Überblick. 2.2. Aufl. URL: http://kai-uwe-carstensen.de/Publikationen/Sprachtechnologie.pdf (letzter Zugriff: 22.02.2018).

DeEpL GmвH (о.J. a): DeepL Übersetzer. URL: https://www.deepl.com/translator (letzter Zugriff: 22.02.2018).

DeepL GmbH (о.J. b): Presseinformationen - Launch des DeepL Übersetzers. URL: https://www.deepl.com/press.html (letzter Zugriff: 22.02.2018).

Google (2016): A Neural Network for Machine Translation, at Production Scale. Unter Mitarbeit von Quoc V. Le und Mike Schuster. URL: https://research.googleblog.com/ 2016/09/a-neural-network-for-machine.html (letzter Zugriff: 25.02.2018).

HACKER, WINFRIED (1994): „Arbeits- und organisationspsychologische Grundlagen der Software-Ergonomie“. In: Eberleh, Edmund/Oberquelle, Horst/Oppermann, REINHARD (Hg.): Einführung in die Software-Ergonomie. Gestaltung graphisch-interaktiver Systeme; Prinzipien, Werkzeuge, Lösungen. 2., völlig neu bearb. Aufl. Berlin: de Gruyter (Mensch-Computer-Kommunikation Grundwissen, 1), 53-93.

Hutchins, William John/SOMers, Harold L. (1992): An introduction to machine translation. London: Academic Press.

JOHNSON, MARK (2009): „How the statistical revolution changes (computational) linguistics“. In: Proceedings of the EACL 2009. Workshop on the Interaction between Linguistics and Computational Linguist, 3-11.

Keller, Nicole (2011): „Neun auf einen Blick. Translation Memory Systeme“. In: MDÜ (5), 16-31.

KRÜGER, RALPH (2017): „Von Netzen und Vektoren. Neuronale maschinelle Übersetzung“. In: $M D \ddot{U}(1), 33-39$.

MAAß, Christiane (2015): Leichte Sprache. Das Regelbuch. Münster: Lit (Barrierefreie Kommunikation; 1).

SCHWAN, BEN (2017): Maschinenintelligenz: Der Besserübersetzer (heise Technology Review). URL: https://heise.de/-3814856 (letzter Zugriff: 26.02.2018). 
Vidulich, Michael A./Tsang, Pamela S. (2012): „Mental Workload and Situation Awareness“. In: SAlvendy, GAVRIEL (Hg.): Handbook of Human Factors and Ergonomics. 4. Aufl. Hoboken, NJ: John Wiley \& Sons, Inc, 243-273.

Villiger, Claudia (2014): „Standardisierung von Sprache als Zugang zu barrierefreier Kommunikation?" In: Jekat, Susanne/Jüngst, Heike Elisabeth/Schubert, Klaus/Villiger, Claudia (Hg.): Sprache barrierefrei gestalten. Perspektiven aus der Angewandten Linguistik. Berlin: Frank \& Timme (TransÜD. Arbeiten zur Theorie und Praxis des Übersetzens und Dolmetschens, 69), 221-265.

Wickens, Christopher D./CARswell, C. Melody (2012): „Information Processing“. In: Salvendy, Gavriel (Hg.): Handbook of Human Factors and Ergonomics. 4. Aufl. Hoboken, NJ: John Wiley \& Sons, Inc, 117-161. URL: http://dx.doi.org/ 10.1002/9781118131350.ch5 (letzter Zugriff: 26.02.2018).

\section{Primärquellen}

LANDESBILDUNGSZENTRUM FÜR HÖRGESCHÄDIGTE HiLdESHEIM (o.J. a): „Koch/Köchin“. URL: https://www.lbzh-hi.niedersachsen.de/startseite/berufliche_bildung_reha/berufsfelder/kochkoechin-156311.html (letzter Zugriff: 28.02.2018).

LANDESBILDUNGSZENTRUM FÜR HÖRGESCHÄDIGTE Hildesheim (o.J. b): „Maler/in und Lackierer/in“. URL: https://www.lbzh-hi.niedersachsen.de/startseite/berufliche_ bildung_reha/berufsfelder/malerin-und-lackiererin-156328.html (letzter Zugriff: 19.02.2018).

LANDESBILDUNGSZENTRUM FÜR HÖRGESCHÄdigte HildeSHeIM (o.J. c): „Näher/in im Damen- und Herrenschneiderhandwerk“. URL: https://www.lbzh-hi.niedersachsen.de/ startseite/berufliche_bildung_reha/berufsfelder/naeher--in-im-damen--und-herrenschneiderhandwerk-156318.html (letzter Zugriff: 19.02.2018).

LANDESBILDUNGSZENTRUM FÜR HÖRgESCHÄDIGTE HildesheIM (2013a): „Maler und Lackierer/in (Fachrichtung Gestaltung und Instandhaltung)“. Fassung in Leichter Sprache. URL: https://www.lbzh-hi.de/maler-und-lackierer.ls.html (letzter Zugriff: 19.02.2018).

LANDESBILDUNGSZENTRUM FÜR HÖRGESCHÄDigTe HildesheIM (2013b): „Näherin im Schneider-Hand-Werk für Damen und Herren“. Fassung in Leichter Sprache. URL: https://www.lbzh-hi.de/naeher.ls.html (letzter Zugriff: 19.02.2018).

\section{Verwendete Software}

ACross (2014-2016). Version 6.3: Across Systems GmbH.

MindReader AutorenunterstütZung (2010). Version 1.0 SP 4: STAR group.

Text-lab. Webanwendung (2017). Version: H\&H Communication GmbH. URL: https://www.text-lab.de/index.php (letzter Zugriff: 28.02.2018). 



\section{Barrierefreiheit und Inklusion am Theater: Das Projekt Inklusives Theater an der Uni Hildesheim}

\section{$1 \quad$ Einleitung}

Die Begriffe Barrierefreiheit und Inklusion werden in zahlreichen Texten, die die Forderung nach einer gleichberechtigten Teilhabe von allen Menschen am politischen und kulturellen Leben formulieren, häufig in einem Zuge genannt. So auch im niedersächsischen Aktionsplan „Inklusion. Für ein barrierefreies Niedersachsen“ (vgl. MS Nds. 2017), der konkrete Maßnahmen benennt, um barrierefreie Zugänge zum Informations-, Unterhaltungs-, und Kulturangebot von Behörden, Medienanstalten sowie Bildungs- und Kultureinrichtungen zu schaffen. Man darf daraus jedoch nicht schließen, dass diese Begriffe einfach synonym zu verwenden wären. Vielmehr handelt es sich um zwei grundlegend verschiedene Ansätze im Umgang mit Behinderung, insbesondere hinsichtlich der Frage, wo in der Gesellschaft die Norm verortet wird.

Bezogen auf den uns hier interessierenden Kulturbereich Theater sollte also die Frage, ob eine Aufführung als barrierefrei oder inklusiv bezeichnet werden kann, mit Blick auf das implizierte Publikum einer Inszenierung beantwortet werden. Wird etwa mit einer bestimmten Inszenierung eines Theaterstücks ursprünglich ein Publikum ohne Behinderungen adressiert und erst nachträglich und für einzelne Aufführungen auch Menschen mit Sinnesbehinderungen zugänglich gemacht, kann nicht von Inklusion gesprochen werden, sondern bestenfalls von einem Streben nach Barrierefreiheit dank des Einsatzes diverser Accessibility-Tools. Am Beispiel des Hildesheimer Projekts Inklusives Theater, das im Fokus dieses Beitrags steht, sollen die Unterschiede zwischen einem barrierefreien und einem inklusiven Ansatz herausgearbeitet und reflektiert werden. Das 2014 ins Leben gerufene Projekt zeichnet sich dadurch aus, dass es sich 
an ein heterogenes Publikum richtet und die Norm bei keiner Teilgruppe ansiedelt: weder bei Menschen ohne, noch bei Menschen mit Sinnesbehinderung (im konkreten Fall bei Menschen mit Hörbeeinträchtigungen), sondern eine Theaterform zu entwickeln sucht, die alle Gruppen gleichberechtigt anspricht und ihnen verschiedene Zugänge bietet.

Der Beitrag wird zunächst eine Klärung der beiden zentralen Begriffe Barrierefreiheit und Inklusion in Angriff nehmen (Punkt 2). Im Anschluss wird die Frage nach der Bedeutung von Gehörlosigkeit fürs Theater (Punkt 3) behandelt, bevor wir uns mit dem translatorischen Konzept der Ko-Translation des Projekts Inklusives Theater befassen (Punkt 4), aus dem bereits drei inklusive Inszenierungen hervorgegangen sind (weitere sind in Planung). Zuletzt wenden wir uns den Ergebnissen aus den an alle drei Inszenierungen anschließenden Publikumsbefragungen $\mathrm{zu}$, die als erkenntnisförderndes Instrument für die Weiterentwicklung des Projekts gewertet werden können (Punkt 5).

\section{Barrierefreiheit und Inklusion im Theaterkontext}

\subsection{Barrierefreiheit am Theater}

Barrierefreiheit zu schaffen bedeutet in dem hier betrachteten Kontext, dass kommunikative Hindernisse durch Hilfsmittel umgangen oder abgebaut werden sollen. Nach Jekat et al. (2014) können diese kommunikativen Barrieren in vier Kategorien eingeteilt werden: 1) Der rezipierenden Person fehlt es an Sprachkenntnis. 2) Die rezipierende Person hat kein ausreichendes Vorwissen, um die Mitteilung zu verstehen. 3) Die Mitteilung ist zu komplex formuliert. 4) Die Kommunikation erfolgt über Sinneskanäle, die nicht oder nur eingeschränkt zur Verfügung stehen (vgl. Jekat et al. 2014: 7).

Die Barrierefreie Kommunikation befasst sich vor allem mit den Barrieren 3 und 4. Stark vorangetrieben wurde die Umsetzung von Barrierefreiheit in Deutschland in den letzten Jahren bei medial gestützten Informationsangeboten. Das Thema Barrierefreiheit hat aber auch im Bereich der außerschulischen Bildung und Teilhabe am kulturellen Leben an Bedeutung gewonnen, wie in Artikel 30 der UN-Behindertenrechtskonvention, insbesondere Absatz 1 und 2 (vgl. BRK 2008: 26f.) gefordert wird. So wurde die Notwendigkeit erkannt, kulturelle 
Angebote wie Museumsausstellungen oder Theateraufführungen für unterschiedliche Zielgruppen zugänglich zu machen und Menschen mit Behinderungen die Gelegenheit zu geben, ihre kreatives Potenzial zu entfalten, selbst wenn in Deutschland bislang noch keine gesetzlichen Regelungen hierzu getroffen wurden. Entsprechend ist die Umsetzung von Barrierefreiheit in diesen Bereichen häufig auf die Initiative und das Engagement von Einzelpersonen zurückzuführen. Dazu können verschiedene Accessibility-Tools eingesetzt werden, wie die Technik der Audiodeskription für Menschen mit Sehbehinderung (s. den Beitrag von Benecke im vorliegenden Band) oder die Gebärdensprachverdolmetschung für gehörlose Personen (s. den Beitrag von Herrmann im vorliegenden Band). Diese intersemiotischen Formen von Translation werden einer bestehenden Inszenierung nachträglich hinzugefügt und können bis zu einem gewissen Grad auch in sie integriert werden. Ugarte Chacón bezweifelt allerdings, dass derartige Translationsprozesse eine neue Ästhetik zeitigen können (vgl. Ugarte Chacón 2015: 41 und 306). Hier soll keinesfalls die Wirksamkeit von Accessibility-Maßnahmen in Frage gestellt oder ihre Wichtigkeit bestritten werden. Sie sind unabdingbar, um sonst nicht zugängliche Bereiche des kulturellen Lebens im Sinne der UN-BRK zu öffnen, und sie leisten damit einen Beitrag zum Abbau von Diskriminierungen. Eine Bedingung für inklusive Inszenierungen ist jedoch die Reflexion der gesellschaftlichen Verhältnisse, der Mehrheitsgesellschaft und der hörend und sehend geprägten Domäne des Theaters. Durch Accessibility-Maßnahmen allein kann dies nicht geleistet werden.

\subsection{Inklusion am Theater}

Wenn es also nicht nur um den Abbau kommunikativer Barrieren geht, sondern um die gleichberechtigte Teilhabe aller Menschen am kulturellen Geschehen, müssen die existierenden Theaterformen überdacht und neue, inklusive Formen geschaffen und erprobt werden. In Abgrenzung zur Barrierefreiheit ist es dabei von Bedeutung, dass sowohl in der Theorie als auch in der Praxis nicht allein individuelle Ausgrenzungsmechanismen adressiert und behoben, sondern gesellschaftliche Veränderungen angestrebt werden, die Inklusion strukturell möglich machen (vgl. Kronauer 2010: 17f.). Ein zentraler Aspekt, den es hierbei zu beachten gilt, ist, dass Ausgrenzung bzw. Exklusion sich innerhalb 
einer Gesellschaft vollzieht, und nicht aus dieser heraus (vgl. Kronauer 2006: 29). Demnach mahnt der Inklusionsdiskurs eine kritische Reflexion der normativ wirkenden Mehrheitsgesellschaft und der durch sie marginalisierten Bevölkerungsgruppen an (vgl. Wünsche 2016: 195). Entsprechend richten sich inklusive Maßnahmen darauf, die Anerkennung von Vielfalt strukturell zu verankern. Dies kann nicht nur durch Einzelmaßnahmen, sondern muss auch durch die Förderung von Strukturen geschehen (vgl. Bentele 2012: 15).

Was bedeutet dies nun für kulturelle Einrichtungen wie das Theater? Das Ziel von Inklusion in diesem Bereich ist weniger die barrierefreie Nachbearbeitung eines für Hörende und Sehende geschriebenen und inszenierten Theaterstücks, damit es auch für Menschen mit Sinnesbehinderungen zugänglich wird, sondern die Erarbeitung von Inszenierungen, die diese Menschen einbezieht und gleichberechtigt alle Zielgruppen anspricht. Damit ist nicht die Umsetzung von Barrierefreiheit für alle erdenklichen Gruppen gemeint, sondern eine Theaterarbeit, die sich an Menschen mit und ohne Sinnesbehinderungen richtet und diese Personengruppen nach Möglichkeit auch in die Produktion dieser Inszenierungen einbindet. Derzeit liegt der Schwerpunkt des Projekts Inklusives Theater bei der Zielgruppe gehörloser, schwerhöriger und hörender Menschen. Eine Ausweitung auf weitere Personengruppen ist für die Zukunft in Planung.

\section{Gehörlosigkeit - Schwerhörigkeit - Hörendsein am Theater}

Wie bereits an verschiedenen Stellen im Band ausgeführt, ist die Zielgruppe hörender, schwerhöriger und gehörloser Menschen äußerst heterogen (vgl. die Beiträge von Hennies und Mälzer/Wünsche im vorliegenden Band). Eine Differenzierung ist nicht nur bezüglich des medizinisch feststellbaren Hörvermögens möglich, sondern auch im Hinblick auf die Muttersprache (Lautsprache oder Gebärdensprache) und die Kultur (hörend oder gehörlos bzw. taub) der einzelnen Person. Grundsätzlich gilt es aber festzuhalten, dass aus dem Zusammentreffen von Gehörlosen und Hörenden häufig eine prekäre Kommunikationssituation resultiert, „bei welcher die sprachliche Verständigung aufgrund der unterschiedlichen kommunikativen Grundvoraussetzungen nur eingeschränkt 
bis gar nicht möglich ist" (Ugarte Chacón 2015: 37). Im Projekt steht der kulturelle Definitionsansatz von Gehörlosigkeit im Vordergrund.

Bei einer heterogenen Zielgruppe, wie sie im Projekt impliziert wird, ist es kaum möglich, von einer einheitlichen Rezeptionssituation für alle zu sprechen, da neben individuellen Unterschieden nicht nur unterschiedliche Kommunikationsvoraussetzungen bestehen, sondern auch verschiedene Muttersprachen verwendet werden. Dabei bleibt es unabdingbar, die existierenden kommunikativen Barrieren abzubauen oder gar nicht erst virulent werden zu lassen. Grundsätzlich lassen sich vier Modi kommunikativer Teilhabe von gehörlosen und schwerhörigen Menschen beschreiben: Lautsprache, Gebärdensprache, Schriftsprache und lautsprachbegleitende Gebärden (LBG) (vgl. Rausch 2011: 65). In den Inszenierungen des Projekts wird bisher auf LBG verzichtet, die anderen drei verbalen Modi kommen in verschiedenen Formen und mit unterschiedlichen Funktionen zum Einsatz. Einen zentralen Raum nimmt die schriftsprachliche Übertitelung ein, da sie von allen anvisierten Teilzielgruppen rezipiert und somit als verbindendes Element genutzt werden kann, das kommunikative Barrieren zumindest bis zu einem gewissen Grad zu umgehen vermag.

Die Verwendung unterschiedlicher Kommunikationsmodi auf der Bühne kann dazu führen, dass die Botschaft von wechselnden Teilen des Publikums nicht verstanden wird - ganz gleich welcher Teilgruppe sie angehören. Verstehen und Nichtverstehen lassen sich also nicht auf das Fehlen oder Vorhandensein eines Hörvermögens zurückführen, sondern sind bei der kulturell bedingten Sprachkenntnis (Laut- oder Gebärdensprache) zu suchen. Der Fokus wird damit von einer defizitären Konstruktion gehörloser Personen als „Hörende, die nicht hören“ (Ugarte Chacón 2015: 129) gelenkt auf kulturelle Unterschiede zwischen Hörenden und Gehörlosen und ihrer Begegnung im Bühnen- und Publikumsraum. Dadurch soll eine gleichberechtigte Rezeptionssituation für alle Personengruppen geschaffen werden, auch wenn diese natürlich nicht identisch sein kann. Entsprechend werden im Projekt Inklusives Theater strukturelle Änderungen im Sinne der Inklusionsdefinition (vgl. Kronauer 2010: 17f.) am Theater angestrebt, das eine nach wie vor hörend geprägte Domäne ist und von Ugarte Chacón als exkludierend beschrieben wird: Denn Theater von gehörlosen 
Personen in Deutscher Gebärdensprache (DGS) ist häufig ausschließlich für gehörlose Menschen konzipiert und Theater von Hörenden ausschließlich für Hörende, selbst wenn auf der Bühne auch gehörlose Schauspieler(innen) auftreten oder DGS verwendet wird (vgl. Ugarte Chacón 2015: 24). In diesem Kontext ist es wichtig, zur Demarginalisierung der DGS beizutragen und ihren Status als eigenständige Sprache anzuerkennen. Viel zu oft nämlich würde im Theater für Hörende „Gebärdensprache [...] zur rein ästhetischen Bewegung, deren Sprachinhalt sekundär ist“ (ebd.). In welcher Mischung bzw. in welcher Relation zueinander die drei Zeichensysteme Lautsprache, Schriftsprache und DGS im Dienste einer inklusiven Inszenierung verwendet werden können, wird im Folgenden theoretisch reflektiert.

\section{Theatertranslation im Dienste der Inklusion}

Für die Konzeption inklusiver Inszenierungen müssen Translationsprozesse verschiedene Bedingungen und Funktionen erfüllen. Insbesondere gilt es, die nötigen organisatorischen und rechtlichen Voraussetzungen für eine Ko-Translation (vgl. Mälzer 2016: 218ff.; 2017: 184f.; Mälzer/Wünsche 2018: 41) zu schaffen, damit die Translation im Entstehungsprozess der Inszenierung verankert werden kann und nicht der fertigen Inszenierung aufgepfropft wird. Nur unter dieser Bedingung kann der Einsatz von Übertiteln im Zusammenspiel mit Gebärdensprache und Lautsprache über ihre funktional-kommunikative Dimension hinaus auch eine ästhetische Funktion auf der Bühne erlangen. Eine weitere Bedingung für die Entstehung einer inklusiven Inszenierung ist die Enthierarchisierung der auf der Bühne verwendeten Kommunikationsmodi: Deutsche Lautsprache (DLS), Deutsche Schriftsprache (DSS) und Deutsche Gebärdensprache (DGS) - d. h. eine Abkehr von der bei barrierefreien Aufführungen üblichen unidirektionalen Übersetzungsrichtung DLS $\rightarrow$ DGS bzw. DLS $\rightarrow$ DSS in Form von Übertiteln. 
4.1 Die translatorisch-kommunikative Funktion der Theatertranslation

Bei einer inklusiven Inszenierung bilden die Übertitel den gemeinsamen Nenner zur Verständnissicherung für das Publikum. Sie sind jedoch nicht nur Zielsprache für schwerhörige und gehörlose Menschen, wie dies etwa bei Gehörlosenübertiteln/SDH (vgl. Mälzer/Wünsche im vorliegenden Band) der Fall ist, sondern stellen ein verbales Zeichensystem unter anderen innerhalb einer mehrsprachigen Theaterform dar, die sich an alle Teile des Publikums gleichberechtigt wendet und zu diesem Zweck die Übersetzungsrichtungen variiert. Die Übertitel werden zum einen genutzt, um Lautsprache (DLS) in Schriftsprache (DSS) zu übersetzen, können aber auch der Translation von Gebärdensprache (DGS) in Schriftsprache (DSS) dienen. Darüber hinaus kann auch zwischen DLS und DGS übersetzt werden. Alle genannten Übersetzungsrichtungen sind zudem umkehrbar. Hieraus ergibt sich ein Translationskonzept, das sich schematisch wie folgt darstellen lässt (vgl. Mälzer 2017: 187; Mälzer/Wünsche 2018: 50):

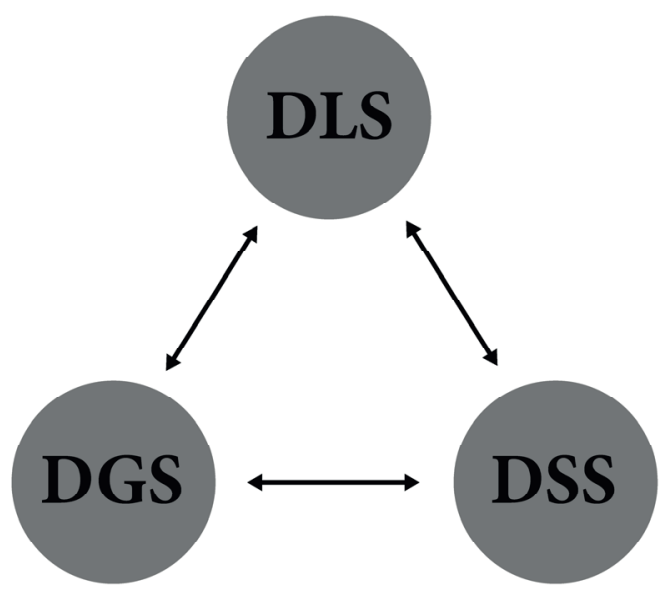

Abb. 1: Zeichensysteme und Übersetzungsrichtungen bei einer inklusiven Inszenierung 
Durch die Enthierarchisierung der verbalen Zeichensysteme und der Übersetzungsrichtungen können alle Teilzielgruppen gleichermaßen mit Nichtverstehen konfrontiert werden und die Erfahrung machen, auf Translation angewiesen zu sein. Auf diese Weise werden eigene und fremde Barrieren deutlich, ohne dass die in unserer Gesellschaft üblichen Hierarchien der verbalen Zeichensysteme Geltung hätten, also der Lautsprache der Vorzug gegeben würde.

\subsection{Die ästhetische Dimension der Übertitelung}

Ein Aspekt, auf den das Projekt Inklusives Theater besonderen Wert legt, ist zudem die Nutzung des ästhetischen Potentials der Theaterübertitelung. Statt wie in barrierefreien Aufführungen als Paratext (Genette 1982), also als Beiwerk zum eigentlichen Basistext, eingesetzt zu werden, gehören Übertitel in inklusiven Inszenierungen zum theatralen Zeichenrepertoire (vgl. Mälzer/Wünsche 2018: 45ff.). Dabei wird das ästhetische Ausdruckpotenzial von Schrift, in Anlehnung an die Comicforschung, die etwa die Expressivität des Letterings untersucht (Schüwer 2002: 209, De Assis 2015), im Bühnenraum ausgelotet und u.a. für die Vermittlung prosodischer und paraverbaler Elemente genutzt. Diese Form der Übertitelung rückt auch in die Nähe des Performance Writing, das Allsop wie folgt definiert: „It is a frame through which a range of sonic, visual, graphic and movement performance are brought into view - the textualities of sonic, graphic and movement performance; the performance of sonic, graphic and movement texts" (Allsop 1999: 77). Aus diesem Blickwinkel gewinnen neben der Materialität von Schrift auch ihre durch technische Medien erweiterten Ausdrucksmöglichkeiten an Bedeutung: insbesondere hinsichtlich Bewegung und Temporalität.

Dass sich die translatorisch-kommunikative Funktion und die ästhetische Dimension von Übertiteln nicht gegenseitig ausschließen, unterstreichen auch verschiedene Translationswissenschaftler(innen). So wendet sich Foerster (2010: 83) von der häufig postulierten Unsichtbarkeit oder Unauffälligkeit von Untertiteln (vgl. u. a. Becquemont 1996) ab, an der sich zu weiten Teilen auch die gängige Übertitelungspraxis (vgl. Griesel 2000: 28) orientiert. Griesel hinterfragt zurecht den Sinn der „Unauffälligkeitsmaxime“ (2016: 338), und McClarty (2012: 138f.) spricht sich gar dezidiert für eine kreative Nutzung von 
Untertiteln aus, die jedoch nur durch eine interdisziplinäre Herangehensweise umgesetzt werden könne. Das Theater erscheint besonders geeignet für eine solche Nutzung, da es ,im Vergleich zu filmischen Medien den Übertiteln weitaus mehr Gestaltungsmöglichkeiten und Projektionsorte zur Verfügung lässt“ (Mälzer/Wünsche 2018: 47). So können Übertitel etwa zur eigenständigen Figur oder zum Requisit werden und/oder der Konstruktion des Bühnenraums dienen.

\subsection{Die Ko-Translation als Voraussetzung für inklusive Inszenierungen}

Um der ästhetischen Dimension von Übertiteln einen Raum zu bieten und die Enthierarchisierung der verbalen Zeichensysteme zu ermöglichen, muss die Inszenierung Hand in Hand mit der Translation entstehen. Es genügt nicht, wie bei einer klassischen fremdsprachigen Theaterübertitelung üblich, kurz vor der Premiere Übersetzer(inne)n die Einrichtung einer Übertitelung anzuvertrauen, sondern die Übersetzer(innen) müssen den Entstehungsprozess der Inszenierung von der Konzeption über den gesamten Zeitraum der Probenarbeit begleiten können: beratend, übersetzend und folglich bis zu einem gewissen Grad mitinszenierend. Es erscheint daher sinnvoll, statt von Translation von KoTranslation (vgl. Mälzer 2016: 218) zu sprechen, im Sinne einer Ko-Produktion, bei der die Regie und die Übersetzer(innen) zwar unterschiedliche Aufgaben übernehmen, ihre Arbeit im Idealfall aber fortlaufend aufeinander abstimmen. Damit die Umsetzung des Translationskonzepts gelingen kann, sind für die Übersetzer(innen) Erfahrungen mit der intralingual-intersemiotischen Translation, profunde Kenntnisse über und ein regelmäßiger Austausch mit Vertreter(inne)n der Zielgruppe(n) unabdingbar. Dieser Austausch kann über die Einbindung von kompetenten Zielgruppenvertreter(inne)n in den Arbeitsprozess erfolgen oder aber im Anschluss an die Aufführungen über die Durchführung empirischer Studien. 


\section{Die Rezeption inklusiver Theaterstücke}

Es wurde bereits angedeutet, dass sich die Rezeptionsformen eines Theaterstücks innerhalb eines heterogenen Publikums zwangsläufig unterscheiden. Von umso größerer Bedeutung ist daher das Feedback des Publikums zu den Aufführungen.

Der Fokus der Rezeptionsstudien lag und liegt daher auf der Frage, inwiefern das oben beschriebene Translationskonzept von der Zielgruppe gehörloser, schwerhöriger und hörender Personen akzeptiert wird und, nach Selbstauskunft der Zuschauer(innen), zum Verständnis des Stücks beiträgt. Konkret galt es herauszufinden, wie die Integration von verschiedenen Kommunikationsmodi und Übersetzungsformen auf der Bühne vom Publikum aufgenommen wird, ob die Aufführungen als hinreichend verständlich eingeschätzt werden und wo noch Optimierungspotential liegt. Die Aspekte, auf die sich die Publikumsbefragungen konzentrierten, waren neben persönlichen Angaben zu Alter, Muttersprache(n), Hörvermögen und Häufigkeit von Theaterbesuchen auch die Verständlichkeit der Aufführung und damit verbunden die Frage, ob die Übertitel den sprechenden oder gebärdenden Schauspieler(inne)n leicht zugeordnet werden konnten und gut lesbar waren. Die Studien wurden mittels Fragebögen durchgeführt, die nach den jeweiligen Aufführungen an das Publikum ausgeteilt und in der Regel vor Ort ausgefüllt wurden.

\subsection{Die Inszenierungen}

Die erste Inszenierung, die im Rahmen des Projekts Inklusives Theater entstanden ist, Club der Dickköpfe und Besserwisser von Klub Kirschrot, wurde von dem Theaterkollektiv in Zusammenarbeit mit einem Team aus Übersetzer(inne)n der Universität Hildesheim am Theaterhaus Hildesheim erarbeitet. Sie wurde 2014/2015 gemeinsam produziert und hatte im Februar 2015 Premiere. Im Sinne der Ko-Translation entwickelten Übertitler(innen) gemeinsam mit dem Kollektiv Inhalt und Form der Übertitel. Darüber hinaus wurden von der Gruppe gedrehte Videos mit Untertiteln versehen, die während der Aufführung projiziert wurden. 
Die zweite Inszenierung, von außen zu nah von BwieZack entstand im Folgejahr ebenfalls als Ko-Translation am Theaterhaus Hildesheim. Sie hatte im Juni 2016 Premiere. Das Publikum wechselte bei den Aufführungen mehrfach den Platz vor und auf der Bühne, zudem gab es interaktive Momente zwischen dem Publikum und den Performerinnen - zwei Aspekte, die für die Übertitelung eine Herausforderung darstellten. Um dem zu begegnen, wurden neben einem fest verankerten Beamer auch Handprojektoren verwendet, die von den Performerinnen frei im Bühnenraum bewegt werden konnten. Als weitere Besonderheit kam hinzu, dass ein Teil der Übertitel live eingetippt und variable Kombinationen von Übertiteln eingeblendet wurden, um Schreibprozesse der Hauptfigur darzustellen und die Interaktion zwischen Schauspielerinnen und dem Publikum einzufangen.

Sowohl in der ersten als auch in der zweiten Inszenierung waren ausschließlich hörende Performerinnen auf der Bühne. Passagen in Gebärdensprache wurden mit der Unterstützung von gehörlosen bzw. gebärdensprachkompetenten Dozierenden und Gebärdensprachlehrer(inne)n einstudiert.

Das dritte Stück, zu dem eine Rezeptionsstudie durchgeführt wurde, war „Mädchen wie die“ von Evan Placey, das in Januar 2018 unter der Regie von Wera Mahne am Jungen Schauspiel Hannover Premiere feierte. Einschränkend muss erwähnt werden, dass bei dieser Inszenierung nicht von einer Ko-Translation im oben beschriebenen Sinne gesprochen werden kann, sondern die Übersetzer(innen) in erster Linie beratend tätig waren. Dennoch war es interessant, beim Publikum ein Feedback zum Zusammenspiel von DGS, DLS und DSS in Form von Übertiteln und weiteren Videoprojektionen einzuholen.

\subsection{Fazit des Publikums: Empfehlungen für inklusive Theaterprojekte}

An den Ergebnissen der drei Studien wird deutlich, dass das Projekt mit seiner Zielsetzung, Translation nicht nur als Mittel zur Schaffung von Barrierefreiheit, sondern auch ästhetisch als theatrales Zeichen in Inszenierungen einzusetzen, bisher erfolgreich war. Aus den Rückmeldungen des Publikums haben sich jedoch auch Kritikpunkte herauskristallisiert, die bei künftigen inklusiven Inszenierungen stärker Beachtung finden sollten. 


\subsubsection{Positive Aspekte}

Grundsätzlich war die Rückmeldung zu allen drei Inszenierungen positiv. Besonders hervorgehoben wurde in allen Befragungswellen der innovative Charakter der Inszenierungen. Dazu zählen vor allem folgende Aspekte: das Zusammenspiel der verschiedenen Zeichenmodalitäten DLS-DGS-DSS auf der Bühne; die kreative, in das Bühnenbild integrierte Übertitelung; die Verwendung und das Sichtbarmachen der Deutschen Gebärdensprache. Die ästhetische Dimension der Translationsformen wird vom Publikum also in allen Inszenierungen offenbar gut angenommen. Im Hinblick auf die kommunikativ-translatorische Funktion stießen vorrangig die ersten beiden Inszenierungen auf positive Resonanz, und zwar vor allem bei gehörlosen und schwerhörigen Befragten. Durch den konstanten Einsatz von mindestens zwei der Zeichenmodalitäten sahen sie das Verständnis der Stücke gewährleistet. Dass es zur dritten Inszenierung in dieser Hinsicht weniger Feedback gab, lag daran, dass der Fokus der Inszenierung auf der ästhetischen Verwendung der Übertitel lag und die Verständnissicherung für alle Zielgruppen in den Hintergrund trat.

\subsubsection{Optimierungspotentiale}

Aus der ersten und dritten Befragungswelle ist festzuhalten, dass der Einsatz von zu lauter Musik oder zu lauten Geräuschen bisweilen als störend empfunden werden kann und dies bei der Konzeption entsprechend zu berücksichtigen ist. Vor allem für Träger(innen) von Hörgeräten, die die akustischen Reize der Umgebung verstärken, können plötzliche laute Geräusche auf der Bühne als unangenehm oder schmerzhaft empfunden werden. Wiederum vornehmlich positiv beurteilt wurde in diesem Zusammenhang das Aufdrehen der Bässe als intersemiotische Teilübersetzung von Musik: Diese wurden dank der entstehenden Vibrationen des Holzbodens im Publikumsraum auch für gehörlose und schwerhörige Menschen haptisch erfahrbar.

Rückmeldungen zur zweiten Inszenierung ließen darauf schließen, dass die Verwendung von aggressivem Vokabular im Übertitel teils auf Ablehnung stieß. Die dritte Inszenierung verwendete zwar eine weitaus größere Anzahl zum Teil auch vulgärer Ausdrücke - allerdings nur in DLS und DGS und nicht im Übertitel. Die Hauptzielgruppe dieser Inszenierung war zudem etwas älter als bei den ersten beiden Inszenierungen. Negative Rückmeldungen zur Vulgarität der 
Sprache gab es aus dieser Befragungswelle nicht. Hieran schließt sich die Frage nach der unterschiedlichen Wirkung von gesprochener/gebärdeter und geschriebener Sprache. Letztere wurde zwar für die Untertitelung von Filmen bereits eingehend betrachtet (vgl. Gottlieb 2002: 191f.; Ivarsson/Carroll 1998: 126f.), für das Theater und hier im Speziellen für die Zielgruppe Kinder und Jugendliche fehlen jedoch eingehendere Untersuchungen.

Aus dem Feedback zur Leserlichkeit der Übertitel in den einzelnen Stücken lassen sich verschiedene Empfehlungen für künftige Inszenierungen ableiten: Im Hinblick auf die formale Gestaltung von Übertiteln spielt offenbar gerade für jüngere Zielgruppen die Frage des Textumfangs der Übertitel eine wichtige Rolle. Dies gilt sowohl für den einzelnen Übertitel als auch für die gesamte Übertitelung. Neben unerwünschten Ermüdungseffekten muss auch die Tatsache in Betracht gezogen werden, dass das Lesen nicht bei allen $\mathrm{Zu}$ schauer(inne)n gleichermaßen beliebt sein muss. In Bezug auf die Projektion der Übertitel während der Aufführungen ist daher zumindest auf ausreichend lange Standzeiten zu achten. Die Erforschung von Standzeiten für verschiedene Zuschauergruppen stellt derzeit allerdings ein Forschungsdesiderat dar.

Optisch sollten die Übertitel so gestaltet sein, dass sie von allen Plätzen im Publikum aus gut gelesen werden können. Besondere Sorgfalt ist bei Inszenierungen geboten, in denen Übertitel an ganz unterschiedlichen Stellen des Bühnenraums projiziert werden. Auch wenn Übertitel mobil, etwa über Handprojektoren, einblendet werden, gilt diesem Aspekt das Augenmerk.

\section{Weiterentwicklungspotenziale des Projekts und sich anknüpfende Forschungsfragen}

In den kommenden Jahren gilt es im Projekt eine ganze Reihe von Fragen zu bearbeiten. Zum einen soll untersucht werden, ob das bisher entwickelte Konzept, das zunächst an Kindern und Jugendlichen erprobt wurde, auch bei einem älteren Publikum auf Akzeptanz stößt. Des Weiteren soll das Konzept verstärkt nicht nur im Rahmen von Stückproduktionen erprobt, sondern auch auf bereits existierende Dramenvorlagen angewendet werden. So soll in den folgenden Produktionen geprüft werden, wie sich kanonische, für ein hörendes Publikum 
geschriebene Dramentexte für eine inklusive Inszenierung eignen. Hieran knüpft sich die Frage, wie sich unter diesen Bedingungen die Zusammenarbeit zwischen Übersetzer(inne)n und Regie bei der Ko-Translation verändern.

Der inklusive Ansatz soll auch verstärkt auf einer weiteren Ebene untersucht werden. So gilt es zu erforschen, wie sich das Zusammenspiel von hörenden, schwerhörigen und gehörlosen Schauspieler(inne)n auf die Probenprozesse auswirkt. Dies kann durch Videographien der Proben und Interviews mit allen Beteiligten analysiert werden, um Empfehlungen für eine inklusive Probenarbeit entwickeln zu können.

Eine weitere Frage wird sein, wie sich das Translationskonzept ggf. verändert, wenn nicht nur im Hinblick auf die Rezeption des Theaterstücks durch das Publikum Barrieren überwunden werden müssen, sondern auch die Kommunikationshürden zwischen den Schauspieler(inne)n respektive den Figuren auf der Bühne thematisiert werden und den Zuschauer(inne)n ggf. ihr Verstehensvorsprung gegenüber den Figuren verdeutlicht werden muss. Dies kann sich auf die Konzeption der Übertitel auswirken, die nicht mehr nur die äußere Kommunikation mit dem Publikum gewährleisten müssen, wenn aus DGS und DLS in DSS übersetzt wird, sondern auch die gestörte innere Kommunikation zwischen den Figuren darstellen.

Interessant wird es außerdem sein, aufgrund diverser Sprachstile und Sprechtempi bei künftigen Dramentexten zu beobachten, welche unterschiedlichen Kürzungsstrategien bei der Übertitelung vorgenommen werden müssen und wie mit nicht zeitgenössischer Sprache umgegangen werden sollte.

Zudem können auch weitere Accessibility-Tools für das Projekt Inklusives Theater untersucht werden. Um nicht nur sprachliche, sondern ggf. auch inhaltliche Barrieren zu umgehen, wären in diesem Zusammenhang bspw. Videoeinführungen zu erforschen, die, wie Audioeinführungen für Blinde (vgl. Mälzer/Wünsche im vorliegenden Band), auch einem Publikum aus gehörlosen, schwerhörigen und hörenden Menschen die Rezeption der Aufführung erleichtern können. 


\section{Literaturverzeichnis}

Allsop, RiC (1999): „Performance Writing“. In: Performing Arts Journal 21, 1, 76-80.

De Assis, ÉRICO GonçAles (2015): „The Letterer as a Translator“. In: MÄlzer, NATHAlie (Hg.) Comics - Übersetzungen und Adaptionen. Berlin: Frank \& Timme, 251-267.

BECQUEMONT, DANIEL (1996): „Le sous-titrage cinématographique: contraintes, sens, servitudes“. In: GAMBIER, Yves (Hg.): Les transferts linguistiques dans les médias audiovisuels. Villeneuve d'Ascq: Presses Universitaires du Septentrion, 145-155.

BENTELE, KATRIN (2012): „Menschenrecht und Ethik - Überlegungen zur Inklusion von Menschen mit einer Hörschädigung“. In: Hintermair, Manfred (Hg.): Inklusion und Hörschädigung. Diskurse über das Dazugehören und Ausgeschlossensein im Kontext besonderer Wahrnehmungsbedingungen. Heidelberg: Median, 13-28.

BRK (2008): Die UN-Behindertenrechtskonvention. Übereinkommen über die Rechte von Menschen mit Behinderungen. In: https://www.behindertenbeauftragte.de/ SharedDocs/Publikationen/UN_Konvention_deutsch.pdf?_blob=publicationFile\&v=2 (letzter Zugriff: 19.03.18).

FOERSTER, ANNE (2010): „Towards a creative approach in subtitling: a case study“. In: Díaz Cintas, Jorge/Matamala, Anna/Neves, Josélia (Hg.): Media for All 2. New Insights into Audiovisual Translation and Media Accessibility. Amsterdam/New York: Rodopi, 81-98.

Genette, GÉrard (1982): Palimpsestes. La littérature au second degré. Paris: Éditions du Seuil.

Gottlieb, Henrik (2002): „Untertitel. Das Visualisieren filmischen Dialogs“. In: FriedRICH, HANS-EDWIN (Hg.): Schrift und Bild im Film. Bielefeld: Aisthesis, 185-214.

Griesel, Yvonne (2016): „Einen offenen Trilog wagen!“ In: RicHTER, JULIA/ZwisCHENBerger, Cornelia/Kremmel, Stefanie/Spitzl, Karlheinz (Hg.): (Neu-) Kompositionen. Aspekte transkultureller Translationswissenschaft. Berlin: Frank \& Timme, 331-348.

GRIESEL, YVONNE (2000): Translation im Theater: die mündliche und schriftliche Übertragung französischsprachiger Inszenierungen ins Deutsche. Frankfurt am Main u. a.: Peter Lang.

IVArsson, JAN/CARroll, MARY (1998): Subtitling. Simrishamn: TransEdit.

Jekat, Susanne J./Jüngst, Heike Elisabeth/Schubert, Klaus/Villiger, Claudia (2014): „Barrieren abbauen. Einleitung“. In: Jekat, Susanne J. et al. (Hg.): Sprache barrierefrei gestalten. Perspektiven aus der Angewandten Linguistik. Berlin: Frank \& Timme, 7-16.

KronAUER, MARTIN (2010): „Einleitung - Oder warum Inklusion und Exklusion wichtige Themen für die Weiterbildung sind“. In: Kronauer, MARTIN (Hg.): Inklusion und Weiterbildung. Reflexionen zur gesellschaftlichen Teilhabe in der Gegenwart. Bielefeld: Bertelsmann, 9-23. 
Kronauer, Martin (2006): „Exklusion“ als Kategorie einer kritischen Gesellschaftsanalyse. Vorschläge für eine anstehende Debatte“. In: Bude, HeInZ/Willisch, ANDREAS (Hg.): Das Problem der Exklusion. Ausgegrenzte, Entbehrliche, Überflüssige. Hamburg: Hamburger Edition, 27-45.

MälZER, NATHAlIE/WÜNSCHE, MARIA (2018): Inklusion am Theater. Übertitel zwischen Ästhetik und Translation. Bern u. a.: Peter Lang.

Mälzer, Nathalie (2017): „Inklusion im Theater“. In: Gerland, Juliane (Hg.): Kultur.Inklusion.Forschung. Weinheim: Beltz Juventa, 182-200.

MÄLZER, NATHALIE (2016): „Audiodeskription Museum - Ein inklusiver Audioguide für Sehende und Blinde“. In: MÄlzer, NATHAlIE (Hg.): Barrierefreie Kommunikation Perspektiven aus Theorie und Praxis. Berlin: Frank \& Timme, 209-229.

MCClarty, REBECCA (2012): „Towards a multidisciplinary approach in creative subtitling“. In: Agost, Rosa/Orero, Pilar/Di Giovanni, Elena (Hg.): Multidisciplinarity in Audiovisual Translation. MonTI 4 2012, Alicante: Publicaciones de la Universidad, 133-153.

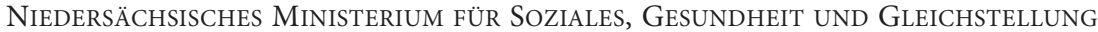
(MS NDS.) (2017): Aktionsplan Inklusion 2017/2018 für ein barrierefreies Niedersachsen. Schritte zur Umsetzung der UN-Behindertenrechtskonvention. URL: https://www.ms.niedersachsen.de/download/114629/AKTIONSPLAN_INKLUSION_ 2017_2018.pdf (letzter Zugriff: 19.03.2017).

RAUSCH, CHRISTFRIED (2011): Hörbehinderung und Teilhabe. Hörschädigung: Einfluss auf Biografie und Potential für den Bildungsprozess. Dissertation. Martin-Luther-Universität Halle-Wittenberg, Philosophische Fakultät III - Erziehungswissenschaften. URL: http://digital.bibliothek.uni-halle.de/download/pdf/1241438?name=H\%C3\% B6rbehinderung\%20und\%20Teilhabe (letzter Zugriff: 19.03.2018).

SCHÜWER, MARTIN (2002): „Erzählen in Comics: Bausteine einer plurimedialen Erzähltheorie“. In: NÜNNING, VERA/NÜNNING, ANSGAR (Hg.): Erzähltheorie transgenerisch, intermedial, interdisziplinär. Trier: WVT, 185-216.

Ugarte Chacón, RAFAel (2015): Theater und Taubheit. Ästhetiken des Zugangs in der Inszenierungskunst. Bielefeld: transcript.

WÜNSCHE, MARIA (2016): „Das Spannungsfeld zwischen Barrierefreiheit und Inklusion am Beispiel der Theaterübertitelung“. In: Mälzer, Nathalie (Hg.): Barrierefreie Kommunikation - Perspektiven aus Theorie und Praxis. Berlin: Frank \& Timme, 193208. 
EEVA RANTAMO/SWENJA SCHUM

\section{Museumstexte - Zum Abbau sprachlicher Barrieren in Museen und Ausstellungen}

\section{$1 \quad$ Einleitung}

Das Museum ist eine Welt aus Texten. Entgegen dem ersten Anschein, der das Augenmerk auf Objekte lenkt und selbst Sprachdokumente wie Bücher und Urkunden zunächst als Objekte präsentiert, übernehmen sehr schnell die mit diesen Objekten verknüpften Texte die Leitung des Gedankens, der Wahrnehmung und der Interpretation des zu Sehenden. Als Institutionen der Wissensund Kulturvermittlung, aber auch als Orte des ästhetischen, ethischen und empathischen Erlebens sind Museen ohne Texte, ohne sprachliche Mitteilung kaum vorstellbar.

Dies meint nicht allein die in den Ausstellungen tatsächlich lesbaren Texte. Auf der Grundlage umfänglicher Besucherbefragungen untersuchte Pierre Bourdieu 1966 den sozialen Charakter europäischer Kunstmuseen (Bourdieu/ Darbel 2006). Diese öffneten und verschlossen sich ihren Besucher(inne)n auch durch die Absenz von Texten, durch die stillschweigend gesetzte Norm, das Publikum bringe sein Verständnis der Kunstwerke, seine ästhetische Erfahrung, die „Liebe zur Kunst“ selbstverständlich mit (Bourdieu/Darbel 2006: 13ff.). Die Forscher konstatierten bereits hier ein „Gefühl des Unbehagens“ auf Seiten vieler Besucher(innen), die dem musealen Raum einen "sakralen Charakter" zuschrieben (Bourdieu/Darbel 2006: 85).

Im Hinblick auf die Barrierefreie Kommunikation erscheinen Texte im $\mathrm{Mu}$ seum so in zweifacher Weise: Einerseits sind sie selbst schon Bestandteil einer museologisch modernen, unterschiedlich weitreichenden sozialen Barrierefreiheit, indem sie sich bemühen, Bildungsprivilegien auszugleichen. Andererseits stellen sie als Texte jedoch selbst Barrieren dar, indem sie schwer zu erkennen, schwer zu lesen und schwer zu verstehen sind. Erste Studien belegen, dass sich 
Besucher(innen) mit besonderen Bedürfnissen aus dem musealen Raum exkludiert fühlen (Rehm 2017; s. exemplarisch Hohmaier 2015 zum Exklusionsempfinden von Jugendlichen mit Förderbedarf). Mit den nationalen wie internationalen rechtlichen Maßnahmen zur Gleichstellung von Menschen mit Behinderungen entstand ein zusätzlicher Druck auf die Museen, im Sinne einer möglichst umfänglichen Barrierefreiheit aktiv zu werden. Abgesehen von grundsätzlich umzusetzenden baurechtlichen Normen trugen die Aktivitäten und Bemühungen um mehr Barrierefreiheit jedoch in den meisten Fällen Projektcharakter - dies vor allem deshalb, weil sie häufig aus zusätzlich eingeworbenen Projektmitteln finanziert werden mussten.

Vor diesem Hintergrund strebt der vorliegende Beitrag einen Überblick über Texte und sprachliche Barrieren in Museen und Ausstellungen an; es sollen zudem Möglichkeiten zum Abbau dieser sprachlichen Barrieren präsentiert werden, die in den letzten Jahren aus verschiedenen Projekten hervorgegangen sind. Einige dieser Projekte werden hier exemplarisch angeführt.

Der Beitrag gliedert sich dabei in drei Teile. Im ersten Teil (Kapitel 2) wird zunächst ein umfassender Textbegriff für das Museum entwickelt: Es soll verdeutlicht werden, dass sich der Besuch einer Ausstellung oder eines musealen Raums aus der Rezeption verschiedener Teiltexte zusammensetzt, welche die Besucher(innen) vor unterschiedliche kommunikative Hürden stellen können. Der Überblick über die verschiedenen Teiltexte im Museum kann anschließend dabei helfen, aufzuzeigen, wo (und in welchen Formen) Konzepte zur Reduzierung kommunikativer Barrieren ansetzen müssen, um diese Barrieren gezielt und systematisch abzubauen.

Im zweiten und dritten Teil des Beitrags (Kapitel 3 und 4) werden anschließend Konzepte und Methoden zur barrierefreien Aufbereitung von Ausstellungen und Museen zusammengetragen: Während im zweiten Teil literaturgestützt gearbeitet wird und der Fokus auf Studien und Erkenntnissen aus dem Bereich der wissenschaftlichen Forschung liegt, präsentieren wir im dritten Teil Ansätze und Methoden aus der museumspädagogischen Berufspraxis aus Deutschland und Finnland. Dabei wurden einige Projekte von der Agentur „Kulturprojekte - Inklusive Kulturarbeit“ durchgeführt, in der eine der Autorinnen dieses Artikels, Eeva Rantamo, tätig ist. 
Das Museum für eine größere Zahl von Besucher(inne)n zu öffnen, liegt im eigenen Interesse der Institution: Im Rahmen vielfältiger öffentlicher Sparbemühungen sind Etats umkämpft und Besucherzahlen sind Argumente. Daneben sehen sich viele Museen und andere Kultureinrichtungen vermehrt als gesellschaftliche Akteure, die durch ihre Arbeit zu gesellschaftlichen Diskussionen beitragen möchten. Alle diese Aufgaben und Aktivitäten erfordern einen bewussten Einsatz verschiedener Textformen, um den Besucher(inne)n auch sprachlich die Tür zu öffnen.

\section{Der Museumsbesuch als Zusammenspiel verschiedener Teiltexte}

Um zu verstehen, an welchen Stellen und in welchen Situationen der Besuch eines Museums oder einer Ausstellung benachteiligte Besucher(innen) vor kommunikative Hürden stellen kann, ist es zunächst funktional, einen Überblick über die verschiedenen Arten von Texten zu gewinnen, mit denen Besucher(innen) vor, während und nach dem Museumsbesuch konfrontiert sind. Wo werden Texte rezipiert, die Informationen vermitteln und so zum Verständnis der Gesamtbotschaft einer Ausstellung beitragen sollen?

Zur Systematisierung soll an dieser Stelle auf das klassische Textmodell nach Genette (1987/2014) zurückgegriffen werden. Genette untersucht literarische Werke und hält fest, dass ein literarischer Text stets von verschiedenen Paratexten flankiert ist. Mit Paratexten meint er ,alle jene Begleittexte, die einem literarischen Werk auf seinem Weg durch die Öffentlichkeit zur Seite gehen“ (Weinrich 2014: 7); Genette selbst beschreibt sie folgendermaßen:

[Ein] Text präsentiert sich jedoch selten nackt, ohne Begleitschutz einiger gleichfalls verbaler oder auch nicht-verbaler Produktionen wie einem Autorennamen, einem Titel, einem Vorwort und Illustrationen. Von ihnen weiß man nicht immer, ob man sie dem Text zurechnen soll; sie umgeben und verlängern ihn jedenfalls, um ihn im üblichen, aber auch im vollsten Sinn des Wortes zu präsentieren: ihn präsent zu machen, und damit seine ,Rezeption' und seinen Konsum (...) ermöglichen. (Genette 1987/2014: 9) 
Die Paratexte unterscheidet Genette weiterhin in Peritexte und Epitexte. Als Peritexte bezeichnet er Teiltexte, die sich im unmittelbaren Umfeld des Haupttextes befinden und mit diesem räumlich verknüpft sind (wie der Titel oder das Vorwort, Kapitelüberschriften oder Anmerkungen; Genette 1987/2014: 12). Unter Epitexten versteht er solche Texte, die sich zwar immer noch im Umfeld des Textes befinden, ihn präsentieren und diskutieren; die Epitexte sind räumlich und zeitlich jedoch vom Haupttext losgelöst (Genette nennt hier zum Beispiel medienvermittelte Interviews oder Gespräche; Genette 1987/2014: 12).

Auch in Museen sind die ausgestellten Werke von verschiedenen Paratexten flankiert; auch hier stehen den Exponaten verschiedene erläuternde Texte zur Seite, welche die Werke präsent machen und ihre Rezeption erleichtern. Diese Texte können in ganz unterschiedlichen Formen realisiert sein: Zum einen werden Exponate in ihrem unmittelbaren Umfeld von geschriebenen Texten (auf Schildern, Schautafeln usw.) begleitet. Weitere geschriebene Texte liegen in Form von Broschüren oder Katalogen vor, die eine Ausstellung begleiten; auch Flyer oder der Internetauftritt eines Museums bereiten auf den Besuch einer Ausstellung vor. Des Weiteren werden die Exponate in einer Ausstellung von gesprochenen Texten flankiert: In Führungen oder Audioguides werden den Besucher(inne)n Informationen und Hintergrundwissen vermittelt, die das Verständnis des Gesehenen unterstützen und festigen sollen. Zu den gesprochenen Texten können weiterhin Präsentationen, Podiumsdiskussionen oder Interviews mit Künstler(inne)n und/oder Kurator(inn)en gezählt werden, in denen das Konzept einer Ausstellung oder eines Museums erläutert wird. Weiterhin sind auch museumspädagogische Angebote wie Workshops etc. relevant. Unter Rückgriff auf die Einteilung nach Genette lassen sich die verschiedenen Teiltexte im Museum folgendermaßen systematisieren: 
Peritexte

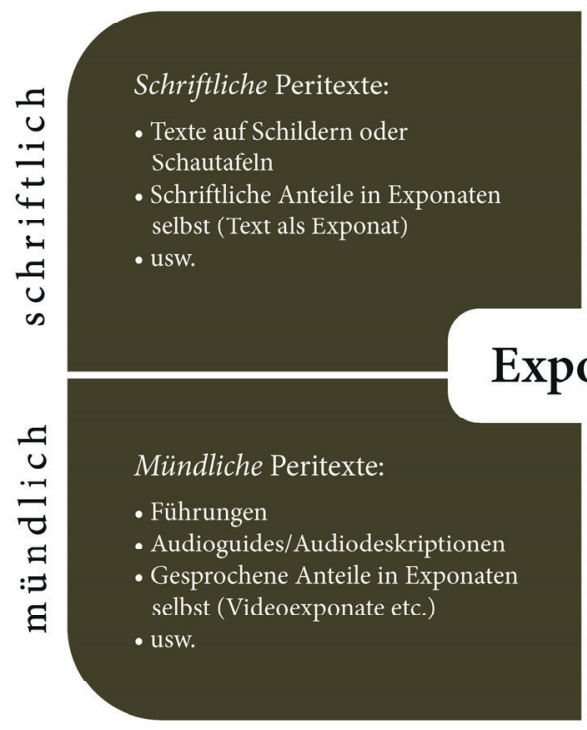

Epitexte

Schriftliche Epitexte:

- Flyer

- Broschüren

- Kataloge

- Internetauftritt des Museums

- usw.

Abb. 1: Paratexte (flankierende Texte) im Museum

Der Übertrag des Konzepts von Genette auf museale Räume zeigt, dass Kunstwerke und Exponate in Museen und Ausstellungen von verschiedenen Texten flankiert sind. Diese Texte können schriftlich und/oder mündlich realisiert sein und über verschiedene Sinneskanäle rezipiert werden, die gemeinsam das individuelle Kulturerlebnis konstruieren. Die Vermittlung des zu Sehenden findet stets im Zusammenspiel mit weiteren Gestaltungselementen des Museumsraums statt (s. exemplarisch Bertron/Schwartz/Frey 2006: 90 zur Bedeutung eines passenden Lichtdesigns, welches Struktur, Erlebbarkeit und Atmosphäre schafft); wir konzentrieren uns im vorliegenden Beitrag aber auf die sprachliche Vermittlung von Informationen in geschriebener und gesprochener Form.

Im folgenden zweiten Teil des Artikels soll der Blick nun auf konkrete Konzepte und Methoden zum Abbau von Barrieren in Museums- und Ausstellungstexten gerichtet werden. Es wird hier zunächst literaturgestützt gearbeitet; so 
soll der Fokus auf wissenschaftlichen Betrachtungen zur Reduzierung kommunikativer Barrieren liegen, während im dritten Teil des Beitrags Projektbeispiele aus der museumspädagogischen Berufspraxis folgen.

\section{Ansätze zum Abbau kommunikativer Barrieren im Museum}

Bis zum Erscheinen dieses Bandes ist die wissenschaftliche Forschungsliteratur zum Abbau sprachlicher Barrieren in Ausstellungen und Museen wenig ausgedehnt. Erste Hinweise auf kommunikative Barrieren und Vorschläge zu deren Abbau liegen hauptsächlich in Form von Berichtsliteratur zu einzelnen Projektarbeiten vor (vgl. exemplarisch Al Masri-Gutternig/Reitstätter 2017; Siegert 2017). Im Sinne einer Systematisierung der vorliegenden Ansätze ordnen und präsentieren wir diese mithilfe der im ersten Teil des Beitrags modifizierten Gliederung von Teiltexten nach Genette.

\section{Reduzierung sprachlicher Barrieren im Bereich der schriftlichen Peritexte}

Zum Bereich der schriftlichen Peritexte gehören all diejenigen geschriebenen Texte, die sich im unmittelbaren Umfeld der Exponate in einer Ausstellung befinden (z. B. Schilder neben den Ausstellungsstücken, Schautafeln etc.). Diese Texte können Besucher(innen) mit besonderen kommunikativen Bedürfnissen dann vor Herausforderungen stellen, wenn diese aus unterschiedlichen Gründen Probleme beim Rezipieren und Verstehen standardsprachlicher und/oder fachsprachlicher Texte haben. Eine Methode zum Abbau solcher Kommunikationsbarrieren ist die Aufbereitung schriftlicher Informationen in einer komplexitätsreduzierten Form, z. B. in Leichter oder Einfacher Sprache, die in den vergangenen Jahren in mehreren Ausstellungen und Museen umgesetzt wurde (z. B. im Salzburg Museum oder im Deutschen Schifffahrtsmuseum in Bremerhaven, s. dazu Al Masri-Gutternig/Reitstätter 2017 bzw. Siegert 2017). Siegert betont, dass Angebote in Leichter Sprache „kein Additiv, sondern integraler Bestandteil der Ausstellungskonzeption" sein sollten, da sonst Akzeptanz für diese Textform fehle (Siegert 2017: 485). Mälzer und Wünsche führen diesen Gedan- 
ken weiter und stellen fest, dass erst die ästhetische Berücksichtigung zugänglichkeitsfördernder Angebote und Maßnahmen zu wirklich inklusiven Momenten führt (vgl. Mälzer/Wünsche zur Inklusion im Theater in diesem Band).

Werden Texte an der Wand angebracht oder auf andere Weise fest installiert, sind generell eine funktionale Positionierung der Texte, eine gute, blend- und reflexfreie Ausleuchtung sowie gute Kontraste und eine angemessene Schriftgröße (insbesondere für Menschen mit Sehbehinderungen) relevant (RNIB 2003: 64). Für diese Zielgruppe können die schriftlichen Peritexte in einer Ausstellung zudem in Braille-Schrift aufbereitet werden; zu bedenken ist hierbei, dass nur eine verhältnismäßig kleine Gruppe von blinden und schwer sehbehinderten Menschen das Lesen der Braille-Schrift erlernt hat. Eine zusätzliche auditive Aufbereitung der Textinhalte ist also unumgänglich, um allen sehbeeinträchtigten Besucher(inne)n Partizipation zu ermöglichen.

\section{Reduzierung sprachlicher Barrieren im Bereich der mündlichen Peritexte}

Exponate sind in einer Ausstellung nicht nur von schriftlichen Peritexten umgeben, sondern werden auch von verschiedenen mündlichen Texten flankiert: In Führungen oder mit Audioguides werden Informationen zum Museum und zu den Ausstellungsstücken bereitgestellt, die insbesondere gehörlose Besucher(innen) oder Besucher(innen) mit einer Hörschädigung von der gleichberechtigten Teilnahme ausschließen, da sie aufgrund ihrer sensorischen Einschränkung gesprochene Sprache nicht oder nur sehr eingeschränkt wahrnehmen können. Viele Museen bieten aus diesem Grund Führungen in Gebärdensprache an.

Einige Museen und Kultureinrichtungen haben ihr Führungsangebot für Menschen mit besonderen kommunikativen Bedürfnissen erweitert und es um Führungen in Leichter und/oder Einfacher Sprache ergänzt. Hierzu ist anzumerken, dass das Konzept der Leichten Sprache ursprünglich als Konzept für die geschriebene Sprache entwickelt wurde, da die umfassende Umsetzung ihrer komplexen Regeln auf Wort-, Satz- und Textebene einen gewissen Vorlauf und Planungsgrad der Texte erfordert (Maaß 2015: 12). Eine spontane, mündliche Produktion regelkonformer Leichte-Sprache-Texte erscheint aus diesem Blickwinkel schwer umsetzbar (Bredel/Maaß 2016: 29). Da die in Besucherführungen gesprochenen Texte jedoch zumeist präzise vorbereitet, nahezu „gescripted“ 
werden, erscheint eine Anwendung des Konzepts auf mündlich realisierte Teiltexte im Museumskontext zumindest denkbar.

Eine große Textgruppe bilden im Bereich der mündlichen Peritexte alle Formen von Audioguides oder Audioführungen. Audioguides sind „akustische Führungen insbesondere durch Museen und Ausstellungen, die mithilfe spezieller tragbarer Geräte abgespielt und in der Regel über Kopfhörer rezipiert werden können“" (Popp 2013: 40). Eggert führt aus, dass es sich bei Audioguides um eine „Hybridform“ aus medialer und personeller Vermittlung handele, „denn durch das Gerät wird der Kommunikationstyp Führung simuliert und modifiziert" (Eggert 2010: 13). Allein die Tatsache, dass ein Informationstext im Audioformat vorliegt, macht ihn jedoch noch nicht barrierefrei (RNIB 2003: 35): $\mathrm{Zu}$ unterscheiden ist das (zunächst technische) Format der Audioguides, welches von allen Besuchergruppen genutzt wird, von speziellen Audiodeskriptionen für blinde und stark sehbeeinträchtigte Besucher(innen). Audiodeskriptionen können Teil eines Audioguide-Textes sein; sie liefern eine orientierende Beschreibung des zu Sehenden, die jedoch möglichst neutral gehalten sein sollte, um gerade dem blinden oder stark sehbeeinträchtigten Publikum nicht $\mathrm{zu}$ viele Interpretationsvorgaben $\mathrm{zu}$ machen („There are, though, dangers in providing recorded information which over-interprets a work - telling people how to look rather than what the work is. A delicate balance has to be struck." RNIB 2003: 39).

Detaillierte Informationen und Anweisungen zu Führungen und Audioguides für blinde und sehbeeinträchtigte Besucher(innen) gibt beispielsweise das RNIB im hier zitierten Talking Images Guide; in diesem Guide wird auch auf Modelle sensorischer Führungen mit ergänzenden Tastmaterialien eingegangen (RNIB 2003: 28ff.).

Im Sinne inklusiver Angebote für ein diverses Publikum mit verschiedenen kommunikativen Kompetenzen und Bedürfnissen wurden in den vergangenen Jahren innovative Konzepte für Audioguides entwickelt, die verschiedene Arten der Vermittlung (Audiodeskription, Soundeffekte, Musik usw.) vereinen. Neves beschreibt, dass solche „angereicherten“ Audioguides die Sinneserfahrungen aller Besucher(innen) verstärken können, die Phantasie und die Neugierde anregen und so zur physischen und kognitiven Auseinandersetzung mit Ausstellungsinhalten einladen: 
[We] understand Enriched Descriptive Guides to be (audio) guides, in which factual information has been 'enriched' through the creative use of description, sound effects and music, to provide thinking prompts that fuel the senses, invite cognitive and/or physical exploration, and capture the uniqueness of the cultural context the guide relates to. Unlike sound painting and descriptive guides that are primarily directed towards blind users, EDGs take all users into account in the belief that this approach will make culture accessible to people of any age, cultural background and personal profile. When combined with other forms of interpretative mediation strategies, EDGs will contribute towards holistic augmented experiences that trigger the imagination, stimulate the acquisition of knowledge and the desire to explore the exhibit or cultural environment through personal and social interaction. (Neves 2016:55)

Die Tatsache, dass viele Museumsbesucher(innen) ein Smartphone mit sich tragen, hat in den letzten Jahren dazu geführt, dass einige größere Museen und Galerien eigene Apps zur Führung durch ihre Ausstellungsräume entwickelt haben und zum kostenlosen Download anbieten (Jankowska et al. 2017: 114). Dass solche Apps auf mitgebrachten Endgeräten auch ein Potenzial zum Abbau sprachlicher Barrieren bergen, zeigt ein Projekt aus Polen: In drei großen Kunstmuseen (dem Nationalmuseum Warschau, dem Nationalmuseum Krakau und dem Museum für Gegenwartskunst in Krakau) wurde eine App namens Open Art getestet, die in Form kurzer Videoclips Beschreibungen zu ausgewählten Werken der Ausstellungen anbietet (Jankowska et al. 2017: 115). Die Videoclips setzen sich dabei aus verschiedenen visuellen Materialien (wie Fotos, kurzen Videoausschnitten, Künstlerportraits und Abbildungen von Archivmaterialien) zusammen und werden durch eine Narration in zwei wählbaren Sprachen ergänzt (Polnisch und Englisch; Jankowska et al. 2017: 115). Wählbar sind zudem eine Untertitelung der gesprochenen Inhalte (auf Polnisch oder Englisch) sowie deren Aufbereitung in (polnischer) Gebärdensprache.

In einer kleinen Pilotstudie mit blinden und sehenden, gehörlosen und hörenden Besucher(inne)n konnten Jankowska et al. zeigen, dass die angebotenen Videoclips sowohl in ihrer Länge, ihrem Inhalt, dessen Aufbereitung und Schwierigkeitsgrad von allen Testgruppen als hilfreich und überwiegend positiv 
bewertet wurden (Jankowska et al. 2017: 122ff.). Auch die Bedienung der App erhielt positive Bewertungen (Jankowska et al. 2017: 128f.); eine blinde Testperson betonte dabei, dass sie die Möglichkeit der Nutzung privater Endgeräte gegenüber den üblichen Audioguides bevorzuge, da sie mit ihrem eigenen Gerät besser umgehen könne und das Erlernen der Handhabung eines fremden Geräts entfalle (Jankowska et al. 2017: 126). Um festzustellen, ob individualisierbare Smartphone-Apps nach dem Vorbild der Open Art-Anwendung in Zukunft eine zweckmäßige Alternative zu herkömmlichen Audioguide-Lösungen darstellen können, müssten diese Ergebnisse jedoch in größeren Testungen ähnlicher Formate bestätigt werden. Zu bedenken wären hierbei neben der Nutzerperspektive auch der Aufwand in Produktion und technischer Umsetzung der Inhalte sowie in der Anpassung musealer Räume auf neue (Technik-)Formate.

\section{Reduzierung sprachlicher Barrieren im Bereich der schriftlichen Epitexte}

Neben den besprochenen Peritexten, die die Exponate in ihrem direkten Umfeld flankieren, rezipieren Besucher(innen) auch außerhalb des unmittelbaren Ausstellungsraums verschiedene Texte, die zum Gesamterlebnis des Museumsbesuchs beitragen. Solche Epitexte sind räumlich und zeitlich losgelöst von den Exponaten und bereiten Besucher(innen) beispielsweise auf den Besuch des Museums vor, indem sie wichtige organisatorische Informationen liefern oder eine erste Vorschau auf die in der Ausstellung gezeigten Objekte ermöglichen.

$\mathrm{Zu}$ den schriftlichen Epitexten, die Besucher(innen) vor oder nach dem $\mathrm{Mu}$ seumsbesuch rezipieren, gehören zunächst Flyer, Broschüren und andere Informations- und Werbematerialien. Nach Föhl (2007: 180) müssen auch solche Marketingaktivitäten bei der Bestrebung nach mehr Barrierefreiheit Beachtung finden. Flyer- und Broschürenformate können (zusätzlich zur Originalversion) in einer komplexitätsreduzierten Variante aufbereitet werden. Als Format bieten sich hier Flyer und Broschüren an, die den standardsprachlichen Text und dessen intralinguale Übersetzung vereinen (z. B. in Form einer Wendebroschüre); so wird einer Stigmatisierung des Publikums entgegengewirkt, das auf Texte in vereinfachter Sprache angewiesen ist. Föhl (2007: 180) betont zudem, dass verschiedene Zielgruppen „ganz spezifische Angewohnheiten [haben], Informationen weiterzugeben bzw. zu rezipieren“ - so kommunizieren Menschen mit einer Hörschädigung ihm zufolge häufig per SMS, während sehbehinderte 
Menschen besondere Anforderungen an Printprodukte stellen (Föhl 2007: 180). Er schlussfolgert, dass vorhandene Marketingmaßnahmen und -materialien für verschiedene Zielgruppen modifiziert werden müssen: „Andernfalls besteht die Gefahr, dass barrierefreie Angebote offeriert, aber nicht wahrgenommen werden, weil das fokussierte Publikum gar nicht erreicht wird“ (Föhl 2007: 180; vgl. auch den Beitrag zu Medienbarrieren von Rink in diesem Band).

Betrachtet man weitere spezielle Zielgruppen wie Forschende mit einem besonderen wissenschaftlichen Interesse, fallen auch wissenschaftliche Kataloge zu bestimmten Sammlungen oder Ausstellungen in die Kategorie der schriftlichen Epitexte. Im Hinblick auf eine Reduzierung von Barrieren kann hier zunächst die Frage gestellt werden, ob diese Texte (auch) ein breiteres Publikum ansprechen und sich (auch) an die museums- und forschungsexterne Öffentlichkeit richten; andere Textkategorien unterliegen den speziellen kommunikativen Bedürfnissen der jeweiligen Fachadressat(inn)en.

Eine besondere Relevanz kommt im Bereich der schriftlichen Epitexte auch dem Internetauftritt eines Museums und der zugänglichen Gestaltung von dessen Web-Oberflächen zu: Der Museumsbesuch beginnt für die meisten Besucher(innen) schon zu Hause mit der Planung des Besuchs, der Vorbereitung der Anfahrt, einem Blick auf die Eintrittspreise und einer ersten thematischen Einstimmung. Werden Informationen auf der Internetseite des Museums nicht in zugänglicher Form aufbereitet, machen sich Besucher(innen) womöglich gar nicht auf den Weg dorthin; eine Exklusion aus dem musealen Raum kann also bereits im Bereich der Online-Epitexte beginnen. Um einer solchen Exklusion entgegenzuwirken, ,können barrierefreie Informationen im Internet vergrößert dargestellt, mit synthetischen Sprachausgaben vorgelesen, mit beliebigen internetfähigen Geräten abgerufen oder auch in verschiedenen multimedialen Formen angeboten werden" (Hellbusch 2007: 205; zu den konkreten Anforderungen und Richtlinien für barrierefreies Webdesign verweisen wir auf die Beiträge von Lang, Hellbusch, Womser-Hacker und Schütt in diesem Band).

Das RNIB betont das Potenzial zugänglicher Online-Informationen besonders für blinde Menschen und Menschen mit Sehbeeinträchtigung: Über die reine Informationsebene hinaus können im Internet Zusatzinformationen (auch in Form von Tondokumenten) und spezielle Lernangebote bereitgestellt 
werden, die von blinden und sehbeeinträchtigten Menschen mithilfe privat installierter Leseprogramme erfasst werden können (RNIB 2003: 35). So stellt das New Yorker Museum of Modern Art (MoMA) mittlerweile diverse Tondokumente und Audiobeschreibungen zu verschiedenen ausgestellten Werken auf seiner Internetseite zur Verfügung; die Dokumente sind dabei auf unterschiedliche Zielgruppen zugeschnitten (einige auf Zielgruppen mit besonderen Bedürfnissen, wie blinde und sehbeeinträchtigte Besucher(innen) oder Kinder; einzelne Audiodokumente beinhalten hingegen auch Interviews mit Künstler(inne)n oder Kurator(inn)en und sind damit für alle Besucher(innen) interessant). Das Potenzial eines barrierefreien Internetauftritts zur Ermöglichung einer individuellen Vor- und Nachbereitung des Museumsbesuchs und zur Förderung zielgruppengerechter Lernprozesse scheint noch nicht vollständig erschlossen zu sein.

\section{Reduzierung sprachlicher Barrieren im Bereich der mündlichen Epitexte}

Die letzte Gruppe von Teiltexten, die im zweiten Abschnitt des Beitrags betrachtet werden soll, sind die mündlichen Epitexte - also gesprochene Texte, die nicht im unmittelbaren Umfeld eines Exponats geäußert werden, sondern die Ausstellung in einem weiteren Kontext umgeben. Zu den mündlichen Epitexten zählen unter anderem Ausstellungspräsentationen, Interviews mit und Einführungen durch Künstler(innen) und/oder Kurator(inn)en, Podiumsdiskussionen, aber auch andere museumspädagogische Angebote wie Workshops.

Um Live-Veranstaltungen zugänglicher $\mathrm{zu}$ gestalten und beispielsweise Menschen mit einer Hörschädigung die Teilnahme zu ermöglichen, können Wortbeiträge durch eine Live-Verdolmetschung in Gebärdensprache ergänzt werden. Des Weiteren ist eine Schriftverdolmetschung der Wortbeiträge möglich (s. hierzu den Beitrag von Witzel im vorliegenden Band). Workshops richten sich häufig bereits an spezielle Zielgruppen und sind dementsprechend aufbereitet; hier sollte bedacht werden, dass Angebote für einzelne, separierte Zielgruppen dem Inklusionsgedanken nicht vollständig gerecht werden.

In der nachstehenden Graphik werden die in diesem Abschnitt gesammelten Methoden und Konzepte zum Abbau sprachlicher Barrieren in Museen und Ausstellungen noch einmal zusammengefasst. 


\section{Peritexte}

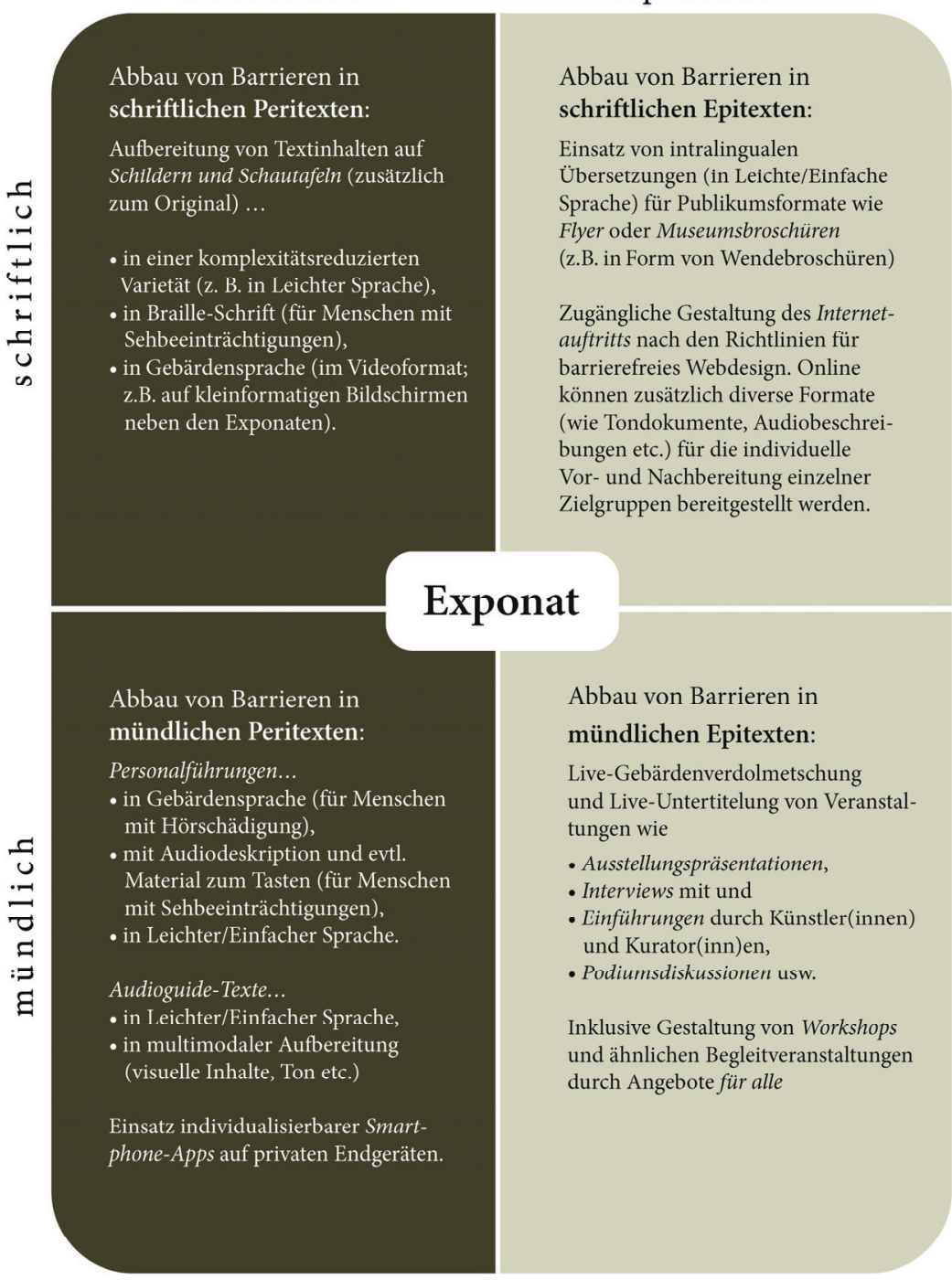

Abb. 2: Möglichkeiten zum Abbau kommunikativer Barrieren im Museum im Überblick 


\section{Projektbeispiele zum barrierefreien Museum aus Deutschland und Finnland}

Auch im berufspraktischen Bereich haben sich in den vergangenen Jahren die ersten innovativen Ansätze zur Gestaltung zugänglicher(er) Ausstellungen entwickelt, die sprachliche Barrieren gezielt abzubauen versuchen. Dies soll im Folgenden anhand ausgewählter Projektbeispiele aus Finnland und Deutschland illustriert werden, die den literaturgestützten Überblick aus dem zweiten Teil des Artikels ergänzen. Die hier verwendeten Bezeichnungen „einfache Sprache“ und „leicht verständliche Sprache“ bezeichnen anwendungs- und adressatenorientierte Konzepte von Leichter Sprache; eine ausführliche Diskussion hierzu findet sich im einleitenden Kapitel von Bock, Fix und Lange (2017: 11ff., bes. 16-19).

Studie und praktische Schlussfolgerungen: Zugänglichkeit für erwachsene Menschen mit Lernschwierigkeiten bzw. mit geistiger Behinderung

Eine von der Stadt Tampere in Finnland beauftragte Untersuchung zur Zugänglichkeit von Kultur und Kunst für erwachsene Menschen mit Lernschwierigkeiten bzw. geistiger Behinderung (Laurila/Haapasalo/Nevalainen 2017; zur Verwendung der Terminologie s. die Beiträge von Schuppener/Bock und Rink im vorliegenden Band) ergab, dass die häufigsten Hindernisse, Kulturangebote zu genießen, neben finanziellen Problemen im Mangel an Begleitung sowie an notwendigen Informationen $\mathrm{zu}$ den Kulturangeboten bestehen (Laurila/Haapasalo/Nevalainen 2017: 4). Dabei liegt eine besondere Herausforderung bei erwachsenen Menschen mit Lernschwierigkeiten bzw. geistiger Behinderung, die weitgehend selbständig agieren. Ihre individuellen Fähigkeiten reichen für ihre gewohnten täglichen Aktivitäten aus, jedoch fallen ihnen die Suche nach neuen Informationen und der eigenständige Besuch von Freizeitaktivitäten schwer (Laurila/Haapasalo/Nevalainen 2017: 2). Fast alle Befragten gaben an, dass es praktisch unmöglich sei, ohne Begleitung allein Kulturangebote zu besuchen (Laurila/Haapasalo/Nevalainen 2017: 11ff.). Die Probleme liegen z. B. in der lautsprachlichen Kommunikation und in Leseschwierigkeiten, die ein selbständiges Verstehen von Museumstexten und -informationen verhindern. 
Entsprechend wurde ein Mangel an verständlichen Informationen zu den Angeboten festgestellt (Laurila/Haapasalo/Nevalainen 2017: 14). Hier wurde der Ausbau von allgemeinen Angebotsinformationen in Leichter Sprache als notwendig herausgestellt. Noch dringlicher aber ist der Studie zufolge der Bedarf am Ausbau von mündlicher Informationsvermittlung, da viele Betroffene Leseprobleme haben oder gar nicht lesen können (Laurila/Haapasalo/Nevalainen 2017: 14).

In der Vergangenheit wurden hier verschiedene Lösungswege beschritten. Laurila, Haapasalo und Nevalainen (2017: 19ff.) stellen heraus, dass vor allem die Zusammenarbeit der Kultureinrichtungen mit den Betroffenen und deren Organisationen notwendig ist. Deshalb wurde der Aufbau eines lokalen Kulturnetzwerkes der Einrichtungen und Vereine für Menschen mit Lernschwierigkeiten bzw. geistiger Behinderung betrieben. In diesem Netzwerk können geschulte „Kulturverantwortliche“ einzelner Einrichtungen über vorhandene Kulturangebote informieren und helfen, diese in Anspruch zu nehmen.

Daneben wurde die Zusammenarbeit mit städtischen Kulturlots(inn)en, d. h. ehrenamtlich tätigen, anforderbaren Begleiter(inne)n, verstärkt. Die Kulturlots(inn)en erhielten eine zusätzliche Schulung zur Begleitung von Menschen mit Lernschwierigkeiten bzw. geistiger Behinderung. Verknüpft wurde dies mit der bestehenden Best Buddies-Vermittlung. Best Buddies ist eine weltweit tätige Non-Profit-Organisation, die sich die soziale Integration von Menschen mit einer geistigen Behinderung zum Ziel gesetzt hat. Es geht dabei um die Förderung von individuellen Freundschaften zwischen Menschen mit und ohne Behinderung und um das Aufbrechen isolierter "Sonderwelten“ (Best Buddies Deutschland: Über uns).

Die Befragten hatten auch Gelegenheit, Wünsche zu äußern (Laurila/Haapasalo/Nevalainen 2017: 18). Auch hier wurde deutlich, dass sich Menschen mit Lernschwierigkeiten bzw. mit geistiger Behinderung weniger Sonderveranstaltungen wünschen, sondern lieber die Möglichkeit hätten, an Kulturveranstaltungen teilzunehmen, die sich an alle richten. Auf großes Interesse stieß auch die Mischung und Überschreitung verschiedener Kunst- und Kultursparten. Genannt wurde zum Beispiel ein Workshop der Tänzerin Marjo Hämäläinen („Lass uns die Malerei tanzen“) in Tampere: Hier erarbeiteten Menschen mit 
Lernschwierigkeiten bzw. mit geistiger Behinderung Tanzaufführungen, nachdem sie Gemälde in einem Kunstmuseum kennen gelernt hatten. Damit entstand eine neue Möglichkeit, eigene Kunsterlebnisse zu verarbeiten und gleichzeitig selbst künstlerisch aktiv zu werden.

\section{Führungen in leicht verständlicher Sprache}

In einer Entwicklungszusammenarbeit mit dem Finnischen Zentrum für Leichte Sprache in Helsinki entwickelte und erprobte die Agentur „Kulturprojekte - Inklusive Kulturarbeit“ mit der Lebenshilfe Köln Leitlinien und organisatorische Prinzipien für Führungen in leicht verständlicher Sprache. Ziel war es, die grundsätzlich ungleiche Kommunikation mit Menschen mit Lernschwierigkeiten bzw. geistiger Behinderung zu überwinden, mit ihren individuell verschiedenen Fähigkeiten und Erfahrungen und ihrem sehr unterschiedlichen Wissen umzugehen. Es zeigte sich, dass eine sehr frühe Zusammenarbeit zwischen der Führerin und ihrer Gruppe der Schlüssel zum Erfolg war. Eine gemeinsame Entwicklung von Zielen, Routen und Zeitplänen sowie vorausgehende Informationen zu den Themen der Führung ermöglichten es der Gruppe, sich gut auf das Erlebnis vorzubereiten, und boten den Organisatoren einen sicheren Rahmen, in dem sie auch spontan auf besondere Bedürfnisse und Ereignisse eingehen konnten (Rantamo 2017: 491ff.).

\section{Einfache Sprache im Museum „kult Westmünsterland“}

Das neu erbaute „kult Westmünsterland“ in Vreden entschloss sich, in seiner Museumsausstellung die erläuternden Texte in Einfacher Sprache zu gestalten. Der Auswahl der sprachlichen Mittel lag eine Betrachtung der erwarteten und anzusprechenden Besuchergruppen zu Grunde. Als ländliche und grenznahe Kultureinrichtung möchte das „kult“ einerseits eine Mehrheit der regionalen Einwohner(innen) ansprechen, darunter auch viele Menschen verschiedener Generationen mit sehr unterschiedlicher Schulbildung, als auch internationale Besucher(innen) mit geringen Deutschkenntnissen, so z. B. aus den benachbarten Niederlanden. In enger Zusammenarbeit mit den Fachwissenschaftler(inne)n des „kult“ wurden durch die Agentur „Kulturprojekte - Inklusive Kulturarbeit" in mehreren Schritten die vorliegenden Textentwürfe in Einfache 
Sprache übertragen. Dabei wurden auch „Test-Leser(innen)“ mit geringen Lesefähigkeiten eingesetzt, denen die behandelten Sachverhalte fremd waren und die ihrerseits Hinweise auf „schwierige“ Wörter und Textpassagen gaben.

Auf diese Weise entstand ein einheitlicher Textkörper, der in inklusiver Absicht einer Mehrheit der künftigen Besucher(innen) sprachlich entgegenkommt, ohne dass diese auf gesonderte Materialien und Handreichungen zugreifen müssten. Die Texte waren auch die Grundlage für Übersetzungen ins Niederländische und Englische.

Bei seiner Ausstellungsgestaltung wendete das „kult Westmünsterland“ ein durchgehendes Sprachkonzept an. In vielen Fällen erfolgt die Textproduktion für eine Museumsausstellung durch verschiedene Autor(inn)en, die mit unterschiedlichen Zielsetzungen die jeweiligen Texte erstellen: Fachwissenschaftler(innen) erstellen Katalog- und Erläuterungstexte, Kurator(inn)en konzipieren Titel und Überschriften, Museumspädagog(inn)en schreiben für Kinder, Werbetexter(innen) betexten Plakate und sonstige Werbemittel. Angebote in Leichter oder Einfacher Sprache werden, ebenso wie fremdsprachliche Übersetzungen, zusätzlich erstellt. Selten scheinen diese Texte sprachlich aufeinander abgestimmt zu sein, noch seltener ist in der Vielzahl ein bewusst gestaltetes, inklusives Konzept erkennbar.

\section{Unterstützte Kommunikation im Museum}

Das Finnische Seefahrtsmuseum (Suomen merimuseo), das Finnische Sportmuseum (Suomen Urheilumuseo) und das Lappländische Regionalmuseum (Lapin maakuntamuseo) verfolgten in Zusammenarbeit mit dem Finnischen Verband für Menschen mit geistiger Behinderung (Kehitysvammaliitto) das Ziel, die kommunikative Zugänglichkeit der Museen zu verbessern. Im Projekt „Kommunikationstafel und leicht verständliche Sprache als Unterstützung für den Besuch im Museum“ (2012-2013) wurden dazu Museumsmaterialien mit Bildsymbolen und Texte in besonders leicht verständlicher Sprache entwickelt.

Leichte und Einfache Sprache können im Allgemeinen all denjenigen Menschen helfen, die Probleme haben, mündliche oder schriftliche Sprache zu verstehen (Bredel/Maaß 2016 und Rink im vorliegenden Band nennen hier beispielsweise Menschen mit kognitiven Einschränkungen, gehörlose Menschen 
oder Migrant(inn)en mit gering ausgeprägten Sprachkenntnissen). Kommunikationstafeln sind ortsbezogene Sprachhilfen für Gäste ohne Lautsprache und für all diejenigen, die Bilder oder Symbole als Unterstützung der Kommunikation benötigen. Die Bilder und Symbole strukturieren das Gesehene und das Gehörte im Museum und bieten so ein Grundvokabular, mit dem man während des Museumsbesuchs und danach diskutieren kann. Für die Kommunikationstafeln wurden hauptsächlich Bilder aus der Arasaac-Bilderdatenbank des „Aragonese Portal of Augmentative and Alternative Communication“ und Fotos aus den Ausstellungen verwendet (Sprachmaterialien für Museumsbesuche 2018).

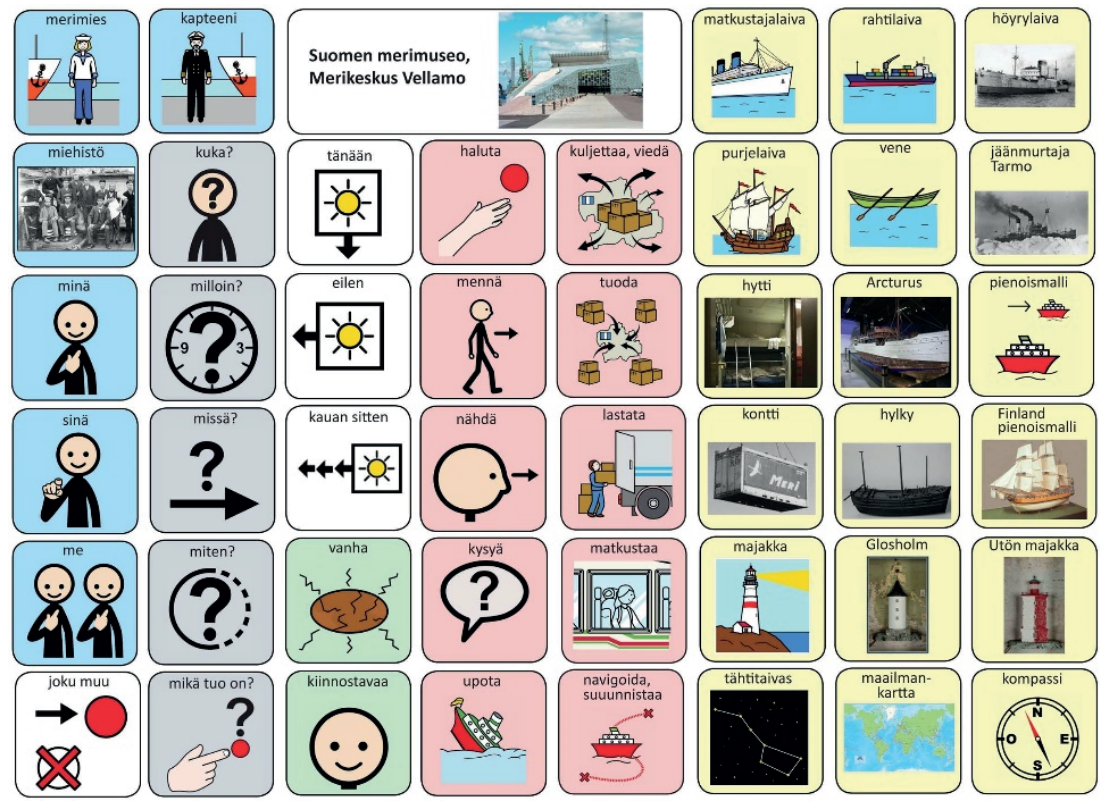

Abb. 3: The Finnish Association on Intellectual and Developmental Disabilities (FAIDD)/Papunet

\section{Kommunikation im Besucherservice}

Die Ergebnisse des Finnischen Projektes zum Einsatz von Kommunikationstafeln waren Anregung, auch in Deutschland auf diese Weise zur Verbesserung der Kommunikation im Museum beizutragen. Für den Landschaftsverband Rheinland (LVR) wurde das gesamte Kassen- und Aufsichtspersonal der LVR- 
Museen von der Agentur „Kulturprojekte - Inklusive Kulturarbeit“ geschult. Das Ziel bestand hier darin, durch verbesserte Kenntnisse und Fähigkeiten des Personals einen Schritt zu einer allgemeinen Barrierefreiheit und zu mehr Inklusion zu gehen. Das Kassen- und Aufsichtspersonal steht täglich im direkten Kontakt mit allen Besucher(inne)n und muss sich unmittelbar mit den Fragen und Schwierigkeiten von Menschen mit besonderen Bedürfnissen auseinandersetzen sowie praktische Hilfe geben. Daher wurde der Schwerpunkt auf die Face-to-Face-Kommunikation gelegt. Dabei standen Situationen im Mittelpunkt, in denen eine sprachliche Kommunikation nur schwer oder gar nicht möglich ist und alle nonverbalen Möglichkeiten wie z. B. auch Gestik oder Zeichnen genutzt werden müssen. Das Konzept der Kommunikationstafeln wurde dabei sehr schnell erlernt und vom Personal mit Begeisterung aufgenommen (Rantamo 2016: 4).

\section{$5 \quad$ Fazit und Ausblick}

Betrachtet man die Vielzahl der möglichen und notwendigen sprachlichen und anderen Hilfsmittel für unterschiedliche Besucher(innen), so zeigt sich der Widerspruch, dass ein Aufbereiten von Informationen für einzelne, voneinander getrennte Zielgruppen dem Inklusionsgedanken nicht völlig gerecht wird: Wenn jede Zielgruppe ihre „eigenen“ Informationen in einer speziell auf sie zugeschnittenen Weise vermittelt bekommt, wird kein gemeinsames Erleben stattfinden. Die Ausstellungstätigkeit des Museums, die sich grundsätzlich an die gesamte Gesellschaft richtet, zerfiele förmlich in eine Reihe von Einzelpräsentationen für unterschiedliche Gruppen.

Die angeführten Beispiele zeigen, dass eine Vernetzung (bzw. eine gemeinsame Organisation des Museumsbesuches) auf allen Ebenen sinnvoll ist. Museum und Besucher(innen) brauchen den engen Kontakt zueinander, ebenso wie sich die Museen organisiert auf ihre Gäste einstellen müssen. Und auch Besucher(innen) können, wo es notwendig ist, ihre Besuche gemeinsam vorbereiten und gestalten. So verhelfen sich alle Beteiligten gegenseitig zu mehr Möglichkeiten. Auf diese Weise kann der scheinbare Widerspruch zwischen der Inklusion, also der gleichzeitigen und gleichberechtigten Teilhabe aller, und der 
notwendigen Differenzierung, also einer Vielzahl verschiedener, unterstützender Angebote für Menschen mit unterschiedlichen Bedürfnissen, aufgehoben werden. In einer gemeinsam erarbeiteten und organisierten Strategie aller Beteiligten liegt der Schlüssel zur Bewältigung der Aufgabe, den Genuss von Wissenschaft und Kunst für alle zu ermöglichen und die Entwicklung von Museumskultur mit allen zu gestalten.

Für die wissenschaftliche Forschung ergibt sich das Forschungsdesiderat, gemeinsam mit der museumspädagogischen Berufspraxis Grundlagen für Sprachkonzepte im Sinne einer besseren Zugänglichkeit in Museen zu entwickeln, welche die in diesem Beitrag genannten Aspekte berücksichtigen und zusammenführen. Eine Evaluation und empirische Überprüfung solcher Sprachkonzepte sollte unter Einbeziehung der jeweils anvisierten Zielgruppen erfolgen. 
Museumstexte - Zum Abbau sprachlicher Barrieren in Museen und Ausstellungen

\section{Literaturverzeichnis}

Al Masri-Gutternig, NAdja/ReitstätTer, Luise (Hrsg.) (2017): Leichte Sprache. Sag es einfach. Sag es laut! Praxisbeispiel Salzburg Museum. Herausgegeben vom Salzburg Museum.

Bertron, Aurelia/Schwartz, Ulrich/Frey, Claudia (2006): Ausstellungen entwerfen. Kompendium für Architekten, Gestalter und Museologen. Basel: Birkhäuser.

Best Buddies Deutschland (2018): Über uns. Online: <http://bestbuddies.de/?page_id=12> (25.06.2018)

Bock, BetTina M./FIX, Ulla/Lange, Daisy (Hrsg.) (2017): „Leichte Sprache“ im Spiegel theoretischer und angewandter Forschung. Kommunikation - Partizipation - Inklusion, Band 1. Berlin: Frank \& Timme.

Bourdieu, Pierre/Darbel, Alain (2006): Die Liebe zur Kunst. Konstanz: UVK Verlagsgesellschaft. Originalausgabe: Bourdieu, Pierre/Darbel, Alain (1966/1969): L'amour de l'art. Les musées d'art européens et leur public. Deuxième édition revue et augmentée. Paris: Les Éditions de Minuit.

Bredel, Ursula/MaAss, Christiane (2016): Leichte Sprache. Theoretische Grundlagen - Orientierung für die Praxis. Berlin: Duden.

EgGert, BARBARA M. (2010): Der Audioguide als Medium der Erwachsenenbildung im Museum. Exemplarische Analysen von Hörtexten hinsichtlich der Sprecherrolle und der Rolle des impliziten Zuhörers. Erwachsenenpädagogischer Report, Band 15. Masterarbeit im Studiengang „Erwachsenenpädagogik/Lebenslanges Lernen“, HumboldtUniversität zu Berlin.

FÖHL, PATRICK S. (2007): „Barrierefreies Museumsmarketing“. In: FÖHL, PATRICK S. et al. (Hrsg.): Das Barrierefreie Museum. Theorie und Praxis einer besseren Zugänglichkeit. Ein Handbuch. Bielefeld: transcript, S. 180-204.

Genette, Gérard (1987/2014): Paratexte. Das Buch vom Beiwerk des Buches. 5. Auflage. Frankfurt am Main: Campus Verlag.

Hellbusch, Jan ERIC (2007): „Barrierefreie Webauftritte der Museen: Eine Einführung.“ In: FöHL, PATRICK S. et al. (Hrsg.): Das Barrierefreie Museum. Theorie und Praxis einer besseren Zugänglichkeit. Ein Handbuch. Bielefeld: transcript, S. 205-226.

Hohmaier, Kathrin (2015): „,Hässlich wie ein modernes Kunstwerk': Die Praxis eines Kunstvermittlungsprojektes für museumsferne Besuchergruppen“. In: DANKO, DAGMar/Moeschler, Olivier/Schumacher, Florian (Hrsg.): Kunst und Öffentlichkeit. Wiesbaden: Springer Fachmedien, S. 167-186.

JANKOWSKA, ANNA et al. (2017): „Smartphone app as a museum guide. Testing the Open Art application with blind, deaf, and sighted users“. In: International Journal of Translation 2017 (19), S. 113-130.

Sprachmaterialien für Museumsbesuche: Kommunikointitauluja museovierailuille. Online: <http://papunet.net/materiaalia/kommunikointitaulut-museoihin> (07.02.2018) 
LaUrila, JaAKKo/HaApasalo, JaAni/NeVAlainen, MarJo (2017): Kulttuurin ja taiteen saavutettavuus aikuisten kehitysvammaisten parissa. Selvitys- ja kehittämistyö Tampereella 2016-2017. Tampereen kaupungin kulttuuripalvelut. Online: <https://www.tampere.fi/tiedostot/k/TWGJElrOV/Kulttuurin_saavutettavuus_ raportti_web.pdf> (23.05.2018)

MaAss, Christiane (2015): Leichte Sprache. Das Regelbuch. Barrierefreie Kommunikation, Band 1. Berlin/Münster: LIT.

Neves, JoséLIA (2016): „Enriched Descriptive Guides for a Multisensory User-Experience in Cultural Contexts“. In: Languages \& The Media 2016: 11th International Conference on Language Transfer in Audiovisual Media. Conference Catalogue, S. 55-56.

Popp, Kathrin (2013): Das Bild zum Sprechen bringen. Eine Soziologie des Audioguides in Kunstausstellungen. Bielefeld: transcript.

RANTAMO, Eeva (2016): Bericht zur Fortbildung „Umgang mit Menschen mit Behinderungen“ 2015-2016. Online: <http://www.inklusive-kulturarbeit.de/fileadmin/ user_upload/Bericht_Fortbildung_LVR-Museumspersonal_2015-2016.pdf> (25.06.2018)

RANTAMO, EEVA (2017): „Kunst- und kulturgeschichtliche Führungen in leicht verständlicher Sprache - Entwicklung einer Methodik in deutsch-finnischer Zusammenarbeit“. In: Bock, BetTina M./Fix, Ulla/LAnge, Daisy (Hrsg.): „Leichte Sprache“ im Spiegel theoretischer und angewandter Forschung. Kommunikation - Partizipation Inklusion, Band 1. Berlin: Frank \& Timme, S. 491-493.

REHM, SUSANNE (2017): „Kulturpädagogik inklusive: Inklusion in kulturellen Kooperationsprojekten - Eine systemische Annäherung“. In: HÜBNER, KERSTIN et al. (Hrsg.): Teilhabe. Versprechen?! Diskurse über Chancen- und Bildungsgerechtigkeit, Kulturelle Bildung und Bildungsbündnisse. Kulturelle Bildung, Bd. 55. München: kopaed, S. 175-182.

RNIB (Royal National Institute of Blind People) (2003): Museums, galleries and heritage sites: improving access for blind and partially sighted people. The Talking Image Guide. Online: <http://www.thetalkingwalls.co.uk/PDF/public_talkingimagesguide.pdf> (21.05.2018)

SiEgERT, STEPHAN (2017): „1975/2015 - Schiffe erzählen Museumsgeschichte - Eine Untersuchung zum Umgang mit Leichter Sprache im Museum - das Beispiel DSM“. In: Bock, Bettina M./FIX, Ulla/LAnge, Daisy (Hrsg.): „Leichte Sprache“ im Spiegel theoretischer und angewandter Forschung. Kommunikation - Partizipation - Inklusion, Band 1. Berlin: Frank \& Timme, S. 485-489.

WeINRICH, HARALD (2014): Vorwort zu Gérard Genette (1987/2014): Paratexte. Das Buch vom Beiwerk des Buches. 5. Auflage. Frankfurt am Main: Campus Verlag, S. 7-8. 
JOHANNES SCHÄDLER

\section{Barrierefreie Verwaltung - Grundlagen und Handlungskonzepte}

\section{$1 \quad$ Einführung}

Die UN-Behindertenrechtskonvention (UN-BRK) und gesetzliche Vorgaben auf Bundes- und Landesebene sehen auch staatliche und andere öffentliche Verwaltungsstellen in der Verantwortung, barrierefrei $\mathrm{zu}$ werden und die gleichberechtigte Nutzung ihrer Leistungen auch für Menschen mit Behinderungen sicherzustellen (s. Lang in diesem Band). Es handelt sich bei Barrierefreiheit um eine relativ neue und begrifflich noch etwas sperrige Anforderung in einem gesellschaftlichen Bereich, der durch anhaltende und intensive Modernisierungsdebatten geprägt ist (vgl. Jann/Wegrich 2010).

Der nachfolgende Text befasst sich damit, was unter der Leitvorstellung einer ,barrierefreien Verwaltung' zu verstehen ist und welche Handlungsschritte von Verantwortlichen unternommen werden können, die sich hier ,auf den Weg machen' wollen. Zunächst wird versucht, den historischen Entwicklungspfad der Beziehung zwischen Behörden und Bürger(inne)n nachzuskizzieren. Dabei wird die These vertreten, dass sich in den zurückliegenden Jahrzehnten das Verwaltungshandeln zunehmend demokratisiert hat. Im Zuge dieser Entwicklung wurden von den Behörden wichtige Kompetenzen im Umgang mit der Vielfalt der Personen entwickelt, die Verwaltungsleistungen nutzen bzw. auf sie angewiesen sind. Diese Modernisierungsprozesse haben dafür gesorgt, dass die Leitvorstellung der , barrierefreien Verwaltung' in vielen Ämtern und Behörden anschlussfähig ist und ihre Einbeziehung in alltäglichen Routinen gelingen kann. Danach werden ausgewählte Konzepte und Erfahrungen dazu vorgestellt, wie Verwaltungsorganisationen zunehmend barrierefrei werden können. 
Im Zuge allgemeiner gesellschaftlicher Demokratisierungsbestrebungen gewannen in den 1970er Jahren Bestrebungen an Kraft, die sich für eine konsequente Abkehr vom „Obrigkeitsstaat mit Untertanengesinnung“ (Jaspers 1966: 155) im Verhältnis zwischen Staat und Bürger(inne)n einsetzten. Bis weit in die Anfangsjahrzehnte hinein, so konstatiert Karl Jaspers in seiner Analyse zur Situation der Bundesrepublik Deutschland (Jaspers 1966), war dieses Verhältnis durch wilhelminische Staatsverehrung und autoritäre Gesinnungen geprägt. Staatliches Handeln war gekennzeichnet durch "Misstrauen gegen das Volk“ (ebd. 175). Der/die Bürger(in) hatte demzufolge den staatlichen Autoritäten verkörpert durch Politik und Behörden - gegenüber Respekt zu zeigen und sich untertänigst an die Vorgaben und Anordnungen zu halten. Dies schlug sich auch in der hoheitlich-hierarchischen Weise nieder, wie in öffentlichen Ämtern und Behörden die Aufgaben verstanden und Anliegen von Bürger(inne)n bearbeitet wurden. Umgekehrt fehlte es auch auf Seiten der Bevölkerung an demokratischem Wissen und staatsbürgerlichem Selbstbewusstsein. Das Grundgesetz - so schrieb Jaspers 1966 - sei im Volk heute ebenso unbekannt wie zur Zeit seiner Annahme (ebd. 177).

In den 1970er Jahren waren Bemühungen zu erkennen, das Verhältnis zwischen der öffentlichen Verwaltung und den Bürger(inne)n von den Relikten eines vordemokratischen Untertanenverhältnisses zu befreien und endlich auf eine neue konzeptionelle Grundlage zu stellen. Leitend hierfür war der Begriff der ,Bürgernähe' (vgl. Grunow 1978), der in den Folgejahren eine rasche Verbreitung fand. Hintergrund waren zum einen die Verwaltungsgebietsreformen, in denen in zahlreichen Bundesländern die bestehenden Gemeinden, Städte und Kreise zu größeren Einheiten zusammengefasst wurden. Mit diesem oft konflikthaften Prozess einher ging die Notwendigkeit, den Interessen der Bürger(innen) auf möglichst einfache Zugänge zu Verwaltungsdienstleistungen nachzukommen, da die Wohnorte nun häufig weiter weg von den Verwaltungszentren lagen.

In den konzeptionellen Ausarbeitungen wurde aber rasch deutlich, dass es nicht nur Entfernungsprobleme waren, die mit dem Begriff Bürgernähe gelöst werden sollten, sondern dass deutlich mehr Dimensionen von Zugänglichkeit 
eine Rolle spielen. Auch ging es um mehr als nur um den Appell an die Beamten und Angestellten in den Behörden, die sie kontaktierenden Bürger(innen) freundlich zu behandeln. Vielmehr wurden neue professionelle Anforderungen an Verwaltungshandeln formuliert, die eine Orientierung an den Bedürfnissen, Problemen und Rechten der Bürger(innen) verlangten. Die Verwaltungswissenschaft erkannte darin die Chance, das Beziehungsverhältnis zwischen Staat und Bürger(in) selbst zum Gegenstand öffentlicher Diskussion zu machen und nach neuen, demokratischen Kriterien auszugestalten (Grunow u.a. 1988). Konkret führte diese Diskussion in vielen Kommunen dazu, dass in den 2000er Jahren „Bürgerämter“ oder „Bürgerbüros“ als niedrigschwellige Anlaufstellen geschaffen wurden. Ziel war es, v.a. alle kurzen und häufig vorkommenden Verwaltungsleistungen an einem Ort flexibel und schnell anzubieten.

Eine etwas andere Akzentuierung erfuhr das Verhältnis zwischen Verwaltungsstellen und Bürger(inne) $n$ in den Konzepten des New Public Management oder in seiner deutschen Ausformulierung des „Neuen Steuerungsmodells“ der Kommunalen Stelle für Verwaltungsvereinfachung (KGST). Oft parallel zur weiteren Entwicklung bürgernaher Verwaltungskonzepte wurde Verwaltungshandeln in einer stärker ökonomischen Perspektive als „Dienstleistung“ verstanden und das Verhältnis zur Bevölkerung innerhalb eines „Kunden-Lieferanten“-Modells (vgl. Jann/Wegrich 2010: 180) entfaltet. Im Vordergrund dieses Modells stand die Bestrebung, die Verwaltungsdienstleistungen möglichst kosteneffizient anzubieten. Dabei wurde vor allem auf Privatisierungen, Personalmanagement, EDV- bzw. Internetgestützte Technologie-Einführungen und die Einführung einer produkt- und output-orientierten Kostenlogik gesetzt. Gleichzeitig sollten die Verwaltungsmitarbeiter(innen) eine Kundenorientierung entwickeln, die sich an Dienstleistungstugenden wie Höflichkeit, Verlässlichkeit, Pünktlichkeit und Einhaltung professioneller Standards ausrichtet.

Nicht ohne Einfluss auf das allgemeine Behördenhandeln gegenüber der Bevölkerung blieb sicherlich das darauffolgende verwaltungspolitische Leitbild des „aktivierenden Staates“, das insbesondere in der sogenannten Leistungsverwaltung das Dienstleistungsmodell fortführte. Im Rahmen von Vertragsmodellen entwickelten sich Vorgaben an die Verwaltung, mit leistungsberechtigten Bürger(inne)n Vereinbarungen auszuhandeln, in denen wechselseitig Rechte und 
Pflichten festgelegt werden (Jann/Wegrich 2010: 181). Die Vorstellung des Bürgers/der Bürgerin als Vertragspartner der Verwaltung kann in ihrer patriarchalpädagogischen Ambivalenz („Fördern und fordern“) und in ihrer Relativierung sozialer Rechte hier nicht weiter ausgeführt werden. In manchen Leistungssystemen, etwa im Bereich der Arbeitslosenhilfe (ALG II) oder auch im Bereich der Eingliederungshilfe für Menschen mit Behinderungen nach $₫ 145$ SGB XII (,Teilhabezielvereinbarung'), wurde der Ansatz institutionalisiert.

Eine weitere Prägung erhielt das Verwaltungshandeln in den 2000er Jahren durch die entschiedener gewordene Debatte um Gleichstellung und Gleichbehandlung von Männern und Frauen (vgl. Krell/Mückenberger/Tondorf 2004). Das Bundesgleichstellungsgesetz (BGG) von 2001 (novelliert 2017) wirkte sich zunächst nur auf die Dienststellen und Behörden des Bundes aus, löste aber darüber hinaus auch auf Landes- und kommunaler Ebene die Forderung nach Gender-MainstreamingProgrammen aus, die sich wiederum auch sensibilisierend auf die Dienstleistungskulturen in den Behörden auswirkten. Um strukturellen Diskriminierungen vorzubeugen, wurden in der Folge neben regelmäßigen Gleichstellungsberichten vielerorts Beschilderungen, Bescheide, Informationsträger, verschiedene Verfahren genderneutral umgeändert und somit respektvoller gemacht. Gleichzeitig ist innerhalb der Verwaltungsstellen der demokratische Anspruch an bürgernahes Verwaltungshandeln damit wohl weiter gewachsen.

Seit einigen Jahren wird öffentliches Verwaltungshandeln damit konfrontiert, dass sich die deutsche Gesellschaft zunehmend kulturell und ethnisch ausdifferenziert und mit der Bevölkerung insgesamt auch die ,Kundschaft ' der Verwaltung vielfältiger wird. Verstärkt sind Verständigungsprobleme zu bewältigen, die auch, aber oft nicht nur, sprachlicher Art sind. Zu ihrer Bewältigung sind auf Seiten der Verwaltung mehr als früher fremdsprachliche Kenntnisse, aber auch interkulturelle Kompetenzen notwendig geworden und mehr als zuvor auch verfügbar. Damit sind auch förderliche Voraussetzungen geschaffen worden, um die Herausforderungen zu bewältigen, die sich mit der gleichberechtigten Behandlung von Menschen mit Behinderungen im Verwaltungsalltag verbinden.

Im Folgen soll skizziert werden, um welche Herausforderungen es sich genauer handelt und wie Umsetzungswege in Verwaltungsstellen aussehen können. Die Ausführungen stützen sich auf verschiedene Projekte des Zentrums für Planung 
und Evaluation Sozialer Dienste (ZPE) der Universität Siegen in kommunalen Verwaltungen im Rahmen des Forschungsschwerpunkts Teilhabeplanung.

Durch die Ratifizierung der UN-Behindertenrechtskonvention wurde 2009 ein menschenrechtsbasiertes Verständnis von Behinderung in das deutsche Rechtssystem übernommen, das sowohl die Bundes- und Landesebene, aber in vielerlei Hinsicht auch die kommunalpolitische Ebene adressiert. In der UN-BRK wird ,Behinderung' nicht als Eigenschaft einer Person verstanden, sondern als Einschränkung der Teilhabemöglichkeiten. Diese Einschränkungen ergeben sich aus einer Wechselwirkung zwischen funktionalen Beeinträchtigungen einer Person und fehlenden Kompensationsmöglichkeiten bzw. Barrieren in ihrer Umgebung. Der Blick auf die Behinderung erweitert sich dadurch zu einem Blick auf die gesellschaftlichen Bedingungen und auf die Bedingungen des ökosozialen Raums, in dem eine Person lebt und sich entwickelt. Unabhängig von Art und Schwere ihrer Beeinträchtigungen wird Menschen mit Behinderungen das Recht auf gleichberechtigte Teilhabe zuerkannt.

Aus einem solchen Behinderungsverständnis leiten sich Aufgaben ab, die das Rechts- und Verwaltungssystem, aber auch den gesellschaftlichen und kulturellen Alltag insgesamt betreffen (vgl. Degener/Diehl 2015). Je weniger Barrieren Menschen mit Beeinträchtigungen im Alltag und im Lebenslauf vorfinden, wenn sie wie andere ihr Leben gestalten wollen, umso weniger wirken sich die individuellen Einschränkungen behindernd aus. Dies wird zum Beispiel in dem etwas plakativen, aber durchaus eingängigen Slogan ,Behindert ist man nicht, behindert wird man' zum Ausdruck gebracht. Artikel 9 der UN-Behindertenrechtskonvention greift diesen Zusammenhang unter der Überschrift ,Zugänglichkeit' explizit auf und verpflichtet alle staatlichen Ebenen dazu, Teilhabebarrieren abzubauen:

Um Menschen mit Behinderungen eine unabhängige Lebensführung und die volle Teilhabe in allen Lebensbereichen zu ermöglichen, treffen die 
Vertragsstaaten geeignete Maßnahmen mit dem Ziel, für Menschen mit Behinderungen den gleichberechtigten Zugang zur physischen Umwelt, zu Transportmitteln, Information und Kommunikation, einschließlich Informations- und Kommunikationstechnologien und -systemen, sowie zu anderen Einrichtungen und Diensten, die der Öffentlichkeit in städtischen und ländlichen Gebieten offenstehen oder für sie bereitgestellt werden, zu gewährleisten (Art. 9, Abs. 1 UN BRK).

Aufgrund der Bedeutung unmittelbarer sozialer und räumlicher Umwelten für die Entstehung von Behinderungen rückt von allen staatlichen Ebenen die kommunale Ebene in den Vordergrund. Nach Art. 28 GG sind die Kommunen verantwortlich für die sogenannte allgemeine Daseinsvorsorge in ihrer Gebietskörperschaft. Die sich daraus ergebenden Aufgaben der Kommunen folgen einer örtlichen Querschnittslogik. Sie erstrecken sich von der Energie und Wasserversorgung, Gesundheitsversorgung, öffentliche Verkehrsmittel, Bildung, Kultur-, Sport- und Freizeitangeboten usw. bis hin zum Vorhalten einer sozialen Infrastruktur zur Versorgung der Bevölkerung mit sozialen Einrichtungen und Diensten verschiedenster Art. In vielen dieser Bereiche kommen Menschen mit Behinderungen - wie alle anderen auch - in persönlichen und schriftlichen Kontakt mit kommunalen Verwaltungsstellen. Die z.T. abstrakten Forderungen der UN-BRK nach Inklusion und gleichberechtigter Teilhabe können dabei rasch konkretisiert und praktisch werden, z. B. wenn

- ein gehörloser oder stark hörbehinderter Mensch einen Personalausweis beantragen möchte oder

- ein Mensch mit einer chronischen psychischen Erkrankung sich über Leistungen der Eingliederungshilfe erkundigen möchte oder

- ein blinder oder hochgradig sehbehinderter Mensch einen Blindenhund anmelden möchte oder

- ein Mensch mit einer Mobilitätsbeeinträchtigung (Rollstuhlfahrer(in)) sich über barrierefreien Wohnraum, d.h. über eine geeignete, für den 
Rollstuhl zugängliche Wohnung in der Stadt erkundigen und sich beraten lassen möchte oder

- ein Mensch mit einer altersbedingten Mobilitätsbeeinträchtigung die Seniorenberatung aufsuchen möchte oder

- ein Mensch mit einer kognitiven Beeinträchtigung Wohngeld beantragen möchte.

Neben kommunalen Stellen sind vor Ort auch Behörden der Landesverwaltung und des Bundes sowie die lokalen Vertretungen der verschiedenen Sozialversicherungsträger (Krankenkassen, Pflegekassen, Bundesagentur für Arbeit, Anlaufstellen der Renten- bzw. Unfallversicherungen) in unmittelbarem Kontakt mit Bürger(inne)n. Um Verwaltungsleistungen auch Menschen mit Behinderungen diskriminierungsfrei zugänglich zu machen, müssen die in den Beispielen anklingenden unterschiedlichen Problemlagen von Menschen mit Behinderungen berücksichtigt werden.

Die UN-BRK beschreibt dabei den Tatbestand der „Diskriminierung aufgrund von Behinderung" als

jede Unterscheidung, Ausschließung oder Beschränkung aufgrund von Behinderung, die zum Ziel oder zur Folge hat, dass das auf die Gleichberechtigung mit anderen gegründete Anerkennen, Genießen oder Ausüben aller Menschenrechte und Grundfreiheiten im politischen, wirtschaftlichen, sozialen, kulturellen, bürgerlichen oder jedem anderen Bereich beeinträchtigt oder vereitelt wird. Sie umfasst alle Formen der Diskriminierung, einschließlich der Versagung angemessener Vorkehrungen (Art 2,3 UN-BRK).

Der Anspruch auf gleiche Behandlung ist bei Menschen mit seelischen oder kognitiven Beeinträchtigungen anders zu realisieren als zum Beispiel bei Menschen mit Sinnesbeeinträchtigung oder einer Mobilitätseinschränkung. Ein vorurteilsfreier, hilfreicher Umgang setzt bei Mitarbeiter(inne)n einer Ver- 
waltungsstelle neben persönlicher Offenheit auch Sensibilität für Diskriminierungsrisiken und ein bestimmtes Wissen voraus, das z. B. über geeignete Fortbildungen erworben werden kann.

\section{Barrierefreiheit und kommunales Verwaltungshandeln}

Im englischen Originaltext der UN-BRK ist der Art. 9 mit „Accessibility“ überschrieben, was in der deutschen Übersetzung einige Unsicherheiten im Hinblick auf die Begriffe „Zugänglichkeit“ und „Barrierefreiheit“ ausgelöst hat. Erfreulicherweise wurde mit der Novellierung des Behindertengleichstellungsgesetzes (BGG) in $\$ 4$ BGG eine differenzierte Ausformulierung des Begriffes der Barrierefreiheit vorgenommen:

Barrierefrei sind bauliche und sonstige Anlagen, Verkehrsmittel, technische Gebrauchsgegenstände, Systeme der Informationsverarbeitung, akustische und visuelle Informationsquellen und Kommunikationseinrichtungen sowie andere gestaltete Lebensbereiche, wenn sie für Menschen mit Behinderungen in der allgemein üblichen Weise, ohne besondere Erschwernis und grundsätzlich ohne fremde Hilfe auffindbar, zugänglich und nutzbar sind. Hierbei ist die Nutzung behinderungsbedingt notwendiger Hilfsmittel zulässig. ( $\$ 4$ BGG)

In dieser Definition sind vier zentrale Dimensionen des Begriffs Barrierefreiheit enthalten:

a) Die Nutzungsmodalitäten (allgemein üblich, ohne Erschwernis und ohne fremde Hilfe),

b) Auffindbarkeit,

c) Zugänglichkeit und

d) Nutzbarkeit

Entlang dieser Dimensionen ist es möglich, den Begriff der Barrierefreiheit zu operationalisieren und ggfs. für Maßnahmen der Organisationsentwicklung in 
Verwaltungsstellen nutzbar zu machen. Dies soll im Weiteren konkretisiert werden. Erfreulicherweise wurden in den vergangenen Jahren auch öffentliche Beratungsangebote aufgebaut, die neben privaten Anbietern interessierten Verwaltungen dabei helfen können, ihre Barrierefreiheit zu verbessern. Von überregionaler Bedeutung und einem breiten Kompetenzspektrum ist etwa die ,Agentur Barrierefrei NRW', siehe: www.ab-nrw.de. Für Beispiele guter Praxis siehe das ,Inklusionskataster NRW ${ }^{\star}$ an der Universität Siegen: www.inklusivegemeinwesen.nrw.de (Abruf am 03.02.2018).

\subsection{Nutzungsmodalitäten von Verwaltungsleistungen}

Die o.g. Definition von Barrierefreiheit bedeutet für Verwaltungsstellen, dass diese bzw. ihre Leistungen von Menschen mit Behinderungen in der allgemein üblichen Weise, ohne besondere Erschwernis und grundsätzlich ohne fremde Hilfe in Anspruch genommen werden können. Bereits im Hinblick auf bauliche Barrieren ist dies vielfach sicherlich eine Anforderung, die nur schrittweise erreicht werden kann. Um dieser Vorgabe gerecht werden zu können, bietet es sich an, dass sich Verwaltungsorganisationen an der Idee des, universellen Designs' orientieren. Dies wird in der UN-BRK definiert

als ein Design von Produkten, Umfeldern, Programmen und Dienstleistungen in der Weise, dass sie von allen Menschen möglichst weitgehend ohne eine Anpassung oder ein spezielles Design genutzt werden können (Art 2, 5. UN-BRK).

Geht man davon aus, dass Menschen mit Beeinträchtigungen die Leistungen von Verwaltungen nutzen können sollen wie alle anderen auch, ergeben sich aus deren Unterschiedlichkeit für die Organisation eine inklusionsorientierten Verwaltungsstelle Konsequenzen in zweierlei Richtungen: Es muss zum einen darum gehen, die allgemeinen Nutzungsbedingungen zu verbessern, und zum anderen darum, spezielle Maßnahmen zu treffen, die auf den Ausgleich nicht kompensierbarer Einschränkungen einzelner Gruppen von Menschen mit Behinderungen abzielen. Die UN-BRK spricht in diesem Zusammenhang von „angemessenen Vorkehrungen“ und meint damit 
notwendige und geeignete Änderungen und Anpassungen, die keine unverhältnismäßige oder unbillige Belastung darstellen und die, wenn sie in einem bestimmten Fall erforderlich sind, vorgenommen werden, um zu gewährleisten, dass Menschen mit Behinderungen gleichberechtigt mit anderen alle Menschenrechte und Grundfreiheiten genießen oder ausüben können (Art 2,4 UN-BRK).

Wie dargestellt geht es darum, die geeigneten Vorkehrungen zu treffen bzw. vorzuhalten, die Menschen mit spezifischen Beeinträchtigungen brauchen, um ihre Angelegenheiten mit der Verwaltung ohne Diskriminierung erledigen zu können. Prinzipiell schließt der Ansatz des „Universellen Designs“ spezielle Hilfsmittel für bestimmte Gruppen von Menschen mit Behinderungen, soweit sie benötigt werden, nicht aus. Allerdings soll Überlegungen zur allgemeinen Nutzbarkeit ohne spezielle Hilfsmittel der Vorrang gegeben werden.

\subsection{Auffindbarkeit}

$\mathrm{Zu}$ den Kerndimensionen einer barrierefreien Verwaltung gehört es, die Auffindbarkeit relevanter Dienststellen für Menschen mit verschiedenen Beeinträchtigungsarten sicherzustellen. Hierfür ist es erforderlich, für jede Verwaltungsstelle ein geeignetes Informations- und Leitsystem zu entwickeln, das aus visuell oder taktil erfassbaren Schildern, taktilen Übersichts- und Leittafeln, akustischen Informationsträgern, aus Brailleschrift oder aus Bodenleitsystemen bestehen kann. Die Vorkehrungen zur Auffindbarkeit betreffen zum einen den Weg zur Verwaltungsstelle. Hier wie auch bei der Sicherstellung von Zugänglichkeit bietet es sich an, sich am Konzept der ,Mobilitätsketten' zu orientieren, d.h. typischerweise genutzte Wege oder nahräumliche Verkehrsstrecken in den Blick zu nehmen, die zur betreffenden Verwaltungsstelle führen (z. B. vom Bahnhof zum Rathaus). Zum anderen ist natürlich die Ausgestaltung eines Informations- und Leitsystems innerhalb der Verwaltungsstelle von wesentlicher Bedeutung. Das zugrundeliegende Konzept sollte sich auf mehrere Informationskanäle stützen, um unterschiedlichen Wahrnehmungsmöglichkeiten von Menschen mit Beeinträchtigungen gerecht zu werden. 
Die Dimension der Auffindbarkeit als Teil von Barrierefreiheit bezieht sich auch auf die Aufgabe, die verschiedenen Ämter und Stellen im Internet auffindbar zu machen. Hierzu gehört es zu prüfen, ob die relevanten Webseiten der Verwaltungsstellen den Kriterien einer barrierefreien Gestaltung entsprechen. Hilfreich ist es, dass unterstützende Technologien genutzt werden können, die verschiedenen Zielgruppen Zugänge eröffnen und Informationen über einzelne Verwaltungsstellen auffindbar machen (z. B. Screen Reader, Bildschirmlupe, Augensteuerung oder andere alternative Steuerungen oder Apps).

\subsection{Zugänglichkeit}

Eine gut zugängliche Verwaltungsstelle stellt einen Mehrwert für alle dar. Aufzüge oder einfach zu öffnende Türen oder automatische Türöffner erleichtern vielen anderen Personengruppen den Behördengang, wie Personen, die vorübergehend in ihrer Mobilität eingeschränkt sind (verletzt oder erkrankt sind), Personen mit Kinderwagen, sonstigen Lasten oder Betagten, die altersbedingte Beeinträchtigungen aufweisen. Es gibt eine Vielzahl an Barrieren oder Hindernissen, die sich in unterschiedliche Dimensionen einteilen lassen. Einige sind offensichtlich, d.h. leicht zu erkennen. Hierzu zählen bauliche, räumliche oder infrastrukturelle Hindernisse, also beispielsweise die unüberwindbare Treppe für Rollstuhlfahrer(innen) oder Bürger(innen) mit Rollatoren oder mit Kinderwagen. Dann gibt es kommunikative Hindernisse, die weniger erkennbar sind, wie Sprachschwierigkeiten, und die zu allgemeinen Verständigungsproblemen führen können.

Des Weiteren gibt es sensorische Barrieren, die ebenso wenig offensichtlich sind. Hierzu zählen unklare, schlecht lesbare Informationen, kontrastarme Farben, fehlende optische oder taktile Hinweise. Für Menschen mit einer Sehbeeinträchtigung können beispielsweise Formulare, wie Anträge, oder auch Hinweisschilder zu klein geschrieben sein. Fehlt zum Beispiel an einem Treppengeländer eine farbliche, kontrastreiche Markierung, stellt dies eine akute Gefahrenquelle dar. 
Ferner sind ,Barrieren im $\operatorname{Kopf}^{f}$ zu nennen, worunter (diskriminierende) Norm- und Wertvorstellungen und Einstellungen der Verwaltungsmitarbeiter(innen) oder auch Wissensdefizite über Folgen von Beeinträchtigungen verstanden werden können.

\subsection{Nutzbarkeit}

Prinzipiell sollte es Menschen mit Behinderungen ermöglicht werden, auch gegenüber Verwaltungsstellen verschiedenster Art ihre vollen Bürgerrechte wahrzunehmen. Dazu zählt, dass sie jede verwaltungsbezogene Dienstleistung beantragen und die damit verbundenen Tätigkeiten eigenständig erledigen können. Eigenständig bedeutet in diesem Sinne wo immer möglich ohne Hilfe Dritter.

Damit stellt sich die Herausforderung für Verwaltungsstellen, die Räume, Strukturen und die Prozesse möglichst so zu gestalten, dass sie nicht nur von ,able-bodied persons;, d.h. von funktionsfähigen Durchschnittserwachsenen, sondern von möglichst allen Menschen unabhängig von Alter- und Körperstrukturen genutzt werden können. Für eine barrierefreie Verwaltung bedeutet dies, dass ein barrierefreier Zugang zu Räumen, Parkplätze für Menschen mit Behinderung und die Gewährleistung der eigenständigen Bewegungsfreiheit in den Verwaltungsgebäuden gegeben sind. Neben der räumlichen Barrierefreiheit ist die barrierefreie Gestaltung der persönlichen Kontakte, z. B. Termine im Amt, Hausbesuche, gemeinsame Ortstermine und Telefonate, relevant. Für alle Bürgerinnen und Bürger ist es wichtig, dass Anträge, Informationsmaterialien und Bescheide verständlich zur Verfügung stehen und ein leicht verständlicher Schriftverkehr gegeben ist. Der Schriftverkehr besteht i.d.R. in Bescheiden, vielfältigen Informationsmaterialien, amtlichen Schreiben oder E-Mails.

Von einer Verwaltungsstelle, die sich auf den Weg der Barrierefreiheit macht, profitieren alle Menschen, die mit ihr zu tun haben. Die Anlässe und die daraus entstehenden Verwaltungsakte sind von Verwaltungstyp zu Verwaltungstyp (Krankenkasse, Arbeitsagentur, Rathaus etc.) oder z. B. auch innerhalb einer Kommunalverwaltung außerordentlich vielfältig und die Unterschiede der Aufgaben der Kfz- Zulassungsbehörde, des Ausländeramts, des Sozialamts oder des 
Gesundheitsamts offensichtlich. Unabhängig von unterschiedlichen Ämterkulturen und Themen gilt aber, dass ein für alle verständliches Verfahren sowohl den Bürgerinnen und Bürgern als auch den Mitarbeitenden der Behörden selbst zu Gute kommt. Im Ergebnis sind weniger Nachfragen und weniger Missverständnisse im betreffenden Verwaltungsvorgang zu erwarten. Gleichzeitig erhöht die Vereinfachung der Sprache das Vertrauen gegenüber Behörden und Institutionen und verringert bestehende Vorbehalte bei der Inanspruchnahme von Leistungen.

Nicht selten ist es so, dass für Menschen mit Behinderung Situationen wie das aktive Aufsuchen einer Verwaltungsstelle neu und ungewohnt sind. In der Vergangenheit wurden diese Angelegenheiten hauptsächlich stellvertretend durch andere getätigt. Gerade deshalb sollten die Verwaltungsmitarbeiter(innen) auf diese neue Situation eingestellt sein und unabhängig von der Art der Behinderung des/der Ratsuchenden offen für individuelle Lösungen sein.

\section{$5 \quad$ Entwicklung einer inklusiven Verwaltungskultur}

Verwaltungsstellen sind Organisationen, die eine spezifische Organisationskultur ausgeprägt haben. Unter Organisationskultur (Schein 1985) sind neben gegenständlichen, sichtbaren Dingen auch typische Wissens- und Verhaltensstrukturen und vor allem tiefsitzende Glaubenssysteme und Haltungen zu verstehen, die sich Verwaltungsmitarbeiter(innen) im Laufe ihrer beruflichen Praxis aneignen. Zur Organisationskultur gehören auch bestimmte für selbstverständlich gehaltene Annahmen, die sich auf den richtigen Umgang mit Menschen mit Behinderungen im Verwaltungsalltag beziehen. Somit stellt sich die Aufgabe, die Herausbildung einer ,inklusiven Kultur ' in der Behörde zu fördern. Eine inklusive Kultur in einer Kommunalverwaltung beinhaltet beispielsweise eine bestimmte proaktiv-empathische Haltung gegenüber einem/r Ratsuchenden mit einer Beeinträchtigung. Diese kann sich etwa in einem ,Mitdenken` äußern, wie trotz einer körperlichen oder geistigen Beeinträchtigung ein eigenständiger Behördengang ermöglicht werden kann oder gegebenenfalls bei nicht ohne Weiteres veränderbaren Barrieren, wie Alternativen aussehen könnten, die nicht diskriminierend sind. Eine in- 
klusive Kultur kann durch kulturbildende Maßnahmen entwickelt werden. Beispiele dafür sind etwa: systematische Begehungen der Verwaltungsräume mit Menschen mit Behinderungen, Einladungen von Selbsthilfeorganisationen zu internen Sitzungen oder die Durchführung von Projekten und gemeinsame Aktivitäten mit Selbsthilfeorganisationen.

Vor allem größere Verwaltungen sind i.d.R. gegliedert in mehrere Fachbereiche, die sich mit ihren Sachgebieten auf vielfältige Lebensbereiche der Bürger(innen) beziehen (u.a. Jugend-, Gesundheit-, Schul-, Sozial-, Verkehrs-, Bauverwaltung usw.). Die einzelnen Fachbereiche und Sachgebiete beziehen sich auf sehr unterschiedliche Handlungsfelder mit unterschiedlichen Kommunikationsformen und demnach auch unterschiedliche Realitätsverständnisse. Jede einzelne Organisationseinheit hat wiederum ihr eigenes Selbstverständnis und folgt bestimmten Eigenlogiken. Soll eine inklusionsorientierte Verwaltung zur übergreifenden Leitvorstellung werden, dann sollten alle Verwaltungsbereiche angesprochen werden, vor allem aber solche mit intensiven Bürgerkontakten und natürlich auch die ,behinderungsspezifischen'Abteilungen.

\section{Inklusionsorientierte Handlungskonzepte für Verwaltungsstellen}

Abschließend sollen exemplarisch zwei Handlungskonzepte für Verwaltungen skizziert werden, mit denen durch systematische Aktivitäten dazu beigetragen werden soll, die Barrierefreiheit einzelner Abteilungen und der Verwaltungsorganisationen insgesamt zu verbessern. Vorgestellt werden soll zum einen das Konzept der Landeshauptstadt Wiesbaden „Leitfaden für eine barrierefreie Verwaltung" (2016), das für eine rein verwaltungsinterne Vorgehensweise steht. Zum anderen soll ein knapper Überblick über die ZPE-Arbeitshilfe „Inklusionsorientierte Verwaltung“ (Konieczny et al. 2012) gegeben werden, deren Ansatz auf Sensibilisierung der Verwaltungsstelle durch Kooperationsaktivitäten mit Personen aus der Behindertenselbsthilfe beruht. 
6.1 "Leitfaden für eine barrierefreie Verwaltung“ (Wiesbaden)

Das Wiesbadener Handlungskonzept für eine barrierefreie Stadtverwaltung wurde im Rahmen eines vom Land Hessen geförderten Vorhabens von einer Projektgruppe des Amtes für Soziale Arbeit erstellt und 2016 mit einem Vorwort des Oberbürgermeisters freigegeben. Der Leitfaden bezieht sich auf die UN-Behindertenrechtskonvention und richtet sich an „alle Menschen, die die Stadtverwaltung kontaktieren [...] insbesondere Menschen mit verschiedensten Behinderungen und Menschen mit Migrationshintergrund“ sowie an alle Mitarbeitenden der Stadtverwaltung (ebd. 7). Im Zuge des Projektes wurden anhand konkreter Beispiele aus verschiedenen Bereichen der Stadtverwaltung Kriterien und Vorschläge erarbeitet, wie Bescheide, Broschüren und andere Informationsmaterialien in einfach verständliche Formen und zugängliche Formate gebracht werden können. Des Weiteren wurden systematisch Informationen zusammengestellt, wie durch bestimmte Vorkehrungen und Verfahren die Kommunikation der Verwaltungsmitarbeitenden mit unterschiedlich beeinträchtigten Personen erleichtert werden kann. Mit diesen Materialien als Basis wurde ein auf eineinhalb Tage angelegtes Fortbildungskonzept für Mitarbeitende der Stadtverwaltung entwickelt, das die Elemente a) Sach- und Fachwissen, b) Bewusstseinsbildung und c) Umgang mit Kundinnen und Kunden umfasst. Auf dieser Grundlage werden nunmehr jährlich und als Teil des städtischen Fortbildungsverzeichnisses Schulungsangebote für Mitarbeitende aus verschiedenen städtischen Ämtern durchgeführt. Die Erfahrungen zeigen, „dass großes Interesse und Informationsbedarf über Maßnahmen jeglicher Art zur Barrierefreiheit bei den Mitarbeitenden besteht. Dazu zählen Audiobescheide, die Möglichkeit der Inanspruchnahme eines Gebärdendolmetschers, Erläuterungen zu Einfacher Sprache, Verhaltensweisen von psychisch/seelisch erkrankten Menschen und deren Umgang etc." (ebd. 21).

\subsection{ZPE-Arbeitshilfe „Inklusionsorientierte Verwaltung“}

Das ZPE-Handlungskonzept wurde 2014 im Rahmen eines Projekts zusammen mit der Kreisverwaltung Olpe erarbeitet (Konieczny et al. 2012). Es zielt darauf ab, die Sensibilität der Verwaltungsmitarbeitenden für Diskriminierungsrisiken und Teilhaberechte von Menschen mit Behinderungen im Verwaltungsalltag zu stärken. 
Das Handlungskonzept basiert darauf, dass Menschen mit verschiedenen Behinderungen als ,Experten in eigener Sache' über Erkundungsgänge aktiv in die Suche nach Barrieren im Verwaltungshandeln einbezogen werden. Gleichzeitig soll es so leichter möglich werden, sachgerechte Lösungen für Anfragen und Probleme zu finden. Durch die Einbeziehungen von Menschen mit Behinderungen in die Identifikation und Bearbeitung von Barrieren soll auch die Kompetenz von Verwaltungspersonal im Umgang mit Menschen mit Behinderungen gestärkt werden.

Angesetzt werden soll an den individuellen Entwicklungsstand einer Verwaltungsorganisation. Bisher unternommene Schritte in Richtung Barrierefreiheit sollen bewusst ermittelt und weiterentwickelt werden, bisher noch nicht erkannte bzw. nicht bearbeitete Barrieren sollen thematisiert und auf Lösungen hin geprüft werden. Es soll damit der Anspruch kommunaler Verwaltungen unterstützt werden, der Vorbildfunktion einer öffentlichen Verwaltung gerecht zu werden.

Das Konzept besteht aus fünf Arbeitsschritten, die im Ergebnis zu ,Handlungsempfehlungen zur Entwicklung einer inklusionsorientierten Verwaltung führen sollen:

1. Einrichtung einer Projektgruppe aus Verwaltung und örtlicher Behindertenselbsthilfe

2. Informationsveranstaltung für alle Abteilungen

3. Schriftliche Befragung von Mitarbeiter(inne)n aller Verwaltungsabteilungen zu Erfahrungen mit Menschen mit Behinderungen und zur Ermittlung von Veränderungsbedarf

4. Verwaltungsbegehungen (,Erkundungen') durch Teams bestehend aus Menschen mit Behinderungen und Mitarbeiter(inne)n der Verwaltung

5. Erarbeitung von konkreten Handlungsempfehlungen durch die Projektgruppe

6. Beschlussfassung über Handlungsempfehlungen durch die Verwaltungsspitze 
Die Informationssammlung des kooperativ angelegten Konzepts beruht auf einer schriftlichen Befragung und auf systematisch durchgeführten Erkundungsgängen. Durch schriftliche Befragung können Ideen und konkrete Vorschläge für Veränderungen breit gesammelt werden. Erfahrungsgemäß regen die Fragestellungen die Mitarbeitenden zum Nachdenken über bisher vielleicht hingenommene oder übersehene Barrieren an und erleichtern es, sich gedanklich in die Situation von Menschen mit unterschiedlichen Behinderungsformen hineinzuversetzen. So können gleichsam auch individuelle oder auch bereichsbezogene Lernprozesse initiiert werden. Vorschläge für einen entsprechenden Fragebogen finden sich im Anhang der Veröffentlichung.

Die Methode der Begehung eignet sich besonders gut für die Identifizierung von Problemen der Auffindbarkeit, Zugänglichkeit und Nutzbarkeit sowohl hinsichtlich der Mobilitätskette zur Behörde sowie innerhalb der Verwaltung (Gebäude) als auch bei verwaltungsbezogenen Dienstabläufen (z. B. bei Telefonaten mit den Ämtern). In Zusammenarbeit mit örtlichen Selbsthilfeorganisationen sollen Personen mit unterschiedlichen Beeinträchtigungen für das Vorhaben gewonnen werden. Bewährt hat sich die Vorgehensweise der aufgabenbezogenen Erkundungen, bei denen - im Sinne eines Rollenspiels unterschiedlich behinderte Personen die Verwaltung aufsuchen, um ausgewählte Dienstleistungen in Anspruch zu nehmen. Es sollte darauf geachtet werden, dass sie und die damit verbundenen Anlaufstellen so gewählt werden, dass möglichst eine große Bandbreite an unterschiedlichen Gebäudeteilen und Dienstleistungen abgedeckt ist. Beispiele für ausgewählte Dienstleistungen sowie Vorschläge für sogenannte Erkundungsbögen finden sich ebenfalls im Anhang der Arbeitshilfe.

Die zu Beginn des Prozesses konstituierte Projektgruppe soll die Befragungs- und Erkundungsergebnisse zusammenfassen und eine Vorlage für einen Aktionsplan mit Handlungsempfehlungen erarbeiten, die sich auf die Beseitigung von als besonders dringlich erachteten Barrieren beziehen. Die Handlungsempfehlungen wären dann den zuständigen Gremien zur Prüfung und Beschlussfassung vorzulegen. 
Das Prinzip der gleichberechtigten Teilhabe aller Menschen unabhängig von Rasse, Geschlecht, Herkunft, Alter oder Behinderung am gesellschaftlichen Leben ist zutiefst demokratischer Natur. Im Rahmen der UN-Behindertenrechtskonvention wurde es gleichsam exemplarisch für Menschen mit Behinderungen als Menschenrecht kodifiziert, das alle Bereiche des öffentlichen Lebens berührt. Die Möglichkeit zur Teilhabe setzt die Möglichkeit des Zugangs zu den Institutionen und Einrichtungen für die Allgemeinheit voraus. Diese normalen Institutionen standen lange Zeit nur Menschen offen, die sich innerhalb eines recht engen Normalitätsspektrums bewegen. Wenn Menschen schwerwiegendere Beeinträchtigungen oder andere psychosoziale Probleme hatten, gehörten sie nicht dazu und blieben ausgegrenzt. Diese Zusammenhänge galten lange auch für öffentliche Verwaltungsstellen und damit einen Bereich, in dem demokratische Grundsätze wie das Recht auf Gleichbehandlung in besonderer Weise für das gesellschaftliche Leben bedeutsam sind. Im Zuge der Umsetzung der UN-Behindertenrechtskonvention ist nun selbstverständlich auch die öffentliche Verwaltung in ihren vielfältigen Ausprägungen gefordert, ihre Prozesse daraufhin zu prüfen, inwieweit sie Menschen wegen körperlicher oder anderer Beeinträchtigungen diskriminieren. Im vorliegenden Text wird argumentiert, dass aufgrund der Modernisierungsprozesse in der Verwaltungspraxis gute Voraussetzungen dafür entstanden sind, dass Inklusion ein fester Bestandteil einer demokratischen Verwaltungsphilosophie werden kann. Beispiele und Handlungsorientierungen dafür sind verfügbar. 
Barrierefreie Verwaltung - Grundlagen und Handlungskonzepte

\section{Literaturverzeichnis}

AgENTUR BARRIEREFREI NRW (2008), URL: www.ab-nrw.de (letzter Zugriff: 03.02.2018).

BGG - Behindertengleichstellungsgesetz hier hinzufügen?

Degener, Therese/Diehl, ElKe (2015): Handbuch Behindertenrechtskonvention. Teilhabe als Menschenrecht - Inklusion als gesellschaftliche Aufgabe. Bundeszentrale für politische Bildung, Schriftenreihe Band 1506, Bonn.

Grunow, Dieter (1978): Alltagskontakte mit der Verwaltung. Capus-Verlag Frankfurt

Grunow, Dieter/Nothbaum-Leiding, Birgit/Wohlfahrt, Norbert (1988): Bürgernahe Verwaltung: Theorie, Empirie, Praxismodelle. Frankfurt: Capus Verlag.

Inklusionskataster NRW an der Universität Siegen (o.J.). URL: www.inklusive-gemeinwesen.nrw.de (letzter Zugriff: 03.02.2018).

JANN, WERNER/WEGRICH, KAI (2010): „Governance und Verwaltungspolitik: Leitbilder und Reformkonzepte“. In: BENZ, ARTHuR/DoOse, NiCOLAI (2010): Governance - regieren in komplexen Regelsystemen. Eine Einführung. 2. Aufl., Basel: Beltz-Verlag, 175-200.

Jaspers, KARL (1966): Wohin treibt die Bundesrepublik Deutschland? Tatsachen - Gefahren-Chancen. München: Piper.

Krell, Gertraude/Mückenberger, Ulrich/Tondorf, Karin (2004): Gender Mainstreaming: Chancengleichheit (nicht nur) für Politik und Verwaltung. Springer Link.

KonieCZny, Eva ET AL. (2012): Inklusionsorientierte Verwaltung. Arbeitshilfe zur Sensibilisierung und Qualifizierung von kommunalen Verwaltungsstellen. ZPE-Schriftenreihe. Siegen: Univ.

LANDESHAUPTSTADT WIESBADEN (2016): Leitfaden für eine barrierefreie Verwaltung. Eigenverlag Amt für Soziale Arbeit - Abteilung Behindertenarbeit Koordinationsstelle für Behindertenarbeit, Wiesbaden.

Schein, Edgar H. (1985): Organizational Culture and Leadership. San Francisco/Calif. u.a.: Jossey-Bass.

UN-BEHINDERTENRECHTSKONVENTION (UN-BRK) (2007): Übereinkommen der Vereinten Nationen über die Rechte von Menschen mit Behinderung (amtliche gemeinsame Übersetzung von Deutschland, Lichtenstein, Österreich und der Schweiz; Originaldokument in englischer Sprache; Originaldokument in französischer Sprache sowie deutsche Übersetzung in Leichter Sprache), URL: https://www.behindertenbeauftragte.de/ SharedDocs/Publikationen/UN_Konvention_deutsch.pdf?_blob=publication File\&v=2 (letzter Zugriff: 05.03.2018). 

THOMAS M. SCHINDLER

\section{Ansätze für barrierefreie Kommunikation im medizinisch-pharmazeutischen Bereich}

Nahezu alle Menschen werden im Laufe ihres Lebens kurzzeitig oder dauerhaft krank und suchen nach medizinisch-pharmazeutischen Informationen, etwa wenn ihre Krankheit durch die Einnahme eines Arzneimittels gelindert oder geheilt werden soll. Doch nicht nur in akuten Krankheitssituationen ist medizinisch-pharmazeutische Information wichtig. Zugang zu und Verständnis von medizinisch-pharmazeutischen Zusammenhängen ist notwendig, um die individuelle Gesundheit zu erhalten und um Erkrankungen vorzubeugen. Die Fähigkeit medizinisch-pharmazeutische Information zu verstehen ist ein wichtiger Teil von Gesundheitskompetenz („health literacy“) und hat somit einen direkten Bezug zur Lebensqualität (hierzu auch Maaß/Rink 2017).

Hier soll es nicht um gesundheitsrelevante Informationen im Allgemeinen, sondern um die deutlich enger gefassten medizinisch-pharmazeutischen Informationen gehen. In Abwesenheit einer allgemein akzeptierten Definition sollen hierunter Informationen und Wissensbestände verstanden werden, die sich mit der Erforschung, Entwicklung, Anwendung und Wirkung von Arzneimitteln befassen. Medizinisch-pharmazeutische Informationen sind gewissermaßen von Barrieren umstellt, denn sie sind inhaltlich komplex, enthalten häufig Zahlen und müssen allgemeinverständlich, aber auch rechtssicher formuliert sein. Jede Verbesserung des Zugangs, jede Verringerung der Hürden wird vielen Menschen zugutekommen.

Nachfolgend soll es um zwei Dokumente gehen, die komplexe medizinischpharmazeutische Daten und Zusammenhänge allgemeinverständlich darstellen sollen: um die Packungsbeilage, also den Beipackzettel für Arzneimittel, und um Lay Summaries, also eine Zusammenfassung der Ergebnisse von klinischen Studien für Laien. Beide Dokumententypen sollen „leicht verständlich“ und in „einfacher Sprache“ formuliert sein und für beide gibt es Vorgaben, wie dies 
konkret zu erreichen sei. In diesen Vorgaben wird der Begriff „einfache Sprache“ („plain language“, „simple language“) jedoch nicht genauer definiert und wird wohl als einfache Variante der Standardsprache verstanden (wie dargestellt bei Bock 2015: 85, Bredel/Maaß 2016: 526ff.). Für beide Dokumente ist die Verpflichtung zu guter Verständlichkeit und zum Gebrauch von einfacher Sprache gesetzlich vorgegeben: für die Packungsbeilage in der EU-Richtlinie ( $\$ 63(2)$, EC 2001/83) und der Lesbarkeitsrichtline (EC 2009, S. 7-10) und für Lay Summaries in der EU-Verordnung (Abschnitte 39, 67, §37, EU 536/2014).

Im medizinisch-pharmazeutischen Bereich hat sich eine umfangreiche Fachsprache entwickelt, die primär der Kommunikation unter Experten und Expertinnen („expert2expert“) dient. Der bio-medizinisch-pharmazeutische Fortschritt und die globale Ausrichtung des pharmazeutischen Geschäftsmodels führen dazu, dass diese Fachsprache äußerst produktiv ist und dass in sie ständig neue Begriffe und Konzepte eingeführt werden. Viele Jahrzehnte lang schloss die Kommunikation unter Fachleuten (also Ärztin - Ärztin, Arzt Pharmazeut, Pharmafirma - Aufsichtsbehörden, Aufsichtsbehörden - Ärzteschaft) die Patient(inn)en nahezu aus. Erst seit den 1970er Jahren kommt es zu einem allmählichen Wandel von einem paternalistischen $\mathrm{zu}$ einem partnerschaftlichen Arzt-Patienten-Verhältnis. Doch ein partnerschaftliches Verhältnis zwischen Ärzt(inn)en, Patient(inn)en, Pharmazeut(inn)en und Pharmafirmen ist nur durch ein Angleichen und Verringern des Informationsgefälles möglich. Nur wenn die Vorzüge und Risiken verschiedener Behandlungsoptionen verstanden werden, können Patient(inn)en die ihrer Lebenssituation angemessene Therapieentscheidung treffen (Bönig 2017, Riedler 2013).

Es ist eine Besonderheit der Kommunikation zwischen Pharmafirmen, Patient(inn)en und Gesundheitsdienstleistern, also z. B. Ärzt(inn)en und Pharmazeut(inn)en, dass sie strikten rechtlichen Vorgaben auf nationaler und internationaler Ebene unterliegt. Auch bei der Zulassung von Arzneimitteln und bei der Überwachung der Arzneimittelsicherheit sind staatliche oder übernationale Aufsichtsbehörden entscheidende Kommunikationspartner. Viele Dokumente, wie etwa die Packungsbeilage, müssen von den Aufsichtsbehörden genehmigt werden, bevor sie öffentlich werden. Bei der Packungsbeilage wird die endgültige Version zwischen Arzneimittelhersteller und Aufsichtsbehörden abgestimmt. 


\section{$1 \quad$ Allgemeine Kennzeichen medizinisch-pharmazeutischer Information}

Medizinisch-pharmazeutische Informationen sind durch eine Reihe von Merkmalen gekennzeichnet, die Zugangsbarrieren darstellen können. Diese sind in Abbildung 1 zusammengefasst.

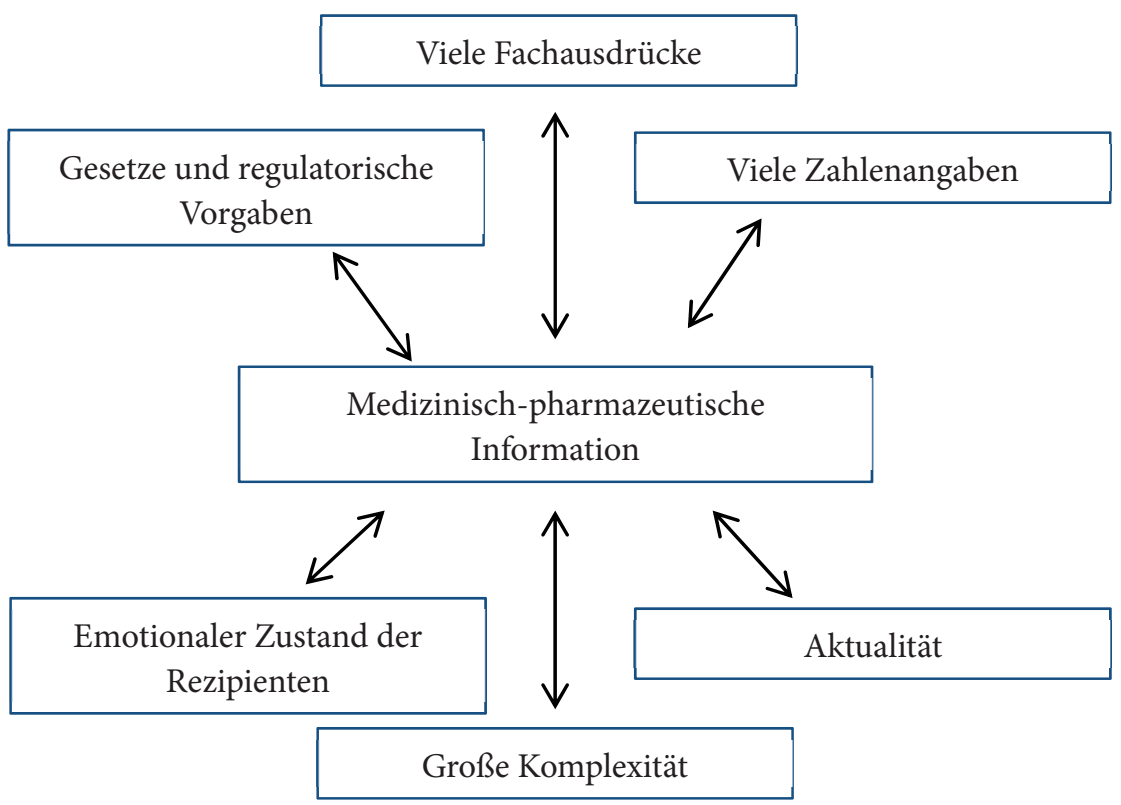

Abb. 1: Merkmale medizinisch-pharmazeutischer Information

Da sie Elemente aus verschiedenen Wissenschaften (Medizin, Biologie, Chemie, Pharmazie, klinische Forschung) enthalten, sind medizinisch-pharmazeutische Informationen in der Regel komplex. Häufig sind sie mit Zahlenangaben gekoppelt, die entscheidende Aspekte zum Ausdruck bringen. Die Ausprägung eines Zahlenwertes entscheidet in vielen Fällen zwischen "gesund“ und „krank“. Viele medizinisch-pharmazeutische Sachverhalte sind in Fachwörtern fixiert, die griechische oder lateinische Wurzeln haben oder aus dem Englischen stammen. 
Die Bereitstellung und die schriftliche Übermittlung medizinisch-pharmazeutischer Information zwischen Herstellern und Verwendern unterliegen strikten gesetzlichen Vorgaben. Die gesetzlichen Regelungen sollen sicherstellen, dass Ärzt(inn)en und Patient(inn)en vollständige und objektive Informationen zur Verfügung gestellt werden. Die rechtlichen Vorgaben erstrecken sich dabei nicht nur auf die Art der Information, die dargestellt werden soll, sondern auch auf den kommunikativen Kontext sowie auf deren Anordnung und Format. Medizinisch-pharmazeutische Informationen werden von den Rezipient(inn)en häufig in einem emotional erregten Zustand gesucht, übermittelt und aufgenommen. Die Emotion rührt aus der unmittelbaren persönlichen Betroffenheit, etwa nach Diagnose einer schwerwiegenden Erkrankung der Rezipient(inn)en oder eines(r) ihrer Angehörigen. Dies ist deshalb von Bedeutung, weil starke Emotionen einen Einfluss auf das Textverstehen haben können (Bohn-Gettler/ Rapp 2011, Langer/Frie 2017).

Um möglichst direkt vom medizinisch-pharmazeutischen Fortschritt zu profitieren, haben viele Rezipient(inn)en von medizinisch-pharmazeutischen Informationen ein besonders großes Interesse an neuen und allerneuesten Ergebnissen und Entwicklungen. Die neuesten Ergebnisse sind jedoch zum Teil mit großer Unsicherheit behaftet, eben weil sie noch nicht zum anerkannten Fachwissen einer Disziplin zählen. Die Unsicherheit bezieht sich nicht nur auf die Bedeutung der Ergebnisse für die medizinische Versorgung, sondern auch auf die Validität der verwendeten Methoden. Mit dieser „Vorläufigkeit“ von neuesten Informationen sind natürlich auch die Expert(inn)en konfrontiert, so dass ein(e) Patient(in), der oder die begeistert mit der Kopie einer brandneuen Publikation zu seiner/ihrer Ärztin bzw. Arzt kommt, wahrscheinlich auf Zurückhaltung trifft, da auch der/die Experte/Expertin nicht in der Lage ist, die Relevanz der Ergebnisse zum aktuellen Zeitpunkt abschließend zu bewerten. 


\section{Die Packungsbeilage (Beipackzettel) als Schlüsseldokument in der medizinisch-pharmazeutischen Kommunikation}

Seit 1992 muss jedes in der Europäischen Union verkaufte Arzneimittel mit einer Packungsbeilage (Beipackzettel, Gebrauchsinformation, umgangssprachlich „Waschzettel“) versehen sein. Wenn Patient(inn)en keine ausführliche Erklärung zu einem Arzneimittel durch den/die verschreibende(n) Arzt/Ärztin oder den/die verkaufende(n) Apotheker(in) erhalten, ist die Packungsbeilage oft ihre einzige Informationsquelle. Die Packungsbeilage, ein 4 bis10-seitiges Dokument, muss den Patient(inn)en alle wichtigen Informationen zu einem Arzneimittel vermitteln, den Zweck, die Wirkungsweise, die damit einhergehenden Risiken (Nebenwirkungen) usw. Wenn Patient(inn)en die Informationen in der Packungsbeilage nicht verstehen, ist nicht nur das Therapieziel gefährdet, unter Umständen bringen sich Patient(inn)en durch fehlerhafte Anwendung eines Arzneimittels sogar in Gefahr.

Eine Packungsbeilage soll mindestens drei Kommunikationsbedürfnisse erfüllen:

- Patient(inn)en erwarten von der Packungsbeilage verständliche Information über die Anwendung und Risiken eines Arzneimittels.

- Für Pharmafirmen müssen Aufbau und Text einer Packungsbeilage eine sichere Anwendung des Arzneimittels ermöglichen, Haftungsrisiken ausschließen und gesetzliche Vorgaben erfüllen.

- Gesetzgeber sowie Zulassungs- und Aufsichtsbehörden wollen durch die einheitliche Gestaltung von Packungsbeilagen den Schutz der Patient(inn)en sicherstellen und gleiche Rahmenbedingungen für alle Marktteilnehmer schaffen.

Diese Kommunikationsziele stehen zum Teil miteinander im Wettbewerb: Verständlichkeit vs. fachliche Richtigkeit, Lesbarkeit und Kürze vs. Aufzählung aller Risiken, gesetzliche Vorgaben vs. Anpassung an ein spezifisches Arzneimittel. 
Die Packungsbeilage ist ein stark reguliertes Dokument. Sowohl der Inhalt als auch die Form und die sprachliche Gestaltung bis hin zu einzelnen Formulierungen müssen gesetzlichen Vorgaben auf nationaler und europäischer Ebene folgen. Auf europäischer Ebene sind die Vorgaben im Gemeinschaftskodex für Humanarzneimittel (2001/83/EG, Titel V) formuliert. In $\$ 59$ wird festgelegt, welche Angaben in der Packungsbeilage gemacht werden müssen und in welcher Reihenfolge diese aufzuführen sind. $\$ 61$ besagt, dass Entwürfe für die Packungsbeilage schon im Genehmigungsverfahren den Behörden eingereicht werden müssen und dass die Behörden die Genehmigung verweigern, wenn nicht alle Aspekte adäquat dargestellt wurden. Schließlich stellt $\$ 63$ fest: „Die Packungsbeilage ist so $\mathrm{zu}$ formulieren und zu konzipieren, dass sie klar und verständlich ist, so dass sich Verwender, erforderlichenfalls mit Hilfe von Angehörigen der Gesundheitsberufe, angemessen verhalten können. Die Packungsbeilage ist gut lesbar in einer Amtssprache bzw. in Amtssprachen des Mitgliedstaats abzufassen [...].“ ( $\$ 63$ 2001/83/EG, Titel V, meine Hervorhebungen).

Um die Packungsbeilagen und die wissenschaftliche Arzneimittelinformation (Fachinformationen) auf europäischer Ebene zu harmonisieren, wurde 1996 eine Arbeitsgruppe („Working group on quality review of documents“) eingerichtet. Sie soll die Europäische Arzneimittelbehörde (European Medicines Agency, EMA) bei den sprachlichen Aspekten dieser Dokumente unterstützen. Die Arbeitsgruppe trifft sich regelmäßig und befasst sich mit der sprachlichen Klarheit, Genauigkeit und Konsistenz von Packungsbeilagen. Sie überprüft die terminologische Konsistenz von Übersetzungen mit dem Original, fördert Aspekte der Lesbarkeit und will zu einem gemeinsamen Verständnis der gesetzlichen Vorgaben und Richtlinien beitragen (EMA 2014: 1-2). Die Arbeitsgruppe hat für bestimmte Inhalte sogenannte "QRD Formatvorlagen“ („QRD templates“) entwickelt, also verbindliche Formulierungen, die in die Packungsbeilagen übernommen werden müssen. Diese Standardisierung der Texte in Packungsbeilagen soll die Harmonisierung der Arzneimittelinformationen in der EU vorantreiben.

Seit 1998 gibt es eine europäische Lesbarkeitsrichtlinie für Packungsbeilagen (Guideline on the readability of labelling and package leaflet, Revision 1, 2009). Sie beschreibt, wie Packungsbeilagen verständlich formuliert werden können 
(Tabelle 2). Die Lesbarkeitsrichtlinie beschreibt auch die Zielgruppe: „Die Packungsbeilage ist gedacht für Patienten und Verbraucher. Wenn eine Packungsbeilage gut gestaltet und in klaren Worten geschrieben ist, maximiert dies die Zahl derer, die diese Information nutzen können, so dass auch ältere Kinder, Jugendliche, Menschen mit geringer Lesefähigkeit und Menschen mit Sehbehinderungen diese Informationen verwenden können." (ebd.: 7, meine Übersetzung und Hervorhebung).

Die Lesbarkeitsrichtlinie schreibt vor, dass Packungsbeilagen durch Patientengruppen auf Lesbarkeit und Auffindbarkeit der Information getestet werden müssen. Es wird genau vorgegeben, wie diese Tests entwickelt, durchgeführt und ausgewertet werden müssen. Seit 2005 müssen alle Packungsbeilagen durch Patientengruppen überprüft werden. Die Unterlagen zu diesen Prüfungen müssen mit dem Zulassungsdossier eingereicht werden. Das Bundesinstitut für Arzneimittel und Medizinprodukte (BfArM) machte 2015 die Anforderungen der europäischen Lesbarkeitsrichtlinie und den QRD-Formatvorlagen auch für deutsche Packungsbeilagen verpflichtend (BfArM 2015).

In Deutschland wird der Inhalt von Packungsbeilagen durch $\$ 11$ des Arzneimittelgesetzes (AMG 2005) geregelt. Es gibt vor, welche Angaben in welcher Reihenfolge in der Packungsbeilage enthalten sein müssen. Die Angaben sind „allgemein verständlich in deutscher Sprache, in gut lesbarer Schrift [...]“ zu halten ( $\$ 11$ AMG, meine Hervorhebung).

Tab. 1: Pflichtangaben, die in der Packungsbeilage gemäß $₫ 11$ AMG enthalten sein müssen

1. Identifizierung des Arzneimittels: die Bezeichnung des Arzneimittels, die Stoff- oder Indikationsgruppe oder die Wirkungsweise

2. Die Anwendungsgebiete

3. Eine Aufzählung von Informationen, die vor der Einnahme des Arzneimittels bekannt sein müssen: Gegenanzeigen, entsprechende Vorsichtsmaßnahmen für die Anwendung, Wechselwirkungen mit anderen Arzneimitteln, Warnhinweise

4. Anleitungen für eine ordnungsgemäße Anwendung, Dosierung, Art der Anwendung, Häufigkeit der Verabreichung, ggf. mit Angabe des genauen Zeitpunkts, Dauer der Behandlung, falls diese festgelegt werden soll, Hinweise für den Fall der Überdosierung, der unterlassenen Einnahme oder Hinweise auf die Gefahr von unerwünschten Folgen des Absetzens, die ausdrückliche Empfehlung, bei Fragen zur Klärung der Anwendung den Arzt oder Apotheker zu befragen

5. Beschreibung der Nebenwirkungen, die bei bestimmungsgemäßem Gebrauch des Arzneimittels eintreten können; bei Nebenwirkungen zu ergreifende Gegenmaßnahmen, soweit dies nach dem jeweiligen Stand der wissenschaftlichen Erkenntnis erforderlich ist; bei allen 
Arzneimitteln, ist zusätzlich ein Standardtext aufzunehmen, durch den die Patienten ausdrücklich aufgefordert werden, jeden Verdachtsfall einer Nebenwirkung ihren Ärzten, Apothekern, Angehörigen von Gesundheitsberufen oder unmittelbar der zuständigen Bundesoberbehörde zu melden

6. Hinweis auf das auf der Verpackung angegebene Verfalldatum sowie Warnung davor, das Arzneimittel nach Ablauf dieses Datums anzuwenden, besondere Vorsichtsmaßnahmen für die Aufbewahrung und die Angabe der Haltbarkeit nach Öffnung des Behältnisses, Warnung vor bestimmten sichtbaren Anzeichen dafür, dass das Arzneimittel nicht mehr zu verwenden ist, vollständige qualitative Zusammensetzung nach Wirkstoffen und sonstigen Bestandteilen, Darreichungsform und Inhalt nach Gewicht, Nennvolumen oder Stückzahl für jede Darreichungsform des Arzneimittels, Name und Anschrift des pharmazeutischen Unternehmers und, soweit vorhanden, seines örtlichen Vertreters, Name und Anschrift des Herstellers oder des Einführers

7. Bei einem Arzneimittel, das unter anderen Bezeichnungen in anderen Mitgliedstaaten der Europäischen Union, ein Verzeichnis der in den einzelnen Mitgliedstaaten genehmigten Bezeichnungen

8. Datum der letzten Überarbeitung der Packungsbeilage

Arzneimittelgesetz in der Fassung der Bekanntmachung vom 12. Dezember 2005 (BGBl. I S. 3394), geändert zuletzt durch Artikel 1 des Gesetzes vom 18. Juli 2017 (BGBl. I S. 2757), modifiziert

Seit der europaweiten Einführung 1992 gab es viele regulatorische Initiativen, Packungsbeilagen möglichst verständlich zu gestalten:

- Genaue gesetzliche Vorgaben zu Inhalt und zur Struktur (EU-Regularien, $A M G)$,

- Festgelegte Texte für bestimmte Abschnitte, (QRD Formatvorlagen),

- Anweisungen zu den sprachlichen Mitteln, die eingesetzt werden sollen (Lesbarkeitsrichtlinie).

- Die Verpflichtung, Packungsbeilagen mit Patient(inn)en zu testen.

Es gibt nun empirische Hinweise darauf, dass diese Initiativen tatsächlich dazu führten, dass Packungsbeilagen von einer Mehrheit der Patient(inn)en verstanden werden. 
In einer Studie aus dem Jahr 2003 legte das wissenschaftliche Institut der AOK die Packungsbeilagen der 100 meistverordneten Arzneimittel 70 Testpersonen vor und ließ sie die Lesbarkeit und Verständlichkeit beurteilen. Außerdem wurden 1900 gesetzlich Versicherte, denen im Vorjahr ein Arzneimittel verschrieben wurde, mittels eines strukturierten Telefoninterviews zu Packungsbeilagen befragt (Nink/Schröder 2005). Wie die Untersuchung zeigt, finden sehr viele der Befragten, nämlich $89.9 \%$ die Packungsbeilage wichtig oder sehr wichtig (Nink/Schröder 2005: 53). Fast alle Versicherten (98\%), lasen die Packungsbeilage zumindest teilweise; 57\% gaben an, sie ganz gelesen zu haben (ebd.: 53). Auch wenn sich 63\% der Versicherten (ebd.: 61) gut informiert fühlten, so haben $28 \%$ der Patient(inn)en die Arzneimittel, die sie verordnet bekamen, aufgrund ihrer Lektüre der Packungsbeilage abgesetzt (ebd.: 56). Ungefähr ein Drittel fühlte sich durch die Informationen im Beipackzettel verunsichert (ebd.: 56). Bei der Beurteilung von Verständlichkeit von Packungsbeilagen waren sowohl die Schulbildung (je höher der Bildungsabschluss, desto weniger Verständnisprobleme) als auch das Alter (je älter, desto mehr Verständnisprobleme) wichtige Einflussfaktoren. Unter den über 60-jährigen Befragten fanden immerhin 23,9\% Packungsbeilagen unverständlich oder sehr unverständlich (ebd.: 69). Dieses Ergebnis ist von Belang, da die Gruppe der über 60-Jährigen den höchsten Anteil an Verschreibungen hat (62\% aller Arzneimittel-Tagesdosen [ebd.: 50]).

Ähnliche Ergebnisse wurden auch in einer neueren Stichprobe (computergestützte Telefoninterviews anhand eines strukturierten Fragebogens) von 2600 Erwachsenen ermittelt. Im Vergleich mit den Informationen auf Lebensmittelverpackungen, den Produktinformationen von Stromanbietern, Mobilfunkbetreibern, Banken und Versicherungen oder den Erläuterungen zur Steuerklärung nehmen Packungsbeilagen bei der Verständlichkeit eine Spitzenstellung ein: 32\% der Befragten fanden sie verständlich, während Produktinformationen von Versicherungen nur von 5\% als verständlich eingestuft wurden (ERGO 2012: 10).

Im Auftrag der Europäischen Kommission untersuchte eine internationale Arbeitsgruppe, ob die Packungsbeilage ihrer Rolle als Informationsquelle über Arzneimittel für Patient(inn)en und Gesundheitsdienstleistende (Ärzten, Apo- 
thekern) gerecht wird. Es sollten die positiven und negativen Aspekte der Packungsbeilagen herausgearbeitet werden und mögliche Verbesserungen benannt werden (van Dijk et al. 2014). Die Studie umfasste eine ausführliche Analyse der Literatur und eine Umfrage mittels (englischem) Fragebogen. Obwohl europaweit angelegt, war der verwertbare Rücklauf sehr bescheiden (49 $\mathrm{Pa}$ tient(inn)en, 33 Gesundheitsdienstleister, 123 Vertreter(innen) von Pharmaunternehmen), so dass keine Repräsentativität erreicht wurde. Es wurde festgestellt, dass sowohl Patient(inn)en als auch Gesundheitsdienstleistende deutlichen Verbesserungsbedarf sahen. So wurde die Gesamtqualität von Packungsbeilagen auf einer Skala von 1 (niedrig) bis 9 (hoch) von Patient(inn)en mit durchschnittlich 5.3 und von Ärzt(inn)en und Pharmazeut(inn)en mit 5.5 bewertet (ebd.: 52). Die Studie kam zum Ergebnis, dass Packungsbeilagen insgesamt zu viel Text enthielten, dass die Texte zu schwierig seien, zu viele medizinische Fachausdrücke enthielten, und dass zu viele Nebenwirkungen genannt würden (ebd.: 70). Außerdem wurde die zu kleine Schriftgröße vieler Packungsbeilagen bemängelt. Neben der Verwendung von "Good Information Design“ zur Verbesserung des Layouts empfehlen die Autoren, die Komplexität bei der Auflistung der Nebenwirkungen zu reduzieren und nur jene Nebenwirkungen zu nennen, die einer sofortigen Behandlung bedürfen. Außerdem sollten Packungsbeilagen nicht nur die Risiken eines Arzneimittels benennen, sondern auch den erwartbaren Nutzen angeben. Aufgrund dieser Ergebnisse hat die Europäische Arzneimittelbehörde EMA kürzlich einen Aktionsplan zur Verbesserung von Packungsbeilagen vorgelegt (EMA 2017).

In einer kommunikationswissenschaftlichen Studie wurde die Verständlichkeit von Packungsbeilagen unter Verwendung der Think-Aloud-Methode mit 10 Freiwilligen untersucht (Loh et al. 2014). Qualitativ decken sich die Ergebnisse weitgehend mit denen von van Dijk et al. (2014), die Autoren konnten jedoch genauer herausarbeiten, welche Abschnitte der Packungsbeilage als besonders problematisch empfunden wurden. Sie berichten, dass die Angaben zu Gegenanzeigen und Nebenwirkungen die Befragten besonders verunsicherten und Misstrauen gegenüber dem Arzneimittel und dem/der verschreibenden Arzt/Ärztin hervorriefen (ebd.: 1184). Auch sie empfehlen, neben einer verständlicheren Beschreibung der Risiken auch die positiven Auswirkungen des Arzneimittels in der Packungsbeilage zu beschreiben (ebd.: 1187). 
Angesichts dieser Datenlage scheint eine vorsichtig positive Bewertung gerechtfertigt. Die Packungsbeilage ist ein Beispiel dafür, wie komplexe medizinischpharmazeutische Informationen durch Richtlinien in verständliche Dokumente überführt werden können. Eine Mehrheit der Versicherten (63\%, Nink/Schröder 2005: 61) fühlte sich durch Packungsbeilagen gut informiert, jedenfalls deutlich besser als durch Produktinformationen von Banken und Versicherungen (32\% vs. 5-6\%, ERGO 2012: 10). Dabei darf jedoch nicht übersehen werden, dass ungefähr jeder fünfte (Nink/Schröder 2005: 69) dieses Dokument nicht versteht und dass insbesondere die Gruppe der über 60-Jährigen Verständnisprobleme hat. Festzuhalten bleibt, dass die ergriffenen Maßnahmen, also gesetzliche Vorgaben, verpflichtende Textvorgaben und Formatvorlagen, eine Richtlinie zum Schreiben dieser Dokumente und das obligatorische Testen mit Patient(inn)en, offensichtlich eine positive Wirkung entfalteten, auch wenn es noch viel Verbesserungspotential gibt.

\section{$5 \quad$ Lay Summaries - laiengerechte Zusammenfassung von Studienergebnissen}

Klinische Studien sind das wichtigste Instrument zur Entwicklung von Arzneimitteln. Bis ein Medikament zugelassen werden kann, müssen viele Studien durchgeführt werden, um zu belegen, dass ein neues Arzneimittel sicher und wirksam ist. Klinische Studien sind komplex und ihre Planung, Durchführung und Berichtung muss einer ganzen Reihe von internationalen und nationalen Rechtsvorschriften folgen. Zwar sind bereits seit einigen Jahren viele Studienergebnisse über Studienregister (z. B. clinicaltrials.gov, clinicaltrialsregister.eu) zugänglich, aber die Informationen richten sich an Fachleute und sind für Laien kaum verständlich. Mit der EUVerordnung zu klinischen Prüfungen, veröffentlicht im Jahre 2014, wurde erstmals europaweit die Pflicht eingeführt, für jede klinische Studie die Ergebnisse in einer für Laien verständlichen Form darzustellen (Artikel 39, 67, \$37 EU-Regulation 536/2014). Damit wird ein Dokument rechtlich verpflichtend, dessen Ziel es ist, die komplexen Daten von klinischen Studien für Laien verständlich darzustellen. 
Diese „Zusammenfassungen für den Laien“ („Lay Summaries“) sollen in einer EU-Datenbank veröffentlicht werden, sobald dieses Portal zur Verfügung steht (geplant Herbst 2019). Die Lay Summaries sollen in einfacher Sprache geschrieben werden und die breite Öffentlichkeit über alle wichtigen Aspekte einer klinischen Studie informieren. Die Informationen sollen faktisch sein und dürfen keinen werbenden Charakter haben. Die Lay Summaries sollen die Hauptziele und die Gründe zur Durchführung der Studie beschreiben. Die demographischen Daten der Prüfungsteilnehmer und die wichtigsten Ergebnisse zur Wirksamkeit und zur Sicherheit sollen angegeben werden (European Commission 2018, Sroka-Saidi et al. 2015, Schindler 2018).

Die geforderten Inhalte einer Lay Summary werden lediglich in Annex V der Verordnung genannt und beschränken sich auf eine Liste mit 10 Elementen. Die Notwendigkeit einer genaueren Spezifizierung hatte auch die Europäische Kommission erkannt und beauftragte im August 2015 die britische Health Research Authority (HRA), Empfehlungen zum Schreiben von Lay Summaries zu erarbeiten. Es wurde eine internationale Kommission gebildet, die alle Akteure mit einbezog. Nach mehreren Monaten wurde ein erster Entwurf verabschiedet, der dann in einer öffentlichen Konsultation zugänglich gemacht wurde. Nach Bewertung der eingegangenen Kommentare wurde ein finaler Entwurf verabschiedet, der dann im August 2017 veröffentlicht wurde; bereits im Februar 2018 wurde eine minimal veränderte Version 2 veröffentlicht (European Commission 2018).

Da für die allgemeine Öffentlichkeit gedacht, sollen Lay Summaries in „einfacher Sprache" geschrieben werden - also so, dass sie für durchschnittliche Leser, besser noch für Leser mit geringer Lesekompetenz, verständlich sind. Ausdrücklich wird verlangt, dass sich die Texte auf der Stufe 2-3 der International Adult Literary Survey (IALS) bewegen sollen (OECD 2000, Kirsch 2001). Durch die Orientierung an Lesern mit geringer Lesekompetenz soll das Dokument für möglichst viele Menschen zugänglich werden.

Die Expertenkommission gibt eine Reihe von Hinweisen, wie die komplizierten Sachverhalte und Ergebnisse einer klinischen Studie in laiengerechter Sprache beschrieben werden sollen. So soll der Text durch Überschriften, Aufzählungszeichen und kurze Abschnitte gegliedert werden. Die Sätze sollen kurz und knapp sein. Statt langer Sätze sollen Aufzählungszeichen („Bullets“) mit kurzen Absätzen verwendet werden. Technische, medizinische oder sonstige 
wissenschaftliche Fachausdrücke sollen vermieden und stattdessen laiengerechte Wörter verwendet werden (z. B. Bluthochdruck statt Hypertension). Ist ein Fachbegriff unumgänglich, soll ein laiengerechter Begriff oder eine laiengerechte Erklärung in Klammern hinzugefügt werden (z. B. Metastasen [Tochtergeschwüre an anderen Stellen des Körpers]). Unnötige Wörter sollen weggelassen und komplizierte durch einfache ersetzt werden. Akronyme und Abkürzungen sollen vermieden werden. Die zugrundeliegenden medizinischen Konzepte sollen klar und leicht verständlich beschrieben werden. Das Layout soll das Lesen erleichtern und den Text zugänglich machen. Um die Übersichtlichkeit zu verbessern, soll möglichst viel „weißer Raum“ um die Absätze sein. Der Einsatz von Grafiken wird empfohlen, es sollen aber nicht zu viele sein und jede Abbildung sollte nur eine Information enthalten. Eine Legende soll die Abbildung umfänglich beschreiben. Es dürfen auch kreative Ansätze bei der Erstellung von Lay Summaries verfolgt werden, so können Bilder, Cartoons und Infografiken verwendet werden (European Commission 2018: 3-7).

Ähnlich wie bei Packungsbeilagen soll eine Kombination von rechtlichen Vorgaben und Expertenempfehlungen, Autor(inn)en dazu befähigen, ein Dokument zu schreiben, das komplexe medizinisch-pharmazeutische Inhalte in laienverständlicher Sprache darstellt. Einige Pharmafirmen haben bereits damit begonnen, Lay Summaries auf ihren Webseiten zu veröffentlichen (z. B. Boehringer Ingelheim Pharma (https://trials.boehringer-ingelheim.com/trial_results.html) oder GlaxoSmithKline (https://www.gsk-clinicalstudyregister.com/)).

\section{Vergleich der Vorgaben für das Schreiben von Packungs- beilagen und Lay Summaries mit den Regeln für Leichte Sprache}

Vergleicht man die Richtlinientexte für das Schreiben von Packungsbeilagen und Lay Summaries mit den Regeln für Leichte Sprache, so lassen sich große Konvergenzen feststellen (Tabelle 2).

Ermittelt wurde, ob es für die Vorgaben, die für das Schreiben von Packungsbeilagen und für Lay Summaries formuliert wurden, korrespondierende Regeln für das Schreiben in Leichter Sprache gibt. Die Zahlen in Klammern in der Spalte 
„Übereinstimmung“ geben die Seitenzahlen an, auf denen die entsprechende Regel in „Die Regeln für Leichte Sprache“ des Netzwerks Leichte Sprache genannt wird. Es wurde eine „mittlere“, „weitgehende“ oder „vollständige“ Übereinstimmung unterschieden; „-“ bedeutet, dass dieses Element nicht aufgeführt wird. Die Kategorien in der linken Spalte wurden von der Lesbarkeitsrichtlinie für Packungsbeilagen (European Commission 2009: 7-10) übernommen.

Tab. 2: Vergleich der Anforderungen zum Schreiben von Packungsbeilagen, Lay Summaries und Texten in Leichter Sprache

\begin{tabular}{|c|c|c|c|}
\hline & $\begin{array}{l}\text { Lesbarkeitsrichtlinie für Pa- } \\
\text { ckungsbeilagen }^{1}\end{array}$ & $\begin{array}{l}\text { Expertenkommission zu Lay } \\
\text { Summaries }^{2}\end{array}$ & $\begin{array}{l}\text { Übereinstim- } \\
\text { mung mit } \\
\text { Vorgaben für } \\
\text { Leichte Spra- } \\
\text { che }^{3}\end{array}$ \\
\hline Zielgruppe & $\begin{array}{l}\text { Patienten, Verbraucher, aber } \\
\text { auch ältere Kinder, Jugendli- } \\
\text { che, Menschen mit geringer } \\
\text { Lesefähigkeit und Menschen } \\
\text { mit Sehbehinderungen }\end{array}$ & $\begin{array}{l}\text { Öffentlichkeit ohne Vorwis- } \\
\text { sen bzgl. Studie, Fachausdrü- } \\
\text { cken oder klinischer For- } \\
\text { schung, Menschen mit } \\
\text { geringer Lesefähigkeit und ge- } \\
\text { ringem Zahlenverständnis }\end{array}$ & mittel (1) \\
\hline $\begin{array}{l}\text { Einbindung der } \\
\text { Zielgruppe in Er- } \\
\text { stellung }\end{array}$ & $\begin{array}{l}\text { Einbindung von Patienten } \\
\text { ist vorgeschrieben, um Les- } \\
\text { barkeit, Klarheit und einfa- } \\
\text { chen Gebrauch zu testen }\end{array}$ & $\begin{array}{l}\text { Einbindung von Patienten, } \\
\text { Patientenorganisationen oder } \\
\text { Bürgern empfohlen, um Ver- } \\
\text { ständlichkeit zu testen }\end{array}$ & $\begin{array}{l}\text { vollständig } \\
\text { (35) }\end{array}$ \\
\hline Länge & - & „so kurz wie möglich“ & - \\
\hline Information & - & $\begin{array}{l}\text { Faktische Informationen, } \\
\text { keine werbenden Inhalte }\end{array}$ & - \\
\hline $\begin{array}{l}\text { Umgang mit } \\
\text { Zahlen }\end{array}$ & - & $\begin{array}{l}\text { Für Menschen mit geringem } \\
\text { Zahlenverständnis verstehbar }\end{array}$ & $\begin{array}{l}\text { weitgehend } \\
(11-14)\end{array}$ \\
\hline \multirow[t]{3}{*}{ Schriftgröße } & $\begin{array}{l}\text { Mindestens } 9 \text { pt Times New } \\
\text { Roman }\end{array}$ & Mindestens $12 \mathrm{pt}$ & $\begin{array}{l}\text { weitgehend } \\
(22,23)\end{array}$ \\
\hline & $\begin{array}{l}\text { Größerer Font für wichtige } \\
\text { Informationen (Überschrif- } \\
\text { ten) }\end{array}$ & - & $\begin{array}{l}\text { weitgehend } \\
\text { (23) }\end{array}$ \\
\hline & $\begin{array}{l}\text { Keine Versalschrift („all } \\
\text { caps“) }\end{array}$ & $\begin{array}{l}\text { Keine Versalschrift („all } \\
\text { caps“) }\end{array}$ & $\begin{array}{l}\text { vollständig } \\
(29)\end{array}$ \\
\hline
\end{tabular}


Ansätze für barrierefreie Kommunikation im medizinisch-pharmazeutischen Bereich

\begin{tabular}{|c|c|c|c|}
\hline & $\begin{array}{l}\text { Lesbarkeitsrichtlinie für } \mathrm{Pa} \text { - } \\
\text { ckungsbeilagen }^{1}\end{array}$ & $\begin{array}{l}\text { Expertenkommission zu Lay } \\
\text { Summaries }^{2}\end{array}$ & $\begin{array}{l}\text { Übereinstim- } \\
\text { mung mit } \\
\text { Vorgaben für } \\
\text { Leichte Spra- } \\
\text { che }^{3}\end{array}$ \\
\hline & $\begin{array}{l}\text { Keine kursiven Wörter und } \\
\text { keine Unterstreichungen }\end{array}$ & Keine Unterstreichungen & $\begin{array}{l}\text { weitgehend } \\
(29)\end{array}$ \\
\hline \multirow[t]{8}{*}{ Design/Layout } & Kein Blocksatz & - & $\begin{array}{l}\text { vollständig } \\
(24)\end{array}$ \\
\hline & - & $\begin{array}{l}\text { Viel „weißer Raum“, Themen } \\
\text { durch Leerzeilen abgrenzen }\end{array}$ & - \\
\hline & - & $\begin{array}{l}\text { Einfache Graphiken empfoh- } \\
\text { len }\end{array}$ & - \\
\hline & $\begin{array}{l}\text { Mindestens 1.5 Zeilenab- } \\
\text { stand }\end{array}$ & - & $\begin{array}{l}\text { weitgehend } \\
(23)\end{array}$ \\
\hline & $\begin{array}{l}\text { Guter Kontrast zwischen } \\
\text { Text und Hintergrund ist } \\
\text { wichtig }\end{array}$ & - & $\begin{array}{l}\text { vollständig } \\
(30)\end{array}$ \\
\hline & Keine Hintergrundbilder & - & $\begin{array}{l}\text { vollständig } \\
(34)\end{array}$ \\
\hline & $\begin{array}{l}\text { Spaltenformat zur einfachen } \\
\text { Navigation im Text }\end{array}$ & - & - \\
\hline & $\begin{array}{l}\text { Zusammengehörige Infor- } \\
\text { mation zusammen lassen }\end{array}$ & - & $\begin{array}{l}\text { vollständig } \\
(20)\end{array}$ \\
\hline \multirow[t]{3}{*}{ Überschriften } & $\begin{array}{l}\text { Überschriften zur Naviga- } \\
\text { tion, nicht mehr als } 2 \text { Hie- } \\
\text { rarchieebenen }\end{array}$ & $\begin{array}{l}\text { Überschriften zur Strukturie- } \\
\text { rung }\end{array}$ & $\begin{array}{l}\text { vollständig } \\
(27)\end{array}$ \\
\hline & $\begin{array}{l}\text { Überschriftenformat einheit- } \\
\text { lich }\end{array}$ & - & $\begin{array}{l}\text { weitgehend } \\
(27)\end{array}$ \\
\hline & $\begin{array}{l}\text { Fettdruck oder Farbe mög- } \\
\text { lich }\end{array}$ & - & $\begin{array}{l}\text { vollständig } \\
(29)\end{array}$ \\
\hline Druckfarbe & $\begin{array}{l}\text { Dunkler Text auf hellem } \\
\text { Hintergrund }\end{array}$ & $\begin{array}{l}\text { Schwarzer Text auf weißem } \\
\text { Hintergrund }\end{array}$ & $\begin{array}{l}\text { vollständig } \\
(30)\end{array}$ \\
\hline Sprache & „einfach zu lesen“ & $\begin{array}{l}\text { Einfaches Vokabular; einfa- } \\
\text { che, alltägliche Sprache }\end{array}$ & $\begin{array}{l}\text { weitgehend } \\
(4,5)\end{array}$ \\
\hline
\end{tabular}


Thomas M. Schindler

\begin{tabular}{|c|c|c|c|}
\hline & $\begin{array}{l}\text { Lesbarkeitsrichtlinie für } \mathrm{Pa} \text { - } \\
\text { ckungsbeilagen }^{1}\end{array}$ & $\begin{array}{l}\text { Expertenkommission zu Lay } \\
\text { Summaries }^{2}\end{array}$ & $\begin{array}{l}\text { Übereinstim- } \\
\text { mung mit } \\
\text { Vorgaben für } \\
\text { Leichte Spra- } \\
\text { che }^{3}\end{array}$ \\
\hline \multirow{4}{*}{ Sprache } & - & $\begin{array}{l}\text { Zugrundeliegenden Konzepte } \\
\text { sollen erklärt werden }\end{array}$ & - \\
\hline & - & $\begin{array}{l}\text { Konsistente Verwendung von } \\
\text { Ausdrücken }\end{array}$ & $\begin{array}{l}\text { vollständig } \\
\text { (5) }\end{array}$ \\
\hline & - & $\begin{array}{l}\text { Keine mehrdeutigen Ausdrü- } \\
\text { cke }\end{array}$ & $\begin{array}{l}\text { weitgehend } \\
\text { (4) }\end{array}$ \\
\hline & - & $\begin{array}{l}\text { Sprache soll Respekt gegen- } \\
\text { über Patienten zum Ausdruck } \\
\text { bringen }\end{array}$ & - \\
\hline \multirow[t]{5}{*}{ Syntax } & $\begin{array}{l}\text { Einfache Wörter mit weni- } \\
\text { gen Silben }\end{array}$ & Keine vielsilbigen Wörter & $\begin{array}{l}\text { vollständig } \\
\text { (5) }\end{array}$ \\
\hline & Keine langen Sätze & $\begin{array}{l}\text { Keine langen Sätzen mit Ne- } \\
\text { bensätzen, kurze Sätze }\end{array}$ & $\begin{array}{l}\text { vollständig } \\
\text { (17) }\end{array}$ \\
\hline & $\begin{array}{l}\text { Keine langen Abschnitte, } \\
\text { besser Aufzählungen }\end{array}$ & Aufzählungen statt Text & mittel (27) \\
\hline & $\begin{array}{l}\text { Anordnung wichtig, die häu- } \\
\text { figsten Nebenwirkungen zu- } \\
\text { erst nennen }\end{array}$ & - & - \\
\hline & $\begin{array}{l}\text { Schwerwiegende Nebenwir- } \\
\text { kungen an den Abschnitts- } \\
\text { anfang }\end{array}$ & - & - \\
\hline \multirow[t]{3}{*}{ Stil } & $\begin{array}{l}\text { Aktive statt passive Formu- } \\
\text { lierungen }\end{array}$ & $\begin{array}{l}\text { Aktive statt passive Formulie- } \\
\text { rungen }\end{array}$ & $\begin{array}{l}\text { weitgehend } \\
(10)\end{array}$ \\
\hline & $\begin{array}{l}\text { Bei Handlungsaufforderun- } \\
\text { gen erst die Anweisungen } \\
\text { geben und dann Begrün- } \\
\text { dung nennen }\end{array}$ & - & - \\
\hline & $\begin{array}{l}\text { Direkte Ansprache der Pati- } \\
\text { enten }\end{array}$ & - & $\begin{array}{l}\text { vollständig } \\
\text { (19) }\end{array}$ \\
\hline
\end{tabular}


Ansätze für barrierefreie Kommunikation im medizinisch-pharmazeutischen Bereich

\begin{tabular}{|c|c|c|c|}
\hline & $\begin{array}{l}\text { Lesbarkeitsrichtlinie für } \mathrm{Pa} \text { - } \\
\text { ckungsbeilagen }^{1}\end{array}$ & $\begin{array}{l}\text { Expertenkommission zu Lay } \\
\text { Summaries }^{2}\end{array}$ & $\begin{array}{l}\text { Übereinstim- } \\
\text { mung mit } \\
\text { Vorgaben für } \\
\text { Leichte Spra- } \\
\text { che }^{3}\end{array}$ \\
\hline \multirow{4}{*}{ Stil } & $\begin{array}{l}\text { Keine Abkürzungen/ } \\
\text { Akronyme, wenn unver- } \\
\text { meidlich, dann erklären }\end{array}$ & $\begin{array}{l}\text { Keine Abkürzungen/ Akro- } \\
\text { nyme }\end{array}$ & $\begin{array}{l}\text { vollständig } \\
\text { (7) }\end{array}$ \\
\hline & $\begin{array}{l}\text { Keine wissenschaftliche } \\
\text { Symbole }\end{array}$ & - & $\begin{array}{l}\text { vollständig } \\
\text { (16) }\end{array}$ \\
\hline & $\begin{array}{l}\text { Medizinische Fachwörter in } \\
\text { Umgangssprache übersetzen; } \\
\text { zuerst Laienausdruck, dahin- } \\
\text { ter das Fachwort; den pas- } \\
\text { sendsten Begriff dann weiter } \\
\text { verwenden }\end{array}$ & $\begin{array}{l}\text { Medizinische Fachwörter in } \\
\text { einfache Sprache übersetzen; } \\
\text { erst den einfachen Ausdruck, } \\
\text { dann das Fachwort; komplexe } \\
\text { Wörter sollen durch einfache } \\
\text { ersetzt werden }\end{array}$ & $\begin{array}{l}\text { weitgehend } \\
\text { (5) }\end{array}$ \\
\hline & - & $\begin{array}{l}\text { Zuerst Überblick, dann die } \\
\text { Details („inverted pyramid } \\
\text { writing style“) }\end{array}$ & - \\
\hline Papierart & $\begin{array}{l}\text { Kein transparentes Papier, } \\
\text { kein Glanzpapier }\end{array}$ & - & $\begin{array}{l}\text { vollständig } \\
\text { (32) }\end{array}$ \\
\hline $\begin{array}{l}\text { Symbole und } \\
\text { Piktogramme }\end{array}$ & $\begin{array}{l}\text { Klar und leicht lesbar, keine } \\
\text { werblichen Aspekte, erleich- } \\
\text { tern Textnavigation }\end{array}$ & $\begin{array}{l}\text { Keine graphischen Zeichen } \\
\text { wie Firmenlogos und Pikto- } \\
\text { gramme }\end{array}$ & $\begin{array}{l}\text { weitgehend } \\
\text { (33) }\end{array}$ \\
\hline Links & - & $\begin{array}{l}\text { Wenige, da sie mit der Zeit } \\
\text { veralten }\end{array}$ & - \\
\hline
\end{tabular}

1 European Commission (2009) Guideline on the readability of the labelling and package leaflet of medicinal products for human use, Revision 1: 7-10.

2 European Commission (2018): Summaries of Clinical Trial Results for Laypersons. Recommendations of the expert group on clinical trials for the implementation of Regulation (EU) No 536/2014 on clinical trials on medicinal products for human use: $3-7$.

3 Netzwerk Leichte Sprache (ohne Datum). Die Regeln für Leichte Sprache.

Die Übereinstimmungen zwischen den Richtlinien betreffen alle Ebenen der Dokumente, Inhalt, Struktur und Layout. Einhellig befürworten sie die Notwendigkeit, die jeweiligen Adressaten zu befragen. Einigkeit besteht in der Betonung von Gliederung durch Überschriften und Hervorhebungen und darüber, dass die Schriftgröße wichtig ist. Auch hinsichtlich der syntaktischen 
Vorgaben sind die Richtlinien nahezu einig: keine langen Sätze, keine langen, vielsilbigen Wörter, kurze Listen statt längerer Absätze etc. Gleiches gilt für das Vokabular: Es sollen keine Fachwörter, Abkürzungen, Akronyme verwendet werden. In keiner der Richtlinien werden linguistische Begründungen für die Empfehlungen gegeben und es wird weder auf wissenschaftliche noch auf empirische Evidenz verwiesen.

Es ist möglich, dass den Verfasserinnen und Verfassern der Expertenempfehlung zu Lay Summaries die Lesbarkeitsrichtlinie für Packungsbeilagen bekannt war. Allerdings wird die Lesbarkeitsrichtlinie in den Expertenempfehlungen weder genannt, noch wird in der Literaturliste darauf verwiesen. Vorstellbar scheint, dass beide Regelsätze unabhängig entwickelt wurden. Es scheint nahezu ausgeschlossen, dass Autor(inn)en der Lesbarkeitsrichtlinie oder der Expertenempfehlungen die Regeln für Leichte Sprache kannten, auch wenn deren Vorläufer („Information für alle“ der ILSMH) bereits 1998 entwickelt worden waren.

Bei der Ähnlichkeit der Richtlinien scheint es sich somit um echte Konvergenz zu handeln. Das Bemühen um Verständlichkeit führte aus sehr unterschiedlichen Kommunikationszusammenhängen zu sehr ähnlichen Vorgaben, wie dies sprachlich erreicht werden soll. Damit ist gezeigt, dass die Empfehlungen zur Vereinfachung unabhängig von der ursprünglichen Kommunikationsabsicht und unabhängig vom textlichen Ausgangsmaterial sind. Gleichzeitig legt die beobachtete Konvergenz eine weitgehend universelle Gültigkeit der Vereinfachungsregeln nahe.

\section{$7 \quad$ Fazit und Ausblick}

Es konnte gezeigt werden, dass komplexe medizinisch-pharmazeutische Informationen für Laien zugänglich gemacht werden können, auch wenn hier noch weitere Bemühungen notwendig sind. Für beide untersuchten Dokumente, die Packungsbeilage für Arzneimittel und die laienverständliche Zusammenfassung der Ergebnisse von klinischen Studien, soll Verständlichkeit durch eine Kombination von gesetzlichen Regeln, verbindlichen Vorgaben zur Struktur, der Ein- 
haltung von Schreibrichtlinien und dem Testen mit der Zielgruppe erreicht werden. Die Richtlinien, die für das Schreiben dieser Dokumente entwickelt wurden, stimmen in vielen Aspekten mit den Regeln für Leichte Sprache überein. Diese Konvergenz untermauert deren Nützlichkeit, um den Zugang zu Informationen über Arzneimittel zu vereinfachen und das Verständnis für medizinisch-pharmazeutische Zusammenhänge zu verbessern. 
Thomas M. Schindler

\section{Literaturverzeichnis}

Bock, BetTina M. (2015): „Leichte Texte schreiben. Zur Wirksamkeit von Regellisten Leichter Sprache in verschiedenen Kommunikationsbereichen und im World Wide Web“. In: trans-kom 8 (1), 79-102.

Bohn-GetTler, Catherine M./Rapp, David N. (2011): „Depending on my mood: mood-driven influences on text comprehension“. In: Journal of Educational Psycho$\log y$ 103(3), 562-577.

BÖNIG, MARKUS (2017): „Zwischen Paternalismus und Selbstbestimmtheit“. In: Monitor Versorgungsforschung 06/17, Juni 2017, 34-37.

Bredel, Ursula/MaAß, Christiane (2016): Leichte Sprache. Theoretische Grundlagen, Orientierung für die Praxis. Duden 2016, Bibliographisches Institut GmbH, Berlin.

BundesinstituT FÜR ARZNEIMITTEL Und MEdizInPROdUKTE (2015): Bekanntmachung von Empfehlungen zur Gestaltung von Packungsbeilagen nach $\$ 11$ des Arzneimittelgesetzes ( $A M G$ ) für Humanarzneimittel (gemäß $\$ 77$ Absatz 1 AMG) und zu den Anforderungen von $\$ 22$ Absatz 7 Satz 2 AMG (Überprüfung der Verständlichkeit von Packungsbeilagen) 14. April 2015. URL:: https://www.bfarm.de/SharedDocs/ Bekanntmachungen/DE/Arzneimittel/natVerf/bm-zul-20150414-packungs beilagen_2015-pdf.pdf;jsessionid=C7C31802115D57A07ED58B93AA88AF78.2_ cid344?_blob=publicationFile\&v=4 (letzter Zugriff: 3.4.2018).

BUNDESMINISTERIUM DER JUSTIZ UND FÜR VERBRAUCHERSCHUTZ (2005): Gesetz über den Verkehr mit Arzneimitteln (Arzneimittelgesetz - AMG). Neugefasst durch Bekanntgabe vom 12.12.2005 I 3394; zuletzt geändert durch Art. 1 G v. 18.7.2017 I 2757. URL: https://www.gesetze-im-internet.de/amg_1976/BJNR024480976.html (letzter Zugriff: 2.4.2018).

ERGO VERSTÄNDLICHKEITSSTUDIE (2012): Was verstehen wir noch? ERGO Versicherungsgruppe AG, Düsseldorf.

EUROPEAN COMMISSION, ENTERPRISE AND INDUSTRY DiRECTORATE-GENERAL (2009): Guideline on the readability of the labelling and package leaflet of medicinal products for human use, Revision 1. 12 January 2009. URL: https://ec.europa.eu/health/sites/ health/files/files/eudralex/vol-2/c/2009_01_12_readability_guideline_final_en.pdf (letzter Zugriff: 3.4.2018).

European COMmission (2014): EU Clinical Trial Regulation 536/2014. URL: https://ec.europa.eu/health//sites/health/files/files/eudralex/vol-1/reg_2014_536/ reg_2014_536_en.pdf (letzter Zugriff: 2.4.2018).

EuRopeAn COMmission (2018): Summaries of Clinical Trial Results for Laypersons. Recommendations of the expert group on clinical trials for the implementation of Regulation (EU) No 536/2014 on clinical trials on medicinal products for human use. URL: https:/ec.europa.eu/health/sites/health/files/files/eudralex/vol-10/2017_01_26_ summaries_of_ct_results_for_laypersons.pdf (letzter Zugriff: 2.4.2018). 
European Medicines Agency (EMA) (2014): Mandate and objectives for the EMA Working Party on Quality Review of Documents (QRD). EMA/403106/2014. URL: http://www.ema.europa.eu/docs/en_GB/document_library/Other/2014/07/ WC500169651.pdf (letzter Zugriff: 3.4.2018).

EUROPEAN MEDICINES AGENCY (EMA) (2017): EMA action plan related to the European Commission's recommendation of product information. EMA/680018/2017.

EUROPÄISCHES PARLAMENT (2001): Richtlinie 2001/83/EG des Europäischen Parlaments und des Rates vom 6. November 2001 zur Schaffung eines Gemeinschaftskodexes für Humanarzneimittel. URL: https://ec.europa.eu/health//sites/health/files/files/ eudralex/vol-1/dir_2001_83_consol_2012/dir_2001_83_cons_2012_de.pdf (letzter Zugriff: 2.4.2018).

KIRSCH, IRWIN (2001): The International Adult Literacy Survey (IALS): Understanding what was measured. Educational Testing Service, Statistics \& Research Division Princeton, Research Report RR-01-25.

LANGER, KATIE/FRIE, MARIA (2017): Emotion, text difficulty, and purpose for reading: how factors influence reading comprehension. College of Saint Benedict and Saint John's University, Celebrating Scholarship \& Creativity Day. 131. URL: http://digitalcommons.csbsju.edu/elce_cscday/131 (letzter Zugriff: 2.4.2018).

Loh, Andreas/Lang, Georg/Box, Gunnar/Haug, Oliver/Hammel, Ludwig/ KeCK, BARbara/Kruk, Uta/ClaUßen, Christina (2014): Gebrauchsinformationen für Arzneimittel. Verständlichkeit einer Gebrauchsinformation aus kommunikationswissenschaftlicher Perspektive. Pharm. Ind. 76, 8, ECV Editio Cantor Verlag Aulendorf, 1182-1188.

MAaß, Christiane/Rink, IsABel (2017): „Leichte Sprache: Verständlichkeit ermöglicht Gesundheitskompetenz“. In: Public Health Forum, 25 (1), 50-53.

NETZWERK LEICHTE SPRACHE (ohne Datum): Regeln für Leichte Sprache. URL: http://www.leichte-sprache.de/dokumente/upload/21dba_regeln_fuer_leichte_ sprache.pdf (letzter Zugriff: 3.4.2018).

Nink, Katrin/Schröder, Helmut (2005): Zu Risiken und Nebenwirkungen: Lesen Sie die Packungsbeilage? Wissenschaftliches Institut der AOK, WIdO-Materialen 53, Bonn.

Organisation for ECONOMIC Co-Operation AND Development (OECD) (2000): Literacy in the Information Age. Final Report of the International Adult Literacy Survey (IALS), Organisation for Economic Co-operation and Development (OECD), Paris, and the Minister of Industry, Canada. URL: http://www.oecd.org/ education/skills-beyond-school/41529765.pdf (letzter Zugriff: 30.3.2018).

Riedler, Katharina (2013): „Historische Betrachtung der Arzt-Patienten-Beziehung mit Blick auf die Aufklärung“. In: Zeitschrift für Gesundheitspolitik, 4/2013, 9-25.

SCHINDler, Thomas M. (2018): Klinische Prüfungen - Ergebnisse endlich auch für Laien. Pharm. Ind. 80, 3, 346-353, ECV Editio Cantor Verlag Aulendorf. 
Thomas M. Schindler

Sroka-Saidi, Kamila/Boggetti, Barbara/Schindler, Thomas M. (2015): „Transferring regulation into practice: The challenges of the new layperson summary of clinical trial results“. In: Medical Writing 24 (1): 24-27. URL: http://journal.emwa. org/plain-language-and-readability/transferring-regulation-into-practice-thechallenges-of-the-new-layperson-summary-of-clinical-trial-results/ (letzter Zugriff: 30.3.2018).

VAn Dijk, Liset/Patrício Monteiro, Susana/Vervolet, Marcia/de Bie, Jolanda/ RAYNOR, DK THEO (2014): Study on the package leaflets and the summaries of product characteristics of medicinal products for human use (PILS-S study). European Union 2014. 
ANTJE BAUMANN

\section{Rechtstexte als Barrieren - Einige Merkmale der Textsorte ,Gesetz' und die Verständlichkeit}

\section{Textsorten in der Sprache des Rechts}

In der Sprache des Rechts gibt es vielerlei Textsorten: Staatsvertrag, Parlamentsprotokoll, Gerichtsurteil, Bescheid, Anklageschrift, Plädoyer, Vorladung, Eid, Zeugenaussage, Verfassungsbeschwerde, Vollstreckungsbefehl, Anzeige, Urkunde, Eintrag (ins Grundbuch, Register etc.), Rechtsgutachten, Urteilskommentierung, Widerspruch, Gesetz, Kommentar, Beschluss, Testament u.v.a.m. (siehe z. B. Hoffmann 1997 und Busse 2000).

Durch ihre fachsprachliche Prägung weichen all diese Textsorten von der Allgemeinsprache ab und werden daher außerhalb des Fachgebiets , Recht ${ }^{\text {' }}$ - wie Textsorten anderer Fachsprachen außerhalb ihres jeweiligen Fachgebiets auch als mehr oder weniger schwerverständlich wahrgenommen bzw. eingestuft.

\subsection{Die Textsorte,Gesetz}

In der Vielfalt von Textsorten im Bereich des Rechts ist die Textsorte,Gesetz jedoch eine spezielle. Denn bei Gesetzen kommt die Schwerverständlichkeit einer besonderen Barriere gleich, weil Gesetze - anders als andere Textsorten des Rechts - die Rechtsordnung und damit prinzipiell alle Staatsbürger und -bürgerinnen betreffen. Würde die Schwerverständlichkeit von Gesetzen nicht nur behauptet (zum Schwerverständlichkeitsstereotyp Warnke 2004), sondern bewiesen, stünde sie in bemerkenswertem Widerspruch zur enormen Bedeutung, die Gesetzen in einer demokratischen Gesellschaft zugeschrieben wird.

Um die (Schwer-)Verständlichkeit von Gesetzen zu qualifizieren, werden im Folgenden Eigenheiten der Textsorte dargestellt, wie sie sich aus praktischer Er- 
fahrung während der sprachlichen Prüfung von Gesetzen innerhalb des Gesetzgebungsprozesses ergeben. Aus dieser Perspektive der Gesetzesredaktion in der „Werkstatt der Gesetzgebung“ sind folgende textinterne und textexterne Merkmale der Textsorte, Gesetz' relevant:

1. Fachsprachlichkeit

2. Institutionalität

3. Mehrfachadressiertheit

4. gemischte Autorenschaft

5. extrem hoher Grad an Intertextualität

6. hoher Grad der Formalisiertheit

7. Spannung zwischen postulierter Exaktheit und realisierter Vagheit

Einige der hier separat aufgeführten Textsorten-Merkmale könnten auch als Facette bzw. als Ergebnis der Fachsprachlichkeit gesehen werden. So gehört zu jeder fachsprachlichen Textsorte eine starke Bezugnahme auf andere fachsprachliche Texte, viele derartige Texte sind formalisiert und etliche an Institutionen gebunden. Jede Art von Fachsprache, die sich einer natürlichen Sprache bedient, trägt damit auch den Widerspruch zwischen behaupteter Genauigkeit und tatsächlicher Ungenauigkeit sprachlicher Elemente in sich. Die Merkmale Intertextualität, Formalisiertheit, Institutionalität und Spannung zwischen postulierter Exaktheit und realisierter Vagheit ließen sich also auch als Untermerkmale bzw. als Folgen der Fachsprachlichkeit fassen.

Im Folgenden werden daher die oben genannten Merkmale nur mit Blick auf ihre spezifische Realisation in der Textsorte, Gesetz ' dargestellt.

\section{Zur Fachsprachlichkeit:}

- In Gesetzen werden neben leicht erkennbaren Fachwörtern (akzessorisch, Exequatur, Rechtshängigkeit etc.) viele Wörter aus der Allgemeinsprache verwendet, die dort jedoch eine andere Bedeutung haben (grundsätzlich, Besitz, Titel, Widerspruch u.v.a.m.). Die zweite Gruppe von Wörtern und Begriffen wird nur von Experten des jeweiligen Fachs als Fachwortschatz erkannt und verstanden. Laien hingegen erscheinen Gesetze aus diesem Grund unglücklicherweise gerade dort 
verständlich, wo sie es nicht sind (siehe Merkmal der Mehrfachadressiertheit).

- Außerdem bleiben fachlich eingeübte, stillschweigende Bezugnahmen auf andere Rechtstexte für Laien meist unsichtbar: zu Definitionen von verwendeten Begriffen in anderen Texten, zu Kommentarliteratur, zu Rechtsprechung etc. (Merkmal der Intertextualität).

Laien erkennen die fachsprachliche Bedeutung einzelner Wörter und Begriffe kaum, weil deren Fachsprachlichkeit nicht durch konstante Markierung (wie z. B. Fremdheit des Wortes) signalisiert wird und sich die gesamte Bedeutung dieser Wörter und Begriffe nur in deren Gebrauch in verschiedenen Texten zeigt. Da der (Bedeutungs-)Unterschied „nur“ im Gebrauch besteht und noch keinen spürbaren lexikographischen Niederschlag gefunden hat, können Laien die Bedeutung vieler Rechtsbegriffe und ganzer Normen nicht oder nur unzureichend erschließen. Daher hemmt die Fachsprachlichkeit dieser Textsorte die Verständlichkeit auf besondere Weise.

\section{Zur Institutionalität:}

- In der Bundesrepublik Deutschland sind die sprachlichen Handlungen ,Recht verfassen' und ,Recht erlassen' an diverse Institutionen gebunden, die ein Text (als Gesetzentwurf) durchläuft und wo er verändert wird, bevor er zum Gesetz wird. Diese Institutionen haben im Laufe von Jahrzehnten Gepflogenheiten (oft schriftlich fixierte Muster oder Regeln) herausgebildet, an welchen Stationen dieses Verfahrens (siehe dazu Busse 2000 oder „Stichwort Gesetzgebung. Von der Idee zum Gesetz" (Deutscher Bundestag 2016)) aus welchem Grund, mit welcher Autorisation und nach welchem Muster diese Texte von welchen Autoren bearbeitet werden (Merkmal der gemischten Autorenschaft). Derartige Gepflogenheiten sind z. B. fixiert im „Handbuch der Rechtsförmlichkeit“" (Bundesministerium der Justiz 2008) oder im 
„Handbuch zur Vorbereitung von Rechts- und Verwaltungsvorschriften“" (Bundesministerium des Innern 2012; siehe Merkmal des hohen Grades der Formalisiertheit).

- Die an Institutionen gebundene Entstehungsweise von Gesetzen macht diese normativ wirkenden Texte zu besonderen Sprechakten: Deklarativa schaffen (sprachliche, metasprachliche oder nichtsprachliche) Tatsachen, indem sie diese benennen. Die Benennung setzt etwas in die Welt, macht es, wirklich', weil die Benennung sowohl vom Sprecher als auch vom Hörer für gültig gehalten wird - weil sie institutionell erfolgte (vgl. Rolf 1997 74; 84). Durch Deklarativa wie Gesetze werden institutionelle Wirklichkeiten erzeugt, verändert oder aufgehoben.

Institutionen sind konstitutiv für die Textsorte ,Gesetz': Die Gepflogenheiten der Institutionen in deren Zusammenspiel (im komplexen Prozess der Gesetzgebung) beeinflussen nicht nur wesentlich die Entstehungsweise von Gesetzen (vielerlei Autoren, die den Entwurf im amtlichen Auftrag nach politischen, zeitlichen und förmlichen Vorgaben bearbeiten), sondern begründen zugleich Eigenheiten von „fertigen“ Gesetzen - und deren Schwerverständlichkeit. Durch Institutionalität erlangen Gesetze „Gültigkeit“ und schaffen Wirklichkeit, worauf sich die ihr (zu Recht) zugewiesene gesamtgesellschaftliche Bedeutung gründet.

Die Institutionalität der Textsorte begrenzt zudem die Möglichkeit, während ihrer Entstehung Einfluss auf die Verständlichkeit von Gesetzen zu nehmen.

\section{Zur Mehrfachadressiertheit:}

- Gesetze unterscheiden sich von anderen Fachtexten dadurch, dass sie nicht nur der effizienten Kommunikation von Experten untereinander dienen, sondern auch von Laien gelesen werden, die sich auch mithilfe 
von Gesetzen über ihre Rechte und Pflichten informieren. In einer Demokratie müssen Bürger und Bürgerinnen das Recht nicht nur verstehen können, um es zu „befolgen“, sondern auch, um es als Souverän verantworten und verändern zu können. Die der Textsorte innewohnende Doppeladressierung (Experten und Laien) unterscheidet die Textsorte wesentlich von anderen und erzeugt Spannung zwischen den Adressaten, die die Verständlichkeit höchst unterschiedlich einschätzen.

- Gesetze sind zudem Arbeitsinstrument oft nicht nur von juristischen, sondern von Experten aus mehreren Disziplinen, da neben juristischen Experten auch Verwaltungsfachleute der ausführenden Behörden, die mit der jeweiligen Spezialmaterie vertraut sind, mit ihnen umgehen (z. B. mit dem Finanzdienstleistungsaufsichtsgesetz oder dem Bundes-Immissionsschutzgesetz). In Gesetzen sind daher oft Elemente verschiedener Fachsprachen zu finden (etwa der Finanzwirtschaft oder der Umwelttechnik), so dass nicht von einer Doppel-, sondern potentiell von einer Mehrfachadressierung auszugehen ist.

Das konstitutive Merkmal der Mehrfachadressiertheit bewirkt, dass der zu erreichende Grad an Verständlichkeit der Textsorte ,Gesetz' nicht auf eine Adressatengruppe allein ausgerichtet sein kann.

\section{Zur gemischten Autorenschaft:}

- In den Institutionen wird ein Gesetzentwurf von vielen an der Gesetzgebung beteiligten Personen (oft unabhängig voneinander) bearbeitet, deren Entscheidungsspielraum oft klein ist, da sie im (amtlichen) Auftrag handeln und dabei entsprechend angewiesen oder kontrolliert werden. Von den vielen Bearbeitungsstufen sind nur einige für die Öffentlichkeit zugänglich, die meisten vollziehen sich ausschließlich innerhalb der jeweiligen Institution - damit hat die Textsorte ,Gesetz ${ }^{c}$ auch eine ungewöhnliche Autorenseite. 
- Die gemischte Autorenschaft, die innerhalb von Institutionen arbeitet, bringt eine partielle „Verdunkelung“ der Textproduktion mit sich: Der Prozess der Gesetzgebung in Bundesministerien, Bundesrat und Bundestag ist äußerst vielgestaltig (kommt in Rechts- und Politikwissenschaften jedoch eher prototypisiert vor) und aus den verschiedenen Institutionen, die ein Gesetzentwurf durchläuft, bevor er als Gesetz verkündet wird, dringt aus Gründen der politischen Praxis nur wenig an die Öffentlichkeit.

Ohne das Wissen über diese Aspekte der Entstehung dieser Textsorte (gemischte Autorenschaft und Institutionalität) können Gesetze für Laien nur sehr begrenzt verständlich (gemacht) werden.

\section{Zum extrem hohen Grad an Intertextualität:}

- Das Bundesrecht umfasst derzeit mehr als 4400 Gesetze und Rechtsverordnungen mit ca. 78000 Einzelvorschriften. Die gesamte Rechtsordnung ist also ein Geflecht von Tausenden einzelnen Rechtsnormen, die auf vielfältige Weise miteinander verbunden sind (vgl. die Bedeutung von ,Text' < lat. textus: ,Geflecht, Gewebe'). In diesem Gewebe ist keine Rechtsnorm je allein, sondern jede verwoben mit anderen und daher nur im Zusammenhang zu verstehen.

- Die Texthaftigkeit der Rechtsordnung wird durch vielerlei Verknüpfungen erreicht: durch Verweisungen (innerhalb ein und desselben Gesetzes, aber auch auf andere Gesetze), durch sachliche oder systematische Bezüge, durch Bezugnahmen auf Definitionen oder Erläuterungen in anderen Texten, auf frühere Fassungen, auf sog. Parallelnormen (die dann oft wortgleich formuliert sind). Außerdem nehmen sehr viele benachbarte Textsorten auf Gesetze Bezug, indem sie sie zitieren, kommentieren, interpretieren oder illustrieren (Urteile, Kommentare, Artikel in Hand- oder Lehrbüchern etc.). 
- Die Texthaftigkeit der Textsorte, Gesetz', wie sie Fachleuten gegenwärtig ist, wird einem Laien erst begreiflich, wenn er z. B. in (meist kostenpflichtigen) juristischen Datenbanken wie im Rechtsportal juris oder in Beck-online über ein Stichwort oder eine Rechtsnorm eine Suche startet, die dank der vielfältigen Verknüpfungen (Verlinkungen) eine Fülle an Textsorten und Informationen erbringt, die miteinander verbunden sind:

- Texte der Rechtsprechung (Urteile und Beschlüsse verschiedener Gerichte),

- Kommentare (der verschiedenen Rechtsnormen),

- Verwaltungsvorschriften,

- Arbeitshilfen (wie Leitlinien oder Unterhaltstabellen),

- Artikel in Lexika, Handbüchern und Fachzeitschriften,

- Pressetexte (wie Nachrichten aus Bundesrat, Bundestag, Bundesministerien, Gerichten und Kammern),

$\circ$ andere Mitteilungen (wie Gutachten und Anfragen) und

- Literaturnachweise.

- Für Laien meist ebenso wenig zugänglich und mit anderer Funktion sind wissenschaftliche Rechtskorpora (zum Juristischen Referenzkorpus JuReKo siehe z. B. Gauer et al. 2016: 129ff.), frei zugänglich und kostenlos, aber mit weniger Verknüpfungen ausgestattet ist nahezu das gesamte aktuelle Bundesrecht auf www.gesetze-im-internet.de (gemeinsames Projekt des Bundesministeriums der Justiz und für Verbraucherschutz und der juris $\mathrm{GmbH}$ ).

- Viele der Bezugnahmen in Gesetzen und Verordnungen sind für Laien unsichtbar. So kann ein Laie einer expliziten Verweisung auf andere Rechtsnormen (z. B. „nach Maßgabe der $\$ \$ 22$ bis 24 des X-Gesetzes“) noch theoretisch folgen, bei einer sog. stillschweigenden Verweisung wird ihm dies jedoch nicht gelingen. Fachleute hingegen stellen systematische Bezüge auch über nur „mitgedachte“ Zusammenhänge her, weil sie das Fach und die dazugehörige Sprache „von innen“ kennen - 
sie denken also Bezüge mit, die auf der sprachlichen Oberfläche nicht abgebildet sind. So erkennen Fachleute z. B. einen Fachbegriff als solchen (Diebstahl, Unterhalt etc.) und stellen einen gedanklichen Bezug zu anderen Fachbegriffen und -inhalten her (Besitz, Eigentum, Unterhaltspflicht etc.) oder sie knüpfen gedanklich die Verbindung zwischen dem Allgemeinen Teil eines Gesetzes und anderen Regelungen desselben Gesetzes oder zwischen dem grundlegenden Strafgesetzbuch und einem Nebenstrafrecht.

- Neben den Verwebungen innerhalb der Rechtsordnung der Bundesrepublik (siehe auch Lang im vorliegenden Band) gibt es auch Verknüpfungen mit jener der Bundesländer und der Europäischen Union.

Die besondere Ausformung der Intertextualität der Textsorte ,Gesetz stellt eine Verstehenshürde für Laien dar, da es sich um extrem viele Verknüpfungen handelt und viele Vertextungen nicht erkennbar sind oder über den jeweiligen Text (d.h. das jeweilige Gesetz) hinausgehen. Einzelne Formulierungen in Gesetzen offenbaren daher ihre Bedeutung nicht isoliert und können auch nicht isoliert verändert bzw. verständlich gemacht werden.

\section{Zum hohen Grad der Formalisiertheit:}

- Gesetze folgen konventionalisierten oder standardisierten Textbauplänen (sog. Makrostrukturen), damit Gesetze und Rechtsverordnungen systematisch gleich aufgebaut sind und aussehen - und der innere $\mathrm{Zu}$ sammenhalt der gesamten Rechtsordnung auch durch die äußere Form der einzelnen Rechtsnormen gewahrt bleibe. Das „Handbuch der Rechtsförmlichkeit" macht - neben Empfehlungen, die tiefer in Rechtsordnung und Systematik eingreifen (z. B. zu Stamm- vs. Änderungsgesetzen, Einfluss von Europarecht, völkerrechtlichen Vereinbarungen etc.) - Vorgaben zur formalen Gestaltung von Rechtsvorschriften, etwa in welchem Verhältnis Gliederungseinheiten (Teil, Abschnitt, Unterabschnitt, Paragraf, Absatz, Satz, Nummer, Buch- 
stabe etc.) zueinander stehen, wie Begriffe zu definieren sind, wo Legaldefinitionen und Begriffsbestimmungen zu finden sind und wo und wie der Anwendungsbereich oder das Inkrafttreten geregelt wird. Neben den Konventionen zu gesetzestechnischen Einzelheiten wie Zitierweise, Abkürzungen, Handhabung von Verweisungen enthält das Handbuch auch Empfehlungen zum Sprach- und Wortgebrauch sowie zur Schreibung von Zahlen, Brüchen, Geldbeträgen etc. in Rechtsvorschriften.

- Auch der Einsatz (typo-)grafischer Elemente ist streng geregelt: Klammern, Schrägstriche, Anführungszeichen sowie Hervorhebungen (Fett- oder Kursivdruck, Randgestaltung) können nur in sehr wenigen und klar bestimmten Fällen genutzt werden.

> Rechtsförmliche Vorgaben schließen etliche verständlichkeitsfördernde Textelemente wie Illustrationen, (typo-)grafische Hervorhebungen, Variationen in der Reihenfolge der sprachlichen Elemente, Redundanz, Paraphrase u.Ä. aus bzw. beschränken deren Gebrauch stark. Durch den hohen Grad an Formalisiertheit wird auch die Verständlichkeit von Gesetzen, die abgesehen von rechtsförmlichen Gliederungseinheiten weitgehend als allgemeinsprachliche Texte erscheinen, begrenzt.

\section{Zur Spannung zwischen postulierter Exaktheit und realisierter Vagheit:}

- Die für Fachsprachen typische Spannung zwischen postulierter Exaktheit und realisierter Vagheit zeigt sich auch in Gesetzen. Präzision wird angestrebt mit (oft vom allgemeinsprachlichen Gebrauch abweichenden) Begriffsbestimmungen und Legaldefinitionen, mit einer rechtsförmlich definierten Gliederung oder mit wirklich eindeutigen Verweisungen. Vagheit entsteht u.a. durch unbestimmte Rechtsbegriffe, Generalklauseln und Ermessensnormen. 
- Spannung wird auch dadurch erzeugt, dass zwar jede Rechtsnorm dem Bestimmtheitsgebot unterliegt, zugleich aber ohne eine gewisse Unbestimmtheit nicht der Rechtsanwendung dienen kann. Rechtsnormen sollen „atmen können“, d.h. sie müssen so formuliert sein, dass sie auch für neue Lebenssachverhalte und Entscheidungssituationen „passen“ auch diese ,grundsätzlich gewünschte Entwicklungsoffenheit von Gesetzen steht in Widerspruch zu dem Wunsch nach Allgemeinverständlichkeit" (Beaucamp 2011: 29f.).

- Vagheit kennzeichnet viele Ausdrücke der natürlichen Sprache (z. B. in sofort, groß, Kind, Wärme). Sie kann durch Konkretisierungen aufgehoben oder gemildert werden, z. B. wenn ein gesetzlich geregelter allgemeiner Grundsatz in einer Verordnung genauer bestimmt oder wenn Wärme in einer Verordnung so definiert wird, dass damit ein bestimmter Temperaturbereich und evtl. sogar Kälte umfasst ist. Es gehört daher zur hohen Kunst der Gesetzgebung, sprachliche Vagheitselemente gezielt so einzusetzen, dass künftige Entwicklungen der niederrangigeren Rechtsetzung und mögliche Zweifelsfälle der Rechtsprechung bewusst überlassen werden (zur "guten“ Vagheit in Gesetzen vgl. Nussbaumer 2005).

Da Ausdrücke der Vagheit in Gesetzen nötig sind, Laien sie aber häufig nicht von unnötigen Vagheitselementen unterscheiden können, wirkt diese Texteigenschaft besonders für Laien verständnishemmend.

Jedes dieser Merkmale schränkt auf seine Weise die Verständlichkeit von Gesetzen ein (ausführlicher zu diesen Textsortenmerkmalen z. B. in Baumann 2015, weitere Merkmale der Textsorte, Gesetz z z. B. in Busse 2000). Die für die Textsorte, Gesetz' spezifische Kombination all dieser Merkmale jedoch schränkt die Verständlichkeit sogar erheblich ein. Da einige der Merkmale unveränderlich sind (Institutionalität, Mehrfachadressiertheit, extreme Intertextualität), bleibt nur wenig Spielraum für die Bearbeitung der weitgehend „fixierten“ Textsorte zugunsten der Verständlichkeit. 
Dabei darf die Sprache des Rechts als theoretisch gut erforscht gelten. Studien der interdisziplinären Arbeitsgruppe Sprache des Rechts der Berlin-Brandenburgischen Akademie der Wissenschaften mündeten 2004 und 2005 in die dreibändige Schriftenreihe „Die Sprache des Rechts“, die den heutigen Forschungsstand zu Sprache und Recht im Wesentlichen darstellt. Allerdings mangelt es an der Verbindung zwischen Theorie und Praxis: Selten gibt es empirische Untersuchungen zur Verständlichkeit von Gesetzestexten (schon älter: Pfeiffer/Strouhal/Wodak 1987; aktuell: das Kooperationsprojekt „Recht verständlich machen " unter www.bundesregierung.de/wirksam-regieren) - und selten kommen theoretische Erkenntnisse der Wissenschaft in der tatsächlichen Praxis der Gesetzgebung zur Anwendung.

Der nur sporadische Austausch von Theorie und Praxis zur Textsorte, Gesetz' mag zum Fortbestehen der widersprüchlichen Behauptungen zu Gesetzen (sie seien schwerverständlich, obwohl grundlegend) beitragen. Als Grund für die wiederkehrende Behauptung der Schwerverständlichkeit wird lückenhaftes und veraltetes Wissen über diese spezielle Textsorte sowohl aufseiten der Textrezipienten als auch der Textproduzenten angenommen. Für die Verständlichkeit der Textsorte ,Gesetz sind jedoch Aktualisierung und Popularisierung des Textsortenwissens nötig.

\subsection{Textsortenkompetenz und Verständlichkeit}

Textsortenkompetenz ist pragmatisch gesehen Voraussetzung für eine angemessene Textrezeption und für eine mögliche Textproduktion. Denn einen Text als Vertreter einer Textsorte zu erkennen heißt auch, seine Merkmale zu kennen und als Verstehenshilfen zu nutzen. Aber nicht nur die Rezeption wird durch diese Kompetenz beeinflusst, sondern auch die Produktion von Texten, da die Kenntnis der Textsortenmerkmale es ermöglicht, diese so zu reproduzieren, wie es die Kommunikationssituation erfordert. Einen Text zu verstehen setzt daher Textsortenkompetenz voraus.

Die typologischen Schwierigkeiten, Texte aufgrund bestimmter Merkmale oder Merkmalskombinationen zu Textsorten oder -klassen zusammenzufassen, spielen für die Verständlichkeit im sprachlichen Alltag üblicherweise keine Rolle. (Zur Textsortenbestimmung siehe neben Große 1974 und Adamzik 2000 
und 2008 z. B. die 3 „klassischen“ Modelle: Matrix verschiedener Merkmalskombinationen [Sandig 1972, Klassifikation nach textinternen und textexternen Faktoren [Linke u. a.: 1994] und kommunikationsbezogener Ansatz [Brinker 2001].) Wenn die meisten Menschen in Deutschland einen Text z. B. als Wettervorhersage, als Backrezept oder als Brief erkennen und ihn als solchen verstehen, liegt das u.a. daran, dass diese Textsorten in unserer Sprachgemeinschaft sehr weit verbreitet und über einen langen Zeitraum mehr oder weniger stabil sind. Mit dem steten Gebrauch derartiger Texte bildet sich also eine Kompetenz hinsichtlich dieser Textsorten aus. Bei Textsorten, die weniger im Alltag vieler Menschen verankert sind, schwindet die dazugehörige Kompetenz. Daher fällt es manchmal schwerer, ein Gedicht als Gedicht zu erkennen, eine Wahlwerbung von einer Zeitungsanzeige zu unterscheiden oder eine Gewinnbenachrichtigung als Warenwerbung zu entlarven.

Da das Wissen über Textsorten in der sprachlichen Tätigkeit erworben wird und diese wiederum an die sich wandelnde außersprachliche Wirklichkeit gebunden ist, sieht eine Bittschrift heute anders aus als im Mittelalter, erreicht uns eine Nachricht vom anderen Ende der Welt schneller und auf anderen Kanälen und schreibt keiner mehr ein Brigadetagebuch. Textsorten verändern sich manche verschwinden ganz, neue tauchen auf - und mit der sich ändernden sprachlichen Tätigkeit ändert sich auch die Textsortenkompetenz.

\subsection{Wenig Wissen über die spezielle Textsorte, Gesetz}

Bei manchen Textsorten jedoch steht das Wissen über sie in einem Missverhältnis zu der ihnen zugeschriebenen Bedeutung: Gesetze sind grundlegend für unsere Gesellschaft, denn sie „regeln“ das Zusammenleben von Menschen, d.h. sie beschreiben Rechte, Pflichten, Ansprüche, Zuständigkeiten etc. von verschiedenen Akteuren sowie Voraussetzungen, Zulässigkeiten und Folgen bestimmter Handlungen als Rechtsnorm. Die gesetzlichen Regelungen (Rechtsnormen) sind wesentlich für funktionierende Beziehungen zwischen den Akteuren. Hier wird im Weisgerberschen Sinn die Welt „gewortet“, d.h. (ein Teil der) Welt wird ins Wort gebracht, versprachlicht, mithilfe der Versprachlichung wird Wirklichkeit erfasst und organisiert, durch Sprache wird Wirklichkeit geschaffen (Weisgerber 1962). Das Verabschieden von Gesetzen ist ein deklarativer Sprech,akt“, 
mit dem im Rahmen einer konventionellen Prozedur (der Gesetzgebung) ein bestimmter Zustand hergestellt wird. Mit dem Inkrafttreten des Gesetzes entfalten die einzelnen Regelungen einschließlich Inkrafttretens- und Übergangsregelungen materielle Wirkungen.

Gesetze bewirken also etwas in der Welt und werden als grundlegend für unser Zusammenleben erachtet. Dennoch ist das Wissen über diese Textsorte schwach ausgeprägt. Sollte man sie nicht ebenso gut kennen wie andere derart wirksame Sprechakte wie z. B. Gerichtsurteile, Eheschließungsformeln, Kriegsoder Liebeserklärungen?

\section{Verständlichkeit von Gesetzen in demokratischen Gesellschaften}

Wenn Textsortenkompetenz Voraussetzung für Verstehen ist (was bei Gesetzen keine Inhaltswiedergabe bedeutete, sondern: sein Verhalten nach dem Verständnis dieses Inhalts auszurichten, sie also zu befolgen, zu ändern etc.), führt nicht ausreichendes Wissen über die Textsorte ,Gesetz' daher zum partiellen Nicht-Verstehen von Gesetzen - mit Auswirkungen für den einzelnen Menschen und das Funktionieren und das Selbstverständnis der gesamten Gesellschaft.

Daher kann es nicht beim allgemeinen Klagen über die Unverständlichkeit von Gesetzen bleiben. Denn dies dauert schon zu lange an (seit es Gesetze gibt) und hat das Recht bisher kaum verständlicher gemacht (zu den verschiedenen Bemühungen in verschiedenen Ländern siehe z. B. Lerch 2002). So wollte z. B. vor mehr als 200 Jahren die österreichische Kaiserin Maria Theresia die Verständlichkeit mithilfe eines „dummen Menschen“ prüfen lassen. Dem [ungarisch] „buta ember“ sollte jede neue gesetzliche Regelung vorgeegt werden. Konnte dieser einfache Mensch ohne juristische Kenntnisse nicht verstehen, was gemeint war, sollte die ministeriale Verwaltung den Entwurf überarbeiten (vgl. Lerch 2004: 237). - Allerdings scheiterte die Kaiserin mit diesem Ansatz.

In einer demokratischen Struktur, für deren Erhalt die Verständlichkeit von Gesetzen eine Voraussetzung ist, muss die Verständlichkeit auf anderen Wegen gesichert oder erzeugt werden. 
Auf Bundesebene gibt es daher innerhalb der Gemeinsamen Geschäftsordnung der Bundesministerien (GGO) ein Verständlichkeitsgebot:

„Gesetzentwürfe müssen sprachlich richtig und möglichst für jedermann verständlich gefasst sein.“ ( $\$ 42$ Absatz 5 GGO)

Dieses Verständlichkeitsgebot leitet sich aus der angestrebten Wirksamkeit des Rechts und dem erwähnten Demokratieprinzip her, da unverständliches Recht unwirksam bleibt bzw. das Volk als Souverän Gesetze nur beherrschen, d.h. befolgen, durchsetzen und verändern kann, wenn es sie versteht. Außerdem leitet sich das Gebot auch aus dem Rechtsstaatsprinzip ab, nach dem die Ausübung aller staatlichen Gewalt an das Recht gebunden ist. Dies soll staatlicher Willkür vorbeugen und den Bürgern Grundrechte gewähren (Willkürverbot). Das Handeln von Regierung und Verwaltung kann durch geltende Gesetze jedoch nur gelenkt und beschränkt werden, wenn diese bestimmt genug sind (Bestimmtheitsgebot).

Schwerverständliche Gesetze erzeugen Misstrauen in Recht und Staat oder zumindest Distanz gegenüber beiden, unverständliche Gesetze können tendenziell sogar staatsgefährdend wirken.

\subsection{Wem würden verständliche Gesetze nützen?}

Zwar sollen Gesetze laut Verständlichkeitsgebot möglichst für jedermann verständlich sein (s.o.). Doch die Unterscheidung zwischen beruflichen Rechtsanwendern und Laien ist wesentlich, denn es ist ein Unterschied, ob Richter, Anwälte oder Justiziare eine Regelung kaum verständlich finden oder ob nichtjuristisch ausgebildete Laien - weil sie betroffen sind oder zur Wahrnehmung ihrer staatsbürgerlichen Rechte und Pflichten - ein Gesetz oder eine Passage darin verstehen wollen. Derzeit haben sowohl berufliche Rechtsanwender als auch Laien Schwierigkeiten, deutsche Gesetze zu verstehen. Schaubild 1 zeigt, wer mit Gesetzen zu welchen Zwecken umgeht. 


\section{Verständliche Gesetze sind /wären gut für:}

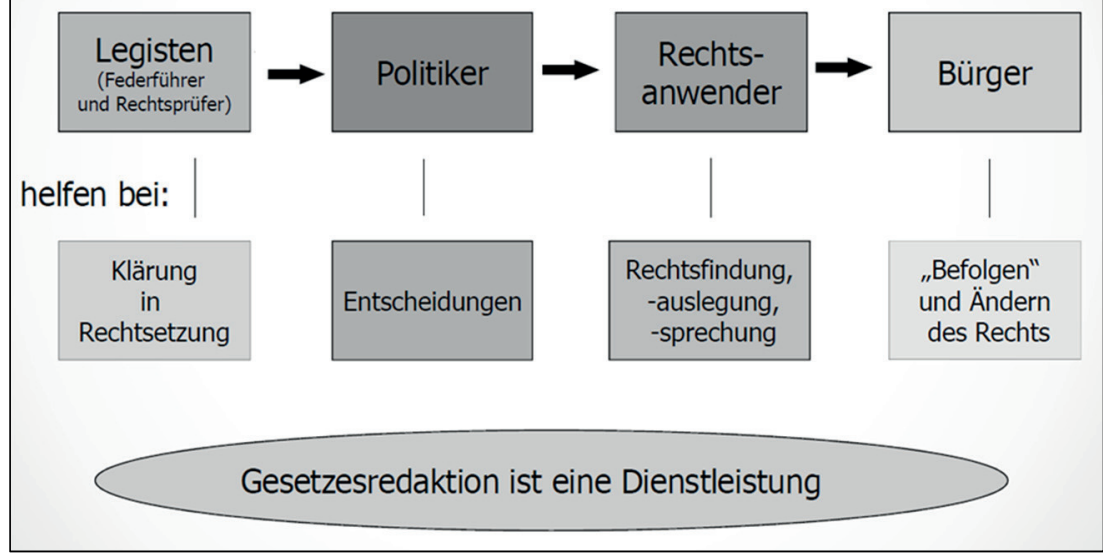

Schaubild 1: Wem nützen verständliche Gesetze? (Quelle: A.B.)

In der Gesetzgebung kommt ein verständlicher Entwurf Legisten und Politikern zugute. So können die einen besser genau das Recht formulieren, das politisch gewollt ist, die anderen besser über neue Gesetze entscheiden. Nach der Verkündung nützt ein verständliches Gesetz allen, die Recht sprechen, auslegen und „übersetzen“ helfen, ebenso den Unternehmen sowie den Bürgerinnen und Bürgern, die sich gesetzeskonform verhalten oder sich informieren möchten. Verständlichere Gesetze würden also sowohl beruflichen Rechtsanwendern als auch Laien die Arbeit bzw. den Zugang zum Recht erleichtern.

Die Gesetzesredaktion mit Verständlichkeitsprüfung ist daher eine Dienstleistung für Demokratie: Sie braucht zunächst Vertrauen, Zeit und Geld - aber auf lange Sicht spart sie auch Zeit und Geld und schafft Vertrauen in die Politik.

Das zeigte sich auch in der Zufriedenheitsbefragung 2015, in der das Statistische Bundesamt im Auftrag der Bundesregierung Privatpersonen und Unternehmen zu ihren Erfahrungen mit Recht, Gesetz und Verwaltung in unterschiedlichen Lebenslagen befragt hat: Während die Bürger und Bürgerinnen über alle Lebenslagen hinweg mit den Leistungen der staatlichen Verwaltung im Wesentlichen zufrieden sind, schnitt die Verständlichkeit (der Formulare und Anträge bzw. des zugrunde liegenden Rechts) von 16 verschiedenen Aspekten 
staatlicher Leistungserbringung am schlechtesten ab (ausführliche Dokumentation der Erhebung unter www.amtlich-einfach.de).

Ein Instrument, mit dem die Bundesregierung verständlichere Gesetze erreichen will, ist die Gesetzesredaktion als Teil der Gesetzgebung.

\subsection{Sprachprüfung im Gesetzgebungsprozess als Teil der Entwurfsarbeit}

Rechtsgrundlage für die Sprachprüfung ist o.g. $\$ 42$ Absatz 5 Satz 1 GGO. Alle neu entstehenden Gesetze und Verordnungen werden, bevor sie ins Parlament gelangen, einer Rechtsprüfung unterzogen, zu der auch eine Sprachprüfung gehört. Diese Gesetzesredaktion inkl. Sprachberatung wird im Bundesministerium der Justiz und für Verbraucherschutz von sprachwissenschaftlich ausgebildeten Fachleuten durchgeführt und steht im gesamten Gesetzgebungsverfahren zur Verfügung:

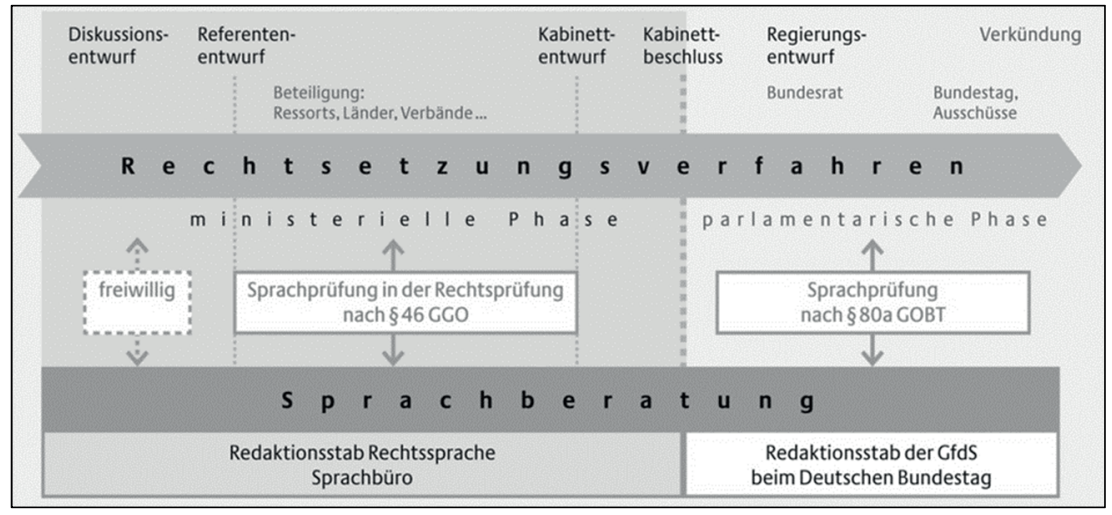

Schaubild 2: Gesetzesredaktion bzw. Sprachberatung im Gesetzgebungsverfahren (Quelle: Bundesjustizministerium)

Sprachprüfung ist Teil der Rechtsprüfung nach $₫ 46$ GGO und damit obligatorisch (in der Rechtsprüfung wird jeder Entwurf daraufhin geprüft, ob er systematisch, rechtsförmlich und sprachlich in die bestehende Rechtsordnung 
passt), in der frühen Phase ist sie freiwillig, im Idealfall findet sie mehrmals statt und ist umso wirksamer, je früher sie einsetzt.

Wie aber gelingt es, einen Entwurf innerhalb des komplexen Prozesses der Gesetzgebung nicht nur auf sprachliche Richtigkeit, sondern auch auf Verständlichkeit zu prüfen?

$\mathrm{Da}$ Verständlichkeit keine absolute Größe und keine reine Texteigenschaft ist, sondern immer in Bezug auf einen jeweiligen Adressaten existiert, wird der unterschiedliche Fokus der verschiedenen am Gesetzgebungsprozess beteiligten Experten bewusst genutzt, indem diese miteinander sprechen und dabei zu einem möglichst auch für Nicht-Experten verständlichen Text kommen. Idealerweise werden die verschiedenen Fachsprachen (neben der juristischen z. B. die des Luftverkehrs, des Finanzwesens, der Umwelttechnologie etc.) mithilfe eines Gesetzesredakteurs so abgewogen, dass der Entwurf auch für die jeweiligen Nicht-Experten und für Bürger verständlich ist. Dieser Abstimmungsprozess gelingt innerhalb der schriftlichen und mündlichen Redaktion nur durch Kooperation und Kommunikation.

Der Gesetzesredakteur kann dank seiner linguistischen Ausbildung und seiner Erfahrung im Gesetzgebungsprozess die Rolle eines Mittlers einnehmen. Ihm ist bewusst, dass verschiedene Fächer mit ihren Terminologien und ihren Gepflogenheiten aufeinandertreffen. Dies vermittelt er in Redaktionssitzungen auch den verschiedenen am Gesetz beteiligten Experten, die als Vertreter des federführenden Bundesministeriums oder als Rechtsprüfer im Bundesjustizministerium jeweils eine andere Perspektive auf den entstehenden Gesetzestext haben.

Arbeit an der Sprache wirft inhaltliche Fragen auf, die sich den Legisten manchmal bisher noch nicht gestellt hatten. Dies kann die Verwendung der Begriffe betreffen (wird z. B. Gleiches ungleich benannt - und verstößt damit gegen ein rechtssystematisches und rechtsförmliches Prinzip?) oder Fragen der Gliederung (ist die Regelung hier an der richtigen Stelle, passt sie unter diese Paragrafen-Überschrift bzw. passt diese Überschrift nach Änderung und Erweiterung des Paragrafen noch?) oder andere Fragen. Die Sprachprüfung macht auf klassische Fehler („Prüfung auf sprachliche Richtigkeit“) aufmerksam, ihre Hauptaufgabe ist jedoch die Verständlichkeitsprüfung. Daher berühren durch Umformulierungen zutage geförderte Fragen oft auch die Bereiche der Logik, 
der Kohärenz und der Bestimmtheit von Regelungen: Ist eine Ausnahme deutlich als solche erkennbar oder als Rückausnahme falsch platziert? Welcher Adressat soll durch die jeweilige Norm angesprochen werden - muss z. B. der Akteur einer Amtshandlung benannt werden oder steht wirklich die Handlung im Vordergrund? Das jeweilige Gesetzgebungsvorhaben wird also systematisch auf vielerlei Ungereimtheiten untersucht und damit sozusagen auf Herz und Nieren geprüft - von der sprachlichen Oberfläche ausgehend bis hin zu rechtssystematischen Fragen. Da diese Fragen materiell-rechtlich relevant sein können, erfordert ihre Lösung die Zusammenarbeit mit den juristischen Experten.

Wie beim Sparring im Sport weist der Gesetzesredakteur also auf Schwächen des Entwurfs hin und er verstärkt - im Zusammenspiel mit den anderen Experten - dessen Stärken. Ein solches Zusammenspiel erfordert allerdings partnerschaftliche und im Gesetzgebungsprozess verankerte Kommunikation. Bei der Reform des Versorgungsausgleichsrechts haben die Sprachwissenschaftlerinnen 2008 z. B. gefragt, ob der Begriff Rechtshängigkeit hier als Fachbegriff wirklich notwendig sei - da diese Regelung zur Klärung der Altersversorgungsansprüche nach einer Scheidung doch auch für Laien verständlich sein sollte. Bis dahin hieß es in $\$ 1587$ Absatz 2 des Bürgerlichen Gesetzbuchs: „Als Ehezeit im Sinne der Vorschriften über den Versorgungsausgleich gilt die Zeit vom Beginn des Monats, in dem die Ehe geschlossen worden ist, bis zum Ende des Monats, der dem Eintritt der Rechtshängigkeit des Scheidungsantrags vorausgeht. "Die Diskussion mit Juristen im Bundesjustizministerium ergab, dass der Begriff hier auflösbar war und so lautet seitdem $\$ 3$ Absatz 1 des Versorgungsausgleichsgesetzes: „Die Ehezeit im Sinne dieses Gesetzes beginnt mit dem ersten Tag des Monats, in dem die Ehe geschlossen worden ist; sie endet am letzten Tag des Monats vor Zustellung des Scheidungsantrags" "(Hervorhebungen: A.B.).

Der Gesetzesredakteur denkt sich also tief in den Entwurf hinein und gibt Hinweise, durch welche (oft kleinen, manchmal aber auch grundlegenden) Eingriffe der Text verständlicher werden könnte. Dabei hat er neben den professionellen Rechtsanwendern, die später das Gesetz anwenden sollen, auch die Bürgerinnen und Bürger im Sinn, um ihnen den Zugang zum Recht so leicht wie möglich zu machen. Und er hat die motivierende Erfahrung gemacht, dass seine Arbeit - den Text so weit wie möglich von verständnishemmenden Elementen zu entlasten und verständnisfördernde Elemente zu stärken - zugleich Experten 
UND Laien nutzt (zur Arbeit von Gesetzesredakteuren z. B. Nussbaumer 2002; 2009, Baumann 2012).

\subsection{Neue Wege zu mehr Verständlichkeit für die „fixierte“ Textsorte ,Gesetz}

Das spezifische Bündel von zum Teil unveränderlichen Textsortenmerkmalen (s.o.) führt dazu, dass Gesetze als schwerverständlich gelten, und bildet einen engen Rahmen, innerhalb dessen überhaupt nur an der Verständlichkeit gearbeitet werden kann. Damit Gesetze dennoch sowohl von beruflichen Rechtsanwendern als auch von juristischen Laien verstanden werden können, bedarf es daher - neben der Gesetzesredaktion während des Gesetzgebungsprozesses weiterer Instrumente, mit denen die Verständlichkeit von Gesetzen gesichert oder erzeugt wird.

\subsubsection{Vor der Gesetzgebung: Linguistische Ausbildung im Jurastudium}

Recht existiert in Sprache. Wer das Recht in seinem Beruf anwenden will, muss Sprache als sein künftiges Hauptarbeitsinstrument professionell beherrschen. Juristen sind als berufliche Rechtsanwender die Experten, die Recht umsetzen, durchsetzen, in Wirklichkeit „übersetzen“, die Recht sprechen und setzen. Dabei müssen sie das versprachlichte Recht für andere Experten, aber auch für juristische Laien mehr oder weniger erklären. Ein Anwalt wird dabei vielleicht mehr erklärende Sprechakte benötigen als ein Richter - in allen juristischen Tätigkeiten jedoch ist der Umgang mit Sprache auf eine ganz andere Weise relevant als in anderen Berufsfeldern, die ebenfalls eine von der Allgemeinsprache abweichende Fachsprache ausgebildet haben. So brauchen Pharmazeuten oder Apotheker nur kleine Ausschnitte ihrer Fachsprache im Kontakt mit Laien, also z. B. gegenüber Patienten, Kunden oder Journalisten, die über keine oder nur einfachste pharmazeutische Kenntnisse verfügen. Auch der Kontakt zwischen Experten der Finanzwirtschaft, der Astrophysik oder der Molekularbiologie und Laien, die Texte aus diesen Bereichen verstehen wollen, dürfte sich in Grenzen halten.

Weil die Sprache des Rechts und insbesondere die der Gesetze so viele Eigenheiten aufweist, die sie von anderen Fachsprachen unterscheidet, sollten 
diese Besonderheiten angehenden Juristen in der universitären Ausbildung vergegenwärtigt werden. Mit der Textsorte ,Gesetz` und der Verständlichkeit von Gesetzen würden neben linguistischen Fragen der Verständlichkeits- und der Fachsprachenforschung auch soziologische Fragestellungen Ausbildungsgegenstand der Rechtswissenschaften.

\subsubsection{In der Gesetzgebung: Schule der Legistik}

Weil der Gesetzgebungsprozess komplex ist, die nationale Gesetzgebung in das Regelwerk der EU gebettet ist und die zu regelnden Sachverhalte kompliziert sind, müssen die in der Gesetzgebung Tätigen (Legisten) mit vielfältigen Fähigkeiten und Kenntnissen ausgestattet sein, um den Anforderungen der heutigen Gesetzgebungspraxis zu genügen. Derzeit werden legistische Fähigkeiten und Kenntnisse aber erst in der „Werkstatt der Gesetzgebung“, also erst in der gesetzgeberischen Arbeit von Bundesregierung und Parlament erworben. Die Bundesregierung hat daher eine Qualifizierungsoffensive zum Aufbau einer Legistenschule eingeleitet, in der künftige Verfasser von Gesetzentwürfen auch Fähigkeiten zur Erstellung verständlicher Gesetzestexte erlernen sollen (Bessere Rechtsetzung 2016: 16).

\subsubsection{Nach bzw. neben der Gesetzgebung}

Rechtslinguistische und soziologische Forschung muss die Gesetzgebung begleiten, um auch „schwere“ Texte wie Gesetze trotz ihrer Beschränkungen (vgl. Fix 2017) verständlich zu machen. Würden derartige Forschungsergebnisse in die Praxis zurückwirken und zugleich zu einem popularisierten Wissen über Gesetzgebung und die Textsorte ,Gesetz führen, profitierten auch diejenigen, die „fertige“ Gesetze verständlicher machen - für konkrete Adressatenkreise und mit verschiedensten Mitteln. So könnten Fragen zu bestimmten Rechtsfragen (und Gesetzen) nicht nur in Broschüren und auf Internetseiten adressatengerecht vermittelt, sondern z. B. auch in Leichte Sprache übersetzt werden. 


\section{$3 \quad$ Fazit}

Die sprachliche Ausformung von Recht stellt eine potentielle Barriere dar, da insbesondere die Textsorte ,Gesetz' Merkmale hat, die die Verständlichkeit einschränken. Die Übersetzung von Recht in den Alltag von Menschen mit verschiedenen Bedürfnissen, Fähigkeiten und Einschränkungen ist zu verbessern

- durch neue Wege zur Verständlichkeit der fixierten Textsorte, Gesetz (neben der Gesetzesredaktion),

- durch politische Bildung, die obligatorische Verständlichkeitshürden der bürgerrelevanten Textsorte, Gesetz' popularisiert (z. B. durch Veranstaltungen wie „MACHT POLITIK SPRACHE ... verständlich? Kann man Gesetze und Amtsschreiben ,leichter machen?" im Rahmen der Initiative DEUTSCH 3.0 des Goethe-Instituts), und

- durch Professionalisierung der beteiligten Akteure (z. B. in einer Legistenschule und im Studiengang Barrierefreie Kommunikation) über einen stärkeren Austausch zwischen Wissenschaft und Praxis. 
Antje Baumann

\section{Literaturverzeichnis}

„amtlich einfach. Staat der kurzen Wege." Initiative der Bundesregierung, Befragung durchgeführt vom Statistischen Bundesamt, Wiesbaden 2018. URL: www.amtlicheinfach.de (letzter Zugriff: 9.3.2018).

„MACHT POLITIK SPRACHE ... verständlich? Kann man Gesetze und Amtsschreiben ,leichter' machen?", Veranstaltung des Bundesjustizministeriums 2014 im Rahmen der Initiative DEUTSCH 3.0 des Goethe-Instituts. URL: http://www.goethe.de/ $\mathrm{lhr} / \mathrm{prj} / \mathrm{d} 30 / \mathrm{ver} / \mathrm{de} 13059114 \mathrm{v} . h t m$ (letzter Zugriff: 6.3.2018).

„Recht verständlich machen“. Kooperationsprojekt des Bundesministeriums für Justiz und Verbraucherschutz und der Projektgruppe ,wirksam regieren' zur Verbesserung der Verständlichkeit von Rechtstexten, Berlin 2017/2018. URL: https://www.bundesregierung.de/Content/DE/StatischeSeiten/Breg/wirksam-regieren/ 2017-06-21-recht-recht-verstaendlich-machen.html;jsessionid=F0CA57447CEB0547E5B7E3F 668E541DA.s7t1?nn=2158204 (letzter Zugriff: 6.3.2018).

AdAMZIK, Kirsten (Hg.) (2000): Textsorten. Reflexionen und Analysen. Tübingen: Stauffenburg.

AdAMZiK, Kirsten (2008): „Textsorten und ihre Beschreibung“. In: NinA JANich (Hg.): Textlinguistik. 15 Einführungen. Tübingen: Narr, 145-176.

Austin, John LANGSHaw (1962): How to do things with words. Harvard University Press, (deutsche Übersetzung 1972: Zur Theorie der Sprechakte).

Baumann, ANTJE (2012): „Sprachberatung in der Gesetzgebung - ein Arbeitsfeld für Pragmatiker". In: KARI KEINÄSTÖ ET AL. (Hg.): Herausforderung Sprache und Kultur. Festschrift für Matti Luukkainen zum 75. Geburtstag. Helsinki, Société Néophilologique, $145-157$.

BAUMANN, ANTJE (2015): „Bedeutung in Gesetzen: Wie man eine spezielle Textsorte mit korpuslinguistischen Mitteln verständlicher machen könnte“. In: VOGEL, FrIEDEMANN (Hg.): Zugänge zur Rechtssemantik. Interdisziplinäre Ansätze im Zeitalter der Mediatisierung zwischen Introspektion und Automaten. Berlin/New York: Walter de Gruyter (linguae \& litterae), 254-274.

BeAucAmp, Guy (2011): „Verständlichkeit und Bestimmtheit - Zwei Welten?“. In: Rechtstheorie 42, 21-62.

Bessere Rechtsetzung 2016: Mehr Zeit für das Wesentliche. Bericht der Bundesregierung 2016 (Mai 2017). URL: https://www.bundesregierung.de/Content/DE/Artikel/ Buerokratieabbau/Anlagen/2017-05-03-jahresbericht-2016.pdf?_blob= publicationFile\&v=7 (letzter Zugriff: 16.3.2018).

BRINKER, KLAUS (2001): Linguistische Textanalyse: eine Einführung in Grundbegriffe und Methoden. Berlin: Erich Schmidt. 
Rechtstexte als Barrieren - Einige Merkmale der Textsorte ,Gesetz` und die Verständlichkeit

Busse, DieTrich (2000): „Textsorten des Bereichs Rechtswesen und Justiz“. In: KLAUS BRINKer U. A. (Hg.): Text- und Gesprächslinguistik. Ein internationales Handbuch zeitgenössischer Forschung. Halbbd. 1. Berlin/New York: de Gruyter, 658-675.

Deutscher Bundestag, Referat ÖfFentlichkeitsarbeit: Stichwort Gesetzgebung. Von der Idee zum Gesetz. (Stand: August 2016). URL: https://www.btg-bestellservice.de/pdf/20264000.pdf (letzter Zugriff: 12.3.2018).

Die Sprache des Rechts. Studien der interdisziplinären Arbeitsgruppe Sprache des Rechts der Berlin-Brandenburgischen Akademie der Wissenschaften. Im Auftrag der BBAW herausgegeben von Kent D. Lerch, Berlin/New York, de Gruyter: 2004 und 2005. (Band 1: Recht verstehen. Verständlichkeit, Missverständlichkeit und Unverständlichkeit von Recht. Band 2: Recht verhandeln. Argumentieren, Begründen und Entscheiden im Diskurs des Rechts. Band 3: Recht vermitteln. Strukturen, Formen und Medien der Kommunikation im Recht.)

FIX, UlLA (2017): „,Schwere“ Texte in ,Leichter Sprache“ - Voraussetzungen, Möglichkeiten und Grenzen (?) aus textlinguistischer Sicht“. In: Bock, BetTinA M./FIX, Ulla/LANGE, DAISY (Hg.): „Leichte Sprache“ im Spiegel theoretischer und angewandter Forschung. Berlin: Frank \& Timme, 163-188.

Gauer, Isabelle; Hamann, Hanjo; Vogel, Friedemann (2016): „Das juristische Referenzkorpus (JuReko) - Computergestützte Rechtslinguistik als empirischer Beitrag zu Gesetzgebung und Justiz“. In: BurR, Elisabeth (Hg.), DHd 2016: Modellierung Vernetzung - Visualisierung. Die Digital Humanities als fächerübergreifendes Forschungsparadigma, Leipzig 2016, 129-131. URL: http://www.dhd2016.de/ boa-large.pdf (letzter Zugriff: 9.4.2018).

Gemeinsame Geschäftsordnung der Bundesministerien; herausgegeben vom Bundesministerium des Innern, Abteilung O - Verwaltungsmodernisierung, September 2011. URL: http://www.bmi.bund.de (letzter Zugriff: 6. März 2018).

Große, ERNST UlRICH (1974): Texttypen. Linguistik gegenwärtiger Kommunikationsakte. Theorie und Deskription. Stuttgart u.a.: Kohlhammer.

Handbuch der Rechtsförmlichkeit - Empfehlungen des Bundesministeriums der Justiz zur einheitlichen rechtsförmlichen Gestaltung von Gesetzen und Rechtsverordnungen; herausg. vom Bundesministerium der Justiz. - 3. Auflage, 2008, Köln. URL: http://hdr.bmj.de/vorwort.html (letzter Zugriff: 6.3.2018).

Handbuch zur Vorbereitung von Rechts- und Verwaltungsvorschriften; herausg. vom Bundesministerium des Innern. Köln, 2012.

HOFFMANN, LUdGER (1997): „Fachtextsorten der Institutionensprache I: das Gesetz“. In: Hoffmann, Lothar/Kalverkämper, Hartwig/Wiegand, Ernst (Hg.): Fachsprachen. Ein internationales Handbuch zur Fachsprachenforschung und Terminologiewissenschaft. Berlin, New York: de Gruyter, 522-528.

LERCH, KENT D. (2002) „Vom Bemühen, die Gesetze verständlicher zu machen. Eine unendliche Geschichte“. In: Rechtshistorisches Journal 20, 635-643. 
Antje Baumann

LERCH, KENT D. (2004): „Gesetze als Gemeingut aller. Der Traum vom verständlichen Gesetz“. In: Sprache des Rechts I, 225-237. URL: https://edoc.bbaw.de/frontdoor/ index/index/docId/1537 (letzter Zugriff: 6.3.2018).

Linke, Angelika/Nussbaumer, Markus/Portmann, Paul R. (1994): Studienbuch Linguistik. Tübingen: Niemeyer.

Nussbaumer, MARKUS (2002): „,Es gibt nichts Gutes, außer man tut es ${ }^{\star}-$ Arbeit an der Verständlichkeit von Gesetzestexten in der Schweizerischen Bundeskanzlei“. In: Hermes, Journal of Linguistics 29, 111-123.

NUSSBAUMER, MARKUS (2005): „Zwischen Rechtsgrundsätzen und Formularsammlung: Gesetze brauchen (gute) Vagheit zum Atmen“. In: BhAtia, V.K./EngberG, J./ GotTI, M./Heller, D. (Hg.):Vagueness In Normative Texts. Bern u.a.: Lang, 49-72.

Nussbaumer, Markus (2009): „Über den Nutzen der Spracharbeit im Prozess der Rechtsetzung“. In: Felder, EKKEHARD/MÜller, MARCUS (Hg.): Knowledge through Language. Theory, Practice and Research Goals of the Research Network, Language and Knowledge' / Wissen durch Sprache. Theorie, Praxis und Erkenntnisinteresse des Forschungsnetzwerkes ,Sprache und Wissen' Berlin/New York: de Gruyter, 503-528. (Sprache und Wissen, 3).

Pfeiffer, OsKar/Strouhal, ERnst/WodaK, Ruth (1987): Recht auf Sprache. Verstehen und Verständlichkeit von Gesetzen. Wien: Orac.

ROLF, ECKARD (1997): Illokutionäre Kräfte. Grundbegriffe der Illokutionslogik. Wiesbaden: VS Verlag für Sozialwissenschaften.

SANDIG, BARBARA (1972): „Zur Differenzierung gebrauchsspezifischer Textsorten im Deutschen“. In: GÜlich, Elisabeth/RAible WolfGAng (Hg.): Textsorten. Differenzierungskriterien aus linguistischer Sicht. Frankfurt/M.: Athenäum, 113-124.

WARNKE, INGO H. (2004): „Schwerverständlichkeitsannahme als Stereotyp - Die Differenz von Kenntnis und Beurteilung der deutschen Gesetzessprache“. In: Sprache des Rechts. Studien der interdisziplinären Arbeitsgruppe der Berlin-Brandenburgischen Akademie der Wissenschaften. Bd. 1: Recht verstehen. Herausg. von Kent Lerch, Berlin/New York, 441-454. URL: http://edoc.bbaw.de/volltexte/2011/1884/pdf/ 441_Warnke_Schwerverstaendlichkeitsannahme_als_Stereotyp.pdf (letzter Zugriff: 6.3.2018).

WeIsGerber, LeO (1962): Die sprachliche Gestaltung der Welt. Düsseldorf: Schwann. 
FRANZISKA HEIDRICH

\section{Fachexterne technische Kommunikation als Barriere}

\section{$1 \quad$ Einleitung}

Der Beitrag befasst sich mit dem Abbau von Barrieren im fachkommunikativen Kontext, hier insbesondere der Technischen Kommunikation. Eine Schwierigkeit bei der Betrachtung von Barrieren bzw. Barrierefreiheit im Kontext der technischen Fachkommunikation besteht in der ausgeprägten Heterogenität der Zielgruppe (vgl. Abschnitt 2). Während Überlegungen zur Barrierefreien Kommunikation meist die Zielgruppen mit spezifischen kommunikativen Einschränkungen fokussieren, kann hier der Rahmen weiter gesetzt werden: Technische Fachkommunikation stellt in der Experten-Laien-Kommunikation per se bereits eine kommunikative Barriere dar, die sich für Zielgruppen mit besonderen Bedürfnissen noch weiter verschärft. Dieser Beitrag befasst sich mit den Barrieren, denen alle fachexternen Zielgruppen der Technischen Fachkommunikation - ungeachtet spezifischer kommunikativer Einschränkungen - gegenüberstehen.

\section{Technische Kommunikation}

Die Fachkommunikation umfasst „zielgerichtete, informative, mit optimierten Kommunikationsmitteln ausgeführte einsprachige und mehrsprachige mündliche und schriftliche Kommunikationshandlungen fachlichen Inhalts, die von Menschen in Ausübung ihrer beruflichen Aufgaben ausgeführt werden" (Schubert 2007: 210; vgl. Hoffmann 1976/1998; Fluck 1976/1996; Roelcke 1999/2010; Rothkegel 2010). Fachkommunikation wird häufig durch bewussten Eingriff gelenkt und dient „der Explizierung, der Mitteilung und dem Aufbau von Wissen" (Schubert 2007: 142). Sie zeichnet sich durch den Bezug zu spezifischen 
Wissensdomänen aus (Rothkegel 2010: 248). Technische Kommunikation ist das Kommunizieren über technische Inhalte. Durch die inhaltliche Ebene des Technischen grenzt sich die Technische Kommunikation von anderen fachlichen Bereichen ab (Krings 1996: 10ff.; Rothkegel 2010: 9). Die Technische Kommunikation umfasst dabei sowohl mündliche als auch schriftliche Kommunikationsprozesse. Ein wesentliches Arbeitsfeld liegt im Bereich der schriftlichen Kommunikationsprozesse der technischen Kommunikation und damit im Wesentlichen der Technischen Dokumentation.

Technische Dokumentation umfasst verschiedene Dokumente über technische Produkte (und Dienstleistungen), die parallel zum Entstehen und zum Lebensweg eines Produktes (Produktlebenszyklus) entstehen. Ein Dokument ist „ein fixierter und zu beliebigen Zeitpunkten wieder rezipierbarer mündlicher oder schriftlicher Text einschließlich eventueller nichtsprachlicher Komponenten“ (Schubert 2007: 7 in Anlehnung an Krings 1996: 12; DIN 6789-1 1990). Eine Dokumentation in Abgrenzung dazu ist die „Summe der für einen bestimmten Zweck vollständig zusammengestellten Dokumente“ (DIN 6789-1 1990). Diese Dokumente enthalten produktbezogene Daten und Informationen für verschiedene Zwecke, sind in strukturierter Form angelegt, können mündlich oder schriftlich ausgeprägt sein, liegen nicht-flüchtig vor und sind immer funktionsorientiert (vgl. Krings 1996: 9; VDI 4500 - Blatt 1; Juhl 2005: 14f.). Häufig steht dabei die Befähigung zu Anschlusshandlungen im Fokus (Juhl 2005: 15). Analog zum Produktlebenszyklus besteht auch ein sog. Informationslebenszyklus. Der Informationslebenszyklus kann z. B. in die Phasen der Erstellung, der Verdichtung und Nutzung, der Bewahrung und der Archivierung unterteilt werden (Thome/Sollbach 2007: 30). In der Technischen Fachkommunikation wird der Informationslebenszyklus häufig als parallel zum Produktlebenszyklus bzw. entlang dessen Phasen ablaufend betrachtet (tekom 2018).

Technische Fachkommunikation ist in verschiedenen Ausprägungen zu finden und schlägt sich mithin auch in verschiedenen Textsorten nieder, die wiederum verschiedenen Textsortenkonventionen unterliegen. Göpferich (1998a: 90ff.) stellt hierzu ein sehr umfassendes Textsortenspektrum auf, das hier als Grundlage dafür dienen soll, die Zielgruppen von Technikkommunikation abzubilden: 
- Juristisch-normative Texte schaffen eine Rechtsgrundlage. Mit ihnen sind Ansprüche verknüpft oder mit ihnen wird eine Vereinheitlichung geschaffen.

- Fortschrittsorientiert-aktualisierende Texte sollen Wissenschaft und Technik vorantreiben.

- Didaktisch-instruktive Texte sollen „den aktuellen Wissensstand zur intellektuellen Bereicherung oder zum Zwecke der praktischen Anwendung" verbreiten (Göpferich 1998a: 91).

- Wissenszusammenstellende Texte vermitteln einen Überblick über das in den anderen Texttypen vermittelte Wissen.

Entlang der Fachtexttypen nimmt der Fachlichkeitsgrad bei gleichzeitiger Vergrößerung des Adressatenkreises (Göpferich 1998a: 91) graduell ab. Graduell, da die Fachlichkeit von Texten nicht streng abgegrenzt bewertet werden kann, denn eine klare Dichotomie im Sinne von ,fachlich vs. nicht fachlich' ist nicht gegeben. Vielmehr kann die Fachlichkeit von Texten auf einer "gleitenden Skala" bewertet werden, „wobei die Skalen von ,(extrem) merkmalreich“ bis ,(extrem) merkmalarm' reichen" (Hoffmann 1998: 163).

Entsprechend der graduellen Fachlichkeit im technischen Textsortenspektrum lassen sich auch die Zielgruppen kategorisieren. Technische Fachkommunikation findet fachintern (innerhalb desselben Fachgebiets), fachextern (zwischen Expert(inn)en und Laien) und interfachlich (zwischen Angehörigen verschiedener Fächer) statt (Möhn 1977: 314; Möhn/Pelka 1984). Bei fachinterner Kommunikation kann bei der Textproduktion davon ausgegangen werden, dass Produzent(in) und Rezipient(in) über dasselbe Fachwissen und dieselben fachsprachlichen Kenntnisse verfügen (Stolze 1999: 126f.). Bei fachexterner Kommunikation kann davon ausgegangen werden, dass weder das Fachwissen noch die fachsprachlichen Kenntnisse bei Produzent(in) und Rezipient(in) gleich sind; es liegt demnach eine zu überwindende Fachsprachenbarriere vor und es muss darüber hinaus ein Wissenstransfer in Richtung der Adressatenschaft stattfinden (ebd. 131). Auch die interfachliche Kommunikation zwischen 
Fachleuten unterschiedlicher Fächer stellt besondere Herausforderungen an die Textproduzent(inn)en: Es bedarf einer Sach- und Verständigungskompetenz, die die Explizierung impliziten Wissens und die „sachadäquate Form der Darstellung prinzipiell in Abhängigkeit vom Adressatenbezug" einschließt (Jäger 1996: 74f.; zit. n. Janich 2012: 13).

In Bezug auf eine Zielgruppenanpassung im Rahmen von Technischer Fachkommunikation, die in aller Regel eine Reduktion von Barrieren erfordert (s. Rink in diesem Band), müssen zunächst die Eigenschaften, Anforderungen und Voraussetzungen einer intendierten Zielgruppe analysiert werden. Hier werden die Fachkenntnisse sowie die Fachsprachenkompetenz ermittelt. Für anwendungsbezogene Endnutzerdokumentationen (Bedienungsanleitungen etc.) im Rahmen der fachexternen Kommunikation muss im Normalfall davon ausgegangen werden, dass die Adressatenschaft über keinerlei Fachwissen und in diesem Zusammenhang auch über ein geringes $\mathrm{Maß}$ an fachsprachlicher Kompetenz verfügt.

\section{Barrieren in der Technischen Kommunikation}

Barrieren sollen hier im übertragenen Sinne verstanden werden als Hürden, die die Kommunikation beeinträchtigen, mithin ist also im Folgenden von kommunikativen Barrieren die Rede, durch die Menschen von kommunikativen Handlungen ausgeschlossen werden. Diese Barrieren können in Wahrnehmungsund Verständnisbarrieren unterteilt werden (Schubert 2016: 18f.; Heidrich/ Schubert 2017: 39). Während bei einer Wahrnehmungsbarriere einer der menschlichen Sinne nicht funktioniert und eine sprachliche Mitteilung, die diesen Sinn anspricht, damit nicht wahrgenommen werden kann, beziehen sich die Verständnisbarrieren auf Mitteilungen, die zwar sinnlich wahrgenommen, aber nicht verstanden werden können (Heidrich/Schubert 2017; Heidrich i. V.). Viele Barrieretypen treten in allen gesellschaftlichen Bereichen auf, wenn Menschen z. B. aufgrund von zu geringer Sprachkompetenz, wegen kognitiver Einschränkungen etc. an Kommunikation nicht oder nicht ausreichend teilhaben können. Auch im Rahmen der Technischen Kommunikation, vor allem in der fachexter- 
nen Kommunikation, können demnach alle Arten von kommunikativen Barrieren auftreten. Darüber hinaus treten aber in der technischen Kommunikation Barrieretypen auf, die für diesen Kommunikationsbereich - auch für Rezipient(inn)en ohne Einschränkungen - ganz spezifisch sind.

Bereits in der gesetzlichen Würdigung der Technischen Dokumentation werden implizit mögliche auftretende Barrieren angesprochen. So legt die EUMaschinenrichtlinie nicht nur fest, dass einer Maschine immer eine Betriebsanleitung beigefügt sein muss, sondern auch, dass diese in der oder den Amtssprachen des Landes vorliegen muss, in dem die Maschine in Verkehr gebracht wird (Maschinenrichtlinie 2006). Dadurch wird bereits eine Verständnisbarriere indirekt adressiert; denn wenn Rezipient(inn)en aufgrund einer sprachlichen Barriere ein Dokument nicht oder nicht ausreichend verstehen, besteht folglich eine kommunikative Barriere. Auch das Produkthaftungsgesetz adressiert implizit kommunikative Barrieren der Technischen Dokumentation. Demnach liegt nämlich ein Mangel an Produktsicherheit auch dann vor, wenn die „Darbietung“ des Produktes in der Technischen Dokumentation ,unvollständig, fehlerhaft, missverständlich, unverständlich“ ist (ProdHaftG 2015). Auch hier liegt bei Nichtbeachtung eine Verständnisbarriere vor. Gemäß der Einteilung von kommunikativen Barrieren in Sinnes-, Fach-, Kultur-, Kognitions-, Sprach- und Fachsprachenbarrieren (Schubert 2016: 17ff.; Rink in diesem Band) liegen in der Technischen Fachkommunikation darüber hinaus vor allem Fach- und Fachsprachenbarrieren, im Sinne der obigen Kategorisierung also Verständnisbarrieren, vor.

Fachsprachen zeichnen sich besonders durch die sprachlichen Merkmale Informationsverdichtung und Anonymisierung aus. Mit der oben beschriebenen gleitenden Skala der Fachlichkeit korreliert zugleich auch die Fachsprachlichkeit von Texten, denn die Fachsprache kann eben nicht als Gegenstück zu Gemeinsprache betrachtet werden. Der Abstraktionsgrad der außersprachlichen Darstellung, das Fachwissen des Autors/der Autorin und das bei der Adressatenschaft vorausgesetzte Wissen sowie die Textfunktion geben Hinweise auf die Fachlichkeit eines Textes (Stolze 1999: 24). Für Fachsprachen charakteristisch ist die Dominanz der Fachlexik, der Nominalstil, das Fehlen von affektiven und wertenden Wörtern und Wendungen, das Fehlen von dialogischen Partien, die Häufung von Funktionsgefügen und eine Tendenz zur Knappheit im Ausdruck 
(Fluck 1976/1996: 47ff.). Einen besonders hohen Stellenwert nimmt die Ökonomie der Aussage ein, was z. B. durch die Nominalisierung oder das Ersetzen von finiten Verben erzielt wird. Nominalisierungen, Appositionen, Partizipialkonstruktionen, satzwertige Infinitive sowie Kondensationsformen wie die Reduzierung von Nebensätzen auf Partizipial- und Gerundialkonstruktionen, Genitiverweiterungen, präpositionale Substantivgruppen, Attribute, Partizipialgruppen, Ellipsen, Aufzählungen oder Asyndeta sollen der Ökonomie der Aussage dienlich sein (Stolze 1999: 94ff.).

Die Verständlichkeit von Fachtexten ist keine rein textimmanente Eigenschaft, sondern orientiert sich vor allem auch an den kognitiven und motivationalen Eigenschaften und den Wissensvoraussetzungen der Rezipient(inn)en, die ihrerseits Einfluss auf den Text nehmen (Groeben 1981; Groeben/Christmann 1989: 168; Christmann 2004: 33f.; Antos/Hasler/Perrin 2011: 643). Aber auch textimmanent lassen sich verständlichkeitsfördernde und -hemmende Elemente ausmachen. Verständlichkeit wird durch inhaltliche, visuelle und sprachliche Faktoren befördert (Lehrndorfer 1996a: 339). Ein verständlicher Text soll im Wesentlichen handlungsorientiert strukturiert, einfach, einheitlich, eindeutig und vollständig sein (Heidrich 2016). Verständlichkeitsfördernd sind auch Mittel der Visualisierung bzw. ihr Zusammenspiel mit dem Text sowie eine unterstützende Makrotypographie, eine durchlaufende access structure und die Anordnung der Textteile (Sauer 1995, 1997). Auch die Motivation der Rezipient(inn)en wird zu den grundlegenden Elementen der Verständlichkeit gezählt (vgl. Groeben 1972; Langer/Schulz von Thun/Tausch 1974/2006; Göpferich 2001, 2002/2006), dazu zählen unter anderem Beispiele und Illustrationen. Das vermittelte Wissen, die Textgestaltung und das Medium sollen an Voraussetzungen und Anforderungen der Adressatenschaft angepasst werden (Göpferich 2001, 2002/2006). Zusammengefasst lassen sich textimmanente Verständlichkeitsmerkmale von technischen Fachtexten den übergeordneten Aspekten Korrektheit, Leserlichkeit, Strukturierung, Vollständigkeit, Einheitlichkeit, Eindeutigkeit, Einfachheit und Kürze zuordnen (Heidrich 2016: 185ff.). Diesen Verständlichkeitsaspekten widersprechen aber in weiten Teilen die oben kurz skizzierten Charakteristika von technischer Fachsprache. Gerade die der Ökonomie der Aussage dienlichen Aspekte der Informationskondensierung und die 
damit einhergehenden grammatischen Mittel behindern die Verständlichkeit und schaffen eine weitere, vom Text ausgehende Verständnisbarriere.

Eine Verständnisbarriere liegt hierbei nicht nur in der fachexternen Kommunikation zwischen Expert(inn)en und Laien und in der interfachlichen Kommunikation zwischen Expert(inn)en unterschiedlicher Fächer vor. Auch fachintern lassen sich Verständlichkeitsprobleme ausmachen. Diese liegen dann nicht mehr in der Fachsprachen- oder Fachbarriere begründet; denn bei fachinterner Kommunikation kann im Normalfall vereinfacht davon ausgegangen werden, dass Produzent(in) und Rezipient(in) über dasselbe Fachwissen und auch über dasselbe fachsprachliche Zeichenrepertoire verfügen. Eine Verständnisbarriere entsteht dann dadurch, dass der sprachliche Ausdruck zu kompliziert, missverständlich, unvollständig etc. ausgeführt ist.

\section{$4 \quad$ Technische Kommunikation barrierefrei}

Entsprechend der oben herausgearbeiteten Barrieretypen im Rahmen der fachexternen, interfachlichen und fachinternen Kommunikation lassen sich verschiedene Methoden identifizieren, die die Reduzierung dieser Verständnisbarrieren zum Ziel haben. Die Reduktion von Barrieren bzw. die Kommunikationsoptimierung in der Fachkommunikation kann z. B. textintern durch die Vereinheitlichung von Terminologie, Syntax und Sprachstil oder durch planmäßigen Aufbau von Makro- und Mikrostruktur realisiert werden (vgl. Baumert 1998/2010; Göpferich 1998b; Schubert 2001: 225ff., 2007: 330; Strohner/Brose 2002/2006; Drewer/Ziegler 2011).

Neben der Standardisierung von Terminologie (unternehmensintern und -übergreifend) haben sich bezüglich der Lenkung des sprachlichen Ausdrucks (bislang weitestgehend branchenintern) die sogenannten kontrollierten oder regulierten Sprachen etabliert, auch vereinzelt als restringierte oder vereinfachte Sprachen bezeichnet. Die terminologische Vielfalt liegt hier wohl zum einen in den heterogenen Entwicklungslinien und zum anderen in unterschiedlichen Übersetzungen der ursprünglichen englischen Benennung controlled languages begründet (Schubert 1999: 434, Anm. 14). Mithilfe regulierter Sprachen wird 
direkt in das Sprachsystem eingegriffen. Es handelt sich hierbei um Sprachformen, deren lexikalische und/oder syntaktische Auswahlmöglichkeiten systematisch eingeschränkt sind. Sie sprechen durch diese Art der Regulierung die Verständnisbarriere in der Fachkommunikation an und finden auch praktisch nur in diesem Bereich Anwendung. Das Ziel älterer regulierter Sprachen, z. B. des Caterpillar Fundamental English, war ursprünglich die Tilgung jeglicher fachinterner Übersetzungen durch die stark erhöhte Verständlichkeit auch für Nicht-Muttersprachler(innen), wobei dieses Ziel nachweislich nicht erreicht wurde (vgl. z. B. Kirkman/Snow/Watson 1987: 160; Lehrndorfer 1996a, b; Kamprath u. a. 1998: 52). Moderne regulierte Sprachen, z. B. das GIFAS Français Rationalisé oder das ASD Simplified Technical English, sind hingegen als Alternative zur Muttersprache etabliert und sollen gegenüber der natürlichen Sprache Inhalte einfacher, eindeutiger und einheitlicher ausdrücken. Damit sind sie der Verständlichkeit in der fachinternen und fachexternen Kommunikation sowie darüber hinaus auch der Übersetzungsfreundlichkeit bei humaner, maschinengestützter und maschineller Übersetzung dienlich (Lehrndorfer 1996b: 13; Göpferich 1998a: 288ff.; Schubert 2007b: 124ff.; Drewer/Ziegler 2011: 206ff.; Heidrich 2016: 125ff.).

Regulierte Sprachen als vereinfachte Varianten einer natürlichen Sprache sollen durch Reduktion der Komplexität, der Ambiguität, des Umfangs und der Redundanz der natürlichen Sprache (Ley 2005: 27) die fachinterne und die fachexterne Kommunikation vereinfachen, sodass nach den Prinzipien einer regulierten Sprache gestaltete Fachtexte sowohl für Expert(inn)en als auch für Laien besser verständlich sind. Etablierte moderne regulierte Sprachen sind das ASD Simplified Technical English und das GIFAS Français Rationalisé (vgl. GIFAS 2010; ASD 2013, 2017). Beide regulierten Sprachen werden im Rahmen der Luftfahrt-, Raumfahrt- und Verteidigungsindustrie eingesetzt. Ihre Existenzberechtigung erlangen sie dadurch, dass in diesen insgesamt sehr stark regulierten Branchen mit sehr hohem Sicherheitsanspruch vor allem die Verständlichkeit von fachinterner Kommunikation einen hohen Stellenwert genießt. So werden die Prinzipien z. B. für die fachinterne Kommunikation zwischen F \& E und Wartung o. ä. eingesetzt (vgl. z. B. Lehrndorfer 1996a, b; Schubert 2001, 2007, 2008). Die anzustrebende erhöhte Einheitlichkeit und Disambiguisierung wird durch Vereinheitlichung und damit einhergehende Konsistenz in Lexik 
und Syntax erzielt. Regulierte Sprachen fußen auf einer reduktiven Umgestaltung von natürlichen Sprachen; d. h. die den Verstehensprozess negativ beeinflussenden Phänomene der natürlichen Sprache werden ausgeschlossen, ohne dass neue sprachliche Phänomene erschaffen werden. Im Einzelnen sind das eine Reduktion des Morphem- oder Lexembestands, der Wortbildungsmöglichkeiten und der syntaktischen Mittel. Häufig wird jedes Lexem auf eine Wortart festgelegt und ambigue syntaktische Konstruktionen werden durch disambiguierte ersetzt (Schubert 2001: 230, 2008: 211; Ley 2005).

Das ASD Simplified Technical English als am weitesten etablierte regulierte Sprache für das Englische enthält neben Schreibregeln auch ein Wörterbuch der bei Anwendung der Spezifikation erlaubten Wörter, wobei das Wörterbuch dem Eineindeutigkeitsprinzip folgt. Eineindeutigkeit in diesem Zusammenhang bedeutet, dass sowohl Synonymie als auch Homonymie ausgeschlossen werden jeder Begriff hat eine einzige Benennung und jede Benennung entspricht einem einzigen Begriff. Die Schreibregeln beziehen sich auf Wortverwendung, Nominalphrasen, Verben, Satzbildung usw. Die laut Wörterbuch verwendbaren Wörter dürfen nur durch fachspezifische Termini ergänzt werden (ASD 2013; Simplified Technical English Maintenance Group 2015).

Bei der Anwendung einer regulierten Sprache findet bewusste Lenkung auf der Ebene des Sprachsystems statt. Dadurch wird ein großer Nachhaltigkeitsgrad für alle noch zu erstellenden Dokumente erzielt. Die erhöhte lexikalische und syntaktische Konsistenz von regulierten Sprachen gegenüber ungelenkter Sprache fördert zudem die effiziente Anwendbarkeit von Content-Management-Systemen. Zugleich führt die erwähnte Konsistenz und Disambiguisierung zu verbesserter Übersetzbarkeit und effizienterer Nutzung von Übersetzungsmanagementsystemen (Kirkman 1983: 9; Kirkman/Snow/Watson 1987: 160; Lehrndorfer 1996b: 13; Göpferich 1998a: 288ff.; Schubert 2007: 124ff.; s. Zehrer in diesem Band). Als Nachteile werden häufig der Initialaufwand und die komplexen Regelwerke verbunden mit einer schwierigen Erlernbarkeit angegeben, was sodann auch zu einer angenommenen geringen Akzeptanz führt (vgl. Lehrndorfer 1996a, b; Schubert 2001). Mit den heutigen Softwareunterstützungssystemen werden allerdings einige dieser Nachteile wieder aufgewogen, da die Regeleinhaltung durch diese Systeme automatisiert und während des Erstellungsprozesses überprüft werden kann. 
Im fachkommunikativen Kontext werden unter Informationsstrukturierung Standardisierungsmethoden verstanden, die darauf abzielen, auf Text- bzw. Teiltextebene Informationen strukturiert darzustellen. Hierunter fallen im Allgemeinen Arbeitstechniken, die es Technischen Redakteur(inn)en ermöglichen, Dokumente so zu gliedern, dass sie leichter verständlich sind. Es wird also auf der Ebene der Textstrukturen lenkend in die Dokumenterstellung eingegriffen. Durch die entstehenden typisierten Informationseinheiten werden Produktion und Rezeption von Dokumenten vereinfacht. Informationen sollen im Dokument leicht aufgefunden und entsprechend ihrer Informationsart leicht identifiziert werden. Die Funktion einer Information wird erkennbar dargestellt und Formulierungen entsprechend gestaltet.

Das Funktionsdesign als in der Technischen Redaktion weit verbreitete Informationsstrukturierungstechnik soll entsprechend der Informationsstrukturierung Dokumente für die Rezipient(inn)en vereinfachen (Lehrndorfer/TjarksSobhani 2001; Schäflein-Armbruster 2004a, b, c, d; Muthig/Schäflein-Armbruster 2008). Im Kern basiert die Technik auf der Strukturierung und Standardisierung von Dokumenten auf allen Beschreibungsebenen. Die Technik ist ursprünglich für die Technische Dokumentation entwickelt worden und basiert auf sprachwissenschaftlichen Erkenntnissen. Aufgrund der Methode werden spezifische firmeneigene Standards entwickelt, wodurch die Methode sehr flexibel anwendbar ist (Schäflein-Armbruster 2004a: 1-46). Der funktionalkommunikative Ansatz des Funktionsdesigns basiert auf der Sprechakttheorie (Schäflein-Armbruster 2004a, b, c, d; vgl. zur Sprechakttheorie Austin 1962/1992; Searle 1969) und nutzt sie insofern, als das Postulat, dass Sprechen immer auch Handeln sei, auf die Technische Dokumentation angewendet wird. Demnach werden auch in der Technischen Dokumentation Sprechakte vollzogen, die einer bestimmten Zielerreichung dienen (Muthig/Schäflein-Armbruster 2008: 43). Die zulässigen sprachlichen Handlungen je nach Dokumentart sowie die konsistente Verwendung, Sequenzierung und Gestaltung dieser Sprechakte werden festgelegt. Dokumentationen sollen konsequent aus funktionalen Elementen aufgebaut und jedes dieser Elemente konsistent definiert werden. Dafür gilt zunächst das Grundprinzip, dass jede Äußerung eine Funktion und einen Inhalt hat, zudem eine optimale Positionierung innerhalb des Dokumentes und 
eine vorgegebene innere Struktur und Formulierung sowie Kennzeichnung, Typografie und Layout (Schmeling 2009: 34).

Der Strukturierung von Dokumenten und Dokumentationen (zur Unterscheidung vgl. Abschnitt 2) liegen vier hierarchische Ebenen zugrunde: die Informationsprodukte, die Sequenzmuster, die Funktionalen Einheiten und die Auszeichnungselemente. Alle vier Ebenen werden den oben beschriebenen Grundprinzipien entsprechend strukturiert und standardisiert, wobei die Funktionalen Einheiten den Kern des Funktionsdesigns bilden. Auf dieser Ebene wird jeder Kommunikationshandlung innerhalb von Dokumenten bzw. Dokumentationen eine festgelegte kommunikative Funktion zugeordnet; die Funktionale Einheit entspricht dabei im Wesentlichen einem Sprechakt. Festgelegt werden für jede Funktionale Einheit ihre Funktion, ihre Anordnung innerhalb des Dokumentes, ihre innere Struktur und Formulierung sowie die Kennzeichnung (vgl. Schmeling 2009: 27). Das Zusammenspiel der Auszeichnung der einzelnen Funktionalen Einheiten miteinander ergibt eine transparente Dokumentstruktur und ermöglicht den selektiven Zugriff auf den Text (Muthig/ Schäflein-Armbruster/Schmeling 2014: o. S.). Für jeden InformationsproduktTypen werden die zulässigen Funktionalen Einheiten festgelegt, deren Anordnung wiederum in Form von Sequenzmustern festgelegt werden kann. Dies ist dann sinnvoll, wenn in einem Informationsprodukt Funktionale Einheiten häufig in immer wiederkehrender Abfolge auftreten. Auf der Ebene der Auszeichnungselemente wird die Auszeichnung von Wörtern oder Wortgruppen reguliert, die keine eigenständige Kommunikationshandlung im Sinne der Funktionalen Einheiten bilden, aber abweichend ausgezeichnet werden sollen. Hier wird z. B. die Darstellung von Displaytexten, Menüpfaden oder Buttons festgelegt (Schmeling 2009: 30).

Die Strukturierung und Standardisierung, die durch das Funktionsdesign erreicht wird, führt dazu, dass Dokumente konsistent und funktional aufgebaut und gestaltet werden, was einen niedrigschwelligen Zugriff und erhöhte, vom Dokument induzierte Verständlichkeit zur Folge haben soll. Durch Informationsstrukturierung gemäß dem Funktionsdesign wird demnach die oben skizzierte Verständnisbarriere adressiert. Eine unabhängig von der Zielgruppe als 
textimmanent angenommene Verständnisbarriere, die durch komplizierte, ambigue und uneinheitliche Strukturierung und Formulierungen hervorgerufen wird, kann hier demnach überwunden werden.

Soll innerhalb von Technischer Dokumentation ein Produktüberblick erreicht werden oder sollen Instruktionen in der Technischen Dokumentation leichter nachvollziehbar gemacht werden, ist es sinnvoll, dies durch den Einsatz von Bildern zu unterstützen. Das Bild sei hier zunächst mit dem weiten Begriff der „nichtsprachlichen Formen der visuellen Kommunikation“ (Ballstaedt 2005/ 2006: 3) definiert. Funktionen von Bildern in der Technischen Fachkommunikation sind das Veranschaulichen, das räumliche Orientieren und das Verdichten von Informationen. Aspekte eines Gegenstands können mithilfe von Bildern hervorgehoben, die genaue Platzierung von Teilprodukten einfach dargestellt und komplexe Objekte durch Abstraktion vereinfacht wiedergegeben werden (Ballstaedt 1996: 192ff.; vgl. Villiger 2017: 316ff.). Damit der Einsatz von Bildern diese Funktionen erfüllen kann, muss ein Zusammenspiel von Text und Bild gewährleistet sein; z. B. indem textuell auf das Bild verwiesen wird (Ballstaedt 2012: 138), wobei Text und Bild häufig kongruent sind und demnach dieselben Begriffe darstellen (ebd., s. auch Alexander in diesem Band). Dadurch wird eine begriffliche und inhaltliche Redundanz hergestellt, die das Verständnis fördert und somit die Verständnisbarriere verringert (Maes/Lenting 1999: 101). Beim komplementären Zusammenspiel von Text und Bild werden hingegen verschiedene Begriffe miteinander verbunden und im Bild dargestellte Sachverhalte werden im Text weiter vertieft (Ballstaedt 2012: 139). Stehen Text und Bild elaborativ nebeneinander, aktivieren sie jeweils unterschiedliche Begriffe, die die Rezipient(inn)en durch Schlussfolgerungen auflösen müssen (ebd.). Diese bedeuten allerdings eine erhöhte kognitive Belastung für die Rezipient(inn)en (vgl. auch Villiger 2017). Abhängig von der Einordnung des Leitmediums ist die Platzierung innerhalb der Dokumentation sowie auch die textuelle Referenz auf das Bild (Villiger 2017: 318). Dabei ist es durchaus nicht selbstverständlich, dass der Text als Leitmedium eingesetzt wird und auch die Art und Weise des $\mathrm{Zu}$ sammenspiels zwischen Text und Bild weist erhebliche Unterschiede, zum Teil innerhalb einer einzigen Dokumentation, auf (ebd.). In instruktiven (Teil-)Texten kann es z. B. wegen der besseren Verarbeitbarkeit sinnvoll sein, das Bild als 
Leitmedium einzusetzen. Dies soll aber nicht bedeuten, dass vollständig auf den Text verzichtet werden kann (Maes/Lenting 1999: 101; Ganier 2004: 22f.; Villiger 2017: 318f.).

Beim Einsatz von Bildern können ebenso wie bei rein textueller Darstellung Verständnisprobleme auftreten. Die Adressatenschaft muss zunächst verstehen, was abgebildet ist und worin die Funktion der Abbildung besteht. Liegt hier eine Mehrdeutigkeit vor, kommt es zu Verständnisproblemen (Ballstaedt 2005/2006: 31ff., s. Alexander in diesem Band). Weitere Einschränkungen kommen z. B. durch die Anordnung von Text und Bild zueinander zum Tragen. Bild und Text werden nur dann effizient zusammen genutzt, wenn sie in räumlicher Nähe zueinander stehen (Ballstaedt 2005/2006: 41, Bredel/Maaß 2016: 282ff.). Bei kongruenter Beziehung müssen beide eindeutig referenziert sein und Benennungen in Text und Bild einander entsprechen. Elaborative Beziehungen zwischen Text und Bild sollten gar gänzlich vermieden werden, weil sie die Rezipient(inn)en überfordern könnten (Ballstaedt 2005/2006: 41f.). Der textuelle Verweis auf ein Bild kann im Basistext, mit einem Bildtitel, einer Legende oder Beschriftungen realisiert werden (Ballstaedt 2005/2006: 42). Ballstaedt legt nahe, „eine didaktisch durchdachte Kombination von Bild und Text schöpf[e] die Stärken beider Kodes aus und dürfte für die meisten Probleme der technischen Vermittlung das Optimum darstellen“ (ebd.: 44).

Die Unterstützung von Texten durch Bilder bzw. die durchdachte Kombination beider Darstellungsformen kann also ebenso wie die oben beschriebenen lenkenden Eingriffe Verständnisbarrieren in der Fachkommunikation erheblich reduzieren.

\section{$5 \quad$ Fazit}

Barrieren in der Technischen Fachkommunikation können auf verschiedenen Faktoren beruhen. Wie oben bereits ausgeführt, können in der Technischen Kommunikation alle Arten von kommunikativen Barrieren auftreten. Die für die Fachkommunikation spezifischen Barrieren sind jedoch diejenigen, die durch das Fehlen des zum Verstehen notwendigen Fachwissens, durch die man- 
gelnde Fachsprachenkompetenz oder durch erhöhte sprachliche Kompliziertheit und textuelle Komplexität entstehen. Durch lenkende Eingriffe wird versucht, Verständnisbarrieren in der Technischen Fachkommunikation zu verringern. Drei von ihnen wurden hier in aller Kürze dargestellt. Die regulierten Sprachen greifen auf der Ebene des Sprachsystems lenkend in die Kommunikationshandlungen ein und verringern damit nachhaltig deren Komplexität, Ambiguität und Uneinheitlichkeit. Die Techniken der Informationsstrukturierung greifen sowohl auf der Ebene der Textstruktur als auch auf darunter liegenden Ebenen in die Textproduktion ein und zielen vor allem auf die Einheitlichkeit und Eindeutigkeit sowie die niederschwellige Zugriffsstruktur von (komplexen) Dokumenten ab. Und die Unterstützung von Kommunikationshandlungen durch Bilder bzw. vielmehr die wechselseitige Unterstützung von Text und Bild dient der weiteren Reduktion von Barrieren in Hinblick auf unterstützende Explizierung komplexer Sachverhalte.

Zielgruppen mit spezifischen Anforderungen in Bezug auf die Rezeption von Texten werden in der Fachkommunikationswissenschaft und -praxis häufig noch nicht bedacht. Da die Technische Fachkommunikation ohnehin vor der Herausforderung steht, fachliche Inhalte für eine sehr heterogene Zielgruppe aufzubereiten, besteht schon eine besondere Schwierigkeit darin, Verständlichkeit für fachliche Laien herzustellen. Die Gestaltung technischer Inhalte für Laien mit stark erhöhten Anforderungen an die Verständlichkeit von Texten und Dokumenten ist eine Herausforderung, der sich Wissenschaft und Praxis noch nicht (ausreichend) stellen. Die wissenschaftliche Erfassung der barrierefreien Technikkommunikation stellt daher ein dringendes Desideratum dar. 


\section{Literaturverzeichnis}

Antos, Gerd/Hasler Ursula/Perrin, Daniel (2011): „Textoptimierung“. In: HABSCHEID, StefAN (Hg.): Textsorten, Handlungsmuster, Oberflächen. Linguistische Typologien der Kommunikation (De Gruyter Lexikon). Berlin/New York: de Gruyter, 638-658.

ASD (2017): „ASD at a Glance“. URL: http://www.asd-europe.org/about-us/asd-at-aglance (letzter Zugriff: 04.04.2018).

ASD AeroSpace and Defense Industries Association of Europe (2013): ASD-STE 100 Simplified Technical English. Specification ASD-STE 100. International Specification for the Preparation of Maintenance Documentation in a Controlled Language. Issue 6. Brüssel.

Austin, JoHn L. (1962/1992): How to do things with words. Nachdruck d. 2. Aufl. v. 1975. Oxford/New York: Oxford Univ. Press.

BAllstaedT, STEFFEN-Peter (1996): „Bildverstehen, Bildverständlichkeit - Forschungsüberblick“. In: KRINGS, HANS P. (Hg.): Wissenschaftliche Grundlagen der Technischen Kommunikation (Forum für Fachsprachen-Forschung 32). Tübingen: Narr, 191-233.

Ballstaedt, STEFFEN-Peter (2005/2006): Visualisierung: Bilder in der technischen Kommunikation. Vorlesungsskript Zertifikatslehrgang Technical Writing/Technische Dokumentation 2005/2006; Fachhochschule Gelsenkirchen/Zürcher Hochschule Winterthur. URL: http://www.ballstaedt-kommunikation.de/wp-content/uploads/Skript_ Bilder_Technischen-Kommunikation.pdf (letzter Zugriff: 04.04.2018).

Ballstaedt, STEFFen-Peter (2012): Visualisieren: Bilder in wissenschaftichen Texten. (UTB Schlüsselkompetenzen 3508.) Konstanz: UVK Verlags-Gesellschaft

BAUMERT, ANDREAS (1998/2010): Gestaltungsrichtlinien. Style Guides planen, erstellen und pflegen. Erstveröffentlichung im doculine Verlag. Hannover: SerWisS - Hochschulschriftenserver der Fachhochschule Hannover. URL: http://serwiss.bib.hshannover.de/files/261/Baumert_Gestaltungsrichtlinien_Style_Guides_1998.PDF (letzter Zugriff: 06.11.2015).

Bredel, Ursula/MaAß, Christiane (2016): Leichte Sprache. Theoretische Grundlagen, Orientierung für die Praxis. Berlin: Duden (= Sprache im Blick).

Christmann, URsula (2004): „Verstehens- und Verständlichkeitsmessung. Methodische Ansätze in der Anwendungsforschung“. In: LERCH, KENT D. (Hg.): Recht verstehen. Verständlichkeit, Missverständlichkeit und Unverständlichkeit von Recht. (Die Sprache des Rechts. Studien der interdisziplinären Arbeitsgruppe Sprache des Rechts der Berlin-Brandenburgischen Akademie der Wissenschaft, 1). Berlin/New York: de Gruyter, 33-62.

DIN 6789-1 (1990): Dokumentationssystematik. Aufbau technischer Produktdokumentationen. Teil 1. Ausgabe 1990-09. Berlin/Wien/Zürich: Beuth. 
Drewer, Petra/Ziegler, Wolfgang (2011): Technische Dokumentation. Übersetzungsgerechte Texterstellung und Content-Management. Würzburg: Vogel.

FLUCK, HANS-RÜDIGER (1976/1996): Fachsprachen. Einführung und Bibliographie (UTB, 483). 5., überarb. u. erw. Aufl. Tübingen u. a.: Francke.

GANIER, FRANCK (2004): „Factors Affecting the Processing of Procedural Instructions: Implications for Document Design“. In: IEEE Transactions on Professional Communication 47 (1), 15-26.

GIFAS (2010): „Présentation générale“. URL: https://www.gifas.asso.fr/presentationgenerale (letzter Zugriff: 28.02.2018).

GÖPFERICH, SUSANNE (1998a): Interkulturelles Technical Writing: Fachliches adressatengerecht vermitteln. Ein Lehr- und Arbeitsbuch (Forum für Fachsprachen-Forschung 40). Tübingen: Narr.

GÖPFERICH, SUSANNE (1998b): „Möglichkeiten der Optimierung von Fachtexten“. In: Hoffmann, Lothar/Kalverkämper, Hartwig/Wiegand, Herbert Ernst (Hg.): Fachsprachen. Ein internationales Handbuch zur Fachsprachenforschung und Terminologiewissenschaft / Languages for Special Purposes. An International Handbook of Special Languages and Terminology Research. New York u. a.: de Gruyter, 888-899.

GÖPfERICH, SUSANNE (2001): „Von Hamburg nach Karlsruhe. Ein kommunikationsorientierter Bezugsrahmen zur Bewertung der Verständlichkeit von Texten“. In: Fachsprache 23 (3-4), 117-138.

GÖPFERICH, SUSANNE (2002/2006): Textproduktion im Zeitalter der Globalisierung: Entwicklung einer Didaktik des Wissenstransfers (Studien zur Translation, 15). Zugl.: Leipzig, Univ., Habil.-Schrift. 2. Aufl. Tübingen: Stauffenburg.

Groeben, Norbert (1972): Die Verständlichkeit von Unterrichtstexten. Dimensionen und Kriterien rezeptiver Lernstadien. Münster: Aschendorff.

GroeBEN, NORBERT (1981): „Verständlichkeitsforschung unter Integrationsperspektive: ein Plädoyer“. In: MANdL, HeInz (Hg.): Zur Psychologie der Textverarbeitung (U\&S Psychologie. Forschung). München/Wien/Baltimore: Urban \& Schwarzenberg, 367385.

Groeben, Norbert/Christmann, Ursula (1989): „Textoptimierung unter Verständlichkeitsperspektive“. In: ANTOS, GERD/KRINGs, HANS P. (Hg.): Textproduktion: ein interdisziplinärer Forschungsüberblick (Konzepte der Sprach- und Literaturwissenschaft, 48). Tübingen: Niemeyer, 165-196.

HEIDRICH, FRANZISKA (2016): Kommunikationsoptimierung im Fachübersetzungsprozess. Ein Modellentwurf. (= TransÜD. Arbeiten zur Theorie und Praxis des Übersetzens und Dolmetschens, 83) Zugl. Dissertation Universität Hildesheim. Berlin: Frank \& Timme.

Heidrich, Franziska (i. V.): „,Gestaltete Sprache“ und ihre Relevanz für die Übersetzungswissenschaft“. In: JÜNGST, HEIKE/LINK, LISA/ZEHRER, CHRISTIANE/SCHUBERT, KLAUS (Hg.): Challenging Boundaries of Translation Studies. 
HeIdrich, Franziska/SCHUbert, Klaus (2017): „Barrieren abbauen durch gestaltete Sprache“. In: Sulikowski, Piotr/SulikowsKa, AnNa/LeSner, Emil (Hg.): Translation Landscapes - Internationale Schriften zur Übersetzungswissenschaft. Band 1 (= TRANSLATOLOGIE - Studien zur Übersetzungswissenschaft, 16). Hamburg: Verlag Dr. Kovač. 38-49.

HofFmann, Lothar (1998): „Fachsprachen und Gemeinsprache“. In: Hoffmann, Lothar/Kalverkämper, Hartwig/Wiegand, Herbert ERnst (Hg.): Fachsprachen. Ein internationales Handbuch zur Fachsprachenforschung und Terminologiewissenschaft / Languages for Special Purposes. An International Handbook of Special Languages and Terminology Research. New York u. a.: de Gruyter, 157-168.

Hoffmann, Lothar (1976): Kommunikationsmittel Fachsprache. (Sammlung Akademie-Verlag 44 Sprache.) 2. Aufl. 1987. Berlin: Akademie-Verlag.

JÄGER, LUDWIG (1996): „Expertenkultur und Sprachkultur. ,Innersprachliche Mehrsprachigkeit' und das Problem der Transparenz des Expertenwissens“. In: BÖKE, KARIN/ Jung, Matthias/Wengeler, Martin (Hg.): Öffentlicher Sprachgebrauch. Praktische, theoretische und historische Perspektiven. Georg Stötzel zum 60. Geburtstag. Opladen: Westdt. Verlag: 68-76.

JANICH, NinA (2012): „Fachsprache, Fachidentität und Verständigungskompetenz - zu einem spannungsreichen Verhältnis“. In: BWP. Berufsbildung in Wissenschaft und Praxis: Sprache und Beruf 2/2012: 10-13.

Juhl, Dietrich (2005): Technische Dokumentation. Praktische Anleitungen und Beispiele. 2., neu bearb. Aufl. Berlin/Heidelberg: Springer.

Kamprath, Christine/Adolphson, Eric/Mitamura, Teruko/Nyberg, Eric (1998): „Controlled Language for Multilingual Document Production: Experience with Caterpillar Technical English“. In: LAnguage Technologies Institute (Hg.): Proceedings of the 2nd International Workshop on Controlled Language Applications. CLAW 98. Carnegie Mellon University, Pittsburgh, Pennsylvania, 21.-22.5.1998, 5161. URL: http://citeseerx.ist.psu.edu/viewdoc/download?doi=10.1.1.44.6405\&rep= rep1\&type=pdf (letzter Zugriff: 04.04.2018).

Kirkman, John (1983): Using „Controlled“ Language in Technical Communication. Manuskript. Ramsbury.

KirkMAN, JOHN/SNOW, Christine/WATSON, IAN (1987): „Controlled English as an alternative to Multiple Translations“. In: IEEE Transactions on Professional Communication 21 (4), 159-161.

KRINGs, Hans P. (1996): „Wieviel Wissenschaft brauchen Technische Redakteure? Zum Verhältnis von Wissenschaft und Praxis in der Technischen Dokumentation“. In: KRINGS, Hans P. (Hg.): Wissenschaftliche Grundlagen der Technischen Kommunikation (Forum für Fachsprachen-Forschung 32). Tübingen: Narr, 5-128.

LANGER, INGHARD/SCHUlZ VON THun, FriedEMANN/TAusCH, REINHARD (1974/2006): Sich verständlich ausdrücken [Anleitungstexte, Unterrichtstexte, Vertragstexte, Amts- 
texte, Versicherungstexte, Wissenschaftstexte u.a.]. 8. Aufl. Erstveröff. u. d. T. Verständlichkeit in Schule, Verwaltung, Politik und Wissenschaft. München u.a.: Ernst Reinhardt.

LEHRndorfer, Anne (1996a): „Kontrollierte Sprache für die Technische Dokumentation. Ein Ansatz für das Deutsche“. In: Krings, Hans P. (Hg.): Wissenschaftliche Grundlagen der technischen Kommunikation (Forum für Fachsprachen-Forschung, 32). Tübingen: Narr, 339-368.

LEHRNDORFER, ANNE (1996b): Kontrolliertes Deutsch. Linguistische und sprachpsychologische Leitlinien für eine (maschinell) kontrollierte Sprache in der Technischen Dokumentation (Tübinger Beiträge zur Linguistik, 145). Tübingen: Narr.

LEHRNDORFER, ANnE/TJARKS-SOBHANI, MARITA (2001): „Schreibprozesssteuerung durch sprachliche Standardisierungen in der technischen Dokumentation“. In: Möhn, Dieter/Ross, Dieter/Tjarks-Sobhani, Marita (Hg.): Mediensprache und Medienlinguistik. Festschrift für Jörg Hennig (Sprache in der Gesellschaft; Beiträge zur Sprachwissenschaft, 26). Frankfurt a. M. u. a.: Lang, 145-164.

LEY, MARTIN (2005): Kontrollierte Textstrukturen. Ein (linguistisches) Informationsmodell für die Technische Kommunikation. Inaugural-Dissertation. Justus-Liebig-Universität, Gießen, Fachbereich Sprache, Literatur, Kultur. URL: http://geb.unigiessen.de/geb/volltexte/2006/2713/pdf/LeyMartin-2006-01-30.pdf (letzter Zugriff: 04.04.2018).

Maes, Alfons/Lenting, Hans (1999): „How to Put the Instructive Space into Words“. In: IEEE Transactions on Professional Communication 42 (2), 100-113.

MASCHINENRICHTLINIE (2006): Richtlinie 2006/42/EG des europäischen Parlaments und des Rates vom 17. Mai 2006 über Maschinen und zur Änderung der Richtlinie 95/16/EG (Neufassung).

MÖHN, DieTER (1977): „Zur Entwicklung neuer Fachsprachen“. In: DeUTSCHE GeSELLSCHaft FÜr Dokumentation E. V. (Hg.): Deutscher Dokumentartag 1976. München, 311-321.

MÖHn, Dieter/Pelka, Roland (1984): Fachsprachen. Eine Einführung. Tübingen: Max Niemeyer.

Muthig, JÜrgen/SCHÄFlein-Armbruster, Robert (2008): „Funktionsdesign - methodische Entwicklung von Standards“. In: MuTHIG, JÜRGEN (Hg.): Standardisierungsmethoden für die Technische Dokumentation (tekom Hochschulschriften, 16). Lübeck: Schmidt-Römhild, 41-74.

Muthig, JÜrgen/Schäflein-Armbruster, Robert/SCHMELING, RolAnd (2014): „Funktionsdesign ${ }^{\circledR}$. Strukturierung“. In: Böcher, Kornelius R./Thiele, Ulrich (Hg.): Technische Dokumentation. WEKA Business Portal. WEKA Media GmbH \& Co. KG. URL: http://www.weka-business-portal.de/modul/index.jsf (letzter Zugriff: 18.02.2014).

ProdHaftG (2015): Produkthaftungsgesetz vom 15. Dezember 1989 (BGBl. I S. 2198), das zuletzt durch Artikel 180 der Verordnung vom 31. August 2015 (BGBl. I S. 1474) 
geändert worden ist. URL: www.gesetze-im-internet.de/bundesrecht/prodhaftg/ gesamt.pdf (letzter Zugriff: 04.04.2018)

Roelcke, Thorsten (1999/2010): Fachsprachen (Grundlagen der Germanistik, 37). 3. Aufl. Berlin: E. Schmidt.

Rothkegel, Annely (2010): Technikkommunikation. Produkte, Texte, Bilder (UTB, 3214). Konstanz: UVK Verl.-Ges.

SAUer, Christoph (1995): „Ein Minimalmodell zur Verständlichkeitsanalyse und -optimierung“. In: SPILleR, BERnd (Hg.): Sprache: Verstehen und Verständlichkeit (forum Angewandte Linguistik, 28). 25. Jahrestagung der Gesellschaft für Angewandte Linguistik GAL e.V. Trier, 1994. Frankfurt a. M. u. a.: Lang, 149-171.

SAUER, CHRISTOPH (1997): „Visualisierung inbegriffen: Textüberarbeitung und Umgestaltung". In: JAKOBS, Eva-MARIA/KNORR, DAgmar (Hg.): Schreiben in den Wissenschaften (Textproduktion und Medium, 1). Interdisziplinäres Kolloquium „Textproduktion im Zeitalter des Computers". Otzenhausen, 1996. Frankfurt am Main u. a.: Lang, 91-106.

Schäflein-Armbruster, Robert (2004a): Planen, Strukturieren, Standardisieren mit Funktionsdesign. Veranstaltungsskript FH Furtwangen. Studiengang Dokumentation und Kommunikation, 29.11.2004. URL: http://web.archive.org/web/200510240 91202/http://www.zindel.de/zindel/pdf/funktionsdesign04/sar_Funktionsdesign_ Man.pdf (letzter Zugriff: 04.10.2012).

SCHÄFlein-Armbruster, Robert (2004b): Problemtypologie. Probleme exakt benennen, gezielt lösen. Veranstaltungsskript FH Furtwangen. Studiengang Dokumentation und Kommunikation.

SCHÄFlein-ARMBruster, Robert (2004c): Sicherheitsgerechte Dokumentation für den Internationalen Markt. Veranstaltungsskript FH Furtwangen. Studiengang Dokumentation und Kommunikation.

SCHÄFlein-ARMBruster, ROBERT (2004d): Standardisierung mit Funktionsdesign. Veranstaltungspräsentation Workshop DokuNord.

SCHMELING, ROLAND (2009): Informationsverarbeitung mit Funktionsdesign. Walldorf: Schmeling + Consultants [Präsentation]. URL: http://www.schmeling-consultants.de/ uploads/media/tekom_Reg_gruppe_Baden_2009-10-21_Informationsverarbeitung mit_Funktionsdesign.pdf (letzter Zugriff: 19.02.2014).

SCHUbERT, Klaus (1999): „Zur Automatisierbarkeit des Übersetzens“. In: GIL, AlbERTO/ Haller, Johann/Steiner, Erich/Gerzymisch-Arbogast, Heidrun (Hg.): Modelle der Translation (Sabest Saarbrücker Beiträge zur Sprach- und Translationswissenschaft 1). Frankfurt am Main u.a.: Lang, 423-441.

SCHUbERT, Klaus (2001): „Gestaltete Sprache. Plansprachen und die regulierten Sprachen der internationalen Fachkommunikation “. In: SCHUberT, KLAUS (Hg.): Planned languages. From concept to reality (Interface. Journal of applied linguistics, 15.1/2). Brüssel: Hogeschool voor Wetenschap en Kunst, 223-257. 
Schubert, Klaus (2007): Wissen, Sprache, Medium, Arbeit (Forum für FachsprachenForschung, 76). Zugl.: Chemnitz, Techn. Univ., Habil.-Schrift. Tübingen: Narr.

SCHUbert, Klaus (2008): „Konstruktion und Reduktion“. In: Krings, Hans P./MaYer, FELIX (Hg.): Sprachenvielfalt im Kontext von Fachkommunikation, Übersetzung und Fremdsprachenunterricht (Forum für Fachsprachenforschung, 83). Berlin: Frank \& Timme, 209-219.

SCHUBERT, KlaUs (2016): „Barriereabbau durch optimierte Kommunikationsmittel. Versuch einer Systematisierung“. In: MäLzer, NATHALIE (Hg.): Barrierefreie Kommunikation - Perspektiven aus Theorie und Praxis (Kommunikation - Partizipation - Inklusion, 2). Berlin: Frank \& Timme, 15-33.

SEArle, John R. (1969): Speech Acts. An Essay in the Philosophy of Language. Cambridge: Cambridge University Press.

Simplified Technical English Maintenance Group (2015): ASD Simplified Technical English. Brüssel. URL: http://www.asd-ste100.org/ (letzter Zugriff: 25.09.2015).

StOlze, Radegundis (1999): Die Fachübersetzung. Eine Einführung. Tübingen: Narr.

Strohner, Hans/Brose, Roselore (2002/2006): „Kommunikation und ihre Optimierung“. In: Strohner, Hans/Brose, Roselore (Hg.): Kommunikationsoptimierung. Verständlicher - instruktiver - überzeugender (Stauffenburg Linguistik, 30). Workshop auf der Tagung der Gesellschaft für angewandte Linguistik. Passau, 2001. 2. Aufl. Tübingen: Stauffenburg, 3-14.

[TEKOM] Gesellschaft FÜr Technische Kommunikation - TeKom E. V. (2018): „Was ist ,Technische Kommunikation'?" URL: http://www.tekom.de/technische-kommunikation/was-ist-technische-kommunikation.html (letzter Zugriff: 28.03.2018).

Thome, GÜNTer/Sollbach, WolfGang (2007): Grundlagen und Modelle des Information Lifecycle Management. Berlin/Heidelberg: Springer.

VDI 4500 (2006): Technische Dokumentation. Begriffsdefinitionen und rechtliche Grundlagen. Blatt 1. Ausgabe deutsch/englisch. Berlin: Beuth.

Villiger, Claudia (2017): „Texte und Visualisierungen im digitalen Nutzungskontext der Technischen Kommunikation“. In: trans-kom 10/3, 315-333. URL http://www.trans-kom.eu/bd10nr03/trans-kom_10_03_03_Villiger_Visualisierungen. 20171221.pdf (letzter Zugriff: 21.02.2018). 
Stimmen aus der Praxis 



\section{Barrierefreie Angebote des NDR und der ARD - Stand, Aufgaben, Ziele}

Der Norddeutsche Rundfunk baut seine barrierefreien Angebote für Menschen mit Behinderungen seit vielen Jahren sukzessive aus.

Arbeitsschwerpunkte sind die Untertitelung und Audiodeskription des Fernsehprogramms, Angebote in Gebärdensprache sowie Leichter Sprache und die Barrierefreiheit des Onlineangebotes. Außerdem hat der NDR innerhalb der ARD die Federführung beim Projekt „Barrierefreier Rundfunk“. Er koordiniert in der ARD den weiteren Ausbau der barrierefreien Angebote und steht in regelmäßigem Austausch mit den Vertreterinnen und Vertretern der Behindertenverbände.

Eine gleichberechtigte Teilhabe am Fernsehen funktioniert nicht, wenn ein Mensch das Programm nur über einen Sinn wahrnehmen kann.

Das Ziel der ARD ist, alle interessierten Menschen mit ihren vielfältigen Angeboten zu erreichen. Allen Bevölkerungsgruppen soll die Teilhabe an der Informationsgesellschaft und den publizistischen Inhalten des ARD-Fernseh-, -Hörfunk- und -Onlineangebots ermöglicht werden. Die Inhalte sollen für möglichst viele Menschen frei zugänglich sein, es gilt, niemanden von der Nutzung auszuschließen. Unabhängig von einer eventuell vorhandenen Behinderung oder Schwäche soll jeder die Möglichkeit haben, sich zu informieren und sich eine eigene Meinung zu bilden, um sich am gesellschaftlichen Diskurs beteiligen zu können.

Die ARD-Intendantinnen und -Intendanten haben deshalb 2011 einen Maßnahmenkatalog zum Ausbau der barrierefreien Angebote beschlossen, den alle Landesrundfunkanstalten seitdem konsequent umgesetzt haben. Denn nur durch barrierefreie Zusatzangebote können Menschen mit einer Hör- oder Sehschädigung den Programmen gleichberechtigt folgen. 


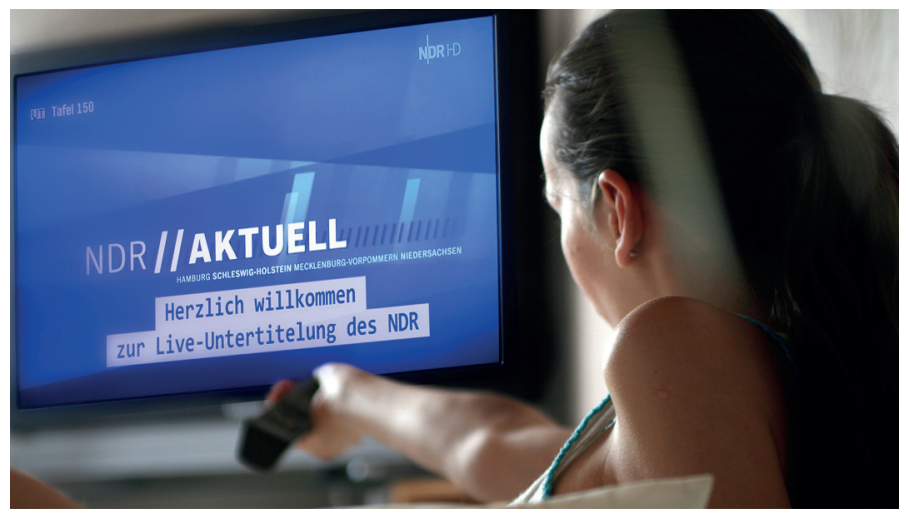

Die Untertitelung von Fernsehsendungen erfolgt auf drei verschiedene Arten: live, semi-live oder vorproduziert. Alle auditiven Inhalte - Sprache, Geräusche und Musik - werden anhand der ARD- und NDR-Standards für Untertitelung in Schriftsprache übersetzt. Untertitel schaffen Barrieren ab. Sie sollen primär hörgeschädigten und gehörlosen Menschen Zugang zu den audiovisuellen Medien ermöglichen und denselben Kenntnisstand vermitteln, den auch Hörende haben. Als Grundlage für die Berechnung der Standzeit eines Untertitels gilt ein Basiswert von 13 bis 15 Zeichen pro Sekunde. Dies entspricht einer durchschnittlichen Lesegeschwindigkeit. Die Synchronität zu Bild und Ton soll bei der Untertitelung möglichst gewahrt bleiben. Ziel ist es zudem, möglichst nah am Originaltext zu bleiben und den kompletten Inhalt wiederzugeben.

Untertitel sind vor allem ein Instrument der Barrierefreiheit. Für Menschen mit Migrationshintergrund können sie zusätzlich durch die Kombination von Hören und Lesen eine Unterstützung beim Erlernen der deutschen Sprache sein. Einen weiteren großen Nutzen haben sie überall dort, wo Fernsehbilder im öffentlichen Raum gezeigt werden, ohne dass der Ton zugeschaltet ist.

Im NDR-Fernsehen gibt es schon seit den 1980er Jahren untertitelte Sendungen. Das Angebot war damals beschränkt auf die Tagesschau um 20 Uhr, Krimis und Spielfilme. Das hat sich nachhaltig verändert. Seit 2007 gibt es im NDR eine eigene Untertitelredaktion. Die Sendung „Das!“, das Magazin mit dem Roten 
Sofa, war im gleichen Jahr die erste Sendung, die im NDR-Fernsehen live untertitelt wurde. Untertitel gehören inzwischen zum Programmauftrag und sind fester Bestandteil der Programmpräsentation. Sukzessive kommen immer neue Sendungen hinzu, sodass inzwischen 82 Prozent des Programms untertitelt sind.

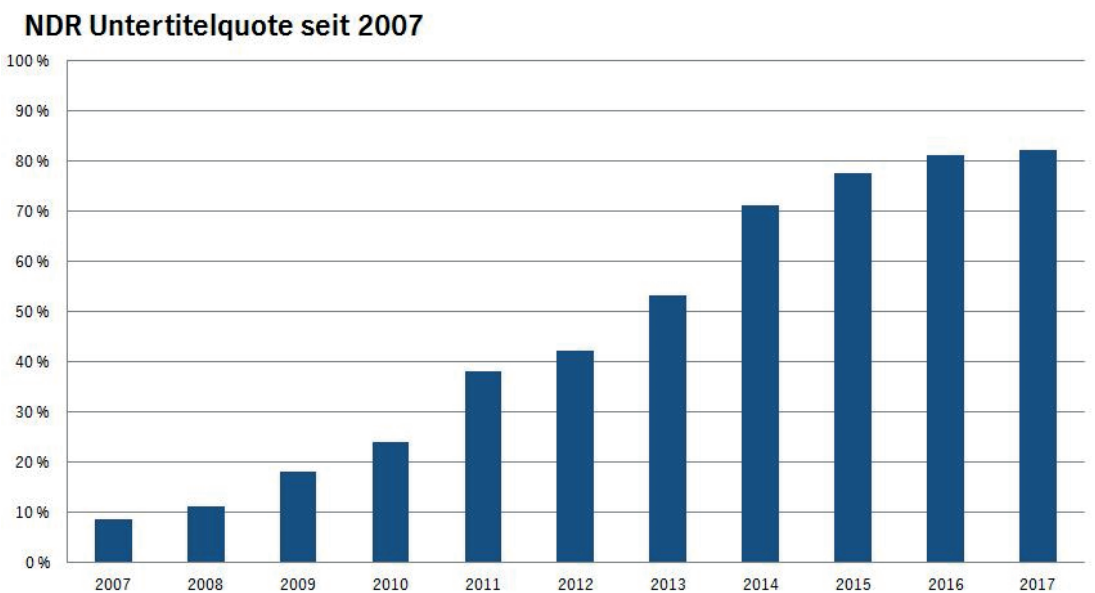

Seit dem 1. Januar 2013 werden alle Erstsendungen im Ersten Programm mit Untertitelung angeboten. Darauf hatten sich die ARD-Intendantinnen und -Intendanten 2011 verständigt. Im Ersten hat sich die Untertitelquote seit 2012 nahezu verdoppelt - sie liegt aktuell bei 96 Prozent. Gleichzeitig haben die neun Landesrundfunkanstalten die Anzahl der untertitelten Sendungen in ihren Dritten Programmen nachhaltig gesteigert. 


\section{Das Erste Untertitelquote seit 2007}

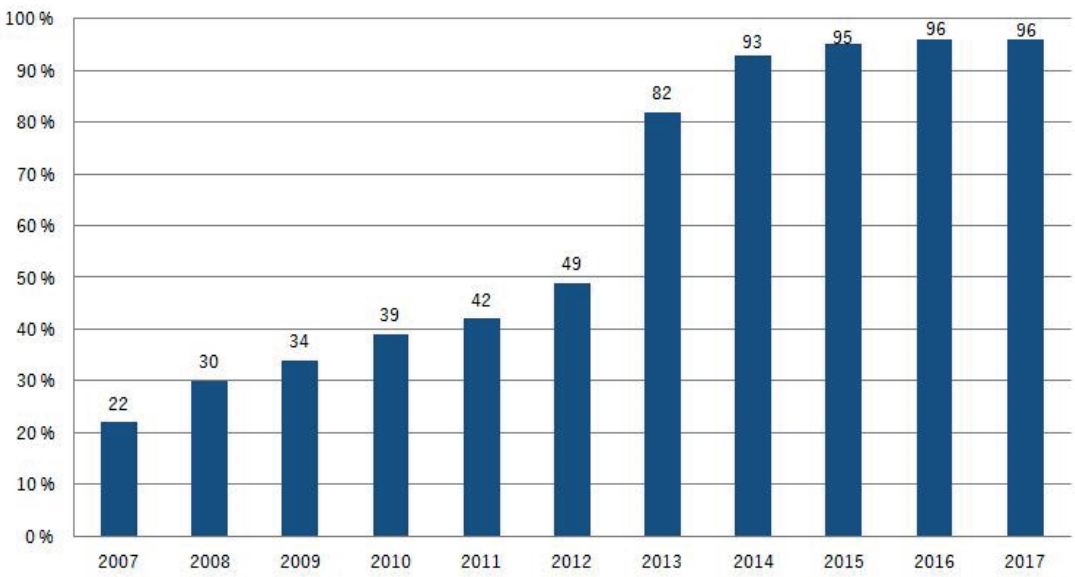

ARD Untertitelquote seit 2012

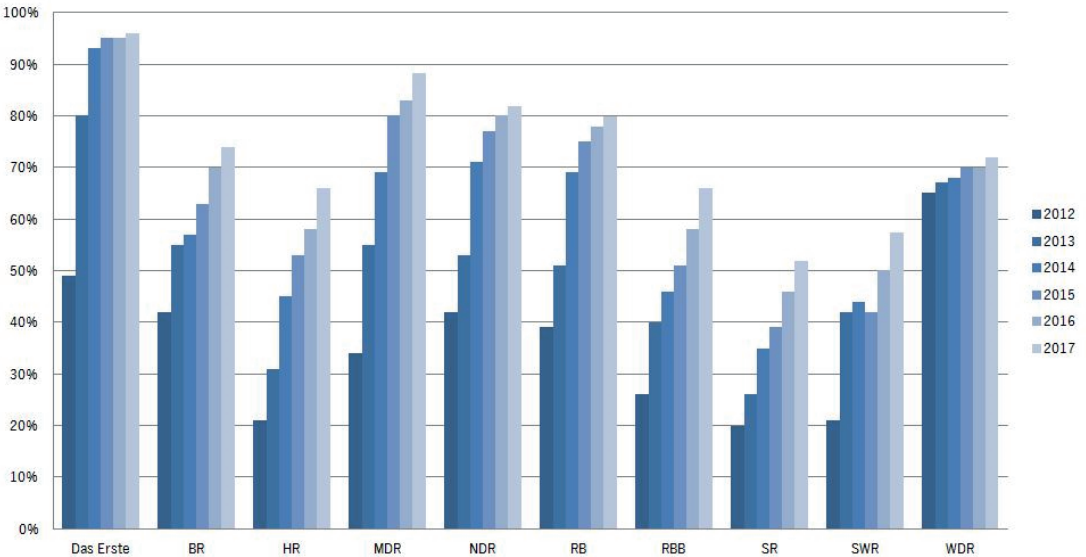

Untertitel werden digital oder im Videotext auf Seite 150 ausgestrahlt. Seit Juni 2016 bietet die ARD für Menschen mit Hörbehinderung außerdem einen Service für mit dem Internet verbundene Fernseher im sogenannten HbbTV- 
Format: Dort können die Untertitel in Größe, Position und Hintergrund an die persönlichen Bedürfnisse angepasst werden. Es besteht die Möglichkeit, zwischen vier Schriftgrößen zu wählen und Untertitel wahlweise am oberen oder unteren Bildrand zu positionieren.

\section{Audiodeskription von Fernsehsendungen}

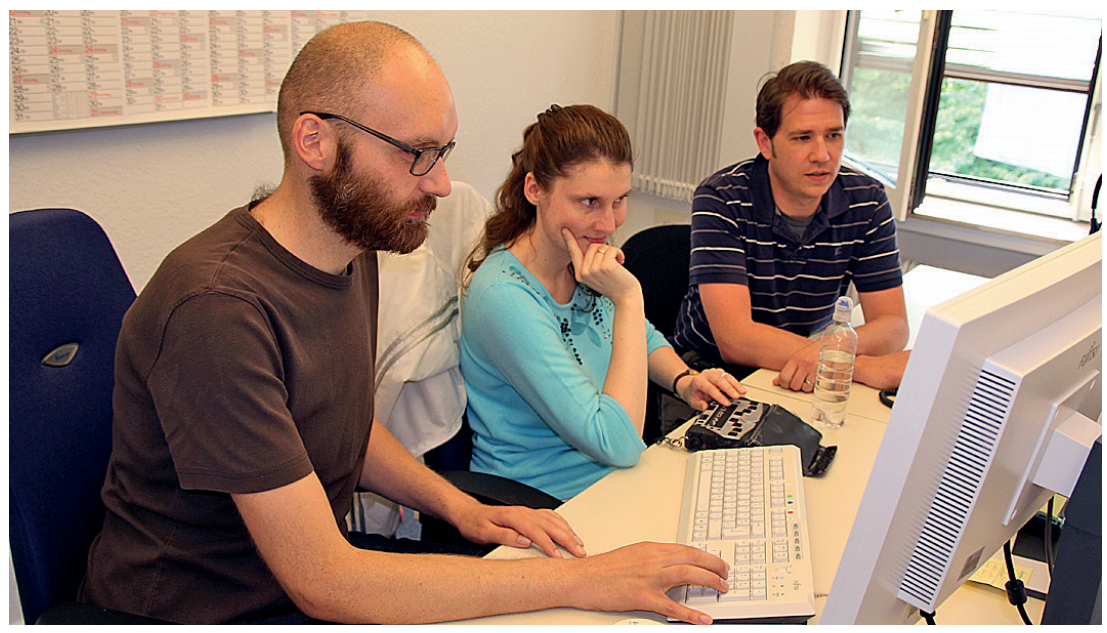

Für ausgewählte Sendungen bietet der NDR außerdem Audiodeskriptionen an und ermöglicht so auch Menschen mit einer Sehbehinderung die Teilhabe an seinem Fernsehprogramm. Für die Hörfilmfassungen gibt es einen gesonderten Tonkanal, der sich mit der Fernbedienung aktivieren lässt.

Die neun Landesrundfunkanstalten der ARD, ZDF, ORF, SRF sowie die Deutsche Hörfilm gGmbH, Hörfilm e.V. und audioskript haben sich 2015 auf Initiative des NDR auf eine Reihe von Grundsätzen für die Erstellung von Audiodeskriptionen im deutschsprachigen Raum verständigt. Das Ziel ist, sehbehinderten Menschen ein barrierefreies Fernseherlebnis zu ermöglichen, das dem Erlebnis der Sehenden entspricht. Der NDR orientiert sich bei der Erstellung seiner Hörfilme an diesen Standards. 
Uschi Heerdegen-Wessel

Die Zahl der Audiodeskriptionen im NDR-Fernsehen wurde in den vergangenen Jahren ebenfalls kontinuierlich gesteigert.

\section{NDR Audiodeskriptionsquote ganzer Sendetag}

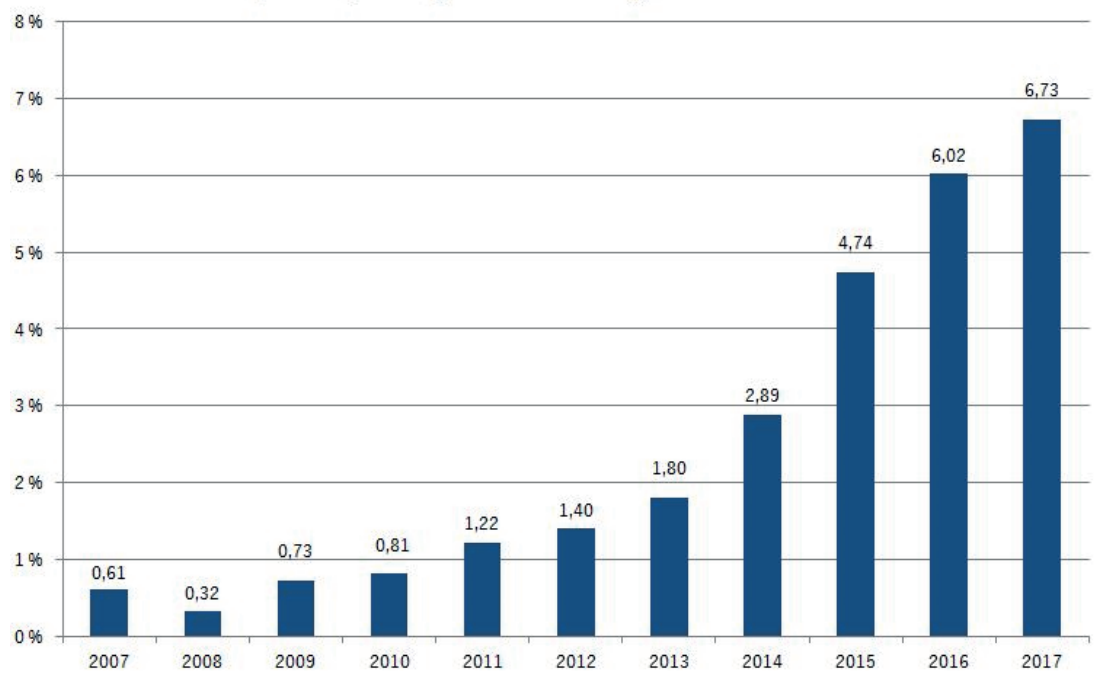




\section{NDR Audiodeskriptionsquote Hauptabend}

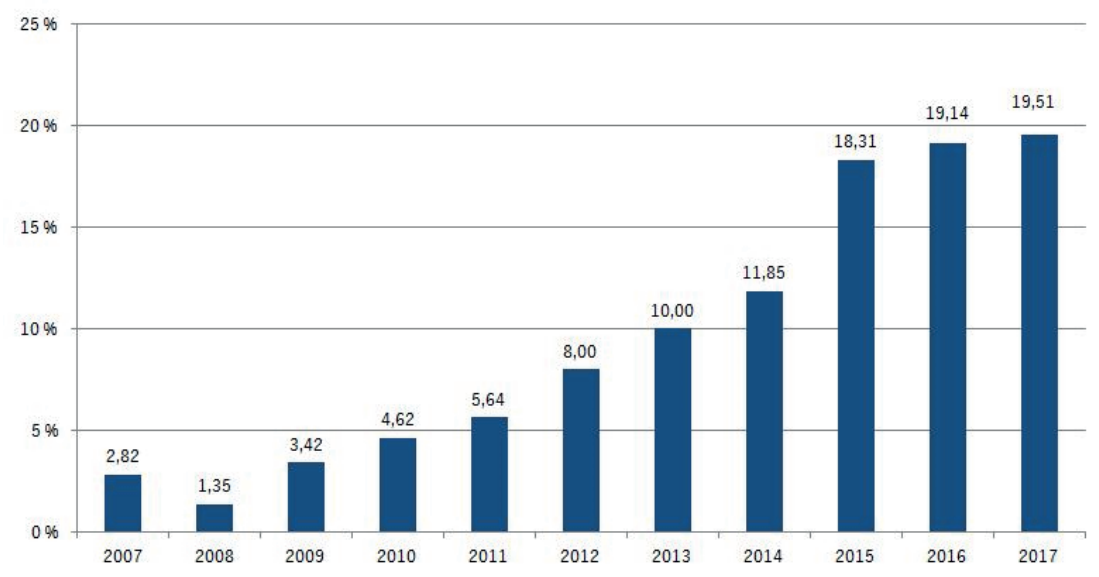

Im Rahmen des ARD-Maßnahmenkatalogs zum Ausbau der barrierefreien Angebote wurde auch beschlossen, das Hörfilmangebot im Ersten Programm auszuweiten. Seit Anfang 2013 werden im Hauptabendprogramm alle fiktionalen Formate (Krimis, Spielfilme, Serien) sowie die Tier- und Naturfilme in einer Hörfilmfassung ausgestrahlt. Anfang 2016 wurde das Audiodeskriptionsangebot im Ersten auch auf das Vorabendprogramm ausgedehnt.

Derzeit liefert Das Erste für rund 18 Prozent seines Programms eine Audiodeskription, 2012 waren es noch gut fünf Prozent. 
Uschi Heerdegen-Wessel

\section{Das Erste Audiodeskriptionsquote ganzer Sendetag}

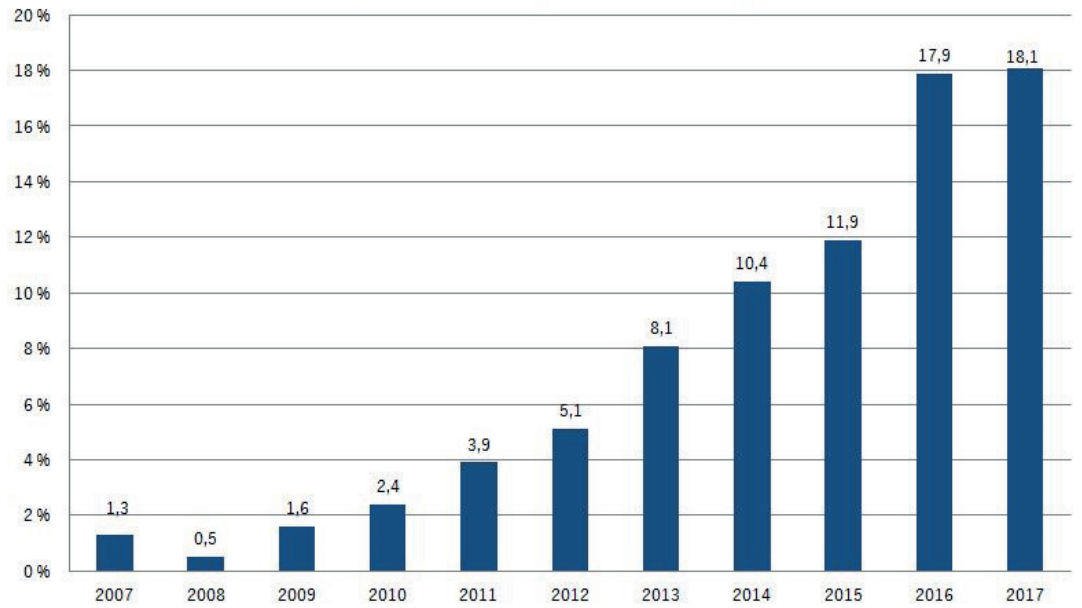

Das Erste Audiodeskriptionsquote Hauptabend

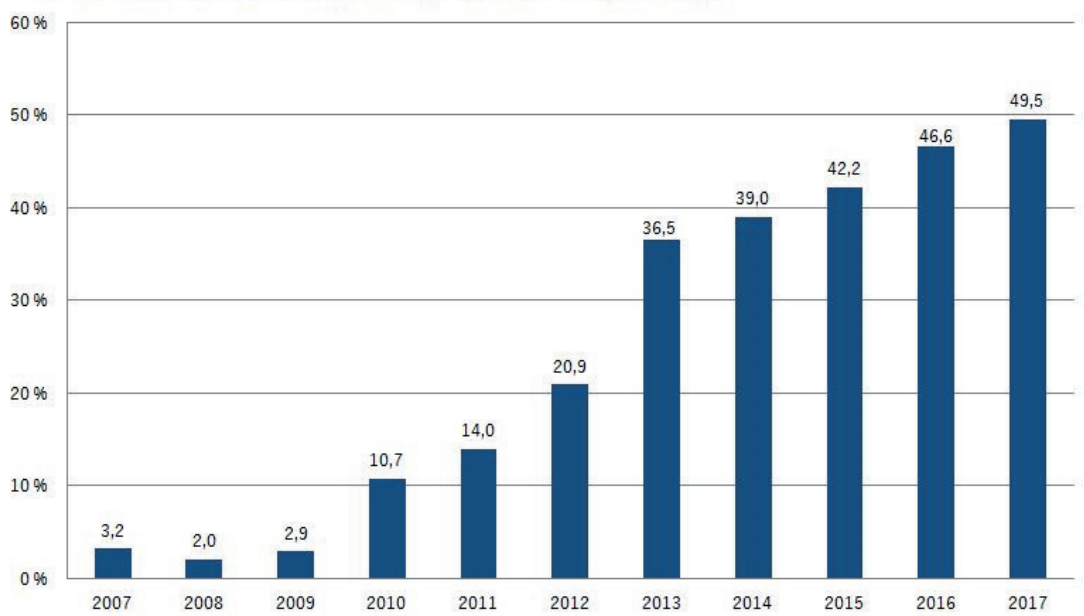


Neben den fiktionalen Formaten und Dokumentationen erstreckt sich Audiodeskription im Ersten inzwischen auch auf prominente Live-Unterhaltungsformate: Der NDR audiodeskribiert die „Eurovision Song Contest“-Abende live sowie die Spielshow „Klein gegen Groß“. Der MDR gestaltet die Verleihung der „Goldenen Henne“ und die „Feste mit Florian Silbereisen“ mit Live-Audiodeskriptionen. Und mit „Verstehen Sie Spaß“ und „Immer wieder sonntags“ ist der SWR 2017 in die Live-Audiodeskription eingestiegen.

Auch die Sport-Audiodeskription gehört mittlerweile zum festen Angebot der ARD: Die sportlichen Live-Großereignisse Fußball-EM 2016, Frauenfußball-EM 2017 sowie Olympische und Paralympische Sommerspiele 2016 waren vollständig audiodeskribiert. Eine umfassende Audiodeskription gab es auch für die Olympischen und Paralympischen Winterspiele 2018.

Die Entwicklung im Ersten hat sich auch positiv auf den Audiodeskriptionsanteil in den Dritten Programmen ausgewirkt, da die Dritten Spielfilme, Reportagen und Dokumentationen aus dem Ersten in ihr Programm übernehmen.

Darüber hinaus haben einige Landesrundfunkanstalten eigene regionale Formate in das Hörfilmangebot aufgenommen: Im Bayerischen Fernsehen werden z. B. die Bayerische Serie am Freitagabend sowie "Dahoam is Dahoam“ in einer Hörfilmfassung angeboten. Auch der MDR hat die Anzahl der Hörfilmneuproduktionen seit 2013 mehr als verdoppelt. Mit der Aufnahme der Reihe "Geschichte Mitteldeutschlands“, einiger Dokumentationen und Unterhaltungssendungen konnte hier vor allem eine Genreerweiterung für Audiodeskription im Programm erreicht werden. Der NDR hat seit 2013 neben den Zulieferungen für Das Erste zahlreiche Sendungen für das NDR-Fernsehen audiodeskribiert, hervorzuheben sind hier die Reihen „Neues aus Büttenwarder“, „Tatortreiniger“ und die „Landpartie“. Der SWR bietet die Mundart-Serie „Die Kirche bleibt im Dorf“ und die Reihe „Lecker aufs Land“ auch in einer Hörfilmfassung an. 


\section{ARD Audiodeskriptionsquote ganzer Sendetag}

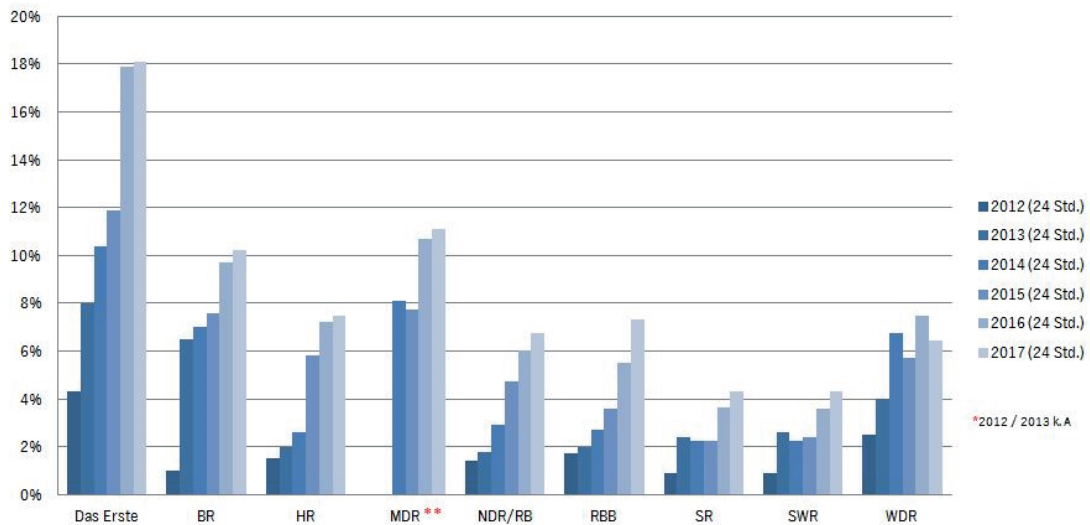

Bei der Erstellung von Hörfilmmanuskripten gibt es in der ARD unterschiedliche Herangehensweisen. Manuskripte können von einem sehenden Autor oder von einem sehenden und einem blinden Autor gemeinsam erstellt werden; seltener wird noch im Dreierteam (zwei sehende und ein blinder Beschreiber) gearbeitet. Von der Anzahl der an der Audiodeskription beteiligten Autoren lassen sich keine Rückschlüsse auf die Qualität des jeweiligen Hörfilms ziehen. Jede Audiodeskription muss für sich sprechen und danach beurteilt werden, ob die geltenden Standards eingehalten werden. Es gibt hervorragende Beschreibungen erfahrener Einzelautoren, die alle Hörfilmrichtlinien vorbildlich erfüllen.

Beim NDR hat sich in den vergangenen Jahren folgendes Prozedere bewährt: Ein Autor erstellt das Manuskript. Gleichzeitig erhält eine blinde Autorin den Originalfilm, um sich auf die Abnahme des fertigen Hörfilmmanuskriptes vorzubereiten. Gemeinsam mit einem NDR Hörfilmredakteur und dem Autor bespricht die blinde Autorin das fertige Manuskript und die drei überarbeiten es für die Aufnahme im Studio. Mit diesem Verfahren konnten Kosten bei der Produktion gesenkt, das Vier-Augen-Prinzip gewahrt und eine professionelle blinde Redakteurin eingebunden werden.

Es ist wünschenswert, dass bei allen Produktionen ein qualifizierter blinder oder sehbehinderter Autor in den Erstellungsprozess einbezogen wird. Er ist der 
erste Hörer und gibt wertvolle Anregungen, denn die Tonspur enthält für blinde Menschen eine Informationsdichte, die für Sehende oftmals nicht unmittelbar nachvollziehbar ist.

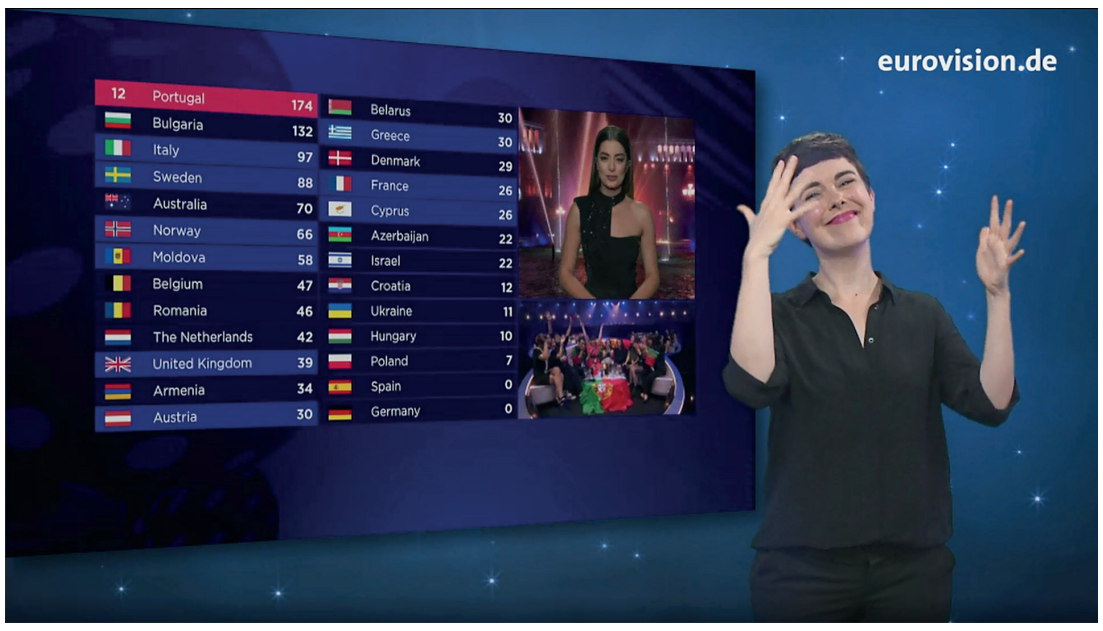

Bei den Gebärdensprachangeboten hat sich die ARD 2013 neben der Tagesschau in Gebärdenfassung auf Phoenix und der Sendung "Sehen statt Hören“ vom Bayerischen Rundfunk einen Ausbau der gebärdeten Sendungen in den Mediatheken zum Ziel gesetzt. Ursprünglich war ein Politmagazin pro Woche vorgesehen, seit 2014 werden zwei Magazine wöchentlich angeboten.

Die aufgeführten Maßnahmen wurden seinerzeit im Einvernehmen mit den Verbänden der Menschen mit Behinderungen beschlossen. Dieser Dialog ist der ARD sehr wichtig, daher finden regelmäßige Treffen insbesondere des NDR als Federführer des Projekts „Barrierefreier Rundfunk“ bei der ARD, aber auch von anderen Sendern mit den Verbänden in ihren jeweiligen Sendegebieten statt.

Untertitel haben eine außerordentlich große und breit gefächerte potentielle Zielgruppe. Denn neben Millionen Schwerhörigen, den Gehörlosen sowie Mig- 
rant(inn)en und jedem anderen, der Deutsch lernt, sind sie auch für alle anderen Menschen eine Option: überall dort, wo der Fernseher ohne Ton läuft oder die Geräuschkulisse es nicht zulässt, dem Fernsehton zu folgen, beim Public Viewing etwa, in Gaststätten oder am Flughafen. Bei der unausweichlichen Priorisierung beim Ausbau der Angebote setzt die ARD angesichts der großen Anzahl der Menschen, die damit erreicht werden und davon profitieren, bei den Untertiteln einen größeren Schwerpunkt als bei den Gebärdensprachangeboten. Auch darüber bestand 2013 Einvernehmen mit den Verbänden, denn die finanziellen Mittel, die der ARD für die Erstellung der barrierefreien Angebote zur Verfügung stehen, sind begrenzt und die Verteilung der Beitragsmittel muss mit größter Sorgfalt und nach klaren Kriterien erfolgen. Dazu zählt auch der Kosten-Nutzen-Faktor. Gleichsam geht die ARD auch konsequent auf die Bedürfnisse numerisch kleinerer Nutzergruppen ein. Folglich sind alle Landesrundfunkanstalten bestrebt, dem Wunsch vieler gehörloser Menschen Rechnung zu tragen und verstärkt additiv Formate in Gebärdensprache anzubieten.

So wird die „Tagesschau“ um 20 Uhr, die Phoenix mit Gebärdensprachdolmetschung sendet, seit Dezember 2012 in die Mediathek des Ersten und der ARD eingestellt. Auch die Politmagazine „Fakt“, „Kontraste“, „Monitor“, „Panorama“, „report Mainz“ und „report München“ werden mit Gebärdenspracheeinblendungen versehen und in dieser Fassung in den Mediatheken on demand bereitgestellt. Seit Jahresbeginn 2018 können Interessierte „Anne Will“, die erfolgreichste politische Gesprächssendung im deutschen Fernsehen, sowie „Hart aber fair" über HbbTV und online live in Gebärdensprache verfolgen.

Einige Landesrundfunkanstalten bieten weitere Sendungen in Gebärdensprache an, z. B. „Sehen statt Hören“ beim BR, die Nachrichtensendungen beim MDR, die Kindernachrichten auf NDR.de, der Wochenzusammenschnitt von „buten un binnen um 6“ in der Mediathek von Radio Bremen oder auch die WDR-Angebote „Bericht aus Brüssel“, „Hier und Heute“, „Sendung mit der Maus“ (die seit Januar 2018 auch in einer Hörfassung und damit nun komplett barrierefrei angeboten wird), „Wissen macht Ah!“ und vieles mehr. 


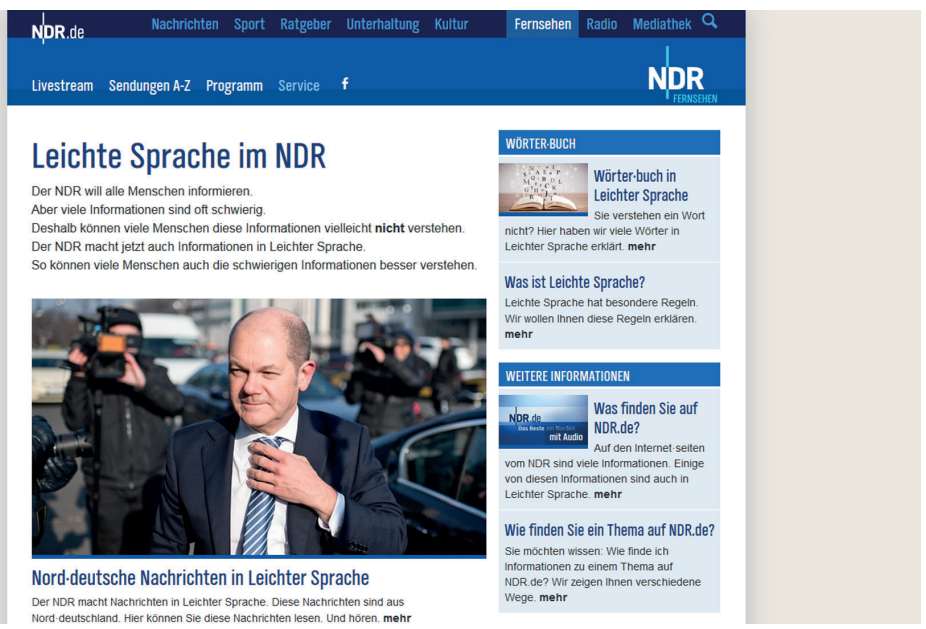

Seit 2015 veröffentlichen die Landesrundfunkanstalten auch Texte in Leichter Sprache. Den Anfang machte der Norddeutsche Rundfunk mit einem Thementag Alphabetisierung: Auf NDR.de wurde ein erstes Informationspaket in Leichter Sprache bereit gestellt. Darin wurde erklärt, weshalb der NDR diesen Thementag macht, das Problem wurde beschrieben und es wurden Hilfsangebote vorgestellt. Gemeinsam mit dem Angebot in Leichter Sprache zum Alphabetisierungstag wurde auch noch ein Serviceangebot publiziert, das den NDR in Leichter Sprache erklärt.

Einen wichtigen Beitrag zur Inklusion der nach Norddeutschland kommenden Flüchtlinge leistet das NDR Onlineangebot „Moin Moin und herzlich willkommen" mit Informationen über das Land auf Deutsch, Englisch und in Leichter Sprache. Es bündelt Links, Adressen und Ansprechpartner zu Themen wie bspw. Sprachkurse oder Wohnungssuche. Außerdem finden sich dort Nachrichten auf Englisch und Arabisch und Kindersendungen mit Untertiteln auf Dari, Arabisch und Kurdisch. Auch dies ist ein Projekt zum Abbau von Barrieren. Leichte Sprache hilft nicht nur den 7,5 Mio. funktionalen Analphabeten in Deutschland. Sie ist für alle, die Deutsch lernen, eine Verständnishilfe. 
Neben Serviceangeboten werden seit Oktober 2015 norddeutsche Nachrichten in Leichter Sprache veröffentlicht. Bis Ende 2017 gab es online ein wöchentliches Nachrichtenformat mit sieben Meldungen aus den Bereichen Politik, Wirtschaft, Kultur, Sport und Vermischtes. Seit Januar 2018 bietet der NDR werktags die Topnachricht aus Norddeutschland in Leichter Sprache an. Es besteht die Möglichkeit, sich die Texte vorlesen zu lassen. Dafür muss lediglich ein auf der jeweiligen Nachrichtenseite angebundenes Audio angeklickt werden. Außerdem gibt es ein stetig wachsendes Onlinewörterbuch.

Auch MDR, RB und WDR bieten auf ihren Websites Informationen oder Nachrichten in Leichter Sprache an. Deutschlandradio und SR veröffentlichen Wochenrückblicke in Einfacher Sprache.

\section{$5 \quad$ Onlineangebote}

Die ARD gestaltet ihre Onlineangebote bereits seit vielen Jahren weitgehend barrierefrei. Die Schrift kann variabel vergrößert werden, die Kontraste sind ausreichend groß, jeder Link ist mit einem Zielverweis gekennzeichnet, visuelle Inhalte (Bilder/Grafiken) sind mit einem beschreibenden Alternativtext versehen und Texte werden in einer Braillezeile wiedergegeben und können so mithilfe einer speziellen Software („screenreader“) vorgelesen werden. In den Mediatheken der ARD wird eine stetig steigende Zahl von Sendungen mit Untertiteln oder als Hörfilm bzw. in Gebärdensprache zum zeitsouveränen Abruf angeboten. In der Das Erste Mediathek stehen nahezu alle Videos zu Sendungen mit Untertitelung zur Verfügung.

\section{Hörfunk}

Barrierefreiheit ist auch den Hörfunkwellen der ARD ein zentrales Anliegen. Das Radio gehört vor allem für blinde und sehbehinderte Menschen zu den wichtigsten Quellen, um sich über das politische und kulturelle Leben zu informieren. Der zeitlich unabhängige Zugriff auf gesendete Hörbeiträge ist für Menschen mit einer Sehschädigung bedeutender Bestandteil ihrer Kommunikation 
mit der Umwelt. Der MDR z. B. bündelt sämtliche Livestreams der MDR-Radioprogramme und der ausschließlich im Internet verbreiteten Hörfunk-Channels in „MDR Audio - Das inklusive Hörangebot“. Darüber hinaus macht die MDRApp „On Demand“ Audios, Podcasts und Tonspuren von Sendungen mit Audiodeskription Blinden, Sehbehinderten und Sehenden gleichermaßen zugänglich. Aktuelle Nachrichten, Wetterinformationen und Verkehrshinweise - auf Wunsch regionalisiert - ergänzen das Angebot.

\section{$7 \quad$ Ausblick}

Der Ausbau der Barrierefreiheit von ARD-Angeboten wird seit Jahren vorangetrieben, und der Prozess ist noch nicht abgeschlossen. An einigen Stellen sind auch Defizite zu benennen: Insbesondere im Bereich der mobilen Endgeräte ist es trotz aller Bemühungen noch nicht gelungen, die öffentlich-rechtlichen Angebote komplett barrierefrei auszugestalten. Dies hängt auch mit externen Faktoren zusammen, z. B. ob das entsprechende Endgerät einen untertitelfähigen Player zur Verfügung stellt oder nicht. In diesem Bereich wird aktiv nach Lösungswegen gesucht. Beim Rundfunk Berlin-Brandenburg z. B. widmen sich die „rbb-Innovationsprojekte“ eingehend der Frage, wie Barrierefreiheit des Fernsehens mit digitalen Technologien noch weiter verbessert werden kann.

Bei der Planung der weiteren Maßnahmen orientiert sich die ARD stark an den Interessen und Bedürfnissen der Zielgruppen und diskutiert entsprechende Schwerpunktsetzungen kontinuierlich mit Verbänden der Menschen mit Behinderungen.

Der Ausbau des barrierefreien Zugangs ist ein kontinuierlicher Prozess, bei dem der NDR und die ARD in vielen Bereichen bereits einen großen Schritt vorangekommen sind. Das wird uns in Gesprächen mit den Verbänden auch regelmäßig bestätigt. Wir wollen den Weg weitergehen und arbeiten konsequent am Abbau noch bestehender Zugangshindernisse, um möglichst vielen Menschen eine Teilhabe zu ermöglichen. 



\author{
LAURA M. SCHWENGBER
}

\title{
Barrierefreiheit bei Kulturevents und Live-Veranstaltungen
}

\section{$1 \quad$ Ein Plädoyer für Zusammenarbeit}

Kultur- und Liveevents stellen die Barrierefreiheit vor besondere Herausforderungen. Nicht nur, dass „live“ das übliche Risiko trägt, dass Beteiligte (und eigentlich Unbeteiligte) etwas völlig ander(e)s tun, als es abgesprochen war, es sind in der Regel auch Künstler(innen) unter den Beteiligten. Künstlerinnen haben oft besondere Anforderungen beispielsweise an Bühnen: Das Licht soll bestimmte Farben abdecken, eine bestimmte Anzahl an Beleuchtungen soll gehängt sein, das Essen ist für eine bestimmte Uhrzeit eingeplant oder der Bühnenbelag muss einen bestimmten Grip haben, um bestimmte Tanzschritte ausführen zu können oder dem Schlagzeuger ausreichend Halt zu geben. Man könnte das als unangenehmes, divenhaftes Verhalten abtun, und manche Vertretenden der Zunft mögen auch eher außergewöhnliche Wünsche haben, tatsächlich tragen alle diese Kleinigkeiten aber dazu bei, dass sich Bühnenarbeitende wohl(er) fühlen, ihren Job machen können, dabei gesund bleiben und den Zuschauenden, wie bestellt, eine gute Show liefern. In der Branche selbst sind diese Absprachen völlig selbstverständlich. Natürlich gibt es eine Dispo, in der genau steht, wer wann eintrifft, wer wo isst und bis wann der Laden geräumt sein muss. Doch während der Mitarbeitende im Büro jedes Verständnis für seinen ergonomischen Schreibtischstuhl bekommt, stoßen Wünsche von Künstlerinnen oft auf Unverständnis bei Außenstehenden. Von außen mag das umständlich wirken, vor Ort erleichtert es die Zusammenarbeit zwischen den Gewerken enorm und ist innerhalb der Branche selbstverständlich.

Auf ähnliches Unverständnis wie das von Laien gegenüber den internen Abläufen auf Großveranstaltungen trifft man, wenn es um die Bedingungen der Barrierefreiheit bei Kulturveranstaltungen geht. Die Gewerke vor Ort sind Expertinnen in ihrem Gebiet. Sie sorgen seit Jahren für das optimale Licht, wissen, 
welche Box wann anfängt zu brummen und welches Kabel wie lang ist. Geht es nun aber um barrierefreie Veranstaltungen, erlebe ich sehr unterschiedliche Reaktionen. Einige davon möchte ich hier exemplarisch darstellen und daraus ableiten, wie Barrierefreiheit bei Kulturevents und Live-Veranstaltungen realisiert werden kann. Dabei lässt sich aus Erfahrung sagen, das dies immer dann besonders gut gelingt, wenn sich alle gegenseitig als Expert(inn)en für ihren Beitrag zum Event wertschätzten.

\section{2}

\section{„Da wird nichts Wichtiges gesagt“ - Licht}

Thema Licht. Der Lichtmeister und Autor dieses Satzes kannte das Theaterstück in- und auswendig. Er kannte jeden Satz, jede Szene und wenn ein Schauspielender ausgefallen wäre, hätte er vermutlich ohne Probleme umgehend einspringen können. Er war ein begnadet guter Lichtmeister: Die Beleuchtung war auf den Punkt und man hätte sich nichts anderes für dieses Stück vorstellen wollen. Ein Geschenk! Und Ergebnis jahrelanger Arbeit. Mit Gebärdensprachdolmetschenden hatte er in diesen Jahren jedoch nie zu tun gehabt. Es habe da wohl mal so ein paar Leute gegeben, die hätten das dann aber mit so Hörgeräten gemacht, das sei spannend gewesen, habe ihn aber nicht weiter beeinflusst. Schwerhörige Menschen kannte er also. Wunderbar! Wenn Sie Vorerfahrungen haben, und sei es mit ihrer altersschwerhörigen Oma, ihrem Nachbarn mit der Gehhilfe oder der blinden Frau, die manchmal vor Ihnen an der Kasse steht, dann nutzen Sie diese! Wenig ist wertvoller als der direkte Kontakt und Ihre eigene Erfahrung, wenn es um Menschen (mit Behinderung) geht. Denn während für eine Rampe über der Stufe am Eingang noch relativ viel Verständnis aufgebracht werden kann, ist das extra Licht für Gebärdensprachdolmetschende eben immer noch oft ein diskutabler Punkt. „An der Stelle wird eh nichts Wichtiges gesagt." Wie oft sagen Sie das zu Ihrer Oma und ab wann schaltet sie ab, wird ungehalten, erzählt dann eben weiter oder etwas völlig anderes? Sie wissen also eigentlich schon, dass dieses Vorgehen gewisse Tücken birgt und es anders vermutlich besser gehen könnte. Wir, als normgerecht hörende Menschen, entscheiden während des Zuhörens, was uns wichtig erscheint, und was nicht. 
Überlassen wir diese Entscheidung und Verantwortung doch ebenso tauben und schwerhörigen Menschen, indem wir zunächst alles zugänglich machen.

Für die Barrierefreiheit auf Kulturevents bedeutet das, dass grundsätzlich alles wichtig ist und die Gebärdensprachdolmetschenden beleuchtet sein müssen, sobald akustische Informationen übermittelt werden. Das Licht darf durchaus mal blau sein oder gedimmt werden, wenn es sonst auf der Bühne völlig dunkel ist. Sprechen Sie das mit Ihren Expertinnen vor Ort ab. Seien Sie mutig und erweitern Sie dieses Konzept auf Menschen mit Sehbeeinträchtigungen oder Menschen im Rollstuhl auf einer Stehparty: „Da sieht man nichts Wichtiges“.

\section{3 „Wir sagen da wirklich keine komplizierten Sachen“- Vorbereitungsmaterial}

In der Regel werden Dolmetschende und Übersetzende Sie im Vorfeld nach Vorbereitungsmaterial fragen. Wir brauchen das, um Ihnen den optimalen Service zu bieten, für den Sie uns buchen und zahlen und auf den Ihre Teilnehmenden einen Anspruch haben. „Ich dachte, Sie haben das studiert?“ ist einer meiner Lieblingssätze zu diesem Thema. Für Außenstehende mag die Frage nach Vorbereitungsmaterial ggf. komisch wirken, schließlich hören Dolmetschende doch, was gesagt wird, können daraus simultan dolmetschen und müssen doch einfach nur wiederholen, was bereits gesagt wurde (ja gut, eben in einer anderen Sprache). Und dann haben sie das auch noch studiert. Oder nicht? Lassen Sie mich an einem typischen Beispiel aus meinem Berufsalltag zeigen, wie schon Kleinigkeiten zu einem Problem werden können:

Sitzung zur Vorbereitung eines Konzertes, ich dolmetsche für den tauben Beratenden. Es fallen Namen von den Mitarbeitenden, die Gewerke werden benannt, immer wieder geht es um Herrn Müller, der nicht anwesend ist, aber des Öfteren im Zusammenhang mit ausstehenden Aufgaben genannt wird. „Macht Müller" ist eine häufige Phrase, die ich an diesem Tag gebärde. Um nicht immer wieder „Herr Müller“ zu wiederholen, platziere ich ihn im Gebärdenraum vor mir, indem ich einmal HERR MÜLLER gebärde und dann auf einen Punkt vor mir zeige. Zeige ich später wieder auf diesen Punkt, wird mein Gegenüber wissen, wer gemeint ist, ebenso als wenn wir in der Lautsprache „er“ sagen und uns 
auf den vorherigen Satz rückbeziehen. Später im Gespräch ist dann noch öfter die Rede vom Stagemanager, der ebenfalls noch viele Aufgaben zu haben scheint. Da auch er öfter benannt wird, bekommt auch er einen Punkt im Raum, einen anderen als Herr Müller, natürlich. Als also nun alle Aufgaben verteilt sind, fällt dem tauben Beratenden auf, dass jeweils Müller und der Stagemanager verhältnismäßig wenige Aufgaben haben. Was sich jedoch später herausstellt, ist, dass beide Männer ein und dieselbe Person sind und durch meine fehlenden Informationen daraus zwei Personen geworden sind. Das ist ausgesprochen unglücklich, jedoch leicht zu vermeiden: Geben Sie uns vorher einfach Auskunft, wenn wir nachfragen. Wir werden Ihnen genau sagen, was wir benötigen. Veranstalten Sie Kulturevents, sind das z. B. Setlisten von Bands, Songtexte und die dazugehörige Musik, Redeskripte, sofern vorhanden, Namen und Funktion von Beteiligten (z. B. Herrn Müller, dem Stagemanager), Fachwörter, Eigennamen und ggf. branchenübliche Fremdwörter. Wir werden Ihnen mitteilen, was wir brauchen.

\section{$4 \quad$ „Und wie viele kommen dann?“ - Werbung und Erwartung}

Eine Erwartung, der ich oft begegne, ist die, dass im Publikum dann hunderte und aberhunderte Menschen mit Behinderung zu finden sind, wenn man eine Rampe an den Eingang stellt. Damit verbunden ist entweder große Sorge („Kommen dann die Normalen noch?“), ein großer Anspruch („Wenn man schon mal was für die macht, muss sich das aber auch lohnen.") und fast immer große Unsicherheit („Brauche ich dann mehr Security oder so?“). Ich bin als Gebärdensprachdolmetscherin seit mehreren Jahren auf den Konzertbühnen Deutschlands unterwegs. Als ich 2011 damit begann, war das in Deutschland fast gänzlich unüblich und es galt jede Menge Pionierarbeit zu leisten. Gleich mein erstes Konzert begann in der Vorbereitung mit der Ansage des Veranstalters, „Schöne Idee, aber nicht bei mir, nicht bei dem Konzert.“ Glücklicherweise stand die Band damals fest hinter der Idee und wollte das unbedingt umsetzen, so dass wir gemeinsam den Veranstaltenden überzeugten sich auf ein „na, dann macht es halt - aber sagt es vorher keinem!“ einzulassen. Ich drehte also ein Video in Gebärdensprache - ohne Ton, ohne Untertitel, ohne Übersetzung - zu 
dem Konzert und lud die taube Community ein. Auf dem Konzert selbst war später eine wunderbare Stimmung, der Abend war für Publikum, Band und Veranstalter ganz zauberhaft und im Jahr darauf bildeten meine Hände den Namen der Band und „Laura M. Schwengber übersetzt Text und Musik in Gebärdensprache" stand ebenso groß auf dem Plakat wie der Name des Gastmusikers. Durch das Erleben des ersten Konzertes hatte ein wunderbarer Wandel stattgefunden. Das Jahr darauf war dann unheimlich unkompliziert.

Lassen Sie uns einen Schritt zurück wagen: Woher wissen Menschen mit Behinderung überhaupt, dass Ihr Event barrierefrei oder barrierearm sein wird? Richtig, aus Ihrer Werbung. Leider ist es nach wie vor nicht selbstverständlich, dass alle Menschen überall Zugang haben und überall willkommen sind. Das gilt nicht nur für Menschen mit Behinderungen. Wenn Ihre Veranstaltung auf bestimmte Bedarfe Rücksicht nimmt, dann ist das großartig und darf angekündigt werden. Sie müssen deshalb kein „Rollstuhlgerechtes Rock im Irgendwo“ veranstalten, aber Sie können beispielsweise gängige Symbole (Rollstuhlfahrende Person, durchgestrichenes Ohr, gebärdende Hände) nutzen, die sie auf Ihre Plakate drucken und auf die Website setzen. Informieren Sie in einem eigenen Menüpunkt am besten über die konkreten Angebote, die Sie machen: Gibt es Gebärdensprachdolmetschende? Wo ist der Eingang für Rollstuhlnutzende? Wie viele barrierefreie Toiletten gibt es? Geben Sie Nachlass für die Begleitperson? Gibt es eine T-Schleife für Hörgerätenutzende? Sie veranstalten überregional? Großartig! Verraten Sie Ihren Besuchenden das nächste barrierefreie Hotel oder Restaurant. Neben den konkreten Hinweisen und den Symbolen sollten Sie außerdem weitere Verbreitungswege nutzen. Schließlich erweitern Sie Ihre Zielgruppe, gönnen Sie sich also auch mehr Kanäle. Googlen Sie Ihre örtlichen Behindertenverbände und informieren Sie diese über Ihr Angebot. Diese haben entweder eigene Verteiler oder nennen Ihnen gute Ansprechpartnerinnen in Ihrer Region. Nutzen Sie diese Kanäle als zusätzliche Ressource, ersetzen Sie damit nicht Ihre übliche Werbung. Sie erreichen viele Menschen mit Behinderungen auch über ganz normale Kanäle: Behinderte Menschen sind auch in den Sozialen Netzwerken oder in der Stadt unterwegs und sehen Ihre Plakatwerbung. 
Wenn Sie gute Angebote und gute Werbung machen, dann läuft der Rest im Prinzip wie üblich: Leute kommen, oder auch nicht. Ein Rezept für die garantiert ausgebuchte Veranstaltung gibt es auch in diesem Bereich nicht. Aber mit barrierearmen Angeboten schaffen Sie die erste und wichtigste Voraussetzung dafür: einen Zugang.

\section{5 „Wir haben nie Rollstuhlfahrende hier, wir brauchen keine Rampe“ - Angebot und Nachfrage}

Es kommen auch keine tauben Menschen, deshalb brauchen Sie keine Gebärdensprachdolmetschenden? Merken wir selber, oder? Es ist natürlich so, dass Menschen, die einen besonderen Bedarf haben, sich vorab bei Ihnen melden und um dieses oder jenes bitten. Gibt es Stufen am Eingang? Gibt es ein laktosefreies Angebot beim Mittagessen? Kann ich meinen Koffer bei Ihnen abstellen? Kann ich meine Fünfjährige mitnehmen? Viele dieser Dinge werden heute selbstverständlich(er) mitgedacht. Denken Sie nur an die Funktion auf Facebook, eine Veranstaltung zu erstellen: Mit einem Klick geben Sie dort an, ob die Veranstaltung kinderfreundlich ist. Nutzen Sie Ihre Kanäle und kommunizieren Sie Ihre barrierefreien Angebote gleich mit. Sind Sie sich unsicher, was Sie eigentlich haben, dann nutzen Sie zum Beispiel online-Checklisten. Geben Sie an, so viel Sie können, und signalisieren Sie ansonsten Offenheit: „Wir haben keine Stufen. Wenn Sie weitere Fragen zur Zugänglichkeit unserer Räume haben, stehen wir Ihnen gern zur Verfügung." Großartig. Und so einfach. Das ist Ihnen zu halbgar, Sie wollen es konkret wissen? Auch gut! Holen Sie sich Expertinnen ins Team und denken Sie die Barrierefreiheit von Anfang an konkret mit. Es gibt einen Bühnenbauer, der Ihnen die Rampe, die er selbst zum Aufbau nutzt, stehen lassen kann und die eine gute Steigung hat? Buchen Sie doch gleich den. Es gibt einen Ort mit ausreichend Türbreite und sonst ähnlichen Konditionen wie die Veranstaltungsräume auf Ihrer Shortlist: Keine Frage, was Sie buchen sollten. Schaffen Sie Zugang, signalisieren Sie Offenheit, nutzen Sie Beratung und die Erfahrung Ihrer Kolleginnen (so wie es auch in anderen professionellen Bereichen üblich und typisch für eine sich ausdifferenzierende Gesellschaft ist). 


\section{$6 \quad$ Ein Wort an die Leute auf der Bühne}

Liebe Musikschaffende, Künstlerinnen und Bühnenarbeitende, ihr seid gebucht auf eine barrierefreie Veranstaltung? Herzlichen Glückwunsch! Danke, dass ihr Euch darauf einlasst. In den ruhigeren Minuten im Backstage haben mir viele Eurer KollegInnen erzählt, welche Gedanken sie sich vor einem Konzert mit Gebärdensprachdolmetschenden auf der Bühne oder mit behinderten Menschen im Publikum gemacht haben. An dieser Stelle ein herzliches Dankeschön an alle, die ihre Erfahrungen mit mir geteilt haben.

Viele berichteten mir davon, dass sie das größte Problem darin sahen, dass sie kaum jemanden kennen, der schon einmal Gebärdensprachdolmetschende auf Konzerten gesehen hat - geschweige denn, mit ihnen gearbeitet. Reagiert das Publikum irgendwie anders? Teile ich mir die Aufmerksamkeit aller mit der dolmetschenden Person auf der Bühne? Werden wir abgelenkt sein? Oder werden die hörenden Menschen sich „wie immer" verhalten und ein Teil des Publikums (die vermutlich Tauben) ganz anders? Können die Dolmetschenden wirklich alles übersetzen? Und machen die das auch? Und eigentlich habe ich auch Fragen, die manche vielleicht als politisch nicht ganz korrekt ansehen würden. Aber die hätte ich eigentlich auch gern beantwortet. Wen kann ich denn sowas fragen?

Die daraus entstehende Unsicherheit wurde oft noch verstärkt, wenn die Barrierefreiheit ausdrücklich angekündigt wird und dadurch zusätzliche Erwartungen entstehen. In der Einleitung zu diesem Kapitel habe ich ausgeführt, dass barrierefreie Veranstaltungen oft dann am besten gelingen, wenn sich alle Beteiligten als Expertinnen für ihre Sache respektieren. Wenn ihr Fragen habt, dann stellt sie uns. Dazu sind wir da. Habt keine Angst vor den vermeintlich unfragbaren Themen: Wir sind es gewohnt, dass Menschen, die diese Fragen viel weniger reflektiert haben als ihr, uns ständig alles Mögliche fragen. Und diese Fragen zu beantworten ist Teil unseres Jobs. Das machen wir gern. 


\section{Reagiert das Publikum irgendwie anders?}

Wenn eine Gebärdensprachdolmetscherin auf der Bühne steht? Ja, vermutlich. Schon allein deshalb, weil dort eben eine weitere Person auf der Bühne steht. $\mathrm{Zu}$ Beginn entsteht oft etwas neugierige Irritation und es ist sinnvoll, nach dem 1., 2. oder 3. Titel kurz ein Wort dazu zu sagen, was die Gebärdensprachdolmetschenden da eigentlich tun. Und das gerne so entspannt, wie sonst auch: „Das ist Max am Schlagzeug! Tina am Bass! Und Laura in der Gebärdensprache!“ Zack, fertig. Menschen mit Behinderung sind genauso vielfältig wie alle anderen Menschen auch. Klar, kann sein, dass einer anders tanzt, als es vermeintlich üblich ist. Eine lauter ruft als die anderen. Oder anders. Alles möglich. Es kann aber auch alles sehr üblich ablaufen. Erlaubt euch, auf Rufe, tanzende Menschen und alles, was sonst noch so passieren kann, einzugehen, wie sonst auch. Ihr macht Musik und euer Publikum möchte dazu tanzen. Offenbar kommt eure Musik gut an. Es ist also ok, sich einfach darüber zu freuen.

Werden die hörenden Menschen sich „wie immer“verhalten und ein Teil des Publikums (die vermutlich Tauben) ganz anders?

Jein: Alle Menschen im Publikum, die sehen können, werden auf das zusätzliche visuelle Angebot in irgendeiner Weise reagieren. Unser Job ist es, eine gute gemeinsame Show zu liefern - dann werden wir ein gemeinsames Publikum erzeugen und noch bevor wir die erste Flasche ausgetrunken haben, werden wir kaum noch unterscheiden können, wer hier eigentlich als hörend oder taub den Saal betreten hat. Mir ist es zum Beispiel schon oft passiert, dass auch die Hörenden nach wenigen Titeln beginnen, mit den Händen in der Luft zu wedeln. Das ist die Gebärde für Applaus und taube Menschen applaudieren so, weil das visuelle Hände wackeln über den Köpfen der Leute eindrucksvoller ist, als das akustische in die Hände klatschen. Viele Hörende finden es spannend, das einfach nachzumachen, und so passiert es oft, dass der Applaus verhältnismäßig still ist - dafür aber visuell umso überzeugender. Freut euch drauf! 
Teile ich mir die Aufmerksamkeit aller mit der dolmetschenden Person auf der Bühne?

Betrachtet uns als Gastmusikanten. Wir spielen ein zusätzliches Instrument und tragen zu Eurer grandiosen Bühnenshow einen weiteren Aspekt bei. Ihr seid die Hauptakteure, bezieht uns gern mit ein: Positionswechsel, gemeinsames Luftgitarre spielen, Publikum zum Klatschen animieren - alles gern! Wir beziehen euch anders herum auch gern ein: Gebärdet die Schlagwörter eures Lieblingsrefrains gern mit, wir zeigen euch, wie. Das sieht am Ende immer großartig aus. Damit ist auch schon die Frage beantwortet, ob die Hörenden sich „wie immer" verhalten werden und nur das taube Publikum sich ganz anders verhalten wird: Im besten Falle können wir nach kürzester Zeit nicht mehr unterscheiden, wer zu welcher Gruppe gehört.

\section{Werden wir selbst abgelenkt sein?}

Ja, vielleicht. Wir machen gern eine gemeinsame Anspielprobe und einen Soundcheck mit euch. Schaut uns zu, checkt einfach einen Moment länger, wenn ihr euch noch daran gewöhnen wollt, und lasst uns einen Teil filmen, so dass ihr nicht während der Show das Gefühl habt, dass ihr das eigentlich auch gern sehen würdet, ständig zu uns schaut und dadurch abgelenkt seid.

Können die Dolmetschenden wirklich alles übersetzen? Und machen die das auch?

Ja, das können wir, sonst sagen wir euch vorher Bescheid und finden mit euch eine Lösung. Und ob wir das auch machen: Ich sag mal, ja :-)

Und eigentlich habe ich auch Fragen, die manche vielleicht als politisch nicht ganz korrekt ansehen würden. Aber die hätte ich eigentlich auch gern beantwortet. Wen kann ich denn sowas fragen?

Auch uns. Niemand kennt alle Begrifflichkeiten, weiß alle Feinheiten und kennt alle Befindlichkeiten. Nutzt unser Expertinnentum. Ihr habt euch auf die Veranstaltung eingelassen - ihr habt jedes Recht auf Informationen und Beantwortung Eurer Fragen und Beseitigung Eurer Unsicherheiten. Wir werden anders 
herum auch ab und zu liebevoll die Gitarre mit dem Bass verwechseln und vielleicht auch den Tontechniker bitten, uns einen schönen Mix zu machen, anstatt selbst den vollen Soundcheck mitzumachen.

\section{$8 \quad$ Zum Schluss eine Bitte}

Zuletzt erlaube ich mir noch einen Wunsch zu äußern: Bitte geht offen mit eurem Feedback um. Ihr seid hier Teil einer in Deutschland neuen Entwicklung und es wäre unheimlich schade, wenn eure Erfahrungen nicht in die weitere Entwicklung einbezogen werden könnten, einfach weil ihr sie für euch behaltet. Sprecht mit uns darüber, mit euren Kolleg(inn)en und gern auch mit allen, die euch danach fragen. Zusammen können wir etwas wunderbares Neues für uns alle schaffen. Danke! 
DANA APEL/JÖRG APEL

„Die Gesellschaft soll uns so akzeptieren, wie wir sind“

(aufgezeichnet von Loraine Keller)

Dana und Jörg Apel sind verheiratet und haben zwei Kinder. Sie sind, wie auch ihre Kinder, gehörlos. Beide unterrichten Gebärdensprache: Jörg Apel gibt Kurse als selbstständiger Dozent für Deutsche Gebärdensprache (DGS) und unterrichtet z. B. an der Universität Hildesheim, an der Hartwig-Claußen-Schule Hannover und in verschiedenen Unternehmen. Dana Apel ist pädagogische Mitarbeiterin am Landesbildungszentrum für Hörgeschädigte in Hildesheim; sie unterstützt gehörlose Schüler(innen) beim Lesen von Texten und erklärt die Unterrichtsinhalte in Gebärdensprache. Sie gibt z. B. auch Lehrer(inne)n und Erzieher(inne)n Unterricht in Deutscher Gebärdensprache.

Dana Apel: Ich bin im Alter von sechs Jahren mit meiner Familie aus der Tschechoslowakei nach Westberlin geflohen. Meine Eltern sind auch gehörlos. Daher habe ich zuerst die tschechische Gebärdensprache gelernt. In Berlin bin ich dann mit der Deutschen Gebärdensprache in Kontakt gekommen: In der Schule und im Kontakt mit meinen Kindheitsfreunden habe ich die Deutsche Gebärdensprache schnell erlernt. Zu Hause haben wir mit einer Mischung aus deutschen und tschechischen Gebärden kommuniziert.

Früher stand die Lautsprache im Zentrum des Unterrichts und es wurde nach der sogenannten oralen Methode unterrichtet. Gebärdensprache durfte in der Schule nicht benutzt werden. Wir mussten die Hände unter dem Tisch halten, damit wir nicht „plaudern“ konnten. In der Schule wurden wir Kinder dazu erzogen, sprechen zu üben und von den Lippen abzusehen. Wir mussten uns in 
der Schule zum Beispiel mit dem Lehrer vor den Spiegel setzen und mit dem Mund die verschiedenen Laute bilden. Wir mussten stundenlang Sätze sprechen üben. Die Unterrichtsinhalte sind daher oft zu kurz gekommen. Das Lippenlesen ist aber sehr anstrengend und es dauert lange, bis man eine gewisse Kompetenz erworben hat. Zudem kann man nur circa ein Drittel der Laute überhaupt absehen und man braucht den Kontext, um das Mundbild interpretieren zu können. Das war eine andere Zeit und die Momente zu Hause - wo immer gebärdet wurde - waren wertvoll. Erst als die Deutsche Gebärdensprache 2002 als eigenständige Sprache anerkannt wurde, hat sich für gehörlose Menschen, auch für mich, gesellschaftlich etwas verändert.

Jörg Apel: Auch bei uns zu Hause war die Situation besonders, denn meine Eltern sind hörend. Aber sie haben sich auf mich eingestellt und haben sogar mit mir zusammen ganz eigene Gebärden erfunden, um gut kommunizieren zu können. Diese Hausgebärden haben wir benutzt, bis ich mit der Zeit immer sicherer in der Deutschen Gebärdensprache wurde und eigene Zusammenhänge herstellen konnte. Die DGS hat sich bei mir vor allem im Kontakt mit anderen Gehörlosen gefestigt.

Ich wurde in Lemshausen bei Göttingen geboren, wo auch meine Verwandtschaft lebt. Es gab jedoch dort keine Gehörlosenschule. Meine Eltern haben sich unter den vier Gehörlosenschulen in Niedersachsen dann für die nächstliegende entschieden: Die Familie zog nach Hildesheim, damit ich die Schule dort besuchen konnte, denn meine Eltern wollten nicht, dass ich in ein Internat muss. Manchmal haben die Lehrerinnen oder Lehrer heimlich gebärdet oder lautsprachbegleitende Gebärden benutzt, um bestimmte Sachverhalte besser erklären zu können. Das hat mir damals sehr geholfen. Die Deutsche Schriftsprache wurde in der Schule im Deutschunterricht gelernt. Dies fällt Gehörlosen jedoch besonders schwer, weil die Grammatik der Deutschen Schriftsprache stark von der der Gebärdensprache abweicht. 


\section{„Früher mussten die Gehörlosen viel einstecken“}

Wenn man die beiden nach ihren Erfahrungen mit Hindernissen im Alltag fragt, ziehen sie eine positive Bilanz im Laufe der Zeit: Früher war es sehr mühsam, wenn man als Gehörloser zum Beispiel aufs Amt musste. Wollte man Papiere beantragen und musste dafür Formulare ausfüllen, so musste man viel Geduld und Zeit mitbringen, um sich verständlich machen zu können. Die Apels mussten dann versuchen zu sprechen, um ihre Belange in so einer Situation durchsetzen zu können. Sie waren sehr geduldig, um den Hörenden mitzuteilen, was sie brauchten. Leider ist es in solchen Situationen trotzdem zu vielen Missverständnissen gekommen, dadurch, dass sie nicht verstanden worden sind. So mussten sie oft sehr viel einstecken, selbst wenn die Hörenden schon viel Akzeptanz mitbrachten und versucht haben, sich auf sie einzustellen und langsam zu sprechen.

Früher gab es noch keine Dolmetscher(innen). Damals haben oftmals noch die Lehrer(innen) aus der Schule und die Familienangehörigen die Gehörlosen bei Arztbesuchen oder Behördengängen begleitet und versucht zu übersetzen. Sie haben bei der Kommunikation und Erledigung von wichtigen Dingen oft geholfen, aber es gab auch Bevormundung und Missverständnisse. Heute ist die Situation nach den Erfahrungen der Apels zum Glück nicht mehr so: Es gibt viel mehr professionelle Dolmetscher(innen) und es gibt mehr Bewusstsein für die Belange von Gehörlosen.

Das Ehepaar Apel berichtet, dass es früher auf der Arbeit gängig war, dass die Gehörlosen von der Kommunikation ausgeschlossen waren. Alle anderen kommunizierten über Lautsprache und die Gehörlosen saßen daneben, haben nichts verstanden und so hatten sie auch in den Pausen das Gefühl, ausgeschlossen zu sein. Deswegen ist die Gemeinschaft für Gehörlose auch so wichtig. Der Austausch miteinander ist essentiell. Das bedeutet, dass sie oft auch heute noch unter sich bleiben wollen. 


\section{Kommunikation als Barriere}

Dana und Jörg Apel: Die Kommunikation ist der Schlüssel und gleichzeitig das Problem. Alle anderen, ob Rollstuhlfahrer, Autisten oder Blinde, können nicht nur miteinander, sondern auch untereinander kommunizieren. Wir Gehörlosen können das nicht und das trennt uns von der Gesellschaft: Die Kommunikation wird zur Barriere. In der Schnelllebigkeit der heutigen Zeit bekommen wir Gehörlosen oft nicht alle Informationen mit. Vieles ändert sich heute zu schnell, sodass Gehörlose befürchten, den Anschluss zu verlieren. Viele Texte sind zu kompliziert, zu schwer. Da hilft uns die Leichte Sprache schon sehr. Zwar haben wir noch keine Formulare oder andere Texte in Leichter Sprache auf einem unserer Behördengänge gesehen, aber zumindest gab es beim Finanzamt einmal ein Formular mit weniger Text in einfacher Sprache. Gehörlose brauchen aber insgesamt mehr Informationen. Heutzutage passieren immer wieder so viele Neuerungen, dass wir Angst haben, dass die Gehörlosengemeinschaft dabei ein bisschen zurückbleibt.

\section{Barrieren im Alltag}

Barrieren im Alltag können Jörg und Dana Apel einige nennen: Es ist zum Beispiel oftmals schwer, mit Hörenden Blickkontakt aufzunehmen, wenn diese zunächst nicht bemerken, dass sie es mit einem/einer Gehörlosen zu tun haben und nicht einfach mit ihnen sprechen können.

Dana Apel: Das habe ich schon mehrmals am Schalter eines Verkehrsunternehmens erlebt. Wenn der Mitarbeiter oder die Mitarbeiterin mich nicht ansieht, wenn ich eine Fahrkarte kaufen möchte, dann kostet es sehr viel Zeit und Geduld, bis ich mich verständlich machen und mein Anliegen vortragen kann. Der andere denkt, ich höre ihn und wendet sich mir nicht zu. In Zukunft wird es vielleicht noch schlimmer, weil alle nur noch auf ihr Smartphone blicken. 
Eine weitere Barriere für Gehörlose ist, dass sie selten ohne Vorplanung etwas erledigen können, wenn für die Kommunikation ein(e) Dolmetscher(in) benötigt wird. Meist dauert es mehrere Wochen, bis man diese(n) zum Beispiel für die Unterstützung beim Arbeitsamt oder einen Arztbesuch überhaupt beanspruchen kann. Dabei muss man vielleicht sehr zeitnah einen Termin wahrnehmen. Damit verbunden ist auch die Barriere Telefon: Es gibt zwar einen Telefondienst, der per Webcam und mit Hilfe eines Dolmetschers/einer Dolmetscherin funktioniert, dieser kostet aber zusätzliches Geld - neben der grundsätzlichen Pauschale für den Dienst bezahlt man auch jede Minute und man ist schon wieder auf fremde Hilfe angewiesen. Selbst Anrufe erledigen können Gehörlose nur per Webcam und mit einem/einer Gesprächspartner(in), der/die die Gebärden verstehen und darauf in irgendeiner Weise reagieren oder antworten kann.

Dana Apel: Das Problem des Telefonierens reicht sogar bis ins Internet: Ich habe es schon häufig erlebt, dass bei Bestellungen im Internet eine Telefonnummer als Pflichtangabe gefordert wurde. Wir haben aber keine Telefonnummer. Wenn daran dann die Bestellung scheitert, ist das schon sehr ärgerlich. In solchen Fällen habe ich schon mehrfach die Unternehmen angeschrieben und darum gebeten, diese Pflichtangabe herauszunehmen. Manche lassen sich darauf ein, andere reagieren gar nicht. Eine Faxnummer wäre viel einfacher, aber da dieses Medium mittlerweile veraltet und nicht mehr gebräuchlich ist, besteht diese Option kaum noch. Auch bei Gewinnspielen gibt es dieses Problem. Ich habe die Lösung eines Rätsels gefunden und möchte mich am Preisausschreiben beteiligen, muss aber bei einer Telefonnummer anrufen! Darf ich als Gehörlose etwa nicht gewinnen?

Jörg Apel: Eine weitere Barriere findet sich bei kulturellen Angeboten, die nicht immer barrierefrei aufbereitet sind: Im Kino haben die Filme meist keine Untertitel. Dann müssen wir erst darauf warten, dass die DVD mit den Untertiteln herauskommt. Allerdings haben die meisten deutschen Filme und Serien auch auf DVD keine Untertitel. Es gibt zwar Dienste, mit denen man im Kino Filme mit Untertiteln sehen kann, aber nicht alle Filme werden angeboten. Greta \& 
Starks ist z. B. eine App, die Audiodeskriptionen und Untertitel zu Filmen zugänglich macht. Das Angebot ist aber begrenzt.

Im Theater wird zwar immer mehr versucht, mit Übertiteln zu arbeiten, aber es gibt ebenfalls noch viel zu wenige Angebote. Man kann auch dort als Gehörloser nicht spontan hingehen, sondern muss erst überprüfen, ob es Übertitel oder Gebärdensprachdolmetscher(innen) geben wird. Hildesheim will sich in Zukunft um neue barrierefreie Angebote bemühen.

Dana Apel: Auf einer Veranstaltung wie dem Stadtfest müsste es eigentlich selbstverständlich sein, dass ein(e) Dolmetscher(in) für Deutsche Gebärdensprache verfügbar ist. Als gehörloser Mensch möchte ich nicht immer warten müssen, bis ein(e) Dolmetscher(in) da ist. Es wäre schön, eben auch spontan dort hingehen können und sich nicht immer die Mühe des Organisierens machen müssen. Ich bin davon überzeugt, dass viel mehr Gehörlose auf kulturellen Veranstaltungen erscheinen und sich den Hörenden gegenüber auch mehr öffnen würden, wenn mehr Dolmetscher(innen) eingesetzt würden.

\section{„Die Gesellschaft soll uns so akzeptieren, wie wir sind!“}

Familie Apel wünscht sich eine Öffnung beider Kulturen, die der Hörenden, aber auch die der Gehörlosen.

Jörg Apel: Ich möchte auch, dass die Gehörlosen sich ändern: Man sieht sie fast ausschließlich in ihrer eigenen kleinen Gruppe. Sie klammern sich noch zu häufig an ihre Gemeinschaft. Im Sinne der Inklusion würde ich mir wünschen, dass sie sich noch mehr öffnen. Manchmal würde ich sie gerne anstoßen und sagen: „Kommt mehr aus Euch heraus, traut Euch, besucht mehr Veranstaltungen!“ Da die Gesellschaft aber von Hörenden dominiert wird, sollten diese auch weiterhin mehr versuchen, Barrieren beim Kommunizieren mit Gehörlosen abzubauen.

Dana Apel: Ich würde gerne mehr Sportangebote wahrnehmen, ohne dabei als nicht zugehörig angesehen zu werden. Es sollte selbstverständlich sein, dass ich 
da hingehe. Die Hörenden sollen sich nicht gehemmt fühlen, wenn ich dabei bin. Es soll so sein, als ob ich nicht gehörlos wäre. Ich wünsche mir hier mehr Sensibilisierung in der Gesellschaft - die Hörenden sollen keine Ängste haben, auf Gehörlose zuzugehen. Man kann sich auch verständigen, indem man über Schriftsprache kommuniziert. Genauso gut geht es aber auch mit intuitiven oder lautsprachbegleitenden Gebärden. Man muss gar nicht die Gebärdensprache beherrschen, um sich mit Gehörlosen verständigen zu können! Hörende können einfach mit Gestik etwas zeigen, sie sollten keine zu komplizierten Wörter verwenden und möglichst langsam sprechen, dann verstehen Gehörlose auch, was die Hörenden mitteilen wollen. Ich möchte, dass die Gesellschaft sich öffnet und uns so akzeptiert, wie wir sind. Dann werden auch wir die Hörenden so annehmen, wie sie sind. Wichtig ist mir, dass die Hörenden mich nicht verändern wollen und mich nicht dauernd fragen: Warum hast du kein Hörgerät oder keine Cochlea-Implantate (CI)? Warum haben deine Kinder keine?

Auf die Tatsache, dass ihre Kinder gehörlos sind, bekamen die Apels oft Betroffenheit und Mitleid als Reaktion. Die Gehörlosigkeit ist für die Familie aber kein Problem.

Dana Apel: Es ist alles in Ordnung, so wie es ist. Wir haben ja mit der Kommunikation in der Familie kein Problem. Wir unterhalten uns in Gebärdensprache. Auch als die Kinder Babys waren, haben wir mit ihnen gebärdet und ihnen bildhaft Geschichten in Gebärdensprache erzählt, so wie die Hörenden es auch machen. Wir bekommen natürlich immer die Reaktion: „Wie funktioniert das? Die Kinder verstehen das doch noch gar nicht!". Wir wollten unseren Kindern auch alle Informationen geben und gebärden eben mit ihnen. Hätten wir ein hörendes Kind, hätten wir es auch angenommen und kommunikativ das Beste daraus gemacht. Unsere beiden gehörlosen Söhne gehen auf ein örtliches Gymnasium und werden durchgehend mit Dolmetschern beschult. Die Dolmetscher sind auch bei Klassenfahrten und Theaterbesuchen dabei. Allgemeinwissen und Bildung sind so wichtig, auch für uns Gehörlose. 


\section{Bestehende Diskriminierung auf dem Weg zu einer inklusiven Gesellschaft}

Heute ist die Diskriminierung vor allem im medizinischen Umfeld immer noch sehr stark. Während Schulen und andere Institutionen sich umstellen und Inklusion möglich machen, fehlt im medizinischen Bereich immer noch Akzeptanz und Toleranz gegenüber Gehörlosen.

Dana Apel: Es ist uns ganz unbegreiflich, dass solche Diskriminierung auch heute noch passiert. Gehörlose mussten besonders früher viel einstecken und es gab kaum ein Bewusstsein darüber, dass wir ebenso das Recht auf Teilhabe am gesellschaftlichen Leben haben. Aber wenn es in der heutigen inklusiv orientierten Gesellschaft noch zu solchen Diskriminierungen kommt, schockt mich das besonders, zum Beispiel, wenn ich zu einem neuen Arzt gehe, der dann „taubstumm“ auf dem Patientenzettel vermerkt. Das ist eine Diskriminierung, denn gehörlose Menschen sind nicht stumm! Eine solch veraltete und diskriminierende Ansicht verletzt mich.

Dana und Jörg Apel: Es gibt Ärzte, die starken Druck auf die Eltern gehörloser Kinder ausüben. Viele Ärzte wollen das Cochlea-Implantat bei gehörlosen Kindern einsetzen - sogar gegen den Willen der Eltern. Diese defizitorientierte Einstellung gegenüber Gehörlosen ist diskriminierend und wir empfinden es als zutiefst anmaßend, wenn Ärzte den Eltern diese Entscheidung absprechen wollen. Die Einsetzung eines Cochlea-Implantats ist immer auch mit Risiken verbunden und es ist deshalb an den Eltern zu entscheiden, ob sie diese eingehen möchten oder nicht. Zudem gibt es keine Garantie, dass das Kind danach voll hören kann und es beginnt nach der Implantierung zunächst einmal eine lange Zeit des „Hören-Übens“. Wir haben uns daher ganz bewusst gegen ein CI bei unseren Kindern entschieden. Wir wollten, dass unsere Kinder Zeit zum Spielen und viele Freiheiten haben. Sie leben als Gehörlose sehr glücklich und zufrieden und würden es nicht anders wollen. 


\section{„Da muss noch viel passieren, aber wir sind auf einem guten Weg!“}

(aufgezeichnet von Loraine Keller)

Ich arbeite schon seit 2008 in der Lebenshilfe Braunschweig. Ich wurde auf dem zweiten Arbeitsmarkt eingestuft und bin im Braunschweiger Büro für Leichte Sprache als Büroassistent tätig. Zu meinen Aufgaben gehört das Vorlesen von Büchern und Nachrichten in einfacher Sprache für andere Kundinnen und Kunden der Lebenshilfe Braunschweig. Im Jahr 2007 wurde bei mir eine leichte Form von Asperger-Autismus festgestellt. Diese Beeinträchtigungen merke ich teilweise schon in meinem Leben. Zum Beispiel habe ich manchmal Probleme mit komplizierten Schreiben von Behörden oder Ärzten. Aber insgesamt komme ich trotzdem in meinem Leben selbstständig sehr gut klar. Nur bei einigen wenigen Sachen benötige ich Unterstützung.

Bevor ich einen Außenarbeitsplatz im Büro für Leichte Sprache bekommen habe, war ich als Leiter der Prüfgruppe für Leichte Sprache tätig. Ich kenne die Texte gut und weiß durch meine Erfahrung genau, was einen qualitativ hochwertigen Leichte-Sprache-Text ausmacht. Durch den ständigen Kontakt mit den Texten und der Prüfgruppe kannte ich mich mit der Leichten Sprache zu gut aus, um Texte zu prüfen. Heute übersetze ich kurze Texte selber in die Leichte Sprache. Ist ein Satz im Ausgangstext zu lang und zu verschachtelt, spalte ich ihn auf und übersetze ihn Stück für Stück. Sind schwierige Wörter in einem Satz enthalten, muss ich als Übersetzer erst einmal prüfen, ob die Zielgruppe diese kennt. Aus meiner Tätigkeit als Prüfgruppenleiter kann ich ziemlich sicher einschätzen, bei welchen Wörtern Erklärungsbedarf besteht. Ich bin mir darüber 
bewusst, wie schwierig es für Übersetzerinnen und Übersetzer ist, das Vorwissen der heterogenen Zielgruppe allein in Bezug auf die Wortebene zu antizipieren und zudem nicht in eine zu kindliche Ausdrucksweise zu verfallen. Ich habe viel Erfahrung mit Menschen mit Beeinträchtigung und kann den vorhandenen Wissensstand der Zielgruppe deshalb sehr gut einschätzen. Natürlich muss auch ich als geübter Übersetzer mit Hilfsmitteln arbeiten. Ich verwende zum Beispiel gerne den Duden, der mir bei unbekannten oder schwierigen Wörtern verlässliche Hilfe bietet. Außerdem nutze ich gerne Suchmaschinen im Internet, um Sachverhalte, bestimmte Wendungen oder Konzepte zu recherchieren.

Das Angebot in Leichter Sprache ist meiner Meinung nach aber noch viel zu klein: Allein die verwaltungsfachsprachlichen Texte der Behörden, mit denen ich auch selbst immer wieder in Kontakt komme, stellen eine Hürde im alltäglichen Leben dar. Oft bin ich mir nicht sicher, ob ich die an mich adressierten Schreiben richtig verstanden habe. Einige Behörden interessieren sich dafür, ihre Formulare und Schreiben verständlicher zu machen. Ich würde ein größeres Angebot von Texten in Leichter Sprache im Bereich der Verwaltung und der Behörden sehr begrüßen. Ausfüllhilfen für Formulare könnten mir und vielen anderen direkt weiterhelfen und den Weg zum selbstständigen Ausfüllen der nötigen Formulare ebnen.

Ich wünsche mir, dass es in Zukunft auch im Freizeitbereich mehr Texte in Leichter Sprache geben wird. Einige der von mir betreuten Seniorinnen und Senioren freuen sich ganz besonders über die Sportnachrichten in Leichter Sprache. Viele von ihnen sind Eintracht-Braunschweig-Fans und generell an Fußball oder weiteren Sportarten interessiert. Ich übersetze eigenständig Artikel über die neuesten Spiele und Meldungen des Vereins in Leichte Sprache, kreiere somit eigene „Spielberichte“ und lese diese leichte Version dann in der Seniorentagesstätte vor. Die große Begeisterung meiner Zuhörerinnen und Zuhörer dort zeigt mir immer wieder, dass es mehr Übersetzungen solcher Nachrichten geben sollte. Daher fordere ich vor allem auch in lebensnahen Bereichen wie Sport und Freizeit mehr Angebote in Leichter Sprache.

Ich wünsche mir mehr Zeitungsartikel in Leichter Sprache. Ich glaube, dass auch Menschen ohne Beeinträchtigung nicht immer die Inhalte der Tageszeitungen verstehen. Ich habe trotz meiner Beeinträchtigungen eine normale 
Schulausbildung abgeschlossen. Nach der Grundschule bin ich auf eine IGS gegangen. Dort habe ich meinen Realschulabschluss gemacht. Ich selbst interessiere mich besonders für die Bereiche Wissenschaft und Philosophie. Was mir inhaltlich keine Probleme bereitet, ist aber meist sprachlich so wenig verständlich formuliert, dass ich beim Lesen von Texten aus diesen Bereichen schnell an meine Grenzen stoße. Aber trotzdem komme ich in meinem Leben selbstständig sehr gut klar. Meine Arbeit macht mir Spaß und den Kontakt mit meinen Kolleginnen und Kollegen genieße ich. Ich wachse mit meinen Aufgaben - jeden Tag ein bisschen mehr. 

Cornelia Plagge

„Das barrierefreie Angebot ist immer noch zu gering“

(aufgezeichnet von Sergio Andres Hernández Garrido)

Cornelia Plagge hat neun Jahre lang als Teil der primären Adressatenschaft von Leichter Sprache die Verständlichkeit von Ausgangstexten geprüft, bevor sie übersetzt wurden. Für ihre Qualifizierung als Prüferin hat sie zunächst einmal freiwillig eine Prüferschulung absolviert. Danach wurde sie in die Prüfgruppe von Texten des Braunschweiger Büros für Leichte Sprache der Lebenshilfe Braunschweig integriert, in der sie zwei Jahre lang aktiv tätig war. Heute setzt sie sich engagiert für die Interessen von Menschen mit Lernschwierigkeiten ein.

In der Prüfgruppe der Lebenshilfe Braunschweig wird sie heutzutage nur gelegentlich eingesetzt, da sich ihr Textverständnis von standardsprachlichen Texten nach ihrer jahrelangen Erfahrung deutlich verbessert hat. „Es hat immer Spaß gemacht, Texte zu prüfen, aber ich bin dafür schon zu gut geworden“, sagt Plagge lachend. In jeder Sitzung mit der Testgruppe lernen die Prüfer(innen) sowohl thematisch als auch sprachlich etwas Neues dazu. Cornelia Plagge ist zwar noch auf das barrierefreie Angebot von Leichter Sprache angewiesen, aber sie ist als Prüferin schon überqualifiziert und deshalb nicht mehr repräsentativer Teil einer Testgruppe der primären Adressatenschaft. Dafür gehört sie aber jetzt zur Expertengruppe, in der sie sich dafür einsetzt, dass Leichte Sprache über die Grenzen von Braunschweig hinaus bekannter wird. Die Expertengruppe vertritt das Büro für Leichte Sprache bei Interviews und Veranstaltungen. Sie sind Experten aus der Praxis für das Prüfen von Texten in Leichter Sprache und repräsentieren zugleich die primäre Adressatenschaft. 
Als Teil der Prüfgruppe hat sich Cornelia Plagge mit Texten beschäftigt, die in Leichte Sprache übersetzt werden sollten. Die Ausgangstexte wurden satzweise oder absatzweise zusammen in der Gruppe gelesen und es wurde dann besprochen, an welchen Stellen Verständnisschwierigkeiten aufgetaucht waren. Diese Schwierigkeiten konnten unterschiedlicher Natur sein: Zum Beispiel konnte auf der Wortebene ein Wort für die Prüfer(innen) zu schwierig oder auf der Satzebene ein Satz zu lang und verschachtelt sein, vielleicht war die Schrift aufgrund der Schriftart oder des Kontrasts mit dem Hintergrund für einen der Prüfer oder eine der Prüferinnen kaum lesbar usw. Die Aufgabe der Testgruppe war es, Verbesserungsvorschläge zu entwerfen, die im Nachhinein in der Übersetzung in Leichte Sprache berücksichtigt wurden. Zusammen haben die Prüfer(innen) an ihren Optimierungen gearbeitet, bis die ganze Gruppe die im Text dargestellten Informationen verstanden hatte, erzählt Plagge.

Cornelia Plagge hat in ihrer Tätigkeit als Textprüferin mit unterschiedlichen Textsorten gearbeitet. Broschüren von Museen, Texte über Politik, über Religion und sogar Stadtführer hat sie schon für die Übersetzung in Leichte Sprache geprüft. Ihre Arbeit als Prüferin hat sich aber nicht darauf beschränkt, in einem Raum zu sitzen und Texte zu verschiedenen Themen zu lesen: Beispielsweise hat die Prüfgruppe mit dem Till-Eulenspiegel Museum in Schöppenstedt (Landkreis Wolfenbüttel) kooperiert und an der Beschilderung in Leichter Sprache gearbeitet. Sie haben darüber hinaus die Übersetzung einzelner Texte geprüft und sind im Anschluss auch zum Museum gefahren, um sich die Dauerausstellung „Ein Erlebnis für alle“ anzuschauen.

Plagge ist der Meinung, dass das Angebot an Leichte-Sprache-Texten in der Gesellschaft noch zu gering sei. „Es müssen noch mehr Texte in Leichte Sprache übersetzt werden“, so Cornelia Plagge. Im Augenblick begegne sie im Alltag noch häufig Texten, die für sie nicht verständlich seien. „Zum Beispiel sind Beipackzettel zu Medikamenten für mich ziemlich schwierig“, sagt sie. „Es würde uns sehr viel helfen, wenn diese in Leichte Sprache übersetzt werden.“

Probleme gebe es aber ihrer Meinung nach nicht nur mit dem zu geringen Angebot von Texten in Leichter Sprache: Insgesamt würden Menschen mit Behinderung immer noch zu wenig in der Gesellschaft berücksichtigt. Durch die Gesetzgebung gebe es zwar mehr barrierefreie Angebote, aber es bleibe doch 
noch viel zu tun. „Ich wünsche mir, dass im Bereich Kultur mehr Veranstaltungen für uns geplant werden. Zum Beispiel im Theater oder im Kino“, so Cornelia Plagge. In Deutschland solle ihrer Meinung noch mehr für Menschen mit Beeinträchtigung getan werden. 

JANNA HINRICHS

\section{Auf dem Weg zur Selbstvertretung von Menschen mit Beeinträchtigungen in der Gesellschaft}

(aufgezeichnet von Loraine Keller)

Ich bin studierte Linguistin und arbeite seit zwei Jahren in der Lebenshilfe Braunschweig. Dort leite ich das Büro für Leichte Sprache, welches durch die finanzielle Unterstützung der Aktion Mensch aufgebaut werden konnte. Obwohl die Lebenshilfe sich schon seit zehn Jahren mit Leichter Sprache beschäftigt und es seit dieser Zeit ebenso eine Prüfgruppe gibt, ist ein speziell auf die Leichte Sprache ausgerichtetes Büro erst vor kurzer Zeit entstanden. Im Büro für Leichte Sprache werden Texte für die Lebenshilfe Braunschweig selbst angefertigt und darüber hinaus Übersetzungen für externe Auftraggeber(innen) erstellt.

Das Büro für Leichte Sprache bietet aber nicht nur Übersetzungen an, sondern führt auch Schulungen durch mit dem Ziel, die Leichte Sprache in Braunschweig und darüber hinaus bekannt zu machen. Bei den Übersetzungen in Leichte Sprache werden je nach Kundenwunsch verschiedene Regelwerke bei der Textproduktion berücksichtigt. Sprachlich orientieren meine Übersetzer(innen) und ich uns also je nach Vorstellung des Auftraggebers/der Auftraggeberin an einem bestimmten Regelwerk - inhaltlich aber steht die Prüfgruppe im Vordergrund, die darüber berät, was relevant bzw. irrelevant ist, welche Sachverhalte, Wörter oder Konzepte erklärt werden sollen und welche Passagen mit in den Leichte-Sprache-Text einbezogen werden müssen bzw. welche Abschnitte herausgelassen werden können. Auf Wunsch können zudem Einzelprüfungen der Texte durchgeführt werden. Anschließend geht der Text noch einmal zum Kunden zurück, eine erneute Korrekturschleife wird durchgeführt, 
beispielsweise um den Text an die Corporate Language der Auftragsfirma anzupassen. Im Ergebnis steht ein Text in Leichter Sprache, der dann vom Auftraggeber/von der Auftraggeberin veröffentlicht wird.

Mit Bezug auf das Niveau der Texte in Leichter Sprache konnte ich in den vergangenen zwei Jahren feststellen, dass dieses insgesamt sehr unterschiedlich ist. So werden verschiedene Zielgruppen mit unterschiedlichen Sprachniveaus angesprochen, ohne dass dabei deutlich markiert ist, wen diese Texte adressieren: Teilweise gibt es Texte, die sich eher an Menschen richten, die gerade einmal lesen und schreiben können; diese gehen meiner Ansicht nach eher in die Richtung der Unterstützten Kommunikation. Teilweise gibt es jedoch auch Texte, die komplexere Themen behandeln und dabei zudem eine komplexere Sprache verwenden; diese Texte werden aber allesamt als Leichte-Sprache-Texte bezeichnet. Das ist durchaus eine schwierige Situation, da es bislang keinen allgemeingültigen Qualitätsstandard für Leichte Sprache gibt. Dieses Dilemma lässt sich nicht leicht lösen, da es sich bei Adressat(inn)en der Leichten Sprache um eine heterogene Gruppe handelt, die zum Teil sehr unterschiedliche Bedarfe und Anforderungen an einen Text stellen. Meiner Erfahrung nach wäre es allerdings ein guter Anfang, wenn man einen Text einfach kennzeichnet und vermerkt, für welche Adressat(inn)engruppe dieser besonders geeignet ist. Das würde ebenso dabei helfen, der Stigmatisierung entgegenzuwirken, die der Leichten Sprache und ihrer Adressatenschaft anhaftet.

Ich habe schon öfter die Erfahrung gemacht, dass Auftraggeber(innen) bereits ein bestimmtes Bild von Texten in Leichter Sprache haben, da sie zuvor einmal einen leichten Text bestimmter Art gesehen haben. Häufig wünschen sich dann die Auftraggeber, dass unser Büro den neuen Ausgangstext auf dieselbe Art übersetzt; das ist nicht immer sinnvoll oder möglich. Die Schwierigkeit besteht dann darin, einen Kompromiss zu finden und einen Text zu produzieren, der einerseits den Vorstellungen des Auftraggebers/der Auftraggeberin sowie andererseits den eigenen Qualitätsstandards gerecht wird.

Mit Bezug auf die Forschung scheint mir, dass Studien zu Leichte-SpracheTexte häufig die Entstehungssituation nicht mit einbeziehen. Texte, die schon veröffentlicht wurden, dahingehend zu überprüfen, ob es sich dabei um qualitativ hochwertige, sozusagen „gute“ Texte in Leichter Sprache handelt, ist pro- 
blematisch, da die Bedingungen, unter denen die Texte entstehen, sehr stark variieren. Dem fertigen Text sieht man allerdings einen speziellen Wunsch des Auftraggebers/der Auftraggeberin oder andere Einflussfaktoren beim Produzieren eines Textes nicht mehr an, weshalb nicht nachvollziehbar ist, warum bestimmte Übersetzungsstrategien gewählt wurden oder auch nicht. Ein Urteil über den bereits produzierten Text greift an dieser Stelle meiner Auffassung nach zu kurz.

Das Büro für Leichte Sprache der Lebenshilfe Braunschweig ist Teil der Empowerment-Bewegung: So gibt es die Verträge für Dienstleistungen der Lebenshilfe in Leichter Sprache, um somit beeinträchtigten Käufer(inne)n den Zugang zu den gewünschten Dienstleistungen zu ermöglichen. Weiterhin werden die Kundinnen und Kunden der Lebenshilfe in Leichter Sprache über ihre Rechte und Pflichten aufgeklärt, damit diese tatsächlich verstanden werden und die Menschen selbst politisch handeln können. Für mich liegt der Fokus Leichter Sprache auf eben diesem Aspekt: Sie soll Menschen mit Beeinträchtigungen dazu befähigen, selbstbestimmt am gesellschaftlichen Leben teilzuhaben und ein unabhängiges Leben zu führen. Es soll ihnen möglich sein, sich weiterhin für ihre Rechte einzusetzen und auf die immer noch bestehenden Barrieren in der Gesellschaft hinzuweisen, damit wir sie gemeinsam immer weiter abbauen können. Leichte Sprache ist dabei kein Allheilmittel, aber doch ein wichtiges Instrument, um die Behindertenrechtsbewegung weiterzuführen. Ganz in diesem Sinne bietet die Lebenshilfe Braunschweig den Menschen vor Ort ambulante und stationäre Angebote der Betreuung und Pflege, die inklusiv gestaltet sind; Menschen mit Beeinträchtigung können dort leben und arbeiten. Die Häuser der Lebenshilfe sind über ganz Braunschweig verteilt, sodass die Menschen, die ihre Hilfe in Anspruch nehmen möchten, nicht in der Peripherie „ausgelagert“ wohnen oder arbeiten, sondern zentral dabei sind und am städtischen Leben teilhaben können. Dabei sind die Beeinträchtigungen der Menschen, die die Unterstützung der Lebenshilfe suchen, sehr vielfältig: Sowohl Menschen mit seelischen oder geistigen als auch mit körperlichen Beeinträchtigungen sind dort willkommen. Genauso wie die Interessen der Menschen verschieden sind, ist ebenfalls das Angebot der Lebenshilfe sehr bunt gestaltet: Allein die Arbeitsmöglichkeiten reichen unter anderem von Saunabau über Kunstatelier bis zu Sozialkaufhaus und Fahrradwerkstatt. Es ist dort möglich, 
sich in ganz unterschiedliche Richtungen zu orientieren und sein Leben individuell zu gestalten. Genauso sind die Wohnangebote der Lebenshilfe auf den individuellen Geschmack und vor allem auf die Bedürfnisse des einzelnen Menschen ausgerichtet. Die Herausforderung, auf jedes Individuum bestmöglich einzugehen, nehmen die Mitarbeiter(innen) der Lebenshilfe tagtäglich neu an.

Für mich ist es essentiell, dass neben dem wegweisenden Kampf für mehr Teilhabe am gesellschaftlichen Leben von Menschen mit Beeinträchtigungen diese ein „normales Leben“ führen können: Dazu gehört zum Beispiel, dass sie in ihrer Freizeit genau die aktuellen Informationen einholen können, die sie haben möchten. Daher ist es für mich besonders wichtig, mit Angeboten in Leichter Sprache nicht zu stark zu didaktisieren: Aktuelle kritische Themen und Nachrichten sollten inhaltlich unverändert bleiben. Vor allem sollten vermehrt Freizeitangebote in Leichter Sprache zur Verfügung gestellt werden. So hat einer meiner Studierenden - ich unterrichte überdies an der Ostfalia-Hochschule in Braunschweig - kürzlich in Leichter Sprache beschrieben, wie man ein Longboard zusammenbaut. Das ist sehr gut bei den Adressat(inn)en angekommen und hat mich begeistert. Spannende und aktuelle Themen des Alltags sollten Menschen mit Beeinträchtigungen zugänglich gemacht werden, um ihnen ebenfalls ein Leben voller Vielfalt und neuer Entdeckungen zu ermöglichen. 
STEFANIE SCHRUHL

\section{Audiodeskription in der Praxis - Bericht einer blinden Rezipientin und Hörfilmautorin}

Mein Name ist Stefanie Schruhl. Ich bin verheiratet, habe eine kleine Tochter und arbeite bundesweit als Filmbeschreiberin für verschiedene Auftraggeber, die Audiodeskriptionen im Bereich TV und Kino erstellen. Eigentlich war ich Diplompsychologin. Doch nach einiger Zeit erfolgloser Versuche eine Festanstellung zu erlangen, entschied ich mich vor sieben Jahren für einen beruflichen Neuanfang. Das Nordteam von Hörfilm e.V., der Vereinigung deutscher Filmbeschreiber, suchte im Frühjahr 2011 eine blinde Autorin und lud mich zu einem Probearbeitstag ein, bei dem wir 10 Filmminuten des Tatortes „Wir sind die Guten" mit den Kommissaren Batic und Leitmayr beschrieben. Das war gleich eine kleine Feuertaufe. Denn bereits die erste Filmsequenz zeigte mir, wie kompliziert bewegte Bilder sein können. In diesem Fall waren die Aufnahmen sowohl farblich verfremdet, als auch von Flecken und Kratzern durchzogen, wie bei einem alten Film, während es auf der Handlungsebene bei einer Wiederbelebung von Kommissar Batic sehr hektisch zuging. Ich fühlte mich von der 1. Minute meines künftigen Autorendaseins an herausgefordert. Die Chemie zwischen mir und den beiden sehenden Kollegen stimmte, so erhielt ich eine Schulung zur Hörfilmautorin beim Bayerischen Rundfunk und bekam nach und nach mehr Aufträge.

Das Nordteam arbeitete wie die zu der Zeit rund ein halbes Dutzend weiteren Beschreiberteams in Deutschland zu dritt. Dabei übernahm der erste sehende Autor die Aufgabe der tatsächlichen Bildbeschreibung für den blinden Kollegen und steuerte die Fernbedienung. Der zweite Sehende schrieb die entstandenen Formulierungen mit und erstellte das Manuskript am Notebook. Außerdem sprach er zur Probe jede fertige Textpassage in den laufenden Film hinein. Der blinde Kollege hatte die Aufgabe des Controllers. Er fragte nach, entschied bei gleichwertigen Informationen, die ihm über Filmbilder gegeben 
wurden, darüber mit, was Priorität hatte, und diktierte dem Schreiber meist den ausformulierten Text der jeweiligen Hörfilmpassage.

Bei dieser Arbeitsweise wird eins klar: Jedes formulierte Wort wird auf die Goldwaage gelegt und von allen Autoren, die mit am Text beteiligt sind, geprüft. Ist es für die Zielgruppe verständlich?

Der größte und steigende Anteil der 1,2 Millionen blinden und sehbehinderten Menschen in Deutschland sind die Altersblinden. Man kann beispielsweise davon ausgehen, dass dieser Personenkreis über eine gute Allgemeinbildung verfügt, Farbwissen hat und weiß, wie ein VW-Käfer aussieht. Aber fügt sich das Wort auf der Waage auch sprachlich harmonisch in den Film ein, oder wirkt es beim Hören zwischen den Filmdialogen, die ja ihre eigene Sprache mitbringen, wie ein Fremdkörper? Man stelle sich zum Beispiel vor, in einer Filmszene prügeln und verfluchen sich Bud Spencer und Terence Hill mit zwei ungehobelten Banditen vor einem Saloon, während die Audiodeskription zwischen den Kraftausdrücken vom güldenen Schein der Sonne über ihnen berichtet. Auch selbst mitgebrachte Dialekteinflüsse muss man häufig zu Hause lassen. In einer norddeutschen Rundfunksendung kann nicht vom Bodenkehren und Wienern die Rede sein. Es kann also durchaus zu hitzigen Debatten kommen, wenn drei $\mathrm{Au}-$ toren an einem Manuskript arbeiten, das ja im Anschluss - noch bevor es beim Profisprecher im Tonstudio landet - auch noch redaktionell unter die Lupe genommen wird. Da muss sich der Einzelne, so perfekt ihm sein eigener Formulierungsansatz auch scheint, im Sinne des Gesamtwerkes und des Arbeitsklimas häufig zurücknehmen.

Und dann ist da auch noch die oftmals geringe Zeitspanne zwischen den Dialogen oder anderen akustischen Filmelementen, in der eigentlich viel mehr passiert, in der doch dem sehbeeinträchtigten Zuschauer viel mehr beschrieben werden müsste. Sie ist die größte Herausforderung bei der Hörfilmerstellung. Denn Bilder sind immer schneller als die gesprochenen Worte, die benötigt werden, um sie in ihrer Komplexität zu vermitteln. Ich erinnere mich daran, wie mir mein sehender Kollege Olaf Koop einen Gladiatorenkampf im Katastrophenfilm ,Pompeii‘ beschrieb, und dass es uns letztendlich tatsächlich gelang, in einer Filmpassage, die uns weniger als 22 Sekunden Raum für eine Beschreibung ließ, 11 Kampfhandlungen unterzubringen. So, dass die Geschichte von Angriff und Verteidigung beinah lückenlos erzählt werden konnte, aber eben 
nur beinah. Gerade im Bereich Action bleibt die Audiodeskription aufgrund der zeitgleichen Filmereignisse immer ein Kompromiss, für den der Rezipient allerdings wahrscheinlich dankbar ist. Denn immerhin hat er durch die Filmbeschreibung nicht den Anschluss verloren und kann der Handlung folgen. Als Beschreiberin lässt mich die Entscheidung dafür, etwas wegzulassen, wenn mir persönlich doch alle Details bekannt sind, jedoch eher unzufrieden mit den Zähnen knirschen.

Ähnlich verhält es sich mit der Filmkomik, für die es manchmal nicht die richtigen Worte gibt, um auch bei der Beschreibung einer witzigen Szene ein Lachen zu erzeugen. So war mein erstes Hörfilmerlebnis der Film ,Tootsie, den wir als Schulklasse aus Blinden und Sehbehinderten im Kino gemeinsam mit sehenden Schülern ansahen. Ich weiß noch, dass mich weniger die Wortwahl in der Audiodeskription zum Lachen brachte, als das Lachen der anderen Kinobesucher. Dass jemand im Film eine Grimasse zieht, klingt eben einfach viel weniger lustig, als es aussieht. Darin liegt eine der scheinbar unüberwindbaren Grenzen in der Filmbeschreibung.

Bis zu meinem 30. Lebensjahr kam ich nur selten in den Genuss einer Audiodeskription. Zum einen gab es nicht sehr viele Angebote, zum anderen wusste man als Fernsehzuschauer häufig nichts davon und stolperte höchstens versehentlich über einen Hörfilm, wenn man mit einem Tastendruck das Stereosignal aufsplittete und zufällig eine Audiodeskription im 2. Tonkanal lief. Die dramatischen Szenen im großen Kino-Blockbuster ,Titanic' erlebte ich durch die verheulte und sehr spärlich gesäte leise Beschreibung meiner Freundin neben mir. Aber mehr als einmal zu flüstern: „Was passiert denn da gerade?“, war eben im stillen Kino nicht drin. Den Teenager-Hype um ,GZSZ machte ich mit. Wobei jedoch mangels Filmbeschreibung das Product Placement keinerlei Auswirkungen auf mich hatte. Aber so ist es nun einmal, wenn die blinden und sehbehinderten Teenager in Deutschland, die ja auch Taschengeld bekommen, als Konsumentengruppe nicht wahrgenommen werden. Sitcoms wie ,Roseanne mochte ich schon wegen ihres Wortwitzes sehr. Doch ich dachte viele Episoden lang, dass es um eine schwarze Familie geht. Immerhin ist Regina Lemnitz ja auch immer die Synchronstimme von Whoopi Goldberg, und die ist nun mal dunkelhäutig. Während meines ersten Theaterbesuches mit Live-Audiodeskription bei dem romantisch-komödiantischen Versdrama ,Cyrano de Bergerac;, 
gab es einen amüsanten Zwischenfall, als die Sprecherin allen blinden Besuchern über Kopfhörer eine Handlung auf der Bühne beschrieb, die zwar in den Proben noch stattgefunden hatte und folgerichtig in ihrem Manuskript stand, die jetzt jedoch durch eine schauspielerische Improvisation ganz anders ausfiel. Was die Sprecherin hörbar zum Schmunzeln brachte, und alle anwesenden Blinden zu lautem Gelächter veranlasste.

Ich ging gern, jedoch nicht oft ins Kino, sah und hörte Filme und Serien ohne Bildbeschreibung, aber dafür mit viel Fantasie, und machte mir selbst einen Reim darauf, was eigentlich passieren müsste, weil ich annahm, etwas von Filmlogik zu verstehen. Doch natürlich kam es ab und an mal vor, dass ich trotz konzentrierten Zuhörens auch nach vielen Filmminuten immer noch völlig im Dunkeln tappte. So verließ ich beispielsweise einmal das Kino, nachdem ich eine halbe Stunde lang verzweifelt versucht hatte, der wummernden akustischen Reizüberflutung in Steven Spielbergs ,Krieg der Welten' einen etwaigen Handlungsstrang abzugewinnen, und begnügte mich dann doch mit dem gleichnamigen Roman von H. G. Wells. Auch die DVD des Kriegsfilms ,Der Soldat James Ryan' gab ich quasi ungesehen beim Verleih zurück, weil sich mir die gesamte Action im Kugelhagel mangels Audiodeskription auch beim besten Willen nicht erschloss.

Leider gibt es auch heute längst nicht zu jedem Film oder kulturellen Großereignis eine entsprechende Bildbeschreibung für Sehbeeinträchtigte, aber in den letzten Jahren zeigt sich immerhin doch eine sehr positive Entwicklung, in der wir als Konsumenten von der Filmindustrie mehr wahrgenommen werden, und in der auch die kulturellen Angebote für Sehbeeinträchtigte zugenommen haben. So muss Dank einer Novellierung des Filmförderungsgesetzes im Jahr 2014 jede filmische Neuproduktion, die in Deutschland eine finanzielle Förderung in Anspruch nehmen möchte, mit einer barrierefreien Filmfassung, und dazu gehört eine Audiodeskription, ausgestattet sein. Was u.a. dazu führte, dass inzwischen bis zu 20 Hörfilme am Tag im Fernsehen ausgestrahlt werden. Dank der Richtlinien zur Erstellung einer Audiodeskription, die im Jahr 2015 in Zusammenarbeit der neun Landesrundfunkanstalten der ARD, ORF, SRF, ZDF sowie der Hörfilm gGmbH, Hörfilm e.V. und audioskript festgelegt wurden, existieren nun auch einheitliche Qualitätsstandards. Diese Richtlinien sorgen dafür, 
dass ein Hörfilm nicht nur vorhanden, sondern auch erträglich, im Idealfall sogar ein schönes Erlebnis für uns sehbeeinträchtigte Kinobesucher und Fernsehzuschauer ist.

Diverse öffentlich-rechtliche Rundfunkanstalten haben neben ihren regulären Ansprechpartnern für die Wünsche und Beschwerden von Zuschauern jetzt auch spezielle Hotlines, die sich mit den Belangen ihrer blinden und sehbehinderten Klientel befassen. Darüber hinaus gibt es unmittelbar vor jedem Hörfilm ein akustisches Signal oder eine sprachunterlegte Texteinblendung, die ihn ankündigen.

Nur das Privatfernsehen lässt sich noch bitten, endlich technisch mit dem 2. Tonkanal aufzurüsten und die blinden und sehbehinderten Fernsehzuschauer als relevante Käufergruppe ernst zu nehmen. Dabei sind Hörfilmfassungen für Formate wie ,Germany's Next Topmodel' oder Boxkämpfe von meiner Klientel unbedingt erwünscht.

Websites wie TV-Butler und EPGDATA, die Textseite der ,Hörzu', haben eine extra Hörfilmliste ihres TV-Programms, und alle deutschsprachigen Hörfilmproduktionen werden in einer regelmäßig aktualisierten öffentlichen Datenbank, die auf der Homepage www.hoerfilmev.de zu finden ist, aufgeführt. Die Datenbank umfasst mittlerweile knapp 10.000 Einträge.

Im Bereich Kino darf der Hörfilm-Fan vor allem aufgrund des Engagements des Filmverleihers ,Universal Pictures' nicht nur deutsche Filme, sondern auch neue internationale Blockbuster mit Audiodeskription genießen. Es gibt Kinos, die sich für deren Empfang eine Drahtlos-Anlage installiert haben, die es blinden und sehbehinderten Besuchern ermöglicht, mittels ausleihbarem Empfangsgerät über Kopfhörer von bestimmten Sitzplätzen aus eine gegebenenfalls vorhandene Audiodeskription während der Filmvorführung mitzuhören. Doch die Erfindung der GRETA-App von ,Greta und Starks', die zudem für HörfilmUser kostenlos ist und unabhängig von der Technologie im Kino funktioniert, war im Sinne der Inklusion die bislang innovativste Lösung. Mit dieser App kann der Sehbeeinträchtigte auf eine Datenbank zugreifen, welche die Tonspur mit der Audiodeskription zum gewünschten Film enthält. Zu Beginn der Vorstellung synchronisiert sich das Smartphone mit dem laufenden Filmton, und man kann in jedem Kino und zu jeder Zeit über Kopfhörer den Hörfilm erleben. 
Zudem darf man auch selbst Filmwünsche äußern, für die noch keine Audiodeskription existiert.

Bedauerlicherweise ist es dem Handel bislang noch nicht gelungen, in seinen Onlineportalen eine Suchfunktion einzurichten, die das Vorhandensein einer Hörfilmfassung auf DVDs und Blu-rays erkennt, so dass ich als Konsumentin gezielt danach suchen könnte. Man muss gewissermaßen die Spürnase eines Trüffelschweins besitzen, um ein geeignetes Produkt zu finden. Filmportale wie Amazon Prime Movie und Netflix bieten inzwischen Hörfilmfassungen, darunter auch englischsprachige Produktionen an, sind jedoch unterschiedlich gut bedienbar.

Es kommt leider auch manchmal vor, dass eine Audiodeskription produziert, aber vom Anbieter nicht eingekauft wird. Sie liegt dann erst einmal auf Eis, bis jemand danach fragt und bereit ist, dafür zu bezahlen, was mich besonders dann ärgert, wenn ich als Hörfilmautorin an der Erstellung beteiligt war. So geschah es zum Beispiel bei oben genanntem Katastrophenfilm ,Pompeii', dessen Termin für den Kinostart einfach vor dem Abschluss der Postproduktion lag, weshalb unser schönes Stück Arbeit allen sehbeeinträchtigten Kinogängern verwehrt blieb. Überhaupt bekommen wir Hörfilmautoren im Bereich der freien Filmproduktionen das Endergebnis unserer Arbeit nur dann zu sehen, wenn wir den Film käuflich erwerben. Es gibt also nicht automatisch und nur weil man an der Postproduktion mitgearbeitet hat ein Gratis-Kinoticket oder eine Beleg-DVD.

Der Beruf des Filmbeschreibers/Hörfilmautors bietet nicht sehr viele Stufen auf der Karriereleiter. Zunächst einmal ist es kein geschützter Begriff. Wer den Job machen und an Aufträge gelangen will, nimmt an einer etwa 4-tägigen Schulung Teil. Mitzubringen sind: Empathie, ein sehr gutes Sprachgefühl/ Sprachkompetenz, die Leidenschaft für Präzision, Kenntnisse über Dramaturgie und filmisches Erzählen, ausdauernde Konzentrationsfähigkeit - und nicht zuletzt ein sehr gutes Allgemeinwissen. Der Rest der Qualifikation liegt im Learning by Doing. Es gibt nach einer Schulung kein „Durchgefallen“. Schlimmstenfalls wird man von den Auftraggebern, die in Deutschland Seminare zum Hörfilmautor anbieten, nicht mit Produktionsangeboten versorgt. Eine mangelhafte Hörfilmproduktion wird höchstens von den blinden und sehbehinderten 
Hörfilmfans in der Mailingliste ,Hörfilmnews' verrissen, die sich dort gelegentlich auch über die Qualität der angebotenen Audiodeskriptionen austauschen. Mehr Qualitätskontrolle gibt es zurzeit noch nicht. Theoretisch darf also jeder einen auf Filmbeschreiber machen, selbst wenn ihm die Begabung dafür eigentlich fehlt, und ich kann nicht viel dagegen tun, außer als blinde Autorin die Zusammenarbeit zu verweigern oder mich als Rezipientin beim Auftraggeber über die schlechte Produktion zu beklagen. Gelegentlich laufen Filme, bei denen ich lieber auf die angebotene Audiodeskription verzichte und den Tonkanal wechsle, weil ich mein Autorenohr nicht abschalten kann. Hierbei ärgert es mich am meisten, wenn ich den Eindruck bekomme, da hat sich jemand keine Mühe gegeben; d. h.: kein Detailreichtum trotz ausreichend Platz zum Beschreiben, fehlende Sprachvarianz im Text, schlecht getimte Einsätze der AD und eine Betonung des Sprechers, die darauf schließen lässt, dass er den Text im Studio runtergelesen hat, ohne den Film mit eingespielt zu bekommen. Im Kampf gegen minderwertige Audiodeskriptionen sollte meiner Meinung nach jedoch mehr getan werden als Autoren zu schulen, auszusieben und Qualitätsstandards zu entwickeln. Es fehlt bislang eine verpflichtende Kontrollinstanz der Qualität und eine Professionalisierung des Filmbeschreiber-Berufes.

Insbesondere wir blinden Beschreiber sind in der Hörfilmbranche, um es einmal auf den Punkt zu bringen, wie die Bauern in einem Schachspiel. Am Anfang werden wir benötigt, um die anderen Figuren, also die sehenden Hörfilmautoren-Newcomer, ins Spiel zu bringen. Wir weihen sie in unsere besondere Wahrnehmung ein und vermitteln ihnen ein Gefühl dafür, welche Informationen wir im Film brauchen. Doch früher oder später, je nach Talent des Autors und Budget des Anbieters, kann die sehende Filmbeschreiber-Spielfigur auch ganz gut ohne Bauer arbeiten, und man wird buchstäblich vom Feld gefegt.

Es gibt einen Interessenskonflikt zwischen sehenden und blinden Hörfilmautoren. Während es für uns inzwischen praktischer wäre, wenn es viele Autoren gäbe, für die wir redaktionell arbeiten könnten, so bedeutete dieser Umstand für den sehenden Kollegen immer: Mehr Konkurrenz.

Als vor wenigen Jahren im Zuge des neu eingeführten Rundfunkbeitrags für Sehgeschädigte immer mehr Audiodeskriptionsanbieter den Markt fluteten, mit ihren längst nicht wirklich vorhandenen Erfahrungen protzten und der Preiskampf begann, löste sich das Modell des Dreierteams binnen weniger Monate 
auf. Immer mehr Produktionen fragten nach einer kostengünstigen Lösung, um eine barrierefreie Filmfassung verkaufen zu können. Auch die öffentlichen Rundfunkanstalten bemühten sich im Zuge der Festlegung des Rundfunkbeitrages für Blinde und Sehbehinderte im Jahr 2013 ihr Hörfilmangebot schnell zu verbreitern, um den Ansprüchen des zahlenden sehbeeinträchtigten Fernsehzuschauers zu entsprechen. Infolge dieser Priorisierung mussten sie Abstriche beim Personalaufkommen für die Audiodeskriptionserstellung machen. Welches Modell hierfür in der Praxis konkret Anwendung fand, hing bei den Rundfunkanstalten vom bewilligten Etat und der Anzahl jährlich geplanter Neuproduktionen ab. Bei den Anbietern des freien Marktes musste nun jeder Autor für sich selbst verhandeln, welches Arbeitsmodell er favorisierte. Letztendlich entschied das Budget der Studios, die häufig nicht nur die Audiodeskription, sondern die gesamte barrierefreie Filmfassung produzierten, darüber, wie viele Autoren und Redakteure bei einem Projekt involviert waren.

Für mich bedeutete diese Umstellung, dass ich nun nicht mehr in einem festen Team mehrere Tage lang an einem Manuskript mitarbeitete. Stattdessen redigierte ich die fertig geschriebenen Texte für so viele Autoren und Anbieter wie möglich, um meine Existenz zu gewährleisten. Denn obwohl sich die erfahreneren Autoren durchaus für uns blinde Controller stark machten, führte der Wettbewerb unter den Anbietern, die ein Hörfilmprojekt an Land ziehen wollten, häufig dazu, dass die einst als Autoren arbeitenden Blinden plötzlich nur noch redaktionell tätig waren. Wir werden inzwischen sehr selten in die Texterstellung mit einbezogen. Es geschieht mittlerweile lediglich bei Anbietern, die noch unerfahren in der Audiodeskriptionserstellung sind und die Anwesenheit eines blinden Autoren als Sicherheitsnetz betrachten, bzw. beim Bayerischen Rundfunk, dem Vorreiter der Audiodeskription, für den die Mitautorenschaft eines Blinden als Qualitätsmarke steht.

Der Idealismus von Anbietern, die noch frisch im Audiodeskriptionsgewerbe sind und es als Ehrensache betrachten, dass blinde Autoren ein Hörfilmmanuskript von Anfang an mit erstellen, legt sich meiner Erfahrung nach immer dann, wenn ihnen die Konkurrenz zum ersten Mal mit einem günstigeren Preisangebot ein Projekt vor der Nase wegschnappt.

Wer in der Medienbranche auf das Thema Audiodeskription stößt und hört, dass man für jedes neue Projekt eine Förderung beantragen kann, denkt gleich, 
dass er da jetzt einen besonders dicken Fisch am Haken hat. Dabei sind die Angelplätze rund um den Teich, in dem das neue Produkt ,Hörfilm' zu schwimmen begann, ziemlich begehrt. Außerdem werden von der FFA nur 50\% der Produktionskosten übernommen und man muss zufüttern. Wer also dem politisch korrekten inklusiven Ansatz auch bei der AD-Erstellung gerecht werden möchte und trotzdem Fische fangen will, nimmt meist Billigfutter. D.h. ein Autor schreibt das Manuskript, und zwar möglichst schnell, der Blinde sieht kurz drüber und wenn der Text auch noch kostensparend eingesprochen werden kann, umso besser. Das geht leider manchmal auch auf Kosten der Qualität des Ergebnisses. Aber ich will hier niemanden verteufeln, weil er auf seine Gewinnspanne schaut. Denn es gibt durchaus Hörfilmanbieter, denen die Audiodeskription, und sei es nur mal für bestimmte Projekte, eine Herzensangelegenheit ist. Was mir dann so etwa einmal alle 2 Jahre die Chance gibt, einen Film mit einem sehenden Autor zu zweit zu beschreiben - eine Arbeitsweise, die ich sehr mag. Denn dann bin ich gleichermaßen Controller und Schreiber. Da ist ein bisschen Multitasking erforderlich, wenn mir meine Sprachausgabe das gerade Geschriebene in den Ohrhörer liest, während ich es fast simultan in den laufenden Film einspreche. Wenn ich meinem Filmbeschreiber mit einem $\mathrm{Ohr}$ lausche, wie die Filmbilder aussehen, und seine Formulierungen so schnell wie möglich notiere, damit kein Fakt in Vergessenheit gerät, hält mich das schön auf Trab. Wenn ich dann noch, wie es bereits einige Male vorkam, mit ins Studio darf, um die Tonregie zu übernehmen, wobei vielleicht noch der eine oder andere Untertitel übrig bleibt, den ich als Zweitstimme einsprechen kann, dann macht mich meine Arbeit richtig glücklich. Dann ist das Produkt Hörfilm fast wie ein eigenes Baby: Man war von vorn bis hinten mit dabei.

Doch für uns Autoren gilt jetzt: Wer etwas vom Fischfang abhaben möchte, sollte auf sich aufmerksam machen und am besten selbst Akquise betreiben.

Zurzeit arbeite ich für 9 Hörfilmautoren, die für 10 Hörfilmanbieter beschreiben, darunter der Norddeutsche Rundfunk. Dort findet meine redaktionelle Mitarbeit tatsächlich noch vor Ort statt. Ich treffe mich mit dem Autor und einem zweiten sehenden Mitarbeiter für die Abnahme eines Hörfilmmanuskripts im Sender. Der Autor liest sein Werk parallel zum laufenden Film ein. Wir unterbrechen, wenn eine Diskussion über Bild oder Sprache nötig ist, und passen den Text gegebenenfalls an; fast wie früher, eine echte Zusammenarbeit 
- nur kürzer. Mit allen anderen Auftraggebern habe ich nur noch per Email zu tun. Den meisten begegne ich höchstens einmal im Jahr, bei der Verleihung des Deutschen Hörfilmpreises im ,Kino International' in Berlin.

Dank dieser schönen Veranstaltung, die vom Deutschen Blinden- und Sehbehindertenverband vor 16 Jahren ins Leben gerufen wurde, ist unsere Arbeit durch die mediale Aufmerksamkeit ein Stück ins Licht der Öffentlichkeit gerückt und es gibt ein bisschen Prestige. Der Preis wird von einer Jury aus Vertretern des Behindertenwesens sowie Politik, Wirtschaft und Film in den 4 Kategorien: ,TV', Kino', ,Dokumentation ' und seit diesem Jahr auch für den besten ,Kinder- und Jugendfilm`vergeben. Darüber hinaus gibt es einen Publikumspreis. Ausgezeichnet mit der Trophäe der Adele werden immer die Produzenten des Films, während schon so mancher Preisträger dabei ertappt wurde oder sich sogar freiwillig outete, dass er gar nicht wusste, was ein Hörfilm eigentlich ist. Aber es ist ein feierliches fröhliches Spektakel, bei dem man ein bisschen Promiluft schnuppern darf und sich auch mit Auftraggebern vernetzen oder den Hörfilm ein bisschen vorantreiben kann. Meine Hörfilmcrew vom NDR wurde bereits $3 \mathrm{x}$ ausgezeichnet und 4 weitere Male war ich mit einem meiner Hörfilmteams nominiert. Als ich bei der ersten Verleihung im pinkfarbenen Abendkleid, die große gerahmte Urkunde in den Händen, auf der Bühne stand und breit ins Blitzlichtgewitter lächelte, schwirrten mir die Worte meiner Mutter durch den Kopf, die ich bislang für mich behalten habe:

„Wann wird dieser Preis eigentlich dotiert?“ 
RUDOLF SCHEPS

\section{„Die Bürokratie im Behindertenrecht muss aufgelockert werden."}

(aufgezeichnet von Sergio Andres Hernández Garrido

und Loraine Keller)

Rudolf Scheps ist seit fast 19 Jahren ehrenamtlich im Blinden- und Sehbehindertenverband Niedersachen e. V. in der Bezirksgruppe Hildesheim tätig. Durch sein jahrelanges Engagement wurde er von seinen Kolleg(inn)en zum ersten Vorsitzenden der Bezirksgruppe des Verbandes gewählt. Als Sehbehinderter setzt er sich im Rahmen seiner Ehrenarbeit für seine Mitmenschen ein: Neben wichtigen Veranstaltungen des Vereins wie beispielsweise der Jahresvollversammlung organisiert er auch Freizeitevents wie das Sommerfest des Vereins, die Weihnachtsfeier und den alljährlichen Tagesausflug. Im Vordergrund steht bei seiner Arbeit aber die Unterstützung der Blinden und Sehbehinderten bei ihren Belangen. Als Vorsitzender des Blinden- und Sehbehindertenverbands ist ihm besonders wichtig, dass diese Gruppe über ihre Rechtslage informiert ist. „Blinde und Sehbehinderte müssen wissen, was ihnen zusteht", so Scheps. Sein Ehrenamt ist ihm so wichtig, dass er dafür Wochenenden in Weiterbildungen und Seminaren opfert. „Ich will mich nicht nur für Blinde und Sehbehinderte einsetzen, sondern ich will auch lernen, wie ich mich für sie einsetzen kann".

Scheps' Ehrenarbeit beschränkt sich nicht nur auf seinen Vorsitz im Blindenund Sehbehindertenverein, denn er ist darüber hinaus auch im Vorstand des Regionalvereins Hannover tätig sowie Mitglied im Behinderten- und Inklusionsbeirat der Stadt Hildesheim. Seine ehrenamtliche Arbeit kombiniert 
Rudolf Scheps mit seiner Tätigkeit als Telefonist im Roemer- und PelizaeusMuseum in Hildesheim. Er ist für Weiterleitung von Telefonaten und die Verwaltung der Infomailpost für die Besucher(innen) zuständig und arbeitet hier mithilfe eines Screen-Readers an seinem Rechner.

\section{Barrieren im Alltag}

Barrieren treten für Blinde und Sehbehinderte vor allem im Bereich Verkehr und Mobilität auf. Beispielsweise sind, wie Scheps ausführt, in der Stadt Hildesheim noch zu wenige Ampelanlagen mit akustischer Hilfe vorhanden. Zudem stellen die grauen Poller für hochgradig Sehbehinderte eine Gefahr dar; durch ihre Behinderung haben viele von ihnen eine eingeschränkte Farbsicht und können nicht kontrastreich sehen, wodurch die Poller schwer vom Boden zu unterscheiden sind. Aber die größte Barriere in der Öffentlichkeit besteht in der Rücksichtlosigkeit der Menschen. Schlechte Erfahrungen hat Scheps bisher vor allem mit Radfahrer(inne)n gemacht. Sie fahren oftmals zu nah am Fußgängerweg entlang; es kam sogar schon vor, dass sie ihm über den Langstock gefahren sind. Ein unangenehmes Aufeinandertreffen ist unvermeidbar, wenn Radfahrer(innen) weder rufend noch klingelnd auf sich aufmerksam machen. „Wir Blinde und Sehbehinderte müssen für andere Verkehrsteilnehmer mitdenken, wenn sie sich auf der Straße uns gegenüber rücksichtslos verhalten“, so Scheps. „Von Radfahrern oder Fußgängern kommen bisweilen verletzende Kommentare wie „Bist du blind!?“ oder „Kannst du nicht aufpassen!?“. Dabei versuchen wir, so vorsichtig wie möglich unterwegs zu sein." Durch solche Erfahrungen ist für Rudolf Scheps der Gang in die Öffentlichkeit mit Anstrengung und mitunter sogar mit Frustration verbunden.

Doch nicht alle Erfahrungen im Alltag sind negativ, wie Rudolf Scheps hervorhebt: Oft bekommt er im Bus einen Sitz angeboten oder wird in der Arztpraxis von einem Mitmenschen zur richtigen Tür geführt. Hilfe sollten Blinde und Sehbehinderte seiner Meinung nach ruhig annehmen, schließlich seien sie immer wieder auf diese Unterstützung angewiesen. Rudolf Scheps nimmt dies mit Humor: „Als Blinder oder Sehbehinderter muss man immer bitte und danke sagen“. Allerdings muss die Hilfe vernünftig angeboten und gegeben werden. 
„Kommunikation ist dabei das A und O. Man sollte zuerst fragen, ob Hilfe gewünscht wird, und nicht automatisch davon ausgehen, dass dies immer so ist“.

Das Internet ist heutzutage auch für Menschen mit Behinderung ein wichtiges Medium für die Informationsbeschaffung. Scheps warnt jedoch anbietende Stellen davor, das Informationsangebot auf dieses Medium zu beschränken, weil dann bestimmte Zielgruppen ausgeschlossen werden, etwa ältere Menschen. Für Scheps selbst und viele andere Blinde und Sehbehinderte ist das Internet ausgesprochen hilfreich, aber in seiner jahrelangen Ehrenarbeit hat er festgestellt, dass es für ältere Menschen - mit oder ohne Sehbeeinträchtigung - oft schwer bedienbar ist. Sie können mit diesem relativ neuen Medium nicht angemessen umgehen, sodass für viele der Zugang zu Informationen über diesen Kanal entfällt. Wenn die Informationen nur noch über das Internet abrufbar sind, führt das zu Problemen. Scheps berichtet etwa über die Schwierigkeiten bei der Bank: „Die Kontoauszüge sind jetzt nur noch im Internet abrufbar. Viele ältere Menschen mit Sehbeeinträchtigung können jetzt nicht mehr ihre Konten verwalten, weil sie teilweise nicht einmal einen Internetzugang haben“.

\section{„Die heutigen Smartphones sind ein unverzichtbarer Baustein der Barrierefreiheit für Blinde und Sehbehinderte“}

Als sehbehinderter Mensch ist Rudolf Scheps auf barrierefreie Angebote angewiesen. Begeistert zeigt er sich von Audiodeskriptionen in Kino und Fernsehen. Dieses barrierefreie Medienangebot funktioniere mittlerweile hervorragend, hier sei eine große Verbesserung zu konstatieren. Auf der Arbeit und im Alltag ist für Scheps ein Screen-Reader unersetzbar. Softwareanwendungen wie Jaws, die er persönlich für die Bedienung seines Rechners nutzt, sind in einer digitalisierten Welt für die Inklusion von Blinden und Sehbehinderten von großem Wert. Die Verbreitung solcher Technologie führt aber seiner Meinung nach dazu, dass die Brailleschrift nach und nach an Bedeutung verliert, da Sprachausgaben schneller und einfacher wahrzunehmen sind. Die Brailleschrift wird zwar heutzutage noch gelehrt, aber sie wird längst nicht mehr so häufig angewendet.

Durch die Sprachausgabe sind Smartphones für Blinde und Sehbehinderte mithin zu einer großen Hilfe im Alltag geworden. Während diese Technologie 
für Menschen mit einer anderen Sinnesbarriere wie etwa für Hörgeschädigte keine Möglichkeit der Informationsbeschaffung oder sogar ein Hindernis darstellt, baut die auditive Wiedergabe von Informationen über Smartphones für Blinde und Sehbehinderte tatsächlich Barrieren ab. Dabei erreichen die Geräte und Anwendungen inzwischen ein hervorragendes Niveau und sind sehr benutzerfreundlich. Dadurch kann Rudolf Scheps mit seinem Smartphone nicht nur per Instant-Messaging kommunizieren, sondern auch jederzeit auf Informationen im Internet zugreifen - wie jeder andere auch: „Die Smartphones sind für uns Blinde und Sehbehinderte unabdingbar geworden."

\section{Der Wunsch nach einer gelockerten Bürokratie}

Scheps ist der Meinung, dass die Bürokratie im Behindertenrecht oftmals eine Barriere ist. Er plädiert dafür, Bürokratie abzubauen und sich stattdessen stärker an den tatsächlichen Erfordernissen zu orientieren. Als Beispiel nennt Scheps, dass inklusive Schulklassen weniger an Auflagen gebunden sein sollten. Es sollte mehr ausprobiert werden, um herauszufinden, unter welchen Umständen die Inklusion im Unterricht funktionieren kann. Auch die Bearbeitung von Anträgen wie zum Beispiel zur Ausstellung eines Behindertenausweises oder die Beantragung von Blindengeld dürfte seiner Meinung nach nicht so kompliziert und langwierig sein, wie das gegenwärtig der Fall ist. Die Bearbeitungsvorgänge sind meistens mit langen Wartezeiten verbunden, auch wenn eine Antwort in manchen Fällen dringlich ist.

Erschwerend kommt dazu, dass viele Blinde und Sehbehinderte über ihre Rechte nicht ausreichend orientiert sind. Werden beispielsweise Anträge in der ersten Instanz nicht bewilligt, denken viele Antragsteller(innen), dass dagegen nichts mehr unternommen werden könne. Scheps hält es für eine ausgesprochen wichtige Aufgabe, Blinde und Sehbehinderte über ihre Rechte zu informieren. Im Falle der Ablehnung eines Antrags müssten sie beispielsweise wissen, dass sie zumeist das Recht auf die Einreichung eines Widerspruchs haben. Rudolf Scheps setzt sich daher jeden Tag mit großer Kraft dafür ein, die bürokratischen Hürden für Blinde und Sehbehinderte zu überwinden und abzubauen. 
OXANA KULIKOVA/GÖKHAN ŞILFELER

\section{Mündliche Einfache Sprache im DaZ-Unterricht}

(aufgezeichnet von Sergio Andres Hernández Garrido)

Oxana Kulikova und Gökhan Şilfeler sind Lehrkräfte für Deutsch als Zweitsprache an der Deutschen Angestellten-Akademie Hannover (DAA) im Standort Hildesheim. Oxana Kulikova kommt ursprünglich aus Russland, wo sie ihr Englisch- und Deutschstudium absolvierte. Nach ihrem Umzug nach Deutschland vor zwanzig Jahren arbeitete sie als Fremdsprachenlehrerin und Übersetzerin für Englisch, Deutsch und Russisch. Seit zwölf Jahren beschäftigt sie sich hauptsächlich mit dem DaZ-Unterricht vom Alphabetisierungskurs bis hin zum C2Niveau an der Volkshochschule Hildesheim und der DAA. Gökhan Şilfeler kommt aus der Türkei und hat in Göttingen Germanistik und Turkologie studiert. Seit 2007 erteilt er EDV- und DaZ-Kurse an der DAA.

\section{Schüler(innen) mit unterschiedlichen Hintergründen}

Kulikova und Şilfeler arbeiten mit einer ausgesprochen heterogenen Schülergruppe. Beispielsweise sitzen in ihren Klassenräumen junge Leute, die aus dem Ausland kommen und in Deutschland eine Ausbildung oder ein Studium anfangen möchten. Studierende mit einem schon abgeschlossenen Bachelor sind teilweise auch unter den Sprachschüler(inne)n, weil sie ein C1- oder C2-Niveau für ihre Weiterbildung in Deutschland anstreben. Aber der Großteil der Unterrichtgruppe besteht aus Migrant(inn)en, die aufgrund des Konfliktes im Nahen Osten aus ihren Herkunftsändern flüchten mussten und jetzt in einer vom Bundesamt für Migration und Flüchtlinge festgelegten Zeit ein B1-Deutschniveau erreichen müssen. 
Die DaZ-Schüler(innen) werden bei der DAA je nach Sprachniveau und -kompetenz in unterschiedliche Kurse eingestuft. Der sogenannte Alpha-Kurs ist für Menschen konzipiert, die entweder keine Schriftsprachkompetenz in der eigenen Muttersprache haben oder ein neues Alphabet lernen müssen, bevor sie mit dem eigentlichen Deutschunterricht anfangen können. Nach Regelungen des BAMF müssen Flüchtlinge dieses Niveaus innerhalb von 900 Unterrichtsstunden abschließen, die im Bedarfsfall um 300 Unterrichtsstunden erweitert werden können. Es schließen sich die Integrationskurse an; hier sollen sie innerhalb von 600 Stunden das Niveau B1 erreichen. Im Anschluss folgen die Orientierungsklassen, die 100 Unterrichtsstunden umfassen und als Vorbereitung für den Einbürgerungstest dienen. Im Vordergrund stehen dabei die Erweiterung des Wortschatzes und des Wissens über Gesellschaft und Politik. Dazu bietet die DAA noch Unterrichtsstunden für die berufsbezogene Sprachförderung, die vom B2- bis hin zum C2-Niveau gehen. Oxana Kulikova und auch Gökhan Şilfeler sind in allen diesen Kurstypen und Niveaustufen als Dozent(inn)en beteiligt.

\section{„Wir müssen uns vieler Hilfsmittel bedienen, um unsere Ziele zu erreichen.“}

Die Zeit, die den Schüler(innen) für die Alpha-, Integrations- und Orientierungskurse zur Verfügung stehen und innerhalb der sie das B1-Niveau erreicht haben sollen, sind vom Bundesamt festgelegt. Der festgelegte Zeitrahmen sei allerdings für dieses Ziel zu eng, so Şilfeler: „Wir arbeiteten unter einem enormen Zeitdruck, denn der zu bearbeitende Stoff ist für die angegebenen Stunden zu viel“. Viele der Sprachschüler(innen) müssen im Alpha-Kurs eine sprachliche Grundkompetenz erwerben, die sie selbst in ihrer eigenen Muttersprache nicht haben. Für andere ist es eine große Herausforderung, eine neue Schrift zu lernen und sie angemessen zu beherrschen. Erst nach diesem Prozess fängt der eigentliche Deutschunterricht an, in dem innerhalb von 600 Stunden das Niveau B1 erreicht werden soll. Für Themen wie ein vertieftes Eingehen auf die deutsche Grammatik, die eigentlich innerhalb der Integrationskurse stattfinden soll, bleibt keine Zeit. 
Gökhan Şilfeler: Der enge zeitliche Rahmen für das Erreichen des B1-Niveaus erschwert unsere Arbeit erheblich. Dabei muss man aber auch bedenken, dass es ebenfalls externe Faktoren außerhalb des Klassenraumes gibt, die einen negativen Einfluss auf den Lernprozess haben können. Manche unserer Schüler sind zum Beispiel aus Syrien geflüchtet und haben dort Familie und Angehörige hinterlassen. Ich kann vollkommen verstehen, wenn die persönliche Situation es manchmal nicht zulässt, dass sie sich im Unterricht oder beim Lernen voll konzentrieren können, weil ihre Gedanken bei ihren Angehörigen in ihren Herkunftsländern sind. Andere besuchen zum ersten Mal in ihrem Leben einen Klassenraum. Sie wissen nicht, wie man eine Sprache lernt oder wie man aus Eigeninitiative zuhause den behandelten Stoff nacharbeitet. In ihrem alltäglichen Leben müssen sie nicht nur die deutsche Sprache erwerben, sondern sich auch ggf. um eine Wohnung oder um bürokratische Angelegenheiten kümmern. Das sind Faktoren, die den Lernprozess unserer Schülerinnen und Schüler beeinflussen. Wir bemühen uns und versuchen alle Thematiken in der festgelegten Zeit zu behandeln. Es ist aber angesichts all dieser Einflussfaktoren eine Herausforderung, unsere Schüler in der nötigen Weise zu motivieren. Der Zeitdruck kommt dann erschwerend hinzu.

Oxana Kulikova und Gökhan Şilfeler müssen diese Schwierigkeiten überwinden und die Ziele in den vom BAMF festgelegten Stunden erreichen. Dafür bedienen sie sich unterschiedlicher Hilfsmittel.

Oxana Kulikova: Ich arbeite zum Beispiel gerne mit Wortkarten in den Vokabelübungen. Auf der Vorderseite sind Abbildungen oder Wörter auf Deutsch, die wir zu einem bestimmten Thema im Unterricht besprechen werden. Ich bereite sie vor der Unterrichtsstunde vor, damit meine Schülerinnen und Schüler die Übersetzung in ihre eigene Muttersprache auf der anderen Seite der Karten schreiben können. Diese Wortkarten bleiben dann im Klassenraum, damit sie jederzeit damit Vokabeln üben können. Dieses Hilfsmittel setze ich vor allem in den Alpha-Kursen ein.

Für mich ist die Kommunikation mit meinen Schülerinnen und Schülern für die Vorbereitung eines Themas sehr wichtig. Für mich ergibt es keinen Sinn, mich nur ans Lehrbuch zu halten und nicht den Wissensstand meiner Klasse zu 
kennen. Zusammen entdecken wir, welche Basis schon vorhanden ist, um danach darauf aufbauen zu können. Als Beispiel kann ich meine Arbeit in einem meiner Orientierungskurse nennen, in dem wir das politische System Deutschlands behandeln mussten. Den meisten der Schülerinnen und Schüler war der Name Angela Merkel bereits bekannt. Ich bin dann von diesem Punkt gestartet und habe versucht, das Politiksystem ausgehend von der Bundeskanzlerin Angela Merkel zu erklären. Daraus ist ein Schaubild entstanden, der jetzt als Lernhilfe im Klassenraum hängt.

Die zwei DaZ-Lehrkräfte sind sich einig, dass Wortschatzlisten mit Übersetzungen in mehrere Sprachen und PowerPoint-Präsentationen als visuelle Unterstützung sehr hilfreich sind; gleiches gilt für die Verwendung der Einfachen Sprache durch die Lehrkräfte in ihren Unterrichtsstunden.

\section{„Wir versuchen, alles sprachlich so einfach wie möglich zu erklären.“}

Die mündliche Einfache Sprache wird von Oxana Kulikova und Gökhan Şilfeler in ihrem Unterricht mit der Intention verwendet, die Sachverhalte so verständlich wie möglich zu erklären und mit dem Lernstoff schneller und effizienter voranzukommen. Vor allem in den Alpha-Kursen versuchen sie, eine Reduktionsvarietät des Deutschen zu verwenden, um mit ihren Schüler(innen)n zu kommunizieren.

Gökhan Şilfeler: Man versucht schon sehr viel in einfacher Sprache zu erklären, vor allem, wenn es um Themen rund um die deutsche Geschichte und Politik geht. Komplexe geschichtliche Ereignisse wie der Mauerfall oder die NS-Zeit sind unserer Erfahrung nach für die meisten unserer Schüler sehr komplex. Durch die Vereinfachung und Erläuterung der Begriffe kommen wir mit dem Thema besser voran.

Oxana Kulikova hat allerdings in höheren Kursen und selbst gelegentlich schon in Alpha-Kursen die Erfahrung gemacht, dass sich ihre Schüler(innen) gegen ein einfacheres Deutsch wehren. 
Oxana Kulikova: Ich mache sehr oft mündliche Übungen, in denen die Schüler Sätze bilden müssen, die vom Aufbau und von den grammatikalischen Strukturen her sehr einfach sind. Zum Beispiel stelle ich die Aufgabe, dass sie über ihren Alltag in Hauptsätzen vom Typ „Ich gehe nach Hause“ berichten müssen. Dabei kam es gelegentlich vor, dass manche meiner Schülerinnen und Schüler die Übung aufgrund des Schwierigkeitsgrades lächerlich fanden und deswegen nicht aktiv mitarbeiten wollten, als ob es eine Art Beleidigung für sie wäre. Auch für Korrekturen sind sie dann nicht offen, weil sie die Ausgangssätze schon als zu einfach empfunden haben.

\section{Die Anwendung von Texten in Leichter Sprache im DaZ-Unterricht}

Als Hilfsmittel im DaZ-Unterricht werden Texte in Leichter Sprache von Oxana Kulikova und Gökhan Şilfeler nicht verwendet. Im Rahmen des Spracherwerbs wäre nach Einschätzung der Lehrkräfte die Verwendung dieses Hilfsmittels teilweise widersprüchlich, da Leichte Sprache eine Reduktionsvarietät des Deutschen ist, die entsprechend ihren Regeln beispielsweise Nebensätze, Passivkonstruktionen oder den Genitiv ausschließt, die aber zum Spracherwerb gehören.

In Orientierungskursen, in denen es hauptsächlich um die Vermittlung von Allgemeinwissen und den Aufbau von Wortschatz geht, könnten Texte in Leichter Sprache allerdings durchaus hilfreich sein, um beispielsweise komplexe Sachverhalte über deutsche Politik und Gesellschaft zu vermitteln. Die in den Lehrbüchern enthaltenden Texte, mit denen im Rahmen dieser Kurse gearbeitet wird, sind nach Einschätzung von Kulikova und Şilfeler für die Schüler(innen) zu kompliziert.

Gökhan Şilfeler: Wir erbringen im Unterricht eine mündliche Übersetzungsleistung in eine verständlichere Form des Deutschen. Die Texte im Lehrbuch werden in ihrer sprachlichen Schwierigkeit dem Ziel des Unterrichts nicht gerecht. Die Teilnehmerinnen und Teilnehmer müssen in nur 100 Stunden für den Einbürgerungstest vorbereitet werden; mit Texten in Leichter Sprache wäre dieses Ziel besser zu erreichen. 
Oxana Kulikova: In dieser Hinsicht wären die Lehrbücher für die Orientierungskurse optimierbar. Man muss es klar sagen - es fehlen uns Texte in Leichter Sprache, um die gesetzten Ziele im Rahmen der Orientierungskurse zu erreichen. 


\section{Über die Beiträgerinnen und Beiträger}

Kerstin Alexander, Hochschule Merseburg, Professorin für Technisches Illustrieren und Grafik-Design. Forschungsinteressen: Barrierefreies Grafikdesign, Typografie und Bildverständlichkeit im Umfeld Leichter Sprache

Dana Apel, Landesbildungszentrum Hildesheim, pädagogische Mitarbeiterin und Gebärdensprachlehrerin, Dozentin für Deutsche Gebärdensprache

Jörg Apel, selbstständiger Dozent für Gebärdensprache. Gebärdensprachlehrer an der Universität Hildesheim und an der Hochschule für angewandte Wissenschaft und Kunst (HAWK) Hildesheim sowie an weiteren Einrichtungen

Antje Baumann, Bundesjustizministerium Berlin, Sprachwissenschaftlerin in der Gesetzesredaktion. Forschungsinteressen: Variationen des Sprachgebrauchs, u.a. in Bezug auf Textsorten, Verständlichkeit und politische Aspekte des Sprachwandels (Text- und Soziolinguistik)

Bernd Benecke, Bayerischer Rundfunk: Leiter des Bereichs Audiodeskription innerhalb der Redaktion Barrierefreie Angebote. Lehrbeauftragter an der Dualen Hochschule Baden-Württemberg und an der Hochschule für Angewandte Sprachen, München. Promotion über „Audiodeskription als partielle Translation“

Uta Benner, Hochschule Landshut, Professorin für Gebärdensprachdolmetschen, Studiengangsleitung des gleichnamigen Studiengangs und Studiendekanin der Fakultät Interdisziplinäre Studien. Forschungsinteresse: Inklusion an Universitäten und Hochschulen, Gebärdensprachdolmetschen

Bettina M. Bock, Universität zu Köln, Juniorporfessorin am Institut für deutsche Sprache und Literatur II Forschungsschwerpunkte: „Leichte Sprache“, Text- und Diskurslinguistik, Verständlichkeitsforschung, Sprachdidaktik und Inklusion 
Ursula Bredel, Universität Hildesheim, Professorin für Deutsche Sprache und ihre Didaktik. Forschungsinteressen: Orthographie/Orthographiedidaktik, Grammatik/Grammatikdidaktik, Leichte Sprache im Rahmen der inklusiven Beschulung

Dino Capovilla, Humboldt-Universität zu Berlin, Juniorprofessor für Blindenund Sehbehindertenpädagogik. Forschungsinteressen: Multi-sensorische Wahrnehmung, Soziale Partizipation, Inklusive Didaktik, Assistive Technologien

Ursula Christmann, (apl.) Professorin am Psychologischen Institut der Universität Heidelberg; Forschungsschwerpunkte: Textverstehen, Psychologie des Lesen, Sprachpsychologie, Kommunikationspsychologie, figurative Sprache

Julia Dobroschke, Fachreferentin im Bereich Barrierefreie Informations- und Kommunikations-Angebote der Deutschen Zentralbücherei für Blinde (DZB)

Kristian Folta-Schoofs, Universität Hildesheim, Professor für Neurodidaktik. Forschungsinteressen: Barrierefreies und inklusiv gestaltetes Lernen, Barrierefreie Kommunikation, Mehrsprachigkeit, Aufmerksamkeit und Arbeitsgedächtnis

Norbert Groeben, Univ.-Prof. (a.D.) Allgemeine u. Kultur-Psychologie Uni Köln, Hon.-Prof. Allg. Literaturwiss. Uni Heidelberg u. Mannheim. Forschungsschwerpunkte: Kognitions-, Sprach- u. Literaturpsychologie; Wissenschaftstheorie und Anthropologie; Literatur- u. Medienwissenschaft

Silke Gutermuth, Johannes Gutenberg-Universität Mainz, wissenschaftliche Mitarbeiterin im TRA\&CO Center am Fachbereich für Translations-, Sprachund Kulturwissenschaft in Germersheim. Forschungsinteressen: Translationsprozessforschung, Eyetracking, Rezeptionsforschung im Leichte-Sprache-Kontext 
Silvia Hansen-Schirra, Johannes Gutenberg-Universität Mainz, Professorin für Englische Sprach- und Übersetzungswissenschaft und Direktorin des TRA\&CO Centers am Fachbereich für Translations-, Sprach- und Kulturwissenschaft in Germersheim. Forschungsinteressen: Empirische Translationswissenschaft, Fachkommunikation, Intralinguale Translation, Maschinelle Übersetzung, Multimediale Translation

Uschi Heerdegen-Wessel, Norddeutscher Rundfunk, Redaktionsleiterin „Barrierefreie Angebote und NDR Text“. Zuständig für die NDR Produktion von Untertiteln, Audiodeskriptionen, Angeboten in Gebärdensprache und Leichter Sprache sowie für die Koordination der ARD Projektgruppe „Barrierefreier Rundfunk“

Franziska Heidrich, Universität Hildesheim, wissenschaftliche Mitarbeiterin am Institut für Übersetzungswissenschaft und Fachkommunikation. Forschungsinteressen: Fachkommunikationswissenschaft, Übersetzungswissenschaft, Gestaltete Sprache, Informationsstrukturierung

Jan Hellbusch, Accessibility Consulting, Dortmund. Tätigkeiten: Erstellung von Gutachten, Workshops und Beratung zur digitalen Barrierefreiheit

Johannes Hennies, Pädagogische Hochschule Heidelberg, Professor für Gehörlosen- und Schwerhörigenpädagogik. Forschungsinteressen: Bimodal-bilinguale Erziehung, Erwerb von Schrift-, Laut- und Gebärdensprache (bei Kindern mit und ohne Hörbehinderung), inklusive Didaktik, Geschichte der Gehörlosen und Schwerhörigen

Annika Herrmann, Institut für Deutsche Gebärdensprache und Kommunikation Gehörloser, Universität Hamburg, Professorin für Gebärdensprachen und Gebärdensprachdolmetschen, Institutsleitung und Studiengangsbeauftragte für die BA und MA Studiengänge Gebärdensprachen und Gebärdensprachdolmetschen. Forschungsinteresse: Gebärdensprachlinguistik, theoretische und experimentelle Gebärdensprachforschung, Gebärdensprachdolmetschen 
Janna Hinrichs, Lebenshilfe Braunschweig, Sprachwissenschaftlerin, Leiterin des Braunschweiger Büros für Leichte Sprache

Thomas Kahlisch, Direktor Deutsche Zentralbücherei für Blinde (DZB), Honorarprofessor an der Universität Leipzig im Lehrgebiet Spezielle Buchwissenschaften, Barrierefreie Mediengestaltung

Oxana Kulikova, Deutsche Angestellten-Akademie Hannover im Standort Hildesheim, Sprachlehrerin für Deutsch als Zweitsprache, lizenzierte Prüferin für die Deutschniveaus B1 bis C2

Alexander Kurch, VerbaVoice GmbH, Schriftdolmetscher und Live-Untertitler für die Bereiche Bildung, Beruf und Events für Deutsch und Englisch, freiberuflicher Optimierer künstlicher Stimmen mittels Sprachsynthese für Audiodeskription, freiberuflicher Dozent und Prüfer für Spracherkennung im Rahmen der Schriftdolmetscher-Ausbildung für blinde und sehende Menschen mit oder ohne Sehbehinderung am SDI München und BFW Würzburg. Forschungsinteressen: Synergien von Barrierefreier Kommunikation in Kombination mit Sprachtechnologien und Maschinellem Lernen, insb.: Spracherkennung, Sprachsynthese und Maschinelle Übersetzung

Katrin Lang, Universität Hildesheim. Tätigkeit in der Gesetzesredaktion des Bundesministeriums der Justiz und für Verbraucherschutz. Aktuell Forschungsprojekt zu Barrierefreier Online-Kommunikation von Behörden an der Forschungsstelle Leichte Sprache. Forschungsinteressen: Barrierefreie Rechtskommunikation

Benedikt Lutz, Donau-Universität Krems, Studiengangsleitungen im Umfeld von Wissens-, Prozess-und Qualitätsmanagement. Schwerpunkte in Forschung und Lehre: Fachkommunikation im beruflichen Umfeld, Wissensmanagement, verständliche Rechts- und Verwaltungssprache 
Christiane Maaß, Universität Hildesheim, Professorin für Medienlinguistik am Institut für Übersetzungswissenschaft und Fachkommunikation; Leiterin der Forschungsstelle Leichte Sprache. Forschungsinteressen: Barrierefreie Kommunikation, insbesondere Leichte Sprache im Rahmen der Sprach- und Übersetzungswissenschaft

Nathalie Mälzer, Universität Hildesheim, Professorin für Transmediale Übersetzung. Forschungsinteressen: Barrierefreie Kommunikation mit den Schwerpunkten Untertitelung und Audiodeskription, Medienübersetzen und Literarisches Übersetzen

Oliver Musenberg, Universität Hildesheim, Professor für Inklusion und Bildung im Institut für Erziehungswissenschaft. Forschungsinteressen: Inklusiver (Fach-)Unterricht; Behinderung in bildungshistorischer Perspektive; Bildungstheorie und „schwere“ Behinderung

Conchita Otero Moreno, Universität Hildesheim, wissenschaftliche Mitarbeiterin am Institut für Übersetzungswissenschaft und Fachkommunikation. Gerichtsdolmetscherin für die spanische Sprache und Mitbegründerin der internationalen Forschungsgruppe Alfaqueque (campus.usal.es/ alfaqueque/). Mehrjährige Forschungs- und Lehraufenthalte an der Universidad de Salamanca (Spanien). Forschungsinteressen: Community Interpreting, Sprach- und Kulturmittlung im internationalen Kontext, Dolmetschwissenschaft

Cornelia Plagge, Lebenshilfe Braunschweig, Prüferin für Texte in Leichte Sprache

Nicola Pridik, Juristin und Inhaberin des Büros für klare Rechtskommunikation in Berlin (www.npridik.de). Interessen: verständliche Aufbereitung von Rechtsinformationen in Wort und Bild, Schwerpunkt Rechtsvisualisierung 
Eeva Rantamo, Kulturprojekte - Inklusive Kulturarbeit Köln, Kulturwissenschaftlerin aus Finnland, Dozentin und Projektleiterin. Beratung von Museen und anderen Kultur- und Bildungseinrichtungen, Analysen und Konzepte zur Inklusion und Barrierefreiheit. Arbeitsschwerpunkt: barrierefreie und interkulturelle Kommunikation

Isabel Rink, Universität Hildesheim, Geschäftsführerin der Forschungsstelle Leichte Sprache, Studiengangskoordinatorin des Masterstudiengangs Barrierefreie Kommunikation und Lehrkraft am Institut für Übersetzungswissenschaft und Fachkommunikation. Forschungsinteressen: Leichte Sprache in der Fachkommunikation, Barrierefreie Kommunikation

Johannes Schädler, Universität Siegen, Professor für Sozialpädagogik mit den Schwerpunkten Inklusion und Planung, Geschäftsführer des Zentrums für Planung und Evaluation Sozialer Dienste (ZPE). Forschungsinteressen: Modernisierung der Behindertenhilfe, Individuelle und örtliche Teilhabeplanung, Soziale Dienste im europäischen Vergleich, Entwicklungszusammenarbeit und Soziale Dienste

Rudolf Scheps, Erster Vorsitzender der Bezirksgruppe Hildesheim des Blindenund Sehbehindertenverbands Niedersachen e.V.; Mitglied im Vorstand des Regionalvereins Hannover und Mitglied im Behinderten- und Inklusionsbeirat der Stadt Hildesheim. Mitarbeiter im Römer- und Pelizaeus-Museum Hildesheim

Thomas M. Schindler, Boehringer Ingelheim Pharma GmbH \& CoKG, Gruppenleiter Medical Writing Europe, Lehrbeauftragter der Hochschule Biberach, Forschungsinteressen: Plain Language und Leichte Sprache in der medizinischpharmazeutischen Kommunikation, Aspekte des Zahlenverständnisses (numeracy) in der Kommunikation von Fachinhalten, Theorie und Praxis des Schreibens wissenschaftlicher und regulatorischer Texte

Stefanie Schruhl, Autorin und Hörfilmautorin für Fernseh- und Kinoproduktionen, dreifache Trägerin des Deutschen Hörfilmpreises 
Swenja Schum, Universität Hildesheim, wissenschaftliche Mitarbeiterin und Lehrkraft am Institut für Übersetzungswissenschaft und Fachkommunikation. Forschungsinteressen: Übersetzungsformen in Museen und Kunstausstellungen im Hinblick auf deren Potenzial zum Abbau verschiedener Sprach- und Sinnesbarrieren

Saskia Schuppener, Universität Leipzig, Professorin für Pädagogik im Förderschwerpunkt geistige Entwicklung. Forschungsschwerpunkte: „Leichte Sprache" als Konzept für Menschen mit Lernschwierigkeiten, inklusive Hochschulentwicklung, herausforderndes Verhalten von Schüler*innen mit dem Förderschwerpunkt geistige Entwicklung, Selbstkonzept \& Identität von Kindern, Jugendlichen und Erwachsenen mit so genannter geistiger Behinderung, Kunst \& Kreativität von Menschen mit Behinderungserfahrungen, berufliche Teilhabe von Menschen mit Lernschwierigkeiten

Marie-Luise Schütt, Universität Hamburg, Postdoktorandin im Projekt ProfaLe „Professionelles Lehrerhandeln zur Förderung fachlichen Lernens unter sich verändernden gesellschaftlichen Bedingungen“ (Qualitätsoffensive Lehrerbildung), Leiterin der Servicestelle InkluSoB - Inklusive Schule ohne Barrieren, Forschungsinteressen: Barrierefreie Schule und Hochschule

Laura M. Schwengber, staatlich geprüfte Dolmetscherin für Deutsche Gebärdensprache, Schwerpunkt im Dolmetschen von Musik- und Kulturveranstaltungen. Referentin zu Themen der Inklusion aus der Perspektive einer nichtbehinderten Person

Volker Sieger, Leiter der Bundesfachstelle Barrierefreiheit, Mitglied des Wissenschaftlichen Beirats für den zweiten Teilhabebericht der Bundesregierung über die Lebenslagen von Menschen mit Beeinträchtigungen (im Januar 2017 vom Bundeskabinett verabschiedet)

Gökhan Şilfeler, Deutsche Angestellten-Akademie Hannover im Standort Hildesheim, Sprachlehrer für Deutsch als Zweitsprache 
Thomas Widmayer, Lebenshilfe Braunschweig, Büroassistent im Braunschweiger Büro für Leichte Sprache

Jutta Witzel, Beraterin, Trainerin für Gesprächsführung, interkulturelle Kommunikation, Umgang mit Konflikten. Zertifizierte Schriftdolmetscherin. Leitung Seminare \& Training am SDI München, Lehrbeauftrage an der Universität Karlsruhe

Christa Womser-Hacker, Universität Hildesheim, Professorin für Informationswissenschaft und Direktorin des Instituts für Informationswissenschaft \& Sprachtechnologie. Forschungsschwerpunkte: (Kulturorientierte) MenschMaschine-Interaktion, (Mehrsprachiges) Information Retrieval, Information Behaviour

Maria Wünsche, wissenschaftliche Mitarbeiterin am Institut für Übersetzungswissenschaft und Fachkommunikation der Universität Hildesheim und freiberufliche Übersetzerin und Theaterübertitlerin. Forschungsinteressen: Verständlichkeit und Translation, Barrierefreie Kommunikation, Theatertranslation

Christiane Zehrer, Projektleiterin in der Softwareentwicklung, Design Thinking Facilitator und Agile Coach. Forschungsinteressen: Softwareunterstützung für barrierefreie Kommunikation, kognitiv-linguistische Ansätze und Multimodalität 


\section{KOMMUNIKATION - PARTIZIPATION - INKLUSION}

Bd. 1 Bettina M. Bock/Ulla Fix/Daisy Lange (Hg.): „Leichte Sprache“ im Spiegel theoretischer und angewandter Forschung. 516 Seiten.ISBN 978-3-7329-0282-8

Bd. 2 Nathalie Mälzer (Hg.): Barrierefreie Kommunikation - Perspektiven aus Theorie und Praxis. 324 Seiten. ISBN 978-3-7329-0231-6

Bd. 3 Christiane Maaß/Isabel Rink (Hg.): Handbuch Barrierefreie Kommunikation. 800 Seiten. ISBN 978-3-7329-0407-5

Bd. 4 Simone Girard-Groeber: Participation by hard-of-hearing students in integration classrooms: Facets of interactional competence. 520 Seiten. ISBN 978-3-7329-0433-4

Bd. 5 Bettina M. Bock: „Leichte Sprache“ - Kein Regelwerk. Sprachwissenschaftliche Ergebnisse und Praxisempfehlungen aus dem LeiSA-Projekt. 100 Seiten. ISBN 978-3-7329-0534-8

Bd. 6 Anne Goldbach/Daniel Bergelt: Leichte Sprache bei der Arbeit. Sozialwissenschaftliche Ergebnisse und Praxisempfehlungen aus dem LeiSA-Projekt. 44 Seiten. ISBN 978-3-7329-0585-0

Bd. 7 Kerstin Alexander (Hg.): Mit Typografie und Bild barrierefrei kommunizieren. Forschungsstand und Studien.

382 Seiten. ISBN 978-3-7329-0584-3 
